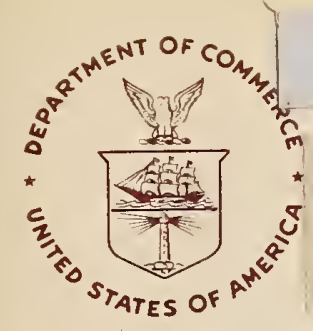

NATL INST OF STANDARDS \& TECH R.I.C.

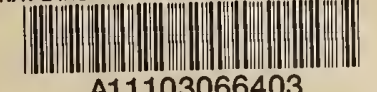

A11103066403

some 1325

U.S. DEPARTMENT OF COMMERCE / National Institute of Standards and Technology

\title{
Tables for the Thermophysical Properties of Methane
}

\author{
Daniel G. Friend \\ James F. Ely \\ Hepburn Ingham
}


he National Institute of Standards and Technology ${ }^{1}$ was established by an act of Congress on March 3, 1901. The Institute's overall goal is to strengthen and advance the Nation's science and technology and facilitate their effective application for public benefit. To this end, the Institute conducts research to assure international competitiveness and leadership of U.S. industry, science and technology. NIST work involves development and transfer of measurements, standards and related science and technology, in support of continually improving U.S. productivity, product quality and reliability, innovation and underlying science and engineering. The Institute's technical work is performed by the National Measurement Laboratory, the National Engineering Laboratory, the National Computer Systems Laboratory, and the Institute for Materials Science and Engineering.

\section{The National Measurement Laboratory}

Provides the national system of physical and chemical measurement; coordinates the system with measurement systems of other nations and furnishes essential services leading to accurate and uniform physical and chemical measurement throughout the Nation's scientific community, industry, and commerce; provides advisory and research services to other Government agencies; conducts physical and chemical research; develops, produces, and distributes Standard Reference Materials; provides calibration services; and manages the National Standard Reference Data System. The Laboratory consists of the following centers:

\section{The National Engineering Laboratory}

Provides technology and technical services to the public and private sectors to address national needs and to solve national problems; conducts research in engineering and applied science in support of these efforts; builds and maintains competence in the necessary disciplines required to carry out this research and technical service; develops engineering data and measurement capabilities; provides engineering measurement traceability services; develops test methods and proposes engineering standards and code changes; develops and proposes new engineering practices; and develops and improves mechanisms to transfer results of its research to the ultimate user. The Laboratory consists of the following centers:

\section{The National Computer Systems Laboratory}

Conducts research and provides scientific and technical services to aid Federal agencies in the selection, acquisition, application, and use of computer technology to improve effectiveness and economy in Government operations in accordance with Public Law 89-306 (40 U.S.C. 759), relevant Executive Orders, and other directives; carries out this mission by managing the Federal Information Processing Standards Program, developing Federal ADP standards guidelines, and managing Federal participation in ADP voluntary standardization activities; provides scientific and technological advisory services and assistance to Federal agencies; and provides the technical foundation for computer-related policies of the Federal Government. The Laboratory consists of the following divisions:
- Basic Standards ${ }^{2}$

- Radiation Research

- Chemical Physics

- Analytical Chemistry
- Computing and Applied Mathematics

- Electronics and Electrical Engineering ${ }^{2}$

- Manufacturing Engineering

- Building Technology

- Fire Research

- Chemical Engineering ${ }^{3}$

\section{The Institute for Materials Science and Engineering}

Conducts research and provides measurements, data, standards, reference materials, quantitative understanding and other technical information fundamental to the processing, structure, properties and performance of materials; addresses the scientific basis for new advanced materials technologies; plans research around cross-cutting scientific themes such as nondestructive evaluation and phase diagram development; oversees Institute-wide technical programs in nuclear reactor radiation research and nondestructive evaluation; and broadly disseminates generic technical information resulting from its programs. The Institute consists of the following divisions:
- Information Systems Engineering

- Systems and Software Technology

- Computer Security

- Systems and Network Architecture

- Advanced Systems

${ }^{1}$ Headquarters and Laboratories at Gaithersburg, MD, unless otherwise noted; mailing address Gaithersburg, MD 20899.

2 Some divisions within the center are located at Boulder, CO 80303.

${ }^{3}$ Located at Boulder, CO, with some elcments at Gaithersburg, MD. 


\section{Tables for the \\ Thermophysical Properties of Methane}

Daniel G. Friend

James F. Ely

Hepburn Ingham

Thermophysics Division

Center for Chemical Engineering

National Engineering Laboratory

National Institute of Standards and Technology

Boulder, Colorado 80303-3328

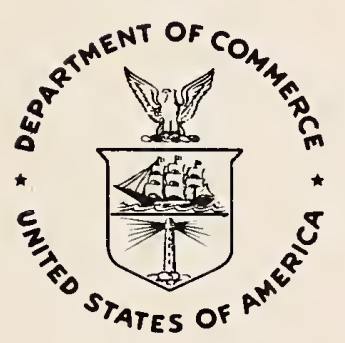

U.S. DEPARTMENT OF COMMERCE, Robert A. Mosbacher, Secretary NATIONAL INSTITUTE OF STANDARDS AND TECHNOLOGY, Raymond G. Kammer, Acting Director 
National Institute of Standards and Technology Technical Note 1325

Natl. Inst. Stand. Technol., Tech. Note 1325, 490 pages (Apr. 1989) CODEN:NTNOEF

U.S. GOVERNMENT PRINTING OFFICE

WASHINGTON: 1989 


\section{CONTENTS}

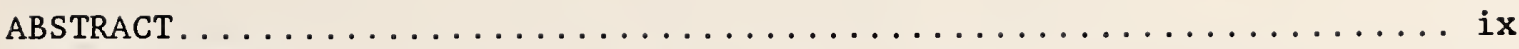

1. INTRODUCTION . . . . . . . . . . . . . . . . . . . . . . . 1

2. THERMODYNAMIC PROPERTIES AND THEIR ALGEBRAIC REPRESENTATION ...... 2

2.1 Fixed Point Constants...................... 2

2.2 Liquid-Vapor Saturation Boundary................. 4

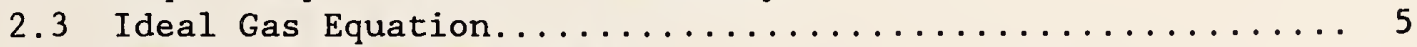

2.4 Residual Helmholtz Energy..................... 6

2.5 Derived Property Equations................... 8

3.TRANSPORT PROPERTIES AND THEIR ALGEBRAIC REPRESENTATION........ 12

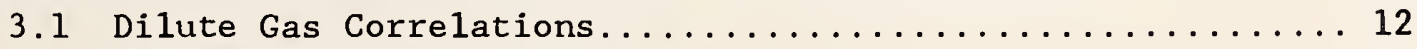

3.2 Excess Property Correlations.................... 14

3.3 Critical Enhancement Correlation................. 15

4.SUMMARY OF UNCERTAINTIES ASSOCIATED WITH THE CORRELATIONS....... 18

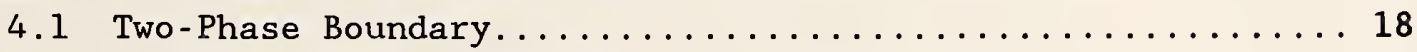

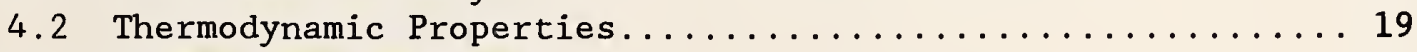

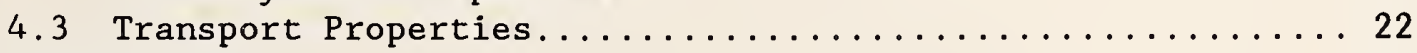

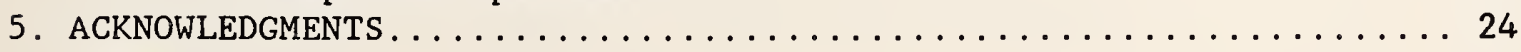

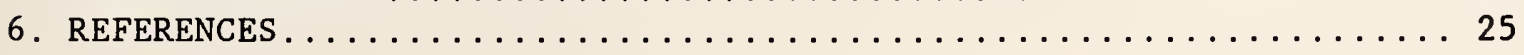

APPENDIX A: TABLES FOR THE COMPARISON OF EXPERIMENTAL DATA AND THE CORRELATIONS ................ 31

APPENDIX B: TABLES FOR THE THERMOPHYSICAL PROPERTIES

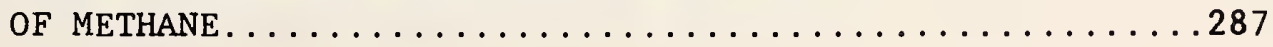

APPENDIX C: LISTING OF A FORTRAN 77 PROGRAM TO CALCULATE THE THERMOPHYSICAL PROPERTIES OF METHANE...........443 



\section{LIST OF TABLES}

1. FIXED POINT CONSTANTS AND OTHER PARAMETERS USED IN THE CORRELATIONS 3

2. COEFFICIENTS FOR LIQUID-VAPOR BOUNDARY CORRELATIONS . . . . . . . 5

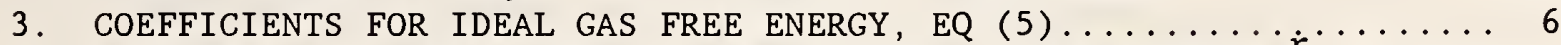

4. EXPONENTS AND COEFFICIENTS FOR THE RESIDUAL FREE ENERGY $\phi^{\text {r }}$ (EQ (6)) 7

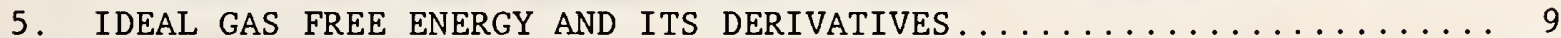

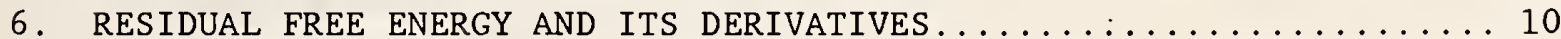

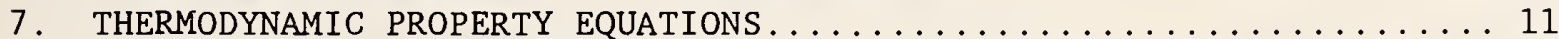

8. COEFFICIENTS FOR DILUTE GAS TRANSPORT PROPERTIES ............. 13

9. COEFFICIENTS FOR EXCESS TRANSPORT PROPERTY CORRELATIONS ......... 15

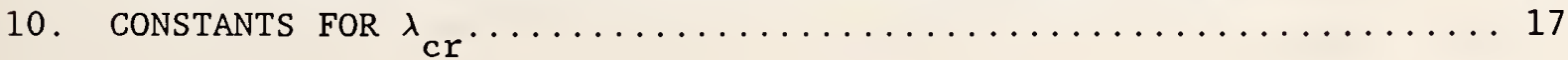

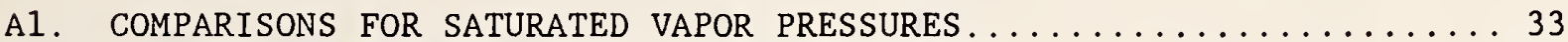

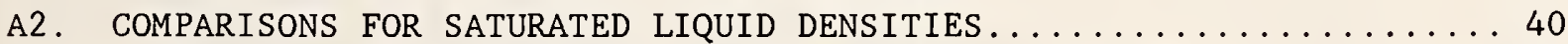

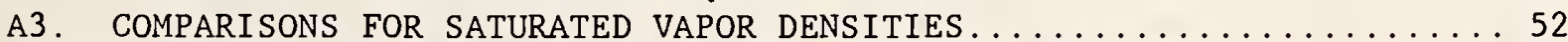

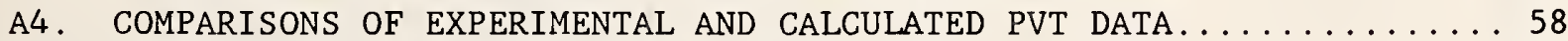

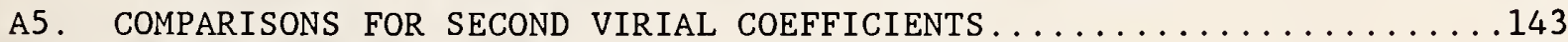

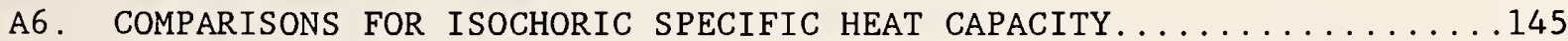

A7. COMPARISONS FOR ISOBARIC SPECIFIC HEAT CAPACITY . . . . . . . . . 156

A8. COMPARISONS FOR SPECIFIC HEAT CAPACITY OF THE SATURATED LIQUID.... 170

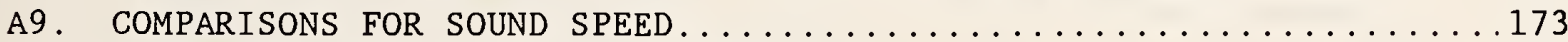

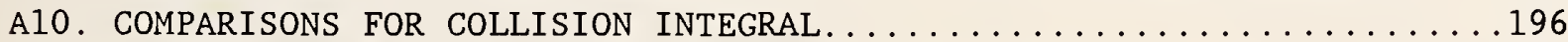

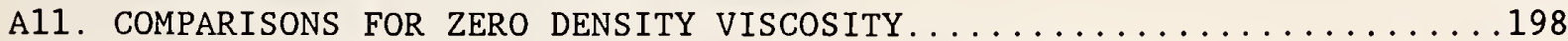

A12. COMPARISONS FOR ZERO DENSITY THERMAL CONDUCTIVITY............204

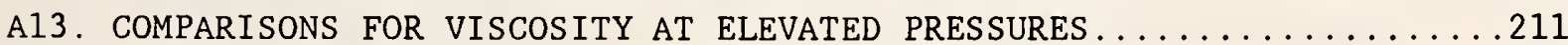

A14. COMPÁRISONS FOR THERMAL CONDUCTIVITY AT ELEVATED PRESSURES $\ldots \ldots \ldots 229$

B1. PROPERTIES OF IDEAL GAS AT 0.1 MPA AND DILUTE GAS

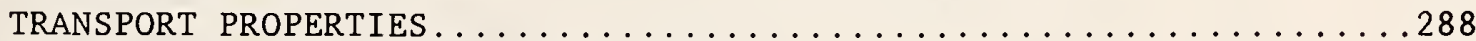

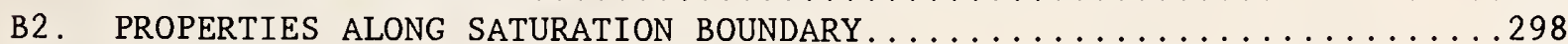

B3. PROPERTIES IN THE SINGLE-PHASE REGION ALONG ISOBARS . . . . . . . . . 301

B4. PROPERTIES IN THE SINGLE-PHASE REGION ALONG ISOTHERMS ..........412 
Symbol

Description

SI Units

REFERENCE

(used in text)

Roman

A Molar Helmholtz energy

$\mathrm{J} \cdot \mathrm{mol}^{-1+}$

eq (4)

$\mathrm{a}, \mathrm{b}$ Exponents in scaled equation

eq (21), table (10)

B Second virial coefficient

$\mathrm{dm}^{3} \cdot \mathrm{mol}^{-1}$

table (7)

$C_{i}$ Coefficients in $\Omega^{(2,2) *}$

eq (10), table (8)

$\mathrm{C}_{\mathrm{P}} \quad$ Isobaric specific heat capacity

$\mathrm{J} \cdot \mathrm{mol}-1 \cdot \mathrm{K}^{-1}$

table (7)

$\mathrm{C}_{\mathrm{V}}$

Isochoric specific heat capacity $\mathrm{J} \cdot \mathrm{mol}^{-1} \cdot \mathrm{K}^{-1}$

table (7)

E Constant in scaled equation

eq (22), table (10)

$F \quad$ Crossover function in $\lambda_{c r}$

eq (17)

$F_{T}, F_{A}, F_{\rho}$ Coefficients in $F$

$f_{\text {int }}, f_{i}$ Contribution from internal modes

eq (17), table (10)

eqs $(11,12)$,

table (8)

G Molar Gibbs energy

$\mathrm{J} \cdot \mathrm{mol}^{-1}$

$G_{i} \quad$ Coefficients in $\rho_{\sigma L}$

$g_{i} \quad$ Coefficients in $\eta_{\text {ex }}$

H Molar enthalpy

$\mathrm{J} \cdot \mathrm{mol}^{-1}$

$\mathrm{H}_{i} \quad$ Coefficients in $\mathrm{P}_{\sigma}$

$J_{i} \quad$ Coefficients in $\rho_{\sigma V}$

$j_{i} \quad$ Coefficients in $\lambda_{\text {ex }}$

$k$ Boltzmann constant

$\mathrm{M}_{\mathrm{r}} \quad$ Relative molecular mass

$\mathrm{N}_{\mathrm{A}} \quad$ Avogadro constant

$n_{i} \quad$ Coefficients in $\phi^{r}$

$P \quad$ Pressure

Q Constant in scaled equation

$Q_{i} \quad$ Coefficients in $\phi^{i d}$

R Molar gas constant

$\mathrm{J} \cdot \mathrm{mol}^{-1} \cdot \mathrm{K}^{-1}$

table (7)

eq (2), table (2)

eq (13), table (9)

tables $(1,7)$

eq (1), table (2)

eq (3), table (2)

eq (15), table (9)

table (1)

table (1)

table (1)

eq (6), table (4)

$\mathrm{MPa}$

$\mathbf{R}$ Constant in scaled equation

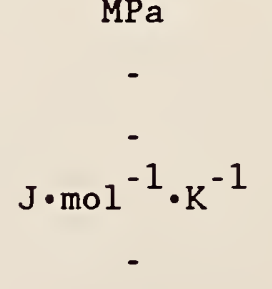

eq (21), table (10)

eq (5), table (3)

table (1)

eq (21), table (10) 


\begin{tabular}{|c|c|c|c|}
\hline Symbol & Description & SI Units & REFERENCE \\
\hline & & (used in text) & \\
\hline $\mathrm{r}_{\mathrm{i}}$ & Exponent of $\delta$ & - & $\begin{array}{l}\text { eqs }(6,13,15) \text {, } \\
\text { tables }(4,9)\end{array}$ \\
\hline $\mathrm{S}$ & Molar entropy & $\mathrm{J} \cdot \mathrm{mol}^{-1} \cdot \mathrm{K}^{-1}$ & table (7) \\
\hline $\mathrm{S}$ & Constant in scaled equation & - & eq (22), table (10) \\
\hline SWEOS & Schmidt-Wagner Equation of State & & \\
\hline$s_{i}$ & Exponent of $\tau$ & - & $\begin{array}{l}\text { eqs }(6,13,15) \text {, } \\
\text { tables }(4,9)\end{array}$ \\
\hline$t$ & Reduced temperature, $\mathrm{kT} / \epsilon$ & - & eqs $(9,10)$ \\
\hline $\mathrm{T}$ & Temperature, IPTS -68 & $\mathrm{~K}$ & - \\
\hline $\mathrm{T}^{*}$ & Reduced temperature, $\left(\mathrm{T}_{\mathrm{c}}-\mathrm{T}\right) / \mathrm{T}_{\mathrm{c}}$ & - & eq $(18)$ \\
\hline $\mathrm{U}$ & Molar internal energy & $\mathrm{J} \cdot \mathrm{mol}^{-1}$ & table (7) \\
\hline $\mathrm{u}$ & Unified atomic mass unit & $\mathrm{kg}$ & table (1) \\
\hline $\mathrm{W}$ & Constant in scaled equation & - & eq (23), table (10) \\
\hline w & Speed of sound & $\mathrm{m} \cdot \mathrm{s}^{-1}$ & table (7) \\
\hline $\mathrm{Z}$ & Compressibility factor, $\mathrm{P} / \mathrm{RT} \rho$ & - & eq (3) \\
\hline \multicolumn{4}{|l|}{ Greek } \\
\hline$\beta$ & Scaling exponent in $\rho_{\sigma \mathrm{L}}, \rho_{\sigma \mathrm{V}}$ & - & $\begin{array}{l}\text { eqs }(2,3) \\
\text { table }(2)\end{array}$ \\
\hline$\Gamma$ & Constant in scaled equation & - & eq $(24)$, table (10) \\
\hline$\delta$ & Reduced density, $\rho / \rho_{c}$ & - & - \\
\hline$\delta_{\sigma}^{*}$ & Reduced density variable & - & eq $(14)$ \\
\hline$\epsilon$ & Scaling exponent in $\mathrm{P}_{\sigma}$ & - & eq (1), table (2) \\
\hline$\epsilon$ & Energy parameter potential & $\mathrm{J}$ & See $\epsilon / k$, below \\
\hline$\epsilon / \mathrm{k}$ & Energy parameter potential & $\mathrm{K}$ & table (1) \\
\hline$\eta$ & Shear viscosity & $\mu \mathrm{Pa} \cdot \mathrm{s}$ & eq (7) \\
\hline$\theta$ & Variable in scaled equation & - & eqs $(21,22)$ \\
\hline$\lambda$ & Thermal conductivity & $\mathrm{mW} \cdot \mathrm{m}^{-1} \cdot \mathrm{K}^{-1}$ & eq $(8)$ \\
\hline$\rho$ & Molar density & $\mathrm{mol} \cdot \mathrm{dm}^{-3}$ & - \\
\hline
\end{tabular}




$\begin{array}{ll}\rho^{*} & \text { Reduced density, }\left(\rho_{\mathrm{c}}-\rho\right) / \rho_{\mathrm{c}} \\ \sigma & \text { Distance parameter in potential } \\ \tau & \text { Reduced inverse temperature, } \mathrm{T}_{\mathrm{c}} / \mathrm{T} \\ \phi & \text { Reduced Helmholtz energy, A/RT } \\ \chi_{\mathrm{T}}^{*} & \text { Reduced compressibility } \\ \Omega & \text { Variable in scaled equation } \\ \Omega^{(2,2) *} & \text { Reduced collision integral }\end{array}$

eq (19)

table (1)

eq (4)

eqs $(20,21,24)$

eqs $(21,23)$

eqs $(9,10)$

\section{Superscripts}

id Ideal gas contribution

eq (4), table (1)

$r$ Residual contribution

eq (4)

\section{Subscripts}
c Value at critical point
cr Critical contribution
ex Excess contribution
$t \quad$ Value at triple point
$t L, t V$ Value at triple point in liquid, vapor
$\sigma \quad$ Value at saturation boundary
$\sigma \mathrm{L}, \sigma \mathrm{V}$ Value in saturated liquid, vapor
$\delta \quad$ Partial derivative with respect to $\delta$
$\tau$ Partial derivative with respect to $\tau$
0 Value at zero density

table (1)

eqs $(8,16)$

eqs $(7,8,13,15)$

table (1)

table (1)

eq (1)

eqs $(2,3)$

tables $(5,6)$

tables $(5,6)$

eqs $(7-9,11)$

+ Throughout this paper, the mole (mol) quantifies the amount of substance whose elementary entities are the molecular constituents $\left(\mathrm{CH}_{4}\right)$ of the methane fluid. 


\title{
TABLES FOR THE THERMOPHYSICAL PROPERTIES OF METHANE.
}

\author{
Daniel G. Friend, James F. Ely and Hepburn Ingham \\ Thermophysics Division \\ National Engineering Laboratory \\ National Institute of Standards and Technology \\ (formerly National Bureau of Standards) \\ Boulder, Colorado 80303
}

The thermophysical properties of methane are tabulated for a large range of fluid states based on recently formulated correlations. For the thermodynamic properties, temperatures from 91 to $600 \mathrm{~K}$ at pressures less than $100 \mathrm{MPa}$ are included. For the viscosity, the corresponding range is $91-400 \mathrm{~K}$ with pressures to $55 \mathrm{MPa}$, while for the thermal conductivity the range is 91 - $600 \mathrm{~K}$ with pressures to $100 \mathrm{MPa}$. In addition to the tables of properties, algebraic expressions and associated tables of coefficients are given to allow additional property calculations. Tables of comparisons between experimental property determinations and the correlations are also given both for primary data used in the formulation of the correlations and for additional data. A listing of a FORTRAN program for the evaluation of methane thermophysical properties using the Schmidt-Wagner equation of state is included.

Key words: correlation; density; equation of state; heat capacity; methane; phase boundary; pressure; speed of sound; thermal conductivity; thermophysical properties; transport properties; virial coefficients; viscosity. 



\section{INTRODUCTION}

We have recently developed correlations for several of the thermophysical properties of methane [1], including an equation of state analogous to that proposed by Schmidt and Wagner [2], equations for the liquid-vapor phase boundary, and correlations for the fluid viscosity and thermal conductivity. Details of the development and error analysis of the formulations have been presented elsewhere [1]. In this paper, we summarize our equations and present extensive tabular comparisons of the correlations with experimental data and tables of properties based on these correlations. We have also included the listing of a FORTRAN program which can be used interactively to calculate properties of methane.

The rationale for these new correlations and the present set of tables was discussed in Ref.[1]. Briefly summarized, since the extensive works of Goodwin [3], Angus et al. [4], and Sychev et al. [5], for example, there have been many new experimental measurements, including accurate phase boundary measurements [6], critical region PVT experiments [7], and extensive thermal conductivity [8] and viscosity measurements [9]. In addition, the form of the residual Helmholtz energy correlation, first proposed by Schmidt and Wagner [2], gives an excellent description of the thermodynamic surface and describes the critical region extremely well for a classical equation of state. The new ancillary equations for the phase boundary incorporate the results of lowest order scaling theory, and both viscosity and thermal conductivity correlations improve the description of the fluid properties when compared to previous correlations. The economic importance of methane, as the major constituent of natural gas, makes the use of improved thermophysical properties correlations especially desirable. Also, the present correlations should prove useful as reference equations for corresponding states models for mixtures and other pure fluids.

The next two sections give, for thermodynamic and transport properties, the algebraic forms for the fluid property correlations with very brief narrative. The List of Symbols and Units given above is a 
useful adjunct to the equations, and tables of necessary coefficients and constants are interspersed throughout the text. Extensive discussion of the development of the equations and evaluation of fitted coefficients was given in Ref.[1]. Tables which follow, in Appendix A, give the comparisons between the correlations and experimental data; these include both primary data, used in the determination of the coefficients in the equation, and secondary data, not explicitly used for any of a number of reasons as discussed in Ref.[1]. The next set of tables, in Appendix $B$, gives values of the thermophysical properties for convenient values of the temperature, density, or pressure. Finally, this report concludes with a listing in Appendix $C$, of a FORTRAN 77 program which allows interactive evaluation of many thermophysical properties for any state point within the range of the correlations.

\section{THERMODYNAMIC PROPERTIES}

\section{AND THEIR ALGEBRAIC REPRESENTATION}

\subsection{Fixed Point Constants}

Values for the critical and triple-point constants, intermolecular potential parameters (for transport properties using the $11-6-8, \gamma=3$ potential), reference values for ideal gas properties, and miscellaneous constants are given in table 1 . Details of the selection process and quoted uncertainties are given in Ref.[1]. The values of the critical constants, including all the quoted digits and disregarding the associated uncertainties, are needed for evaluating the correlating equations below. The fundamental constants in table 1 agree with the values most recently recommended by CODATA [10]. 
TABLE 1

FIXED POINT CONSTANTS AND OTHER PARAMETERS USED IN THE CORRELATIONS

\begin{tabular}{|c|c|}
\hline Triple Point: & $\begin{aligned} \mathrm{T}_{\mathrm{t}} & =90.6854 \pm 0.0003 \mathrm{~K} \\
\mathrm{P}_{\mathrm{t}} & =11.696 \pm 0.002 \mathrm{kPa} \\
\rho_{\mathrm{tL}} & =28.145 \pm 0.005 \mathrm{~mol} \cdot \mathrm{dm}^{-3} \\
\rho_{\mathrm{tv}} & =15.66 \pm 0.05 \mathrm{~mol} \cdot \mathrm{m}^{-3}\end{aligned}$ \\
\hline Critical Point: & $\begin{array}{l}\mathrm{T}_{\mathrm{c}}=190.551 \pm 0.01 \mathrm{~K} \\
\mathrm{P}_{\mathrm{c}}=4.5992 \pm 0.003 \mathrm{MPa} \\
\rho_{\mathrm{c}}=10.139 \pm 0.01 \mathrm{mo} \cdot \mathrm{dm}^{-3} \\
\mathrm{Z}_{\mathrm{c}}=0.28631 \pm 0.0005\end{array}$ \\
\hline $\begin{array}{l}\text { Intermolecular } \\
\text { Potential } \\
\text { Parameters: }\end{array}$ & $\epsilon / \mathrm{k}=174 \mathrm{~K} \quad \sigma=0.36652 \mathrm{~nm}$ \\
\hline $\begin{array}{l}\text { Ideal Gas } \\
\text { Reference Point } \\
\text { Values: }\end{array}$ & $\begin{array}{l}\text { (at } 298.15 \mathrm{~K} \text { and } 0.101325 \mathrm{MPa}) \\
\mathrm{s}^{\mathrm{id}}=186.266 \mathrm{~J} \cdot \mathrm{K}^{-1} \cdot \mathrm{mol}^{-1} \\
\mathrm{H}^{\mathrm{id}}=10.0177 \mathrm{~kJ} \cdot \mathrm{mol}^{-1}\end{array}$ \\
\hline Miscellaneous: & $\begin{array}{l}\text { Relative molecular mass...M } \mathrm{M}_{\mathrm{r}}=16.043 \\
\text { Universal gas constant...R }=8.314510 \mathrm{~J} \cdot \mathrm{mol}^{-1} \cdot \mathrm{K}^{-1} \\
\text { Boltzmann constant... } \mathrm{k}=1.380658 \times 10^{-23} \mathrm{~J} \cdot \mathrm{K}^{-1} \\
\text { Avogadro constant... } \mathrm{N}_{\mathrm{A}}=6.0221367 \times 10^{23} \mathrm{~mol}^{-1} \\
\text { Unified atomic mass unit..u }=1.6605402 \times 10^{-27} \mathrm{~kg}\end{array}$ \\
\hline
\end{tabular}




\subsection{Liquid-Vapor Saturation Boundary}

The three equations for the pressure, liquid density, and vapor density along the two-phase boundary are described in this section. Although the full equation of state can be used with the Maxwell construction method to evaluate these quantities, these equations include theoretically inspired scaling terms and are more accurate in the critical region. The measurements of Kleinrahm and Wagner [6] were the primary data used to establish these correlations.

For the saturated vapor pressure $\mathrm{P}_{\sigma}$, the equation

$$
\mathrm{P}_{\sigma}(\mathrm{T})=\mathrm{P}_{c} \operatorname{EXP}\left[\mathrm{H}_{1} \mathrm{~T}^{*} /\left(1-\mathrm{T}^{*}\right)+\mathrm{H}_{2} \mathrm{~T}^{*}+\mathrm{H}_{3} \mathrm{~T}^{* \epsilon}+\mathrm{H}_{4} \mathrm{~T}^{* 2}+\mathrm{H}_{5} \mathrm{~T}^{* 3}\right] \text {, }
$$

where $\mathrm{T}^{*}=\left(\mathrm{T}_{\mathrm{c}}-\mathrm{T}\right) / \mathrm{T}_{\mathrm{c}}$, is used. The necessary constants and critical exponent are given in table 2 .

The densities of the saturated liquid $\rho_{\sigma \mathrm{L}}$ and saturated vapor $\rho_{\sigma \mathrm{V}}$ are given by

$$
\rho_{\sigma L}(T)=\rho_{c}\left[1+\frac{G_{1} T^{* \beta}+G_{2} T^{* 2}+G_{3} T^{* 3}}{1+G_{4} T^{*(1-\beta)}}\right]
$$

and

$$
\rho_{\sigma \mathrm{V}}(\mathrm{T})=\frac{\mathrm{P}_{\sigma}(\mathrm{T})}{\mathrm{R} \mathrm{T}}\left\{1+\mathrm{P}_{\sigma}(\mathrm{T}) \tau^{8} \frac{\mathrm{Z}_{\mathrm{c}}-1}{\mathrm{P}_{\mathrm{c}}}\left[1+\frac{\mathrm{J}_{0} \mathrm{~T}^{* \beta}+\mathrm{J}_{1} \mathrm{~T}^{* 2 \beta}+\mathrm{J}_{2}\left(\mathrm{~T}^{*}+\mathrm{T}^{* 4}\right)+\mathrm{J}_{3} \mathrm{~T}^{* 2}}{1+\mathrm{J}_{4} \mathrm{~T}^{*}}\right]\right\}^{-1}
$$

respectively. In eq (3), $\mathrm{Z}_{c}=\mathrm{P}_{c} /\left(\mathrm{RT}_{\mathrm{c}} \rho_{c}\right)$ is the critical compressibility factor given in table $1, \tau=T_{c} / T$, and $P_{\sigma}(T)$ must be evaluated from eq ( 1 ). The constants are again found in table 2. Tables A1, A2, and A3 in Appendix A give comparisons between experimental phase boundary measurements and the quantities calculated by eqs (1-3). 
TABLE 2

COEFFICIENTS FOR LIQUID-VAPOR BOUNDARY CORRELATIONS

\begin{tabular}{|l|l|l|}
\hline \multicolumn{2}{|l|}{$\begin{array}{l}\text { Saturated Vapor } \\
\text { Pressure eq (1) }\end{array}$} & \multicolumn{2}{|l|}{$\begin{array}{l}\text { Saturated Liquid } \\
\text { Density eq (2) }\end{array}$} & $\begin{array}{l}\text { Saturated Vapor Density } \\
\text { eq (3) }\end{array}$ \\
\hline$\epsilon=1.90$ & $\beta=0.355$ & $\beta=0.355$ \\
$\mathrm{H}_{1}=-6.589879$ & $\mathrm{G}_{1}=1.838982$ & $\mathrm{~J}_{0}=-0.7377483$ \\
$\mathrm{H}_{2}=0.6355175$ & $\mathrm{G}_{2}=-0.7727452$ & $\mathrm{~J}_{1}^{0}=-1.241532$ \\
$\mathrm{H}_{3}=11.31028$ & $\mathrm{G}_{3}=0.5592446$ & $\mathrm{~J}_{2}=-1.649972$ \\
$\mathrm{H}_{4}=-10.38720$ & $\mathrm{G}_{4}=-0.3807793$ & $\mathrm{~J}_{3}^{2}=2.281949$ \\
$\mathrm{H}_{5}=3.393075$ & & $\mathrm{~J}_{4}=1.439570$ \\
\hline
\end{tabular}

\subsection{Ideal Gas Equation}

The thermodynamic surface is described by an equation for the molar Helmholtz energy, A. Thus, the important derived properties can be obtained by simple, analytical differentiation. The Helmholtz energy is divided into ideal gas and residual constituents according to

$$
A(\rho, T)=A^{i d}+A^{r}=\operatorname{RT} \phi=\operatorname{RT}\left(\phi^{i d}+\phi^{r}\right) .
$$

All the derived thermodynamic properties can similarly be divided into ideal and residual contributions as discussed below.

Our ideal gas correlation is based on Goodwin's fit [11] of the spectroscopically derived data of McDowell and Kruse [12]. With the definitions $\delta=\rho / \rho_{c}$ and $\tau=\mathrm{T}_{\mathrm{c}} / \mathrm{T}$, the dimensionless ideal gas contribution to the Helmholtz energy is written as

$$
\begin{aligned}
\phi^{\mathrm{id}}(\delta, \tau)= & \mathrm{A}^{\mathrm{id}} / \mathrm{RT} \\
= & \mathrm{Q}_{1}+\ln \delta+\mathrm{Q}_{2} \ln \tau+\mathrm{Q}_{3} \tau^{-1 / 3}+ \\
& \mathrm{Q}_{4} \tau^{-2 / 3}+\mathrm{Q}_{5} \tau^{-1}+\mathrm{Q}_{6} \ln \left(1-\mathrm{e}_{7}\right)
\end{aligned}
$$


with the coefficients $Q_{i}$ given in table 3. Equation (5) must be evaluated at the experimental density and temperature, although for many thermodynamic properties, there is no density (or pressure) dependence in the required derivatives, as discussed below. The constants in table 3 incorporate integration constants necessary to give the standard values of the entropy and enthalpy reported in table 1. Reference [11] gives a table of comparisons between the spectroscopic data and the correlations; these comparisons are not repeated in this paper.

TABLE 3

COEFFICIENTS FOR IDEAL GAS FREE ENERGY, eq (5)

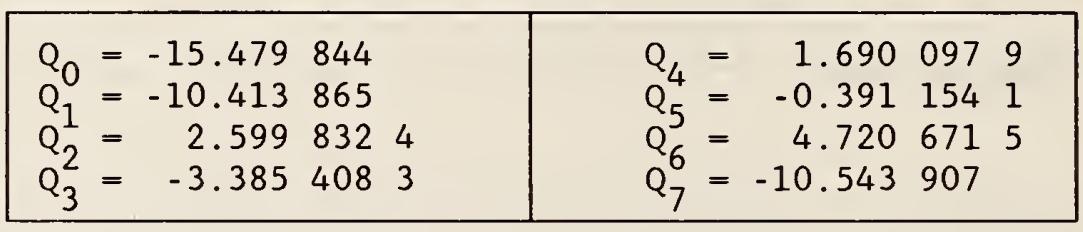

\subsection{Residual Helmholtz Energy}

The residual term in eq (5) for the Helmholtz energy was correlated by a 32 -term function introduced by Schmidt and Wagner [2]. The resulting equation of state has been called the SWEOS and the coefficients for methane were obtained by multiproperty least-squares fitting as described in Ref.[1]. Explicitly, we write

$$
\phi^{r}=\sum_{i=1}^{13} n_{i} \delta^{r}{ }^{s}{ }^{s}+e^{-\delta^{2}} \sum_{i=14}^{24} n_{i} \delta^{r}{ }_{i}{ }_{\tau}^{s_{i}}+e^{-\delta^{4}} \sum_{i=25}^{32} n_{i} \delta^{r_{i}}{ }_{\tau}^{s_{i}}
$$

with $\delta=\rho / \rho_{c}$ and $\tau=\mathrm{T}_{c} / \mathrm{T}$ as before. The coefficients $\mathrm{n}_{i}$ and the exponents $r_{i}$ and $s_{i}$ are given in table 4 . 
TABLE 4

EXPONENTS AND COEFFICIENTS FOR THE RESIDUAL FREE ENERGY $\phi^{\Upsilon}$ (eq (6))

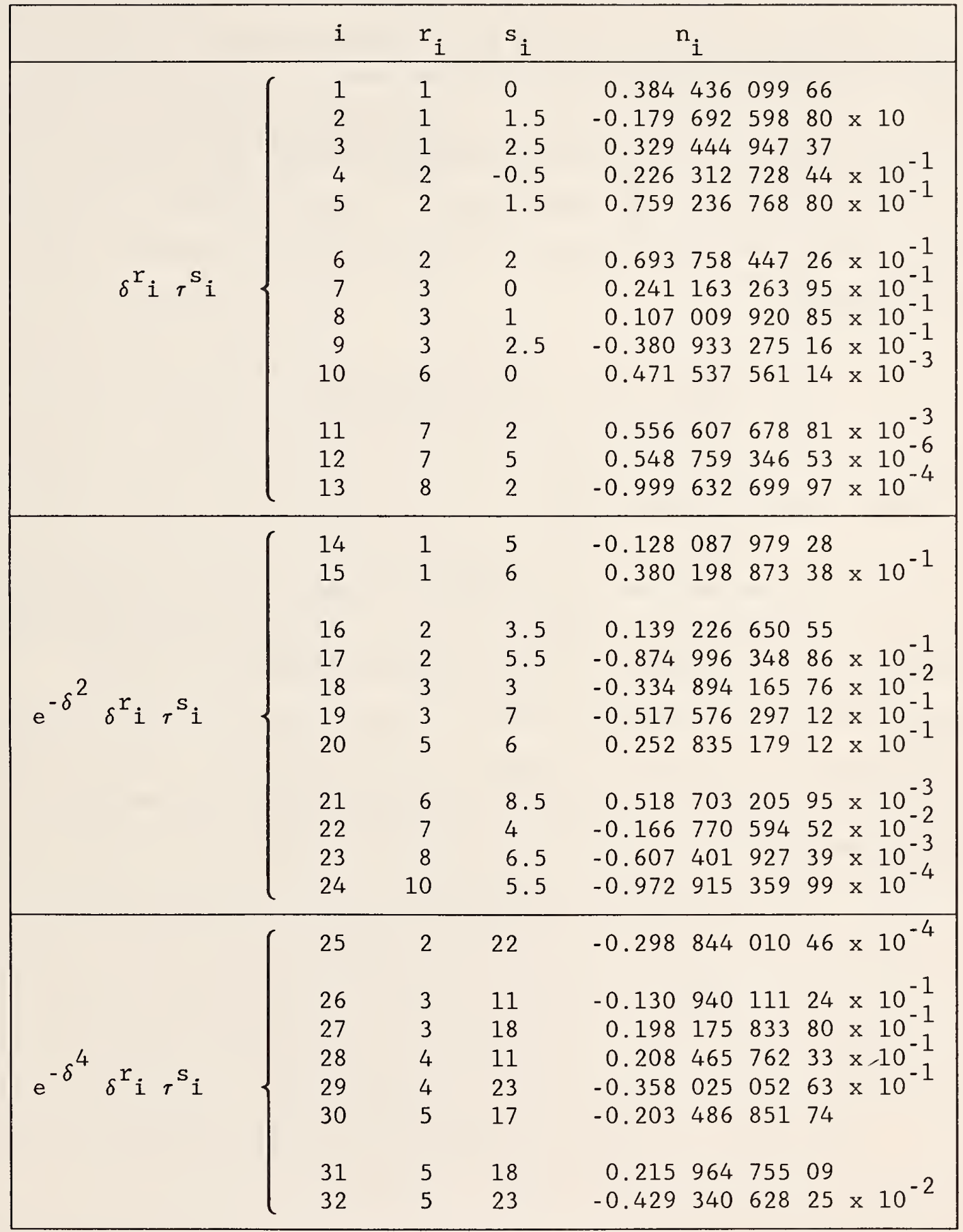


One can evaluate many of the thermodynamic properties of methane by taking the appropriate derivatives of the Helmholtz energy as given in eqs (4-6). In tables 5 and 6 we have collected the coefficients necessary to calculate the six lowest order derivatives for the ideal gas and residual contributions to the free energy. In our notation, the first isothermal (reduced) density derivative of the (reduced) residual free energy is denoted

$$
\phi_{\delta}^{\mathrm{r}}=\left.\frac{\partial \phi^{\mathrm{r}}(\delta, \tau)}{\partial \delta}\right|_{\tau}=\left.\frac{\rho_{\mathrm{C}}}{\mathrm{RT}} \frac{\partial \mathrm{A}^{\mathrm{r}}(\rho, \mathrm{T})}{\partial \rho}\right|_{\mathrm{T}}
$$

and similarly for other derivatives.

To calculate derivatives of the ideal gas contribution to the free energy, one is guided by the form of eq (5). The left-most column of table 5 lists the factors necessary for the various derivatives, and the remaining columns give the coefficients of these factors directly beneath the heading which indicates the quantity to be calculated. The resulting terms are to be added as in eq (7). The values of the $Q_{i}$ parameters are obtained from table 4. 
TABLE 5

IDEAL GAS FREE ENERGY AND ITS DERIVATIVES

\begin{tabular}{|c|c|c|c|c|c|c|}
\hline & $\begin{array}{c}\phi^{\text {id }} \\
\text { eq (5) }\end{array}$ & $\begin{array}{c}\delta \phi_{\delta}{ }^{i d} \\
(=1)\end{array}$ & $\tau \phi_{\tau}^{i \mathrm{~d}}$ & $\begin{array}{l}\delta^{2} \phi_{\delta \delta}{ }^{i d} \\
(=-1)\end{array}$ & $\tau^{2} \phi_{\tau \tau} i d$ & $\begin{array}{c}\delta \tau \phi_{\delta \tau}{ }^{i d} \\
(=0)\end{array}$ \\
\hline 1 & $\mathrm{Q}_{1}$ & 1 & $\overline{Q_{2}}$ & -1 & $-Q_{2}$ & 0 \\
\hline $\ln \delta$ & 1 & 0 & 0 & 0 & 0 & 0 \\
\hline $\ln \tau$ & $\mathrm{Q}_{2}$ & 0 & 0 & 0 & 0 & 0 \\
\hline$\tau^{-1 / 3}$ & $\mathrm{Q}_{3}$ & 0 & $-Q_{3} / 3$ & 0 & $4 Q_{3} / 9$ & 0 \\
\hline$\tau^{-2 / 3}$ & $Q_{4}$ & 0 & $-2 Q_{4} / 3$ & 0 & $10 Q_{4} / 9$ & 0 \\
\hline$\tau^{-1}$ & $Q_{5}$ & 0 & $-Q_{5}$ & 0 & $2 Q_{5}$ & 0 \\
\hline $\ln \left(1-e^{Q_{7}^{\tau}}\right)$ & $\mathrm{Q}_{6}$ & 0 & 0 & 0 & 0 & 0 \\
\hline$\left(e^{-Q_{7}^{\tau}-1}\right)^{-1}$ & 0 & 0 & $-Q_{6} Q_{7}^{\tau}$ & 0 & 0 & 0 \\
\hline$e^{Q_{7}^{\tau}}\left(e^{Q_{7}^{\tau}}-1\right)^{-2}$ & 0 & 0 & 0 & 0 & $-Q_{6} Q_{7}{ }^{2} \tau^{2}$ & 0 \\
\hline
\end{tabular}

For derivatives of the residual free energy, eq (2) and tables 4 and 6 can be used. As in eq (6), the derivatives are obtained by summing 32 terms of three general types. Each of the terms has factors consisting of powers of the reduced density and temperature with the explicit exponents $r_{i}$ and $s_{i}$ and coefficients $n_{i}$ given for each value of $i$ in table 4. The additional exponential factor, with its argument either the second or fourth power of the density, is indicated in the column heading of table 6 where appropriate. The remaining coefficients relevant to the derivative being calculated are given in the appropriate row in that table. 
TABLE 6

RESIDUAL FREE ENERGY AND ITS DERIVATIVES

\begin{tabular}{|c|c|c|c|}
\hline & $\begin{array}{c}n_{i} \delta{ }_{i} s_{i} \\
(i=1 \text { to 13) }\end{array}$ & $\begin{array}{c}e^{-\delta} n_{i} \delta r_{i} s_{i} \\
(i=14 \text { to 24) }\end{array}$ & $\begin{array}{c}e^{-\delta} n_{i} \delta r_{i} s_{i} \\
(i=25 \text { to 32) }\end{array}$ \\
\hline \hline$\phi^{r}$ & 1 & 1 & 1 \\
\hline$\delta \phi_{\delta}^{r}$ & $r_{i}$ & $r_{i}-2 \delta^{2}$ & $r_{i}-4 \delta^{4}$ \\
\hline$\tau \phi_{\tau}^{r}$ & $s_{i}$ & $s_{i}$ & $s_{i}$ \\
\hline$\delta \phi_{\delta \delta}^{r}$ & $r_{i}\left(r_{i}-1\right)$ & {$\left[r_{i}\left(r_{i}-1\right)\right.$} & $-2\left(2 r_{i}+1\right) \delta^{2}$ \\
\hline$\tau^{2} \phi_{\tau \tau}^{r}$ & $s_{i}\left(s_{i}-1\right)$ & $s_{i}\left(s_{i}-1\right)$ & $r_{i}\left(r_{i}-1\right)$ \\
\hline$\delta \tau \phi_{\delta \tau}^{r}$ & $r_{i} s_{i}$ & $s_{i}\left(r_{i}-2 \delta^{2}\right)$ & $s_{i}\left(s_{i}-1\right)$ \\
\hline
\end{tabular}

In table 7, the most common thermodynamic quantities of interest have been expressed in terms of the reduced derivatives of the Helmholtz energy as discussed above. All nominally extensive quantities (that is, the various thermodynamic potentials and heat capacities) are given on a permole basis. It is straightforward to use these tables to calculate the most useful thermodynamic properties of methane. In tables A4-A9 of Appendix A, we give comparisons of experimental data and correlated values for the PVT surface, second virial coefficient, isochoric specific heat capacity, isobaric specific heat capacity, specific heat capacity of the liquid along the saturation boundary, and speed of sound. 
THERMODYNAMIC PROPERTY EQUATIONS

\begin{tabular}{|c|c|}
\hline Pressure: & $\mathrm{P}(\rho, \mathrm{T})=\rho \mathrm{RT}\left(1+\delta \phi_{\delta}^{\mathrm{r}}\right)$ \\
\hline Internal Energy: & $\mathrm{U}(\rho, \mathrm{T})=\mathrm{RT}\left(\tau \phi_{\tau}^{\mathrm{id}}+\tau \phi_{\tau}^{\mathrm{r}}\right)$ \\
\hline Enthalpy: & $\mathrm{H}(\rho, \mathrm{T})=\mathrm{RT}\left(1+\tau \phi_{\tau}^{\mathrm{id}}+\tau \phi_{\tau}^{\mathrm{r}}+\delta \phi_{\delta}^{\mathrm{r}}\right)$ \\
\hline Gibbs Energy: & $\mathrm{G}(\rho, \mathrm{T})=\mathrm{RT}\left(1+\phi^{\mathrm{id}}+\phi^{\mathrm{r}}+\delta \phi_{\delta}^{\mathrm{r}}\right)$ \\
\hline He1mho1tz Energy: & $\mathrm{A}(\rho, \mathrm{T})=\mathrm{RT}\left(\phi^{\mathrm{id}}+\phi^{\mathrm{r}}\right)$ \\
\hline Entropy: & $\mathrm{S}(\rho, \mathrm{T})=-\mathrm{R}\left(\phi^{\mathrm{id}}+\phi^{\mathrm{r}}-\tau \phi_{\tau}^{\mathrm{id}}-\tau \phi_{\tau}^{\mathrm{r}}\right)$ \\
\hline $\begin{array}{l}\text { Isochoric Specific } \\
\text { Heat Capacity: }\end{array}$ & $\mathrm{C}_{\mathrm{V}}(\rho, \mathrm{T})=-\mathrm{R}\left(\tau^{2} \phi_{\tau \tau}^{\mathrm{id}}+\tau^{2} \phi_{\tau \tau}^{\mathrm{r}}\right)$ \\
\hline $\begin{array}{l}\text { Isobaric Specific } \\
\text { Heat Capacity: }\end{array}$ & $\mathrm{C}_{\mathrm{p}}(\rho, \mathrm{T})=\mathrm{C}_{\mathrm{V}}(\rho, \mathrm{T})+\mathrm{R} \frac{\left(1+\delta \phi_{\delta}^{\mathrm{r}}-\delta \tau \phi_{\delta}^{\mathrm{r}}\right)^{2}}{1+2 \delta \phi_{\delta}^{\mathrm{r}}+\delta^{2} \phi_{\delta \delta}^{\mathrm{r}}}$ \\
\hline $\begin{array}{l}\text { Saturated Liquid } \\
\text { Specific Heat Capacity: }\end{array}$ & $\begin{aligned} \mathrm{C}_{\sigma \mathrm{L}}(\mathrm{T})= & \mathrm{C}_{\mathrm{V}}\left(\rho_{\sigma \mathrm{L}}, \mathrm{T}\right) \\
& -\mathrm{R}\left(1+\delta \phi_{\delta}^{\mathrm{r}}-\delta \tau \phi_{\delta \tau}^{\mathrm{r}}\right) \frac{\mathrm{T}}{\rho_{\sigma \mathrm{L}}} \frac{\mathrm{d} \rho_{\sigma \mathrm{L}}}{\mathrm{dT}}\end{aligned}$ \\
\hline Speed of Sound: & $w^{2}(\rho, T)=\frac{R T}{u_{A}{ }_{A}^{M}} \frac{C_{p}(\rho, T)}{C_{v}(\rho, T)}\left(1+2 \delta \phi_{\delta}^{r}+\delta^{2} \phi_{\delta \delta}^{r}\right)$ \\
\hline $\begin{array}{l}\text { Second Virial } \\
\text { Coefficient: }\end{array}$ & $\mathrm{B}(\mathrm{T})=\frac{1}{\rho} \lim _{\delta \rightarrow 0} \phi_{\delta}^{\mathrm{r}}$ \\
\hline
\end{tabular}




\section{TRANSPORT PROPERTIES}

\section{AND THEIR ALGEBRAIC REPRESENTATION}

The viscosity and thermal conductivity are correlated as the sums of terms representing the temperature dependent dilute gas contributions, the temperature and density dependent excess contributions and, for the thermal conductivity, a contribution from the critical enhancement. The viscosity is given by

$$
\eta(\rho, \mathrm{T})=\eta_{0}(\mathrm{~T})+\eta_{\mathrm{ex}}(\rho, \mathrm{T})
$$

and the expression for thermal conductivity is

$$
\lambda(\rho, \mathrm{T})=\lambda_{0}(\mathrm{~T})+\lambda_{\mathrm{ex}}(\rho, \mathrm{T})+\lambda_{\mathrm{cr}}(\rho, \mathrm{T})
$$

These terms are described in this section.

\subsection{Dilute Gas Correlations}

Our expression for the dilute gas viscosity is from the Chapman-Enskog theory [13]. Using the appropriate constants from table 1, we find

$$
\eta_{0}(\mathrm{~T})=10.50 \sqrt{\mathrm{t}} / \Omega^{(2,2) *}(\mathrm{t}) \mu \mathrm{Pa} \cdot \mathrm{s}
$$

where the reduced temperature $t=k \mathrm{~T} / \epsilon$. The collision integral $\Omega(2,2) *$ has been approximated for the 11-6-8, $\gamma=3$ intermolecular potential function [14],

$$
\Omega^{(2,2) *}=\left[\sum_{i=1}^{9} C_{i} t^{[(i-1) / 3-1]}\right]^{-1},
$$

with coefficients $C_{i}$ listed in table 8 . The collision integrals tabulated 
in Ref.[14] are compared to eq (10) in table A10 of Appendix A;

experimental values of $\eta_{0}$ are compared to values calculated from eq ( 9 ) in table All.

TABLE 8

COEFFICIENTS FOR DILUTE GAS TRANSPORT PROPERTIES

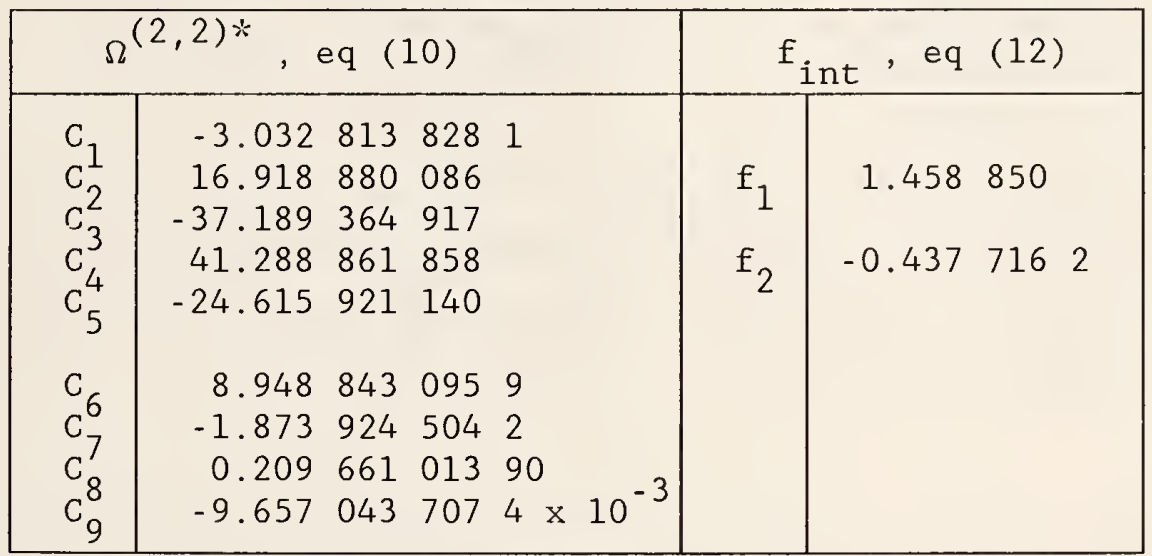

For the thermal conductivity $\lambda_{0}$ of the dilute gas, we have chosen a modified Eucken model [15] with resulting equation

$$
\lambda_{0}(\mathrm{~T})=0.51826 \eta_{0}(\mathrm{~T})\left[3.75-\mathrm{f}_{\mathrm{int}}\left(\tau^{2} \phi_{\tau \tau}^{\mathrm{id}}+1.5\right)\right] \mathrm{mW} \cdot \mathrm{m}^{-1} \cdot \mathrm{K}^{-1}
$$

In eq (11), $\mathrm{f}_{\text {int }}$ is the dimensionless function defined by

$$
\mathrm{f}_{\text {int }}=\mathrm{f}_{1}+\left(\mathrm{f}_{2} / \mathrm{t}\right)
$$

with the fitted coefficients $\mathrm{f}$ also given in table 8 , and the viscosity should be expressed in $\mu \mathrm{Pa} \cdot \mathrm{s}$, as in eq (9). The quantity $\tau^{2} \phi_{\tau \tau}^{\text {id }}$ in eq (12) can be evaluated from tables 3 and 5. Table A12 of Appendix A gives comparisons between experimental values for the dilute gas thermal conductivity and values calculated from eq (11). 
For the excess viscosity, we use the rational polynomial

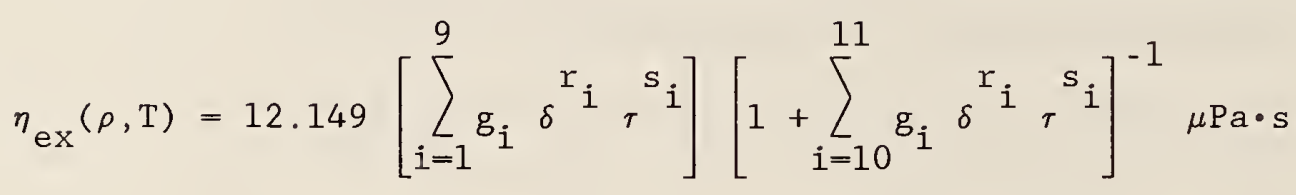

where the exponents $r_{i}$ and $s_{i}$ and the dimensionless fitted coefficients, $g_{i}$, are given in table 9. Comparisons between experimental viscosities and viscosities calculated using eq (7) with eqs (9) and (13) are given in table A13 of Appendix A.

The excess thermal conductivity $\lambda_{\text {ex }}$ has been correlated to a polynomial in $\delta$ and $\tau$ with a factor in the final term of $\left(\delta_{\sigma}^{*}\right)^{-1}$, defined by

$$
\delta_{\sigma}^{*}(\mathrm{~T})=\left\{\begin{array}{cl}
\rho_{\sigma \mathrm{V}}(\mathrm{T}) / \rho_{\mathrm{c}}, & \text { if } \mathrm{T}<\mathrm{T}_{\mathrm{c}} \text { and } \rho<\rho_{\mathrm{c}} \\
1 \quad, \text { otherwise, }
\end{array}\right.
$$

in the final term. In eq (14), $\rho_{\sigma \mathrm{V}}$ is the density of the saturated vapor and can be calculated from eq (3). The quantity $\delta_{\sigma}{ }^{*}(\mathrm{~T})$ differs from 1 only in the vapor phase below the critical temperature. Thus we write

$$
\lambda_{\text {ex }}(\rho, T)=6.29638\left[\sum_{i=1}^{6} j_{i} \delta{ }^{\mathrm{r}_{i}}{ }^{\mathrm{s}^{\mathrm{i}}}+\mathrm{j}_{7} \delta^{2} / \delta_{\sigma}^{*}\right] \mathrm{mW} \cdot \mathrm{m}^{-1} \cdot \mathrm{K}^{-1}
$$

The exponents and dimensionless fitting coefficients for eq (15) are included in table 9 . 
TABLE 9

COEFFICIENTS FOR EXCESS TRANSPORT PROPERTY CORRELATIONS

\begin{tabular}{|c|c|c|c|c|c|c|c|c|c|c|}
\hline & \multicolumn{5}{|c|}{$\eta_{\text {ex }}$ eq (13) } & \multicolumn{5}{|c|}{$\lambda_{\text {ex }}$, eq (15) } \\
\hline $\mathrm{i}$ & $r_{i}$ & & g & & & $\mathrm{r}$ & $s_{i}$ & j & $j_{i}$ & \\
\hline 1 & 1 & 0 & 0.412 & 501 & 37 & 1 & 0 & 2.414 & 920 & \\
\hline 2 & 1 & 1 & -0.143 & 909 & 12 & 3 & 0 & 0.551 & 663 & 31 \\
\hline 3 & 2 & 0 & 0.103 & 669 & 93 & 4 & 0 & -0.528 & 377 & 34 \\
\hline 4 & 2 & 1 & 0.402 & 874 & 64 & 4 & 1 & 0.073 & 809 & 553 \\
\hline 5 & 2 & 1.5 & -0.249 & 035 & 24 & 5 & 0 & 0.244 & 655 & 07 \\
\hline 6 & 3 & 0 & -0.129 & 531 & & 5 & 1 & -0.047 & 613 & 626 \\
\hline 7 & 3 & 2 & 0.065 & 757 & 76 & $* 2$ & 0 & 1.555 & 461 & \\
\hline 8 & 4 & 0 & 0.025 & 666 & 28 & \multirow{4}{*}{\multicolumn{5}{|c|}{${ }^{*}$ Term divided by $\delta_{\sigma}^{*}$}} \\
\hline 9 & 4 & 1 & -0.037 & 165 & 26 & & & & & \\
\hline 10 & 1 & 0 & -0.387 & 983 & & & & & & \\
\hline 11 & 1 & 1 & 0.035 & 338 & 15 & & & & & \\
\hline
\end{tabular}

\subsection{Critical Enhancement Correlation}

The remaining term in eq (8) is the critical enhancement of the thermal conductivity; it is easily observed in a broad region around the critical point. We have correlated this using a function very similar to that developed by Sengers et a1. [16]. After evaluating the universal, fluid dependent, and correlated constants, we write

$$
\lambda_{c r}(\rho, \mathrm{T})=\frac{91.855}{\eta(\rho, \mathrm{T}) \tau^{2}}\left[1+\delta \phi_{\delta}^{\mathrm{r}}-\delta \tau \phi_{\delta \tau}^{\mathrm{r}}\right]^{2} \chi_{\mathrm{T}}^{* 0.4681} \mathrm{~F}\left(\mathrm{~T}^{*}, \rho^{*}\right) \frac{\mathrm{mW}}{\mathrm{m} \cdot \mathrm{K}}
$$

where the viscosity is expressed in $\mu \mathrm{Pa} \cdot \mathrm{s}$ in eq (16). The empirical dimensionless crossover or damping function $F$ is defined by

$$
\mathrm{F}\left(\mathrm{T}^{*}, \rho^{*}\right)=\mathrm{e}^{-\left[\mathrm{F}_{\mathrm{T}}\left|\mathrm{T}^{*}\right|^{1 / 2}+\mathrm{F}_{\rho} \rho^{* 2}+\mathrm{F}_{\mathrm{A}} \rho^{*}\right]}
$$

where $\mathrm{F}_{\mathrm{T}}, \mathrm{F}_{\mathrm{A}}$, and $\mathrm{F}_{\rho}$ are constants in table 10 and $\mathrm{T}^{*}$ and $\rho^{*}$ measure deviations from the critical point according to 


$$
\mathrm{T}^{*}=\left(\mathrm{T}_{\mathrm{c}}-\mathrm{T}\right) / \mathrm{T}_{\mathrm{c}} \quad(=1-1 / \tau)
$$

and

$$
\rho^{*}=\left(\rho_{\mathrm{c}}-\rho\right) / \rho_{\mathrm{c}} \quad(=1-\delta)
$$

The final factor in eq (16) is the reduced and symmetrized compressibility $x_{\mathrm{T}}{ }^{*}$. Throughout most of the phase diagram, this can be evaluated classically using

$$
x_{\mathrm{T}}^{*}=0.28631 \delta \tau\left[1+2 \delta \phi_{\delta}^{\mathrm{r}}+\delta^{2} \phi_{\delta \delta}^{\mathrm{r}}\right]^{-1},
$$

where the derivatives in eq (20) are found from tables 4 and 6 . However, the classical quantity in eq $(20)$ is not very accurate in the region very close to the critical point, so in the small region defined by $185 \mathrm{~K} \leq \mathrm{T} \leq$ $196 \mathrm{~K}$ and $7.6 \mathrm{~mol} \cdot \mathrm{dm}^{-3} \leq \rho \leq 12.7 \mathrm{~mol} \cdot \mathrm{dm}^{-3}$, we recommend evaluating the compressibility using the MLSG [17] scaled equation of state. In this region, then,

$$
\chi_{\mathrm{T}}^{*}(\rho, \mathrm{T})=\mathrm{Q}\left|\rho^{*}\right|^{-\mathrm{a}} \theta^{\mathrm{b}}[\theta+\Omega(\theta+\mathrm{R})]^{-1} .
$$

where the variables $\theta$ and $\Omega$ are defined as

$$
\theta= \begin{cases}1+\mathrm{E}\left(1+\mathrm{S} \mathrm{T}^{*}\left|\rho^{*}\right|^{-1 / \beta}\right)^{2 \beta}, & \text { if } \mathrm{T}^{*}<-\left|\rho^{*}\right|^{1 / \beta} / \mathrm{S} \\ 1, & \text { if } \mathrm{T}^{*}>-\left|\rho^{*}\right|^{1 / \beta} / \mathrm{S}\end{cases}
$$

and

$$
\Omega=\mathrm{WT}^{*}\left|\rho^{*}\right|^{-1 / \beta}
$$

and the remaining constants in eq (21) can be found in table 10. Finally, along the critical isochore, if $185 \mathrm{~K} \leq \mathrm{T} \leq 196 \mathrm{~K}$, we can use

$$
\chi_{\mathrm{T}}^{*}\left(\rho=\rho_{\mathrm{C}}, \mathrm{T}\right)=\Gamma\left|\mathrm{T}^{*}\right|^{-\gamma}
$$


to avoid spurious singularities in eq (21). This completes the description of the thermal conductivity enhancement and the correlation for the methane fluid transport properties. Table A14 of Appendix A gives tabular comparisons between experimental values of the thermal conductivity coefficient and those calculated from eq (8) using eqs (11, 12, 15-17).

TABLE 10

CONSTANTS FOR $\lambda_{\mathrm{cr}}$

\begin{tabular}{|l|l|}
\hline Fitted coefficients: & $\mathrm{F}_{\mathrm{T}}=2.646$ \\
& $\mathrm{~F}_{\rho}=2.678$ \\
& $\mathrm{~F}_{\mathrm{A}}=-0.637$ \\
\hline Critical exponent: & $\beta=0.355$ \\
\hline Universal constants: & $\mathrm{a}=3.352$ \\
& $\mathrm{~b}=0.732$ \\
& $\mathrm{E}=0.287$ \\
$\mathrm{R}=0.535$ \\
\hline Fluid dependent constants & $\mathrm{Q}=0.1133$ \\
derived from Ref. [16] : & $\mathrm{S}=-6.098$ \\
& $\mathrm{~W}=-1.401$ \\
& $\Gamma=0.0801$ \\
\hline
\end{tabular}




\section{SUMMARY OF UNCERTAINTIES}

\section{ASSOCIATED WITH THE CORRELATIONS}

We have judged the uncertainties associated with the correlations in various regions of the phase diagram. These judgements are based on an examination of the deviations of our correlations from experimental data using tables such as included in Appendix $A$ and deviation plots as in Ref.[1], an assessment of the experiments themselves and their reported accuracies, and comparisons of these correlations with previously published equations. In this section, we will summarize the results of our assesment so that the equations and the tables in Appendix B can be used appropriately; details concerning the establishment of these uncertainties are given in Ref.[1]. In this section, the terms "uncertainty", "error", and "accuracy" are used interchangeably; the deviations from the true value, of quantities calculated from the correlations, will not exceed the stated uncertainty.

4.1 Two-Phase Boundary

First, for the pressure along the liquid-vapor saturation boundary, we have considered the uncertainties of both the triple-point and critical point pressures (table 1), the quality of the measurements of Ref.[6], and the disconcerting disagreement between the earlier benchmark data. We make the subjective judgment that either the ancillary eq ( 1 ) or the SWEOS will provide values of the saturation pressures accurate within 0.06 percent above $\mathrm{T}=120 \mathrm{~K}$ and accurate within 0.3 percent below this temperature.

For the density of the saturated liquid, again, either the ancillary equation or the value of the density from the SWEOS can be used with an estimated accuracy well under 0.2 percent throughout the range from the triple point to just below the critical point. We estimate that between $190.5 \mathrm{~K}$ and $\mathrm{T}_{\mathrm{c}}$ the uncertainty in the saturated liquid density associated with these equations may exceed 1 percent. 
We have considered the data and conclusions of Refs. $[3,4,6]$, and estimate that the error associated with either eq (3) or the SwEos for the density of the saturated vapor does not exceed 1 percent from the triple point temperature to $91 \mathrm{~K}, 2$ percent above $\mathrm{T}=190.5 \mathrm{~K}$, and is well under 0.5 percent for the remainder of the two-phase boundary.

\subsection{Thermodynamic Properties}

In this section, we discuss the uncertainties associated with the full equation of state. The PVT surface is considered first, and both the calculation of pressure with temperature and density as independent variables, and the calculation of density with temperature and pressure as independent variables are discussed. For this discussion, the thermodynamic space is divided into temperature regions: $\mathrm{T}_{t}-185 \mathrm{~K}, 185$ $195 \mathrm{~K}, 195-300 \mathrm{~K}$, and $300-600 \mathrm{~K}$. Following the treatment of the PVT surface, uncertainties associated with the calculation of the second virial coefficient, isochoric specific heat capacity, isobaric specific heat capacity, specific heat capacity of the saturated liquid, and speed of sound are discussed in turn.

We offer the following guidelines when calculating pressures from the SWEOS when given densities and temperatures between the triple point and $185 \mathrm{~K}$. For the vapor phase, the errors should not exceed 0.2 percent. For the liquid below $150 \mathrm{~K}$ and below $1 \mathrm{MPa}$, the percentage uncertainty is extremely large, but the actual pressure should not be in error by more than $0.1 \mathrm{MPa}$. Most of the remaining data indicate that the error should be less than 5 percent. An exception occurs below $120 \mathrm{~K}$ or very close to the saturation line, where the uncertainty approaches 10 percent. Most of the primary PVT data (from Goodwin [38]) in this temperature region and below about $30 \mathrm{MPa}$ and $28.5 \mathrm{~mol} \cdot \mathrm{dm}^{-3}$ exhibit deviations from the correlation well below 1 percent. Calculation of an accurate density from a given temperature and pressure is a much easier task in the region below $185 \mathrm{~K}$. The general uncertainty is well below 1 percent. In the vapor phase, all points at temperatures below $180 \mathrm{~K}$ or pressures below $3.5 \mathrm{MPa}$ show absolute 
deviations below 0.5 percent. A few vapor points closer to the critical point in both temperature and pressure show higher deviations. We estimate that for the liquid in this range, a 0.2 percent accuracy in the determination of the density from the SWEOS is expected.

Our comparisons suggest that in calculating the pressure using the SWEOS in the temperature range 185 - $195 \mathrm{~K}$, accuracies of better than 0.1 percent are expected at up to $6 \mathrm{MPa}$. The accuracy is expected to deteriorate to about 2 percent at higher pressures. Our study suggests that in this temperature range the SWEOS should return a density well within 0.5 percent accuracy. The exception is quite close to the critical point, $190.4 \mathrm{~K}<\mathrm{T}<190.6 \mathrm{~K}$ and $4.4 \mathrm{MPa}<\mathrm{P}<4.8 \mathrm{MPa}$, where errors of nearly 5 percent are possible.

In the temperature range 195 to $300 \mathrm{~K}$, we find the largest quantity of PVT data with a very large range in pressures. In predicting pressures from the present SWEOS in this temperature region and below $10 \mathrm{MPa}$ (or 15 $\mathrm{mol} \cdot \mathrm{dm}^{-3}$ ) an accuracy of 0.5 percent is expected; above these pressures, 5 percent errors are possible. For prediction of densities, in our judgment, 0.2 percent is the general uncertainty associated with the SWEOS for most of this region, except for a deterioration to 0.5 percent above $20 \mathrm{~mol} \cdot \mathrm{dm}^{-3}$ and below $210 \mathrm{~K}$. The correlation can be extrapolated to pressures above $100 \mathrm{MPa}$ in this temperature range (to about $600 \mathrm{MPa}$ ), but the estimated uncertainty becomes closer to 8 percent in pressure and 1 percent in density.

We summarize our observations of the data and the SWEOS for predicting pressures at temperatures above $300 \mathrm{~K}$ as follows. For pressures below 40 $\mathrm{MPa}$ and temperatures to $600 \mathrm{~K}$, the expected accuracy is 0.2 percent. In the pressure range 40 - $200 \mathrm{MPa}$ the accuracy is near 1 percent, but for higher pressures the uncertainty increases with increasing pressure and may reach 20 percent at $1000 \mathrm{MPa}$. In the highest pressure range, above 800 $\mathrm{MPa}$, it is also prudent to restrict the temperature to $450 \mathrm{~K}$ because there are no data at significantly higher temperatures. We have extended the correlation above the $100 \mathrm{MPa}$ cutoff in this temperature range, but the quality of the equation clearly deteriorates at high pressures; pressures 
above $100 \mathrm{MPa}$ are not included in the tables of Appendix B. For the calculation of densities for temperatures below $350 \mathrm{~K}$ and densities below $15 \mathrm{~mol} \cdot \mathrm{dm}^{-3}$, an accuracy of 0.2 percent should be associated with the SWEOS. For temperatures up to $600 \mathrm{~K}$ or densities to $20 \mathrm{~mol} \cdot \mathrm{dm}^{-3}$, the error increases to 0.5 percent. The error increases to 1 percent up to 25 $\mathrm{mol} \cdot \mathrm{dm}^{-3}$ and 5 percent at higher densities. Extrapolation of the SWEOS to state points above $450 \mathrm{~K}$ for the density range above $20 \mathrm{~mol} \cdot \mathrm{dm}^{-3}$ is not advised.

We estimate an uncertainty in the correlation for the second virial coefficient of about 1 percent throughout the range, from the triple point to $625 \mathrm{~K}$.

In our judgment, a 2 percent uncertainty should be expected when calculating the isochoric specific heat capacity from the SWEOS outside the critical region, and the accuracy approaches 5 percent for temperatures between $180 \mathrm{~K}$ and $200 \mathrm{~K}$ for densities up to $14 \mathrm{~mol} \cdot \mathrm{dm}^{-3}$. The asymptotically critical behavior of $\mathrm{C}_{\mathrm{V}}$ is not well described by this (or any) classical equation of state.

We estimate that the SWEOS will give isobaric heat specific capacities within about 2 percent for the liquid and supercritical fluid from $115 \mathrm{~K}$ to about $300 \mathrm{~K}$ for pressures to about $15 \mathrm{MPa}$. The uncertainty is assumed to be worse outside these ranges, and in the general critical region, 20 percent errors may be possible. Very close to the critical point, the behavior of this classical SWEOS will not describe the heat capacities accurately.

The accuracy of the SWEOS in predicting saturated liquid specific heat capacities is 2 percent from the triple point to $186 \mathrm{~K}$ and 5 percent for temperatures approaching the critical point.

For predicting the speed of sound in methane, we can summarize our observations by the following subjective guidelines for the use of the SWEOS. Below $180 \mathrm{~K}$ or above $195 \mathrm{~K}$, with pressures below about $20 \mathrm{MPa}$, for the saturated liquid, compressed liquid, vapor phase, and supercritical 
fluid, accuracies of 0.6 percent can be anticipated. Between $180 \mathrm{~K}$ and 188 $\mathrm{K}$, or above $20 \mathrm{MPa}$ (to about $35 \mathrm{MPa}$ ), the accuracy decreases to 1.5 percent. In the general region of the critical point, $188 \mathrm{~K}$ to $195 \mathrm{~K}$ and 4.5 $\mathrm{MPa}$ to $4.7 \mathrm{MPa}$, the uncertainties can exceed 5 percent. Our classical equation of state cannot give accurate results for derivative properties at the critical point or for state points extremely close to the critical point (approximately 190 to $191 \mathrm{~K}$ and 4.55 to $4.65 \mathrm{MPa}$ ). In particular, theory indicates that the speed of sound should vanish at the critical point; in our formulation this speed is about $231 \mathrm{~m} \cdot \mathrm{s}^{-1}$ This discrepancy between theory and our correlation occurs principally because the very small divergence of the isochoric specific heat capacity $\left(-\left|\mathrm{T}-\mathrm{T}_{\mathrm{c}}\right|^{-\alpha}\right.$ where $\alpha$ is about 0.1 ) is not predicted by a classical equation of state; in our formulation the value of $\mathrm{C}_{\mathrm{v}}$ at the critical point is about $45 \mathrm{~J} \cdot \mathrm{mol}{ }^{-1} \cdot \mathrm{K}^{-1}$. In addition, because of truncation error, the nominally zero value of $\partial \mathrm{P} /\left.\partial \rho\right|_{\mathrm{T}}$ is equal to about $10^{-8} \mathrm{~J} \cdot \mathrm{mol}^{-1}$ and the related $\mathrm{C}_{\mathrm{P}^{\prime}}$, which should diverge strongly, is found to be about $10^{13} \mathrm{~J} \cdot \mathrm{mol}^{-1} \cdot \mathrm{K}^{-1}$ at the critical point.

The comparisons with experimental data (and the process of determining the coefficients of the SWEOS) probe only four derivatives of the dimensionless, residual Helmholtz energy, namely $\frac{\mathrm{r}}{\delta}, \phi \frac{\mathrm{r}}{\delta} \delta, \phi_{\tau \tau}^{\mathrm{r}}$, and $\frac{\mathrm{r}}{\delta} \tau$. While this should describe the actual surface quite well, additional uncertainties will enter any calculation which requires other derivatives or integrals of these directly accessible functions. We hesitate to make any quantitative predictions of the errors involved in calculating any thermodynamic quantities not explicitly discussed in this section, but we do conjecture that these errors will be comparable to those found with any other precise equation of state for methane.

\subsection{Transport Properties}

From the dispersion of the data and correlations, the quoted experimental accuracies associated with the data, and a study of the use of the 11-6-8 intermolecular potential in the Chapman-Enskog dilute gas 
theory, we estimate that the correlation of eqs (9) and (10) will give dilute gas viscosities for methane with associated accuracies of 0.5 percent from the $270 \mathrm{~K}$ to $600 \mathrm{~K}$ and 1 percent above that range. For lower temperatures, and especially below the critical point, the data are extremely sparse or nonexistent. We anticipate that, with this potential function, the theory will extrapolate well, but we increase our error estimate to 2 percent for temperatures from the triple point to $270 \mathrm{~K}$.

We next examine the viscosity of methane at elevated pressures. For the vapor phase, below the critical temperature, we estimate that the error varies from 2 percent, as above, to more than 5 percent as the saturated vapor line is approached. For the liquid below the critical temperature, the data indicate an accuracy of about 3 percent from the phase boundary to the compressed liquid at pressures to $30 \mathrm{MPa}$. Above the critical temperature, in the temperature range from $\mathrm{T}_{c}$ to $270 \mathrm{~K}$, the data indicate an accuracy of about 5 percent for densities up to $10 \mathrm{~mol} \cdot \mathrm{dm}^{-3}$. In the same temperature range, but for higher densities and for pressures up to about $30 \mathrm{MPa}$, the viscosity increases and the estimated uncertainty drops to about 2 percent. For the low density region, to $10 \mathrm{~mol} \cdot \mathrm{dm}^{-3}$, we estimate that the uncertainty associated with the correlation is 1 percent in the higher temperature range, to $450 \mathrm{~K}$. For pressures up to $55 \mathrm{MPa}$ and temperatures to $450 \mathrm{~K}$, the error is about 2 percent. The correlation attempts to describe the rapidly rising excess viscosity as the melting line is approached. It has a zero in the denominator, corresponding to a singularity in the correlation within the fluid region; this occurs well outside the range of any data. In addition to the usual warnings concerning extrapolation beyond the range of the correlating equation, any user is cautioned about this singularity, which can be a problem only at pressures exceeding $200 \mathrm{MPa}$.

For the thermal conductivity of the dilute methane gas, we estimate that the accuracy of the correlation is about 2.5 percent for temperatures between 130 and $625 \mathrm{~K}$. For temperatures below $130 \mathrm{~K}$, where the vapor pressure is about $0.37 \mathrm{MPa}$, the current correlation could give errors in excess of 10 percent for the thermal conductivity of dilute methane. 
We estimate that the accuracy of the correlation for the total thermal conductivity of methane is about 2 percent for most of the range between $110 \mathrm{~K}$ and $725 \mathrm{~K}$ and for pressures to $70 \mathrm{MPa}$ (or densities to about 29 $\mathrm{mol} \cdot \mathrm{dm}^{-3}$ ). The exceptions occur around the critical point and near both saturation boundaries, where 5 percent errors are typical, and greater errors could occur in the very close to the critical point. For the vapor at lower temperatures and the dense liquid near the triple point, an uncertainty of nearly 10 percent is possible.

\section{ACKNOWLEDGMENTS}

We thank R.D. McCarty for helpful discussions and W. Wagner for providing a copy of his experimental tables prior to publication. We also thank R.T Jacobsen and M.W. Chase for their careful reviews of parts of the manuscript. 


\section{REFERENCES}

[1] D.G. Friend, J.F. Ely, H. Ingham, "Thermophysical Properties of Methane," to be published J. Phys. Chem. Ref. Data (1989).

[2] R. Schmidt and W. Wagner, Fluid Phase Equil. 19, 175 (1985).

[3] R.D. Goodwin, Nat. Bur. Stand. (U.S.) Technical Note 653 (1974).

[4] S. Angus, B. Armstrong, and K.M. de Reuck, International Thermodynamic Tables of the Fluid State Vol. 5 Methane, International Union of Pure and Applied Chemistry, Chemical Data Series No. 16, Pergamon Press, Oxford (1978).

[5] V.V. Sychev, A.A. Vasserman, V.A. Zagoruchenko, A.D. Kozlov, G.A. Spiridonov, and V.A. Tsymarny, Thermodynamic Properties of Methane (Hemisphere Publishing, Washington (1987), T.B. Selover Jr., English Language Ed.

[6] R. Kleinrahm and W. Wagner, J. Chem. Thermo. 18, 739 (1986).

[7] R. Kleinrahm, W. Duschek, and W. Wagner, J. Chem. Thermo. 18, 1103 (1986).

[8] H.M. Roder, Int. J. Thermo. 6, 119 (1984) and Nat. Bur. Stand. (U.S.) Interagency Report NBSIR 84-3006 (1984).

[9] D.E. Diller, Physica 104A, 417 (1980) and Personal Communication, Nat. Inst. Stand. Tech., Boulder, CO 80303.

[10] E.R. Cohen and B. N. Taylor, J. Res. Nat. Bur. Stand. (U.S.) 92, 85 (1987).

[11] R.D. Goodwin, J. Res. Nat. Bur. Stand. (U.S.) 75A, 15 (1971).

[12] R.S. McDowe11 and F.H. Kruse, J. Chem. Eng. Data 8, 547 (1963).

[13] J.O. Hirschfelder, C.F. Curtiss, and R.B. Bird, Molecular Theory of Gases and Liquids, Wiley, New York (1967).

[14] M. Klein, H.J.M. Hanley, F.J. Smith, and P. Holland, Nat. Stand. Ref. Data Ser., Nat. Bur. Stand. (U.S.), 47, (1974).

[15] R.C. Reid, J.M. Prausnitz, and T.K. Sherwood, The Properties of Gases and Liquids, McGraw-Hill, New York, 3rd Ed. (1977).

[16] J.V. Sengers, R.S. Basu, and J.M.H. Levelt Sengers, National Aeronautics and Space Administration (U.S.) NASA Contractor Report 3424 (1981). 
[17] M. Vicentini-Missoni, J.M.H. Levelt Sengers, and M.S. Green, J. Res. Nat. Bur. Stand. (U.S.) 73A, 563 (1969) and Phys. Rev. Lett. 22, 389 (1969).

[18] R. Prydz and R.D. Goodwin, J. Chem. Thermo. 4, 127 (1972); R.D.Goodwin and R. Prydz, J. Res. Nat. Bur. Stand. (U.S.) 76A, 81 (1972).

[19] W.M. Haynes, Personal Communication, Nat. Inst. Stand. Tech., Boulder, CO 80303 .

[20] J.E. Orrit and J.M. Laupretre, Adv. Cryo. Eng. 23, 573 (1978).

[21] W.M. Haynes and M.J. Hiza, J. Chem. Thermo. 9, 179 (1977).

[22] C.R. McClune, Cryo. 16, 289 (1976).

[23] M.J. Terry, J.T. Lynch, M. Bunclark, K.R. Mansell, and L.A.K. Staveley, J. Chem. Thermo. 1, 413 (1969).

[24] J. Klosek and C. McKinley, Proc. 1st Int. Conf. on LNG, Inst. Gas Tech., Chicago, Sess. 5, Pap. 22 (1968).

[25] J.B. Rodosevich and R.C. Miller, A. I. Ch. E. J. 19, 729 (1973).

[26] O.B. Verbeke, Personal Communication to R.D. Goodwin, National Bureau of Standards, Boulder, CO. (1973). Reported as ID 7 in table 1c (p.46) of Ref. 3 .

[27] A.J. Vennix, T.W. Leland Jr., and R. Kobayashi, J. Chem. Eng. Data 15, $238(1970)$.

[28] R.D. Goodwin, Reported as ID 101-114 in table 1c (p.46) of Ref. 3.

[29] F.P. Ricci and E. Scafe, Phys. Lett. 29A, 650 (1969).

[30] R.D. Goodwin, Reported as ID 601-604 in table lc (p.46) of Ref. 3 .

[31] R.D. Goodwin, Reported as ID 1 in table 1c (p.46) of Ref. 3.

[32] V. Jansoone, H. Gielen, J. de Boelpaep, and O.B. Verbeke, Physica $46,213(1970)$.

[33] H.J. Achtermann, T.K. Bose, H. Rögener, and J.M. St-Arnaud, Int. J. Thermo. 7, 709 (1986).

[34] H.J. Achtermann, F. Klobasa, and H. Rögener, Brennst.-Warme-Kraft 34, 266 and 311 (1982).

[35] V.M. Cheng, Ph.D. Thesis (Dept. Aero. and Mech. Sci.), Princeton University (1972).

[36] D.R. Douslin, R.H. Harrison, R.T. Moore, and J.P. McCullough, J. Chem. Eng. Data 9, 358 (1964). 
[37] B.E. Gammon and D.R. Douslin, J. Chem. Phys. 64, 203 (1976).

[38] R.D. Goodwin, Ref. 3, data appear in table 4 (pp. 69-78); some data also published in R.D. Goodwin and R. Prydz, J. Res. Nat. Bur. Stand. (U.S.) 76A, 81 (1972).

[39] R.D. Goodwin, Ref. 3, table 4 (p. 53).

[40] H.M. Kvalnes and V.L. Gaddy, J. Am. Chem. Soc. 53, 394 (1931).

[41] J. Mo1lerup, J. Chem. Thermo. 17, 489 (1985).

[42] E.C. Morris, Int. J. Thermo. 5, 281 (1984).

[43] S.L. Robertson and S.E. Babb Jr., J. Chem. Phys. 51, 1357 (1969).

[44] A. Sivaraman and B.E. Gammon, "Speed-of-Sound Measurements in Natural Gas Fluids," Gas Research Institute Report 86-0043 (1986).

[45] N.J. Trappeniers, T. Wassenaar, and J.C. Abels, Physica A98, 289 (1979).

[46] A. Van Itterbeek, O. Verbeke, and K. Staes, Physica 29, 742 (1963).

[47] A.J. Vennix, Ph.D. Thesis (Dept. Chem. Eng.), Rice University (1966) and A.J. Vennix, T.W. Leland Jr., and R. Kobayashi, J. Chem. Eng. Data 15,238 (1970).

[48] M.A. Byrne, M.R. Jones, and L.A.K. Staveley, Trans. Faraday Soc. 64, 1747 (1968).

[49] G.A. Pope, Ph.D. Thesis (Dept. Chem. Eng.), Rice University (1971).

[50] A.E. Hoover, I. Nagata, T.W. Leland Jr., and R. Kobayashi, J. Chem. Phys. 48, 2633 (1968).

[51] J.M.H. Levelt Sengers, M. Klein, and J.S. Gallagher, in Amer. Inst. Phys. Handbook, 3rd ed., McGraw Hil1, N.Y. (1972), p. 4-204.

[52] B.A. Younglove, J. Res. Nat. Bur. Stand. (U.S.) 78A, 401 (1974).

[53] H.M. Roder, J. Res. Nat. Bur. Stand. (U.S.) 80A, 739 (1976).

[54] M.L. Jones Jr., D.T. Mage, R.C. Faulkner Jr. and D.L. Katz, Chem. Eng. Prog. Symp. Ser. 59, 52 (1963).

[55] P.H.G. Van Kasteren and H. Zeldenrust, Ind. Engin. Chem. Fund. 18, 333 (1979).

[56] V.G. Baidakov, A.M. Kaverin, and V.P. Skripov, J. Chem. Thermo. 14, 1003 (1982).

[57] Y.P. Blagoi, A.E. Butko, S.A. Mikhailenko and V.V. Yakuba, Zh. Fiz. Khim. 41, 1699 (1967). 
[58] G.C. Straty, Cryo. 14, 367 (1974).

[59] W. Van Dae1, A. Van Itterbeek, J. Thoen, and A. Cops, Physica 31, 1643 (1965).

[60] A. Van Itterbeek, J. Thoen, A. Cops, and W. Van Dael, Physica 35, 162 (1967).

[61] J.M. Hellemans, J. Kestin, and S.T. Ro, Physica 65, 376 (1973).

[62] R.A. Dawe, G.C. Maitland, M. Rigby, and E.B. Smith, Trans. Faraday Soc. 66,1955 (1970).

[63] J. Kestin, S.T. Ro, and W.A. Wakeham, Trans. Faraday Soc. 67, 2308 (1971).

[64] D.L. Timrot, M.A. Serednitskaya, and M.S. Bespalov, Sov. Phys. Dok1. 20,107 (1975).

[65] J. Kestin and J. Yata, J. Chem Phys. 49, 4780 (1968).

[66] A.G. De Rocco and J.0. Halford, J. Chem. Phys. 28, 1152 (1958).

[67] G.C. Maitland and E.B. Smith, J. Chem. Soc. Faraday Trans. I70, 1191 (1973).

[68] Y. Abe, J. Kestin, H.E. Khalifa, and W.A. Wakeham, Physica 93A, 155 (1978).

[69] A.G. Clarke and E.B. Smith, J. Chem. Phys. 51, 4156 (1969).

[70] J.G. Giddings, J.T.F. Kao, and R. Kobayashi, J. Chem. Phys. 45, 578 (1966).

[71] L.T. Carmichael, V.M. Berry, and B.H. Sage, J. Chem. Eng. Data 10, 57 (1965).

[72] A.A. Clifford, J. Kestin, and W. A. Wakeham, Physica 97A, 287 (1979).

[73] M.J. Assael and W.A. Wakeham, J. Chem. Soc. Faraday Trans. I77, 697 (1981).

[74] H.L. Johnston and E.R. Grilly, J. Chem. Phys. 14, 233 (1946).

[75] X.Y. Zheng, S. Yamamota, H. Yosida, H. Masuoka, and M. Yorizane, J. Chem. Eng. Japan 17, 237 (1984).

[76] W.B. Mann and B.G. Dickins, Proc. Roy. Soc. A134, 77 (1931).

[77] D. Misic and G. Thodos, Physica 32, 885 (1966).

[78] L.T. Carmichael, H.H. Reamer, and B.H. Sage, J. Chem. Eng. Data 11, 52 (1966). 
[79] C.E. Baker and R.S. Brokaw, J. Chem. Phys. 43, 3519 (1965).

[80] V.P. Sokolova and I.F. Golubev, Teploenergetika 14, 91 (1967).

[81] B. Le Neindre, R. Tufeu, P. Bury, P. Johannin, and B. Vodar, Proceedings of the Eighth Conference on Thermal Conductivity C.Y. Ho and R.E. Taylor, eds., Plenum Press, N.Y.(1969) p.229.

[82] M. Yorizane, S. Yoshimura, H. Masuoka, and H. Yoshida, Ind. Engin. Chem. Fund. 22, 454 (1983).

[83] Y. Tanaka, M. Noguchi, H. Kubota, and T. Makita, J. Chem. Eng. Japan 12,171 (1979).

[84] A.K. Barua, M. Afzal, G.P. Flynn, and J. Ross, J. Chem. Phys. 41, 374 (1964).

[85] J.P. Boon, J.C. Legros, and G. Thomaes, Physica 33, 547 (1967).

[86] M.H. Gonzalez, R.F. Bukacek, and A.L. Lee, Soc. Petr. Eng. J. 7, 75 (1967).

[87] W.M. Haynes, Physica 70, 410 (1973).

[88] J. Hellemans, H. Zink, and O. Van Paeme1, Physica 46, 395 (1970).

[89] E.T.S. Huang, G.W. Swift, and F. Kurata, A. I. Ch. E. J. 12, 932 (1966).

[90] L.D. Ikenberry and S.A. Rice, J. Chem. Phys. 39, 1561 (1963).

[91] U.V. Mardolcar and C.A. Nieto de Castro, Ber. Bunsen. Phys. Chem. 91, 152 (1987).

[92] R.C. Prasad, N. Mani, and J.E.S. Venart, Int. J. Thermo. 5, 265 (1984).

$-29-$ 

APPENDIX A

TABLES FOR THE COMPARISON OF EXPERIMENTAL DATA AND THE CORRELATIONS

A1. COMPARISONS FOR SATURATED VAPOR PRESSURES $\ldots \ldots \ldots \ldots \ldots \ldots \ldots \ldots$

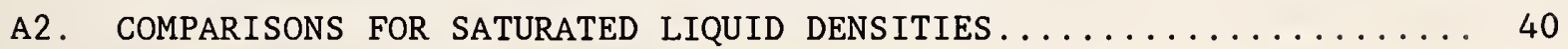

A3. COMPARISONS FOR SATURATED VAPOR DENSITIES ............. 52

A4. COMPARISONS OF EXPERIMENTAL AND CALCULATED PVT DATA......... 58

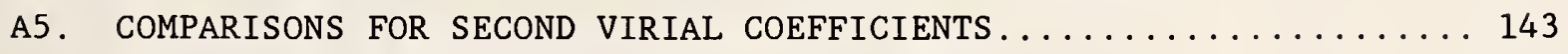

A6. COMPARISONS FOR ISOCHORIC SPECIFIC HEAT CAPACITY.......... 145

A7. COMPARISONS FOR ISOBARIC SPECIFIC HEAT CAPACITY........... 156

A8. COMPARISONS FOR SPECIFIC HEAT CAPACITY OF THE SATURATED LIQUID. . 170

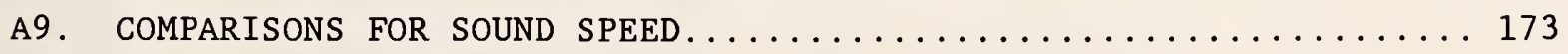

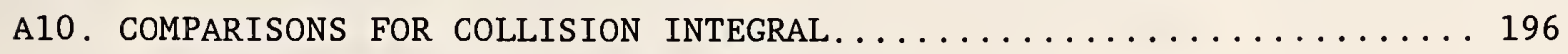

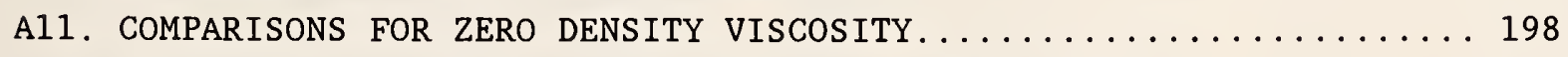

A12. COMPARISONS FOR ZERO DENSITY THERMAL CONDUCTIVITY.......... 204

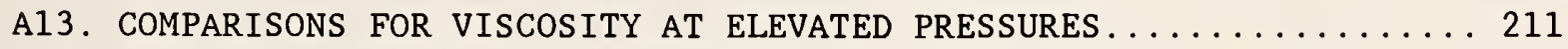

A14. COMPARISONS FOR THERMAL CONDUCTIVITY AT ELEVATED PRESSURES..... 229 
We make several prefatory notes to the fourteen tables in this Appendix. Additional specific notes can be found at the beginning of most of the tables. In several instances, only first authors are given; complete citations can be found in the numbered references. Deviations are given on a percentage basis according to $\mathrm{Dev}=100(\mathrm{Cal}-\mathrm{Exp}) /$ Exp. The weights (Wt) refer to weighting of individual data points in the least squares algorithms for the determination of the coefficients of the correlating equations as discussed in detail in Ref.[1]. The summary statistics given for each reference and for the cumulative experimental data are defined as follows,

$$
\begin{gathered}
\mathrm{AAD}=\frac{1}{N} \sum_{i}\left|\mathrm{Cal}_{i}-\operatorname{Exp}_{i}\right| \\
\text { BIAS }=\frac{1}{N} \sum_{i}\left(\mathrm{Cal}_{i}-\operatorname{Exp}_{i}\right) \\
\mathrm{RMS}=\left[\frac{1}{N} \sum_{i}\left(\mathrm{Cal}_{i}-\operatorname{Exp}_{i}\right)^{2}-\mathrm{BIAS}^{2}\right]^{1 / 2} .
\end{gathered}
$$

The quantity $\mathrm{N}$ above represents the number of points in the data set, and the summation over the index $i$ is over all $\mathrm{N}$ points. These statistics give dimensioned quantities; the analogously defined dimensionless statistics are based on percentage deviations. Thus, AAD -8, BIAS -8 , and RMS -8 are defined as above, but with the quantity $\left(\mathrm{Cal}_{\mathbf{i}}-\operatorname{Exp}_{\mathbf{i}}\right)$ replaced by $100\left(\mathrm{Cal}_{\mathbf{i}}-\operatorname{Exp}_{\mathbf{i}}\right)$. Some of the entries in the tables have digits beyond the range of significance, including trailing zeroes; these serve only to maintain uniform appearance of the columns and the quantities can be truncated according to the error estimates in the original experimental publications, the discussion in Ref.[1], or the uncertainties quoted in Sec. 4 above. 
TABLE A1

COMPARISONS FOR SATURATED VAPOR PRESSURES

Notes for Table Al. The numeral 1 associated with quantities refers to comparisons with eq (1); the numeral 2 refers to comparisons with the SWEOS. Similarly, Wt1 refers to weighting for the development of eq (1), and Wt2 refers to weighting of the Gibbs condition for the development of the SWEOS. For the points generated from eq (1), in the last part of the table, statistics relative to eq (1) have no meaning; these points are therefore excluded from the totals.

Data from Kleinrahm and Wagner [6]

\begin{tabular}{|c|c|c|c|c|c|c|c|}
\hline $\mathrm{T}$ & $P_{\sigma}, \exp$ & $P_{\sigma}$, eq $(1)$ & Devl & $\mathrm{P}_{\sigma}$, SWEOS & Dev2 & Wt1 & Wt2 \\
\hline K & $\mathrm{MPa}$ & $\mathrm{MPa}$ & 8 & $\mathrm{MPa}$ & 8 & & \\
\hline 90.685 & 0.117 00E-01 & $0.11693 \mathrm{E}-01$ & -0.060 & 0.116 93E-01 & -0.061 & 1.0 & 0.0 \\
\hline 91.000 & $0.12170 \mathrm{E}-01$ & $0.12175 \mathrm{E}-01$ & 0.043 & $0.12175 \mathrm{E}-01$ & 0.040 & 1.0 & 0.0 \\
\hline 95.000 & 0.198 30E-01 & $0.198 \quad 34 \mathrm{E}-01$ & 0.019 & 0.198 32E-01 & 0.011 & 1.0 & 0 \\
\hline 100.000 & 0.344 00E-01 & $0.34408 \mathrm{E}-01$ & 0.022 & 0.344 08E-01 & 0.024 & 1.0 & 0.0 \\
\hline 105.000 & 0.564 30E-01 & $0.56426 \mathrm{E}-01$ & -0.007 & $0.56433 \mathrm{E}-01$ & 0.006 & 1.0 & 0.0 \\
\hline 10.000 & 0.882 20E-01 & 0.882 03E-01 & -0.019 & 0.882 19E-01 & -0.001 & 1.0 & 0.0 \\
\hline 111.631 & $0.10110 \mathrm{E}+00$ & $0.10111 \mathrm{E}+00$ & 0.006 & $0.101 \quad 13 E+00$ & 0.025 & 1.0 & 0.0 \\
\hline 115.000 & $0.13234 \mathrm{E}+00$ & $0.13232 E+00$ & -0.015 & $0.13234 \mathrm{E}+00$ & 0.003 & 1.0 & 0.0 \\
\hline 120.000 & $0.19156 \mathrm{E}+00$ & $0.19158 \mathrm{E}+00$ & 0.010 & $0.19161 \mathrm{E}+00$ & 0.024 & 1.0 & 0.0 \\
\hline 125.000 & $0.26898 \mathrm{E}+00$ & $0.26896 \mathrm{E}+00$ & -0.006 & $0.26899 \mathrm{E}+00$ & 0.002 & 1.0 & 0.0 \\
\hline 130.000 & $0.36762 E+00$ & $0.36760 \mathrm{E}+00$ & -0.006 & $0.367 \quad 60 \mathrm{E}+00$ & -0.005 & 1.0 & 0.0 \\
\hline 135.000 & $0.49070 \mathrm{E}+00$ & $0.49071 \mathrm{E}+00$ & 0.003 & $0.49068 \mathrm{E}+00$ & -0.003 & 1.0 & 0.0 \\
\hline 140.000 & $0.64162 \mathrm{E}+00$ & $0.64165 \mathrm{E}+00$ & 0.004 & $0.64156 \mathrm{E}+00$ & -0.010 & 1.0 & 0.0 \\
\hline 145.000 & $0.82373 E+00$ & $0.82379 \mathrm{E}+00$ & 0.008 & $0.82369 \mathrm{E}+00$ & -0.005 & 1.0 & 0.0 \\
\hline 150.000 & $0.10406 \mathrm{E}+01$ & $0.10406 \mathrm{E}+01$ & 0.007 & $0.10405 E+01$ & -0.007 & 1.0 & 0.0 \\
\hline 155.000 & $0.12957 \mathrm{E}+01$ & $0.12958 \mathrm{E}+01$ & 0.008 & $0.129 \quad 56 \mathrm{E}+01$ & -0.005 & 1.0 & 0.0 \\
\hline 160.000 & $0.15929 \mathrm{E}+01$ & $0.15930 \mathrm{E}+01$ & 0.004 & $0.159 \quad 28 \mathrm{E}+01$ & -0.007 & 1.0 & 0.0 \\
\hline 165.000 & $0.19362 E+01$ & $0.19361 E+01$ & -0.007 & $0.19359 E+01$ & -0.014 & 1.0 & 0.0 \\
\hline 170.000 & $0.23296 \mathrm{E}+01$ & $0.23294 \mathrm{E}+01$ & -0.011 & $0.23293 E+01$ & -0.015 & 1.0 & 0.0 \\
\hline 175.000 & $0.27779 \mathrm{E}+01$ & $0.277 \quad 76 \mathrm{E}+01$ & -0.010 & $0.27776 \mathrm{E}+01$ & -0.011 & 1.0 & 0.0 \\
\hline 180.000 & $0.32868 \mathrm{E}+01$ & $0.328 \quad 65 \mathrm{E}+01$ & -0.009 & $0.328 \quad 63 E+01$ & -0.017 & 1.0 & 0.0 \\
\hline 182.000 & $0.35089 \mathrm{E}+01$ & $0.35087 \mathrm{E}+01$ & -0.005 & $0.35084 E+01$ & -0.014 & 1.0 & 0.0 \\
\hline 184.000 & $0.37423 \mathrm{E}+01$ & $0.37423 E+01$ & 0.000 & $0.374 \quad 19 \mathrm{E}+01$ & -0.011 & 1.0 & 0.0 \\
\hline 186.000 & $0.39878 \mathrm{E}+01$ & $0.398 \quad 81 E+01$ & 0.007 & $0.398 \quad 76 \mathrm{E}+01$ & -0.006 & 1.0 & 0.0 \\
\hline 187.000 & $0.41154 \mathrm{E}+01$ & $0.41159 \mathrm{E}+01$ & 0.010 & $0.41153 \mathrm{E}+01$ & -0.003 & 1.0 & 0.0 \\
\hline
\end{tabular}


Data from Kleinrahm and Wagner [6] (continued)

\begin{tabular}{|c|c|c|c|c|c|c|c|c|}
\hline $\mathrm{T}$ & $\mathrm{P}_{\sigma}, \exp$ & $\mathrm{P}_{\sigma}$, eq (1) & Devl & $\mathrm{P}_{\sigma}$ & SWEOS & Dev2 & Wt1 & Wt2 \\
\hline K & $\mathrm{MPa}$ & $\mathrm{MPa}$ & 8 & & $\mathrm{~Pa}$ & 8 & & \\
\hline 188.000 & $0.42466 \mathrm{E}+01$ & $0.42471 E+01$ & 0.013 & 0.424 & $65 E+01$ & 0.000 & 1.0 & 0.0 \\
\hline 189.000 & $0.438 \quad 14 E+01$ & $0.438 \quad 20 \mathrm{E}+01$ & 0.014 & 0.438 & $15 E+01$ & 0.003 & 1.0 & 0.0 \\
\hline 189.500 & $0.44503 E+01$ & $0.44509 \mathrm{E}+01$ & 0.013 & 0.445 & $05 E+01$ & 0.004 & 1.0 & 0.0 \\
\hline 189.800 & $0.44922 \mathrm{E}+01$ & $0.44927 \mathrm{E}+01$ & 0.011 & 0.449 & $24 \mathrm{E}+01$ & 0.004 & 1.0 & 0.0 \\
\hline 190.000 & $0.45204 \mathrm{E}+01$ & $0.45208 \mathrm{E}+01$ & 0.010 & 0.452 & $05 E+01$ & 0.003 & 1.0 & 0.0 \\
\hline 190.100 & $0.45345 \mathrm{E}+01$ & $0.45349 \mathrm{E}+01$ & 0.009 & 0.453 & $47 E+01$ & 0.003 & 1.0 & 0.0 \\
\hline 190.200 & $0.45488 \mathrm{E}+01$ & $0.45491 \mathrm{E}+01$ & 0.007 & 0.454 & $89 E+01$ & 0.003 & 1.0 & 0.0 \\
\hline 190.300 & $0.45630 \mathrm{E}+01$ & $0.45633 E+01$ & 0.006 & 0.456 & $31 E+01$ & 0.002 & 1.0 & 0.0 \\
\hline 190.400 & $0.457 \quad 74 \mathrm{E}+01$ & $0.457 \quad 76 \mathrm{E}+01$ & 0.004 & 0.457 & $75 E+01$ & 0.002 & 1.0 & 0.0 \\
\hline 190.450 & $0.458 \quad 46 \mathrm{E}+01$ & $0.458 \quad 47 \mathrm{E}+01$ & 0.003 & 0.458 & $46 E+01$ & 0.001 & 1.0 & 0.0 \\
\hline 190.500 & $0.459 \quad 18 \mathrm{E}+01$ & $0.459 \quad 19 \mathrm{E}+01$ & 0.002 & 0.459 & $18 \mathrm{E}+01$ & 0.001 & 1.0 & 0.0 \\
\hline 190.530 & $0.45961 \mathrm{E}+01$ & $0.45962 \mathrm{E}+01$ & 0.001 & 0.459 & $62 E+01$ & 0.000 & 1.0 & 0.0 \\
\hline 190.551 & $0.45992 \mathrm{E}+01$ & $0.45992 \mathrm{E}+01$ & 0.000 & 0.459 & $92 E+01$ & 0.000 & 1.0 & 0.0 \\
\hline
\end{tabular}

Number of Points (Ref.6) 38

$\begin{array}{lllllll}\text { AAD1- } 8 & 0.011, & \text { BIAS1-8 } & 0.002, & \text { RMS1-8 } & 0.015 & \\ \text { AAD1 } & 0.171, & \text { BIAS1 } & 0.105, & \text { RMS1 } & 0.233 & \mathrm{kPa} \\ \text { AAD2-8 } & 0.009, & \text { BIAS2-8 } & -0.001, & \text { RMS2-8 } & 0.015 & \\ \text { AAD2 } & 0.118, & \text { BIAS2 } & -0.050, & \text { RMS2 } & 0.177 & \mathrm{kPa}\end{array}$

Data from Prydz and Goodwin [18]

\begin{tabular}{|c|c|c|c|c|c|c|c|}
\hline $\mathrm{T}$ & $\mathrm{P}_{\sigma}, \exp$ & $\mathrm{P}_{\sigma}$, eq $(1)$ & Dev1 & $\mathrm{P}_{\sigma}$, SWEOS & Dev2 & Wt1 & Wt2 \\
\hline $\mathrm{K}$ & $\mathrm{MPa}$ & $\mathrm{MPa}$ & 8 & $\mathrm{MPa}$ & 8 & & \\
\hline 91.000 & 0.122 30E-01 & $0.12175 \mathrm{E}-01$ & -0.448 & $0.12175 E-01$ & -0.451 & 0.0 & 0.0 \\
\hline 92.000 & $0.13880 \mathrm{E}-01$ & $0.138 \quad 14 \mathrm{E}-01$ & -0.473 & $0.138 \quad 14 E-01$ & -0.477 & 0.0 & 0.0 \\
\hline 93.000 & 0.157 00E-01 & $0.156 \quad 28 \mathrm{E}-01$ & -0.458 & 0.156 27E-01 & -0.465 & 0.0 & 0.0 \\
\hline 94.000 & 0.177 00E-01 & $0.176 \quad 30 \mathrm{E}-01$ & -0.396 & 0.176 29E-01 & -0.404 & 0.0 & 0.0 \\
\hline 95.000 & 0.199 10E-01 & $0.198 \quad 34 \mathrm{E}-01$ & -0.383 & 0.198 32E-01 & -0.391 & 0.0 & 0.0 \\
\hline
\end{tabular}


Data from Prydz and Goodwin [18] (continued)

\begin{tabular}{|c|c|c|c|c|c|c|c|c|c|}
\hline $\mathrm{T}$ & $\mathrm{P}_{\sigma}, \exp$ & $\mathrm{P}_{\sigma}$ & eq (1) & Dev1 & $\mathrm{P}_{\sigma}$ & SWEOS & Dev2 & Wt1 & Wt2 \\
\hline $\mathrm{K}$ & $\mathrm{MPa}$ & & $\mathrm{MPa}$ & 8 & M & $\mathrm{Pa}$ & 8 & & \\
\hline 96.000 & 0.223 40E-01 & 0.222 & $54 \mathrm{E}-01$ & -0.385 & 0.222 & $52 \mathrm{E}-01$ & -0.392 & 0.0 & 0.0 \\
\hline 97.000 & 0.250 O0E-01 & 0.249 & 06E-01 & -0.377 & 0.249 & $04 \mathrm{E}-01$ & -0.382 & 0.0 & 0.0 \\
\hline 98.000 & 0.279 O0E-01 & 0.278 & $04 \mathrm{E}-01$ & -0.343 & 0.278 & $04 \mathrm{E}-01$ & -0.346 & 0.0 & 0.0 \\
\hline 99.000 & $0.31070 \mathrm{E}-01$ & 0.309 & $66 \mathrm{E}-01$ & -0.334 & 0.309 & $66 \mathrm{E}-01$ & -0.335 & 0.0 & 0.0 \\
\hline 100.000 & 0.345 10E-01 & 0.344 & $08 \mathrm{E}-01$ & -0.297 & 0.344 & $08 \mathrm{E}-01$ & -0.295 & 0.0 & 0.0 \\
\hline 101.000 & $0.38260 \mathrm{E}-01$ & 0.381 & $46 \mathrm{E}-01$ & -0.298 & 0.381 & $47 \mathrm{E}-01$ & -0.294 & 0.0 & 0.0 \\
\hline 102.000 & 0.423 20E-01 & 0.421 & 99E-01 & -0.287 & 0.422 & 01E-01 & -0.280 & 0.0 & 0.0 \\
\hline 103.000 & 0.467 10E-01 & 0.465 & $84 \mathrm{E}-01$ & -0.270 & 0.465 & $88 \mathrm{E}-01$ & -0.262 & 0.0 & 0.0 \\
\hline 104.000 & 0.514 40E-01 & 0.513 & $20 E-01$ & -0.233 & 0.513 & $25 \mathrm{E}-01$ & -0.223 & 0.0 & 0.0 \\
\hline 105.000 & $0.56560 \mathrm{E}-01$ & 0.564 & $26 \mathrm{E}-01$ & -0.237 & 0.564 & $33 \mathrm{E}-01$ & -0.224 & 0.0 & 0.0 \\
\hline 106.000 & $0.62060 \mathrm{E}-01$ & 0.619 & $22 E-01$ & -0.223 & 0.619 & $31 E-01$ & -0.208 & 0.0 & 0.0 \\
\hline 107.000 & 0.679 90E-01 & 0.678 & $27 E-01$ & -0.240 & 0.678 & $38 \mathrm{E}-01$ & -0.224 & 0.0 & 0.0 \\
\hline 108.000 & 0.743 20E-01 & 0.741 & $62 E-01$ & -0.213 & 0.741 & $74 \mathrm{E}-01$ & -0.196 & 0.0 & 0.0 \\
\hline 109.000 & 0.811 30E-01 & 0.809 & $47 E-01$ & -0.226 & 0.809 & $61 E-01$ & -0.208 & 0.0 & 0.0 \\
\hline 110.000 & $0.88390 \mathrm{E}-01$ & 0.882 & $03 E-01$ & -0.211 & 0.882 & $19 \mathrm{E}-01$ & -0.193 & 0.0 & 0.0 \\
\hline 111.000 & 961 50E-01 & 0.959 & $52 E-01$ & -0.205 & 0.959 & $71 \mathrm{E}-01$ & -0.187 & 0.0 & 0.0 \\
\hline 111.000 & 0.961 60E-01 & 0.959 & 52E-01 & -0.216 & 0.959 & $71 E-01$ & -0.197 & 0.0 & 0.0 \\
\hline 112.000 & $0.104 \quad 48 \mathrm{E}+00$ & 0.104 & $22 \mathrm{E}+00$ & -0.252 & 0.104 & $24 \mathrm{E}+00$ & -0.233 & 0.0 & 0.0 \\
\hline 113.000 & $0.11328 \mathrm{E}+00$ & 0.113 & $02 E+00$ & -0.232 & 0.113 & $04 \mathrm{E}+00$ & -0.213 & 0.0 & 0.0 \\
\hline 114.000 & $0.12265 \mathrm{E}+00$ & 0.122 & $38 \mathrm{E}+00$ & -0.222 & 0.122 & $40 \mathrm{E}+00$ & -0.203 & 0.0 & 0.0 \\
\hline 115.000 & $0.13255 \mathrm{E}+00$ & 0.132 & $32 E+00$ & -0.174 & 0.132 & $34 E+00$ & -0.155 & 0.0 & 0.0 \\
\hline 115.000 & $0.13262 \mathrm{E}+00$ & 0.132 & $32 E+00$ & -0.227 & 0.132 & $34 \mathrm{E}+00$ & -0.208 & 0.0 & 0.0 \\
\hline 116.000 & $0.143 \quad 11 \mathrm{E}+00$ & 0.142 & $87 E+00$ & -0.170 & 0.142 & $89 E+00$ & -0.152 & 0.0 & 0.0 \\
\hline 116.000 & $0.143 \quad 17 \mathrm{E}+00$ & 0.142 & $87 \mathrm{E}+00$ & -0.212 & 0.142 & $89 \mathrm{E}+00$ & -0.194 & 0.0 & 0.0 \\
\hline 117.000 & $0.154 \quad 34 \mathrm{E}+00$ & 0.154 & $04 E+00$ & -0.193 & 0.154 & $07 E+00$ & .0 .175 & 0.0 & 0.0 \\
\hline 118.000 & $0.166 \quad 16 \mathrm{E}+00$ & 0.165 & $87 E+00$ & -0.174 & 0.165 & $90 \mathrm{E}+00$ & -0.158 & 0.0 & 0.0 \\
\hline 119.000 & $0.17871 \mathrm{E}+00$ & 0.178 & $37 E+00$ & -0.188 & 0.178 & $40 E+00$ & -0.172 & 0.0 & 0.0 \\
\hline 120.000 & $0.19191 \mathrm{E}+00$ & 0.191 & $58 \mathrm{E}+00$ & -0.173 & 0.191 & $61 E+00$ & -0.158 & 0.0 & 0.0 \\
\hline 121.000 & $0.20581 \mathrm{E}+00$ & 0.205 & $51 E+00$ & -0.147 & 0.205 & $54 \mathrm{E}+00$ & -0.134 & 0.0 & 0.0 \\
\hline 122.000 & $0.220 \quad 52 \mathrm{E}+00$ & 0.220 & $18 \mathrm{E}+00$ & -0.152 & 0.220 & $21 \mathrm{E}+00$ & -0.140 & 0.0 & 0.0 \\
\hline 123.000 & $0.23598 \mathrm{E}+00$ & 0.235 & $64 E+00$ & -0.145 & 0.235 & $66 E+00$ & -0.134 & 0.0 & 0.0 \\
\hline 124.000 & $0.25228 \mathrm{E}+00$ & 0.251 & $89 E+00$ & -0.155 & 0.251 & $91 E+00$ & -0.146 & 0.0 & 0.0 \\
\hline 125.000 & $0.26933 \mathrm{E}+00$ & 0.268 & $96 \mathrm{E}+00$ & -0.136 & 0.268 & $99 E+00$ & -0.128 & 0.0 & 0.0 \\
\hline 126.000 & $0.28731 \mathrm{E}+00$ & 0.286 & $89 E+00$ & -0.147 & 0.286 & $91 E+00$ & -0.140 & 0.0 & 0.0 \\
\hline 127.000 & $0.30608 \mathrm{E}+00$ & 0.305 & $69 E+00$ & -0.128 & 0.305 & $70 E+00$ & -0.123 & 0.0 & 0.0 \\
\hline
\end{tabular}


Data from Prydz and Goodwin [18] (continued)

\begin{tabular}{|c|c|c|c|c|c|c|c|c|c|}
\hline $\mathrm{T}$ & $\mathrm{P}_{\sigma}, \exp$ & $\mathrm{P}_{\sigma}$ & eq (1) & Devl & $\mathrm{P}_{\sigma}$ & SWEOS & Dev2 & Wt1 & Wt2 \\
\hline K & $\mathrm{MPa}$ & & $\mathrm{MPa}$ & 8 & & $\mathrm{~Pa}$ & 8 & & \\
\hline 28.000 & $0.32580 \mathrm{E}+00$ & 0.325 & $39 E+00$ & -0.126 & 0.325 & $40 E+00$ & -0.122 & 0.0 & 0.0 \\
\hline 129.000 & $0.346 \quad 44 \mathrm{E}+00$ & 0.346 & $02 \mathrm{E}+00$ & -0.122 & 0.346 & $02 E+00$ & -0.120 & 0.0 & 0.0 \\
\hline 130.000 & $0.368 \quad 02 E+00$ & 0.367 & $60 \mathrm{E}+00$ & -0.115 & 0.367 & $60 E+00$ & -0.114 & 0.0 & 0.0 \\
\hline 131.000 & $0.39061 \mathrm{E}+00$ & 0.390 & $16 \mathrm{E}+00$ & -0.116 & 0.390 & $16 \mathrm{E}+00$ & -0.117 & 0.0 & 0.0 \\
\hline 132.000 & $0.41418 \mathrm{E}+00$ & 0.413 & $72 \mathrm{E}+00$ & -0.111 & 0.413 & $71 E+00$ & -0.113 & 0.0 & 0.0 \\
\hline 33.000 & $0.43880 \mathrm{E}+00$ & .438 & $32 E+00$ & -0.110 & 0.438 & $30 E+00$ & -0.113 & 0.0 & 0.0 \\
\hline 134.000 & $0.464 \quad 47 \mathrm{E}+00$ & 0.463 & $97 \mathrm{E}+00$ & -0.107 & 0.463 & $95 E+00$ & -0.111 & 0.0 & 0.0 \\
\hline 135.000 & $0.49126 \mathrm{E}+00$ & 0.490 & $71 E+00$ & -0.111 & 0.490 & $68 E+00$ & -0.117 & 0.0 & 0.0 \\
\hline 136.000 & $0.51908 \mathrm{E}+00$ & 0.518 & $57 \mathrm{E}+00$ & -0.099 & 0.518 & $53 E+00$ & -0.106 & 0.0 & 0.0 \\
\hline 137.000 & $0.548 \quad 10 \mathrm{E}+00$ & 0.547 & $56 \mathrm{E}+00$ & -0.098 & 0.547 & $51 E+00$ & -0.108 & 0.0 & 0.0 \\
\hline 38.000 & $0.578 \quad 34 \mathrm{E}+00$ & 0.577 & $72 E+00$ & -0.107 & 0.577 & $66 \mathrm{E}+00$ & -0.118 & 0.0 & .0 \\
\hline 139.000 & $0.60964 \mathrm{E}+00$ & 0.609 & $07 \mathrm{E}+00$ & -0.093 & 0.609 & $00 E+00$ & -0.105 & 0.0 & 0.0 \\
\hline 140.000 & $0.64219 \mathrm{E}+00$ & 0.641 & $65 \mathrm{E}+00$ & -0.085 & 0.641 & $56 \mathrm{E}+00$ & -0.099 & 0.0 & 0.0 \\
\hline 141.000 & $0.67606 \mathrm{E}+00$ & 0.675 & $47 E+00$ & -0.088 & 0.675 & $36 E+00$ & -0.103 & 0.0 & 0.0 \\
\hline 142.000 & $0.71116 \mathrm{E}+00$ & 0.710 & $56 \mathrm{E}+00$ & -0.084 & 0.710 & $44 E+00$ & -0.101 & 0.0 & 0.0 \\
\hline 143.000 & $.74758 \mathrm{E}+00$ & .746 & $97 E+00$ & -0.082 & 0.746 & $82 E+00$ & -0.101 & 0.0 & 0.0 \\
\hline 144.000 & $0.785 \quad 25 \mathrm{E}+00$ & 0.784 & $70 E+00$ & -0.070 & 0.784 & $60 \mathrm{E}+00$ & -0.082 & 0.0 & 0.0 \\
\hline 145.000 & $0.82439 \mathrm{E}+00$ & 0.823 & $79 E+00$ & -0.072 & 0.823 & $69 E+00$ & -0.085 & 0.0 & 0.0 \\
\hline 146.000 & $0.86491 \mathrm{E}+00$ & 0.864 & $27 E+00$ & -0.073 & 0.864 & $16 \mathrm{E}+00$ & -0.087 & 0.0 & 0.0 \\
\hline 147.000 & $0.90679 \mathrm{E}+00$ & 0.906 & $17 \mathrm{E}+00$ & -0.068 & 0.906 & $05 E+00$ & -0.082 & 0.0 & 0.0 \\
\hline 148.000 & $0.95021 \mathrm{E}+00$ & 0.949 & $51 E+00$ & -0.073 & 0.949 & $38 E+00$ & -0.087 & 0.0 & 0.0 \\
\hline 149.000 & $0.99501 \mathrm{E}+00$ & 0.994 & $33 E+00$ & -0.068 & 0.994 & $19 E+00$ & -0.082 & 0.0 & 0.0 \\
\hline 150.000 & $0.104 \quad 14 \mathrm{E}+01$ & 0.104 & $06 \mathrm{E}+01$ & -0.074 & 0.104 & $05 E+01$ & -0.088 & 0.0 & 0.0 \\
\hline 151.000 & $0.10892 \mathrm{E}+01$ & 0.108 & $85 E+01$ & -0.065 & 0.108 & $83 E+01$ & -0.080 & 0.0 & 0.0 \\
\hline 152.000 & $0.11386 \mathrm{E}+01$ & 0.113 & $79 E+01$ & -0.064 & 0.113 & $77 E+01$ & -0.078 & 0.0 & 0.0 \\
\hline 153.000 & $0.11898 \mathrm{E}+01$ & 0.118 & $89 E+01$ & -0.072 & 0.118 & $87 E+01$ & -0.086 & 0.0 & 0.0 \\
\hline 154.000 & $0.12424 \mathrm{E}+01$ & 0.124 & $15 E+01$ & -0.068 & 0.124 & $14 \mathrm{E}+01$ & -0.082 & 0.0 & 0.0 \\
\hline 55.000 & $0.12967 \mathrm{E}+01$ & 0.129 & $58 E+01$ & -0.068 & 0.129 & $56 \mathrm{E}+01$ & -0.082 & 0.0 & 0.0 \\
\hline 156.000 & $0.13526 \mathrm{E}+01$ & 0.135 & $17 \mathrm{E}+01$ & -0.064 & 0.135 & $16 \mathrm{E}+01$ & -0.077 & 0.0 & 0.0 \\
\hline 157.000 & $0.14104 E+01$ & 0.140 & $94 \mathrm{E}+01$ & -0.068 & 0.140 & $92 \mathrm{E}+01$ & -0.080 & 0.0 & 0.0 \\
\hline 158.000 & $0.14698 \mathrm{E}+01$ & 0.146 & $88 \mathrm{E}+01$ & -0.070 & 0.146 & $86 E+01$ & -0.082 & 0.0 & 0.0 \\
\hline 159.000 & $0.15310 \mathrm{E}+01$ & 0.153 & $00 E+01$ & -0.070 & 0.152 & $98 \mathrm{E}+01$ & -0.081 & 0.0 & 0.0 \\
\hline 160.000 & $0.159 \quad 41 E+01$ & 0.159 & $30 E+01$ & -0.069 & 0.159 & $28 E+01$ & -0.080 & 0.0 & 0.0 \\
\hline 161.000 & $0.16588 \mathrm{E}+01$ & 0.165 & $78 E+01$ & -0.064 & 0.165 & $76 \mathrm{E}+01$ & -0.074 & 0.0 & 0.0 \\
\hline 162.000 & $0.17257 \mathrm{E}+01$ & 0.172 & $45 E+01$ & -0.073 & 0.172 & $43 E+01$ & -0.083 & 0.0 & 0.0 \\
\hline
\end{tabular}


Data from Prydz and Goodwin [18] (continued)

\begin{tabular}{|c|c|c|c|c|c|c|c|}
\hline $\mathrm{T}$ & $\mathrm{P}_{\sigma}, \exp$ & $\mathrm{P}_{\sigma}$, eq (1) & Devl & $\mathrm{P}_{\sigma}$, SWEOS & Dev2 & Wt1 & Wt2 \\
\hline K & $\mathrm{MPa}$ & $\mathrm{MPa}$ & 8 & $\mathrm{MPa}$ & 8 & & \\
\hline 163.000 & $0.179 \quad 42 \mathrm{E}+01$ & $0.17930 \mathrm{E}+01$ & -0.063 & $0.17929 \mathrm{E}+01$ & -0.072 & 0.0 & 0.0 \\
\hline 164.000 & $0.186 \quad 49 \mathrm{E}+01$ & $0.18636 \mathrm{E}+01$ & -0.069 & $0.18634 \mathrm{E}+01$ & -0.077 & 0.0 & 0.0 \\
\hline 165.000 & $0.19373 \mathrm{E}+01$ & $0.19361 \mathrm{E}+01$ & -0.062 & $0.19359 \mathrm{E}+01$ & -0.069 & 0.0 & 0.0 \\
\hline 166.000 & $0.20120 \mathrm{E}+01$ & $0.20106 \mathrm{E}+01$ & -0.070 & $0.20104 \mathrm{E}+01$ & -0.076 & 0.0 & 0.0 \\
\hline 167.000 & $0.208 \quad 83 \mathrm{E}+01$ & $0.20871 \mathrm{E}+01$ & -0.057 & $0.20870 \mathrm{E}+01$ & -0.062 & 0.0 & 0.0 \\
\hline 168.000 & $0.21674 \mathrm{E}+01$ & $0.216 \quad 57 \mathrm{E}+01$ & -0.079 & $0.21656 \mathrm{E}+01$ & -0.083 & 0.0 & 0.0 \\
\hline 169.000 & $0.22481 \mathrm{E}+01$ & $0.22465 \mathrm{E}+01$ & -0.072 & $0.22464 \mathrm{E}+01$ & -0.075 & 0.0 & 0.0 \\
\hline 170.000 & $0.23310 \mathrm{E}+01$ & $0.23294 \mathrm{E}+01$ & -0.071 & $0.23293 \mathrm{E}+01$ & -0.074 & 0.0 & 0.0 \\
\hline 171.000 & $0.24160 \mathrm{E}+01$ & $0.241 \quad 45 \mathrm{E}+01$ & -0.064 & $0.241 \quad 44 \mathrm{E}+01$ & -0.066 & 0.0 & 0.0 \\
\hline 172.000 & $0.250 \quad 33 E+01$ & $0.25018 \mathrm{E}+01$ & -0.059 & $0.250 \quad 17 \mathrm{E}+01$ & -0.061 & 0.0 & 0.0 \\
\hline 73.000 & $0.25930 \mathrm{E}+01$ & $0.25914 \mathrm{E}+01$ & -0.060 & $0.25913 E+01$ & -0.062 & 0.0 & 0.0 \\
\hline 174.000 & $0.26849 \mathrm{E}+01$ & $0.268 \quad 33 E+01$ & -0.058 & $0.268 \quad 33 \mathrm{E}+01$ & -0.060 & 0.0 & 0.0 \\
\hline 175.000 & $0.27791 \mathrm{E}+01$ & $0.277 \quad 76 \mathrm{E}+01$ & -0.053 & $0.27776 \mathrm{E}+01$ & -0.054 & 0.0 & 0.0 \\
\hline 176.000 & $0.287 \quad 61 \mathrm{E}+01$ & $0.287 \quad 44 \mathrm{E}+01$ & -0.060 & $0.287 \quad 43 \mathrm{E}+01$ & -0.062 & 0.0 & 0.0 \\
\hline 177.000 & $0.29750 \mathrm{E}+01$ & $0.29736 \mathrm{E}+01$ & -0.049 & $0.29735 \mathrm{E}+01$ & -0.051 & 0.0 & 0.0 \\
\hline 178.000 & $0.30770 \mathrm{E}+01$ & $0.30753 \mathrm{E}+01$ & -0.054 & $0.307 \quad 52 E+01$ & -0.057 & 0.0 & 0.0 \\
\hline 179.000 & $0.318 \quad 09 \mathrm{E}+01$ & $0.31796 \mathrm{E}+01$ & -0.041 & $0.31795 \mathrm{E}+01$ & -0.044 & 0.0 & 0.0 \\
\hline 180.000 & $0.328 \quad 82 \mathrm{E}+01$ & $0.32865 \mathrm{E}+01$ & -0.052 & $0.32863 \mathrm{E}+01$ & -0.059 & 0.0 & 0.0 \\
\hline 180.000 & $0.32884 \mathrm{E}+01$ & $0.32865 \mathrm{E}+01$ & -0.058 & $0.32863 E+01$ & -0.065 & 0.0 & 0.0 \\
\hline 181.000 & $0.33977 \mathrm{E}+01$ & $0.33962 \mathrm{E}+01$ & -0.042 & $0.33959 \mathrm{E}+01$ & -0.051 & 0.0 & 0.0 \\
\hline 182.000 & $0.35098 \mathrm{E}+01$ & $0.350 \quad 87 \mathrm{E}+01$ & -0.032 & $0.35084 \mathrm{E}+01$ & -0.041 & 0.0 & 0.0 \\
\hline 182.000 & $0.35100 \mathrm{E}+01$ & $0.350 \quad 87 \mathrm{E}+01$ & -0.038 & $0.35084 E+01$ & -0.047 & 0.0 & 0.0 \\
\hline 183.000 & $0.36256 \mathrm{E}+01$ & $0.362 \quad 40 \mathrm{E}+01$ & -0.045 & $0.362 \quad 36 \mathrm{E}+01$ & -0.055 & 0.0 & 0.0 \\
\hline 184.000 & $0.37435 \mathrm{E}+01$ & $0.374 \quad 23 E+01$ & -0.032 & $0.374 \quad 19 E+01$ & -0.044 & 0.0 & 0.0 \\
\hline 185.000 & $0.386 \quad 47 \mathrm{E}+01$ & $0.38636 \mathrm{E}+01$ & -0.029 & $0.38631 E+01$ & -0.041 & 0.0 & 0.0 \\
\hline 186.000 & $0.39889 \mathrm{E}+01$ & $0.39881 \mathrm{E}+01$ & -0.020 & $0.39876 \mathrm{E}+01$ & -0.033 & 0.0 & 0.0 \\
\hline 187.000 & $0.41166 \mathrm{E}+01$ & $0.41159 \mathrm{E}+01$ & -0.018 & $0.41153 E+01$ & -0.031 & 0.0 & 0.0 \\
\hline 188.000 & $0.42474 \mathrm{E}+01$ & $0.42471 \mathrm{E}+01$ & -0.008 & $0.42465 \mathrm{E}+01$ & -0.021 & 0.0 & 0.0 \\
\hline 189.000 & $0.438 \quad 24 E+01$ & $0.438 \quad 20 \mathrm{E}+01$ & -0.009 & $0.438 \quad 15 \mathrm{E}+01$ & -0.020 & 0.0 & 0.0 \\
\hline 190.000 & $0.45210 \mathrm{E}+01$ & $0.45208 \mathrm{E}+01$ & -0.003 & $0.45205 E+01$ & -0.010 & 0.0 & 0.0 \\
\hline
\end{tabular}

Number of Points (Ref.18) 105

$\begin{array}{lllllll}\text { AAD1- } 8 & 0.137, & \text { BIAS1-8 } & -0.137, & \text { RMS1-8 } & 0.108 & \\ \text { AAD1 } & 0.679, & \text { BIAS1 } & -0.679, & \text { RMS1 } & 0.514 & \mathrm{kPa} \\ \text { AAD2-8 } & 0.139, & \text { BIAS2-8 } & -0.139, & \text { RMS2-8 } & 0.103 & \\ \text { AAD2 } & 0.771, & \text { BIAS2 } & -0.771, & \text { RMS2 } & 0.587 & \mathrm{kPa}\end{array}$


Data from Haynes [19]

\begin{tabular}{|c|c|c|c|c|c|c|c|}
\hline $\mathrm{T}$ & $\mathrm{P} \sigma, \exp$ & $\mathrm{P} \sigma$, eq (1) & Devl & P $\sigma$, SWEOS & Dev2 & Wtl & Wt2 \\
\hline K & $\mathrm{MPa}$ & $\mathrm{MPa}$ & of & $\mathrm{MPa}$ & 8 & & \\
\hline 95.000 & $0.198 \quad 70 \mathrm{E}-01$ & $0.198 \quad 34 \mathrm{E}-01$ & -0.182 & 0.198 32E-01 & -0.190 & 0.0 & 0 \\
\hline 100.000 & $0.34480 \mathrm{E}-01$ & $0.34408 \mathrm{E}-01$ & -0.210 & $0.34408 \mathrm{E}-01$ & -0.208 & 0.0 & . \\
\hline 105.000 & $0.565 \quad 10 \mathrm{E}-01$ & $0.56426 \mathrm{E}-01$ & -0.148 & $0.56433 E-01$ & -0.136 & 0.0 & . \\
\hline 110.000 & $0.883 \quad 40 \mathrm{E}-01$ & 0.882 03E-01 & -0.155 & $0.882 \quad 19 \mathrm{E}-01$ & -0.137 & 0.0 & 0 . \\
\hline 115.000 & $0.132 \quad 48 \mathrm{E}+00$ & $0.13232 \mathrm{E}+00$ & -0.121 & $0.13234 \mathrm{E}+00$ & -0.103 & 0.0 & 0.0 \\
\hline 120.000 & $0.19167 \mathrm{E}+00$ & $0.19158 \mathrm{E}+00$ & -0.048 & $0.19161 \mathrm{E}+00$ & -0.033 & 0.0 & \\
\hline 125.000 & $0.26908 E+00$ & $0.26896 \mathrm{E}+00$ & -0.044 & $0.26899 E+00$ & -0.035 & 0.0 & \\
\hline 130.000 & $0.36760 E+00$ & $0.36760 \mathrm{E}+00$ & -0.001 & $0.367 \quad 60 \mathrm{E}+00$ & 0.000 & 0.0 & \\
\hline 135.000 & $0.490 \quad 69 \mathrm{E}+00$ & $0.49071 E+00$ & 0.005 & $0.49068 \mathrm{E}+00$ & -0.001 & 0.0 & \\
\hline 140.000 & $0.64155 \mathrm{E}+00$ & $0.64165 \mathrm{E}+00$ & 0.015 & $0.64156 \mathrm{E}+00$ & 0.001 & 0.0 & 0 \\
\hline
\end{tabular}

Number of Points (Ref. 19) 10

$\begin{array}{lllllll}\text { AAD1- } 8 & 0.093, & \text { BIAS1-8 } & -0.089, & \text { RMS1-8 } & 0.080 & \\ \text { AAD1 } & 0.082, & \text { BIAS1 } & -0.058, & \text { RMS1 } & 0.075 & \mathrm{kPa} \\ \text { AAD2-8 } & 0.084, & \text { BIAS2-8 } & -0.084, & \text { RMS2-8 } & 0.077 & \\ \text { AAD2 } & 0.061, & \text { BIAS2 } & -0.060, & \text { RMS2 } & 0.048 & \mathrm{kPa}\end{array}$

Data from eq (1)

\begin{tabular}{|c|c|c|c|c|c|c|c|c|}
\hline $\mathrm{T}$ & $\mathrm{P}_{\sigma}$, exp & $\mathrm{P}_{\sigma}$, eq (1) & Dev1 & $\mathrm{P}_{\sigma}$ & SWEOS & Dev2 & $W t]$ & Wt2 \\
\hline K & $\mathrm{MPa}$ & $\mathrm{MPa}$ & 8 & & $\mathrm{MPa}$ & 8 & & \\
\hline 91.0 & $1.21752 \mathrm{E}-02$ & $1.21752 \mathrm{E}-02$ & - & 1.217 & 49E-02 & -0.003 & - & 46595.385 \\
\hline 96.0 & $2.225 \quad 40 \mathrm{E}-02$ & $2.225 \quad 40 \mathrm{E}-02$ & - & 2.225 & $25 E-02$ & -0.007 & - & 25531.209 \\
\hline 101.0 & $3.81459 \mathrm{E}-02$ & 3.814 59E-02 & - & 3.814 & $75 E-02$ & 0.004 & - & 14913.515 \\
\hline 106.0 & 6.192 18E-02 & $6.19218 \mathrm{E}-02$ & - & 6.193 & 06E-02 & 0.014 & - & 9199.929 \\
\hline 111.0 & 9.595 25E-02 & $9.59525 \mathrm{E}-02$ & - & 9.597 & 06E-02 & 0.019 & - & 5947.908 \\
\hline 116.0 & $1.428 \quad 67 \mathrm{E}-01$ & $1.428 \quad 67 \mathrm{E}-01$ & - & 1.428 & 93E-01 & 0.018 & - & 4004.906 \\
\hline 121.0 & $2.055 \quad 07 \mathrm{E}-01$ & $2.055 \quad 07 E-01$ & - & 2.055 & $35 \mathrm{E}-01$ & 0.013 & - & 2793.881 \\
\hline 126.0 & $2.868 \quad 87 \mathrm{E}-01$ & $2.868 \quad 87 E-01$ & - & 2.869 & 07E-01 & 0.007 & - & 2010.580 \\
\hline 131.0 & $3.90156 \mathrm{E}-01$ & $3.90156 \mathrm{E}-01$ & - & 3.901 & $55 \mathrm{E}-01$ & 0.000 & - & 1487.113 \\
\hline 136.0 & $5.185 \quad 69 \mathrm{E}-01$ & $5.18569 \mathrm{E}-01$ & - & 5.185 & $28 \mathrm{E}-01$ & -0.008 & - & 1127.035 \\
\hline
\end{tabular}


Data from eq (1) (continued)

$\begin{array}{cccccccc}\mathrm{T} & \mathrm{P}_{\sigma}, \exp & \mathrm{P}_{\sigma}, \text { eq }(1) & \text { Dev1 } & \mathrm{P}_{\sigma}, \text { SWEOS } & \text { Dev2 } & \text { Wt1 } & \text { Wt2 } \\ \mathrm{K} & \mathrm{MPa} & \mathrm{MPa} & \& & \mathrm{MPa} & \& & \end{array}$

\begin{tabular}{|c|c|c|c|c|c|c|c|c|c|c|}
\hline 141.0 & 6.754 & $67 E-01$ & 6.754 & $67 \mathrm{E}-01$ & - & 6.753 & $61 E-01$ & -0.016 & - & 1500.000 \\
\hline 146.0 & 8.642 & $75 \mathrm{E}-01$ & 8.642 & $75 E-01$ & - & 8.641 & $59 \mathrm{E}-01$ & -0.013 & - & 1500.000 \\
\hline 151.0 & 1.088 & $50 E+00$ & 1.088 & $50 E+00$ & - & 1.088 & $34 E+00$ & -0.014 & - & 1500.000 \\
\hline 156.0 & 1.351 & $74 \mathrm{E}+00$ & 1.351 & $74 \mathrm{E}+00$ & - & 1.351 & $57 E+00$ & -0.013 & - & 1500.000 \\
\hline 161.0 & 1.657 & $78 \mathrm{E}+00$ & 1.657 & $78 E+00$ & - & 1.657 & $60 \mathrm{E}+00$ & -0.010 & - & 1500.000 \\
\hline 166.0 & 2.010 & $57 \mathrm{E}+00$ & 2.010 & $57 E+00$ & - & 2.010 & $44 E+00$ & -0.006 & F & 3750.000 \\
\hline 171.0 & 2.414 & $45 E+00$ & 2.414 & $45 E+00$ & - & 2.414 & $39 \mathrm{E}+00$ & -0.002 & - & 3750.000 \\
\hline 176.0 & 2.874 & $35 E+00$ & 2.874 & $35 E+00$ & - & 2.874 & $31 E+00$ & -0.001 & - & 3750.000 \\
\hline 181.0 & 3.396 & $22 \mathrm{E}+00$ & 3.396 & $22 E+00$ & - & 3.395 & $94 E+00$ & -0.008 & - & 3750.000 \\
\hline 186.0 & 3.988 & $10 E+00$ & 3.988 & $10 E+00$ & - & 3.987 & $57 E+00$ & -0.013 & - & 3750.000 \\
\hline
\end{tabular}

Number of Points (eq (1)) 20

\begin{tabular}{|c|c|c|c|c|c|}
\hline $\mathrm{AAD} 1-8$ & - & BIAS $1-8$ & - & RMS1-8 & - \\
\hline $\mathrm{AAD} 1$ & - & BIAS1 & - & RMS1 & - \\
\hline $\mathrm{AAD} 2-8$ & 0.010 , & BIAS2- 8 & -0.002 , & RMS2-8 & 0.011 \\
\hline $\mathrm{AAD} 2$ & 0.096, & BIAS2 & -0.086 & RMS2 & 0.132 \\
\hline
\end{tabular}

Total Points 153 for Ancillary Equation

$\begin{array}{lllllll}\text { AAD1- } 8 & 0.103, & \text { BIAS1-8 } & -0.099, & \text { RMS1-8 } & 0.109 & \\ \text { AAD1 } & 0.514, & \text { BIAS1 } & -0.444, & \text { RMS1 } & 0.563 & k P a\end{array}$

Total Points 173 for Equation of State

AAD2- $8 \quad 0.092, \quad$ BIAS2-8 $-0.090, \quad$ RMS2-8 0.104

AAD2 $0.508, \quad$ BIAS2 $-0.492, \quad$ RMS2 $0.581 \mathrm{kPa}$ 
COMPARISONS FOR SATURATED LIQUID DENSITIES

Note: The numeral 1 associated with quantities refers to comparisons with eq (2); the numeral 2 refers to comparisons with the SWEOS. Similarly, Wtl refers to weighting for the development of eq (2), and Wt2 refers to weighting of the Gibbs condition for the development of the SWEOS. For the points generated from eq (2), in the last part of the table, statistics relative to eq (2) have no meaning; these points are therefore excluded from the totals.

Data from Kleinrahm and Wagner [6]

\begin{tabular}{|c|c|c|c|c|c|c|c|}
\hline $\mathrm{T}$ & $\rho_{\sigma \mathrm{L}}, \exp$ & $\rho_{\sigma \mathrm{L}}$, eq (2) & Devl & $\rho_{\sigma L}$, SWEOS & Dev2 & Wt1 & Wt2 \\
\hline K & $\mathrm{mol} \cdot \mathrm{dm}^{-3}$ & $\mathrm{~mol} \cdot \mathrm{dm}^{-3}$ & 8 & $\mathrm{~mol} \cdot \mathrm{dm}^{-3}$ & 8 & & \\
\hline 90.685 & 28.1411 & 28.1474 & 0.022 & 28.1452 & 0.015 & 1.0 & 0.0 \\
\hline 91.000 & 28.1144 & 28.1208 & 0.023 & 28.1192 & 0.017 & 1.0 & 0.0 \\
\hline 95.000 & 27.7811 & 27.7811 & 0.000 & 27.7860 & 0.018 & 1.0 & 0.0 \\
\hline 100.000 & 27.3571 & 27.3518 & -0.019 & 27.3624 & 0.019 & 1.0 & 0.0 \\
\hline 105.000 & 26.9223 & 26.9157 & -0.025 & 26.9295 & 0.027 & 1.0 & 0.0 \\
\hline 110.000 & 26.4772 & 26.4709 & -0.024 & 26.4858 & 0.032 & 1.0 & 0.0 \\
\hline 111.631 & 26.3286 & 26.3236 & -0.019 & 26.3384 & 0.037 & 1.0 & 0.0 \\
\hline 115.000 & 26.0197 & 26.0154 & -0.016 & 26.0298 & 0.039 & 1.0 & 0.0 \\
\hline 120.000 & 25.5490 & 25.5473 & -0.007 & 25.5600 & 0.043 & 1.0 & 0.0 \\
\hline 125.000 & 25.0629 & 25.0641 & 0.005 & 25.0744 & 0.046 & 1.0 & 0.0 \\
\hline 130.000 & 24.5594 & 24.5632 & 0.015 & 24.5708 & 0.046 & 1.0 & 0.0 \\
\hline 135.000 & 24.0353 & 24.0415 & 0.026 & 24.0466 & 0.047 & 1.0 & 0.0 \\
\hline 140.000 & 23.4889 & 23.4956 & 0.028 & 23.4984 & 0.040 & 1.0 & 0.0 \\
\hline 145.000 & 22.9145 & 22.9210 & 0.028 & 22.9221 & 0.033 & 1.0 & 0.0 \\
\hline 150.000 & 22.3071 & 22.3123 & 0.023 & 22.3124 & 0.024 & 1.0 & 0.0 \\
\hline 155.000 & 21.6599 & 21.6624 & 0.012 & 21.6622 & 0.011 & 1.0 & 0.0 \\
\hline 160.000 & 20.9618 & 20.9618 & 0.000 & 20.9617 & -0.001 & 1.0 & 0.0 \\
\hline 165.000 & 20.2000 & 20.1966 & -0.017 & 20.1966 & -0.017 & 1.0 & 0.0 \\
\hline 170.000 & 19.3516 & 19.3457 & -0.030 & 19.3451 & -0.034 & 1.0 & 0.0 \\
\hline 175.000 & 18.3815 & 18.3730 & -0.046 & 18.3706 & -0.059 & 1.0 & 0.0 \\
\hline 180.000 & 17.2151 & 17.2070 & -0.047 & 17.2044 & -0.062 & 1.0 & 0.0 \\
\hline 182.000 & 16.6600 & 16.6537 & -0.038 & 16.6536 & -0.039 & 1.0 & 0.0 \\
\hline 184.000 & 16.0238 & 16.0205 & -0.020 & 16.0263 & 0.016 & 1.0 & 0.0 \\
\hline 186.000 & 15.2610 & 15.2622 & 0.008 & 15.2793 & 0.120 & 1.0 & 0.0 \\
\hline 187.000 & 14.8040 & 14.8072 & 0.021 & 14.8325 & 0.193 & 1.0 & 0.0 \\
\hline
\end{tabular}


Data from Kleinrahm and Wagner [6] continued

$\begin{array}{cccccccc}\mathrm{T} & \rho_{\sigma \mathrm{L}}, \exp & \rho_{\sigma \mathrm{L}}, \mathrm{eq}(2) & \text { Devl } & \rho_{\sigma \mathrm{L}}, \text { SWEOS } & \text { Dev2 } & \text { Wt1 } & \text { Wt2 } \\ \mathrm{K} & \mathrm{mol} \cdot \mathrm{dm}^{-3} & \mathrm{~mol} \cdot \mathrm{dm}^{-3} & 8 & \mathrm{~mol} \cdot \mathrm{dm}^{-3} & 8 & & \\ 188.000 & 14.2617 & 14.2672 & 0.039 & 14.3028 & 0.288 & 1.0 & 0.0 \\ 189.000 & 13.5704 & 13.5766 & 0.046 & 13.6234 & 0.391 & 1.0 & 0.0 \\ 189.500 & 13.1152 & 13.1218 & 0.050 & 13.1727 & 0.439 & 1.0 & 0.0 \\ 189.800 & 12.7744 & 12.7795 & 0.040 & 12.8303 & 0.438 & 1.0 & 0.0 \\ 190.000 & 12.4960 & 12.5000 & 0.032 & 12.5476 & 0.413 & 1.0 & 0.0 \\ 190.100 & 12.3325 & 12.3356 & 0.025 & 12.3796 & 0.382 & 1.0 & 0.0 \\ 190.200 & 12.1436 & 12.1463 & 0.022 & 12.1838 & 0.331 & 1.0 & 0.0 \\ 190.300 & 11.9180 & 11.9187 & 0.006 & 11.9449 & 0.226 & 1.0 & 0.0 \\ 190.400 & 11.6225 & 11.6228 & 0.002 & 11.6265 & 0.034 & 1.0 & 0.0 \\ 190.450 & 11.4243 & 11.4242 & -0.001 & 11.4071 & -0.150 & 1.0 & 0.0 \\ 190.500 & 11.1494 & 11.1463 & -0.027 & 11.0923 & -0.513 & 1.0 & 0.0 \\ 190.530 & 10.8882 & 10.8736 & -0.135 & 10.7796 & -0.997 & 1.0 & 0.0 \\ 190.551 & 10.1390 & 10.1390 & 0.000 & 10.1390 & 0.000 & 1.0 & 0.0\end{array}$

Number of Points (Ref. 6) 38

$\begin{array}{lllllll}\text { AAD1- } 8 & 0.025, & \text { BIAS1-8 } & 0.000, & \text { RMS1-8 } & 0.034 & \\ \text { AAD1 } & 0.004, & \text { BIAS1 } & 0.000, & \text { RMS1 } & 0.005 & \mathrm{~mol} \cdot \mathrm{dm}^{-3} \\ \text { AAD2-8 } & 0.148, & \text { BIAS2-8 } & 0.050, & \text { RMS2-8 } & 0.249 & \\ \text { AAD2 } & 0.020, & \text { BIAS2 } & 0.009, & \text { RMS2 } & 0.030 & \mathrm{~mol} \cdot \mathrm{dm}^{-3}\end{array}$

Data from Orrit and Laupretre [20]

\begin{tabular}{cccccccc}
\multicolumn{1}{c}{$\rho_{\sigma \mathrm{L}}, \exp$} & $\rho_{\sigma \mathrm{L}}, \operatorname{eq}(2)$ & Dev1 & $\rho_{\sigma \mathrm{L}}$, SWEOS & Dev2 & Wt1 & Wt2 \\
$\mathrm{K}$ & $\mathrm{mol} \cdot \mathrm{dm}^{-3}$ & $\mathrm{~mol} \cdot \mathrm{dm}^{-3}$ & $\&$ & $\mathrm{~mol} \cdot \mathrm{dm}^{-3}$ & o & & \\
99.447 & 27.4263 & 27.3996 & -0.097 & 27.4097 & -0.061 & 0.0 & 0.0 \\
104.630 & 26.9731 & 26.9482 & -0.092 & 26.9618 & -0.042 & 0.0 & 0.0 \\
109.832 & 26.5081 & 26.4860 & -0.083 & 26.5009 & -0.027 & 0.0 & 0.0 \\
115.147 & 26.0219 & 26.0019 & -0.077 & 26.0162 & -0.022 & 0.0 & 0.0 \\
117.812 & 25.7545 & 25.7539 & -0.002 & 25.7674 & 0.050 & 0.0 & 0.0
\end{tabular}


Data from Orrit and Laupretre [20] continued

\begin{tabular}{|c|c|c|c|c|c|c|c|}
\hline $\mathrm{T}$ & $\rho_{\sigma \mathrm{L}}, \exp$ & $\rho_{\sigma \mathrm{I}}, \mathrm{eq}(2)$ & Devl & $\rho_{\sigma L}$, SWEOS & Dev2 & Wt1 & Wt2 \\
\hline K & $\mathrm{mol} \cdot \mathrm{dm}^{-3}$ & $\mathrm{~mol} \cdot \mathrm{dm}^{-3}$ & 8 & $\mathrm{~mol} \cdot \mathrm{dm}^{-3}$ & 8 & & \\
\hline 120.577 & 25.5183 & 25.4924 & -0.102 & 25.5048 & -0.053 & 0.0 & 0.0 \\
\hline 123.300 & 25.2303 & 25.2302 & 0.000 & 25.2414 & 0.044 & 0.0 & 0.0 \\
\hline 126.026 & 24.9872 & 24.9628 & -0.097 & 24.9726 & -0.059 & 0.0 & 0.0 \\
\hline 128.614 & 24.7142 & 24.7040 & -0.041 & 24.7123 & -0.008 & 0.0 & 0.0 \\
\hline 131.494 & 24.4343 & 24.4096 & -0.101 & 24.4165 & -0.073 & 0.0 & 0.0 \\
\hline 134.235 & 24.1376 & 24.1228 & -0.061 & 24.1282 & -0.039 & 0.0 & 0.0 \\
\hline 137.004 & 23.8590 & 23.8259 & -0.139 & 23.8299 & -0.122 & 0.0 & 0.0 \\
\hline 139.730 & 23.5405 & 23.5258 & -0.063 & 23.5287 & -0.050 & 0.0 & 0.0 \\
\hline 142.412 & 23.2494 & 23.2223 & -0.117 & 23.2242 & -0.108 & 0.0 & 0.0 \\
\hline 145.146 & 22.9265 & 22.9037 & -0.099 & 22.9048 & -0.095 & 0.0 & 0.0 \\
\hline 147.933 & 22.5955 & 22.5685 & -0.120 & 22.5689 & -0.118 & 0.0 & 0.0 \\
\hline 153.472 & 21.9111 & 21.8659 & -0.206 & 21.8657 & -0.207 & 0.0 & 0.0 \\
\hline 150.627 & 22.2515 & 22.2332 & -0.082 & 22.2333 & -0.082 & 0.0 & 0.0 \\
\hline 155.955 & 21.5415 & 21.5329 & -0.040 & 21.5327 & -0.041 & 0.0 & 0.0 \\
\hline 159.051 & 21.1301 & 21.0992 & -0.146 & 21.0991 & -0.147 & 0.0 & 0.0 \\
\hline
\end{tabular}

Number of Points (Ref. 20) 20

$\begin{array}{lllllll}\text { AAD1-8 } & 0.088, & \text { BIAS1-8 } & -0.088, & \text { RMS1-8 } & 0.047 & \\ \text { AAD1 } & 0.021, & \text { BIAS1 } & -0.021, & \text { RMS1 } & 0.011 & \mathrm{~mol} \cdot \mathrm{dm}^{-3} \\ \text { AAD2- } 8 & 0.072, & \text { BIAS2 - } 8 & -0.063, & \text { RMS2-8 } & 0.059 & \\ \text { AAD2 } & 0.017, & \text { BIAS2 } & -0.015, & \text { RMS2 } & 0.013 & \mathrm{~mol} \cdot \mathrm{dm}^{-3}\end{array}$

Data from Haynes and Hiza [21]

$\begin{array}{cccccccc}\mathrm{T} & \rho_{\sigma \mathrm{L}}, \exp & \rho_{\sigma \mathrm{I}}, \mathrm{eq}(2) & \text { Devl } & \rho_{\sigma \mathrm{L}}, \text { SWEOS } & \text { Dev2 } & \text { Wt1 } & \text { Wt2 } \\ \mathrm{K} & \mathrm{mol} \cdot \mathrm{dm}^{-3} & \mathrm{~mol} \cdot \mathrm{dm}^{-3} & 8 & \mathrm{~mol} \cdot \mathrm{dm}^{-3} & 8 & & \\ 105.000 & 26.9458 & 26.9157 & -0.112 & 26.9295 & -0.061 & 0.0 & 0.0 \\ 110.000 & 26.4985 & 26.4709 & -0.104 & 26.4858 & -0.048 & 0.0 & 0.0 \\ 115.000 & 26.0443 & 26.0154 & -0.111 & 26.0298 & -0.056 & 0.0 & 0.0 \\ 120.000 & 25.5721 & 25.5473 & -0.097 & 25.5600 & -0.047 & 0.0 & 0.0 \\ 125.000 & 25.0845 & 25.0641 & -0.081 & 25.0744 & -0.040 & 0.0 & 0.0\end{array}$


Data from Haynes and Hiza [21] continued

$\begin{array}{cccccccc}\mathrm{T} & \rho_{\sigma \mathrm{L}}, \exp & \rho_{\sigma \mathrm{L}}, \mathrm{eq}(2) & \text { Dev1 } & \rho_{\sigma \mathrm{L}}, \mathrm{SWEOS} & \text { Dev2 } & \text { Wt1 } & \text { Wt2 } \\ \mathrm{K} & \mathrm{mol} \cdot \mathrm{dm}^{-3} & \mathrm{~mol} \cdot \mathrm{dm}^{-3} & 8 & \mathrm{~mol} \cdot \mathrm{dm}^{-3} & 8 & & \\ 130.000 & 24.5775 & 24.5632 & -0.058 & 24.5708 & -0.027 & 0.0 & 0.0 \\ 135.000 & 24.0540 & 24.0415 & -0.052 & 24.0466 & -0.031 & 0.0 & 0.0 \\ 140.000 & 23.5067 & 23.4956 & -0.047 & 23.4984 & -0.035 & 0.0 & 0.0 \\ 145.000 & 22.9312 & 22.9210 & -0.045 & 22.9221 & -0.040 & 0.0 & 0.0 \\ 150.000 & 22.3218 & 22.3123 & -0.043 & 22.3124 & -0.042 & 0.0 & 0.0 \\ 160.000 & 20.9876 & 20.9618 & -0.123 & 20.9617 & -0.124 & 0.0 & 0.0\end{array}$

Number of Points (Ref. 21) 11

$\begin{array}{lllllll}\text { AAD1- } 8 & 0.079, & \text { BIAS1-8 } & -0.079, & \text { RMS1-8 } & 0.030 & \\ \text { AAD1 } & 0.020, & \text { BIAS1 } & -0.020, & \text { RMS1 } & 0.008 & \mathrm{~mol} \cdot \mathrm{dm}^{-3} \\ \text { AAD2-8 } & 0.050, & \text { BIAS2-8 } & -0.050, & \text { RMS2-8 } & 0.025 & \\ \text { AAD2 } & 0.012, & \text { BIAS2 } & -0.012, & \text { RMS2 } & 0.005 & \mathrm{~mol} \cdot \mathrm{dm}^{-3}\end{array}$

Data from McClune [22]

\begin{tabular}{|c|c|c|c|c|c|c|c|}
\hline $\mathrm{T}$ & $\rho_{\sigma \mathrm{L}}, \exp$ & $\rho_{\sigma L}$, eq (2) & Dev1 & $\rho_{\sigma L}$, SWEOS & Dev2 & Wt1 & Wt2 \\
\hline K & $\mathrm{mol} \cdot \mathrm{dm}^{-3}$ & $\mathrm{~mol} \cdot \mathrm{dm}^{-3}$ & 8 & $\mathrm{~mol} \cdot \mathrm{dm}^{-3}$ & 8 & & \\
\hline 93.150 & 27.9493 & 27.9385 & -0.039 & 27.9407 & -0.031 & 0.0 & 0.0 \\
\hline 93.150 & 27.9493 & 27.9385 & -0.039 & 27.9407 & -0.031 & 0.0 & 0.0 \\
\hline 98.150 & 27.5267 & 27.5113 & -0.056 & 27.5201 & -0.024 & 0.0 & 0.0 \\
\hline 103.150 & 27.0978 & 27.0779 & -0.073 & 27.0908 & -0.026 & 0.0 & 0.0 \\
\hline 108.150 & 26.6521 & 26.6366 & -0.058 & 26.6513 & -0.003 & 0.0 & 0.0 \\
\hline 113.150 & 26.1977 & 26.1853 & -0.047 & 26.2000 & 0.009 & 0.0 & 0.0 \\
\hline 118.150 & 25.7340 & 25.7221 & -0.046 & 25.7355 & 0.006 & 0.0 & 0.0 \\
\hline 123.150 & 25.2615 & 25.2448 & -0.066 & 25.2560 & -0.022 & 0.0 & 0.0 \\
\hline
\end{tabular}

Number of Points (Ref.22) 7

$\begin{array}{lllllll}\text { AAD1- } 8 & 0.055, & \text { BIAS1-8 } & -0.055, & \text { RMS1-8 } & 0.011 & \\ \text { AAD1 } & 0.015, & \text { BIAS1 } & -0.015, & \text { RMS1 } & 0.003 & \mathrm{~mol} \cdot \mathrm{dm}^{-3} \\ \text { AAD2-8 } & 0.017, & \text { BIAS2-8 } & -0.013, & \text { RMS2 -8 } & 0.015 & \\ \text { AAD2 } & 0.005, & \text { BIAS2 } & -0.004, & \text { RMS2 } & 0.004 & \mathrm{~mol} \cdot \mathrm{dm}^{-3}\end{array}$


Data from Terry et al. [23]

\begin{tabular}{|c|c|c|c|c|c|c|c|}
\hline $\mathrm{T}$ & $\rho_{\sigma L}, \exp$ & $\rho_{\sigma \mathrm{L}}, \mathrm{eq}(2)$ & Dev1 & $\rho_{\sigma L}$, SWEOS & Dev2 & Wtl & Wt2 \\
\hline K & $\mathrm{mol} \cdot \mathrm{dm}^{-3}$ & $\mathrm{~mol} \cdot \mathrm{dm}^{-3}$ & 8 & $\mathrm{~mol} \cdot \mathrm{dm}^{-3}$ & 8 & & \\
\hline 92.029 & 28.0346 & 28.0336 & -0.003 & 28.0339 & -0.003 & 0.0 & 0.0 \\
\hline 93.090 & 27.9519 & 27.9436 & -0.030 & 27.9457 & -0.022 & 0.0 & 0.0 \\
\hline 93.654 & 27.8975 & 27.8957 & -0.006 & 27.8986 & 0.004 & 0.0 & 0.0 \\
\hline 95.743 & 27.7233 & 27.7177 & -0.020 & 27.7236 & 0.001 & 0.0 & 0.0 \\
\hline 95.905 & 27.7225 & 27.7038 & -0.067 & 27.7099 & -0.045 & 0.0 & 0.0 \\
\hline 100.939 & 27.2831 & 27.2705 & -0.046 & 27.2818 & -0.005 & 0.0 & 0.0 \\
\hline 102.616 & 27.1502 & 27.1246 & -0.094 & 27.1371 & -0.048 & 0.0 & 0.0 \\
\hline 107.338 & 26.7229 & 26.7089 & -0.053 & 26.7234 & 0.002 & 0.0 & 0.0 \\
\hline 108.806 & 26.6082 & 26.5780 & -0.114 & 26.5928 & -0.058 & 0.0 & 0.0 \\
\hline 112.156 & 26.2882 & 26.2759 & -0.047 & 26.2907 & 0.010 & 0.0 & 0.0 \\
\hline 114.003 & 26.1369 & 26.1072 & -0.114 & 26.1218 & -0.058 & 0.0 & 0.0 \\
\hline 117.523 & 25.7883 & 25.7810 & -0.028 & 25.7946 & 0.024 & 0.0 & 0.0 \\
\hline 119.319 & 25.6420 & 25.6119 & -0.117 & 25.6248 & -0.067 & 0.0 & 0.0 \\
\hline 123.040 & 25.2307 & 25.2555 & 0.098 & 25.2667 & 0.143 & 0.0 & 0.0 \\
\hline 125.765 & 25.0206 & 24.9887 & -0.128 & 24.9986 & -0.088 & 0.0 & 0.0 \\
\hline 127.426 & 24.8201 & 24.8234 & 0.013 & 24.8324 & 0.050 & 0.0 & 0.0 \\
\hline 130.078 & 24.5872 & 24.5552 & -0.130 & 24.5628 & -0.099 & 0.0 & 0.0 \\
\hline 132.228 & 24.3426 & 24.3335 & -0.037 & 24.3399 & -0.011 & 0.0 & 0.0 \\
\hline 136.122 & 23.9493 & 23.9213 & -0.117 & 23.9258 & -0.098 & 0.0 & 0.0 \\
\hline 137.671 & 23.8061 & 23.7532 & -0.222 & 23.7569 & -0.207 & 0.0 & 0.0 \\
\hline 140.482 & 23.4704 & 23.4415 & -0.123 & 23.4441 & -0.112 & 0.0 & 0.0 \\
\hline 142.092 & 23.2555 & 23.2589 & 0.015 & 23.2610 & 0.023 & 0.0 & 0.0 \\
\hline 145.195 & 22.9151 & 22.8979 & -0.075 & 22.8990 & -0.070 & 0.0 & 0.0 \\
\hline 147.541 & 22.6171 & 22.6163 & -0.004 & 22.6168 & -0.001 & 0.0 & 0.0 \\
\hline 151.369 & 22.1523 & 22.1388 & -0.061 & 22.1388 & -0.061 & 0.0 & 0.0 \\
\hline
\end{tabular}

Number of Points (Ref. 23) 25

$\begin{array}{lllllll}\text { AAD1-8 } & 0.070, & \text { BIAS1-8 } & -0.060, & \text { RMS1 }-8 & 0.064 & \\ \text { AAD1 } & 0.018, & \text { BIAS1 } & -0.015, & \text { RMS1 } & 0.016 & \mathrm{~mol} \cdot \mathrm{dm}^{-3} \\ \text { AAD2-8 } & 0.052, & \text { BIAS2-8 } & -0.032, & \text { RMS2-8 } & 0.065 & \\ \text { AAD2 } & 0.013, & \text { BIAS2 } & -0.008, & \text { RMS2 } & 0.016 & \mathrm{~mol} \cdot \mathrm{dm}^{-3}\end{array}$ 
Data from Klosek and McKinley [24]

\begin{tabular}{|c|c|c|c|c|c|c|c|}
\hline $\mathrm{T}$ & $\rho_{\sigma \mathrm{L}}, \exp$ & $\rho_{\sigma \mathrm{L}}$, eq(2) & Devl & $\rho_{\sigma \mathrm{L}}$, SWEOS & Dev2 & Wel & Wt2 \\
\hline K & $\mathrm{mol} \cdot \mathrm{dm}^{-3}$ & $\mathrm{~mol} \cdot \mathrm{dm}^{-3}$ & 8 & $\mathrm{~mol} \cdot \mathrm{dm}^{-3}$ & 8 & & \\
\hline 94.261 & 27.8437 & 27.8441 & 0.001 & 27.8479 & 0.015 & 0.0 & 0.0 \\
\hline 99.817 & 27.3539 & 27.3676 & 0.050 & 27.3780 & 0.088 & 0.0 & 0.0 \\
\hline 105.372 & 26.8631 & 26.8829 & 0.074 & 26.8968 & 0.126 & 0.0 & 0.0 \\
\hline 110.928 & 26.3635 & 26.3872 & 0.090 & 26.4021 & 0.146 & 0.0 & 0.0 \\
\hline 116.483 & 25.8654 & 25.8780 & 0.049 & 25.8920 & 0.103 & 0.0 & 0.0 \\
\hline 122.039 & 25.3377 & 25.3522 & 0.057 & 25.3640 & 0.104 & 0.0 & 0.0 \\
\hline 127.594 & 24.7887 & 24.8066 & 0.072 & 24.8155 & 0.108 & 0.0 & 0.0 \\
\hline 133.150 & 24.2373 & 24.2372 & 0.000 & 24.2431 & 0.024 & 0.0 & 0.0 \\
\hline
\end{tabular}

Number of Points (Ref. 24) 8

AAD1- $80.049, \quad$ BIAS1- $80.049, \quad$ RMS1-8 0.031

AAD1 0.013 , BIAS1 0.013 , RMS1 $0.008 \mathrm{~mol} \cdot \mathrm{dm}^{-3}$

$\begin{array}{lllllll}\mathrm{AAD2}-8 & 0.089, & \text { BIAS2-8 } & 0.089, & \text { RMS2 - } 8 & 0.043 & \\ \mathrm{AAD} & 0.023, & \text { BIAS2 } & 0.023, & \text { RMS2 } & 0.011 & \mathrm{~mol} \cdot \mathrm{dm}^{-3}\end{array}$

Data from Rodosevich and Miller [25]

$\begin{array}{cccccccc}\mathrm{T} & \rho_{\sigma \mathrm{L}}, \exp & \rho_{\sigma \mathrm{L}}, \mathrm{eq}(2) & \operatorname{Devl} & \rho_{\sigma \mathrm{L}}, \mathrm{SWEOS} & \mathrm{Dev2} & \text { WtI } & \text { Wt2 } \\ \mathrm{K} & \mathrm{mol} \cdot \mathrm{dm}^{-3} & \mathrm{~mol} \cdot \mathrm{dm}^{-3} & 8 & \mathrm{~mol} \cdot \mathrm{dm}^{-3} & 8 & & \\ 91.000 & 28.1175 & 28.1208 & 0.012 & 28.1192 & 0.006 & 0.0 & 0.0 \\ 100.150 & 27.3545 & 27.3388 & -0.057 & 27.3495 & -0.018 & 0.0 & 0.0 \\ 108.050 & 26.6638 & 26.6455 & -0.069 & 26.6602 & -0.014 & 0.0 & 0.0 \\ 114.940 & 26.0396 & 26.0210 & -0.071 & 26.0354 & -0.016 & 0.0 & 0.0\end{array}$

Number of Points (Ref. 25) 4

$\begin{array}{lllllll}\text { AAD1- } 8 & 0.052, & \text { BIAS1- } 8 & -0.046, & \text { RMS1- } 8 & 0.034 & \\ \text { AAD1 } & 0.014, & \text { BIAS1 } & -0.012, & \text { RMS1 } & 0.009 & \mathrm{~mol} \cdot \mathrm{dm}^{-3} \\ \text { AAD2-8 } & 0.014, & \text { BIAS2-8 } & -0.011, & \text { RMS2-8 } & 0.010 & \\ \text { AAD2 } & 0.004, & \text { BIAS2 } & -0.003, & \text { RMS2 } & 0.003 & \mathrm{~mol} \cdot \mathrm{dm}^{-3}\end{array}$ 
Data from Verbeke [26]

$\begin{array}{cccccccc}\mathrm{T} & \rho_{\sigma \mathrm{L}}, \exp & \rho_{\sigma \mathrm{L}}, \mathrm{eq}(2) & \operatorname{Devl} & \rho_{\sigma \mathrm{L}}, \text { SWEOS } & \text { Dev2 } & \text { Wt1 } & \text { Wt2 } \\ \mathrm{K} & \mathrm{mol} \cdot \mathrm{dm}^{-3} & \mathrm{~mol} \cdot \mathrm{dm}^{-3} & 8 & \mathrm{~mol} \cdot \mathrm{dm}^{-3} & 8 & & \\ 190.050 & 12.4400 & 12.4204 & -0.158 & 12.4664 & 0.212 & 0.0 & 0.0 \\ 190.150 & 12.2700 & 12.2447 & -0.206 & 12.2859 & 0.129 & 0.0 & 0.0 \\ 190.250 & 12.0700 & 12.0385 & -0.261 & 12.0712 & 0.010 & 0.0 & 0.0 \\ 190.350 & 11.7900 & 11.7826 & -0.063 & 11.7997 & 0.082 & 0.0 & 0.0 \\ 190.450 & 11.4100 & 11.4242 & 0.125 & 11.4071 & -0.025 & 0.0 & 0.0\end{array}$

Number of Points (Ref. 26) 5

AAD1- $80.163, \quad$ BIAS1-8 $-0.113, \quad$ RMS1-8 0.135

AAD1 0.020, BIAS1 $-0.014, \quad$ RMS1 $0.016 \mathrm{~mol} \cdot \mathrm{dm}^{-3}$

$\begin{array}{lllllll}\text { AAD2 }-q & 0.092, & \text { BIAS2 - } 8 & 0.082, & \text { RMS2 - } 8 & 0.085 & \\ \text { AAD2 } & 0.011, & \text { BIAS2 } & 0.010, & \text { RMS2 } & 0.010 & \mathrm{~mol} \cdot \mathrm{dm}^{-3}\end{array}$

Data from Vennix et al. [27]

$\begin{array}{cccccccc}\mathrm{T} & \rho_{\sigma \mathrm{L}}, \exp & \rho_{\sigma \mathrm{L}}, \operatorname{eq}(2) & \text { Dev1 } & \rho_{\sigma \mathrm{L}}, \text { SWEOS } & \text { Dev2 } & \text { Wt1 } & \text { Wt2 } \\ \mathrm{K} & \mathrm{mol} \cdot \mathrm{dm}^{-3} & \mathrm{~mol} \cdot \mathrm{dm}^{-3} & q & \mathrm{~mol} \cdot \mathrm{dm}^{-3} & 8 & & \\ 138.951 & 23.6246 & 23.6124 & -0.052 & 23.6155 & -0.038 & 0.0 & 0.0 \\ 158.829 & 21.2348 & 21.1311 & -0.488 & 21.1309 & -0.489 & 0.0 & 0.0 \\ 166.766 & 20.0075 & 19.9073 & -0.501 & 19.9072 & -0.501 & 0.0 & 0.0 \\ 174.773 & 18.5009 & 18.4206 & -0.434 & 18.4183 & -0.446 & 0.0 & 0.0 \\ 178.726 & 17.5690 & 17.5291 & -0.227 & 17.5260 & -0.245 & 0.0 & 0.0 \\ 182.608 & 16.5275 & 16.4711 & -0.341 & 16.4723 & -0.334 & 0.0 & 0.0 \\ 186.771 & 14.9754 & 14.9175 & -0.387 & 14.9408 & -0.231 & 0.0 & 0.0 \\ 188.852 & 13.7549 & 13.6933 & -0.448 & 13.7385 & -0.119 & 0.0 & 0.0 \\ 189.882 & 12.6809 & 12.6713 & -0.076 & 12.7213 & 0.319 & 0.0 & 0.0\end{array}$

Number of Points (Ref. 27) 9

$\begin{array}{lllllll}\text { AAD1- } 8 & 0.328, & \text { BIAS1- } 8 & -0.328, & \text { RMS1- } 8 & 0.161 & \\ \text { AAD1 } & 0.058, & \text { BIAS1 } & -0.058, & \text { RMS1 } & 0.032 & \mathrm{~mol} \cdot \mathrm{dm}^{-3} \\ \text { AAD2- } 8 & 0.302, & \text { BIAS2-8 } & -0.232, & \text { RMS2-8 } & 0.247 & \\ \text { AAD2 } & 0.054, & \text { BIAS2 } & -0.045, & \text { RMS2 } & 0.044 & \mathrm{~mol} \cdot \mathrm{dm}^{-3}\end{array}$


Data from Goodwin [28]

\begin{tabular}{|c|c|c|c|c|c|c|c|}
\hline $\mathrm{T}$ & $\rho_{\sigma L}, \exp$ & $\rho_{\sigma L}$, eq (2) & Dev1 & $\rho_{\sigma L}$, SWEOS & Dev2 & Wt1 & Wt2 \\
\hline K & $\mathrm{mol} \cdot \mathrm{dm}^{-3}$ & $\mathrm{~mol} \cdot \mathrm{dm}^{-3}$ & 8 & $\mathrm{~mol} \cdot \mathrm{dm}^{-3}$ & 8 & & \\
\hline 130.000 & 24.5580 & 24.5632 & 0.021 & 24.5708 & 0.052 & 0.0 & 0.0 \\
\hline 135.000 & 24.0410 & 24.0415 & 0.002 & 24.0466 & 0.023 & 0.0 & 0.0 \\
\hline 140.000 & 23.5000 & 23.4956 & -0.019 & 23.4984 & -0.007 & 0.0 & 0.0 \\
\hline 145.000 & 22.9320 & 22.9210 & -0.048 & 22.9221 & -0.043 & 0.0 & 0.0 \\
\hline 150.000 & 22.3290 & 22.3123 & -0.075 & 22.3124 & -0.074 & 0.0 & 0.0 \\
\hline 155.000 & 21.6860 & 21.6624 & -0.109 & 21.6622 & -0.110 & 0.0 & 0.0 \\
\hline 160.000 & 20.9910 & 20.9618 & -0.139 & 20.9617 & -0.140 & 0.0 & 0.0 \\
\hline 165.000 & 20.2340 & 20.1966 & -0.185 & 20.1966 & -0.185 & 0.0 & 0.0 \\
\hline 170.000 & 19.3870 & 19.3457 & -0.213 & 19.3451 & -0.216 & 0.0 & 0.0 \\
\hline 175.000 & 18.4170 & 18.3730 & -0.239 & 18.3706 & -0.252 & 0.0 & 0.0 \\
\hline 180.000 & 17.2490 & 17.2070 & -0.243 & 17.2044 & -0.258 & 0.0 & 0.0 \\
\hline 184.000 & 16.0540 & 16.0205 & -0.209 & 16.0263 & -0.172 & 0.0 & 0.0 \\
\hline 186.000 & 15.2860 & 15.2622 & -0.156 & 15.2793 & -0.044 & 0.0 & 0.0 \\
\hline 188.000 & 14.2840 & 14.2672 & -0.118 & 14.3028 & 0.131 & 0.0 & 0.0 \\
\hline
\end{tabular}

Number of Points (Ref. 28) 14

$\begin{array}{lllllll}\text { AAD1- } 8 & 0.127, & \text { BIAS1- } 8 & -0.124, & \text { RMS1- } 8 & 0.086 & \\ \text { AAD } & 0.024, & \text { BIAS1 } & -0.023, & \text { RMS1 } & 0.016 & \mathrm{~mol} \cdot \mathrm{dm}^{-3} \\ \text { AAD2-8 } & 0.122, & \text { BIAS2-8 } & -0.093, & \text { RMS2-8 } & 0.114 & \\ \text { AAD } & 0.023, & \text { BIAS2 } & -0.018, & \text { RMS2 } & 0.021 & \mathrm{~mol} \cdot \mathrm{dm}^{-3}\end{array}$

Data from Ricci and Scafe [29]

$\begin{array}{cccccccc}\mathrm{T} & \rho_{\sigma \mathrm{L}}, \exp & \rho_{\sigma \mathrm{L}}, \operatorname{eq}(2) & \text { Devl } & \rho_{\sigma \mathrm{L}}, \text { SWEOS } & \text { Dev2 } & \text { Wt1 } & \text { Wt2 } \\ \mathrm{K} & \mathrm{mol} \cdot \mathrm{dm}^{-3} & \mathrm{~mol} \cdot \mathrm{dm}^{-3} & 8 & \mathrm{~mol} \cdot \mathrm{dm}^{-3} & 8 & & \\ 185.030 & 15.7100 & 15.6501 & -0.381 & 15.6609 & -0.313 & 0.0 & 0.0 \\ 186.030 & 15.3020 & 15.2495 & -0.343 & 15.2668 & -0.230 & 0.0 & 0.0 \\ 187.031 & 14.8360 & 14.7919 & -0.297 & 14.8176 & -0.124 & 0.0 & 0.0 \\ 188.031 & 14.2850 & 14.2486 & -0.255 & 14.2845 & -0.004 & 0.0 & 0.0 \\ 189.032 & 13.5780 & 13.5505 & -0.202 & 13.5976 & 0.145 & 0.0 & 0.0 \\ 190.032 & 12.4740 & 12.4496 & -0.196 & 12.4963 & 0.178 & 0.0 & 0.0\end{array}$


Data from Ricci and Scafe [29] continued

Number of Points (Ref. 29) 6

AAD1-8 $0.279, \quad$ BIAS1-8 $-0.279, \quad$ RMS1-8 0.069

AAD1 0.041 , BIAS1 -0.041, RMS1 $0.013 \mathrm{~mol} \cdot \mathrm{dm}^{-3}$

$\begin{array}{lllllll}\text { AAD2-8 } & 0.166, & \text { BIAS2 - } 8 & -0.058, & \text { RMS2 - } 8 & 0.182 & \\ \text { AAD2 } & 0.024, & \text { BIAS2 } & -0.010, & \text { RMS2 } & 0.027 & \mathrm{~mol} \cdot \mathrm{dm}^{-3}\end{array}$

Data from Goodwin [30]

$\begin{array}{cccccccc}\mathrm{T} & \rho_{\sigma \mathrm{L}}, \exp & \rho_{\sigma \mathrm{L}}, \mathrm{eq}(2) & \text { Dev1 } & \rho_{\sigma \mathrm{L}}, \mathrm{SWEOS} & \text { Dev2 } & \text { Wt1 } & \text { Wt2 } \\ \mathrm{K} & \mathrm{mol} \cdot \mathrm{dm}^{-3} & \mathrm{~mol} \cdot \mathrm{dm}^{-3} & 8 & \mathrm{~mol} \cdot \mathrm{dm}^{-3} & 8 & & \\ 150.000 & 22.3320 & 22.3123 & -0.088 & 22.3124 & -0.088 & 0.0 & 0.0 \\ 175.000 & 18.4200 & 18.3730 & -0.255 & 18.3706 & -0.268 & 0.0 & 0.0 \\ 180.000 & 17.2540 & 17.2070 & -0.272 & 17.2044 & -0.287 & 0.0 & 0.0 \\ 184.000 & 16.0600 & 16.0205 & -0.246 & 16.0263 & -0.210 & 0.0 & 0.0\end{array}$

Number of Points (Ref. 30) 4

$\begin{array}{lllllll}\text { AAD1- } 8 & 0.215, & \text { BIAS1- } 8 & -0.215, & \text { RMS1-8 } & 0.074 & \\ \text { AAD1 } & 0.038, & \text { BIAS1 } & -0.038, & \text { RMS1 } & 0.011 & \mathrm{~mol} \cdot \mathrm{dm}^{-3} \\ \text { AAD2- } 8 & 0.213, & \text { BIAS2- } 8 & -0.213, & \text { RMS2-8 } & 0.078 & \\ \text { AAD2 } & 0.038, & \text { BIAS2 } & -0.038, & \text { RMS2 } & 0.012 & \mathrm{~mol} \cdot \mathrm{dm}^{-3}\end{array}$

Data from Goodwin [31]

\begin{tabular}{cccccccc}
\multicolumn{1}{c}{ T } & $\rho_{\sigma L}, \exp$ & $\rho_{\sigma L}, \operatorname{eq}(2)$ & Dev1 & $\rho_{\sigma L}$, SWEOS & Dev2 & Wt1 & Wt2 \\
$\mathrm{K}$ & $\mathrm{mol} \cdot \mathrm{dm}^{-3}$ & $\mathrm{~mol} \cdot \mathrm{dm}^{-3}$ & $\&$ & $\mathrm{~mol} \cdot \mathrm{dm}^{-3}$ & 8 & & \\
93.512 & 27.9100 & 27.9078 & -0.008 & 27.9105 & 0.002 & 0.0 & 0.0 \\
97.173 & 27.6050 & 27.5952 & -0.035 & 27.6029 & -0.007 & 0.0 & 0.0 \\
101.434 & 27.2430 & 27.2275 & -0.057 & 27.2392 & -0.014 & 0.0 & 0.0 \\
105.165 & 26.9160 & 26.9011 & -0.055 & 26.9150 & -0.004 & 0.0 & 0.0 \\
109.611 & 26.5270 & 26.5058 & -0.080 & 26.5207 & -0.024 & 0.0 & 0.0
\end{tabular}


Data from Goodwin [31] (continued)

\begin{tabular}{|c|c|c|c|c|c|c|c|}
\hline $\mathbf{T}$ & $\rho_{\sigma \mathrm{L}}, \exp$ & $\rho_{\sigma \mathrm{L}}$, eq (2) & Dev1 & $\rho_{\sigma \mathrm{L}}$, SWEOS & Dev2 & Wt1 & Wt2 \\
\hline K & $\mathrm{mol} \cdot \mathrm{dm}^{-3}$ & $\mathrm{~mol} \cdot \mathrm{dm}^{-3}$ & 8 & $\mathrm{~mol} \cdot \mathrm{dm}^{-3}$ & 8 & & \\
\hline 113.772 & 26.1460 & 26.1284 & -0.067 & 26.1430 & -0.011 & 0.0 & 0.0 \\
\hline 117.746 & 25.7820 & 25.7601 & -0.085 & 25.7736 & -0.033 & 0.0 & 0.0 \\
\hline 121.893 & 25.3880 & 25.3663 & -0.086 & 25.3781 & -0.039 & 0.0 & 0.0 \\
\hline 125.825 & 24.9990 & 24.9827 & -0.065 & 24.9926 & -0.026 & 0.0 & 0.0 \\
\hline 129.657 & 24.6110 & 24.5982 & -0.052 & 24.6060 & -0.020 & 0.0 & 0.0 \\
\hline 133.773 & 24.1860 & 24.1717 & -0.059 & 24.1773 & -0.036 & 0.0 & 0.0 \\
\hline 133.878 & 24.1760 & 24.1606 & -0.064 & 24.1661 & -0.041 & 0.0 & 0.0 \\
\hline 139.352 & 23.5780 & 23.5679 & -0.043 & 23.5709 & -0.030 & 0.0 & 0.0 \\
\hline 145.448 & 22.8800 & 22.8679 & -0.053 & 22.8689 & -0.048 & 0.0 & 0.0 \\
\hline 151.553 & 22.1300 & 22.1152 & -0.067 & 22.1152 & -0.067 & 0.0 & 0.0 \\
\hline 157.199 & 21.3790 & 21.3612 & -0.083 & 21.3610 & -0.084 & 0.0 & 0.0 \\
\hline 163.659 & 20.4280 & 20.4092 & -0.092 & 20.4092 & -0.092 & 0.0 & 0.0 \\
\hline 169.326 & 19.4920 & 19.4665 & -0.131 & 19.4660 & -0.133 & 0.0 & 0.0 \\
\hline 175.053 & 18.3900 & 18.3618 & -0.153 & 18.3594 & -0.166 & 0.0 & 0.0 \\
\hline
\end{tabular}

Number of Points (Ref. 31) 19

AAD1-8 $0.070, \quad$ BIAS1-8 $-0.070, \quad$ RMS1-8 0.031

AAD1 0.016, BIAS1 -0.016 , RMS1 $0.006 \mathrm{~mol} \cdot \mathrm{dm}^{-3}$

$\begin{array}{lllllll}\text { AAD2-8 } & 0.046, & \text { BIAS2-8 } & -0.046, & \text { RMS2-8 } & 0.044 & \\ \text { AAD2 } & 0.010, & \text { BIAS2 } & -0.010, & \text { RMS2 } & 0.008 & \mathrm{~mol} \cdot \mathrm{dm}^{-3}\end{array}$

Data from Jansoone et a1. [32]

$\begin{array}{cccccccc}\mathrm{T} & \rho_{\sigma \mathrm{L}}, \exp & \rho_{\sigma \mathrm{L}}, \operatorname{eq}(2) & \operatorname{Dev1} & \rho_{\sigma \mathrm{L}}, \mathrm{SWEOS} & \mathrm{Dev2} & \text { Wt1 } & \text { Wt2 } \\ \mathrm{K} & \mathrm{mol} \cdot \mathrm{dm}^{-3} & \mathrm{~mol} \cdot \mathrm{dm}^{-3} & 8 & \mathrm{~mol} \cdot \mathrm{dm}^{-3} & 8 & & \\ 187.469 & 14.5820 & 14.5672 & -0.102 & 14.5971 & 0.103 & 0.0 & 0.0 \\ 189.311 & 13.3000 & 13.3068 & 0.051 & 13.3566 & 0.425 & 0.0 & 0.0 \\ 189.707 & 12.8790 & 12.8935 & 0.112 & 12.9448 & 0.511 & 0.0 & 0.0 \\ 190.048 & 12.3970 & 12.4237 & 0.215 & 12.4698 & 0.587 & 0.0 & 0.0 \\ 190.316 & 11.8240 & 11.8772 & 0.450 & 11.9008 & 0.650 & 0.0 & 0.0 \\ 190.442 & 11.3670 & 11.4597 & 0.815 & 11.4466 & 0.701 & 0.0 & 0.0 \\ 190.515 & 10.7960 & 11.0288 & 2.157 & 10.9573 & 1.494 & 0.0 & 0.0\end{array}$


Data from Jansoone et al. [32] (continued)

Number of Points (Ref. 32) 7

$\begin{array}{lllllll}\text { AAD1-8 } & 0.557, & \text { BIAS1-8 } & 0.528, & \text { RMS1-8 } & 0.721 & \\ \text { AAD1 } & 0.063, & \text { BIAS1 } & 0.059, & \text { RMS1 } & 0.078 & \mathrm{~mol} \cdot \mathrm{dm}^{-3} \\ \text { AAD2-8 } & 0.639, & \text { BIAS2-8 } & 0.639, & \text { RMS2-8 } & 0.394 & \\ \text { AAD2 } & 0.076, & \text { BIAS2 } & 0.076, & \text { RMS2 } & 0.041 & \mathrm{~mol} \cdot \mathrm{dm}^{-3}\end{array}$

Data from eq (2)

\begin{tabular}{|c|c|c|c|c|c|c|}
\hline $\mathrm{T}$ & $\rho_{\sigma \mathrm{L}}, \exp$ & $\rho_{\sigma \mathrm{L}}, \mathrm{eq}(2)$ & Dev1 & $\rho_{\sigma \mathrm{L}}$, SWEOS & Dev2 & Wt1 \\
\hline K & $\mathrm{mol} \cdot \mathrm{dm}^{-3}$ & $\mathrm{~mol} \cdot \mathrm{dm}^{-3}$ & 8 & $\mathrm{~mol} \cdot \mathrm{dm}^{-3}$ & 8 & \\
\hline 91.0 & 28.12075 & 28.12075 & - & 28.11919 & -0.006 & 17.390 \\
\hline 96.0 & 27.69569 & 27.69569 & - & 27.70194 & 0.023 & 10.963 \\
\hline 101.0 & 27.26517 & 27.26517 & - & 27.27660 & 0.042 & 7.354 \\
\hline 106.0 & 26.82746 & 26.82746 & - & 26.84162 & 0.053 & 5.201 \\
\hline 111.0 & 26.38069 & 26.38069 & - & 26.39559 & 0.056 & 3.850 \\
\hline 116.0 & 25.92291 & 25.92291 & - & 25.93700 & 0.054 & 2.964 \\
\hline 121.0 & 25.45195 & 25.45195 & - & 25.46417 & 0.048 & 2.362 \\
\hline 126.0 & 24.96542 & 24.96542 & - & 24.97517 & 0.039 & 1.941 \\
\hline 131.0 & 24.46062 & 24.46062 & - & 24.46769 & 0.029 & 1.639 \\
\hline 136.0 & 23.93442 & 23.93442 & - & 23.93895 & 0.019 & 49.669 \\
\hline 141.0 & 23.38312 & 23.38312 & - & 23.38551 & 0.010 & 44.004 \\
\hline 146.0 & 22.80217 & 22.80217 & - & 22.80303 & 0.004 & 199.223 \\
\hline 151.0 & 22.18586 & 22.18586 & - & 22.18588 & 0.000 & 184.268 \\
\hline 156.0 & 21.52672 & 21.52672 & - & 21.52651 & -0.001 & 174.141 \\
\hline 161.0 & 20.81447 & 20.81447 & - & 20.81438 & 0.000 & 168.363 \\
\hline 166.0 & 20.03418 & 20.03418 & - & 20.03409 & 0.000 & 167.007 \\
\hline 171.0 & 19.16248 & 19.16248 & - & 19.16155 & -0.005 & 170.897 \\
\hline 176.0 & 18.15860 & 18.15860 & - & 18.15590 & -0.015 & 182.282 \\
\hline 181.0 & 16.93856 & 16.93856 & - & 16.93686 & -0.010 & 206.862 \\
\hline 186.0 & 15.26221 & 15.26221 & - & 15.27930 & 0.112 & 260.343 \\
\hline
\end{tabular}

Number of Points (eq. (2)) 20

$\begin{array}{lrlllll}\text { AAD1-8 } & -, & \text { BIAS1-8 } & -, ; & \text { RMS1-8 } & - & \\ \text { AAD1 } & -, & \text { BIAS1 } & -, & \text { RMS1 } & - & \\ \text { AAD2-8 } & 0.026, & \text { BIAS2-8 } & 0.023, & \text { RMS2-8 } & 0.031 & \\ \text { AAD2 } & 0.006, & \text { BIAS2 } & 0.005, & \text { RMS2 } & 0.006 & \mathrm{~mol} \cdot \mathrm{dm}^{-3}\end{array}$


Total Points 177 for Ancillary Equation

AAD1-8 0.111, BIAS1-8 $-0.055, \quad$ RMS1- 80.214

AAD1 0.020 , BIAS1 -0.013 , RMS1 $0.029 \mathrm{~mol} \cdot \mathrm{dm}^{-3}$

Total Points 197 for Equation of State

AAD2-8 0.114, BIAS2-8 $-0.001, \quad$ RMS2-8 0.211

AAD2 0.019, BIAS2 -0.003, RMS2 $0.033 \mathrm{~mol} \cdot \mathrm{dm}^{-3}$

Total Points 76 from Refs. 6, 20-22 for Ancillary Equation

AADl- $8 \quad 0.052$, BIAS1- 8 $\quad-0.040, \quad$ RMS1-8 0.054

AAD1 0.012 , BIAS1 -0.010 , RMS1 $0.012 \mathrm{~mol} \cdot \mathrm{dm}^{-3}$

Total Points 76 from Refs. 6, 20-22 for Equation of State

AAD1-8 0.102, BIAS1-8 $0.000, \quad$ RMS1-8 0.186

AAD1 0.017, BIAS1 -0.002 , RMS1 $0.025 \mathrm{mo} 1 \cdot \mathrm{dm}^{-3}$ 
COMPARISONS FOR SATURATED VAPOR DENSITIES

Notes for Table A3. The numeral 1 associated with quantities refers to comparisons with eq (3); the numeral 2 refers to comparisons with the SWEOS. Similarly, Wt1 refers to weighting for the development of eq (3), and Wt2 refers to weighting of the saturated vapor densities in the development of the SWEOS. For the points generated from eq (3), in the last part of the table, statistics relative to eq (3) have no meaning; these points are therefore excluded from the totals.

Data from Kleinrahm and Wagner [6]

\begin{tabular}{|c|c|c|c|c|c|c|c|}
\hline $\mathrm{T}$ & $\rho_{\sigma \mathrm{V}}, \exp$ & $\rho_{\sigma \mathrm{v}}$, eq (3) & Devl & $\rho_{\sigma \mathrm{V}}$, SWEOS & Dev2 & Wt1 & Wt2 \\
\hline K & $\mathrm{mol} \cdot \mathrm{dm}^{-3}$ & $\mathrm{~mol} \cdot \mathrm{dm}^{-3}$ & 8 & $\mathrm{~mol} \cdot \mathrm{dm}^{-3}$ & 8 & & \\
\hline 90.685 & 0.01563 & 0.01564 & 0.034 & 0.01565 & 0.155 & 1.0 & 0.0 \\
\hline 91.000 & 0.01623 & 0.01623 & -0.012 & 0.01625 & 0.104 & 1.0 & 0.0 \\
\hline 95.000 & $0.025 \quad 43$ & 0.02542 & -0.041 & 0.02543 & 0.018 & 1.0 & 0.0 \\
\hline 100.000 & 0.04216 & 0.04214 & -0.047 & 0.04215 & -0.025 & 1.0 & 0.0 \\
\hline 105.000 & 0.06628 & 0.06630 & 0.028 & 0.06630 & 0.035 & 1.0 & 0.0 \\
\hline 110.000 & 0.09977 & 0.09981 & 0.045 & 0.09982 & 0.053 & 1.0 & 0.0 \\
\hline 111.631 & 0.11310 & 0.11312 & 0.017 & 0.11313 & 0.028 & 1.0 & 0.0 \\
\hline 115.000 & 0.14475 & 0.14479 & 0.029 & 0.14483 & 0.052 & 1.0 & 0.0 \\
\hline 120.000 & 0.20349 & 0.20354 & 0.026 & 0.20363 & 0.070 & 1.0 & 0.0 \\
\hline 125.000 & 0.27863 & 0.27862 & -0.003 & $0.278 \quad 81$ & 0.063 & 1.0 & 0.0 \\
\hline 130.000 & 0.37302 & 0.37291 & -0.030 & 0.37322 & 0.052 & 1.0 & 0.0 \\
\hline 135.000 & 0.49001 & 0.48971 & -0.062 & 0.49014 & 0.027 & 1.0 & 0.0 \\
\hline 140.000 & 0.63324 & 0.63289 & -0.055 & 0.63343 & 0.030 & 1.0 & 0.0 \\
\hline 145.000 & 0.80750 & 0.80715 & -0.043 & 0.80771 & 0.025 & 1.0 & 0.0 \\
\hline 150.000 & 1.01833 & 1.01830 & -0.003 & 1.01876 & 0.042 & 1.0 & 0.0 \\
\hline 155.000 & $1.273 \quad 35$ & 1.27383 & 0.037 & 1.27406 & 0.056 & 1.0 & 0.0 \\
\hline 160.000 & 1.58293 & 1.58383 & 0.057 & 1.58375 & 0.052 & 1.0 & 0.0 \\
\hline 165.000 & 1.96149 & 1.96264 & 0.059 & 1.96228 & 0.040 & 1.0 & 0.0 \\
\hline 170.000 & 2.43079 & $2.432 \quad 20$ & 0.058 & 2.43173 & 0.039 & 1.0 & 0.0 \\
\hline 175.000 & 3.02890 & 3.02952 & 0.020 & 3.02925 & 0.012 & 1.0 & 0.0 \\
\hline 180.000 & 3.82869 & 3.82739 & -0.034 & $3.827 \quad 36$ & -0.035 & 1.0 & 0.0 \\
\hline 182.000 & $4.238 \quad 42$ & 4.23604 & -0.056 & 4.23579 & -0.062 & 1.0 & 0.0 \\
\hline 184.000 & 4.72987 & $4.726 \quad 28$ & -0.076 & $4.725 \quad 28$ & -0.097 & 1.0 & 0.0 \\
\hline 186.000 & 5.34768 & 5.34367 & -0.075 & 5.34076 & -0.129 & 1.0 & 0.0 \\
\hline 187.000 & 5.73294 & 5.72903 & -0.068 & $5.724 \quad 44$ & -0.148 & 1.0 & 0.0 \\
\hline
\end{tabular}


Data from Kleinrahm and Wagner [6] (continued)

$\mathrm{T} \quad \rho_{\sigma \mathrm{V}}, \exp \rho_{\sigma \mathrm{v}}, \operatorname{eq}(3)$ Dev1 $\rho_{\sigma \mathrm{v}}$, SWEOS Dev2 Wt1 Wt2

$\mathrm{K} \quad \mathrm{mol} \cdot \mathrm{dm}^{-3} \quad \mathrm{~mol} \cdot \mathrm{dm}^{-3} \quad$ o $\quad \mathrm{mol} \cdot \mathrm{dm}^{-3} \quad$ o

$\begin{array}{rrrrrrrrr}188.000 & 6.20244 & 6.19974 & -0.044 & 6.19276 & -0.156 & 1.0 & 0.0 \\ 189.000 & 6.82073 & 6.821 & 13 & 0.006 & 6.81137 & -0.137 & 1.0 & 0.0 \\ 189.500 & 7.23774 & 7.24106 & 0.046 & 7.23089 & -0.095 & 1.0 & 0.0 \\ 189.800 & 7.55657 & 7.56203 & 0.072 & 7.55344 & -0.041 & 1.0 & 0.0 \\ 190.000 & 7.82023 & 7.82692 & 0.085 & 7.82173 & 0.019 & 1.0 & 0.0 \\ 190.100 & 7.97669 & 7.98380 & 0.089 & 7.98191 & 0.065 & 1.0 & 0.0 \\ 190.200 & 8.15683 & 8.16549 & 0.106 & 8.16894 & 0.148 & 1.0 & 0.0 \\ 190.300 & 8.37562 & 8.38508 & 0.113 & 8.39775 & 0.264 & 1.0 & 0.0 \\ 190.400 & 8.66047 & 8.67258 & 0.140 & 8.70306 & 0.492 & 1.0 & 0.0 \\ 190.450 & 8.85495 & 8.86651 & 0.131 & 8.91333 & 0.659 & 1.0 & 0.0 \\ 190.500 & 9.12922 & 9.13930 & 0.110 & 9.21475 & 0.937 & 1.0 & 0.0 \\ 190.530 & 9.39600 & 9.40846 & 0.133 & 9.51412 & 1.257 & 1.0 & 0.0 \\ 190.551 & 10.13900 & 10.13900 & 0.000 & 10.13900 & 0.000 & 1.0 & 0.0\end{array}$

Number of Points (Ref. 6) 38

$\begin{array}{lllllll}\text { AAD1-8 } & 0.055, & \text { BIAS1-8 } & 0.021, & \text { RMS1-8 } & 0.063 & \\ \text { AAD1 } & 0.003, & \text { BIAS1 } & 0.002, & \text { RMS1 } & 0.004 & \mathrm{mo1} \cdot \mathrm{dm}^{-3} \\ \text { AAD2-8 } & 0.150, & \text { BIAS2-8 } & 0.102, & \text { RMS2-8 } & 0.282 & \\ \text { AAD2 } & 0.011, & \text { BIAS2 } & 0.008, & \text { RMS2 } & 0.026 & \mathrm{~mol} \cdot \mathrm{dm}^{-3}\end{array}$

Data from Goodwin [3]

T $\quad \rho_{\sigma \mathrm{V}}, \exp \quad \rho_{\sigma \mathrm{V}}, \operatorname{eq}(3)$ Dev1 $\rho_{\sigma \mathrm{V}}$, SWEOS Dev2 Wt1 Wt2

$\mathrm{K} \quad \mathrm{mol} \cdot \mathrm{dm}^{-3} \quad \mathrm{~mol} \cdot \mathrm{dm}^{-3} \quad$ o $\quad \mathrm{mol} \cdot \mathrm{dm}^{-3} \quad$ \&

\begin{tabular}{|c|c|c|c|c|c|c|c|}
\hline 94.000 & 0.02290 & 0.02281 & -0.384 & 0.0228 & -0.313 & 0.0 & 0.0 \\
\hline 100.000 & $0.042 \quad 20$ & 0.04214 & -0.141 & 0.0421 & -0.120 & 0.0 & 0.0 \\
\hline 106.000 & 0.07220 & 0.07219 & -0.016 & 0.07219 & -0.010 & 0.0 & 0.0 \\
\hline 112.000 & 0.11620 & 0.11631 & 0.091 & 0.1163 & 0.104 & 0.0 & 0.0 \\
\hline 118.000 & 0.17810 & 0.17823 & 0.074 & 0.1782 & 0.109 & 0.0 & 0.0 \\
\hline 124.000 & 0.26210 & 0.26217 & 0.026 & 0.2623 & 0.087 & 0.0 & 0.0 \\
\hline 130.000 & 0.37300 & 0.37291 & -0.024 & 0.3732 & 0.058 & 0.0 & 0.0 \\
\hline 136.000 & 0.51640 & 0.51610 & -0.059 & 0.5165 & 0.030 & 0.0 & 0.0 \\
\hline 142.000 & 0.69920 & 0.69857 & -0.090 & 0.6991 & -0.011 & 0.0 & 0.0 \\
\hline 148.000 & 0.93010 & 0.92898 & -0.121 & 0.9294 & -0.065 & 0.0 & 0.0 \\
\hline
\end{tabular}


Data from Goodwin [3] (continued)

\begin{tabular}{|c|c|c|c|c|c|c|}
\hline $\mathrm{T}$ & $\rho_{\sigma \mathrm{v}}, \exp$ & $\rho_{\sigma \mathrm{V}}$, eq $(3)$ & Devl & $\rho_{\sigma \mathrm{V}}$, SWEOS & Dev2 & Wt1 \\
\hline K & $\mathrm{mol} \cdot \mathrm{dm}^{-3}$ & $\mathrm{~mol} \cdot \mathrm{dm}^{-3}$ & 8 & $\mathrm{~mol} \cdot \mathrm{dm}^{-3}$ & 8 & \\
\hline 154.000 & 1.22090 & 1.21873 & -0.178 & 1.21902 & -0.154 & 0.0 \\
\hline 160.000 & 1.58520 & 1.58383 & -0.087 & 1.58375 & -0.091 & 0.0 \\
\hline 169.067 & 2.34410 & 2.33630 & -0.333 & 2.33583 & -0.353 & 0.0 \\
\hline 169.270 & 2.36400 & 2.35681 & -0.304 & 2.35634 & -0.324 & 0.0 \\
\hline 169.417 & 2.38100 & 2.37178 & -0.387 & 2.37131 & -0.407 & 0.0 \\
\hline 169.601 & 2.40060 & 2.39067 & -0.414 & 2.39020 & -0.433 & 0.0 \\
\hline 169.794 & $2.418 \quad 80$ & 2.41066 & -0.336 & 2.41019 & -0.356 & 0.0 \\
\hline 173.088 & 2.79160 & 2.78259 & -0.323 & 2.78221 & -0.336 & 0.0 \\
\hline 173.290 & 2.81470 & 2.80749 & -0.256 & $2.807 \quad 12$ & -0.269 & 0.0 \\
\hline 173.489 & 2.84000 & 2.83228 & -0.272 & 2.83192 & -0.284 & 0.0 \\
\hline 173.473 & 2.84230 & 2.83028 & -0.423 & 2.82992 & -0.436 & 0.0 \\
\hline 173.675 & 2.86430 & 2.85569 & -0.301 & 2.85534 & -0.313 & 0.0 \\
\hline 173.857 & 2.88770 & $2.878 \quad 83$ & -0.307 & $2.878 \quad 49$ & -0.319 & 0.0 \\
\hline 177.094 & 3.34340 & 3.33277 & -0.318 & 3.33264 & -0.322 & 0.0 \\
\hline 177.328 & 3.37950 & 3.36912 & -0.307 & 3.36901 & -0.310 & 0.0 \\
\hline 181.389 & 4.12690 & 4.10390 & -0.557 & 4.10376 & -0.561 & 0.0 \\
\hline 181.768 & 4.20420 & 4.18503 & -0.456 & $4.184 \quad 83$ & -0.461 & 0.0 \\
\hline 183.514 & 4.61890 & 4.59776 & -0.458 & 4.59702 & -0.474 & 0.0 \\
\hline 184.285 & 4.82630 & 4.80497 & -0.442 & 4.80378 & -0.467 & 0.0 \\
\hline 184.510 & 4.88760 & 4.86898 & -0.381 & 4.86763 & -0.409 & 0.0 \\
\hline $\begin{array}{l}186.319 \\
188.545\end{array}$ & $\begin{array}{l}5.47950 \\
6.52780\end{array}$ & $\begin{array}{l}5.45946 \\
6.512 \quad 37\end{array}$ & $\begin{array}{l}-0.366 \\
-0.236\end{array}$ & $\begin{array}{l}5.45609 \\
6.503 \\
83\end{array}$ & $\begin{array}{l}-0.427 \\
-0.367\end{array}$ & $\begin{array}{l}0.0 \\
0.0\end{array}$ \\
\hline
\end{tabular}

Number of Points (Ref. 3) 32

$\begin{array}{lllllll}\text { AAD1- } 8 & 0.265, & \text { BIAS1-8 } & -0.253, & \text { RMS1-8 } & 0.168 & \\ \text { AAD1 } & 0.008, & \text { BIAS1 } & -0.008, & \text { RMS1 } & 0.007 & \mathrm{~mol} \cdot \mathrm{dm}^{-3} \\ \text { AAD2-8 } & 0.274, & \text { BIAS2 -8 } & -0.250, & \text { RMS2-8 } & 0.194 & \\ \text { AAD2 } & 0.009, & \text { BIAS2 } & -0.009, & \text { RMS2 } & 0.008 & \mathrm{~mol} \cdot \mathrm{dm}^{-3}\end{array}$


Data from Ricci and Scafe [29]

$\begin{array}{cccccccc}\mathrm{T} & \rho_{\sigma \mathrm{V}}, \exp & \rho_{\sigma \mathrm{V}}, \operatorname{eq}(3) & \text { Dev1 } & \rho_{\sigma \mathrm{V}}, \text { SWEOS } & \text { Dev2 } & \text { Wt1 } & \text { Wt2 } \\ \mathrm{K} & \mathrm{mol} \cdot \mathrm{dm}^{-3} & \mathrm{~mol} \cdot \mathrm{dm}^{-3} & 8 & \mathrm{~mol} \cdot \mathrm{dm}^{-3} & \text { \& } & & \\ & & & & & & & \\ 85.030 & 5.03800 & 5.02389 & -0.280 & 5.02210 & -0.316 & 0.0 & 0.0 \\ 86.030 & 5.38400 & 5.35431 & -0.552 & 5.35136 & -0.606 & 0.0 & 0.0 \\ 87.031 & 5.78600 & 5.74213 & -0.758 & 5.73748 & -0.839 & 0.0 & 0.0 \\ 88.031 & 6.27500 & 6.21623 & -0.937 & 6.20916 & -1.049 & 0.0 & 0.0 \\ 89.032 & 6.91800 & 6.84503 & -1.055 & 6.83519 & -1.197 & 0.0 & 0.0 \\ 90.032 & 7.96000 & 7.87493 & -1.069 & 7.87063 & -1.123 & 0.0 & 0.0\end{array}$

Number of Points (Ref. 29) 6

$\begin{array}{lllllll}\text { AAD1- } 8 & 0.775, & \text { BIAS1-8 } & -0.775, & \text { RMS1-8 } & 0.285 & \\ \text { AAD1 } & 0.051, & \text { BIAS1 } & -0.051, & \text { RMS1 } & 0.024 & \mathrm{~mol} \cdot \mathrm{dm}^{-3} \\ \text { AAD2-8 } & 0.855, & \text { BIAS2-8 } & -0.855, & \text { RMS2-8 } & 0.311 & \\ \text { AAD2 } & 0.055, & \text { BIAS2 } & -0.055, & \text { RMS2 } & 0.026 & \mathrm{~mol} \cdot \mathrm{dm}^{-3}\end{array}$

Data from Jansoone et al. [32]

$\begin{array}{cccccccc}\mathrm{T} & \rho_{\sigma \mathrm{V}}, \exp & \rho_{\sigma \mathrm{V}}, \operatorname{eq}(3) & \text { Dev1 } & \rho_{\sigma \mathrm{v}}, \text { SWEOS } & \text { Dev2 } & \text { Wt1 } & \text { Wt2 } \\ \mathrm{K} & \mathrm{mol} \cdot \mathrm{dm}^{-3} & \mathrm{~mol} \cdot \mathrm{dm}^{-3} & 8 & \mathrm{~mol} \cdot \mathrm{dm}^{-3} & 8 & & \\ 189.785 & 7.55100 & 7.54414 & -0.091 & 7.53540 & -0.207 & 0.0 & 0.0 \\ 190.066 & 7.93500 & 7.92811 & -0.087 & 7.92493 & -0.127 & 0.0 & 0.0 \\ 190.299 & 8.39200 & 8.38263 & -0.112 & 8.39517 & 0.038 & 0.0 & 0.0 \\ 190.500 & 9.16100 & 9.13930 & -0.237 & 9.21475 & 0.587 & 0.0 & 0.0 \\ 190.524 & 9.68600 & 9.34056 & -3.566 & 9.43901 & -2.550 & 0.0 & 0.0\end{array}$

Number of Points (Ref. 32) 5

$\begin{array}{lllllll}\text { AAD1- } 8 & 0.819, & \text { BIAS1-8 } & -0.819, & \text { RMS1- } 8 & 1.375 & \\ \text { AAD1 } & 0.078, & \text { BIAS1 } & -0.078, & \text { RMS1 } & 0.134 & \mathrm{~mol} \cdot \mathrm{dm}^{-3} \\ \text { AAD2- } 8 & 0.702, & \text { BIAS2 }-8 & -0.452, & \text { RMS2-8 } & 1.085 & \\ \text { AAD2 } & 0.066, & \text { BIAS2 } & -0.043, & \text { RMS2 } & 0.105 & \mathrm{~mol} \cdot \mathrm{dm}^{-3}\end{array}$


Data from Verbeke [26]

$\begin{array}{cccccccc}\mathrm{T} & \rho_{\sigma \mathrm{V}}, \exp & \rho_{\sigma \mathrm{V}}, \operatorname{eq}(3) & \text { Devl } & \rho_{\sigma \mathrm{V}}, \text { SWEOS } & \text { Dev2 } & \text { Wt1 } & \text { Wt2 } \\ \mathrm{K} & \mathrm{mol} \cdot \mathrm{dm}^{-3} & \mathrm{~mol} \cdot \mathrm{dm}^{-3} & 8 & \mathrm{~mol} \cdot \mathrm{dm}^{-3} & 8 & & \\ 190.070 & 8.00000 & 7.93453 & -0.818 & 7.93148 & -0.856 & 0.0 & 0.0 \\ 190.170 & 8.17000 & 8.10778 & -0.762 & 8.10933 & -0.743 & 0.0 & 0.0 \\ 190.270 & 8.36000 & 8.31399 & -0.550 & 8.32330 & -0.439 & 0.0 & 0.0 \\ 190.370 & 8.62000 & 8.57586 & -0.512 & 8.59953 & -0.237 & 0.0 & 0.0 \\ 190.470 & 9.00000 & 8.96186 & -0.424 & 9.01802 & 0.200 & 0.0 & 0.0\end{array}$

Number of Points (Ref. 26) 5

$\begin{array}{lllllll}\text { AAD1- } 8 & 0.613, & \text { BIAS1- } 8 & -0.613, & \text { RMS1- } 8 & 0.151 & \\ \text { AAD1 } & 0.051, & \text { BIAS1 } & -0.051, & \text { RMS1 } & 0.011 & \mathrm{~mol} \cdot \mathrm{dm}^{-3} \\ \text { AAD2-8 } & 0.495, & \text { BIAS2-8 } & -0.415, & \text { RMS2-8 } & 0.378 & \\ \text { AAD2 } & 0.041, & \text { BIAS2 } & -0.034, & \text { RMS2 } & 0.031 & \mathrm{~mol} \cdot \mathrm{dm}^{-3}\end{array}$

Data from eq (3)

\begin{tabular}{|c|c|c|c|c|c|c|c|c|}
\hline $\mathrm{T}$ & $\rho_{\sigma \mathrm{V}}, \exp$ & $\rho_{\sigma \mathrm{v}}, \mathrm{eq}(3)$ & Devl & $\rho_{\sigma \mathrm{V}}$, & SWEOS & Dev2 & Wt1 & Wt2 \\
\hline K & $\mathrm{mol} \cdot \mathrm{dm}^{-3}$ & $\mathrm{~mol} \cdot \mathrm{dm}^{-3}$ & 8 & mol & $\cdot \mathrm{dm}^{-3}$ & 8 & & \\
\hline 91.0 & $1.62281 \mathrm{E}-02$ & $1.62281 \mathrm{E}-02$ & - & 1.624 & $68 \mathrm{E}-02$ & 0.115 & - & 46595.385 \\
\hline 96.0 & $2.825 \quad 47 \mathrm{E}-02$ & $2.825 \quad 47 \mathrm{E}-02$ & - & 2.826 & $86 \mathrm{E}-02$ & 0.049 & - & 25531.209 \\
\hline 101.0 & $4.63183 \mathrm{E}-02$ & $4.63183 \mathrm{E}-02$ & - & 4.632 & $59 E-02$ & 0.017 & - & 14913.515 \\
\hline 106.0 & 7.218 88E-02 & $7.218 \quad 88 \mathrm{E}-02$ & - & 7.219 & $25 E-02$ & 0.005 & - & 9199.929 \\
\hline 111.0 & $1.07822 \mathrm{E}-01$ & $1.07822 \mathrm{E}-01$ & - & 1.078 & $33 E-01$ & 0.010 & - & 5947.908 \\
\hline 116.0 & $1.55363 \mathrm{E}-01$ & $1.55363 \mathrm{E}-01$ & - & 1.554 & 04E-01 & 0.027 & - & 4004.90 \\
\hline 121.0 & $2.17165 \mathrm{E}-01$ & $2.17165 \mathrm{E}-01$ & - & 2.172 & $70 E-01$ & 0.048 & - & 2793.88 \\
\hline 126.0 & $2.95844 \mathrm{E}-01$ & $2.958 \quad 44 \mathrm{E}-01$ & - & 2.960 & $50 E-01$ & 0.070 & - & 2010.58 \\
\hline 131.0 & $3.94353 \mathrm{E}-01$ & $3.94353 \mathrm{E}-01$ & - & 3.946 & $86 \mathrm{E}-01$ & 0.084 & - & 1487.11 \\
\hline 136.0 & $5.16096 \mathrm{E}-01$ & $5.16096 \mathrm{E}-01$ & - & 5.165 & $55 E-01$ & 0.089 & - & 1127.03 \\
\hline 141.0 & $6.65093 \mathrm{E}-01$ & $6.65093 \mathrm{E}-01$ & - & 6.656 & $38 \mathrm{E}-01$ & 0.082 & - & 1500.00 \\
\hline 146.0 & 8.462 19E-01 & 8.462 19E-01 & - & 8.467 & $63 E-01$ & 0.064 & - & 1500.00 \\
\hline 151.0 & $1.06557 \mathrm{E}+00$ & $1.06557 \mathrm{E}+00$ & - & 1.066 & $00 E+00$ & 0.040 & - & 1500.00 \\
\hline 156. & $1.33108 \mathrm{E}+00$ & $1.33108 \mathrm{E}+00$ & - & 1.331 & $27 \mathrm{E}+00$ & 0.014 & - & 1500.00 \\
\hline 161.0 & $1.65352 \mathrm{E}+00$ & $1.65352 \mathrm{E}+00$ & - & 1.653 & $39 E+00$ & -0.008 & - & 1500.00 \\
\hline
\end{tabular}


Data from eq. (3) (continued)

\begin{tabular}{|c|c|c|c|c|c|c|}
\hline$\rho_{\sigma \mathrm{V}}, \exp$ & $\rho_{\sigma \mathrm{v}}, \mathrm{eq}(3)$ & Dev1 & $\rho_{\sigma \mathrm{V}}$, SWEOS & Dev2 & Wtl & Wt2 \\
\hline $\mathrm{mol} \cdot \mathrm{dm}^{-3}$ & $\mathrm{~mol} \cdot \mathrm{dm}^{-3}$ & 8 & $\mathrm{~mol} \cdot \mathrm{dm}^{-3}$ & 8 & & \\
\hline $2.048 \quad 40 \mathrm{E}+00$ & $2.048 \quad 40 \mathrm{E}+00$ & - & $2.04800 \mathrm{E}+00$ & -0.020 & - & 3750.000 \\
\hline $2.53984 \mathrm{E}+00$ & $2.53984 E+00$ & - & $2.53939 \mathrm{E}+00$ & -0.018 & - & 3750.000 \\
\hline $3.16962 \mathrm{E}+00$ & $3.16962 \mathrm{E}+00$ & - & $3.169 \quad 42 \mathrm{E}+00$ & -0.006 & - & 3750.000 \\
\hline $4.02330 \mathrm{E}+00$ & $4.02330 \mathrm{E}+00$ & - & $4.02321 E+00$ & -0.002 & - & 3750.000 \\
\hline $5.34367 \mathrm{E}+00$ & $5.34367 \mathrm{E}+00$ & - & $5.34076 \mathrm{E}+00$ & -0.054 & - & 3750.000 \\
\hline
\end{tabular}

Number of Points (eq (3)) 20

$\begin{array}{lrlrllll}\text { AAD1- } 8 & - & \text { BIAS1-8 } & - & , & \text { RMS1-8 } & - & \\ \text { AAD1 } & -, & \text { BIAS1 } & - & \text { RMS1 } & - & \\ \text { AAD2-8 } & 0.041, & \text { BIAS2-8 } & 0.030, & \text { RMS2-8 } & 0.043 & \\ \text { AAD2 } & 0.354, & \text { BIAS2 } & -0.064, & \text { RMS2 } & 0.707 & \mathrm{~mol} \cdot \mathrm{dm}^{-3}\end{array}$

Total Points 86 for Ancillary Equation

$\begin{array}{lllllll}\text { AAD1- } 8 & 0.260, & \text { BIAS1-8 } & -0.222, & \text { RMS1-8 } & 0.454 & \\ \text { AAD1 } & 0.015, & \text { BIAS1 } & -0.013, & \text { RMS1 } & 0.041 & \mathrm{~mol} \cdot \mathrm{dm}^{-3}\end{array}$

Total Points 106 for Equation of State

$\begin{array}{lllllll}\text { AAD2- } 8 & 0.250, & \text { BIAS2-8 } & -0.123, & \text { RMS2 - } 8 & 0.418 & \\ \text { AAD2 } & 0.012, & \text { BIAS2 } & -0.011, & \text { RMS2 } & 0.037 & \mathrm{~mol} \cdot \mathrm{dm}^{-3}\end{array}$ 


\section{COMPARISONS OF EXPERIMENTAL AND CALCULATED PVT DATA}

Notes for Table A4. The first column marked Dev in the tables refers to deviations between the experimental pressure and the pressure calculated using the SWEOS and the experimental temperature and density. The statistics for these deviations are given under the Pressure heading. The second Dev column refers to deviations between the experimental density and the density calculated using the SWEOS and the experimental temperature and pressure. The statistics for these deviations are given under the Density heading. The weights in the tables are for the least squares determination of the coefficients in the equation of state. Some of the tabulated densities in Ref.[37] (and included in this table) were near the two-phase boundary and are based on alternative correlations rather than being direct experimental determinations. This also applies to the data Ref. [44] at temperatures above $250 \mathrm{~K}$.

Data from Achtermann et al. [33]

$\begin{array}{cccccccc}\mathrm{T} & \mathrm{P}, \text { exp } & \mathrm{P}, \mathrm{cal} & \text { Dev } & \rho, \text { exp } & \rho, \mathrm{cal} & \text { Dev } & \text { Wt } \\ \mathrm{K} & \mathrm{MPa} & \mathrm{MPa} & 8 & \mathrm{~mol} \cdot \mathrm{dm}^{-3} & \mathrm{~mol} \cdot \mathrm{dm}^{-3} & 8 & \\ 323.15 & 1.070 & 1.069 & -0.011 & 0.4035 & 0.4036 & 0.011 & 0.2263 \\ 323.15 & 1.550 & 1.550 & -0.017 & 0.5882 & 0.5883 & 0.017 & 0.1566 \\ 323.15 & 2.021 & 2.021 & -0.019 & 0.7716 & 0.7717 & 0.019 & 0.1204 \\ 323.15 & 2.467 & 2.467 & -0.026 & 0.9471 & 0.9474 & 0.027 & 0.0989 \\ 323.15 & 3.047 & 3.046 & -0.033 & 1.1779 & 1.1783 & 0.034 & 0.0803 \\ 323.15 & 3.559 & 3.558 & -0.038 & 1.3844 & 1.3849 & 0.040 & 0.0690 \\ 323.15 & 4.026 & 4.024 & -0.047 & 1.5746 & 1.5753 & 0.049 & 0.0611 \\ 323.15 & 4.512 & 4.509 & -0.049 & 1.7746 & 1.7755 & 0.052 & 0.0546 \\ 323.15 & 5.026 & 5.023 & -0.058 & 1.9886 & 1.9898 & 0.062 & 0.0492 \\ 323.15 & 5.528 & 5.524 & -0.061 & 2.1993 & 2.2007 & 0.065 & 0.0448 \\ 323.15 & 6.000 & 5.996 & -0.064 & 2.3996 & 2.4013 & 0.068 & 0.0413 \\ 323.15 & 6.546 & 6.541 & -0.066 & 2.6329 & 2.6348 & 0.071 & 0.0379 \\ 323.15 & 7.037 & 7.032 & -0.068 & 2.8448 & 2.8469 & 0.073 & 0.0353 \\ 323.15 & 8.049 & 8.043 & -0.069 & 3.2863 & 3.2888 & 0.075 & 0.0309 \\ 323.15 & 9.027 & 9.021 & -0.066 & 3.7180 & 3.7206 & 0.072 & 0.0276 \\ 323.15 & 10.067 & 10.062 & -0.056 & 4.1816 & 4.1841 & 0.060 & 0.0247 \\ 323.15 & 11.072 & 11.068 & -0.042 & 4.6321 & 4.6341 & 0.045 & 0.0224 \\ 323.15 & 12.070 & 12.067 & -0.027 & 5.0801 & 5.0816 & 0.029 & 0.0205 \\ 323.15 & 13.054 & 13.052 & -0.011 & 5.5210 & 5.5216 & 0.011 & 0.0189 \\ 323.15 & 14.079 & 14.080 & 0.006 & 5.9777 & 5.9774 & -0.006 & 0.0174\end{array}$


Data from Achtermann et al. [33] (continued)

$\begin{array}{cccccccc}\mathrm{T} & \text { P,exp } & \text { P,cal } & \text { Dev } & \rho, \exp & \rho, \mathrm{cal} & \text { Dev } & \text { Wt } \\ \mathrm{K} & \mathrm{MPa} & \mathrm{MPa} & 8 & \mathrm{~mol} \cdot \mathrm{dm}^{-3} & \mathrm{~mol} \cdot \mathrm{dm}^{-3} & 8 & \\ 323.15 & 15.092 & 15.095 & 0.022 & 6.4243 & 6.4229 & -0.022 & 0.0161 \\ 323.15 & 16.068 & 16.073 & 0.029 & 6.8484 & 6.8464 & -0.029 & 0.0150 \\ 323.15 & 17.081 & 17.088 & 0.042 & 7.2813 & 7.2783 & -0.042 & 0.0140 \\ 323.15 & 18.084 & 18.093 & 0.050 & 7.7011 & 7.6973 & -0.049 & 0.0131 \\ 323.15 & 18.988 & 18.999 & 0.055 & 8.0706 & 8.0664 & -0.052 & 0.0123 \\ 323.15 & 20.142 & 20.154 & 0.060 & 8.5292 & 8.5245 & -0.055 & 0.0114 \\ 323.15 & 21.125 & 21.138 & 0.063 & 8.9078 & 8.9028 & -0.056 & 0.0108 \\ 323.15 & 22.103 & 22.116 & 0.061 & 9.2728 & 9.2679 & -0.053 & 0.0101 \\ 323.15 & 23.109 & 23.123 & 0.058 & 9.6360 & 9.6312 & -0.050 & 0.0095 \\ 323.15 & 24.101 & 24.113 & 0.054 & 9.9816 & 9.9771 & -0.045 & 0.0090 \\ 323.15 & 25.107 & 25.118 & 0.046 & 10.3196 & 10.3158 & -0.037 & 0.0085 \\ 323.15 & 26.126 & 26.136 & 0.040 & 10.6497 & 10.6464 & -0.031 & 0.0080 \\ 323.15 & 27.139 & 27.148 & 0.033 & 10.9656 & 10.9628 & -0.025 & 0.0076 \\ 323.15 & 28.112 & 28.120 & 0.028 & 11.2581 & 11.2557 & -0.021 & 0.0072 \\ 323.15 & 28.668 & 28.675 & 0.024 & 11.4203 & 11.4183 & -0.017 & 0.0070\end{array}$

Number of Points (Ref. 33) 35

$\begin{array}{llrlrlrl}\text { PRESSURE : } & \text { AAD }-8 & 0.043 & \text { BIAS }-8 & -0.004 & \text { RMS }-8 & 0.046 & \\ & \text { AAD } & 0.006 & \text { BIAS } & 0.003 & \text { RMS } & 0.007 & \mathrm{MPa} \\ \text { DENSITY : } & \text { AAD }-8 & 0.042 & \text { BIAS }-8 & 0.008 & \text { RMS }-8 & 0.046 & \\ & \text { AAD } & 0.002 & \text { BIAS } & -0.001 & \text { RMS } & 0.003 & \mathrm{~mol} \cdot \mathrm{dm}^{-3}\end{array}$

Data from Achtermann et al. [34]

$\begin{array}{cccccccc}\text { T } & \text { P,exp } & \text { P,cal } & \text { Dev } & \rho, \exp & \rho, \text { cal } & \text { Dev } & \text { Wt } \\ \text { K } & \text { MPa } & \text { MPa } & 8 & \mathrm{~mol} \cdot \mathrm{dm}^{-3} & \mathrm{~mol} \cdot \mathrm{dm}^{-3} & 8 & \\ & & & & & & \\ 273.15 & 0.102 & 0.102 & -0.072 & 0.0449 & 0.0449 & 0.072 & 0.0000 \\ 273.15 & 0.625 & 0.625 & -0.010 & 0.2794 & 0.2794 & 0.010 & 0.0000 \\ 273.15 & 1.086 & 1.086 & 0.004 & 0.4909 & 0.4909 & -0.004 & 0.0000 \\ 273.15 & 1.590 & 1.589 & -0.010 & 0.7273 & 0.7274 & 0.010 & 0.0000 \\ 273.15 & 2.102 & 2.102 & -0.014 & 0.9739 & 0.9740 & 0.015 & 0.0000\end{array}$


Data from Achtermann et al. [34] (continued)

\begin{tabular}{|c|c|c|c|c|c|c|c|}
\hline $\mathrm{T}$ & $P, \exp$ & P,cal & Dev & $\rho, \exp$ & $\rho$, cal & Dev & Wt \\
\hline K & $\mathrm{MPa}$ & $\mathrm{MPa}$ & 8 & $\mathrm{~mol} \cdot \mathrm{dm}^{-3}$ & $\mathrm{~mol} \cdot \mathrm{dm}^{-3}$ & 8 & \\
\hline 273.15 & 2.568 & 2.567 & -0.018 & 1.2038 & 1.2040 & 0.019 & 0.0000 \\
\hline 273.15 & 3.061 & 3.060 & -0.029 & 1.4527 & 1.4532 & 0.032 & 0.0000 \\
\hline 273.15 & 3.542 & 3.541 & -0.024 & 1.7018 & 1.7022 & 0.026 & 0.0000 \\
\hline 273.15 & 4.046 & 4.045 & -0.026 & 1.9691 & 1.9697 & 0.029 & 0.0000 \\
\hline 273.15 & 4.466 & 4.465 & -0.033 & 2.1968 & 2.1976 & 0.036 & 0.0000 \\
\hline 273.15 & 5.026 & 5.024 & -0.044 & 2.5074 & 2.5086 & 0.049 & 0.0000 \\
\hline 273.15 & 5.492 & 5.490 & -0.047 & 2.7729 & 2.7744 & 0.053 & 0.0000 \\
\hline 273.15 & 6.013 & 6.008 & -0.077 & 3.0748 & 3.0775 & 0.088 & 0.0000 \\
\hline 273.15 & 6.544 & 6.539 & -0.086 & 3.3911 & 3.3945 & 0.100 & 0.0000 \\
\hline 273.15 & 6.967 & 6.961 & -0.088 & 3.6477 & 3.6515 & 0.103 & 0.0000 \\
\hline 273.15 & 7.191 & 7.184 & -0.096 & 3.7853 & 3.7896 & 0.113 & 0.0000 \\
\hline 273.15 & 8.586 & 8.585 & -0.007 & 4.6745 & 4.6749 & 0.008 & 0.0000 \\
\hline 273.15 & 7.297 & 7.294 & -0.050 & 3.8534 & 3.8556 & 0.058 & 0.0000 \\
\hline 273.15 & 6.185 & 6.180 & -0.077 & 3.1764 & 3.1792 & 0.089 & 0.0000 \\
\hline 273.15 & 5.224 & 5.220 & -0.088 & 2.6184 & 2.6210 & 0.100 & 0.0000 \\
\hline 273.15 & 4.398 & 4.394 & -0.089 & 2.1584 & 2.1605 & 0.100 & 0.0000 \\
\hline 273.15 & 3.692 & 3.688 & -0.087 & 1.7792 & 1.7809 & 0.096 & 0.0000 \\
\hline 273.15 & 3.090 & 3.087 & -0.079 & 1.4667 & 1.4680 & 0.085 & 0.0000 \\
\hline 273.15 & 2.580 & 2.578 & -0.069 & 1.2090 & 1.2099 & 0.074 & 0.0000 \\
\hline 273.15 & 2.149 & 2.148 & -0.066 & 0.9966 & 0.9973 & 0.070 & 0.0000 \\
\hline 273.15 & 1.788 & 1.787 & -0.060 & 0.8215 & 0.8220 & 0.062 & 0.0000 \\
\hline 273.15 & 1.485 & 1.484 & -0.050 & 0.6772 & 0.6776 & 0.052 & 0.0000 \\
\hline 273.15 & 1.231 & 1.231 & -0.049 & 0.5582 & 0.5585 & 0.050 & 0.0000 \\
\hline 273.15 & 1.020 & 1.020 & -0.053 & 0.4601 & 0.4603 & 0.054 & 0.0000 \\
\hline 273.15 & 0.844 & 0.844 & -0.035 & 0.3793 & 0.3794 & 0.035 & 0.0000 \\
\hline 273.15 & 0.699 & 0.698 & -0.053 & 0.3126 & 0.3128 & 0.054 & 0.0000 \\
\hline 273.15 & 0.577 & 0.577 & -0.045 & 0.2577 & 0.2578 & 0.045 & 0.0000 \\
\hline 273.15 & 0.477 & 0.477 & -0.038 & 0.2124 & 0.2125 & 0.039 & 0.0000 \\
\hline 273.15 & 0.394 & 0.394 & -0.036 & 0.1751 & 0.1752 & 0.037 & 0.0000 \\
\hline 273.15 & 0.325 & 0.325 & -0.048 & 0.1443 & 0.1444 & 0.048 & 0.0000 \\
\hline 273.15 & 0.269 & 0.269 & 0.027 & 0.1190 & 0.1190 & -0.027 & 0.0000 \\
\hline 273.15 & 0.221 & 0.222 & 0.052 & 0.0981 & 0.0980 & -0.052 & 0.0000 \\
\hline 273.15 & 0.183 & 0.183 & -0.027 & 0.0808 & 0.0808 & 0.027 & 0.0000 \\
\hline 283.15 & 0.236 & 0.236 & -0.027 & 0.1008 & 0.1008 & 0.027 & 0.0000 \\
\hline 283.15 & 0.331 & 0.331 & 0.006 & 0.1418 & 0.1418 & -0.006 & 0.0000 \\
\hline
\end{tabular}


Data from Achtermann et al. [34] (continued)

\begin{tabular}{|c|c|c|c|c|c|c|c|}
\hline $\mathrm{T}$ & $P, \exp$ & P,cal & Dev & $\rho, \exp$ & $\rho, \mathrm{cal}$ & Dev & Wt \\
\hline $\mathrm{K}$ & $\mathrm{MPa}$ & $\mathrm{MPa}$ & 8 & $\mathrm{~mol} \cdot \mathrm{dm}^{-3}$ & $\mathrm{~mol} \cdot \mathrm{dm}^{-3}$ & 8 & \\
\hline 283.15 & 0.426 & 0.426 & -0.010 & 0.1826 & 0.1826 & 0.010 & 0.0000 \\
\hline 283.15 & 0.526 & 0.526 & -0.029 & 0.2258 & 0.2259 & 0.030 & 0.0000 \\
\hline 283.15 & 0.623 & 0.623 & -0.035 & 0.2682 & 0.2683 & 0.035 & 0.0000 \\
\hline 283.15 & 0.718 & 0.718 & -0.023 & 0.3096 & 0.3097 & 0.023 & 0.0000 \\
\hline 283.15 & 0.913 & 0.913 & -0.032 & 0.3954 & 0.3955 & 0.033 & 0.0000 \\
\hline 283.15 & 1.109 & 1.109 & -0.037 & 0.4821 & 0.4823 & 0.038 & 0.0000 \\
\hline 283.15 & 1.280 & 1.279 & -0.052 & 0.5582 & 0.5585 & 0.053 & 0.0000 \\
\hline 283.15 & 1.611 & 1.610 & -0.047 & 0.7078 & 0.7081 & 0.049 & 0.0000 \\
\hline 283.15 & 1.638 & 1.637 & -0.054 & 0.7198 & 0.7202 & 0.056 & 0.0000 \\
\hline 283.15 & 2.100 & 2.098 & -0.060 & 0.9320 & 0.9326 & 0.063 & 0.0000 \\
\hline 283.15 & 2.156 & 2.155 & -0.056 & 0.9582 & 0.9588 & 0.058 & 0.0000 \\
\hline 283.15 & 2.591 & 2.590 & -0.062 & 1.1626 & 1.1634 & 0.066 & 0.0000 \\
\hline 283.15 & 3.070 & 3.067 & -0.074 & 1.3914 & 1.3925 & 0.079 & 0.0000 \\
\hline 283.15 & 3.154 & 3.152 & -0.063 & 1.4324 & 1.4334 & 0.067 & 0.0000 \\
\hline 283.15 & 3.555 & 3.552 & -0.075 & 1.6284 & 1.6297 & 0.081 & 0.0000 \\
\hline 283.15 & 4.043 & 4.040 & -0.074 & 1.8717 & 1.8732 & 0.081 & 0.0000 \\
\hline 283.15 & 4.105 & 4.103 & -0.065 & 1.9034 & 1.9048 & 0.071 & 0.0000 \\
\hline 283.15 & 4.541 & 4.538 & -0.069 & 2.1255 & 2.1271 & 0.075 & 0.0000 \\
\hline 283.15 & 5.014 & 5.010 & -0.069 & 2.3707 & 2.3725 & 0.076 & 0.0000 \\
\hline 283.15 & 5.515 & 5.512 & -0.064 & 2.6359 & 2.6378 & 0.072 & 0.0000 \\
\hline 283.15 & 5.884 & 5.880 & -0.066 & 2.8343 & 2.8364 & 0.074 & 0.0000 \\
\hline 283.15 & 5.999 & 5.995 & -0.069 & 2.8965 & 2.8987 & 0.077 & 0.0000 \\
\hline 283.15 & 6.509 & 6.504 & -0.068 & 3.1761 & 3.1786 & 0.077 & 0.0000 \\
\hline 283.15 & 6.722 & 6.718 & -0.059 & 3.2951 & 3.2973 & 0.067 & 0.0000 \\
\hline 283.15 & 6.991 & 6.988 & -0.052 & 3.4461 & 3.4482 & 0.060 & 0.0000 \\
\hline 283.15 & 7.477 & 7.473 & -0.046 & 3.7216 & 3.7236 & 0.053 & 0.0000 \\
\hline 283.15 & 7.531 & 7.528 & -0.036 & 3.7529 & 3.7544 & 0.041 & 0.0000 \\
\hline 283.15 & 8.466 & 8.467 & 0.009 & 4.2973 & 4.2969 & -0.010 & 0.0000 \\
\hline 283.15 & 7.160 & 7.158 & -0.021 & 3.5425 & 3.5434 & 0.024 & 0.0000 \\
\hline 283.15 & 6.041 & 6.038 & -0.037 & 2.9201 & 2.9213 & 0.042 & 0.0000 \\
\hline 283.15 & 5.082 & 5.080 & -0.038 & 2.4072 & 2.4082 & 0.042 & 0.0000 \\
\hline 283.15 & 4.264 & 4.262 & -0.035 & 1.9843 & 1.9851 & 0.039 & 0.0000 \\
\hline 283.15 & 3.568 & 3.567 & -0.030 & 1.6357 & 1.6362 & 0.032 & 0.0000 \\
\hline 283.15 & 2.979 & 2.978 & -0.023 & 1.3483 & 1.3486 & 0.025 & 0.0000 \\
\hline 283.15 & 2.482 & 2.482 & -0.021 & 1.1114 & 1.1116 & 0.022 & 0.0000 \\
\hline
\end{tabular}


Data from Achtermann et a1. [34] (continued)

\begin{tabular}{|c|c|c|c|c|c|c|c|}
\hline $\mathrm{T}$ & $P, \exp$ & P,cal & Dev & $\rho, \exp$ & $\rho, \mathrm{cal}$ & Dev & Wt \\
\hline K & $\mathrm{MPa}$ & $\mathrm{MPa}$ & 8 & $\mathrm{~mol} \cdot \mathrm{dm}^{-3}$ & $\mathrm{~mol} \cdot \mathrm{dm}^{-3}$ & 8 & \\
\hline 283.15 & 2.064 & 2.064 & -0.005 & 0.9162 & 0.9162 & 0.005 & 0.0000 \\
\hline 283.15 & 1.714 & 1.714 & -0.005 & 0.7552 & 0.7552 & 0.005 & 0.0000 \\
\hline 283.15 & 1.422 & 1.422 & -0.004 & 0.6225 & 0.6225 & 0.004 & 0.0000 \\
\hline 283.15 & 1.178 & 1.178 & 0.012 & 0.5132 & 0.5131 & -0.012 & 0.0000 \\
\hline 283.15 & 0.976 & 0.975 & -0.001 & 0.4230 & 0.4230 & 0.001 & 0.0000 \\
\hline 283.15 & 0.807 & 0.807 & 0.023 & 0.3487 & 0.3486 & -0.024 & 0.0000 \\
\hline 283.15 & 0.667 & 0.667 & -0.013 & 0.2874 & 0.2874 & 0.013 & 0.0000 \\
\hline 283.15 & 0.551 & 0.551 & -0.007 & 0.2369 & 0.2369 & 0.007 & 0.0000 \\
\hline 283.15 & 0.455 & 0.455 & 0.012 & 0.1953 & 0.1953 & -0.013 & 0.0000 \\
\hline 283.15 & 0.376 & 0.376 & 0.025 & 0.1610 & 0.1610 & -0.025 & 0.0000 \\
\hline 283.15 & 0.310 & 0.310 & -0.019 & 0.1327 & 0.1327 & 0.019 & 0.0000 \\
\hline 283.15 & 0.256 & 0.256 & 0.018 & 0.1094 & 0.1094 & -0.018 & 0.0000 \\
\hline 283.15 & 0.211 & 0.211 & 0.031 & 0.0902 & 0.0902 & -0.031 & 0.0000 \\
\hline 283.15 & 0.174 & 0.174 & -0.050 & 0.0743 & 0.0743 & 0.050 & 0.0000 \\
\hline 283.15 & 0.144 & 0.144 & 0.084 & 0.0613 & 0.0612 & -0.085 & 0.0000 \\
\hline 293.15 & 0.098 & 0.098 & 0.035 & 0.0405 & 0.0405 & -0.035 & 0.0000 \\
\hline 293.15 & 0.243 & 0.243 & -0.029 & 0.1002 & 0.1002 & 0.029 & 0.0000 \\
\hline 293.15 & 0.353 & 0.353 & -0.013 & 0.1458 & 0.1458 & 0.013 & 0.0000 \\
\hline 293.15 & 0.433 & 0.433 & 0.006 & 0.1793 & 0.1793 & -0.006 & 0.0000 \\
\hline 293.15 & 0.524 & 0.524 & -0.012 & 0.2171 & 0.2171 & 0.012 & 0.0000 \\
\hline 293.15 & 0.637 & 0.637 & -0.020 & 0.2644 & 0.2645 & 0.020 & 0.0000 \\
\hline 293.15 & 0.734 & 0.734 & -0.016 & 0.3051 & 0.3051 & 0.016 & 0.0000 \\
\hline 293.15 & 0.931 & 0.931 & -0.019 & 0.3886 & 0.3887 & 0.020 & 0.0000 \\
\hline 293.15 & 1.137 & 1.137 & -0.024 & 0.4763 & 0.4764 & 0.024 & 0.0000 \\
\hline 293.15 & 1.312 & 1.312 & -0.032 & 0.5515 & 0.5517 & 0.033 & 0.0000 \\
\hline 293.15 & 1.621 & 1.621 & -0.036 & 0.6853 & 0.6856 & 0.037 & 0.0000 \\
\hline 293.15 & 1.908 & 1.908 & -0.038 & 0.8110 & 0.8113 & 0.040 & 0.0000 \\
\hline 293.15 & 2.313 & 2.312 & -0.035 & 0.9903 & 0.9907 & 0.037 & 0.0000 \\
\hline 293.15 & 2.642 & 2.641 & -0.042 & 1.1384 & 1.1389 & 0.044 & 0.0000 \\
\hline 293.15 & 3.080 & 3.079 & -0.037 & 1.3380 & 1.3385 & 0.039 & 0.0000 \\
\hline 293.15 & 3.565 & 3.564 & -0.038 & 1.5629 & 1.5635 & 0.041 & 0.0000 \\
\hline 293.15 & 4.064 & 4.063 & -0.038 & 1.7983 & 1.7990 & 0.040 & 0.0000 \\
\hline 293.15 & 4.265 & 4.263 & -0.049 & 1.8940 & 1.8950 & 0.053 & 0.0000 \\
\hline 293.15 & 4.540 & 4.538 & -0.048 & 2.0267 & 2.0278 & 0.052 & 0.0000 \\
\hline 293.15 & 5.024 & 5.022 & -0.050 & 2.2625 & 2.2637 & 0.055 & 0.0000 \\
\hline
\end{tabular}


Data from Achtermann et al. [34] (continued)

\begin{tabular}{|c|c|c|c|c|c|c|c|}
\hline $\mathrm{T}$ & $P, \exp$ & $\mathrm{P}, \mathrm{cal}$ & Dev & $\rho, \exp$ & $\rho$, cal & Dev & Wt \\
\hline $\mathrm{K}$ & $\mathrm{MPa}$ & $\mathrm{MPa}$ & 8 & $\mathrm{~mol} \cdot \mathrm{dm}^{-3}$ & $\mathrm{~mol} \cdot \mathrm{dm}^{-3}$ & 8 & \\
\hline 293.15 & 5.514 & 5.511 & -0.042 & 2.5052 & 2.5063 & 0.046 & 0.0000 \\
\hline 293.15 & 6.005 & 6.002 & -0.049 & 2.7521 & 2.7536 & 0.054 & 0.0000 \\
\hline 293.15 & 6.493 & 6.490 & -0.039 & 3.0016 & 3.0029 & 0.043 & 0.0000 \\
\hline 293.15 & 6.979 & 6.977 & -0.036 & 3.2536 & 3.2549 & 0.040 & 0.0000 \\
\hline 293.15 & 7.466 & 7.463 & -0.036 & 3.5087 & 3.5101 & 0.041 & 0.0000 \\
\hline 293.15 & 7.705 & 7.702 & -0.037 & 3.6353 & 3.6368 & 0.042 & 0.0000 \\
\hline 293.15 & 8.409 & 8.406 & -0.036 & 4.0120 & 4.0136 & 0.040 & 0.0000 \\
\hline 293.15 & 7.083 & 7.079 & -0.055 & 3.3073 & 3.3094 & 0.062 & 0.0000 \\
\hline 293.15 & 5.954 & 5.951 & -0.061 & 2.7263 & 2.7281 & 0.068 & 0.0000 \\
\hline 293.15 & 4.994 & 4.991 & -0.061 & 2.2474 & 2.2489 & 0.066 & 0.0000 \\
\hline 293.15 & 4.179 & 4.176 & -0.054 & 1.8526 & 1.8537 & 0.058 & 0.0000 \\
\hline 293.15 & 3.489 & 3.487 & -0.046 & 1.5271 & 1.5278 & 0.049 & 0.0000 \\
\hline 293.15 & 2.907 & 2.906 & -0.044 & 1.2588 & 1.2594 & 0.046 & 0.0000 \\
\hline 293.15 & 2.419 & 2.418 & -0.059 & 1.0377 & 1.0383 & 0.062 & 0.0000 \\
\hline 293.15 & 2.009 & 2.008 & -0.051 & 0.8554 & 0.8559 & 0.053 & 0.0000 \\
\hline 293.15 & 1.667 & 1.666 & -0.047 & 0.7051 & 0.7054 & 0.048 & 0.0000 \\
\hline 293.15 & 1.381 & 1.381 & -0.040 & 0.5812 & 0.5814 & 0.041 & 0.0000 \\
\hline 293.15 & 1.144 & 1.143 & -0.031 & 0.4791 & 0.4793 & 0.032 & 0.0000 \\
\hline 293.15 & 0.946 & 0.946 & -0.022 & 0.3949 & 0.3950 & 0.023 & 0.0000 \\
\hline 293.15 & 0.782 & 0.782 & -0.023 & 0.3255 & 0.3256 & 0.023 & 0.0000 \\
\hline 293.15 & 0.646 & 0.646 & -0.035 & 0.2683 & 0.2684 & 0.035 & 0.0000 \\
\hline 293.15 & 0.534 & 0.534 & -0.011 & 0.2212 & 0.2212 & 0.012 & 0.0000 \\
\hline 293.15 & 0.441 & 0.441 & -0.027 & 0.1823 & 0.1823 & 0.027 & 0.0000 \\
\hline 293.15 & 0.364 & 0.364 & -0.009 & 0.1503 & 0.1503 & 0.009 & 0.0000 \\
\hline 293.15 & 0.300 & 0.300 & -0.034 & 0.1239 & 0.1239 & 0.034 & 0.0000 \\
\hline 293.15 & 0.248 & 0.248 & -0.016 & 0.1021 & 0.1021 & 0.017 & 0.0000 \\
\hline 293.15 & 0.204 & 0.205 & 0.026 & 0.0842 & 0.0842 & -0.026 & 0.0000 \\
\hline 293.15 & 0.169 & 0.169 & 0.017 & 0.0694 & 0.0694 & -0.017 & 0.0000 \\
\hline 293.15 & 0.139 & 0.139 & 0.001 & 0.0572 & 0.0572 & -0.001 & 0.0000 \\
\hline
\end{tabular}

Number of Points (Ref. 34) 139

$\begin{array}{llllrlll}\text { PRESSURE : } & \text { AAD }-8 & 0.040 & \text { BIAS }-8 & -0.034 & \text { RMS }-8 & 0.030 & \\ & \text { AAD } & 0.001 & \text { BIAS } & -0.001 & \text { RMS } & 0.002 & \mathrm{MPa} \\ \text { DENSITY : } & \text { AAD }-8 & 0.043 & \text { BIAS }-8 & 0.037 & \text { RMS }-8 & 0.033 & \\ & \text { AAD } & 0.001 & \text { BIAS } & 0.001 & \text { RMS } & 0.001 & \mathrm{~mol} \cdot \mathrm{dm}^{-3}\end{array}$


Data from Cheng [35]

\begin{tabular}{|c|c|c|c|c|c|c|c|}
\hline $\mathrm{T}$ & $P, \exp$ & P,cal & Dev & $\rho, \exp$ & $\rho$, cal & Dev & Wt \\
\hline $\mathrm{K}$ & $\mathrm{MPa}$ & $\mathrm{MPa}$ & 8 & $\mathrm{~mol} \cdot \mathrm{dm}^{-3}$ & $\mathrm{~mol} \cdot \mathrm{dm}^{-3}$ & 8 & \\
\hline 111.23 & 28.700 & 33.440 & 16.514 & 27.9096 & 27.7278 & -0.651 & 0.0136 \\
\hline 111.23 & 40.200 & 44.534 & 10.780 & 28.3046 & 28.1549 & -0.529 & 0.2020 \\
\hline 111.23 & 51.900 & 57.667 & 11.113 & 28.7274 & 28.5470 & -0.628 & 0.1794 \\
\hline 111.23 & 72.600 & 79.897 & 10.052 & 29.3600 & 29.1621 & -0.674 & 0.1516 \\
\hline 121.19 & 21.800 & 22.251 & 2.068 & 26.7237 & 26.7019 & -0.081 & 0.0183 \\
\hline 121.19 & 33.500 & 34.321 & 2.452 & 27.2628 & 27.2285 & -0.126 & 0.0155 \\
\hline 121.19 & 74.600 & 75.071 & 0.631 & 28.6780 & 28.6640 & -0.049 & 0.1730 \\
\hline 121.19 & 94.200 & 94.341 & 0.150 & 29.2141 & 29.2104 & -0.013 & 0.1506 \\
\hline 121.19 & 114.400 & 113.820 & -0.507 & 29.7000 & 29.7138 & 0.046 & 0.1336 \\
\hline 131.79 & 46.500 & 48.655 & 4.634 & 27.1518 & 27.0645 & -0.322 & 0.2489 \\
\hline 131.79 & 60.700 & 63.311 & 4.301 & 27.7008 & 27.6082 & -0.335 & 0.2133 \\
\hline 131.79 & 74.800 & 77.646 & 3.805 & 28.1770 & 28.0865 & -0.321 & 0.1877 \\
\hline 131.79 & 89.200 & 91.881 & 3.006 & 28.6041 & 28.5267 & -0.271 & 0.1680 \\
\hline 131.79 & 104.800 & 107.675 & 2.743 & 29.0360 & 28.9603 & -0.261 & 0.1507 \\
\hline 143.82 & 48.300 & 49.093 & 1.642 & 26.4131 & 26.3774 & -0.135 & 0.2861 \\
\hline 143.82 & 70.500 & 70.968 & 0.664 & 27.2926 & 27.2756 & -0.062 & 0.2236 \\
\hline 143.82 & 96.800 & 96.598 & -0.208 & 28.1373 & 28.1434 & 0.022 & 0.1792 \\
\hline 156.96 & 49.500 & 48.046 & -2.937 & 25.5297 & 25.6039 & 0.291 & 0.3400 \\
\hline 156.96 & 61.700 & 61.235 & -0.754 & 26.1575 & 26.1780 & 0.078 & 0.2839 \\
\hline 156.96 & 76.900 & 75.511 & -1.806 & 26.7451 & 26.7982 & 0.199 & 0.2417 \\
\hline 156.96 & 95.100 & 93.085 & -2.119 & 27.3748 & 27.4417 & 0.244 & 0.2049 \\
\hline 167.61 & 41.500 & 41.589 & 0.214 & 24.4618 & 24.4562 & -0.023 & 0.4340 \\
\hline 167.61 & 56.000 & 55.754 & -0.439 & 25.2653 & 25.2779 & 0.050 & 0.3423 \\
\hline 167.61 & 75.100 & 74.609 & -0.654 & 26.1370 & 26.1575 & 0.078 & 0.2687 \\
\hline 167.61 & 93.600 & 92.773 & -0.883 & 26.8384 & 26.8679 & 0.110 & 0.2234 \\
\hline 179.29 & 48.800 & 49.037 & 0.486 & 24.1604 & 24.1455 & -0.062 & 0.4358 \\
\hline 179.29 & 75.800 & 75.419 & -0.503 & 25.5493 & 25.5664 & 0.067 & 0.2951 \\
\hline 179.29 & 100.300 & 99.289 & -1.008 & 26.5111 & 26.5477 & 0.138 & 0.2296 \\
\hline 192.87 & 33.400 & 34.083 & 2.045 & 22.0605 & 21.9933 & -0.304 & 0.0015 \\
\hline 192.87 & 52.900 & 53.319 & 0.792 & 23.5849 & 23.5573 & -0.117 & 0.0009 \\
\hline 192.87 & 71.400 & 71.610 & 0.294 & 24.6427 & 24.6319 & -0.044 & 0.0007 \\
\hline 192 & 97.900 & 97.370 & -0.542 & 25.7998 & 25.8208 & 0.081 & 0.0005 \\
\hline 212.00 & 40.100 & 39.993 & -0.268 & 21.2404 & 21.2514 & 0.052 & 0.4820 \\
\hline 212.00 & 58.600 & 58.230 & -0.632 & 22.7739 & 22.7998 & 0.114 & 0.3094 \\
\hline 212.00 & 74.600 & 73.651 & -1.273 & 23.7417 & 23.7949 & 0.224 & 0.2378 \\
\hline
\end{tabular}


Data from Cheng [35] (continued)

\begin{tabular}{|c|c|c|c|c|c|c|c|}
\hline $\mathrm{T}$ & $P, \exp$ & P,cal & Dev & $\rho, \exp$ & $\rho$, cal & Dev & Wt \\
\hline K & $\mathrm{MPa}$ & $\mathrm{MPa}$ & 8 & $\mathrm{~mol} \cdot \mathrm{dm}^{-3}$ & $\mathrm{~mol} \cdot \mathrm{dm}^{-3}$ & 8 & \\
\hline 212.00 & 95.000 & 93.595 & -1.479 & 24.7464 & 24.8095 & 0.255 & 0.1832 \\
\hline 225.07 & 42.800 & 42.659 & -0.330 & 20.6101 & 20.6252 & 0.073 & 0.5187 \\
\hline 225.07 & 62.500 & 61.376 & -1.799 & 22.2519 & 22.3331 & 0.365 & 0.3269 \\
\hline 225.07 & 82.300 & 80.755 & -1.877 & 23.4797 & 23.5647 & 0.362 & 0.2362 \\
\hline 225.07 & 108.700 & 106.345 & -2.167 & 24.7219 & 24.8216 & 0.403 & 0.1728 \\
\hline 237.57 & 30.200 & 30.295 & 0.314 & 17.9437 & 17.9260 & -0.098 & 0.0034 \\
\hline 237.57 & 60.600 & 60.209 & -0.645 & 21.4638 & 21.4951 & 0.146 & 0.3722 \\
\hline 237.57 & 94.600 & 93.635 & -1.021 & 23.5793 & 23.6283 & 0.208 & 0.2156 \\
\hline 249.43 & 41.100 & 41.437 & 0.820 & 18.8076 & 18.7619 & -0.243 & 0.7017 \\
\hline 249.43 & 58.100 & 57.853 & -0.425 & 20.6016 & 20.6238 & 0.108 & 0.4320 \\
\hline 249.43 & 74.400 & 73.885 & -0.692 & 21.8579 & 21.8932 & 0.161 & 0.3118 \\
\hline 249.43 & 94.200 & 93.131 & -1.135 & 23.0256 & 23.0828 & 0.249 & 0.2327 \\
\hline 261.55 & 40.700 & 40.850 & 0.369 & 17.9147 & 17.8923 & -0.125 & 0.8045 \\
\hline 261.55 & 53.300 & 53.246 & -0.102 & 19.4666 & 19.4724 & 0.030 & 0.5348 \\
\hline 261.55 & 68.000 & 67.150 & -1.250 & 20.7512 & 20.8194 & 0.329 & 0.3842 \\
\hline 261.55 & 82.600 & 81.065 & -1.858 & 21.7628 & 21.8625 & 0.458 & 0.2981 \\
\hline 261.55 & 100.100 & 97.875 & -2.222 & 22.7583 & 22.8763 & 0.518 & 0.2337 \\
\hline 278.71 & 34.800 & 35.181 & 1.093 & 15.7754 & 15.6977 & -0.492 & 0.0040 \\
\hline 278.71 & 50.000 & 50.473 & 0.945 & 18.1752 & 18.1163 & -0.324 & 0.4391 \\
\hline 278.71 & 62.400 & 62.920 & 0.834 & 19.5160 & 19.4667 & -0.253 & 0.3145 \\
\hline 278.71 & 77.400 & 77.392 & -0.011 & 20.7211 & 20.7217 & 0.003 & 0.2339 \\
\hline 278.71 & 99.900 & 99.329 & -0.571 & 22.1288 & 22.1607 & 0.144 & 0.1668 \\
\hline 293.41 & 46.500 & 46.390 & -0.236 & 16.7983 & 16.8144 & 0.096 & 0.5525 \\
\hline 293.41 & 64.000 & 63.485 & -0.805 & 18.8466 & 18.8972 & 0.269 & 0.3379 \\
\hline 293.41 & 79.100 & 78.868 & -0.293 & 20.1776 & 20.1952 & 0.087 & 0.2462 \\
\hline 293.41 & 94.700 & 93.829 & -0.920 & 21.2089 & 21.2631 & 0.256 & 0.1930 \\
\hline 309.29 & 36.900 & 36.877 & -0.061 & 14.2227 & 14.2275 & 0.033 & 0.8834 \\
\hline 309.29 & 51.600 & 51.431 & -0.327 & 16.6694 & 16.6923 & 0.137 & 0.5110 \\
\hline 309.29 & 66.700 & 67.047 & 0.521 & 18.4570 & 18.4231 & -0.184 & 0.3384 \\
\hline 309.29 & 84.700 & 84.449 & -0.297 & 19.9283 & 19.9468 & 0.093 & 0.2406 \\
\hline 309.29 & 105.800 & 105.101 & -0.661 & 21.2721 & 21.3122 & 0.189 & 0.1769 \\
\hline
\end{tabular}

Number of Points (Ref. 35) 66

$\begin{array}{llllrlrl}\text { PRESSURE : } & \text { AAD }-8 & 1.803 & \text { BIAS }-8 & 0.721 & \text { RMS }-8 & 3.315 & \\ & \text { AAD } & 1.080 & \text { BIAS } & 0.205 & \text { RMS } & 1.739 & \mathrm{MPa} \\ \text { DENSITY : } & \text { AAD }-8 & 0.204 & \text { BIAS }-8 & 0.001 & \text { RMS }-8 & 0.261 & \\ & \text { AAD } & 0.049 & \text { BIAS } & -0.002 & \text { RMS } & 0.065 & \mathrm{~mol} \cdot \mathrm{dm}^{-3}\end{array}$


Data from Douslin et al. [36]

\begin{tabular}{|c|c|c|c|c|c|c|c|}
\hline $\mathrm{T}$ & $P, \exp$ & $P, c a l$ & Dev & $\rho, \exp$ & $\rho, \mathrm{cal}$ & Dev & Wt \\
\hline $\mathrm{K}$ & $\mathrm{MPa}$ & $\mathrm{MPa}$ & 8 & $\mathrm{~mol} \cdot \mathrm{dm}^{-3}$ & $\mathrm{~mol} \cdot \mathrm{dm}^{-3}$ & 8 & \\
\hline 273.15 & 1.638 & 1.637 & -0.029 & 0.7500 & 0.7502 & 0.030 & 0.0150 \\
\hline 298.14 & 1.802 & 1.801 & -0.028 & 0.7500 & 0.7502 & 0.029 & 0.0135 \\
\hline 303.14 & 1.835 & 1.834 & -0.021 & 0.7500 & 0.7502 & 0.021 & 0.0133 \\
\hline 323.14 & 1.966 & 1.965 & -0.008 & 0.7500 & 0.7501 & 0.008 & 0.0124 \\
\hline 348.14 & 2.129 & 2.129 & -0.005 & 0.7500 & 0.7500 & 0.006 & 0.0114 \\
\hline 373.15 & 2.293 & 2.293 & -0.002 & 0.7500 & 0.7500 & 0.002 & 0.0106 \\
\hline 398.16 & 2.456 & 2.456 & -0.003 & 0.7500 & 0.7500 & 0.003 & 0.0098 \\
\hline 273.15 & 2.156 & 2.155 & -0.040 & 1.0000 & 1.0004 & 0.042 & 0.0114 \\
\hline 298.14 & 2.379 & 2.378 & -0.033 & 1.0000 & 1.0003 & 0.034 & 0.0103 \\
\hline 303.14 & 2.423 & 2.422 & -0.036 & 1.0000 & 1.0004 & 0.037 & 0.0101 \\
\hline 323.14 & 2.601 & 2.600 & -0.023 & 1.0000 & 1.0002 & 0.024 & 0.0094 \\
\hline 348.14 & 2.822 & 2.822 & -0.018 & 1.0000 & 1.0002 & 0.018 & 0.0086 \\
\hline 373.15 & 3.043 & 3.043 & -0.012 & 1.0000 & 1.0001 & 0.012 & 0.0080 \\
\hline 398.16 & 3.264 & 3.263 & -0.007 & 1.0000 & 1.0001 & 0.007 & 0.0074 \\
\hline 273.15 & 3.154 & 3.152 & -0.052 & 1.5000 & 1.5008 & 0.056 & 0.0079 \\
\hline 298.14 & 3.499 & 3.498 & -0.046 & 1.5000 & 1.5007 & 0.048 & 0.0071 \\
\hline 303.14 & 3.569 & 3.567 & -0.053 & 1.5000 & 1.5008 & 0.056 & 0.0069 \\
\hline 323.14 & 3.843 & 3.842 & -0.029 & 1.5000 & 1.5005 & 0.031 & 0.0064 \\
\hline 348.14 & 4.185 & 4.184 & -0.024 & 1.5000 & 1.5004 & 0.025 & 0.0058 \\
\hline 373.15 & 4.527 & 4.526 & -0.020 & 1.5000 & 1.5003 & 0.020 & 0.0054 \\
\hline 398.16 & 4.868 & 4.867 & -0.021 & 1.5000 & 1.5003 & 0.021 & 0.0050 \\
\hline 273.15 & 4.105 & 4.102 & -0.068 & 2.0000 & 2.0015 & 0.076 & 0.0061 \\
\hline 298.14 & 4.580 & 4.578 & -0.057 & 2.0000 & 2.0012 & 0.062 & 0.0054 \\
\hline 303.14 & 4.675 & 4.672 & -0.059 & 2.0000 & 2.0013 & 0.064 & 0.0053 \\
\hline 323.14 & 5.053 & 5.050 & -0.052 & 2.0000 & 2.0011 & 0.055 & 0.0049 \\
\hline 348.14 & 5.522 & 5.521 & -0.025 & 2.0000 & 2.0005 & 0.026 & 0.0044 \\
\hline 373.15 & 5.992 & 5.990 & -0.041 & 2.0000 & 2.0008 & 0.042 & 0.0041 \\
\hline 398.16 & 6.458 & 6.457 & -0.021 & 2.0000 & 2.0004 & 0.022 & 0.0038 \\
\hline 273.15 & 5.014 & 5.010 & -0.069 & 2.5000 & 2.5019 & 0.077 & 0.0051 \\
\hline 298.14 & 5.626 & 5.623 & -0.061 & 2.5000 & 2.5017 & 0.067 & 0.0045 \\
\hline 303.14 & 5.748 & 5.745 & -0.064 & 2.5000 & 2.5017 & 0.069 & 0.0044 \\
\hline 323.14 & 6.234 & 6.231 & -0.050 & 2.5000 & 2.5013 & 0.054 & 0.0040 \\
\hline 348.14 & 6.839 & 6.836 & -0.040 & 2.5000 & 2.5010 & 0.041 & 0.0036 \\
\hline 373.15 & 7.442 & 7.439 & -0.047 & 2.5000 & 2.5012 & 0.049 & 0.0033 \\
\hline 398.16 & 8.041 & 8.039 & -0.023 & 2.5000 & 2.5006 & 0.023 & 0.0030 \\
\hline
\end{tabular}


Data from Douslin et al. [36] (continued)

\begin{tabular}{|c|c|c|c|c|c|c|c|}
\hline $\mathrm{T}$ & $P, \exp$ & P,cal & Dev & $\rho, \exp$ & $\rho$, cal & Dev & Wt \\
\hline K & $\mathrm{MPa}$ & $\mathrm{MPa}$ & 8 & $\mathrm{~mol} \cdot \mathrm{dm}^{-3}$ & $\mathrm{~mol} \cdot \mathrm{dm}^{-3}$ & 8 & \\
\hline 273.15 & 5.884 & 5.881 & -0.055 & 3.0000 & 3.0019 & 0.063 & 0.0044 \\
\hline 298.14 & 6.641 & 6.638 & -0.057 & 3.0000 & 3.0019 & 0.063 & 0.0038 \\
\hline 303.14 & 6.794 & 6.788 & -0.083 & 3.0000 & 3.0027 & 0.091 & 0.0037 \\
\hline 323.14 & 7.393 & 7.389 & -0.051 & 3.0000 & 3.0016 & 0.055 & 0.0034 \\
\hline 348.14 & 8.139 & 8.136 & -0.043 & 3.0000 & 3.0014 & 0.045 & 0.0030 \\
\hline 373.15 & 8.884 & 8.879 & -0.052 & 3.0000 & 3.0016 & 0.054 & 0.0027 \\
\hline 398.16 & 9.623 & 9.619 & -0.039 & 3.0000 & 3.0012 & 0.040 & 0.0025 \\
\hline 273.15 & 6.722 & 6.719 & -0.050 & 3.5000 & 3.5020 & 0.058 & 0.0038 \\
\hline 298.14 & 7.631 & 7.627 & -0.046 & 3.5000 & 3.5018 & 0.051 & 0.0033 \\
\hline 303.14 & 7.815 & 7.808 & -0.085 & 3.5000 & 3.5033 & 0.094 & 0.0032 \\
\hline 323.14 & 8.532 & 8.528 & -0.048 & 3.5000 & 3.5018 & 0.052 & 0.0029 \\
\hline 348.14 & 9.428 & 9.424 & -0.043 & 3.5000 & 3.5016 & 0.046 & 0.0026 \\
\hline 373.15 & 10.320 & 10.315 & -0.054 & 3.5000 & 3.5019 & 0.055 & 0.0024 \\
\hline 398.16 & 11.207 & 11.202 & -0.048 & 3.5000 & 3.5017 & 0.049 & 0.0022 \\
\hline 273.15 & 7.531 & 7.529 & -0.027 & 4.0000 & 4.0013 & 0.031 & 0.0034 \\
\hline 298.14 & 8.599 & 8.596 & -0.036 & 4.0000 & 4.0016 & 0.041 & 0.0029 \\
\hline 303.14 & 8.814 & 8.809 & -0.065 & 4.0000 & 4.0029 & 0.072 & 0.0029 \\
\hline 323.14 & 9.658 & 9.655 & -0.041 & 4.0000 & 4.0018 & 0.044 & 0.0026 \\
\hline 348.14 & 10.709 & 10.706 & -0.031 & 4.0000 & 4.0013 & 0.032 & 0.0023 \\
\hline 373.15 & 11.758 & 11.752 & -0.048 & 4.0000 & 4.0020 & 0.049 & 0.0021 \\
\hline 398.16 & 12.799 & 12.793 & -0.048 & 4.0000 & 019 & 0.048 & 0.0019 \\
\hline 273.15 & 8.316 & 8.315 & -0.005 & 4.5000 & 4.5003 & 0.006 & 0.0031 \\
\hline 298.14 & 9.551 & 9.549 & -0.020 & 4.5000 & 4.5010 & 0.022 & 0.0027 \\
\hline 303.14 & 9.800 & 9.795 & -0.056 & 4.5000 & 4.5028 & 0.062 & 0.0026 \\
\hline 323.14 & 10.778 & 10.773 & -0.049 & 4.5000 & 4.5024 & 0.053 & 0.0023 \\
\hline 348.14 & 11.992 & 11.988 & -0.035 & 4.5000 & 16 & 0. & 0.0020 \\
\hline 373.15 & 13.201 & 13.196 & -0.039 & 4.5000 & 4.5018 & 0.039 & 0.0018 \\
\hline 398.16 & 14.408 & 14.399 & -0.066 & 4.5000 & 4.5029 & 0.065 & 0.0017 \\
\hline 273.15 & 9.080 & 9.083 & 0.030 & 5.0000 & 4.9982 & -0.036 & 0.0029 \\
\hline 298.14 & 10.491 & 10.491 & 0.003 & 5.0000 & 4.9998 & -0.003 & 0.0024 \\
\hline 303.14 & 10.774 & 10.772 & -0.027 & 5.0000 & 5.0015 & 0.029 & 0.0023 \\
\hline 323.14 & 11.891 & 11.888 & -0.030 & 5.0000 & 5.0016 & 0.032 & 0.0021 \\
\hline 348.14 & 13.278 & 13.274 & -0.029 & 5.0000 & 5.0015 & 0.030 & 0.0018 \\
\hline 373.15 & 14.658 & 14.653 & -0.036 & 5.0000 & 5.0018 & 0.036 & 0.0016 \\
\hline 398.16 & 16.035 & 16.025 & -0.060 & 5.0000 & 5.0029 & 0.058 & 0.0015 \\
\hline
\end{tabular}


Data from Douslin et a1. [36] (continued)

\begin{tabular}{|c|c|c|c|c|c|c|c|}
\hline $\mathrm{T}$ & $P, \exp$ & P,cal & Dev & $\rho, \exp$ & $\rho, \mathrm{cal}$ & Dev & Wt \\
\hline K & $\mathrm{MPa}$ & $\mathrm{MPa}$ & 8 & $\mathrm{~mol} \cdot \mathrm{dm}^{-3}$ & $\mathrm{~mol} \cdot \mathrm{dm}^{-3}$ & 8 & \\
\hline 273.15 & 9.832 & 9.837 & 0.051 & 5.5000 & 5.4967 & -0.061 & 0.0027 \\
\hline 298.14 & 11.426 & 11.428 & 0.013 & 5.5000 & 5.4992 & -0.014 & 0.0022 \\
\hline 303.14 & 11.745 & 11.744 & -0.007 & 5.5000 & 5.5004 & 0.007 & 0.0021 \\
\hline 323.14 & 13.003 & 13.005 & 0.014 & 5.5000 & 5.4992 & -0.015 & 0.0019 \\
\hline 348.14 & 14.575 & 14.571 & -0.024 & 5.5000 & 5.5014 & 0.025 & 0.0017 \\
\hline 373.15 & 16.132 & 16.129 & -0.021 & 5.5000 & 5.5012 & 0.021 & 0.0015 \\
\hline 398.16 & 17.689 & 17.678 & -0.059 & 5.5000 & 5.5031 & 0.057 & 0.0013 \\
\hline 273.15 & 10.568 & 10.580 & 0.118 & 6.0000 & 5.9916 & -0.141 & 0.0025 \\
\hline 298.14 & 12.358 & 12.363 & 0.034 & 6.0000 & 5.9978 & -0.037 & 0.0020 \\
\hline 303.14 & 12.716 & 12.717 & 0.010 & 6.0000 & 5.9993 & -0.011 & 0.0020 \\
\hline 323.14 & 14.126 & 14.130 & 0.025 & 6.0000 & 5.9984 & -0.026 & 0.0017 \\
\hline 348.14 & 15.890 & 15.885 & -0.032 & 6.0000 & 6.0019 & 0.032 & 0.0015 \\
\hline 373.15 & 17.631 & 17.629 & -0.009 & 6.0000 & 6.0005 & 0.009 & 0.0013 \\
\hline 398.16 & 19.370 & 19.365 & -0.026 & 6.0000 & 6.0015 & 0.025 & 0.0012 \\
\hline 273.15 & 11.306 & 11.320 & 0.124 & 6.5000 & 6.4905 & -0.146 & 0.0023 \\
\hline 298.14 & 13.294 & 13.302 & 0.058 & 6.5000 & 6.4959 & -0.063 & 0.0019 \\
\hline 303.14 & 13.692 & 13.696 & 0.031 & 6.5000 & 6.4978 & -0.033 & 0.0018 \\
\hline 323.14 & 15.269 & 15.268 & -0.010 & 6.5000 & 6.5007 & 0.010 & 0.0016 \\
\hline 348.14 & 17.220 & 17.220 & 0.004 & 6.5000 & 6.4997 & -0.004 & 0.0014 \\
\hline 373.15 & 19.159 & 19.162 & 0.015 & 6.5000 & 6.4990 & -0.015 & 0.0012 \\
\hline 398.16 & 21.101 & 21.093 & -0.042 & 6.5000 & 6.5 & 0.0 & 0.0011 \\
\hline 273.15 & 12.049 & 12.059 & 0.079 & 7.0000 & 6.9936 & -0.092 & 0.0021 \\
\hline 298.14 & 14.241 & 14.251 & 0.067 & 7.0000 & 6.9950 & -0.071 & 0.0017 \\
\hline 303.14 & 14.681 & 14.687 & 0.041 & 7.0000 & 6.9970 & -0.043 & 0.0017 \\
\hline 323.14 & 16.421 & 16.425 & 0.025 & 7.0000 & 6.9982 & -0.026 & 0.0015 \\
\hline 348.14 & 18.587 & 18.585 & -0.008 & 7.0 & 7.0 & 0.008 & 0.0013 \\
\hline 373.15 & 20.735 & 20.732 & -0.011 & 7.0000 & 7.0007 & 0.010 & 0.0011 \\
\hline 398.16 & 22.871 & 22.869 & -0.008 & 7.0000 & 7.0005 & 0.007 & 0.0010 \\
\hline 273.15 & 12.785 & 12.803 & 0.141 & 7.5000 & 7.4880 & -0.160 & 0.0020 \\
\hline 298.14 & 15.204 & 15.215 & 0.070 & 7.5000 & 7.4945 & -0.073 & 0.0016 \\
\hline 303.14 & 15.678 & 15.695 & 0.105 & 7.5000 & 7.4919 & -0.108 & 0.0016 \\
\hline 323.14 & 17.597 & 17.608 & 0.064 & 7.5000 & 7.4953 & -0.063 & 0.0014 \\
\hline 348.14 & 19.981 & 19.986 & 0.024 & 7.5000 & 7.4983 & -0.023 & 0.0012 \\
\hline 373.15 & 22.344 & 22.349 & 0.025 & 7.5000 & 7.4983 & -0.022 & 0.0010 \\
\hline 398.16 & 24.705 & 24.701 & -0.017 & 7.5000 & 7.5011 & 0.015 & 0.0009 \\
\hline
\end{tabular}


Data from Douslin et a1. [36] (continued)

\begin{tabular}{|c|c|c|c|c|c|c|c|}
\hline $\mathrm{T}$ & $P, \exp$ & P,cal & Dev & $\rho, \exp$ & $\rho, \mathrm{cal}$ & Dev & Wt \\
\hline K & $\mathrm{MPa}$ & $\mathrm{MPa}$ & 8 & $\mathrm{~mol} \cdot \mathrm{dm}^{-3}$ & $\mathrm{~mol} \cdot \mathrm{dm}^{-3}$ & 8 & \\
\hline 273.15 & 13.541 & 13.558 & 0.129 & 8.0000 & 7.9886 & -0.143 & 0.0019 \\
\hline 298.14 & 16.187 & 16.200 & 0.079 & 8.0000 & 7.9936 & -0.080 & 0.0015 \\
\hline 303.14 & 16.713 & 16.726 & 0.076 & 8.0000 & 7.9939 & -0.076 & 0.0014 \\
\hline 323.14 & 18.814 & 18.823 & 0.047 & 8.0000 & 7.9964 & -0.045 & 0.0012 \\
\hline 348.14 & 21.422 & 21.430 & 0.036 & 8.0000 & 7.9974 & -0.033 & 0.0011 \\
\hline 373.15 & 24.008 & 24.021 & 0.055 & 8.0000 & 7.9961 & -0.048 & 0.0009 \\
\hline 398.16 & 26.602 & 26.599 & -0.013 & 8.0000 & 8.0009 & 0.011 & 0.0008 \\
\hline 273.15 & 14.309 & 14.330 & 0.147 & 8.5000 & 8.4866 & -0.158 & 0.0017 \\
\hline 298.14 & 17.198 & 17.214 & 0.091 & 8.5000 & 8.4924 & -0.089 & 0.0014 \\
\hline 303.14 & 17.774 & 17.788 & 0.082 & 8.5000 & 8.4933 & -0.079 & 0.0013 \\
\hline 323.14 & 20.067 & 20.078 & 0.054 & 8.5000 & 8.4958 & -0.050 & 0.0011 \\
\hline 348.14 & 22.919 & 22.925 & 0.025 & 8.5000 & 8.4982 & -0.022 & 0.0010 \\
\hline 373.15 & 25.743 & 25.755 & 0.049 & 8.5000 & 8.4964 & -0.042 & 0.0009 \\
\hline 398.16 & 28.576 & 28.571 & -0.017 & 8.5000 & 8.5012 & 0.014 & 0.0008 \\
\hline 273.15 & 15.109 & 15.124 & 0.099 & 9.0000 & 8.9908 & -0.102 & 0.0016 \\
\hline 298.14 & 18.247 & 18.262 & 0.084 & 9.0000 & 8.9928 & -0.080 & 0.0013 \\
\hline 303.14 & 18.873 & 18.888 & 0.077 & 9.0000 & 8.9935 & -0.072 & 0.0012 \\
\hline 323.14 & 21.376 & 21.381 & 0.023 & 9.0000 & 8.9981 & -0.021 & 0.0011 \\
\hline 348.14 & 24.477 & 24.480 & 0.016 & 9.0000 & 8.9988 & -0.014 & 0.0009 \\
\hline 373.15 & 27.543 & 27.562 & 0.069 & 9.0000 & 8.9949 & -0.057 & 0.0008 \\
\hline 398.16 & 30.621 & 30.628 & 0.022 & 9.0000 & 8.9984 & -0.018 & 0.0007 \\
\hline 273.15 & 15.931 & 15.949 & 0.113 & 9.5000 & 9.4893 & -0.112 & 0.0015 \\
\hline 298.14 & 19.342 & 19.354 & 0.062 & 9.5000 & 9.4946 & -0.057 & 0.0012 \\
\hline 303.14 & 20.012 & 20.033 & 0.109 & 9.5000 & 9.4907 & -0.098 & 0.0011 \\
\hline 323.14 & 22.737 & 22.740 & 0.016 & 9.5000 & 9.4987 & -0.014 & 0.0010 \\
\hline 348.14 & 26.102 & 26.106 & 0.013 & 9.5000 & 9.4990 & -0.011 & 0.0008 \\
\hline 373.15 & 29.448 & 29.452 & 0.014 & 9.5000 & 9.4989 & -0.012 & 0.0007 \\
\hline 398.16 & 32.786 & 32.780 & -0.020 & 9.5000 & 9.5015 & 0.016 & 0.0006 \\
\hline 273.15 & 16.798 & 16.810 & 0.075 & 10.0000 & 9.9928 & -0.072 & 0.0014 \\
\hline 298.14 & 20.483 & 20.498 & 0.078 & 10.0000 & 9.9932 & -0.068 & 0.0011 \\
\hline 303.14 & 21.210 & 21.234 & 0.112 & 10.0000 & 9.9903 & -0.097 & 0.0010 \\
\hline 323.14 & 24.153 & 24.166 & 0.051 & 10.0000 & 9.9958 & -0.042 & 0.0009 \\
\hline 348.14 & 27.805 & 27.811 & 0.024 & 10.0000 & 9.9981 & -0.019 & 0.0008 \\
\hline 373.15 & 31.425 & 31.436 & 0.034 & 10.0000 & 9.9974 & -0.026 & 0.0007 \\
\hline 398.16 & 35.049 & 35.040 & -0.027 & 10.0000 & 10.0021 & 0.021 & 0.0059 \\
\hline
\end{tabular}


Data from Douslin et al. [36] (continued)

\begin{tabular}{|c|c|c|c|c|c|c|c|}
\hline $\mathrm{T}$ & $P, \exp$ & P,cal & Dev & $\rho, \exp$ & $\rho$, cal & Dev & Wt \\
\hline K & $\mathrm{MPa}$ & $\mathrm{MPa}$ & 8 & $\mathrm{~mol} \cdot \mathrm{dm}^{-3}$ & $\mathrm{~mol} \cdot \mathrm{dm}^{-3}$ & 8 & \\
\hline 273.15 & 17.700 & 17.719 & 0.104 & 10.5000 & 10.4902 & -0.094 & 0.0013 \\
\hline 298.14 & 21.694 & 21.704 & 0.047 & 10.5000 & 10.4959 & -0.039 & 0.0010 \\
\hline 303.14 & 22.480 & 22.499 & 0.082 & 10.5000 & 10.4929 & -0.068 & 0.0010 \\
\hline 323.14 & 25.657 & 25.668 & 0.042 & 10.5000 & 10.4965 & -0.033 & 0.0008 \\
\hline 348.14 & 29.601 & 29.608 & 0.024 & 10.5000 & 10.4981 & -0.018 & 0.0007 \\
\hline 373.15 & 33.510 & 33.525 & 0.045 & 10.5000 & 10.4965 & -0.034 & 0.0006 \\
\hline 398.16 & 37.430 & 37.420 & -0.027 & 10.5000 & 10.5021 & 0.020 & 0.0054 \\
\hline 273.15 & 18.678 & 18.682 & 0.023 & 11.0000 & 10.9978 & -0.020 & 0.0012 \\
\hline 298.14 & 22.969 & 22.982 & 0.057 & 11.0000 & 10.9950 & -0.045 & 0.0009 \\
\hline 303.14 & 23.829 & 23.840 & 0.043 & 11.0000 & 10.9963 & -0.034 & 0.0009 \\
\hline 323.14 & 27.254 & 27.259 & 0.017 & 11.0000 & 10.95 & -0.0 & 0.0008 \\
\hline 348.14 & 31.503 & 31.509 & 0.020 & 11.0000 & 10.9984 & -0.015 & 0.0006 \\
\hline 373.15 & 35.730 & 35.734 & 0.013 & 11.0000 & 10.9990 & -0.009 & 0.0055 \\
\hline 398.16 & 39.952 & 39.935 & -0.044 & 11.0000 & 11.0034 & 0.031 & 0.0049 \\
\hline 273.15 & 19.705 & 19.713 & 0.038 & 11.5000 & 11.4965 & -0.031 & 0.0011 \\
\hline 298.14 & 24.345 & 24.344 & -0.006 & 11.5000 & 11.5005 & 0.004 & 0.0008 \\
\hline 303.14 & 25.251 & 25.268 & 0.067 & 11.5000 & 11.4943 & -0.050 & 0.0008 \\
\hline 323.14 & 28.954 & 28.950 & -0.014 & 11.5000 & 11.5011 & 0.010 & 0.0007 \\
\hline 348.14 & 33.510 & 33.528 & 0.053 & 11.5000 & 11.4958 & -0.037 & 0.0006 \\
\hline 373.15 & 38.087 & 38.077 & -0.025 & 11.5000 & 11.5020 & 0.017 & 0.0051 \\
\hline 73.15 & 20.821 & 20.821 & 0.000 & 12.0000 & 12.0000 & 0.000 & 0.0010 \\
\hline 298.14 & 25.795 & 25.803 & 0.029 & 12.0000 & 11.9975 & -0.021 & 0.0008 \\
\hline 303.14 & 26.788 & 26.796 & 0.029 & 12.0000 & 11.9975 & -0.021 & 0.0007 \\
\hline 323.14 & 30.755 & 30.757 & 0.004 & 12.0000 & 11.9997 & -0.003 & 0.0006 \\
\hline 348.14 & 35.665 & 35.679 & 0.039 & 12.0000 & 11.9969 & -0.026 & 0.0053 \\
\hline 15 & 40.593 & 40.569 & -0.059 & 12.0000 & 12.0047 & 0.0 & 0.0046 \\
\hline 273.15 & 22.028 & 22.021 & -0.030 & 12.5000 & 12.5026 & 0.021 & 0.0009 \\
\hline 298.14 & 27.379 & 27.373 & -0.023 & 12.5000 & 12.5019 & 0.015 & 0.0007 \\
\hline 303.14 & 28.425 & 28.440 & 0.052 & 12.5000 & 12.4957 & -0.034 & 0.0007 \\
\hline 323.14 & 32.697 & 32.693 & -0.012 & 12.5000 & 12.5010 & 0.008 & 0.0006 \\
\hline 348.14 & 37.999 & 37.978 & -0.0 & 12.5000 & 12.5044 & 0.0 & 0.0048 \\
\hline & & 3.924 & -0.006 & 1.0000 & 1.0001 & 0.006 & 0.0061 \\
\hline 473.19 & 7.854 & 7.852 & -0.031 & 2.0000 & 2.0006 & 0.030 & 0.0031 \\
\hline 473.19 & 11.832 & 11.826 & -0.056 & 3.0000 & 3.0017 & 0.055 & 0.0020 \\
\hline 473.19 & 15.904 & 15.893 & -0.064 & 4.0000 & 4.0025 & 0.062 & 0.0015 \\
\hline
\end{tabular}


Data from Douslin et al. [36] (continued)

\begin{tabular}{|c|c|c|c|c|c|c|c|}
\hline $\mathbf{T}$ & $P, \exp$ & P,cal & Dev & $\rho, \exp$ & $\rho$, cal & Dev & Wt \\
\hline K & $\mathrm{MPa}$ & $\mathrm{MPa}$ & 8 & $\mathrm{~mol} \cdot \mathrm{dm}^{-3}$ & $\mathrm{~mol} \cdot \mathrm{dm}^{-3}$ & 8 & \\
\hline 473.19 & 20.121 & 20.108 & -0.063 & 5.0000 & 5.0030 & 0.059 & 0.0012 \\
\hline 473.19 & 24.542 & 24.528 & -0.055 & 6.0000 & 6.0030 & 0.050 & 0.0009 \\
\hline 473.19 & 29.239 & 29.222 & -0.058 & 7.0000 & 7.0035 & 0.050 & 0.0008 \\
\hline 473.19 & 34.287 & 34.263 & -0.072 & 8.0000 & 8.0047 & 0.059 & 0.0006 \\
\hline 473.19 & 39.759 & 39.738 & -0.053 & 9.0000 & 9.0037 & 0.041 & 0.0052 \\
\hline 523.21 & 4.363 & 4.363 & -0.012 & 1.0000 & 1.0001 & 0.012 & 0.0055 \\
\hline 523.21 & 8.780 & 8.777 & -0.038 & 2.0000 & 2.0007 & 0.037 & 0.0027 \\
\hline 523.21 & 13.294 & 13.287 & -0.054 & 3.0000 & 3.0016 & 0.052 & 0.0018 \\
\hline 523.21 & 17.960 & 17.945 & -0.087 & 4.0000 & 4.0033 & 0.082 & 0.0013 \\
\hline 523.21 & 22.835 & 22.808 & -0.119 & 5.0000 & 5.0054 & 0.109 & 0.0010 \\
\hline 523.21 & 27.975 & 27.941 & -0.122 & 6.0000 & 6.0065 & 0.108 & 0.0008 \\
\hline 523.21 & 33.457 & 33.419 & -0.114 & 7.0000 & 7.0067 & 0.096 & 0.0007 \\
\hline 523.21 & 39.363 & 39.324 & -0.100 & 8.0000 & 8.0064 & 0.080 & 0.0054 \\
\hline 573.22 & 4.802 & 4.801 & -0.017 & 1.0000 & 1.0002 & 0.017 & 0.0050 \\
\hline 573.22 & 9.703 & 9.699 & -0.037 & 2.0000 & 2.0007 & 0.036 & 0.0024 \\
\hline 573.22 & 14.751 & 14.743 & -0.055 & 3.0000 & 3.0016 & 0.053 & 0.0016 \\
\hline 573.22 & 20.005 & 19.986 & -0.093 & 4.0000 & 4.0035 & 0.087 & 0.0012 \\
\hline 573.22 & 25.527 & 25.493 & -0.131 & 5.0000 & 5.0059 & 0.118 & 0.0009 \\
\hline 573.22 & 31.384 & 31.335 & -0.156 & 6.0000 & 6.0081 & 0.135 & 0.0007 \\
\hline 573.22 & 37.639 & 37.592 & -0.125 & 7.0000 & 7.0072 & 0.103 & 0.0058 \\
\hline 623.22 & 5.240 & 5.238 & -0.032 & 1.0000 & 1.0003 & 0.031 & 0.0046 \\
\hline 623.22 & 10.625 & 10.619 & -0.056 & 2.0000 & 2.0011 & 0.055 & 0.0022 \\
\hline 623.22 & 16.207 & 16.193 & -0.085 & 3.0000 & 3.0024 & 0.081 & 0.0014 \\
\hline 623.22 & 22.049 & 22.020 & -0.133 & 4.0000 & 4.0049 & 0.123 & 0.0010 \\
\hline 623.22 & 28.216 & 28.167 & -0.173 & 5.0000 & 5.0077 & 0.154 & 0.0008 \\
\hline 623.22 & 34.777 & 34.713 & -0.183 & 6.0000 & 6.0094 & 0.156 & 0.0006 \\
\hline
\end{tabular}

Number of Points (Ref. 36) 201

$\begin{array}{llllrlll}\text { PRESSURE: } & \text { AAD }-8 & 0.048 & \text { BIAS }-8 & -0.009 & \text { RMS }-8 & 0.059 & \\ & \text { AAD } & 0.009 & \text { BIAS } & -0.001 & \text { RMS } & 0.013 & \mathrm{MPa} \\ \text { DENSITY : } & \text { AAD }-8 & 0.046 & \text { BIAS }-8 & 0.009 & \text { RMS }-8 & 0.057 & \\ & \text { AAD } & 0.003 & \text { BIAS } & 0.000 & \text { RMS } & 0.004 & \mathrm{~mol} \cdot \mathrm{dm}^{-3}\end{array}$


Data from Gammon and Douslin [37]

\begin{tabular}{|c|c|c|c|c|c|c|c|}
\hline $\mathbf{T}$ & $P, \exp$ & P,cal & Dev & $\rho, \exp$ & $\rho$, cal & Dev & Wt \\
\hline K & $\mathrm{MPa}$ & $\mathrm{MPa}$ & 8 & $\mathrm{~mol} \cdot \mathrm{dm}^{-3}$ & $\mathrm{~mol} \cdot \mathrm{dm}^{-3}$ & 8 & \\
\hline 323.15 & 24.595 & 24.602 & 0.027 & 10.1474 & 10.1452 & -0.022 & 0.0001 \\
\hline 298.15 & 2.378 & 2.377 & -0.032 & 0.9998 & 1.0001 & 0.033 & 0.0010 \\
\hline 298.15 & 5.627 & 5.623 & -0.061 & 2.5000 & 2.5017 & 0.067 & 0.0004 \\
\hline 298.15 & 10.491 & 10.491 & -0.003 & 4.9996 & 4.9998 & 0.003 & 0.0002 \\
\hline 298.15 & 15.205 & 15.216 & 0.072 & 7.5002 & 7.4946 & -0.075 & 0.0002 \\
\hline 298.15 & 19.418 & 19.432 & 0.072 & 9.5344 & 9.5282 & -0.065 & 0.0001 \\
\hline 298.15 & 20.702 & 20.715 & 0.061 & 10.0914 & 10.0860 & -0.053 & 0.0001 \\
\hline 273.15 & 16.178 & 16.195 & 0.102 & 9.6452 & 9.6355 & -0.100 & 0.0001 \\
\hline 248.15 & 12.816 & 12.870 & 0.423 & 9.8009 & 9.7544 & -0.474 & 0.0002 \\
\hline 223.15 & 9.320 & 9.363 & 0.463 & 9.9547 & 9.8857 & -0.693 & 0.0003 \\
\hline 198.15 & 5.704 & 5.711 & 0.118 & 10.1081 & 10.0518 & -0.557 & 0.0006 \\
\hline 193.05 & 0.782 & 0.782 & 0.014 & 0.5168 & 0.5167 & -0.015 & 0.0032 \\
\hline 193.05 & 1.563 & 1.565 & 0.069 & 1.1071 & 1.1062 & -0.079 & 0.0016 \\
\hline 193.05 & 2.244 & 2.247 & 0.128 & 1.7074 & 1.7047 & -0.160 & 0.0012 \\
\hline 193.05 & 2.820 & 2.824 & 0.142 & 2.3052 & 2.3007 & -0.197 & 0.0010 \\
\hline 93.05 & 3.306 & 3.311 & 0.172 & 2.9109 & 2.9031 & -0.267 & 0.0009 \\
\hline 193.05 & 3.703 & 3.710 & 0.195 & 3.5146 & 3.5025 & -0.346 & 0.0008 \\
\hline 193.05 & 4.025 & 4.034 & 0.216 & 4.1234 & 4.1050 & -0.445 & 0.0008 \\
\hline 193.05 & 4.281 & 4.290 & 0.215 & 4.7353 & 4.7103 & -0.527 & 0.0007 \\
\hline 193.05 & 4.478 & 4.486 & 0.185 & 5.3479 & 5.3181 & -0.557 & 0.0007 \\
\hline 193.05 & 4.626 & 4.634 & 0.166 & 5.9688 & 5.9 & -0.633 & 0.0007 \\
\hline 193.05 & 4.729 & 4.735 & 0.131 & 6.5599 & 6.5175 & -0.646 & 0.0007 \\
\hline 193.05 & 4.806 & 4.810 & 0.089 & 7.1780 & 7.1360 & -0.585 & 0.0007 \\
\hline 193.05 & 4.859 & 4.862 & 0.047 & 7.7943 & 7.7612 & -0.424 & 0.0007 \\
\hline 193.05 & 4.897 & 4.897 & 0.010 & 8.4106 & 8.4004 & -0.122 & 0.0007 \\
\hline 193.05 & 4.924 & 4.924 & -0.009 & 9.0278 & 9.0390 & 0.124 & 0.0007 \\
\hline 193.05 & 4.946 & 4.946 & -0.004 & 9.6448 & 9.6509 & 0.063 & 0.0007 \\
\hline 193.05 & 4.962 & 4.963 & 0.017 & 10.1433 & 10.1192 & -0.237 & 0.0007 \\
\hline 193.05 & 4.966 & 4.967 & 0.020 & 10.2656 & 10.2373 & -0.276 & 0.0007 \\
\hline 193.05 & 4.988 & 4.990 & 0.049 & 10.8790 & 10.8200 & -0.543 & 0.0007 \\
\hline 193.05 & 5.016 & 5.0 & 0.089 & 11.4941 & 11.4142 & -0.695 & 0.0007 \\
\hline & 4.587 & 4.591 & 0.099 & 6.5658 & 6.5274 & -0.584 & 0.0000 \\
\hline 191.45 & 4.646 & 4.649 & 0.053 & 7.1849 & 7.1510 & -0.471 & 0.0000 \\
\hline 191.45 & 4.683 & 4.684 & 0.008 & 7.8031 & 7.7947 & -0.108 & 0.0000 \\
\hline 191.45 & 4.705 & 4.704 & -0.019 & 8.4225 & 8.4593 & 0.437 & 0.0000 \\
\hline
\end{tabular}


Data from Gammon and Douslin [37] (continued)

\begin{tabular}{|c|c|c|c|c|c|c|c|}
\hline $\mathrm{T}$ & $P, \exp$ & P,cal & Dev & $\rho, \exp$ & $\rho$, cal & Dev & Wt \\
\hline K & $\mathrm{MPa}$ & $\mathrm{MPa}$ & 8 & $\mathrm{~mol} \cdot \mathrm{dm}^{-3}$ & $\mathrm{~mol} \cdot \mathrm{dm}^{-3}$ & 8 & \\
\hline 191.45 & 4.717 & 4.716 & -0.026 & 9.0460 & 9.1297 & 0.926 & 0.0000 \\
\hline 191.45 & 4.725 & 4.724 & -0.018 & 9.6692 & 9.7436 & 0.770 & 0.0000 \\
\hline 191.45 & 4.730 & 4.730 & 0.002 & 10.1732 & 10.1671 & -0.060 & 0.0000 \\
\hline 191.45 & 4.731 & 4.731 & 0.005 & 10.2951 & 10.2770 & -0.176 & 0.0000 \\
\hline 191.45 & 4.738 & 4.739 & 0.031 & 10.9028 & 10.8054 & -0.893 & 0.0000 \\
\hline 191.45 & 4.748 & 4.751 & 0.062 & 11.5131 & 11.3877 & -1.090 & 0.0000 \\
\hline 190.85 & 4.533 & 4.537 & 0.087 & 6.5680 & 6.5310 & -0.564 & 0.0000 \\
\hline 190.85 & 4.586 & 4.588 & 0.042 & 7.1876 & 7.1567 & -0.431 & 0.0000 \\
\hline 190.85 & 4.616 & 4.616 & 0.001 & 7.8070 & 7.8050 & -0.026 & 0.0000 \\
\hline 190.85 & 4.632 & 4.631 & -0.018 & 8.4302 & 8.4869 & 0.672 & 0.0000 \\
\hline 190.85 & 4.638 & 4.637 & -0.019 & 9.0767 & 9.2125 & 1.496 & 0.0000 \\
\hline 190.85 & 4.641 & 4.641 & -0.012 & 9.7495 & 9.8937 & 1.479 & 0.0000 \\
\hline 190.85 & 4.643 & 4.643 & -0.002 & 10.2668 & 10.2893 & 0.220 & 0.0000 \\
\hline 190.85 & 4.643 & 4.643 & 0.002 & 10.3899 & 10.3650 & -0.239 & 0.0000 \\
\hline 190.85 & 4.646 & 4.646 & 0.015 & 10.9572 & 10.8366 & -1.101 & 0.0000 \\
\hline 190.85 & 4.650 & 4.651 & 0.033 & 11.5304 & 11.4050 & -1.088 & 0.0000 \\
\hline 190.63 & 4.513 & 4.517 & 0.078 & 6.5688 & 6.5346 & -0.520 & 0.0000 \\
\hline 190.63 & 4.564 & 4.565 & 0.039 & 7.1886 & 7.1586 & -0.418 & 0.0000 \\
\hline 190.63 & 4.592 & 4.592 & 0.000 & 7.8086 & 7.8086 & 0.000 & 0.0000 \\
\hline 190.63 & 4.605 & 4.604 & -0.015 & 8.4349 & 8.4958 & 0.722 & 0.0000 \\
\hline 190.63 & 4.609 & 4.609 & -0.009 & 9.1376 & 9.2686 & 1.433 & 0.0000 \\
\hline 190.63 & 4.610 & 4.610 & 0.004 & 10.0202 & 9.8346 & -1.852 & 0.0000 \\
\hline 190.63 & 4.611 & 4.611 & 0.005 & 10.6490 & 10.4918 & -1.476 & 0.0000 \\
\hline 190.63 & 4.611 & 4.611 & 0.010 & 10.7652 & 10.4918 & -2.540 & 0.0000 \\
\hline 190.63 & 4.612 & 4.612 & 0.002 & 11.1070 & 11.0773 & -0.267 & 0.0000 \\
\hline 190.63 & 4.615 & 4.615 & 0.015 & 11.5501 & 11.4704 & -0.690 & 0.0000 \\
\hline 190.56 & 4.507 & 4.510 & 0.083 & 6.5691 & 6.5324 & -0.558 & 0.0000 \\
\hline 190.56 & 4.556 & 4.558 & 0.038 & 7.1890 & 7.1593 & -0.413 & 0.0000 \\
\hline 190.56 & 4.584 & 4.584 & 0.000 & 7.8092 & 7.8088 & -0.005 & 0.0000 \\
\hline 190.56 & 4.596 & 4.595 & -0.012 & 8.4377 & 8.4896 & 0.615 & 0.0000 \\
\hline 190.56 & 4.600 & 4.600 & -0.003 & 9.1450 & 9.2024 & 0.628 & 0.0000 \\
\hline 190.56 & 4.601 & 4.601 & 0.000 & 10.1650 & 10.1392 & -0.254 & 0.0000 \\
\hline 190.56 & 4.601 & 4.601 & -0.010 & 10.4400 & 10.9536 & 4.919 & 0.0000 \\
\hline 190.56 & 4.601 & 4.601 & -0.005 & 10.5100 & 10.8181 & 2.932 & 0.0000 \\
\hline 190.56 & 4.602 & 4.601 & -0.012 & 11.0320 & 11.2174 & 1.680 & 0.0000 \\
\hline
\end{tabular}


Data from Gammon and Douslin [37] (continued)

\begin{tabular}{|c|c|c|c|c|c|c|c|}
\hline $\mathrm{T}$ & $P, \exp$ & $\mathrm{P}, \mathrm{cal}$ & Dev & $\rho, \exp$ & $\rho, \mathrm{cal}$ & Dev & Wt \\
\hline K & $\mathrm{MPa}$ & $\mathrm{MPa}$ & 8 & $\mathrm{~mol} \cdot \mathrm{dm}^{-3}$ & $\mathrm{~mol} \cdot \mathrm{dm}^{-3}$ & 8 & \\
\hline 190.56 & 4.603 & 4.604 & 0.006 & 11.5780 & 11.5414 & -0.316 & 0.0000 \\
\hline 190.50 & 0.771 & 0.771 & 0.039 & 0.5172 & 0.5170 & -0.041 & 0.0032 \\
\hline 190.50 & 1.538 & 1.539 & 0.085 & 1.1081 & 1.1070 & -0.098 & 0.0017 \\
\hline 190.50 & 2.203 & 2.206 & 0.126 & 1.7090 & 1.7063 & -0.159 & 0.0012 \\
\hline 190.50 & 2.760 & 2.765 & 0.161 & 2.3075 & 2.3023 & -0.226 & 0.0010 \\
\hline 190.50 & 3.226 & 3.232 & 0.189 & 2.9139 & 2.9052 & -0.300 & 0.0000 \\
\hline 190.50 & 3.603 & 3.610 & 0.203 & 3.5185 & 3.5054 & -0.372 & 0.0000 \\
\hline 190.50 & 3.902 & 3.910 & 0.210 & 4.1282 & 4.1093 & -0.457 & 0.0000 \\
\hline 190.50 & 4.133 & 4.141 & 0.195 & 4.7411 & 4.7164 & -0.521 & 0.0000 \\
\hline 190.50 & 4.304 & 4.311 & 0.163 & 5.3548 & 5.3249 & -0.559 & 0.0000 \\
\hline 190.50 & 4.425 & 4.430 & 0.124 & 5.9769 & 5.9421 & -0.582 & 0.0000 \\
\hline 190.50 & 4.501 & 4.505 & 0.083 & 6.5693 & 6.5320 & -0.567 & 0.0000 \\
\hline 190.50 & 4.550 & 4.552 & 0.037 & 7.1893 & 7.1598 & -0.411 & 0.0000 \\
\hline 190.50 & 4.577 & 4.577 & 0.001 & 7.8097 & 7.8080 & -0.021 & 0.0000 \\
\hline 190.50 & 4.589 & 4.588 & -0.010 & 8.4412 & 8.4898 & 0.575 & 0.0000 \\
\hline 190.43 & 4.495 & 4.499 & 0.079 & 6.5696 & 6.5338 & -0.545 & 0.0000 \\
\hline 190.43 & 4.543 & 4.545 & 0.035 & 7.1896 & 7.1607 & -0.402 & 0.0000 \\
\hline 190.43 & 4.569 & 4.569 & 0.001 & 7.8103 & 7.8086 & -0.022 & 0.0000 \\
\hline 190.43 & 4.580 & 4.579 & -0.008 & 8.4416 & 8.4823 & 0.483 & 0.0000 \\
\hline 190.25 & 4.287 & 4.293 & 0.157 & 5.3555 & 5.3261 & -0.548 & 0.0000 \\
\hline 190.25 & 4.405 & 4.410 & 0.118 & 5.9777 & 5.9436 & -0.570 & 0.0000 \\
\hline 190.25 & 4.479 & 4.482 & 0.078 & 6.5703 & 6.5340 & -0.553 & 0.0000 \\
\hline 190.25 & 4.525 & 4.526 & 0.034 & 7.1905 & 7.1610 & -0.410 & 0.0000 \\
\hline 190.25 & 4.549 & 4.549 & 0.002 & 7.8122 & 7.8073 & -0.063 & 0.0000 \\
\hline 189.65 & 3.861 & 3.869 & 0.202 & 4.1298 & 4.1112 & -0.450 & 0.0000 \\
\hline 189.65 & 4.083 & 4.091 & 0.188 & 4.7430 & 4.7183 & -0.521 & 0.0000 \\
\hline 189.65 & 4.245 & 4.252 & 0.148 & 5.3571 & 5.3283 & -0.537 & 0.0000 \\
\hline 189.65 & 4.357 & 4.362 & 0.108 & 5.9796 & 5.9464 & -0.555 & 0.0000 \\
\hline 189.65 & 4.424 & 4.427 & 0.069 & 6.5726 & 6.5365 & -0.549 & 0.0000 \\
\hline 189.65 & 4.463 & 4.465 & 0.030 & 7.1938 & 7.1614 & -0.450 & 0.0000 \\
\hline 188.15 & 0.761 & 0.761 & 0.062 & 0.5176 & 0.5173 & -0.066 & 0.0016 \\
\hline 188.15 & 1.515 & 1.516 & 0.090 & 1.1089 & 1.1077 & -0.104 & 0.0008 \\
\hline 188.15 & 2.164 & 2.168 & 0.158 & 1.7104 & 1.7070 & -0.201 & 0.0006 \\
\hline 188.15 & 2.705 & 2.710 & 0.178 & 2.3096 & 2.3038 & -0.253 & 0.0005 \\
\hline 188.15 & 3.153 & 3.159 & 0.204 & 2.9168 & 2.9071 & -0.333 & 0.0000 \\
\hline
\end{tabular}


Data from Gammon and Douslin [37] (continued)

\begin{tabular}{|c|c|c|c|c|c|c|c|}
\hline $\mathrm{T}$ & $P, \exp$ & P,cal & Dev & $\rho, \exp$ & $\rho$, cal & Dev & Wt \\
\hline K & $\mathrm{MPa}$ & $\mathrm{MPa}$ & 8 & $\mathrm{~mol} \cdot \mathrm{dm}^{-3}$ & $\mathrm{~mol} \cdot \mathrm{dm}^{-3}$ & 8 & \\
\hline 188.15 & 3.509 & 3.516 & 0.215 & 3.5221 & 3.5077 & -0.409 & 0.0000 \\
\hline 188.15 & 3.787 & 3.795 & 0.209 & 4.1327 & 4.1127 & -0.485 & 0.0000 \\
\hline 188.15 & 3.995 & 4.002 & 0.177 & 4.7465 & 4.7217 & -0.522 & 0.0000 \\
\hline 188.15 & 4.140 & 4.146 & 0.132 & 5.3612 & 5.3325 & -0.535 & 0.0000 \\
\hline 188.15 & 4.235 & 4.239 & 0.086 & 5.9846 & 5.9525 & -0.536 & 0.0000 \\
\hline 183.15 & 0.738 & 0.739 & 0.102 & 0.5184 & 0.5178 & -0.109 & 0.0017 \\
\hline 183.15 & 1.465 & 1.466 & 0.104 & 1.1108 & 1.1095 & -0.121 & 0.0009 \\
\hline 183.15 & 2.083 & 2.086 & 0.156 & 1.7135 & 1.7100 & -0.204 & 0.0006 \\
\hline 183.15 & 2.587 & 2.592 & 0.200 & 2.3140 & 2.3072 & -0.296 & 0.0005 \\
\hline 183.15 & 2.994 & 3.001 & 0.222 & 2.9228 & 2.9116 & -0.384 & 0.0005 \\
\hline 183.15 & 3.307 & 3.314 & 0.215 & 3.5298 & 3.5138 & -0.454 & 0.0000 \\
\hline 183.15 & 3.537 & 3.543 & 0.175 & 4.1424 & 4.1224 & -0.483 & 0.0000 \\
\hline 173.15 & 0.694 & 0.694 & 0.048 & 0.5192 & 0.5189 & -0.052 & 0.0018 \\
\hline 173.15 & 1.363 & 1.365 & 0.140 & 1.1143 & 1.1124 & -0.168 & 0.0010 \\
\hline 173.15 & 1.914 & 1.918 & 0.206 & 1.7193 & 1.7145 & -0.282 & 0.0007 \\
\hline 173.15 & 2.344 & 2.349 & 0.235 & 2.3220 & 2.3131 & -0.382 & 0.0006 \\
\hline 163.15 & 0.649 & 0.650 & 0.053 & 0.5204 & 0.5201 & -0.058 & 0.0019 \\
\hline 163.15 & 1.259 & 1.262 & 0.168 & 1.1175 & 1.1152 & -0.208 & 0.0011 \\
\hline 163.15 & 1.740 & 1.743 & 0.190 & 1.7223 & 1.7175 & -0.280 & 0.0008 \\
\hline 153.15 & 0.604 & 0.604 & 0.063 & 0.5215 & 0.5211 & -0.070 & 0.0021 \\
\hline 143.15 & 0.557 & 0.558 & 0.068 & 0.5223 & 0.5219 & -0.077 & 0.0023 \\
\hline 190.50 & 4.592 & 4.592 & -0.009 & 11.1700 & 11.3105 & 1.258 & 0.0000 \\
\hline 190.50 & 4.594 & 4.594 & -0.005 & 11.5750 & 11.6031 & 0.242 & 0.0000 \\
\hline 190.43 & 4.582 & 4.582 & -0.009 & 11.5100 & 11.5827 & 0.631 & 0.0000 \\
\hline 190.43 & 4.584 & 4.582 & -0.032 & 11.5750 & 11.7644 & 1.636 & 0.0000 \\
\hline 190.25 & 4.557 & 4.556 & -0.033 & 12.0406 & 12.1414 & 0.837 & 0.0000 \\
\hline 189.65 & 4.472 & 4.469 & -0.076 & 12.9613 & 13.0260 & 0.499 & 0.0000 \\
\hline 188.15 & 4.267 & 4.263 & -0.094 & 14.1950 & 14.2176 & 0.159 & 0.0000 \\
\hline 185.91 & 3.977 & 3.981 & 0.111 & 15.3297 & 15.3186 & -0.072 & 0.0000 \\
\hline 183.15 & 3.642 & 3.663 & 0.571 & 16.3353 & 16.3056 & -0.182 & 0.0000 \\
\hline 183.15 & 3.642 & 3.663 & 0.563 & 16.3353 & 16.3061 & -0.179 & 0.0000 \\
\hline 178.15 & 3.092 & 3.138 & 1.512 & 17.7013 & 17.6660 & -0.199 & 0.0001 \\
\hline 173.15 & 2.606 & 2.668 & 2.360 & 18.7794 & 18.7493 & -0.160 & 0.0000 \\
\hline 170.75 & 2.394 & 2.460 & 2.767 & 19.2357 & 19.2083 & -0.142 & 0.0000 \\
\hline 163.15 & 1.804 & 1.883 & 4.381 & 20.5098 & 20.4886 & -0.103 & 0.0000 \\
\hline
\end{tabular}


Data from Gammon and Douslin [37] (continued)

\begin{tabular}{|c|c|c|c|c|c|c|c|}
\hline $\mathrm{T}$ & $P, \exp$ & P,cal & Dev & $\rho, \exp$ & $\rho$, cal & Dev & Wt \\
\hline $\mathrm{K}$ & $\mathrm{MPa}$ & $\mathrm{MPa}$ & 8 & $\mathrm{~mol} \cdot \mathrm{dm}^{-3}$ & $\mathrm{~mol} \cdot \mathrm{dm}^{-3}$ & 8 & \\
\hline 155.44 & 1.321 & 1.412 & 6.862 & 21.6202 & 21.6029 & -0.080 & 0.0000 \\
\hline 153.15 & 1.197 & 1.291 & 7.792 & 21.9245 & 21.9082 & -0.074 & 0.0000 \\
\hline 143.15 & 0.753 & 0.849 & 12.744 & 23.1510 & 23.1390 & -0.052 & 0.0000 \\
\hline 140.02 & 0.643 & 0.738 & 14.700 & 23.5070 & 23.4962 & -0.046 & 0.0000 \\
\hline 133.15 & 0.443 & 0.529 & 19.524 & 24.2514 & 24.2432 & -0.034 & 0.0001 \\
\hline 125.09 & 0.271 & 0.348 & 28.161 & 25.0715 & 25.0655 & -0.024 & 0.0001 \\
\hline 123.15 & 0.239 & 0.313 & 30.974 & 25.2616 & 25.2561 & -0.022 & 0.0001 \\
\hline 113.15 & 0.115 & 0.186 & 61.893 & 26.2044 & 26.2001 & -0.017 & 0.0001 \\
\hline 190.37 & 4.572 & 4.572 & 0.000 & 8.4443 & 8.4441 & -0.003 & 0.0000 \\
\hline 190.35 & 4.569 & 4.570 & 0.006 & 8.4332 & 8.4024 & -0.365 & 0.0000 \\
\hline 190.03 & 4.523 & 4.524 & 0.011 & 7.8149 & 7.7887 & -0.335 & 0.0000 \\
\hline 189.50 & 4.448 & 4.449 & 0.034 & 7.1947 & 7.1554 & -0.546 & 0.0000 \\
\hline 188.70 & 4.336 & 4.339 & 0.063 & 6.5765 & 6.5362 & -0.614 & 0.0000 \\
\hline 187.60 & 4.189 & 4.193 & 0.092 & 5.9865 & 5.9491 & -0.624 & 0.0000 \\
\hline 186.10 & 3.993 & 3.999 & 0.137 & 5.3671 & 5.3312 & -0.669 & 0.0000 \\
\hline 184.15 & 3.751 & 3.758 & 0.170 & 4.7560 & 4.7259 & -0.634 & 0.0000 \\
\hline 181.55 & 3.451 & 3.456 & 0.142 & 4.1324 & 4.1151 & -0.418 & 0.0000 \\
\hline 174.25 & 2.699 & 2.703 & 0.169 & 2.9218 & 2.9118 & -0.341 & 0.0006 \\
\hline 169.15 & 2.241 & 2.245 & 0.190 & 2.3197 & 2.3121 & -0.326 & 0.0007 \\
\hline 162.65 & 1.730 & 1.733 & 0.190 & 1.7217 & 1.7168 & -0.282 & 0.0008 \\
\hline 155.05 & 1.173 & 1.175 & 0.172 & 1.1192 & 1.1167 & -0.221 & 0.0011 \\
\hline 138.15 & 0.533 & 0.533 & 0.071 & 0.5217 & 0.5213 & -0.081 & 0.0024 \\
\hline 190.85 & 4.533 & 4.537 & 0.089 & 6.5670 & 6.5291 & -0.577 & 0.0000 \\
\hline 190.85 & 4.586 & 4.588 & 0.041 & 7.1850 & 7.1551 & -0.416 & 0.0000 \\
\hline 190.85 & 4.616 & 4.616 & 0.001 & 7.8010 & 7.7989 & -0.027 & 0.0000 \\
\hline 190.85 & 4.631 & 4.631 & -0.018 & 8.4170 & 8.4730 & 0.665 & 0.0000 \\
\hline 190.85 & 4.638 & 4.637 & -0.021 & 9.0330 & 9.1792 & 1.619 & 0.0000 \\
\hline 190.85 & 4.641 & 4.641 & -0.014 & 9.6500 & 9.8155 & 1.715 & 0.0000 \\
\hline 190.85 & 4.643 & 4.642 & -0.005 & 10.1480 & 10.2117 & 0.628 & 0.0000 \\
\hline 190.85 & 4.643 & 4.643 & -0.002 & 10.2700 & 10.2893 & 0.188 & 0.0000 \\
\hline 190.85 & 4.645 & 4.646 & 0.013 & 10.8860 & 10.7816 & -0.959 & 0.0000 \\
\hline 190.85 & 4.650 & 4.651 & 0.032 & 11.5030 & 11.3776 & -1.090 & 0.0000 \\
\hline 190.43 & 4.495 & 4.498 & 0.080 & 6.5680 & 6.5318 & -0.551 & 0.0000 \\
\hline 190.43 & 4.543 & 4.545 & 0.036 & 7.1870 & 7.1571 & -0.416 & 0.0000 \\
\hline 190.43 & 4.569 & 4.569 & 0.001 & 7.8030 & 7.8008 & -0.028 & 0.0000 \\
\hline
\end{tabular}


Data from Gammon and Douslin [37] (continued)

\begin{tabular}{|c|c|c|c|c|c|c|c|}
\hline $\mathrm{T}$ & $P, \exp$ & P, cal & Dev & $\rho, \exp$ & $\rho$, cal & Dev & Wt \\
\hline $\mathrm{K}$ & $\mathrm{MPa}$ & $\mathrm{MPa}$ & 8 & $\mathrm{~mol} \cdot \mathrm{dm}^{-3}$ & $\mathrm{~mol} \cdot \mathrm{dm}^{-3}$ & 8 & \\
\hline 190.43 & 4.580 & 4.579 & -0.008 & 8.4190 & 8.4589 & 0.474 & 0.0000 \\
\hline 190.56 & 4.507 & 4.510 & 0.082 & 6.5680 & 6.5315 & -0.556 & 0.0000 \\
\hline 190.56 & 4.556 & 4.558 & 0.036 & 7.1860 & 7.1576 & -0.396 & 0.0000 \\
\hline 190.56 & 4.583 & 4.583 & 0.000 & 7.8020 & 7.8016 & -0.005 & 0.0000 \\
\hline 190.56 & 4.596 & 4.595 & -0.012 & 8.4180 & 8.4701 & 0.619 & 0.0000 \\
\hline 190.56 & 4.600 & 4.599 & -0.006 & 9.0350 & 9.1223 & 0.967 & 0.0000 \\
\hline 190.56 & 4.600 & 4.600 & 0.004 & 9.6510 & 9.4440 & -2.145 & 0.0000 \\
\hline 190.56 & 4.601 & 4.601 & -0.004 & 10.1500 & 10.7195 & 5.610 & 0.0000 \\
\hline 190.56 & 4.601 & 4.601 & 0.000 & 10.2720 & 10.1392 & -1.293 & 0.0000 \\
\hline 190.56 & 4.601 & 4.601 & -0.011 & 10.8880 & 11.1261 & 2.186 & 0.0000 \\
\hline 190.56 & 4.603 & 4.603 & 0.001 & 11.5050 & 11.5014 & -0.031 & 0.0000 \\
\hline 189.65 & 4.424 & 4.427 & 0.068 & 6.5710 & 6.5354 & -0.542 & 0.0000 \\
\hline 189.65 & 4.463 & 4.464 & 0.031 & 7.1900 & 7.1567 & -0.463 & 0.0000 \\
\hline 190.63 & 4.513 & 4.517 & 0.078 & 6.5670 & 6.5327 & -0.522 & 0.0000 \\
\hline 190.63 & 4.563 & 4.565 & 0.038 & 7.1860 & 7.1569 & -0.405 & 0.0000 \\
\hline 190.63 & 4.591 & 4.591 & 0.000 & 7.8020 & 7.8017 & -0.004 & 0.0000 \\
\hline 190.63 & 4.604 & 4.604 & -0.015 & 8.4180 & 8.4778 & 0.710 & 0.0000 \\
\hline 190.63 & 4.609 & 4.609 & -0.013 & 9.0350 & 9.2010 & 1.838 & 0.0000 \\
\hline 190.63 & 4.610 & 4.610 & -0.001 & 9.6510 & 9.6743 & 0.241 & 0.0000 \\
\hline 190.63 & 4.611 & 4.611 & -0.004 & 10.1490 & 10.3218 & 1.702 & 0.0000 \\
\hline 190.63 & 4.611 & 4.611 & 0.001 & 10.2720 & 10.2259 & -0.449 & 0.0000 \\
\hline 190.63 & 4.612 & 4.612 & -0.006 & 10.8870 & 10.9885 & 0.932 & 0.0000 \\
\hline 190.63 & 4.614 & 4.615 & 0.013 & 11.5040 & 11.4316 & -0.629 & 0.0000 \\
\hline 190.25 & 4.478 & 4.482 & 0.077 & 6.5690 & 6.5329 & -0.549 & 0.0000 \\
\hline 190.25 & 4.525 & 4.526 & 0.032 & 7.1870 & 7.1591 & -0.388 & 0.0000 \\
\hline 190.25 & 4.548 & 4.549 & 0.003 & 7.8040 & 7.7986 & -0.069 & 0.0000 \\
\hline 191.45 & 4.587 & 4.591 & 0.098 & 6.5640 & 6.5258 & -0.583 & 0.0000 \\
\hline 191.45 & 4.646 & 4.649 & 0.055 & 7.1830 & 7.1483 & -0.483 & 0.0000 \\
\hline 191.45 & 4.683 & 4.683 & 0.008 & 7.7990 & 7.7900 & -0.115 & 0.0000 \\
\hline 191.45 & 4.704 & 4.704 & -0.019 & 8.4140 & 8.4509 & 0.438 & 0.0000 \\
\hline 191.45 & 4.717 & 4.715 & -0.027 & 9.0300 & 9.1159 & 0.951 & 0.0000 \\
\hline 191.45 & 4.724 & 4.724 & -0.018 & 9.6460 & 9.7179 & 0.746 & 0.0000 \\
\hline 191.45 & 4.729 & 4.729 & 0.001 & 10.1450 & 10.1415 & -0.035 & 0.0000 \\
\hline 191.45 & 4.731 & 4.731 & 0.004 & 10.2670 & 10.2518 & -0.148 & 0.0000 \\
\hline 191.45 & 4.738 & 4.739 & 0.030 & 10.8820 & 10.7848 & -0.893 & 0.0000 \\
\hline
\end{tabular}


Data from Gammon and Douslin [37] (continued)

$\begin{array}{ccccrrrr}\text { T } & \text { P,exp } & \text { P, cal } & \text { Dev } & \rho, \exp & \rho, \mathrm{cal} & \text { Dev } & \text { Wt } \\ \mathrm{K} & \mathrm{MPa} & \mathrm{MPa} & \text { \& } & \mathrm{mol} \cdot \mathrm{dm}^{-3} & \mathrm{~mol} \cdot \mathrm{dm}^{-3} & \text { \& } & \\ 191.45 & 4.748 & 4.751 & 0.061 & 11.4990 & 11.3741 & -1.087 & 0.0000 \\ 190.50 & 4.501 & 4.505 & 0.083 & 6.5680 & 6.5311 & -0.562 & 0.0000 \\ 190.50 & 4.550 & 4.552 & 0.037 & 7.1860 & 7.1563 & -0.414 & 0.0000 \\ 190.50 & 4.577 & 4.577 & 0.001 & 7.8030 & 7.8006 & -0.030 & 0.0000 \\ 190.50 & 4.588 & 4.588 & -0.011 & 8.4190 & 8.4685 & 0.588 & 0.0000 \\ 190.50 & 4.591 & 4.592 & 0.004 & 9.0350 & 8.9704 & -0.715 & 0.0000 \\ 190.50 & 4.594 & 4.593 & -0.006 & 11.5050 & 11.5478 & 0.372 & 0.0000\end{array}$

Number of Points (Ref. 37)

217

$\begin{array}{llllrlll}\text { PRESSURE : } & \text { AAD }-8 & 0.963 & \text { BIAS }-8 & 0.956 & \text { RMS }-8 & 5.384 & \\ & \text { AAD } & 0.007 & \text { BIAS } & 0.007 & \text { RMS } & 0.018 & \mathrm{MPa} \\ \text { DENSITY : } & \text { AAD- } 8 & 0.533 & \text { BIAS }-8 & -0.084 & \text { RMS }-8 & 0.842 & \\ & \text { AAD } & 0.046 & \text { BIAS } & -0.001 & \text { RMS } & 0.084 & \mathrm{~mol} \cdot \mathrm{dm}^{-3}\end{array}$

Data from Goodwin [38]

$\begin{array}{cccccccc}\text { T } & \text { P,exp } & \text { P, cal } & \text { Dev } & \rho, \exp & \rho, \mathrm{ca} 1 & \text { Dev } & \text { Wt } \\ \mathrm{K} & \mathrm{MPa} & \mathrm{MPa} & 8 & \mathrm{~mol} \cdot \mathrm{dm}^{-3} & \mathrm{~mol} \cdot \mathrm{dm}^{-3} & 8 & \\ 176.00 & 2.796 & 2.798 & 0.060 & 2.9954 & 2.9918 & -0.120 & 0.0544 \\ 180.00 & 2.933 & 2.935 & 0.066 & 2.9936 & 2.9899 & -0.123 & 0.0510 \\ 184.00 & 3.068 & 3.070 & 0.052 & 2.9918 & 2.9891 & -0.092 & 0.0000 \\ 188.00 & 3.201 & 3.202 & 0.042 & 2.9901 & 2.9880 & -0.070 & 0.0000 \\ 192.00 & 3.332 & 3.333 & 0.032 & 2.9883 & 2.9868 & -0.051 & 0.0000 \\ & & & & & & & \\ 196.00 & 3.461 & 3.462 & 0.022 & 2.9866 & 2.9856 & -0.033 & 24.7201 \\ 200.00 & 3.589 & 3.590 & 0.017 & 2.9848 & 2.9840 & -0.026 & 23.6115 \\ 204.00 & 3.716 & 3.716 & 0.016 & 2.9831 & 2.9823 & -0.024 & 22.6052 \\ 208.00 & 3.842 & 3.842 & 0.014 & 2.9813 & 2.9807 & -0.020 & 21.6863 \\ 212.00 & 3.967 & 3.967 & 0.017 & 2.9796 & 2.9789 & -0.023 & 20.8446 \\ & & & & & & & \\ 216.00 & 4.091 & 4.091 & 0.014 & 2.9779 & 2.9773 & -0.019 & 20.0692 \\ 220.00 & 4.214 & 4.215 & 0.010 & 2.9761 & 2.9757 & -0.013 & 19.3528 \\ 225.00 & 4.367 & 4.368 & 0.012 & 2.9739 & 2.9735 & -0.015 & 18.5313 \\ 230.00 & 4.520 & 4.520 & 0.009 & 2.9717 & 2.9714 & -0.011 & 17.7803 \\ 240.00 & 4.822 & 4.822 & -0.003 & 2.9675 & 2.9676 & 0.004 & 16.4562\end{array}$


Data from Goodwin [38] (continued)

\begin{tabular}{|c|c|c|c|c|c|c|c|}
\hline $\mathrm{T}$ & $P, \exp$ & $\mathrm{P}, \mathrm{cal}$ & Dev & $\rho, \exp$ & $\rho, \mathrm{cal}$ & Dev & Wt \\
\hline K & $\mathrm{MPa}$ & $\mathrm{MPa}$ & 8 & $\mathrm{~mol} \cdot \mathrm{dm}^{-3}$ & $\mathrm{~mol} \cdot \mathrm{dm}^{-3}$ & 8 & \\
\hline 250.00 & 5.120 & 5.120 & -0.004 & 2.9630 & 2.9632 & 0.005 & 15.3274 \\
\hline 260.00 & 5.416 & 5.415 & -0.010 & 2.9585 & 2.9589 & 0.012 & 14.3516 \\
\hline 270.00 & 5.709 & 5.708 & -0.012 & 2.9540 & 2.9544 & 0.014 & 0.0450 \\
\hline 280.00 & 6.000 & 5.998 & -0.018 & 2.9495 & 2.9501 & 0.020 & 0.0425 \\
\hline 290.00 & 6.287 & 6.287 & -0.005 & 2.9450 & 2.9452 & 0.006 & 0.0403 \\
\hline 300.00 & 6.575 & 6.572 & -0.035 & 2.9402 & 2.9414 & 0.039 & 0.0383 \\
\hline 184.00 & 3.516 & 3.531 & 0.444 & 3.9782 & 3.9350 & -1.085 & 0.0000 \\
\hline 188.00 & 3.710 & 3.724 & 0.374 & 3.9756 & 3.9430 & -0.819 & 0.0000 \\
\hline 192.00 & 3.899 & 3.912 & 0.327 & 3.9731 & 3.9469 & -0.657 & 0.0000 \\
\hline 196.00 & 4.090 & 4.097 & 0.165 & 3.9705 & 3.9581 & -0.310 & 22.0316 \\
\hline 200.00 & 4.275 & 4.280 & 0.110 & 3.9683 & 3.9605 & -0.195 & 20.8002 \\
\hline 204.00 & 4.456 & 4.460 & 0.104 & 3.9654 & 3.9584 & -0.176 & 19.7117 \\
\hline 208.00 & 4.635 & 4.639 & 0.094 & 3.9629 & 3.9568 & -0.153 & 18.7340 \\
\hline 212.00 & 4.812 & 4.817 & 0.091 & 3.9604 & 3.9548 & -0.142 & 17.8526 \\
\hline 216.00 & 4.988 & 4.993 & 0.087 & 3.9579 & 3.9526 & -0.132 & 17.0531 \\
\hline 220.00 & 5.163 & 5.168 & 0.087 & 3.9554 & 3.9503 & -0.128 & 16.3251 \\
\hline 225.00 & 5.380 & 5.385 & 0.085 & 3.9522 & 3.9474 & -0.121 & 15.5011 \\
\hline 230.00 & 5.595 & 5.600 & 0.088 & 3.9491 & 3.9443 & -0.121 & 14.7598 \\
\hline 240.00 & 6.020 & 6.026 & 0.089 & 3.9427 & 3.9381 & -0.116 & 13.4790 \\
\hline 250.00 & 6.441 & 6.446 & 0.088 & 3.9364 & 3.9320 & -0.112 & 12.4111 \\
\hline 260.00 & 6.857 & 6.862 & 0.082 & 3.9300 & 3.9260 & -0.100 & 11.5065 \\
\hline 270.00 & 7.268 & 7.274 & 0.082 & 3.9235 & 3.9196 & -0.098 & 0.0358 \\
\hline 280.00 & 7.676 & 7.682 & 0.083 & 3.9170 & 3.9132 & -0.096 & 0.0335 \\
\hline 290.00 & 8.080 & 8.087 & 0.075 & 3.9104 & 3.9071 & -0.085 & 0.0316 \\
\hline 300.00 & 8.482 & 8.488 & 0.068 & 3.9038 & 3.9008 & -0.075 & 0.0298 \\
\hline 188.00 & 4.028 & 4.041 & 0.343 & 4.9304 & 4.8761 & -1.101 & 0.0000 \\
\hline 192.00 & 4.277 & 4.292 & 0.354 & 4.9270 & 4.8800 & -0.952 & 0.0000 \\
\hline 196.00 & 4.522 & 4.537 & 0.335 & 4.9236 & 4.8845 & -0.793 & 20.7942 \\
\hline 200.00 & 4.764 & 4.778 & 0.297 & 4.9202 & 4.8888 & -0.638 & 19.4180 \\
\hline 204.00 & 5.004 & 5.016 & 0.250 & 4.9169 & 4.8925 & -0.496 & 18.2088 \\
\hline 208.00 & 5.241 & 5.251 & 0.200 & 4.9135 & 4.8953 & -0.371 & 17.1392 \\
\hline 212.00 & 5.474 & 5.484 & 0.182 & 4.9102 & 4.8945 & -0.320 & 16.1910 \\
\hline 216.00 & 5.705 & 5.715 & 0.178 & 4.9069 & 4.8922 & -0.298 & 15.3436 \\
\hline 220.00 & 5.933 & 5.945 & 0.195 & 4.9035 & 4.8882 & -0.313 & 14.5828 \\
\hline 225.00 & 6.218 & 6.229 & 0.176 & 4.8994 & 4.8862 & -0.270 & 13.7288 \\
\hline
\end{tabular}


Data from Goodwin [38] (continued)

\begin{tabular}{|c|c|c|c|c|c|c|c|}
\hline $\mathrm{T}$ & $P, \exp$ & P,cal & Dev & $\rho, \exp$ & $\rho, \mathrm{cal}$ & Dev & Wt \\
\hline K & $\mathrm{MPa}$ & $\mathrm{MPa}$ & 8 & $\mathrm{~mol} \cdot \mathrm{dm}^{-3}$ & $\mathrm{~mol} \cdot \mathrm{dm}^{-3}$ & 8 & \\
\hline 230.00 & 6.500 & 6.511 & 0.171 & 4.8952 & 4.8829 & -0.251 & 12.9716 \\
\hline 240.00 & 7.056 & 7.068 & 0.166 & 4.8868 & 4.8757 & -0.228 & 11.6871 \\
\hline 250.00 & 7.606 & 7.618 & 0.158 & 4.8784 & 4.8683 & -0.206 & 10.6391 \\
\hline 260.00 & 8.149 & 8.162 & 0.153 & 4.8699 & 4.8606 & -0.191 & 9.7686 \\
\hline 270.00 & 8.687 & 8.699 & 0.145 & 4.8614 & 4.8529 & -0.174 & 0.0301 \\
\hline 280.00 & 9.219 & 9.232 & 0.137 & 4.8527 & 4.8450 & -0.160 & 0.0280 \\
\hline 290.00 & 9.747 & 9.759 & 0.125 & 4.8441 & 4.8372 & -0.142 & 0.0262 \\
\hline 300.00 & 10.270 & 10.282 & 0.117 & 4.8354 & 4.8291 & -0.130 & 0.0246 \\
\hline 192.00 & 4.537 & 4.546 & 0.193 & 5.9478 & 5.9012 & -0.784 & 0.0000 \\
\hline 196.00 & 4.849 & 4.860 & 0.216 & 5.9435 & 5.9027 & -0.686 & 20.0683 \\
\hline 200.00 & 5.155 & 5.167 & 0.231 & 5.9392 & 5.9024 & -0.620 & 18.5422 \\
\hline 204.00 & 5.458 & 5.470 & 0.230 & 5.9350 & 5.9028 & -0.542 & 17.2123 \\
\hline 208.00 & 5.758 & 5.770 & 0.215 & 5.9307 & 5.9034 & -0.460 & 16.0467 \\
\hline 212.00 & 6.054 & 6.067 & 0.213 & 5.9264 & 5.9016 & -0.419 & 15.0219 \\
\hline 216.00 & 6.347 & 6.361 & 0.215 & 5.9222 & 5.8988 & -0.395 & 14.1148 \\
\hline 220.00 & 6.638 & 6.653 & 0.225 & 5.9179 & 5.8949 & -0.389 & 13.3081 \\
\hline 225.00 & 6.999 & 7.014 & 0.224 & 5.9126 & 5.8910 & -0.364 & 12.4163 \\
\hline 230.00 & 7.357 & 7.373 & 0.225 & 5.9072 & 5.8867 & -0.347 & 11.6346 \\
\hline 240.00 & 8.064 & 8.083 & 0.231 & 5.8965 & 5.8772 & -0.328 & 10.3317 \\
\hline 250.00 & 8.763 & 8.783 & 0.228 & 5.8857 & 5.8680 & -0.302 & 9.2910 \\
\hline 260.00 & 9.454 & 9.475 & 0.220 & 5.8749 & 5.8586 & -0.277 & 8.4427 \\
\hline 270.00 & 10.138 & 10.160 & 0.209 & 5.8640 & 5.8493 & -0.251 & 0.0258 \\
\hline 280.00 & 10.816 & 10.837 & 0.200 & 5.8531 & 5.8395 & -0.232 & 0.0238 \\
\hline 290.00 & 11.487 & 11.509 & 0.184 & 5.8422 & 5.8300 & -0.208 & 0.0221 \\
\hline 300.00 & 12.153 & 12.174 & 0.167 & 5.8312 & 5.8205 & -0.184 & 0.0207 \\
\hline 192.00 & 4.685 & 4.687 & 0.050 & 6.9818 & 6.9571 & -0.354 & 0.0000 \\
\hline 196.00 & 5.066 & 5.072 & 0.124 & 6.9765 & 6.9384 & -0.546 & 19.6268 \\
\hline 200.00 & 5.441 & 5.450 & 0.168 & 6.9712 & 6.9321 & -0.561 & 17.9586 \\
\hline 204.00 & 5.811 & 5.822 & 0.189 & 6.9659 & 6.9295 & -0.524 & 16.5074 \\
\hline 208.00 & 6.177 & 6.190 & 0.203 & 6.9607 & 6.9267 & -0.489 & 15.2439 \\
\hline 212.00 & 6.541 & 6.555 & 0.208 & 6.9555 & 6.9243 & -0.448 & 14.1394 \\
\hline 216.00 & 6.901 & 6.917 & 0.220 & 6.9502 & 6.9202 & -0.432 & 13.1713 \\
\hline 220.00 & 7.259 & 7.276 & 0.229 & 6.9450 & 6.9159 & -0.418 & 12.3179 \\
\hline 225.00 & 7.703 & 7.722 & 0.239 & 6.9384 & 6.9104 & -0.403 & 11.3861 \\
\hline 230.00 & 8.144 & 8.164 & 0.251 & 6.9317 & 6.9042 & -0.397 & 10.5795 \\
\hline
\end{tabular}


Data from Goodwin [38] (continued)

\begin{tabular}{|c|c|c|c|c|c|c|c|}
\hline $\mathrm{T}$ & $P, \exp$ & $\mathrm{P}, \mathrm{cal}$ & Dev & $\rho, \exp$ & $\rho, \mathrm{cal}$ & Dev & Wt \\
\hline K & $\mathrm{MPa}$ & $\mathrm{MPa}$ & 8 & $\mathrm{~mol} \cdot \mathrm{dm}^{-3}$ & $\mathrm{~mol} \cdot \mathrm{dm}^{-3}$ & 8 & \\
\hline 240.00 & 9.016 & 9.040 & 0.272 & 6.9186 & 6.8917 & -0.388 & 9.2574 \\
\hline 250.00 & 9.879 & 9.906 & 0.271 & 6.9053 & 6.8806 & -0.357 & 8.2230 \\
\hline 260.00 & 10.732 & 10.761 & 0.272 & 6.8920 & 6.8688 & -0.337 & 7.3956 \\
\hline 270.00 & 11.579 & 11.608 & 0.250 & 6.8786 & 6.8583 & -0.295 & 0.0224 \\
\hline 280.00 & 12.417 & 12.446 & 0.234 & 6.8653 & 6.8471 & -0.265 & 0.0205 \\
\hline 290.00 & 13.249 & 13.277 & 0.214 & 6.8521 & 6.8360 & -0.234 & 0.019 \\
\hline 300.00 & 14.074 & 14.101 & 0.191 & 6.8389 & 6.8250 & -0.204 & 0.0176 \\
\hline 192.00 & 4.753 & 4.754 & 0.018 & 7.9789 & 7.9598 & -0.238 & 0.000 \\
\hline 196.00 & 5.201 & 5.206 & 0.101 & 7.9726 & 7.9253 & -0.593 & 19.318 \\
\hline 200.00 & 5.642 & 5.651 & 0.176 & 7.9664 & 7.9110 & -0.695 & 17.520 \\
\hline 204.00 & 6.078 & 6.092 & 0.223 & 7.9601 & 7.9055 & -0.687 & 15.954 \\
\hline 208.00 & 6.512 & 6.528 & 0.248 & 7.9539 & 7.9033 & -0.636 & 14.593 \\
\hline 212.00 & 6.944 & 6.962 & 0.250 & 7.9476 & 7.9031 & -0.560 & 13.411 \\
\hline 216.00 & 7.373 & 7.392 & 0.263 & 7.9413 & 7.8994 & -0.528 & 12.384 \\
\hline 220.00 & 7.800 & 7.821 & 0.273 & 7.9350 & 7.8952 & -0.502 & 11.488 \\
\hline 25.00 & 8.329 & 8.353 & 0.288 & 7.9271 & 7.8 & -0.484 & 10.521 \\
\hline 230.00 & 8.857 & 8.882 & 0.291 & 7.9192 & 7.8832 & -0.454 & 9.693 \\
\hline 240.00 & 9.901 & 9.931 & 0.305 & 7.9034 & 7.8699 & -0.424 & 8.360 \\
\hline 250.00 & 10.936 & 10.969 & 0.303 & 7.8876 & 7.8570 & -0.387 & 7.340 \\
\hline 260.00 & 11.961 & 11.996 & 0.292 & 7.8718 & 7.8443 & -0.349 & 6.537 \\
\hline 270.00 & 12.978 & 13.014 & 0.276 & & 7. & -0.313 & 0.019 \\
\hline 280.00 & 13.986 & 14.022 & 0.257 & 7.8405 & 7.8186 & -0.279 & 0.017 \\
\hline 290.00 & 14.985 & 15.021 & 0.237 & 7.8251 & 7.8056 & -0.249 & 0.016 \\
\hline 300.00 & 15.977 & 16.012 & 0.217 & 7.8100 & 7.7927 & -0.221 & 0.015 \\
\hline 192.00 & 4.789 & 4.788 & -0.012 & 9.0890 & 9.1140 & 0.274 & 0.000 \\
\hline 196.00 & 5.308 & 5.311 & 0.051 & 9.08 & 9.0 & -0.361 & 19.003 \\
\hline 200.00 & 5.824 & 5.831 & 0.118 & 9.0743 & 9.0286 & -0.504 & 17.038 \\
\hline 204.00 & 6.336 & 6.348 & 0.187 & 9.0669 & 9.0135 & -0.589 & 15.332 \\
\hline 208.00 & 6.850 & 6.864 & 0.205 & 9.0595 & 9.0121 & -0.523 & 13.856 \\
\hline 212.00 & 7.361 & 7.379 & 0.231 & 9.0520 & 9.0063 & -0.505 & 12.591 \\
\hline 216.00 & 7.871 & 7.891 & 0.254 & 9.0445 & 9.0000 & -0.492 & 11.506 \\
\hline 220.00 & 8.380 & 8.402 & 0.262 & 9.0369 & 8.9953 & -0.461 & 10.570 \\
\hline 225.00 & 9.013 & 9.038 & 0.280 & 9.0275 & 8.9873 & -0.445 & 9.577 \\
\hline 230.00 & 9.644 & 9.671 & 0.286 & 9.0180 & 8.9801 & -0.420 & 8.740 \\
\hline 240.00 & 10.897 & 10.930 & 0.300 & 8.9990 & 8.9638 & -0.392 & 7.420 \\
\hline
\end{tabular}


Data from Goodwin [38] (continued)

\begin{tabular}{|c|c|c|c|c|c|c|c|}
\hline $\mathrm{T}$ & $P, \exp$ & $\mathrm{P}, \mathrm{cal}$ & Dev & $\rho, \exp$ & $\rho$, cal & Dev & Wt \\
\hline K & $\mathrm{MPa}$ & $\mathrm{MPa}$ & 8 & $\mathrm{~mol} \cdot \mathrm{dm}^{-3}$ & $\mathrm{~mol} \cdot \mathrm{dm}^{-3}$ & 8 & \\
\hline 250.00 & 12.140 & 12.177 & 0.306 & 8.9801 & 8.9473 & -0.365 & 6.4348 \\
\hline 260.00 & 13.373 & 13.413 & 0.304 & 8.9614 & 8.9310 & -0.339 & 5.6753 \\
\hline 270.00 & 14.597 & 14.639 & 0.288 & 8.9429 & 8.9156 & -0.305 & 0.0169 \\
\hline 280.00 & 15.811 & 15.855 & 0.274 & 8.9249 & 8.9001 & -0.278 & 0.0153 \\
\hline 290.00 & 17.017 & 17.060 & 0.254 & 8.9072 & 8.8850 & -0.249 & 0.0140 \\
\hline 300.00 & 18.215 & 18.256 & 0.228 & 8.8900 & 8.8707 & -0.216 & 0.0129 \\
\hline 192.00 & 4.810 & 4.811 & 0.017 & 10.2380 & 10.1955 & -0.415 & 0.0000 \\
\hline 196.00 & 5.402 & 5.404 & 0.031 & 10.2296 & 10.2096 & -0.196 & 18.6281 \\
\hline 200.00 & 5.998 & 6.003 & 0.075 & 10.2210 & 10.1922 & -0.282 & 16.4207 \\
\hline 204.00 & 6.598 & 6.606 & 0.118 & 10.2123 & 10.1790 & -0.326 & 14.5265 \\
\hline 208.00 & 7.199 & 7.210 & 0.151 & 10.2036 & 10.1690 & -0.339 & 12.9269 \\
\hline 212.00 & 7.801 & 7.815 & 0.175 & 10.1947 & 10.1604 & -0.336 & 11.5846 \\
\hline 216.00 & 8.404 & 8.420 & 0.193 & 10.1858 & 10.1523 & -0.329 & 10.4578 \\
\hline 220.00 & 9.006 & 9.024 & 0.199 & 10.1769 & 10.1454 & -0.309 & 9.5067 \\
\hline 225.00 & 9.757 & 9.778 & 0.217 & 10.1657 & 10.1346 & -0.305 & 8.5167 \\
\hline 230.00 & 10.507 & 10.530 & 0.224 & 10.1545 & 10.1248 & -0.292 & 7.6992 \\
\hline 240.00 & 11.998 & 12.028 & 0.244 & 10.1321 & 10.1033 & -0.284 & 6.4401 \\
\hline 250.00 & 13.481 & 13.516 & 0.257 & 10.1100 & 10.0822 & -0.275 & 5.5235 \\
\hline 260.00 & 14.954 & 14.993 & 0.255 & 10.0882 & 10.0624 & -0.256 & 4.8311 \\
\hline 270.00 & 16.417 & 16.458 & 0.255 & 10.0671 & 10.0425 & -0.244 & 0.0143 \\
\hline 280.00 & 17.870 & 17.913 & 0.242 & 10.0466 & 10.0242 & -0.223 & 0.0129 \\
\hline 290.00 & 19.313 & 19.358 & 0.229 & 10.0271 & 10.0066 & -0.204 & 0.0117 \\
\hline 300.00 & 20.747 & 20.791 & 0.211 & 10.0081 & 9.9898 & -0.183 & 0.0107 \\
\hline 192.00 & 4.832 & 4.831 & -0.026 & 11.1113 & 11.1567 & 0.408 & 0.0000 \\
\hline 196.00 & 5.485 & 5.484 & -0.035 & 11.1019 & 11.1205 & 0.167 & 18.1729 \\
\hline 200.00 & 6.151 & 6.150 & -0.018 & 11. & 11 & 0. & 15. \\
\hline 204.00 & 6.825 & 6.825 & -0.005 & 11.0825 & 11.0837 & 0.011 & 13.6401 \\
\hline 208.00 & 7.502 & 7.503 & 0.021 & 11.0725 & 11.0682 & -0.039 & 11.9618 \\
\hline 212.00 & 8.177 & 8.184 & 0.085 & 11.0625 & 11.0472 & -0.138 & 10.5985 \\
\hline 216.00 & 8.861 & 8.866 & 0.061 & 11.0523 & 11.0424 & -0.089 & 9.4759 \\
\hline 220.00 & 9.542 & 9.549 & 0.076 & 11.0422 & 11.0309 & -0.102 & 8.5500 \\
\hline 225.00 & 10.391 & 10.401 & 0.098 & 11.0294 & 11.0162 & -0.120 & 7.6027 \\
\hline 230.00 & 11.240 & 11.253 & 0.115 & 11.0167 & 11.0022 & -0.132 & 6.8329 \\
\hline 240.00 & 12.934 & 12.951 & 0.133 & 10.9914 & 10.9763 & -0.138 & 5.6668 \\
\hline 250.00 & 14.617 & 14.641 & 0.161 & 10.9667 & 10.9497 & -0.155 & 4.8327 \\
\hline
\end{tabular}


Data from Goodwin [38] (continued)

\begin{tabular}{|c|c|c|c|c|c|c|c|}
\hline $\mathrm{T}$ & $P, \exp$ & P,cal & Dev & $\rho, \exp$ & $\rho$, cal & Dev & Wt \\
\hline K & $\mathrm{MPa}$ & $\mathrm{MPa}$ & 8 & $\mathrm{~mol} \cdot \mathrm{dm}^{-3}$ & $\mathrm{~mol} \cdot \mathrm{dm}^{-3}$ & 8 & \\
\hline 260.00 & 16.291 & 16.319 & 0.175 & 10.9428 & 10.9254 & -0.159 & 4.2102 \\
\hline 270.00 & 17.955 & 17.987 & 0.179 & 10.9198 & 10.9028 & -0.156 & 0.0124 \\
\hline 280.00 & 19.607 & 19.643 & 0.182 & 10.8978 & 10.8811 & -0.154 & 0.0112 \\
\hline 290.00 & 21.250 & 21.287 & 0.177 & 10.8769 & 10.8612 & -0.145 & 0.0101 \\
\hline 300.00 & 22.883 & 22.920 & 0.164 & 10.8570 & 10.8428 & -0.131 & 0.0093 \\
\hline 192.00 & 4.869 & 4.869 & 0.003 & 12.0697 & 12.0670 & -0.022 & 0.0000 \\
\hline 196.00 & 5.603 & 5.604 & 0.021 & 12.0591 & 12.0517 & -0.062 & 17.2314 \\
\hline 200.00 & 6.357 & 6.359 & 0.021 & 12.0482 & 12.0429 & -0.044 & 14.4081 \\
\hline 204.00 & 7.122 & 7.123 & 0.027 & 12.0370 & 12.0317 & -0.044 & 12.2259 \\
\hline 208.00 & 7.891 & 7.894 & 0.038 & 12.0256 & 12.0191 & -0.054 & 10.5450 \\
\hline 212.00 & 8.665 & 8.669 & 0.039 & 12.0142 & 12.0083 & -0.049 & 9.2315 \\
\hline 216.00 & 9.443 & 9.445 & 0.026 & 12.0026 & 11.9989 & -0.031 & 8.1869 \\
\hline 220.00 & 10.220 & 10.223 & 0.029 & 11.9912 & 11.9874 & -0.032 & 7.3423 \\
\hline 225.00 & 11.191 & 11.196 & 0.041 & 11.9767 & 11.9717 & -0.042 & 6.4936 \\
\hline 230.00 & 12.162 & 12.168 & 0.054 & 11.9624 & 11.9562 & -0.052 & 5.8141 \\
\hline 240.00 & 14.099 & 14.110 & 0.079 & 11.9342 & 11.9258 & -0.071 & 4.7997 \\
\hline 250.00 & 16.026 & 16.043 & 0.109 & 11.9069 & 11.8960 & -0.091 & 4.0834 \\
\hline 260.00 & 17.943 & 17.966 & 0.130 & 11.8808 & 11.8684 & -0.104 & 3.5529 \\
\hline 270.00 & 19.849 & 19.877 & 0.142 & 11.8562 & 11.8432 & -0.110 & 0.0105 \\
\hline 280.00 & 21.744 & 21.776 & 0.147 & 11.8330 & 11.8198 & -0.111 & 0.0094 \\
\hline 290.00 & 23.629 & 23.663 & 0.143 & 11.8112 & 11.7988 & -0.105 & 0.0085 \\
\hline 300.00 & 25.503 & 25.537 & 0.133 & 11.7906 & 11.7792 & -0.097 & 0.0078 \\
\hline 192.00 & 4.946 & 4.948 & 0.052 & 12.9815 & 12.9612 & -0.157 & 0.0000 \\
\hline 196.00 & 5.779 & 5.785 & 0.106 & 12.9695 & 12.9450 & -0.189 & 15.3489 \\
\hline 200.00 & 6.633 & 6.640 & 0.111 & 12.9570 & 12.9371 & -0.154 & 12.4178 \\
\hline 204.00 & 7.499 & 7.506 & 0.085 & 12.9442 & 12.9313 & -0.100 & 10.3465 \\
\hline 208.00 & 8.373 & 8.377 & 0.058 & 12.9313 & 12.9234 & -0.061 & 8.8342 \\
\hline 212.00 & 9.250 & 9.253 & 0.036 & 12.9184 & 12.9138 & -0.035 & 7.6910 \\
\hline 216.00 & 10.130 & 10.132 & 0.011 & 12.9053 & 12.9040 & -0.010 & 6.8008 \\
\hline 220.00 & 11.012 & 11.011 & -0.003 & 12.8923 & 12.8926 & 0.002 & 6.0902 \\
\hline 225.00 & 12.111 & 12.112 & 0.004 & 12.8761 & 12.8757 & -0.003 & 5.3835 \\
\hline 230.00 & 13.212 & 13.213 & 0.008 & 12.8600 & 12.8591 & -0.007 & 4.8216 \\
\hline 240.00 & 15.406 & 15.411 & 0.032 & 12.8286 & 12.8255 & -0.024 & 3.9876 \\
\hline 250.00 & 17.590 & 17.601 & 0.060 & 12.7988 & 12.7932 & -0.044 & 3.4004 \\
\hline 260.00 & 19.764 & 19.779 & 0.079 & 12.7708 & 12.7637 & -0.056 & 2.9657 \\
\hline
\end{tabular}


Data from Goodwin [38] (continued)

\begin{tabular}{|c|c|c|c|c|c|c|c|}
\hline $\mathrm{T}$ & $P, \exp$ & P,cal & Dev & $\rho, \exp$ & $\rho$, cal & Dev & Wt \\
\hline K & $\mathrm{MPa}$ & $\mathrm{MPa}$ & 8 & $\mathrm{~mol} \cdot \mathrm{dm}^{-3}$ & $\mathrm{~mol} \cdot \mathrm{dm}^{-3}$ & 8 & \\
\hline 270.00 & 21.926 & 21.946 & 0.091 & 12.7448 & 12.7369 & -0.062 & 0.008 \\
\hline 280.00 & 24.075 & 24.099 & 0.099 & 12.7206 & 12.7121 & -0.066 & 0.007 \\
\hline 290.00 & 26.213 & 26.239 & 0.097 & 12.6980 & 12.6898 & -0.065 & 0.007 \\
\hline 300.00 & 28.339 & 28.363 & 0.087 & 12.6767 & 12.6694 & -0.057 & 0.006 \\
\hline 192.00 & 5.205 & 5.224 & 0.368 & 14.2336 & 14.1765 & -0.401 & 0.000 \\
\hline 196.00 & 6.221 & 6.235 & 0.223 & 14.2188 & 14.1907 & -0.198 & 10.812 \\
\hline 200.00 & 7.245 & 7.258 & 0.179 & 14.2038 & 14.1836 & -0.142 & 8.702 \\
\hline 204.00 & 8.279 & 8.289 & 0.130 & 14.1886 & 14.1750 & -0.096 & 7.280 \\
\hline 208.00 & 9.318 & 9.325 & 0.083 & 14.1733 & 14.1651 & -0.058 & 6.258 \\
\hline 212.00 & 10.361 & 10.365 & 0.039 & 14.1580 & 14.1543 & -0.026 & 5.488 \\
\hline 216.00 & 11.404 & 11.406 & 0.019 & 14.1427 & 14.1409 & -0.012 & 4.888 \\
\hline 220.00 & 12.448 & 12.449 & 0.006 & 14.1275 & 14.1269 & -0.004 & 4.407 \\
\hline 225.00 & 13.752 & 13.752 & 0.003 & 14.1089 & 14.1086 & -0.002 & 3.927 \\
\hline 230.00 & 15.053 & 15.055 & 0.009 & 14.0906 & 14.0898 & -0.005 & 3.542 \\
\hline 240.00 & 17.651 & 17.655 & 0.024 & 14.0557 & 14.0537 & -0.014 & 2.967 \\
\hline 250.00 & 20.235 & 20.244 & 0.045 & 14.0234 & 14.0196 & -0.027 & 2.556 \\
\hline 260.00 & 22.806 & 22.821 & 0.066 & 13.9939 & 13.9886 & -0.038 & 2.248 \\
\hline 270.00 & 25.363 & 25.383 & 0.076 & 13.9669 & 13.9607 & -0.044 & 0.006 \\
\hline 280.00 & 27.907 & 27.929 & 0.078 & 13.9421 & 13.9358 & -0.045 & 0.006 \\
\hline 290.00 & 30.433 & 30.458 & 0.080 & 13.9190 & 13.9126 & -0.046 & 0.005 \\
\hline 300.00 & 32.948 & 32.969 & 0.062 & 13.8972 & 13.8923 & -0.035 & 0.00 \\
\hline 190 & 5. & 5.211 & 0.473 & 15.3796 & 396 & -0.260 & 0.000 \\
\hline 194.00 & 6.379 & 6.404 & 0.394 & 15.3621 & 15.3305 & -0.205 & 7.322 \\
\hline 198.00 & 7.586 & 7.607 & 0.275 & 15.3444 & 15.3231 & -0.139 & 6.033 \\
\hline 202.00 & 8.801 & 8.816 & 0.171 & 15.3267 & 15.3136 & -0.085 & 5.139 \\
\hline 206.00 & 10.020 & 10.029 & 0.091 & 15.3088 & 15. & -0.045 & 4.482 \\
\hline 210.00 & 11.238 & 11.244 & 0.058 & 15.2911 & 15.2867 & -0.029 & 3.978 \\
\hline 215.00 & 12.760 & 12.764 & 0.037 & 15.2692 & 15.2665 & -0.018 & 3.493 \\
\hline 220.00 & 14.279 & 14.284 & 0.033 & 15.2478 & 15.2422 & -0.016 & 3.117 \\
\hline 230.00 & 17.313 & 17.318 & 0.030 & 15.2072 & 15.2049 & -0.015 & 2.572 \\
\hline 240.00 & 20.329 & 20.340 & 0.056 & 15.1701 & 15.1660 & -0.027 & 2.19 \\
\hline 250. & 23.331 & 23.348 & 0.070 & 15.1369 & 15.1317 & -0.034 & 1.919 \\
\hline 260.00 & 26.318 & 26.338 & 0.077 & 15.1069 & 15.1012 & -0.038 & 1.707 \\
\hline 270.00 & 29.286 & 29.309 & 0.079 & 15.0797 & 15.0738 & -0.039 & 0.005 \\
\hline 280.00 & 32.240 & 32.260 & 0.062 & 15.0546 & 15.0500 & -0.031 & 0.004 \\
\hline
\end{tabular}


Data from Goodwin [38] (continued)

\begin{tabular}{|c|c|c|c|c|c|c|c|}
\hline $\mathrm{T}$ & $P, \exp$ & P,cal & Dev & $\rho, \exp$ & $\rho$, cal & Dev & Wt \\
\hline K & $\mathrm{MPa}$ & $\mathrm{MPa}$ & 8 & $\mathrm{~mol} \cdot \mathrm{dm}^{-3}$ & $\mathrm{~mol} \cdot \mathrm{dm}^{-3}$ & 8 & \\
\hline 290.00 & 35.168 & 35.189 & 0.061 & 15.0313 & 15.0268 & -0.030 & 0.0430 \\
\hline 186.00 & 4.562 & 4.581 & 0.423 & 16.2733 & 16.2502 & -0.142 & 0.0000 \\
\hline 190.00 & 5.908 & 5.931 & 0.396 & 16.2543 & 16.2319 & -0.138 & 0.0000 \\
\hline 194.00 & 7.268 & 7.292 & 0.325 & 16.2348 & 16.2159 & -0.116 & 4.7079 \\
\hline 198.00 & 8.636 & 8.658 & 0.251 & 16.2151 & 16.2002 & -0.092 & 4.0524 \\
\hline 202.00 & 10.005 & 10.027 & 0.225 & 16.1953 & 16.1817 & -0.084 & 3.5641 \\
\hline 206.00 & 11.375 & 11.398 & 0.198 & 16.1755 & 16.1633 & -0.076 & 3.1859 \\
\hline 210.00 & 12.745 & 12.768 & 0.179 & 16.1562 & 16.1450 & -0.069 & 2.8837 \\
\hline 215.00 & 14.440 & 14.480 & 0.276 & 16.1328 & 16.1152 & -0.109 & 2.5822 \\
\hline 220.00 & 16.161 & 16.188 & 0.172 & 16.1098 & 16.0987 & -0.069 & 2.3408 \\
\hline 230.00 & 19.563 & 19.595 & 0.166 & 16.0677 & 16.0567 & -0.068 & 1.9781 \\
\hline 240.00 & 22.944 & 22.984 & 0.176 & 16.0302 & 16.0184 & -0.074 & 1.7178 \\
\hline 250.00 & 26.311 & 26.354 & 0.162 & 15.9971 & 15.9861 & -0.069 & 1.5211 \\
\hline 260.00 & 29.654 & 29.702 & 0.162 & 15.9676 & 15.9564 & -0.070 & 1.3671 \\
\hline 270.00 & 32.976 & 33.026 & 0.153 & 15.9408 & 15.9301 & -0.067 & 0.0041 \\
\hline 182.00 & 4.132 & 4.164 & 0.761 & 17.2858 & 17.2607 & -0.145 & 0.0000 \\
\hline 186.00 & 5.695 & 5.724 & 0.505 & 17.2636 & 17.2444 & -0.111 & 0.0000 \\
\hline 190.00 & 7.268 & 7.292 & 0.334 & 17.2416 & 17.2277 & -0.081 & 16.4604 \\
\hline 194.00 & 8.835 & 8.863 & 0.325 & 17.2194 & 17.2048 & -0.085 & 2.8998 \\
\hline 198.00 & 10.408 & 10.436 & 0.266 & 17.1973 & 17.1847 & -0.073 & 2.5967 \\
\hline 202.00 & 11.977 & 12.007 & 0.245 & 17.1754 & 17.16 & -0.071 & 2.3554 \\
\hline 206.00 & 13.546 & 13.575 & 0.214 & 17.1539 & 17.1429 & -0.064 & 2.1583 \\
\hline 210.00 & 15.108 & 15.141 & 0.219 & 17.1331 & 17.1215 & -0.068 & 1.9940 \\
\hline 215.00 & 17.060 & 17.094 & 0.196 & 17.1084 & 17.0976 & -0.063 & 1.8231 \\
\hline 220.00 & 19.003 & 19.040 & 0.192 & 17.0848 & 17.0740 & -0.063 & 1.6815 \\
\hline 230.00 & 22.872 & 22.915 & 0.188 & 17.0424 & 17.0313 & -0.065 & 1.4589 \\
\hline 240.00 & 26.719 & 26.766 & 0.176 & 17.0056 & 16.9948 & -0.063 & 1.2916 \\
\hline 250.00 & 30.544 & 30.589 & 0.146 & 16.9731 & 16.9639 & -0.054 & 1.1609 \\
\hline 260.00 & 34.336 & 34.382 & 0.136 & 16.9441 & 16.9353 & -0.052 & 1.0558 \\
\hline 176.00 & 3.217 & 3.267 & 1.543 & 18.3840 & 18.3568 & -0.148 & 14.9925 \\
\hline 180.00 & 5.044 & 5.087 & 0.848 & 18.3570 & 18.3368 & -0.110 & 0.0000 \\
\hline 184.00 & 6.869 & 6.914 & 0.648 & 18.3320 & 18.3134 & -0.102 & 0.0000 \\
\hline 188.00 & 8.691 & 8.740 & 0.572 & 18.3070 & 18.2883 & -0.102 & 10.2954 \\
\hline 192.00 & 10.509 & 10.560 & 0.490 & 18.2810 & 18.2633 & -0.097 & 1.8752 \\
\hline 196.00 & 12.323 & 12.377 & 0.440 & 18.2560 & 18.2389 & -0.094 & 1.7245 \\
\hline
\end{tabular}


Data from Goodwin [38] (continued)

\begin{tabular}{|c|c|c|c|c|c|c|c|}
\hline $\mathrm{T}$ & $P, \exp$ & P,cal & Dev & $\rho, \exp$ & $\rho$, cal & Dev & Wt \\
\hline K & $\mathrm{MPa}$ & $\mathrm{MPa}$ & 8 & $\mathrm{~mol} \cdot \mathrm{dm}^{-3}$ & $\mathrm{~mol} \cdot \mathrm{dm}^{-3}$ & 8 & \\
\hline 200.00 & 14.131 & 14.189 & 0.407 & 18.2320 & 18.2151 & -0.093 & 1.598 \\
\hline 205.00 & 16.386 & 16.444 & 0.353 & 18.2030 & 18.1874 & -0.086 & 1.4674 \\
\hline 210.00 & 18.631 & 18.687 & 0.298 & 18.1750 & 18.1611 & -0.076 & 1. 3585 \\
\hline 215.00 & 20.869 & 20.925 & 0.270 & 18.1500 & 18.1368 & -0.073 & 1.265 \\
\hline 220.00 & 23.100 & 23.155 & 0.240 & 18.1270 & 18.1149 & -0.067 & 1.1854 \\
\hline 225.00 & 25.324 & 25.379 & 0.217 & 18.1060 & 18.0947 & -0.063 & 1.115 \\
\hline 230.00 & 27.539 & 27.590 & 0.185 & 18.0860 & 18.0760 & -0.055 & 1.054 \\
\hline 235.00 & 29.748 & 29.796 & 0.163 & 18.0680 & 18.0590 & -0.050 & 0.999 \\
\hline 240.00 & 31.949 & 31.998 & 0.153 & 18.0520 & 18.0434 & -0.048 & 0.950 \\
\hline 245.00 & 34.140 & 34.184 & 0.128 & 18.0360 & 18.0286 & -0.041 & 0.906 \\
\hline 170.00 & 2.637 & 2.753 & 4.431 & 19.5060 & 19.4631 & -0.220 & 9.488 \\
\hline 174.00 & 4.774 & 4.887 & 2.363 & 19.4750 & 19.4379 & -0.190 & 8.502 \\
\hline 178.00 & 6.901 & 7.021 & 1.743 & 19.4460 & 19.4101 & -0.184 & 7.723 \\
\hline 182.00 & 9.012 & 9.146 & 1.487 & 19.4170 & 19.3802 & -0.189 & 7.096 \\
\hline 186.00 & 11.113 & 11.259 & 1.312 & 19.3880 & 19.3509 & -0.191 & 6.580 \\
\hline 190.00 & 13.206 & 13.357 & 1.144 & 19.3590 & 19.3231 & -0.186 & 6.147 \\
\hline 194.00 & 15.291 & 15.449 & 1.031 & 19.3320 & 19.2968 & -0.182 & 1.154 \\
\hline 198.00 & 17.367 & 17.534 & 0.963 & 19.3070 & 19.2717 & -0.183 & 1.089 \\
\hline 202.00 & 19.435 & 19.608 & 0.891 & 19.2830 & 19.2484 & -0.180 & 1.032 \\
\hline 205.00 & 20.984 & 21.158 & 0.831 & 19.2660 & 19.2324 & -0.174 & 0.993 \\
\hline 210.00 & 23.558 & 23.734 & 0.7 & 19.2400 & 19.2080 & -0.167 & 0.93 \\
\hline 215.00 & 26.121 & 26.297 & 0.677 & 19.2160 & 19.1857 & -0.158 & 0.884 \\
\hline 220.00 & 28.676 & 28.855 & 0.624 & 19.1950 & 19.1658 & -0.152 & 0.838 \\
\hline 225.00 & 31.221 & 31.397 & 0.562 & 19.1750 & 19.1478 & -0.142 & 0.797 \\
\hline 230.00 & 33.754 & 33.930 & 0.521 & 19.1570 & 19.1309 & -0.136 & 0.761 \\
\hline 164.00 & 2.029 & 2.115 & 4.230 & 20.4250 & 20.4016 & -0.115 & 6.774 \\
\hline 168.00 & 4.469 & 4.532 & 1.405 & 20.3890 & 20.3734 & -0.077 & 6.204 \\
\hline 172.00 & 6.882 & 6.949 & 0.975 & 20.3570 & 20.3416 & -0.076 & 5.731 \\
\hline 176.00 & 9.270 & 9.345 & 0.807 & 20.3240 & 20.3080 & -0.079 & 5.343 \\
\hline 180.00 & 11.639 & 11.724 & 0.733 & 20.2920 & 20.2748 & -0.085 & 5.014 \\
\hline 184.00 & 13.997 & 14.082 & 0.608 & 20.2600 & 20.2438 & -0.080 & 4.732 \\
\hline 188.00 & 16.342 & 16.432 & 0.553 & 20.2310 & 20.2147 & -0.080 & 4.483 \\
\hline 192.00 & 18.678 & 18.783 & 0.566 & 20.2060 & 20.1879 & -0.090 & 0.851 \\
\hline 196.00 & 21.008 & 21.095 & 0.411 & 20.1780 & 20.1639 & -0.070 & 0.813 \\
\hline 200.00 & 23.332 & 23.415 & 0.354 & 20.1550 & 20.1421 & -0.064 & 0.777 \\
\hline
\end{tabular}


Data from Goodwin [38] (continued)

\begin{tabular}{|c|c|c|c|c|c|c|c|}
\hline $\mathrm{T}$ & $P, \exp$ & $\mathrm{P}, \mathrm{cal}$ & Dev & $\rho, \exp$ & $\rho$, cal & Dev & Wt \\
\hline K & $\mathrm{MPa}$ & $\mathrm{MPa}$ & 8 & $\mathrm{~mol} \cdot \mathrm{dm}^{-3}$ & $\mathrm{~mol} \cdot \mathrm{dm}^{-3}$ & 8 & \\
\hline 204.00 & 25.649 & 25.727 & 0.303 & 20.1340 & 20.1224 & -0.058 & 0.7448 \\
\hline 208.00 & 27.960 & 28.033 & 0.262 & 20.1150 & 20.1045 & -0.052 & 0.7149 \\
\hline 212.00 & 30.263 & 30.327 & 0.212 & 20.0970 & 20.0881 & -0.044 & 0.6876 \\
\hline 216.00 & 32.557 & 32.610 & 0.163 & 20.0800 & 20.0729 & -0.035 & 0.6626 \\
\hline 220.00 & 34.843 & 34.890 & 0.135 & 20.0650 & 20.0589 & -0.030 & 0.6392 \\
\hline 158.00 & 1.984 & 2.061 & 3.912 & 21.3710 & 21.3554 & -0.073 & 4.7922 \\
\hline 162.00 & 4.754 & 4.809 & 1.155 & 21.3320 & 21.3217 & -0.048 & 4.4733 \\
\hline 166.00 & 7.475 & 7.533 & 0.775 & 21.2940 & 21.2838 & -0.048 & 4.2049 \\
\hline 170.00 & 10.160 & 10.238 & 0.769 & 21.2580 & 21.2450 & -0.061 & 3.9735 \\
\hline 174.00 & 12.829 & 12.917 & 0.691 & 21.2230 & 21.2090 & -0.066 & 3.7727 \\
\hline 178.00 & 15.481 & 15.571 & 0.585 & 21.1890 & 21.1754 & -0.064 & 3.5961 \\
\hline 182.00 & 18.122 & 18.214 & 0.507 & 21.1580 & 21.1448 & -0.063 & 3.4368 \\
\hline 186.00 & 20.756 & 20.839 & 0.402 & 21.1290 & 21.1175 & -0.054 & 3.2930 \\
\hline 190.00 & 23.382 & 23.457 & 0.321 & 21.1030 & 21.0931 & -0.047 & 3.1609 \\
\hline 194.00 & 26.003 & 26.070 & 0.258 & 21.0800 & 21.0715 & -0.041 & 0.6078 \\
\hline 198.00 & 28.612 & 28.672 & 0.210 & 21.0590 & 21.0516 & -0.035 & 0.5853 \\
\hline 202.00 & 31.214 & 31.265 & 0.165 & 21.0400 & 21.0339 & -0.029 & 0.5645 \\
\hline 206.00 & 33.804 & 33.843 & 0.116 & 21.0220 & 21.0175 & -0.021 & 0.5453 \\
\hline 152.00 & 1.459 & 1.545 & 5.871 & 22.1250 & 22.1110 & -0.063 & 3.7607 \\
\hline 156.00 & 4.509 & 4.560 & 1.124 & 22.0800 & 22.0722 & -0.035 & 3.5533 \\
\hline 160.00 & 7.492 & 7.566 & 0.987 & 22.0400 & 22.0292 & -0.049 & 3.3697 \\
\hline 164.00 & 10.440 & 10.539 & 0.949 & 22.0010 & 21.9872 & -0.063 & 3.2104 \\
\hline 168.00 & 13.369 & 13.471 & 0.760 & 21.9620 & 21.9485 & -0.061 & 3.0715 \\
\hline 172.00 & 16.277 & 16.407 & 0.797 & 21.9290 & 21.9125 & -0.075 & 2.9423 \\
\hline 176.00 & 19.176 & 19.271 & 0.498 & 21.8920 & 21.8803 & -0.053 & 2.8317 \\
\hline 180.00 & 22.065 & 22.153 & 0.400 & 21.8620 & 21.8516 & -0.048 & 2.7257 \\
\hline 184.00 & 24.952 & 25.033 & 0.325 & 21.8360 & 21.8268 & -0.042 & 2.6265 \\
\hline 188.00 & 27.831 & 27.897 & 0.237 & 21.8120 & 21.8048 & -0.033 & 2.5348 \\
\hline 192.00 & 30.702 & 30.756 & 0.178 & 21.7910 & 21.7852 & -0.027 & 0.4898 \\
\hline 196.00 & 33.558 & 33.604 & 0.139 & 21.7720 & 21.7672 & -0.022 & 0.4738 \\
\hline 146.00 & 1.313 & 1.383 & 5.358 & 22.8730 & 22.8636 & -0.041 & 2.9682 \\
\hline 140.00 & 1.226 & 1.278 & 4.205 & 23.5700 & 23.5643 & -0.024 & 2.4063 \\
\hline 150.00 & 4.670 & 4.690 & 0.427 & 22.8240 & 22.8215 & -0.011 & 2.8326 \\
\hline 154.00 & 7.946 & 8.000 & 0.674 & 22.7820 & 22.7755 & -0.028 & 2.7080 \\
\hline 158.00 & 11.174 & 11.248 & 0.671 & 22.7390 & 22.7303 & -0.038 & 2.6005 \\
\hline
\end{tabular}


Data from Goodwin [38] (continued)

\begin{tabular}{|c|c|c|c|c|c|c|c|}
\hline $\mathrm{T}$ & $P, \exp$ & P,cal & Dev & $\rho, \exp$ & $\rho$, cal & Dev & Wt \\
\hline $\mathrm{K}$ & $\mathrm{MPa}$ & $\mathrm{MPa}$ & 8 & $\mathrm{~mol} \cdot \mathrm{dm}^{-3}$ & $\mathrm{~mol} \cdot \mathrm{dm}^{-3}$ & 8 & \\
\hline 62.00 & 14.381 & 14.461 & 0.559 & 22.6980 & 22.6890 & -0.040 & 2.5038 \\
\hline 166.00 & 17.571 & 17.649 & 0.444 & 22.6600 & 22.6516 & -0.037 & 2.4150 \\
\hline 170.00 & 20.755 & 20.825 & 0.335 & 22.6260 & 22.6188 & -0.032 & 2.3320 \\
\hline 174.00 & 23.933 & 23.993 & 0.250 & 22.5960 & 22.5900 & -0.027 & 2.2539 \\
\hline 178.00 & 27.111 & 27.157 & 0.170 & 22.5700 & 22.5655 & -0.020 & 2.1800 \\
\hline 182.00 & 30.277 & 30.302 & 0.082 & 22.5460 & 22.5436 & -0.010 & 2.1112 \\
\hline 186.00 & 33.428 & 33.440 & 0.035 & 22.5250 & 22.5239 & -0.005 & 2.0461 \\
\hline 144.00 & 4.891 & 4.882 & -0.181 & 23.5180 & 23.5189 & 0.004 & 2.3137 \\
\hline 148.00 & 8.450 & 8.476 & 0.311 & 23.4720 & 23.4693 & -0.011 & 2.2280 \\
\hline 152.00 & 11.953 & 12.005 & 0.438 & 23.4260 & 23.4208 & -0.022 & 2.1526 \\
\hline 156.00 & 15.435 & 15.499 & 0.414 & 23.3830 & 23.3769 & -0.026 & 2.0832 \\
\hline 160.00 & 18.907 & 18.962 & 0.291 & 23.3430 & 23.3379 & -0.022 & 2.0189 \\
\hline 164.00 & 22.376 & 22.419 & 0.192 & 23.3080 & 23.3041 & -0.017 & 1.9573 \\
\hline 168.00 & 25.844 & 25.877 & 0.128 & 23.2780 & 23.2751 & -0.012 & 1.8981 \\
\hline 172.00 & 29.304 & 29.330 & 0.090 & 23.2520 & 23.2498 & -0.010 & 1.8416 \\
\hline 176.00 & 32.758 & 32.760 & 0.005 & 23.2280 & 23.2279 & -0.001 & 1.7886 \\
\hline 134.00 & 0.686 & 0.773 & 12.615 & 24.1830 & 24.1747 & -0.034 & 27.0410 \\
\hline 138.00 & 4.638 & 4.625 & -0.286 & 24.1250 & 24.1262 & 0.005 & 1.9628 \\
\hline 142.00 & 8.463 & 8.494 & 0.370 & 24.0760 & 24.0732 & -0.012 & 1.8992 \\
\hline 146.00 & 12.224 & 12.286 & 0.505 & 24.0270 & 24.0216 & -0.022 & 1.8431 \\
\hline 150.00 & 15.966 & 16.035 & 0.434 & 23.9810 & 23.9751 & -0.024 & 1.7911 \\
\hline 154.00 & 19.704 & 19.770 & 0.335 & 23.9400 & 23.9346 & -0.023 & 1.7413 \\
\hline 134.00 & 0.584 & 0.679 & 16.256 & 24.1740 & 24.1648 & -0.038 & 27.1442 \\
\hline 136.00 & 2.577 & 2.572 & -0.207 & 24.1410 & 24.1415 & 0.002 & 2.0050 \\
\hline 138.00 & 4.526 & 4.517 & -0.185 & 24.1150 & 24.1158 & 0.003 & 1.9707 \\
\hline 140.00 & 6.451 & 6.454 & 0.049 & 24.0900 & 24.0897 & -0.001 & 1.9380 \\
\hline 142.00 & 8.335 & 8.383 & 0.578 & 24.0660 & 24.0617 & -0.018 & 1.9066 \\
\hline 144.00 & 10.213 & 10.292 & 0.773 & 24.0420 & 24.0350 & -0.029 & 1.8771 \\
\hline 146.00 & 12.094 & 12.171 & 0.632 & 24.0170 & 24.0103 & -0.028 & 1.8501 \\
\hline 148.00 & 13.970 & 14.053 & 0.594 & 23.9940 & 23.9869 & -0.030 & 1.8232 \\
\hline 150.00 & 15.837 & 15.917 & 0.500 & 23.9710 & 23.9643 & -0.028 & 1.7977 \\
\hline 152.00 & 17.705 & 17.785 & 0.451 & 23.9500 & 23.9433 & -0.028 & 1.7722 \\
\hline 154 & 19.575 & 19.660 & 0.437 & 23.9310 & 23.9240 & -0.029 & 1.7468 \\
\hline 156 & 21.442 & 21.518 & 0.357 & 23.9120 & 23.9058 & -0.026 & 1.7226 \\
\hline 158.00 & 23.289 & 23.359 & 0.303 & 23.8930 & 23.8874 & -0.024 & 1.6996 \\
\hline
\end{tabular}


Data from Goodwin [38] (continued)

\begin{tabular}{|c|c|c|c|c|c|c|c|}
\hline $\mathrm{T}$ & $P, \exp$ & P,cal & Dev & $\rho, \exp$ & $\rho$, cal & Dev & Wt \\
\hline K & $\mathrm{MPa}$ & $\mathrm{MPa}$ & 8 & $\mathrm{~mol} \cdot \mathrm{dm}^{-3}$ & $\mathrm{~mol} \cdot \mathrm{dm}^{-3}$ & 8 & \\
\hline 160.00 & 25.164 & 25.222 & 0.231 & 23.8770 & 23.8724 & -0.019 & 1.6758 \\
\hline 162.00 & 27.032 & 27.081 & 0.184 & 23.8620 & 23.8581 & -0.016 & 1.6525 \\
\hline 164.00 & 28.896 & 28.952 & 0.194 & 23.8490 & 23.8447 & -0.018 & 1.6292 \\
\hline 166.00 & 30.769 & 30.809 & 0.129 & 23.8360 & 23.8330 & -0.013 & 1.6068 \\
\hline 168.00 & 32.639 & 32.665 & 0.080 & 23.8240 & 23.8220 & -0.008 & 1.5848 \\
\hline 170.00 & 34.499 & 34.508 & 0.028 & 23.8120 & 23.8113 & -0.003 & 1.5636 \\
\hline 130.00 & 0.729 & 0.760 & 4.250 & 24.6050 & 24.6023 & -0.011 & 1.8011 \\
\hline 132.00 & 2.823 & 2.760 & -2.227 & 24.5720 & 24.5774 & 0.022 & 1.7764 \\
\hline 134.00 & 4.878 & 4.820 & -1.195 & 24.5460 & 24.5509 & 0.020 & 1.7485 \\
\hline 136.00 & 6.889 & 6.846 & -0.635 & 24.5190 & 24.5226 & 0.015 & 1.7232 \\
\hline 138.00 & 8.864 & 8.874 & 0.111 & 24.4940 & 24.4932 & -0.003 & 1.6982 \\
\hline 140.00 & 10.843 & 10.880 & 0.348 & 24.4690 & 24.4660 & -0.012 & 1.6746 \\
\hline 142.00 & 12.818 & 12.852 & 0.272 & 24.4430 & 24.4402 & -0.011 & 1.6530 \\
\hline 144.00 & 14.786 & 14.829 & 0.290 & 24.4190 & 24.4156 & -0.014 & 1.6314 \\
\hline 146.00 & 16.752 & 16.796 & 0.267 & 24.3960 & 24.3925 & -0.014 & 1.6103 \\
\hline 148.00 & 18.722 & 18.757 & 0.185 & 24.3740 & 24.3714 & -0.011 & 1.5898 \\
\hline 150.00 & 20.692 & 20.724 & 0.153 & 24.3540 & 24.3516 & -0.010 & 1.5691 \\
\hline 152.00 & 22.664 & 22.685 & 0.091 & 24.3350 & 24.3335 & -0.006 & 1.5489 \\
\hline 154.00 & 24.612 & 24.641 & 0.120 & 24.3170 & 24.3148 & -0.009 & 1.5291 \\
\hline 156.00 & 26.591 & 26.608 & 0.064 & 24.3010 & 24.2998 & -0.005 & 1.5091 \\
\hline 158.00 & 28.567 & 28.570 & 0.012 & 24.2860 & 24.2858 & -0.001 & 1.4894 \\
\hline 160.00 & 30.546 & 30.545 & -0.002 & 24.2730 & 24.2730 & 0.000 & 1.4697 \\
\hline 162.00 & 32.515 & 32.505 & -0.033 & 24.2600 & 24.2607 & 0.003 & 1.4507 \\
\hline 164.00 & 34.492 & 34.464 & -0.083 & 24.2480 & 24.2500 & 0.008 & 1.4319 \\
\hline 126.00 & 0.479 & 0.549 & 14.402 & 24.9960 & 24.9905 & -0.022 & 21.6203 \\
\hline 128.00 & 2.674 & 2.617 & -2.151 & 24.9600 & 24.96 & 0.018 & 1.6028 \\
\hline 130.00 & 4.828 & 4.774 & -1.109 & 24.9330 & 24.9371 & 0.017 & 1.5799 \\
\hline 132.00 & 6.944 & 6.894 & -0.719 & 24.9050 & 24.9088 & 0.015 & 1.5591 \\
\hline 134.00 & 9.021 & 9.017 & -0.044 & 24.8790 & 24.8793 & 0.001 & 1.5384 \\
\hline 136.00 & 11.090 & 11.102 & 0.105 & 24.8520 & 24.8511 & -0.003 & 1.5196 \\
\hline 138.00 & 13.149 & 13.176 & 0.209 & 24.8260 & 24.8240 & -0.008 & 1.5012 \\
\hline 140.00 & 15.205 & 15.241 & 0.239 & 24.8010 & 24.7984 & -0.011 & 1.4834 \\
\hline 142.00 & 17.262 & 17.296 & 0.200 & 24.7770 & 24.7745 & -0.010 & 1.4659 \\
\hline 144.00 & 19.322 & 19.343 & 0.109 & 24.7540 & 24.7525 & -0.006 & 1.4488 \\
\hline 146.00 & 21.386 & 21.411 & 0.119 & 24.7340 & 24.7322 & -0.007 & 1.4311 \\
\hline
\end{tabular}


Data from Goodwin [38] (continued)

\begin{tabular}{|c|c|c|c|c|c|c|c|}
\hline $\mathrm{T}$ & $P, \exp$ & P,cal & Dev & $\rho, \exp$ & $\rho, c a l$ & Dev & Wt \\
\hline K & $\mathrm{MPa}$ & $\mathrm{MPa}$ & 8 & $\mathrm{~mol} \cdot \mathrm{dm}^{-3}$ & $\mathrm{~mol} \cdot \mathrm{dm}^{-3}$ & 8 & \\
\hline 148.00 & 23.459 & 23.473 & 0.058 & 24.7150 & 24.7141 & -0.004 & 1.4137 \\
\hline 148.00 & 23.456 & 23.458 & 0.008 & 24.7140 & 24.7139 & -0.001 & 1.4142 \\
\hline 150.00 & 25.529 & 25.529 & -0.002 & 24.6970 & 24.6970 & 0.000 & 1.3966 \\
\hline 152.00 & 27.602 & 27.595 & -0.026 & 24.6810 & 24.6815 & 0.002 & 1.3793 \\
\hline 154.00 & 29.668 & 29.658 & -0.035 & 24.6660 & 24.6667 & 0.003 & 1.3623 \\
\hline 156.00 & 31.735 & 31.718 & -0.052 & 24.6520 & 24.6531 & 0.004 & 1.3455 \\
\hline 158.00 & 33.803 & 33.776 & -0.080 & 24.6390 & 24.6407 & 0.007 & 1.3290 \\
\hline 122.00 & 0.348 & 0.471 & 35.275 & 25.3860 & 25.3771 & -0.035 & 19.4872 \\
\hline 124.00 & 2.617 & 2.618 & 0.034 & 25.3480 & 25.3479 & 0.000 & 1.4471 \\
\hline 126.00 & 4.881 & 4.876 & -0.116 & 25.3200 & 25.3204 & 0.002 & 1.4283 \\
\hline 128.00 & 7.036 & 7.122 & 1.213 & 25.2930 & 25.2870 & -0.024 & 1.4102 \\
\hline 130.00 & 9.222 & 9.341 & 1.297 & 25.2660 & 25.2577 & -0.033 & 1.3933 \\
\hline 132.00 & 11.404 & 11.535 & 1.148 & 25.2390 & 25.2301 & -0.035 & 1.3774 \\
\hline 134.00 & 13.579 & 13.702 & 0.910 & 25.2120 & 25.2037 & -0.033 & 1.3624 \\
\hline 134.00 & 13.581 & 13.687 & 0.780 & 25.2110 & 25.2038 & -0.028 & 1.3629 \\
\hline $136.00^{\circ}$ & 15.717 & 15.828 & 0.707 & 25 . & 25 & -0.0 & 1.3487 \\
\hline 138.00 & 17.880 & 17.989 & 0.610 & 25.1600 & 25.1528 & -0.029 & 1.3339 \\
\hline 140.00 & 20.043 & 20.141 & 0.487 & 25.1370 & 25.1306 & -0.025 & 1.3193 \\
\hline 142.00 & 22.214 & 22.299 & 0.381 & 25.1160 & 25.1105 & -0.022 & 1.3046 \\
\hline 144.00 & 24.387 & 24.466 & 0.326 & 25.0970 & 25.0919 & -0.020 & 1.2897 \\
\hline 146.00 & 26.564 & 26.642 & 0.294 & 25.0800 & 25.07 & -0.020 & 1.2745 \\
\hline 148.00 & 28.746 & 28.814 & 0.236 & 25.0640 & 25.0598 & -0.017 & 1.2596 \\
\hline 150.00 & 30.928 & 30.982 & 0.173 & 25.0490 & 25.0457 & -0.013 & 1.2449 \\
\hline 152.00 & 33.099 & 33.146 & 0.143 & 25.0350 & 25. & -0 . & 1.2304 \\
\hline 154.00 & 35.273 & 35.308 & 0.101 & 25.0220 & 25.0199 & -0.009 & 0.0081 \\
\hline 118.00 & 0.474 & 0.575 & 21.302 & 25.7770 & 25.7703 & -0.026 & 17.5736 \\
\hline 120.00 & 2.849 & 2.830 & -0.649 & 25.7390 & 25.7402 & 0.005 & 1.3061 \\
\hline 122.00 & 5.181 & 5.192 & 0.204 & 25.7100 & 25.7093 & -0.003 & 1.2907 \\
\hline 124.00 & 7.430 & 7.540 & 1.482 & 25.6820 & 25.6749 & -0.028 & 1.2760 \\
\hline 126.00 & 9.720 & 9.860 & 1.446 & 25.6540 & 25.6451 & -0.035 & 1.2622 \\
\hline 128.00 & 12.001 & 12.135 & 1.118 & 25.6250 & $25.6]$ & -0.033 & 1.2497 \\
\hline 130.00 & 14.285 & 14.413 & 0.902 & 25.5980 & 25.5900 & -0.031 & 1.2371 \\
\hline 132.00 & 16. & 16.712 & 0.948 & 25.5740 & 25.5643 & -0.038 & 1.2239 \\
\hline 134.00 & 18.832 & 18.984 & 0.808 & 25.5500 & 25.5407 & -0.036 & 1.2113 \\
\hline 136.00 & 21.106 & 21.196 & 0.426 & 25.5240 & 25.5186 & -0.021 & 1.2002 \\
\hline
\end{tabular}


Data from Goodwin [38] (continued)

\begin{tabular}{|c|c|c|c|c|c|c|c|}
\hline $\mathrm{T}$ & $P, \exp$ & P,cal & Dev & $\rho, \exp$ & $\rho$, cal & Dev & Wt \\
\hline $\mathrm{K}$ & $\mathrm{MPa}$ & $\mathrm{MPa}$ & 8 & $\mathrm{~mol} \cdot \mathrm{dm}^{-3}$ & $\mathrm{~mol} \cdot \mathrm{dm}^{-3}$ & 8 & \\
\hline 138.00 & 23.385 & 23.465 & 0.340 & 25.5030 & 25.4983 & -0.019 & 1.1877 \\
\hline 138.00 & 23.345 & 23.465 & 0.516 & 25.5030 & 25.4958 & -0.028 & 1.1877 \\
\hline 140.00 & 25.633 & 25.743 & 0.427 & 25.4840 & 25.4775 & -0.025 & 1.1749 \\
\hline 142.00 & 27.933 & 28.014 & 0.288 & 25.4660 & 25.4613 & -0.018 & 1.1623 \\
\hline 144.00 & 30.221 & 30.296 & 0.248 & 25.4500 & 25.4457 & -0.017 & 1.149 \\
\hline 146.00 & 32.523 & 32.574 & 0.157 & 25.4350 & 25.4321 & -0.011 & 1.1369 \\
\hline 148.00 & 34.815 & 34.866 & 0.146 & 25.4220 & 25.4191 & -0.011 & 1.1240 \\
\hline 114.00 & 0.411 & 0.446 & 8.623 & 26.1420 & 26.1398 & -0.008 & 16.0215 \\
\hline 116.00 & 2.890 & 2.794 & -3.317 & 26.1030 & 26.1088 & 0.022 & 1.1919 \\
\hline 118.00 & 5.297 & 5.240 & -1.070 & 26.0720 & 26.0754 & 0.013 & 1.179 \\
\hline 120.00 & 7.636 & 7.705 & 0.908 & 26.0440 & 26.0399 & -0.016 & 1.1668 \\
\hline 122.00 & 10.016 & 10.106 & 0.892 & 26.0140 & 26.0087 & -0.020 & 1.1557 \\
\hline 124.00 & 12.393 & 12.491 & 0.795 & 25.9850 & 25.9792 & -0.022 & 1.1449 \\
\hline 126.00 & 14.766 & 14.846 & 0.547 & 25.9560 & 25.9513 & -0.018 & 1.1348 \\
\hline 128.00 & 17.134 & 17.205 & 0.417 & 25.9290 & 25.9249 & -0.016 & 1.1245 \\
\hline 130.00 & 19.512 & 19.569 & 0.292 & 25.9040 & 25.9008 & -0.012 & 1.1140 \\
\hline 132.00 & 21.896 & 21.940 & 0.203 & 25.8810 & 25.8785 & -0.010 & 1.1033 \\
\hline 132.00 & 21.853 & 21.922 & 0.314 & 25.8800 & 25.8761 & -0.015 & 1.1037 \\
\hline 134.00 & 24.244 & 24.300 & 0.232 & 25.8590 & 25.8559 & -0.012 & 1.0928 \\
\hline 136.00 & 26.644 & 26.688 & 0.165 & 25.8400 & 25.8376 & -0.009 & 1.0816 \\
\hline 138.00 & 29.047 & 29.068 & 0.071 & 25.8 & 25. & -0.0 & 1.0706 \\
\hline 140.00 & 31.458 & 31.479 & 0.067 & 25.8070 & 25.8059 & -0.004 & 1.0591 \\
\hline 142.00 & 33.859 & 33.866 & 0.020 & 25.7920 & 25.7916 & -0.001 & 1.0480 \\
\hline 110.00 & 0.594 & 0.672 & 13.244 & 26.5190 & 26.5145 & -0.017 & 14.5524 \\
\hline 112.00 & 3.184 & 3.134 & -1.578 & 26.4800 & 26.4828 & 0.011 & 1.0832 \\
\hline 14.00 & 5.644 & 5.703 & 1.033 & 26.4490 & 26.4 & -0. & 1.0727 \\
\hline 116.00 & 8.105 & 8.256 & 1.857 & 26.4190 & 26.4107 & -0.031 & 1.0626 \\
\hline 118.00 & 10.587 & 10.757 & 1.607 & 26.3880 & 26.3787 & -0.035 & 1.0536 \\
\hline 120.00 & 13.066 & 13.224 & 1.211 & 26.3570 & 26.3484 & -0.033 & 1.0451 \\
\hline 122.00 & 15.540 & 15.676 & 0.874 & 26.3270 & 26.3197 & -0.028 & 1.0369 \\
\hline 124.00 & 18.015 & 18.132 & 0.645 & 26.2990 & 26.2928 & -0.023 & 1.028 \\
\hline 126.00 & 20.498 & 20.612 & 0.557 & 26.2740 & 26.2680 & -0.023 & 1.0194 \\
\hline 128.00 & 22.989 & 23.099 & 0.476 & 26.2510 & 26.2453 & -0.022 & 1.0103 \\
\hline 130.00 & 25.491 & 25.594 & 0.406 & 26.2300 & 26.2246 & -0.020 & 1.0009 \\
\hline 132.00 & 28.002 & 28.100 & 0.347 & 26.2110 & 26.2060 & -0.019 & 0.9913 \\
\hline
\end{tabular}


Data from Goodwin [38] (continued)

\begin{tabular}{|c|c|c|c|c|c|c|c|}
\hline $\mathrm{T}$ & $P, \exp$ & P,cal & Dev & $\rho, \exp$ & $\rho$, cal & Dev & Wt \\
\hline K & $\mathrm{MPa}$ & $\mathrm{MPa}$ & 8 & $\mathrm{~mol} \cdot \mathrm{dm}^{-3}$ & $\mathrm{~mol} \cdot \mathrm{dm}^{-3}$ & 8 & \\
\hline 134.00 & 30.521 & 30.597 & 0.247 & 26.1930 & 26.1892 & -0.015 & 0.9818 \\
\hline 134.00 & 30.497 & 30.577 & 0.263 & 26.1920 & 26.1879 & -0.016 & 0.9821 \\
\hline 136.00 & 33.019 & 33.086 & 0.206 & 26.1760 & 26.1726 & -0.013 & 0.9724 \\
\hline 106.00 & 1.202 & 1.190 & -1.023 & 26.9010 & 26.9016 & 0.002 & 0.9905 \\
\hline 108.00 & 3.899 & 3.809 & -2.291 & 26.8640 & 26.8686 & 0.017 & 0.9830 \\
\hline 110.00 & 6.459 & 6.507 & 0.749 & 26.8330 & 26.8305 & -0.009 & 0.9741 \\
\hline 112.00 & 9.030 & 9.150 & 1. 324 & 26.8010 & 26.7949 & -0.023 & 0.9662 \\
\hline 114.00 & 11.612 & 11.735 & 1.056 & 26.7680 & 26.7618 & -0.023 & 0.9592 \\
\hline 116.00 & 14.187 & 14.303 & 0.817 & 26.7360 & 26.7302 & -0.022 & 0.9524 \\
\hline 118.00 & 16.765 & 16.875 & 0.652 & 26.7060 & 26.7006 & -0.020 & 0.9455 \\
\hline 120.00 & 19.354 & 19.471 & 0.606 & 26.6790 & 26.6732 & -0.022 & 0.9380 \\
\hline 122.00 & 21.954 & 22.054 & 0.452 & 26.6530 & 26.6482 & -0.018 & 0.9307 \\
\hline 124.00 & 24.582 & 24.664 & 0.333 & 26.6300 & 26.6260 & -0.015 & 0.9229 \\
\hline 124.00 & 24.568 & 24.684 & 0.475 & 26.6310 & 26.6254 & -0.021 & 0.9226 \\
\hline 126.00 & 27.205 & 27.304 & 0.364 & 26.6100 & 26.6053 & -0.018 & 0.9146 \\
\hline 128.00 & 29.851 & 29.955 & 0.349 & 26.5920 & 26.5871 & -0.019 & 0.9060 \\
\hline 130.00 & 32.502 & 32.576 & 0.228 & 26.5740 & 26. & -0 & 0.8979 \\
\hline 132.00 & 35.142 & 35.232 & 0.256 & 26.5590 & 26.55 & -0.016 & 0.0059 \\
\hline 102.00 & 0.847 & 0.888 & 4.818 & 27.2320 & 27.2300 & -0.007 & 0.9166 \\
\hline 104.00 & 3.649 & 3.565 & -2.295 & 27.1920 & 27.1961 & 0.015 & 0.9108 \\
\hline 106.00 & 6.303 & 6.367 & 1.008 & 27.1600 & 27.1569 & -0.011 & 0.9031 \\
\hline 108.00 & 8.951 & 9.109 & 1.756 & 27.1270 & 27.1195 & -0.028 & 0.8963 \\
\hline 110.00 & 11.622 & 11.790 & 1.447 & 27.0930 & 27.0850 & -0.029 & 0.8904 \\
\hline 112.00 & 14.294 & 14.453 & 1.113 & 27.0600 & 27.0525 & -0.028 & 0.8847 \\
\hline 114.00 & 16.961 & 17.119 & 0.931 & 27.0290 & 27.0216 & -0.027 & 0.8788 \\
\hline 116.00 & 19.648 & 19.812 & 0.833 & 27.0010 & 26.9934 & -0.028 & 0.8724 \\
\hline 116.00 & 19.647 & 19.812 & 0.838 & 27.0010 & 26.9934 & -0.028 & 0.8724 \\
\hline 118.00 & 22.357 & 22.532 & 0.785 & 26.9760 & 26.9679 & -0.030 & 0.8656 \\
\hline 120.00 & 25.086 & 25.239 & 0.608 & 26.9520 & 26.9450 & -0.026 & 0.8589 \\
\hline 122.00 & 27.828 & 27.977 & 0.535 & 26.9310 & 26.9243 & -0.025 & 0.8518 \\
\hline 124.00 & 30.590 & 30.725 & 0.442 & 26.9120 & 26.9059 & -0.023 & 0.8444 \\
\hline 126.00 & 33.342 & 33.486 & 0.432 & 26.8950 & 26.8886 & -0.024 & 0.8369 \\
\hline 98.00 & 1.244 & 1.232 & -0.913 & 27.5880 & 27.5885 & 0.002 & 0.8411 \\
\hline 100.00 & 4.141 & 4.034 & -2.568 & 27.5480 & 27.5528 & 0.017 & 0.8361 \\
\hline 102.00 & 6.870 & 6.970 & 1.467 & 27.5160 & 27.5115 & -0.016 & 0.8294 \\
\hline 104.00 & 9.627 & 9.797 & 1.765 & 27.4810 & 27.4734 & -0.027 & 0.8241 \\
\hline
\end{tabular}


Data from Goodwin [38] (continued)

\begin{tabular}{|c|c|c|c|c|c|c|c|}
\hline $\mathrm{T}$ & $P, \exp$ & $\mathrm{P}, \mathrm{cal}$ & Dev & $\rho, \exp$ & $\rho, \mathrm{cal}$ & Dev & Wt \\
\hline K & $\mathrm{MPa}$ & $\mathrm{MPa}$ & 8 & $\mathrm{~mol} \cdot \mathrm{dm}^{-3}$ & $\mathrm{~mol} \cdot \mathrm{dm}^{-3}$ & 8 & \\
\hline 106.00 & 12.400 & 12.603 & 1.638 & 27.4470 & 27.4380 & -0.033 & 0.8190 \\
\hline 108.00 & 15.176 & 15.367 & 1.264 & 27.4130 & 27.4046 & -0.031 & 0.8143 \\
\hline 110.00 & 17.965 & 18.158 & 1.075 & 27.3820 & 27.3736 & -0.031 & 0.8092 \\
\hline 112.00 & 20.779 & 20.954 & 0.839 & 27.3530 & 27.3454 & -0.028 & 0.8039 \\
\hline 114.00 & 23.615 & 23.803 & 0.796 & 27.3280 & 27.3199 & -0.030 & 0.7980 \\
\hline 116.00 & 26.475 & 26.660 & 0.698 & 27.3050 & 27.2971 & -0.029 & 0.7919 \\
\hline 116.00 & 26.489 & 26.660 & 0.644 & 27.3050 & 27.2977 & -0.027 & 0.7919 \\
\hline 118.00 & 29.361 & 29.551 & 0.647 & 27.2850 & 27.2770 & -0.029 & 0.7854 \\
\hline 120.00 & 32.245 & 32.430 & 0.576 & 27.2660 & 27.2582 & -0.029 & 0.7790 \\
\hline 94.00 & 0.751 & 0.722 & -3.843 & 27.9000 & 27.9012 & 0.004 & 0.7844 \\
\hline 96.00 & 3.752 & 3.604 & -3.930 & 27.8580 & 27.8643 & 0.022 & 0.7804 \\
\hline 98.00 & 6.552 & 6.650 & 1.496 & 27.8250 & 27.8209 & -0.015 & 0.7745 \\
\hline 100.00 & 9.406 & 9.578 & 1.828 & 27.7890 & 27.7818 & -0.026 & 0.7699 \\
\hline 102.00 & 12.273 & 12.460 & 1.531 & 27.7530 & 27.7452 & -0.028 & 0.7658 \\
\hline 104.00 & 15.138 & 15.345 & 1.368 & 27.7190 & 27.7104 & -0.031 & 0.7615 \\
\hline 106.00 & 18.022 & 18.210 & 1.042 & 27.6860 & 27.6783 & -0.028 & 0.7574 \\
\hline 108.00 & 20.935 & 21.128 & 0.922 & 27.6570 & 27.6491 & -0.029 & 0.7526 \\
\hline 110.00 & 23.873 & 24.053 & 0.755 & 27.6300 & 27.6227 & -0.027 & 0.7477 \\
\hline 112.00 & 26.839 & 27.036 & 0.731 & 27.6070 & 27.5991 & -0.029 & 0.7421 \\
\hline 114.00 & 29.826 & 30.028 & 0.678 & 27.5860 & 27.5779 & -0.029 & 0.7365 \\
\hline 116.00 & 32.835 & 33.033 & 0.603 & 27.5670 & 27.5591 & -0.029 & 0.7306 \\
\hline 92.00 & 4.412 & 4.258 & -3.488 & 28.2090 & 28.2151 & 0.022 & 0.7184 \\
\hline 94.00 & 7.285 & 7.428 & 1.954 & 28.1750 & 28.1694 & -0.020 & 0.7135 \\
\hline 96.00 & 10.254 & 10.443 & 1.840 & 28.1370 & 28.1296 & -0.026 & 0.7101 \\
\hline 98.00 & 13.235 & 13.459 & 1.689 & 28.1010 & 28.0923 & -0.031 & 0.7065 \\
\hline 100.00 & 16.218 & 16.451 & 1.437 & 28.0660 & 28.0570 & -0.032 & 0.7030 \\
\hline 100.00 & 16.219 & 16.451 & 1.431 & 28.0660 & 28.0570 & -0.032 & 0.7030 \\
\hline 102.00 & 19.224 & 19.473 & 1.293 & 28.0340 & 28.0244 & -0.034 & 0.6992 \\
\hline 104.00 & 22.269 & 22.500 & 1.039 & 28.0040 & 27.9951 & -0.032 & 0.6953 \\
\hline 106.00 & 25.355 & 25.587 & 0.913 & 27.9780 & 27.9692 & -0.032 & 0.6908 \\
\hline 108.00 & 28.464 & 28.708 & 0.859 & 27.9550 & 27.9458 & -0.033 & 0.6859 \\
\hline 110.00 & 31.602 & 31.868 & 0.842 & 27.9350 & 27.9250 & -0.036 & 0.6807 \\
\hline 112.00 & 34.753 & 35.013 & 0.750 & 27.9160 & 27.9063 & -0.035 & 0.6756 \\
\hline 94.00 & 12.813 & 13.088 & 2.141 & 28.3910 & 28.3808 & -0.036 & 0.6644 \\
\hline 96.00 & 15.889 & 16.157 & 1.682 & 28.3540 & 28.3441 & -0.035 & 0.6617 \\
\hline
\end{tabular}


Data from Goodwin [38] (continued)

\begin{tabular}{rccccccc}
\multicolumn{1}{r}{$\mathrm{T}$} & $\mathrm{P}, \exp$ & $\mathrm{P}, \mathrm{cal}$ & $\mathrm{Dev}$ & $\rho, \exp$ & $\rho, \mathrm{cal}$ & Dev & Wt \\
$\mathrm{K}$ & $\mathrm{MPa}$ & $\mathrm{MPa}$ & 8 & $\mathrm{~mol} \cdot \mathrm{dm}^{-3}$ & $\mathrm{~mol} \cdot \mathrm{dm}^{-3}$ & 8 & \\
98.00 & 18.989 & 19.282 & 1.545 & 28.3210 & 28.3102 & -0.038 & 0.6584 \\
100.00 & 22.134 & 22.412 & 1.256 & 28.2900 & 28.2798 & -0.036 & 0.6550 \\
102.00 & 25.322 & 25.631 & 1.219 & 28.2640 & 28.2528 & -0.040 & 0.6508 \\
104.00 & 28.544 & 28.859 & 1.102 & 28.2400 & 28.2287 & -0.040 & 0.6465 \\
106.00 & 31.805 & 32.125 & 1.006 & 28.2190 & 28.2075 & -0.041 & 0.6419 \\
108.00 & 35.057 & 35.404 & 0.991 & 28.2000 & 28.1877 & -0.044 & 0.0042 \\
96.50 & 22.494 & 22.708 & 0.954 & 28.5520 & 28.5445 & -0.026 & 0.6194 \\
97.00 & 23.313 & 23.534 & 0.950 & 28.5450 & 28.5373 & -0.027 & 0.6185 \\
98.00 & 24.964 & 25.177 & 0.850 & 28.5310 & 28.5236 & -0.026 & 0.6168 \\
100.00 & 28.284 & 28.539 & 0.902 & 28.5070 & 28.4982 & -0.031 & 0.6128 \\
100.00 & 28.293 & 28.539 & 0.869 & 28.5070 & 28.4985 & -0.030 & 0.6128 \\
102.00 & 31.643 & 31.942 & 0.943 & 28.4860 & 28.4758 & -0.036 & 0.6085 \\
104.00 & 35.009 & 35.327 & 0.908 & 28.4660 & 28.4552 & -0.038 & 0.0040
\end{tabular}

Number of Points (Ref. 38) 554

$\begin{array}{llllrlll}\text { PRESSURE : } & \text { AAD }-8 & 0.717 & \text { BIAS }-8 & 0.585 & \text { RMS }-8 & 2.258 & \\ & \text { AAD } & 0.060 & \text { BIAS } & 0.055 & \text { RMS } & 0.071 & \mathrm{MPa} \\ \text { DENSITY : } & \text { AAD }-8 & 0.117 & \text { BIAS }-8 & -0.112 & \text { RMS }-8 & 0.170 & \\ & \text { AAD } & 0.012 & \text { BIAS } & -0.012 & \text { RMS } & 0.012 & \mathrm{~mol} \cdot \mathrm{dm}^{-3}\end{array}$

Data from Goodwin [39]

$\begin{array}{cccccccc}\mathrm{T} & \mathrm{P}, \exp & \mathrm{P}, \mathrm{cal} & \text { Dev } & \rho, \exp & \rho, \mathrm{cal} & \text { Dev } & \text { Wt } \\ \mathrm{K} & \mathrm{MPa} & \mathrm{MPa} & \text { \& } & \mathrm{mol} \cdot \mathrm{dm}^{-3} & \mathrm{~mol} \cdot \mathrm{dm}^{-3} & 8 & \\ 150.00 & 1.026 & 1.025 & -0.054 & 1.0000 & 1.0007 & 0.069 & 52.0934 \\ 155.00 & 1.074 & 1.074 & -0.029 & 1.0000 & 1.0004 & 0.036 & 49.2938 \\ 160.00 & 1.122 & 1.122 & -0.014 & 1.0000 & 1.0002 & 0.017 & 46.8494 \\ 165.00 & 1.170 & 1.170 & -0.004 & 1.0000 & 1.0000 & 0.004 & 44.6701 \\ 170.00 & 1.217 & 1.217 & 0.019 & 1.0000 & 0.9998 & -0.022 & 42.7086 \\ 175.00 & 1.263 & 1.264 & 0.032 & 1.0000 & 0.9996 & -0.038 & 40.9250 \\ 180.00 & 1.310 & 1.310 & 0.049 & 1.0000 & 0.9994 & -0.056 & 39.2963 \\ 185.00 & 1.356 & 1.357 & 0.055 & 1.0000 & 0.9994 & -0.063 & 37.7994 \\ 190.00 & 1.402 & 1.403 & 0.061 & 1.0000 & 0.9993 & -0.069 & 36.4197 \\ 195.00 & 1.448 & 1.449 & 0.068 & 1.0000 & 0.9992 & -0.077 & 35.1437\end{array}$


Data from Goodwin [39] (continued)

\begin{tabular}{|c|c|c|c|c|c|c|c|}
\hline $\mathrm{T}$ & $P, \exp$ & $\mathrm{P}, \mathrm{cal}$ & Dev & $\rho, \exp$ & $\rho, \mathrm{cal}$ & Dev & Wt \\
\hline K & $\mathrm{MPa}$ & $\mathrm{MPa}$ & 8 & $\mathrm{~mol} \cdot \mathrm{dm}^{-3}$ & $\mathrm{~mol} \cdot \mathrm{dm}^{-3}$ & 8 & \\
\hline 200.00 & 1.494 & 1.495 & 0.071 & 1.0000 & 0.9992 & -0.079 & 33.9585 \\
\hline 205.00 & 1.539 & 1.540 & 0.071 & 1.0000 & 0.9992 & -0.079 & 32.8545 \\
\hline 210.00 & 1.585 & 1.586 & 0.063 & 1.0000 & 0.9993 & -0.069 & 31.8223 \\
\hline 215.00 & 1.631 & 1.632 & 0.060 & 1.0000 & 0.9993 & -0.066 & 30.8569 \\
\hline 220.00 & 1.676 & 1.677 & 0.052 & 1.0000 & 0.9994 & -0.057 & 29.9501 \\
\hline 225.00 & 1.722 & 1.722 & 0.044 & 1.0000 & 0.9995 & -0.048 & 29.0973 \\
\hline 230.00 & 1.767 & 1.767 & 0.038 & 1.0000 & 0.9996 & -0.041 & 28.2939 \\
\hline 235.00 & 1.812 & 1.813 & 0.033 & 1.0000 & 0.9996 & -0.036 & 27.5355 \\
\hline 240.00 & 1.857 & 1.858 & 0.024 & 1.0000 & 0.9997 & -0.026 & 26.8176 \\
\hline 245.00 & 1.902 & 1.903 & 0.018 & 1.0000 & 0.9998 & -0.020 & 26.1377 \\
\hline 250.00 & 1.947 & 1.948 & 0.009 & 1.0000 & 0.9999 & -0.010 & 25.4921 \\
\hline 255.00 & 1.992 & 1.992 & 0.003 & 1.0000 & 1.0000 & -0.003 & 24.8789 \\
\hline 260.00 & 2.037 & 2.037 & -0.001 & 1.0000 & 1.0000 & 0.001 & 24.2957 \\
\hline 265.00 & 2.082 & 2.082 & -0.007 & 1.0000 & 1.0001 & 0.007 & 23.7397 \\
\hline 270.00 & 2.127 & 2.127 & -0.015 & 1.0000 & 1.0002 & 0.016 & 0.1160 \\
\hline 170.00 & 2.062 & 2.067 & 0.260 & 2.0000 & 1.9921 & -0.393 & 27.5425 \\
\hline 175.00 & 2.166 & 2.172 & 0.276 & 2.0000 & 1.9920 & -0.400 & 25.8633 \\
\hline 180.00 & 2.270 & 2.276 & 0.277 & 2.0000 & 1.9922 & -0.389 & 24.4071 \\
\hline 185.00 & 2.372 & 2.378 & 0.272 & 2.0000 & 1.9926 & -0.371 & 23.1233 \\
\hline 190.00 & 2.474 & 2.480 & 0.262 & 2.0000 & 1.9930 & -0.349 & 21.9789 \\
\hline 195.00 & 2.575 & 2.581 & 0.254 & 2.0000 & 1.9934 & -0.330 & 20.9509 \\
\hline 200.00 & 2.675 & 2.681 & 0.240 & 2.0000 & 1.9939 & -0.306 & 20.0206 \\
\hline 205.00 & 2.775 & 2.781 & 0.223 & 2.0000 & 1.9944 & -0.281 & 19.1741 \\
\hline 210.00 & 2.874 & 2.880 & 0.207 & 2.0000 & 1.9949 & -0.256 & 18.4006 \\
\hline 215.00 & 2.973 & 2.978 & 0.187 & 2.0000 & 1.9955 & -0.227 & 17.6899 \\
\hline 220.00 & 3.072 & 3.077 & 0.166 & 2.0000 & 1.9960 & -0.200 & 17.0351 \\
\hline 225.00 & 3.170 & 3.174 & 0.145 & 2.0000 & 1.9965 & -0.173 & 16.4292 \\
\hline 230.00 & 3.268 & 3.272 & 0.127 & 2.0000 & 1.9970 & -0.150 & 15.8672 \\
\hline 235.00 & 3.365 & 3.369 & 0.107 & 2.0000 & 1.9975 & -0.124 & 15.3439 \\
\hline 240.00 & 3.463 & 3.466 & 0.085 & 2.0000 & 1.9980 & -0.099 & 14.8554 \\
\hline 245.00 & 3.560 & 3.563 & 0.066 & 2.0000 & 1.9985 & -0.076 & 14.3984 \\
\hline 250.00 & 3.657 & 3.659 & 0.050 & 2.0000 & 1.9989 & -0.057 & 13.9699 \\
\hline 255.00 & 3.754 & 3.755 & 0.034 & 2.0000 & 1.9992 & -0.039 & 13.5672 \\
\hline 260.00 & 3.850 & 3.851 & 0.020 & 2.0000 & 1.9996 & -0.022 & 13.1879 \\
\hline 265.00 & 3.947 & 3.947 & 0.006 & 2.0000 & 1.9999 & -0.006 & 12.8300 \\
\hline 270.00 & 4.043 & 4.042 & -0.006 & 2.0000 & 2.0001 & 0.007 & 0.0625 \\
\hline
\end{tabular}


Data from Goodwin [39] (continued)

Number of Points (Ref. 39) 46

$\begin{array}{llllrlll}\text { PRESSURE : } & \text { AAD }-8 & 0.091 & \text { BIAS }-\frac{8}{8} & 0.085 & \text { RMS }-8 & 0.095 & \\ & \text { AAD } & 0.002 & \text { BIAS } & 0.002 & \text { RMS } & 0.002 & \mathrm{MPa} \\ \text { DENSITY : } & \text { AAD }-8 & 0.114 & \text { BIAS }-\frac{8}{8} & -0.108 & \text { RMS }-8 & 0.130 & \\ & \text { AAD } & 0.002 & \text { BIAS } & -0.002 & \text { RMS } & 0.003 & \mathrm{~mol} \cdot \mathrm{dm}^{-3}\end{array}$

Data from Kleinrahm et al. [7]

\begin{tabular}{|c|c|c|c|c|c|c|c|}
\hline $\mathrm{T}$ & $P, \exp$ & P,cal & Dev & $\rho, \exp$ & $\rho, \mathrm{cal}$ & Dev & Wt \\
\hline K & $\mathrm{MPa}$ & $\mathrm{MPa}$ & 8 & $\mathrm{~mol} \cdot \mathrm{dm}^{-3}$ & $\mathrm{~mol} \cdot \mathrm{dm}^{-3}$ & 8 & \\
\hline 180.00 & 3.286 & 3.286 & 0.000 & 3.8273 & 3.8272 & -0.001 & 1.453 \\
\hline 180.00 & 3.282 & 3.282 & 0.001 & 3.8140 & 3.8139 & -0.002 & 1.454 \\
\hline 182.00 & 3.508 & 3.508 & 0.007 & 4.2332 & 4.2323 & -0.022 & 1.382 \\
\hline 184.00 & 3.741 & 3.742 & 0.016 & 4.7252 & 4.7223 & -0.060 & 1.316 \\
\hline $186.00^{\circ}$ & 3.986 & 3.987 & 0.022 & 5.3386 & 5.3329 & -0.106 & 1.252 \\
\hline 186.00 & 3.989 & 3.981 & -0.184 & 15.2634 & 15.2826 & 0.125 & 0.722 \\
\hline 186.00 & 3.989 & 3.982 & -0.184 & 15.2647 & 15.2838 & 0.125 & 0.721 \\
\hline 187.00 & 4.114 & 4.115 & 0.022 & 5.7225 & 5.7149 & -0.133 & 1.221 \\
\hline 188.00 & 4.246 & 4.247 & 0.020 & 6.1997 & 6.1897 & -0.161 & 1.190 \\
\hline 188.00 & 4.245 & 4.246 & 0.020 & 6.1893 & 6.1796 & -0.157 & 1.19 \\
\hline 189.00 & 4.381 & 4.382 & 0.014 & 6.8197 & 6.8077 & -0.175 & 1.10 \\
\hline 189.00 & 4.381 & 4.382 & 0.014 & 6.8158 & 6.8040 & -0.172 & $1.15 \mathrm{c}$ \\
\hline 189.00 & 4.380 & 4.381 & 0.014 & 6.8030 & 6.7915 & -0.169 & 1.15 \\
\hline 189.00 & 4.383 & 4.377 & -0.118 & 13.5833 & 13.6338 & 0.372 & 1.107 \\
\hline 189.00 & 4.380 & 4.381 & 0.014 & 6.8028 & 6.7911 & -0.171 & 1.155 \\
\hline 189.00 & 4.383 & 4.378 & -0.119 & 13.5905 & 13.6406 & 0.368 & 1.106 \\
\hline 189.00 & 4.380 & 4.381 & 0.014 & 6.7987 & 6.7875 & -0.165 & 1.15 \\
\hline 189.00 & 4.385 & 4.379 & -0.118 & 13.6039 & 13.6526 & 0.358 & 1.104 \\
\hline 189.00 & 4.379 & 4.380 & 0.014 & 6.7843 & 6.7735 & -0.160 & 1.159 \\
\hline 189.00 & 4.386 & 4.381 & -0.116 & 13.6144 & 13.6617 & 0.348 & 1.102 \\
\hline 189.00 & 4.379 & 4.380 & 0.014 & 6.7838 & 6.7729 & -0.161 & 1.150 \\
\hline 189.00 & 4.379 & 4.380 & 0.014 & 6.7823 & 6.7715 & -0.160 & $1.15 \mathrm{~s}$ \\
\hline 189.00 & 4.379 & 4.379 & 0.014 & 6.7688 & 6.7583 & -0.156 & 1.159 \\
\hline 189.00 & 4.378 & 4.379 & 0.014 & 6.7665 & 6.7556 & -0.160 & 1.159 \\
\hline 189.00 & 4.378 & 4.378 & 0.013 & 6.7543 & 6.7442 & -0.149 & 1.159 \\
\hline
\end{tabular}


Data from Kleinrahm et al. [7] (continued)

\begin{tabular}{|c|c|c|c|c|c|c|c|}
\hline $\mathrm{T}$ & $P, \exp$ & $\mathrm{P}, \mathrm{cal}$ & Dev & $\rho, \exp$ & $\rho$, cal & Dev & Wt \\
\hline $\mathrm{K}$ & $\mathrm{MPa}$ & $\mathrm{MPa}$ & 8 & $\mathrm{~mol} \cdot \mathrm{dm}^{-3}$ & $\mathrm{~mol} \cdot \mathrm{dm}^{-3}$ & 8 & \\
\hline 189.00 & 4.377 & 4.378 & 0.014 & 6.7486 & 6.7384 & -0.150 & 1.1594 \\
\hline 189.00 & 4.376 & 4.377 & 0.014 & 6.7307 & 6.7205 & -0.150 & 1.1595 \\
\hline 189.50 & 4.450 & 4.450 & 0.009 & 7.2303 & 7.2199 & -0.145 & 1.1429 \\
\hline 189.50 & 4.450 & 4.450 & 0.008 & 7.2124 & 7.2034 & -0.125 & 1.1429 \\
\hline 189.50 & 4.449 & 4.449 & 0.007 & 7.1939 & 7.1860 & -0.109 & 1.1430 \\
\hline 189.50 & 4.451 & 4.448 & -0.074 & 13.1312 & 13.1832 & 0.396 & 1.1232 \\
\hline 189.50 & 4.452 & 4.448 & -0.075 & 13.1386 & 13.1902 & 0.393 & 1.1226 \\
\hline 189.80 & 4.492 & 4.492 & 0.004 & 7.5430 & 7.5357 & -0.098 & 1.1330 \\
\hline 189.80 & 4.491 & 4.491 & 0.003 & 7.5151 & 7.5095 & -0.073 & 1.1331 \\
\hline 189.80 & 4.491 & 4.491 & 0.003 & 7.5131 & 7.5077 & -0.071 & 1.1331 \\
\hline 189.80 & 4.493 & 4.491 & -0.046 & 12.7972 & 12.8460 & 0.382 & 1.1234 \\
\hline 189.80 & 4.490 & 4.490 & 0.003 & 7.4848 & 7.4803 & -0.060 & 1.1332 \\
\hline 189.80 & 4.494 & 4.491 & -0.047 & 12.8086 & 12.8566 & 0.375 & 1.1229 \\
\hline 190.00 & 4.520 & 4.520 & 0.003 & 7.8171 & 7.8109 & -0.079 & 1.1262 \\
\hline 190.00 & 4.520 & 4.520 & 0.002 & 7.8018 & 7.7972 & -0.058 & 1.1263 \\
\hline 190.00 & 4.519 & 4.519 & 0.001 & 7.7588 & 7.7562 & -0.034 & 1.1264 \\
\hline 190.00 & 4.521 & 4.520 & -0.028 & 12.5246 & 12.5673 & 0.341 & 1.1208 \\
\hline 190.00 & 4.519 & 4.519 & 0.001 & 7.7547 & 7.7529 & -0.023 & 1.1264 \\
\hline 190.00 & 4.522 & 4.521 & -0.028 & 12.5478 & 12.5888 & 0.326 & 1.1203 \\
\hline 190.00 & 4.519 & 4.519 & 0.000 & 7.7206 & 7.7202 & -0.005 & 1.1265 \\
\hline 190.00 & 4.517 & 4.517 & -0.001 & 7.6754 & 7.6765 & 0.014 & 1.1267 \\
\hline 190.00 & 4.517 & 4.517 & -0.001 & 7.6746 & 7.6761 & 0.020 & 1.1267 \\
\hline 190.00 & 4.517 & 4.517 & -0.001 & 7.6390 & 7.6409 & 0.025 & 1.1268 \\
\hline 190.00 & 4.515 & 4.515 & -0.002 & 7.5902 & 7.5934 & 0.042 & 1.1270 \\
\hline 190.10 & 4.534 & 4.534 & 0.000 & 7.9530 & 7.9516 & -0.018 & 1.1229 \\
\hline 190.10 & 4.533 & 4.533 & -0.001 & 7.9038 & 7.9056 & 0.024 & 1.1230 \\
\hline 190.10 & 4.533 & 4.533 & -0.002 & 7.8535 & 7.8579 & 0.057 & 1.1232 \\
\hline 190.10 & 4.536 & 4.535 & -0.018 & 12.3832 & 12.4172 & 0.275 & 1.1185 \\
\hline 190.10 & 4.536 & 4.535 & -0.018 & 12.4007 & 12.4339 & 0.268 & 1.1182 \\
\hline 190.20 & 4.549 & 4.549 & -0.001 & 8.1454 & $8: 1492$ & 0.047 & 1.1194 \\
\hline 190.20 & 4.548 & 4.548 & -0.003 & 8.0911 & 8.0998 & 0.107 & 1.1195 \\
\hline 190.20 & 4.549 & 4.549 & -0.011 & 12.1679 & 12.1958 & 0.230 & 1.1166 \\
\hline 190.20 & 4.548 & 4.547 & -0.004 & 8.0542 & 8.0661 & 0.147 & 1.1196 \\
\hline 190.20 & 4.549 & 4.549 & -0.010 & 12.1930 & 12.2179 & 0.204 & 1.1164 \\
\hline 190.20 & 4.550 & 4.550 & -0.009 & 12.2303 & 12.2521 & 0.178 & 1.1159 \\
\hline 190.30 & 4.563 & 4.563 & -0.003 & 8.3497 & 8.3653 & 0.187 & 1.1160 \\
\hline
\end{tabular}


Data from Kleinrahm et al. [7] (continued)

\begin{tabular}{|c|c|c|c|c|c|c|c|}
\hline $\mathrm{T}$ & $P, \exp$ & P,cal & Dev & $\rho, \exp$ & $\rho$, cal & Dev & Wt \\
\hline $\mathrm{K}$ & $\mathrm{MPa}$ & $\mathrm{MPa}$ & 8 & $\mathrm{~mol} \cdot \mathrm{dm}^{-3}$ & $\mathrm{~mol} \cdot \mathrm{dm}^{-3}$ & 8 & \\
\hline 190.30 & 4.562 & 4.562 & -0.005 & 8.2945 & 8.3159 & 0.259 & 1.1161 \\
\hline 190.30 & 4.563 & 4.563 & -0.003 & 11.9523 & 11.9637 & 0.096 & 1.1139 \\
\hline 190.30 & 4.562 & 4.562 & -0.005 & 8.2935 & 8.3149 & 0.258 & 1.1161 \\
\hline 190.30 & 4.564 & 4.564 & -0.002 & 11.9815 & 11.9896 & 0.068 & 1.1137 \\
\hline 190.30 & 4.562 & 4.562 & -0.006 & 8.2513 & 8.2751 & 0.289 & 1.1162 \\
\hline 190.30 & 4.564 & 4.564 & -0.002 & 12.0102 & 12.0169 & 0.056 & 1.1135 \\
\hline 190.30 & 4.561 & 4.561 & -0.007 & 8.1952 & 8.2227 & 0.337 & 1.1163 \\
\hline 190.40 & 4.577 & 4.577 & -0.004 & 8.6113 & 8.6423 & 0.360 & 1.1125 \\
\hline 190.40 & 4.577 & 4.577 & -0.005 & 8.5691 & 8.6054 & 0.424 & 1.1125 \\
\hline 190.40 & 4.578 & 4.578 & 0.003 & 11.6797 & 11.6626 & -0.146 & 1.1109 \\
\hline 190.40 & 4.577 & 4.577 & -0.005 & 8.5692 & 8.6037 & 0.403 & 1.1125 \\
\hline 190.40 & 4.578 & 4.578 & 0.003 & 11.6847 & 11.6656 & -0.164 & 1.1109 \\
\hline 190.40 & 4.577 & 4.576 & -0.005 & 8.5346 & 8.5700 & 0.415 & 1.1126 \\
\hline 190.40 & 4.578 & 4.578 & 0.003 & 11.7123 & 11.6921 & -0.173 & 1.1108 \\
\hline 190.40 & 4.576 & 4.576 & -0.007 & 8.4785 & 8.5178 & 0.464 & 1.1127 \\
\hline 190.40 & 4.578 & 4.578 & 0.003 & 11.7585 & 11.7425 & -0.136 & 1.1106 \\
\hline 190.45 & 4.585 & 4.584 & -0.004 & 8.8037 & 8.8536 & 0.568 & 1.1107 \\
\hline 190.45 & 4.584 & 4.584 & -0.005 & 8.7457 & 8.8017 & 0.640 & 1.1108 \\
\hline 190.45 & 4.585 & 4.585 & 0.005 & 11.5101 & 11.4657 & -0.386 & 1.1094 \\
\hline 190.45 & 4.584 & 4.584 & -0.007 & 8.6968 & 8.7582 & 0.707 & 1.1108 \\
\hline 190.45 & 4.585 & 4.585 & 0.006 & 11.5532 & 11.5069 & -0.401 & 1.1093 \\
\hline 190.45 & 4.584 & 4.583 & -0.009 & 8.6074 & 8.6764 & 0.801 & 1.1109 \\
\hline 190.45 & 4.585 & 4.586 & 0.007 & 11.6058 & 11.5590 & -0.403 & 1.1092 \\
\hline 190.50 & 4.592 & 4.592 & -0.003 & 9.0651 & 9.1299 & 0.715 & 1.1089 \\
\hline 190.50 & 4.592 & 4.592 & 0.004 & 11.2133 & 11.1347 & -0.701 & 1.1080 \\
\hline 190.50 & 4.592 & 4.591 & -0.004 & 8.9909 & 9.06 & 0.824 & 1.1089 \\
\hline 190.50 & 4.592 & 4.592 & 0.006 & 11.3065 & 11.2207 & -0.759 & 1.1078 \\
\hline 190.50 & 4.591 & 4.591 & -0.006 & 8.9139 & 9.0038 & 1.009 & 1.1090 \\
\hline 190.50 & 4.592 & 4.593 & 0.007 & 11.3787 & 11.2962 & -0.725 & 1.1077 \\
\hline 190.50 & 4.591 & 4.591 & -0.007 & 8.8552 & 8.9428 & 0.989 & 1.1090 \\
\hline 190.50 & 4.592 & 4.593 & 0.008 & 11.4310 & 11.3476 & -0.729 & 1.1076 \\
\hline 190.50 & 4.591 & 4.591 & -0.009 & 8.7960 & 8.8903 & 1.072 & 1.1091 \\
\hline 190.53 & 4.596 & 4.596 & -0.002 & 9.2923 & 9.3855 & 1.004 & 1.1078 \\
\hline 190.53 & 4.596 & 4.596 & 0.004 & 11.0271 & 10.9014 & -1.140 & 1.1070 \\
\hline 190.53 & 4.596 & 4.596 & -0.004 & 9.1939 & 9.2931 & 1.079 & 1.1078 \\
\hline
\end{tabular}


Data from Kleinrahm et al. [7] (continued)

\begin{tabular}{|c|c|c|c|c|c|c|c|}
\hline $\mathrm{T}$ & $P, \exp$ & P,cal & Dev & $\rho, \exp$ & $\rho$, cal & Dev & Wt \\
\hline K & $\mathrm{MPa}$ & $\mathrm{MPa}$ & 8 & $\mathrm{~mol} \cdot \mathrm{dm}^{-3}$ & $\mathrm{~mol} \cdot \mathrm{dm}^{-3}$ & 8 & \\
\hline 190.53 & 4.596 & 4.597 & 0.005 & 11.1245 & 10.9995 & -1.124 & 1.1069 \\
\hline 190.53 & 4.596 & 4.596 & -0.005 & 9.1130 & 9.2232 & 1.210 & 1.1079 \\
\hline 190.53 & 4.597 & 4.597 & 0.007 & 11.2014 & 11.0814 & -1.071 & 1.1068 \\
\hline 190.53 & 4.596 & 4.595 & -0.006 & 9.0545 & 9.1660 & 1.231 & 1.1079 \\
\hline 190.53 & 4.597 & 4.597 & 0.008 & 11.3041 & 11.1964 & -0.953 & 1.1067 \\
\hline 189.00 & 3.669 & 3.670 & 0.015 & 3.7558 & 3.7547 & -0.031 & 4.1515 \\
\hline 189.00 & 3.935 & 3.937 & 0.045 & 4.3927 & 4.3877 & -0.113 & 4.0086 \\
\hline 189.00 & 3.935 & 3.937 & 0.044 & 4.3931 & 4.3882 & -0.111 & 4.0086 \\
\hline 189.00 & 3.937 & 3.938 & 0.045 & 4.3966 & 4.3917 & -0.112 & 4.0080 \\
\hline 189.00 & 4.115 & 4.117 & 0.046 & 4.9766 & 4.9693 & -0.147 & 3.9381 \\
\hline 189.00 & 4.246 & 4.247 & 0.036 & 5.5754 & 5.5668 & -0.154 & 3.8992 \\
\hline 189.00 & 4.332 & 4.333 & 0.019 & 6.1936 & 6.1860 & -0.123 & 3.8769 \\
\hline 189.00 & 4.363 & 4.363 & 0.010 & 6.5300 & 6.5242 & -0.088 & 3.8686 \\
\hline 189.00 & 4.398 & 4.393 & -0.117 & 13.7266 & 13.7680 & 0.301 & 3.5996 \\
\hline 189.00 & 4.424 & 4.419 & -0.108 & 13.9141 & 13.9447 & 0.220 & 3.4452 \\
\hline 189.00 & 4.460 & 4.456 & -0.094 & 14.1253 & 14.1465 & 0.150 & 3.2276 \\
\hline 189.00 & 4.526 & 4.523 & -0.067 & 14.4189 & 14.4303 & 0.079 & 2.8674 \\
\hline 189.00 & 4.704 & 4.704 & -0.004 & 14.9556 & 14.9561 & 0.003 & 2.1654 \\
\hline 189.00 & 4.729 & 4.729 & 0.002 & 15.0158 & 15.0156 & -0.002 & 2.0910 \\
\hline 189.00 & 5.006 & 5.009 & 0.053 & 15.5378 & 15.5337 & -0.027 & 1.5272 \\
\hline 189.00 & 5.061 & 5.064 & 0.059 & 15.6221 & 15.6177 & -0.028 & 1.4506 \\
\hline 189.00 & 5.062 & 5.065 & 0.059 & 15.6243 & 15.6199 & -0.029 & 1.4486 \\
\hline 189.00 & 5.063 & 5.067 & 0.059 & 15.6260 & 15.6216 & -0.028 & 1.4471 \\
\hline 189.00 & 5.505 & 5.511 & 0.094 & 16.1752 & 16.1698 & -0.034 & 1.0390 \\
\hline 190.55 & 3.736 & 3.736 & 0.019 & 3.7521 & 3.7507 & -0.038 & 4.0531 \\
\hline 190.55 & 4.015 & 4.017 & 0.045 & 4.3825 & 4.3778 & -0.107 & 3.9002 \\
\hline 190.55 & 4.016 & 4.018 & 0.046 & 4.3840 & 4. 3792 & -0.109 & 3.9000 \\
\hline 190.55 & 4.017 & 4.019 & 0.043 & 4.3862 & 4.3817 & -0.101 & 3.8995 \\
\hline 190.55 & 4.217 & 4.220 & 0.052 & 4.9880 & 4.9803 & -0.155 & 3.8172 \\
\hline 190.55 & 4.368 & 4.370 & 0.047 & 5.6108 & 5.6006 & -0.182 & 3.7685 \\
\hline 190.55 & 4.463 & 4.464 & 0.027 & 6.1780 & 6.1691 & -0.144 & 3.7411 \\
\hline 190.55 & 4.535 & 4.535 & 0.005 & 6.8560 & 6.8533 & -0.039 & 3.7194 \\
\hline 190.55 & 4.573 & 4.572 & -0.013 & 7.4905 & 7.5055 & 0.201 & 3.7052 \\
\hline 190.55 & 4.593 & 4.592 & -0.016 & 8.2224 & 8.2710 & 0.591 & 3.6952 \\
\hline 190.55 & 4.593 & 4.592 & -0.015 & 8.2268 & 8.2745 & 0.580 & 3.6951 \\
\hline
\end{tabular}


Data from Kleinrahm et a1. [7] (continued)

\begin{tabular}{|c|c|c|c|c|c|c|c|}
\hline $\mathrm{T}$ & $P, \exp$ & $P, \mathrm{cal}$ & Dev & $\rho, \exp$ & $\rho, \mathrm{cal}$ & Dev & Wt \\
\hline K & $\mathrm{MPa}$ & $\mathrm{MPa}$ & 8 & $\mathrm{~mol} \cdot \mathrm{dm}^{-3}$ & $\mathrm{~mol} \cdot \mathrm{dm}^{-3}$ & 8 & \\
\hline 190.55 & 4.594 & 4.593 & -0.016 & 8.2945 & 8.3503 & 0.673 & 3.6945 \\
\hline 190.55 & 4.595 & 4.595 & -0.015 & 8.4257 & 8.4923 & 0.790 & 3.6934 \\
\hline 190.55 & 4.597 & 4.596 & -0.012 & 8.5869 & 8.6570 & 0.816 & 3.6924 \\
\hline 190.55 & 4.598 & 4.597 & -0.008 & 8.7544 & 8.8219 & 0.771 & 3.6916 \\
\hline 190.55 & 4.599 & 4.599 & 0.000 & 9.3499 & 9.3421 & -0.084 & 3.6898 \\
\hline 190.55 & 4.600 & 4.600 & 0.001 & 9.8684 & 9.7154 & -1.550 & 3.6889 \\
\hline 190.55 & 4.600 & 4.600 & 0.001 & 10.4590 & 10.0083 & -4.309 & 3.6882 \\
\hline 190.55 & 4.600 & 4.600 & 0.003 & 10.5041 & 9.7155 & -7.507 & 3.6881 \\
\hline 190.55 & 4.600 & 4.600 & 0.003 & 10.7635 & 10.5900 & -1.612 & 3.6877 \\
\hline 190.55 & 4.601 & 4.601 & 0.009 & 11.3080 & 11.1972 & -0.979 & 3.6857 \\
\hline 190.55 & 4.601 & 4.601 & 0.010 & 11.3083 & 11.1972 & -0.982 & 3.6857 \\
\hline 190.55 & 4.601 & 4.601 & 0.009 & 11.3101 & 11.2030 & -0.947 & 3.6857 \\
\hline 190.55 & 4.606 & 4.607 & 0.016 & 11.8901 & 11.8407 & -0.415 & 3.6777 \\
\hline 190.55 & 4.609 & 4.610 & 0.018 & 12.0818 & 12.0438 & -0.315 & 3.6714 \\
\hline 190.55 & 4.627 & 4.628 & 0.009 & 12.5952 & 12.5864 & -0.069 & 3.6302 \\
\hline 190.55 & 4.672 & 4.672 & -0.003 & 13.1974 & 13.1987 & 0.009 & 3.4780 \\
\hline 190.55 & 4.764 & 4.764 & -0.002 & 13.8216 & 13.8222 & 0.004 & 3.0837 \\
\hline 190.55 & 4.896 & 4.896 & 0.010 & 14.3460 & 14.3444 & -0.011 & 2.5578 \\
\hline 190.55 & 4.925 & 4.926 & 0.017 & 14.4376 & 14.4351 & -0.017 & 2.4568 \\
\hline 190.55 & 5.135 & 5.137 & 0.044 & 14.9548 & 14.9502 & -0.031 & 1.8966 \\
\hline 190.55 & 5.441 & 5.444 & 0.066 & 15.4796 & 15.4744 & -0.034 & 1.4155 \\
\hline 190.55 & 5.442 & 5.445 & 0.066 & 15.4813 & 15.4761 & -0.034 & 1.4141 \\
\hline 190.55 & 5.442 & 5.446 & 0.067 & 15.4818 & 15.4766 & -0.034 & 1.4137 \\
\hline 190.55 & 5.977 & 5.981 & 0.073 & 16.1186 & 16.1142 & -0.027 & 0.9846 \\
\hline 193.00 & 3.839 & 3.840 & 0.017 & 3.7459 & 3.7447 & -0.032 & 3.9082 \\
\hline 193.00 & 4.140 & 4.142 & 0.044 & 4.3693 & 4.3650 & -0.098 & 3.7420 \\
\hline 193.00 & 4.141 & 4.143 & 0.045 & 4.3708 & 4.3665 & -0.099 & 3.7417 \\
\hline 193.00 & 4.141 & 4.143 & 0.045 & 4.3710 & 4.3666 & -0.100 & 3.7416 \\
\hline 193.00 & 4.368 & 4.371 & 0.060 & 4.9772 & 4.9692 & -0.160 & 3.6437 \\
\hline 193.00 & 4.543 & 4.546 & 0.061 & 5.5930 & 5.5816 & -0.204 & 3.5816 \\
\hline 193.00 & 4.663 & 4.665 & 0.051 & 6.1558 & 6.1426 & -0.214 & 3.5437 \\
\hline 193.00 & 4.769 & 4.771 & 0.030 & 6.8657 & 6.8539 & -0.172 & 3.5095 \\
\hline 193.00 & 4.835 & 4.835 & 0.003 & 7.5080 & 7.5063 & -0.023 & 3.4856 \\
\hline 193.00 & 4.880 & 4.879 & -0.018 & 8.1773 & 8.1939 & 0.203 & 3.4651 \\
\hline 193.00 & 4.906 & 4.904 & -0.030 & 8.7009 & 8.7366 & 0.410 & 3.4513 \\
\hline
\end{tabular}


Data from Kleinrahm et al. [7] (continued)

$\begin{array}{cccccccc}\mathrm{T} & \text { P,exp } & \text { P,cal } & \text { Dev } & \rho, \exp & \rho, \mathrm{cal} & \text { Dev } & \text { Wt } \\ \mathrm{K} & \mathrm{MPa} & \mathrm{MPa} & 8 & \mathrm{~mol} \cdot \mathrm{dm}^{-3} & \mathrm{~mol} \cdot \mathrm{dm}^{-3} & 8 & \\ 193.00 & 4.928 & 4.926 & -0.032 & 9.2741 & 9.3200 & 0.495 & 3.4375 \\ 193.00 & 4.930 & 4.928 & -0.034 & 9.3316 & 9.3806 & 0.525 & 3.4361 \\ 193.00 & 4.944 & 4.942 & -0.026 & 9.7518 & 9.7913 & 0.406 & 3.4261 \\ 193.00 & 4.954 & 4.953 & -0.015 & 10.0802 & 10.1024 & 0.221 & 3.4180 \\ 193.00 & 4.968 & 4.968 & -0.001 & 10.5090 & 10.5104 & 0.014 & 3.4057 \\ & & & & & & & \\ 193.00 & 4.996 & 4.997 & 0.023 & 11.2215 & 11.1975 & -0.214 & 3.3760 \\ 193.00 & 4.996 & 4.998 & 0.025 & 11.2217 & 11.1956 & -0.233 & 3.3760 \\ 193.00 & 4.997 & 4.998 & 0.025 & 11.2253 & 11.1994 & -0.230 & 3.3758 \\ 193.00 & 5.029 & 5.031 & 0.037 & 11.8110 & 11.7831 & -0.236 & 3.3303 \\ 193.00 & 5.042 & 5.044 & 0.043 & 11.9880 & 11.9596 & -0.237 & 3.3094 \\ 193.00 & 5.089 & 5.091 & 0.046 & 12.4960 & 12.4747 & -0.170 & 3.2159 \\ 193.00 & 5.173 & 5.175 & 0.048 & 13.1022 & 13.0878 & -0.110 & 3.0004 \\ 193.00 & 5.330 & 5.333 & 0.056 & 13.8020 & 13.7915 & -0.076 & 2.5546 \\ 193.00 & 5.522 & 5.526 & 0.064 & 14.3568 & 14.3483 & -0.059 & 2.0887 \\ 193.00 & 5.522 & 5.526 & 0.068 & 14.3570 & 14.3479 & -0.063 & 2.0885 \\ 193.00 & 5.816 & 5.820 & 0.066 & 14.9440 & 14.9376 & -0.043 & 1.5960 \\ 193.00 & 6.109 & 6.113 & 0.060 & 15.3769 & 15.3721 & -0.031 & 1.2821 \\ 193.00 & 6.123 & 6.127 & 0.061 & 15.3958 & 15.3909 & -0.032 & 1.2697 \\ 193.00 & 6.123 & 6.127 & 0.070 & 15.3963 & 15.3907 & -0.036 & 1.2694 \\ 193.00 & 6.127 & 6.130 & 0.063 & 15.4002 & 15.3953 & -0.032 & 1.2668 \\ 193.00 & 6.704 & 6.707 & 0.042 & 16.0263 & 16.0237 & -0.016 & 0.9138\end{array}$

Number of Points (Ref.7) 187

$\begin{array}{llllrlrl}\text { PRESSURE: } & \text { AAD- }-8 & 0.026 & \text { BIAS- }-8 & 0.005 & \text { RMS }-8 & 0.041 & \\ & \text { AAD } & 0.001 & \text { BIAS } & 0.000 & \text { RMS } & 0.002 & \mathrm{MPa} \\ \text { DENSITY : } & \text { AAD- } 8 & 0.347 & \text { BIAS- } 8 & -0.043 & \text { RMS }-8 & 0.766 & \\ & \text { AAD } & 0.035 & \text { BIAS } & -0.005 & \text { RMS } & 0.079 & \mathrm{~mol} \cdot \mathrm{dm}^{-3}\end{array}$


Data from Kvalnes and Gaddy [40]

\begin{tabular}{|c|c|c|c|c|c|c|c|}
\hline $\mathrm{T}$ & $P, \exp$ & P,cal & Dev & $\rho, \exp$ & $\rho$, cal & Dev & Wt \\
\hline K & $\mathrm{MPa}$ & $\mathrm{MPa}$ & 8 & $\mathrm{~mol} \cdot \mathrm{dm}^{-3}$ & $\mathrm{~mol} \cdot \mathrm{dm}^{-3}$ & 8 & \\
\hline 203.08 & 0.101 & 0.101 & -0.022 & 0.0604 & 0.0604 & 0.023 & 0.2381 \\
\hline 223.25 & 0.101 & 0.101 & 0.079 & 0.0549 & 0.0548 & -0.080 & 0.2378 \\
\hline 248.18 & 0.101 & 0.101 & 0.051 & 0.0493 & 0.0493 & -0.051 & 0.2377 \\
\hline 273.15 & 0.101 & 0.101 & 0.020 & 0.0447 & 0.0447 & -0.020 & 0.2376 \\
\hline 298.14 & 0.101 & 0.101 & 0.032 & 0.0410 & 0.0409 & -0.032 & 0.2375 \\
\hline 323.14 & 0.101 & 0.101 & 0.020 & 0.0378 & 0.0378 & -0.020 & 0.2375 \\
\hline 373.15 & 0.101 & 0.101 & 0.022 & 0.0327 & 0.0327 & -0.022 & 0.2374 \\
\hline 423.17 & 0.101 & 0.101 & 0.033 & 0.0288 & 0.0288 & -0.033 & 0.2373 \\
\hline 473.19 & 0.101 & 0.101 & 0.038 & 0.0258 & 0.0258 & -0.038 & 0.2373 \\
\hline 203.08 & 2.026 & 2.022 & -0.214 & 1.3818 & 1.3853 & 0.251 & 0.0128 \\
\hline 223.25 & 2.026 & 2.026 & -0.010 & 1.2084 & 1.2085 & 0.011 & 0.0125 \\
\hline 298.14 & 2.026 & 2.029 & 0.100 & 0.8479 & 0.8470 & -0.103 & 0.0121 \\
\hline 203.08 & 3.040 & 3.026 & -0.465 & 2.2701 & 2.2841 & 0.616 & 0.0089 \\
\hline 223.25 & 3.040 & 3.033 & -0.211 & 1.9191 & 1.9239 & 0.251 & 0.0086 \\
\hline 248.18 & 3.040 & 3.039 & -0.037 & 1.6396 & 1.6402 & 0.041 & 0.0083 \\
\hline 273.15 & 3.040 & 3.039 & -0.013 & 1.4422 & 1.4424 & 0.014 & 0.0082 \\
\hline 323.14 & 3.040 & 3.040 & 0.013 & 1.1757 & 1.1755 & -0.013 & 0.0081 \\
\hline 373.15 & 3.040 & 3.044 & 0.137 & 1.0004 & 0.9990 & -0.139 & 0.0080 \\
\hline 423.17 & 3.040 & 3.044 & 0.150 & 0.8729 & 0.8716 & -0.151 & 0.0079 \\
\hline 473.19 & 3.040 & 3.042 & 0.076 & 0.7750 & 0.7744 & -0.076 & 0.0079 \\
\hline 203.08 & 4.053 & 4.030 & -0.573 & 3.4113 & 3.4421 & 0.905 & 0.0071 \\
\hline 223.25 & 4.053 & 4.041 & -0.298 & 2.7324 & 2.7429 & 0.385 & 0.0066 \\
\hline 248.18 & 4.053 & 4.048 & -0.120 & 2.2722 & 2.2753 & 0.139 & 0.0064 \\
\hline 273.15 & 4.053 & 4.053 & 0.000 & 1.9734 & 1.9734 & 0.000 & 0.0062 \\
\hline 298.14 & 4.053 & 4.051 & -0.040 & 1.7541 & 1.7549 & 0.043 & 0.0061 \\
\hline 323.14 & 4.053 & 4.050 & -0.073 & 1.5853 & 1.5865 & 0.077 & 0.0061 \\
\hline 203.08 & 5.066 & 5.028 & -0.755 & 5.0533 & 5.1342 & 1.600 & 0.0061 \\
\hline 223.25 & 5.066 & 5.052 & -0.284 & 3.6845 & 3.6992 & 0.400 & 0.0055 \\
\hline 273.15 & 5.066 & 5.066 & 0.002 & 2.5315 & 2.5315 & -0.002 & 0.0050 \\
\hline 373.15 & 5.066 & 5.070 & 0.080 & 1.6853 & 1.6839 & -0.082 & 0.0048 \\
\hline 423.17 & 5.066 & 5.068 & 0.033 & 1.4597 & 1.4592 & -0.033 & 0.0048 \\
\hline 473.19 & 5.066 & 5.068 & 0.034 & 1.2919 & 1.2914 & -0.034 & 0.0047 \\
\hline 203.08 & 6.079 & 5.994 & -1.413 & 7.9718 & 8.3547 & 4.804 & 0.0000 \\
\hline 223.25 & 6.079 & 6.076 & -0.066 & 4.8339 & 4.8389 & 0.102 & 0.0047 \\
\hline 248.18 & 6.079 & 6.070 & -0.152 & 3.7047 & 3.7118 & 0.191 & 0.0044 \\
\hline
\end{tabular}


Data from Kvalnes and Gaddy [40] (continued)

\begin{tabular}{|c|c|c|c|c|c|c|c|}
\hline $\mathrm{T}$ & $P, \exp$ & $\mathrm{P}, \mathrm{cal}$ & Dev & $\rho, \exp$ & $\rho, \mathrm{cal}$ & Dev & Wt \\
\hline K & $\mathrm{MPa}$ & $\mathrm{MPa}$ & 8 & $\mathrm{~mol} \cdot \mathrm{dm}^{-3}$ & $\mathrm{~mol} \cdot \mathrm{dm}^{-3}$ & 8 & \\
\hline 273.15 & 6.079 & 6.078 & -0.019 & 3.1161 & 3.1168 & 0.022 & 0.0042 \\
\hline 298.14 & 6.079 & 6.069 & -0.164 & 2.7184 & 2.7233 & 0.181 & 0.0041 \\
\hline 323.14 & 6.079 & 6.079 & -0.010 & 2.4349 & 2.4352 & 0.010 & 0.0041 \\
\hline 423.17 & 6.079 & 6.076 & -0.054 & 1.7532 & 1.7542 & 0.054 & 0.0040 \\
\hline 473.19 & 6.079 & 6.083 & 0.058 & 1.5505 & 1.5496 & -0.058 & 0.0040 \\
\hline 203.08 & 6.586 & 6.418 & -2.554 & 9.9825 & 10.7430 & 7.618 & 0.0000 \\
\hline 203.08 & 7.093 & 6.898 & -2.739 & 11.8896 & 12.4424 & 4.649 & 0.0000 \\
\hline 223.25 & 7.093 & 7.076 & -0.237 & 6.1880 & 6.2129 & 0.401 & 0.0041 \\
\hline 203.08 & 8.106 & 7.910 & -2.415 & 13.9974 & 14.2613 & 1.885 & 0.0027 \\
\hline 223.25 & 8.106 & 8.073 & -0.412 & 7.7710 & 7.8270 & 0.721 & 0.0036 \\
\hline 248.18 & 8.106 & 8.091 & -0.180 & 5.3793 & 5.3922 & 0.241 & 0.0034 \\
\hline 273.15 & 8.106 & 8.103 & -0.040 & 4.3636 & 4.3657 & 0.048 & 0.0032 \\
\hline 298.14 & 8.106 & 8.092 & -0.168 & 3.7389 & 3.7459 & 0.188 & 0.0031 \\
\hline 323.14 & 8.106 & 8.099 & -0.085 & 3.3109 & 3.3139 & 0.091 & 0.0031 \\
\hline 223.25 & 9.119 & 9.057 & -0.680 & 9.4350 & 9.5372 & 1.084 & 0.0032 \\
\hline 203.08 & .132 & 9.971 & -1.591 & 15.9266 & 16. & 0.644 & 0.0013 \\
\hline 223.25 & 10.132 & 10.035 & -0.961 & 10.9398 & 11.0745 & 1.231 & 0.0027 \\
\hline 248.18 & 10.132 & 10.110 & -0.219 & 7.2518 & 7.2729 & 0.291 & 0.0027 \\
\hline 273.15 & 10.132 & 10.127 & -0.051 & 5.6949 & 5.6983 & 0.061 & 0.0026 \\
\hline 298.14 & 10.132 & 10.114 & -0.186 & 4.7990 & 4.8090 & 0.209 & 0.0025 \\
\hline 323.14 & 10.132 & 10.113 & -0.191 & 4.2048 & 4.2 & 0.206 & 0.0025 \\
\hline 203.08 & 12.159 & 11.801 & -2.941 & 16.9027 & 17.0572 & 0.914 & 0.0009 \\
\hline 223.25 & 12.159 & 11.974 & -1.520 & 13.1053 & 13.2641 & 1.212 & 0.0018 \\
\hline 248.18 & 12.159 & 12.113 & -0.376 & 9.1316 & 9.1728 & 0.452 & 0.0021 \\
\hline 273.15 & 12.159 & 12.144 & -0.120 & 7.0576 & 7.0675 & 0.140 & 0.0021 \\
\hline 298.14 & 12.159 & 12.139 & -0.163 & 5.8806 & 5.89 & 0. & 0.0021 \\
\hline 323.14 & 12.159 & 12.138 & -0.176 & 5.1120 & 5.1216 & 0.188 & 0.0020 \\
\hline 203.08 & 14.185 & 13.801 & -2.713 & 17.6716 & 17.7979 & 0.715 & 0.0006 \\
\hline 223.25 & 14.185 & 13.983 & -1.425 & 14.5471 & 14.6645 & 0.807 & 0.0012 \\
\hline 248.18 & 14.185 & 14.097 & -0.624 & 10.7931 & 10.8604 & 0.624 & 0.0017 \\
\hline 273.15 & 14.185 & 14.168 & -0.122 & 8.3962 & 8.4074 & 0.133 & 0.0018 \\
\hline 298.14 & 14.185 & 14.164 & -0.155 & 6.9544 & 6.9659 & 0.165 & 0.0018 \\
\hline 323.14 & 14.185 & 14.165 & -0.145 & 6.0156 & 6.0247 & 0.151 & 0.0017 \\
\hline 203.08 & 16.212 & 15.811 & -2.475 & 18.2771 & 18.3842 & 0.586 & 0.0005 \\
\hline 223.25 & 16.212 & 15.959 & -1.560 & 15.5520 & 15.6617 & 0.705 & 0.0009 \\
\hline
\end{tabular}


Data from Kvalnes and Gaddy [40] (continued)

\begin{tabular}{|c|c|c|c|c|c|c|c|}
\hline $\mathbf{T}$ & $P, \exp$ & P,cal & Dev & $\rho, \exp$ & $\rho, \mathrm{cal}$ & Dev & Wt \\
\hline K & $\mathrm{MPa}$ & $\mathrm{MPa}$ & 8 & $\mathrm{~mol} \cdot \mathrm{dm}^{-3}$ & $\mathrm{~mol} \cdot \mathrm{dm}^{-3}$ & 8 & \\
\hline 248.18 & 16.212 & 16.064 & -0.911 & 12.1465 & 12.2367 & 0.743 & 0.0013 \\
\hline 273.15 & 16.212 & 16.181 & -0.193 & 9.6370 & 9.6553 & 0.190 & 0.0015 \\
\hline 298.14 & 16.212 & 16.192 & -0.125 & 7.9959 & 8.0060 & 0.127 & 0.0015 \\
\hline 323.14 & 16.212 & 16.197 & -0.094 & 6.9022 & 6.9087 & 0.095 & 0.0015 \\
\hline 203.08 & 18.238 & 17.796 & -2.427 & 18.7731 & 18.8735 & 0.534 & 0.0004 \\
\hline 223.25 & 18.238 & 17.996 & -1.331 & 16.3483 & 16.4319 & 0.511 & 0.0007 \\
\hline 248.18 & 18.238 & 18.046 & -1.057 & 13.2422 & 13.3369 & 0.715 & 0.0011 \\
\hline 273.15 & 18.238 & 18.210 & -0.155 & 10.7591 & 10.7737 & 0.136 & 0.0012 \\
\hline 298.14 & 18.238 & 18.207 & -0.171 & 8.9743 & 8.9888 & 0.162 & 0.0013 \\
\hline 323.14 & 18.238 & 18.236 & -0.012 & 7.7605 & 7.7613 & 0.011 & 0.0013 \\
\hline 373.15 & 18.238 & 18.222 & -0.093 & 6.1946 & 6.2002 & 0.090 & 0.0013 \\
\hline 203.08 & 20.265 & 19.834 & -2.128 & 19.2104 & 19.2956 & 0.443 & 0.0003 \\
\hline 223.25 & 20.265 & 19.970 & -1.455 & 16.9754 & 17.0600 & 0.498 & 0.0005 \\
\hline 248.18 & 20.265 & 20.077 & -0.927 & 14.1547 & 14.2305 & 0.536 & 0.0008 \\
\hline 273.15 & 20.265 & 20.192 & -0.359 & 11.7211 & 11.7539 & 0.280 & 0.0010 \\
\hline 298.14 & 20.265 & 20.231 & -0.167 & 9.8855 & 9.9000 & 0.147 & 0.0011 \\
\hline 323.14 & 20.265 & 20.257 & -0.039 & 8.5699 & 8.5730 & 0.036 & 0.0011 \\
\hline 373.15 & 20.265 & 20.226 & -0.193 & 6.8403 & 6.8527 & 0.181 & 0.0011 \\
\hline 423.17 & 20.265 & 20.223 & -0.209 & 5.7691 & 5.7804 & 0.196 & 0.0011 \\
\hline 473.19 & 20.265 & 20.264 & -0.004 & 5.0362 & 5.0364 & 0.004 & 0.0011 \\
\hline 203.08 & 25.331 & 24.836 & -1.957 & 20.0834 & 20.1587 & 0.375 & 0.0003 \\
\hline 223.25 & 25.331 & 25.035 & -1.169 & 18.2033 & 18.2634 & 0.331 & 0.0004 \\
\hline 248.18 & 25.331 & 25.075 & -1.013 & 15.8229 & 15.8932 & 0.444 & 0.0005 \\
\hline 273.15 & 25.331 & 25.244 & -0.345 & 13.6614 & 13.6897 & 0.207 & 0.0007 \\
\hline 298.14 & 25.331 & 25.228 & -0.407 & 11.8074 & 11.8424 & 0.296 & 0.0008 \\
\hline 323.14 & 25.331 & 25.286 & -0.180 & 10.3753 & 10.3903 & 0.144 & 0.0008 \\
\hline 373.15 & 25.331 & 25.284 & -0.186 & 8.3661 & 8.3795 & 0.161 & 0.0009 \\
\hline 473.19 & 25.331 & 25.327 & -0.019 & 6.1747 & 6.1757 & 0.017 & 0.0009 \\
\hline 203.08 & 30.398 & 29.824 & -1.887 & 20.7751 & 20.8465 & 0.344 & 0.0002 \\
\hline 223.25 & 30.398 & 29.983 & -1.365 & 19.0983 & 19.1644 & 0.346 & 0.0003 \\
\hline 248.18 & 30.398 & 30.131 & -0.878 & 17.0282 & 17.0832 & 0.323 & 0.0004 \\
\hline 273.15 & 30.398 & 30.351 & -0.154 & 15.0985 & 15.1100 & 0.076 & 0.0005 \\
\hline 298.14 & 30.398 & 30.281 & -0.382 & 13.3339 & 13.3649 & 0.233 & 0.0006 \\
\hline 323.14 & 30.398 & 30.340 & -0.188 & 11.8878 & 11.9033 & 0.130 & 0.0006 \\
\hline 373.15 & 30.398 & 30.363 & -0.114 & 9.7327 & 9.7415 & 0.090 & 0.0007 \\
\hline
\end{tabular}


Data from Kvalnes and Gaddy [40] (continued)

\begin{tabular}{|c|c|c|c|c|c|c|c|}
\hline $\mathrm{T}$ & $P, \exp$ & P,cal & Dev & $\rho, \exp$ & $\rho$, cal & Dev & Wt \\
\hline K & $\mathrm{MPa}$ & $\mathrm{MPa}$ & 8 & $\mathrm{~mol} \cdot \mathrm{dm}^{-3}$ & $\mathrm{~mol} \cdot \mathrm{dm}^{-3}$ & 8 & \\
\hline 423.17 & 30.398 & 30.321 & -0.252 & 8.2645 & 8.2818 & 0.209 & 0.0007 \\
\hline 473.19 & 30.398 & 30.391 & -0.021 & 7.2389 & 7.2402 & 0.018 & 0.0007 \\
\hline 203.08 & 40.530 & 39.804 & -1.792 & 21.8555 & 21.9233 & 0.310 & 0.0014 \\
\hline 223.25 & 40.530 & 40.012 & -1.279 & 20.4443 & 20.5027 & 0.286 & 0.0018 \\
\hline 248.18 & 40.530 & 40.102 & -1.055 & 18.7101 & 18.7693 & 0.316 & 0.0024 \\
\hline 273.15 & 40.530 & 40.410 & -0.296 & 17.0890 & 17.1083 & 0.113 & 0.0030 \\
\hline 298.14 & 40.530 & 40.425 & -0.259 & 15.5568 & 15.5757 & 0.121 & 0.0036 \\
\hline 323.14 & 40.530 & 40.427 & -0.255 & 14.1884 & 14.2081 & 0.139 & 0.0040 \\
\hline 373.15 & 40.530 & 40.479 & -0.125 & 11.9825 & 11.9924 & 0.082 & 0.0046 \\
\hline 423.17 & 40.530 & 40.529 & -0.001 & 10.3595 & 10.3596 & 0.001 & 0.0049 \\
\hline 473.19 & 40.530 & 40.506 & -0.058 & 9.1334 & 9.1375 & 0.045 & 0.0051 \\
\hline 203.08 & 50.662 & 49.319 & -2.651 & 22.6623 & 22.7642 & 0.449 & 0.0011 \\
\hline 223.25 & 50.662 & 49.860 & -1.584 & 21.4329 & 21.5039 & 0.332 & 0.0014 \\
\hline 248.18 & 50.662 & 50.183 & -0.947 & 19.9277 & 19.9781 & 0.253 & 0.0017 \\
\hline 273.15 & 50.662 & 50.566 & -0.190 & 18.5015 & 18.5131 & 0.063 & 0.0021 \\
\hline 298.14 & 50.662 & 50.485 & -0.351 & 17.1164 & 17.1403 & 0.139 & 0.0025 \\
\hline 323.14 & 50.662 & 50.455 & -0.410 & 15.8521 & 15.8819 & 0.189 & 0.0028 \\
\hline 373.15 & 50.662 & 50.647 & -0.030 & 13.7377 & 13.7401 & 0.017 & 0.0033 \\
\hline 423.17 & 50.662 & 50.652 & -0.021 & 12.0596 & 12.0612 & 0.013 & 0.0036 \\
\hline 473.19 & 50.662 & 50.667 & 0.010 & 10.7489 & 10.7482 & -0.007 & 0.0038 \\
\hline 203.08 & 60.795 & 59.213 & -2.603 & 23. & 23. & 0.4 & 0.0009 \\
\hline 223.25 & 60.795 & 59.670 & -1.851 & 22.2294 & 22.3120 & 0.372 & 0.0011 \\
\hline 248.18 & 60.795 & 60.029 & -1.260 & 20.8623 & 20.9275 & 0.313 & 0.0014 \\
\hline 273.15 & 60.795 & 60.540 & -0.419 & 19.5733 & 19.5978 & 0.125 & 0.0016 \\
\hline 298.14 & 60.795 & 60.439 & -0.585 & 18.3049 & 18.3427 & 0.207 & 0.0019 \\
\hline 323.14 & 60.795 & 60.499 & -0.487 & 17.1425 & 17.1763 & 0.198 & 0.0021 \\
\hline 373.15 & 60.795 & 60.800 & 0.009 & 15.1351 & 15.1345 & -0.004 & 0.0025 \\
\hline 423.17 & 60.795 & 60.738 & -0.093 & 13.4603 & 13.4675 & 0.054 & 0.0028 \\
\hline 473.19 & 60.795 & 60.847 & 0.085 & 12.1247 & 12.1182 & -0.054 & 0.0030 \\
\hline 203.08 & 70.928 & 69.131 & -2.533 & 23.9593 & 24.0597 & 0.419 & 0.0008 \\
\hline 223.25 & 70.928 & 69.689 & -1.746 & 22.9158 & 22.9937 & 0.340 & 0.0009 \\
\hline 248.18 & 70.928 & 69.916 & -1.427 & 21.6406 & 21.7134 & 0.336 & 0.0011 \\
\hline 203.08 & 81.060 & 78.381 & -3.305 & 24.4532 & 24.5866 & 0.546 & 0.0007 \\
\hline 223.25 & 81.060 & 78.917 & -2.643 & 23.4668 & 23.5857 & 0.507 & 0.0008 \\
\hline 248.18 & 81.060 & 79.631 & -1.764 & 22.2968 & 22.3861 & 0.401 & 0.0009 \\
\hline
\end{tabular}


Data from Kvalnes and Gaddy [40] (continued)

\begin{tabular}{|c|c|c|c|c|c|c|c|}
\hline $\mathrm{T}$ & $P, \exp$ & P,cal & Dev & $\rho, \exp$ & $\rho$, cal & Dev & Wt \\
\hline K & $\mathrm{MPa}$ & $\mathrm{MPa}$ & 8 & $\mathrm{~mol} \cdot \mathrm{dm}^{-3}$ & $\mathrm{~mol} \cdot \mathrm{dm}^{-3}$ & 8 & \\
\hline 273.15 & 81.060 & 80.241 & -1.010 & 21.1776 & 21.2342 & 0.267 & 0.0011 \\
\hline 298.14 & 81.060 & 80.515 & -0.672 & 20.0986 & 20.1396 & 0.204 & 0.0012 \\
\hline 323.14 & 81.060 & 80.330 & -0.901 & 19.0498 & 19.1089 & 0.310 & 0.0014 \\
\hline 373.15 & 81.060 & 81.013 & -0.058 & 17.2471 & 17.2513 & 0.024 & 0.0016 \\
\hline 423.17 & 81.060 & 81.168 & 0.134 & 15.6726 & 15.6626 & -0.064 & 0.0018 \\
\hline 473.19 & 81.060 & 81.307 & 0.305 & 14.3402 & 14.3169 & -0.163 & 0.0020 \\
\hline 203.08 & 91.192 & 88.026 & -3.472 & 24.9162 & 25.0585 & 0.571 & 0.0006 \\
\hline 223.25 & 91.192 & 88.642 & -2.797 & 23.9838 & 24.1105 & 0.528 & 0.0007 \\
\hline 248.18 & 91.192 & 89.104 & -2.291 & 22.8600 & 22.9759 & 0.507 & 0.0008 \\
\hline 203.08 & 101.325 & 97.494 & -3.781 & 25.3295 & 25.4869 & 0.621 & 0.0006 \\
\hline 223.25 & 101.325 & 98.496 & -2.792 & 24.4555 & 24.5827 & 0.520 & 0.0006 \\
\hline 248.18 & 101.325 & 99.324 & -1.975 & 23.4023 & 23.5018 & 0.425 & 0.0007 \\
\hline 273.15 & 101.325 & 99.432 & -1.869 & 22.3609 & 22.4640 & 0.461 & 0.0008 \\
\hline 298.14 & 101.325 & 100.156 & -1.154 & 21.4062 & 21.4747 & 0.320 & 0.0009 \\
\hline 323.14 & 101.325 & 100.284 & -1.027 & 20.4723 & 20.5374 & 0.318 & 0.0010 \\
\hline 373.15 & 101.325 & 101.337 & 0.012 & 18.8247 & 18.8238 & -0.005 & 0.0012 \\
\hline 423.17 & 101.325 & 101.519 & 0.192 & 17.3361 & 17.3220 & -0.081 & 0.0013 \\
\hline 473.19 & 101.325 & 101.808 & 0.476 & 16.0518 & 16.0158 & -0.224 & 0.0014 \\
\hline Number 0 & ff Points & $(\operatorname{Ref} .40)$ & 158 & & & & \\
\hline \multirow[t]{2}{*}{ PRESSURE } & AAD - 8 & 0.714 & BIAS - 8 & -0.6 & RMS - 8 & 0.925 & \\
\hline & $\mathrm{AAD}$ & 0.331 & BIAS & -0.3 & RMS & 0.666 & $\mathrm{MPa}$ \\
\hline \multirow[t]{2}{*}{ DENSITY } & $A A D-8$ & 0.379 & BIAS - 8 & 0.3 & RMS - 8 & 0.827 & \\
\hline & AAD & 0.049 & BIAS & 0.0 & RMS & 0.089 & $\mathrm{~mol} \cdot \mathrm{dn}$ \\
\hline
\end{tabular}

Data from Mollerup [41]

$\begin{array}{cccccccc}\mathrm{T} & \mathrm{P}, \exp & \mathrm{P}, \mathrm{cal} & \text { Dev } & \rho, \exp & \rho, \mathrm{cal} & \text { Dev } & \text { Wt } \\ \mathrm{K} & \mathrm{MPa} & \mathrm{MPa} & \& & \mathrm{~mol} \cdot \mathrm{dm}^{-3} & \mathrm{~mol} \cdot \mathrm{dm}^{-3} & 8 & \\ 310.00 & 0.154 & 0.154 & 0.001 & 0.0600 & 0.0600 & -0.001 & 1.5600 \\ 310.00 & 0.435 & 0.435 & -0.006 & 0.1700 & 0.1700 & 0.006 & 0.5541 \\ 310.00 & 0.714 & 0.714 & -0.013 & 0.2800 & 0.2800 & 0.013 & 0.3385 \\ 310.00 & 0.990 & 0.990 & -0.017 & 0.3900 & 0.3901 & 0.018 & 0.2445 \\ 310.00 & 2.484 & 2.483 & -0.045 & 1.0000 & 1.0005 & 0.047 & 0.0985\end{array}$


Data from Mollerup [41] (continued)

$\begin{array}{crrrrrrr}\mathrm{T} & \text { P,exp } & \text { P,cal } & \text { Dev } & \rho, \text { exp } & \rho, \text { cal } & \text { Dev } & \text { Wt } \\ \text { K } & \text { MPa } & \text { MPa } & \text { 8 } & \text { mol. } \mathrm{dm}^{-3} & \text { mol. } \mathrm{dm}^{-3} & 8 & \\ 310.00 & 4.806 & 4.802 & -0.076 & 2.0000 & 2.0016 & 0.081 & 0.0516 \\ 310.00 & 7.000 & 6.995 & -0.081 & 3.0000 & 3.0027 & 0.088 & 0.0358 \\ 310.00 & 9.105 & 9.099 & -0.061 & 4.0000 & 4.0027 & 0.067 & 0.0276 \\ 310.00 & 11.158 & 11.155 & -0.023 & 5.0000 & 5.0013 & 0.025 & 0.0225 \\ 310.00 & 13.200 & 13.202 & 0.019 & 6.0000 & 5.9988 & -0.020 & 0.0188 \\ & & & & & & & \\ 310.00 & 15.276 & 15.284 & 0.053 & 7.0000 & 6.9962 & -0.054 & 0.0160 \\ 310.00 & 17.434 & 17.447 & 0.070 & 8.0000 & 7.9945 & -0.068 & 0.0137 \\ 310.00 & 19.731 & 19.744 & 0.066 & 9.0000 & 8.9946 & -0.060 & 0.0117 \\ 310.00 & 22.231 & 22.241 & 0.042 & 10.0000 & 9.9964 & -0.036 & 0.0099 \\ 310.00 & 25.013 & 25.014 & 0.005 & 11.0000 & 10.9996 & -0.004 & 0.0083 \\ & & & & & & & \\ 310.00 & 28.168 & 28.157 & -0.040 & 12.0000 & 12.0033 & 0.028 & 0.0069 \\ 310.00 & 31.809 & 31.782 & -0.086 & 13.0000 & 13.0070 & 0.054 & 0.0057 \\ 310.00 & 36.073 & 36.024 & -0.134 & 14.0000 & 14.0105 & 0.075 & 0.0461 \\ 310.00 & 41.123 & 41.045 & -0.190 & 15.0000 & 15.0142 & 0.095 & 0.0371 \\ 310.00 & 47.157 & 47.034 & -0.262 & 16.0000 & 16.0188 & 0.118 & 0.0296 \\ & & & & & & & \\ 310.00 & 54.412 & 54.216 & -0.360 & 17.0000 & 17.0249 & 0.146 & 0.0236 \\ 310.00 & 63.170 & 62.857 & -0.495 & 18.0000 & 18.0329 & 0.183 & 0.0187 \\ 310.00 & 73.762 & 73.269 & -0.668 & 19.0000 & 19.0430 & 0.226 & 0.0148\end{array}$

Number of Points (Ref. 41) 23

$\begin{array}{llllrlll}\text { PRESSURE : } & \text { AAD }-8 & 0.122 & \text { BIAS }-8 & -0.100 & \text { RMS }-8 & 0.181 & \\ & \text { AAD } & 0.059 & \text { BIAS } & -0.055 & \text { RMS } & 0.121 & \text { MPa } \\ \text { DENSITY : } & \text { AAD }-8 & 0.066 & \text { BIAS }-8 & 0.045 & \text { RMS }-8 & 0.074 & \\ & \text { AAD } & 0.008 & \text { BIAS } & 0.006 & \text { RMS } & 0.012 & \mathrm{~mol} \cdot \mathrm{dm}^{-3}\end{array}$

\begin{tabular}{|c|c|c|c|c|c|c|c|}
\hline \multicolumn{8}{|c|}{ Data from Morris [42] } \\
\hline $\mathrm{T}$ & $P, \exp$ & P,cal & Dev & $\rho, \exp$ & $\rho, \mathrm{cal}$ & Dev & Wt \\
\hline K & $\mathrm{MPa}$ & $\mathrm{MPa}$ & $q$ & $\mathrm{~mol} \cdot \mathrm{dm}^{-3}$ & $\mathrm{~mol} \cdot \mathrm{dm}^{-3}$ & 8 & \\
\hline 250.00 & 171.797 & 168.904 & -1.684 & 26.0000 & 26.0864 & 0.332 & 0.0038 \\
\hline 250.00 & 208.651 & 205.372 & -1.572 & 27.0000 & 27.0816 & 0.302 & 0.0031 \\
\hline 250.00 & 252.321 & 249.031 & -1.304 & 28.0000 & 28.0687 & 0.245 & 0.0025 \\
\hline 250.00 & 303.683 & 300.983 & -0.889 & 29.0000 & 29.0476 & 0.164 & 0.0020 \\
\hline 250.00 & 363.664 & 362.425 & -0.341 & 30.0000 & 30.0186 & 0.062 & 0.0017 \\
\hline
\end{tabular}


Data from Morris [42] (continued)

\begin{tabular}{|c|c|c|c|c|c|c|c|}
\hline $\mathrm{T}$ & $P, \exp$ & P,cal & Dev & $\rho, \exp$ & $\rho, \mathrm{cal}$ & Dev & Wt \\
\hline K & $\mathrm{MPa}$ & $\mathrm{MPa}$ & 8 & $\mathrm{~mol} \cdot \mathrm{dm}^{-3}$ & $\mathrm{~mol} \cdot \mathrm{dm}^{-3}$ & 8 & \\
\hline 250.00 & 433.220 & 434.637 & 0.327 & 31.0000 & 30.9818 & .0 .059 & 0.0014 \\
\hline 250.00 & 513.328 & 518.973 & 1.100 & 32.0000 & 31.9376 & -0.195 & 0.0011 \\
\hline 265.91 & 189.546 & 186.791 & -1.453 & 26.0000 & 26.0778 & 0.299 & 0.0036 \\
\hline 265.91 & 228.152 & 225.295 & -1.253 & 27.0000 & 27.0676 & 0.250 & 0.0029 \\
\hline 265.91 & 273.660 & 271.212 & -0.894 & 28.0000 & 28.0488 & 0.174 & 0.0024 \\
\hline 265.91 & 326.938 & 325.678 & -0.385 & 29.0000 & 29.0213 & 0.073 & 0.0019 \\
\hline 265.91 & 388.902 & 389.929 & 0.264 & 30.0000 & 29.9852 & -0.049 & 0.0016 \\
\hline 265.91 & 460.501 & 465.293 & 1.041 & 31.0000 & 30.9409 & -0.191 & 0.0013 \\
\hline 265.91 & 542.705 & 553.181 & 1.930 & 32.0000 & 31.8884 & -0.349 & 0.0011 \\
\hline 281.82 & 206.953 & 204.514 & -1.179 & 26.0000 & 26.0654 & 0.252 & 0.0034 \\
\hline 281.82 & 247.258 & 245.062 & -0.888 & 27.0000 & 27.0495 & 0.183 & 0.0028 \\
\hline 281.82 & 294.549 & 293.257 & -0.439 & 28.0000 & 28.0246 & 0.088 & 0.0023 \\
\hline 281.82 & 349.689 & 350.267 & 0.165 & 29.0000 & 28.9907 & -0.032 & 0.0018 \\
\hline 281.82 & 413.585 & 417.367 & 0.915 & 30.0000 & 29.9477 & -0.174 & 0.0015 \\
\hline 281.82 & 487.179 & 495.937 & 1.798 & 31.0000 & 30.8959 & -0.336 & 0.0013 \\
\hline 281.82 & 571.437 & 587.447 & 2.802 & 32.0000 & 31.8355 & -0.514 & 0.0011 \\
\hline 297.73 & 188.335 & 186.301 & -1.080 & 25.0000 & 25.0618 & 0.247 & 0.0040 \\
\hline 297.73 & 224.024 & 222.104 & -0.857 & 26.0000 & 26.0490 & 0.188 & 0.0033 \\
\hline 297.73 & 265.977 & 264.708 & -0.477 & 27.0000 & 27.0273 & 0.101 & 0.0026 \\
\hline 297.73 & 315.000 & 315.199 & 0.063 & 28.0000 & 27.9964 & -0.013 & 0.0022 \\
\hline 297.73 & 371.949 & 374.779 & 0.761 & 29.0000 & 28.9560 & -0.152 & 0.0018 \\
\hline 297.73 & 437.726 & 444.767 & 1.609 & 30.0000 & 29.9063 & -0.312 & 0.0015 \\
\hline 297.73 & 513.267 & 526.592 & 2.596 & 31.0000 & 30.8472 & -0.493 & 0.0012 \\
\hline 297.73 & 599.534 & 621.785 & 3.711 & 32.0000 & 31.7792 & -0.690 & 0.0010 \\
\hline 313.64 & 145.261 & 143.802 & -1.005 & 23.0000 & 23.0600 & 0.261 & 0.0059 \\
\hline 313.64 & 171.962 & 170.302 & -0.965 & 24.0000 & 24.0571 & 0.238 & 0.0047 \\
\hline 313.64 & 203.566 & 201.936 & -0.801 & 25.0000 & 25.0471 & 0.188 & 0.0038 \\
\hline 313.64 & 240.772 & 239.589 & -0.491 & 26.0000 & 26.0288 & 0.111 & 0.0031 \\
\hline 313.64 & 284.327 & 284.258 & -0.024 & 27.0000 & 27.0014 & 0.005 & 0.0025 \\
\hline 313.64 & 335.034 & 337.062 & 0.605 & 28.0000 & 27.9645 & -0.127 & 0.0021 \\
\hline 313.64 & 393.743 & 399.239 & 1.396 & 29.0000 & 28.9178 & -0.283 & 0.0017 \\
\hline 313.64 & 461.351 & 472.149 & 2.340 & 30.0000 & 29.8614 & -0.462 & 0.0014 \\
\hline 313.64 & 538.790 & 557.273 & 3.430 & 31.0000 & 30.7954 & -0.660 & 0.0012 \\
\hline 313.64 & 627.020 & 656.206 & 4.655 & 32.0000 & 31.7200 & -0.875 & 0.0010 \\
\hline 329.55 & 157.527 & 156.197 & -0.845 & 23.0000 & 23.0517 & 0.225 & 0.0056 \\
\hline
\end{tabular}


Data from Morris [42] (continued)

\begin{tabular}{|c|c|c|c|c|c|c|c|}
\hline$T$ & $P, \exp$ & P,cal & Dev & $\rho, \exp$ & $\rho$, cal & Dev & Wt \\
\hline K & $\mathrm{MPa}$ & $\mathrm{MPa}$ & 8 & $\mathrm{~mol} \cdot \mathrm{dm}^{-3}$ & $\mathrm{~mol} \cdot \mathrm{dm}^{-3}$ & 8 & \\
\hline 329.55 & 185.533 & 184.185 & -0.726 & 24.0000 & 24.0440 & 0.183 & 0.0045 \\
\hline 329.55 & 218.529 & 217.480 & -0.480 & 25.0000 & 25.0289 & 0.116 & 0.0036 \\
\hline 329.55 & 257.213 & 256.988 & -0.088 & 26.0000 & 26.0052 & 0.020 & 0.0030 \\
\hline 329.55 & 302.329 & 303.734 & 0.465 & 27.0000 & 26.9722 & -0.103 & 0.0024 \\
\hline 329.55 & 354.675 & 358.867 & 1.182 & 28.0000 & 27.9294 & -0.252 & 0.0020 \\
\hline 329.55 & 415.099 & 423.664 & 2.063 & 29.0000 & 28.8766 & -0.425 & 0.0016 \\
\hline 329.55 & 484.492 & 499.528 & 3.103 & 30.0000 & 29.8138 & -0.621 & 0.0013 \\
\hline 329.55 & 563.783 & 587.993 & 4.294 & 31.0000 & 30.7410 & -0.835 & 0.0011 \\
\hline 329.55 & 653.930 & 690.716 & 5.625 & 32.0000 & 31.6585 & -1.067 & 0.0009 \\
\hline 345.45 & 169.604 & 168.516 & -0.641 & 23.0000 & 23.0401 & 0.174 & 0.0053 \\
\hline 345.45 & 198.885 & 197.993 & -0.448 & 24.0000 & 24.0277 & 0.116 & 0.0043 \\
\hline 345.45 & 233.242 & 232.950 & -0.125 & 25.0000 & 25.0077 & 0.031 & 0.0035 \\
\hline 345.45 & 273.368 & 274.319 & 0.348 & 26.0000 & 25.9788 & -0.082 & 0.0028 \\
\hline 345.45 & 320.006 & 323.152 & 0.983 & 27.0000 & 26.9403 & -0.221 & 0.0023 \\
\hline 345.45 & 373.952 & 380.631 & 1.786 & 28.0000 & 27.8917 & -0.387 & 0.0019 \\
\hline 345.45 & 436.048 & 448.069 & 2.757 & 29.0000 & 28.8329 & -0.576 & 0.0016 \\
\hline 345.45 & 507.182 & 526.917 & 3.891 & 30.0000 & 29.7638 & -0.787 & 0.0013 \\
\hline 345.45 & 588.281 & 618.760 & 5.181 & 31.0000 & 30.6845 & -1.018 & 0.0011 \\
\hline 345.45 & 680.299 & 725.319 & 6.618 & 32.0000 & 31.5952 & -1.265 & 0.0009 \\
\hline 361.36 & 155.482 & 154.623 & -0.552 & 22.0000 & 22.0357 & 0.162 & 0.0062 \\
\hline 361.36 & 181.501 & 180.770 & -0.403 & 23.0000 & 23.0257 & 0.112 & 0.0050 \\
\hline 361.36 & 212.032 & 211.735 & -0.140 & 24.0000 & 24.0088 & 0.037 & 0.0041 \\
\hline 361.36 & 247.720 & 248.358 & 0.258 & 25.0000 & 24.9839 & -0.064 & 0.0033 \\
\hline 361.36 & 289.256 & 291.594 & 0.808 & 26.0000 & 25.9499 & -0.193 & 0.0027 \\
\hline 361.36 & 337.383 & 342.525 & 1.524 & 27.0000 & 26.9061 & -0.348 & 0.0022 \\
\hline 361.36 & 392.890 & 402.364 & 2.411 & 28.0000 & 27.8519 & -0.529 & 0.0018 \\
\hline 361.36 & 456.619 & 472.464 & 3.470 & 29.0000 & 28.7873 & -0.734 & 0.0015 \\
\hline 361.36 & 529.454 & 554.321 & 4.697 & 30.0000 & 29.7121 & -0.960 & 0.0013 \\
\hline 361.36 & 612.319 & 649.577 & 6.085 & 31.0000 & 30.6265 & -1.205 & 0.0010 \\
\hline 377.27 & 123.138 & 122.570 & -0.461 & 20.0000 & 20.0313 & 0.157 & 0.0089 \\
\hline 377.27 & 142.890 & 142.241 & -0.454 & 21.0000 & 21.0303 & 0.144 & 0.0072 \\
\hline 377.27 & 166.066 & 165.492 & -0.346 & 22.0000 & 22.0227 & 0.103 & 0.0059 \\
\hline 377.27 & 193.231 & 192.969 & -0.135 & 23.0000 & 23.0088 & 0.038 & 0.0048 \\
\hline 377.27 & 224.988 & 225.424 & 0.194 & 24.0000 & 23.9876 & -0.052 & 0.0039 \\
\hline 377.27 & 261.980 & 263.716 & 0.662 & 25.0000 & 24.9581 & -0.168 & 0.0032 \\
\hline
\end{tabular}


Data from Morris [42] (continued)

\begin{tabular}{|c|c|c|c|c|c|c|c|}
\hline $\mathrm{T}$ & $P, \exp$ & P,cal & Dev & $\rho, \exp$ & $\rho, \mathrm{cal}$ & Dev & Wt \\
\hline K & $\mathrm{MPa}$ & $\mathrm{MPa}$ & 8 & $\mathrm{~mol} \cdot \mathrm{dm}^{-3}$ & $\mathrm{~mol} \cdot \mathrm{dm}^{-3}$ & 8 & \\
\hline 377.27 & 304.898 & 308.825 & 1.288 & 26.0000 & 25.9191 & -0.311 & 0.0026 \\
\hline 377.27 & 354.480 & 361.862 & 2.083 & 27.0000 & 26.8701 & -0.481 & 0.0021 \\
\hline 377.27 & 411.515 & 424.076 & 3.052 & 28.0000 & 27.8104 & -0.677 & 0.0018 \\
\hline 377.27 & 476.842 & 496.858 & 4.198 & 29.0000 & 28.7401 & -0.896 & 0.0015 \\
\hline 377.27 & 551.340 & 581.750 & 5.516 & 30.0000 & 29.6590 & -1.137 & 0.0012 \\
\hline 377.27 & 635.931 & 680.448 & 7.000 & 31.0000 & 30.5673 & -1.396 & 0.0010 \\
\hline 393.18 & 131.568 & 131.135 & -0.329 & 20.0000 & 20.0226 & 0.113 & 0.0084 \\
\hline 393.18 & 152.288 & 151.874 & -0.272 & 21.0000 & 21.0184 & 0.088 & 0.0068 \\
\hline 393.18 & 176.513 & 176.314 & -0.112 & 22.0000 & 22.0 & 0.034 & 0.0056 \\
\hline 393.18 & 204.805 & 205.123 & 0.155 & 23.0000 & 22.9898 & -0.044 & 0.0045 \\
\hline 393.18 & 237.766 & 239.068 & 0.548 & 24.0000 & 23.9 & -0.148 & 0.0037 \\
\hline 393.18 & 276.039 & 279.032 & 1.085 & 25.0000 & 24.9305 & -0.278 & 0.0030 \\
\hline 393.18 & 320.311 & 326.021 & 1.783 & 26.0000 & 25.8867 & -0.436 & 0.0025 \\
\hline 393.18 & 371.320 & 381.175 & 2.654 & 27.0000 & 26.8326 & -0.620 & 0.0020 \\
\hline 393.18 & 429.852 & 445.776 & 3.704 & 28.0000 & 27.7677 & -0.830 & 0.0017 \\
\hline 393.18 & 496.743 & 521.257 & 4.935 & 29. & 28. & -1 & 14 \\
\hline 393.18 & 572.870 & 609.208 & 6.343 & 30.0000 & 29.6 & -1.317 & 0.0012 \\
\hline 393.18 & 659.151 & 711.378 & 7.923 & 31.0000 & 30.5074 & -1.589 & 0.0010 \\
\hline 409.09 & 139.898 & 139.665 & -0.167 & 20.0000 & 20.0116 & 0.058 & 0.0080 \\
\hline 409.09 & 161.574 & 161.468 & -0.066 & 21.0000 & 21.0045 & 0.021 & 0.0065 \\
\hline 409.09 & 186.832 & 187.098 & 0.142 & 22.0 & 21. & -0.044 & 0.0053 \\
\hline 409.09 & 216.234 & 217.238 & 0.464 & 23.0000 & 22.9692 & -0.134 & 0.0043 \\
\hline 409.09 & 250.380 & 252.676 & 0.917 & 24.0000 & 23.9399 & -0.250 & 0.0036 \\
\hline 409.09 & 289.910 & 294.316 & 1.520 & 25.0000 & 24.9015 & -0.394 & 0.0029 \\
\hline 409.09 & 335.512 & 343.190 & 2.288 & 26.0000 & 25.8531 & -0.565 & 0.0024 \\
\hline 409.09 & 387.922 & 400.469 & 3.234 & 27.0000 & 26.7940 & -0.763 & 0.0020 \\
\hline 409.09 & 447.923 & 467.471 & 4.364 & 28.0000 & 27.7240 & -0.986 & 0.0016 \\
\hline 409.09 & 516.347 & 545.668 & 5.678 & 29.0000 & 28.6428 & -1.232 & 0.0014 \\
\hline 409.09 & 594.071 & 636.698 & 7.175 & 30.0 & 29.5504 & -1.499 & 0.0011 \\
\hline 409.09 & 682.008 & 742.367 & 8.850 & 31.0000 & 30.4470 & -1.784 & 0.0009 \\
\hline
\end{tabular}

Number of Points (Ref. 42) 105

$\begin{array}{llrlrlrl}\text { PRESSURE: } & \text { AAD }-8 & 1.920 & \text { BIAS }-8 & 1.448 & \text { RMS }-8 & 2.381 & \\ & \text { AAD } & 9.234 & \text { BIAS } & 8.254 & \text { RMS } & 13.539 & \mathrm{MPa} \\ \text { DENSITY : } & \text { AAD }-8 & 0.404 & \text { BIAS }-8 & -0.296 & \text { RMS }-8 & 0.490 & \\ & \text { AAD } & 0.117 & \text { BIAS } & -0.090 & \text { RMS } & 0.146 & \mathrm{~mol} \cdot \mathrm{dm}^{-3}\end{array}$


Data from Robertson and Babb [43]

\begin{tabular}{|c|c|c|c|c|c|c|c|}
\hline $\mathrm{T}$ & $P, \exp$ & P, cal & Dev & $\rho, \exp$ & $\rho$, cal & Dev & Wt \\
\hline $\mathrm{K}$ & $\mathrm{MPa}$ & $\mathrm{MPa}$ & 8 & $\mathrm{~mol} \cdot \mathrm{dm}^{-3}$ & $\mathrm{~mol} \cdot \mathrm{dm}^{-3}$ & 용 & \\
\hline 308.15 & 150.000 & 146.393 & -2.404 & 23.2830 & 23.4257 & 0.613 & 0.0565 \\
\hline 308.15 & 200.000 & 196.116 & -1.942 & 24.9870 & 25.1008 & 0.455 & 0.0390 \\
\hline 308.15 & 250.000 & 246.617 & -1.353 & 26.3150 & 26.3940 & 0.300 & 0.0295 \\
\hline 308.15 & 300.000 & 298.063 & -0.646 & 27.4150 & 27.4527 & 0.137 & 0.0236 \\
\hline 308.15 & 350.000 & 350.634 & 0.181 & 28.3630 & 28.3524 & -0.037 & 0.0195 \\
\hline 308.15 & 400.000 & 404.294 & 1.073 & 29.2000 & 29.1370 & -0.216 & 0.0166 \\
\hline 308.15 & 450.000 & 458.607 & 1.913 & 29.9470 & 29.8343 & -0.376 & 0.0144 \\
\hline 308.15 & 500.000 & 513.768 & 2.754 & 30.6260 & 30.4630 & -0.532 & 0.0126 \\
\hline 308.15 & 550.000 & 570.413 & 3.711 & 31.2570 & 31.0365 & -0.706 & 0.0113 \\
\hline 308.15 & 600.000 & 627.104 & 4.517 & 31.8340 & 31.5643 & -0.847 & 0.0102 \\
\hline 8.15 & 650.000 & 685.244 & 5.422 & 32.3790 & 32.0538 & -1 & 0.0092 \\
\hline 308.15 & 700.000 & 743.943 & 6.278 & 32.8890 & 32.5107 & -1.150 & 0.0084 \\
\hline 308.15 & 750.000 & 803.580 & 7.144 & 33.3720 & 32.9396 & -1.296 & 0.0078 \\
\hline 308.15 & 800.000 & 863.680 & 7.960 & 33.8280 & 33.3439 & -1.431 & 0.0072 \\
\hline 308.15 & 850.000 & 924.468 & 8.761 & 34.2620 & 33.7267 & -1.562 & 0.0067 \\
\hline 08.15 & 900.000 & 986.160 & 9.573 & 34.6780 & 34 & -1. & 063 \\
\hline 308.15 & 950.000 & 1048.447 & 10.363 & 35.0760 & 34.4370 & -1.822 & 0.0059 \\
\hline 308.15 & 1000.000 & 1111.850 & 11.185 & 35.4610 & 34.7683 & -1.954 & 0.0055 \\
\hline 373.15 & 150.000 & 148.343 & -1.105 & 21.3950 & 21.4682 & 0.342 & 0.0673 \\
\hline 373.15 & 200.000 & 198.476 & -0.762 & 23.2870 & 23.3361 & 0.211 & 0.0454 \\
\hline 373.15 & 250. & 250. & & & & -0 & 336 \\
\hline 373.15 & 300.000 & 302 & 0.9 & 25 & & -0.219 & 0.0264 \\
\hline 373.15 & 350.000 & 356.570 & 1.877 & 26.9950 & 26.8782 & -0.433 & 0.0215 \\
\hline 373.15 & 400.000 & 411.449 & 2.862 & 27.8940 & 27.7167 & -0.636 & 0.0181 \\
\hline 373.15 & 450.000 & 467.275 & 3.839 & 28.6940 & 28.4570 & -0.826 & 0.0155 \\
\hline 373.15 & 00 & 524. & 4.8 & & & -1 & 36 \\
\hline 373.15 & 550.000 & 582.028 & 5.823 & 30.0810 & 29.7 & -1.192 & 0.0120 \\
\hline 373.15 & 600.000 & 641.355 & 6.893 & 30.6980 & 30.2740 & -1.381 & 0.0107 \\
\hline 373.15 & 650.000 & 701.979 & 7.997 & 31.2750 & 30.7833 & -1.572 & 0.0097 \\
\hline 373.15 & 700.000 & 763.076 & 9.011 & 31.8110 & 31.2569 & -1.742 & 0.0088 \\
\hline 373.15 & 750.000 & 825 & 10 & 32 & & -1.922 & 0.0080 \\
\hline 373.15 & 800.000 & 888.271 & 11.034 & & 32.1 & -2.071 & 0.0074 \\
\hline 373.15 & 850.000 & 952.608 & 12.072 & 33.2520 & 32.5085 & -2.236 & 0.0069 \\
\hline 373.15 & 900.000 & 1016.834 & 12.982 & 33.6810 & 32.8805 & -2.377 & 0.0064 \\
\hline 373.15 & 950.000 & 1082.015 & 13.896 & 34.0920 & 33.2340 & -2.517 & 0.006 \\
\hline
\end{tabular}


Data from Robertson and Babb [43] (continued)

\begin{tabular}{|c|c|c|c|c|c|c|c|}
\hline $\mathrm{T}$ & $P, \exp$ & P,cal & Dev & $\rho, \exp$ & $\rho$, cal & Dev & Wt \\
\hline $\mathrm{K}$ & $\mathrm{MPa}$ & $\mathrm{MPa}$ & 8 & $\mathrm{~mol} \cdot \mathrm{dm}^{-3}$ & $\mathrm{~mol} \cdot \mathrm{dm}^{-3}$ & 8 & \\
\hline 73.15 & 400.000 & 418.622 & 4.655 & 26.1050 & 25.7974 & -1.178 & 0.0203 \\
\hline 473.15 & 450.000 & 476.456 & 5.879 & 26.9770 & 26. & -1.425 & 0.0172 \\
\hline 473.15 & 500.000 & 535.369 & 7.074 & 27.7600 & 27.3012 & -1.653 & 0.0149 \\
\hline 473.15 & 550.000 & 595.615 & 8.294 & 28.4750 & 27.9409 & -1.876 & 0.0131 \\
\hline 473.15 & 600.000 & 657.070 & 9.512 & 29.1330 & 28.5242 & -2.090 & 0.0116 \\
\hline 473.15 & 650.000 & 719.455 & 10.685 & 29.7410 & 29. & -2.288 & 0.0104 \\
\hline 473.15 & 700.000 & 784.058 & 12.008 & 30.3180 & 29.5572 & -2.509 & 0.0094 \\
\hline 473.15 & 750.000 & 849.146 & 13.220 & 30.8540 & 30.0199 & -2.703 & 0.0085 \\
\hline 473.15 & 800.000 & 915.944 & 14.493 & 31.3640 & 30.4532 & -2.904 & 0.0078 \\
\hline 473.15 & 850.000 & 983.869 & 15.749 & 31.8470 & 30.8608 & -3.097 & 0.0072 \\
\hline 473.15 & 900.000 & 1052.419 & 16.935 & 32.3030 & 31.2456 & -3.273 & 0.0066 \\
\hline 473.15 & 950.000 & 1121.878 & 18.092 & 32.7370 & 31.6103 & -3.442 & 0.0061 \\
\hline 473.15 & 1000.000 & 1192.522 & 19.252 & 33.1530 & 31.9570 & -3.608 & 0.0057 \\
\hline Number o: & of Points & (Ref. 43) & 53 & & & & \\
\hline \multirow[t]{2}{*}{ PRESSURE } & $A A D-8$ & 7.024 & BIAS - $\frac{8}{8}$ & 6.714 & RMS - 8 & 5.581 & \\
\hline & AAD & 52.863 & BIAS & 52.260 & RMS & 51.540 & $\mathrm{MPa}$ \\
\hline \multirow[t]{2}{*}{ DENSITY } & AAD -8 & 1.394 & BIAS - 8 & -1.317 & RMS - 8 & 1.070 & \\
\hline & AAD & 0.437 & BIAS & -0.418 & RMS & 0.347 & $\mathrm{~mol} \cdot \mathrm{dm}$ \\
\hline
\end{tabular}

Data from Sivaraman and Gammon [44]

$\begin{array}{cccccccc}\text { T } & \text { P,exp } & \text { P,cal } & \text { Dev } & \rho, \exp & \rho, \text { cal } & \text { Dev } & \text { Wt } \\ \text { K } & \text { MPa } & \text { MPa } & 8 & \text { mol. } \mathrm{dm}^{-3} & \text { mol. } \mathrm{dm}^{-3} & 8 & \\ 193.05 & 1.456 & 1.454 & -0.077 & 1.0186 & 1.0195 & 0.088 & 0.0000 \\ 193.05 & 3.446 & 3.443 & -0.090 & 3.0974 & 3.1019 & 0.145 & 0.0000 \\ 193.05 & 4.429 & 4.427 & -0.051 & 5.1435 & 5.1509 & 0.144 & 0.0000 \\ 193.05 & 4.818 & 4.821 & 0.046 & 7.2853 & 7.2617 & -0.324 & 0.0000 \\ 193.05 & 4.937 & 4.978 & 0.834 & 10.5749 & 9.3913 & -11.193 & 0.0000 \\ 193.05 & 5.019 & 5.016 & -0.058 & 11.4240 & 11.4766 & 0.460 & 0.0000 \\ 193.05 & 5.174 & 5.173 & -0.032 & 13.0245 & 13.0344 & 0.076 & 0.0000 \\ 193.05 & 5.643 & 5.604 & -0.696 & 14.5064 & 14.5900 & 0.577 & 0.0000 \\ 195.15 & 1.475 & 1.474 & -0.072 & 1.0183 & 1.0191 & 0.082 & 0.0000 \\ 195.15 & 3.517 & 3.514 & -0.099 & 3.0961 & 3.1010 & 0.157 & 0.0000\end{array}$


Data from Sivaraman and Gammon [44] (continued)

\begin{tabular}{|c|c|c|c|c|c|c|c|}
\hline $\mathrm{T}$ & $P, \exp$ & P,cal & Dev & $\rho, \exp$ & $\rho$, cal & Dev & Wt \\
\hline K & $\mathrm{MPa}$ & $\mathrm{MPa}$ & 8 & $\mathrm{~mol} \cdot \mathrm{dm}^{-3}$ & $\mathrm{~mol} \cdot \mathrm{dm}^{-3}$ & 8 & \\
\hline 193.05 & 5.019 & 5.016 & -0.058 & 11.4240 & 11.4766 & 0.460 & 0.0000 \\
\hline 193.05 & 5.174 & 5.173 & -0.032 & 13.0245 & 13.0344 & 0.076 & 0.0000 \\
\hline 193.05 & 5.643 & 5.604 & -0.696 & 14.5064 & 14.5900 & 0.577 & 0.0000 \\
\hline 195.15 & 1.475 & 1.474 & -0.072 & 1.0183 & 1.0191 & 0.082 & 0.0000 \\
\hline 195.15 & 3.517 & 3.514 & -0.099 & 3.0961 & 3.1010 & 0.157 & 0.0000 \\
\hline 95.15 & 4.564 & 4.564 & -0.015 & 5.1447 & 5.1467 & 0.039 & 0.0000 \\
\hline 195.15 & 5.028 & 5.035 & 0.126 & 7.2887 & 7.2401 & -0.667 & 0.0000 \\
\hline 195.15 & 5.217 & 5.244 & 0.510 & 9.7392 & 9.3377 & -4.122 & 0.0000 \\
\hline 195.15 & 5.379 & 5.398 & 0.347 & 11.6649 & 11.4880 & -1.516 & 0.000 \\
\hline 95.15 & 5.617 & 5.604 & -0.232 & 12.9654 & 13.0226 & 0.441 & 0.0000 \\
\hline 5.15 & 6.197 & 6.155 & -0.683 & 14.4934 & 14.5701 & 0.529 & 0.0000 \\
\hline 200.15 & 1.520 & 1.519 & -0.070 & 1.0171 & 1.0179 & 0.079 & 0.0000 \\
\hline 200.15 & 3.683 & 3.680 & -0.093 & 3.0919 & 3.0963 & 0.142 & 0.0000 \\
\hline 200.15 & 4.882 & 4.883 & 0.026 & 5.1398 & 5.1368 & -0.059 & 0.000 \\
\hline 200.15 & 5.344 & 5.352 & 0.140 & 6.5245 & 6.4968 & -0.424 & 0.000 \\
\hline 200.15 & 5.884 & 5.902 & 0.299 & 9.4139 & 9.2974 & -1.237 & 0.0000 \\
\hline 200.15 & 6.252 & 6.250 & -0.033 & 11.4638 & 11.4734 & 0.084 & 0.0000 \\
\hline 200.15 & 6.684 & 6.654 & -0.451 & 12.9066 & 12.9870 & 0.623 & 0.0000 \\
\hline 200.15 & 7.523 & 7.480 & -0.572 & 14.4680 & 14.5259 & 0.400 & 0.0000 \\
\hline 210.15 & 1.610 & 1.608 & -0.065 & 1.0147 & 1.0154 & 0.072 & 0.0000 \\
\hline 0.15 & 3.982 & 3.978 & -0.097 & 3.0540 & 3.0 & 0.1 & 0.0000 \\
\hline 210.15 & 5.498 & 5.500 & 0.040 & 5.1195 & 5.1157 & -0.074 & 0.0000 \\
\hline 210.15 & 6.475 & 6.489 & 0.211 & 7.2171 & 7.1821 & -0.486 & 0.0000 \\
\hline 210.15 & 7.202 & 7.215 & 0.176 & 9.2742 & 9.2367 & -0.404 & 0.0000 \\
\hline 210.15 & 7.973 & 7.959 & -0.175 & 11.2795 & 11.3115 & 0.284 & 0.0000 \\
\hline 210.15 & 8.839 & 8.802 & -0.427 & 12.8555 & 12. & 0.439 & 0.000 \\
\hline 210.15 & 10.170 & 10.137 & -0.331 & 14.4030 & 14.4332 & 0.210 & 0.0000 \\
\hline 223.15 & 1.725 & 1.724 & -0.049 & 1.0117 & 1.0122 & 0.054 & 0.0000 \\
\hline 223.15 & 4.378 & 4.373 & -0.096 & 3.0313 & 3.0351 & 0.127 & 0.0000 \\
\hline 223.15 & 6.273 & 6.275 & 0.034 & 5.0921 & 5.0894 & -0.053 & 0.0000 \\
\hline 223.15 & 7.678 & 7.692 & 0.185 & 7.1588 & 7.1357 & -0.322 & 0.0000 \\
\hline 223.15 & 8.859 & 8.872 & 0.152 & 9.1478 & 9.1251 & -0.248 & 0.0000 \\
\hline 223.15 & 10.227 & 10.216 & -0.113 & 11.2105 & 11.2260 & 0.138 & 0.0000 \\
\hline 223.15 & 11.815 & 11.784 & -0.264 & 12.9553 & 12.9837 & 0.220 & 0.0000 \\
\hline 248.15 & 1.942 & 1.941 & -0.041 & 1.0056 & 1.0060 & 0.044 & 0.0000 \\
\hline
\end{tabular}


Data from Sivaraman and Gammon [44] (continued)

\begin{tabular}{|c|c|c|c|c|c|c|c|}
\hline $\mathrm{T}$ & $P, \exp$ & P,cal & Dev & $\rho, \exp$ & $\rho, \mathrm{cal}$ & Dev & wt \\
\hline K & $\mathrm{MPa}$ & $\mathrm{MPa}$ & 8 & $\mathrm{~mol} \cdot \mathrm{dm}^{-3}$ & $\mathrm{~mol} \cdot \mathrm{dm}^{-3}$ & 8 & \\
\hline 248.15 & 5.107 & 5.103 & -0.086 & 2.9907 & 2.9938 & 0.104 & 0.0000 \\
\hline 248.15 & 7.644 & 7.645 & 0.016 & 4.9901 & 4.9891 & -0.021 & 0.0000 \\
\hline 248.15 & 9.877 & 9.891 & 0.146 & 7.0451 & 7.0314 & -0.195 & 0.0000 \\
\hline 248.15 & 11.983 & 12.001 & 0.151 & 9.0327 & 9.0162 & -0.183 & 0.0000 \\
\hline 248.15 & 14.470 & 14.474 & 0.022 & 11.0784 & 11.0761 & -0.021 & 0.000 \\
\hline 273.15 & 2.155 & 2.154 & -0.029 & 0.9997 & 1.0000 & 0.030 & 0.000 \\
\hline 273.15 & 5.883 & 5.878 & -0.074 & 2.9984 & 3.0009 & 0.085 & 0.000 \\
\hline 273.15 & 9.037 & 9.037 & 0.004 & 4.9698 & 4.9696 & -0.004 & 0.0000 \\
\hline 273.15 & 11.986 & 11.999 & 0.105 & 6.9596 & 6.9511 & -0.122 & 0.000 \\
\hline 273.15 & 15.110 & 15.130 & 0.135 & 9.0038 & 8.9912 & -0.140 & 0.000 \\
\hline 273.15 & 18.678 & 18.685 & 0.036 & 11.0011 & 10.9977 & -0.031 & 0.000 \\
\hline 298.15 & 2.377 & 2.378 & 0.030 & 1.0000 & 0.9997 & -0.031 & 0.000 \\
\hline 298.15 & 4.581 & 4.578 & -0.055 & 2.0000 & 2.0012 & 0.059 & 0.000 \\
\hline 298.15 & 6.641 & 6.638 & -0.048 & 3.0000 & 3.0016 & 0.053 & 0.000 \\
\hline 298.15 & 8.599 & 8.596 & -0.035 & 3.9999 & 4.0015 & 0.039 & 0.000 \\
\hline 298.15 & 10.493 & 10.491 & -0.011 & 4.9998 & 5.0004 & 0.012 & 0.0000 \\
\hline 298.15 & 12.360 & 12.362 & 0.016 & 5.9994 & 5.9984 & -0.017 & 0.000 \\
\hline 298.15 & 14.242 & 14.249 & 0.048 & 6.9988 & 6.9952 & -0.051 & 0.000 \\
\hline 298.15 & 16.188 & 16.201 & 0.080 & 8.0002 & 7.9937 & -0.081 & 0.000 \\
\hline 298.15 & 18.247 & 18.264 & 0.090 & 9.0002 & 8.9925 & -0.085 & 0.000 \\
\hline 298.15 & 20.484 & 20.500 & 0.080 & 10.0003 & 9.9933 & -0.070 & 0.000 \\
\hline 298.15 & 22.973 & 22.985 & 0.051 & 11.0005 & 10.9960 & -0.041 & 0.000 \\
\hline 323.15 & 2.600 & 2.602 & 0.068 & 1.0006 & 0.9999 & -0.070 & 0.000 \\
\hline 323.15 & 5.052 & 5.052 & -0.002 & 2.0007 & 2.0007 & 0.002 & 0.000 \\
\hline 323.15 & 7.392 & 7.391 & -0.015 & 3.0008 & 3.0013 & 0.016 & 0.000 \\
\hline 323.15 & 9.659 & 9.658 & -0.001 & 4.0016 & 4.0016 & 0.001 & 0.000 \\
\hline 323.15 & 11.891 & 11.894 & 0.024 & 5.0026 & 5.0013 & -0.025 & 0.000 \\
\hline 323.15 & 14.126 & 14.135 & 0.066 & 6.0023 & 5.9982 & -0.068 & 0.0000 \\
\hline 323.15 & 16.421 & 16.434 & 0.078 & 7.0036 & 6.9981 & -0.079 & 0.000 \\
\hline 323.15 & 18.815 & 18.834 & 0.104 & 8.0042 & 7.9962 & -0.099 & 0.000 \\
\hline 323.15 & 21.377 & 21.395 & 0.082 & 9.0047 & 8.9981 & -0.073 & 0.0000 \\
\hline 323.15 & 24.156 & 24.183 & 0.112 & 10.0053 & 9.9960 & -0.093 & 0.0000 \\
\hline 348.15 & 2.821 & 2.822 & 0.034 & 1.0002 & 0.9998 & -0.035 & 0.0000 \\
\hline 348.15 & 5.522 & 5.525 & 0.062 & 2.0015 & 2.0002 & -0.064 & 0.0000 \\
\hline 348.15 & 11.993 & 12.000 & 0.058 & 4.5045 & 4.5018 & -0.060 & 0.000 \\
\hline
\end{tabular}


Data from Sivaraman and Gammon [44] (continued)

\begin{tabular}{|c|c|c|c|c|c|c|c|}
\hline $\mathbf{T}$ & $P, \exp$ & P,cal & Dev & $\rho, \exp$ & $\rho, \mathrm{cal}$ & Dev & Wt \\
\hline K & $\mathrm{MPa}$ & $\mathrm{MPa}$ & 8 & $\mathrm{~mol} \cdot \mathrm{dm}^{-3}$ & $\mathrm{~mol} \cdot \mathrm{dm}^{-3}$ & 8 & \\
\hline 348.15 & 13.280 & 13.288 & 0.060 & 5.0052 & 5.0021 & -0.061 & 0.0000 \\
\hline 348.15 & 15.891 & 15.902 & 0.074 & 6.0066 & 6.0022 & -0.074 & 0.0000 \\
\hline 348.15 & 18.588 & 18.607 & 0.101 & 7.0077 & 7.0009 & -0.097 & 0.0000 \\
\hline 348.15 & 21.423 & 21.456 & 0.155 & 8.0089 & 7.9976 & -0.141 & 0.0000 \\
\hline 348.15 & 24.479 & 24.513 & 0.138 & 9.0099 & 8.9993 & -0.118 & 0.0000 \\
\hline 373.15 & 3.043 & 3.046 & 0.085 & 1.0011 & 1.0002 & -0.087 & 0.0000 \\
\hline 373.15 & 5.993 & 5.996 & 0.051 & 2.0023 & 2.0012 & -0.053 & 0.0000 \\
\hline 373.15 & 8.884 & 8.891 & 0.085 & 3.0043 & 3.0017 & -0.088 & 0.0000 \\
\hline 373.15 & 11.760 & 11.770 & 0.087 & 4.0064 & 4.0029 & -0.088 & 0.0000 \\
\hline 373.15 & 14.663 & 14.679 & 0.108 & 5.0087 & 5.0033 & -0.108 & 0.0000 \\
\hline 373.15 & 17.631 & 17.662 & 0.176 & 6.0109 & 6.0007 & -0.170 & 0.0000 \\
\hline 373.15 & 20.742 & 20.773 & 0.146 & 7.0126 & 7.0031 & -0.135 & 0.0000 \\
\hline 373.15 & 24.007 & 24.070 & 0.264 & 8.0144 & 7.9958 & -0.232 & 0.0000 \\
\hline 373.15 & 27.542 & 27.620 & 0.281 & 9.0155 & 8.9945 & -0.232 & 0.0000 \\
\hline 398.15 & 3.234 & 3.267 & 1.037 & 1.0012 & 0.9908 & -1.040 & 0.0000 \\
\hline 398.15 & 6.458 & 6.468 & 0.156 & 2.0037 & 2.0005 & -0.158 & 0.0000 \\
\hline 398.15 & 9.623 & 9.639 & 0.170 & 3.0064 & 3.0012 & -0.172 & 0.0000 \\
\hline 398.15 & 12.800 & 12.823 & 0.178 & 4.0095 & 4.0024 & -0.178 & 0.0000 \\
\hline 398.15 & 16.037 & 16.066 & 0.184 & 5.0128 & 5.0038 & -0.180 & 0.0000 \\
\hline 398.15 & 19.368 & 19.418 & 0.257 & 6.0156 & 6.0010 & -0.242 & 0.0000 \\
\hline 398.15 & 22.875 & 22.933 & 0.252 & 7.0180 & 7.0020 & -0.227 & 0.0000 \\
\hline 398.15 & 26.602 & 26.675 & 0.273 & 8.0199 & 8.0012 & -0.234 & 0.0000 \\
\hline 423.15 & 3.484 & 3.491 & 0.193 & 1.0020 & 1.0001 & -0.194 & 0.0000 \\
\hline 423.15 & 6.925 & 6.939 & 0.207 & 2.0049 & 2.0007 & -0.209 & 0.0000 \\
\hline 423.15 & 10.361 & 10.386 & 0.241 & 3.0087 & 3.0015 & -0.241 & 0.0000 \\
\hline 423.15 & 13.838 & 13.874 & 0.260 & 4.0129 & 4.0026 & -0.256 & 0.0000 \\
\hline 423.15 & 17.401 & 17.453 & 0.298 & 5.0173 & 5.0030 & -0.285 & 0.0000 \\
\hline 423.15 & 21.100 & 21.171 & 0.335 & 6.0209 & 6.0023 & -0.310 & 0.0000 \\
\hline 423.15 & 24.997 & 25.088 & 0.366 & 7.0236 & 7.0009 & -0.323 & 0.0000 \\
\hline
\end{tabular}

Number of Points (Ref. 44) 104

$\begin{array}{llllrlrl}\text { PRESSURE : } & \text { AAD }-8 & 0.158 & \text { BIAS }-8 & 0.055 & \text { RMS }-8 & 0.229 & \\ & \text { AAD } & 0.016 & \text { BIAS } & 0.010 & \text { RMS } & 0.023 & \mathrm{MPa} \\ \text { DENSITY : } & \text { AAD }-8 & 0.337 & \text { BIAS }-8 & -0.218 & \text { RMS }-8 & 1.189 & \\ & \text { AAD } & 0.030 & \text { BIAS } & -0.018 & \text { RMS } & 0.125 & \mathrm{~mol}^{-8} \mathrm{dm}^{-3}\end{array}$


Data from Trappeniers et al. [45]

\begin{tabular}{|c|c|c|c|c|c|c|c|}
\hline $\mathrm{T}$ & $P, \exp$ & P, cal & Dev & $\rho, \exp$ & $\rho$, cal & Dev & Wt \\
\hline K & $\mathrm{MPa}$ & $\mathrm{MPa}$ & 8 & $\mathrm{~mol} \cdot \mathrm{dm}^{-3}$ & $\mathrm{~mol} \cdot \mathrm{dm}^{-3}$ & 8 & \\
\hline 273.15 & 1.794 & 1.794 & -0.007 & 0.8250 & 0.8251 & 0.008 & 102.6723 \\
\hline 285.65 & 1.885 & 1.885 & -0.003 & 0.8250 & 0.8250 & 0.004 & 97.4853 \\
\hline 298.15 & 1.976 & 1.976 & -0.002 & 0.8250 & 0.8250 & 0.003 & 92.8060 \\
\hline 323.15 & 2.157 & 2.157 & 0.005 & 0.8250 & 0.8250 & -0.005 & 84.7006 \\
\hline 348.15 & 2.338 & 2.338 & 0.005 & 0.8250 & 0.8250 & -0.006 & 77.9150 \\
\hline 373.15 & 2.518 & 2.518 & 0.012 & 0.8250 & 0.8249 & -0.013 & 72.1521 \\
\hline 398.15 & 2.698 & 2.698 & 0.011 & 0.8250 & 0.8249 & -0.011 & 67.1909 \\
\hline 423.15 & 2.878 & 2.878 & 0.012 & 0.8250 & 0.8249 & -0.012 & 62.8762 \\
\hline 273.15 & 2.226 & 2.224 & -0.065 & 1.0340 & 1.0347 & 0.069 & 83.1956 \\
\hline 285.65 & 2.341 & 2.340 & -0.060 & 1.0340 & 1.0347 & 0.063 & 78.8476 \\
\hline 298.15 & 2.457 & 2.455 & -0.057 & 1.0340 & 1.0346 & 0.059 & 74.9408 \\
\hline 323.15 & 2.687 & 2.686 & -0.050 & 1.0340 & 1.0345 & 0.052 & 68.2048 \\
\hline 348.15 & 2.917 & 2.915 & -0.043 & 1.0340 & 1.0345 & 0.045 & 62.5998 \\
\hline 373.15 & 3.146 & 3.144 & -0.042 & 1.0340 & 1.0344 & 0.042 & 57.8583 \\
\hline 398.15 & 3.374 & 3.373 & -0.041 & 1.0340 & 1.0344 & 0.042 & 53.7940 \\
\hline 423.15 & 3.603 & 3.601 & -0.046 & 1.0340 & 1.0345 & 0.046 & 50.2694 \\
\hline 73.15 & 2.648 & 2.646 & -0.082 & 1.2430 & 1.2441 & 0.088 & 786 \\
\hline 285.65 & 2.789 & 2.787 & -0.082 & 1.2430 & 1.2441 & 0.087 & 66.4800 \\
\hline 298.15 & 2.930 & 2.927 & -0.080 & 1.2430 & 1.2440 & 0.084 & 63.0814 \\
\hline 323.15 & 3.210 & 3.208 & -0.072 & 1.2430 & 1.2439 & 0.074 & 57.2518 \\
\hline 348.15 & 3.490 & 3.487 & -0.065 & 1.2430 & 1.2438 & 0.067 & 52.4271 \\
\hline 373.15 & 3.769 & 3.766 & -0.066 & 1.2430 & 1.24 & 0.067 & 48.3639 \\
\hline 398.15 & 4.047 & 4.044 & -0.062 & 1.2430 & 1.2438 & 0.063 & 44.8957 \\
\hline 423.15 & 4.325 & 4.322 & -0.067 & 1.2430 & 1.2438 & 0.068 & 41.8972 \\
\hline 273.15 & 3.050 & 3.047 & -0.105 & 1.4460 & 1.4476 & 0.113 & 61.2998 \\
\hline 285.65 & 3.216 & 3.213 & -0.099 & 1.4460 & 1.4475 & 0.106 & 57.8828 \\
\hline 298.15 & 3.382 & 3.379 & -0.096 & 1.4460 & 1.4475 & 0.101 & 54.8358 \\
\hline 323.15 & 3.712 & 3.709 & -0.084 & 1.4460 & 1.4473 & 0.088 & 49.6336 \\
\hline 348.15 & 4.042 & 4.038 & -0.083 & 1.4460 & 1.4472 & 0.085 & 45.3493 \\
\hline 373.15 & 4.370 & 4.367 & -0.081 & 1.4460 & 1.4472 & 0.083 & 41.7586 \\
\hline 398.15 & 4.698 & 4.694 & -0.083 & 1.4460 & 1.4472 & 0.084 & 38.7035 \\
\hline 423.15 & 5.025 & 5.021 & -0.092 & 1.4460 & 1.4473 & 0.093 & 36.0704 \\
\hline 273.15 & 3.463 & 3.461 & -0.054 & 1.6600 & 1.6610 & 0.059 & 54.2274 \\
\hline 285.65 & 3.656 & 3.655 & -0.047 & 1.6600 & 1.6608 & 0.051 & 51.1061 \\
\hline 298.15 & 3.849 & 3.848 & -0.045 & 1.6600 & 1.6608 & 0.048 & 48.3328 \\
\hline 323.15 & 4.233 & 4.232 & -0.036 & 1.6600 & 1.6606 & 0.038 & 43.6213 \\
\hline
\end{tabular}


Data from Trappeniers et al. [45] (continued)

\begin{tabular}{|c|c|c|c|c|c|c|c|}
\hline $\mathrm{T}$ & $P, \exp$ & $\mathrm{P}, \mathrm{cal}$ & Dev & $\rho, \exp$ & $\rho, \mathrm{cal}$ & Dev & Wt \\
\hline K & $\mathrm{MPa}$ & $\mathrm{MPa}$ & 8 & $\mathrm{~mol} \cdot \mathrm{dm}^{-3}$ & $\mathrm{~mol} \cdot \mathrm{dm}^{-3}$ & 8 & \\
\hline 348.15 & 4.616 & 4.615 & -0.028 & 1.6600 & 1.6605 & 0.029 & 39.7645 \\
\hline 373.15 & 4.997 & 4.996 & -0.022 & 1.6600 & 1.6604 & 0.023 & 36.5463 \\
\hline 398.15 & 5.378 & 5.377 & -0.025 & 1.6600 & 1.6604 & 0.025 & 33.8178 \\
\hline 423.15 & 5.758 & 5.756 & -0.036 & 1.6600 & 1.6606 & 0.036 & 31.4736 \\
\hline 273.15 & 3.867 & 3.863 & -0.082 & 1.8720 & 1.8737 & 0.090 & 48.7882 \\
\hline 285.65 & 4.075 & 4.085 & 0.235 & 1.8720 & 1.8672 & -0.254 & 0.0000 \\
\hline 298.15 & 4.308 & 4.305 & -0.071 & 1.8720 & 1.8734 & 0.076 & 43.3282 \\
\hline 323.15 & 4.747 & 4.744 & -0.058 & 1.8720 & 1.8731 & 0.061 & 38.9941 \\
\hline 348.15 & 5.183 & 5.181 & -0.041 & 1.8720 & 1.8728 & 0.043 & 35.4665 \\
\hline 373.15 & 5.619 & 5.616 & -0.041 & 1.8720 & 1.8728 & 0.042 & 32.5336 \\
\hline 398.15 & 6.053 & 6.050 & -0.045 & 1.8720 & 1.8729 & 0.045 & 30.0568 \\
\hline 423.15 & 6.487 & 6.483 & -0.059 & 1.8720 & 1.8731 & 0.060 & 27.9352 \\
\hline 273.15 & 4.153 & 4.149 & -0.110 & 2.0250 & 2.0275 & 0.121 & 45.5632 \\
\hline 285.65 & 4.395 & 4.390 & -0.104 & 2.0250 & 2.0273 & 0.114 & 42.7980 \\
\hline 298.15 & 4.635 & 4.631 & -0.095 & 2.0250 & 2.0271 & 0.103 & 40.3591 \\
\hline 323.15 & 5.115 & 5.110 & -0.084 & 2.0250 & 2.0268 & 0.089 & 36.2472 \\
\hline 348.15 & 5.592 & 5.587 & -0.079 & 2.0250 & 2.0267 & 0.082 & 32.9115 \\
\hline 373.15 & 6.067 & 6.062 & -0.082 & 2.0250 & 2.0267 & 0.085 & 30.1484 \\
\hline 398.15 & 6.542 & 6.536 & -0.090 & 2.0250 & 2.0269 & 0.092 & 27.8210 \\
\hline 423.15 & 7.015 & 7.008 & -0.097 & 2.0250 & 2.0270 & 0.098 & 25.8334 \\
\hline 273.15 & 4.248 & 4.243 & -0.108 & 2.0760 & 2.0785 & 0.120 & 44.5954 \\
\hline 285.65 & 4.496 & 4.491 & -0.101 & 2.0760 & 2.0783 & 0.111 & 41.8698 \\
\hline 298.15 & 4.744 & 4.739 & -0.101 & 2.0760 & 2.0783 & 0.109 & 39.4658 \\
\hline 323.15 & 5.236 & 5.232 & -0.082 & 2.0760 & 2.0778 & 0.087 & 35.4221 \\
\hline 348.15 & 5.726 & 5.722 & -0.074 & 2.0760 & 2.0776 & 0.077 & 32.1450 \\
\hline 373.15 & 6.215 & 6.211 & -0.076 & 2.0760 & 2.0776 & 0.079 & 29.4331 \\
\hline 398.15 & 6.703 & 6.697 & -0.081 & 2.0760 & 2.0777 & 0.083 & 27.1511 \\
\hline 423.15 & 7.190 & 7.183 & -0.094 & 2.0760 & 2.0780 & 0.095 & 25.2026 \\
\hline 273.15 & 4.526 & 4.522 & -0.096 & 2.2280 & 2.2304 & 0.107 & 41.9729 \\
\hline 285.65 & 4.795 & 4.790 & -0.089 & 2.2280 & 2.2302 & 0.098 & 39.3525 \\
\hline 298.15 & 5.063 & 5.059 & -0.086 & 2.2280 & 2.2301 & 0.093 & 37.0484 \\
\hline 323.15 & 5.596 & 5.592 & -0.075 & 2.2280 & 2.2298 & 0.080 & 33.1831 \\
\hline 348.15 & 6.128 & 6.123 & -0.073 & 2.2280 & 2.2297 & 0.076 & 30.0630 \\
\hline 373.15 & 6.657 & 6.652 & -0.073 & 2.2280 & 2.2297 & 0.075 & 27.4901 \\
\hline 398.15 & 7.185 & 7.179 & -0.081 & 2.2280 & 2.2298 & 0.083 & 25.3299 \\
\hline
\end{tabular}


Data from Trappeniers et al. [45] (continued)

\begin{tabular}{|c|c|c|c|c|c|c|c|}
\hline $\mathrm{T}$ & $P, \exp$ & P, cal & Dev & $\rho, \exp$ & $\rho$, cal & Dev & Wt \\
\hline K & $\mathrm{MPa}$ & $\mathrm{MPa}$ & 8 & $\mathrm{~mol} \cdot \mathrm{dm}^{-3}$ & $\mathrm{~mol} \cdot \mathrm{dm}^{-3}$ & 8 & \\
\hline 423.15 & 7.711 & 7.704 & -0.088 & 2.2280 & 2.2300 & 0.088 & 23.4906 \\
\hline 273.15 & 4.633 & 4.628 & -0.090 & 2.2870 & 2.2893 & 0.101 & 41.0478 \\
\hline 285.65 & 4.910 & 4.906 & -0.082 & 2.2870 & 2.2891 & 0.090 & 38.4648 \\
\hline 298.15 & 5.186 & 5.182 & -0.079 & 2.2870 & 2.2890 & 0.086 & 36.1953 \\
\hline 323.15 & 5.735 & 5.731 & -0.063 & 2.2870 & 2.2885 & 0.067 & 32.3941 \\
\hline 348.15 & 6.282 & 6.278 & -0.055 & 2.2870 & 2.2883 & 0.058 & 29.3305 \\
\hline 373.15 & 6.827 & 6.823 & -0.059 & 2.2870 & 2.2884 & 0.060 & 26.8058 \\
\hline 398.15 & 7.370 & 7.366 & -0.063 & 2.2870 & 2.2885 & 0.064 & 24.6892 \\
\hline 423.15 & 7.912 & 7.907 & -0.074 & 2.2870 & 2.2887 & 0.074 & 22.8875 \\
\hline 273.15 & 4.893 & 4.889 & -0.074 & 2.4320 & 2.4340 & 0.083 & 38.9627 \\
\hline 285.65 & 5.190 & 5.187 & -0.065 & 2.4320 & 2.4338 & 0.072 & 36.4623 \\
\hline 298.15 & 5.486 & 5.483 & -0.060 & 2.4320 & 2.4336 & 0.066 & 34.2712 \\
\hline 323.15 & 6.075 & 6.072 & -0.052 & 2.4320 & 2.4333 & 0.055 & 30.6113 \\
\hline 348.15 & 6.662 & 6.659 & -0.054 & 2.4320 & 2.4334 & 0.056 & 27.6716 \\
\hline 373.15 & 7.247 & 7.242 & -0.059 & 2.4320 & 2.4335 & 0.061 & 25.2574 \\
\hline 398.15 & 7.829 & 7.824 & -0.063 & 2.4320 & 2.4336 & 0.064 & 23.2384 \\
\hline 423.15 & 8.421 & 8.404 & -0.206 & 2.4320 & 2.4371 & 0.208 & 21.5089 \\
\hline 273.15 & 5.248 & 5.244 & -0.081 & 2.6320 & 2.6344 & 0.092 & 36.4508 \\
\hline 285.65 & 5.574 & 5.569 & -0.077 & 2.6320 & 2.6343 & 0.086 & 34.0478 \\
\hline 298.15 & 5.898 & 5.894 & -0.071 & 2.6320 & 2.6341 & 0.078 & 31.9507 \\
\hline 323.15 & 6.543 & 6.539 & -0.065 & 2.6320 & 2.6338 & 0.069 & 28.4619 \\
\hline 348.15 & 7.186 & 7.181 & -0.065 & 2.6320 & 2.6338 & 0.068 & 25.6739 \\
\hline 373.15 & 7.825 & 7.820 & -0.065 & 2.6320 & 2.6338 & 0.067 & 23.3937 \\
\hline 398.15 & 8.462 & 8.456 & -0.074 & 2.6320 & 2.6340 & 0.075 & 21.4919 \\
\hline 423.15 & 9.099 & 9.090 & -0.091 & 2.6320 & 2.6344 & 0.091 & 19.8809 \\
\hline 273.15 & 5.601 & 5.596 & -0.094 & 2.8340 & 2.8370 & 0.107 & 34.2644 \\
\hline 285.65 & 5.956 & 5.951 & -0.087 & 2.8340 & 2.8368 & 0.098 & 31.9463 \\
\hline 298.15 & 6.310 & 6.304 & -0.086 & 2.8340 & 2.8367 & 0.095 & 29.9290 \\
\hline 323.15 & 7.013 & 7.007 & -0.090 & 2.8340 & 2.8367 & 0.096 & 26.5876 \\
\hline 348.15 & 7.713 & 7.706 & -0.091 & 2.8340 & 2.8367 & 0.095 & 23.9317 \\
\hline 373.15 & 8.410 & 8.401 & -0.099 & 2.8340 & 2.8369 & 0.102 & 21.7673 \\
\hline 398.15 & 9.104 & 9.094 & -0.103 & 2.8340 & 2.8370 & 0.105 & 19.9692 \\
\hline 423.15 & 9.796 & 9.785 & -0.119 & 2.8340 & 2.8374 & 0.120 & 18.4499 \\
\hline 273.15 & 5.828 & 5.825 & -0.064 & 2.9670 & 2.9692 & 0.073 & 32.9898 \\
\hline 285.65 & 6.204 & 6.199 & -0.076 & 2.9670 & 2.9695 & 0.085 & 30.7169 \\
\hline
\end{tabular}


Data from Trappeniers et al. [45] (continued)

\begin{tabular}{|c|c|c|c|c|c|c|c|}
\hline $\mathrm{T}$ & $P, \exp$ & P,cal & Dev & $\rho, \exp$ & $\rho$, cal & Dev & Wt \\
\hline $\mathrm{K}$ & $\mathrm{MPa}$ & $\mathrm{MPa}$ & 8 & $\mathrm{~mol} \cdot \mathrm{dm}^{-3}$ & $\mathrm{~mol} \cdot \mathrm{dm}^{-3}$ & 8 & \\
\hline 348.15 & 8.243 & 8.237 & -0.071 & 3.0390 & 3.0413 & 0.075 & 22.3971 \\
\hline 373.15 & 8.998 & 8.991 & -0.081 & 3.0390 & 3.0415 & 0.083 & 20.3353 \\
\hline 398.15 & 9.750 & 9.742 & -0.086 & 3.0390 & 3.0417 & 0.087 & 18.6283 \\
\hline 423.15 & 10.501 & 10.491 & -0.095 & 3.0390 & 3.0419 & 0.095 & 17.1905 \\
\hline 273.15 & 6.306 & 6.302 & -0.058 & 3.2490 & 3.2512 & 0.067 & 30.6103 \\
\hline 285.65 & 6.723 & 6.719 & -0.062 & 3.2490 & 3.2513 & 0.070 & 28.4281 \\
\hline 298.15 & 7.138 & 7.134 & -0.064 & 3.2490 & 3.2513 & 0.072 & 26.5433 \\
\hline 323.15 & 7.964 & 7.959 & -0.064 & 3.2490 & 3.2513 & 0.069 & 23.4503 \\
\hline 348.15 & 8.785 & 8.778 & -0.069 & 3.2490 & 3.2514 & 0.073 & 21.0150 \\
\hline 373.15 & 9.601 & 9.594 & -0.071 & 3.2490 & 3.2514 & 0.073 & 19.0472 \\
\hline 398.15 & 10.415 & 10.406 & -0.084 & 3.2490 & 3.2517 & 0.085 & 17.4218 \\
\hline 423.15 & 11.226 & 11.215 & -0.098 & 3.2490 & 3.2522 & 0.098 & 16.0567 \\
\hline 273.15 & 6.330 & 6.327 & -0.050 & 3.2640 & 3.2659 & 0.058 & 30.4962 \\
\hline 285.65 & 6.750 & 6.746 & -0.060 & 3.2640 & 3.2662 & 0.068 & 28.3171 \\
\hline 298.15 & 7.168 & 7.163 & -0.066 & 3.2640 & 3.2664 & 0.073 & 26.4360 \\
\hline 323.15 & 7.998 & 7.993 & -0.068 & 3.2640 & 3.2664 & 0.073 & 23.3505 \\
\hline 348.15 & 8.823 & 8.817 & -0.072 & 3.2640 & 3.2665 & 0.075 & 20.9225 \\
\hline 373.15 & 9.644 & 9.637 & -0.073 & 3.2640 & 3.2664 & 0.075 & 18.9609 \\
\hline 398.15 & 10.463 & 10.454 & -0.084 & 3.2640 & 3.2668 & 0.085 & 17.3412 \\
\hline 423.15 & 11.278 & 11.267 & -0.094 & 3.2640 & 3.2671 & 0.094 & 15.9814 \\
\hline 273.15 & 6.622 & 6.618 & -0.054 & 3.4390 & 3.4412 & 0.063 & 29.2175 \\
\hline 285.65 & 7.068 & 7.064 & -0.062 & 3.4390 & 3.4414 & 0.070 & 27.0861 \\
\hline 298.15 & 7.513 & 7.508 & -0.062 & 3.4390 & 3.4414 & 0.069 & 25.2520 \\
\hline 323.15 & 8.397 & 8.390 & -0.075 & 3.4390 & 3.4418 & 0.080 & 22.2517 \\
\hline 348.15 & 9.275 & 9.267 & -0.080 & 3.4390 & 3.4419 & 0.084 & 19.9013 \\
\hline 373.15 & 10.148 & 10.140 & -0.080 & 3.4390 & 3.4418 & 0.082 & 18.0090 \\
\hline 398.15 & 11.018 & 11.008 & -0.089 & 3.4390 & 3.4421 & 0.090 & 16.4507 \\
\hline 423.15 & 11.885 & 11.873 & -0.103 & 3.4390 & 3.4425 & 0.103 & 15.1450 \\
\hline 273.15 & 6.824 & 6.821 & -0.043 & 3.5620 & 3.5638 & 0.050 & 28.3923 \\
\hline 285.65 & 7.290 & 7.286 & -0.055 & 3.5620 & 3.5642 & 0.063 & 26.2901 \\
\hline 298.15 & 7.754 & 7.749 & -0.063 & 3.5620 & 3.5645 & 0.070 & 24.4847 \\
\hline 323.15 & 8.674 & 8.669 & -0.062 & 3.5620 & 3.5644 & 0.067 & 21.5420 \\
\hline 348 . & 9.590 & 9.583 & -0.072 & 3.5620 & 3.5647 & 0.076 & 19.2414 \\
\hline 373. & 10.501 & 10.493 & -0.079 & 3.5620 & 3.5649 & 0.081 & 17.3932 \\
\hline 398.15 & 11.408 & 11.398 & -0.088 & 3.5620 & 3.5652 & 0.089 & 15.8748 \\
\hline
\end{tabular}


Data from Trappeniers et al. [45] (continued)

\begin{tabular}{|c|c|c|c|c|c|c|c|}
\hline $\mathrm{T}$ & $P, \exp$ & P,cal & Dev & $\rho, \exp$ & $\rho$, cal & Dev & Wt \\
\hline K & $\mathrm{MPa}$ & $\mathrm{MPa}$ & 8 & $\mathrm{~mol} \cdot \mathrm{dm}^{-3}$ & $\mathrm{~mol} \cdot \mathrm{dm}^{-3}$ & 8 & \\
\hline 423.15 & 12.313 & 12.300 & -0.105 & 3.5620 & 3.5657 & 0.104 & 14.6042 \\
\hline 273.15 & 7.300 & 7.298 & -0.027 & 3.8560 & 3.8572 & 0.031 & 26.6178 \\
\hline 285.65 & 7.813 & 7.810 & -0.039 & 3.8560 & 3.8577 & 0.044 & 24.5797 \\
\hline 298.15 & 8.323 & 8.319 & -0.045 & 3.8560 & 3.8579 & 0.050 & 22.8378 \\
\hline 323.15 & 9.336 & 9.332 & -0.047 & 3.8560 & 3.8579 & 0.050 & 20.0151 \\
\hline 348.15 & 10.343 & 10.337 & -0.055 & 3.8560 & 3.8582 & 0.058 & 17.8234 \\
\hline 373.15 & 11.346 & 11.338 & -0.075 & 3.8560 & 3.8589 & 0.076 & 16.0710 \\
\hline 398.15 & 12.344 & 12.333 & -0.086 & 3.8560 & 3.8593 & 0.087 & 14.6384 \\
\hline 423.15 & 13.338 & 13.325 & -0.101 & 3.8560 & 3.8599 & 0.100 & 13.4444 \\
\hline 273.15 & 7.771 & 7.770 & -0.015 & 4.1520 & 4.1527 & 0.018 & 25.0653 \\
\hline 285.65 & 8.333 & 8.330 & -0.032 & 4.1520 & 4.1535 & 0.037 & 23.0816 \\
\hline 298.15 & 8.891 & 8.888 & -0.042 & 4.1520 & 4.1540 & 0.047 & 21.3945 \\
\hline 348.15 & 11.102 & 11.096 & -0.059 & 4.1520 & 4.1546 & 0.062 & 16.5816 \\
\hline 323.15 & 10.000 & 9.996 & -0.048 & 4.1520 & 4.1541 & 0.052 & 18.6773 \\
\hline 373.15 & 12.200 & 12.190 & -0.078 & 4.1520 & 4.1553 & 0.080 & 14.9151 \\
\hline 98.15 & 13.292 & 13.279 & -0.095 & 4.1520 & 4.1560 & 0.095 & 13.5583 \\
\hline 23.15 & 14.381 & 14.363 & -0.119 & 4.1520 & 4.1568 & 0.117 & 12.4314 \\
\hline 273 & 8.192 & 8.192 & 0.004 & 4.4210 & 4.4208 & -0.004 & 23.8185 \\
\hline 285 & 8.799 & 8.798 & -0.011 & 4.4210 & 4.4216 & 0.013 & 21.8790 \\
\hline 298.15 & 9.401 & 9.400 & -0.018 & 4.4210 & 4.4219 & 0.020 & 20.2367 \\
\hline 323.15 & 10.600 & 10.597 & -0.030 & 4.4210 & 4.4224 & 0.033 & 17.6040 \\
\hline 348.15 & 11.790 & 11.785 & -0.043 & 4.4210 & 4.4230 & 0.045 & 15.5862 \\
\hline 373.15 & 12.975 & 12.967 & -0.061 & 4.4210 & 4.4237 & 0.062 & 13.9894 \\
\hline 398.15 & 14.155 & 14.143 & -0.082 & 4.4210 & 4.4246 & 0.082 & 12.6941 \\
\hline 423.15 & 15.328 & 15.315 & -0.088 & 4.4210 & 4.4248 & 0.086 & 11.6229 \\
\hline 273.15 & 8.240 & 8.241 & 0.008 & 4.4520 & 4.4516 & -0.010 & 23.6838 \\
\hline 285 & 8.852 & 8.851 & -0.008 & 4.4520 & 4.4524 & 0.010 & 21.7487 \\
\hline 298.15 & 9.461 & 9.459 & -0.020 & 4.4520 & 4.4530 & 0.022 & 20.1106 \\
\hline 323.15 & 10.669 & 10.666 & -0.030 & 4.4520 & 4.4534 & 0.032 & 17.4876 \\
\hline 348.15 & 11.870 & 11.865 & -0.045 & 4.4520 & 4.4541 & 0.047 & 15.4781 \\
\hline 373.15 & 13.066 & 13.057 & -0.068 & 4.4520 & 4.4550 & 0.068 & 13.8887 \\
\hline 398 & 14.256 & 14.243 & -0.085 & 4.4520 & 4.4558 & 0.085 & 12.6003 \\
\hline 423.15 & 15.441 & 15.425 & -0.103 & 4.4520 & 4.4565 & 0.101 & 11.5344 \\
\hline 273.15 & 8.714 & 8.715 & 0.017 & 4.7590 & 4.7581 & -0.020 & 22.4271 \\
\hline 285.65 & 9.378 & 9.378 & 0.002 & 4.7590 & 4.7589 & -0.002 & 20.5363 \\
\hline
\end{tabular}


Data from Trappeniers et al. [45] (continued)

\begin{tabular}{|c|c|c|c|c|c|c|c|}
\hline $\mathrm{T}$ & $P, \exp$ & P,cal & Dev & $\rho, \exp$ & $\rho$, cal & Dev & Wt \\
\hline K & $\mathrm{MPa}$ & $\mathrm{MPa}$ & 8 & $\mathrm{~mol} \cdot \mathrm{dm}^{-3}$ & $\mathrm{~mol} \cdot \mathrm{dm}^{-3}$ & 8 & \\
\hline 298.15 & 10.040 & 10.039 & -0.015 & 4.7590 & 4.7598 & 0.017 & 18.9427 \\
\hline 323.15 & 11.354 & 11.351 & -0.027 & 4.7590 & 4.7604 & 0.029 & 16.4069 \\
\hline 348.15 & 12.660 & 12.654 & -0.047 & 4.7590 & 4.7613 & 0.049 & 14.4768 \\
\hline 373.15 & 13.958 & 13.949 & -0.068 & 4.7590 & 4.7623 & 0.069 & 12.9588 \\
\hline 398.15 & 15.252 & 15.238 & -0.095 & 4.7590 & 4.7634 & 0.093 & 11.7330 \\
\hline 423.15 & 16.540 & 16.521 & -0.113 & 4.7590 & 4.7642 & 0.110 & 10.7229 \\
\hline 273.15 & 8.740 & 8.743 & 0.029 & 4.7770 & 4.7753 & -0.035 & 22.3593 \\
\hline 285.65 & 9.408 & 9.409 & 0.009 & 4.7770 & 4.7765 & -0.010 & 20.4701 \\
\hline 298.15 & 10.073 & 10.073 & -0.003 & 4.7770 & 4.7772 & 0.004 & 18.8794 \\
\hline 323.15 & 11.393 & 11.391 & -0.018 & 4.7770 & 4.7779 & 0.019 & 16.3481 \\
\hline 348.15 & 12.705 & 12.700 & -0.036 & 4.7770 & 4.7788 & 0.037 & 14.4225 \\
\hline 373.15 & 14.008 & 14.001 & -0.050 & 4.7770 & 4.7794 & 0.051 & 12.9087 \\
\hline 398.15 & 15.307 & 15.296 & -0.071 & 4.7770 & 4.7803 & 0.070 & 11.6866 \\
\hline 423.15 & 16.600 & 16.586 & -0.088 & 4.7770 & 4.7811 & 0.085 & 10.6796 \\
\hline 273.15 & 9.136 & 9.141 & 0.046 & 5.0380 & 5.0352 & -0.056 & 21.4016 \\
\hline 285.65 & 9.851 & 9.854 & 0.025 & 5.0380 & 5.0366 & -0.028 & 19.5460 \\
\hline 298.15 & 10.562 & 10.563 & 0.013 & 5.0380 & 5.0373 & -0.014 & 17.9899 \\
\hline 323.15 & 11.974 & 11.973 & -0.009 & 5.0380 & 5.0385 & 0.009 & 15.5254 \\
\hline 348.15 & 13.377 & 13.373 & -0.033 & 5.0380 & 5.0397 & 0.034 & 13.6613 \\
\hline 373.15 & 14.773 & 14.765 & -0.059 & 5.0380 & 5.0410 & 0.059 & 12.2019 \\
\hline 398.15 & 16.162 & 16.149 & -0.082 & 5.0380 & 5.0420 & 0.080 & 11.0285 \\
\hline 423.15 & 17.546 & 17.528 & -0.104 & 5.0380 & 5.0430 & 0.100 & 10.0644 \\
\hline 273.15 & 9.282 & 9.286 & 0.039 & 5.1340 & 5.1316 & .0 .046 & 21.0679 \\
\hline 285.65 & 10.015 & 10.017 & 0.016 & 5.1340 & 5.1331 & -0.018 & 19.2241 \\
\hline 298.15 & 10.743 & 10.743 & 0.002 & 5.1340 & 5.1339 & -0.003 & 17.6802 \\
\hline 323.15 & 12.189 & 12.187 & -0.018 & 5.1340 & 5.1350 & 0.019 & 15.2397 \\
\hline 348.15 & 13.627 & 13.621 & -0.043 & 5.1340 & 5.1362 & 0.044 & 13.3974 \\
\hline 373.15 & 15.056 & 15.046 & -0.062 & 5.1340 & 5.1372 & 0.062 & 11.9578 \\
\hline 398.15 & 16.478 & 16.465 & -0.080 & 5.1340 & 5.1380 & 0.078 & 10.8018 \\
\hline 423.15 & 17.894 & 17.877 & -0.097 & 5.1340 & 5.1388 & 0.093 & 9.8529 \\
\hline 273.15 & 9.832 & 9.839 & 0.072 & 5.5020 & 5.4973 & -0.086 & 19.8837 \\
\hline 285.65 & 10.633 & 10.638 & 0.047 & 5.5020 & 5.4990 & -0.054 & 18.0820 \\
\hline 298.15 & 11.428 & 11.432 & 0.034 & 5.5020 & 5.4999 & -0.038 & 16.5821 \\
\hline 323.15 & 13.009 & 13.010 & 0.009 & 5.5020 & 5.5015 & -0.009 & 14.2269 \\
\hline 348.15 & 14.580 & 14.577 & -0.022 & 5.5020 & 5.5032 & 0.022 & 12.4625 \\
\hline
\end{tabular}


Data from Trappeniers et al. [45] (continued)

\begin{tabular}{|c|c|c|c|c|c|c|c|}
\hline $\mathrm{T}$ & $P, \exp$ & P, cal & Dev & $\rho, \exp$ & $\rho, \mathrm{cal}$ & Dev & Wt \\
\hline K & $\mathrm{MPa}$ & $\mathrm{MPa}$ & 8 & $\mathrm{~mol} \cdot \mathrm{dm}^{-3}$ & $\mathrm{~mol} \cdot \mathrm{dm}^{-3}$ & 8 & \\
\hline 373.15 & 16.142 & 16.135 & -0.045 & 5.5020 & 5.5044 & 0.044 & 11.0923 \\
\hline 398.15 & 17.696 & 17.684 & -0.065 & 5.5020 & 5.5055 & 0.063 & 9.9975 \\
\hline 423.15 & 19.244 & 19.227 & -0.087 & 5.5020 & 5.5065 & 0.082 & 9.1023 \\
\hline 273.15 & 10.364 & 10.373 & 0.084 & 5.8600 & 5.8541 & -0.100 & 18.8412 \\
\hline 285.65 & 11.233 & 11.239 & 0.059 & 5.8600 & 5.8561 & -0.067 & 17.0782 \\
\hline 298.15 & 12.096 & 12.101 & 0.042 & 5.8600 & 5.8573 & -0.046 & 15.6179 \\
\hline 323.15 & 13.813 & 13.814 & 0.012 & 5.8600 & 5.8593 & -0.013 & 13.3403 \\
\hline 348.15 & 15.519 & 15.515 & -0.023 & 5.8600 & 5.8613 & 0.023 & 11.6465 \\
\hline 373.15 & 17.215 & 17.206 & -0.048 & 5.8600 & 5.8628 & 0.047 & 10.3388 \\
\hline 398.15 & 18.902 & 18.888 & -0.071 & 5.8600 & 5.8640 & 0.068 & 9.2987 \\
\hline 423.15 & 20.583 & 20.563 & -0.099 & 5.8600 & 5.8654 & 0.093 & 8.4514 \\
\hline 273.15 & 10.861 & 10.873 & 0.116 & 6.1980 & 6.1895 & -0.137 & 17.9411 \\
\hline 285.65 & 11.796 & 11.806 & 0.087 & 6.1980 & 6.1919 & -0.099 & 16.2124 \\
\hline 298.15 & 12.725 & 12.734 & 0.071 & 6.1980 & 6.7 & -0.077 & 14.7880 \\
\hline 323.15 & 14.574 & 14.579 & 0.036 & 6.1980 & 6.1957 & -0.037 & 12.5795 \\
\hline 348.15 & 16.412 & 16.411 & -0.005 & 6.1980 & 6.1983 & 0.005 & 10.9481 \\
\hline 373.15 & 18.237 & 18.232 & -0.029 & 6.1980 & 6.1998 & 0.028 & 9.6957 \\
\hline 398.15 & 20.053 & 20.043 & -0.051 & 6.1980 & 6.2010 & 0.048 & 8.7037 \\
\hline 423.15 & 21.864 & 21.846 & -0.080 & 6.1980 & 6.2026 & 0.074 & 7.8983 \\
\hline 273.15 & 10.993 & 11.005 & 0.108 & 6.2870 & 6.2790 & -0.128 & 17.7137 \\
\hline 85.65 & 11.945 & 11. & 0.090 & 6. & & -0 . & 15.9951 \\
\hline 298.15 & 12.892 & 12.901 & 0.072 & 6.2 & 6. & -0 & 14.5800 \\
\hline 323.15 & 14.777 & 14.782 & 0.034 & 6.2870 & 6.2848 & -0.035 & 12.3892 \\
\hline 348.15 & 16.649 & 16.649 & -0.005 & 6.2870 & 6.2873 & 0.005 & 10.7740 \\
\hline 373.15 & 18.511 & 18.504 & -0.035 & 6.2870 & 6.2891 & 0.034 & 9.5353 \\
\hline 398.15 & 20.361 & 20.351 & -0.054 & 6. & & 0. & 8.5557 \\
\hline 423.15 & 22.205 & 22.188 & -0.076 & 6. & 6.2 & 0.070 & 7.7611 \\
\hline 273.15 & 11.358 & 11.371 & 0.122 & 6.5350 & 6.5256 & -0.144 & 17.1058 \\
\hline 285.65 & 12.360 & 12.372 & 0.097 & 6.5350 & 6.5279 & -0.109 & 15.4115 \\
\hline 298.15 & 13.358 & 13.368 & 0.076 & 6.5350 & 6.5296 & -0.082 & 14.0217 \\
\hline 323.15 & 15.343 & 15.349 & 0.038 & 6.5350 & 6.5324 & -0.039 & 11.8801 \\
\hline 348.15 & 17.315 & 17.315 & 0.004 & 6.5350 & 6.5348 & -0.003 & 10.3089 \\
\hline 373.15 & 19.276 & 19.270 & -0.032 & 6.5350 & 6.5370 & 0.030 & 9.1081 \\
\hline 398.15 & 21.227 & 21.215 & -0.061 & 6.5350 & 6.5387 & 0.056 & 8.1611 \\
\hline 423.15 & 23.170 & 23.150 & -0.084 & 6.5350 & 6.5400 & 0.077 & 7. 3951 \\
\hline
\end{tabular}


Data from Trappeniers et al. [45] (continued)

\begin{tabular}{|c|c|c|c|c|c|c|c|}
\hline $\mathrm{T}$ & $P, \exp$ & P,cal & Dev & $\rho, \exp$ & $\rho, c a l$ & Dev & Wt \\
\hline K & $\mathrm{MPa}$ & $\mathrm{MPa}$ & 8 & $\mathrm{~mol} \cdot \mathrm{dm}^{-3}$ & $\mathrm{~mol} \cdot \mathrm{dm}^{-3}$ & 8 & \\
\hline 273.15 & 11.738 & 11.753 & 0.128 & 6.7930 & 6.7829 & -0.149 & 16.5030 \\
\hline 285.65 & 12.793 & 12.807 & 0.108 & 6.7930 & 6.7848 & -0.120 & 14.8353 \\
\hline 298.15 & 13.846 & 13.857 & 0.081 & 6.7930 & 6.7871 & -0.087 & 13.4715 \\
\hline 323.15 & 15.937 & 15.944 & 0.044 & 6.7930 & 6.7900 & -0.044 & 11.3799 \\
\hline 348.15 & 18.017 & 18.017 & 0.000 & 6.7930 & 6.7930 & 0.000 & 9.8526 \\
\hline 373.15 & 20.084 & 20.077 & -0.035 & 6.7930 & 6.7952 & 0.032 & 8.6902 \\
\hline 398.15 & 22.139 & 22.126 & -0.058 & 6.7930 & 6.7966 & 0.053 & 7.7764 \\
\hline 423.15 & 24.186 & 24.166 & -0.081 & 6.7930 & 6.7979 & 0.072 & 7.0389 \\
\hline 273.15 & 11.839 & 11.855 & 0.133 & 6.8620 & 6.8514 & -0.155 & 16.3469 \\
\hline 285.65 & 12.911 & 12.924 & 0.099 & 6.8620 & 6.8544 & -0.111 & 14.6850 \\
\hline 298.15 & 13.977 & 13.988 & 0.081 & 6.8620 & 6.8561 & -0.086 & 13.3289 \\
\hline 323.15 & 16.097 & 16.104 & 0.044 & 6.8620 & 6.8590 & -0.044 & 11.2506 \\
\hline 348.15 & 18.205 & 18.206 & 0.006 & 6.8620 & 6.8616 & -0.006 & 9.7353 \\
\hline 373.15 & 20.300 & 20.295 & -0.028 & 6.8620 & 6.8638 & 0.027 & 8.5829 \\
\hline 398.15 & 22.385 & 22.372 & -0.056 & 6.8620 & 6.8655 & 0.051 & 7.6776 \\
\hline 423.15 & 24.462 & 24.441 & -0.087 & 6.8620 & 6.8673 & 0.078 & 6.9473 \\
\hline 273.15 & 12.489 & 12.508 & 0.147 & 7.3020 & 7.2897 & -0.168 & 15.3909 \\
\hline 285.65 & 13.654 & 13.672 & 0.133 & 7.3020 & 7.2914 & -0.145 & 13.7754 \\
\hline 298.15 & 14.816 & 14.831 & 0.105 & 7.3020 & 7.2939 & -0.111 & 12.4635 \\
\hline 323.15 & 17.126 & 17.137 & 0.062 & 7.3020 & 7.2975 & -0.061 & 10.4685 \\
\hline 348.15 & 19.424 & 19.427 & 0.017 & 7.3020 & 7.3008 & -0.016 & 9.0257 \\
\hline 373.15 & 21.761 & 21.703 & -0.265 & 7.3 & 7.3 & 0.243 & 7.9266 \\
\hline 398.15 & 23.976 & 23.967 & -0.034 & 7.3020 & 7.3042 & 0.031 & 7.0837 \\
\hline 423.15 & 26.237 & 26.221 & -0.062 & 7.3020 & 7.3060 & 0.055 & 6.3990 \\
\hline 273.15 & 13.271 & 13.293 & 0.167 & 7.8250 & 7.8104 & -0.187 & 14.3315 \\
\hline 285.65 & 14.554 & 14.575 & 0.146 & 7.8250 & 7.8128 & -0.155 & 12.7714 \\
\hline 298.15 & 15.833 & 15.853 & 0.127 & 7.8250 & 7.8148 & -0.130 & 11.5144 \\
\hline 323.15 & 18.380 & 18.395 & 0.081 & 7.8250 & 7.8189 & -0.078 & 9.6183 \\
\hline 348.15 & 20.912 & 20.919 & 0.034 & 7.8250 & 7.8226 & -0.031 & 8.2597 \\
\hline 373.15 & 23.431 & 23.429 & -0.008 & 7.8250 & 7.8256 & 0.007 & 7.2401 \\
\hline 398.15 & 25.931 & 25.926 & -0.022 & 7.8250 & 7.8265 & 0.019 & 6.4480 \\
\hline 423.15 & 28.422 & 28.410 & -0.041 & 7.8250 & 7.8277 & 0.035 & 5.8143 \\
\hline 273.15 & 14.050 & 14.073 & 0.166 & 8.3350 & 8.3200 & -0.180 & 13.3587 \\
\hline 285.65 & 15.454 & 15.477 & 0.151 & 8.3350 & 8.3220 & -0.157 & 11.8574 \\
\hline 298.15 & 16.855 & 16.876 & 0.128 & 8.3350 & 8.3244 & -0.128 & 10.6554 \\
\hline 323.15 & 19.643 & 19.660 & 0.087 & 8.3350 & 8.3282 & -0.081 & 8.8572 \\
\hline
\end{tabular}


Data from Trappeniers et al. [45] (continued)

\begin{tabular}{|c|c|c|c|c|c|c|c|}
\hline $\mathrm{T}$ & $P, \exp$ & $\mathrm{P}, \mathrm{cal}$ & Dev & $\rho, \exp$ & $\rho, c a l$ & Dev & Wt \\
\hline K & $\mathrm{MPa}$ & $\mathrm{MPa}$ & 8 & $\mathrm{~mol} \cdot \mathrm{dm}^{-3}$ & $\mathrm{~mol} \cdot \mathrm{dm}^{-3}$ & 8 & \\
\hline 348.15 & 22.417 & 22.426 & 0.038 & 8.3350 & 8.3321 & -0.034 & 7.5793 \\
\hline 373.15 & 25.176 & 25.176 & -0.003 & 8.3350 & 8.3352 & 0.003 & 6.6265 \\
\hline 398.15 & 27.916 & 27.910 & -0.020 & 8.3350 & 8.3364 & 0.017 & 5.8896 \\
\hline 423.15 & 30.642 & 30.632 & -0.036 & 8.3350 & 8.3375 & 0.029 & 5.3025 \\
\hline 273.15 & 14.802 & 14.826 & 0.159 & 8.8140 & 8.7992 & -0.168 & 12.4877 \\
\hline 285.65 & 16.326 & 16.350 & 0.146 & 8.8140 & 8.8011 & -0.146 & 11.0456 \\
\hline 298.15 & 17.847 & 17.869 & 0.122 & 8.8140 & 8.8036 & -0.118 & 9.8981 \\
\hline 323.15 & 20.874 & 20.891 & 0.083 & 8.8140 & 8.8073 & -0.075 & 8.1934 \\
\hline 348.15 & 23.887 & 23.895 & 0.034 & 8.8140 & 8.8114 & -0.030 & 6.9909 \\
\hline 373.15 & 26.882 & 26.881 & -0.003 & 8.8140 & 8.8142 & 0.003 & 6.0991 \\
\hline 398.15 & 29.860 & 29.851 & -0.029 & 8.8140 & 8.8161 & 0.024 & 5.4119 \\
\hline 423.15 & 32.823 & 32.806 & -0.052 & 8.8140 & 8.8177 & 0.042 & 4.8661 \\
\hline 273.15 & 15.535 & 15.560 & 0.166 & 9.2670 & 9.2514 & -0.169 & 11.6951 \\
\hline 285.65 & 17.177 & 17.203 & 0.153 & 9.2670 & 9.2533 & -0.148 & 10.3134 \\
\hline 298.15 & 18.816 & 18.841 & 0.130 & 9.2670 & 9.2558 & -0.121 & 9.2199 \\
\hline 323.15 & 22.079 & 22.100 & 0.097 & 9.2670 & 9.2591 & -0.085 & 7.6057 \\
\hline 348.15 & 25.327 & 25.340 & 0.053 & 9.2670 & 9.2629 & -0.045 & 6.4741 \\
\hline 373.15 & 28.558 & 28.561 & 0.010 & 9.2670 & 9.2662 & -0.008 & 5.6385 \\
\hline 398.15 & 31.773 & 31.763 & -0.030 & 9.2670 & 9.2692 & 0.024 & 4.9965 \\
\hline 423.15 & 34.971 & 34.950 & -0.062 & 9.2670 & 9.2715 & 0.048 & 4.4881 \\
\hline 273.15 & 15.582 & 15.607 & 0.156 & 9.2950 & 9.2803 & -0.158 & 11.6463 \\
\hline 285.65 & 17.232 & 17.257 & 0.146 & 9.2950 & 9.2819 & -0.141 & 10.2687 \\
\hline 298.15 & 18.877 & 18.902 & 0.130 & 9.2950 & 9.2838 & -0.120 & 9.1791 \\
\hline 323.15 & 22.156 & 22.177 & 0.093 & 9.2950 & 9.2874 & -0.081 & 7.5704 \\
\hline 348.15 & 25.419 & 25.431 & 0.049 & 9.2950 & 9.2912 & -0.041 & 6.4432 \\
\hline 373.15 & 28.663 & 28.667 & 0.012 & 9.2950 & 9.2941 & -0.010 & 5.6111 \\
\hline 398.15 & 31.891 & 31.884 & -0.022 & 9.2950 & 9.2966 & 0.017 & 4.9721 \\
\hline 423.15 & 35.102 & 35.085 & -0.047 & 9.2950 & 9.2984 & 0.036 & 2.9773 \\
\hline 273.15 & 16.369 & 16.392 & 0.136 & 9.7600 & 9.7471 & -0.132 & 10.8579 \\
\hline 285.65 & 18.147 & 18.170 & 0.125 & 9.7600 & 9.7487 & -0.116 & 9.5477 \\
\hline 298.15 & 19.921 & 19.943 & 0.112 & 9.7600 & 9.7502 & -0.100 & 8.5169 \\
\hline 323.15 & 23.455 & 23.474 & 0.080 & 9.7600 & 9.7534 & -0.068 & 7.0035 \\
\hline 348.15 & 26.973 & 26.983 & 0.039 & 9.7600 & 9.7569 & -0.031 & 5.9489 \\
\hline 373.15 & 30.470 & 30.471 & 0.002 & 9.7600 & 9.7599 & -0.001 & 5.1735 \\
\hline 398.15 & 33.950 & 33.939 & -0.031 & 9.7600 & 9.7623 & 0.024 & 4.5797 \\
\hline
\end{tabular}


Data from Trappeniers et al. [45] (continued)

\begin{tabular}{|c|c|c|c|c|c|c|c|}
\hline $\mathrm{T}$ & $P, \exp$ & P,cal & Dev & $\rho, \exp$ & $\rho, \mathrm{cal}$ & Dev & Wt \\
\hline K & $\mathrm{MPa}$ & $\mathrm{MPa}$ & 8 & $\mathrm{~mol} \cdot \mathrm{dm}^{-3}$ & $\mathrm{~mol} \cdot \mathrm{dm}^{-3}$ & 8 & \\
\hline 423.15 & 37.408 & 37.390 & -0.048 & 9.7600 & 9.7635 & 0.036 & 2.7403 \\
\hline 273.15 & 16.812 & 16.833 & 0.130 & 10.0130 & 10.0007 & -0.123 & 10.4388 \\
\hline 285.65 & 18.661 & 18.684 & 0.123 & 10.0130 & 10.0018 & -0.112 & 9.1675 \\
\hline 298.15 & 20.509 & 20.530 & 0.105 & 10.0130 & 10.0038 & -0.092 & 8.1695 \\
\hline 323.15 & 24.187 & 24.205 & 0.074 & 10.0130 & 10.0069 & -0.061 & 6.7088 \\
\hline 348.15 & 27.848 & 27.858 & 0.035 & 10.0130 & 10.0103 & -0.027 & 5.6936 \\
\hline 373.15 & 31.487 & 31.489 & 0.005 & 10.0130 & 10.0126 & -0.004 & 4.9487 \\
\hline 398.15 & 35.111 & 35.099 & -0.035 & 10.0130 & 10.0157 & 0.027 & 2.9191 \\
\hline 423.15 & 38.713 & 38.690 & -0.058 & 10.0130 & 10.0173 & 0.043 & 2.6191 \\
\hline 273.15 & 18.199 & 18.218 & 0.102 & 10.7630 & 10.7534 & -0.089 & 9.2324 \\
\hline 285.65 & 20.275 & 20.295 & 0.102 & 10. & 10. & -0.086 & 8.0848 \\
\hline 298.15 & 22.347 & 22.368 & 0.091 & 10.7630 & 10.7550 & -0.074 & 7.1896 \\
\hline 323.15 & 26.479 & 26.495 & 0.060 & 10.7630 & 10.7580 & -0.047 & 5.8874 \\
\hline 348.15 & 30.586 & 30.596 & 0.032 & 10.7630 & 10.7604 & -0.024 & 4.988 \\
\hline 373.15 & 34.672 & 34.672 & -0.003 & 10.7630 & 10.7632 & 0.002 & 4.330 \\
\hline 398.15 & 38.736 & 38.723 & -0.0 & 10 & 10. & & 2.5528 \\
\hline 423.15 & 42.777 & 42.754 & -0.054 & 10. & 10. & 38 & 2.2893 \\
\hline 273.15 & 19.771 & 19.785 & 0.075 & 11.5340 & 11.5271 & -0.060 & 8.0511 \\
\hline 285.65 & 22.098 & 22.116 & 0.080 & 11.5340 & 11.5269 & -0.062 & 7.0412 \\
\hline 298.15 & 24.423 & 24.441 & 0.077 & 11.5340 & 11.5274 & -0.057 & 6.256 \\
\hline 323 & $29 . C$ & 29. & 0.0 & 11. & 11. & -0 . & 5.119 \\
\hline 348 & 33.6 & 33.671 & 0.0 & 11.5340 & 11.5316 & -0.021 & 4.3363 \\
\hline 373.15 & 38.241 & 38.242 & 0.001 & 11.5340 & 11.5339 & -0.001 & 2.509 \\
\hline 398.15 & 42.799 & 42.784 & -0.034 & 11.5340 & 11.5367 & 0.023 & 2.219 \\
\hline 423.15 & 47.325 & 47.301 & -0.051 & 11.5340 & 11.5379 & 0.034 & 1.990 \\
\hline 273.15 & 21.491 & 21.498 & 0.031 & 12.2870 & 12.2843 & -0.022 & 6.964 \\
\hline 285.65 & 24.088 & 24.097 & 0.040 & 12.2870 & 12.2835 & -0.028 & 6.093 \\
\hline 298.15 & 26.680 & 26.691 & 0.042 & 12.2870 & 12.2834 & -0.029 & 5.418 \\
\hline 323.15 & 31.837 & 31.853 & 0.051 & 12.2870 & 12.2829 & -0.034 & 4.439 \\
\hline 348.15 & 36.971 & 36.981 & 0.027 & 12.2870 & 12.2848 & -0.018 & 2.510 \\
\hline 373.15 & 42.073 & 42.074 & 0.001 & 12.2870 & 12.2869 & -0.001 & 2.181 \\
\hline 398.15 & 47.151 & 47.134 & -0.036 & 12.2870 & 12.2898 & 0.023 & 1.931 \\
\hline 423.15 & 52.196 & 52.165 & -0.060 & 12.2870 & 12.2916 & 0.038 & 1.733 \\
\hline 273.15 & 23.308 & 23.310 & 0.010 & 12.9940 & 12.9931 & -0.007 & 6.017 \\
\hline 285.65 & 26.178 & 26.184 & 0.022 & 12.9940 & 12.9922 & -0.014 & 5.276 \\
\hline
\end{tabular}


Data from Trappeniers et al. [45] (continued)

\begin{tabular}{|c|c|c|c|c|c|c|c|}
\hline $\mathrm{T}$ & $P, \exp$ & $\mathrm{P}, \mathrm{cal}$ & Dev & $\rho, \exp$ & $\rho, \mathrm{cal}$ & Dev & Wt \\
\hline K & $\mathrm{MPa}$ & $\mathrm{MPa}$ & 8 & $\mathrm{~mol} \cdot \mathrm{dm}^{-3}$ & $\mathrm{~mol} \cdot \mathrm{dm}^{-3}$ & 8 & \\
\hline 298.15 & 29.043 & 29.050 & 0.023 & 12.9940 & 12.9921 & -0.015 & 4.7004 \\
\hline 323.15 & 34.746 & 34.753 & 0.021 & 12.9940 & 12.9923 & -0.013 & 3.8634 \\
\hline 348.15 & 40.417 & 40.415 & -0.006 & 12.9940 & 12.9945 & 0.003 & 2.1897 \\
\hline 373.15 & 46.053 & 46.036 & -0.036 & 12.9940 & 12.9969 & 0.022 & 1.9068 \\
\hline 398.15 & 51.648 & 51.620 & -0.056 & 12.9940 & 12.9983 & 0.033 & 1.6903 \\
\hline 423.15 & 57.210 & 57.170 & -0.069 & 12.9940 & 12.9994 & 0.041 & 1.5191 \\
\hline 273.15 & 24.678 & 24.677 & -0.004 & 13.4740 & 13.4744 & 0.003 & 5.4204 \\
\hline 285.65 & 27.745 & 27.749 & 0.015 & 13.4740 & 13.4728 & -0.009 & 4.7642 \\
\hline 298.15 & 30.809 & 30.813 & 0.012 & 13.4740 & 13.4731 & -0.007 & 4.2522 \\
\hline 323.15 & 36.907 & 36.906 & -0.003 & 13.4740 & 13.4742 & 0.002 & 2.3372 \\
\hline 348.15 & 42.958 & 42.953 & -0.011 & 13.4740 & 13.4749 & 0.007 & 1.9917 \\
\hline 373.15 & 48.980 & 48.956 & -0.050 & 13.4740 & 13.4779 & 0.029 & 1.7374 \\
\hline 398.15 & 54.953 & 54.917 & -0.066 & 13.4740 & 13.4791 & 0.038 & 1.5423 \\
\hline 423.15 & 60.890 & 60.842 & -0.080 & 13.4740 & 13.4801 & 0.046 & 1.3877 \\
\hline 273.15 & 25.373 & 25.373 & 0.000 & 13.7030 & 13.7030 & 0.000 & 5.1501 \\
\hline 285.65 & 28.542 & 28.544 & 0.004 & 13.7030 & 13.7027 & -0.002 & 4.5323 \\
\hline 298.15 & 31.704 & 31.705 & 0.004 & 13.7030 & 13.7027 & -0.002 & 4.0498 \\
\hline 323.15 & 37.994 & 37.992 & -0.007 & 13.7030 & 13.7035 & 0.004 & 2.2297 \\
\hline 348.15 & 44.243 & 44.230 & -0.031 & 13.7030 & 13.7054 & 0.018 & 1.9024 \\
\hline 373.15 & 50.449 & 50.421 & -0.056 & 13.7030 & 13.7074 & 0.032 & 1.6612 \\
\hline 398.15 & 56.614 & 56.569 & -0.080 & 13.7030 & 13.7092 & 0.045 & 1.4758 \\
\hline 423.15 & 62.732 & 62.678 & -0.086 & 13.7030 & 13.7096 & 0.048 & 1.3286 \\
\hline 273.15 & 28.274 & 28.265 & -0.029 & 14.5600 & 14.5622 & 0.015 & 4.2232 \\
\hline 285.65 & 31.837 & 31.828 & -0.027 & 14.5600 & 14.5620 & 0.014 & 3.7389 \\
\hline 298.15 & 35.391 & 35.378 & -0.038 & 14.5600 & 14.5629 & 0.020 & 2.2381 \\
\hline 323.15 & 42.458 & 42.431 & -0.063 & 14.5600 & 14.5648 & 0.033 & 1.8624 \\
\hline 348.15 & 49.462 & 49.424 & -0.076 & 14.5600 & 14.5658 & 0.040 & 1.5979 \\
\hline 373.15 & 56.415 & 56.361 & -0.095 & 14.5600 & 14.5672 & 0.050 & 1.4012 \\
\hline 398.15 & 63.315 & 63.248 & -0.106 & 14.5600 & 14.5681 & 0.056 & 1.2489 \\
\hline 423.15 & 70.160 & 70.090 & -0.099 & 14.5600 & 14.5676 & 0.052 & 1.1274 \\
\hline 273.15 & 32.762 & 32.750 & -0.038 & 15.6530 & 15.6557 & 0.018 & 3.2389 \\
\hline 285.65 & 36.889 & 36.868 & -0.058 & 15.6530 & 15.6572 & 0.027 & 1.9296 \\
\hline 298.15 & 40.998 & 40.966 & -0.078 & 15.6530 & 15.6587 & 0.036 & 1.7459 \\
\hline 323.15 & 49.160 & 49.102 & -0.118 & 15.6530 & 15.6616 & 0.055 & 1.4698 \\
\hline 348.15 & 57.235 & 57.160 & -0.131 & 15.6530 & 15.6627 & 0.062 & 1.2718 \\
\hline
\end{tabular}


Data from Trappeniers et al. [45] (continued)

\begin{tabular}{|c|c|c|c|c|c|c|c|}
\hline $\mathrm{T}$ & $P, \exp$ & P,cal & Dev & $\rho, \exp$ & $\rho, \mathrm{cal}$ & Dev & Wt \\
\hline $\mathrm{K}$ & $\mathrm{MPa}$ & $\mathrm{MPa}$ & 8 & $\mathrm{~mol} \cdot \mathrm{dm}^{-3}$ & $\mathrm{~mol} \cdot \mathrm{dm}^{-3}$ & 8 & \\
\hline 373.15 & 65.243 & 65.149 & -0.145 & 15.6530 & 15.6638 & 0.069 & 1.1225 \\
\hline 398.15 & 73.175 & 73.076 & -0.135 & 15.6530 & 15.6631 & 0.065 & 1.0055 \\
\hline 423.15 & 81.041 & 80.951 & -0.111 & 15.6530 & 15.6614 & 0.054 & 0.9113 \\
\hline 273.15 & 38.578 & 38.545 & -0.086 & 16.7770 & 16.7828 & 0.034 & 1.6288 \\
\hline 285.65 & 43.355 & 43.305 & -0.114 & 16.7770 & 16.7847 & 0.046 & 1.4711 \\
\hline 298.15 & 48.110 & 48.037 & -0.151 & 16.7770 & 16.7874 & 0.062 & 1.3427 \\
\hline 323.15 & 57.533 & 57.421 & -0.196 & 16.7770 & 16.7908 & 0.082 & 1.1455 \\
\hline 348.15 & 66.849 & 66.705 & -0.214 & 16.7770 & 16.7924 & 0.092 & 1.0008 \\
\hline 373.15 & 76.067 & 75.905 & -0.213 & 16.7770 & 16.7925 & 0.092 & 0.8897 \\
\hline 398.15 & 85.185 & 85.031 & -0.180 & 16.7770 & 16.7902 & 0.079 & 0.8016 \\
\hline 423.15 & 94.210 & 94.096 & -0.121 & 16.7770 & 16.7860 & 0.053 & 0.7299 \\
\hline 273.15 & 39.645 & 39.600 & -0.112 & 16.9560 & 16.9634 & 0.044 & 1.5566 \\
\hline 285.65 & 44.532 & 44.470 & -0.139 & 16.9560 & 16.9653 & 0.055 & 1.4083 \\
\hline 298.15 & 49.396 & 49.310 & -0.175 & 16.9560 & 16.9679 & 0.070 & 1.2871 \\
\hline 323.15 & 59.033 & 58.905 & -0.217 & 16.9560 & 16.9712 & 0.090 & 1.1005 \\
\hline 348.15 & 68.564 & 68.398 & -0.241 & 16.9560 & 16.9732 & 0.102 & 0.9630 \\
\hline 373.15 & 77.991 & 77.804 & -0.240 & 16.9560 & 16.9734 & 0.103 & 0.8572 \\
\hline 398.15 & 87.304 & 87.134 & -0.195 & 16.9560 & 16.9702 & 0.084 & 0.7730 \\
\hline 273.15 & 45.790 & 45.689 & -0.221 & 17.8750 & 17.8889 & 0.078 & 1.2322 \\
\hline 285.65 & 51.285 & 51.151 & -0.261 & 17.8750 & 17.8918 & 0.094 & 1.1246 \\
\hline 298.15 & 56.750 & 56.577 & -0.305 & 17.8750 & 17.8950 & 0.112 & 1.0351 \\
\hline 323.15 & 67.554 & 67.324 & -0.340 & 17.8750 & 17.8981 & 0.129 & 0.8949 \\
\hline 348.15 & 78.223 & 77.951 & -0.349 & 17.8750 & 17.8993 & 0.136 & 0.7895 \\
\hline 373.15 & 88.749 & 88.476 & -0.308 & 17.8750 & 17.8968 & 0.122 & 0.7071 \\
\hline 398.15 & 99.153 & 98.916 & -0.239 & 17.8750 & 17.8921 & 0.096 & 0.6408 \\
\hline 423.15 & 109.438 & 109.287 & -0.139 & 17.8750 & 17.8851 & 0.056 & 0.5863 \\
\hline 273.15 & 52.050 & 51.879 & -0.329 & 18.6570 & 18.6769 & 0.107 & 1.0103 \\
\hline 285.65 & 58.119 & 57.891 & -0.393 & 18.6570 & 18.6815 & 0.131 & 0.9285 \\
\hline 298.15 & 64.122 & 63.858 & -0.412 & 18.6570 & 18.6833 & 0.141 & 0.8597 \\
\hline 323.15 & 76.008 & 75.673 & -0.441 & 18.6570 & 18.6862 & 0.156 & 0.7500 \\
\hline 348.15 & 87.717 & 87.351 & -0.417 & 18.6570 & 18.6854 & 0.152 & 0.6662 \\
\hline 373.15 & 99.272 & 98.917 & -0.358 & 18.6570 & 18.6819 & 0.133 & 0.5998 \\
\hline 398.15 & 110.653 & 110.390 & -0.238 & 18.6570 & 18.6739 & 0.090 & 0.5459 \\
\hline 273.15 & 54.294 & 54.090 & -0.375 & 18.9080 & 18.9304 & 0.119 & 0.9481 \\
\hline 285.65 & 60.544 & 60.287 & -0.424 & 18.9080 & 18.9342 & 0.138 & 0.8732 \\
\hline
\end{tabular}


Data from Trappeniers et a1. [45] (continued)

\begin{tabular}{|c|c|c|c|c|c|c|c|}
\hline $\mathrm{T}$ & $P, \exp$ & P,cal & Dev & $\rho, \exp$ & $\rho$, cal & Dev & Wt \\
\hline K & $\mathrm{MPa}$ & $\mathrm{MPa}$ & 8 & $\mathrm{~mol} \cdot \mathrm{dm}^{-3}$ & $\mathrm{~mol} \cdot \mathrm{dm}^{-3}$ & 8 & \\
\hline 298.15 & 66.744 & 66.437 & -0.460 & 18.9080 & 18.9371 & 0.154 & 0.8100 \\
\hline 323.15 & 78.994 & 78.613 & -0.482 & 18.9080 & 18.9397 & 0.168 & 0.7086 \\
\hline 348.15 & 91.058 & 90.648 & -0.451 & 18.9080 & 18.9385 & 0.161 & 0.6308 \\
\hline 373.15 & 102.949 & 102.566 & -0.373 & 18.9080 & 18.9338 & 0.136 & 0.5689 \\
\hline 398.15 & 114.685 & 114.388 & -0.259 & 18.9080 & 18.9262 & 0.096 & 0.5184 \\
\hline 423.15 & 126.275 & 126.133 & -0.112 & 18.9080 & 18.9160 & 0.042 & 0.4764 \\
\hline 273.15 & 64.911 & 64.537 & -0.576 & 19.9440 & 19.9773 & 0.167 & 0.7308 \\
\hline 285.65 & 72.005 & 71.549 & -0.633 & 19.9440 & 19.9818 & 0.189 & 0.6788 \\
\hline 298.15 & 79. & 78.505 & -0.649 & 19.9440 & 19.9838 & 0.200 & 0.6341 \\
\hline 323.15 & 92.860 & 92.272 & -0.634 & 19.9440 & 19.9846 & 0.203 & 0.5610 \\
\hline 348 & 106.475 & 105.877 & -0.562 & 19.9440 & 19.9812 & 0.186 & 0.5036 \\
\hline 373.15 & 119.862 & 119.351 & -0.427 & 19.9440 & 19.9729 & 0.145 & 0.4572 \\
\hline 398.15 & 133.045 & 132.721 & -0.244 & 19.9440 & 19.9608 & 0.084 & 0.4189 \\
\hline 423.15 & 146.052 & 146.006 & -0.031 & 19.9440 & 19.9462 & 0.011 & 0.3866 \\
\hline 273.15 & 70.125 & 69.657 & -0.668 & 20.3810 & 20.4191 & 0.187 & 0.6556 \\
\hline 28 & 77.578 & 77.037 & -0.697 & 20.3810 & 20.4221 & 0.202 & 0.6109 \\
\hline 298 & 84.960 & 84.359 & -0.707 & 20.3810 & 20.4239 & 0.210 & 0.5722 \\
\hline 323.15 & 99.515 & 98.848 & -0.670 & 20.3810 & 20.4234 & 0.208 & 0.5085 \\
\hline 348.15 & 113.813 & 113.168 & -0.567 & 20.3810 & 20.4181 & 0.182 & 0.4581 \\
\hline 373.15 & 127.867 & 127.352 & -0.402 & 20.3810 & 20.4080 & 0.133 & 0.4170 \\
\hline 398.15 & 141.711 & 141.428 & -0.200 & 20.3810 & 20.3947 & 0.067 & 0.3829 \\
\hline 273.15 & 95.434 & 94.444 & -1.036 & 22.0790 & 22.1362 & 0.259 & 0.4334 \\
\hline 285.65 & 104.481 & 103.419 & -1.016 & 22.0790 & 22.1369 & 0.262 & 0.4083 \\
\hline 298.15 & 113.423 & 112.323 & -0.970 & 22.0790 & 22.1359 & 0.258 & 0.3861 \\
\hline 323.15 & 131.013 & 129.949 & -0.812 & 22.0790 & 22.1288 & 0.226 & 0.3485 \\
\hline 348.15 & 148.245 & 147.381 & -0.583 & 22.0790 & 22.1160 & 0.168 & 0.3177 \\
\hline 373.15 & 165.122 & 164.662 & -0.279 & 22.0790 & 22.0972 & 0.082 & 0.2920 \\
\hline 398 & 181.755 & 181.825 & 0.038 & 22.0790 & 22.0764 & -0.012 & 0.2702 \\
\hline 273 & 132.872 & 131.161 & -1.288 & 23.8640 & 23.9340 & 0.293 & 0.2851 \\
\hline 285 & 143.821 & 141.692 & -1.481 & 23.8640 & 23.9470 & 0.348 & 0.2715 \\
\hline 298.15 & 154.629 & 153.018 & -1.042 & 23.8640 & 23.9239 & 0.251 & 0.2583 \\
\hline 323.15 & 175.817 & 174.600 & -0.692 & 23.8640 & 23.9056 & 0.174 & 0.2362 \\
\hline 348 & 196.524 & 195.978 & -0.278 & 23.8640 & 23.8813 & 0.072 & 0.2176 \\
\hline 373. & 216.79 & 217.200 & 0.188 & 23.8640 & 23.8520 & -0.050 & 0.2018 \\
\hline 398.15 & 236.696 & 238.305 & 0.680 & 23.8640 & 23.8197 & -0.186 & 0.1881 \\
\hline
\end{tabular}


Data from Trappeniers et al. [45] (continued)

$\begin{array}{crrrrrrr}\text { T } & \text { P,exp } & \text { P,cal } & \text { Dev } & \rho, \exp & \rho, \text { cal } & \text { Dev } & \text { Wt } \\ \text { K } & \mathrm{MPa} & \mathrm{MPa} & \text { o } & \mathrm{mol} \cdot \mathrm{dm}^{-3} & \mathrm{~mol} \cdot \mathrm{dm}^{-3} & 8 & \\ & & & & & & & \\ 273.15 & 183.811 & 181.441 & -1.289 & 25.6140 & 25.6841 & 0.274 & 0.1924 \\ 285.65 & 196.882 & 194.750 & -1.083 & 25.6140 & 25.6745 & 0.236 & 0.1841 \\ 298.15 & 209.721 & 207.981 & -0.830 & 25.6140 & 25.6614 & 0.185 & 0.1766 \\ 323.15 & 234.829 & 234.252 & -0.246 & 25.6140 & 25.6286 & 0.057 & 0.1631 \\ 348.15 & 259.361 & 260.330 & 0.374 & 25.6140 & 25.5911 & -0.089 & 0.1516\end{array}$

Number of Points (Ref.45) 472

$\begin{array}{llrlrlll}\text { PRESSURE : } & \text { AAD }-8 & 0.123 & \text { BIAS }-8 & -0.084 & \text { RMS }-8 & 0.209 & \\ & \text { AAD } & 0.088 & \text { BIAS } & -0.070 & \text { RMS } & 0.284 & \mathrm{MPa} \\ \text { DENSITY : } & \text { AAD }-8 & 0.074 & \text { BIAS }-8 & 0.039 & \text { RMS }-8 & 0.082 & \\ & \text { AAD } & 0.007 & \text { BIAS } & 0.004 & \text { RMS } & 0.013 & \mathrm{mo} 1 \cdot \mathrm{dm}^{-3}\end{array}$

\begin{tabular}{crrrrrrr}
\hline Data from Van Itterbeek et al. [46] & & & \\
T & P,exp & P,cal & Dev & $\rho, \exp$ & $\rho, c a 1$ & Dev & Wt \\
& & & & & & \\
$\mathrm{K}$ & $\mathrm{MPa}$ & $\mathrm{MPa}$ & 8 & $\mathrm{~mol} \cdot \mathrm{dm}^{-3}$ & $\mathrm{~mol} \cdot \mathrm{dm}^{-3}$ & 8 & \\
& & & & & & \\
114.52 & 0.894 & 1.160 & 29.684 & 26.1376 & 26.1213 & -0.062 & 0.0001 \\
114.52 & 4.752 & 4.946 & 4.082 & 26.3609 & 26.3498 & -0.042 & 0.0001 \\
114.52 & 7.542 & 7.638 & 1.275 & 26.5104 & 26.5052 & -0.020 & 0.0001 \\
114.52 & 10.382 & 10.424 & 0.398 & 26.6581 & 26.6560 & -0.008 & 0.0001 \\
114.52 & 13.326 & 13.312 & -0.100 & 26.8046 & 26.8053 & 0.002 & 0.0001 \\
& & & & & & & \\
114.52 & 16.344 & 16.378 & 0.211 & 26.9535 & 26.9518 & -0.006 & 0.0001 \\
114.52 & 19.666 & 19.599 & -0.338 & 27.1032 & 27.1062 & 0.011 & 0.0001 \\
114.52 & 23.245 & 23.219 & -0.111 & 27.2643 & 27.2654 & 0.004 & 0.0001 \\
114.52 & 27.155 & 27.140 & -0.056 & 27.4311 & 27.4317 & 0.002 & 0.0001 \\
114.52 & 31.299 & 31.523 & 0.718 & 27.6091 & 27.6001 & -0.032 & 0.0000 \\
119.42 & 0.833 & 1.149 & 37.950 & 25.6812 & 25.6598 & -0.083 & 0.0001 \\
119.42 & 3.027 & 3.477 & 14.846 & 25.8345 & 25.8055 & -0.112 & 0.0001 \\
119.42 & 6.215 & 6.638 & 6.817 & 26.0308 & 26.0052 & -0.098 & 0.0001 \\
119.42 & 10.401 & 10.733 & 3.194 & 26.2681 & 26.2495 & -0.071 & 0.0001 \\
119.42 & 14.909 & 15.100 & 1.279 & 26.5034 & 26.4935 & -0.037 & 0.0001
\end{tabular}


Data from Van Itterbeek et al. [46] (continued)

\begin{tabular}{|c|c|c|c|c|c|c|c|}
\hline $\mathrm{T}$ & $P, \exp$ & $\mathrm{P}, \mathrm{cal}$ & Dev & $\rho, \exp$ & $\rho, \mathrm{cal}$ & Dev & Wt \\
\hline K & $\mathrm{MPa}$ & $\mathrm{MPa}$ & 8 & $\mathrm{~mol} \cdot \mathrm{dm}^{-3}$ & $\mathrm{~mol} \cdot \mathrm{dm}^{-3}$ & 8 & \\
\hline 119.42 & 19.837 & 19.972 & 0.682 & 26.7480 & 26.7414 & -0.025 & 0.0001 \\
\hline 119.42 & 25.375 & 25.475 & 0.394 & 27.0051 & 27.0006 & -0.017 & 0.0001 \\
\hline 119.42 & 31.691 & 31.198 & -1.556 & 27.2546 & 27.2754 & 0.076 & 0.0001 \\
\hline 124.92 & 0.980 & 1.819 & 85.668 & 25.2010 & 25.1375 & -0.252 & 0.0001 \\
\hline 124.92 & 4.496 & 5.182 & 15.250 & 25.4421 & 25.3945 & -0.187 & 0.0001 \\
\hline 124.92 & 8.419 & 8.952 & 6.335 & 25.6911 & 25.6571 & -0.132 & 0.0001 \\
\hline 124.92 & 12.519 & 13.004 & 3.872 & 25.9383 & 25.9097 & -0.110 & 0.0001 \\
\hline 124.92 & 16.883 & 17.136 & 1.498 & 26.1725 & 26.1587 & -0.053 & 0.0001 \\
\hline 124.92 & 21.799 & 21.948 & 0.687 & 26.4264 & 26.4187 & -0.029 & 0.0001 \\
\hline 124.92 & 27.364 & 27.467 & 0.378 & 26.6966 & 26.6917 & -0.018 & 0.0001 \\
\hline 130.99 & 1.502 & 1.792 & 19.298 & 24.5924 & 24.5673 & -0.102 & 0.0001 \\
\hline 130.99 & 4.625 & 4.754 & 2.803 & 24.8361 & 24.8258 & -0.041 & 0.0001 \\
\hline 130.99 & 7.934 & 8.684 & 9.448 & 25.1307 & 25.0767 & -0.215 & 0.0001 \\
\hline 130.99 & 11.520 & 11.904 & 3.331 & 25.3524 & 25.3268 & -0.101 & 0.0001 \\
\hline 130.99 & 15.774 & 15.495 & -1.770 & 25.5826 & 25.5999 & 0.067 & 0.0001 \\
\hline 130.99 & 20.071 & 19.558 & -2.558 & 25.8251 & 25.8545 & 0.114 & 0.0001 \\
\hline 130.99 & 24.931 & 24.300 & -2.530 & 26.0879 & 26.1214 & 0.128 & 0.0001 \\
\hline 130.99 & 30.287 & 29.714 & -1.895 & 26.3658 & 26.3940 & 0.107 & 0.0001 \\
\hline 134.33 & 0.689 & 1.266 & 83.767 & 24.1949 & 24.1394 & -0.229 & 0.0013 \\
\hline 134.33 & 2.712 & 3.289 & 21.270 & 24.3807 & 24.3290 & -0.212 & 0.0001 \\
\hline 134.33 & 5.783 & 6.388 & 10.464 & 24.6433 & 24.5939 & -0.200 & 0.0001 \\
\hline 134.33 & 9.130 & 9.740 & 6.686 & 24.9029 & 24.8572 & -0.183 & 0.0001 \\
\hline 134.33 & 12.783 & 13.258 & 3.716 & 25.1534 & 25.1208 & -0.130 & 0.0001 \\
\hline 134.33 & 16.625 & 17.093 & 2.813 & 25.4059 & 25.3761 & -0.117 & 0.0001 \\
\hline 134.33 & 20.947 & 21.320 & 1.783 & 25.6634 & 25.6414 & -0.086 & 0.0001 \\
\hline 134.33 & 25.768 & 26.084 & 1.229 & 25.9323 & 25.9150 & -0.067 & 0.0001 \\
\hline 134.33 & 31.029 & 31.541 & 1.650 & 26.2171 & 26.1913 & -0.098 & 0.0001 \\
\hline 139.06 & 1.252 & 1.820 & 45.318 & 23.7344 & 23.6737 & -0.256 & 0.0002 \\
\hline 139.06 & 3.825 & 4.420 & 15.546 & 23.9958 & 23.9382 & -0.240 & 0.0001 \\
\hline 139.06 & 6.749 & 7.333 & 8.658 & 24.2618 & 24.2104 & -0.212 & 0.0001 \\
\hline 139.06 & 9.876 & 10.475 & 6.061 & 24.5236 & 24.4755 & -0.196 & 0.0001 \\
\hline 139.06 & 13.230 & 13.827 & 4.515 & 24.7801 & 24.7359 & -0.178 & 0.0001 \\
\hline 139.06 & 16.900 & 17.415 & 3.045 & 25.0332 & 24.9981 & -0.140 & 0.0001 \\
\hline 139.06 & 20.966 & 21.404 & 2.087 & 25.2934 & 25.2658 & -0.109 & 0.0001 \\
\hline 139.06 & 25.497 & 25.770 & 1.073 & 25.5572 & 25.5412 & -0.062 & 0.0001 \\
\hline
\end{tabular}


Data from Van Itterbeek et al. [46] (continued)

\begin{tabular}{|c|c|c|c|c|c|c|c|}
\hline $\mathrm{T}$ & $P, \exp$ & P,cal & Dev & $\rho, \exp$ & $\rho$, cal & Dev & Wt \\
\hline K & $\mathrm{MPa}$ & $\mathrm{MPa}$ & 8 & $\mathrm{~mol} \cdot \mathrm{dm}^{-3}$ & $\mathrm{~mol} \cdot \mathrm{dm}^{-3}$ & 8 & \\
\hline 139.06 & 30.600 & 30.952 & 1.149 & 25.8465 & 25.8276 & -0.073 & 0.0001 \\
\hline 145.56 & 1.300 & 1.826 & 40.516 & 22.9848 & 22.9165 & -0.297 & 0.0001 \\
\hline 145.56 & 3.528 & 4.119 & 16.770 & 23.2623 & 23.1936 & -0.296 & 0.0002 \\
\hline 145.56 & 6.037 & 6.627 & 9.784 & 23.5355 & 23.4736 & -0.263 & 0.0001 \\
\hline 145.56 & 8.736 & 9.460 & 8.288 & 23.8146 & 23.7458 & -0.289 & 0.0001 \\
\hline 145.56 & 11.719 & 12.461 & 6.333 & 24.0836 & 24.0193 & -0.267 & 0.0001 \\
\hline 145.56 & 14.913 & 15.735 & 5.509 & 24.3522 & 24.2870 & -0.268 & 0.0001 \\
\hline 145.56 & 18.402 & 19.290 & 4.829 & 24.6202 & 24.5553 & -0.264 & 0.0001 \\
\hline 145.56 & 22.258 & 23.265 & 4.525 & 24.8961 & 24.8283 & -0.272 & 0.0001 \\
\hline 145.56 & 26.589 & 27.697 & 4.168 & 25.1794 & 25.1107 & -0.273 & 0.0001 \\
\hline 145.56 & 31.458 & 32.637 & 3.746 & 25.4706 & 25.4032 & -0.264 & 0.0001 \\
\hline 150.75 & 3.102 & 3.779 & 21.826 & 22.6157 & 22.5220 & -0.414 & 0.0001 \\
\hline 150.75 & 5.188 & 5.938 & 14.450 & 22.8933 & 22.8002 & -0.406 & 0.0002 \\
\hline 150.75 & 7.511 & 8.297 & 10.462 & 23.1664 & 23.0784 & -0.380 & 0.0002 \\
\hline 150.75 & 10.039 & 10.879 & 8.363 & 23.4373 & 23.3520 & -0.364 & 0.0001 \\
\hline 150.75 & 12.807 & 13.617 & 6.322 & 23.6995 & 23.6243 & -0.317 & 0.0001 \\
\hline 150.75 & 15.745 & 16.618 & 5.542 & 23.9630 & 23.8887 & -0.310 & 0.0001 \\
\hline 150.75 & 19.000 & 19.859 & 4.520 & 24.2248 & 24.1575 & -0.278 & 0.0001 \\
\hline 150.75 & 22.614 & 23.517 & 3.997 & 24.4972 & 24.4319 & -0.266 & 0.0001 \\
\hline 150.75 & 26.640 & 27.539 & 3.371 & 24.7733 & 24.7135 & -0.241 & 0.0001 \\
\hline 150.75 & 30.986 & 32.023 & 3.346 & 25.0576 & 24.9939 & -0.254 & 0.0001 \\
\hline 158.24 & 2.289 & 3.073 & 34.213 & 21.5318 & 21.3814 & -0.698 & 0.0001 \\
\hline 158.24 & 3.963 & 4.812 & 21.438 & 21.8341 & 21.6913 & -0.654 & 0.0003 \\
\hline 158.24 & 5.895 & 6.788 & 15.154 & 22.1371 & 22.0047 & -0.598 & 0.0002 \\
\hline 158.24 & 8.025 & 8.989 & 12.005 & 22.4371 & 22.3099 & -0.567 & 0.0002 \\
\hline 158.24 & 10.390 & 11.379 & 9.520 & 22.7293 & 22.6121 & -0.516 & 0.0002 \\
\hline 158.24 & 12.936 & 13.985 & 8.111 & 23.0171 & 22.9046 & -0.489 & 0.0002 \\
\hline 158.24 & 15.762 & 16.813 & 6.667 & 23.3008 & 23.1984 & -0.439 & 0.0001 \\
\hline 158.24 & 18.908 & 19.904 & 5.269 & 23.5838 & 23.4953 & -0.375 & 0.0001 \\
\hline 158.24 & 22.362 & 23.344 & 4.389 & 23.8721 & 23.7923 & -0.334 & 0.0001 \\
\hline 158.24 & 26.172 & 27.213 & 3.977 & 24.1692 & 24.0918 & -0.320 & 0.0001 \\
\hline 158.24 & 30.459 & 31.495 & 3.401 & 24.4708 & 24.4002 & -0.289 & 0.0001 \\
\hline 165.82 & 3.056 & 3.428 & 12.197 & 20.4637 & 20.3671 & -0.472 & 0.0002 \\
\hline 165 & 4.220 & 4.701 & 11.393 & 20.7641 & 20.6554 & -0.523 & 0.0004 \\
\hline 165.82 & 5.627 & 6.145 & 9.204 & 21.0624 & 20.9598 & -0.487 & 0.0003 \\
\hline
\end{tabular}


Data from Van Itterbeek et al. [46] (continued)

\begin{tabular}{|c|c|c|c|c|c|c|c|}
\hline $\mathrm{T}$ & $P, \exp$ & P,cal & Dev & $\rho, \exp$ & $\rho$, cal & Dev & Wt \\
\hline $\mathrm{K}$ & $\mathrm{MPa}$ & $\mathrm{MPa}$ & 8 & $\mathrm{~mol} \cdot \mathrm{dm}^{-3}$ & $\mathrm{~mol} \cdot \mathrm{dm}^{-3}$ & 8 & \\
\hline 165.82 & 7.146 & 7.760 & 8.591 & 21.3570 & 21.2492 & -0.505 & 0.000 \\
\hline 165.82 & 8.784 & 9.569 & 8.939 & 21.6507 & 21.5273 & -0.570 & 0.000 \\
\hline 165.82 & 10.628 & 11.527 & 8.466 & 21.9356 & 21.8084 & -0.580 & 0.0002 \\
\hline 165.82 & 12.548 & 13.687 & 9.073 & 22.2193 & 22.0733 & -0.657 & 0.0002 \\
\hline 165.82 & 14.671 & 16.054 & 9.427 & 22.5012 & 22.3398 & -0.718 & 0.000 \\
\hline 165.82 & 16.984 & 18.612 & 9.589 & 22.7790 & 22.6051 & -0.763 & 0.00 \\
\hline 165.82 & 19.490 & 21.436 & 9.983 & 23.0595 & 22.8689 & -0.827 & 0.000 \\
\hline 165.82 & 22.218 & 24.545 & 10.473 & 23.3427 & 23.1331 & -0.898 & 0.000 \\
\hline 165.82 & 25.243 & 27.980 & 10.841 & 23.6301 & 23.4031 & -0.960 & 0.00 \\
\hline 165.82 & 28.886 & 31.669 & 9.634 & 23.9143 & 23.7020 & -0.888 & 0.000 \\
\hline 171.02 & 3.051 & 3.192 & 4.632 & 19.4606 & 19.4090 & -0.265 & 0.00 \\
\hline 171.02 & 3.962 & 4.085 & 3.117 & 19.7578 & 19.7192 & -0.195 & 0.000 \\
\hline 171.02 & 4.988 & 5.167 & 3.588 & 20.0678 & 20.0196 & -0.241 & 0.000 \\
\hline 171.02 & 6.186 & 6.398 & 3.437 & 20.3741 & 20.3241 & -0.245 & 0.000 \\
\hline 171.02 & 7.532 & 7.792 & 3.455 & 20.6778 & 20.6240 & -0.260 & 0.00 \\
\hline 171.02 & 9.017 & 9.363 & 3.830 & 20. & 20.9167 & -0.3 & 0. \\
\hline 171.02 & 10.629 & 11.079 & 4.230 & 21.2743 & 21.2004 & -0.348 & 0.000 \\
\hline 171.02 & 12.408 & 12.955 & 4.407 & 21.5629 & 21.4818 & -0.376 & 0.000 \\
\hline 171.02 & 14.286 & 15.010 & 5.070 & 21.8484 & 21.7510 & -0.446 & 0.000 \\
\hline 171.02 & 16.366 & 17.222 & 5.226 & 22.1273 & 22.0225 & -0.474 & 0.000 \\
\hline 171.02 & 18.630 & 19.697 & 5.727 & 22.4120 & 22.2925 & -0.533 & 0.000 \\
\hline 171.02 & 21.083 & 22.342 & 5.969 & 22.6901 & 22.5608 & -0.570 & 0.000 \\
\hline 171.02 & 23.817 & 25.314 & 6.287 & 22.9769 & 22.8355 & -0.615 & 0.000 \\
\hline 171.02 & 26.800 & 28.592 & 6.688 & 23.2672 & 23.1115 & -0.669 & 0.000 \\
\hline 171.02 & 30.081 & 32.194 & 7.025 & 23.5605 & 23.3913 & -0.718 & 0.000 \\
\hline 172.81 & 2.692 & 2.762 & 2.606 & 18. & 18.871 & -0.172 & 0.000 \\
\hline 172.81 & 3.418 & 3.510 & 2.709 & 19.2171 & 19.1811 & -0.187 & 0.000 \\
\hline 172.81 & 4.311 & 4.432 & 2.801 & 19.5431 & 19.5033 & -0.203 & 0.000 \\
\hline 172.81 & 5.364 & 5.511 & 2.735 & 19.8681 & 19.8267 & -0.208 & 0.000 \\
\hline 172.81 & 6.553 & 6.740 & 2.855 & 20.1873 & 20.1416 & -0.227 & 0.000 \\
\hline 172.81 & 7.894 & 8.130 & 2.984 & 20.5019 & 20.4514 & -0.246 & 0.000 \\
\hline 172.81 & 9.396 & 9.690 & 3.127 & 20.8125 & 20.7569 & -0.267 & 0.000 \\
\hline 172.81 & 11.031 & 11.402 & 3.366 & 21.1153 & 21.0525 & -0.297 & 0.000 \\
\hline 172.81 & 12.896 & 13.293 & 3.078 & 21.4142 & 21.3540 & -0.281 & 0.0002 \\
\hline 172.81 & 14.873 & 15.388 & 3.462 & 21.7122 & 21.6418 & -0.324 & 0.0002 \\
\hline
\end{tabular}


Data from Van Itterbeek et al. [46] (continued)

\begin{tabular}{|c|c|c|c|c|c|c|c|}
\hline $\mathrm{T}$ & $P, \exp$ & P, cal & Dev & $\rho, \exp$ & $\rho, \mathrm{cal}$ & Dev & Wt \\
\hline K & $\mathrm{MPa}$ & $\mathrm{MPa}$ & 8 & $\mathrm{~mol} \cdot \mathrm{dm}^{-3}$ & $\mathrm{~mol} \cdot \mathrm{dm}^{-3}$ & 8 & \\
\hline 172.81 & 16.946 & 17.676 & 4.311 & 22.0066 & 21.9157 & -0.413 & 0.0002 \\
\hline 172.81 & 19.340 & 20.202 & 4.457 & 22.3015 & 22.2039 & -0.438 & 0.0002 \\
\hline 172.81 & 21.909 & 23.007 & 5.012 & 22.5999 & 22.4864 & -0.502 & 0.0002 \\
\hline 172.81 & 24.749 & 26.093 & 5.429 & 22.8995 & 22.7724 & -0.555 & 0.0001 \\
\hline 172.81 & 27.916 & 29.514 & 5.725 & 23.2035 & 23.0648 & -0.598 & 0.0001 \\
\hline 172.81 & 31.343 & 33.285 & 6.197 & 23.5106 & 23.3557 & -0.659 & 0.0001 \\
\hline 179.53 & 3.829 & 3.941 & 2.941 & 17.8514 & 17.7783 & -0.409 & 0.0012 \\
\hline 179.53 & 4.391 & 4.519 & 2.906 & 18.1841 & 18.1158 & -0.376 & 0.0010 \\
\hline 179.53 & 5.037 & 5.212 & 3.465 & 18.5168 & 18.4383 & -0.424 & 0.0008 \\
\hline 179.53 & 5.837 & 6.014 & 3.045 & 18.8423 & 18.7746 & -0.360 & 0.0007 \\
\hline 179.53 & 6.735 & 6.951 & 3.204 & 19.1674 & 19.0967 & -0.369 & 0.0006 \\
\hline 179.53 & 7.761 & 8.010 & 3.208 & 19.4852 & 19.4144 & -0.363 & 0.0005 \\
\hline 179.53 & 8.912 & 9.218 & 3.435 & 19.8016 & 19.7253 & -0.385 & 0.0004 \\
\hline 179.53 & 10.223 & 10.561 & 3.307 & 20.1110 & 20.0366 & -0.370 & 0.0004 \\
\hline 179.53 & 11.656 & 12.063 & 3.494 & 20.4182 & 20.3384 & -0.391 & 0.0003 \\
\hline 179.53 & 13.222 & 13.733 & 3.860 & 20.7228 & 20.6332 & -0.432 & 0.0003 \\
\hline 179.53 & 14.960 & 15.562 & 4.023 & 21.0225 & 20.9274 & -0.453 & 0.0003 \\
\hline 179.53 & 16.854 & 17.575 & 4.276 & 21.3201 & 21.2170 & -0.484 & 0.0002 \\
\hline 179.53 & 18.944 & 19.797 & 4.500 & 21.6179 & 21.5070 & -0.513 & 0.0002 \\
\hline 179.53 & 21.248 & 22.247 & 4.702 & 21.9164 & 21.7980 & -0.540 & 0.0002 \\
\hline 179.53 & 23.756 & 24.947 & 5.013 & 22.2163 & 22.0874 & -0.580 & 0.0002 \\
\hline 179.53 & 26.506 & 27.912 & 5.306 & 22.5175 & 22.3780 & -0.620 & 0.0001 \\
\hline 179.53 & 29.518 & 31.194 & 5.677 & 22.8227 & 22.6702 & -0.668 & 0.0001 \\
\hline 188.22 & 5.326 & 5.388 & 1.160 & 16.3228 & 16.2594 & -0.388 & 0.0000 \\
\hline 188.22 & 5.703 & 5.760 & 0.986 & 16.6622 & 16.6149 & -0.284 & 0.0000 \\
\hline 188.22 & 6.139 & 6.208 & 1.137 & 17.0016 & 16.9527 & -0.288 & 0.0000 \\
\hline 188.22 & 6.663 & 6.740 & 1.159 & 17.3388 & 17.2933 & -0.263 & 0.0000 \\
\hline 188.22 & 7.275 & 7.363 & 1.205 & 17.6728 & 17.6289 & -0.249 & 0.0009 \\
\hline 188.22 & 7.965 & 8.082 & 1.474 & 18.0034 & 17.9528 & -0.281 & 0.0008 \\
\hline 188.22 & 8.768 & 8.909 & 1.609 & 18.3308 & 18.2781 & -0.287 & 0.0007 \\
\hline 188.22 & 8.844 & 8.990 & 1.649 & 18.3604 & 18.3066 & -0.293 & 0.0007 \\
\hline 188.22 & 9.743 & 9.941 & 2.033 & 18.6839 & 18.6200 & -0.342 & 0.0006 \\
\hline 188.22 & 10.795 & 11.003 & 1.921 & 19.0009 & 18.9421 & -0.309 & 0.0005 \\
\hline 188.22 & 11.951 & 12.194 & 2.036 & 19.3151 & 19.2539 & -0.317 & 0.0004 \\
\hline 188.22 & 13.134 & 13.539 & 3.079 & 19.6298 & 19.5389 & -0.463 & 0.0004 \\
\hline
\end{tabular}


Data from Van Itterbeek et al. [46] (continued)

$\begin{array}{cccccccc}\text { T } & \text { P,exp } & \text { P,cal } & \text { Dev } & \rho, \exp & \rho, \mathrm{cal} & \text { Dev } & \text { Wt } \\ \text { K } & \mathrm{MPa} & \mathrm{MPa} & \text { \& } & \mathrm{mol} \cdot \mathrm{dm}^{-3} & \mathrm{~mol} \cdot \mathrm{dm}^{-3} & 8 & \\ & & & & & & & \\ 188.22 & 14.506 & 15.018 & 3.529 & 19.9390 & 19.8358 & -0.518 & 0.0003 \\ 188.22 & 16.146 & 16.683 & 3.326 & 20.2511 & 20.1541 & -0.479 & 0.0003 \\ 188.22 & 17.851 & 18.406 & 3.107 & 20.5427 & 20.4519 & -0.442 & 0.0003 \\ 188.22 & 19.721 & 20.387 & 3.376 & 20.8468 & 20.7479 & -0.475 & 0.0002 \\ 188.22 & 21.779 & 22.559 & 3.581 & 21.1497 & 21.0442 & -0.499 & 0.0002 \\ 188.22 & 24.026 & 24.964 & 3.903 & 21.4551 & 21.3393 & -0.539 & 0.0002 \\ 188.22 & 26.498 & 27.576 & 4.068 & 21.7581 & 21.6363 & -0.560 & 0.0002 \\ 188.22 & 29.174 & 30.366 & 4.084 & 22.0546 & 21.9310 & -0.560 & 0.0002\end{array}$

Number of Points (Ref. 46) 163

$\begin{array}{llrlrlrl}\text { PRESSURE: } & \text { AAD }-8 & 7.170 & \text { BIAS }-8 & 7.037 & \text { RMS }-8 & 11.241 & \\ & \text { AAD } & 0.636 & \text { BIAS } & 0.604 & \text { RMS } & 0.548 & \mathrm{MPa} \\ \text { DENSITY : } & \text { AAD }-8 & 0.323 & \text { BIAS- } 8 & -0.317 & \text { RMS }-8 & 0.217 & \\ & \text { AAD } & 0.071 & \text { BIAS } & -0.070 & \text { RMS } & 0.049 & \mathrm{~mol} \cdot \mathrm{dm}^{-3}\end{array}$

\begin{tabular}{|c|c|c|c|c|c|c|c|}
\hline Data from & Vennix & 7] & & & & & \\
\hline $\mathrm{T}$ & $P, \exp$ & P,cal & Dev & $\rho, \exp$ & $\rho$, cal & Dev & Wt \\
\hline K & $\mathrm{MPa}$ & $\mathrm{MPa}$ & 8 & $\mathrm{~mol} \cdot \mathrm{dm}^{-3}$ & $\mathrm{~mol} \cdot \mathrm{dm}^{-3}$ & 8 & \\
\hline 172.53 & 2.466 & 2.469 & 0.130 & 2.5593 & 2.5533 & -0.233 & 0.0602 \\
\hline 174.69 & 2.528 & 2.532 & 0.128 & 2.5593 & 2.5536 & -0.222 & 0.0583 \\
\hline 177.20 & 2.599 & 2.602 & 0.119 & 2.5586 & 2.5535 & -0.200 & 0.0562 \\
\hline 183.18 & 2.767 & 2.769 & 0.080 & 2.5580 & 2.5548 & -0.125 & 0.0518 \\
\hline 191.85 & 3.004 & 3.005 & 0.037 & 2.5568 & 2.5554 & -0.054 & 0.0000 \\
\hline 203.08 & 3.305 & 3.305 & 0.000 & 2.5555 & 2.5555 & 0.000 & 0.0831 \\
\hline 213.04 & 3.567 & 3.566 & -0.026 & 2.5543 & 2.5552 & 0.035 & 0.0758 \\
\hline 217.86 & 3.693 & 3.692 & -0.038 & 2.5537 & 2.5549 & 0.048 & 0.0727 \\
\hline 223.83 & 3.848 & 3.846 & -0.053 & 2.5530 & 2.5547 & 0.067 & 0.0692 \\
\hline 233.55 & 4.099 & 4.095 & -0.089 & 2.5518 & 2.5546 & 0.109 & 0.0643 \\
\hline 239.80 & 4.259 & 4.255 & -0.096 & 2.5512 & 2.5541 & 0.115 & 0.0614 \\
\hline 252.83 & 4.590 & 4.585 & -0.094 & 2.5499 & 2.5527 & 0.111 & 0.0563 \\
\hline 263.40 & 4.856 & 4.851 & -0.100 & 2.5487 & 2.5516 & 0.115 & 0.0528 \\
\hline 273.38 & 5.106 & 5.100 & -0.108 & 2.5474 & 2.5505 & 0.122 & 0.0499 \\
\hline 190.43 & 4.472 & 4.488 & 0.358 & 6.4670 & 6.3251 & -2.193 & 0.0000 \\
\hline
\end{tabular}


Data from Vennix [47] (continued)

\begin{tabular}{|c|c|c|c|c|c|c|c|}
\hline $\mathrm{T}$ & $P, \exp$ & P,cal & Dev & $\rho, \exp$ & $\rho$, cal & Dev & Wt \\
\hline K & $\mathrm{MPa}$ & $\mathrm{MPa}$ & 8 & $\mathrm{~mol} \cdot \mathrm{dm}^{-3}$ & $\mathrm{~mol} \cdot \mathrm{dm}^{-3}$ & 8 & \\
\hline 208.57 & 6.027 & 6.045 & 0.304 & 6.4614 & 6.4174 & -0.682 & 0.0515 \\
\hline 223.23 & 7.235 & 7.254 & 0.271 & 6.4571 & 6.4274 & -0.461 & 0.0405 \\
\hline 234.44 & 8.143 & 8.163 & 0.240 & 6.4539 & 6.4307 & -0.360 & 0.0347 \\
\hline 243.73 & 8.891 & 8.908 & 0.191 & 6.4508 & 6.4337 & -0.265 & 0.0310 \\
\hline 243.77 & 8.894 & 8.911 & 0.198 & 6.4508 & 6.4331 & -0.274 & 0.0310 \\
\hline 251.38 & 9.502 & 9.517 & 0.160 & 6.4483 & 6.4348 & -0.210 & 0.0285 \\
\hline 262.19 & 10.360 & 10.373 & 0.122 & 6.4452 & 6.4354 & -0.152 & 0.0255 \\
\hline 272.15 & 11.148 & 11.156 & 0.072 & 6.4421 & 6.4366 & -0.085 & 0.0233 \\
\hline 272.18 & 11.149 & 11.158 & 0.079 & 6.4421 & 6.4361 & -0.093 & 0.0233 \\
\hline 190.05 & 4.499 & 4.508 & 0.203 & 7.2329 & 7.0527 & -2.491 & 0.0000 \\
\hline 190.75 & 4.571 & 4.580 & 0.203 & 7.2329 & 7.0830 & -2.072 & 0.0000 \\
\hline 193.32 & 4.834 & 4.843 & 0.199 & 7.2316 & 7.1396 & -1.272 & 0.0695 \\
\hline 197.74 & 5.272 & 5.285 & 0.233 & 7.2304 & 7.1617 & -0.951 & 0.0626 \\
\hline 202.75 & 5.763 & 5.777 & 0.248 & 7.2285 & 7.1743 & -0.750 & 0.0560 \\
\hline 208.22 & 6.291 & 6.307 & 0.260 & 7.2266 & 7.1808 & -0.634 & 0.0500 \\
\hline 212.16 & 6.669 & 6.686 & 0.257 & 7.2254 & 7.1850 & -0.559 & 0.0463 \\
\hline 217.14 & 7.142 & 7.161 & 0.259 & 7.2235 & 7.1872 & -0.502 & 0.0423 \\
\hline 222.93 & 7.691 & 7.710 & 0.247 & 7.2217 & 7.1906 & -0.431 & 0.0384 \\
\hline 230.05 & 8.361 & 8.381 & 0.234 & 7.2192 & 7.1926 & -0.369 & 0.0343 \\
\hline 237.34 & 9.043 & 9.063 & 0.216 & 7.2167 & 7.1940 & -0.314 & 0.0310 \\
\hline 242.71 & 9.543 & 9.563 & 0.203 & 7.2148 & 7.1945 & -0.282 & 0.0289 \\
\hline 248.44 & 10.076 & 10.094 & 0.186 & 7.2129 & 7.1951 & -0.247 & 0.0269 \\
\hline 250.19 & 10.238 & 10.256 & 0.183 & 7.2123 & 7.1951 & -0.239 & 0.0264 \\
\hline 253.45 & 10.538 & 10.556 & 0.175 & 7.2111 & 7.1949 & -0.224 & 0.0254 \\
\hline 258.64 & 11.017 & 11.035 & 0.157 & 7.2092 & 7.1952 & -0.194 & 0.0240 \\
\hline 263.05 & 11.423 & 11.439 & 0.143 & 7.2079 & 7.1954 & -0.174 & 0.0229 \\
\hline 268.30 & 11.905 & 11.920 & 0.126 & 7.2061 & 7.1954 & -0.148 & 0.0218 \\
\hline 273.17 & 12.350 & 12.364 & 0.110 & 7.2042 & 7.1951 & -0.127 & 0.0208 \\
\hline 191.55 & 4.732 & 4.740 & 0.176 & 9.8370 & 9.2264 & -6.207 & 0.0000 \\
\hline 192.94 & 4.928 & 4.936 & 0.164 & 9.8364 & 9.5852 & -2.554 & 0.0000 \\
\hline 195.24 & 5.256 & 5.264 & 0.141 & 9.8351 & 9.7252 & -1.117 & 0.0641 \\
\hline 197.56 & 5.587 & 5.595 & 0.136 & 9.8345 & 9.7629 & -0.728 & 0.0598 \\
\hline 200.41 & 5.998 & 6.003 & 0.082 & 9.8333 & 9.8022 & -0.316 & 0.0548 \\
\hline 202.83 & 6.342 & 6.350 & 0.127 & 9.8320 & 9.7922 & -0.405 & 0.0511 \\
\hline 206.37 & 6.850 & 6.859 & 0.130 & 9.8301 & 9.7972 & -0.335 & 0.0462 \\
\hline
\end{tabular}


Data from Vennix [47] (continued)

\begin{tabular}{|c|c|c|c|c|c|c|c|}
\hline $\mathrm{T}$ & $P, \exp$ & P,cal & Dev & $\rho, \exp$ & $\rho, \mathrm{cal}$ & Dev & Wt \\
\hline K & $\mathrm{MPa}$ & $\mathrm{MPa}$ & 8 & $\mathrm{~mol} \cdot \mathrm{dm}^{-3}$ & $\mathrm{~mol} \cdot \mathrm{dm}^{-3}$ & 8 & \\
\hline 213.50 & 7.877 & 7.888 & 0.137 & 9.8270 & 9.8011 & -0.264 & 0.0383 \\
\hline 223.65 & 9.339 & 9.352 & 0.139 & 9.8220 & 9.8014 & -0.210 & 0.0303 \\
\hline 236.40 & 11.177 & 11.190 & 0.122 & 9.8158 & 9.8008 & -0.153 & 0.0238 \\
\hline 248.15 & 12.867 & 12.881 & 0.105 & 9.8108 & 9.7993 & -0.118 & 0.0197 \\
\hline 255.16 & 13.875 & 13.887 & 0.089 & 9.8071 & 9.7978 & -0.095 & 0.0179 \\
\hline 273.17 & 16.450 & 16.462 & 0.067 & 9.7990 & 9.7926 & -0.065 & 0.0144 \\
\hline 190.84 & 4.634 & 4.641 & 0.149 & 10.2128 & 8.8339 & -13.501 & 0.0000 \\
\hline 191.04 & 4.662 & 4.670 & 0.184 & 10.2128 & 9.0079 & -11.798 & 0.0000 \\
\hline 191.23 & 4.690 & 4.698 & 0.170 & 10.2128 & 9.3273 & -8.670 & 0.0000 \\
\hline 191.43 & 4.719 & 4.727 & 0.174 & 10.2128 & 9.4927 & -7.051 & 0.0000 \\
\hline 191.82 & 4.776 & 4.784 & 0.171 & 10.2128 & 9.7203 & -4.822 & 0.0000 \\
\hline 192.08 & 4.813 & 4.822 & 0.182 & 10.2128 & 9.7765 & -4.272 & 0.0000 \\
\hline 193.52 & 5.026 & 5.035 & 0.165 & 10.2115 & 10.0120 & -1.954 & 0.0673 \\
\hline 195.16 & 5.270 & 5.277 & 0.130 & 10.2109 & 10.1103 & -0.985 & 0.0639 \\
\hline 197.93 & 5.684 & 5.690 & 0.107 & 10.2096 & 10.1575 & -0.510 & 0.058 \\
\hline 200.57 & 6.081 & 6.087 & 0.098 & 10.2084 & 10.1 & -0 . & 0.0538 \\
\hline 204.04 & 6.604 & 6.610 & 0.093 & 10.2065 & 10.1804 & -0.256 & 0.0484 \\
\hline 207.85 & 7.180 & 7.188 & 0.116 & 10.2047 & 10.1781 & -0.261 & 0.0433 \\
\hline 207.85 & 7.183 & 7.188 & 0.068 & 10.2047 & 10.1890 & -0.154 & 0.0432 \\
\hline 213.04 & 7.969 & 7.977 & 0.098 & 10.2022 & 10.1837 & -0.181 & 0.0375 \\
\hline 233.39 & 11.069 & 11.0 & 0.081 & 10.1922 & 10.1 & -0 . & 0.0239 \\
\hline 252.89 & 14.039 & 14.048 & 0.063 & 10.1829 & 10.1762 & -0.066 & 0.017 \\
\hline 273.02 & 17.093 & 17.098 & 0.035 & 10.1729 & 10.1696 & -0.033 & 0.0136 \\
\hline 273.06 & 17.099 & 17.105 & 0.036 & 10.1729 & 10.1695 & -0.034 & 0.0136 \\
\hline 191.85 & 4.798 & 4.808 & 0.194 & 11.1543 & 10.7425 & -3.692 & 0.0000 \\
\hline 191.87 & 4.801 & 4.812 & 0.224 & 11.1543 & 10. & -4.243 & 0.0000 \\
\hline 193.25 & 5.025 & 5.035 & 0.199 & 11.1537 & 10.9489 & -1.836 & 0.067 \\
\hline 195.11 & 5.3 & 5.3 & 0. & 11.1 & 11.0706 & -0.740 & 0.0626 \\
\hline 197.30 & 5.699 & 5.706 & 0.119 & 11.1518 & 11.0999 & -0.466 & 0.0577 \\
\hline 199.22 & 6.023 & 6.028 & 0.094 & 11.1506 & 11.1178 & -0.294 & 0.0537 \\
\hline 200.90 & 6.309 & 6.314 & 0.077 & 11.1500 & 11.1269 & -0.207 & 0.0505 \\
\hline 203.06 & 6.678 & 6.683 & 0.066 & 11.1487 & 11.1315 & -0.155 & 0.0467 \\
\hline 207.56 & 7.452 & 7.455 & 0.045 & 11.1462 & 11.1369 & -0.083 & 0.0401 \\
\hline 213.20 & 8.427 & 8.429 & 0.032 & 11.1431 & 11.1376 & -0.049 & 0.0338 \\
\hline 213.20 & 8.428 & 8.431 & 0.028 & 11.1431 & 11.1384 & -0.042 & 0.0338 \\
\hline
\end{tabular}


Data from Vennix [47] (continued)

\begin{tabular}{|c|c|c|c|c|c|c|c|}
\hline $\mathrm{T}$ & $P, \exp$ & P,cal & Dev & $\rho, \exp$ & $\rho, \mathrm{cal}$ & Dev & Wt \\
\hline K & $\mathrm{MPa}$ & $\mathrm{MPa}$ & 8 & $\mathrm{~mol} \cdot \mathrm{dm}^{-3}$ & $\mathrm{~mol} \cdot \mathrm{dm}^{-3}$ & q & \\
\hline 213.21 & 8.429 & 8.432 & 0.031 & 11.1431 & 11.1378 & -0.047 & .0337 \\
\hline 223.18 & 10.166 & 10.168 & 0.014 & 11.1375 & 11.1355 & -0.018 & 0.0260 \\
\hline 233.16 & 11.912 & 11.912 & -0.003 & 11.1325 & 11.1329 & 0.003 & 0.0210 \\
\hline 243.14 & 13.660 & 13.658 & -0.013 & 11.1269 & 11.1283 & 0.013 & 0.0175 \\
\hline 253.15 & 15.412 & 15.413 & 0.002 & 11.1219 & 11.1217 & -0.002 & 0.0150 \\
\hline 3.12 & 17.155 & 7.156 & 0.002 & 11.1163 & 11.1161 & -0.002 & 0.0131 \\
\hline 73.09 & 18.898 & 18.895 & -0.012 & 11.1113 & 11.1124 & 0.010 & 0.0116 \\
\hline 273.15 & 18.893 & 18.906 & 0.068 & 11.1113 & 11.1049 & -0.057 & 116 \\
\hline 192.50 & 4.929 & 4.940 & 0.214 & 11.7625 & 11.5548 & -1.766 & 0.0000 \\
\hline 195.84 & 5.526 & 5.533 & 0.124 & 11.7607 & 11.7079 & -0.449 & 0.0592 \\
\hline 8.1 & 5.95 & 5.956 & 0.082 & L.7594 & 11.7 & -0.224 & 0.0535 \\
\hline 03.43 & 6.919 & 6.922 & 0.037 & 11. & 11.7 & $-0 .(1$ & 0.0433 \\
\hline 12.03 & 8.531 & 8.530 & -0.005 & 11. & 11.7 & 0. & 0.0321 \\
\hline 212.04 & 8.531 & 8.531 & -0.005 & 11.751 .3 & 11.7521 & 0.007 & 0.0321 \\
\hline 222.63 & 10.541 & 10.536 & -0.041 & 11.7451 & 11.7505 & 0.046 & 0.0239 \\
\hline 3.40 & 1 & 12.589 & -0.050 & 5 & & 0 . & 88 \\
\hline 33.4 & 12.5 & 2.5 & -0.062 & 95 & 11. & 0 . & 188 \\
\hline 237.03 & 13.292 & 13.282 & -0.074 & 11.7370 & 11.7 & 0. & 0.0175 \\
\hline 237.03 & 13.292 & 13.283 & -0.071 & 11.7 & 11. & 0.067 & 0.0175 \\
\hline 243.38 & 14.505 & 14.495 & -0.069 & 11.7332 & 11.7405 & 0.062 & 0.0157 \\
\hline+3. & & 0106 & & & & & \\
\hline 53. & 1 & 1 & $-0 . c$ & 3 & & 0 . & 134 \\
\hline 263.45 & 18.340 & 18.332 & -0.042 & 11.7220 & 11.7260 & 0.034 & 0.0117 \\
\hline 268.30 & 19.268 & 19.259 & -0.051 & & & & \\
\hline 268.30 & 19.268 & 19.259 & -0.048 & 11.7195 & 11.7240 & 0.038 & 0.0110 \\
\hline 72 . & & & & & & & \\
\hline 272.70 & 20.105 & 20.096 & -0.044 & 11. & 11 & 0.034 & 0.0104 \\
\hline 272.70 & 20.105 & 20.097 & -0.0 & & & & 0.0104 \\
\hline 190.70 & 4.66 & 4.673 & 0.280 & 12. & 12 & -1 & 0.0000 \\
\hline 191.45 & 4.810 & 4.824 & 0.287 & 12.8636 & 12.7111 & -1.186 & 0.0000 \\
\hline & & & & & & & \\
\hline & & & & & & -0 . & 0.0507 \\
\hline & 7.3 & 1.3 & 0.0 & 12. & 12.8 & -0.110 & 0.0358 \\
\hline & 8 & & 0.017 & & 12. & -0.018 & $0.02 \varepsilon$ \\
\hline 218.62 & 10.668 & 10.662 & -0.062 & 12.8468 & 12.8539 & 0.055 & 0.0213 \\
\hline
\end{tabular}


Data from Vennix [47] (continued)

\begin{tabular}{|c|c|c|c|c|c|c|c|}
\hline $\mathrm{T}$ & $\mathrm{P}, \exp$ & P,cal & Dev & $\rho, \exp$ & $\rho$, cal & Dev & Wt \\
\hline K & $\mathrm{MPa}$ & $\mathrm{MPa}$ & 8 & $\mathrm{~mol} \cdot \mathrm{dm}^{-3}$ & $\mathrm{~mol} \cdot \mathrm{dm}^{-3}$ & 8 & \\
\hline 228.10 & 12.775 & 12.764 & -0.090 & 12.8406 & 12.8500 & 0.073 & 0.0168 \\
\hline 238.55 & 15.109 & 15.094 & -0.099 & 12.8343 & 12.8440 & 0.076 & 0.0136 \\
\hline 245.92 & 16.755 & 16.741 & -0.079 & 12.8294 & 12.8368 & 0.058 & 0.0120 \\
\hline 252.97 & 18.335 & 18.319 & -0.087 & 12.8250 & 12.8330 & 0.062 & 0.0108 \\
\hline 260.11 & 19.930 & 19.914 & -0.080 & 12.8206 & 12.8278 & 0.056 & 0.009 \\
\hline 267.31 & 21.537 & 21.523 & -0.067 & 12.8163 & 12.8222 & 0.046 & 0.008 \\
\hline 274.15 & 23.058 & 23.047 & -0.048 & 12.8119 & 12.8160 & 0.032 & 0.0082 \\
\hline 188.97 & 4.386 & 4.389 & 0.083 & 13.7516 & 13.7216 & -0.218 & 0.0000 \\
\hline 189.26 & 4.450 & 4.455 & 0.098 & 13.7510 & 13.7181 & -0.239 & 0.0000 \\
\hline 189.77 & 4.564 & 4.570 & 0.140 & 13.7510 & 13.7088 & -0.307 & 0.0000 \\
\hline 190.1 & 4.655 & 4.6 & 0.168 & 13. & 13.7 & -0.342 & 0.000 \\
\hline 190.45 & 4.718 & 4.726 & 0.171 & 13.7504 & 13.7046 & -0.333 & 0.0000 \\
\hline 190.74 & 4.784 & 4.793 & 0.182 & 13.7504 & 13.7040 & -0.338 & 0.0000 \\
\hline 191.07 & 4.862 & 4.870 & 0.181 & 13.7497 & 13.7058 & -0.320 & 0.0000 \\
\hline 192.89 & 5.282 & 5.293 & 0.213 & 13.7485 & 13.7064 & -0.306 & 0.000 \\
\hline $195.05^{\circ}$ & 5.789 & 5.8 & 0.211 & 13 & 13. & -0.256 & 0.045 \\
\hline 195.07 & 5.793 & 5.806 & 0.220 & 13.7473 & 13.7106 & -0.267 & 0.045 \\
\hline 197.10 & 6.279 & 6.291 & 0.195 & 13.7460 & 13.7169 & -0.212 & 0.039 \\
\hline 200.01 & 6.981 & 6.992 & 0.155 & 13.7441 & 13.7234 & -0.150 & 0.0338 \\
\hline 203.32 & 7.790 & 7.797 & 0.092 & 13.7416 & 13.7305 & -0.081 & 0.028 \\
\hline 207.20 & 8.744 & 8.748 & 0.051 & 13.7392 & 13.7 & -0.042 & 0.024 \\
\hline 213.32 & 10.271 & 10.264 & -0.060 & 13.7348 & 13.7410 & 0.045 & 0.019 \\
\hline 222.79 & 12.643 & 12.631 & -0.095 & 13.7286 & 13.7376 & 0.065 & 0.015 \\
\hline 227.85 & 13.917 & 13.903 & -0.106 & 13.7254 & 13.7352 & 0.071 & 0.013 \\
\hline 233.27 & 15.288 & 15.271 & -0.113 & 13.7217 & 13.7318 & 0.074 & 0.012 \\
\hline 238.51 & 16.611 & 16.594 & -0.101 & 13.7180 & 13.7269 & 0.065 & 0.011 \\
\hline 242.99 & 17.743 & 17.726 & -0.094 & 13.7148 & 13.7229 & 0.059 & 0.010 \\
\hline 248.77 & 19.203 & 19.190 & -0.067 & 13.7111 & 13.7169 & 0.042 & 0.009 \\
\hline 253.11 & 20.301 & 20.290 & -0.051 & 13.7086 & 13.7129 & 0.032 & 0.008 \\
\hline 258.31 & 21.615 & 21.605 & -0.043 & 13.7049 & 13.7085 & 0.026 & 0.008 \\
\hline 262.93 & 22.781 & 22.772 & -0.041 & 13.7018 & 13.7052 & 0.025 & 0.007 \\
\hline 267.82 & 24.012 & 24.005 & -0.028 & 13.6986 & 13.7009 & 0.017 & 0.007 \\
\hline 267.89 & 24.029 & 24.022 & -0.028 & 13.6986 & 13.7009 & 0.017 & 0.007 \\
\hline 273.15 & 25.355 & 25.348 & -0.027 & 13.6949 & 13.6971 & 0.016 & 0.006 \\
\hline 187.65 & 4.327 & 4.336 & 0.199 & 14.9705 & 14.9463 & -0.162 & 0.000 \\
\hline
\end{tabular}


Data from Vennix [47] (continued)

\begin{tabular}{|c|c|c|c|c|c|c|c|}
\hline $\mathrm{T}$ & $P, \exp$ & P,cal & Dev & $\rho, \exp$ & $\rho, \mathrm{cal}$ & Dev & Wt \\
\hline K & $\mathrm{MPa}$ & $\mathrm{MPa}$ & 8 & $\mathrm{~mol} \cdot \mathrm{dm}^{-3}$ & $\mathrm{~mol} \cdot \mathrm{dm}^{-3}$ & 8 & \\
\hline 188.93 & 4.677 & 4.689 & 0.264 & 14.9699 & 14.9402 & -0.199 & 0.0000 \\
\hline 191.45 & 5.379 & 5.396 & 0.324 & 14.9680 & 14.9350 & -0.220 & 0.0000 \\
\hline 194.80 & 6.326 & 6.347 & 0.332 & 14.9655 & 14.9344 & -0.207 & 0.0282 \\
\hline 199.61 & 7.710 & 7.729 & 0.240 & 14.9618 & 14.9409 & -0.140 & 0.0220 \\
\hline 203.05 & 8.714 & 8.729 & 0.181 & 14.9593 & 14.9441 & -0.101 & 0.0191 \\
\hline 207.63 & 10.057 & 10.068 & 0.106 & 14.9562 & 14.9476 & -0.058 & 0.0161 \\
\hline 212.93 & 11.627 & 11.633 & 0.056 & 14.9524 & 14.9480 & -0.030 & 0.0137 \\
\hline 221.36 & 14.131 & 14.134 & 0.022 & 14.9462 & 14.9445 & -0.011 & 0.0111 \\
\hline 228.64 & 16.302 & 16.305 & 0.019 & 14.9406 & 14.9391 & -0.010 & 0.0095 \\
\hline 236.60 & 18.680 & 18.687 & 0.035 & 14.9350 & 14.9323 & -0.018 & 0.0083 \\
\hline 245.09 & 21.214 & 21.226 & 0.057 & 14.9287 & 14.9244 & -0.029 & 0.0072 \\
\hline 253.07 & 23.597 & 23.611 & 0.060 & 14.9225 & 14.9180 & -0.030 & 0.0065 \\
\hline 260.84 & 25.904 & 25.928 & 0.094 & 14.9169 & 14.9098 & -0.048 & 0.0059 \\
\hline 268.82 & 28.272 & 28.304 & 0.116 & 14.9113 & 14.9026 & -0.059 & 0.0054 \\
\hline 275.15 & 30.147 & 30.180 & 0.107 & 14.9063 & 14.8982 & -0.054 & 0.0051 \\
\hline 183.35 & 3.825 & 3.877 & 1.346 & 16.5234 & 16.4599 & -0.385 & 0.0000 \\
\hline 185.27 & 4.488 & 4.548 & 1.328 & 16.5215 & 16.4568 & -0.391 & 0.0000 \\
\hline 189.26 & 5.897 & 5.964 & 1.139 & 16.5184 & 16.4601 & -0.353 & 0.0000 \\
\hline 194.42 & 7.751 & 7.819 & 0.878 & 16.5140 & 16.4669 & -0.286 & 0.0134 \\
\hline 198.42 & 9.196 & 9.271 & 0.818 & 16.5109 & 16.4657 & -0.274 & 0.0116 \\
\hline 201.04 & 10.164 & 10.228 & 0.634 & 16.5084 & 16.4728 & -0.216 & 0.0107 \\
\hline 207.77 & 12.617 & 12.695 & 0.619 & 16.5028 & 16.4668 & -0.218 & 0.0089 \\
\hline 213.67 & 14.788 & 14.869 & 0.547 & 16.4978 & 16.4652 & -0.197 & 0.0078 \\
\hline 222.89 & 18.182 & 18.279 & 0.531 & 16.4903 & 16.4576 & -0.198 & 0.0065 \\
\hline 232.81 & 21.835 & 21.946 & 0.510 & 16.4822 & 16.4500 & -0.195 & 0.0056 \\
\hline 241.29 & 24.958 & 25.076 & 0.476 & 16.4754 & 16.4448 & -0.186 & 0.0049 \\
\hline 254.53 & 29.803 & 29.938 & 0.454 & 16.4648 & 16.4348 & -0.182 & 0.0042 \\
\hline 261.83 & 32.459 & 32.601 & 0.437 & 16.4586 & 16.4294 & -0.177 & 0.0039 \\
\hline 267.88 & 34.658 & 34.804 & 0.420 & 16.4536 & 16.4253 & -0.172 & 0.0037 \\
\hline 272.98 & 36.500 & 36.650 & 0.413 & 16.4492 & 16.4212 & -0.170 & 0.1773 \\
\hline 179.14 & 3.304 & 3.368 & 1.913 & 17.5640 & 17.5143 & -0.283 & 0.1481 \\
\hline 179.83 & 3.585 & 3.650 & 1.818 & 17.5634 & 17.5140 & -0.281 & 0.0143 \\
\hline 181.01 & 4.065 & 4.134 & 1.689 & 17.5622 & 17.5132 & -0.279 & 0.0000 \\
\hline 184.44 & 5.477 & 5.554 & 1.402 & 17.5590 & 17.5120 & -0.268 & 0.0000 \\
\hline 196.38 & 10.460 & 10.577 & 1.118 & 17.5485 & 17.4999 & -0.277 & 0.0077 \\
\hline
\end{tabular}


Data from Vennix [47] (continued)

\begin{tabular}{|c|c|c|c|c|c|c|c|}
\hline $\mathrm{T}$ & $P, \exp$ & P,cal & Dev & $\rho, \exp$ & $\rho$, cal & Dev & Wt \\
\hline K & $\mathrm{MPa}$ & $\mathrm{MPa}$ & 8 & $\mathrm{~mol} \cdot \mathrm{dm}^{-3}$ & $\mathrm{~mol} \cdot \mathrm{dm}^{-3}$ & 8 & \\
\hline 200.54 & 12.224 & 12.340 & 0.949 & 17.5447 & 17.5012 & -0.248 & 0.0070 \\
\hline 208.82 & 15.743 & 15.872 & 0.821 & 17.5379 & 17.4971 & -0.232 & 0.0058 \\
\hline 217.04 & 19.228 & 19.378 & 0.781 & 17.5304 & 17.4893 & -0.234 & 0.0051 \\
\hline 225.02 & 22.597 & 22.775 & 0.787 & 17.5235 & 17.4802 & -0.247 & 0.0045 \\
\hline 233.08 & 25.996 & 26.192 & 0.755 & 17.5160 & 17.4729 & -0.246 & 0.0040 \\
\hline 241.00 & 29.322 & 29.538 & 0.736 & 17.5092 & 17.4660 & -0.247 & 0.0037 \\
\hline 250.74 & 33.392 & 33.628 & 0.705 & 17.5005 & 17.4578 & -0.244 & 0.0033 \\
\hline 258.65 & 36.660 & 36.930 & 0.737 & 17.4936 & 17.4480 & -0.261 & 0.1540 \\
\hline 266.75 & 40.009 & 40.295 & 0.713 & 17.4868 & 17.4419 & -0.257 & 0.1436 \\
\hline 274.30 & 43.114 & 43.408 & 0.683 & 17.4799 & 17.4362 & -0.250 & 0.135 \\
\hline 182.83 & 6.552 & 6.741 & 2.882 & 18.4888 & 18.4116 & -0.417 & 0.000 \\
\hline 182.83 & 6.549 & 6.741 & 2.925 & 18.4888 & 18.4105 & -0.423 & 0.0000 \\
\hline 175.63 & 3.138 & 3.303 & 5.268 & 18.4956 & 18.4080 & -0.474 & 0.0946 \\
\hline 178.08 & 4.293 & 4.466 & 4.028 & 18.4931 & 18.4100 & -0.449 & 0.008 \\
\hline 188.29 & 9.165 & 9.372 & 2.258 & 18.4838 & 18.4112 & -0.393 & 0.006 \\
\hline 193.63 & 11.730 & 11.957 & 1.928 & 18.4788 & 18.4089 & -0.379 & 0.005 \\
\hline 198.67 & 14.156 & 14.399 & 1.719 & 18.4738 & 18.4 & -0.368 & 0.005 \\
\hline 203.16 & 16.318 & 16.577 & 1.587 & 18.4695 & 18.4028 & -0.361 & 0.0046 \\
\hline 208.21 & 18.751 & 19.031 & 1.491 & 18.4651 & 18.3989 & -0.359 & 0.0042 \\
\hline 212.42 & 20.769 & 21.066 & 1.430 & 18.4607 & 18.3946 & -0.358 & 0.0039 \\
\hline 218.76 & 23.805 & 24.125 & 1.346 & 18.2251 & 18.3897 & -0.354 & 0.003 \\
\hline 223.74 & 26.187 & 26.5 & 1.275 & 18 & 18. & -0. & 0.0034 \\
\hline 229.13 & 28.748 & 29.106 & 1.242 & 18.4452 & 18.3807 & -0.350 & 0.0032 \\
\hline 233.40 & 30.768 & 31.140 & 1.209 & 18.4414 & 18.3772 & -0.348 & 0.0030 \\
\hline 237.61 & 32.760 & 33.146 & 1.178 & 18.4377 & 18.3739 & -0.346 & 0.0029 \\
\hline 243.05 & 35.325 & 35.724 & 1.128 & 18.4321 & 18.3695 & -0.339 & 0.1378 \\
\hline 248.04 & 37.659 & 38.0 & 1.1 & 18.4 & 18 & -0 . & 0.1316 \\
\hline 253.13 & 40.035 & 40.464 & 1.071 & 18.4227 & 18.3611 & -0.335 & 0.1259 \\
\hline 263.28 & 44.752 & 45.203 & 1.007 & 18.4134 & 18.3536 & -0.325 & 0.1160 \\
\hline 272.93 & 49.184 & 49.666 & 0.980 & 18.4047 & 18.3450 & -0.324 & 0.1080 \\
\hline 172.57 & 5.501 & 5.835 & 6.070 & 19.9962 & 19.9059 & -0.452 & 0.0045 \\
\hline 178.48 & 9.008 & 9.383 & 4.159 & 19.9899 & 19.9012 & -0.444 & 0.0039 \\
\hline 186.85 & 13.976 & 14.405 & 3.065 & 19.9812 & 19.8944 & -0.434 & 0.0034 \\
\hline 196.09 & 19.431 & 19.925 & 2.540 & 19.9719 & 19.8849 & -0.436 & 0.0029 \\
\hline 204.18 & 24.179 & 24.721 & 2.243 & 19.9631 & 19.8769 & -0.432 & 0.0026 \\
\hline 211.52 & 28.463 & 29.044 & 2.041 & 19.9557 & 19.8707 & -0.426 & 0.0024 \\
\hline
\end{tabular}


Data from Vennix [47] (continued)

\begin{tabular}{|c|c|c|c|c|c|c|c|}
\hline $\mathrm{T}$ & $P, \exp$ & P,cal & Dev & $\rho, \exp$ & $\rho$, cal & Dev & Wt \\
\hline $\mathrm{K}$ & $\mathrm{MPa}$ & $\mathrm{MPa}$ & $q$ & $\mathrm{~mol} \cdot \mathrm{dm}^{-3}$ & $\mathrm{~mol} \cdot \mathrm{dm}^{-3}$ & 8 & \\
\hline 219.06 & 32.825 & 33.441 & 1.877 & 19.9476 & 19.8641 & -0.419 & 0.0022 \\
\hline 227.10 & 37.434 & 38.096 & 1.768 & 19.9395 & 19.8562 & -0.418 & 0.1034 \\
\hline 235.30 & 42.100 & 42.797 & 1.655 & 19.9314 & 19.8495 & -0.411 & 0.0964 \\
\hline 244.17 & 47.089 & 47.834 & 1.581 & 19.9220 & 19.8402 & -0.411 & 0.0899 \\
\hline 253.83 & 52.486 & 53.260 & 1.475 & 19.9120 & 19.8326 & -0.399 & 0.0839 \\
\hline 259.34 & 55.544 & 56.336 & 1.426 & 19.9064 & 19.8280 & -0.394 & 0.0808 \\
\hline 267.73 & 60.105 & 60.981 & 1.456 & 19.8977 & 19.8154 & -0.413 & 0.0766 \\
\hline 274.49 & 63.813 & 64.695 & 1.383 & 19.8909 & 19.8112 & -0.401 & 0.0736 \\
\hline 172.77 & 11.383 & 11.966 & 5.119 & 21.2132 & 21.1176 & -0.451 & 0.0026 \\
\hline 176.89 & 14.280 & 14.906 & 4.377 & 21.2088 & 21.1124 & -0.454 & 0.0024 \\
\hline 181.67 & 17.634 & 18.294 & 3.745 & 21.2032 & 21.1079 & -0.450 & 0.0023 \\
\hline 189.78 & 23.252 & 24.001 & 3.218 & 21.1939 & 21.0960 & -0.462 & 0.0020 \\
\hline 197.32 & 28.439 & 29.245 & 2.836 & 21.1858 & 21.0885 & -0.459 & 0.0019 \\
\hline 204.17 & 33.127 & 33.968 & 2.540 & 21.1783 & 21.0833 & -0.449 & 0.0018 \\
\hline 212.64 & 38.858 & 39.743 & 2.278 & 21.1689 & 21.0761 & -0.438 & 0.0817 \\
\hline 228.43 & 49.346 & 50.311 & 1.956 & 21.1509 & 21.0613 & -0.424 & 0.0722 \\
\hline 235.78 & 54.155 & 55.158 & 1.851 & 21.1428 & 21.0542 & -0.419 & 0.0686 \\
\hline 247.38 & 61.653 & 62.722 & 1.734 & 21.1303 & 21.0425 & -0.415 & 0.0636 \\
\hline 252.99 & 65.231 & 66.342 & 1.703 & 21.1241 & 21.0359 & -0.418 & 0.0615 \\
\hline 168.75 & 16.942 & 17.767 & 4.870 & 22.4109 & 22.3163 & -0.422 & 0.0017 \\
\hline 180.12 & 26.268 & 27.188 & 3.500 & 22.3972 & 22.3039 & -0.416 & 0.0015 \\
\hline 188.53 & 33.045 & 34.037 & 3.003 & 22.3872 & 22.2941 & -0.416 & 0.0014 \\
\hline 196.79 & 39.639 & 40.662 & 2.579 & 22.3772 & 22.2876 & -0.400 & 0.0650 \\
\hline 204.37 & 45.592 & 46.648 & 2.316 & 22.3679 & 22.2806 & -0.390 & 0.0613 \\
\hline 211.26 & 50.940 & 52.030 & 2.138 & 22.3598 & 22.2740 & -0.384 & 0.0583 \\
\hline 218.29 & 56.352 & 57.469 & 1.983 & 22.3517 & 22.2677 & -0.376 & 0.0556 \\
\hline 226.29 & 62.408 & 63.563 & 1.852 & 22.3417 & 22.2590 & -0.370 & 0.0528 \\
\hline 234.42 & 68.503 & 69.708 & 1.758 & 22.3324 & 22.2502 & -0.368 & 0.0503 \\
\hline
\end{tabular}

Number of Points (Ref. 47) 254

(

$\begin{array}{llllrlll}\text { PRESSURE: } & \text { AAD }-8 & 0.676 & \text { BIAS }-8 & 0.653 & \text { RMS }-8 & 1.068 & \\ & \text { AAD } & 0.154 & \text { BIAS } & 0.151 & \text { RMS } & 0.287 & \mathrm{MPa} \\ \text { DENSITY : } & \text { AAD- } 8 & 0.552 & \text { BIAS }-8 & -0.533 & \text { RMS }-8 & 1.467 & \\ & \text { AAD } & 0.066 & \text { BIAS } & -0.064 & \text { RMS } & 0.150 & \mathrm{~mol} \cdot \mathrm{dm}^{-3}\end{array}$


Total Points 2777

$\begin{array}{llllrlrl}\text { PRESSURE : } & \text { AAD }-8 & 1.028 & \text { BIAS }-8 & 0.811 & \text { RMS - } 8 & 3.917 & \\ & \text { AAD } & 1.484 & \text { BIAS } & 1.345 & \text { RMS } & 10.521 & \mathrm{MPa} \\ \text { DENSITY : } & \text { AAD }-8 & 0.260 & \text { BIAS }-8 & -0.115 & \text { RMS }-8 & 0.703 & \\ & \text { AAD } & 0.038 & \text { BIAS } & -0.021 & \text { RMS } & 0.107 & \mathrm{~mol} \cdot \mathrm{dm}^{-3}\end{array}$

Total Points 2574 with pressures less than $100 \mathrm{MPa}$

$\begin{array}{llllrlrl}\text { PRESSURE : } & \text { AAD- } 8 & 0.871 & \text { BIAS }-8 & 0.690 & \text { RMS }-8 & 3.856 & \\ & \text { AAD } & 0.116 & \text { BIAS } & 0.053 & \text { RMS } & 0.381 & \mathrm{MPa} \\ \text { DENSITY : } & \text { AAD- } 8 & 0.232 & \text { BIAS-8 } & -0.088 & \text { RMS }-8 & 0.683 & \\ & \text { AAD } & 0.026 & \text { BIAS } & -0.011 & \text { RMS } & 0.073 & \mathrm{~mol} \cdot \mathrm{dm}^{-3}\end{array}$


TABLE A5

COMPARISONS FOR SECOND VIRIAL COEFFICIENTS

Notes for Table A5. Data reported from Ref.[51] were from a correlation and are not direct experimental measurements.

\begin{tabular}{|c|c|c|c|c|c|}
\hline REF & $\mathrm{T}$ & $\begin{array}{c}B, \exp \\
\mathrm{dm}^{3} \cdot \mathrm{mol}^{-1}\end{array}$ & $\begin{array}{c}\mathrm{B}, \mathrm{cal} \\
\mathrm{dm}^{3} \cdot \mathrm{mol}^{-1}\end{array}$ & $\begin{array}{l}\text { Dev } \\
\&\end{array}$ & Wt \\
\hline $\begin{array}{l}48 \\
48 \\
48 \\
48 \\
48\end{array}$ & $\begin{array}{l}110.830 \\
112.430 \\
114.440 \\
116.780 \\
121.240\end{array}$ & $\begin{array}{l}-0.33010 \\
-0.31990 \\
-0.30780 \\
-0.29550 \\
-0.27450\end{array}$ & 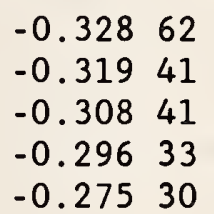 & $\begin{array}{r}-0.448 \\
-0.153 \\
0.197 \\
0.281 \\
0.292\end{array}$ & $\begin{array}{l}3514.1 \\
3626.1 \\
3768.7 \\
3925.5 \\
4225.9\end{array}$ \\
\hline $\begin{array}{l}49 \\
48 \\
50 \\
49 \\
49\end{array}$ & $\begin{array}{l}126.571 \\
128.830 \\
131.920 \\
135.984 \\
147.581\end{array}$ & 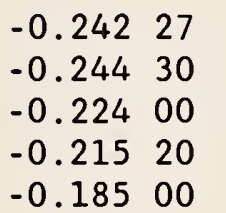 & 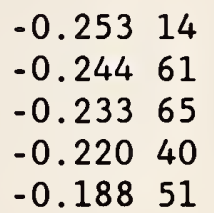 & $\begin{array}{l}4.488 \\
0.125 \\
4.310 \\
2.418 \\
1.898\end{array}$ & $\begin{array}{r}0.0 \\
4748.3 \\
0.0 \\
0.0 \\
627.0\end{array}$ \\
\hline $\begin{array}{l}49 \\
51 \\
49 \\
51 \\
48\end{array}$ & $\begin{array}{l}158.916 \\
168.000 \\
173.507 \\
176.000 \\
178.444\end{array}$ & 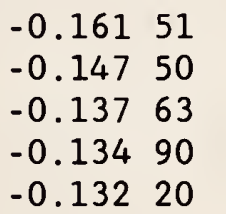 & 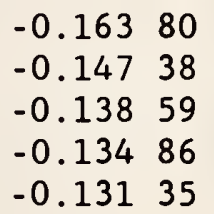 & $\begin{array}{r}1.417 \\
-0.080 \\
0.699 \\
-0.028 \\
-0.646\end{array}$ & $\begin{array}{r}718.2 \\
7864.4 \\
842.8 \\
8599.0 \\
8774.6\end{array}$ \\
\hline $\begin{array}{l}51 \\
50 \\
49 \\
51 \\
51\end{array}$ & $\begin{array}{l}184.000 \\
191.090 \\
191.129 \\
192.000 \\
200.000\end{array}$ & 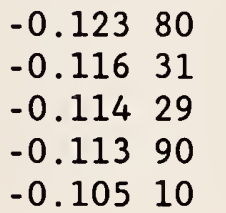 & 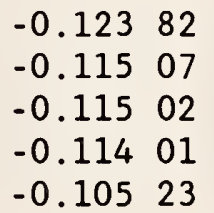 & $\begin{array}{r}0.017 \\
-1.068 \\
0.641 \\
0.094 \\
0.119\end{array}$ & $\begin{array}{r}9369.9 \\
997.3 \\
1015.0 \\
10184.4 \\
11037.1\end{array}$ \\
\hline $\begin{array}{l}50 \\
48 \\
51 \\
50 \\
51\end{array}$ & $\begin{array}{l}200.030 \\
202.520 \\
210.000 \\
215.030 \\
220.000\end{array}$ & 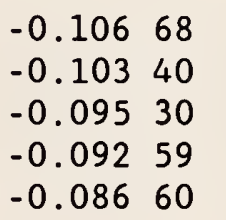 & 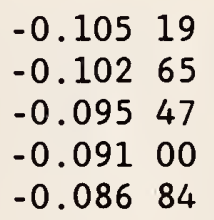 & $\begin{array}{r}-1.393 \\
-0.728 \\
0.173 \\
-1.722 \\
0.271\end{array}$ & $\begin{array}{r}1087.4 \\
11218.6 \\
12172.1 \\
0.0 \\
13394.9\end{array}$ \\
\hline $\begin{array}{l}48 \\
51 \\
51 \\
50 \\
48\end{array}$ & $\begin{array}{l}221.130 \\
230.000 \\
240.000 \\
240.020 \\
243.820\end{array}$ & 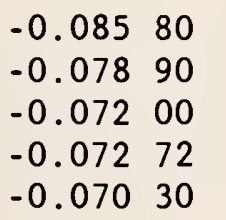 & $\begin{array}{l}-0.08592 \\
-0.079 \\
-0.072 \\
-0.072 \\
-0.069 \\
-025\end{array}$ & $\begin{array}{r}0.142 \\
0.316 \\
0.362 \\
-0.649 \\
-0.691\end{array}$ & $\begin{array}{r}13519.8 \\
14702.1 \\
16111.1 \\
1595.2 \\
16500.7\end{array}$ \\
\hline $\begin{array}{l}51 \\
36 \\
50 \\
48 \\
51\end{array}$ & $\begin{array}{l}260.000 \\
273.150 \\
273.150 \\
273.170 \\
280.000\end{array}$ & $\begin{array}{l}-0.06020 \\
-0.053 \\
-0.053 \\
-0.053 \\
-0.050\end{array}$ & $\begin{array}{l}-0.06043 \\
-0.05380 \\
-0.05380 \\
-0.05379 \\
-0.05064\end{array}$ & $\begin{array}{l}0.381 \\
0.835 \\
0.967 \\
0.160 \\
0.275\end{array}$ & $\begin{array}{r}7225.9 \\
8153.7 \\
816.4 \\
8100.6 \\
8613.9\end{array}$ \\
\hline
\end{tabular}


Table A5 (continued)

\begin{tabular}{|c|c|c|c|c|c|}
\hline \multirow[t]{2}{*}{ REF } & $\mathrm{T}$ & $B, \exp$ & B, cal & Dev & Wt \\
\hline & $\mathrm{K}$ & $\mathrm{dm}^{3} \cdot \mathrm{mol}^{-1}$ & $\mathrm{dm}^{3} \cdot \mathrm{mol}^{-1}$ & 8 & \\
\hline 36 & 298.142 & -0.04282 & -0.04312 & 0.701 & 10158.8 \\
\hline 51 & 300.000 & -0.04230 & -0.04241 & 0.264 & 10283.7 \\
\hline 36 & 303.141 & -0.04091 & -0.04124 & 0.802 & 10633.1 \\
\hline 51 & 320.000 & -0.03540 & -0.03541 & 0.026 & 1228.8 \\
\hline 36 & 323.140 & -0.03423 & -0.03440 & 0.508 & 12708.1 \\
\hline 51 & 340.000 & -0.02940 & -0.02938 & -0.053 & 1479.6 \\
\hline 36 & 348.143 & -0.02706 & -0.02717 & 0.396 & 16075.4 \\
\hline 51 & 360.000 & -0.02420 & -0.02415 & -0.198 & 1797.5 \\
\hline 36 & 373.150 & -0.02100 & -0.02107 & 0.354 & 20714.3 \\
\hline 51 & 380.000 & -0.01970 & -0.01957 & -0.653 & 2208.1 \\
\hline 36 & 398.160 & -0.01587 & -0.01588 & 0.087 & 27410.2 \\
\hline 51 & 400.000 & -0.01570 & $-0.015 \quad 53$ & -1.067 & 2770.7 \\
\hline 36 & 423.170 & -0.01140 & -0.01142 & 0.153 & 38157.9 \\
\hline 36 & 448.182 & -0.00756 & -0.00754 & -0.270 & 57539.6 \\
\hline 51. & 450.000 & -0.00740 & -0.00728 & -1.652 & 5878.4 \\
\hline 36 & 473.193 & -0.00416 & $-0.004 \quad 15$ & -0.318 & 104566.8 \\
\hline 36 & 498.203 & -0.00116 & -0.00116 & -0.219 & 374982.4 \\
\hline 36 & 523.211 & 0.00149 & 0.00149 & 0.179 & 291939.7 \\
\hline 36 & 548.218 & 0.00389 & 0.00386 & -0.888 & 111824.9 \\
\hline 36 & 573.223 & 0.00598 & 0.00597 & -0.120 & 72742.4 \\
\hline 36 & 598.226 & 0.00788 & 0.00788 & -0.012 & 55203.0 \\
\hline 36 & 623.227 & 0.00966 & 0.00960 & -0.594 & 45031.0 \\
\hline
\end{tabular}

Number of Points (Total) 57

$\begin{array}{lrlrlll}\text { AAD }-8 & 0.667 & \text { BIAS }-8 & 0.188 & \text { RMS }-8 & 1.086 & \\ \text { AAD } & 0.001 & \text { BIAS } & -0.001 & \text { RMS } & 0.002 & \mathrm{dm}^{3} \cdot \mathrm{mol}^{-1}\end{array}$


Notes for Table A6. Since the experiment directly measured neither the pressure nor density, but calculated both from a filling pressure and the equation of state of coodvin (Ref. [3]), we have included 5 types of deviations in the tables. Our tabulated temperatures, densities, pressures, and experimental heat capacities are identical to those given by Roder [53]. The column Dev refers to deviations between the experimental isochoric specific heat capacity and that calculated from the tabulated temperature and density; this latter quantity is presented in our table as $C_{V}$, cal. The column marked Devl measures deviations between the densities tabulated by Roder [53] and those calculated from our SWEOS from his tabulated temperature and pressure. Dev2 similarly compares pressures tabulated in Ref. [53] and those calculated from the tabulated temperature and density. These two deviations represent direct comparisons between the current SWEOS and the equation of state in Ref. [3]. Dev3 gives the deviations between the experimental heat capacities and those calculated form the tabulated temperature and pressure. Finally, Dev4 gives the deviations between the heat capacities calculated from tabulated temperature and density and those calculated from the tabulated temperature and pressure.

Data are from Younglove [52] and Roder [53] as adjusted by Roder [53]

\begin{tabular}{|c|c|c|c|c|c|c|c|c|c|c|}
\hline $\mathrm{T}$ & $\rho$ & $C_{v}, \exp$ & $C_{v}, \mathrm{cal}$ & Dev & Wt & $P$ & Devl & Dev2 & Dev3 & Dev4 \\
\hline K & $\mathrm{mol} \cdot \mathrm{dm}^{-3}$ & $\mathrm{~J} \cdot \mathrm{mol}^{-}$ & $1 \cdot \mathrm{K}^{-1}$ & 8 & & $\mathrm{MPa}$ & 8 & 8 & 8 & 8 \\
\hline 3.601 & 7.992 & 29.341 & 29.203 & -0.469 & 38.5 & 13.5573 & -0.30 & 0.27 & -0.481 & -0.013 \\
\hline 5.349 & 7.996 & 29.282 & 29.199 & -0.282 & 1828.1 & 12.7921 & -0.34 & 0.30 & -0.297 & -0.015 \\
\hline 9.066 & 8.000 & 29.486 & 29.251 & -0.796 & 1704.7 & 12.0212 & -0.39 & 0.32 & -0.813 & -0.018 \\
\hline 2.815 & 8.003 & 29.560 & 29.347 & -0.720 & 1614.4 & 11.3576 & -0.42 & 0.34 & -0.740 & -0.020 \\
\hline 5.476 & 8.007 & 29.555 & 29.530 & -0.084 & 1521.3 & 10.5762 & -0.47 & 0.35 & -0.108 & -0.024 \\
\hline .733 & 8.007 & 29.514 & 29.553 & 0.133 & 1516.1 & 10.4969 & -0.47 & 0.35 & 0.109 & -0.024 \\
\hline 8.128 & 8.010 & 29.637 & 29.801 & 0.553 & 1422.5 & 9.7912 & -0.50 & 0.35 & 0.526 & -0.028 \\
\hline 7.534 & 8.011 & 29.854 & 29.827 & -0.089 & 1395.1 & 9.7276 & -0.51 & 0.36 & -0.118 & -0.028 \\
\hline 0.453 & 8.014 & 29.853 & 30.197 & 1.152 & 1312.0 & 8.9684 & -0.53 & 0.34 & 1.119 & -0.032 \\
\hline 373 & 8.014 & 30.094 & 30.202 & 0.358 & 1290.5 & 8.9599 & -0.53 & 0.34 & 0.325 & -0.032 \\
\hline 937 & 8.018 & 30.399 & 30.727 & 1.080 & 1183.5 & 8.1593 & -0.55 & 0.32 & 1.042 & -0.037 \\
\hline 2.993 & 8.018 & 30.413 & 30.723 & 1.018 & 1183.1 & 8.1654 & -0.55 & 0.32 & 0.980 & -0.037 \\
\hline 5.402 & 8.022 & 31.042 & 31.474 & 1.393 & 1059.1 & 7.3443 & -0.55 & 0.27 & 1.349 & -0.043 \\
\hline 854 & 8.025 & 32.420 & 32.656 & 0.728 & 904.4 & 6.5226 & -0.47 & 0.18 & 0.679 & -0.049 \\
\hline 168 & 8.025 & 32.410 & 32.592 & 0.562 & 907.7 & 6.5569 & -0.48 & 0.19 & 0.513 & -0.049 \\
\hline 822 & 10.119 & 29.665 & 29.744 & 0.265 & 44.1 & 20.4199 & -0.05 & 0.06 & 0.263 & -0.002 \\
\hline 146 & 10.124 & 29.524 & 29.615 & 0.308 & 42.5 & 19.4252 & -0.07 & 0.08 & 0.306 & -0.002 \\
\hline .516 & 10.129 & 29.531 & 29.517 & -0.048 & 40.5 & 18.4348 & -0.09 & 0.10 & -0.051 & -0.003 \\
\hline 154 & 10.141 & 29.232 & 29.427 & 0.668 & 1831.5 & 15.9810 & -0.13 & 0.14 & 0.665 & -0.003 \\
\hline 9.403 & 10.145 & 29.307 & 29.468 & 0.549 & 1729.9 & 14.9654 & -0.14 & 0.14 & 0.547 & -0.002 \\
\hline
\end{tabular}


Table A6 (continued)

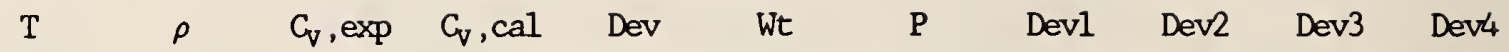

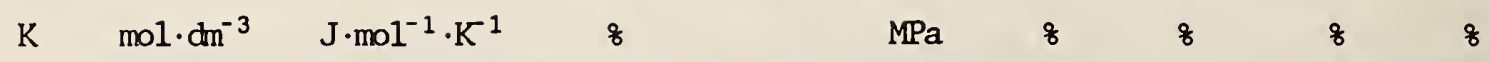

\begin{tabular}{|c|c|c|c|c|c|c|c|c|c|c|}
\hline 251.488 & 10.151 & 29.316 & 88 & 0.927 & 1623.6 & 13.7727 & -0.15 & 0.15 & 0.925 & 002 \\
\hline 244.636 & 10.156 & 29.506 & 29.764 & 0.875 & 1515.7 & 12.7389 & -0.16 & 0.15 & 0.875 & -0.001 \\
\hline 37.756 & 10.161 & 9.623 & 30.021 & 1.344 & 1419.3 & 11.7001 & -0.16 & 0.13 & 1.344 & 0.000 \\
\hline 30.790 & 10.166 & 0.028 & 30.376 & .158 & 1300.9 & 10.6480 & -0.14 & 0.11 & 1.159 & 0.001 \\
\hline 23.396 & 0.171 & 30.879 & 30.881 & 0.005 & 152.4 & 9.5315 & -0.09 & 0.06 & .007 & 0.002 \\
\hline 6.467 & 6 & .281 & 31.527 & 0.786 & 053.8 & 860 & 0.00 & 0.00 & 0.786 & 0.000 \\
\hline 16.813 & 10.176 & .717 & 31.489 & 0.719 & 028.8 & 8.5382 & -0.01 & 0.00 & -0.718 & 0.000 \\
\hline .4 .044 & 10.178 & 1.996 & 31.815 & 0.567 & 985.1 & 8.1207 & 0.05 & -0.03 & -0.569 & -0.002 \\
\hline 9.853 & 10.180 & .524 & 32.437 & 0.267 & 916.3 & 7.4892 & 0.17 & -0.08 & -0.278 & -0.011 \\
\hline 9.057 & 10.181 & 2.947 & 32.580 & 1.115 & 886.5 & 7.3695 & 0.19 & -0.09 & -1.128 & -0.014 \\
\hline 6 & . & 1 & 32. & 0.350 & 921.4 & 7. & 0.17 & 8 & 0. & -0.012 \\
\hline 4 & 10. & 7 & 32. & .212 & 903.3 & 64 & 0.20 & 0.09 & 0. & -0.015 \\
\hline 5 & 10. & .274 & 33. & -0.846 & 775.5 & 152 & 0.46 & 0.16 & -0.901 & -0.055 \\
\hline 3.731 & 10 & .864 & 33.869 & 0.015 & 796.9 & 6.5686 & 0.43 & -0.15 & -0 . & -0.050 \\
\hline 2.601 & 10 & 34.297 & 34.251 & -0.135 & 768.5 & 6.3990 & 0.52 & -0.17 & -0.201 & -0.067 \\
\hline 4 & L. & 2 & 99 & 0.664 & 44.1 & 79 & 0.07 & -0.10 & 0 . & 0.003 \\
\hline 6.5 & 11.759 & .448 & 61 & 0.383 & 39.0 & 99 & 0.04 & -0.05 & 0. & 0.001 \\
\hline 7 & 8 & 2 & 29 & 0.313 & 321.8 & 60 & 0.03 & 3 & 0. & 0.000 \\
\hline 3 & 11. & 77 & 29. & 0.705 & 703.6 & 322 & 0.02 & -0.02 & 0.705 & 0.000 \\
\hline 17 & 11. & 21 & 45 & -0.244 & 077.7 & 9.6540 & 0.14 & -0.12 & -0.260 & -0.016 \\
\hline 4 & 6 & & & 18 & 682.5 & & 0.01 & 0 & & 0.000 \\
\hline 2.330 & 11.791 & 27 & 31.567 & -0.190 & 991.5 & 216 & 0.21 & -0.16 & -0.222 & -0.032 \\
\hline 212.697 & 11.791 & 7 & 31.527 & -0.097 & 999.4 & 8.6908 & 0.20 & -0.15 & -0.126 & -0.029 \\
\hline 240 & 11. & 3 & 10 & 19 & 1480.5 & 57 & 02 & -0 & 19 & -0.001 \\
\hline 239. & 11. & 29. & 29.8 & 0.237 & 1427.1 & 13. & 0.02 & -0.03 & 0.236 & -0.001 \\
\hline 15 & & & & .400 & 916.6 & & 0. & 0.19 & & -0.055 \\
\hline 208.469 & 11.795 & 2 & 32. & -0.336 & 924.2 & 39 & 0.28 & -0.19 & -0.388 & -0.052 \\
\hline 204.724 & 11.798 & 5 & 32. & -0.526 & 853.7 & 15 & 0.37 & -0.21 & 0.615 & -0.090 \\
\hline 205. & 11.798 & & & -0.468 & 862.0 & & 0.35 & -0.21 & -0.551 & -0.084 \\
\hline 233.566 & 11.800 & 29.846 & 30.078 & 0.777 & 1348.9 & 12.6929 & 0.04 & -0.04 & 0.775 & -0.002 \\
\hline 2 . & 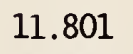 & & & & 806.1 & & 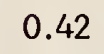 & -0 & & -0.129 \\
\hline 2. & 11.801 & & 33. & -0.899 & 806.4 & 6.7965 & 0.41 & -0.22 & -1.018 & -0.120 \\
\hline & 11.801 & & 30.163 & 1.001 & 1325.2 & 12.3302 & 0.05 & -0.05 & 0.998 & -0.003 \\
\hline 200.564 & 11.802 & 34.091 & 33.749 & -1.004 & 761.9 & 6.4166 & 0.47 & -0.22 & -1.169 & -0.167 \\
\hline 200.945 & 11.802 & 34.008 & 33.630 & -1.113 & 768.5 & 6.4874 & 0.46 & -0.22 & -1.269 & -0.158 \\
\hline
\end{tabular}


Table A6 (continued)

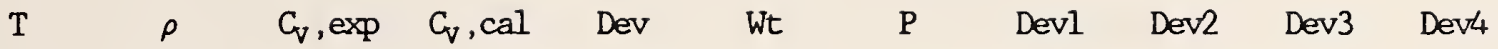

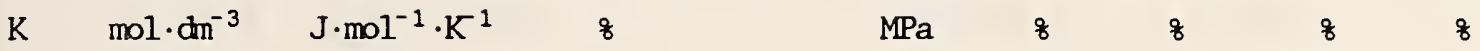

\begin{tabular}{|c|c|c|c|c|c|c|c|c|c|c|}
\hline 198.915 & 11.804 & 34.646 & 34.333 & -0.902 & 725.8 & 6.1116 & 0.51 & -0.21 & -1.112 & -0.211 \\
\hline 197.280 & 11.805 & 35.386 & 35.052 & -0.944 & 0.0 & 5.8105 & 0.53 & -0.19 & -1.203 & -0.261 \\
\hline 26.191 & 11.806 & 30.035 & 30.451 & 1.384 & 1248.1 & 11.2792 & 0.08 & -0.07 & 1.378 & -0.006 \\
\hline 23.653 & 11.808 & 30.288 & 30.607 & 1.053 & 1199.9 & 10.7937 & 0.10 & -0.09 & 1.045 & -0.008 \\
\hline 18.787 & 11.813 & 30.582 & 30.955 & 1.221 & 1125.8 & 9.8645 & 0.13 & -0.11 & 1.207 & -0.014 \\
\hline 5.626 & 1.815 & 0.797 & 31.225 & 1.390 & 1078.0 & 9.2626 & 0.17 & -0.14 & 1.368 & -0.022 \\
\hline 11.370 & 11.819 & 31.543 & 31.665 & 0.387 & 987.6 & 8.4547 & 0.23 & -0.17 & 0.351 & -0.036 \\
\hline 03.924 & 11.826 & 32.837 & 32.840 & 0.010 & 848.3 & 7.0511 & 0.38 & -0.22 & -0.091 & -0.101 \\
\hline 37.427 & 13.094 & 30.056 & 30.070 & 0.046 & 43.4 & 29.2487 & 0.02 & -0.04 & 0.047 & 0.001 \\
\hline 30.591 & 13.101 & 29.801 & 29.904 & 0.344 & 42.1 & 27.6775 & 0.01 & -0.02 & 0.345 & 0.000 \\
\hline 3.783 & 3.109 & 9.617 & 29.762 & 0.489 & 40.6 & 26.1074 & .01 & .02 & 0.489 & 0.000 \\
\hline 76.944 & 13.117 & 9.613 & 29.646 & 0.112 & 38.7 & 24.5256 & .03 & .05 & 0.111 & -0.001 \\
\hline 76.780 & 13.117 & 29.807 & 29.644 & -0.548 & 38.2 & 24.4876 & 0.03 & 0.05 & -0.548 & -0.001 \\
\hline 62.566 & 13.133 & 29.446 & 29.508 & 0.209 & 1756.4 & 21.1874 & -0.07 & 0.10 & 0.208 & 0.000 \\
\hline+8.387 & 13.149 & 29.481 & 29.549 & 0.229 & 1565.8 & 17.8847 & -0.10 & 0.14 & 0.232 & 0.003 \\
\hline 1.245 & .157 & 9.485 & 29. & 0.579 & 1475.4 & 16.2201 & 0.11 & 0.15 & 0. & 0.005 \\
\hline 34.072 & 13.164 & 29.642 & 29. & 0.651 & 1373.2 & 14.5496 & -0 . & 0.15 & 0.659 & 0.008 \\
\hline 26.524 & 13.173 & 29.927 & 30.114 & 0.623 & 1260.8 & 12.7957 & -0.12 & 0.16 & 0.637 & 0.013 \\
\hline 19.265 & 13.180 & 30.289 & 30.486 & 0.649 & 1152.4 & 11.1153 & -0.13 & 0.16 & 0.668 & 0.019 \\
\hline 12.016 & 13.188 & 30.879 & 30.990 & 0.360 & 1036.2 & 9.4478 & -0.15 & 0.16 & 0.388 & 0.028 \\
\hline 4.751 & .196 & .761 & 31. & -0.100 & 913.2 & 7.7933 & -0 & 0.16 & -0 . & 0.047 \\
\hline 30.167 & 13.402 & 9.622 & 29. & 0.329 & 39.6 & 26.1595 & -0.0 & 0.05 & 0.328 & -0.001 \\
\hline 63 & 13.411 & 29.475 & 29. & 0.446 & 37.9 & 24.3748 & -0.05 & 0.09 & 0.445 & -0.001 \\
\hline 65.168 & 13.420 & 29.421 & 29.529 & 0.368 & 1794.8 & 22.5388 & -0.08 & 0.12 & 0.367 & -0.001 \\
\hline 57.310 & 13.429 & 29.215 & 29. & 0.966 & 1712.3 & 20.6355 & -0 & 0.15 & 0.967 & 0.000 \\
\hline+9.756 & 13.437 & 0 & 29. & 73 & 1584.5 & 18. & -0. & 0.16 & & 0.002 \\
\hline 42.161 & 13.446 & 29.393 & 29.609 & 0.733 & 1496.0 & 16.9600 & -0 & 0.18 & 0.739 & 0.006 \\
\hline 27 & 13.455 & 29.418 & 29. & 1.205 & 1399.4 & 15. & -0 & 0.20 & 1.215 & 0.010 \\
\hline 226.091 & 13.464 & 29.853 & 30.059 & 0.690 & 1262.0 & 13.0669 & -0.15 & 0.21 & 0.707 & 0.017 \\
\hline 218.423 & 13.473 & 30.077 & 30.435 & 1.189 & 1159.3 & 11.2192 & -0.17 & 0.22 & 1.215 & 0.026 \\
\hline 99 & 13.479 & & & 1.053 & 1066.5 & 9.8001 & & 0.22 & 1.089 & 0.035 \\
\hline 210.717 & 13.481 & 30.583 & 30.961 & 1.235 & 1042.8 & 9.3752 & -0.19 & 0.23 & 1.275 & 0.039 \\
\hline 203.443 & 13.489 & 31.440 & 31.699 & 0.825 & 919.6 & 7.6538 & -0.23 & 0.23 & 0.893 & 0.067 \\
\hline 202.980 & 13.490 & 31.670 & 31.760 & 0.284 & 902.3 & 7.5453 & -0.23 & 0.23 & 0.355 & 0.071 \\
\hline 194.862 & 13.498 & 33.868 & 33.421 & -1.319 & 0.0 & 5.6658 & -0.31 & 0.21 & -1.138 & 0.184 \\
\hline
\end{tabular}


Table A6 (continued)

\begin{tabular}{|c|c|c|c|c|c|c|c|c|c|c|}
\hline $\mathrm{T}$ & $\rho$ & $C_{v}, \exp$ & $\mathrm{C}_{\mathrm{v}}, \mathrm{cal}$ & Dev & Wt & $\mathrm{P}$ & Devl & Dev2 & Dev3 & Dev4 \\
\hline $\mathrm{K}$ & $\mathrm{mol} \cdot \mathrm{dm}^{-3}$ & $\mathrm{~J} \cdot \mathrm{mol}^{-}$ & $K^{-1}$ & 8 & & $\mathrm{MPa}$ & 8 & 8 & 8 & 8 \\
\hline 9.349 & 4.301 & 30.189 & 30.001 & 0.622 & 40.7 & 1.7855 & -0.02 & 0.03 & -0.623 & -0.001 \\
\hline 281.439 & 14.311 & 29.734 & 29.826 & 0.309 & 39.7 & 29.6385 & -0.04 & 0.07 & 0.307 & 0.002 \\
\hline 277.494 & 14.317 & 29.565 & 29.750 & 0.625 & 39.0 & 28.5645 & -0.06 & 0.11 & .623 & -0.003 \\
\hline 273.495 & 14.322 & 29.585 & 29.681 & 0.325 & 37.8 & 27.4733 & -0.07 & 0.13 & 0.323 & 0.003 \\
\hline 269.538 & 14.327 & 29.398 & 29.622 & 0.763 & 1858.1 & 26.3919 & -0.08 & 0.15 & 0.761 & -0.003 \\
\hline 65.537 & 4.333 & 29.395 & 29.5 & 0.605 & 03.0 & 25.2966 & -0.10 & 0.18 & 0.602 & -0.003 \\
\hline 262.143 & 14.338 & 29.372 & 29.539 & 0.568 & 1759.4 & 24.3660 & -0.11 & 0.21 & 0.566 & -0.002 \\
\hline 255.197 & 14.347 & 29.214 & 29.496 & 0.965 & 1684.0 & 22.4587 & -0.13 & 0.24 & 0.964 & -0.001 \\
\hline 248.293 & 14.356 & 29.190 & 29.493 & 1.037 & 1595.5 & 20.5599 & -0.15 & 0.27 & .039 & 0.002 \\
\hline 242.493 & 14.363 & 29.277 & 29.525 & 0.848 & 1511.9 & 18.9632 & -0.16 & 0.29 & 0.854 & 0.005 \\
\hline 241.372 & 4.365 & 29.337 & 29 & .678 & 1.7 & 18.6545 & -0.17 & 0.30 & 0.684 & 0.006 \\
\hline 234.287 & 14.373 & 29.495 & 29.636 & 0.479 & 1389.3 & 16.7043 & -0.18 & 0.31 & 0.491 & 0.012 \\
\hline 234.417 & 14.374 & 29.475 & 29.634 & 0.539 & 1392.8 & 16.7399 & -0.19 & 0.33 & 0.551 & 0.012 \\
\hline 227.167 & 14.383 & 29.714 & 29.805 & 0.306 & 1286.0 & 14.7466 & -0.20 & 0.34 & .326 & 0.020 \\
\hline 225.394 & 14.385 & 29.674 & 29.859 & 0.623 & 1269.0 & 14.2599 & -0.20 & 0.35 & 0.646 & 0.023 \\
\hline 20.066 & 4.35 & 7 & & 930 & 6 & $\infty$ & -0.22 & 0.36 & 1 & 0.031 \\
\hline 217.103 & 14.395 & 29.907 & 30.186 & 0.932 & 1157.9 & 11.9904 & -0.22 & 0.36 & 0.968 & 0.035 \\
\hline 212.954 & 14.401 & 30.188 & 30.403 & 0.711 & 1093.1 & 10.8603 & -0.24 & 0.38 & 0. & 0.045 \\
\hline 208.733 & 14.406 & 30.531 & 30.671 & 0.457 & 1026.4 & 9.7159 & -0.25 & 0.39 & 0.513 & 0.056 \\
\hline 205.816 & 14.410 & 30.756 & 30.891 & 0.440 & 983.1 & 8.9293 & -0.26 & 0.40 & 0.507 & 0.066 \\
\hline 3 & 4.4 & 3 & & & & & -0 . & & & 089 \\
\hline 198. & 14.419 & 31.5 & 31 & 12 & 86 & 40 & -0.28 & 0.37 & 4 & 0.102 \\
\hline 195.039 & 14.422 & 32.331 & 32. & -0.380 & 798.8 & 6.0693 & -0.25 & 0.30 & -0 . & 0.121 \\
\hline 191.539 & 14.427 & 33.603 & 33.005 & -1.779 & 0.0 & 5.1655 & -0.19 & 0.20 & -1 & 0.130 \\
\hline 265.198 & 15.950 & 29.596 & 29.712 & 0.390 & 1774.3 & 31.3546 & -0. & 0.20 & 0. & -0.006 \\
\hline 259 & 5.9 & - & & 56 & 2 & 43 & -0 & & & 006 \\
\hline 253.443 & 15.969 & 29.4 & & 0.273 & 1631.3 & 27.3593 & -0.13 & 0.29 & 7 & -0.006 \\
\hline 249.308 & 15.973 & 28.662 & 29. & 3.009 & 0.0 & 25.9480 & -0.12 & 0.28 & 3.004 & -0.004 \\
\hline 247.521 & 15.979 & 29.445 & 29.513 & 0.230 & 1558.5 & 25.3373 & -0.15 & 0.34 & 0.226 & -0.005 \\
\hline 239.424 & 15.989 & 29.394 & 29.488 & 0.319 & 1461.8 & 22.5652 & -0.15 & 0.35 & 0.318 & -0.001 \\
\hline 241.503 & 10.307 & 29.466 & & & 1480.5 & & & & & -0.002 \\
\hline 270.623 & 16.003 & 29.602 & 29.813 & 0.714 & 37.0 & 33.4902 & -0.06 & 0.14 & 0.710 & -0.004 \\
\hline 229.421 & 16.005 & 29.529 & 29.532 & 0.010 & 1328.3 & 19.1327 & -0.16 & 0.40 & 0.015 & 0.006 \\
\hline 229.453 & 16.008 & 29.483 & 29.531 & 0.164 & 1332.8 & 19.1436 & -0.18 & 0.44 & 0.170 & 0.006 \\
\hline 264.035 & 16.014 & 29.704 & 29.701 & -0.011 & 1746.0 & 31.2435 & -0.08 & 0.20 & -0.016 & -0.006 \\
\hline
\end{tabular}


Table A6 (continued)

\begin{tabular}{|c|c|c|c|c|c|c|c|c|c|c|}
\hline$T$ & $\rho$ & $c_{v}, \exp$ & $\mathrm{C}_{\mathrm{v}}, \mathrm{cal}$ & Dev & Wt & $\mathrm{P}$ & Devl & Dev2 & Dev3 & Dev4 \\
\hline K & $\mathrm{mol} \cdot \mathrm{dm}^{-3}$ & $\mathrm{~J} \cdot \mathrm{mol}^{-}$ & $\cdot \mathrm{K}^{1}$ & 8 & & $\mathrm{MPa}$ & 8 & 8 & 8 & 8 \\
\hline 23.903 & 6.017 & 29.506 & 29.600 & 0.317 & 1266.1 & 17.2378 & -0.19 & 0.47 & 0.330 & 0.012 \\
\hline 23.224 & 16.018 & 29.500 & 29.611 & 0.375 & 1258.8 & 17.0049 & -0.19 & 0.46 & 0.388 & 0.013 \\
\hline 57.424 & 16.025 & 29.450 & 29.609 & 0.540 & 1687.0 & 28.9799 & -0.11 & 0.25 & 0.534 & -0.006 \\
\hline 217.884 & 16.026 & 29.449 & 29.718 & 0.912 & 1202.3 & 15.1722 & -0.19 & 0.47 & 0.932 & 0.019 \\
\hline 250.777 & 16.036 & 29.381 & 29.540 & 0.543 & 1607.2 & 26.6958 & -0.13 & 0.30 & 0.537 & -0.005 \\
\hline 209.230 & 6.037 & 29.880 & 29.989 & 0.365 & 1075.9 & 12.2102 & -0.17 & 0.43 & 0.394 & 0.029 \\
\hline 244.048 & 16.047 & 29.337 & 29.499 & 0.552 & 1525.5 & 24.3763 & -0.15 & 0.35 & 0.548 & -0.004 \\
\hline 200.031 & 16.052 & 30.364 & 30.460 & 0.316 & 951.2 & 9.0844 & -0.16 & 0.41 & 0.358 & 0.043 \\
\hline 199.628 & 16.058 & $30.4 / 48$ & 30.484 & 0.117 & 942.2 & 8.9482 & -0.19 & 0.50 & 0.169 & 0.052 \\
\hline 237.251 & 16.058 & 29.456 & 29.490 & 0.114 & 1429.0 & 22.0273 & -0.16 & 0.39 & 0.114 & 0.000 \\
\hline 7.622 & 6.061 & .772 & 30.625 & .476 & 904.0 & 8.2721 & -0.1 & 0.48 & -0.422 & 0.055 \\
\hline 195.615 & 16.064 & 30.656 & 30.785 & 0.420 & 891.9 & 7.5981 & -0.18 & 0.46 & 0.479 & 0.058 \\
\hline 192.871 & 16.065 & 31.025 & 31.041 & 0.052 & 0.0 & 6.6817 & -0.14 & 0.36 & 0.1 & 0.053 \\
\hline 193.605 & 16.067 & 30.778 & 30.966 & 0.612 & 866.6 & 6.9263 & -0.1 & 0.42 & 0.672 & 0.060 \\
\hline 230.437 & 16.069 & 29.349 & 29.520 & 0.581 & 1356.5 & 19.6687 & -0.18 & 0.43 & 0.586 & 0.005 \\
\hline 1.595 & 5.070 & .347 & 31. & .551 & 0.0 & 6.2 & -0.1 & 0.37 & 0.492 & .059 \\
\hline 189.591 & 16.073 & 31.445 & 31.415 & -0.097 & 0.0 & 5.5958 & -0.12 & 0.31 & -0.040 & 0.056 \\
\hline 187.587 & 16.076 & 31.801 & 31.697 & -0.328 & 0.0 & 4.9389 & -0.08 & 0.22 & -0.283 & 0.045 \\
\hline 185.501 & 16.079 & 32.221 & 32.048 & -0.537 & 0.0 & 4.2614 & -0.02 & 0.07 & -0 . & 0.016 \\
\hline 223.900 & 16.080 & 29.472 & 29.593 & 0.409 & 1268.9 & 17.4046 & -0.19 & 0.46 & 20 & 0.011 \\
\hline 3.352 & 5.081 & .495 & 29 & 359 & 126 & 17. & -0.1 & .46 & & .012 \\
\hline 216.494 & 16.091 & 29.582 & 29.740 & 0.533 & 1176.3 & 14.8411 & -0.18 & 0.46 & 53 & 0.020 \\
\hline 216.509 & 16.091 & 29.546 & 29.739 & 0.655 & 1179.3 & 14.8464 & -0.18 & 0.46 & 0.675 & 0.020 \\
\hline 209.569 & 16.102 & 29.912 & 29.956 & 0.147 & 1077.2 & 12.4502 & -0.18 & 0.46 & 0.176 & 0.029 \\
\hline 209.408 & 16.102 & 29.858 & 29.962 & 0.349 & 1079.3 & 12.3948 & -0.18 & 0.46 & 0.378 & 0.029 \\
\hline 202.632 & 16.113 & 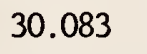 & 30 & 4 & 994.6 & 71 & -0.1 & 45 & & .040 \\
\hline 202.277 & 16.113 & 30.311 & 30.294 & -0.057 & 976.5 & 9.9456 & -0.17 & 0.44 & -0.017 & 0.039 \\
\hline 199.140 & 16.118 & 30.308 & 30.486 & 0.587 & 946.0 & 8.8745 & -0.16 & 0.42 & 0.631 & 0.044 \\
\hline 195.637 & 16.124 & 30.676 & 30.746 & 0.227 & 891.0 & 7.6852 & -0.15 & 0.40 & 0.276 & 0.049 \\
\hline 195.118 & 16.124 & 30.702 & 30.789 & 0.285 & 884.7 & 7.5098 & -0.14 & 0.38 & 0.332 & 0.047 \\
\hline 191.577 & שת. & 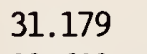 & & . & .0 & & & $a^{2}$ & & G. \\
\hline 188.669 & 16.134 & 31.621 & 31.480 . & -0.446 & 0.0 & 5.3493 & -0.08 & 0.21 & -0.407 & 0.039 \\
\hline 187.947 & 16.135 & 31.669 & 31.581 & -0.279 & 0.0 & 5.1103 & -0.06 & 0.18 & -0.245 & 0.034 \\
\hline 259.304 & 16.612 & 29.709 & 29.707 & -0.008 & 1682.7 & 32.3473 & -0.08 & 0.20 & -0.014 & -0.006 \\
\hline 253.921 & 16.622 & 29.624 & 29.634 & 0.033 & 1621.8 & 30.3550 & -0.10 & 0.26 & 0.026 & -0.007 \\
\hline
\end{tabular}


Table A6 (continued)

\begin{tabular}{|c|c|c|c|c|c|c|c|c|c|c|}
\hline $\mathrm{T}$ & $\rho$ & $C_{v}, \exp$ & $\mathrm{C}_{\mathrm{v}}, \mathrm{cal}$ & Dev & Wt & $P$ & Devl & Dev2 & Dev3 & Dev4 \\
\hline K & $\mathrm{mol} \cdot \mathrm{dm}^{-3}$ & $\mathrm{~J} \cdot \mathrm{mol}^{-}$ & $\cdot \mathrm{K}^{1}$ & 8 & & $\mathrm{MPa}$ & 8 & 8 & 8 & 8 \\
\hline 248.519 & 16.632 & 29.472 & 29.575 & 0.351 & 1568.4 & 28.3488 & -0.12 & 0.31 & $0.34 / 4$ & -0.007 \\
\hline 243.097 & 16.641 & 29.579 & 29.533 & -0.156 & 1489.1 & 26.3288 & -0.13 & 0.34 & -0.162 & -0.006 \\
\hline 237.657 & 16.651 & 29.416 & 29.508 & 0.314 & 1437.8 & 24.2969 & -0.15 & 0.39 & 0.309 & -0.005 \\
\hline 232.337 & 16.661 & 29.337 & 29.504 & 0.570 & 1380.5 & 22.3052 & -0.16 & 0.43 & 0.568 & -0.002 \\
\hline 230.407 & 16.664 & 29.196 & 29.508 & 1.070 & 1370.2 & 21.5817 & -0.16 & 0.43 & 1.069 & -0.001 \\
\hline 227.102 & 16.670 & 29.097 & 23 & 1.462 & 1339.4 & 20.3420 & -0.17 & 0.45 & 1.464 & 0.002 \\
\hline 225.003 & 16.673 & 29.481 & 29. & 0.189 & 1280.9 & 19.5541 & -0.16 & 0.45 & 0.193 & 0.003 \\
\hline 219.582 & 16.683 & 29.444 & 29.595 & 0.512 & 1221.9 & 17.5183 & -0.17 & 0.48 & 0.521 & 0.009 \\
\hline 216.658 & 16.688 & 29.633 & 29.640 & 0.025 & 1174.1 & 16.4203 & -0.17 & 0.49 & 0.037 & 0.012 \\
\hline 215.024 & 16.691 & 29.546 & 29.671 & 0.422 & 1162.9 & 15.8067 & -0.17 & 0.49 & 0.436 & 0.014 \\
\hline 14.143 & 6.692 & 29.5 & 29 & 0.466 & 1152.8 & 15.4759 & -0 . & 0.48 & 0.480 & .015 \\
\hline 211.348 & 16.697 & 29.684 & 29.753 & 0.232 & 1112.5 & 14.4271 & -0.17 & 0.49 & 0.250 & .018 \\
\hline 210.401 & 16.699 & 29.578 & 29.777 & 0.674 & 1110.1 & 14.0717 & -0.17 & 0.49 & 0.694 & 0.020 \\
\hline 208.680 & 16.701 & 29.876 & 29.826 & -0.166 & 1070.4 & 13.4263 & -0.16 & 0.48 & -0.145 & 0.021 \\
\hline 206.062 & 16.706 & 29.891 & 29.911 & 0.066 & 1042.2 & 12.4457 & -0.16 & 0.48 & 0.091 & 0.025 \\
\hline 206.122 & 6.7 & 29.7 & & 4 & 1055.2 & 12.4681 & -0 . & 0.50 & 0.690 & .026 \\
\hline 202.937 & 16.710 & 29.921 & 30 & 0.366 & 1008.2 & 11.2772 & -0. & 0.45 & 0.393 & .027 \\
\hline 201.094 & 16.7 & 29.8 & 30 & 0.791 & 992.6 & 10.5894 & -0 . & 0.46 & 0.821 & 0.030 \\
\hline 195.445 & 16.7 & 30.354 & 30. & 0.220 & 907.8 & 8.4891 & -0.13 & 0.41 & 0.256 & 0.036 \\
\hline 194.733 & 16.723 & 30.360 & 30.468 & 0.356 & 900.7 & 8.2252 & -0.12 & 0.39 & 0.391 & 0.034 \\
\hline 190.103 & $\cdots$ & 30.8 & & & & 6.5 & & & & .039 \\
\hline 187.826 & 16.73 & 31.032 & 31. & 0.046 & 0.0 & 5.6863 & -0.0 & 0.30 & 0.082 & 0.036 \\
\hline 184.764 & 16.741 & 31.395 & 31. & 0.026 & 0.0 & 4.5748 & -0. & 0.24 & 0.060 & 0.034 \\
\hline 252.926 & 16.8 & 29.4 & 29 & 0.571 & 1623.5 & 31.2877 & -0.0 & 0.21 & 0.565 & -0.006 \\
\hline 245.293 & 16.908 & 29.446 & 29.576 & 0.442 & 1530.1 & 28.3488 & -0.10 & 0.27 & 0.436 & -0.007 \\
\hline 235.598 & 16.9 & 20. & & & 1401.6 & 73 & . & & & 0.005 \\
\hline 227.768 & 16.940 & 29.468 & 29.520 & 75 & 1314.3 & 21.5545 & -0.1 & 0.39 & 0.174 & -0.001 \\
\hline 219.901 & 16.954 & 29.531 & 29.572 & 38 & 1218.4 & 18.4899 & -0.14 & 0.42 & 0.142 & 0.004 \\
\hline 211.988 & 16.96 & 29.7 & 29. & -0.238 & 1113.6 & 15.4046 & -0.14 & 0.43 & -0.226 & 0.012 \\
\hline 203.953 & 16.982 & 29.834 & 29.909 & 0.251 & 1024.4 & 12.2749 & -0.13 & 0.41 & 0.271 & 0.020 \\
\hline 196.587 & 16.9 & Ju. & & & & 9.4174 & -0.11 & 0.40 & 0.422 & 0.028 \\
\hline 193.924 & 17.001 & 30.219 & 30.377 & 0.522 & 901.2 & 8.3890 & -0.11 & 0.39 & 0.553 & 0.030 \\
\hline 187.325 & 17.011 & 30.929 & 30.880 & -0.1 & 0.0 & 5.8591 & -0.07 & 0.30 & -0.127 & 0.031 \\
\hline 240.990 & 17.941 & 29.691 & 29.682 & -0.029 & 1452.2 & 31.7566 & -0.07 & 0.21 & -0.036 & -0.007 \\
\hline 234.304 & 17.955 & 29.518 & 29.626 & 0.365 & 1387.4 & 28.8038 & -0.08 & 0.26 & 0.358 & -0.007 \\
\hline
\end{tabular}


Table A6 (continued)

\begin{tabular}{|c|c|c|c|c|c|c|c|c|c|c|}
\hline $\mathrm{T}$ & $\rho$ & $C_{v}, \exp$ & $\mathrm{C}_{\mathrm{V}}, \mathrm{cal}$ & Dev & Wt & $P$ & Devl & Dev2 & Dev3 & Dev4 \\
\hline $\mathrm{K}$ & $\mathrm{mol} \cdot \mathrm{dm}^{-3}$ & $\mathrm{~J} \cdot \mathrm{mol}$ & ${ }^{1} \cdot \mathrm{K}^{1}$ & 8 & & $\mathrm{MPa}$ & 8 & 8 & 8 & 8 \\
\hline 28.488 & 7.967 & 29.563 & 29.594 & 0.105 & 1314.4 & 26.2230 & -0.09 & 0.30 & 0.098 & -0.007 \\
\hline 222.620 & 17.979 & 29.618 & 29.581 & -0.125 & 1242.1 & 23.6084 & -0.10 & 0.34 & -0.130 & -0.005 \\
\hline 216.641 & 17.992 & 29.533 & 29.592 & 0.199 & 1181.8 & 20.9345 & -0.10 & 0.38 & 0.196 & -0.003 \\
\hline 210.370 & 18.005 & 29.581 & 29.634 & 0.180 & 1109.6 & 18.1216 & -0.10 & 0.40 & 0.180 & 0.001 \\
\hline 204.379 & 18.017 & 29.725 & 29.713 & -0.041 & 1036.2 & 15.4279 & -0.10 & 0.40 & -0.036 & 0.005 \\
\hline 98.35 & 8.029 & 29.850 & 29.8 & 1 & 966.8 & 12.7163 & -0.09 & 0.38 & -0.022 & 0.010 \\
\hline 192.299 & 18.041 & 30.133 & 30. & -0.325 & 890.8 & 9.9914 & -0.08 & 0.37 & 0.310 & 0.014 \\
\hline 186.224 & 18.053 & 30.360 & 30 & -0.125 & 1233.1 & 7.2642 & -0.07 & 0.40 & -0.105 & 0.020 \\
\hline 181.649 & 18.065 & 30.767 & 30.621 & -0.474 & 0.0 & 5.2194 & -0.09 & 0.62 & -0.439 & 0.036 \\
\hline 178.581 & 18.071 & 31.036 & 30.878 & -0.509 & 10.8 & 3.8554 & -0.11 & 0.85 & -0.459 & 0.050 \\
\hline 22.829 & 19.346 & .5 & 29. & 0.900 & 1250.7 & 1.3168 & -0 . & 0.10 & .897 & -0.004 \\
\hline 223.411 & 19.3448 & 29.6 & 29. & 0.596 & 1249.5 & 48 & -0.02 & 0.07 & .594 & -0.003 \\
\hline 221.388 & 19.349 & 29.3 & 29. & 1.591 & 1251.7 & 30.5529 & -0.02 & 0.09 & .588 & -0.003 \\
\hline 20.207 & 19.352 & 29.696 & 29. & 0.326 & 1208.6 & 29.9262 & -0.02 & 0.10 & 0.322 & -0.004 \\
\hline 220.387 & 19.356 & 29.677 & 29.795 & 0.397 & 1212.1 & 30.0506 & -0.02 & 0.10 & 0.393 & -0.004 \\
\hline 03 & 9.360 & 7 & & & & & & 4 & & -0.005 \\
\hline 217.573 & 19.363 & 29.586 & 29.780 & 0.656 & 1188.0 & 28.5526 & -0.03 & 0.12 & 0.652 & -0.004 \\
\hline 215.238 & 19.364 & 29.794 & 29.769 & -0.084 & 1146.3 & 27.2787 & -0 . & 0.12 & -0.087 & -0.004 \\
\hline 214.493 & 19.366 & 29.584 & 29.766 & 0.617 & 1154.1 & 26.8804 & -0 & 0.13 & 0.613 & -0.004 \\
\hline 214.522 & 19.370 & 29.610 & 29.767 & 0.532 & 1152.4 & 26.9228 & -0.03 & 0.12 & 0.528 & -0.004 \\
\hline 1.785 & 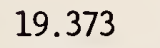 & 2 & & & & $\infty$ & & & & -0.004 \\
\hline 211.480 & 19.378 & 29.665 & 29. & 0.317 & 1115.3 & 25.2928 & -0.03 & 0.15 & 0.313 & -0.004 \\
\hline 209.060 & 19.379 & 29.638 & 29.755 & 0.394 & 1091.4 & 23.9668 & -0.03 & 0.14 & .390 & -0.004 \\
\hline 208.755 & 19.380 & 29.6 & 29. & 0.322 & 1086.6 & 23.8025 & -0 & 0.15 & .318 & -0.004 \\
\hline 208.615 & 19.385 & 29.669 & 29.755 & 0.291 & 1084.4 & 23.7528 & -0.03 & 0.16 & 0.287 & -0.004 \\
\hline 205.568 & 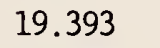 & & & & & & & & & -0.004 \\
\hline 202.844 & 19.395 & 29.5 & 29. & 0.601 & 1030.0 & 20.6 & -0 & 0.18 & 0.598 & -0.003 \\
\hline 202.557 & 19.395 & 29.748 & 29. & 0.046 & 1015.9 & 20.4 & -0 & 0.16 & 0.043 & -0.003 \\
\hline 202.661 & 19.400 & 29.781 & 29. & -0.064 & 1014.7 & 20.5377 & -0.04 & 0.18 & -0.067 & -0.003 \\
\hline 199.616 & 19.407 & 29.719 & 29.775 & 0.187 & 987.8 & 18.8860 & -0.03 & 0.17 & 0.185 & -0.002 \\
\hline 196.210 & 9.4 & 2 & & & & & & 0.10 & & -0.001 \\
\hline 195.819 & 19.412 & 29.7 & 29. & & 946.2 & 16.7984 & -0 & 0.18 & 0.075 & -0.001 \\
\hline 196.693 & 19.414 & 29.778 & 29.794 & & 954.8 & 17.2961 & -0 & 0.17 & 0.052 & -0.001 \\
\hline 193.640 & 19.422 & 29.818 & 29.822 & 0.015 & 922.3 & 15.6310 & -0.03 & 0.18 & 0.014 & -0.001 \\
\hline 189.912 & 19.426 & 29.755 & 29.872 & 0.392 & 1335.1 & 13.5728 & -0.03 & 0.16 & 0.392 & 0.000 \\
\hline
\end{tabular}


Table A6 (continued)

\begin{tabular}{|c|c|c|c|c|c|c|c|c|c|c|}
\hline $\mathrm{T}$ & $\rho$ & $C_{v}, \exp$ & $\mathrm{C}_{\mathrm{v}}, \mathrm{cal}$ & Dev & Wt & $P$ & Devl & Dev2 & Dev3 & lev4 \\
\hline K & $\mathrm{mol} \cdot \mathrm{dm}^{-3}$ & $\mathrm{~J} \cdot \mathrm{mol}^{-}$ & $1 \cdot K^{1}$ & 8 & & $\mathrm{MPa}$ & 8 & 8 & 8 & 8 \\
\hline 89.464 & 19.427 & 29.826 & 29.879 & 0.177 & 1322.5 & 13.3278 & -0.03 & 0.16 & 0.177 & 0.000 \\
\hline 189.446 & 19.427 & 29.896 & 29.879 & -0.057 & 1316.2 & 13.3178 & -0.03 & 0.16 & -0.056 & 0.000 \\
\hline 190.680 & 19.429 & 29.878 & 29.860 & -0.060 & 890.2 & 14.0129 & -0.03 & 0.18 & -0.060 & 0.000 \\
\hline 187.628 & 19.436 & 29.890 & 29.911 & 0.069 & 1290.9 & 12.3409 & -0.02 & 0.16 & 0.070 & 0.001 \\
\hline 183.556 & 19.441 & 30.224 & 30.002 & -0.736 & 1207.7 & 10.0867 & -0.02 & 0.17 & -0.734 & 0.002 \\
\hline 33.084 & 9.442 & .061 & 30. & .156 & 1214.1 & 9.8271 & -0.02 & 0.17 & -0.154 & .002 \\
\hline 183.063 & 19.442 & 30.107 & 30.015 & -0.306 & 1210.2 & 9.8159 & -0.02 & 0.16 & -0.304 & 0.002 \\
\hline 184.637 & 19.443 & 30.060 & 29.974 & -0.285 & 1235.4 & 10.6985 & -0.02 & 0.16 & -0.284 & 0.002 \\
\hline 181.613 & 19.451 & 30.105 & 30.055 & -0.166 & 1190.8 & 9.0348 & -0.03 & 0.22 & -0.163 & 0.003 \\
\hline 180.587 & 19.453 & 30.258 & 30.0 & -0.565 & 1165.5 & 8.4696 & -0.03 & 0.23 & -0.562 & 0.004 \\
\hline 77.160 & 9.4 & 0.226 & & 1 & 122.9 & 59 & -0.03 & 0.31 & .035 & .006 \\
\hline 176.684 & 19.457 & 30.313 & 30. & -0.262 & 1110.5 & 6.3037 & -0.03 & 0.33 & -0.255 & 0.007 \\
\hline 176.618 & 19.457 & 30.236 & 30.236 & 0.002 & 1115.1 & 6.2669 & -0.03 & 0.33 & 0.008 & 0.007 \\
\hline 178.550 & 19.458 & 30.384 & 30.158 & -0.745 & 1129.5 & 7.3475 & -0.03 & 0.29 & -0.740 & 0.005 \\
\hline 174.095 & 19.464 & 30.202 & 30.354 & 0.504 & 1085.1 & & -0.05 & & 0.517 & 0.013 \\
\hline 4.742 & 9.4 & & & & 470.4 & & & & & .012 \\
\hline 172.603 & 19.472 & 30.453 & 30. & -0.068 & 10.5 & 4.0666 & -0.06 & 0.86 & -0.050 & 0.018 \\
\hline 182.089 & 20.470 & 30.087 & 30. & -0.238 & 1198.6 & 13.9877 & -0.01 & 0.05 & -0.239 & 0.000 \\
\hline 178.506 & 20.479 & 30.163 & 30. & -0.347 & 1145.1 & 11.7168 & 0.00 & 0.02 & -0.347 & 0.000 \\
\hline 174.898 & 20.489 & 30.217 & 30. & -0.321 & 1094.3 & 9.4196 & -0.01 & 0.06 & -0.321 & 0.000 \\
\hline 12.195 & 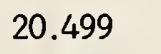 & & & & כ & & -u. & & & 0.001 \\
\hline 170.403 & 20.504 & 30.2 & 30 & -0 & 1032.1 & 6.5423 & -0.03 & 0.44 & -0.212 & 0.003 \\
\hline 200.078 & 21.167 & 29.929 & 30. & 0.908 & 978.9 & 31.0692 & 0.03 & -0.16 & 0.915 & 0.007 \\
\hline 198.229 & 21.171 & 29.957 & 30.192 & 0.784 & 958.8 & 29.8244 & 0.04 & -0.21 & 0.793 & 0.009 \\
\hline 194.626 & 21.184 & 30.043 & 30.179 & 0.453 & 918.4 & 27.3861 & 0.03 & -0.16 & 0.459 & 0.006 \\
\hline 191.134 & 2. & & & & 893.9 & & 0. & & & 0.008 \\
\hline 189.153 & 21.201 & 30.0 & 30 & 92 & 1299.1 & 23.6 & 0.03 & -0.18 & 98 & 0.006 \\
\hline 185.182 & 21.211 & 30.004 & 30.161 & 0.522 & 1247.4 & 20.9182 & 0.04 & -0.28 & 0.530 & 0.008 \\
\hline 183.640 & 21.218 & 30.035 & 30.162 & 0.422 & 1223.8 & 19.8512 & 0.03 & -0.21 & 0.427 & 0.006 \\
\hline 178.582 & 21.231 & 30.088 & 30.173 & 0.281 & 1151.7 & 16.3306 & 0.04 & -0.36 & 0.288 & 0.007 \\
\hline 170.031 & 2. & & & & & & & & & .006 \\
\hline 172.488 & 21.251 & 30.155 & 30. & 0.197 & 1067.8 & 12.0442 & 0.03 & -0.36 & 0.201 & 0.004 \\
\hline 171.921 & 21.252 & 30.323 & 30.220 & -0.339 & 1049.2 & 11.6427 & 0.04 & -0.41 & -0.335 & 0.004 \\
\hline 166.828 & 21.269 & 30.358 & 30.294 & -0.210 & 984.1 & 8.0183 & 0.02 & -0.30 & -0.209 & 0.001 \\
\hline 165.189 & 21.272 & 30.406 & 30.327 & -0.258 & 961.4 & 6.8445 & 0.02 & -0.40 & -0.258 & 0.000 \\
\hline
\end{tabular}


Table A6 (continued)

\begin{tabular}{|c|c|c|c|c|c|c|c|c|c|c|}
\hline $\mathrm{T}$ & $\rho$ & $C_{v}, \exp$ & $\mathrm{C}_{\mathrm{v}}, \mathrm{cal}$ & Dev & Wt & $\mathbf{P}$ & Devl & Dev2 & Dev3 & Dev4 \\
\hline K & $\mathrm{mol} \cdot \mathrm{dm}^{-3}$ & $\mathrm{~J} \cdot \mathrm{mol}^{-}$ & $K^{-1}$ & 8 & & $\mathrm{MPa}$ & 8 & 8 & 8 & 8 \\
\hline 61.140 & 1.286 & 30.358 & 30.436 & 0.256 & 9.2 & 3.9294 & -0.01 & 0.19 & 0.257 & 0.000 \\
\hline 187.701 & 21.988 & 30.282 & 30.456 & 0.573 & 1259.5 & 29.3776 & 0.03 & -0.23 & .583 & 0.010 \\
\hline 183.514 & 22.003 & 30.245 & 30.441 & 0.649 & 1205.5 & 26.2195 & 0.03 & -0.23 & 0.658 & 0.009 \\
\hline 179.314 & 22.017 & 30.429 & 30.430 & 0.003 & 1136.2 & 23.0213 & 0.03 & -0.28 & 0.013 & .010 \\
\hline 175.011 & 22.032 & 30.383 & 30.425 & 0.137 & 1084.1 & 19.7127 & 0.03 & -0.32 & 0.146 & 0.009 \\
\hline 70.767 & 2.046 & .536 & 30.428 & .354 & 1020.8 & 16.4170 & 0.04 & 0.40 & -0.345 & 0.009 \\
\hline 166.497 & 22.060 & 30.671 & 30.444 & .740 & 960.8 & 13.0668 & 0.04 & -0.50 & -0.732 & .008 \\
\hline 162.200 & 22.075 & 30.653 & 30.480 & -0.565 & 911.5 & 9.6595 & 0.03 & -0.52 & -0.559 & .005 \\
\hline 157.876 & 22.090 & 30.769 & 30.544 & -0.732 & 855.8 & 6.1932 & 0.02 & -0.41 & 0.730 & .002 \\
\hline 153.984 & 22.102 & 30.845 & 30.635 & -0.682 & 0.1 & 3.0412 & 0.00 & -0.01 & -0.682 & 0.000 \\
\hline 75.530 & 2.813 & .720 & & 0.180 & 067.5 & 27.7047 & 0 . & -0.47 & 0.200 & .020 \\
\hline 175.122 & 22.815 & 30.620 & 30.774 & $0.50 \alpha_{4}$ & 1069.2 & 27.3613 & 0.05 & -0.46 & 0.523 & 0.019 \\
\hline 169.076 & 22.838 & 30.651 & 30.757 & 0.345 & 992.9 & 22.2365 & 0.06 & -0.58 & 0.366 & .020 \\
\hline 168.648 & 22.839 & 30.749 & 30. & 0.022 & 981.6 & 21.8708 & 0 . & 0.62 & 0.043 & .021 \\
\hline 162.555 & 22.862 & 30.764 & 30.752 & -0.040 & 909.2 & 16.6099 & 0.06 & -0.82 & -0.020 & 0.021 \\
\hline 52.130 & 2.864 & 0.799 & & .152 & 90 & 16 & 0 . & -0.81 & 0 . & .020 \\
\hline 156.936 & 22.881 & 30.901 & 30.770 & -0.425 & 838.3 & 11.6734 & 0.0 & 1.24 & -0.405 & 0.021 \\
\hline 155.969 & 22.887 & 30.957 & 30.777 & -0.581 & 824.8 & 10.8146 & 0. & 1.11 & -0.565 & 0.017 \\
\hline 155.536 & 22.888 & 30.956 & 30 & -0.568 & 820.2 & 10.4300 & 0. & 1.19 & -0.551 & 0.017 \\
\hline 148.828 & 22.913 & 31.148 & 30.871 & -0.888 & 74.0 & 4.3941 & 0.04 & 1.76 & -0.881 & 0.007 \\
\hline 164.346 & 3.368 & $093 /$ & & 56 & 0 & 23 & & -0.40 & 71 & .015 \\
\hline 160.058 & 23.385 & 31.014 & 31.005 & -0.028 & 866.9 & 19.5801 & 0. & -0.54 & -0.011 & 0.017 \\
\hline 156.170 & 23.403 & 31.053 & 31.007 & -0.148 & 822.1 & 15.9591 & 0. & -0.53 & -0.134 & 0.013 \\
\hline 152.695 & 23.417 & 31.101 & & -0.269 & 782.4 & 12.6822 & 0. & -0.64 & -0.256 & 0.012 \\
\hline 150.090 & 23.431 & 31.142 & 31.035 & -0.343 & 753.2 & 10.1996 & 0.02 & -0.39 & -0.337 & 0.006 \\
\hline 148.358 & 3.438 & 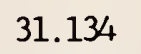 & & -0.266 & & 8. & & & 1 & .005 \\
\hline 146.608 & 23.445 & 31.162 & 31.073 & -0.287 & 7166.1 & 6.8455 & 0.01 & -0.34 & -0.284 & 0.003 \\
\hline 164.072 & 23.885 & 31.111 & 31.320 & 0.671 & 906.7 & 29.5630 & 0. & -0.22 & 0.681 & 0.010 \\
\hline 160.011 & 23.903 & 31.1 & & & & 25.6422 & & & & 0.012 \\
\hline 155.908 & 23.921 & 31.397 & 31.300 & -0.308 & 801.9 & 21.6240 & 0.03 & -0.42 & -0.294 & 0.014 \\
\hline 151.599 & 3.9 & . & & -0.014 & & 10. & & & & 0.017 \\
\hline 147.448 & 23.957 & 31.480 & 31. & -0.539 & 7110.5 & 13.1452 & 0.04 & -0.83 & -0.522 & 0.017 \\
\hline 143.270 & 23.975 & 31.525 & 31.342 & -0.580 & 6681.4 & 8.8550 & 0.0 & 1.10 & -0.566 & 0.014 \\
\hline 139.073 & 23.993 & 31.633 & 31.405 & -0.720 & 62.4 & 4.4671 & 0. & 1.41 & -0.712 & 0.008 \\
\hline 154.473 & 24.651 & 31.706 & 31.784 & 0.247 & 772.0 & 30.0452 & 0.02 & -0.25 & 0.258 & 0.011 \\
\hline
\end{tabular}


Table A6 (continued)

\begin{tabular}{|c|c|c|c|c|c|c|c|c|c|c|}
\hline $\mathrm{T}$ & $\rho$ & $C_{v}, \exp$ & $\mathrm{C}_{\mathrm{v}}, \mathrm{cal}$ & Dev & Wt & $\mathbf{P}$ & Devl & Dev2 & Dev3 & Dev4 \\
\hline $\mathrm{K}$ & $\mathrm{mol} \cdot \mathrm{dm}^{-3}$ & $\mathrm{~J} \cdot \mathrm{mol}^{-}$ & ${ }^{1} \cdot \mathrm{K}^{1}$ & 8 & & $\mathrm{MPa}$ & 8 & 8 & 8 & 8 \\
\hline 153.185 & 24.657 & 31.595 & 31.782 & 0.591 & 764.0 & 28.6816 & 0.02 & -0.31 & 0.605 & 0.013 \\
\hline 149.124 & 24.676 & 31.598 & 31.777 & 0.565 & 7226.0 & 24.3346 & 0.03 & -0.51 & 0.585 & 0.019 \\
\hline 147.886 & 24.682 & 31.651 & 31.776 & 0.396 & 7079.7 & 22.9952 & 0.04 & -0.57 & 0.416 & 0.021 \\
\hline 143.713 & 24.702 & 31.844 & 31.781 & -0.199 & 6594.8 & 18.4271 & 0.04 & -0.83 & -0.175 & 0.024 \\
\hline 142.547 & 24.708 & 31.794 & 31.785 & -0.030 & 6504.3 & 17.1361 & 0.04 & -0.88 & -0.006 & 0.024 \\
\hline 139.337 & 24.723 & 31.826 & 31.801 & -0.078 & 6192.4 & 13.5454 & 0.05 & 1.18 & -0.054 & 0.025 \\
\hline 138.242 & 24.728 & 32.209 & 31.809 & -1.240 & 5953.1 & 12.3089 & 0.05 & 1.32 & -1.216 & 0.025 \\
\hline 137.159 & 24.733 & 31.953 & 31.820 & -0.417 & 5947.5 & 11.0794 & 0.05 & 1.47 & -0.393 & 0.025 \\
\hline 134.136 & 24.749 & 32.039 & 31.860 & -0.558 & 5649.2 & 7.6115 & 0.04 & 1.65 & -0.540 & 0.018 \\
\hline 132.694 & 24.754 & 32.346 & 31.885 & -1.426 & 5423.4 & 5.9387 & 0.04 & 2.29 & -1.408 & 0.019 \\
\hline 131.693 & 24.759 & 32.305 & 31.906 & -1.236 & 5351.8 & 4.7717 & 0.04 & 2.57 & -1.220 & 0.016 \\
\hline 130.508 & 24.772 & 32.470 & 31.938 & -1.640 & 52.0 & 3.3808 & 0.00 & -0.45 & -1.638 & 0.002 \\
\hline 130.274 & 25.807 & 32.609 & 32.642 & 0.102 & 5139.3 & 18.3466 & 0.02 & -0.46 & 0.115 & 0.012 \\
\hline 127.262 & 25.823 & 32.654 & 32.675 & 0.064 & 4882.1 & 14.4728 & 0.03 & -0.81 & 0.081 & 0.017 \\
\hline 124.223 & 25.840 & 32.844 & 32.723 & -0.369 & 4590.9 & 10.4959 & 0.03 & 1.13 & -0.353 & 0.016 \\
\hline 121.160 & 25.856 & 33.035 & 32.789 & -0.745 & 4309.8 & 6.4101 & 0.03 & 1.76 & -0.730 & .015 \\
\hline 127.100 & 26.539 & 33.110 & 33.300 & 0.573 & 4741.4 & 27.6133 & 0.02 & -0.43 & 0.589 & 0.016 \\
\hline 127.123 & 26.539 & 33.141 & 33.300 & 0.478 & 4734.7 & 27.6449 & 0.02 & -0.42 & 0.494 & 0.016 \\
\hline 122.094 & 26.569 & 33.244 & 33.350 & 0.319 & 4326.9 & 20.7254 & 0.04 & 1.10 & 0.350 & 0.031 \\
\hline 122.089 & 26.569 & 33.348 & 33.350 & 0.006 & 4300.8 & 20.7187 & 0.04 & 1.11 & 0.037 & 0.031 \\
\hline 117.051 & 26.599 & 33.396 & 33.426 & 0.091 & 3927.5 & 13.5645 & 0.06 & 2.19 & & 0.039 \\
\hline 116.030 & 26.605 & 33.471 & 33.446 & -0.075 & 3839.6 & 12.0862 & 0.06 & 2.53 & -0.036 & 0.039 \\
\hline 111.917 & 26.629 & 33.691 & 33.537 & -0.457 & 3515.7 & 6.0116 & 0.06 & 4.88 & -0.424 & 0.034 \\
\hline 111.855 & 26.629 & 33.625 & 33.538 & -0.258 & 3524.7 & 5.9191 & 0.06 & 5.06 & -0.224 & 0.034 \\
\hline 110.867 & 26.635 & 33.783 & 33.563 & -0.650 & 34.3 & 4.4383 & 0.06 & 6.48 & -0.619 & 0.032 \\
\hline 117.361 & 27.268 & 33.843 & 34.045 & 0.598 & 3850.3 & 28.2504 & 0.03 & -0.60 & 0.620 & 0.021 \\
\hline 115.142 & 27.283 & 33.956 & 34.070 & 0.336 & 3676.1 & 24.9294 & 0.04 & -0.93 & 0.365 & 0.029 \\
\hline 112.446 & 27.300 & 34.003 & 34.100 & 0.284 & 3489.0 & 20.8258 & 0.05 & 1.56 & 0.324 & 0.040 \\
\hline 110.058 & 27.315 & 34.021 & 34.125 & 0.304 & 3331.9 & 17.1238 & 0.06 & 2.27 & 0.351 & 0.046 \\
\hline 107.466 & 27.332 & 34.088 & 34.147 & 0.174 & 3157.3 & 13.0295 & 0.07 & 3.23 & 0.222 & 0.048 \\
\hline 105.029 & 27.347 & 34.237 & 34.156 & & 2983.6 & 9.1061 & 0.07 & 4.91 & -0 . & 0.049 \\
\hline 102.424 & 27.365 & 34.360 & 34.144 & -0.628 & 2810.5 & 4.8191 & 0.07 & 8.14 & -0.589 & 0.040 \\
\hline 106.655 & 28.040 & 34.664 & 34.825 & 0.466 & 3010.2 & 28.4769 & 0.00 & -0.05 & 0.467 & 0.002 \\
\hline 105.104 & 28.051 & 34.616 & 34.813 & 0.569 & 2926.5 & 25.9193 & 0.01 & -0.34 & 0.579 & 0.010 \\
\hline 103.902 & 28.060 & 34.807 & 34.797 & -0.028 & 2826.8 & 23.9163 & 0.02 & -0.54 & -0.014 & 0.014 \\
\hline 02.319 & 28.070 & & & & 274 & 21.2466 & 0.03 & 1.05 & 0.066 & 0.024 \\
\hline 101.123 & 28.079 & 34.886 & 34.726 & -0.458 & 2658.3 & 19.2058 & 0.03 & 1.29 & -0.431 & 0.027 \\
\hline
\end{tabular}


Table A6 (continued)

Number of Points (Total) 337

\begin{tabular}{|c|c|c|c|c|c|c|c|c|}
\hline$C_{V}(T, \rho)$, & Dev : & $\begin{array}{l}A A D-8 \\
A A D\end{array}$ & $\begin{array}{l}0.462 \\
0.142\end{array}$ & $\begin{array}{l}\text { BIAS- } 8 \\
\text { BIAS }\end{array}$ & $\begin{array}{l}0.165 \\
0.047\end{array}$ & $\begin{array}{l}\text { RMS } 8 \\
\text { RMS }\end{array}$ & $\begin{array}{l}0.571 \\
0.176\end{array}$ & $\mathrm{~J} \cdot \mathrm{mol}^{-1} \cdot \mathrm{K}^{-1}$ \\
\hline$\rho(\mathrm{T}, \mathrm{P})$ & Devl : & $\begin{array}{l}A A D-8 \\
A A D\end{array}$ & $\begin{array}{l}0.119 \\
0.017\end{array}$ & $\begin{array}{l}\text { BIAS }-\frac{8}{8} \\
\text { BIAS }\end{array}$ & $\begin{array}{l}-0.052 \\
-0.007\end{array}$ & $\begin{array}{l}\text { RMS } 8 \\
\text { RMS }\end{array}$ & $\begin{array}{l}0.162 \\
0.020\end{array}$ & $\mathrm{~mol} \cdot \mathrm{dm}^{-3}$ \\
\hline $\mathrm{P}(\mathrm{T}, \rho)$ & Dev2 : & $\begin{array}{l}A A D-8 \\
A A D\end{array}$ & $\begin{array}{l}0.463 \\
0.059\end{array}$ & $\begin{array}{l}\text { BIAS- } \frac{8}{8} \\
\text { BIAS }\end{array}$ & $\begin{array}{l}-0.105 \\
-0.007\end{array}$ & $\begin{array}{l}\text { RMS } 8 \\
\text { RMS }\end{array}$ & $\begin{array}{l}0.916 \\
0.088\end{array}$ & $\mathrm{MPa}$ \\
\hline $\mathrm{C}_{\mathrm{V}}(\mathrm{T}, \mathrm{P})$, & Dev3 : & $\begin{array}{l}A A D-8 \\
A A D\end{array}$ & $\begin{array}{l}0.468 \\
0.144\end{array}$ & $\begin{array}{l}\text { BIAS- } 8 \\
\text { BIAS }\end{array}$ & $\begin{array}{l}0.171 \\
0.049\end{array}$ & $\begin{array}{l}\text { RMS\& } \\
\text { RMS }\end{array}$ & $\begin{array}{l}0.574 \\
0.177\end{array}$ & $\mathrm{~J} \cdot \mathrm{mol}^{-1} \cdot \mathrm{K}^{1}$ \\
\hline $\begin{array}{l}C_{V}(T, P) \\
C_{V}(T, \rho)\end{array}$ & , Dev4: & $\begin{array}{l}A A D-8 \\
A A D\end{array}$ & $\begin{array}{l}0.021 \\
0.007\end{array}$ & $\begin{array}{l}\text { BIAS-8 } \\
\text { BIAS }\end{array}$ & $\begin{array}{l}0.006 \\
0.002\end{array}$ & $\begin{array}{l}\text { RMS } 8 \\
\text { RMS }\end{array}$ & $\begin{array}{l}0.037 \\
0.012\end{array}$ & $\mathrm{~J} \cdot \mathrm{mol}^{-1} \cdot \mathrm{K}^{-1}$ \\
\hline
\end{tabular}


Notes for Table A7. Entries for the first 3 columns, the temperature, pressure, and isobaric specific heat capacity, are directly from the experimental data. The primary isobaric specific heat capacity data were exclusively from Ref. [54]; 12 points from this reference, indicated by negative weights, were excluded from the primary data set.

Data from Jones et al. [54]

$\begin{array}{crrrrr}\mathrm{T} & \mathrm{P} & \mathrm{C} \text {, exp } & \mathrm{C}_{\mathrm{p}}, \mathrm{Cal} & \mathrm{Dev} & \text { Wt } \\ \mathrm{K} & \mathrm{MPa} & \mathrm{J} \cdot \mathrm{mol}^{-1} \cdot \mathrm{K}^{-1} & \mathrm{~J} \cdot \mathrm{mol}^{-1} \cdot \mathrm{K}^{-1} & 8 & \\ 116.483 & 1.034 & 55.420 & 56.387 & 1.745 & 467.80 \\ 116.483 & 2.068 & 55.360 & 56.165 & 1.454 & 93.76 \\ 116.483 & 2.413 & 55.360 & 56.094 & 1.325 & 93.76 \\ 116.483 & 3.103 & 55.290 & 55.955 & 1.202 & 93.99 \\ 116.483 & 3.792 & 55.220 & 55.820 & 1.087 & 94.23 \\ & & & & & \\ 116.483 & 4.137 & 55.160 & 55.755 & 1.079 & 94.43 \\ 116.483 & 4.309 & 55.090 & 55.723 & 1.148 & 94.66 \\ 116.483 & 4.688 & 55.090 & 55.653 & 1.021 & 94.66 \\ 116.483 & 5.516 & 55.020 & 55.504 & 0.879 & 94.90 \\ 116.483 & 6.895 & 54.950 & 55.268 & 0.579 & 95.14 \\ 116.483 & 8.274 & 54.890 & 55.047 & 0.286 & 95.34 \\ 116.483 & 10.342 & 54.550 & 54.739 & 0.346 & 96.51 \\ 116.483 & 13.790 & 54.010 & 54.281 & 0.501 & 98.41 \\ 122.039 & 1.034 & 56.570 & 57.103 & 0.943 & 409.96 \\ 122.039 & 2.068 & 56.500 & 56.828 & 0.580 & 82.19 \\ 122.039 & 2.413 & 56.430 & 56.739 & 0.548 & 82.39 \\ 122.039 & 3.103 & 56.300 & 56.568 & 0.476 & 82.77 \\ 122.039 & 3.792 & 56.160 & 56.403 & 0.433 & 83.17 \\ 122.039 & 4.137 & 56.030 & 56.323 & 0.523 & 83.55 \\ 122.039 & 4.309 & 55.960 & 56.283 & 0.578 & 83.76 \\ 122.039 & 4.688 & 55.960 & 56.198 & 0.425 & 83.76 \\ 122.039 & 5.516 & 55.830 & 56.017 & 0.335 & 84.14 \\ 122.039 & 6.895 & 55.690 & 55.732 & 0.076 & 84.56 \\ 122.039 & 8.274 & 55.420 & 55.467 & 0.084 & 85.37 \\ 122.039 & 10.342 & 54.890 & 55.100 & 0.382 & 87.00 \\ 122.039 & 13.790 & 54.350 & 54.560 & 0.386 & 88.71 \\ 127.594 & 1.034 & 57.910 & 57.982 & 0.125 & 358.60 \\ 127.594 & 2.068 & 57.510 & 57.635 & 0.217 & 72.71 \\ 127.594 & 2.413 & 57.440 & 57.524 & 0.146 & 72.88 \\ 127.594 & 3.103 & 57.370 & 57.310 & -0.105 & 73.06\end{array}$


Data from Jones et al. [54] (continued)

\begin{tabular}{|c|c|c|c|c|c|}
\hline $\mathrm{T}$ & $P$ & $C_{p}, \exp$ & $C_{p}, c a 1$ & Dev & Wt \\
\hline K & $\mathrm{MPa}$ & $\mathrm{J} \cdot \mathrm{mol}^{-1} \cdot \mathrm{K}^{-1}$ & $\mathrm{~J} \cdot \mathrm{mol}^{-1} \cdot \mathrm{K}^{-1}$ & 8 & \\
\hline 127.594 & 3.792 & 57.040 & 57.105 & 0.115 & 73.89 \\
\hline 127.594 & 4.137 & 56.970 & 57.006 & 0.064 & 74.07 \\
\hline 127.594 & 4.309 & 56.900 & 56.958 & 0.101 & 74.25 \\
\hline 127.594 & 4.688 & 56.770 & 56.852 & 0.145 & 74.59 \\
\hline 127.594 & 5.516 & 56.500 & 56.630 & 0.231 & 75.29 \\
\hline 127.594 & 6.895 & 56.370 & 56.284 & -0.153 & 75.63 \\
\hline 127.594 & 8.274 & 56.030 & 55.963 & -0.120 & 76.54 \\
\hline 127.594 & 10.342 & 55.360 & 55.523 & 0.295 & 78.38 \\
\hline 127.594 & 13.790 & 54.750 & 54.885 & 0.246 & 80.1 \\
\hline 133.150 & 1.034 & 59.390 & 59.086 & -0.511 & 313.65 \\
\hline 133.150 & 2.068 & 58.780 & 58.640 & -0.238 & 64.02 \\
\hline 133.150 & 2.413 & 58.580 & 58.499 & -0.139 & 64.45 \\
\hline 133.150 & 3.103 & 56.380 & 58.227 & 3.277 & -0.00 \\
\hline 133.150 & 3.792 & 58.040 & 57.970 & -0.120 & 65.64 \\
\hline 133.150 & 4.137 & 57.910 & 57.846 & -0.111 & 65.94 \\
\hline 133.150 & 4.309 & 57.780 & 57.785 & 0.009 & 66.23 \\
\hline 133.150 & 4.688 & 57.710 & 57.654 & -0.097 & 66.39 \\
\hline 133.150 & 5.516 & 57.310 & 57.378 & 0.119 & 67.31 \\
\hline 133.150 & 6.895 & 57.100 & 56.952 & -0.259 & 67.79 \\
\hline 133.150 & 8.274 & 56.700 & 56.561 & -0.245 & 68.74 \\
\hline 133.150 & 10.342 & 55.960 & 56.032 & 0.128 & 70.55 \\
\hline 133.150 & 13.790 & 55.160 & 55.273 & 0.206 & 72.58 \\
\hline 138.706 & 1.034 & 61.140 & 60.496 & -1.054 & 273.15 \\
\hline 138.706 & 2.068 & 60.130 & 59.909 & -0.368 & 56.46 \\
\hline 138.706 & 2.413 & 59.930 & 59.725 & -0.342 & 56.83 \\
\hline 138.706 & 3.103 & 59.660 & 59.374 & -0.479 & 57.34 \\
\hline 138.706 & 3.792 & 59.460 & 59.044 & -0.699 & 57.73 \\
\hline 138.706 & 4.137 & 59.190 & 58.886 & -0.513 & 58.25 \\
\hline 138.706 & 4.309 & 58.990 & 58.809 & -0.307 & 58.64 \\
\hline 138.706 & 4.688 & 58.850 & 58.643 & -0.352 & 58.91 \\
\hline 138.706 & 5.516 & 58.520 & 58.296 & -0.382 & 59.57 \\
\hline 138.706 & 6.895 & 57.840 & 57.766 & -0.129 & 60.97 \\
\hline 138.706 & 8.274 & 57.370 & 57.285 & -0.149 & 61.96 \\
\hline 138.706 & 10.342 & 56.570 & 56.642 & 0.128 & 63.70 \\
\hline 138.706 & 13.790 & 55.560 & 55.737 & 0.319 & 66.01 \\
\hline
\end{tabular}


Data from Jones et a1. [54] (continued)

\begin{tabular}{|c|c|c|c|c|c|}
\hline $\mathrm{T}$ & $P$ & $C_{p}, \exp$ & $C_{p}, c a 1$ & Dev & Wt \\
\hline K & $\mathrm{MPa}$ & $\mathrm{J} \cdot \mathrm{mol}^{-1} \cdot \mathrm{K}^{-1}$ & $\mathrm{~J} \cdot \mathrm{mol}^{-1} \cdot \mathrm{K}^{-1}$ & 8 & \\
\hline 144.261 & 1.034 & 63.420 & 62.334 & -1.713 & 100.73 \\
\hline 144.261 & 2.068 & 61.740 & 61.536 & -0.330 & 21.25 \\
\hline 144.261 & 2.413 & 61.610 & 61.290 & -0.519 & 21.34 \\
\hline 144.261 & 3.103 & 61.000 & 60.825 & -0.287 & 21.76 \\
\hline 144.261 & 3.792 & 60.530 & 60.392 & -0.228 & 51.56 \\
\hline 144.261 & 4.137 & 60.400 & 60.186 & -0.354 & 51.78 \\
\hline 144.261 & 4.309 & 60.190 & 60.086 & -0.172 & 52.14 \\
\hline 144.261 & 4.688 & 60.060 & 59.872 & -0.313 & 52.36 \\
\hline 144.261 & 5.516 & 59.720 & 59.428 & -0.489 & 52.95 \\
\hline 144.261 & 6.895 & 58.850 & 58.758 & -0.157 & 54.51 \\
\hline 144.261 & 8.274 & 58.110 & 58.159 & 0.085 & 55.89 \\
\hline 144.261 & 10.342 & 57.370 & 57.372 & 0.004 & 57.33 \\
\hline 144.261 & 13.790 & 56.100 & 56.285 & 0.330 & 59.92 \\
\hline 149.817 & 2.068 & 63.760 & 63.677 & -0.130 & 18.50 \\
\hline 149.817 & 2.413 & 63.690 & 63.335 & -0.558 & 18.54 \\
\hline 149.817 & 3.103 & 62.880 & 62.695 & -0.294 & 19.01 \\
\hline 149.817 & 3.792 & 62.140 & 62.110 & -0.048 & 45.41 \\
\hline 149.817 & 4.137 & 61.810 & 61.835 & 0.040 & 45.90 \\
\hline 149.817 & 4.309 & 61.670 & 61.702 & 0.052 & 46.10 \\
\hline 149.817 & 4.688 & 61.270 & 61.418 & 0.241 & 46.70 \\
\hline 149.817 & 5.516 & 60.930 & 60.837 & -0.153 & 47.22 \\
\hline 149.817 & 6.895 & 59.790 & 59.973 & 0.307 & 49.02 \\
\hline 149.817 & 8.274 & 59.120 & 59.218 & 0.165 & 50.13 \\
\hline 149.817 & 10.342 & 58.250 & 58.242 & -0.014 & 51.62 \\
\hline 149.817 & 13.790 & 56.700 & 56.928 & 0.402 & 54.45 \\
\hline 155.372 & 1.034 & 42.120 & 43.390 & 3.016 & 13.01 \\
\hline 155.372 & 2.068 & 66.580 & 66.606 & 0.039 & 15.79 \\
\hline 155.372 & 2.413 & 66.380 & 66.102 & -0.419 & 15.88 \\
\hline 155.372 & 3.103 & 65.230 & 65.179 & -0.078 & 16.45 \\
\hline 155.372 & 3.792 & 64.160 & 64.353 & 0.301 & 39.65 \\
\hline 155.372 & 4.137 & 63.890 & 63.971 & 0.127 & 39.99 \\
\hline 155.372 & 4.309 & 63.620 & 63.788 & 0.263 & 40.32 \\
\hline 155.372 & 4.688 & 63.080 & 63.399 & 0.505 & 41.01 \\
\hline 155.372 & 5.516 & 62.410 & 62.614 & 0.328 & 41.89 \\
\hline 155.372 & 6.895 & 61.200 & 61.476 & 0.451 & 43.55 \\
\hline
\end{tabular}


Data from Jones et al. [54] (continued)

\begin{tabular}{|c|c|c|c|c|c|}
\hline $\mathrm{T}$ & $\mathbf{P}$ & $C_{p}, \exp$ & $C_{p}, c a l$ & Dev & Wt \\
\hline K & $\mathrm{MPa}$ & $\mathrm{J} \cdot \mathrm{mol}^{-1} \cdot \mathrm{K}^{-1}$ & $\mathrm{~J} \cdot \mathrm{mol}^{-1} \cdot \mathrm{K}^{-1}$ & 8 & \\
\hline 155.372 & 8.274 & 60.260 & 60.504 & 0.404 & 44.91 \\
\hline 155.372 & 10.342 & 59.320 & 59.279 & -0.070 & 46.33 \\
\hline 155.372 & 13.790 & 57.510 & 57.676 & 0.289 & 49.26 \\
\hline 160.928 & 1.034 & 40.510 & 41.624 & 2.751 & 13.11 \\
\hline 160.928 & 2.068 & 71.350 & 70.876 & -0.665 & 12.83 \\
\hline 160.928 & 2.413 & 69.870 & 70.068 & 0.283 & 13.38 \\
\hline 160.928 & 3.103 & 68.590 & 68.633 & 0.063 & 13.88 \\
\hline 160.928 & 3.792 & 67.050 & 67.396 & 0.516 & 33.88 \\
\hline 160.928 & 4.137 & 66.910 & 66.836 & -0.110 & 34.02 \\
\hline 160.928 & 4.309 & 66.380 & 66.570 & 0.286 & 34.57 \\
\hline 160.928 & 4.688 & 65.500 & 66.012 & 0.782 & 35.4 \\
\hline 160.928 & 5.516 & 64.630 & 64.911 & 0.435 & 36.4 \\
\hline 160.928 & 6.895 & 63.080 & 63.362 & 0.448 & 38.25 \\
\hline 160.928 & 8.274 & 61.740 & 62.082 & 0.553 & 39.9 \\
\hline 160.928 & 10.342 & 60.530 & 60.518 & -0.020 & 41.5 \\
\hline 160.928 & 13.790 & 58.450 & 58.545 & 0.162 & 44.4 \\
\hline 166.483 & 1.034 & 39.370 & 40.416 & 2.656 & 12.9 \\
\hline 166.483 & 2.413 & 76.050 & 76.317 & 0.351 & 10.5 \\
\hline 166.483 & 3.103 & 73.770 & 73.806 & 0.049 & 11.2 \\
\hline 166.483 & 3.792 & 71.620 & 71.770 & 0.209 & 27.7 \\
\hline 166.483 & 4.137 & 70.940 & 70.883 & -0.080 & 28.31 \\
\hline 166.483 & 4.309 & 70.680 & 70.469 & -0.298 & 28.5 \\
\hline 166.483 & 4.688 & 69.200 & 69.614 & 0.599 & 29.7 \\
\hline 166.483 & 5.516 & 68.320 & 67.978 & -0.500 & 30.5 \\
\hline 166.483 & 6.895 & 65.770 & 65.781 & 0.017 & 32.9 \\
\hline 166.483 & 8.274 & 63.820 & 64.043 & 0.350 & 34.9 \\
\hline 166.483 & 10.342 & 62.010 & 62.006 & -0.006 & 36.9 \\
\hline 166.483 & 13.790 & 59.520 & 59.550 & 0.051 & 40.1 \\
\hline 172.039 & 1.034 & 38.560 & 39.487 & 2.403 & 12.6 \\
\hline 172.039 & 2.068 & 55.490 & 55.055 & -0.785 & 1.2 \\
\hline 172.039 & 3.103 & 82.830 & 82.634 & -0.236 & 8.3 \\
\hline 172.039 & 3.792 & 78.940 & 78.698 & -0.307 & 21.4 \\
\hline 172.039 & 4.137 & 77.660 & 77.104 & -0.716 & 22.1 \\
\hline 172.039 & 4.309 & 77.190 & 76.381 & -1.049 & 22.4 \\
\hline 172.039 & 4.688 & 75.710 & 74.928 & -1.032 & 23.3 \\
\hline
\end{tabular}


Data from Jones et al. [54] (continued)

\begin{tabular}{|c|c|c|c|c|c|}
\hline $\mathrm{T}$ & $\mathbf{P}$ & $C_{p}, \exp$ & $C_{p}, c a l$ & Dev & Wt \\
\hline K & $\mathrm{MPa}$ & $\mathrm{J} \cdot \mathrm{mol}^{-1} \cdot \mathrm{K}^{-1}$ & $\mathrm{~J} \cdot \mathrm{mol}^{-1} \cdot \mathrm{K}^{-1}$ & 8 & \\
\hline 177.594 & 1.034 & 38.020 & 38.743 & 1.902 & 12.24 \\
\hline 177.594 & 2.068 & 50.650 & 50.001 & -1.281 & 1.39 \\
\hline 177.594 & 2.413 & 58.180 & 57.990 & -0.327 & 1.06 \\
\hline 177.594 & 3.103 & 106.080 & 102.644 & -3.239 & -0.00 \\
\hline 177.594 & 3.792 & 93.720 & 91.854 & -1.991 & 14.30 \\
\hline 177.594 & 4.137 & 89.080 & 88.205 & -0.982 & 15.82 \\
\hline 177.594 & 4.309 & 87.940 & 86.653 & -1.464 & 16.23 \\
\hline 177.594 & 4.688 & 84.580 & 83.704 & -1.036 & 17.54 \\
\hline 177.594 & 5.516 & 80.210 & 78.825 & -1.727 & 19.50 \\
\hline 177.594 & 6.895 & 74.370 & 73.408 & -1.294 & 22.66 \\
\hline 177.594 & 8.274 & 69.730 & 69.770 & 0.058 & 25.77 \\
\hline 177.594 & 10.342 & 65.430 & 66.026 & 0.911 & 29.24 \\
\hline 177.594 & 13.790 & 62.010 & 62.060 & 0.080 & 32.54 \\
\hline 183.150 & 1.034 & 37.620 & 38.139 & 1.380 & 11.76 \\
\hline 183.150 & 2.068 & 47.360 & 46.952 & -0.862 & 1.49 \\
\hline 183.150 & 2.413 & 52.740 & 52.149 & -1.121 & 1.21 \\
\hline 183.150 & 3.103 & 75.040 & 74.155 & -1.179 & 0.01 \\
\hline 183.150 & 3.792 & 132.480 & 132.968 & 0.369 & 0.00 \\
\hline 183.150 & 4.137 & 114.410 & 116.342 & 1.688 & 0.00 \\
\hline 183.150 & 4.309 & 109.370 & 110.792 & 1.301 & 0.00 \\
\hline 183.150 & 4.688 & 102.920 & 101.852 & -1.037 & 0.00 \\
\hline 183.150 & 5.516 & 89.750 & 90.150 & 0.446 & 0.00 \\
\hline 183.150 & 6.895 & 81.090 & 79.939 & -1.419 & 0.01 \\
\hline 183.150 & 8.274 & 74.300 & 74.136 & -0.220 & 21.36 \\
\hline 183.150 & 10.342 & 67.790 & 68.781 & 1.461 & 25.63 \\
\hline 183.150 & 13.790 & 63.490 & 63.618 & 0.202 & 29.20 \\
\hline 188.706 & 1.034 & 37.290 & 37.646 & 0.954 & 11.28 \\
\hline 188.706 & 2.068 & 44.940 & 44.836 & -0.232 & 1.56 \\
\hline 188.706 & 2.413 & 49.240 & 48.624 & -1.252 & 1.30 \\
\hline 188.706 & 3.103 & 62.010 & 61.310 & -1.128 & 0.01 \\
\hline 188.706 & 3.792 & 100.240 & 97.671 & -2.563 & 0.00 \\
\hline 188.706 & 4.137 & 164.660 & 174.573 & 6.020 & -0.00 \\
\hline 188.706 & 4.309 & 352.170 & 410.075 & 16.442 & -0.00 \\
\hline 188.706 & 4.688 & 184.480 & 178.776 & -3.092 & 0.00 \\
\hline 188.706 & 5.516 & 115.420 & 115.902 & 0.417 & 0.00 \\
\hline 188.706 & 6.895 & 90.900 & 90.598 & -0.332 & 0.00 \\
\hline 188.706 & 8.274 & 80.480 & 80.302 & -0.221 & 17.16 \\
\hline 188.706 & 10.342 & 70.810 & 72.260 & 2.048 & 22.15 \\
\hline 188.706 & 13.790 & 65.030 & 65.420 & 0.599 & 26.24 \\
\hline
\end{tabular}


Data from Jones et a1. [54] (continued)

\begin{tabular}{|c|c|c|c|c|c|}
\hline $\mathrm{T}$ & $P$ & $C_{p}, \exp$ & $C_{p}, c a l$ & Dev & Wt \\
\hline K & $\mathrm{MPa}$ & $\mathrm{J} \cdot \mathrm{mol}^{-1} \cdot \mathrm{K}^{-1}$ & $\mathrm{~J} \cdot \mathrm{mol}^{-1} \cdot \mathrm{K}^{-1}$ & 8 & \\
\hline 194.261 & 1.034 & 37.020 & 37.243 & 0.602 & 162.12 \\
\hline 194.261 & 2.068 & 43.260 & 43.262 & 0.005 & 23.84 \\
\hline 194.261 & 2.413 & 46.690 & 46.200 & -1.050 & 20.50 \\
\hline 194.261 & 3.103 & 54.950 & 54.871 & -0.144 & 14.84 \\
\hline 194.261 & 3.792 & 73.300 & 72.008 & -1.762 & 8.36 \\
\hline 194.261 & 4.137 & 91.500 & 89.294 & -2.411 & 1.34 \\
\hline 194.261 & 4.309 & 101.180 & 103.278 & 2.073 & 1.10 \\
\hline 194.261 & 4.688 & 193.210 & 172.350 & -10.797 & -0.00 \\
\hline 194.261 & 5.516 & 240.850 & 243.740 & 1.200 & -0.00 \\
\hline 194.261 & 6.895 & 107.090 & 110.944 & 3.599 & 0.98 \\
\hline 194.261 & 8.274 & 88.680 & 89.537 & 0.966 & 5.72 \\
\hline 194.261 & 10.342 & 75.180 & 76.723 & 2.053 & 7.95 \\
\hline 194.261 & 13.790 & 66.850 & 67.494 & 0.963 & 10.05 \\
\hline 199.817 & 1.034 & 36.750 & 36.913 & 0.444 & 155.58 \\
\hline 199.817 & 2.068 & 41.920 & 42.048 & 0.305 & 23.99 \\
\hline 199.817 & 2.413 & 44.680 & 44.416 & -0.591 & 21.15 \\
\hline 199.817 & 3.103 & 50.790 & 50.874 & 0.165 & 16.40 \\
\hline 199.817 & 3.792 & 62.010 & 61.517 & -0.794 & 11.03 \\
\hline 199.817 & 4.137 & 71.820 & 69.973 & -2.571 & 2.06 \\
\hline 199.817 & 4.309 & 77.330 & 75.553 & -2.298 & 1.78 \\
\hline 199.817 & 4.688 & 94.460 & 93.311 & -1.216 & 1.19 \\
\hline 199.817 & 5.516 & 231.440 & 217.015 & -6.233 & -0.00 \\
\hline 199.817 & 6.895 & 148.070 & 155.359 & 4.922 & -0.00 \\
\hline 199.817 & 8.274 & 102.250 & 103.941 & 1.654 & 4.07 \\
\hline 199.817 & 10.342 & 81.760 & 82.477 & 0.877 & 6.36 \\
\hline 199.817 & 13.790 & 68.860 & 69.859 & 1.451 & 8.95 \\
\hline 205.372 & 1.034 & 36.550 & 36.646 & 0.261 & 148.98 \\
\hline 205.372 & 2.068 & 40.850 & 41.090 & 0.587 & 23.92 \\
\hline 205.372 & 2.413 & 43.130 & 43.051 & -0.182 & 21.48 \\
\hline 205.372 & 3.103 & 47.900 & 48.116 & 0.451 & 17.45 \\
\hline 205.372 & 3.792 & 55.760 & 55.601 & -0.286 & 12.90 \\
\hline 205.372 & 4.137 & 61.940 & 60.863 & -1.738 & 2.62 \\
\hline 205.372 & 4.309 & 65.640 & 64.047 & -2.427 & 2.33 \\
\hline 205.372 & 4.688 & 75.380 & 72.907 & -3.281 & 1.77 \\
\hline 205.372 & 5.516 & 111.050 & 108.656 & -2.156 & 0.82 \\
\hline
\end{tabular}


Data from Jones et al. [54] (continued)

\begin{tabular}{|c|c|c|c|c|c|}
\hline $\mathrm{T}$ & $\mathbf{P}$ & $C_{p}, \exp$ & $C_{p}, c a l$ & Dev & Wt \\
\hline K & $\mathrm{MPa}$ & $\mathrm{J} \cdot \mathrm{mol}^{-1} \cdot \mathrm{K}^{-1}$ & $\mathrm{~J} \cdot \mathrm{mol}^{-1} \cdot \mathrm{K}^{-1}$ & 8 & \\
\hline 205.372 & 6.895 & 194.830 & 202.733 & 4.057 & -0.00 \\
\hline 205.372 & 8.274 & 123.010 & 124.144 & 0.922 & 2.66 \\
\hline 205.372 & 10.342 & 90.490 & 89.615 & -0.967 & 4.92 \\
\hline 205.372 & 13.790 & 70.940 & 72.496 & 2.193 & 7.99 \\
\hline 210.928 & 1.034 & 36.410 & 36.430 & 0.054 & 142.41 \\
\hline 210.928 & 2.068 & 40.040 & 40.323 & 0.707 & 23.60 \\
\hline 210.928 & 2.413 & 41.790 & 41.982 & 0.459 & 21.69 \\
\hline 210.928 & 3.103 & 45.820 & 46.095 & 0.600 & 18.07 \\
\hline 210.928 & 3.792 & 51.600 & 51.743 & 0.278 & 14.27 \\
\hline 210.928 & 4.137 & 55.630 & 55.429 & -0.360 & 3.07 \\
\hline 210.928 & 4.309 & 58.250 & 57.554 & -1.194 & 2.80 \\
\hline 210.928 & 4.688 & 64.960 & 63.092 & -2.875 & 2.26 \\
\hline 210.928 & 5.516 & 84.310 & 81.295 & -3.576 & 1.34 \\
\hline 210.928 & 6.895 & 139.000 & 141.210 & 1.590 & 0.49 \\
\hline 210.928 & 8.274 & 138.460 & 140.483 & 1.461 & 1.99 \\
\hline 210.928 & 10.342 & 97.880 & 97.331 & -0.561 & 3.99 \\
\hline 210.928 & 13.790 & 73.560 & 75.300 & 2.366 & 7.05 \\
\hline 216.483 & 1.034 & 36.280 & 36.258 & -0.061 & 136.24 \\
\hline 216.483 & 2.068 & 39.370 & 39.704 & 0.848 & 23.18 \\
\hline 216.483 & 2.413 & 40.850 & 41.130 & 0.685 & 21.55 \\
\hline 216.483 & 3.103 & 44.270 & 44.557 & 0.649 & 18.38 \\
\hline 216.483 & 3.792 & 48.770 & 49.020 & 0.513 & 15.16 \\
\hline 216.483 & 4.137 & 51.600 & 51.790 & 0.368 & 3.39 \\
\hline 216.483 & 4.309 & 53.280 & 53.338 & 0.109 & 3.18 \\
\hline 216.483 & 4.688 & 57.980 & 57.220 & -1.311 & 2.69 \\
\hline 216.483 & 5.516 & 69.940 & 68.677 & -1.806 & 1.85 \\
\hline 216.483 & 6.895 & 103.260 & 100.997 & -2.192 & 05 \\
\hline 216.483 & 8.274 & 127.710 & 129.476 & 1.383 & 2.22 \\
\hline 216.483 & 10.342 & 103.120 & 103.472 & 0.341 & 3.41 \\
\hline 216.483 & 13.790 & 76.180 & 78.021 & 2.417 & 6.24 \\
\hline 222.039 & 1.034 & 36.080 & 36.125 & 0.124 & 131.01 \\
\hline 222.039 & 2.068 & 38.900 & 39.202 & 0.776 & 22.58 \\
\hline 222.039 & 2.413 & 40.110 & 40.444 & 0.833 & 21.25 \\
\hline 222.039 & 3.103 & 43.130 & 43.357 & 0.527 & 18.40 \\
\hline 222.039 & 3.792 & 46.690 & 47.000 & 0.665 & 15.72 \\
\hline
\end{tabular}


Data from Jones et al. [54] (continued)

\begin{tabular}{|c|c|c|c|c|c|}
\hline $\mathrm{T}$ & $P$ & $C_{p}, \exp$ & $C_{p}, c a 1$ & Dev & Wt \\
\hline K & $\mathrm{MPa}$ & $\mathrm{J} \cdot \mathrm{mol}^{-1} \cdot \mathrm{K}^{-1}$ & $\mathrm{~J} \cdot \mathrm{mol}^{-1} \cdot \mathrm{K}^{-1}$ & 8 & \\
\hline 222.039 & 4.137 & 48.910 & 49.181 & 0.553 & 3.58 \\
\hline 222.039 & 4.309 & 50.180 & 50.374 & 0.386 & 3.41 \\
\hline 222.039 & 4.688 & 53.280 & 53.289 & 0.016 & 3.02 \\
\hline 222.039 & 5.516 & 61.740 & 61.339 & -0.649 & 2.25 \\
\hline 222.039 & 6.895 & 85.320 & 81.518 & -4.456 & -0.00 \\
\hline 222.039 & 8.274 & 105.340 & 105.634 & 0.279 & 3.11 \\
\hline 222.039 & 10.342 & 103.660 & 104.748 & 1.050 & 3.21 \\
\hline 222.039 & 13.790 & 79.010 & 80.282 & 1.610 & 5.52 \\
\hline 227.594 & 1.034 & 36.010 & 36.025 & 0.043 & 125.25 \\
\hline 227.594 & 2.068 & 38.500 & 38.794 & 0.764 & 21.95 \\
\hline 227.594 & 2.413 & 39.570 & 39.889 & 0.806 & 20.79 \\
\hline 227.594 & 3.103 & 42.190 & 42.404 & 0.508 & 18.31 \\
\hline 227.594 & 3.792 & 45.080 & 45.453 & 0.827 & 16.05 \\
\hline 227.594 & 4.137 & 46.890 & 47.227 & 0.719 & 3.71 \\
\hline 227.594 & 4.309 & 47.770 & 48.183 & 0.865 & 3.58 \\
\hline 227.594 & 4.688 & 50.320 & 50.476 & 0.310 & 3.22 \\
\hline 227.594 & 5.516 & 56.500 & 56.530 & 0.052 & 2.56 \\
\hline 227.594 & 6.895 & 74.100 & 70.501 & -4.856 & -0.00 \\
\hline 227.594 & 8.274 & 87.940 & 87.938 & -0.002 & 4.24 \\
\hline 227.594 & 10.342 & 97.680 & 99.199 & 1.555 & 3.44 \\
\hline 227.594 & 13.790 & 81.220 & 81.671 & 0.555 & 4.97 \\
\hline 233.150 & 1.034 & 35.940 & 35.956 & 0.045 & 119.87 \\
\hline 233.150 & 2.068 & 38.230 & 38.464 & 0.613 & 21.22 \\
\hline 233.150 & 2.413 & 39.100 & 39.438 & 0.865 & 20.29 \\
\hline 233.150 & 3.103 & 41.380 & 41.639 & 0.627 & 18.13 \\
\hline 233.150 & 3.792 & 44.000 & 44.239 & 0.543 & 16.05 \\
\hline 233.150 & 4.137 & 45.480 & 45.720 & 0.528 & 3.76 \\
\hline 233.150 & 4.309 & 46.290 & 46.508 & 0.472 & 3.63 \\
\hline 233.150 & 4.688 & 48.030 & 48.374 & 0.716 & 3.37 \\
\hline 233.150 & 5.516 & 53.070 & 53.139 & 0.130 & 2.77 \\
\hline 233.150 & 6.895 & 65.770 & 63.501 & -3.450 & -0.00 \\
\hline 233.150 & 8.274 & 76.520 & 76.295 & -0.294 & 5.33 \\
\hline 233.150 & 10.342 & 88.210 & 90.061 & 2.098 & 4.02 \\
\hline 233.150 & 13.790 & 80.890 & 81.812 & 1.139 & 4.77 \\
\hline 238.706 & 1.034 & 35.940 & 35.914 & -0.073 & 114.42 \\
\hline
\end{tabular}


Data from Jones et al. [54] (continued)

\begin{tabular}{|c|c|c|c|c|c|}
\hline $\mathrm{T}$ & P & $C_{p}, \exp$ & $C_{p}, c a l$ & Dev & Wt \\
\hline K & $\mathrm{MPa}$ & $\mathrm{J} \cdot \mathrm{mol}^{-1} \cdot \mathrm{K}^{-1}$ & $\mathrm{~J} \cdot \mathrm{mol}^{-1} \cdot \mathrm{K}^{-1}$ & 8 & \\
\hline 238.706 & 2.068 & 37.960 & 38.199 & 0.631 & 20.53 \\
\hline 238.706 & 2.413 & 38.760 & 39.073 & 0.808 & 19.70 \\
\hline 238.706 & 3.103 & 40.780 & 41.020 & 0.590 & 17.82 \\
\hline 238.706 & 3.792 & 43.060 & 43.272 & 0.493 & 15.99 \\
\hline 238.706 & 4.137 & 44.270 & 44.533 & 0.594 & 3.78 \\
\hline 238.706 & 4.309 & 45.080 & 45.198 & 0.262 & 3.65 \\
\hline 238.706 & 4.688 & 46.420 & 46.754 & 0.720 & 3.44 \\
\hline 238.706 & 5.516 & 50.790 & 50.632 & -0.311 & 2.88 \\
\hline 238.706 & 6.895 & 59.660 & 58.696 & -1.616 & 2.09 \\
\hline 238.706 & 8.274 & 68.860 & 68.431 & -0.624 & 6.28 \\
\hline 238.706 & 10.342 & 79.480 & 81.165 & 2.120 & 4.72 \\
\hline 238.706 & 13.790 & 78.870 & 80.474 & 2.034 & 4.79 \\
\hline 244.261 & 1.034 & 35.880 & 35.896 & 0.046 & 109.69 \\
\hline 244.261 & 2.068 & 37.760 & 37.990 & 0.609 & 19.83 \\
\hline 244.261 & 2.413 & 38.500 & 38.780 & 0.727 & 19.08 \\
\hline 244.261 & 3.103 & 40.240 & 40.519 & 0.692 & 17.48 \\
\hline 244.261 & 3.792 & 42.260 & 42.495 & 0.555 & 15.86 \\
\hline 244.261 & 4.137 & 43.400 & 43.585 & 0.426 & 3.76 \\
\hline 244.261 & 4.309 & 43.740 & 44.156 & 0.951 & 3.70 \\
\hline 244.261 & 4.688 & 45.410 & 45.480 & 0.155 & 3.44 \\
\hline 244.261 & 5.516 & 48.440 & 48.717 & 0.572 & 3.02 \\
\hline 244.261 & 6.895 & 55.560 & 55.218 & -0.616 & 2.30 \\
\hline 244.261 & 8.274 & 63.220 & 62.883 & -0.533 & 7.11 \\
\hline 244.261 & 10.342 & 72.350 & 73.831 & 2.047 & 5.44 \\
\hline 244.261 & 13.790 & 76.320 & 77.831 & 1.979 & 4.89 \\
\hline 249.817 & 1.034 & 35.940 & 35.901 & -0.107 & 104.56 \\
\hline 249.817 & 2.068 & 37.620 & 37.828 & 0.554 & 19.10 \\
\hline 249.817 & 2.413 & 38.290 & 38.547 & 0.671 & 18.44 \\
\hline 249.817 & 3.103 & 39.840 & 40.112 & 0.683 & 17.05 \\
\hline 249.817 & 3.792 & 41.590 & 41.864 & 0.660 & 15.65 \\
\hline 249.817 & 4.137 & 42.730 & 42.820 & 0.211 & 3.71 \\
\hline 249.817 & 4.309 & 43.130 & 43.317 & 0.434 & 3.64 \\
\hline 249.817 & 4.688 & 44.340 & 44.463 & 0.277 & 3.45 \\
\hline 249.817 & 5.516 & 46.960 & 47.219 & 0.551 & 3.07 \\
\hline 249.817 & 6.895 & 52.740 & 52.602 & -0.262 & 2.44 \\
\hline
\end{tabular}


Data from Jones et al. [54] (continued)

\begin{tabular}{|c|c|c|c|c|c|}
\hline $\mathrm{T}$ & $P$ & $C_{p}, \exp$ & $C_{p}, c a 1$ & Dev & Wt \\
\hline K & $\mathrm{MPa}$ & $\mathrm{J} \cdot \mathrm{mol}^{-1} \cdot \mathrm{K}^{-1}$ & $\mathrm{~J} \cdot \mathrm{mol}^{-1} \cdot \mathrm{K}^{-1}$ & 8 & \\
\hline 249.817 & 8.274 & 59.050 & 58.814 & -0.399 & 7.79 \\
\hline 249.817 & 10.342 & 66.780 & 68.064 & 1.923 & 6.10 \\
\hline 249.817 & 13.790 & 73.360 & 74.419 & 1.443 & 5.06 \\
\hline 255.372 & 1.034 & 35.940 & 35.927 & -0.035 & 100.10 \\
\hline 255.372 & 2.068 & 37.550 & 37.709 & 0.422 & 18.35 \\
\hline 255.372 & 2.413 & 38.160 & 38.366 & 0.539 & 17.78 \\
\hline 255.372 & 3.103 & 39.570 & 39.784 & 0.542 & 16.54 \\
\hline 255.372 & 3.792 & 41.120 & 41.353 & 0.566 & 15.33 \\
\hline 255.372 & 4.137 & 42.060 & 42.200 & 0.332 & 14.65 \\
\hline 255.372 & 4.309 & 42.390 & 42.638 & 0.585 & 14.43 \\
\hline 255.372 & 4.688 & 43.470 & 43.642 & 0.396 & 13.72 \\
\hline 255.372 & 5.516 & 45.680 & 46.026 & 0.758 & 12.44 \\
\hline 255.372 & 6.895 & 50.650 & 50.580 & -0.137 & 10.13 \\
\hline 255.372 & 8.274 & 55.830 & 55.736 & -0.168 & 8.34 \\
\hline 255.372 & 10.342 & 62.340 & 63.565 & 1.966 & 6.69 \\
\hline 255.372 & 13.790 & 70.140 & 70.805 & 0.948 & 5.29 \\
\hline 260.928 & 1.034 & 36.010 & 35.973 & -0.103 & 95.55 \\
\hline 260.928 & 2.068 & 37.490 & 37.625 & 0.361 & 17.64 \\
\hline 260.928 & 2.413 & 38.020 & 38.229 & 0.550 & 17.16 \\
\hline 260.928 & 3.103 & 39.370 & 39.523 & 0.389 & 16.01 \\
\hline 260.928 & 3.792 & 40.780 & 40.938 & 0.388 & 14.93 \\
\hline 260.928 & 4.137 & 41.590 & 41.696 & 0.254 & 14.36 \\
\hline 260.928 & 4.309 & 41.790 & 42.086 & 0.708 & 14.22 \\
\hline 260.928 & 4.688 & 42.790 & 42.976 & 0.434 & 13.57 \\
\hline 260.928 & 5.516 & 44.940 & 45.066 & 0.281 & 12.31 \\
\hline 260.928 & 6.895 & 49.110 & 48.985 & -0.255 & 10.32 \\
\hline 260.928 & 8.274 & 53.340 & 53.348 & 0.015 & 8.75 \\
\hline 260.928 & 10.342 & 58.920 & 60.030 & 1.884 & 7.18 \\
\hline 260.928 & 13.790 & 67.050 & 67.359 & 0.461 & 5.55 \\
\hline 266.483 & 1.034 & 36.080 & 36.037 & -0.120 & 91.29 \\
\hline 266.483 & 2.068 & 37.420 & 37.575 & 0.414 & 16.98 \\
\hline 266.483 & 2.413 & 37.960 & 38.133 & 0.455 & 16.51 \\
\hline 266.483 & 3.103 & 39.170 & 39.319 & 0.381 & 15.51 \\
\hline 266.483 & 3.792 & 40.440 & 40.604 & 0.407 & 14.56 \\
\hline 266.483 & 4.137 & 41.050 & 41.287 & 0.578 & 14.13 \\
\hline
\end{tabular}


Data from Jones et al. [54] (continued)

\begin{tabular}{|c|c|c|c|c|c|}
\hline $\mathrm{T}$ & $\mathbf{P}$ & $C_{p}, \exp$ & $\mathrm{C}_{\mathrm{p}}, \mathrm{cal}$ & Dev & Wt \\
\hline K & $\mathrm{MPa}$ & $\mathrm{J} \cdot \mathrm{mol}^{-1} \cdot \mathrm{K}^{-1}$ & $\mathrm{~J} \cdot \mathrm{mol}^{-1} \cdot \mathrm{K}^{-1}$ & 8 & \\
\hline 266.483 & 4.309 & 41.380 & 41.638 & 0.623 & 13.91 \\
\hline 266.483 & 4.688 & 42.190 & 42.434 & 0.578 & 13.38 \\
\hline 266.483 & 5.516 & 44.270 & 44.287 & 0.038 & 12.16 \\
\hline 266.483 & 6.895 & 47.830 & 47.707 & -0.257 & 10.43 \\
\hline 266.483 & 8.274 & 51.390 & 51.460 & 0.136 & 9.04 \\
\hline 266.483 & 10.342 & 56.430 & 57.222 & 1.404 & 7.50 \\
\hline 266.483 & 13.790 & 64.090 & 64.255 & 0.257 & 5.82 \\
\hline 272.039 & 1.034 & 36.140 & 36.118 & -0.062 & 5.82 \\
\hline 272.039 & 2.068 & 37.420 & 37.554 & 0.359 & 1.09 \\
\hline 272.039 & 2.413 & 37.890 & 38.071 & 0.478 & 1.06 \\
\hline 272.039 & 3.103 & 39.030 & 39.164 & 0.344 & 1.00 \\
\hline 272.039 & 3.792 & 40.240 & 40.339 & 0.245 & 0.94 \\
\hline 272.039 & 4.137 & 40.780 & 40.959 & 0.438 & 0.92 \\
\hline 272.039 & 4.309 & 41.050 & 41.276 & 0.550 & 0.90 \\
\hline 272.039 & 4.688 & 41.850 & 41.993 & 0.343 & 0.87 \\
\hline 272.039 & 5.516 & 44.000 & 43.652 & -0.792 & 0.79 \\
\hline 272.039 & 6.895 & 46.890 & 46.672 & -0.466 & 0.69 \\
\hline 272.039 & 8.274 & 49.980 & 49.944 & -0.071 & 0.61 \\
\hline 272.039 & 10.342 & 54.550 & 54.965 & 0.762 & 0.51 \\
\hline 272.039 & 13.790 & 61.540 & 61.539 & -0.001 & 0.40 \\
\hline 277.594 & 1.034 & 36.210 & 36.215 & 0.013 & 5.57 \\
\hline 277.594 & 2.068 & 37.420 & 37.560 & 0.375 & 1.04 \\
\hline 277.594 & 2.413 & 37.890 & 38.041 & 0.398 & 1.02 \\
\hline 277.594 & 3.103 & 38.970 & 39.053 & 0.212 & 0.96 \\
\hline 277.594 & 3.792 & 40.170 & 40.131 & -0.096 & 0.91 \\
\hline 277.594 & 4.137 & 40.640 & 40.697 & 0.141 & 0.89 \\
\hline 277.594 & 4.309 & 40.850 & 40.986 & 0.334 & 0.88 \\
\hline 277.594 & 4.688 & 41.590 & 41.638 & 0.116 & 0.85 \\
\hline 277.594 & 5.516 & 43.600 & 43.134 & -1.069 & 0.77 \\
\hline 277.594 & 6.895 & 46.020 & 45.827 & -0.419 & 0.69 \\
\hline 277.594 & 8.274 & 48.910 & 48.715 & -0.400 & 0.61 \\
\hline 277.594 & 10.342 & 53.140 & 53.133 & -0.012 & 0.52 \\
\hline 277.594 & 13.790 & 59.460 & 59.201 & -0.435 & 0.42 \\
\hline 283.150 & 1.034 & 36.280 & 36.327 & 0.131 & 5.34 \\
\hline 283.150 & 2.068 & 37.350 & 37.590 & 0.644 & 1.01 \\
\hline
\end{tabular}


Data from Jones et al. [54] (continued)

$\begin{array}{cccccc}\mathrm{T} & \mathrm{P} & \mathrm{C}_{\mathrm{p}}, \exp & \mathrm{C}_{\mathrm{p}}, \mathrm{cal} & \mathrm{Dev} & \text { Wt } \\ \mathrm{K} & \mathrm{MPa} & \mathrm{J} \cdot \mathrm{mol}^{-1} \cdot \mathrm{K}^{-1} & \mathrm{~J} \cdot \mathrm{mol}^{-1} \cdot \mathrm{K}^{-1} & 8 & \\ 283.150 & 2.413 & 37.820 & 38.039 & 0.579 & 0.98 \\ 283.150 & 3.103 & 38.900 & 38.979 & 0.203 & 0.93 \\ 283.150 & 3.792 & 40.110 & 39.974 & -0.339 & 0.87 \\ 283.150 & 4.137 & 40.510 & 40.494 & -0.039 & 0.86 \\ 283.150 & 4.309 & 40.780 & 40.759 & -0.052 & 0.85 \\ 283.150 & 4.688 & 41.520 & 41.354 & -0.399 & 0.82 \\ 283.150 & 5.516 & 42.930 & 42.713 & -0.506 & 0.76 \\ 283.150 & 6.895 & 45.480 & 45.136 & -0.757 & 0.68 \\ 283.150 & 8.274 & 48.030 & 47.708 & -0.671 & 0.61 \\ 283.150 & 10.342 & 52.070 & 51.632 & -0.841 & 0.52 \\ 283.150 & 13.790 & 57.780 & 57.203 & -0.999 & 0.42\end{array}$

Number of Points (Ref. 54, Primary) 388
$A A D-8$
0.669
BIAS - 8
0.160 RMS - 8
0.931
AAD
0.445 BIAS
0.069 RMS
$0.749 \mathrm{~J} \cdot \mathrm{mol}^{-1} \cdot \mathrm{K}^{-1}$

Number of Points (Ref. 54, Tota1) 400
$A A D-8$
0.821
BIAS - 8
0.162 RMS - 8
1.514
AAD
0.772 BIAS
0.165
RMS
3.345
$\mathrm{J} \cdot \mathrm{mol}^{-1} \cdot \mathrm{K}^{-1}$

Data from Van Kasteren and Zeldenrust [55]

$\mathrm{T}$

$\mathrm{K}$

$$
\begin{aligned}
& 121.1 \\
& 121.1 \\
& 121.1 \\
& 121.0 \\
& 136.1 \\
& 136.2 \\
& 136.2 \\
& 151.3 \\
& 151.3 \\
& 151.2
\end{aligned}
$$

$$
P
$$

$\mathrm{MPa}$

5.00

5.00

5.00

5.00

5.00

5.00

5.00

5.00

5.00

5.00
$C_{p}, \exp \quad C_{p}, c a l$

$$
\mathrm{J} \cdot \mathrm{mol}^{-1} \cdot \mathrm{K}^{-1} \quad \mathrm{~J} \cdot \mathrm{mol}^{-1} \cdot \mathrm{K}^{-1}
$$

55.188

55.060

54.979

55.156

57.338

57.402

57.241

61.268

61.044

60.963

56.032
56.032
56.032
56.022
58.033

58.051
58.051
61.654
61.654
61.622

61.622
Dev Wt

$1.530 \quad 0.00$

$1.766 \quad 0.00$

$1.916 \quad 0.00$

$1.570 \quad 0.00$

$1.213 \quad 0.00$

$\begin{array}{ll}1.130 & 0.00\end{array}$

$1.415 \quad 0.00$

$0.630 \quad 0.00$

$1.000 \quad 0.00$

$1.081 \quad 0.00$ 
Data from Van Kasteren and Zeldenrust [55]

\begin{tabular}{|c|c|c|c|c|c|}
\hline $\mathrm{T}$ & $\mathbf{P}$ & $C_{p}, \exp$ & $C_{p}, c a l$ & Dev & Wt \\
\hline K & $\mathrm{MPa}$ & $\mathrm{J} \cdot \mathrm{mol}^{-1} \cdot \mathrm{K}^{-1}$ & $\mathrm{~J} \cdot \mathrm{mol}^{-1} \cdot \mathrm{K}^{-1}$ & 8 & \\
\hline 165.1 & 5.00 & 67.846 & 68.011 & 0.244 & 0.00 \\
\hline 165.1 & 5.00 & 67.830 & 68.011 & 0.267 & 0.00 \\
\hline 165.1 & 5.00 & 68.054 & 68.011 & -0.063 & 0.00 \\
\hline 165.0 & 5.00 & 67.734 & 67.946 & 0.313 & 0.00 \\
\hline 175.6 & 5.00 & 78.579 & 78.367 & -0.270 & 0.00 \\
\hline 175.7 & 5.00 & 79.092 & 78.516 & -0.728 & 0.00 \\
\hline 175.6 & 5.00 & 78.707 & 78.367 & -0.432 & 0.00 \\
\hline 175.5 & 5.00 & 79.076 & 78.219 & -1.084 & 0.00 \\
\hline 182.7 & 5.00 & 94.622 & 94.846 & 0.237 & 0.00 \\
\hline 182.7 & 5.00 & 94.573 & 94.846 & 0.289 & 0.00 \\
\hline 182.7 & 5.00 & 94.654 & 94.846 & 0.203 & 0.00 \\
\hline 184.2 & 5.00 & 101.841 & 01.159 & -0.670 & 0.00 \\
\hline 186.4 & 5.00 & 116.248 & 14.823 & -1.226 & 0.00 \\
\hline 188.5 & 5.00 & 140.408 & 38.418 & -1.417 & 0.00 \\
\hline 191.0 & 5.00 & 251.298 & 18.727 & -12.961 & 0.00 \\
\hline 192.7 & 5.00 & 558.746 & 11.247 & 9.396 & 0.00 \\
\hline 192.6 & 5.00 & 549.681 & 46.601 & -0.560 & 0.00 \\
\hline 195.4 & 5.00 & 279.662 & 64.189 & -5.533 & 0.00 \\
\hline 198.0 & 5.00 & 149.408 & 46.547 & -1.915 & 0.00 \\
\hline 201.5 & 5.00 & 103.959 & 02.911 & -1.008 & 0.00 \\
\hline 201.5 & 5.00 & 103.927 & 02.911 & -0.978 & 0.00 \\
\hline 209.5 & 5.00 & 73.108 & 71.555 & -2.124 & 0.00 \\
\hline 209.5 & 5.00 & 73.108 & 71.555 & -2.124 & 0.00 \\
\hline 220.0 & 5.00 & 57.659 & 57.625 & -0.060 & 0.00 \\
\hline 220.0 & 5.00 & 57.851 & 57.625 & -0.391 & 0.00 \\
\hline 230.5 & 5.00 & 50.712 & 51.165 & 0.894 & 0.00 \\
\hline 240.0 & 5.00 & 47.359 & 47.755 & 0.836 & 0.00 \\
\hline 240.4 & 5.00 & 47.311 & 47.642 & 0.699 & 0.00 \\
\hline 250.6 & 5.00 & 44.985 & 45.313 & 0.729 & 0.00 \\
\hline 144.4 & 3.20 & 59.850 & 60.803 & 1.592 & 0.00 \\
\hline 144.3 & 3.20 & 60.690 & 60.773 & 0.137 & 0.00 \\
\hline 144.4 & 3.20 & 59.960 & 60.803 & 1.405 & 0.00 \\
\hline 146.2 & 3.20 & 61.600 & 61.353 & -0.402 & 0.00 \\
\hline 146.5 & 3.20 & 60.890 & 61.449 & 0.918 & 0.00 \\
\hline 154.9 & 3.20 & 63.990 & 64.819 & 1.296 & 0.00 \\
\hline
\end{tabular}


Data from Van Kasteren and Zeldenrust [55] (continued)

$\mathrm{T}$

K

154.8

164.9

164.9

174.3

185.5

193.2

193.1

202.9

202.9
$\mathbf{P}$

$\mathrm{MPa}$

3.20

3.20

3.20

3.20

3.20

3.20

3.20

3.20

3. 20
$C_{p}, \exp$

$\mathrm{J} \cdot \mathrm{mol}^{-1} \cdot \mathrm{K}^{-1}$

64.140

71.560

71.310

89.160

72.530

57.960

58.070

50.180

50.000
$\mathrm{C}_{\mathrm{p}}, \mathrm{cal}$

$\mathrm{J} \cdot \mathrm{mol}^{-1} \cdot \mathrm{K}^{-1}$

64.769

71.818

71.818

87.547

71.596

57.691

57.803

50.219

50.219

Dev Wt

Number of Points (Ref. 55) 54

$\begin{array}{lllllll}\text { AAD - } 8 & 1.356 & \text { BIAS }-8 & -0.050 & \text { RMS }-\frac{8}{8} & 2.524 & \\ \text { AAD } & 2.530 & \text { BIAS } & -0.021 & \text { RMS } & 8.717 & \mathrm{~J} \cdot \mathrm{mol}^{-1} \cdot \mathrm{K}^{-1}\end{array}$

Total Points 454
$A A D-q$
0.885 BIAS -8
0.137 RMS -8
1.668
$A A D$
0.981 BIAS
0.143 RMS
$4.347 \mathrm{~J} \cdot \mathrm{mol}^{-1} \cdot \mathrm{K}^{-1}$ 
COMPARISONS FOR SPECIFIC HEAT CAPACITY OF THE SATURATED LIQUID

Notes for Table A8. The densities above were calculated from the experimental temperatures and the ancillary eq (2) for the density of the saturated liquid. These densities were used to calculate the heat capacity along the saturation boundary according to the equation given in Table 7 .

Data are from Younglove [52] and Roder [53] as adjusted by Roder [53].

\begin{tabular}{|c|c|c|c|c|c|}
\hline $\mathrm{T}$ & $\rho$ & $C_{\sigma L}, \exp$ & $\mathrm{C}_{\sigma \mathrm{L}}, \mathrm{cal}$ & Dev & Wt \\
\hline K & $\mathrm{mol} \cdot \mathrm{dm}^{-3}$ & $\mathrm{~J} \cdot \mathrm{mol}^{-1} \cdot \mathrm{K}^{-1}$ & $\mathrm{~J} \cdot \mathrm{mol}^{-1} \cdot \mathrm{K}^{-1}$ & 8 & \\
\hline 95.402 & 27.7468 & 54.206 & 54.130 & -0.141 & 2004.81 \\
\hline 95.846 & 27.7089 & 54.066 & 54.209 & 0.264 & 1996.77 \\
\hline 99.021 & 27.4363 & 54.590 & 54.680 & 0.165 & 1887.59 \\
\hline 101.651 & 27.2086 & 54.782 & 54.981 & 0.363 & 1812.19 \\
\hline 101.961 & 27.1817 & 54.691 & 55.013 & 0.589 & 1807.43 \\
\hline 102.574 & 27.1282 & 54.820 & 55.074 & 0.464 & 1787.90 \\
\hline 106.891 & 26.7486 & 55.505 & 55.462 & -0.077 & 1666.02 \\
\hline 108.219 & 26.6304 & 55.459 & 55.573 & 0.206 & 1638.81 \\
\hline 108.800 & 26.5785 & 55.187 & 55.622 & 0.788 & 0.00 \\
\hline 112.604 & 26.2351 & 55.960 & 55.946 & -0.026 & 1536.67 \\
\hline 115.165 & 26.0002 & 56.292 & 56.178 & -0.202 & 1480.87 \\
\hline 116.044 & 25.9188 & 56.582 & 56.263 & -0.564 & 1457.92 \\
\hline 118.479 & 25.6912 & 56.761 & 56.509 & -0.443 & 1412.62 \\
\hline 119.117 & 25.6310 & 56.685 & 56.578 & -0.189 & 1404.24 \\
\hline 122.089 & 25.3474 & 57.292 & 56.919 & -0.650 & 1343.73 \\
\hline 122.474 & 25.3103 & 57.137 & 56.967 & -0.298 & 1341.72 \\
\hline 123.367 & 25.2237 & 56.845 & 57.079 & 0.411 & 1335.66 \\
\hline 125.766 & 24.9886 & 57.514 & 57.400 & -0.198 & 1286.54 \\
\hline 130.765 & 24.4848 & 58.092 & 58.175 & 0.142 & 1210.31 \\
\hline 132.274 & 24.3287 & 58.640 & 58.439 & -0.343 & 1181.30 \\
\hline 132.751 & 24.2790 & 58.441 & 58.526 & 0.145 & 1179.95 \\
\hline 135.455 & 23.9929 & 58.701 & 59.048 & 0.591 & 1145.11 \\
\hline 137.805 & 23.7385 & 59.285 & 59.546 & 0.441 & 1109.70 \\
\hline 139.970 & 23.4989 & 59.463 & 60.046 & 0.980 & 1085.54 \\
\hline 144.305 & 23.0028 & 60.317 & 61.176 & 1.424 & 1032.07 \\
\hline 144.401 & 22.9915 & 60.686 & 61.203 & 0.852 & 1024.84 \\
\hline 148.500 & 22.4989 & 61.780 & 62.463 & 1.106 & 975.23 \\
\hline 148.881 & 22.4518 & 61.751 & 62.591 & 1.361 & 973.01 \\
\hline 150.824 & 22.2082 & 63.536 & 63.275 & -0.411 & 0.00 \\
\hline 152.809 & 21.9528 & 63.632 & 64.033 & 0.630 & 918.27 \\
\hline
\end{tabular}


Table A8 (continued)

\begin{tabular}{|c|c|c|c|c|c|}
\hline $\mathrm{T}$ & $\rho$ & $C_{\sigma L}, \exp$ & $\mathrm{C}_{\sigma \mathrm{L}}, \mathrm{cal}$ & Dev & Wt \\
\hline $\mathrm{K}$ & $\mathrm{mol} \cdot \mathrm{dm}^{-3}$ & $\mathrm{~J} \cdot \mathrm{mol}^{-1} \cdot \mathrm{K}^{-1}$ & $\mathrm{~J} \cdot \mathrm{mol}^{-1} \cdot \mathrm{K}^{-1}$ & 8 & \\
\hline 153.157 & 21.9073 & 63.781 & 64.172 & 0.613 & 913.99 \\
\hline 155.542 & 21.5891 & 64.754 & 65.189 & 0.672 & 886.59 \\
\hline 157.043 & 21.3829 & 65.156 & 65.888 & 1.123 & 873.29 \\
\hline 157.355 & 21.3395 & 65.288 & 66.039 & 1.151 & 869.94 \\
\hline 159.127 & 21.0883 & 66.400 & 66.946 & 0.822 & 846.84 \\
\hline 161.181 & 20.7875 & 67.440 & 68.103 & 0.983 & 825.06 \\
\hline 161.483 & 20.7424 & 67.604 & 68.284 & 1.006 & 821.86 \\
\hline 163.136 & 20.4906 & 67.864 & 69.331 & 2.162 & 0.00 \\
\hline 163.782 & 20.3900 & 69.643 & 69.768 & 0.179 & 789.49 \\
\hline 165.128 & 20.1760 & 70.569 & 70.734 & 0.233 & 775.06 \\
\hline 165.249 & 20.1565 & 70.232 & 70.824 & 0.843 & 778.55 \\
\hline 165.548 & 20.1080 & 70.442 & 71.052 & 0.866 & 775.40 \\
\hline 166.549 & 19.9435 & 71.232 & 71.846 & 0.861 & 764.24 \\
\hline 169.253 & 19.4795 & 73.657 & 74.282 & 0.849 & 733.94 \\
\hline 169.565 & 19.4239 & 74.109 & 74.595 & 0.656 & 729.00 \\
\hline 170.157 & 19.3173 & 74.426 & 75.211 & 1.054 & 725.27 \\
\hline 170.197 & 19.3100 & 74.846 & 75.253 & 0.544 & 721.05 \\
\hline 170.251 & 19.3002 & 75.280 & 75.311 & 0.041 & 716.76 \\
\hline 171.097 & 19.1444 & 76.029 & 76.249 & 0.290 & 709.01 \\
\hline 173.180 & 18.7445 & 78.254 & 78.869 & 0.786 & 688.53 \\
\hline 173.480 & 18.6848 & 78.910 & 79.289 & 0.481 & 682.86 \\
\hline 173.505 & 18.6798 & 78.214 & 79.325 & 1.420 & 689.15 \\
\hline 174.032 & 18.5734 & 79.372 & 80.094 & 0.910 & 679.30 \\
\hline 174.429 & 18.4920 & 80.836 & 80.700 & -0.168 & 667.15 \\
\hline 175.228 & 18.3248 & 81.869 & 81.999 & 0.158 & 659.75 \\
\hline 176.295 & 18.0938 & 84.010 & 83.917 & -0.111 & 644.79 \\
\hline 177.029 & 17.9294 & 85.230 & 85.379 & 0.175 & 637.38 \\
\hline 177.420 & 17.8397 & 87.184 & 86.213 & -1.114 & 0.00 \\
\hline 177.902 & 17.7272 & 86.393 & 87.299 & 1.049 & 631.65 \\
\hline 178.557 & 17.5703 & 88.222 & 88.890 & 0.757 & 620.93 \\
\hline 180.613 & 17.0442 & 94.968 & 94.981 & 0.014 & 5868.77 \\
\hline 181.034 & 16.9292 & 96.269 & 96.493 & 0.232 & 5817.64 \\
\hline 181.645 & 16.7569 & 98.591 & 98.894 & 0.308 & 5725.55 \\
\hline 182.572 & 16.4822 & 103.209 & 103.107 & -0.099 & 5547.41 \\
\hline 184.222 & 15.9437 & 113.503 & 113.028 & -0.418 & 5223.69 \\
\hline
\end{tabular}


Table A8 (continued)

$\begin{array}{cccccr}\mathrm{T} & \rho & \mathrm{C}_{\sigma \mathrm{L}}, \exp & \mathrm{C}_{\sigma \mathrm{L}}, \mathrm{Cal} & \text { Dev } & \text { Wt } \\ \mathrm{K} & \mathrm{mol} \cdot \mathrm{dm}^{-3} & \mathrm{~J} \cdot \mathrm{mol}^{-1} \cdot \mathrm{K}^{-1} & \mathrm{~J} \cdot \mathrm{mol}^{-1} \cdot \mathrm{K}^{-1} & \text { o } & \\ 185.271 & 15.5577 & 121.885 & 121.882 & -0.003 & 5021.62 \\ 186.127 & 15.2079 & 132.650 & 131.567 & -0.816 & 0.00 \\ 187.577 & 14.5088 & 152.131 & 157.745 & 3.690 & 0.00 \\ 187.633 & 14.4781 & 157.474 & 159.174 & 1.079 & 0.00\end{array}$

Number of Points (Refs. 52 and 53) 69
AAD - z
0.626
BIAS - 8
0.444
RMS - \&
0.716
$A A D$
0.496
BIAS
0.354
RMS
$0.791 \mathrm{~J} \cdot \mathrm{mol}^{-1} \cdot \mathrm{K}^{-1}$ 


\section{COMPARISONS FOR SOUND SPEED}

Notes for Table A9. The temperatures and pressures refer to experimentally reported conditions, except that, for data reported on the saturation boundary (in Refs. 57-59), an iteration of the SWEOS equation of state was used to calculate a pressure and density. Other reported densities were also from this SWEOS. These quantities were used to determine the "experimental" value for $\partial \mathrm{P} /\left.\partial \rho\right|_{\mathrm{T}}$ from the experimental sound speed and calculated values for the isobaric and isochoric specific heat capacities (according to $\partial \mathrm{P} /\left.\partial \rho\right|_{\mathrm{T}}=\mathrm{u} \mathrm{N} \mathrm{N}_{\mathrm{A}} \mathrm{M} \mathrm{w}^{2} \mathrm{C}_{\mathrm{V}} / \mathrm{C}_{\mathrm{P}}$ ). This derivative was used to determine the coefficients in the final SWEOS according to the weights in the final column of the table. Calculated values of the sound speed and derivative were from the final SWEOS. Values missing in the tables or statistics have been omitted for any of several reasons including recent acquisition, replicate points, critical region data, etc.

Data from Baidakov et al. [56]

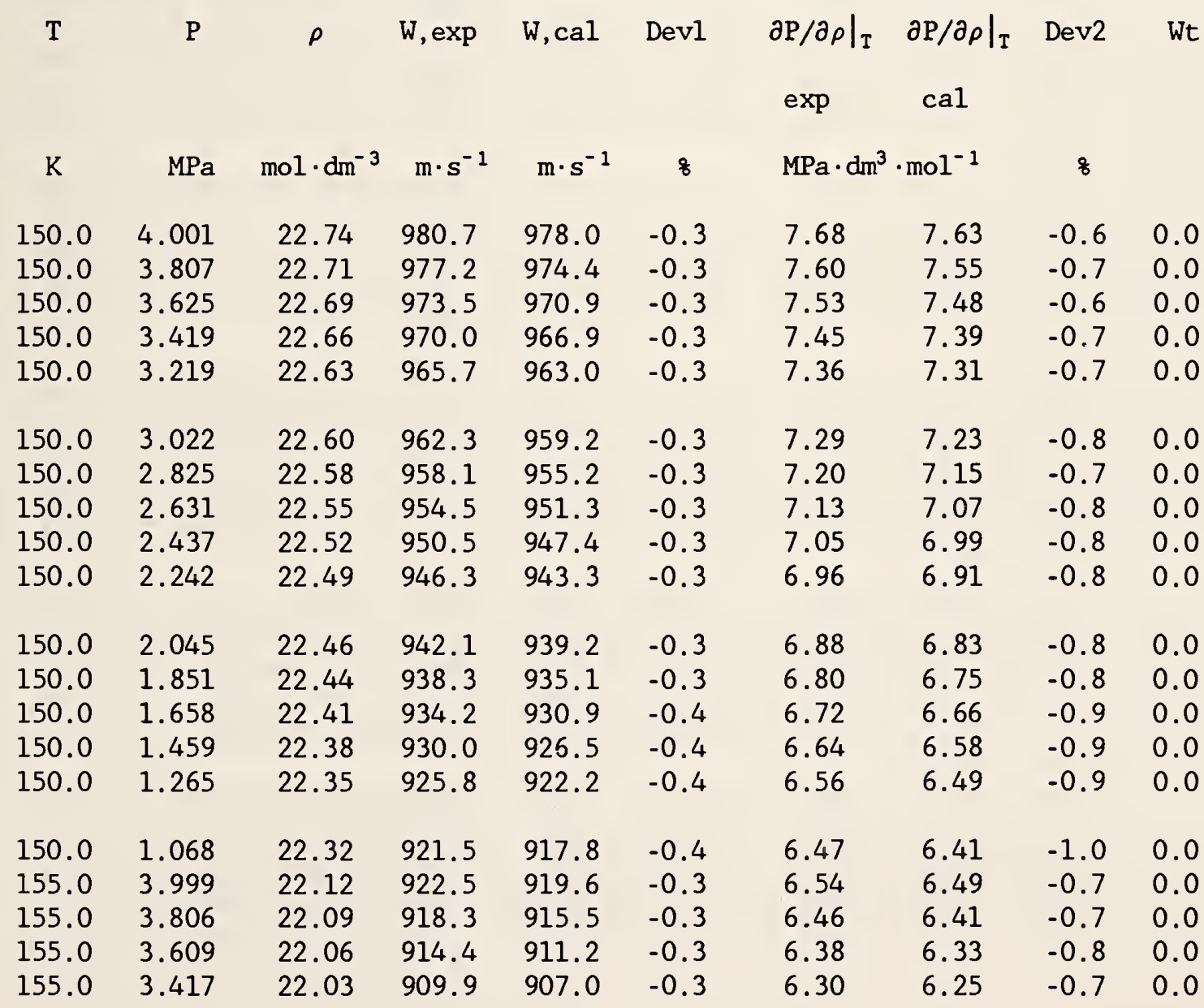


Data from Baidakov et al. [56] (continued)

\begin{tabular}{|c|c|c|c|c|c|c|c|c|c|}
\hline $\mathrm{T}$ & $P$ & $\rho$ & $W, \exp$ & W, cal & Devl & $\begin{array}{c}\partial \mathrm{P} /\left.\partial \rho\right|_{\mathrm{T}} \\
\exp \end{array}$ & $\begin{array}{c}\partial \mathrm{P} /\left.\partial \rho\right|_{\mathrm{T}} \\
\text { cal }\end{array}$ & Dev2 & Wt \\
\hline K & $\mathrm{MPa}$ & $\mathrm{mol} \cdot \mathrm{dm}^{-3}$ & $\mathrm{~m} \cdot \mathrm{s}^{-1}$ & $m \cdot s^{-1}$ & 8 & $\mathrm{MPa} \cdot \mathrm{dm}^{3}$ & $\mathrm{~mol}^{-1}$ & 8 & \\
\hline 155.0 & 3.218 & 22.00 & 905.8 & 902.5 & -0.4 & 6.22 & 6.17 & -0.8 & 0.0 \\
\hline 155.0 & 3.022 & 21.97 & 901.3 & 898.1 & -0.4 & 6.13 & 6.08 & -0.8 & 0.0 \\
\hline 155.0 & 2.827 & 21.93 & 896.8 & 893.6 & -0.4 & 6.05 & 6.00 & -0.8 & 0.0 \\
\hline 155.0 & 2.632 & 21.90 & 892.2 & 889.0 & -0.4 & 5.97 & 5.92 & -0.8 & .0 \\
\hline 155.0 & 2.436 & 21.87 & 887.7 & 884.4 & -0.4 & 5.88 & 5.83 & -0.9 & 0.0 \\
\hline 155.0 & 2.244 & 21.83 & 883.0 & 879.7 & -0.4 & 5.80 & 5.75 & -0.9 & 0.0 \\
\hline 155.0 & 2.047 & 21.80 & 878.3 & 874.9 & -0.4 & 5.71 & 5.66 & -0.9 & 0.0 \\
\hline 155.0 & 1.850 & 21.76 & 873.4 & 870.0 & -0.4 & - & - & - & 0.0 \\
\hline 155.0 & 1.656 & 21.73 & 868.5 & 865.0 & -0.4 & 5.54 & 5.48 & -0.9 & 0.0 \\
\hline 155.0 & 1.459 & 21.69 & 863.7 & 860.0 & -0.4 & 5.45 & 5.40 & -1.0 & 0.0 \\
\hline 160.0 & 3.998 & 21.46 & 861.2 & 858.2 & -0.3 & 5.46 & 5.41 & -0.8 & 0.0 \\
\hline 160.0 & 3.981 & 21.45 & 861.1 & 857.8 & -0.4 & 5.45 & 5.41 & -0.9 & 0.0 \\
\hline 160.0 & 3.805 & 21.42 & 856.5 & 853.4 & -0.4 & 5.37 & 5.33 & -0.8 & 0.0 \\
\hline 160.0 & 3.787 & 21.42 & 856.4 & 852.9 & -0.4 & 5.37 & 5.32 & -0.9 & 0.0 \\
\hline 160.0 & 3.621 & 21.39 & 852.2 & 848.7 & -0.4 & 5.30 & 5.25 & -0.9 & 0.0 \\
\hline 160.0 & 3.608 & 21.38 & 851.9 & 848.4 & -0.4 & 5.29 & 5.24 & -0.9 & 0.0 \\
\hline 160.0 & 3.588 & 21.38 & 851.3 & 847.9 & -0.4 & 5.28 & 5.23 & -0.9 & 0.0 \\
\hline 160.0 & 3.419 & 21.35 & 847.3 & 843.5 & -0.4 & 5.21 & 5.16 & -1.0 & 0.0 \\
\hline 160.0 & 3.230 & 21.31 & 842.3 & 838.6 & -0.4 & 5.13 & 5.08 & -1.0 & 0.0 \\
\hline 160.0 & 3.033 & 21.27 & 837.0 & 833.3 & -0.4 & 5.04 & 4.99 & -1.0 & 0.0 \\
\hline 160.0 & 2.826 & 21.23 & 831.3 & 827.7 & -0.4 & 4.94 & 4.89 & -1.0 & 0.0 \\
\hline 160.0 & 2.613 & 21.19 & 825.4 & 821.8 & -0.4 & 4.84 & 4.80 & -1.0 & 0.0 \\
\hline 160.0 & 2.434 & 21.15 & 820.4 & 816.7 & -0.5 & 4.76 & 4.71 & -1.0 & 0.0 \\
\hline 160.0 & 2.240 & 21.11 & 814.8 & 811.1 & -0.5 & 4.72 & 4.62 & -2.0 & 0.0 \\
\hline 160.0 & 2.043 & 21.06 & 809.0 & 805.3 & -0.5 & 4.58 & 4.53 & -1.0 & 0.0 \\
\hline 160.0 & 1.850 & 21.02 & 803 & 79 & -0.5 & 4.49 & 4.44 & -1.1 & 0.0 \\
\hline 160.0 & 1.737 & 20.99 & 799.8 & 796.0 & -0.5 & 4.43 & 4.38 & -1.1 & 0.0 \\
\hline 160.0 & 1.655 & 20.98 & 797.3 & 793.5 & -0.5 & 4.39 & 4.34 & -1.1 & 0.0 \\
\hline 160.0 & 1.644 & 20.97 & 797.2 & 793.1 & -0.5 & 4.39 & 4.34 & -1.2 & 0.0 \\
\hline 163.0 & 4.000 & 21.03 & 823.3 & 819.5 & -0.5 & 4.84 & 4.79 & -1.0 & 0.0 \\
\hline 163.0 & 3.806 & 20.99 & 818.0 & 814.2 & -0.5 & 4.75 & 4.70 & -1.0 & 0.0 \\
\hline 163.0 & 3.613 & 20.95 & 812.7 & 808.8 & -0.5 & 4.66 & 4.61 & -1.0 & 0.0 \\
\hline 163.0 & 3.422 & 20.91 & 807.3 & 803.3 & -0.5 & 4.57 & 4.53 & -1.1 & 0.0 \\
\hline 163.0 & 3.223 & 20.86 & 801.5 & 797.5 & -0.5 & 4.48 & 4.43 & -1.1 & 0.0 \\
\hline 163.0 & 3.024 & 20.82 & 795.5 & 791.6 & -0.5 & 4.39 & 4.34 & -1.1 & 0.0 \\
\hline
\end{tabular}


Data from Baidakov et al. [56] (continued)

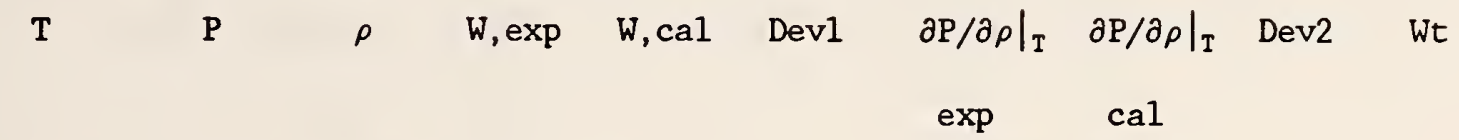

\begin{tabular}{|c|c|c|c|c|c|c|c|c|c|}
\hline K & $\mathrm{MPa}$ & $\mathrm{mol} \cdot \mathrm{dm}^{-3}$ & $\mathrm{~m} \cdot \mathrm{s}^{-1}$ & $m \cdot s^{-1}$ & 8 & \multicolumn{2}{|c|}{$\mathrm{MPa} \cdot \mathrm{dm}^{3} \cdot \mathrm{mol}^{-1}$} & \multicolumn{2}{|l|}{8} \\
\hline 163.0 & 2.826 & 20.77 & 789.6 & 785.5 & -0.5 & 4.30 & 4.25 & -1.1 & 0.0 \\
\hline 163.0 & 2.632 & 20.72 & 783.7 & 779.5 & -0.5 & 4.21 & 4.16 & -1.2 & 0.0 \\
\hline 163.0 & 2.435 & 20.68 & 777.3 & 773.2 & -0.5 & 4.11 & 4.06 & -1.2 & 0.0 \\
\hline 163.0 & 2.244 & 20.63 & 771.2 & 766.9 & -0.6 & 4.02 & 3.97 & -1.2 & 0.0 \\
\hline 163.0 & 2.051 & 20.58 & 764.7 & 760.5 & -0.6 & 3.92 & 3.87 & -1.2 & 0.0 \\
\hline 163.0 & 1.854 & 20.53 & 757.9 & 753.7 & -0.6 & 3.82 & 3.77 & -1.2 & 0.0 \\
\hline 167.0 & 3.995 & 20.42 & 769.5 & 765.0 & -0.6 & 4.03 & 3.98 & -1.2 & 0.0 \\
\hline 167.0 & 3.802 & 20.37 & 763.1 & 758.9 & -0.6 & 3.93 & 3.89 & -1.2 & 0.0 \\
\hline 167.0 & 3.616 & 20.32 & 757.2 & 752.8 & -0.6 & 3.85 & 3.80 & -1.2 & 0.0 \\
\hline 167.0 & 3.490 & 20.28 & 752.9 & 748.6 & -0.6 & 3.78 & 3.74 & -1.2 & 0.0 \\
\hline 167.0 & 3.423 & 20.27 & 750.6 & 746.3 & -0.6 & 3.75 & 3.70 & -1.2 & 0.0 \\
\hline 167.0 & 3.219 & 20.21 & 743.8 & 739.3 & -0.6 & 3.65 & 3.60 & -1.3 & 0.0 \\
\hline 167.0 & 3.036 & 20.16 & 737.1 & 732.8 & -0.6 & 3.56 & 3.51 & -1.2 & 0.0 \\
\hline 167.0 & 2.823 & 20.10 & 729.7 & 725.1 & -0.6 & 3.45 & 3.41 & -1.3 & 0.0 \\
\hline 167.0 & 2.623 & 20.04 & 722.2 & 717.6 & -0.6 & 3.35 & 3.31 & -1.4 & 0.0 \\
\hline 167.0 & 2.462 & 19.99 & 715.8 & 711.4 & -0.6 & 3.27 & 3.22 & -1.3 & 0.0 \\
\hline 167.0 & 2.435 & 19.98 & 714.9 & 710.3 & -0.6 & 3.25 & 3.21 & -1.4 & 0.0 \\
\hline 167.0 & 2.325 & 19.95 & 710.3 & 706.0 & -0.6 & 3.19 & 3.15 & -1.3 & 0.0 \\
\hline 167.0 & 2.267 & 19.93 & 708.2 & 703.6 & -0.6 & 3.16 & 3.12 & -1.4 & 0.0 \\
\hline 167.0 & 2.240 & 19.92 & 707.1 & 702.5 & -0.6 & 3.15 & 3.11 & -1.4 & 0.0 \\
\hline 167.0 & 2.121 & 19.88 & 702.2 & 697.7 & -0.6 & 3.09 & 3.04 & -1.4 & 0.0 \\
\hline 167.0 & 2.104 & 19.87 & 701.5 & 697.0 & -0.6 & 3.08 & 3.04 & -1.4 & 0.0 \\
\hline 170.0 & 4.000 & 19.91 & 726.4 & 721.8 & -0.6 & 3.43 & 3.39 & -1.3 & 0.0 \\
\hline 170.0 & 3.810 & 19.86 & 719.3 & 714.9 & -0.6 & 3.34 & 3.30 & -1.3 & 0.0 \\
\hline 170.0 & 3.608 & 19.79 & 711.9 & 707.4 & -0.6 & 3.24 & 3.20 & -1.3 & 0.0 \\
\hline 170.0 & 3.412 & 19.73 & 704.2 & 699.8 & -0.6 & 3.14 & 3.10 & -1.3 & 0.0 \\
\hline 170.0 & 3.217 & 19.67 & 696.7 & 692.1 & -0.7 & 3.04 & 3.00 & -1.4 & 0.0 \\
\hline 170.0 & 3.027 & 19.60 & 688.8 & 684.3 & -0.7 & 2.94 & 2.90 & -1.4 & 0.0 \\
\hline 170.0 & 2.829 & 19.53 & 680.4 & 675.8 & -0.7 & 2.83 & 2.79 & -1.4 & 0.0 \\
\hline 170.0 & 2.629 & 19.46 & 671.4 & 667.0 & -0.7 & 2.72 & 2.69 & -1.4 & 0.0 \\
\hline 170.0 & 2.434 & 19.39 & 662.4 & 658.1 & -0.7 & 2.62 & 2.58 & -1.4 & 0.0 \\
\hline 170.0 & 2.354 & 19.35 & 658.6 & 654.3 & -0.7 & 2.57 & 2.53 & -1.4 & 0.0 \\
\hline 173.0 & 4.001 & 19.36 & 680.2 & 675.6 & -0.7 & 2.86 & 2.82 & -1.4 & 0.0 \\
\hline 173.0 & 3.806 & 19.29 & 671.9 & 667.4 & -0.7 & 2.75 & 2.72 & -1.4 & 0.0 \\
\hline 173.0 & 3.610 & 19.22 & 663.2 & 658.8 & -0.7 & 2.65 & 2.61 & -1.3 & 0.0 \\
\hline
\end{tabular}


Data from Baidakov et al. [56] (continued)

\begin{tabular}{|c|c|c|c|c|c|c|c|c|c|}
\hline $\mathrm{T}$ & $\mathrm{P}$ & $\rho$ & $W, \exp$ & $\mathrm{W}, \mathrm{cal}$ & Devl & $\begin{array}{c}\partial \mathrm{P} /\left.\partial \rho\right|_{\mathrm{T}} \\
\exp \end{array}$ & $\begin{array}{c}\partial \mathrm{P} /\left.\partial \rho\right|_{\mathrm{T}} \\
\text { cal }\end{array}$ & Dev2 & Wt \\
\hline K & $\mathrm{MPa}$ & $\mathrm{mol} \cdot \mathrm{dm}^{-3}$ & $\mathrm{~m} \cdot \mathrm{s}^{-1}$ & $\mathrm{~m} \cdot \mathrm{s}^{-1}$ & 8 & $\mathrm{MPa} \cdot \mathrm{dm}^{3}$ & $\mathrm{~mol}^{-1}$ & 8 & \\
\hline 173.0 & 3.419 & 19.14 & 654.7 & 650.2 & -0.7 & 2.55 & 2.51 & -1.4 & 0.0 \\
\hline 173.0 & 3.221 & 19.06 & 645.2 & 640.9 & -0.7 & 2.44 & 2.40 & -1.4 & 0.0 \\
\hline 173.0 & 3.024 & 18.98 & 635.4 & 631.2 & -0.7 & 2.33 & 2.29 & -1.4 & 0.0 \\
\hline 173.0 & 2.824 & 18.89 & 624.9 & 620.9 & -0.6 & 2.21 & 2.18 & -1.3 & 0.0 \\
\hline 173.0 & 2.628 & 18.80 & 614.0 & 610.3 & -0.6 & 2.09 & 2.07 & -1.2 & 0.0 \\
\hline 177.0 & 4.003 & 18.52 & 612.2 & 607.8 & -0.7 & 2.10 & 2.07 & -1.4 & .0 \\
\hline 177.0 & 3.807 & 18.42 & 601.4 & 597.4 & -0.7 & 1.99 & 1.96 & -1.3 & 0.0 \\
\hline 177.0 & 3.617 & 18.32 & 590.4 & 586.8 & -0.6 & 1.88 & 1.85 & -1.2 & .0 \\
\hline 177.0 & 3.610 & 18.32 & 590.0 & 586.3 & -0.6 & 1.87 & 1.85 & -1.2 & 0.0 \\
\hline 177.0 & 3.414 & 18.21 & 578.0 & 574.7 & -0.6 & 1.75 & 1.74 & -1.1 & 0.0 \\
\hline 177.0 & 3.219 & 18.09 & 565.0 & 562.4 & -0.5 & 1.63 & 1.62 & -0.9 & 0.0 \\
\hline 177.0 & 3.023 & 17.97 & 551.0 & 549.2 & -0.3 & 1.50 & 1.50 & -0.6 & 0.0 \\
\hline 177.0 & 2.982 & 17.94 & 547.9 & 546.3 & -0.3 & 1.48 & 1.47 & -0.6 & 0.0 \\
\hline 180.0 & 4.000 & 17.76 & 553.3 & 550.0 & -0.6 & 1.54 & 1.52 & -1.2 & 0.0 \\
\hline 180.0 & 3.907 & 17.70 & 547.0 & 543.8 & -0.6 & 1.49 & 1.47 & -1.1 & 0.0 \\
\hline 180.0 & 3.808 & 17.63 & 540.0 & 537.0 & -0.5 & 1.42 & 1.41 & -1.1 & 0.0 \\
\hline 180.0 & 3.716 & 17.56 & 532.9 & 530.5 & -0.4 & 1.36 & 1.35 & -0.9 & 0.0 \\
\hline 180.0 & 3.613 & 17.48 & 524.3 & 522.9 & -0.3 & 1.29 & 1.29 & -0.5 & 0.0 \\
\hline 180.0 & 3.520 & 17.41 & 516.8 & 515.7 & -0.2 & 1.23 & 1.23 & -0.4 & 0.0 \\
\hline 180.0 & 3.416 & 17.32 & 507.4 & 507.3 & 0.0 & 1.16 & 1.16 & 0.0 & 0.0 \\
\hline 180.0 & 3.346 & 17.26 & 500.9 & 501.4 & 0.1 & 1.11 & 1.11 & 0.2 & 0.0 \\
\hline 180.0 & 3.338 & 17.25 & 500.0 & 500.7 & 0.1 & 1.10 & 1.10 & 0.3 & 0.0 \\
\hline 183.0 & 3.996 & 16.79 & 482.4 & 481.6 & -0.2 & 0.98 & 0.98 & -0.3 & 0.0 \\
\hline 183.0 & 3.977 & 16.78 & 481.2 & 479.9 & -0.3 & 0.97 & 0.96 & -0.5 & 0.0 \\
\hline 183.0 & 3.912 & 16.71 & 474.4 & 473.9 & -0.1 & 0.92 & 0.92 & -0.2 & 0.0 \\
\hline 183.0 & 3.808 & 16.59 & 462.1 & 463.6 & 0.3 & 0.84 & 0.85 & 0.7 & 0.0 \\
\hline 183.0 & 3.731 & 16.49 & 452.8 & 455.5 & 0.6 & 0.78 & 0.79 & 1.3 & 0.0 \\
\hline 183.0 & 3.690 & 16.44 & 447.1 & 450.9 & 0.9 & 0.75 & 0.76 & 1.8 & 0.0 \\
\hline 183.0 & 3.649 & 16.39 & 441.7 & 446.2 & 1.0 & 0.71 & 0.73 & 2.1 & 0.0 \\
\hline
\end{tabular}

Number of Points (Ref. 56) 119

$\begin{array}{llllllll}\text { SOUND SPEED: } & \text { AAD }-8 & 0.475 & \text { BIAS }-8 & -0.425 & \text { RMS }-8 & 0.267 & \\ \text { AAD } & 3.504 & \text { BIAS } & -3.277 & \text { RMS } & 1.589 & \mathrm{~m} \cdot \mathrm{s}^{-1}\end{array}$

Number of Points (Ref. 56) 118

$\begin{array}{llllllll}\text { DERIVATIVE: } & \text { AAD-8 } & 1.037 & \text { BIAS }- & -0.929 & \text { RMS }-8 & 0.567 & \\ & \text { AAD } & 0.040 & \text { BIAS } & -0.039 & \text { RMS } & 0.018 & \mathrm{MPa} \cdot \mathrm{dm}^{3} \cdot \mathrm{mol}^{-1}\end{array}$ 
Data from Blagoi et al. [57]

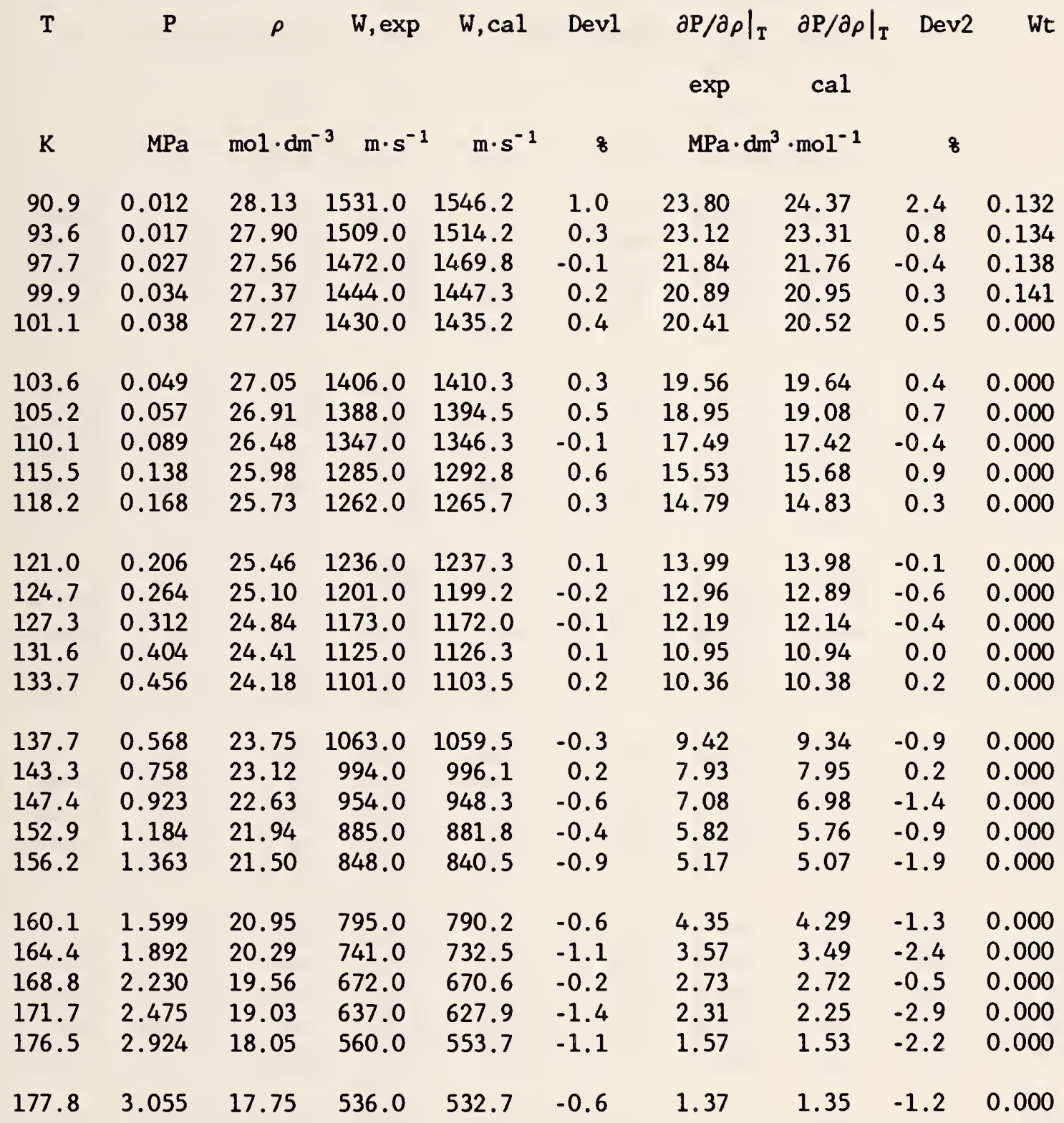

Number of Points (Ref. 57) 26 on saturation boundary

$\begin{array}{lllllll}\text { SOUND SPEED: } & \text { AAD- } 8 & 0.461 & \text { BIAS }-8 & -0.134 & \text { RMS }-8 & 0.572 \\ & \text { AAD } & 4.506 & \text { BIAS } & -0.030 & \text { RMS } & 5.524 \mathrm{~m} \cdot \mathrm{s}^{-1} \\ \text { DERIVATIVE: } & \text { AAD-8 } & 0.928 & \text { BIAS }-8 & -0.411 & \text { RMS }-8 & 1.147 \\ & \text { AAD } & 0.086 & \text { BIAS } & 0.018 & \text { RMS } & 0.135 \mathrm{MPa} \cdot \mathrm{dm}^{3} \cdot \mathrm{mol}^{-1}\end{array}$


Data from Gammon and Douslin [37]

\begin{tabular}{|c|c|c|c|c|c|c|c|c|c|}
\hline $\mathrm{T}$ & $\mathbf{P}$ & $\rho$ & $W, \exp$ & W, cal & Devl & $\begin{array}{c}\partial \mathrm{P} /\left.\partial \rho\right|_{\mathrm{T}} \\
\exp \end{array}$ & $\begin{array}{c}\partial \mathrm{P} /\left.\partial \rho\right|_{\mathrm{T}} \\
\mathrm{cal}\end{array}$ & Dev2 & Wt \\
\hline K & $\mathrm{MPa}$ & $\mathrm{mol} \cdot \mathrm{dm}^{-3}$ & $\mathrm{~m} \cdot \mathrm{s}^{-1}$ & $\mathrm{~m} \cdot \mathrm{s}^{-1}$ & 8 & $\mathrm{MPa} \cdot \mathrm{dm}$ & $\mathrm{mol}^{-1}$ & 8 & \\
\hline 3.15 & 24.595 & 10.14 & 574.3 & 573.3 & -0.2 & 2.98 & 2.97 & -0.4 & 0.00 \\
\hline 8.15 & 2.378 & 1.00 & 441.9 & 442.0 & 0.0 & 2.28 & 2.28 & 0.0 & 0.857 \\
\hline 8.15 & 5.627 & 2.50 & 437.1 & 437.0 & 0.0 & 2.06 & 2.06 & -0.1 & 0.586 \\
\hline 3.15 & 10.491 & 5.00 & 444.6 & 444.6 & 0.0 & 1.88 & 1.88 & 0.0 & 0.48 \\
\hline 8.15 & 15.205 & 7.49 & 474.5 & 474.1 & -0.1 & 1.95 & 1.95 & -0.2 & 0.00 \\
\hline 3.15 & 19.418 & 9.53 & 518.3 & 517.5 & -0.1 & 2.25 & 2.24 & -0.3 & 0.000 \\
\hline 8.15 & 20.702 & 10.08 & 533.9 & 532.9 & -0.2 & 2.38 & 2.37 & -0.4 & 0.000 \\
\hline 3.15 & 16.178 & 9.63 & 478.4 & 477.4 & -0.2 & 1.71 & 1.70 & -0.4 & 0.000 \\
\hline 8.15 & 12.816 & 9.75 & 430.9 & 429.5 & -0.3 & 1.17 & 1.16 & -0.6 & 0.00 \\
\hline 3.15 & 9.320 & 9.88 & 371.6 & 370.1 & -0.4 & 0.63 & 0.62 & -0.6 & 0.000 \\
\hline 3.15 & 5.704 & 10.05 & 282.6 & 282.5 & 0.0 & 0.12 & 0.12 & -0.1 & 0.00 \\
\hline 3.05 & 0.782 & 0.52 & 353.2 & 353.1 & 0.0 & 1.42 & 1.42 & 0.0 & 3.56 \\
\hline 3.05 & 1.563 & 1.11 & 340.6 & 340.4 & -0.1 & 1.23 & 1.23 & -0.2 & 2.48 \\
\hline 3.05 & 2.244 & 1.70 & 328.7 & 328.4 & -0.1 & 1.05 & 1.05 & -0.2 & 2.03 \\
\hline 3.05 & 2.820 & 2.30 & 317.7 & 317.5 & -0.1 & 0.89 & 0.88 & -0.2 & 1.78 \\
\hline .05 & 3.306 & 2.90 & 307.5 & 307.4 & 0.0 & 0.73 & 0.73 & -0.1 & 1.625 \\
\hline .05 & 3.703 & 3.50 & 298.3 & 298.4 & 0.1 & 0.60 & 0.60 & 0.1 & 1.51 \\
\hline 3.05 & 4.025 & 4.10 & 289.9 & 290.3 & 0.1 & 0.47 & 0.48 & 0.2 & 1.42 \\
\hline 3.05 & 4.281 & 4.71 & 282.4 & 282.9 & 0.2 & 0.37 & 0.37 & 0.4 & 1.36 \\
\hline 3.05 & 4.478 & 5.32 & 275.6 & 276.3 & 0.2 & 0.28 & 0.28 & 0.2 & 1.31 \\
\hline .05 & 4.626 & 5.93 & 269.5 & 270.2 & 0.3 & 0.20 & 0.21 & 0.4 & 1.28 \\
\hline .05 & 4.729 & 6.51 & 264.2 & 265.0 & 0.3 & 0.15 & 0.15 & 0.4 & 0.000 \\
\hline 3.05 & 4.806 & 7.13 & 258.9 & 260.0 & 0.4 & 0.10 & 0.10 & 1.0 & 0.000 \\
\hline 3.05 & 4.859 & 7.75 & 254.1 & 255.6 & 0.6 & 0.07 & 0.07 & 0.8 & 0.000 \\
\hline 3.05 & 4.897 & 8.39 & 249.9 & 252.0 & 0.9 & 0.05 & 0.05 & 1.0 & 0.00 \\
\hline 05 & 4.924 & 9.03 & 246.7 & 249.7 & 1.2 & 0.04 & 0.04 & 2.9 & 0.0 \\
\hline 05 & 4.946 & 9.64 & 245.7 & 249.3 & 1.5 & 0.03 & 0.03 & 2.9 & 0.000 \\
\hline .05 & 4.962 & 10.12 & 246.8 & 250.6 & 1.5 & 0.03 & 0.03 & 4.4 & 0.000 \\
\hline 3.05 & 4.966 & 10.24 & 247.5 & 251.3 & 1.5 & 0.03 & 0.04 & 3.1 & 0.000 \\
\hline 3.05 & 4.988 & 10.83 & 253.1 & 256.4 & 1.3 & 0.04 & 0.04 & 3.0 & 0.000 \\
\hline 05 & 5.016 & 11.43 & 263.1 & 265.7 & 1.0 & 0.05 & 0.05 & 3.2 & 0.00 \\
\hline 45 & 4.587 & 6.52 & 258.4 & 259.2 & 0.3 & 0.12 & 0.12 & 0.6 & 0.00 \\
\hline 45 & 4.646 & 7.15 & 251.8 & 253.3 & 0.6 & 0.07 & 0.07 & 0.8 & 0.000 \\
\hline 45 & 4.683 & 7.79 & 244.5 & 247.8 & 1.4 & 0.04 & 0.04 & 2.6 & 0.000 \\
\hline 1.45 & 4.705 & 8.45 & 236.8 & 243.0 & 2.6 & 0.02 & 0.02 & 3.6 & 0.000 \\
\hline
\end{tabular}


Data from Gammon and Douslin [37] (continued)

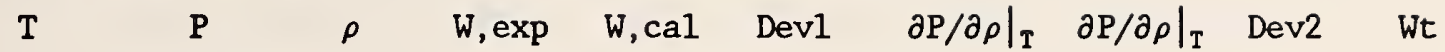
$\exp \quad \mathrm{cal}$

\begin{tabular}{|c|c|c|c|c|c|c|c|c|c|}
\hline $\mathrm{K}$ & $\mathrm{MPa}$ & $\mathrm{mol} \cdot \mathrm{dm}^{-3}$ & $\mathrm{~m} \cdot \mathrm{s}^{-1}$ & $\mathrm{~m} \cdot \mathrm{s}^{-1}$ & 8 & \multicolumn{2}{|c|}{$\mathrm{MPa} \cdot \mathrm{dm}^{3} \cdot \mathrm{mol}^{-1}$} & \multicolumn{2}{|l|}{8} \\
\hline 191.45 & 4.717 & 9.12 & 229.5 & 239.4 & 4.3 & 0.01 & 0.01 & 11.5 & 0.000 \\
\hline 191.45 & 4.725 & 9.74 & 224.5 & 237.9 & 6.0 & 0.01 & 0.01 & 16.6 & 0.000 \\
\hline 191.45 & 4.730 & 10.17 & 223.7 & 238.4 & 6.5 & 0.01 & 0.01 & 17.2 & 0.000 \\
\hline 191.45 & 4.731 & 10.28 & 224.1 & 238.7 & 6.5 & 0.01 & 0.01 & 19.6 & 0.000 \\
\hline 191.45 & 4.738 & 10.82 & 229.2 & 242.3 & 5.7 & 0.01 & 0.01 & 13.1 & 0.000 \\
\hline 191.45 & 4.748 & 11.40 & 240.2 & 250.2 & 4.2 & 0.02 & 0.02 & 6.8 & 0.000 \\
\hline 190.85 & 4.533 & 6.53 & 256.1 & 257.0 & 0.3 & 0.11 & 0.11 & 0.7 & 0.000 \\
\hline 190.85 & 4.586 & 7.15 & 248.6 & 250.7 & 0.8 & 0.06 & 0.06 & 1.3 & 0.000 \\
\hline 190.85 & 4.616 & 7.80 & 239.8 & 244.8 & 2.1 & 0.03 & 0.03 & 3.0 & 0.000 \\
\hline 30.85 & 4.632 & 8.48 & 228.9 & 239.4 & 4.6 & 0.01 & 0.01 & 11.3 & 0.000 \\
\hline 190.85 & 4.638 & 9.21 & 215.8 & 235.0 & 8.9 & 0.00 & 0.01 & 18.5 & 0.000 \\
\hline 190.85 & 4.641 & 9.89 & 205.1 & 233.2 & 13.7 & 0.00 & 0.00 & 26.9 & 0.000 \\
\hline 190.85 & 4.643 & 10.29 & 204.4 & 233.7 & 14.3 & 0.00 & 0.00 & 30.4 & 0.000 \\
\hline 190.85 & 4.643 & 10.37 & 205.1 & 233.9 & 14.1 & 0.00 & 0.00 & 34.4 & 0.000 \\
\hline 190.85 & 4.646 & 10.86 & 212.9 & 237.0 & 11.3 & 0.00 & 0.01 & 14.2 & 0.000 \\
\hline 190.85 & 4.650 & 11.42 & 225.9 & 244.5 & 8.3 & 0.01 & 0.01 & 14.4 & 0.000 \\
\hline 190.63 & 4.513 & 6.53 & 255.2 & 256.1 & 0.3 & 0.10 & 0.10 & 0.5 & 0.000 \\
\hline 190.63 & 4.564 & 7.15 & 247.4 & 249.7 & 1.0 & 0.06 & 0.06 & 1.5 & 0.000 \\
\hline 190.63 & 4.592 & 7.80 & 237.7 & 243.6 & 2.5 & 0.03 & 0.03 & 4.4 & 0.000 \\
\hline 190.63 & 4.605 & 8.49 & 224.5 & 238.0 & 6.0 & 0.01 & 0.01 & 10.8 & 0.000 \\
\hline 190.63 & 4.609 & 9.28 & 204.1 & 233.2 & 14.2 & 0.00 & 0.00 & 36.6 & 0.000 \\
\hline 190.63 & 4.610 & 9.86 & 191.3 & 231.5 & 21.0 & 0.00 & 0.00 & 10.8 & 0.000 \\
\hline 190.63 & 4.611 & 10.53 & 195.2 & 232.6 & 19.2 & 0.00 & 0.00 & 36.8 & 0.000 \\
\hline 190.63 & 4.611 & 10.51 & 195.5 & 232.6 & 18.9 & 0.00 & 0.00 & 33.9 & 0.000 \\
\hline 190.63 & 4.612 & 11.08 & 205.9 & 237.2 & 15.2 & 0.00 & 0.00 & 22.4 & 0.000 \\
\hline 190.63 & 4.615 & 11.48 & 219.1 & 243.3 & 11.1 & 0.01 & 0.01 & 17.6 & 0.000 \\
\hline 190.56 & 4.507 & 6.53 & 255.0 & 255.8 & 0.3 & 0.10 & 0.10 & 0.4 & 0.000 \\
\hline 190.56 & 4.556 & 7.16 & 246.9 & 249.4 & 1.0 & 0.06 & 0.06 & 2.8 & 0.000 \\
\hline 190.56 & 4.584 & 7.80 & 237.0 & 243.3 & 2.7 & 0.03 & 0.03 & 4.0 & 0.000 \\
\hline 190.56 & 4.596 & 8.48 & 222.8 & 237.6 & 6.7 & 0.01 & 0.01 & 14.0 & 0.000 \\
\hline 190.56 & 4.600 & 9.21 & 199.1 & 233.0 & 17.0 & 0.00 & 0.00 & 11.5 & 0.000 \\
\hline 190.56 & 4.601 & 10.23 & 184.2 & 231.0 & 25.4 & 0.00 & 0.00 & 127.9 & 0.000 \\
\hline 190.56 & 4.601 & 10.95 & 198.0 & 235.1 & 18.8 & 0.00 & 0.00 & 79.3 & 0.000 \\
\hline 190.56 & 4.601 & 10.83 & 196.6 & 234.0 & 19.0 & 0.00 & 0.00 & 22.3 & 0.000 \\
\hline 190.56 & 4.602 & 11.24 & 205.4 & 238.6 & 16.2 & 0.00 & 0.00 & 30.4 & 0.000 \\
\hline
\end{tabular}


Data from Gammon and Douslin [37] (continued)

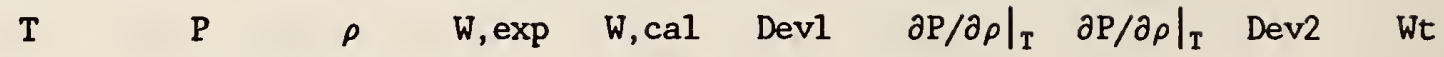

\begin{tabular}{|c|c|c|c|c|c|c|c|c|c|}
\hline \multirow[b]{2}{*}{$\mathrm{K}$} & \multirow[b]{2}{*}{$\mathrm{MPa}$} & \multirow[b]{2}{*}{$\mathrm{mol} \cdot \mathrm{dm}^{-3}$} & \multirow[b]{2}{*}{$\mathrm{m} \cdot \mathrm{s}^{-1}$} & \multirow[b]{2}{*}{$\mathrm{m} \cdot \mathrm{s}^{-1}$} & \multirow[b]{2}{*}{8} & \multicolumn{2}{|r|}{ cal } & \multirow[b]{2}{*}{8} & \\
\hline & & & & & & \multicolumn{2}{|c|}{$\mathrm{MPa} \cdot \mathrm{dm}^{3} \cdot \mathrm{mol}^{-1}$} & & \\
\hline 190.56 & 4.603 & 11.55 & 217.0 & 243.9 & 12.4 & 0.01 & 0.01 & 32.8 & 0.000 \\
\hline 190.50 & 0.771 & 0.52 & 350.5 & 350.4 & 0.0 & 1.40 & 1.40 & -0.1 & 3.549 \\
\hline 190.50 & 1.538 & 1.11 & 337.6 & 337.3 & -0.1 & 1.21 & 1.20 & -0.1 & 2.466 \\
\hline 190.50 & 2.203 & 1.71 & 325.3 & 325.0 & -0.1 & 1.02 & 1.02 & -0.2 & 2.023 \\
\hline 190.50 & 2.760 & 2.30 & 313.9 & 313.7 & -0.1 & 0.85 & 0.85 & -0.2 & 1.775 \\
\hline 190.50 & 3.226 & 2.91 & 303.2 & 303.2 & 0.0 & 0.70 & 0.70 & -0.1 & 0.000 \\
\hline 190.50 & 3.603 & 3.51 & 293.5 & 293.7 & 0.0 & 0.56 & 0.56 & 0.0 & 0.000 \\
\hline 190.50 & 3.902 & 4.11 & 284.7 & 284.9 & 0.1 & 0.44 & 0.44 & 0.2 & 0.000 \\
\hline 190.50 & 4.133 & 4.72 & 276.5 & 276.8 & 0.1 & 0.33 & 0.33 & 0.2 & 0.000 \\
\hline 190.50 & 4.304 & 5.32 & 269.0 & 269.3 & 0.1 & 0.24 & 0.24 & 0.0 & 0.000 \\
\hline 190.50 & 4.425 & 5.94 & 261.7 & 262.1 & 0.1 & 0.16 & 0.16 & 0.4 & 0.000 \\
\hline 190.50 & 4.501 & 6.53 & 254.7 & 255.6 & 0.4 & 0.10 & 0.10 & 0.4 & 0.000 \\
\hline 190.50 & 4.550 & 7.15 & 246.6 & 249.1 & 1.0 & 0.06 & 0.06 & 2.9 & 0.000 \\
\hline 190.50 & 4.577 & 7.80 & 236.3 & 243.0 & 2.8 & 0.03 & 0.03 & 4.4 & 0.000 \\
\hline 190.50 & 4.589 & 8.48 & 221.0 & 237.3 & 7.4 & 0.01 & 0.01 & 17.4 & 0.000 \\
\hline 190.50 & 4.592 & 9.04 & 200.0 & 233.5 & 16.8 & 0.00 & 0.00 & 28.7 & 0.000 \\
\hline 190.43 & 4.495 & 6.53 & 254.4 & 255.3 & 0.4 & 0.10 & 0.10 & 1.0 & 0.000 \\
\hline 190.43 & 4.543 & 7.16 & 246.1 & 248.8 & 1.1 & 0.05 & 0.06 & 2.4 & 0.000 \\
\hline 190.43 & 4.569 & 7.80 & 235.5 & 242.6 & 3.0 & 0.02 & 0.03 & 8.4 & 0.000 \\
\hline 190.43 & 4.580 & 8.48 & 218.3 & 236.9 & 8.5 & 0.01 & 0.01 & 20.7 & 0.000 \\
\hline 190.25 & 4.287 & 5.33 & 268.3 & 268.5 & 0.1 & 0.23 & 0.23 & 0.2 & 0.000 \\
\hline 190.25 & 4.405 & 5.94 & 260.9 & 261.2 & 0.1 & 0.16 & 0.16 & 0.0 & 0.000 \\
\hline 190.25 & 4.479 & 6.53 & 253.7 & 254.6 & 0.4 & 0.10 & 0.10 & 0.7 & 0.000 \\
\hline 190.25 & 4.525 & 7.16 & 245.0 & 248.0 & 1.2 & 0.05 & 0.05 & 2.1 & 0.000 \\
\hline 190.25 & 4.549 & 7.80 & 233.2 & 241.7 & 3.6 & 0.02 & 0.02 & 5.3 & 0.000 \\
\hline 189.65 & 3.861 & 4.11 & 282.9 & 283.1 & 0.1 & 0.42 & 0.42 & 0.0 & 0.000 \\
\hline 189.65 & 4.083 & 4.72 & 274.5 & 274.7 & 0.1 & 0.31 & 0.31 & 0.1 & 0.000 \\
\hline 189.65 & 4.245 & 5.33 & 266.7 & 266.8 & 0.0 & 0.22 & 0.22 & -0.2 & 0.000 \\
\hline 189.65 & 4.357 & 5.94 & 258.9 & 259.2 & 0.1 & 0.14 & 0.14 & -0.1 & 0.000 \\
\hline 189.65 & 4.424 & 6.53 & 251.0 & 252.3 & 0.5 & 0.09 & 0.09 & 0.7 & 0.000 \\
\hline 189.65 & 4.463 & 7.16 & 240.5 & 245.3 & 2.0 & 0.04 & 0.04 & 4.0 & 0.000 \\
\hline 188.15 & 0.761 & 0.52 & 348.0 & 347.9 & 0.0 & 1.38 & 1.38 & -0.1 & 0.012 \\
\hline 188.15 & 1.515 & 1.11 & 334.8 & 334.5 & -0.1 & 1.18 & 1.18 & -0.2 & 0.008 \\
\hline 188.15 & 2.164 & 1.71 & 322.1 & 321.8 & -0.1 & 0.99 & 0.99 & -0.2 & 0.007 \\
\hline 188.15 & 2.705 & 2.30 & 310.3 & 310.1 & -0.1 & 0.82 & 0.82 & -0.1 & 0.006 \\
\hline
\end{tabular}


Data from Gammon and Douslin [37] (continued)

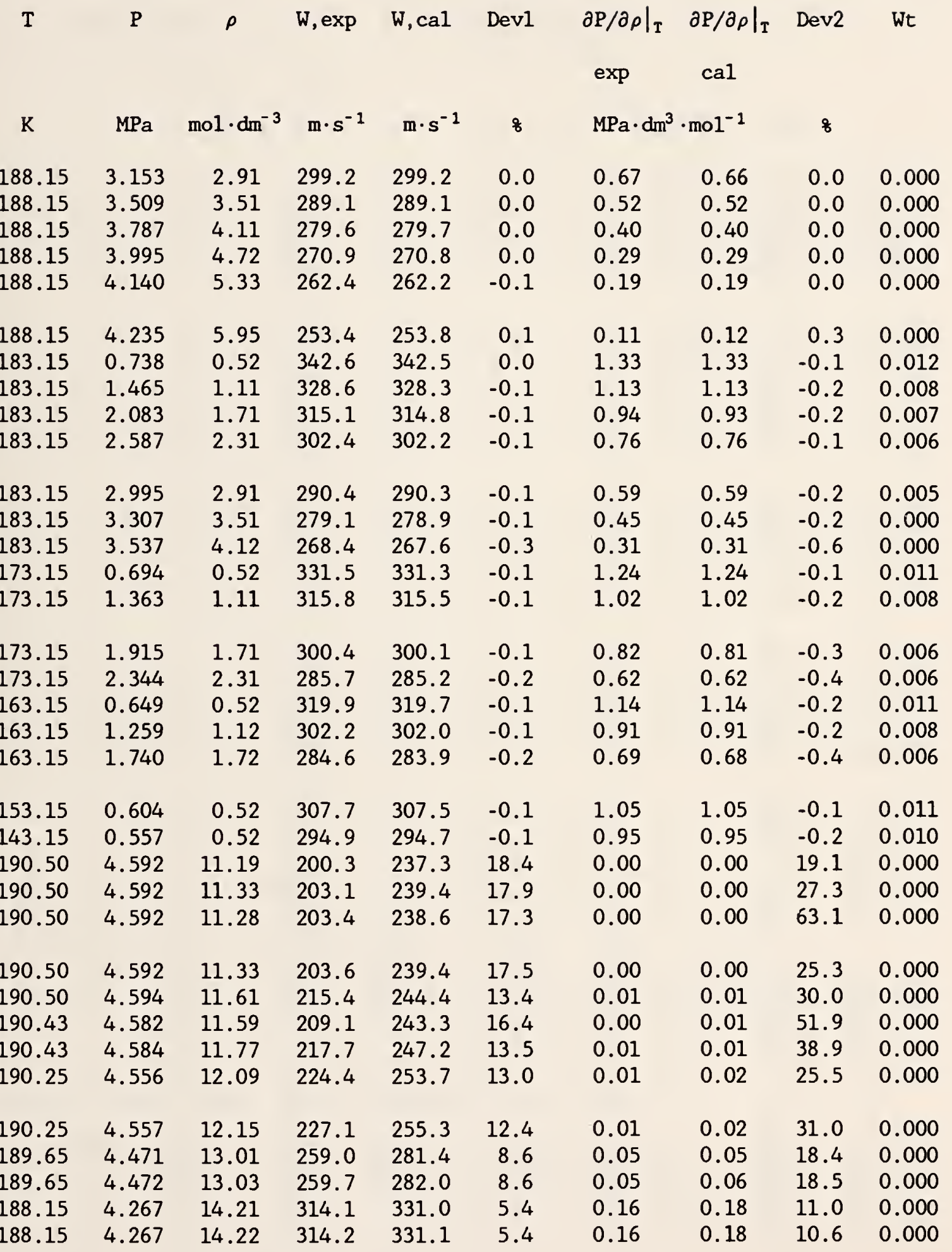


Data from Gammon and Douslin [37] (continued)

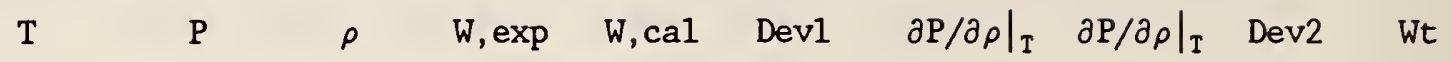

\begin{tabular}{|c|c|c|c|c|c|c|c|c|c|}
\hline \multirow{3}{*}{$\begin{array}{c}K \\
185.91\end{array}$} & \multirow{3}{*}{$\begin{array}{c}\mathrm{MPa} \\
3.977\end{array}$} & \multirow{3}{*}{$\begin{array}{c}\mathrm{mol} \cdot \mathrm{dm}^{-3} \\
15.32\end{array}$} & \multirow{3}{*}{$\begin{array}{l}\mathrm{m} \cdot \mathrm{s}^{-1} \\
374.2\end{array}$} & \multirow{3}{*}{$\begin{array}{c}\mathrm{m} \cdot \mathrm{s}^{-1} \\
385.6\end{array}$} & \multirow{3}{*}{$\begin{array}{c}8 \\
3.0\end{array}$} & \multicolumn{2}{|r|}{ cal } & \multirow[b]{2}{*}{8} & \\
\hline & & & & & & \multicolumn{2}{|c|}{$\mathrm{MPa} \cdot \mathrm{dm}^{3} \cdot \mathrm{mol}^{-1}$} & & \\
\hline & & & & & & 0.37 & 0.40 & 6.0 & 0.000 \\
\hline 183.15 & 3.642 & 16.31 & 434.9 & 440.5 & 1.3 & 0.68 & 0.69 & 2.5 & 0.000 \\
\hline 183.15 & 3.642 & 16.31 & 435.0 & 440.5 & 1.3 & 0.68 & 0.69 & 2.6 & 0.000 \\
\hline 178.15 & 3.092 & 17.67 & 528.4 & 527.1 & -0.2 & 1.31 & 1.31 & -0.5 & 9.928 \\
\hline 173.15 & 2.606 & 18.75 & 610.0 & 606.1 & -0.6 & 2.05 & 2.02 & -1.3 & 5.181 \\
\hline 170.75 & 2.394 & 19.21 & 646.5 & 642.1 & -0.7 & 2.43 & 2.40 & -1.4 & 4.019 \\
\hline 163.15 & 1.804 & 20.49 & 754.1 & 749.6 & -0.6 & 3.77 & 3.72 & -1.3 & 2.608 \\
\hline 155.44 & 1.321 & 21.60 & 854.4 & 850.2 & -0.5 & 5.29 & 5.23 & -1.1 & 2.024 \\
\hline 153.15 & 1.197 & 21.91 & 882.8 & 878.7 & -0.5 & 5.77 & 5.71 & -1.1 & 1.908 \\
\hline 143.15 & 0.753 & 23.14 & 1001.9 & 997.9 & -0.4 & 8.06 & 7.98 & -1.0 & 1.544 \\
\hline 140.02 & 0.643 & 23.50 & 1037.6 & 1033.5 & -0.4 & 8.84 & 8.75 & -1.0 & 1.461 \\
\hline 133.15 & 0.443 & 24.24 & 1113.9 & 1109.5 & -0.4 & 10.64 & 10.53 & -1.0 & 1.311 \\
\hline 125.09 & 0.271 & 25.06 & 1200.3 & 1195.1 & -0.4 & 12.92 & 12.77 & -1.1 & 1.174 \\
\hline 123.15 & 0.239 & 25.26 & 1220.6 & 1215.2 & -0.4 & 13.49 & 13.34 & -1.1 & 1.146 \\
\hline 113.15 & 0.115 & 26.20 & 1322.7 & 1316.2 & -0.5 & 16.64 & 16.43 & -1.3 & 1.022 \\
\hline 190.43 & 4.582 & 8.78 & 206.8 & 234.7 & 13.5 & 0.00 & 0.00 & 38.7 & 0.000 \\
\hline 190.37 & 4.572 & 8.44 & 215.8 & 236.8 & 9.8 & 0.01 & 0.01 & 17.3 & 0.000 \\
\hline 190.35 & 4.569 & 8.40 & 215.6 & 237.0 & 9.9 & 0.01 & 0.01 & 24.2 & 0.000 \\
\hline 190.25 & 4.556 & 8.22 & 220.8 & 237.9 & 7.7 & 0.01 & 0.01 & 21.7 & 0.000 \\
\hline 190.03 & 4.523 & 7.79 & 229.7 & 240.7 & 4.8 & 0.02 & 0.02 & 12.0 & 0.000 \\
\hline 189.97 & 4.516 & 7.74 & 230.0 & 240.9 & 4.7 & 0.02 & 0.02 & 9.8 & 0.000 \\
\hline 189.65 & 4.471 & 7.36 & 235.9 & 243.1 & 3.1 & 0.03 & 0.03 & 6.1 & 0.000 \\
\hline 189.65 & 4.471 & 7.36 & 235.9 & 243.2 & 3.1 & 0.03 & 0.03 & 6.6 & 0.000 \\
\hline 189.50 & 4.448 & 7.15 & 239.3 & 244.7 & 2.3 & 0.04 & 0.04 & 3.5 & 0.000 \\
\hline 189.40 & 4.436 & 7.12 & 239.1 & 244.6 & 2.3 & 0.04 & 0.04 & 4.3 & 0.000 \\
\hline 188.70 & 4.336 & 6.54 & 246.2 & 248.4 & 0.9 & 0.07 & 0.07 & 1.6 & 0.000 \\
\hline 188.60 & 4.326 & 6.52 & 245.9 & 248.3 & 0.9 & 0.07 & 0.07 & 1.4 & 0.000 \\
\hline 188.15 & 4.266 & 6.26 & 248.2 & 249.7 & 0.6 & 0.08 & 0.08 & 1.4 & 0.000 \\
\hline 187.60 & 4.189 & 5.95 & 251.3 & 251.9 & 0.2 & 0.10 & 0.11 & 0.3 & 0.000 \\
\hline 187.45 & 4.173 & 5.92 & 251.2 & 251.8 & 0.2 & 0.11 & 0.11 & 0.1 & 0.000 \\
\hline 186.10 & 3.993 & 5.33 & 256.1 & 255.7 & -0.2 & 0.16 & 0.16 & -0.6 & 0.000 \\
\hline 185.90 & 3.974 & 5.30 & 256.0 & 255.5 & -0.2 & 0.16 & 0.16 & -0.4 & 0.000 \\
\hline 184.15 & 3.751 & 4.73 & 260.5 & 259.5 & -0.4 & 0.22 & 0.21 & -0.7 & 0.000 \\
\hline 183.90 & 3.729 & 4.70 & 260.4 & 259.3 & -0.4 & 0.22 & 0.22 & -0.9 & 0.000 \\
\hline 183.15 & 3.641 & 4.50 & 261.7 & 260.6 & -0.4 & 0.24 & 0.24 & -0.7 & 0.000 \\
\hline
\end{tabular}


Data from Gammon and Douslin [37] (continued)

$\begin{array}{lllllllll}\mathrm{T} & \mathrm{P} & \rho & \mathrm{W}, \exp & \mathrm{W}, \mathrm{cal} & \operatorname{Dev} 1 & \partial \mathrm{P} /\left.\partial \rho\right|_{\mathrm{T}} & \partial \mathrm{P} /\left.\partial \rho\right|_{\mathrm{T}} & \text { Dev2 }\end{array}$

$\begin{array}{cccccccccc}\mathrm{K} & \mathrm{MPa} & \mathrm{mol} \cdot \mathrm{dm}^{-3} & \mathrm{~m} \cdot \mathrm{s}^{-1} & \mathrm{~m} \cdot \mathrm{s}^{-1} & 8 & \mathrm{MPa} \cdot \mathrm{dm}^{3} \cdot \mathrm{mol}^{-1} & \text { o } & \\ 181.55 & 3.451 & 4.12 & 264.7 & 263.5 & -0.5 & 0.29 & 0.28 & -1.1 & 0.000 \\ 181.30 & 3.429 & 4.08 & 264.8 & 263.4 & -0.5 & 0.29 & 0.29 & -1.1 & 0.000 \\ 178.25 & 3.098 & 3.51 & 269.0 & 267.7 & -0.5 & 0.37 & 0.37 & -0.9 & 0.005 \\ 178.05 & 3.080 & 3.48 & 269.1 & 267.8 & -0.5 & 0.37 & 0.37 & -0.9 & 0.005 \\ 174.25 & 2.699 & 2.91 & 273.7 & 272.5 & -0.4 & 0.46 & 0.46 & -1.0 & 0.005 \\ 173.95 & 2.678 & 2.89 & 273.6 & 272.4 & -0.5 & 0.46 & 0.46 & -0.9 & 0.005 \\ 173.15 & 2.605 & 2.79 & 274.4 & 273.2 & -0.5 & 0.48 & 0.48 & -1.0 & 0.005 \\ 169.15 & 2.241 & 2.31 & 278.6 & 277.6 & -0.4 & 0.57 & 0.56 & -0.7 & 0.005 \\ 168.55 & 2.209 & 2.28 & 278.3 & 277.3 & -0.4 & 0.57 & 0.56 & -0.8 & 0.005 \\ 163.15 & 1.803 & 1.81 & 281.9 & 281.0 & -0.3 & 0.65 & 0.65 & -0.7 & 0.006 \\ 162.65 & 1.730 & 1.72 & 283.8 & 283.1 & -0.3 & 0.68 & 0.68 & -0.5 & 0.006 \\ 161.45 & 1.687 & 1.68 & 282.8 & 282.0 & -0.3 & 0.68 & 0.67 & -0.6 & 0.006 \\ 155.05 & 1.173 & 1.12 & 290.5 & 290.2 & -0.1 & 0.82 & 0.82 & -0.2 & 0.007 \\ 153.15 & 1.196 & 1.17 & 285.8 & 285.2 & -0.2 & 0.77 & 0.77 & -0.4 & 0.007 \\ 150.65 & 1.071 & 1.05 & 286.3 & 285.8 & -0.2 & 0.80 & 0.79 & -0.4 & 0.007 \\ & & & & & & & & & \\ 148.15 & 0.956 & 0.94 & 286.6 & 286.1 & -0.2 & 0.82 & 0.81 & -0.3 & 0.008 \\ 143.15 & 0.752 & 0.74 & 286.6 & 286.2 & -0.1 & 0.85 & 0.85 & -0.3 & 0.009 \\ 138.15 & 0.533 & 0.52 & 288.2 & 288.0 & -0.1 & 0.90 & 0.90 & -0.2 & 0.010 \\ 133.15 & 0.442 & 0.44 & 284.6 & 284.2 & -0.1 & 0.89 & 0.88 & -0.3 & 0.011 \\ 123.15 & 0.238 & 0.25 & 279.9 & 279.4 & -0.2 & 0.89 & 0.89 & -0.3 & 0.014 \\ 113.15 & 0.114 & 0.13 & 272.8 & 271.8 & -0.4 & 0.87 & 0.87 & -0.8 & 0.019\end{array}$

Number of Points (Ref.37) 196

$\begin{array}{llrlrlrl}\text { SOUND SPEED: } & \text { AAD- } 8 & 3.379 & \text { BIAS }-8 & 3.196 & \text { RMS }-8 & 5.712 & \\ & \text { AAD } & 7.556 & \text { BIAS } & 6.649 & \text { RMS } & 11.888 & \mathrm{~m} \cdot \mathrm{s}^{-1} \\ \text { DERIVATIVE: } & \text { AAD-8 } & 7.363 & \text { BIAS-8 } & 6.963 & \text { RMS- } 8 & 15.048 & \\ & \text { AAD } & 0.007 & \text { BIAS } & -0.005 & \text { RMS } & 0.025 & \mathrm{MPa} \cdot \mathrm{dm}^{3} \cdot \mathrm{mol}^{-1}\end{array}$

Number of Points (Ref. 37) 138 outside critical region

$\begin{array}{lllllll}\text { SOUND SPEED: } & \text { AAD }-8 & 0.844 & \text { BIAS }-8 & 0.584 & \text { RMS }-8 & 1.752 \\ \text { AAD } & 2.489 & \text { BIAS } & 1.200 & \text { RMS } & 4.765 & \mathrm{~m} \cdot \mathrm{s}^{-1}\end{array}$ 
Data from Sivaramin and Gammon [44]

\begin{tabular}{|c|c|c|c|c|c|c|c|c|}
\hline $\mathrm{T}$ & $\mathbf{P}$ & $\rho$ & $W, \exp$ & W, cal & Dev1 & $\begin{array}{c}\partial \mathrm{P} /\left.\partial \rho\right|_{\mathrm{I}} \\
\exp \end{array}$ & $\begin{array}{c}\partial \mathrm{P} /\left.\partial \rho\right|_{\mathrm{T}} \\
\mathrm{cal}\end{array}$ & Dev2 \\
\hline K & $\mathrm{MPa}$ & $\mathrm{mol} \cdot \mathrm{dm}^{-3}$ & $\mathrm{~m} \cdot \mathrm{s}^{-1}$ & $\mathrm{~m} \cdot \mathrm{s}^{-1}$ & 8 & $\mathrm{MPa} \cdot \mathrm{dm}^{3}$ & $\cdot \mathrm{mol}^{-1}$ & 8 \\
\hline 193.05 & 1.4555 & - & 342.31 & 342.20 & -0.032 & - & - & - \\
\hline 193.05 & 3.4464 & - & 304.34 & 304.34 & 0.000 & - & - & - \\
\hline 193.05 & 4.4289 & - & 277.41 & 278.04 & 0.228 & - & - & - \\
\hline 193.05 & 4.8182 & - & 257.92 & 259.05 & 0.439 & - & - & - \\
\hline 193.05 & 4.9370 & - & 245.84 & 249.23 & 1.378 & - & - & - \\
\hline 193.05 & 5.0192 & - & 264.32 & 266.94 & 0.992 & - & - & - \\
\hline 193.05 & 5.1745 & - & 313.12 & 315.90 & 0.889 & - & - & - \\
\hline 193.05 & 5.6433 & - & 392.17 & 393.83 & 0.424 & - & - & - \\
\hline 195.15 & 1.4748 & - & 344.79 & 344.64 & -0.042 & - & - & - \\
\hline 195.15 & 3.5174 & - & 307.89 & 307.88 & -0.001 & - & - & - \\
\hline 195.15 & 4.5645 & - & 282.46 & 283.24 & 0.276 & - & - & - \\
\hline 195.15 & 5.0281 & - & 266.32 & 267.32 & 0.377 & - & - & - \\
\hline 195.15 & 5.2175 & - & 261.11 & 261.87 & 0.294 & - & - & - \\
\hline 195.15 & 5.3794 & - & 283.72 & 284.34 & 0.218 & - & - & - \\
\hline 195.15 & 5.6169 & - & 330.36 & 331.88 & 0.460 & - & - & - \\
\hline 195.15 & 6.1971 & - & 406.00 & 406.25 & 0.062 & - & - & - \\
\hline 200.15 & 1.5200 & - & 350.48 & 350.36 & -0.034 & - & - & - \\
\hline 200.15 & 3.6831 & - & 316.08 & 316.10 & 0.004 & - & - & - \\
\hline 200.15 & 4.8817 & - & 293.83 & 294.72 & 0.303 & - & - & - \\
\hline 200.15 & 5.3444 & - & 285.38 & 286.65 & 0.443 & - & - & - \\
\hline 200.15 & 5.8840 & - & 286.39 & 286.68 & 0.101 & - & - & - \\
\hline 200.15 & 6.2521 & - & 315.80 & 316.11 & 0.097 & - & - & - \\
\hline 200.15 & 6.6840 & - & 361.70 & 361.84 & 0.041 & - & - & - \\
\hline 200.15 & 7.5229 & - & 433.29 & 431.98 & -0.301 & - & - & - \\
\hline 210.15 & 1.6096 & - & 361.65 & 361.42 & -0.064 & - & - & - \\
\hline 210.15 & 3.9818 & - & 331.92 & 331.97 & 0.016 & - & - & - \\
\hline 210.15 & 5.4978 & - & 314.59 & 315.28 & 0.221 & - & - & - \\
\hline 210.15 & 6.4755 & - & 310.70 & 311.65 & 0.306 & - & - & - \\
\hline 210.15 & 7.2020 & - & 322.91 & 322.90 & -0.001 & - & - & - \\
\hline 210.15 & 7.9731 & - & 357.87 & 357.09 & -0.218 & - & - & - \\
\hline 210.15 & 8.8393 & - & 407.26 & 405.64 & -0.398 & - & - & - \\
\hline 210.15 & 10.1705 & - & 476.05 & 473.81 & -0.470 & - & - & - \\
\hline 223.15 & 1.7246 & - & 375.27 & 375.11 & -0.043 & - & - & - \\
\hline 223.15 & 4.3776 & - & 350.79 & 350.77 & -0.004 & - & - & - \\
\hline 223.15 & 6.2730 & - & 338.77 & 339.21 & 0.131 & - & - & - \\
\hline
\end{tabular}


Data from Sivaramin and Gammon [44] (continued)

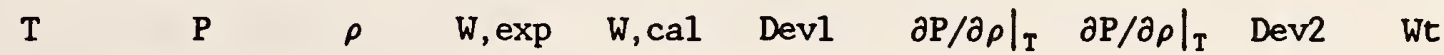
$\exp \quad c a l$

\begin{tabular}{|c|c|c|c|c|c|c|c|c|}
\hline K & $\mathrm{MPa}$ & $\mathrm{mol} \cdot \mathrm{dm}^{-3}$ & $3 \mathrm{~m} \cdot \mathrm{s}^{-1}$ & $\mathrm{~m} \cdot \mathrm{s}^{-1}$ & 8 & $\mathrm{MPa} \cdot \mathrm{dm}^{3} \cdot \mathrm{mol}$ & & 8 \\
\hline 223.15 & 7.6782 & - & 341.08 & 341.36 & 0.082 & - & - & - \\
\hline 223.15 & 8.8588 & - & 360.17 & 358.52 & -0.458 & - & - & - \\
\hline 223.15 & 10.2272 & - & 400.90 & 398.98 & -0.478 & - & - & - \\
\hline 223.15 & 11.8153 & - & 453.11 & 456.85 & 0.825 & - & - & - \\
\hline 248.15 & 1.9419 & - & 399.71 & 399.49 & -0.055 & - & - & - \\
\hline 248.15 & 5.1074 & - & 383.14 & 383.12 & -0.005 & - & - & - \\
\hline 248.15 & 7.6438 & - & 379.16 & 379.36 & 0.055 & - & - & - \\
\hline 248.15 & 9.8765 & - & 389.83 & 389.51 & -0.081 & - & - & - \\
\hline 248.15 & 11.9829 & - & 417.23 & 415.15 & -0.497 & - & - & - \\
\hline 248.15 & 14.4705 & - & 464.55 & 463.08 & -0.317 & - & - & - \\
\hline 273.15 & 2.1550 & - & 421.74 & 421.67 & -0.017 & - & - & - \\
\hline 273.15 & 5.8826 & - & 411.50 & 411.42 & -0.019 & - & - & - \\
\hline 273.15 & 9.0367 & - & 413.83 & 413.86 & 0.007 & - & - & - \\
\hline 273.15 & 11.9865 & - & 430.46 & 429.89 & -0.133 & - & - & - \\
\hline 273.15 & 15.1102 & - & 463.69 & 462.90 & -0.171 & - & - & - \\
\hline
\end{tabular}

$\begin{array}{rrllllllll}273.15 & 18.6778 & - & 516.55 & 515.58 & -0.189 & - & - & - & - \\ 298.15 & 2.3772 & - & 441.86 & 441.95 & 0.022 & - & - & - & - \\ 298.15 & 4.5805 & - & 438.02 & 437.88 & -0.032 & - & - & - & - \\ 298.15 & 6.6411 & - & 437.04 & 436.87 & -0.039 & - & - & - & - \\ 298.15 & 8.5992 & - & 439.19 & 439.07 & -0.027 & - & - & - & - \\ & & & & & & & & & \\ 298.15 & 10.4926 & - & 444.59 & 444.61 & 0.004 & - & - & - & - \\ 298.15 & 12.3602 & - & 453.65 & 453.61 & -0.007 & - & - & - & - \\ 298.15 & 14.2422 & - & 466.56 & 466.31 & -0.053 & - & - & - & - \\ 298.15 & 16.1884 & - & 483.45 & 483.05 & -0.083 & - & - & - & - \\ 298.15 & 18.2474 & - & 504.86 & 504.20 & -0.131 & - & - & - & - \\ & & & & & & & & & \\ 298.15 & 20.4839 & - & 531.11 & 530.26 & -0.160 & - & - & - & - \\ 298.15 & 22.9728 & - & 562.60 & 561.69 & -0.162 & - & - & - & - \\ 323.15 & 2.5998 & - & 460.66 & 460.70 & 0.010 & - & - & - & - \\ 323.15 & 5.0523 & - & 459.14 & 458.86 & -0.061 & - & - & - & - \\ 323.15 & 7.3921 & - & 460.30 & 460.03 & -0.058 & - & - & - & - \\ 323.15 & 9.6586 & - & 464.64 & 464.41 & -0.049 & - & - & - & - \\ 323.15 & 11.8912 & - & 472.31 & 472.12 & -0.039 & - & - & - & - \\ 323.15 & 14.1263 & - & 483.53 & 483.31 & -0.045 & - & - & - & -\end{array}$


Data from Sivaramin and Gammon [44] (continued)

$\mathrm{T} \quad \mathrm{P} \quad \rho \quad \mathrm{W}, \exp \mathrm{W}, \mathrm{cal} \quad \operatorname{Dev1} \quad \partial \mathrm{P} /\left.\partial \rho\right|_{\mathrm{T}} \partial \mathrm{P} /\left.\partial \rho\right|_{\mathrm{I}}$ Dev2 $\mathrm{Wt}$ $\exp \quad$ cal

$\mathrm{K} \quad \mathrm{MPa} \quad \mathrm{mol} \cdot \mathrm{dm}^{-3} \mathrm{~m} \cdot \mathrm{s}^{-1} \mathrm{~m} \cdot \mathrm{s}^{-1}$ \& $\quad \mathrm{MPa} \cdot \mathrm{dm}^{3} \cdot \mathrm{mol}^{-1} \quad$ o

$\begin{array}{rrrrrr}323.15 & 16.4214 & - & 498.55 & 498.27 & -0.055 \\ 323.15 & 18.8148 & - & 517.76 & 517.22 & -0.105 \\ 323.15 & 21.3771 & - & 541.31 & 540.62 & -0.126 \\ 323.15 & 24.1556 & - & 569.45 & 568.68 & -0.135 \\ 348.15 & 2.8212 & - & 478.08 & 478.19 & 0.023\end{array}$

348.15

$5.5215 \quad-\quad 478.58$

$478.28-0.063$

348.15

$11.9927-492.36$

$13.2802-497.55$

$491.99-0.074$

348.15

$15.8908-510.84$

497.23

$-0.065$

348.15

18.5881

527.81

510.36

527.21

$-0.093$

348.15

$21.4232-548.62$

348.15

24.4792

548.02

$-0.114$

348.15

24.4792

573.98

373.15

3.0434

573.27

$-0.109$

373.15

5.9932

494.48

494.64

$-0.124$

373.15

$8.8836-501.54$

496.45

501.22

0.033

$-0.035$

373.15

11.7603

509.58

373.15

14.6627

509.17

$-0.063$

373.15

17.6313

520.99

373.15

20.7423

373.15

24.006

535.78

520.46

$-0.081$

535.22

$-0.102$

554.36

553.81

$-0.104$

576.81

576.19

$-0.098$

$-0.108$

373.15

27.542

603.75

398.15

3.2337

602.97

$-0.129$

398.15

$6.4583-513.95$

510.24

0.003

398.15

$9.6228-520.31$

513.61

$-0.065$

398.15

$12.8003-529.93$

519.91

$-0.077$

$529.37-0.106$

398.15

16.0370

542.82

398.15

19.3679

542.18

$-0.119$

398.15

22.8752

559.18

558.45

$-0.130$

398.15

26.6023

579.33

578.53

$-0.139$

423.15

3.4839

603.37

602.55

$-0.136$

$525.18 \quad 0.022$

423.15

6.9249

530.37

529.96

$-0.077$

423.15

10.3612

538.16

537.66

$-0.092$

423.15

13.8385

549.13

548.51

$-0.112$

423.15

17.4008

563.41

562.69

$-0.127$

423.15

21.1001

581.48

580.38

$-0.188$

423.15

24.9969

602.88

$601.80-0.179$ 
Data from Sivaramin and Gammon [44] (continued)

Number of Points (Ref.44) 104

$\begin{array}{lllrlrl}\text { AAD- } 8 & 0.164 & \text { BIAS }-8 & 0.005 & \text { RMS }-8 & 0.272 & \\ \text { AAD } & 0.643 & \text { BIAS } & -0.124 & \text { RMS } & 0.939 & \mathrm{~m} \cdot \mathrm{s}^{-1}\end{array}$

Data from Straty [58]

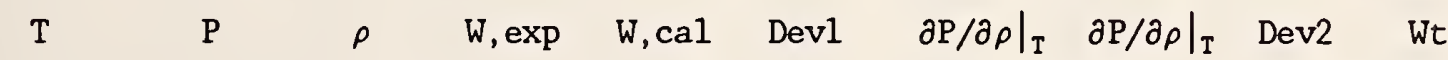
$\exp \quad$ cal

\begin{tabular}{rrrrrrrrrr}
\multicolumn{1}{c}{$\mathrm{K}$} & $\mathrm{MPa}$ & $\mathrm{mol} \cdot \mathrm{dm}^{-3}$ & $\mathrm{~m} \cdot \mathrm{s}^{-1}$ & $\mathrm{~m} \cdot \mathrm{s}^{-1}$ & o & $\mathrm{MPa} \cdot \mathrm{dm}^{3} \cdot \mathrm{mol}^{-1}$ & 8 \\
91.00 & 0.012 & 28.12 & 1538.1 & 1544.9 & 0.4 & 24.03 & 24.33 & 1.3 & 8.604 \\
92.00 & 0.014 & 28.04 & 1528.7 & 1532.8 & 0.3 & 23.74 & 23.93 & 0.8 & 8.658 \\
96.00 & 0.022 & 27.70 & 1490.6 & 1487.7 & -0.2 & 22.48 & 22.39 & -0.4 & 8.913 \\
100.00 & 0.034 & 27.36 & 1452.2 & 1446.2 & -0.4 & 21.13 & 20.92 & -1.0 & 9.213 \\
101.73 & 0.041 & 27.21 & 1435.5 & 1428.9 & -0.5 & 20.53 & 20.30 & -1.1 & -0.000 \\
104.00 & 0.051 & 27.02 & 1413.4 & 1406.3 & -0.5 & 19.74 & 19.50 & -1.2 & 9.553 \\
108.00 & 0.074 & 26.66 & 1373.8 & 1367.0 & -0.5 & 18.36 & 18.12 & -1.3 & 9.933 \\
112.00 & 0.104 & 26.30 & 1334.1 & 1327.6 & -0.5 & 17.01 & 16.80 & -1.3 & 10.349 \\
116.00 & 0.143 & 25.94 & 1294.0 & 1287.8 & -0.5 & 15.71 & 15.52 & -1.2 & 10.807 \\
120.00 & 0.192 & 25.56 & 1252.9 & 1247.5 & -0.4 & 14.45 & 14.28 & -1.1 & 2.263 \\
124.00 & 0.252 & 25.17 & 1211.3 & 1206.4 & -0.4 & 13.23 & 13.09 & -1.1 & 2.377 \\
128.00 & 0.325 & 24.77 & 1169.1 & 1164.6 & -0.4 & 12.06 & 11.94 & -1.0 & 2.503 \\
132.00 & 0.414 & 24.36 & 1126.0 & 1121.9 & -0.4 & 10.94 & 10.84 & -1.0 & 2.646 \\
136.00 & 0.518 & 23.94 & 1082.2 & 1078.4 & -0.4 & 9.86 & 9.77 & -0.9 & 2.809 \\
140.00 & 0.642 & 23.50 & 1037.4 & 1033.8 & -0.4 & 8.84 & 8.76 & -0.9 & 2.997 \\
& & & & & & & & & \\
144.00 & 0.785 & 23.04 & 991.7 & 988.0 & -0.4 & 7.85 & 7.78 & -0.9 & 3.218 \\
120.00 & 0.192 & 25.56 & 1252.8 & 1247.5 & -0.4 & 14.44 & 14.28 & -1.1 & -0.000 \\
140.00 & 0.642 & 23.50 & 1037.4 & 1033.8 & -0.4 & 8.84 & 8.76 & -0.9 & -0.001 \\
148.00 & 0.949 & 22.56 & 944.8 & 941.1 & -0.4 & 6.91 & 6.85 & -1.0 & 3.482 \\
150.00 & 1.040 & 22.31 & 921.0 & 917.2 & -0.4 & 6.46 & 6.40 & -1.0 & 3.635 \\
152.00 & 1.138 & 22.06 & 896.6 & 892.9 & -0.4 & 6.02 & 5.96 & -1.0 & 3.807 \\
156.00 & 1.352 & 21.53 & 846.9 & 843.1 & -0.5 & 5.17 & 5.11 & -1.1 & 4.219 \\
160.00 & 1.593 & 20.96 & 795.5 & 791.5 & -0.5 & 4.36 & 4.31 & -1.1 & 4.767 \\
160.00 & 1.593 & 20.96 & 795.5 & 791.5 & -0.5 & 4.36 & 4.31 & -1.1 & -0.001 \\
164.00 & 1.863 & 20.36 & 742.2 & 738.0 & -0.6 & 3.61 & 3.56 & -1.3 & 5.545
\end{tabular}


Data from Straty [58] (continued)

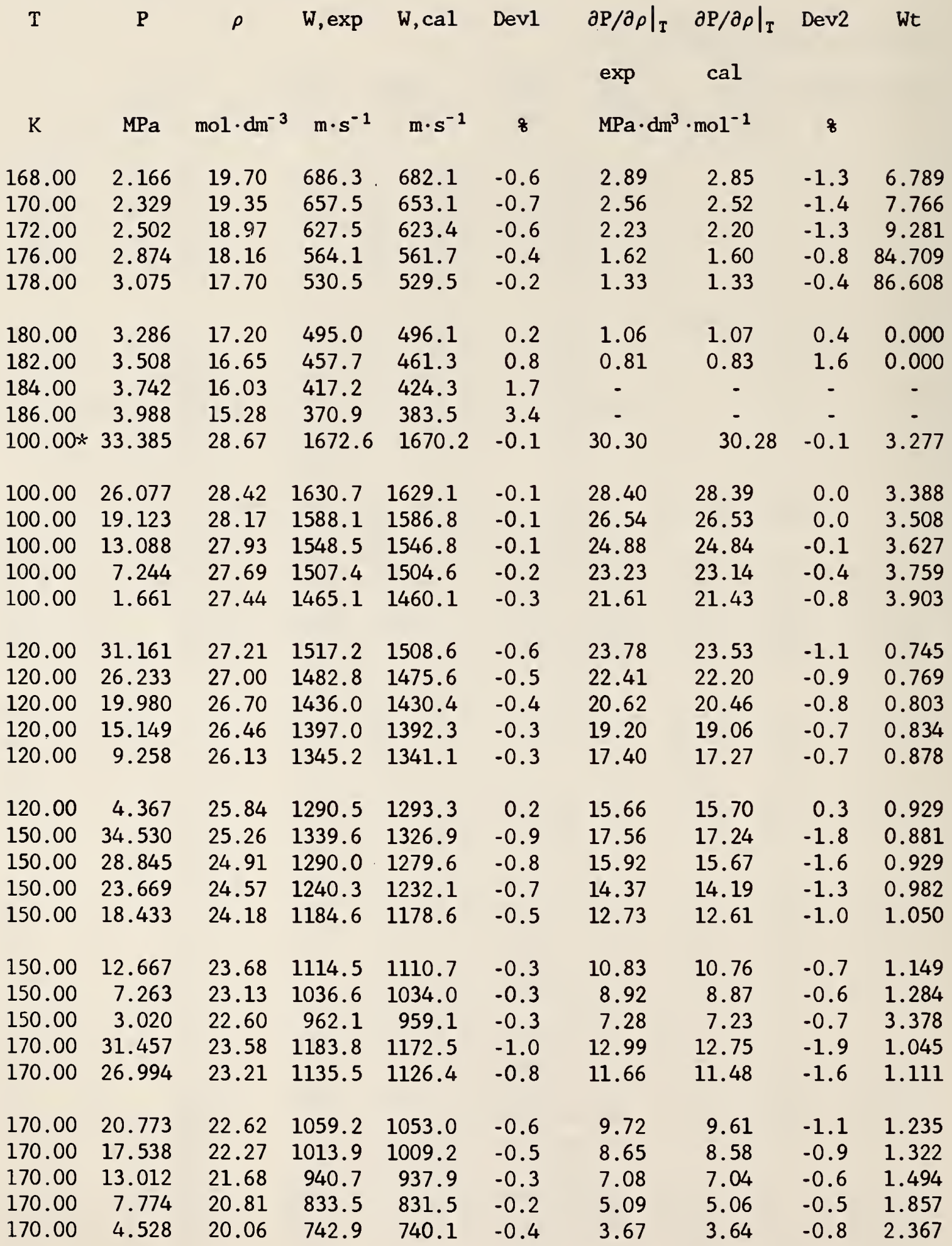


Data from Straty [58] (continued)

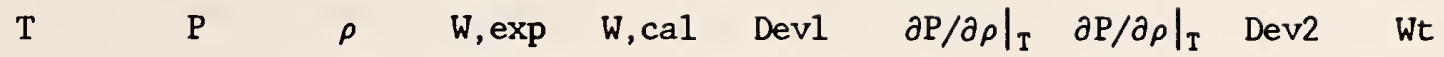

\begin{tabular}{|c|c|c|c|c|c|c|c|c|c|}
\hline \multirow[b]{2}{*}{ K } & \multirow[b]{2}{*}{$\mathrm{MPa}$} & \multirow[b]{2}{*}{$\mathrm{mol} \cdot \mathrm{dm}^{-3}$} & \multirow[b]{2}{*}{$m \cdot s^{-1}$} & \multirow[b]{2}{*}{$\mathrm{m} \cdot \mathrm{s}^{-1}$} & \multicolumn{3}{|c|}{$\exp \quad c a 1$} & \multirow[b]{2}{*}{8} & \\
\hline & & & & & 8 & $\mathrm{MPa} \cdot \mathrm{dm}$ & $\cdot \mathrm{mol}^{-1}$ & & \\
\hline 184.00 & 34.254 & 22.75 & 1129.0 & 1117.6 & -1.0 & 11.68 & 11.45 & -2.0 & 4.466 \\
\hline 184.00 & 27.883 & 22.15 & 1055.1 & 1046.9 & -0.8 & 9.81 & 9.66 & -1.5 & 4.946 \\
\hline 184.00 & 22.511 & 21.54 & 982.8 & 977.2 & -0.6 & 8.14 & 8.06 & -1.1 & 5.533 \\
\hline 184.00 & 17.862 & 20.90 & 909.0 & 905.6 & -0.4 & 6.62 & 6.58 & -0.7 & 6.306 \\
\hline 184.00 & 13.839 & 20.21 & 831.9 & 830.0 & -0.2 & 5.22 & 5.19 & -0.4 & 7.413 \\
\hline 184.00 & 10.436 & 19.46 & 751.2 & 749.2 & -0.3 & 3.93 & 3.91 & -0.5 & 9.183 \\
\hline 184.00 & 7.722 & 18.65 & 666.6 & 664.2 & -0.4 & 2.79 & 2.77 & -0.7 & 12.672 \\
\hline 184.00 & 5.770 & 17.79 & 579.9 & 579.7 & 0.0 & 1.84 & 1.84 & 0.0 & 0.000 \\
\hline 184.00 & 5.301 & 17.52 & 552.8 & 553.9 & 0.2 & 1.58 & 1.59 & 0.4 & 0.000 \\
\hline 210.00 & 34.073 & 20.74 & 987.0 & 979.0 & -0.8 & 8.56 & 8.43 & -1.6 & 0.182 \\
\hline 210.00 & 27.598 & 19.88 & 898.0 & 893.4 & -0.5 & 6.72 & 6.65 & -1.0 & 0.213 \\
\hline 210.00 & 22.264 & 18.96 & 809.8 & 807.7 & -0.3 & 5.13 & 5.10 & -0.5 & 0.258 \\
\hline 210.00 & 17.486 & 17.86 & 711.5 & 710.9 & -0.1 & 3.63 & 3.62 & -0.1 & 0.346 \\
\hline 210.00 & 14.832 & 17.02 & 643.9 & 643.2 & -0.1 & 2.76 & 2.75 & -0.2 & 0.472 \\
\hline 210.00 & 12.703 & 16.12 & 577.5 & 576.9 & -0.1 & 2.02 & 2.02 & -0.2 & 0.901 \\
\hline 210.00 & 10.944 & 15.08 & 511.5 & 509.6 & -0.4 & 1.41 & 1.40 & -0.7 & 0.817 \\
\hline 210.00 & 12.705 & 16.12 & 578.0 & 576.9 & -0.2 & 2.03 & 2.02 & -0.3 & -0.001 \\
\hline 210.00 & 10.951 & 15.09 & 511.5 & 509.9 & -0.3 & 1.41 & 1.40 & -0.6 & -0.001 \\
\hline 210.00 & 10.173 & 14.47 & 477.0 & 475.2 & -0.4 & 1.14 & 1.13 & -0.7 & 0.607 \\
\hline 210.00 & 9.646 & 13.96 & 451.2 & 449.6 & -0.4 & 0.95 & 0.95 & -0.7 & 0.533 \\
\hline 240.00 & 34.638 & 18.49 & 863.5 & 858.7 & -0.6 & 6.40 & 6.33 & -1.1 & 0.226 \\
\hline 240.00 & 29.111 & 17.51 & 782.7 & 780.0 & -0.3 & 5.00 & 4.96 & -0.7 & 0.274 \\
\hline 240.00 & 24.656 & 16.49 & 707.0 & 705.7 & -0.2 & 3.86 & 3.84 & -0.4 & 0.348 \\
\hline 240.00 & 20.885 & 15.36 & 632.6 & 632.2 & -0.1 & 2.89 & 2.89 & -0.1 & 0.500 \\
\hline 240.00 & 24.662 & 16.49 & 706.8 & 705.8 & -0.1 & 3.85 & 3.84 & -0.3 & -0.000 \\
\hline 240.00 & 20.886 & 15.36 & 632.8 & 632.2 & -0.1 & 2.90 & 2.89 & -0.2 & -0.000 \\
\hline 240.00 & 18.890 & 14.61 & 588.8 & 588.4 & -0.1 & 2.40 & 2.40 & -0.2 & 0.746 \\
\hline 240.00 & 16.841 & 13.65 & 540.2 & 539.4 & -0.2 & 1.91 & 1.91 & -0.3 & 1.662 \\
\hline 240.00 & 15.186 & 12.68 & 498.4 & 497.3 & -0.2 & 1.55 & 1.54 & -0.4 & 0.711 \\
\hline 240.00 & 14.071 & 11.90 & 469.8 & 468.4 & -0.3 & 1.33 & 1.32 & -0.6 & 0.582 \\
\hline 270.00 & 34.769 & 16.30 & 771.5 & 769.1 & -0.3 & 5.12 & 5.09 & -0.6 & 0.001 \\
\hline 270.00 & 30.486 & 15.37 & 711.2 & 709.9 & -0.2 & 4.20 & 4.19 & -0.4 & 0.001 \\
\hline 270.00 & 25.972 & 14.15 & 641.6 & 641.0 & -0.1 & 3.27 & 3.27 & -0.2 & 0.002 \\
\hline 270.00 & 21.837 & 12.70 & 572.8 & 572.3 & -0.1 & 2.49 & 2.49 & -0.2 & 0.004 \\
\hline 300.00 & 34.838 & 14.33 & 713.9 & 712.2 & -0.2 & 4.52 & 4.50 & -0.5 & 0.001 \\
\hline
\end{tabular}


Data from Straty [58] (continued)

\begin{tabular}{|c|c|c|c|c|c|c|c|c|c|}
\hline $\mathrm{T}$ & $\mathbf{P}$ & $\rho$ & $W, \exp$ & W, cal & Devl & $\begin{array}{l}\partial \mathrm{P} /\left.\partial \rho\right|_{\mathrm{T}} \\
\exp \end{array}$ & $\begin{array}{c}\partial \mathrm{P} /\left.\partial \rho\right|_{\mathrm{T}} \\
\mathrm{cal}\end{array}$ & Dev2 & Wt \\
\hline K & $\mathrm{MPa}$ & $\mathrm{mol} \cdot \mathrm{dm}^{-3}$ & $m \cdot s^{-1}$ & $\mathrm{~m} \cdot \mathrm{s}^{-1}$ & 8 & $\mathrm{MPa} \cdot \mathrm{dm}^{3}$ & $\cdot \mathrm{mol}^{-1}$ & 8 & \\
\hline 00.00 & 29.732 & 13.07 & 649.5 & 648.3 & -0.2 & 3.64 & 3.63 & -0.4 & 0.002 \\
\hline 300.00 & 27.295 & 12.36 & 618.0 & 616.8 & -0.2 & 3.26 & 3.24 & -0.4 & 0.002 \\
\hline 300.00 & 24.427 & 11.41 & 580.4 & 579.5 & -0.2 & 2.84 & 2.83 & -0.4 & 0.003 \\
\hline 300.00 & 20.983 & 10.09 & 537.3 & 536.0 & -0.2 & 2.42 & 2.41 & -0.5 & 0.007 \\
\hline
\end{tabular}

Number of Points (Ref. 58) 99 Data above the starred temperature for this reference were at saturation boundary.

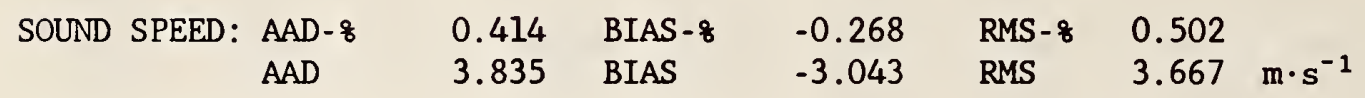

Number of Points (Ref. 58) 91 Selected data (omitting those with negative weights). These 91 points contribute to total below.

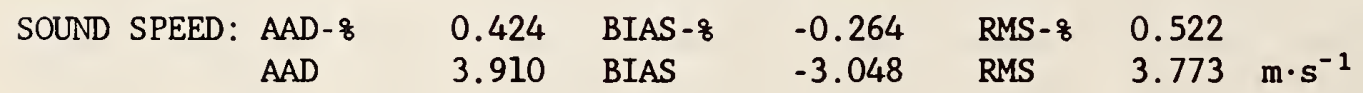

Number of Points (Ref. 58) 97

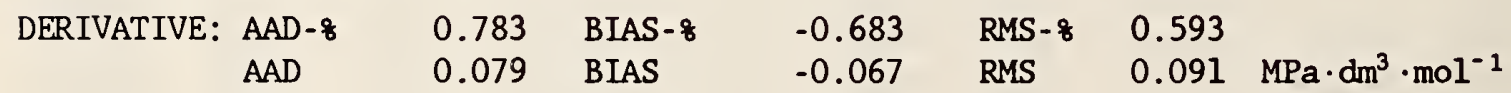


Data from Van Dael et al. [59]

\begin{tabular}{|c|c|c|c|c|c|c|c|c|c|}
\hline $\mathrm{T}$ & $\mathbf{P}$ & $\rho$ & $W, \exp$ & $\mathrm{W}, \mathrm{cal}$ & Devl & $\begin{array}{c}\partial \mathrm{P} /\left.\partial \rho\right|_{\mathrm{T}} \\
\exp \end{array}$ & $\begin{array}{c}\partial \mathrm{P} /\left.\partial \rho\right|_{\mathrm{T}} \\
\mathrm{cal}\end{array}$ & Dev2 & Wt \\
\hline K & $\mathrm{MPa}$ & $\mathrm{mol} \cdot \mathrm{dm}^{-3}$ & $\mathrm{~m} \cdot \mathrm{s}^{-1}$ & $\mathrm{~m} \cdot \mathrm{s}^{-1}$ & 8 & $\mathrm{MPa} \cdot \mathrm{dm}^{3}$ & $\cdot \mathrm{mol}^{-1}$ & 8 & \\
\hline 94.05 & 0.018 & 27.86 & 1509.0 & 1509.1 & 0.0 & 23.11 & 23.13 & 0.1 & 0.001 \\
\hline 94.36 & 0.018 & 27.84 & 1507.0 & 1505.6 & -0.1 & 23.04 & 23.02 & -0.1 & 0.001 \\
\hline 96.17 & 0.023 & 27.69 & 1489.8 & 1485.9 & -0.3 & 22.45 & 22.33 & -0.5 & 0.001 \\
\hline 98.50 & 0.029 & 27.49 & 1466.6 & 1461.5 & -0.3 & 21.64 & 21.46 & -0.8 & 0.001 \\
\hline 101.79 & 0.041 & 27.21 & 1433.9 & 1428.3 & -0.4 & 20.48 & 20.27 & -1.0 & 0.001 \\
\hline 05.45 & 0.059 & 26.89 & 1400.0 & 1392.0 & -0.6 & 19.26 & 18.99 & -1.4 & 0.001 \\
\hline 109.09 & 0.082 & 26.57 & 1363.8 & 1356.2 & -0.6 & 18.01 & 17.76 & -1.4 & 0.001 \\
\hline 112.79 & 0.111 & 26.23 & 1326.8 & 1319.7 & -0.5 & 16.77 & 16.54 & -1.4 & 0.001 \\
\hline 116.07 & 0.144 & 25.93 & 1293.3 & 1287.1 & -0.5 & 15.69 & 15.50 & -1.2 & 0.001 \\
\hline 119.78 & 0.189 & 25.58 & 1256.0 & 1249.7 & -0.5 & 14.54 & 14.35 & -1.3 & 0.001 \\
\hline 23.81 & 0.249 & 25.19 & 1214.5 & 1208.4 & -0.5 & 13.31 & 13.15 & -1.3 & 0.001 \\
\hline 127.96 & 0.325 & 24.78 & 1170.3 & 1165.0 & -0.4 & 12.09 & 11.95 & -1.1 & 0.001 \\
\hline 131.97 & 0.413 & 24.37 & 1127.2 & 1122.3 & -0.4 & 10.97 & 10.84 & -1.1 & 0.001 \\
\hline 136.17 & 0.523 & 23.92 & 1081.0 & 1076.5 & -0.4 & 9.83 & 9.73 & -1.0 & 0.001 \\
\hline 140.23 & 0.649 & 23.47 & 1035.3 & 1031.2 & -0.4 & 8.79 & 8.70 & -1.0 & 0.001 \\
\hline 144.49 & 0.804 & 22.98 & 986.7 & 982.4 & -0.4 & 7.74 & 7.66 & -1.1 & 0.001 \\
\hline 149.01 & 0.995 & 22.44 & 933.3 & 929.1 & -0.5 & 6.69 & 6.62 & -1.1 & 0.001 \\
\hline 153.78 & 1.230 & 21.83 & 875.7 & 870.9 & -0.5 & 5.65 & 5.58 & -1.3 & 0.001 \\
\hline 158.02 & 1.470 & 21.25 & 822.2 & 817.3 & -0.6 & 4.77 & 4.70 & -1.3 & 0.001 \\
\hline 162.81 & 1.780 & 20.54 & 759.1 & 754.1 & -0.7 & 3.83 & 3.78 & -1.4 & 0.002 \\
\hline 167.50 & 2.126 & 19.78 & 694.3 & 689.2 & -0.7 & 2.99 & 2.94 & -1.6 & 0.002 \\
\hline 172.02 & 2.503 & 18.97 & 628.2 & 623.1 & -0.8 & 2.23 & 2.20 & -1.6 & 0.003 \\
\hline 176.44 & 2.918 & 18.06 & 558.1 & 554.7 & -0.6 & 1.56 & 1.54 & -1.2 & 0.008 \\
\hline 179.47 & 3.229 & 17.34 & 506.0 & 505.1 & -0.2 & 1.14 & 1.14 & -0.4 & 0.004 \\
\hline 182.51 & 3.567 & - & 449.0 & 452.1 & 0.7 & - & - & - & - \\
\hline & 3.979 & - & 374.3 & 385.0 & 2.9 & - & - & 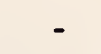 & - \\
\hline & 4.187 & - & 331.5 & 346.9 & 4.7 & - & - & - & - \\
\hline 189.60 & 4.464 & - & 273.0 & 283.3 & 3.8 & - & - & - & - \\
\hline
\end{tabular}

Number of Points (Ref. 59) 28 on saturation boundary

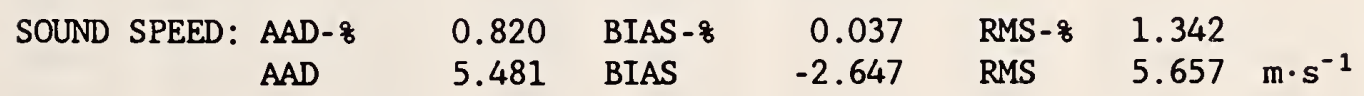

Number of Points (Ref. 59) 24

$\begin{array}{llllllll}\text { DERIVATIVE: } & \text { AAD- } 8 & 1.074 & \text { BIAS- } 8 & -1.064 & \text { RMS }-8 & 0.430 & \\ & \text { AAD } & 0.115 & \text { BIAS } & -0.112 & \text { RMS } & 0.080 & \mathrm{MPa} \cdot \mathrm{dm}^{3} \cdot \mathrm{mol}^{-1}\end{array}$ 
Data from Van Itterbeek et al. [60]

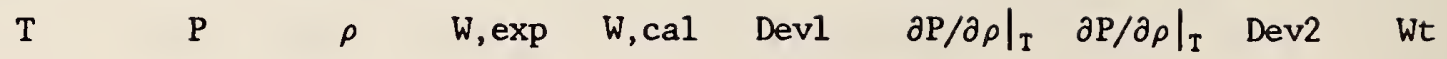
$\exp \quad c a 1$

$\mathrm{K} \quad \mathrm{MPa} \quad \mathrm{mol} \cdot \mathrm{dm}^{-3} \mathrm{~m} \cdot \mathrm{s}^{-1} \mathrm{~m} \cdot \mathrm{s}^{-1} \quad$ \& $\quad \mathrm{MPa} \cdot \mathrm{dm}^{3} \cdot \mathrm{mol}^{-1} \quad$ \&

\begin{tabular}{|c|c|c|c|c|c|c|c|c|c|}
\hline 111.33 & 0.112 & 26.37 & 1340.9 & 1334.3 & -0.5 & 17.24 & 17.02 & -1.3 & 0.000 \\
\hline 111.33 & 0.505 & 26.39 & 1345.0 & 1338.3 & -0.5 & 17.38 & 17.15 & -1.3 & 0.000 \\
\hline 111.33 & 0.981 & 26.42 & 1349.6 & 1343.1 & -0.5 & 17.53 & 17.31 & -1.2 & 0.000 \\
\hline 111.33 & 1.973 & 26.47 & 1358.7 & 1352.8 & -0.4 & 17.84 & 17.64 & -1.1 & 0.000 \\
\hline 111.33 & 2.937 & 26.53 & 1367.0 & 1362.0 & -0.4 & 18.12 & 17.95 & -0.9 & 0.000 \\
\hline 11.33 & 3.932 & 26.58 & 1376.6 & 1371.4 & -0.4 & 18.45 & 18.27 & -1.0 & 0.000 \\
\hline 111.33 & 4.913 & 26.64 & 1384.8 & 1380.3 & -0.3 & 18.74 & 18.59 & -0.8 & 0.000 \\
\hline 11.33 & 5.884 & 26.69 & 1393.1 & 1389.1 & -0.3 & 19.03 & 18.89 & -0.7 & 0.000 \\
\hline 111.33 & 6.815 & 26.74 & 1402.1 & 1397.3 & -0.3 & 19.34 & 19.18 & -0.8 & 0.000 \\
\hline 111.33 & 7.569 & 26.78 & 1408.5 & 1403.8 & -0.3 & 19.57 & 19.42 & -0.8 & 0.000 \\
\hline 25.09 & 0.281 & 25.07 & 1200.6 & 1195.3 & -0.4 & 12.93 & 12.78 & -1.2 & 0.000 \\
\hline 125.09 & 0.530 & 25.09 & 1202.9 & 1198.4 & -0.4 & 13.00 & 12.87 & -1.0 & 0.000 \\
\hline 125.09 & 1.010 & 25.12 & 1209.0 & 1204.4 & -0.4 & 13.17 & 13.04 & -1.0 & 0.000 \\
\hline 125.09 & 1.991 & 25.20 & 1220.8 & 1216.5 & -0.4 & 13.51 & 13.38 & -0.9 & 0.000 \\
\hline 125.09 & 2.958 & 25.27 & 1232.1 & 1227.9 & -0.3 & 13.84 & 13.72 & -0.9 & 0.000 \\
\hline 25.09 & 3.917 & 25.34 & 1242.7 & 1239.0 & -0.3 & 14.16 & 14.05 & -0.8 & 0.000 \\
\hline 125.09 & 4.864 & 25.40 & 1253.8 & 1249.6 & -0.3 & 14.49 & 14.37 & -0.8 & 0.000 \\
\hline 125.09 & 5.924 & 25.48 & 1265.1 & 1261.2 & -0.3 & 14.84 & 14.73 & -0.8 & 0.000 \\
\hline 125.09 & 6.846 & 25.54 & 1275.0 & 1270.9 & -0.3 & 15.14 & 15.03 & -0.8 & 0.000 \\
\hline 125.09 & 7.796 & 25.60 & 1285.0 & 1280.8 & -0.3 & 15.46 & 15.34 & -0.8 & 0.000 \\
\hline 125.09 & 8.775 & 25.66 & 1295.0 & 1290.7 & -0.3 & 15.77 & 15.65 & -0.8 & 0.000 \\
\hline 125.09 & 9.788 & 25.73 & 1305.2 & 1300.7 & -0.3 & 16.10 & 15.98 & -0.8 & 0.000 \\
\hline 140.02 & 0.647 & 23.50 & 1038.5 & 1033.6 & -0.5 & 8.85 & 8.75 & -1.1 & 0.000 \\
\hline 140.02 & 1.112 & 23.55 & 1045.0 & 1041.6 & -0.3 & 9.01 & 8.94 & -0.8 & 0.000 \\
\hline 140.02 & 1.988 & 23.65 & 1059.5 & 1056.3 & -0.3 & 9.35 & 9.28 & -0.8 & 0.000 \\
\hline 140.02 & 2.981 & 23.75 & 1075.0 & 1072.1 & -0.3 & 9.73 & 9.66 & -0.7 & 0.000 \\
\hline 140.02 & 3.934 & 23.85 & 1089.1 & 1086.7 & -0.2 & 10.07 & 10.02 & -0.6 & 0.000 \\
\hline 140.02 & 4.931 & 23.95 & 1104.0 & 1101.3 & -0.2 & 10.44 & 10.38 & -0.6 & 0.000 \\
\hline 140.02 & 5.899 & 24.04 & 1117.9 & 1114.9 & -0.3 & 10.80 & 10.73 & -0.6 & 0.000 \\
\hline 140.02 & 6.835 & 24.12 & 1130.6 & 1127.6 & -0.3 & 11.13 & 11.06 & -0.6 & 0.000 \\
\hline 140.02 & 7.893 & 24.22 & 1145.3 & 1141.5 & -0.3 & 11.52 & 11.43 & -0.7 & 0.000 \\
\hline 140.02 & 8.836 & 24.30 & 1157.0 & 1153.4 & -0.3 & 11.83 & 11.75 & -0.7 & 0.000 \\
\hline 140.02 & 9.788 & 24.38 & 1169.5 & 1165.1 & -0.4 & 12.17 & 12.08 & -0.8 & 0.000 \\
\hline 140.02 & 10.609 & 24.45 & 1178.7 & 1174.9 & -0.3 & 12.43 & 12.35 & -0.7 & 0.000 \\
\hline 140.02 & 11.034 & 24.48 & 1183.9 & 1179.8 & -0.3 & 12.58 & 12.49 & -0.7 & 0.000 \\
\hline
\end{tabular}


Data from Van Itterbeek et al. [60] (continued)

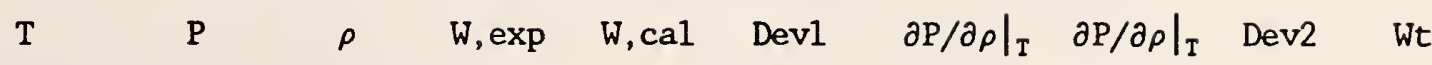

$\exp \quad$ cal

$\mathrm{K} \quad \mathrm{MPa} \quad \mathrm{mol} \cdot \mathrm{dm}^{-3} \mathrm{~m} \cdot \mathrm{s}^{-1} \mathrm{~m} \cdot \mathrm{s}^{-1} \quad$ \& $\quad \mathrm{MPa} \cdot \mathrm{dm}^{3} \cdot \mathrm{mol}^{-1} \quad$ \&

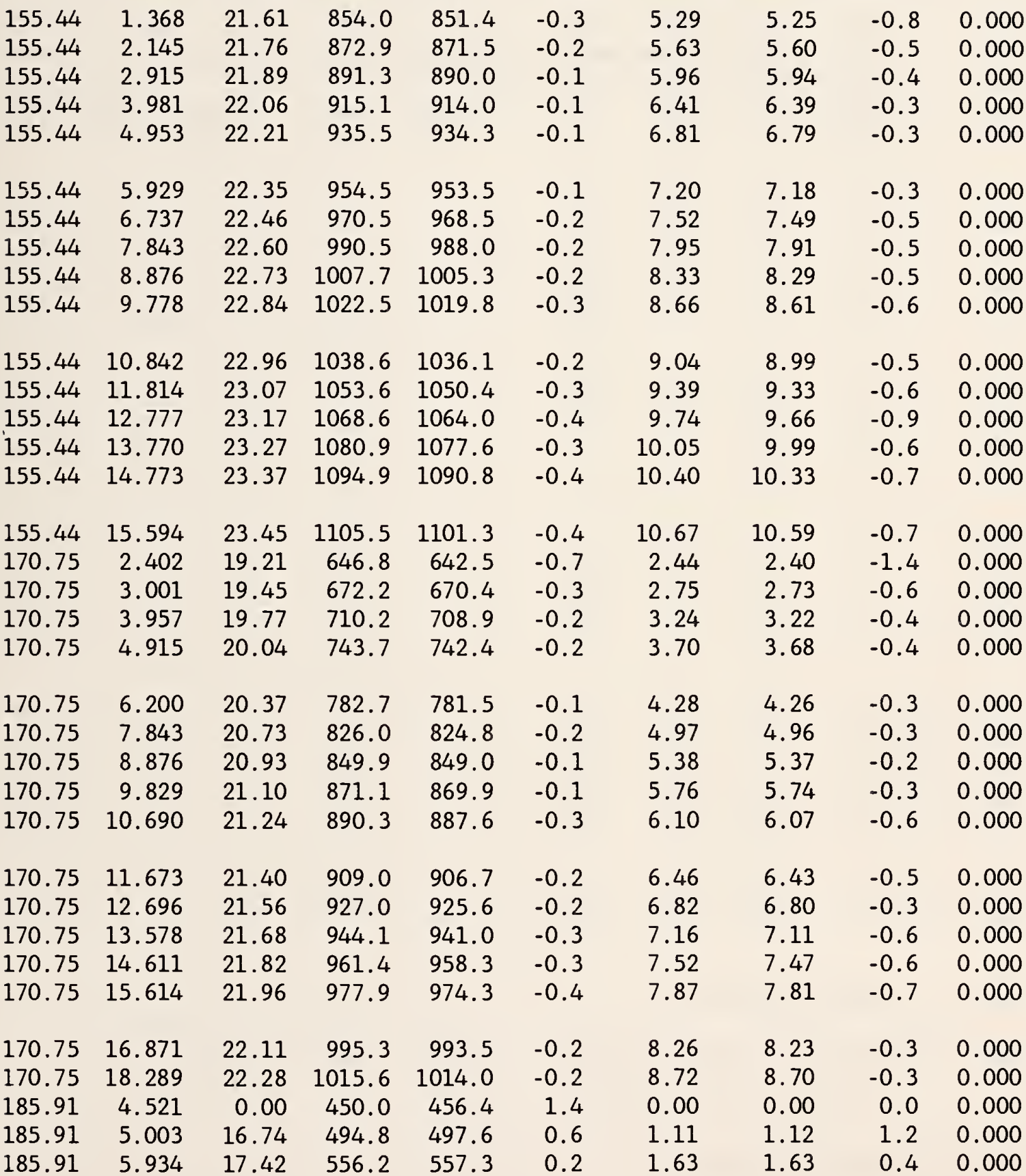


Data from Van Itterbeek et al. [60] (continued)

\begin{tabular}{|c|c|c|c|c|c|c|c|c|c|}
\hline $\mathrm{T}$ & $\mathbf{P}$ & $\rho$ & $W, \exp$ & W, cal & Dev1 & $\begin{array}{c}\partial \mathrm{P} /\left.\partial \rho\right|_{\mathrm{T}} \\
\exp \end{array}$ & $\begin{array}{c}\partial \mathrm{P} /\left.\partial \rho\right|_{\mathrm{T}} \\
\mathrm{cal}\end{array}$ & Dev2 & Wt \\
\hline K & $\mathrm{MPa}$ & $\mathrm{mol} \cdot \mathrm{dm}^{-3}$ & $\mathrm{~m} \cdot \mathrm{s}^{-1}$ & $\mathrm{~m} \cdot \mathrm{s}^{-1}$ & 8 & $\mathrm{MPa} \cdot \mathrm{dm}^{3}$ & $\mathrm{~mol}^{-1}$ & 8 & \\
\hline 185.91 & 6.886 & 17.93 & 603.0 & 604.8 & 0.3 & 2.10 & 2.11 & 0.6 & 0.000 \\
\hline 185.91 & 7.893 & 18.36 & 646.6 & 646.5 & 0.0 & 2.58 & 2.58 & 0.0 & 0.000 \\
\hline 185.91 & 8.927 & 18.73 & 685.6 & 683.3 & -0.3 & 3.05 & 3.03 & -0.6 & 0.000 \\
\hline 185.91 & 9.808 & 19.01 & 707.4 & 711.3 & 0.5 & 3.36 & 3.39 & 1.1 & 0.000 \\
\hline 185.91 & 10.811 & 19.29 & 733.7 & 740.0 & 0.9 & 3.73 & 3.80 & 1.8 & 0.000 \\
\hline 185.91 & 11.825 & 19.54 & 766.8 & 766.6 & 0.0 & 4.19 & 4.19 & 0.0 & 0.000 \\
\hline 185.91 & 13.253 & 19.86 & 800.6 & 800.5 & 0.0 & 4.72 & 4.72 & 0.0 & 0.000 \\
\hline 185.91 & 14.814 & 20.17 & 836.3 & 833.9 & -0.3 & 5.31 & 5.28 & -0.5 & 0.000 \\
\hline 185.91 & 15.888 & 20.37 & 856.1 & 855.2 & -0.1 & 5.66 & 5.65 & -0.2 & 0.000 \\
\hline 185.91 & 17.357 & 20.62 & 884.2 & 882.3 & -0.2 & 6.18 & 6.15 & -0.4 & 0.000 \\
\hline 185.91 & 18.340 & 20.78 & 901.8 & 899.3 & -0.3 & 6.51 & 6.48 & -0.5 & 0.000 \\
\hline 190.03 & 4.553 & 0.00 & 264.2 & 288.5 & 9.2 & 0.00 & 0.00 & 0.0 & 0.000 \\
\hline 190.03 & 4.775 & 0.00 & 340.2 & 359.2 & 5.6 & 0.00 & 0.00 & 0.0 & 0.000 \\
\hline 190.03 & 5.295 & 0.00 & 415.6 & 430.5 & 3.6 & 0.00 & 0.00 & 0.0 & 0.000 \\
\hline 190.03 & 5.953 & 16.26 & 482.3 & 486.4 & 0.9 & 1.04 & 1.06 & 1.8 & 0.000 \\
\hline 190.03 & 6.840 & 16.96 & 539.8 & 541.9 & 0.4 & 1.51 & 1.52 & 0.8 & 0.000 \\
\hline 190.03 & 7.792 & 17.50 & 590.9 & 589.0 & -0.3 & 1.99 & 1.98 & -0.6 & 0.000 \\
\hline 190.03 & 8.856 & 17.99 & 634.5 & 632.8 & -0.3 & 2.46 & 2.45 & -0.5 & 0.000 \\
\hline 190.03 & 10.102 & 18.45 & 677.7 & 676.4 & -0.2 & 2.99 & 2.98 & -0.3 & 0.000 \\
\hline 190.03 & 11.348 & 18.83 & 715.7 & 714.1 & -0.2 & 3.49 & 3.47 & -0.4 & 0.000 \\
\hline 190.03 & 12.574 & 19.16 & 748.8 & 747.1 & -0.2 & 3.96 & 3.94 & -0.4 & 0.000 \\
\hline 190.03 & 13.790 & 19.46 & 778.2 & 776.7 & -0.2 & 4.40 & 4.39 & -0.3 & 0.000 \\
\hline 190.03 & 15.037 & 19.73 & 807.2 & 804.5 & -0.3 & 4.86 & 4.83 & -0.6 & 0.000 \\
\hline 190.03 & 16.334 & 19.98 & 833.3 & 831.2 & -0.3 & 5.31 & 5.28 & -0.5 & 0.000 \\
\hline 190.03 & 17.509 & 20.20 & 855.1 & 853.7 & -0.2 & 5.69 & 5.68 & -0.3 & 0.000 \\
\hline 190.03 & 18.735 & 20.41 & 878.5 & 875.8 & -0.3 & 6.12 & 6.08 & -0.6 & 0.000 \\
\hline 190.03 & 19.971 & 20.60 & 900.5 & 896.8 & -0.4 & 6.53 & 6.48 & -0.8 & 0.000 \\
\hline
\end{tabular}

Number of Points (Ref. 60) 97

$\begin{array}{cllllll}\text { SOUND SPEED: AAD-8 } & 0.492 & \text { BIAS }-8 & -0.007 & \text { RMS }-8 & 1.205 & \\ \text { AAD } & 3.540 & \text { BIAS } & -1.751 & \text { RMS } & 4.508 & \mathrm{~m} \cdot \mathrm{s}^{-1}\end{array}$

Number of Points (Ref. 60) 93

$\begin{array}{llllllll}\text { DERIVATIVE: } & \text { AAD }-8 & 0.663 & \text { BIAS }-8 & -0.496 & \text { RMS }-8 & 0.548 & \\ & \text { AAD } & 0.064 & \text { BIAS } & -0.060 & \text { RMS } & 0.059 & \mathrm{MPa} \cdot \mathrm{dm}^{3} \cdot \mathrm{mol}^{-1}\end{array}$ 
Data from Van Itterbeek et al. [60] (continued)

Number of Points Total 661

$\begin{array}{lllllll}\text { SOUND SPEED: } & \text { AAD-8 } & 1.297 & \text { BIAS }-8 & 0.831 & \text { RMS- } 8 & 3.524 \\ \text { AAD } & 4.440 & \text { BIAS } & 0.573 & \text { RMS } & 8.170 \mathrm{~m} \cdot \mathrm{s}^{-1}\end{array}$

Number of Points Total 603 excluding critical region data of Ref. [37]

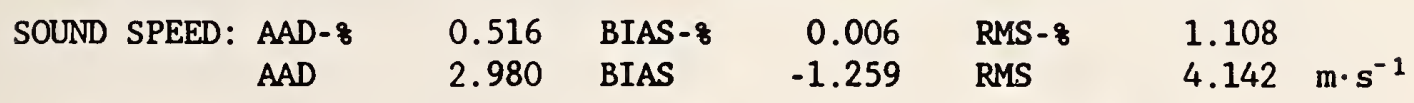


TABLE A10

COMPARISONS FOR COLLISION INTEGRAL

Notes for Table Al0. The temperature in kelvins corresponds to the given reduced temperature for the methane fluid $(t=k T / \epsilon)$. The collision integral $\Omega^{(2,2) *}$, int is from the integration results of Ref.[14]; the "cor" result' is from the correlation of eq (10).

\begin{tabular}{|c|c|c|c|c|}
\hline \multirow[t]{2}{*}{$t$} & $\mathrm{~T}$ & $\Omega^{(2,2) *}$ & $\Omega^{(2,2) *}$ & Dev \\
\hline & K & int & cor & 8 \\
\hline 0.50 & 87.000 & $2.136 \quad 15$ & 2.13873 & 0.121 \\
\hline 0.55 & 95.700 & 2.05191 & $2.053 \quad 45$ & 0.075 \\
\hline 0.60 & 104.400 & 1.97292 & 1.97511 & 0.111 \\
\hline 0.65 & 113.100 & 1.90393 & 1.90350 & -0.023 \\
\hline 0.70 & 121.800 & 1.83999 & 1.83822 & -0.096 \\
\hline 0.75 & 130.500 & $1.778 \quad 44$ & $1.778 \quad 77$ & 0.019 \\
\hline 0.80 & 139.200 & 1.72505 & 1.72465 & -0.023 \\
\hline 0.85 & 147.900 & $1.677 \quad 59$ & $1.675 \quad 33$ & -0.135 \\
\hline 0.90 & 156.600 & 1.63261 & 1.63031 & -0.141 \\
\hline 0.95 & 165.300 & 1.59074 & 1.58915 & -0.100 \\
\hline 1.00 & 174.000 & 1.55245 & 1.55143 & -0.065 \\
\hline 1.10 & 191.400 & 1.48592 & 1.48491 & -0.068 \\
\hline 1.20 & 208.800 & $1.428 \quad 35$ & $1.428 \quad 28$ & -0.005 \\
\hline 1.30 & 226.200 & $1.379 \quad 36$ & 1.37963 & 0.020 \\
\hline 1.40 & 243.600 & 1.33706 & 1.33748 & 0.031 \\
\hline 1.50 & 261.000 & $1.300 \quad 37$ & 1.30065 & 0.022 \\
\hline 1.60 & 278.400 & $1.267 \quad 48$ & $1.268 \quad 24$ & 0.060 \\
\hline 1.70 & 295.800 & $1.238 \quad 42$ & 1.23951 & 0.088 \\
\hline 1.80 & 313.200 & 1.21281 & 1.21390 & 0.090 \\
\hline 1.90 & 330.600 & 1.19000 & 1.19092 & 0.077 \\
\hline 2.00 & 348.000 & 1.16943 & $1.170 \quad 20$ & 0.066 \\
\hline 2.20 & 382.800 & 1.13394 & $1.134 \quad 34$ & 0.035 \\
\hline 2.40 & 417.600 & 1.10430 & $1.104 \quad 39$ & 0.008 \\
\hline 2.60 & 452.400 & 1.07911 & 1.07900 & -0.010 \\
\hline 2.80 & 487.200 & 1.05760 & 1.05719 & -0.039 \\
\hline 3.00 & 522.000 & 1.03897 & $1.038 \quad 24$ & -0.070 \\
\hline 3.20 & 556.800 & 1.02262 & 1.02161 & -0.098 \\
\hline 3.40 & 591.600 & 1.00809 & $1.006 \quad 89$ & -0.119 \\
\hline 3.60 & 626.400 & 0.99507 & 0.99374 & -0.134 \\
\hline 3.80 & 661.200 & $0.983 \quad 32$ & 0.98191 & -0.143 \\
\hline
\end{tabular}


Table Al0 (continued)

\begin{tabular}{|c|c|c|c|c|}
\hline \multirow[t]{2}{*}{$t$} & $\mathrm{~T}$ & $\Omega^{(2,2) *}$ & $\Omega^{(2,2) *}$ & Dev \\
\hline & K & int & $\operatorname{cor}$ & 8 \\
\hline 4.00 & 696.000 & 0.97266 & 0.97120 & -0.150 \\
\hline 4.50 & 783.000 & 0.94972 & $0.948 \quad 32$ & -0.147 \\
\hline 5.00 & 870.000 & 0.93087 & 0.92962 & -0.135 \\
\hline 5.50 & 957.000 & 0.91494 & 0.91391 & -0.112 \\
\hline 6.00 & 1044.000 & 0.90118 & 0.90043 & -0.083 \\
\hline 6.50 & 1131.000 & 0.88909 & 0.88865 & -0.050 \\
\hline
\end{tabular}

Number of Points (Ref. 14) 36

$\begin{array}{lllrll}\text { AAD }-8 & 0.077 & \text { BIAS }-8 & -0.031 & \text { RMS }-8 & 0.084 \\ \text { AAD } & 0.001 & \text { BIAS } & 0.000 & \text { RMS } & 0.001\end{array}$




\section{COMPARISONS FOR ZERO DENSITY VISCOSITY}

Notes for Table All. The column $\eta$,exp refers to the reported experimental value at the lowest pressure at the given temperature. These pressures were often at $0.101325 \mathrm{MPa}(1 \mathrm{~atm})$. In the column $\eta_{0}$, exp, the reported viscosities were corrected to zero pressure by subtracting the value of the excess viscosity at the reported conditions and calculated from the current correlation. The deviations and statistics refer to these corrected values of $\eta_{0}$. When the experimental data have already been extrapolated to zero pressure, (that is for Refs. 62, 65, 66), there are no entries in the $\eta_{0}$, exp column. The weights refer to weighting in the determination of the intermolecular parameters $\epsilon$ and $\sigma$.

Data from Hellemans et al. [61]

$\begin{array}{crrrrr}\mathrm{T} & \eta \exp & \eta_{0}, \exp & \eta_{0}, \mathrm{cal} & \text { Dev } & \text { Wt } \\ \mathrm{K} & \mu \mathrm{Pa} \cdot \mathrm{s} & \mu \mathrm{Pa} \cdot \mathrm{s} & \mu \mathrm{Pa} \cdot \mathrm{s} & \text { \& } & \\ 298.15 & 11.092 & 11.076 & 11.121 & 0.407 & 1.0 \\ 323.15 & 11.941 & 11.926 & 11.920 & -0.053 & 1.0 \\ 373.15 & 13.514 & 13.501 & 13.445 & -0.410 & 1.0 \\ 423.15 & 14.896 & 14.884 & 14.885 & 0.006 & 1.0 \\ 468.15 & 16.116 & 16.105 & 16.115 & 0.064 & \end{array}$

Number of points $(\operatorname{Ref.61)~} 5$

$\begin{array}{lllrlrl}\text { AAD }-8 & 0.188 & \text { BIAS }-8 & 0.003 & \text { RMS }-8 & 0.261 & \\ \text { AAD } & 0.024 & \text { BIAS } & -0.001 & \text { RMS } & 0.032 & \mu \mathrm{Pa} \cdot \mathrm{s}\end{array}$

Data from Dawe et a1. [62]

$\begin{array}{rccccc}\mathrm{T} & \eta, \exp & \eta_{0}, \exp & \eta_{0}, \mathrm{cal} & \text { Dev } & \text { Wt } \\ \mathrm{K} & \mu \mathrm{Pa} \cdot \mathrm{s} & \mu \mathrm{Pa} \cdot \mathrm{s} & \mu \mathrm{Pa} \cdot \mathrm{s} & 8 & \\ 293.0 & 11.00 & - & 10.954 & -0.421 & 0.0 \\ 403.0 & 14.39 & - & 14.314 & -0.525 & 0.0 \\ 497.0 & 16.92 & - & 16.875 & -0.266 & 0.0 \\ 601.0 & 19.45 & - & 19.452 & 0.011 & 0.0 \\ 676.0 & 21.13 & - & 21.178 & 0.229 & 0.0 \\ 749.0 & 22.68 & - & & & 0.0 \\ 823.0 & 24.26 & - & 22.771 & 0.403 & 0.0 \\ 900.0 & 25.78 & - & 24.312 & 0.214 & 0.0 \\ 1050.0 & 28.55 & - & 25.847 & 0.259 & 0.431 \\ \end{array}$


Data from Dawe et al. [62]

Number of points (Ref.62) 9
$A A D-8$
0.306
BIAS - 8
0.037
RMS - 8
0.337
AAD
0.061
BIAS
0.024
RMS
$0.065 \mu \mathrm{Pa} \cdot \mathrm{s}$

$\overline{\text { Data from Kestin et al. [63] }}$

$\begin{array}{crrccc}\mathrm{T} & \eta \exp & \eta_{0}, \exp & \eta_{0}, \mathrm{cal} & \text { Dev } & \text { Wt } \\ \mathrm{K} & \mu \mathrm{Pa} \cdot \mathrm{s} & \mu \mathrm{Pa} \cdot \mathrm{s} & \mu \mathrm{Pa} \cdot \mathrm{s} & 8 & \\ 296.22 & 11.023 & 11.007 & 11.059 & 0.471 & 0.0 \\ 302.44 & 11.226 & 11.210 & 11.260 & 0.445 & 0.0\end{array}$

Number of points (Ref.63) 2

$\begin{array}{lllllll}\text { AAD }-8 & 0.458 & \text { BIAS }-8 & 0.458 & \text { RMS }-8 & 0.013 & \\ \text { AAD } & 0.051 & \text { BIAS } & 0.051 & \text { RMS } & 0.001 & \mu \mathrm{Pa} \cdot \mathrm{s}\end{array}$

Data from Timrot et al. [64]

$\begin{array}{cccccc}\mathrm{T} & \eta, \exp & \eta_{0}, \exp & \eta_{0}, \mathrm{cal} & \text { Dev } & \text { Wt } \\ \mathrm{K} & \mu \mathrm{Pa} \cdot \mathrm{s} & \mu \mathrm{Pa} \cdot \mathrm{s} & \mu \mathrm{Pa} \cdot \mathrm{s} & 8 & \\ 296.85 & 11.13 & 11.125 & 11.079 & -0.416 & 0.0 \\ 325.74 & 12.03 & 12.026 & 12.001 & -0.204 & 0.0 \\ 375.85 & 13.53 & 13.526 & 13.525 & -0.007 & 0.0 \\ 423.45 & 14.93 & 14.927 & 14.893 & -0.223 & 0.0 \\ 471.05 & 16.22 & 16.217 & 16.193 & -0.149 & 0.0 \\ 523.75 & 17.59 & 17.587 & & & 0.0 \\ 569.35 & 18.73 & 18.727 & 17.561 & -0.148 & 0.0 \\ 622.45 & 19.96 & 19.957 & 18.693 & -0.186 & 0.0 \\ 675.25 & 21.18 & 21.178 & 21.162 & -0.076 & 0.07\end{array}$

Number of points (Ref.64) 9

$\begin{array}{lllllll}\text { AAD }-8 & 0.157 & \text { BIAS }-8 & -0.157 & \text { RMS }-8 & 0.118 & \\ \text { AAD } & 0.023 & \text { BIAS } & -0.023 & \text { RMS } & 0.014 & \mu \mathrm{Pa} \cdot \mathrm{s}\end{array}$


Data from Kestin and Yata [65]

$\begin{array}{cccccc}\mathrm{T} & \eta \text {, exp } & \eta_{0}, \exp & \eta_{0}, \mathrm{cal} & \text { Dev } & \text { Wt } \\ \mathrm{K} & \mu \mathrm{Pa} \cdot \mathrm{s} & \mu \mathrm{Pa} \cdot \mathrm{s} & \mu \mathrm{Pa} \cdot \mathrm{s} & 8 & \\ 293.15 & 10.924 & - & 10.959 & 0.316 & 0.0 \\ 303.15 & 11.258 & - & 11.283 & 0.222 & 0.0\end{array}$

Number of points (Ref.65) 2
AAD- $8 \quad 0.269$
BIAS - 8
0.269
RMS - 8
0.047
AAD
0.030
BIAS
RMS
$0.005 \quad \mu \mathrm{Pa} \cdot \mathrm{s}$

Data from De Rocco and Halford [66]

\begin{tabular}{|c|c|c|c|c|c|}
\hline $\mathrm{T}$ & $\eta$, exp & $\eta_{0}, \exp$ & $\eta_{0}, \mathrm{cal}$ & Dev & Wt \\
\hline K & $\mu \mathrm{Pa} \cdot \mathrm{s}$ & $\mu \mathrm{Pa} \cdot \mathrm{s}$ & $\mu \mathrm{Pa} \cdot \mathrm{s}$ & 8 & \\
\hline 210.70 & 8.10 & - & 8.122 & 0.2711 & 0.0 \\
\hline 250.10 & 9.67 & - & 9.514 & -1.6134 & 0.0 \\
\hline 273.16 & 10.31 & - & 10.298 & -0.1205 & 0.0 \\
\hline 292.70 & 11.03 & - & 10.944 & -0.7810 & 0.0 \\
\hline 300.70 & 11.25 & - & 11.204 & -0.4099 & 0.0 \\
\hline 308.20 & 11.55 & - & 11.445 & -0.9066 & 0.0 \\
\hline 320.70 & 11.89 & - & 11.843 & -0.3983 & 0.0 \\
\hline 328.70 & 12.20 & - & 12.094 & -0.8711 & 0.0 \\
\hline 337.30 & 12.53 & - & 12.361 & -1.3494 & 0.0 \\
\hline 351.10 & 12.81 & - & 12.784 & -0.2040 & 0.0 \\
\hline 363.10 & 13.22 & - & 13.146 & -0.5597 & 0.0 \\
\hline 373.20 & 13.55 & - & 13.447 & -0.7612 & 0.0 \\
\hline 387.90 & 13.91 & - & 13.879 & -0.2266 & 0.0 \\
\hline 394.40 & 14.19 & - & 14.067 & -0.8666 & 0.0 \\
\hline 407.20 & 14.49 & - & 14.434 & -0.3842 & 0.0 \\
\hline 424.40 & 15.06 & - & 14.920 & -0.9311 & 0.0 \\
\hline 439.90 & 15.41 & - & 15.350 & -0.3921 & 0.0 \\
\hline 451.60 & 15.74 & - & 15.669 & -0.4486 & 0.0 \\
\hline 459.40 & 15.94 & - & 15.881 & -0.3735 & 0.0 \\
\hline 473.16 & 16.35 & - & 16.249 & -0.6193 & 0.0 \\
\hline
\end{tabular}

Number of points (Ref.66) 20
AAD- $8 \quad 0.624$
BIAS - $8 \quad-0.597$
RMS - 8
0.420
AAD
0.080
BIAS $\quad-0.078$
RMS
$0.048 \quad \mu \mathrm{Pa} \cdot \mathrm{s}$ 
Data from Maitland and Smith [67]

\begin{tabular}{rrrrrr}
$\mathrm{T}$ & \multicolumn{1}{r}{ exp } & $\eta_{0}, \exp$ & $\eta_{0}, \mathrm{cal}$ & $\mathrm{Dev}$ & Wt \\
$\mathrm{K}$ & $\mu \mathrm{Pa} \cdot \mathrm{s}$ & $\mu \mathrm{Pa} \cdot \mathrm{s}$ & $\mu \mathrm{Pa} \cdot \mathrm{s}$ & 8 & \\
295.0 & 11.09 & 11.0747 & 11.0189 & -0.5036 & 0.0 \\
394.0 & 14.08 & 14.0635 & 14.0555 & -0.0572 & 0.0 \\
547.0 & 18.21 & 18.1926 & 18.1436 & -0.2691 & 0.0 \\
698.0 & 21.63 & 21.6120 & 21.6668 & 0.2533 & 0.0 \\
873.0 & 25.42 & 25.4016 & 25.3158 & -0.3379 & 0.0 \\
873.0 & 25.23 & 25.2116 & 25.3158 & 0.4132 & 0.0 \\
1022.0 & 27.83 & 27.8114 & 28.1600 & 1.2536 &
\end{tabular}

Number of points (Ref.67) 7
$A A D-8$
0.441
BIAS - $8 \quad 0.107$
RMS - 8
0.557
AAD
0.101
BIAS
0.044
RMS
$0.139 \mu \mathrm{Pa} \cdot \mathrm{s}$

Data from Abe et al. [68]

T

K

$$
\begin{array}{ll}
\eta, \exp & \eta_{0}, \exp \\
\mu \mathrm{Pa} \cdot \mathrm{s} & \mu \mathrm{Pa} \cdot \mathrm{s}
\end{array}
$$

298.15

333.15

373.15

418.15

468.15
11.0842

12.2655

13.4667

14.7679

16.0889 $\eta_{0}, \mathrm{cal}$

$\mu \mathrm{Pa} \cdot \mathrm{s}$

11.1213

12.2323

13.4454

14.7444

16.1152
Dev

8

Number of points (Ref.68) 5
AAD - $q$
0.217
BIAS - $8 \quad-0.018$
RMS - 8
0.228
AAD
0.028
BIAS $\quad-0.003$
$0.029 \mu \mathrm{Pa} \cdot \mathrm{s}$

0.3346

$-0.2701$

$-0.1582$

$-0.1586$

0.1636 
Data from Clarke and Smith [69]

\begin{tabular}{rrrrrr}
$\mathrm{T}$ & $\eta, \exp$ & $\eta_{0}, \exp$ & \multicolumn{1}{c}{$\eta_{0}, \mathrm{cal}$} & \multicolumn{1}{c}{ Dev } & Wt \\
$\mathrm{K}$ & $\mu \mathrm{Pa} \cdot \mathrm{s}$ & $\mu \mathrm{Pa} \cdot \mathrm{s}$ & $\mu \mathrm{Pa} \cdot \mathrm{s}$ & \multicolumn{1}{c}{} & \\
113.8 & 4.47 & 4.4656 & 4.4739 & 0.1853 & 0.0 \\
140.1 & 5.50 & 5.4905 & 5.4799 & -0.1931 & 0.0 \\
171.4 & 6.68 & 6.6710 & 6.6702 & -0.0126 & 0.0 \\
200.3 & 7.76 & 7.7516 & 7.7435 & -0.1042 & 0.0 \\
234.1 & 8.95 & 8.9423 & 8.9568 & 0.1614 & 0.0 \\
273.0 & 10.29 & 10.2831 & 10.2922 & 0.0891 & 0.0 \\
299.1 & 11.14 & 11.1307 & 11.1521 & 0.1922 & 0.0 \\
333.2 & 12.23 & 12.2214 & 12.2339 & 0.1021 & 0.0 \\
373.9 & 13.48 & 13.4721 & 13.4676 & -0.0338 & 0.0
\end{tabular}

Number of points (Ref.69) 10
$A A D-8$
0.119
BIAS - 8
0.043
RMS - 8
0.128
AAD
0.010
BIAS
0.005
RMS
$0.010 \mu \mathrm{Pa} \cdot \mathrm{s}$

$\overline{\text { Data from Giddings et a1. [70] }}$

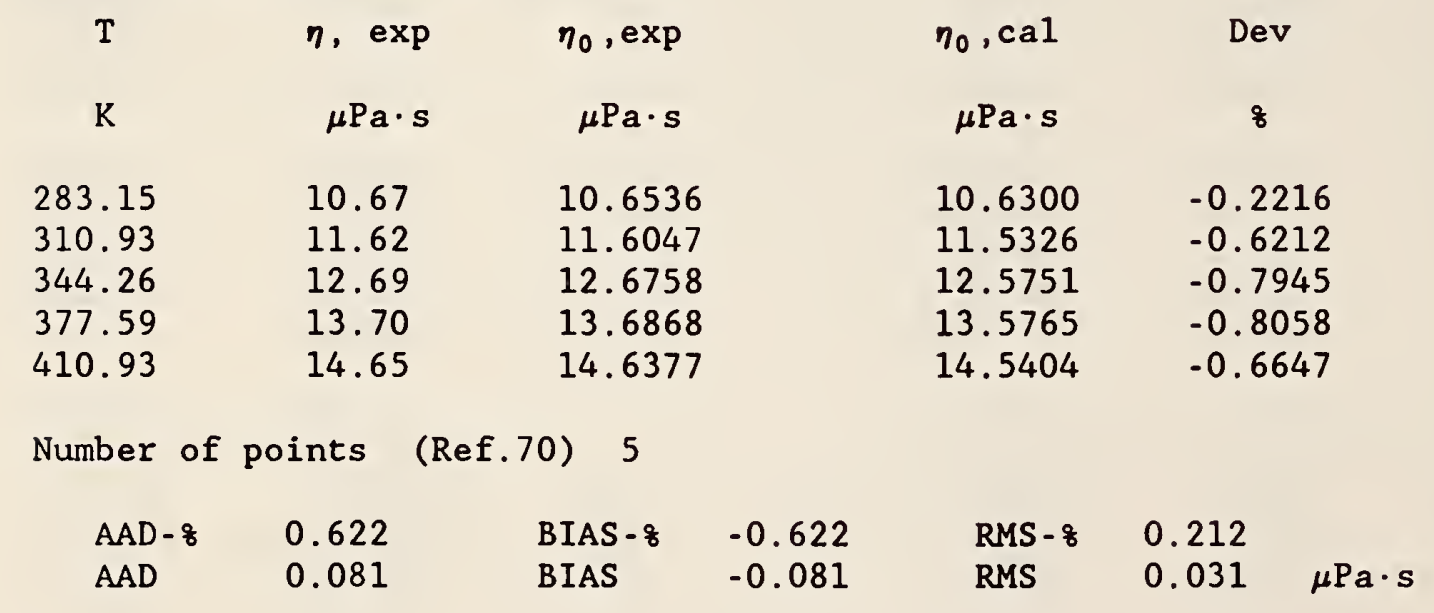


Data from Carmichael et al. [71]

$\mathrm{T} \quad \eta, \exp \quad \eta_{0}, \exp$

$\mathrm{K}$

$\mu \mathrm{Pa} \cdot \mathrm{s} \quad \mu \mathrm{Pa} \cdot \mathrm{s}$

$\eta_{0}, \mathrm{cal}$

Dev

Wt

277.594

10.530

10.5117

11.5667

11.585

13.6145

377.594

13.631

15.5817

15.597

16.5198

$$
\mu \mathrm{Pa} \cdot \mathrm{s}
$$

8

$\begin{array}{ll}10.4456 & -0.6280 \\ 11.5325 & -0.2956 \\ 13.5766 & -0.2777 \\ 15.4692 & -0.7219 \\ 16.3663 & -0.9287\end{array}$

0.0

0.0

0.0

0.0

477.594

16.543

16.3663

0.0

Number of points (Ref.71) 5

$\begin{array}{lllllll}\text { AAD }-8 & 0.570 & \text { BIAS }-8 & -0.570 & \text { RMS }-8 & 0.251 & \\ \text { AAD } & 0.081 & \text { BIAS } & -0.081 & \text { RMS } & 0.046 & \mu \mathrm{Pa} \cdot \mathrm{s}\end{array}$

Number of Points Total

78

$\begin{array}{lllllll}\text { AAD }-8 & 0.388 & \text { BIAS }-8 & -0.211 & \text { RMS }-8 & 0.456 & \\ \text { AAD } & 0.056 & \text { BIAS } & -0.024 & \text { RMS } & 0.073 & \mu \mathrm{Pa} \cdot \mathrm{s}\end{array}$


Notes for Table A12. The column $\lambda$,exp refers to the reported experimental value at the lowest pressure at the given temperature. These pressures were often at $0.101325 \mathrm{MPa}(1 \mathrm{~atm})$. In the column $\lambda_{0}$, exp, the reported thermal conductivities were corrected to zero pressure by subtracting the value of the excess thermal conductivity at the reported conditions and calculated from the current correlation. The deviations and statistics refer to these corrected values of $\lambda_{0}$. When the experimental data have already been extrapolated to zero pressure, (that is for Refs. 8, 72, 73, and 76), there are no entries in the $\lambda_{0}$,exp column. The weights refer to weighting in the determination of the intermolecular parameters in the damping function, eq (12).

Data from Roder [8]

$\begin{array}{rccccc}\mathrm{T} & \lambda, \exp & \lambda_{0}, \exp & \lambda_{0}, \mathrm{cal} & \mathrm{Dev} & \text { Wt } \\ \mathrm{K} & \mathrm{mW} \cdot \mathrm{m}^{-1} \cdot \mathrm{K}^{-1} & \mathrm{~mW} \cdot \mathrm{m}^{-1} \cdot \mathrm{K}^{-1} & \mathrm{~mW} \cdot \mathrm{m}^{-1} \cdot \mathrm{K}^{-1} & 8 & \\ 133.0 & 13.92 & - & 13.72 & -1.458 & 1.0 \\ 143.0 & 14.97 & - & 14.90 & -0.448 & 1.0 \\ 155.0 & 16.40 & - & 16.32 & -0.459 & 1.0 \\ 163.0 & 17.33 & - & 17.27 & -0.339 & 1.0 \\ 175.0 & 18.70 & - & 18.69 & -0.060 & 1.0 \\ 183.0 & 19.62 & - & 19.63 & 0.067 & 1.0 \\ 197.0 & 21.31 & - & 21.29 & -0.103 & 1.0 \\ 215.0 & 23.12 & - & 23.43 & 1.339 & 1.0 \\ 235.0 & 25.60 & - & 25.85 & 0.975 & 1.0 \\ 255.0 & 28.03 & - & 28.34 & 1.101 & 1.0 \\ 275.0 & 30.54 & - & 30.92 & 1.253 & 1.0 \\ 295.0 & 33.01 & - & 33.62 & 1.854 & 1.0 \\ 310.0 & 35.36 & - & 35.73 & 1.048 & 1.0\end{array}$

Number of points (Ref. 8) 13

$\begin{array}{lllllll}\text { AAD }-8 & 0.808 & \text { BIAS }-8 & 0.367 & \text { RMS }-8 & 0.919 & \\ \text { AAD } & 0.206 & \text { BIAS } & 0.139 & \text { RMS } & 0.235 & \mathrm{~mW} \cdot \mathrm{m}^{-1} \cdot \mathrm{K}^{-1}\end{array}$


Data from Clifford et al. [72]

$\begin{array}{cccccc}\mathrm{T} & \lambda, \exp & \lambda_{0}, \exp & \lambda_{0}, \mathrm{cal} & \text { Dev } & \text { Wt } \\ \mathrm{K} & \mathrm{mW} \cdot \mathrm{m}^{-1} \cdot \mathrm{K}^{-1} & \mathrm{~mW} \cdot \mathrm{m}^{-1} \cdot \mathrm{K}^{-1} & \mathrm{~mW} \cdot \mathrm{m}^{-1} \cdot \mathrm{K}^{-1} & 8 & \\ 300.65 & 34.93 & - & 34.41 & -1.496 & 0.0\end{array}$

Number of points (Ref.72) 1

$\begin{array}{lllllll}\text { AAD }-8 & 1.496 & \text { BIAS }-8 & -1.496 & \text { RMS }-8 & - & \\ \text { AAD } & 0.523 & \text { BIAS } & -0.523 & \text { RMS } & - & \mathrm{mW} \cdot \mathrm{m}^{-1} \cdot \mathrm{K}^{-1}\end{array}$

$\overline{\text { Data from Assael and Wakeham [73] }}$

$\begin{array}{cccccc}\mathrm{T} & \lambda, \exp & \lambda_{0}, \exp & \lambda_{0}, \mathrm{cal} & \text { Dev } & \text { Wt } \\ \mathrm{K} & \mathrm{mW} \cdot \mathrm{m}^{-1} \cdot \mathrm{K}^{-1} & \mathrm{~mW} \cdot \mathrm{m}^{-1} \cdot \mathrm{K}^{-1} & \mathrm{~mW} \cdot \mathrm{m}^{-1} \cdot \mathrm{K}^{-1} & 8 & \\ 308.15 & 35.35 & - & 35.47 & 0.329 & 0.0\end{array}$

Number of points (Ref.73) 1

$\begin{array}{llllll}\text { AAD }-8 & 0.329 & \text { BIAS }-8 & 0.329 & \text { RMS - 8 } & - \\ \text { AAD } & 0.116 & \text { BIAS } & 0.116 & \text { RMS } & -\mathrm{mW} \cdot \mathrm{m}^{-1} \cdot \mathrm{K}^{-1}\end{array}$

Data from Johnston and Grilly [74]

$\begin{array}{cccccc}\mathrm{T} & \lambda, \exp & \lambda_{0}, \exp & \lambda_{0}, \mathrm{cal} & \text { Dev } & \text { Wt } \\ \mathrm{K} & \mathrm{mW} \cdot \mathrm{m}^{-1} \cdot \mathrm{K}^{-1} & \mathrm{~mW} \cdot \mathrm{m}^{-1} \cdot \mathrm{K}^{-1} & \mathrm{~mW} \cdot \mathrm{m}^{-1} \cdot \mathrm{K}^{-1} & \&\end{array}$

$\begin{array}{rrrrrr}96.76 & 10.2550 & 10.2161 & 9.4505 & -7.494 & 0.0 \\ 125.35 & 13.3679 & 13.3413 & 12.8104 & -3.979 & 0.0 \\ 140.49 & 15.0959 & 15.0703 & 14.6053 & -3.085 & 0.0 \\ 155.29 & 16.7569 & 16.7310 & 16.3591 & -2.223 & 0.0 \\ 169.93 & 18.4347 & 18.4069 & 18.0901 & -1.721 & 0.0 \\ 185.41 & 20.1041 & 20.0700 & 19.9178 & -0.758 & 0.0 \\ 201.24 & 21.9451 & 21.9155 & 21.7905 & -0.571 & 0.0 \\ 216.69 & 23.7819 & 23.7583 & 23.6321 & -0.531 & 0.0 \\ 231.71 & 25.5935 & 25.5734 & 25.4474 & -0.493 & 0.0 \\ 246.86 & 27.4219 & 27.4042 & 27.3154 & -0.324 & 0.0\end{array}$


Data from Johnston and Grilly [74] (continued)

\begin{tabular}{ccrrrr}
$\mathrm{T}$ & \multicolumn{1}{c}{$\lambda, \exp$} & $\lambda_{0}, \exp$ & $\lambda_{0}, \mathrm{cal}$ & Dev & Wt \\
$\mathrm{K}$ & $\mathrm{mW} \cdot \mathrm{m}^{-1} \cdot \mathrm{K}^{-1}$ & $\mathrm{~mW} \cdot \mathrm{m}^{-1} \cdot \mathrm{K}^{-1}$ & $\mathrm{~mW} \cdot \mathrm{m}^{-1} \cdot \mathrm{K}^{-1}$ & 8 & \\
262.85 & 29.3926 & 29.3768 & 29.3402 & -0.124 & 0.0 \\
278.72 & 31.4218 & 31.4075 & 31.4154 & 0.025 & 0.0 \\
294.49 & 33.5808 & 33.5677 & 33.5515 & -0.048 & 0.0 \\
324.00 & 37.5974 & 37.5861 & 37.7669 & 0.481 & 0.0 \\
338.60 & 39.9112 & 39.9006 & 39.9620 & 0.154 & 0.0 \\
353.96 & 42.4509 & 42.4410 & 42.3495 & -0.215 & 0.0 \\
368.33 & 44.8901 & 44.8808 & 44.6538 & -0.506 & 0.0 \\
383.57 & 47.5972 & 47.5883 & 47.1688 & -0.882 & 0.0
\end{tabular}

Number of points (Ref.74) 18

$\begin{array}{lllllll}\text { AAD }-8 & 1.312 & \text { BIAS }-8 & -1.239 & \text { RMS }-8 & 1.896 & \\ \text { AAD } & 0.228 & \text { BIAS } & -0.200 & \text { RMS } & 0.230 & \mathrm{~mW} \cdot \mathrm{m}^{-1} \cdot \mathrm{K}^{-1}\end{array}$

$\overline{\text { Data from Zheng et a1. [75] }}$

$\begin{array}{cccccc}\mathrm{T} & \lambda, \exp & \lambda_{0}, \exp & \lambda_{0}, \mathrm{cal} & \text { Dev } & \text { Wt } \\ \mathrm{K} & \mathrm{mW} \cdot \mathrm{m}^{-1} \cdot \mathrm{K}^{-1} & \mathrm{~mW} \cdot \mathrm{m}^{-1} \cdot \mathrm{K}^{-1} & \mathrm{~mW} \cdot \mathrm{m}^{-1} \cdot \mathrm{K}^{-1} & 8 & \\ 299.45 & 34.74 & 34.68 & 34.24 & -1.233 & 0.0\end{array}$

Number of points (Ref.75) 1

$\begin{array}{lllllll}\text { AAD }-8 & 1.233 & \text { BIAS }-8 & -1.233 & \text { RMS }-8 & - & \\ \text { AAD } & 0.427 & \text { BIAS } & -0.427 & \text { RMS } & - & \mathrm{mW} \cdot \mathrm{m}^{-1} \cdot \mathrm{K}^{-1}\end{array}$

$\overline{\text { Data from Mann and Dickins [76] }}$

$\begin{array}{cccccc}\mathrm{T} & \lambda, \exp & \lambda_{0}, \exp & \lambda_{0}, \mathrm{cal} & \text { Dev } & \text { Wt } \\ \mathrm{K} & \mathrm{mW} \cdot \mathrm{m}^{-1} \cdot \mathrm{K}^{-1} & \mathrm{~mW} \cdot \mathrm{m}^{-1} \cdot \mathrm{K}^{-1} & \mathrm{~mW} \cdot \mathrm{m}^{-1} \cdot \mathrm{K}^{-1} & 8 & \\ 275.18 & 30.480 & - & 30.946 & 1.530 & 0.0 \\ 276.18 & 30.619 & - & 31.078 & 1.501 & 0.0 \\ 277.17 & 30.853 & - & 31.210 & 1.156 & 0.0 \\ 279.17 & 31.079 & - & 31.475 & 1.275 & 0.0 \\ 281.18 & 31.372 & - & 31.744 & 1.184 & 0.0 \\ 283.18 & 31.665 & - & 32.012 & 1.095 & 0.0\end{array}$


Data from Mann and Dickins [76] (continued)

Number of points (Ref.76) 6
AAD - 8
1.290
BIAS - 8
1.290
RMS - $8 \quad 0.168$
AAD
0.400
BIAS
0.400
RMS
$0.047 \mathrm{~mW} \cdot \mathrm{m}^{-1} \cdot \mathrm{K}^{-1}$

\begin{tabular}{cccccc}
\hline Data from Misic and Thodos $[77]$ & & & \\
T & $\lambda, \exp$ & \multicolumn{1}{c}{$\lambda_{0}, \exp$} & $\lambda_{0}, \mathrm{cal}$ & Dev & Wt \\
$\mathrm{K}$ & $\mathrm{mW} \cdot \mathrm{m}^{-1} \cdot \mathrm{K}^{-1}$ & $\mathrm{~mW} \cdot \mathrm{m}^{-1} \cdot \mathrm{K}^{-1}$ & $\mathrm{~mW} \cdot \mathrm{m}^{-1} \cdot \mathrm{K}^{-1}$ & 8 & \\
& & & & & \\
275.05 & 31.4637 & 31.3798 & 30.9292 & -1.436 & 0.0 \\
297.75 & 34.3506 & 34.2750 & 34.0029 & -0.794 & 0.0 \\
316.75 & 37.0702 & 37.0002 & 36.7040 & -0.801 & 0.0 \\
348.25 & 41.8818 & 41.8195 & 41.4527 & -0.877 & 0.0
\end{tabular}

Number of points (Ref.77) 4
AAD - 8
0.977
BIAS - 8
$-0.977$
RMS - $8 \quad 0.267$
AAD
0.346
BIAS
$-0.346$
RMS
$0.069 \mathrm{~mW} \cdot \mathrm{m}^{-1} \cdot \mathrm{K}^{-1}$

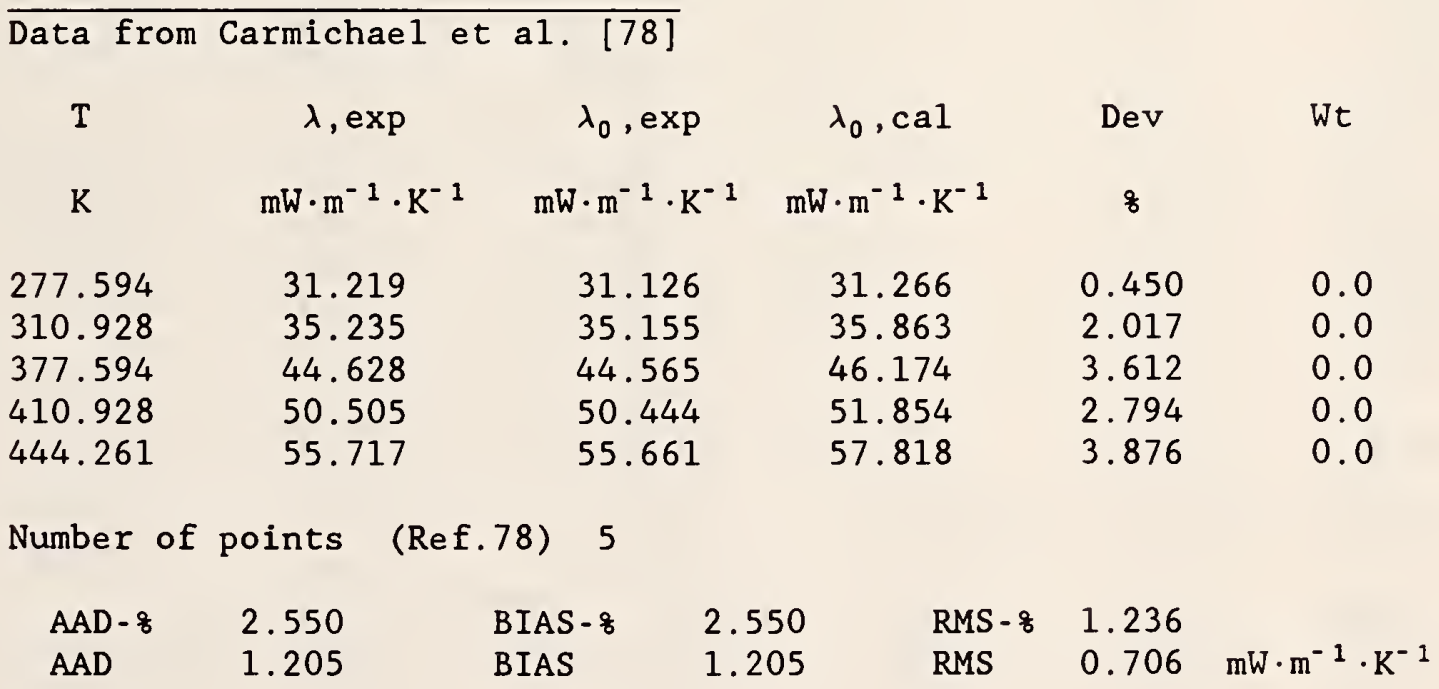


Data from Baker and Brokaw [79]

\begin{tabular}{cccccc}
$\mathrm{T}$ & \multicolumn{1}{c}{$\lambda, \exp$} & $\lambda_{0}, \exp$ & $\lambda_{0}, \mathrm{cal}$ & Dev & Wt \\
$\mathrm{K}$ & $\mathrm{mW} \cdot \mathrm{m}^{-1} \cdot \mathrm{K}^{-1}$ & $\mathrm{~mW} \cdot \mathrm{m}^{-1} \cdot \mathrm{K}^{-1}$ & $\mathrm{~mW} \cdot \mathrm{m}^{-1} \cdot \mathrm{K}^{-1}$ & \& & \\
& & & & & \\
299.8 & 33.7649 & 33.6912 & 34.2885 & 1.773 & 0.0 \\
333.0 & 38.1623 & 38.0977 & 39.1114 & 2.661 & 0.0 \\
375.5 & 44.5805 & 44.5246 & 45.8281 & 2.928 & 0.0 \\
423.3 & 52.3711 & 52.3226 & 54.0374 & 3.277 & 0.0 \\
472.3 & 60.4504 & 60.4076 & 63.0125 & 4.312 & 0.0
\end{tabular}

Number of points (Ref.79) 5

$\begin{array}{lllllll}\text { AAD - } 8 & 2.990 & \text { BIAS }-8 & 2.990 & \text { RMS }-8 & 0.828 & \\ \text { AAD } & 1.447 & \text { BIAS } & 1.447 & \text { RMS } & 0.685 & \mathrm{~mW} \cdot \mathrm{m}^{-1} \cdot \mathrm{K}^{-1}\end{array}$

$\overline{\text { Data from Sokolova and Golubev [80] }}$

\begin{tabular}{|c|c|c|c|c|c|}
\hline $\mathrm{T}$ & $\lambda, \exp$ & $\lambda_{0}, \exp$ & $\lambda_{0}, \mathrm{cal}$ & Dev & Wt \\
\hline K & $\mathrm{mW} \cdot \mathrm{m}^{-1} \cdot \mathrm{K}^{-1}$ & $\mathrm{~mW} \cdot \mathrm{m}^{-1} \cdot \mathrm{K}^{-1}$ & $\mathrm{~mW} \cdot \mathrm{m}^{-1} \cdot \mathrm{K}^{-1}$ & 8 & \\
\hline 238.7 & 26.08 & 25.98 & 26.30 & 1.245 & 0.0 \\
\hline 231.8 & 25.25 & 25.15 & 25.46 & 1.244 & 0.0 \\
\hline 224.8 & 24.45 & 24.34 & 24.61 & 1.102 & 0.0 \\
\hline 216.4 & 23.47 & 23.35 & 23.60 & 1.048 & 0.0 \\
\hline 210.0 & 22.71 & 22.59 & 22.83 & 1.093 & 0.0 \\
\hline 183.2 & 19.68 & 19.53 & 19.66 & 0.662 & 0.0 \\
\hline 176.8 & 19.01 & 18.86 & 18.90 & 0.206 & 0.0 \\
\hline 165.3 & 17.75 & 17.60 & 17.54 & -0.343 & 0.0 \\
\hline 152.6 & 16.39 & 16.24 & 16.04 & -1.213 & 0.0 \\
\hline 140.3 & 15.07 & 14.90 & 14.58 & -2.160 & 0.0 \\
\hline 118.8 & 12.71 & 12.48 & 12.04 & -3.556 & \\
\hline
\end{tabular}

Number of points (Ref.80) 11

$\begin{array}{lllrlll}\text { AAD - } 8 & 1.261 & \text { BIAS }-8 & -0.061 & \text { RMS }-8 & 1.536 & \\ \text { AAD } & 0.235 & \text { BIAS } & 0.049 & \text { RMS } & 0.256 & \mathrm{~mW} \cdot \mathrm{m}^{-1} \cdot \mathrm{K}^{-1}\end{array}$


Data from Le Neindre et al. [81]

$\begin{array}{cccccc}\mathrm{T} & \lambda, \exp & \lambda_{0}, \exp & \lambda_{0}, \mathrm{cal} & \text { Dev } & \text { Wt } \\ \mathrm{K} & \mathrm{mW} \cdot \mathrm{m}^{-1} \cdot \mathrm{K}^{-1} & \mathrm{~mW} \cdot \mathrm{m}^{-1} \cdot \mathrm{K}^{-1} & \mathrm{~mW} \cdot \mathrm{m}^{-1} \cdot \mathrm{K}^{-1} & \&\end{array}$

$\begin{array}{llllll}307.35 & 35.6 & 35.53 & 35.35 & -0.498 & 0.0 \\ 298.65 & 34.5 & 34.40 & 34.13 & -0.791 & 0.0 \\ 368.85 & 45.1 & 45.04 & 44.74 & -0.678 & 1.0 \\ 407.95 & 51.4 & 51.35 & 51.33 & -0.031 & 1.0 \\ 420.25 & 53.9 & 53.83 & 53.50 & -0.628 & 1.0 \\ & & & & & \\ 465.55 & 62.3 & 62.24 & 61.75 & -0.789 & 1.0 \\ 523.35 & 73.2 & 73.15 & 72.77 & -0.520 & 1.0 \\ 571.85 & 82.8 & 82.77 & 82.25 & -0.618 & 1.0 \\ 575.75 & 84.6 & 84.57 & 83.02 & -1.826 & 1.0 \\ 621.45 & 94.0 & 93.96 & 92.05 & -2.031 & 1.0\end{array}$

Number of points (Ref.81) 10

$\begin{array}{lllllll}\text { AAD }-8 & 0.841 & \text { BIAS }-8 & -0.841 & \text { RMS }-8 & 0.582 & \\ \text { AAD } & 0.594 & \text { BIAS } & -0.594 & \text { RMS } & 0.588 & \mathrm{~mW} \cdot \mathrm{m}^{-1} \cdot \mathrm{K}^{-1}\end{array}$

\begin{tabular}{cccccc}
\hline Data from Yorizane et al. [82] & & & \\
$\mathrm{T}$ & $\lambda$, exp & $\lambda_{0}$, exp & $\lambda_{0}, \mathrm{cal}$ & Dev & Wt \\
$\mathrm{K}$ & $\mathrm{mW} \cdot \mathrm{m}^{-1} \cdot \mathrm{K}^{-1}$ & $\mathrm{~mW} \cdot \mathrm{m}^{-1} \cdot \mathrm{K}^{-1}$ & $\mathrm{~mW} \cdot \mathrm{m}^{-1} \cdot \mathrm{K}^{-1}$ & 8 & \\
323.35 & 37.7 & 37.63 & 37.67 & 0.098 & 0.0 \\
319.95 & 37.5 & 37.43 & 37.17 & -0.700 & 0.0 \\
316.75 & 37.3 & 37.23 & 36.70 & -1.418 & 0.0 \\
312.85 & 36.7 & 36.63 & 36.14 & -1.342 & 0.0 \\
309.35 & 36.3 & 36.23 & 35.64 & -1.635 & 0.0 \\
308.25 & 35.7 & 35.63 & 35.48 & -0.419 & 0.0 \\
303.15 & 35.1 & 35.03 & 34.76 & -0.771 & 0.0 \\
297.75 & 34.4 & 34.33 & 34.00 & -0.943 & 0.0 \\
303.15 & 35.1 & 35.03 & 34.76 & -0.771 & 0.0 \\
309.85 & 36.6 & 36.53 & 35.71 & -2.248 & 0.0 \\
316.75 & 37.2 & 37.13 & 36.70 & -1.153 & 0.0 \\
317.75 & 37.2 & 37.13 & 36.85 & -0.762 & 0.0 \\
323.45 & 37.7 & 37.63 & 37.69 & -0.138 & 0.0
\end{tabular}

Number of points (Ref.82) 13

$\begin{array}{lllllll}\text { AAD - } 8 & 0.954 & \text { BIAS }-8 & -0.917 & \text { RMS }-8 & 0.637 & \\ \text { AAD } & 0.347 & \text { BIAS } & -0.333 & \text { RMS } & 0.234 & \mathrm{~mW} \cdot \mathrm{m}^{-1} \cdot \mathrm{K}^{-1}\end{array}$


Data from Tanaka et al. [83]

$\begin{array}{cccccc}\mathrm{T} & \lambda, \exp & \lambda_{0}, \exp & \lambda_{0}, \mathrm{cal} & \text { Dev } & \text { Wt } \\ \mathrm{K} & \mathrm{mW} \cdot \mathrm{m}^{-1} \cdot \mathrm{K}^{-1} & \mathrm{~mW} \cdot \mathrm{m}^{-1} \cdot \mathrm{K}^{-1} & \mathrm{~mW} \cdot \mathrm{m}^{-1} \cdot \mathrm{K}^{-1} & 8 & \\ 298.15 & 33.9 & 33.83 & 34.06 & 0.685 & 0.0 \\ 323.15 & 37.4 & 37.33 & 37.64 & 0.824 & 0.0 \\ 348.15 & 41.4 & 41.34 & 41.44 & 0.236 & 0.0\end{array}$

Number of points (Ref.83) 3
$A A D-8$
0.582
BIAS - 8
0.582
$\begin{array}{ll}\text { RMS - } 8 & 0.251\end{array}$
$\mathrm{AAD}$
0.212
BIAS
0.212
RMS
$0.087 \mathrm{~mW} \cdot \mathrm{m}^{-1} \cdot \mathrm{K}^{-1}$

Number of Points Total with positive weight 21
$A A D-8$
0.839
BIAS - $8 \quad-0.112$
RMS - $8 \quad 1.025$
AAD
0.389
BIAS
$-0.176$
RMS
$0.586 \mathrm{~mW} \cdot \mathrm{m}^{-1} \cdot \mathrm{K}^{-1}$

Number of Points Total referenced in Ref.[1] 74

$\begin{array}{lllllll}\text { AAD }-8 & 1.076 & \text { BIAS }-8 & -0.449 & \text { RMS }-8 & 1.456 & \\ \text { AAD } & 0.315 & \text { BIAS } & -0.135 & \text { RMS } & 0.413 & \mathrm{~mW} \cdot \mathrm{m}^{-1} \cdot \mathrm{K}^{-1}\end{array}$

Number of Points Total

91

$\begin{array}{lllrlll}\text { AAD - } 8 & 1.241 & \text { BIAS }-8 & -0.084 & \text { RMS }-8 & 1.705 & \\ \text { AAD } & 0.424 & \text { BIAS } & 0.028 & \text { RMS } & 0.639 & \mathrm{~mW} \cdot \mathrm{m}^{-1} \cdot \mathrm{K}^{-1}\end{array}$


Notes for Table A13. The quantities $T, P$, and $\eta$,exp refer to experimental quantities; the density was evaluated from the SWEOS. For the data along the saturated liquid boundary of Boon [85] and Haynes [87], however, both the pressure and density were calculated from the ancillary equations for the phase boundary. The tabulated densities were then used as input to calculate the viscosity.

Data from Barua et al. [84]

\begin{tabular}{|c|c|c|c|c|c|c|}
\hline $\mathrm{T}$ & $\mathrm{P}$ & $\rho$ & $\eta, \exp$ & $\eta, c a l$ & Dev & Wt \\
\hline K & $\mathrm{MPa}$ & $\mathrm{mol} \cdot \mathrm{dm}^{-3}$ & $\mu \mathrm{Pa} \cdot \mathrm{s}$ & $\mu \mathrm{Pa} \cdot \mathrm{s}$ & 8 & \\
\hline 223.15 & 2.0265 & 1.2092 & 8.820 & 9.0509 & 2.618 & 0.0 \\
\hline 223.15 & 6.0795 & 4.8458 & 10.875 & 11.3032 & 3.938 & 0.0 \\
\hline 223.15 & 8.1065 & 7.8446 & 13.528 & 14.1882 & 4.880 & 0.0 \\
\hline 223.15 & 12.1590 & 13.2838 & 21.641 & 22.5027 & 3.982 & 0.0 \\
\hline 223.15 & 14.1855 & 14.6807 & 25.013 & 25.5006 & 1.949 & 0.0 \\
\hline 223.15 & 16.2120 & 15.6757 & 27.310 & 27.9289 & 2.266 & 0.0 \\
\hline 248.15 & 1.0133 & 0.5081 & 9.555 & 9.6414 & 0.904 & 0.0 \\
\hline 248.15 & 3.0398 & 1.6405 & 9.980 & 10.1550 & 1.754 & 0.0 \\
\hline 248.15 & 6.0795 & 3.7125 & 11.117 & 11.3922 & 2.476 & 0.0 \\
\hline 248.15 & 8.1060 & 5.3936 & 12.390 & 12.6976 & 2.483 & 0.0 \\
\hline 248.15 & 10.1325 & 7.2752 & 14.144 & 14.5088 & 2.579 & 0.0 \\
\hline 248.15 & 14.1855 & 10.8636 & 18.692 & 19.1512 & 2.456 & 0.0 \\
\hline 248.15 & 16.2120 & 12.2398 & 20.865 & 21.4262 & 2.690 & 0.0 \\
\hline 273.15 & 1.4196 & 0.6469 & 10.410 & 10.5567 & 1.409 & 0.0 \\
\hline 273.15 & 2.6223 & 1.2312 & 10.644 & 10.8216 & 1.669 & 0.0 \\
\hline 273.15 & 4.9426 & 2.4619 & 11.264 & 11.4766 & 1.888 & 0.0 \\
\hline 273.15 & 7.0137 & 3.6802 & 12.010 & 12.2591 & 2.074 & 0.0 \\
\hline 273.15 & 9.8903 & 5.5360 & 13.454 & 13.7217 & 1.989 & 0.0 \\
\hline 273.15 & 13.3516 & 7.8639 & 15.799 & 16.0592 & 1.647 & 0.0 \\
\hline 273.15 & 16.8899 & 10.0448 & 18.487 & 18.8258 & 1.832 & 0.0 \\
\hline 298.15 & 2.8999 & 1.2307 & 11.479 & 11.6574 & 1.554 & 0.0 \\
\hline 298.15 & 5.5455 & 2.4624 & 12.123 & 12.3235 & 1.654 & 0.0 \\
\hline 298.15 & 7.9804 & 3.6810 & 12.923 & 13.1148 & 1.484 & 0.0 \\
\hline 298.15 & 11.5004 & 5.5387 & 14.384 & 14.5871 & 1.412 & 0.0 \\
\hline 298.15 & 15.9404 & 7.8691 & 16.731 & 16.9271 & 1.172 & 0.0 \\
\hline 298.15 & 17.7552 & 8.7601 & 17.709 & 17.9814 & 1.538 & 0.0 \\
\hline 348.15 & 2.8989 & 1.0281 & 13.037 & 13.1487 & 0.857 & 0.0 \\
\hline 348.15 & 5.5648 & 2.0165 & 13.504 & 13.6689 & 1.221 & 0.0 \\
\hline 348.15 & 8.1384 & 3.0010 & 14.119 & 14.2697 & 1.067 & 0.0 \\
\hline 348.15 & 12.1185 & 4.5508 & 15.222 & 15.3883 & 1.092 & 0.0 \\
\hline
\end{tabular}


Data from Barua et al. [84] (continued)

\begin{tabular}{cr}
$T$ & \multicolumn{1}{c}{$P$} \\
$K$ & \multicolumn{1}{c}{$M P a$} \\
348.15 & 14.6800 \\
348.15 & 17.6539 \\
423.15 & 1.1703 \\
423.15 & 2.8513 \\
423.15 & 5.6793 \\
& \\
423.15 & 8.5052 \\
423.15 & 12.7163 \\
423.15 & 15.7844 \\
423.15 & 17.2253
\end{tabular}

$\begin{array}{ccccc}\rho & \eta, \exp & \eta, \mathrm{cal} & \text { Dev } & \text { Wt } \\ \mathrm{mol} \cdot \mathrm{dm}^{-3} & \mu \mathrm{Pa} \cdot \mathrm{s} & \mu \mathrm{Pa} \cdot \mathrm{s} & \text { 8 } & \\ & & & & \\ 5.5415 & 16.066 & 16.2187 & 0.950 & 0.0 \\ 6.6599 & 17.100 & 17.2700 & 0.994 & 0.0 \\ 0.3339 & 15.063 & 15.0284 & -0.230 & 0.0 \\ 0.8172 & 15.233 & 15.2521 & 0.125 & 0.0 \\ 1.6377 & 15.620 & 15.6752 & 0.354 & 0.0 \\ & & & & \\ 2.4615 & 16.055 & 16.1555 & 0.626 & 0.0 \\ 3.6816 & 16.831 & 16.9709 & 0.831 & 0.0 \\ 4.5531 & 17.496 & 17.6313 & 0.773 & 0.0 \\ 4.9545 & 17.827 & 17.9579 & 0.734 & 0.0\end{array}$

Number of points (Ref.84) 39

$\begin{array}{llllll}\text { AAD }-8 & 1.696 & \text { BIAS }-8 & 1.684 & \text { RMS }-8 & 1.026 \\ \text { AAD } & 0.255 & \text { BIAS } & 0.253 & \text { RMS } & 0.182 \mu \mathrm{Pa} \cdot \mathrm{s}\end{array}$

\begin{tabular}{ccccccc}
\hline Data from & Boon et a1. [85] & & & & & \\
$\mathrm{T}$ & $\mathrm{P}$ & $\rho$ & $\eta, \exp$ & $\eta, \mathrm{cal}$ & Dev & Wt \\
$\mathrm{K}$ & $\mathrm{MPa}$ & $\mathrm{mol} \cdot \mathrm{dm}^{-3}$ & $\mu \mathrm{Pa} \cdot \mathrm{s}$ & $\mu \mathrm{Pa} \cdot \mathrm{s}$ & 8 & \\
& & & & & & \\
91.01 & 0.0122 & 28.1199 & 199.50 & 200.3647 & 0.433 & 0.0 \\
93.77 & 0.0172 & 27.8858 & 182.50 & 185.5583 & 1.676 & 0.0 \\
97.34 & 0.0259 & 27.5809 & 162.40 & 168.4102 & 3.701 & 0.0 \\
100.88 & 0.0377 & 27.2756 & 146.70 & 153.4119 & 4.575 & 0.0 \\
103.84 & 0.0505 & 27.0175 & 138.40 & 142.2355 & 2.771 & 0.0 \\
107.05 & 0.0681 & 26.7344 & 127.20 & 131.3626 & 3.272 & 0.0 \\
109.86 & 0.0872 & 26.4835 & 120.10 & 122.7949 & 2.244 & 0.0 \\
114.15 & 0.1238 & 26.0937 & 112.30 & 111.2087 & -0.972 & 0.0
\end{tabular}

Number of points (Ref.85) 8

$\begin{array}{llllll}\text { AAD }-8 & 2.456 & \text { BIAS }-8 & 2.213 & \text { RMS }-8 & 1.689 \\ \text { AAD } & 3.554 & \text { BIAS } & 3.281 & \text { RMS } & 2.389 \mu \mathrm{Pa} \cdot \mathrm{s}\end{array}$


Data from Carmichael et a1. [71]

\begin{tabular}{|c|c|c|c|c|c|c|}
\hline $\mathrm{T}$ & $\mathbf{P}$ & $\rho$ & $\eta, \exp$ & $\eta, \mathrm{cal}$ & Dev & Wt \\
\hline K & $\mathrm{MPa}$ & $\mathrm{mol} \cdot \mathrm{dm}^{-3}$ & $\mu \mathrm{Pa} \cdot \mathrm{s}$ & $\mu \mathrm{Pa} \cdot \mathrm{s}$ & 8 & \\
\hline 277.594 & 0.1117 & 0.0485 & 10.530 & 10.4640 & -0.627 & 1.0 \\
\hline 277.594 & 6.9685 & 3.5515 & 12.447 & 12.3242 & -0.986 & 1.0 \\
\hline 277.594 & 14.1301 & 8.0623 & 16.990 & 16.4423 & -3.223 & 1.0 \\
\hline 277.594 & 14.1473 & 8.0730 & 17.008 & 16.4547 & -3.253 & 1.0 \\
\hline 277.594 & 14.1632 & 8.0828 & 16.881 & 16.4661 & -2.458 & 1.0 \\
\hline 277.594 & 27.7051 & 14.0602 & 26.417 & 25.9290 & -1.847 & 1.0 \\
\hline 277.594 & 27.7361 & 14.0691 & 26.438 & 25.9478 & -1.854 & 1.0 \\
\hline 277.594 & 27.7430 & 14.0711 & 26.436 & 25.9519 & -1.831 & 1.0 \\
\hline 277.594 & 35.6368 & 15.9423 & 30.814 & 30.3397 & -1.539 & 1.0 \\
\hline 277.594 & 35.6547 & 15.9458 & 30.770 & 30.3489 & -1.368 & 1.0 \\
\hline 277.594 & 35.6713 & 15.9491 & 30.833 & 30.3575 & -1.542 & 1.0 \\
\hline 310.928 & 0.1207 & 0.0468 & 11.685 & 11.5508 & -1.148 & 1.0 \\
\hline 310.928 & 0.1227 & 0.0476 & 11.602 & 11.5511 & -0.439 & 1.0 \\
\hline 310.928 & 0.1241 & 0.0481 & 11.615 & 11.5513 & -0.548 & 1.0 \\
\hline 310.928 & 0.1262 & 0.0489 & 11.684 & 11.5516 & -1.133 & 1.0 \\
\hline 310.928 & 0.1317 & 0.0510 & 11.609 & 11.5525 & -0.487 & 1.0 \\
\hline 310.928 & 6.2839 & 2.6594 & 12.829 & 12.8643 & 0.275 & 1.0 \\
\hline 310.928 & 10.2077 & 4.5153 & 14.318 & 14.1618 & -1.091 & 1.0 \\
\hline 310.928 & 10.2070 & 4.5149 & 14.328 & 14.1615 & -1.162 & 1.0 \\
\hline 310.928 & 13.4034 & 6.0653 & 15.587 & 15.4942 & -0.595 & 1.0 \\
\hline 310.928 & 27.4438 & 11.7304 & 22.952 & 22.6524 & -1.305 & 1.0 \\
\hline 310.928 & 27.4652 & 11.7370 & 22.924 & 22.6632 & -1.138 & 1.0 \\
\hline 310.928 & 27.4679 & 11.7378 & 22.741 & 22.6645 & -0.336 & 1.0 \\
\hline 310.928 & 27.5127 & 11.7515 & 22.697 & 22.6871 & -0.044 & 1.0 \\
\hline 310.928 & 34.5757 & 13.6235 & 26.489 & 26.0815 & -1.538 & 1.0 \\
\hline 310.928 & 34.6129 & 13.6320 & 26.454 & 26.0985 & -1.344 & 1.0 \\
\hline 310.928 & 34.6447 & 13.6393 & 26.414 & 26.1130 & -1.139 & 1.0 \\
\hline 310.928 & 35.1866 & 13.7622 & 26.436 & 26.3598 & -0.288 & 1.0 \\
\hline 310.928 & 35.2107 & 13.7676 & 26.439 & 26.3708 & -0.258 & 1.0 \\
\hline 377.594 & 0.1269 & 0.0405 & 13.631 & 13.5932 & -0.277 & 1.0 \\
\hline 377.594 & 7.0540 & 2.3327 & 14.639 & 14.7501 & 0.759 & 1.0 \\
\hline 377.594 & 7.0547 & 2.3329 & 14.657 & 14.7503 & 0.636 & 1.0 \\
\hline 377.594 & 13.8770 & 4.6575 & 16.389 & 16.3790 & -0.061 & 1.0 \\
\hline 377.594 & 14.0611 & 4.7195 & 16.368 & 16.4290 & 0.373 & 1.0 \\
\hline 377.594 & 14.0694 & 4.7223 & 16.363 & 16.4312 & 0.417 & 1.0 \\
\hline
\end{tabular}


Data from Carmichael [71] (continued)

\begin{tabular}{|c|c|c|c|c|c|c|}
\hline $\mathrm{T}$ & $P$ & $\rho$ & $\eta, \exp$ & $\eta, \mathrm{cal}$ & Dev & Wt \\
\hline K & $\mathrm{MPa}$ & $\mathrm{mol} \cdot \mathrm{dm}^{-3}$ & $\mu \mathrm{Pa} \cdot \mathrm{s}$ & $\mu \mathrm{Pa} \cdot \mathrm{s}$ & 8 & \\
\hline 377.594 & 27.6741 & 8.8847 & 20.850 & 20.6467 & -0.975 & 1.0 \\
\hline 377.594 & 27.6844 & 8.8874 & 20.940 & 20.6501 & -1.385 & 1.0 \\
\hline 377.594 & 27.6913 & 8.8893 & 20.658 & 20.6523 & -0.027 & 1.0 \\
\hline 377.594 & 27.9085 & 8.9471 & 20.743 & 20.7239 & -0.092 & 1.0 \\
\hline 377.594 & 27.9340 & 8.9539 & 20.849 & 20.7323 & -0.560 & 1.0 \\
\hline 377.594 & 27.9954 & 8.9702 & 20.776 & 20.7525 & -0.113 & 1.0 \\
\hline 377.594 & 35.0494 & 10.6868 & 23.350 & 23.0703 & -1.198 & 1.0 \\
\hline 444.261 & 0.1338 & 0.0362 & 15.597 & 15.4845 & -0.721 & 1.0 \\
\hline 444.261 & 6.9913 & 1.9112 & 16.377 & 16.4191 & 0.257 & 1.0 \\
\hline 444.261 & 6.9933 & 1.9118 & 16.357 & 16.4194 & 0.381 & 1.0 \\
\hline 444.261 & 6.9961 & 1.9126 & 16.358 & 16.4198 & 0.378 & 1.0 \\
\hline 444.261 & 14.0446 & 3.8254 & 17.589 & 17.6695 & 0.458 & 1.0 \\
\hline 444.261 & 14.0466 & 3.8259 & 17.573 & 17.6699 & 0.552 & 1.0 \\
\hline 444.261 & 28.6221 & 7.4148 & 20.976 & 20.8781 & -0.467 & 1.0 \\
\hline 444.261 & 28.6621 & 7.4236 & 20.918 & 20.8876 & -0.145 & 1.0 \\
\hline 444.261 & 28.6704 & 7.4255 & 20.915 & 20.8895 & -0.122 & 1.0 \\
\hline 444.261 & 34.8177 & 8.7200 & 22.208 & 22.3553 & 0.663 & 1.0 \\
\hline 444.261 & 34.8212 & 8.7207 & 22.172 & 22.3561 & 0.830 & 1.0 \\
\hline 444.261 & 35.2997 & 8.8158 & 22.513 & 22.4709 & -0.187 & 1.0 \\
\hline 444.261 & 35.3259 & 8.8210 & 22.499 & 22.4771 & -0.097 & 1.0 \\
\hline 444.261 & 35.3438 & 8.8245 & 22.581 & 22.4814 & -0.441 & 1.0 \\
\hline 477.594 & 0.2158 & 0.0544 & 16.543 & 16.3896 & -0.927 & 1.0 \\
\hline 477.594 & 7.0795 & 1.7857 & 17.242 & 17.2527 & 0.062 & 1.0 \\
\hline 477.594 & 7.0823 & 1.7864 & 17.253 & 17.2531 & 0.001 & 1.0 \\
\hline 477.594 & 7.0857 & 1.7873 & 17.214 & 17.2536 & 0.230 & 1.0 \\
\hline 477.594 & 14.0639 & 3.5158 & 18.338 & 18.3564 & 0.100 & 1.0 \\
\hline 477.594 & 14.0666 & 3.5165 & 18.372 & 18.3569 & -0.082 & 1.0 \\
\hline 477.594 & 27.8368 & 6.6392 & 20.909 & 20.9922 & 0.398 & 1.0 \\
\hline 477.594 & 27.8464 & 6.6412 & 20.921 & 20.9942 & 0.350 & 1.0 \\
\hline 477.594 & 27.9616 & 6.6651 & 21.023 & 21.0178 & -0.025 & 1.0 \\
\hline 477.594 & 34.9349 & 8.0423 & 22.285 & 22.4699 & 0.830 & 1.0 \\
\hline 477.594 & 34.9549 & 8.0461 & 22.296 & 22.4741 & 0.799 & 1.0 \\
\hline
\end{tabular}

Number of points (Ref.71) 67

$\begin{array}{llllll}\text { AAD }-8 & 0.764 & \text { BIAS }-8 & -0.503 & \text { RMS }-8 & 0.905 \\ \text { AAD } & 0.156 & \text { BIAS } & -0.108 & \text { RMS } & 0.193 \mu \mathrm{Pa} \cdot \mathrm{s}\end{array}$


Data from Diller [9]

\begin{tabular}{|c|c|c|c|c|c|c|}
\hline $\mathrm{T}$ & $P$ & $\rho$ & $\eta, \exp$ & $\eta, \mathrm{cal}$ & Dev & Wt \\
\hline K & $\mathrm{MPa}$ & $\mathrm{mol} \cdot \mathrm{dm}^{-3}$ & $\mu \mathrm{Pa} \cdot \mathrm{s}$ & $\mu \mathrm{Pa} \cdot \mathrm{s}$ & 8 & \\
\hline 300.0 & 27.5171 & 12.4236 & 23.08 & 23.4859 & 1.759 & 0.0 \\
\hline 300.0 & 24.6130 & 11.4741 & 21.63 & 21.8836 & 1.172 & 0.0 \\
\hline 300.0 & 21.9816 & 10.4929 & 20.11 & 20.3771 & 1.328 & 0.0 \\
\hline 300.0 & 19.8617 & 9.6115 & 18.89 & 19.1409 & 1.328 & 0.0 \\
\hline 300.0 & 17.5856 & 8.5754 & 17.60 & 17.8172 & 1.234 & 0.0 \\
\hline 300.0 & 14.2203 & 6.9008 & 16.17 & 15.9469 & -1.380 & 0.0 \\
\hline 300.0 & 10.6642 & 5.0361 & 14.10 & 14.2183 & 0.839 & 0.0 \\
\hline 300.0 & 8.5932 & 3.9579 & 13.43 & 13.3756 & -0.405 & 0.0 \\
\hline 300.0 & 6.5757 & 2.9418 & 12.57 & 12.6805 & 0.879 & 0.0 \\
\hline 300.0 & 4.4641 & 1.9307 & 12.13 & 12.0809 & -0.404 & 0.0 \\
\hline 300.0 & 2.3501 & 0.9808 & 11.67 & 11.5988 & -0.610 & 0.0 \\
\hline 250.0 & 33.1075 & 17.4623 & 33.91 & 33.8731 & -0.109 & .0 \\
\hline 250.0 & 30.2114 & 16.8947 & 32.12 & 32.1340 & 0.043 & 0.0 \\
\hline 250.0 & 26.2641 & 15.9738 & 29.56 & 29.5642 & 0.014 & 0.0 \\
\hline 250.0 & 23.4267 & 15.1618 & 27.44 & 27.5203 & 0.293 & 0.0 \\
\hline 250.0 & 19.9095 & 13.8848 & 24.55 & 24.6567 & 0.435 & 0.0 \\
\hline 250.0 & 15.6382 & 11.6497 & 20.33 & 20.4779 & 0.727 & 0.0 \\
\hline 250.0 & 14.2168 & 10.6548 & 19.30 & 18.8990 & -2.078 & 0.0 \\
\hline 250.0 & 12.8282 & 9.5433 & 17.24 & 17.3106 & 0.410 & 0.0 \\
\hline 250.0 & 11.4851 & 8.3599 & 15.87 & 15.8044 & -0.413 & 0.0 \\
\hline 250.0 & 10.0994 & 7.0840 & 14.56 & 14.3750 & -1.270 & 0.0 \\
\hline 250.0 & 8.6876 & 5.8009 & 13.12 & 13.1252 & 0.040 & 0.0 \\
\hline 250.0 & 7.2482 & 4.5728 & 12.31 & 12.0926 & -1.766 & 0.0 \\
\hline 250.0 & 5.8005 & 3.4501 & 11.36 & 11.2803 & -0.701 & 0.0 \\
\hline 250.0 & 4.5051 & 2.5444 & 10.84 & 10.7125 & -1.177 & 0.0 \\
\hline 250.0 & 3.0433 & 1.6256 & 10.57 & 10.2130 & -3.378 & 0.0 \\
\hline 212.0 & 25.9756 & 19.4383 & 40.14 & 40.0453 & -0.236 & 1.0 \\
\hline 212.0 & 24.0602 & 19.1042 & 39.18 & 38.6561 & -1.337 & 1.0 \\
\hline 212.0 & 22.0279 & 18.7101 & 37.20 & 37.1058 & -0.253 & 1.0 \\
\hline 212.0 & 19.8848 & 18.2372 & 35.20 & 35.3592 & 0.452 & 1.0 \\
\hline 212.0 & 18.0669 & 17.7740 & 33.66 & 33.7563 & 0.286 & 0.0 \\
\hline 212.0 & 17.8173 & 17.7049 & 33.45 & 33.5253 & 0.225 & 0.0 \\
\hline 212.0 & 15.8212 & 17.0886 & 31.53 & 31.5564 & 0.084 & 0.0 \\
\hline 212.0 & 15.1417 & 16.8471 & 30.36 & 30.8256 & 1.534 & 0.0 \\
\hline 212.0 & 12.5149 & 15.6714 & 27.22 & 27.5556 & 1.233 & 0.0 \\
\hline
\end{tabular}


Data from Diller [9] (continued)

\begin{tabular}{|c|c|c|c|c|c|c|}
\hline $\mathrm{T}$ & $\mathbf{P}$ & $\rho$ & $\eta, \exp$ & $\eta, c a l$ & Dev & Wt \\
\hline K & $\mathrm{MPa}$ & $\mathrm{mol} \cdot \mathrm{dm}^{-3}$ & $\mu \mathrm{Pa} \cdot \mathrm{s}$ & $\mu \mathrm{Pa} \cdot \mathrm{s}$ & 8 & \\
\hline 212.0 & 12.3572 & 15.5834 & 26.86 & 27.3283 & 1.743 & 0.0 \\
\hline 212.0 & 10.7324 & 14.4780 & 24.30 & 24.6547 & 1.460 & 0.0 \\
\hline 212.0 & 9.8615 & 13.6563 & 22.68 & 22.8642 & 0.812 & 0.0 \\
\hline 212.0 & 9.2284 & 12.8844 & 20.78 & 21.3169 & 2.584 & 0.0 \\
\hline 212.0 & 8.6517 & 11.9841 & 19.32 & 19.6613 & 1.767 & 0.0 \\
\hline 212.0 & 8.2518 & 11.2078 & 18.35 & 18.3512 & 0.006 & 0.0 \\
\hline 212.0 & 7.6827 & 9.8580 & 15.71 & 16.3074 & 3.803 & 0.0 \\
\hline 212.0 & 7.3221 & 8.9005 & 14.48 & 15.0219 & 3.742 & 0.0 \\
\hline 212.0 & 6.9239 & 7.8512 & 13.38 & 13.7564 & 2.813 & 0.0 \\
\hline 212.0 & 6.5161 & 6.8677 & 12.19 & 12.6967 & 4.156 & 0.0 \\
\hline 212.0 & 6.0626 & 5.9185 & 11.59 & 11.7826 & 1.662 & 0.0 \\
\hline 212.0 & 5.2844 & 4.6049 & 10.31 & 10.6825 & 3.613 & 0.0 \\
\hline 212.0 & 4.7580 & 3.8854 & 9.69 & 10.1567 & 4.816 & 0.0 \\
\hline 212.0 & 3.8811 & 2.8909 & 9.41 & 9.5148 & 1.114 & 0.0 \\
\hline 212.0 & 2.6830 & 1.8045 & 9.07 & 8.9211 & -1.642 & 0.0 \\
\hline 212.0 & 1.3573 & 0.8328 & 8.69 & 8.4809 & -2.406 & 0.0 \\
\hline 200.0 & 26.0666 & 20.5452 & 45.55 & 44.9282 & -1.365 & 1.0 \\
\hline 200.0 & 22.7861 & 20.0554 & 43.26 & 42.5112 & -1.731 & 1.0 \\
\hline 200.0 & 17.1539 & 18.9907 & 38.47 & 37.8847 & -1.522 & 1.0 \\
\hline 200.0 & 13.4880 & 18.0190 & 34.49 & 34.2569 & -0.676 & 1.0 \\
\hline 200.0 & 10.7512 & 16.9746 & 31.18 & 30.8498 & -1.059 & 0.0 \\
\hline 200.0 & 8.9805 & 15.9606 & 28.11 & 27.9351 & -0.622 & 0.0 \\
\hline 200.0 & 7.9300 & 15.0569 & 26.03 & 25.6097 & -1.615 & 0.0 \\
\hline 200.0 & 7.0972 & 13.9399 & 23.25 & 23.0368 & -0.917 & 0.0 \\
\hline 200.0 & 6.0940 & 10.7792 & 17.73 & 17.1987 & -2.997 & 0.0 \\
\hline 200.0 & 5.7235 & 8.3884 & 13.97 & 13.9010 & -0.494 & 0.0 \\
\hline 200.0 & 5.3374 & 6.5189 & 12.28 & 11.8645 & -3.383 & 0.0 \\
\hline 200.0 & 5.0575 & 5.6154 & 11.44 & 11.0312 & -3.573 & 0.0 \\
\hline 200.0 & 4.6874 & 4.7241 & 10.71 & 10.2986 & -3.841 & 0.0 \\
\hline 200.0 & 4.1792 & 3.8062 & 10.06 & 9.6321 & -4.253 & 0.0 \\
\hline 200.0 & 3.5092 & 2.8863 & 9.58 & 9.0494 & -5.538 & 0.0 \\
\hline 200.0 & 2.5405 & 1.8678 & 9.05 & 8.4990 & -6.088 & 0.0 \\
\hline 180.0 & 29.6172 & 22.6414 & 58.06 & 57.9165 & -0.247 & 1.0 \\
\hline 180.0 & 26.0926 & 22.2956 & 55.35 & 55.2777 & -0.131 & 1.0 \\
\hline 180.0 & 22.5630 & 21.9099 & 52.91 & 52.5713 & -0.640 & 1.0 \\
\hline
\end{tabular}


Data from Diller [9] (continued)

\begin{tabular}{|c|c|c|c|c|c|c|}
\hline $\mathrm{T}$ & $\mathbf{P}$ & $\rho$ & $\eta, \exp$ & $\eta, \mathrm{cal}$ & Dev & Wt \\
\hline K & $\mathrm{MPa}$ & $\mathrm{mol} \cdot \mathrm{dm}^{-3}$ & $\mu \mathrm{Pa} \cdot \mathrm{s}$ & $\mu \mathrm{Pa} \cdot \mathrm{s}$ & 8 & \\
\hline 180.0 & 18.9364 & 21.4591 & 50.00 & 49.6796 & -0.641 & 1.0 \\
\hline 180.0 & 18.0006 & 21.3314 & 49.05 & 48.9073 & -0.291 & 1.0 \\
\hline 180.0 & 15.5309 & 20.9659 & 47.34 & 46.7988 & -1.143 & 1.0 \\
\hline 180.0 & 14.3643 & 20.7761 & 46.07 & 45.7585 & -0.676 & 1.0 \\
\hline 180.0 & 10.9669 & 20.1355 & 42.77 & 42.4934 & -0.647 & 1.0 \\
\hline 180.0 & 6.6784 & 18.9933 & 37.64 & 37.4444 & -0.520 & 1.0 \\
\hline 180.0 & 4.7313 & 18.1821 & 34.47 & 34.3347 & -0.392 & 1.0 \\
\hline 180.0 & 3.5334 & 17.4191 & 31.78 & 31.7013 & -0.248 & 0.0 \\
\hline 170.0 & 31.3603 & 23.5728 & 65.87 & 66.1360 & 0.404 & 1.0 \\
\hline 170.0 & 28.0109 & 23.2992 & 63.50 & 63.4795 & -0.032 & 1.0 \\
\hline 170.0 & 24.6337 & 22.9996 & 61.26 & 60.7878 & -0.771 & 1.0 \\
\hline 170.0 & 21.4364 & 22.6889 & 58.20 & 58.2058 & 0.010 & 1.0 \\
\hline 170.0 & 17.7783 & 22.2923 & 55.66 & 55.1763 & -0.869 & 1.0 \\
\hline 170.0 & 14.7678 & 21.9226 & 53.31 & 52.5838 & -1.362 & 1.0 \\
\hline 170.0 & 14.6458 & 21.9066 & 53.26 & 52.4762 & -1.472 & 1.0 \\
\hline 170.0 & 11.6174 & 21.4778 & 50.26 & 49.7178 & -1.079 & 1.0 \\
\hline 170.0 & 8.5905 & 20.9691 & 47.07 & 46.7281 & -0.726 & 1.0 \\
\hline 170.0 & 5.9150 & 20.4137 & 43.65 & 43.7607 & 0.254 & 1.0 \\
\hline 170.0 & 3.2114 & 19.6654 & 40.24 & 40.1690 & -0.176 & 1.0 \\
\hline 170.0 & 2.3339 & 19.3469 & 38.78 & 38.7617 & -0.047 & 1.0 \\
\hline 170.0 & 2.3338 & 19.3468 & 38.72 & 38.7616 & 0.107 & 1.0 \\
\hline 140.0 & 32.1731 & 25.8442 & 97.32 & 99.9805 & 2.734 & 1.0 \\
\hline 140.0 & 28.6862 & 25.6534 & 93.95 & 96.0911 & 2.279 & 1.0 \\
\hline 140.0 & 25.5140 & 25.4705 & 91.10 & 92.6413 & 1.692 & 1.0 \\
\hline 140.0 & 21.9776 & 25.2547 & 88.47 & 88.8758 & 0.459 & 1.0 \\
\hline 140.0 & 18.3655 & 25.0192 & 85.03 & 85.0952 & 0.077 & 1.0 \\
\hline 140.0 & 16.7132 & 24.9056 & 82.61 & 83.3812 & 0.934 & 1.0 \\
\hline 140.0 & 14.8198 & 24.7704 & 81.70 & 81.4242 & -0.338 & 1.0 \\
\hline 140.0 & 14.3898 & 24.7389 & 81.05 & 80.9804 & -0.086 & 1.0 \\
\hline 140.0 & 11.6975 & 24.5340 & 78.46 & 78.2020 & -0.329 & 1.0 \\
\hline 140.0 & 11.5486 & 24.5223 & 78.50 & 78.0482 & -0.576 & 1.0 \\
\hline 140.0 & 8.7964 & 24.2966 & 75.33 & 75.1958 & -0.178 & 1.0 \\
\hline 140.0 & 8.1090 & 24.2375 & 75.22 & 74.4791 & -0.985 & 1.0 \\
\hline 140.0 & 5.9313 & 24.0417 & 71.93 & 72.1916 & 0.364 & 1.0 \\
\hline 140.0 & 4.6192 & 23.9169 & 71.39 & 70.7963 & -0.832 & 1.0 \\
\hline
\end{tabular}


Data from Diller [9] (continued)

\begin{tabular}{|c|c|c|c|c|c|c|}
\hline $\mathrm{T}$ & $P$ & $\rho$ & $\eta, \exp$ & $\eta$, cal & Dev & Wt \\
\hline K & $\mathrm{MPa}$ & $\mathrm{mol} \cdot \mathrm{dm}^{-3}$ & $\mu \mathrm{Pa} \cdot \mathrm{s}$ & $\mu \mathrm{Pa} \cdot \mathrm{s}$ & 8 & \\
\hline 140.0 & 3.6012 & 23.8159 & 69.54 & 69.7021 & 0.233 & 1.0 \\
\hline 140.0 & 3.5782 & 23.8136 & 69.59 & 69.6772 & 0.125 & 1.0 \\
\hline 140.0 & 1.0162 & 23.5408 & 67.74 & 66.8629 & -1.295 & 1.0 \\
\hline 140.0 & 0.6435 & 23.4986 & 66.18 & 66.4447 & 0.400 & 1.0 \\
\hline 120.0 & 29.6804 & 27.1490 & 135.66 & 139.1141 & 2.546 & 1.0 \\
\hline 120.0 & 26.0938 & 26.9908 & 132.02 & 133.5790 & 1.181 & 1.0 \\
\hline 120.0 & 22.8894 & 26.8433 & 126.93 & 128.8088 & 1.480 & 1.0 \\
\hline 120.0 & 18.5450 & 26.6331 & 121.87 & 122.5704 & 0.575 & 1.0 \\
\hline 120.0 & 14.9691 & 26.4501 & 117.36 & 117.6036 & 0.208 & 1.0 \\
\hline 120.0 & 11.8634 & 26.2826 & 112.56 & 113.3914 & 0.739 & 1.0 \\
\hline 120.0 & 10.0661 & 26.1816 & 110.98 & 110.9899 & 0.009 & 1.0 \\
\hline 120.0 & 8.2936 & 26.0789 & 109.04 & 108.6433 & -0.364 & 1.0 \\
\hline 120.0 & 6.4887 & 25.9707 & 105.48 & 106.2723 & 0.751 & 1.0 \\
\hline 120.0 & 4.7333 & 25.8617 & 103.04 & 103.9804 & 0.913 & 1.0 \\
\hline 120.0 & 3.0145 & 25.7511 & 101.69 & 101.7460 & 0.055 & 1.0 \\
\hline 120.0 & 1.1885 & 25.6289 & 99.49 & 99.3782 & -0.112 & 1.0 \\
\hline 100.0 & 31.3341 & 28.6020 & 218.49 & 220.8303 & 1.071 & 1.0 \\
\hline 100.0 & 29.1106 & 28.5266 & 215.48 & 215.6186 & 0.064 & 1.0 \\
\hline 100.0 & 27.6657 & 28.4768 & 212.13 & 212.3065 & 0.083 & 1.0 \\
\hline 100.0 & 26.8786 & 28.4494 & 210.75 & 210.5254 & -0.107 & 1.0 \\
\hline 100.0 & 25.2648 & 28.3926 & 206.80 & 206.9247 & 0.060 & 1.0 \\
\hline 100.0 & 24.0528 & 28.3494 & 205.58 & 204.2630 & -0.641 & 1.0 \\
\hline 100.0 & 23.3380 & 28.3236 & 203.68 & 202.7097 & -0.476 & 1.0 \\
\hline 100.0 & 22.0477 & 28.2767 & 201.56 & 199.9366 & -0.805 & 1.0 \\
\hline 100.0 & 20.4257 & 28.2169 & 198.40 & 196.5039 & -0.956 & 1.0 \\
\hline 100.0 & 18.5381 & 28.1460 & 193.94 & 192.5810 & -0.701 & 1.0 \\
\hline 100.0 & 16.5683 & 28.0705 & 190.05 & 188.5651 & -0.781 & 1.0 \\
\hline 100.0 & 12.5966 & 27.9134 & 181.94 & 180.6900 & -0.687 & 1.0 \\
\hline 100.0 & 9.8678 & 27.8011 & 174.37 & 175.4359 & 0.611 & 1.0 \\
\hline 100.0 & 9.4111 & 27.7820 & 175.95 & 174.5682 & -0.785 & 1.0 \\
\hline 100.0 & 7.3829 & 27.6956 & 169.50 & 170.7511 & 0.738 & 1.0 \\
\hline 100.0 & 5.8976 & 27.6310 & 169.32 & 167.9918 & -0.784 & 1.0 \\
\hline 100.0 & 4.5249 & 27.5700 & 164.74 & 165.4672 & 0.441 & 1.0 \\
\hline 100.0 & 3.3040 & 27.5149 & 161.63 & 163.2414 & 0.997 & 1.0 \\
\hline 100.0 & 2.3281 & 27.4701 & 162.62 & 161.4742 & -0.705 & 1.0 \\
\hline 100.0 & 1.7964 & 27.4455 & 159.99 & 160.5160 & 0.329 & 1.0 \\
\hline
\end{tabular}


Data from Diller et al. [9] (continued)

Number of points (Ref. 9) 141

$\begin{array}{lllrll}\text { AAD }-8 & 1.101 & \text { BIAS }-8 & -0.089 & \text { RMS }-8 & 1.598 \\ \text { AAD } & 0.489 & \text { BIAS } & 0.014 & \text { RMS } & 0.749 \mu \mathrm{Pa} \cdot \mathrm{s}\end{array}$

$\overline{\text { Data from Giddings et al. [70] }}$

\begin{tabular}{|c|c|c|c|c|c|c|}
\hline $\mathrm{T}$ & $P$ & $\rho$ & $\eta, \exp$ & $\eta, \mathrm{cal}$ & Dev & Wt \\
\hline K & $\mathrm{MPa}$ & $\mathrm{mol} \cdot \mathrm{dm}^{-3}$ & $\mu \mathrm{Pa} \cdot \mathrm{s}$ & $\mu \mathrm{Pa} \cdot \mathrm{s}$ & 8 & \\
\hline 283.15 & 0.1013 & 0.0431 & 10.67 & 10.6464 & -0.221 & 4.0 \\
\hline 283.15 & 0.6890 & 0.2969 & 10.74 & 10.7460 & 0.056 & 4.0 \\
\hline 283.15 & 1.3790 & 0.6031 & 10.86 & 10.8733 & 0.123 & 4.0 \\
\hline 283.15 & 2.0680 & 0.9180 & 10.90 & 11.0126 & 1.033 & 4.0 \\
\hline 283.15 & 2.7581 & 1.2427 & 11.14 & 11.1650 & 0.224 & 4.0 \\
\hline 283.15 & 3.4471 & 1.5766 & 11.31 & 11.3312 & 0.188 & 4.0 \\
\hline 283.15 & 4.1371 & 1.9208 & 11.48 & 11.5127 & 0.285 & 4.0 \\
\hline 283.15 & 5.5161 & 2.6383 & 11.93 & 11.9244 & -0.047 & 4.0 \\
\hline 283.15 & 6.8952 & 3.3941 & 12.37 & 12.4083 & 0.309 & 4.0 \\
\hline 283.15 & 8.6187 & 4.3867 & 13.12 & 13.1240 & 0.031 & 4.0 \\
\hline 283.15 & 10.3220 & 5.4071 & 14.08 & 13.9584 & -0.864 & 4.0 \\
\hline 283.15 & 12.0658 & 6.4699 & 14.96 & 14.9390 & -0.141 & 4.0 \\
\hline 283.15 & 13.7893 & 7.5111 & 16.00 & 16.0161 & 0.100 & 4.0 \\
\hline 283.15 & 17.3377 & 9.5126 & 18.32 & 18.4396 & 0.653 & 4.0 \\
\hline 283.15 & 20.6804 & 11.1214 & 20.70 & 20.7672 & 0.324 & 4.0 \\
\hline 283.15 & 27.5786 & 13.6062 & 25.05 & 25.1734 & 0.493 & 4.0 \\
\hline 283.15 & 34.4738 & 15.3290 & 28.80 & 28.9703 & 0.591 & 4.0 \\
\hline 283.15 & 41.3680 & 16.6170 & 32.25 & 32.3405 & 0.281 & 4.0 \\
\hline 283.15 & 48.2631 & 17.6396 & 35.50 & 35.4399 & -0.169 & 4.0 \\
\hline 283.15 & 55.1583 & 18.4868 & 38.50 & 38.3703 & -0.337 & 0.0 \\
\hline 310.93 & 0.1013 & 0.0392 & 11.62 & 11.5479 & -0.620 & 4.0 \\
\hline 310.93 & 0.6890 & 0.2693 & 11.68 & 11.6402 & -0.340 & 4.0 \\
\hline 310.93 & 1.3790 & 0.5445 & 11.79 & 11.7565 & -0.284 & 4.0 \\
\hline 310.93 & 2.0680 & 0.8249 & 11.90 & 11.8814 & -0.156 & 4.0 \\
\hline 310.93 & 2.7581 & 1.1113 & 12.00 & 12.0159 & 0.133 & 4.0 \\
\hline 310.93 & 3.4471 & 1.4029 & 12.16 & 12.1599 & -0.001 & 4.0 \\
\hline 310.93 & 4.1371 & 1.7004 & 12.31 & 12.3144 & 0.035 & 4.0 \\
\hline 310.93 & 5.5161 & 2.3108 & 12.65 & 12.6551 & 0.041 & 4.0 \\
\hline 310.93 & 6.8952 & 2.9409 & 13.03 & 13.0411 & 0.085 & 4.0 \\
\hline 310.93 & 8.6187 & 3.7515 & 13.68 & 13.5898 & -0.659 & 4.0 \\
\hline
\end{tabular}


Data from Giddings et al. [70] (continued)

\begin{tabular}{|c|c|c|c|c|c|c|}
\hline $\mathrm{T}$ & $P$ & $\rho$ & $\eta, \exp$ & $\eta, c a l$ & Dev & Wt \\
\hline $\mathrm{K}$ & $\mathrm{MPa}$ & $\mathrm{mol} \cdot \mathrm{dm}^{-3}$ & $\mu \mathrm{Pa} \cdot \mathrm{s}$ & $\mu \mathrm{Pa} \cdot \mathrm{s}$ & 8 & \\
\hline 310.93 & 10.3422 & 4.5803 & 14.22 & 14.2131 & -0.048 & 4.0 \\
\hline 310.93 & 12.0658 & 5.4174 & 14.94 & 14.9087 & -0.210 & 4.0 \\
\hline 310.93 & 13.7893 & 6.2509 & 15.71 & 15.6699 & -0.256 & 4.0 \\
\hline 310.93 & 17.3377 & 7.9075 & 17.52 & 17.3976 & -0.699 & 4.0 \\
\hline 310.93 & 20.6804 & 9.3362 & 19.30 & 19.1372 & -0.844 & 4.0 \\
\hline 310.93 & 27.5786 & 11.7715 & 22.80 & 22.7201 & -0.350 & 4.0 \\
\hline 310.93 & 34.4738 & 13.5999 & 26.10 & 26.0347 & -0.250 & 4.0 \\
\hline 310.93 & 41.3680 & 15.0082 & 29.20 & 29.0564 & -0.492 & 4.0 \\
\hline 310.93 & 48.2631 & 16.1369 & 31.80 & 31.8533 & 0.168 & 4.0 \\
\hline 310.93 & 55.1583 & 17.0733 & 34.20 & 34.4918 & 0.853 & 4.0 \\
\hline 344.26 & 0.1013 & 0.0354 & 12.69 & 12.5893 & -0.794 & 4.0 \\
\hline 344.26 & 0.6890 & 0.2423 & 12.75 & 12.6742 & -0.595 & 4.0 \\
\hline 344.26 & 1.3790 & 0.4883 & 12.84 & 12.7796 & -0.470 & 4.0 \\
\hline 344.26 & 2.0680 & 0.7370 & 12.94 & 12.8914 & -0.375 & 4.0 \\
\hline 344.26 & 2.7581 & 0.9893 & 13.05 & 13.0100 & -0.307 & 4.0 \\
\hline 344.26 & 3.4471 & 1.2442 & 13.15 & 13.1352 & -0.113 & 4.0 \\
\hline 344.26 & 4.1371 & 1.5023 & 13.28 & 13.2675 & -0.094 & 4.0 \\
\hline 344.26 & 5.5161 & 2.0262 & 13.55 & 13.5535 & 0.026 & 4.0 \\
\hline 344.26 & 6.8952 & 2.5595 & 13.86 & 13.8686 & 0.062 & 4.0 \\
\hline 344.26 & 8.6187 & 3.2362 & 14.29 & 14.3039 & 0.097 & 4.0 \\
\hline 344.26 & 10.3422 & 3.9200 & 14.79 & 14.7849 & -0.035 & 4.0 \\
\hline 344.26 & 12.0658 & 4.6059 & 15.33 & 15.3097 & -0.133 & 4.0 \\
\hline 344.26 & 13.7893 & 5.2881 & 15.89 & 15.8749 & -0.095 & 4.0 \\
\hline 344.26 & 17.3377 & 6.6588 & 17.12 & 17.1463 & 0.154 & 4.0 \\
\hline 344.26 & 20.6804 & 7.8789 & 18.42 & 18.4396 & 0.106 & 4.0 \\
\hline 344.26 & 27.5786 & 10.0969 & 21.23 & 21.2238 & -0.029 & 4.0 \\
\hline 344.26 & 34.4738 & 11.8972 & 23.94 & 23.9618 & 0.091 & 4.0 \\
\hline 344.26 & 41.3680 & 13.3519 & 26.50 & 26.5568 & 0.214 & 4.0 \\
\hline 344.26 & 48.2631 & 14.5478 & 29.10 & 29.0044 & -0.328 & 4.0 \\
\hline 344.26 & 55.1583 & 15.5526 & 31.30 & 31.3296 & 0.095 & 4.0 \\
\hline 377.59 & 0.1013 & 0.0323 & 13.70 & 13.5897 & -0.805 & 4.0 \\
\hline 377.59 & 0.6890 & 0.2204 & 13.76 & 13.6683 & -0.667 & 4.0 \\
\hline 377.59 & 1.3790 & 0.4430 & 13.84 & 13.7649 & -0.542 & 4.0 \\
\hline 377.59 & 2.0680 & 0.6671 & 13.93 & 13.8663 & -0.457 & 4.0 \\
\hline 377.59 & 2.7581 & 0.8933 & 14.03 & 13.9729 & -0.407 & 4.0 \\
\hline
\end{tabular}


Data from Giddings et al. [70] (continued)

\begin{tabular}{|c|c|c|c|c|c|c|}
\hline $\mathrm{T}$ & $\mathbf{P}$ & $\rho$ & $\eta, \exp$ & $\eta, \mathrm{cal}$ & Dev & Wt \\
\hline K & $\mathrm{MPa}$ & $\mathrm{mol} \cdot \mathrm{dm}^{-3}$ & $\mu \mathrm{Pa} \cdot \mathrm{s}$ & $\mu \mathrm{Pa} \cdot \mathrm{s}$ & 8 & \\
\hline 377.59 & 3.4471 & 1.1207 & 14.12 & 14.0842 & -0.254 & 4.0 \\
\hline 377.59 & 4.1371 & 1.3500 & 14.23 & 14.2008 & -0.205 & 4.0 \\
\hline 377.59 & 5.5161 & 1.8122 & 14.45 & 14.4490 & -0.007 & 4.0 \\
\hline 377.59 & 6.8952 & 2.2788 & 14.71 & 14.7178 & 0.053 & 4.0 \\
\hline 377.59 & 8.6187 & 2.8661 & 15.07 & 15.0822 & 0.081 & 4.0 \\
\hline 377.59 & 10.3422 & 3.4554 & 15.47 & 15.4777 & 0.050 & 4.0 \\
\hline 377.59 & 12.0658 & 4.0438 & 15.80 & 15.9027 & 0.650 & 4.0 \\
\hline 377.59 & 13.7893 & 4.6280 & 16.36 & 16.3552 & -0.029 & 4.0 \\
\hline 377.59 & 17.3377 & 5.8055 & 17.34 & 17.3621 & 0.127 & 4.0 \\
\hline 377.59 & 20.6804 & 6.8670 & 18.39 & 18.3834 & -0.036 & 4.0 \\
\hline 377.59 & 27.5786 & 8.8593 & 20.62 & 20.6153 & -0.023 & 4.0 \\
\hline 377.59 & 34.4738 & 10.5579 & 22.86 & 22.8828 & 0.100 & 4.0 \\
\hline 377.59 & 41.3680 & 11.9874 & 25.10 & 25.0967 & -0.013 & 4.0 \\
\hline 377.59 & 48.2631 & 13.1961 & 27.20 & 27.2273 & 0.100 & 4.0 \\
\hline 377.59 & 55.1583 & 14.2300 & 29.40 & 29.2743 & -0.428 & 4.0 \\
\hline 410.93 & 0.1013 & 0.0297 & 14.65 & 14.5527 & -0.664 & 4.0 \\
\hline 410.93 & 0.6890 & 0.2022 & 14.70 & 14.6258 & -0.505 & 4.0 \\
\hline 410.93 & 1.3790 & 0.4057 & 14.78 & 14.7151 & -0.439 & 4.0 \\
\hline 410.93 & 2.0680 & 0.6099 & 14.86 & 14.8081 & -0.349 & 4.0 \\
\hline 410.93 & 2.7581 & 0.8154 & 14.94 & 14.9050 & -0.234 & 4.0 \\
\hline 410.93 & 3.4471 & 1.0213 & 15.03 & 15.0056 & -0.162 & 4.0 \\
\hline 410.93 & 4.1371 & 1.2282 & 15.12 & 15.1102 & -0.065 & 4.0 \\
\hline 410.93 & 5.5161 & 1.6436 & 15.32 & 15.3307 & 0.070 & 4.0 \\
\hline 410.93 & 6.8952 & 2.0607 & 15.54 & 15.5663 & 0.170 & 4.0 \\
\hline 410.93 & 8.6187 & 2.5829 & 15.84 & 15.8818 & 0.264 & 4.0 \\
\hline 410.93 & 10.3422 & 3.1046 & 16.17 & 16.2197 & 0.308 & 4.0 \\
\hline 410.93 & 12.0658 & 3.6239 & 16.33 & 16.5790 & 1.525 & 4.0 \\
\hline 410.93 & 13.7893 & 4.1387 & 16.91 & 16.9582 & 0.285 & 4.0 \\
\hline 410.93 & 17.3377 & 5.1773 & 17.74 & 17.7943 & 0.306 & 4.0 \\
\hline 410.93 & 20.6804 & 6.1195 & 18.61 & 18.6377 & 0.149 & 4.0 \\
\hline 410.93 & 27.5786 & 7.9189 & 20.49 & 20.4876 & -0.012 & 4.0 \\
\hline 410.93 & 34.4738 & 9.5003 & 22.40 & 22.3977 & -0.010 & 4.0 \\
\hline 410.93 & 41.3680 & 10.8712 & 24.30 & 24.2988 & -0.005 & 4.0 \\
\hline 410.93 & 48.2631 & 12.0584 & 26.10 & 26.1577 & 0.221 & 4.0 \\
\hline 410.93 & 55.1583 & 13.0925 & 28.00 & 27.9639 & -0.129 & 4.0 \\
\hline
\end{tabular}

Number of points (Ref.70) 100

$\begin{array}{llllll}\text { AAD }-8 & 0.281 & \text { BIAS }-8 & -0.055 & \text { RMS }-8 & 0.386 \\ \text { AAD } & 0.049 & \text { BIAS } & -0.006 & \text { RMS } & 0.071 \mu \mathrm{Pa} \cdot \mathrm{s}\end{array}$


Data from Gonzalez et al. [86]

\begin{tabular}{|c|c|c|c|c|c|c|}
\hline $\mathrm{T}$ & $\mathbf{P}$ & $\rho$ & $\eta, \exp$ & $\eta, \mathrm{cal}$ & Dev & Wt \\
\hline K & $\mathrm{MPa}$ & $\mathrm{mol} \cdot \mathrm{dm}^{-3}$ & $\mu \mathrm{Pa} \cdot \mathrm{s}$ & $\mu \mathrm{Pa} \cdot \mathrm{s}$ & 8 & \\
\hline 310.928 & 1.3789 & 0.5444 & 12.01 & 11.7564 & -2.112 & 0.0 \\
\hline 310.928 & 2.7579 & 1.1112 & 12.10 & 12.0158 & -0.696 & 0.0 \\
\hline 310.928 & 4.1368 & 1.7002 & 12.54 & 12.3142 & -1.800 & 0.0 \\
\hline 310.928 & 5.5158 & 2.3107 & 12.76 & 12.6550 & -0.823 & 0.0 \\
\hline 310.928 & 6.8947 & 2.9407 & 13.16 & 13.0409 & -0.905 & 0.0 \\
\hline 310.928 & 10.3421 & 4.5803 & 14.46 & 14.2131 & -1.708 & 0.0 \\
\hline 310.928 & 13.7895 & 6.2511 & 15.95 & 15.6699 & -1.756 & 0.0 \\
\hline 310.928 & 20.6842 & 9.3378 & 19.56 & 19.1393 & -2.151 & 0.0 \\
\hline 310.928 & 27.5789 & 11.7717 & 23.14 & 22.7204 & -1.813 & 0.0 \\
\hline 310.928 & 34.4737 & 13.6000 & 26.65 & 26.0348 & -2.309 & 0.0 \\
\hline 310.928 & 41.3684 & 15.0083 & 29.90 & 29.0567 & -2.820 & 0.0 \\
\hline 310.928 & 55.1579 & 17.0733 & 35.47 & 34.4919 & -2.758 & 0.0 \\
\hline 344.261 & 1.3789 & 0.4882 & 13.08 & 12.7797 & -2.296 & 0.0 \\
\hline 344.261 & 2.7579 & 0.9892 & 13.28 & 13.0100 & -2.033 & 0.0 \\
\hline 344.261 & 4.1368 & 1.5022 & 13.46 & 13.2675 & -1.430 & 0.0 \\
\hline 344.261 & 5.5158 & 2.0260 & 13.73 & 13.5534 & -1.286 & 0.0 \\
\hline 344.261 & 6.8947 & 2.5593 & 14.08 & 13.8685 & -1.502 & 0.0 \\
\hline 344.261 & 10.3421 & 3.9200 & 14.90 & 14.7849 & -0.773 & 0.0 \\
\hline 344.261 & 13.7895 & 5.2882 & 15.95 & 15.8750 & -0.470 & 0.0 \\
\hline 344.261 & 20.6842 & 7.8802 & 18.68 & 18.4411 & -1.279 & 0.0 \\
\hline 344.261 & 27.5789 & 10.0969 & 21.63 & 21.2239 & -1.878 & 0.0 \\
\hline 344.261 & 34.4737 & 11.8971 & 24.46 & 23.9617 & -2.037 & 0.0 \\
\hline 344.261 & 41.3684 & 13.3519 & 27.50 & 26.5569 & -3.430 & 0.0 \\
\hline 344.261 & 55.1579 & 15.5525 & 32.22 & 31.3294 & -2.764 & 0.0 \\
\hline 377.594 & 1.3789 & 0.4430 & 14.05 & 13.7650 & -2.028 & 0.0 \\
\hline 377.594 & 2.7579 & 0.8932 & 14.22 & 13.9729 & -1.737 & 0.0 \\
\hline 377.594 & 4.1368 & 1.3499 & 14.40 & 14.2008 & -1.383 & 0.0 \\
\hline 377.594 & 5.5158 & 1.8120 & 14.63 & 14.4491 & -1.237 & 0.0 \\
\hline 377.594 & 6.8947 & 2.2786 & 14.81 & 14.7178 & -0.623 & 0.0 \\
\hline 377.594 & 13.7895 & 4.6280 & 16.46 & 16.3553 & -0.636 & 0.0 \\
\hline 377.594 & 20.6842 & 6.8680 & 18.60 & 18.3846 & -1.158 & 0.0 \\
\hline 377.594 & 27.5789 & 8.8593 & 20.64 & 20.6153 & -0.120 & 0.0 \\
\hline 377.594 & 34.4737 & 10.5577 & 23.27 & 22.8827 & -1.665 & 0.0 \\
\hline 377.594 & 41.3684 & 11.9874 & 25.56 & 25.0967 & -1.812 & 0.0 \\
\hline 377.594 & 55.1579 & 14.2298 & 30.03 & 29.2740 & -2.517 & 0.0 \\
\hline
\end{tabular}


Data from Gonzalez et al. [86] (continued)

$\begin{array}{ccccccc}\mathrm{T} & \mathrm{P} & \rho & \eta, \exp & \eta, \mathrm{ca} 1 & \text { Dev } & \text { Wt } \\ \mathrm{K} & \mathrm{MPa} & \mathrm{mol} \cdot \mathrm{dm}^{-3} & \mu \mathrm{Pa} \cdot \mathrm{s} & \mu \mathrm{Pa} \cdot \mathrm{s} & \text { o } & \\ 410.928 & 1.3789 & 0.4057 & 15.09 & 14.7150 & -2.485 & 0.0 \\ 410.928 & 2.7579 & 0.8153 & 15.26 & 14.9049 & -2.327 & 0.0 \\ 410.928 & 4.1368 & 1.2281 & 15.34 & 15.1101 & -1.499 & 0.0 \\ 410.928 & 5.5158 & 1.6435 & 15.50 & 15.3306 & -1.093 & 0.0 \\ 410.928 & 6.8947 & 2.0605 & 15.66 & 15.5662 & -0.599 & 0.0 \\ 410.928 & 10.3421 & 3.1046 & 16.33 & 16.2197 & -0.676 & 0.0 \\ 410.928 & 13.7895 & 4.1388 & 17.02 & 16.9582 & -0.363 & 0.0 \\ 410.928 & 20.6842 & 6.1206 & 18.82 & 18.6386 & -0.964 & 0.0 \\ 410.928 & 34.4737 & 9.5003 & 22.83 & 22.3976 & -1.894 & 0.0 \\ 410.928 & 41.3684 & 10.8713 & 24.86 & 24.2989 & -2.257 & 0.0 \\ & & & & & & \\ 410.928 & 55.1579 & 13.0925 & 29.08 & 27.9638 & -3.838 & 0.0 \\ 444.261 & 1.3789 & 0.3744 & 16.00 & 15.6322 & -2.299 & 0.0 \\ 444.261 & 4.1368 & 1.1280 & 16.37 & 15.9943 & -2.295 & 0.0 \\ 444.261 & 6.8947 & 1.8847 & 16.72 & 16.4039 & -1.891 & 0.0 \\ 444.261 & 13.7895 & 3.7574 & 17.85 & 17.6198 & -1.289 & 0.0 \\ 444.261 & 20.6842 & 5.5417 & 19.33 & 19.0567 & -1.414 & 0.0 \\ 444.261 & 27.5789 & 7.1813 & 20.92 & 20.6325 & -1.374 & 0.0 \\ 444.261 & 34.4737 & 8.6512 & 22.63 & 22.2728 & -1.579 & 0.0\end{array}$

Number of points (Ref.86) 53

$\begin{array}{llllll}\text { AAD }-8 & 1.658 & \text { BIAS }-\frac{8}{8} & -1.658 & \text { RMS }-\frac{8}{8} & 0.768 \\ \text { AAD } & 0.335 & \text { BIAS } & -0.335 & \text { RMS } & 0.248 \mu \mathrm{Pa} \cdot \mathrm{s}\end{array}$

$\overline{\text { Data from Haynes [87] }}$

$\begin{array}{rcccccc}\mathrm{T} & \mathrm{P} & \rho & \eta, \exp & \eta, \mathrm{cal} & \text { Dev } & \text { Wt } \\ \mathrm{K} & \mathrm{MPa} & \mathrm{mol} \cdot \mathrm{dm}^{-3} & \mu \mathrm{Pa} \cdot \mathrm{s} & \mu \mathrm{Pa} \cdot \mathrm{s} & 8 & \\ & & & & & & \\ 95.0 & 0.01983 & 27.78109 & 178.44724 & 179.40555 & 0.5370 & 2.0 \\ 100.0 & 0.03441 & 27.35180 & 156.97692 & 156.96669 & -0.0065 & 2.0 \\ 105.0 & 0.05643 & 26.91566 & 138.38713 & 138.16422 & -0.1611 & 2.0 \\ 110.0 & 0.08820 & 26.47086 & 122.58596 & 122.38943 & -0.1603 & 2.0 \\ 115.0 & 0.13232 & 26.01545 & 109.07683 & 109.10539 & 0.0262 & 2.0\end{array}$


Data from Haynes [87] (continued)

\begin{tabular}{ccccccc}
$\mathrm{T}$ & $\mathrm{P}$ & $\rho$ & $\eta, \exp$ & $\eta, \mathrm{cal}$ & $\mathrm{Dev}$ & Wt \\
$\mathrm{K}$ & $\mathrm{MPa}$ & $\mathrm{mol} \cdot \mathrm{dm}^{-3}$ & $\mu \mathrm{Pa} \cdot \mathrm{s}$ & $\mu \mathrm{Pa} \cdot \mathrm{s}$ & \multicolumn{1}{c}{} \\
& & & & & \\
120.0 & 0.19158 & 25.54730 & 97.56533 & 97.85224 & 0.2941 & 2.0 \\
125.0 & 0.26896 & 25.06409 & 88.35418 & 88.24503 & -0.1235 & 2.0 \\
130.0 & 0.36760 & 24.56319 & 81.04580 & 79.96689 & -1.3312 & 2.0 \\
135.0 & 0.49072 & 24.04154 & 73.34020 & 72.75987 & -0.7913 & 2.0 \\
140.0 & 0.64165 & 23.49559 & 67.33754 & 66.41512 & -1.3699 & 2.0 \\
& & & & & & \\
145.0 & 0.82379 & 22.92098 & 61.23474 & 60.76358 & -0.7694 & 2.0 \\
150.0 & 1.04065 & 22.31228 & 55.73642 & 55.66745 & -0.1238 & 2.0 \\
155.0 & 1.29580 & 21.66242 & 51.43842 & 51.01256 & -0.8279 & 2.0 \\
160.0 & 1.59296 & 20.96180 & 46.54146 & 46.70134 & 0.3435 & 2.0 \\
165.0 & 1.93607 & 20.19664 & 41.94595 & 42.64546 & 1.6677 & 2.0 \\
& & & & & & \\
170.0 & 2.32936 & 19.34572 & 37.64927 & 38.75676 & 2.9416 & 2.0 \\
175.0 & 2.77762 & 18.37296 & 34.35499 & 34.93156 & 1.6783 & 2.0 \\
180.0 & 3.28655 & 17.20702 & 30.95908 & 31.01327 & 0.1750 & 2.0 \\
185.0 & 3.86361 & 15.66142 & 26.15027 & 26.65399 & 1.9263 & 2.0 \\
190.0 & 4.52082 & 12.49998 & 19.76671 & 19.75384 & -0.0651 & 2.0
\end{tabular}

Number of points (Ref.87) 20

$\begin{array}{lllllll}\text { AAD }-8 & 0.766 & \text { BIAS }-8 & 0.193 & \text { RMS }-8 & 1.081 \\ \text { AAD } & 0.424 & \text { BIAS } & 0.014 & \text { RMS } & 0.556 \mu \mathrm{Pa} \cdot s\end{array}$

\begin{tabular}{ccccccc}
\hline Data from Hellemans et al. $[88]$ & & & & \\
$\mathrm{T}$ & $\mathrm{P}$ & $\rho$ & $\eta, \exp$ & $\eta, \mathrm{cal}$ & Dev & Wt \\
& $\mathrm{MPa}$ & $\mathrm{mol} \cdot \mathrm{dm}^{-3}$ & $\mu \mathrm{Pa} \cdot \mathrm{s}$ & $\mu \mathrm{Pa} \cdot \mathrm{s}$ & o & \\
$\mathrm{K}$ & & & & & & \\
96.8 & 0.0405 & 27.6352 & 169.0 & 171.2001 & 1.302 & 0.0 \\
96.8 & 0.2837 & 27.6462 & 170.0 & 171.6593 & 0.976 & 0.0 \\
96.8 & 1.9657 & 27.7211 & 173.0 & 174.8539 & 1.072 & 0.0 \\
96.8 & 1.8238 & 27.7149 & 180.0 & 174.5834 & -3.009 & 0.0 \\
96.8 & 5.8262 & 27.8869 & 180.0 & 182.3155 & 1.286 & 0.0 \\
96.8 & 7.8122 & 27.9690 & 191.0 & 186.2324 & -2.496 & 0.0 \\
96.8 & 9.3523 & 28.0314 & 188.0 & 189.3108 & 0.697 & 0.0 \\
106.0 & 0.0808 & 26.8426 & 133.0 & 135.2417 & 1.685 & 0.0 \\
106.0 & 1.9252 & 26.9391 & 135.0 & 138.2104 & 2.378 & 0.0 \\
106.0 & 4.0327 & 27.0458 & 138.0 & 141.6371 & 2.636 & 0.0
\end{tabular}


Data from Hellemans et al. [88] (continued)

\begin{tabular}{|c|c|c|c|c|c|c|}
\hline $\mathbf{T}$ & $P$ & $\rho$ & $\eta, \exp$ & $\eta, c a l$ & Dev & Wt \\
\hline K & $\mathrm{MPa}$ & $\mathrm{mol} \cdot \mathrm{dm}^{-3}$ & $\mu \mathrm{Pa} \cdot \mathrm{s}$ & $\mu \mathrm{Pa} \cdot \mathrm{s}$ & 8 & \\
\hline 106.0 & 5.8465 & 27.1349 & 141.0 & 144.6196 & 2.567 & 0.0 \\
\hline 106.0 & 7.9439 & 27.2349 & 145.0 & 148.1123 & 2.146 & 0.0 \\
\hline 106.0 & 9.5448 & 27.3093 & 149.0 & 150.8128 & 1.217 & 0.0 \\
\hline 115.3 & 0.1517 & 26.0031 & 107.0 & 108.7093 & 1.597 & 0.0 \\
\hline 115.3 & 2.1380 & 26.1265 & 110.0 & 111.4579 & 1.325 & 0.0 \\
\hline 115.3 & 4.0023 & 26.2380 & 112.0 & 114.0509 & 1.831 & 0.0 \\
\hline 115.3 & 6.0592 & 26.3565 & 117.0 & 116.9325 & -0.058 & 0.0 \\
\hline 115.3 & 7.8122 & 26.4540 & 119.0 & 119.4099 & 0.344 & 0.0 \\
\hline 115.3 & 0.1224 & 26.5783 & 123.0 & 122.7114 & -0.235 & 0.0 \\
\hline 125.0 & 0.2736 & 25.0747 & 90.0 & 88.4116 & -1.765 & 0.0 \\
\hline 125.0 & 3.8098 & 25.3383 & 93.6 & 92.7271 & -0.933 & 0.0 \\
\hline 125.0 & 6.1504 & 25.5004 & 95.8 & 95.5786 & -0.231 & 0.0 \\
\hline 125.0 & 7.7108 & 25.6039 & 100.7 & 97.4845 & -3.193 & 0.0 \\
\hline 125.0 & 9.8083 & 25.7378 & 98.3 & 100.0576 & 1.788 & 0.0 \\
\hline 138.7 & 0.6181 & 23.6455 & 69.5 & 68.0467 & -2.091 & 0.0 \\
\hline 138.7 & 1.9252 & 23.7854 & 71.2 & 69.5065 & -2.378 & 0.0 \\
\hline 138.7 & 3.9415 & 23.9875 & 73.6 & 71.7128 & -2.564 & 0.0 \\
\hline 138.7 & 5.8465 & 24.1657 & 76.5 & 73.7595 & -3.582 & 0.0 \\
\hline 138.7 & 7.9540 & 24.3504 & 78.8 & 75.9945 & -3.560 & 0.0 \\
\hline 138.7 & 9.4435 & 24.4743 & 80.2 & 77.5619 & -3.289 & 0.0 \\
\hline 145.6 & 0.8613 & 22.8527 & 63.5 & 60.1486 & -5.278 & 0.0 \\
\hline 145.6 & 1.1146 & 22.8866 & 63.7 & 60.4347 & -5.126 & 0.0 \\
\hline 145.6 & 1.9961 & 23.0012 & 66.0 & 61.4168 & -6.944 & 0.0 \\
\hline 145.6 & 3.9821 & 23.2416 & 68.3 & 63.5643 & -6.934 & 0.0 \\
\hline 145.6 & 5.8465 & 23.4487 & 71.3 & 65.5170 & -8.111 & 0.0 \\
\hline 145.6 & 7.7919 & 23.6491 & 72.5 & 67.5067 & -6.887 & 0.0 \\
\hline 145.6 & 9.5955 & 23.8230 & 75.8 & 69.3193 & -8.550 & 0.0 \\
\hline 156.3 & 1.3780 & 21.4872 & 48.8 & 49.8724 & 2.198 & 0.0 \\
\hline 156.3 & 2.5129 & 21.7009 & 49.1 & 51.2355 & 4.349 & 0.0 \\
\hline 156.3 & 4.0530 & 21.9621 & 51.5 & 52.9799 & 2.874 & 0.0 \\
\hline 156.3 & 6.0187 & 22.2588 & 52.1 & 55.0765 & 5.713 & 0.0 \\
\hline 156.3 & 8.0959 & 22.5388 & 51.5 & 57.1789 & 11.027 & 0.0 \\
\hline 156.3 & 9.7069 & 22.7374 & 53.3 & 58.7506 & 10.226 & 0.0 \\
\hline 171.4 & 2.4619 & 19.0923 & 35.5 & 37.7034 & 6.207 & 0.0 \\
\hline 171.4 & 3.9314 & 19.6399 & 36.8 & 40.0699 & 8.886 & 0.0 \\
\hline
\end{tabular}


Data from Hellemans et al. [88] (continued)

\begin{tabular}{ccccccc}
$\mathrm{T}$ & $\mathrm{P}$ & $\rho$ & $\eta, \exp$ & $\eta, \mathrm{ca} 1$ & Dev & Wt \\
$\mathrm{K}$ & $\mathrm{MPa}$ & $\mathrm{mol} \cdot \mathrm{dm}^{-3}$ & $\mu \mathrm{Pa} \cdot \mathrm{s}$ & $\mu \mathrm{Pa} \cdot \mathrm{s}$ & \multicolumn{1}{c}{} \\
171.4 & 5.9072 & 20.1983 & 38.1 & 42.6942 & 12.058 & 0.0 \\
171.4 & 7.6906 & 20.6046 & 39.6 & 44.7578 & 13.025 & 0.0 \\
171.4 & 9.6360 & 20.9816 & 40.2 & 46.8052 & 16.431 & 0.0 \\
183.0 & 3.6477 & 16.3848 & 27.0 & 28.5960 & 5.911 & 0.0 \\
183.0 & 5.5222 & 17.8916 & 28.4 & 33.3737 & 17.513 & 0.0 \\
183.0 & 7.7716 & 18.8385 & 29.9 & 36.8849 & 23.361 & 0.0 \\
183.0 & 9.9602 & 19.4847 & 30.7 & 39.5636 & 28.872 & 0.0 \\
187.3 & 4.1645 & 14.7229 & 22.5 & 24.3686 & 8.305 & 0.0 \\
187.3 & 5.8870 & 17.0254 & 23.7 & 30.6393 & 29.280 & 0.0 \\
187.3 & 7.8628 & 18.0876 & 23.9 & 34.1682 & 42.963 & 0.0 \\
187.3 & 9.7069 & 18.7569 & 25.3 & 36.6581 & 44.894 & 0.0
\end{tabular}

Number of points (Ref.88) 56

$\begin{array}{llllll}\text { AAD }-q & 7.074 & \text { BIAS }-8 & 4.317 & \text { RMS }-q & 11.118 \\ \text { AAD } & 3.405 & \text { BIAS } & 1.160 & \text { RMS } & 4.006 \mu \mathrm{Pa} \cdot \mathrm{s}\end{array}$

$\overline{\text { Data from Huang et a1. [89] }}$

$\begin{array}{ccccccc}\mathrm{T} & \mathrm{P} & \rho & \eta, \exp & \eta, \mathrm{cal} & \text { Dev } & \text { Wt } \\ \mathrm{K} & \mathrm{MPa} & \mathrm{mol} \cdot \mathrm{dm}^{-3} & \mu \mathrm{Pa} \cdot \mathrm{s} & \mu \mathrm{Pa} \cdot \mathrm{s} & \text { 8 } & \\ 173.15 & 2.6067 & 18.7496 & 38.5 & 36.3444 & -5.599 & 0.0 \\ 173.15 & 4.0279 & 19.3416 & 40.5 & 38.7816 & -4.243 & 0.0 \\ 173.15 & 6.7132 & 20.1296 & 43.8 & 42.3765 & -3.250 & 0.0 \\ 173.15 & 10.0699 & 20.8403 & 47.7 & 46.0341 & -3.492 & 0.0 \\ 173.15 & 13.4265 & 21.3968 & 51.2 & 49.2358 & -3.836 & 0.0 \\ & & & & & & \\ 173.15 & 20.1397 & 22.2632 & 57.3 & 54.9746 & -4.058 & 0.0 \\ 173.15 & 26.8529 & 22.9416 & 62.7 & 60.3075 & -3.816 & 0.0 \\ 173.15 & 33.5662 & 23.5072 & 67.6 & 65.5140 & -3.086 & 0.0 \\ 153.15 & 1.1975 & 21.9082 & 54.2 & 52.6900 & -2.786 & 0.0 \\ 153.15 & 4.0279 & 22.3571 & 57.0 & 55.8884 & -1.950 & 0.0 \\ 153.15 & & & & & & \\ 153.15 & 10.0699 & 23.1097 & 63.1 & 62.0007 & -1.742 & 0.0 \\ 153.15 & 13.4265 & 23.4536 & 66.4 & 65.1794 & -1.838 & 0.0 \\ 153.15 & 20.1397 & 24.0428 & 72.7 & 71.3503 & -1.857 & 0.0 \\ 153.15 & 26.8529 & 24.5415 & 78.8 & 77.4837 & -1.670 & 0.0\end{array}$


Data from Huang et al. [89] (continued)

\begin{tabular}{|c|c|c|c|c|c|c|}
\hline $\mathrm{T}$ & $P$ & $\rho$ & $\eta, \exp$ & $\eta$, cal & Dev & Wt \\
\hline K & $\mathrm{MPa}$ & $\mathrm{mol} \cdot \mathrm{dm}^{-3}$ & $\mu \mathrm{Pa} \cdot \mathrm{s}$ & $\mu \mathrm{Pa} \cdot \mathrm{s}$ & 8 & \\
\hline 153.15 & 33.5662 & 24.9774 & 84.7 & 83.7509 & -1.121 & \\
\hline 133.15 & 0.4426 & 24.2432 & 76.7 & 75.3883 & -1.710 & 0 \\
\hline 133.15 & 4.0279 & 24.5639 & 81.0 & 79.4553 & -1.907 & 0 . \\
\hline 133.15 & 6.7132 & 24.7821 & 84.0 & 82.4523 & -1.842 & 0 \\
\hline 133.15 & 10.0699 & 25.0340 & 87.8 & 86.1768 & -1.849 & 0.0 \\
\hline 133.15 & 13.4265 & 25.2667 & 91.7 & 89.9079 & -1.954 & \\
\hline 133.15 & 20.1397 & 25.6872 & 99.0 & 97.4929 & -1.522 & \\
\hline 133.15 & 26.8529 & 26.0613 & 106.0 & 105.3840 & -0.581 & \\
\hline 133.15 & 33.5662 & 26.4001 & 114.0 & 113.7365 & -0.231 & \\
\hline 123.15 & 0.2384 & 25.2560 & 94.0 & 91.8162 & -2.323 & \\
\hline 123.15 & 4.0279 & 25.5268 & 99.0 & 96.5397 & -2.485 & \\
\hline 123.15 & 6.7132 & 25.7047 & 102.0 & 99.8924 & -2.066 & \\
\hline 123.15 & 10.0699 & 25.9135 & 107.0 & 104.1160 & -2.695 & \\
\hline 123.15 & 13.4265 & 26.1094 & 111.0 & 108.3998 & -2.342 & \\
\hline 123.15 & 20.1397 & 26.4698 & 120.0 & 117.2396 & -2.300 & 0 \\
\hline 123.15 & 26.8529 & 26.7963 & 129.0 & 126.5817 & -1.875 & \\
\hline 123.15 & 33.5662 & 27.0959 & 137.0 & 136.5954 & -0.295 & \\
\hline 113.15 & 0.1151 & 26.2001 & 114.0 & 114.1058 & 0.093 & \\
\hline 113.15 & 4.0279 & 26.4292 & 119.0 & 119.7182 & 0.604 & \\
\hline 113.15 & 6.7132 & 26.5772 & 123.0 & 123.6223 & 0.506 & \\
\hline 113.15 & 10.0699 & 26.7530 & 128.0 & 128.5843 & 0.456 & \\
\hline 113.15 & 13.4265 & 26.9200 & 133.0 & 133.6591 & 0.496 & \\
\hline 113.15 & 20.1397 & 27.2312 & 142.0 & 144.2348 & 1.574 & \\
\hline 113.15 & 26.8529 & 27.5175 & 152.0 & 155.5272 & 2.321 & 0 . \\
\hline 113.15 & 33.5662 & 27.7832 & 162.0 & 167.7264 & 3.535 & \\
\hline 103.15 & 0.0479 & 27.0909 & 144.0 & 145.1668 & 0.810 & \\
\hline 103.15 & 4.0279 & 27.2857 & 151.0 & 151.9432 & 0.625 & \\
\hline 103.15 & 6.7132 & 27.4107 & 156.0 & 156.6087 & 0.390 & 0 \\
\hline 103.15 & 10.0699 & 27.5607 & 162.0 & 162.5659 & 0.349 & 0 \\
\hline 103.15 & 13.4265 & 27.7043 & 168.0 & 168.6842 & 0.407 & $0 . c$ \\
\hline 103.15 & 20.1397 & 27.9751 & 181.0 & 181.4927 & 0.272 & \\
\hline 103.15 & 26.8529 & 28.2271 & 193.0 & 195.2203 & 1.150 & \\
\hline 103.15 & 33.5662 & 28.4633 & 206.0 & 210.0740 & 1.978 & 0. \\
\hline
\end{tabular}

Number of points (Ref.89) 48

$\begin{array}{llllll}\text { AAD }-8 & 1.873 & \text { BIAS }-8 & -1.225 & \text { RMS }-8 & 1.885 \\ \text { AAD } & 1.635 & \text { BIAS } & -0.596 & \text { RMS } & 1.843 \mu \mathrm{Pa} \cdot \mathrm{s}\end{array}$


Data from Kestin and Yata [65]

$\begin{array}{ccccccc}\mathrm{T} & \mathrm{P} & \rho & \eta, \exp & \eta, \mathrm{cal} & \text { Dev } & \text { Wt } \\ \mathrm{K} & \mathrm{MPa} & \mathrm{mol} \cdot \mathrm{dm}^{-3} & \mu \mathrm{Pa} \cdot \mathrm{s} & \mu \mathrm{Pa} \cdot \mathrm{s} & 8 & \\ 293.15 & 2.5524 & 1.0984 & 11.321 & 11.4289 & 0.953 & 0.0 \\ 293.15 & 2.0255 & 0.8631 & 11.217 & 11.3196 & 0.914 & 0.0 \\ 293.15 & 1.5219 & 0.6424 & 11.137 & 11.2213 & 0.757 & 0.0 \\ 293.15 & 1.0153 & 0.4245 & 11.054 & 11.1283 & 0.672 & 0.0 \\ 293.15 & 0.5087 & 0.2107 & 10.986 & 11.0409 & 0.500 & 0.0 \\ & & & & & & \\ 293.15 & 0.1064 & 0.0437 & 10.936 & 10.9754 & 0.360 & 0.0 \\ 293.15 & 0.0500 & 0.0205 & 10.924 & 10.9664 & 0.388 & 0.0 \\ 303.15 & 2.2443 & 0.9238 & 11.590 & 11.6751 & 0.734 & 0.0 \\ 303.15 & 1.9221 & 0.7870 & 11.526 & 11.6125 & 0.750 & 0.0 \\ 303.15 & 1.5280 & 0.6216 & 11.462 & 11.5389 & 0.671 & 0.0 \\ 303.15 & 1.0072 & 0.4063 & 11.387 & 11.4466 & 0.524 & 0.0 \\ 303.15 & 0.5147 & 0.2059 & 11.318 & 11.3642 & 0.408 & 0.0 \\ 303.15 & 0.5137 & 0.2055 & 11.318 & 11.3641 & 0.407 & 0.0 \\ 303.15 & 0.5066 & 0.2027 & 11.322 & 11.3629 & 0.361 & 0.0 \\ 303.15 & 0.1155 & 0.0459 & 11.276 & 11.3008 & 0.220 & 0.0 \\ 303.15 & 0.1125 & 0.0447 & 11.268 & 11.3003 & 0.287 & 0.0 \\ 303.15 & 0.1054 & 0.0419 & 11.267 & 11.2992 & 0.286 & 0.0 \\ 303.15 & 0.0500 & 0.0199 & 11.258 & 11.2907 & 0.290 & 0.0\end{array}$

Number of points (Ref.65) 18
AAD - $8 \quad 0.527$
AAD $\quad 0.059$
BIAS - 8
0.527
RMS - \&
0.222
BIAS
0.059 RMS
$0.025 \mu \mathrm{Pa} \cdot \mathrm{s}$

Number of Points Total with positive weight 267

$\begin{array}{llllll}\text { AAD }-8 & 0.559 & \text { BIAS }-8 & -0.161 & \text { RMS }-8 & 0.791 \\ \text { AAD } & 0.292 & \text { BIAS } & -0.025 & \text { RMS } & 0.559 \mu \mathrm{Pa} \cdot \mathrm{s}\end{array}$

Number of Points Total referenced in Ref. [1] 532

$\begin{array}{lllllll}\text { AAD }-8 & 1.710 & \text { BIAS }-8 & 0.246 & \text { RMS }-8 & 4.122 \\ \text { AAD } & 0.786 & \text { BIAS } & 0.092 & \text { RMS } & 1.613 \mu \mathrm{Pa} \cdot \mathrm{s}\end{array}$

Number of Points Total

550

$\begin{array}{lllllll}\text { AAD }-8 & 1.672 & \text { BIAS }-8 & 0.255 & \text { RMS }-8 & 4.055 \\ \text { AAD } & 0.762 & \text { BIAS } & 0.092 & \text { RMS } & 1.586 \mu \mathrm{Pa} \cdot \mathrm{s}\end{array}$


Notes for Table A14. The quantities T, P, and $\lambda$,exp refer to experimental quantities; the density was evaluated from the SWEOS. The tabulated densities were then used as input to calculate the thermal conductivity.

Data from Assael and Wakeham [73]

$\begin{array}{ccccccc}\mathrm{T} & \mathrm{P} & \rho & \lambda, \exp & \lambda, \mathrm{cal} & \text { Dev } & \text { Wt } \\ \mathrm{K} & \mathrm{MPa} & \mathrm{mol} \cdot \mathrm{dm}^{-3} & \mathrm{~mW} \cdot \mathrm{m}^{-1} \cdot \mathrm{K}^{-1} & 8\end{array}$

$\begin{array}{lllllll}307.48 & 1.84 & 0.741 & 36.45 & 36.60 & 0.412 & 0.0 \\ 307.27 & 2.60 & 1.060 & 37.01 & 37.14 & 0.351 & 0.0 \\ 307.16 & 3.31 & 1.364 & 37.62 & 37.68 & 0.159 & 0.0 \\ 307.03 & 3.93 & 1.636 & 38.09 & 38.19 & 0.263 & 0.0 \\ 306.96 & 4.49 & 1.885 & 38.62 & 38.67 & 0.129 & 0.0 \\ 306.82 & & & & & & \\ 306.73 & 5.06 & 2.144 & 39.22 & 39.19 & -0.076 & 0.0 \\ 306.62 & 5.79 & 2.480 & 39.99 & 39.90 & -0.225 & 0.0 \\ 306.58 & 6.48 & 2.804 & 40.78 & 40.61 & -0.417 & 0.0 \\ 306.87 & 7.12 & 3.109 & 41.53 & 41.31 & -0.530 & 0.0 \\ 306.73 & 7.81 & 3.436 & 42.71 & 42.15 & -1.311 & 0.0 \\ 306.66 & 8.36 & 3.706 & 43.39 & 42.81 & -1.337 & 0.0 \\ 306.52 & 8.79 & 3.918 & 43.99 & 43.35 & -1.455 & 0.0 \\ & 9.27 & 4.157 & 44.65 & 43.97 & -1.523 & 0.0\end{array}$

Number of Points (Ref. 73) 13

$\begin{array}{llllll}\text { AAD }-8 & 0.630 & \text { BIAS }-8 & -0.428 & \text { RMS }-8 & 0.706 \\ \text { AAD } & 0.266 & \text { BIAS } & -0.191 & \text { RMS } & 0.303 \mathrm{~mW} \cdot \mathrm{m}^{-1} \cdot \mathrm{K}^{-1}\end{array}$

Data from Clifford et al. [72]

$\begin{array}{ccccccc}\mathrm{T} & \mathrm{P} & \rho & \lambda, \exp & \lambda, \mathrm{cal} & \text { Dev } & \text { Wt } \\ \mathrm{K} & \mathrm{MPa} & \mathrm{mol} \cdot \mathrm{dm}^{-3} & \mathrm{~mW} \cdot \mathrm{m}^{-1} \cdot \mathrm{K}^{-1} & & \\ 301.10 & 1.61 & 0.661 & 35.85 & 35.57 & -0.781 & 0.0 \\ 300.98 & 2.10 & 0.869 & 36.06 & 35.91 & -0.416 & 0.0 \\ 300.90 & 2.36 & 0.982 & 36.35 & 36.10 & -0.688 & 0.0 \\ 300.89 & 3.06 & 1.288 & 36.98 & 36.66 & -0.865 & 0.0 \\ 300.64 & 3.60 & 1.531 & 37.29 & 37.09 & -0.536 & 0.0\end{array}$


Data from Clifford et al. [72] (continued)

\begin{tabular}{|c|c|c|c|c|c|c|}
\hline $\mathrm{T}$ & $P$ & $\rho$ & $\lambda, \exp$ & $\lambda, \mathrm{cal}$ & Dev & Wt \\
\hline K & $\mathrm{MPa}$ & $\mathrm{mol} \cdot \mathrm{dm}^{-3}$ & \multicolumn{2}{|c|}{$\mathrm{mW} \cdot \mathrm{m}^{-1} \cdot \mathrm{K}^{-1}$} & 8 & \\
\hline 300.54 & 4.08 & 1.749 & 37.72 & 37.50 & -0.583 & 0.0 \\
\hline 300.35 & 4.56 & 1.972 & 38.22 & 37.93 & -0.759 & 0.0 \\
\hline 300.30 & 5.04 & 2.198 & 38.73 & 38.39 & -0.878 & 0.0 \\
\hline 300.15 & 5.87 & 2.595 & 39.67 & 39.24 & -1.084 & 0.0 \\
\hline 300.05 & 6.58 & 2.943 & 40.39 & 40.03 & -0.891 & 0.0 \\
\hline 299.95 & 7.42 & 3.363 & 41.57 & 41.02 & -1.323 & .0 \\
\hline 299.85 & 8.11 & 3.714 & 42.90 & 41.89 & -2.354 & 0.0 \\
\hline 299.73 & 9.18 & 4.267 & 44.00 & 43.35 & -1.477 & 0.0 \\
\hline 299.61 & 10.18 & 4.793 & 45.58 & 44.82 & -1.667 & 0.0 \\
\hline 299.49 & 11.15 & 5.308 & 47.04 & 46.33 & -1.509 & 0.0 \\
\hline 299.40 & 12.13 & 5.830 & 48.68 & 47.94 & -1.520 & .0 \\
\hline 299.32 & 13.08 & 6.334 & 50.26 & 49.55 & -1.413 & 0.0 \\
\hline 299.16 & 14.12 & 6.886 & 51.84 & 51.38 & -0.887 & 0.0 \\
\hline 299.09 & 15.15 & 7.421 & 53.68 & 53.21 & -0.876 & 0.0 \\
\hline 299.05 & 15.72 & 7.712 & 54.55 & 54.23 & -0.587 & 0.0 \\
\hline 298.93 & 18.14 & 8.897 & 58.83 & 58.51 & -0.544 & 0.0 \\
\hline 298.88 & 19.60 & 9.564 & 61.32 & 61.01 & -0.506 & 0.0 \\
\hline 298.80 & 21.17 & 10.240 & 63.53 & 63.60 & 0.110 & 0.0 \\
\hline 298.78 & 22.81 & 10.893 & 66.30 & 66.17 & -0.196 & 0.0 \\
\hline 298.77 & 24.20 & 11.408 & 68.31 & 68.26 & -0.073 & 0.0 \\
\hline 298.73 & 25.48 & 11.854 & 70.68 & 70.12 & -0.792 & .0 \\
\hline 298.73 & 27.17 & 12.399 & 72.69 & 72.47 & -0.303 & 0.0 \\
\hline 298.65 & 28.64 & 12.844 & 74.64 & 74.45 & -0.255 & 0.0 \\
\hline 298.66 & 30.09 & 13.249 & 76.69 & 76.33 & -0.469 & 0.0 \\
\hline 298.65 & 31.41 & 13.597 & 78.35 & 77.99 & -0.459 & 0.0 \\
\hline 298.65 & 32.85 & 13.953 & 79.91 & 79.75 & -0.200 & 0.0 \\
\hline 298.65 & 33.90 & 14.200 & 81.48 & 81.01 & -0.577 & .0 \\
\hline 298.67 & 35.35 & 14.522 & 82.76 & 82.70 & -0.072 & \\
\hline
\end{tabular}

Number of Points (Ref. 72) 33

$\begin{array}{llllll}\text { AAD }-8 & 0.777 & \text { BIAS }-8 & -0.771 & \text { RMS }-8 & 0.531 \\ \text { AAD } & 0.378 & \text { BIAS } & -0.374 & \text { RMS } & 0.233 \mathrm{~mW} \cdot \mathrm{m}^{-1} \cdot \mathrm{K}^{-1}\end{array}$


Data from Ikenberry and Rice [90]

\begin{tabular}{|c|c|c|c|c|c|c|}
\hline $\mathrm{T}$ & $\mathrm{P}$ & $\rho$ & $\lambda, \exp$ & $\lambda, \mathrm{ca} 1$ & Dev & Wt \\
\hline K & $\mathrm{MPa}$ & $\mathrm{mol} \cdot \mathrm{dm}^{-3}$ & \multicolumn{2}{|c|}{$\mathrm{mW} \cdot \mathrm{m}^{-1} \cdot \mathrm{K}^{-1}$} & 8 & \\
\hline 98.90 & 39.82 & 28.949 & 287.36 & 234.92 & -18.249 & 0.0 \\
\hline 98.93 & 30.44 & 28.645 & 267.61 & 227.58 & -14.958 & 0.0 \\
\hline 98.99 & 20.31 & 28.286 & 225.48 & 219.25 & -2.763 & 0.0 \\
\hline 99.01 & 10.13 & 27.890 & 216.52 & 210.41 & -2.822 & 0.0 \\
\hline 99.02 & 5.12 & 27.677 & 211.71 & 205.82 & -2.782 & 0.0 \\
\hline 99.02 & 2.56 & 27.563 & 209.16 & 203.41 & -2.749 & 0.0 \\
\hline 99.03 & 0.16 & 27.451 & 206.90 & 201.09 & -2.808 & 0.0 \\
\hline 125.47 & 50.67 & 27.624 & 218.99 & 215.69 & -1.507 & 0.0 \\
\hline 125.49 & 40.68 & 27.235 & 210.12 & 206.97 & -1.499 & 0.0 \\
\hline 125.55 & 30.41 & 26.788 & 200.33 & 197.43 & -1.448 & 0.0 \\
\hline 125.53 & 20.41 & 26.300 & 189.91 & 187.57 & -1.232 & 0.0 \\
\hline 125.54 & 10.15 & 25.712 & 178.24 & 176.45 & -1.004 & 0.0 \\
\hline 125.61 & 5.08 & 25.371 & 171.38 & 170.35 & -0.601 & 0.0 \\
\hline 125.62 & 2.43 & 25.178 & 168.03 & 167.02 & -0.601 & 0.0 \\
\hline 150.23 & 50.74 & 26.087 & 192.38 & 189.73 & -1.377 & 0.0 \\
\hline 150.25 & 40.54 & 25.577 & 182.84 & 179.88 & -1.619 & 0.0 \\
\hline 150.28 & 30.44 & 24.995 & 171.67 & 169.34 & -1.357 & 0.0 \\
\hline 150.28 & 20.48 & 24.312 & 159.54 & 157.91 & -1.022 & 0.0 \\
\hline 150.28 & 10.13 & 23.409 & 145.60 & 144.17 & -0.982 & 0.0 \\
\hline 150.29 & 5.04 & 22.834 & 137.40 & 136.18 & -0.888 & 0.0 \\
\hline 150.30 & 2.62 & 22.510 & 132.88 & 131.91 & -0.730 & 0.0 \\
\hline 175.13 & 50.67 & 24.523 & 166.52 & 165.89 & -0.378 & 0.0 \\
\hline 175.14 & 40.58 & 23.879 & 156.19 & 155.42 & -0.493 & 0.0 \\
\hline 175.17 & 30.41 & 23.095 & 144.43 & 143.74 & -0.478 & 0.0 \\
\hline 175.15 & 20.41 & 22.107 & 130.92 & 130.56 & -0.275 & 0.0 \\
\hline 175.16 & 10.13 & 20.596 & 114.06 & 113.25 & -0.710 & 0.0 \\
\hline 175.15 & 5.13 & 19.345 & 102.76 & 101.19 & -1.528 & 0.0 \\
\hline 200.20 & 50.67 & 22.946 & 145.31 & 145.38 & 0.048 & 0.0 \\
\hline 200.20 & 40.41 & 22.115 & 134.22 & 134.22 & 0.000 & 0.0 \\
\hline 200.21 & 30.41 & 21.084 & 122.05 & 121.89 & -0.131 & 0.0 \\
\hline 200.22 & 20.28 & 19.601 & 106.86 & 106.75 & -0.103 & 0.0 \\
\hline 200.18 & 13.01 & 17.837 & 92.80 & 92.23 & -0.614 & 0.0 \\
\hline 200.18 & 10.17 & 16.652 & 85.73 & 84.40 & -1.551 & 0.0 \\
\hline 200.18 & 7.63 & 14.653 & 78.28 & 74.66 & -4.624 & 0.0 \\
\hline 200.10 & 5.11 & 5.735 & 41.92 & 42.01 & 0.215 & 0.0 \\
\hline
\end{tabular}


Data from Ikenberry and Rice [90] (continued)

\begin{tabular}{|c|c|c|c|c|c|c|}
\hline $\mathrm{T}$ & $\mathbf{P}$ & $\rho$ & $\lambda, \exp$ & $\lambda, \mathrm{cal}$ & Dev & Wt \\
\hline K & $\mathrm{MPa}$ & $\mathrm{mol} \cdot \mathrm{dm}^{-3}$ & \multicolumn{2}{|c|}{$\mathrm{mW} \cdot \mathrm{m}^{-1} \cdot \mathrm{K}^{-1}$} & 8 & \\
\hline 200.08 & 2.48 & 1.813 & 25.02 & 25.46 & 1.759 & 0.0 \\
\hline 200.08 & 0.28 & 0.174 & 21.59 & 22.00 & 1.899 & 0.0 \\
\hline 235.01 & 50.74 & 20.786 & 123.34 & 123.08 & -0.211 & 0.0 \\
\hline 235.00 & 40.54 & 19.681 & 112.13 & 111.74 & -0.348 & 0.0 \\
\hline 235.00 & 30.68 & 18.228 & 99.50 & 99.03 & -0.472 & 0.0 \\
\hline 234.99 & 20.28 & 15.717 & 82.30 & 81.92 & -0.462 & 0.0 \\
\hline 235.00 & 13.06 & 11.882 & 64.89 & 64.29 & -0.925 & 0.0 \\
\hline 234.98 & 10.10 & 8.769 & 53.18 & 52.69 & -0.921 & 0.0 \\
\hline 234.96 & 5.07 & 3.294 & 32.89 & 32.91 & 0.061 & 0.0 \\
\hline 234.95 & 2.49 & 1.419 & 27.91 & 28.44 & 1.899 & 0.0 \\
\hline
\end{tabular}

Number of Points (Ref. 90) 45

$\begin{array}{llllll}\text { AAD }-8 & 1.847 & \text { BIAS }-8 & -1.585 & \text { RMS }-8 & 3.472 \\ \text { AAD } & 3.683 & \text { BIAS } & -3.614 & \text { RMS } & 9.466 \mathrm{~mW} \cdot \mathrm{m}^{-1} \cdot \mathrm{K}^{-1}\end{array}$

Data from Le Neindre et a1. [81]

$\begin{array}{ccclccc}\mathrm{T} & \mathrm{P} & \rho & \lambda, \exp & \lambda, \mathrm{cal} & \text { Dev } & \text { Wt } \\ \mathrm{K} & \mathrm{MPa} & \mathrm{mol} \cdot \mathrm{dm}^{-3} & \mathrm{~mW} \cdot \mathrm{m}^{-1} \cdot \mathrm{K}^{-1} & & \\ 307.35 & & & & & \\ 298.65 & 0.10 & 0.039 & 35.60 & 35.42 & -0.506 & 0.0 \\ 304.45 & 0.14 & 0.057 & 34.50 & 34.23 & -0.783 & 0.0 \\ 298.55 & 1.00 & 0.401 & 35.80 & 35.60 & -0.559 & 0.0 \\ 303.85 & 1.00 & 0.410 & 35.00 & 34.79 & -0.600 & 0.0 \\ 298.45 & 4.40 & 1.870 & 38.80 & 38.21 & -1.521 & 0.0 \\ 306.35 & 5.00 & 2.197 & 38.40 & 38.14 & -0.677 & 0.0 \\ 302.85 & 5.80 & 2.489 & 40.70 & 39.86 & -2.064 & 0.0 \\ 298.85 & 7.30 & 3.254 & 41.90 & 41.15 & -1.790 & 0.0 \\ 304.75 & 10.10 & 4.772 & 45.50 & 44.66 & -1.846 & 0.0 \\ 301.45 & 10.50 & 4.817 & 46.80 & 45.57 & -2.628 & 0.0 \\ 304.15 & 10.50 & 4.908 & 46.30 & 45.39 & -1.965 & 0.0 \\ 299.35 & 13.70 & 6.462 & 51.30 & 50.58 & -1.404 & 0.0 \\ 303.45 & 14.70 & 7.178 & 53.20 & 52.40 & -1.504 & 0.0 \\ 302.75 & 16.30 & 7.780 & 55.70 & 55.01 & -1.239 & 0.0 \\ & 18.50 & 8.847 & 59.30 & 58.79 & -0.860 & 0.0\end{array}$


Data from Le Neindre et al. [81] (continued)

\begin{tabular}{|c|c|c|c|c|c|c|}
\hline $\mathrm{T}$ & $\mathbf{P}$ & $\rho$ & $\lambda, \exp$ & $\lambda, \mathrm{cal}$ & Dev & Wt \\
\hline $\mathrm{K}$ & $\mathrm{MPa}$ & $\mathrm{mol} \cdot \mathrm{dm}^{-3}$ & \multicolumn{2}{|c|}{$\mathrm{mW} \cdot \mathrm{m}^{-1} \cdot \mathrm{K}^{-1}$} & 8 & \\
\hline 298.75 & 20.40 & 9.920 & 62.60 & 62.35 & -0.399 & 0.0 \\
\hline 302.05 & 21.60 & 10.214 & 64.30 & 63.88 & -0.653 & 0.0 \\
\hline 303.25 & 22.30 & 10.417 & 65.40 & 64.82 & -0.887 & 0.0 \\
\hline 302.75 & 26.90 & 12.057 & 71.60 & 71.48 & -0.168 & 0.0 \\
\hline 298.45 & 30.60 & 13.399 & 77.00 & 77.01 & 0.013 & 0.0 \\
\hline 302.55 & 32.30 & 13.577 & 78.70 & 78.39 & -0.394 & 0.0 \\
\hline 302.35 & 38.60 & 14.970 & 86.30 & 85.64 & -0.765 & 0.0 \\
\hline 298.65 & 40.70 & 15.577 & 89.00 & 88.66 & -0.382 & 0.0 \\
\hline 302.35 & 45.40 & 16.155 & 93.80 & 92.73 & -1.141 & 0.0 \\
\hline 298.75 & 50.90 & 17.140 & 99.70 & 98.92 & -0.782 & 0.0 \\
\hline 302.25 & 51.40 & 17.024 & 99.90 & 98.57 & -1.331 & 0.0 \\
\hline 302.05 & 58.20 & 17.868 & 106.60 & 104.83 & -1.660 & 0.0 \\
\hline 298.65 & 60.90 & 18.329 & 108.80 & 108.07 & -0.671 & 0.0 \\
\hline 301.75 & 69.00 & 18.982 & 115.90 & 114.12 & -1.536 & 0.0 \\
\hline 298.65 & 71.50 & 19.345 & 118.40 & 117.02 & -1.166 & 0.0 \\
\hline 301.65 & 78.50 & 19.793 & 123.20 & 121.75 & -1.177 & 0.0 \\
\hline 298.65 & 82.10 & 20.196 & 126.80 & 125.41 & -1.096 & 0.0 \\
\hline 301.45 & 87.00 & 20.431 & 129.50 & 128.30 & -0.927 & 0.0 \\
\hline 298.55 & 93.90 & 21.008 & 134.40 & 134.28 & -0.089 & 0.0 \\
\hline 301.25 & 95.90 & 21.027 & 136.40 & 134.89 & -1.107 & 0.0 \\
\hline 298.45 & 101.70 & 21.485 & 141.10 & 139.92 & -0.836 & 0.0 \\
\hline 301.15 & 103.60 & 21.491 & 141.90 & 140.40 & -1.057 & 0.0 \\
\hline 301.15 & 106.90 & 21.677 & 143.50 & 142.70 & -0.557 & 0.0 \\
\hline 301.35 & 116.20 & 22.161 & 150.60 & 148.98 & -1.076 & 0.0 \\
\hline 368.85 & 0.10 & 0.033 & 45.10 & 44.79 & -0.687 & 0.0 \\
\hline 368.75 & 1.10 & 0.362 & 45.60 & 45.30 & -0.658 & 0.0 \\
\hline 368.75 & 4.80 & 1.615 & 47.50 & 47.51 & 0.021 & 0.0 \\
\hline 369.05 & 10.20 & 3.511 & 52.00 & 51.64 & -0.692 & 0.0 \\
\hline 369.25 & 20.60 & 7.065 & 61.90 & 61.85 & -0.081 & 0.0 \\
\hline 368.85 & 31.00 & 10.047 & 72.00 & 72.49 & 0.681 & 0.0 \\
\hline 368.65 & 41.80 & 12.409 & 81.90 & 82.34 & 0.537 & 0.0 \\
\hline 368.55 & 51.40 & 14.028 & 90.00 & 90.18 & 0.200 & 0.0 \\
\hline 368.75 & 60.90 & 15.311 & 97.20 & 97.36 & 0.165 & 0.0 \\
\hline 368.75 & 71.60 & 16.509 & 105.10 & 104.96 & -0.133 & 0.0 \\
\hline 368.75 & 81.50 & 17.442 & 112.00 & 111.63 & -0.330 & 0.0 \\
\hline
\end{tabular}


Data from Le Neindre et al. [81] (continued)

\begin{tabular}{|c|c|c|c|c|c|c|}
\hline $\mathrm{T}$ & $P$ & $\rho$ & $\lambda, \exp$ & $\lambda, \mathrm{cal}$ & Dev & We \\
\hline K & $\mathrm{MPa}$ & $\mathrm{mol} \cdot \mathrm{dm}^{-3}$ & \multicolumn{2}{|c|}{$\mathrm{mW} \cdot \mathrm{m}^{-1} \cdot \mathrm{K}^{-1}$} & 8 & \\
\hline 368.55 & 92.10 & 18.311 & 119.20 & 118.50 & -0.587 & 0.0 \\
\hline 368.35 & 102.30 & 19.045 & 125.20 & 124.87 & -0.264 & 0.0 \\
\hline 407.95 & 0.10 & 0.029 & 51.40 & 51.38 & -0.039 & 0.0 \\
\hline 407.65 & 3.30 & 0.986 & 53.00 & 52.90 & -0.189 & 0.0 \\
\hline 406.85 & 10.50 & 3.191 & 58.00 & 57.21 & -1.362 & 0.0 \\
\hline 406.25 & 17.60 & 5.332 & 63.30 & 62.60 & -1.106 & 0.0 \\
\hline 405.85 & 24.30 & 7.207 & 68.70 & 68.28 & -0.611 & 0.0 \\
\hline 405.65 & 30.80 & 8.828 & 74.00 & 73.84 & -0.216 & 0.0 \\
\hline 406.65 & 38.50 & 10.453 & 80.70 & 80.18 & -0.644 & 0.0 \\
\hline 406.15 & 45.40 & 11.736 & 86.00 & 85.43 & -0.663 & 0.0 \\
\hline 405.65 & 52.50 & 12.879 & 91.30 & 90.53 & -0.843 & 0.0 \\
\hline 405.15 & 59.50 & 13.862 & 96.30 & 95.33 & -1.007 & 0.0 \\
\hline 404.35 & 67.20 & 14.820 & 101.80 & 100.38 & -1.395 & 0.0 \\
\hline 403.75 & 77.30 & 15.895 & 108.20 & 106.75 & -1.340 & 0.0 \\
\hline 402.85 & 83.30 & 16.479 & 112.00 & 110.43 & -1.402 & 0.0 \\
\hline 404.25 & 95.90 & 17.468 & 119.50 & 117.78 & -1.439 & 0.0 \\
\hline 404.25 & 104.50 & 18.088 & 124.30 & 122.66 & -1.319 & 0.0 \\
\hline 404.35 & 114.70 & 18.749 & 130.20 & 128.30 & -1.459 & 0.0 \\
\hline 420.25 & 0.14 & 0.040 & 53.90 & 53.56 & -0.631 & 0.0 \\
\hline 420.25 & 1.00 & 0.287 & 54.40 & 53.95 & -0.827 & 0.0 \\
\hline 420.45 & 5.00 & 1.450 & 56.30 & 55.99 & -0.551 & 0.0 \\
\hline 420.55 & 10.20 & 2.977 & 59.60 & 59.11 & -0.822 & 0.0 \\
\hline 420.45 & 20.60 & 5.918 & 67.10 & 66.76 & -0.507 & 0.0 \\
\hline 420.35 & 31.00 & 8.487 & 75.10 & 75.12 & 0.027 & 0.0 \\
\hline 420.25 & 40.80 & 10.492 & 82.50 & 82.65 & 0.182 & 0.0 \\
\hline 420.25 & 51.30 & 12.245 & 90.00 & 90.09 & 0.100 & 0.0 \\
\hline 420.25 & 60.90 & 13.569 & 96.60 & 96.42 & -0.186 & 0.0 \\
\hline 420.15 & 71.40 & 14.791 & 104.00 & 102.95 & -1.010 & 0.0 \\
\hline 420.05 & 81.60 & 15.804 & 110.00 & 109.00 & -0.909 & 0.0 \\
\hline 419.95 & 92.10 & 16.710 & 116.50 & 115.00 & -1.288 & 0.0 \\
\hline 419.85 & 101.70 & 17.442 & 121.40 & 120.31 & -0.898 & 0.0 \\
\hline 465.55 & 0.14 & 0.036 & 62.30 & 61.81 & -0.787 & 0.0 \\
\hline 465.55 & 1.00 & 0.259 & 62.80 & 62.16 & -1.019 & 0.0 \\
\hline 465.55 & 5.00 & 1.297 & 64.20 & 63.91 & -0.452 & 0.0 \\
\hline 466.05 & 10.20 & 2.638 & 67.00 & 66.64 & -0.537 & 0.0 \\
\hline
\end{tabular}


Data from Le Neindre et al. [81] (continued)

\begin{tabular}{|c|c|c|c|c|c|c|}
\hline $\mathrm{T}$ & $P$ & $\rho$ & $\lambda, \exp$ & $\lambda, \mathrm{cal}$ & Dev & Wt \\
\hline K & $\mathrm{MPa}$ & $\mathrm{mol} \cdot \mathrm{dm}^{-3}$ & \multicolumn{2}{|c|}{$\mathrm{mW} \cdot \mathrm{m}^{-1} \cdot \mathrm{K}^{-1}$} & 8 & \\
\hline 465.85 & 20.80 & 5.257 & 73.20 & 73.02 & -0.246 & 0.0 \\
\hline 465.65 & 30.80 & 7.460 & 79.60 & 79.68 & 0.101 & 0.0 \\
\hline 465.55 & 40.80 & 9.350 & 86.10 & 86.29 & 0.221 & 0.0 \\
\hline 465.45 & 51.10 & 10.996 & 92.30 & 92.74 & 0.477 & 0.0 \\
\hline 465.35 & 60.80 & 12.312 & 98.20 & 98.45 & 0.255 & 0.0 \\
\hline 465.25 & 71.00 & 13.501 & 104.20 & 104.14 & -0.058 & 0.0 \\
\hline 465.15 & 81.70 & 14.578 & 110.20 & 109.85 & -0.318 & 0.0 \\
\hline 465.15 & 92.20 & 15.500 & 116.10 & 115.25 & -0.732 & 0.0 \\
\hline 464.95 & 102.50 & 16.305 & 121.30 & 120.39 & -0.750 & 0.0 \\
\hline 483.25 & 0.55 & 0.137 & 65.20 & 65.29 & 0.138 & 0.0 \\
\hline 486.85 & 1.20 & 0.297 & 66.40 & 66.23 & -0.256 & 0.0 \\
\hline 483.15 & 3.40 & 0.848 & 66.60 & 66.43 & -0.255 & 0.0 \\
\hline 486.55 & 6.80 & 1.681 & 69.10 & 68.57 & -0.767 & 0.0 \\
\hline 482.85 & 7.30 & 1.819 & 68.60 & 68.13 & -0.685 & 0.0 \\
\hline 482.65 & 11.80 & 2.927 & 70.80 & 70.37 & -0.607 & 0.0 \\
\hline 487.05 & 15.10 & 3.682 & 73.50 & 72.92 & -0.789 & 0.0 \\
\hline 486.65 & 21.60 & 5.169 & 77.20 & 76.66 & -0.699 & 0.0 \\
\hline 485.25 & 28.40 & 6.632 & 81.10 & 80.67 & -0.530 & 0.0 \\
\hline 484.85 & 35.90 & 8.083 & 85.60 & 85.33 & -0.315 & 0.0 \\
\hline 484.55 & 42.70 & 9.264 & 89.80 & 89.48 & -0.356 & 0.0 \\
\hline 484.35 & 54.10 & 10.980 & 96.30 & 96.16 & -0.145 & 0.0 \\
\hline 484.35 & 55.10 & 11.116 & 96.80 & 96.73 & -0.072 & 0.0 \\
\hline 484.05 & 65.70 & 12.449 & 102.50 & 102.53 & 0.029 & 0.0 \\
\hline 483.95 & 70.60 & 12.999 & 105.20 & 105.12 & -0.076 & 0.0 \\
\hline 483.95 & 72.60 & 13.213 & 106.30 & 106.17 & -0.122 & 0.0 \\
\hline 483.75 & 75.50 & 13.517 & 107.80 & 107.65 & -0.139 & 0.0 \\
\hline 483.65 & 79.40 & 13.905 & 109.90 & 109.64 & -0.237 & 0.0 \\
\hline 483.55 & 84.30 & 14.365 & 112.50 & 112.09 & -0.364 & 0.0 \\
\hline 483.55 & 87.30 & 14.633 & 114.20 & 113.58 & -0.543 & 0.0 \\
\hline 483.45 & 95.10 & 15.288 & 118.30 & 117.38 & -0.778 & 0.0 \\
\hline 483.45 & 104.90 & 16.033 & 122.90 & 122.05 & -0.692 & 0.0 \\
\hline 483.45 & 109.80 & 16.378 & 125.10 & 124.34 & -0.608 & 0.0 \\
\hline 483.65 & 112.80 & 16.577 & 125.40 & 125.74 & 0.271 & 0.0 \\
\hline 523.35 & 0.14 & 0.032 & 73.20 & 72.82 & -0.519 & 0.0 \\
\hline 523.35 & 1.00 & 0.230 & 73.70 & 73.13 & -0.773 & 0.0 \\
\hline
\end{tabular}


Data from Le Neindre et al. [81] (continued)

\begin{tabular}{|c|c|c|c|c|c|c|}
\hline $\mathrm{T}$ & $\mathbf{P}$ & $\rho$ & $\lambda, \exp$ & $\lambda, \mathrm{cal}$ & Dev & Wt \\
\hline K & $\mathrm{MPa}$ & $\mathrm{mol} \cdot \mathrm{dm}^{-3}$ & \multicolumn{2}{|c|}{$\mathrm{mW} \cdot \mathrm{m}^{-1} \cdot \mathrm{K}^{-1}$} & 8 & \\
\hline 523.25 & 5.00 & 1.145 & 74.70 & 74.63 & -0.094 & 0.0 \\
\hline 523.25 & 10.40 & 2.363 & 76.80 & 76.94 & 0.182 & 0.0 \\
\hline 523.15 & 20.40 & 4.512 & 81.60 & 81.87 & 0.331 & 0.0 \\
\hline 523.05 & 30.70 & 6.515 & 87.00 & 87.48 & 0.552 & 0.0 \\
\hline 522.95 & 40.80 & 8.242 & 92.20 & 93.08 & 0.954 & 0.0 \\
\hline 522.85 & 50.90 & 9.745 & 97.70 & 98.52 & 0.839 & 0.0 \\
\hline 522.65 & 60.70 & 11.019 & 102.90 & 103.56 & 0.641 & 0.0 \\
\hline 522.65 & 71.40 & 12.228 & 108.30 & 108.83 & 0.489 & 0.0 \\
\hline 522.55 & 82.40 & 13.316 & 114.00 & 114.00 & 0.000 & 0.0 \\
\hline 522.45 & 94.90 & 14.397 & 120.20 & 119.66 & -0.449 & 0.0 \\
\hline 571.85 & 0.10 & 0.021 & 82.80 & 82.29 & -0.616 & 0.0 \\
\hline 571.65 & 5.30 & 1.106 & 85.30 & 84.02 & -1.501 & 0.0 \\
\hline 571.45 & 10.50 & 2.168 & 87.30 & 85.96 & -1.535 & 0.0 \\
\hline 571.35 & 20.70 & 4.147 & 91.60 & 90.35 & -1.365 & 0.0 \\
\hline 571.15 & 30.30 & 5.850 & 95.90 & 94.85 & -1.095 & 0.0 \\
\hline 570.85 & 40.50 & 7.472 & 100.60 & 99.77 & -0.825 & 0.0 \\
\hline 570.85 & 50.90 & 8.928 & 105.40 & 104.77 & -0.598 & 0.0 \\
\hline 570.65 & 60.50 & 10.120 & 109.80 & 109.21 & -0.537 & 0.0 \\
\hline 570.55 & 72.60 & 11.438 & 115.20 & 114.59 & -0.530 & 0.0 \\
\hline 570.45 & 83.70 & 12.499 & 120.00 & 119.33 & -0.558 & 0.0 \\
\hline 570.35 & 95.40 & 13.490 & 125.20 & 124.15 & -0.839 & 0.0 \\
\hline 570.25 & 107.90 & 14.431 & 130.40 & 129.14 & -0.966 & 0.0 \\
\hline 570.15 & 117.40 & 15.078 & 134.30 & 132.84 & -1.087 & 0.0 \\
\hline 570.05 & 124.70 & 15.542 & 137.50 & 135.63 & -1.360 & 0.0 \\
\hline 575.75 & 0.10 & 0.021 & 84.60 & 83.06 & -1.820 & 0.0 \\
\hline 575.75 & 1.00 & 0.209 & 85.20 & 83.35 & -2.171 & 0.0 \\
\hline 575.75 & 5.10 & 1.057 & 86.10 & 84.74 & -1.580 & 0.0 \\
\hline 575.35 & 10.10 & 2.072 & 87.40 & 86.54 & -0.984 & 0.0 \\
\hline 575.65 & 20.50 & 4.077 & 91.80 & 91.02 & -0.850 & 0.0 \\
\hline 575.55 & 30.60 & 5.852 & 96.20 & 95.72 & -0.499 & 0.0 \\
\hline 575.45 & 40.80 & 7.455 & 100.70 & 100.61 & -0.089 & 0.0 \\
\hline 575.35 & 50.70 & 8.833 & 105.50 & 105.30 & -0.190 & 0.0 \\
\hline 575.35 & 60.60 & 10.054 & 110.30 & 109.86 & -0.399 & 0.0 \\
\hline 575.25 & 69.20 & 11.005 & 114.20 & 113.68 & -0.455 & 0.0 \\
\hline 575.25 & 80.60 & 12.130 & 119.10 & 118.57 & -0.445 & 0.0 \\
\hline
\end{tabular}


Data from Le Neindre et al. [81] (continued)

\begin{tabular}{|c|c|c|c|c|c|c|}
\hline $\mathrm{T}$ & $\mathbf{P}$ & $\rho$ & $\lambda, \exp$ & $\lambda, \mathrm{cal}$ & Dev & Wt \\
\hline K & $\mathrm{MPa}$ & $\mathrm{mol} \cdot \mathrm{dm}^{-3}$ & \multicolumn{2}{|c|}{$\mathrm{mW} \cdot \mathrm{m}^{-1} \cdot \mathrm{K}^{-1}$} & 8 & \\
\hline 575.15 & 91.60 & 13.094 & 123.80 & 123.10 & -0.565 & 0.0 \\
\hline 575.05 & 103.50 & 14.024 & 129.00 & 127.86 & -0.884 & 0.0 \\
\hline 621.45 & 0.14 & 0.027 & 94.00 & 92.09 & -2.032 & 0.0 \\
\hline 621.45 & 1.00 & 0.193 & 94.50 & 92.35 & -2.275 & 0.0 \\
\hline 621.45 & 5.00 & 0.958 & 95.40 & 93.59 & -1.897 & 0.0 \\
\hline 621.45 & 10.20 & 1.929 & 96.80 & 95.36 & -1.488 & 0.0 \\
\hline 621.25 & 20.30 & 3.722 & 100.20 & 99.15 & -1.048 & 0.0 \\
\hline 621.25 & 30.50 & 5.382 & 104.00 & 103.38 & -0.596 & 0.0 \\
\hline 621.15 & 40.50 & 6.852 & 108.40 & 107.66 & -0.683 & 0.0 \\
\hline 621.05 & 50.70 & 8.195 & 112.30 & 112.02 & -0.249 & 0.0 \\
\hline 620.95 & 60.90 & 9.395 & 116.70 & 116.28 & -0.360 & 0.0 \\
\hline 620.95 & 68.40 & 10.196 & 119.90 & 119.34 & -0.467 & 0.0 \\
\hline 620.95 & 81.40 & 11.442 & 125.40 & 124.48 & -0.734 & 0.0 \\
\hline 620.95 & 92.10 & 12.354 & 129.40 & 128.55 & -0.657 & 0.0 \\
\hline 620.75 & 102.20 & 13.139 & 133.10 & 132.27 & -0.624 & 0.0 \\
\hline 648.65 & 0.50 & 0.093 & 98.70 & 97.57 & -1.145 & 0.0 \\
\hline 648.55 & 3.40 & 0.626 & 100.50 & 98.39 & -2.100 & 0.0 \\
\hline 648.45 & 7.00 & 1.277 & 101.80 & 99.49 & -2.269 & 0.0 \\
\hline 648.45 & 11.20 & 2.021 & 103.10 & 100.87 & -2.163 & 0.0 \\
\hline 648.25 & 21.10 & 3.689 & 106.20 & 104.40 & -1.695 & 0.0 \\
\hline 648.15 & 30.50 & 5.150 & 109.70 & 108.05 & -1.504 & 0.0 \\
\hline 647.95 & 40.70 & 6.592 & 113.40 & 112.13 & -1.120 & 0.0 \\
\hline 647.95 & 50.30 & 7.816 & 117.30 & 116.02 & -1.091 & 0.0 \\
\hline 647.85 & 59.40 & 8.868 & 120.60 & 119.64 & -0.796 & 0.0 \\
\hline 647.75 & 71.60 & 10.132 & 125.10 & 124.35 & -0.600 & 0.0 \\
\hline 647.65 & 83.20 & 11.200 & 129.30 & 128.68 & -0.480 & 0.0 \\
\hline 647.65 & 95.10 & 12.179 & 134.30 & 132.99 & -0.975 & 0.0 \\
\hline 647.75 & 117.10 & 13.741 & 141.50 & 140.64 & -0.608 & 0.0 \\
\hline 647.85 & 119.40 & 13.887 & 142.30 & 141.43 & -0.611 & 0.0 \\
\hline 648.05 & 121.10 & 13.992 & 142.50 & 142.03 & -0.330 & 0.0 \\
\hline 726.55 & 1.00 & 0.165 & 114.50 & 113.00 & -1.310 & 0.0 \\
\hline 726.45 & 3.60 & 0.590 & 115.30 & 113.65 & -1.431 & 0.0 \\
\hline 725.15 & 40.10 & 5.822 & 126.10 & 125.00 & -0.872 & 0.0 \\
\hline 725.05 & 50.70 & 7.060 & 128.90 & 128.67 & -0.178 & 0.0 \\
\hline 725.15 & 60.80 & 8.131 & 131.30 & 132.18 & 0.670 & 0.0 \\
\hline 725.25 & 72.00 & 9.207 & 134.80 & 136.00 & 0.890 & 0.0 \\
\hline 725.45 & 81.00 & 9.996 & 137.90 & 139.01 & 0.805 & 0.0 \\
\hline 725.75 & 89.00 & 10.646 & 141.70 & 141.65 & -0.035 & 0.0 \\
\hline
\end{tabular}


Data from Le Neindre et a1. [81] (continued)

Number of Points (Ref. 81) 193

$\begin{array}{llllll}\text { AAD }-8 & 0.778 & \text { BIAS }-8 & -0.677 & \text { RMS }-8 & 0.664 \\ \text { AAD } & 0.735 & \text { BIAS } & -0.632 & \text { RMS } & 0.657 \mathrm{~mW} \cdot \mathrm{m}^{-1} \cdot \mathrm{K}^{-1}\end{array}$

$\overline{\text { Data from Mardolcar and de Castro [91] }}$

\begin{tabular}{|c|c|c|c|c|c|c|}
\hline $\mathrm{T}$ & $P$ & $\rho$ & $\lambda, \exp$ & $\lambda, \mathrm{cal}$ & Dev & Wt \\
\hline K & $\mathrm{MPa}$ & $\mathrm{mol} \cdot \mathrm{dm}^{-3}$ & \multicolumn{2}{|c|}{$\mathrm{mW} \cdot \mathrm{m}^{-1} \cdot \mathrm{K}^{-1}$} & 8 & \\
\hline 110.868 & 0.204 & 26.414 & 184.60 & 185.17 & 0.309 & 9.7 \\
\hline 111.002 & 1.100 & 26.453 & 185.59 & 185.97 & 0.205 & 9.7 \\
\hline 110.971 & 2.236 & 26.521 & 187.00 & 187.26 & 0.139 & 9.7 \\
\hline 111.202 & 3.270 & 26.558 & 188.06 & 188.07 & 0.005 & 9.7 \\
\hline 111.024 & 4.269 & 26.628 & 189.03 & 189.37 & 0.180 & 9.6 \\
\hline 110.897 & 5.269 & 26.692 & 190.10 & 190.60 & 0.263 & 9.6 \\
\hline 111.038 & 6.337 & 26.737 & 191.04 & 191.53 & 0.256 & 9.6 \\
\hline 110.718 & 7.404 & 26.819 & 192.55 & 193.06 & 0.265 & 9.6 \\
\hline 110.952 & 8.645 & 26.862 & 194.09 & 194.02 & -0.036 & 9.5 \\
\hline 124.806 & 0.721 & 25.129 & 164.41 & 165.97 & 0.949 & 10.1 \\
\hline 124.985 & 1.755 & 25.190 & 166.20 & 167.05 & 0.511 & 10.1 \\
\hline 125.059 & 2.719 & 25.254 & 167.39 & 168.18 & 0.472 & 10.1 \\
\hline 125.034 & 3.822 & 25.336 & 168.97 & 169.60 & 0.373 & 10.0 \\
\hline 124.867 & 4.924 & 25.429 & 170.41 & 171.18 & 0.452 & 10.0 \\
\hline 125.196 & 5.957 & 25.469 & 172.13 & 171.99 & -0.081 & 10.0 \\
\hline 124.728 & 7.060 & 25.586 & 173.44 & 173.94 & 0.288 & 9.9 \\
\hline 124.784 & 8.369 & 25.666 & 175.10 & 175.40 & 0.171 & 9.9 \\
\hline 155.480 & 1.514 & 21.634 & 122.11 & 121.93 & -0.147 & 11.3 \\
\hline 155.251 & 2.306 & 21.810 & 122.85 & 123.98 & 0.920 & 11.3 \\
\hline 154.953 & 2.891 & 21.950 & 125.47 & 125.62 & 0.120 & 11.2 \\
\hline 155.192 & 3.374 & 21.997 & 125.94 & 126.23 & 0.230 & 11.2 \\
\hline 154.969 & 4.132 & 22.145 & 127.52 & 128.01 & 0.384 & 11.1 \\
\hline 155.034 & 4.924 & 22.255 & 129.08 & 129.40 & 0.248 & 11.1 \\
\hline 155.285 & 5.648 & 22.328 & 130.18 & 130.36 & 0.138 & 11.0 \\
\hline 154.726 & 6.302 & 22.484 & 131.09 & 132.28 & 0.908 & 11.0 \\
\hline 154.995 & 7.129 & 22.562 & 132.64 & 133.33 & 0.520 & 11.0 \\
\hline 154.598 & 7.887 & 22.702 & 134.03 & 135.11 & 0.806 & 10.9 \\
\hline 155.167 & 8.576 & 22.724 & 135.51 & 135.50 & -0.007 & 10.9 \\
\hline 180.336 & 3.753 & 17.485 & 89.69 & 87.39 & -2.564 & 12.5 \\
\hline 180.509 & 4.132 & 17.703 & 91.15 & 88.90 & -2.468 & 12.5 \\
\hline
\end{tabular}


Data from Mardolcar and de Castro [91] (continued)

$\begin{array}{cccccrc}\mathrm{T} & \mathrm{P} & \rho & \lambda, \exp & \lambda, \mathrm{cal} & \text { Dev } & \text { Wt } \\ \mathrm{K} & \mathrm{MPa} & \mathrm{mol} \cdot \mathrm{dm}^{-3} & \mathrm{~mW} \cdot \mathrm{m}^{-1} \cdot \mathrm{K}^{-1} & & \\ 180.132 & 4.580 & 18.070 & 93.03 & 91.49 & -1.655 & 12.4 \\ 180.172 & 5.027 & 18.290 & 93.96 & 93.16 & -0.851 & 12.4 \\ 180.255 & 5.578 & 18.523 & 95.12 & 94.98 & -0.147 & 12.4 \\ 180.139 & 6.095 & 18.756 & 97.01 & 96.84 & -0.175 & 12.3 \\ 180.058 & 6.784 & 19.019 & 99.01 & 99.03 & 0.020 & 12.2 \\ 179.989 & 7.335 & 19.211 & 100.49 & 100.67 & 0.179 & 12.2 \\ 179.905 & 8.231 & 19.487 & 102.61 & 103.12 & 0.497 & 12.1\end{array}$

Number of Points (Ref. 91) 37

$\begin{array}{llllll}\text { AAD }-8 & 0.485 & \text { BIAS }-8 & 0.045 & \text { RMS }-\frac{8}{8} & 0.763 \\ \text { AAD } & 0.602 & \text { BIAS } & 0.192 & \text { RMS } & 0.801 \mathrm{~mW} \cdot \mathrm{m}^{-1} \cdot \mathrm{K}^{-1}\end{array}$

Data from Prasad et al. [92]

$\begin{array}{ccccccc}\mathrm{T} & \mathrm{P} & \rho & \lambda, \exp & \lambda, \mathrm{cal} & \text { Dev } & \text { Wt } \\ \mathrm{K} & \mathrm{MPa} & \mathrm{mol} \cdot \mathrm{dm}^{-3} & & \mathrm{~mW} \cdot \mathrm{m}^{-1} \cdot \mathrm{K}^{-1} & & \\ 139.920 & 59.998 & 27.104 & 204.88 & 208.52 & 1.777 & 0.0 \\ 140.070 & 59.998 & 27.095 & 204.70 & 208.36 & 1.788 & 0.0 \\ 139.950 & 50.001 & 26.696 & 196.45 & 199.67 & 1.639 & 0.0 \\ 139.970 & 50.001 & 26.694 & 195.83 & 199.65 & 1.951 & 0.0 \\ 140.000 & 40.004 & 26.240 & 187.30 & 190.31 & 1.607 & 0.0 \\ 140.010 & 40.004 & 26.239 & 187.37 & 190.30 & 1.564 & 0.0 \\ 140.090 & 30.006 & 25.720 & 176.87 & 180.25 & 1.911 & 0.0 \\ 140.000 & 30.006 & 25.727 & 177.60 & 180.35 & 1.548 & 0.0 \\ 120.080 & 10.004 & 26.171 & 179.56 & 183.48 & 2.183 & 0.0 \\ 120.010 & 10.004 & 26.177 & 179.65 & 183.57 & 2.182 & 0.0 \\ 120.080 & 10.004 & 26.171 & 179.74 & 183.48 & 2.081 & 0.0 \\ 119.900 & 20.002 & 26.713 & 190.16 & 194.09 & 2.067 & 0.0 \\ 119.890 & 20.002 & 26.713 & 190.80 & 194.10 & 1.730 & 0.0 \\ 120.040 & 29.999 & 27.160 & 199.96 & 203.48 & 1.760 & 0.0 \\ 119.910 & 29.999 & 27.169 & 200.32 & 203.63 & 1.652 & 0.0 \\ 119.940 & 40.004 & 27.575 & 208.87 & 212.56 & 1.767 & 0.0 \\ 119.860 & 40.004 & 27.580 & 209.33 & 212.65 & 1.586 & 0.0 \\ 121.010 & 50.001 & 27.877 & 212.99 & 219.91 & 3.249 & 0.0 \\ 139.900 & 20.002 & 25.136 & 166.86 & 169.61 & 1.648 & 0.0 \\ 139.930 & 20.002 & 25.134 & 166.32 & 169.58 & 1.960 & 0.0\end{array}$


Data from Prasad et al. [92] (continued)

\begin{tabular}{|c|c|c|c|c|c|c|}
\hline $\mathrm{T}$ & $\mathrm{P}$ & $\rho$ & $\lambda, \exp$ & $\lambda, \mathrm{cal}$ & Dev & Wt \\
\hline K & $\mathrm{MPa}$ & $\mathrm{mol} \cdot \mathrm{dm}^{-3}$ & \multicolumn{2}{|c|}{$\mathrm{mW} \cdot \mathrm{mx}^{-1} \cdot \mathrm{K}^{-1}$} & q & \\
\hline 139.890 & 10.004 & 24.408 & 154.05 & 157.42 & 2.188 & 0.0 \\
\hline 139.890 & 10.004 & 24.408 & 154.20 & 157.42 & 2.088 & 0.0 \\
\hline 139.890 & 10.004 & 24.408 & 153.85 & 157.42 & 2.320 & 0.0 \\
\hline 139.920 & 4.999 & 23.962 & 147.09 & 150.46 & 2.291 & 0.0 \\
\hline 139.900 & 4.999 & 23.964 & 147.19 & 150.49 & 2.242 & 0.0 \\
\hline 139.950 & 2.496 & 23.707 & 143.24 & 146.66 & 2.388 & 0.0 \\
\hline 139.950 & 2.496 & 23.707 & 143.39 & 146.66 & 2.280 & 0.0 \\
\hline 159.930 & 2.496 & 21.172 & 115.33 & 117.32 & 1.725 & 0.0 \\
\hline 159.940 & 2.496 & 21.170 & 115.08 & 117.30 & 1.929 & 0.0 \\
\hline 159.900 & 20.002 & 23.447 & 143.77 & 146.36 & 1.801 & 0.0 \\
\hline 159.890 & 20.002 & 23.448 & 143.74 & 146.37 & 1.830 & 0.0 \\
\hline 159.960 & 29.999 & 24.237 & 156.17 & 158.49 & 1.486 & 0.0 \\
\hline 159.980 & 29.999 & 24.236 & 155.82 & 158.47 & 1.701 & 0.0 \\
\hline 159.980 & 10.004 & 22.375 & 129.31 & 131.69 & 1.841 & 0.0 \\
\hline 159.980 & 10.004 & 22.375 & 129.16 & 131.69 & 1.959 & 0.0 \\
\hline 159.980 & 4.999 & 21.638 & 120.29 & 122.64 & 1.954 & 0.0 \\
\hline 160.010 & 4.999 & 21.634 & 119.94 & 122.60 & 2.218 & 0.0 \\
\hline 160.040 & 2.496 & 21.155 & 115.18 & 117.15 & 1.710 & 0.0 \\
\hline 160.000 & 2.496 & 21.161 & 114.93 & 117.21 & 1.984 & 0.0 \\
\hline 160.000 & 40.004 & 24.884 & 166.74 & 169.33 & 1.553 & 0.0 \\
\hline 160.000 & 40.004 & 24.884 & 166.64 & 169.33 & 1.614 & 0.0 \\
\hline 160.000 & 49.994 & 25.438 & 176.81 & 179.32 & 1.420 & 0.0 \\
\hline 159.950 & 49.994 & 25.441 & 176.98 & 179.37 & 1.350 & 0.0 \\
\hline 159.980 & 59.998 & 25.925 & 185.53 & 188.69 & 1.703 & 0.0 \\
\hline 159.970 & 59.998 & 25.926 & 185.80 & 188.70 & 1.561 & 0.0 \\
\hline 160.050 & 69.996 & 26.357 & 193.60 & 197.48 & 2.004 & 0.0 \\
\hline 159.940 & 69.996 & 26.363 & 194.17 & 197.59 & 1.761 & 0.0 \\
\hline 179.960 & 69.996 & 25.262 & 176.98 & 179.87 & 1.633 & 0.0 \\
\hline 180.000 & 69.996 & 25.260 & 177.06 & 179.84 & 1.570 & 0.0 \\
\hline 179.910 & 59.998 & 24.758 & 168.28 & 170.76 & 1.474 & 0.0 \\
\hline 179.960 & 50.001 & 24.178 & 158.37 & 160.98 & 1.648 & 0.0 \\
\hline 179.920 & 40.004 & 23.506 & 148.17 & 150.48 & 1.559 & 0.0 \\
\hline 179.950 & 29.999 & 22.681 & 136.53 & 138.74 & 1.619 & 0.0 \\
\hline 180.000 & 20.002 & 21.598 & 122.91 & 125.06 & 1.749 & 0.0 \\
\hline 179.980 & 20.002 & 21.600 & 122.79 & 125.08 & 1.865 & 0.0 \\
\hline
\end{tabular}


Data from Prasad et al. [92] (continued)

\begin{tabular}{|c|c|c|c|c|c|c|}
\hline $\mathrm{T}$ & $\mathrm{P}$ & $\rho$ & $\lambda, \exp$ & $\lambda, \mathrm{cal}$ & Dev & Wt \\
\hline K & $\mathrm{MPa}$ & $\mathrm{mol} \cdot \mathrm{dm}^{-3}$ & \multicolumn{2}{|c|}{$\mathrm{mW} \cdot \mathrm{m}^{-1} \cdot \mathrm{K}^{-1}$} & 8 & \\
\hline 179.980 & 9.997 & 19.923 & 106.00 & 107.18 & 1.113 & 0.0 \\
\hline 179.980 & 9.997 & 19.923 & 105.83 & 107.18 & 1.276 & 0.0 \\
\hline 179.980 & 4.999 & 18.320 & 93.92 & 93.36 & -0.596 & 0.0 \\
\hline 179.990 & 4.999 & 18.318 & 94.16 & 93.34 & -0.871 & 0.0 \\
\hline 199.990 & 69.996 & 24.174 & 161.18 & 164.15 & 1.843 & 0.0 \\
\hline 200.010 & 59.998 & 23.588 & 152.39 & 154.82 & 1.595 & 0.0 \\
\hline 200.010 & 59.998 & 23.588 & 152.44 & 154.82 & 1.561 & 0.0 \\
\hline 200.010 & 50.001 & 22.909 & 142.54 & 144.83 & 1.607 & 0.0 \\
\hline 200.000 & 40.004 & 22.093 & 131.59 & 133.90 & 1.755 & 0.0 \\
\hline 199.990 & 29.999 & 21.054 & 119.76 & 121.52 & 1.470 & 0.0 \\
\hline 199.990 & 20.002 & 19.575 & 104.91 & 106.48 & 1.497 & 0.0 \\
\hline 200.000 & 10.004 & 16.597 & 84.95 & 84.07 & -1.036 & 0.0 \\
\hline 199.990 & 10.004 & 16.599 & 84.65 & 84.08 & -0.673 & 0.0 \\
\hline 199.990 & 4.999 & 5.458 & 40.15 & 40.35 & 0.498 & 0.0 \\
\hline 220.020 & 69.996 & 23.105 & 147.79 & 150.52 & 1.847 & 0.0 \\
\hline 220.000 & 69.996 & 23.106 & 147.89 & 150.53 & 1.785 & 0.0 \\
\hline 220.050 & 59.998 & 22.436 & 139.11 & 141.15 & 1.466 & 0.0 \\
\hline 220.010 & 50.001 & 21.648 & 128.97 & 131.08 & 1.636 & 0.0 \\
\hline 220.050 & 40.004 & 20.670 & 118.07 & 119.89 & 1.541 & 0.0 \\
\hline 220.110 & 29.999 & 19.367 & 105.42 & 106.96 & 1.461 & 0.0 \\
\hline 220.000 & 20.002 & 17.358 & 89.67 & 90.80 & 1.260 & 0.0 \\
\hline 220.030 & 9.997 & 11.685 & 66.15 & 63.42 & -4.127 & 0.0 \\
\hline 240.020 & 69.996 & 22.063 & 137.42 & 138.98 & 1.135 & 0.0 \\
\hline 240.060 & 59.998 & 21.308 & 128.56 & 129.68 & 0.871 & 0.0 \\
\hline 240.000 & 50.001 & 20.407 & 118.30 & 119.66 & 1.150 & 0.0 \\
\hline 240.000 & 40.004 & 19.264 & 106.96 & 108.47 & 1.412 & 0.0 \\
\hline 240.000 & 29.999 & 17.684 & 94.38 & 95.42 & 1.102 & 0.0 \\
\hline 240.060 & 20.002 & 15.039 & 78.35 & 78.67 & 0.408 & 0.0 \\
\hline 260.030 & 69.996 & 21.051 & 128.14 & 129.39 & 0.975 & 0.0 \\
\hline 260.000 & 59.998 & 20.217 & 119.20 & 120.31 & 0.931 & 0.0 \\
\hline 260.000 & 50.001 & 19.200 & 109.25 & 110.44 & 1.089 & 0.0 \\
\hline 260.030 & 40.004 & 17.889 & 98.59 & 99.43 & 0.852 & 0.0 \\
\hline 260.050 & 30.006 & 16.034 & 86.20 & 86.59 & 0.452 & 0.0 \\
\hline 300.180 & 69.996 & 19.143 & 114.95 & 115.36 & 0.357 & 0.0 \\
\hline 299.330 & 69.996 & 19.182 & 115.72 & 115.60 & -0.104 & 0.0 \\
\hline
\end{tabular}


Data from Prasad et al. [92] (continued)

\begin{tabular}{|c|c|c|c|c|c|c|}
\hline $\mathrm{T}$ & $\mathbf{P}$ & $\rho$ & $\lambda, \exp$ & $\lambda, \mathrm{cal}$ & Dev & Wt \\
\hline K & $\mathrm{MPa}$ & $\mathrm{mol} \cdot \mathrm{dm}^{-3}$ & \multicolumn{2}{|c|}{$\mathrm{mW} \cdot \mathrm{m}^{-1} \cdot \mathrm{K}^{-1}$} & 8 & \\
\hline 300.070 & 69.996 & 19.148 & 114.75 & 115.39 & 0.558 & 0.0 \\
\hline 300.080 & 69.996 & 19.148 & 114.55 & 115.39 & 0.733 & 0.0 \\
\hline 300.000 & 59.998 & 18.167 & 106.00 & 106.93 & 0.877 & 0.0 \\
\hline 300.010 & 59.998 & 18.167 & 106.39 & 106.93 & 0.508 & 0.0 \\
\hline 300.000 & 49.994 & 16.952 & 97.09 & 97.76 & 0.690 & 0.0 \\
\hline 300.030 & 49.994 & 16.950 & 97.22 & 97.75 & 0.545 & 0.0 \\
\hline 300.030 & 40.004 & 15.370 & 86.59 & 87.62 & 1.190 & 0.0 \\
\hline 300.050 & 40.004 & 15.369 & 86.64 & 87.62 & 1.131 & 0.0 \\
\hline 299.970 & 29.999 & 13.141 & 74.99 & 75.98 & 1.320 & 0.0 \\
\hline 299.970 & 29.999 & 13.141 & 75.09 & 75.98 & 1.185 & 0.0 \\
\hline 299.960 & 20.002 & 9.675 & 60.81 & 61.56 & 1.233 & 0.0 \\
\hline 300.040 & 20.002 & 9.670 & 61.01 & 61.55 & 0.885 & 0.0 \\
\hline 300.000 & 9.997 & 4.686 & 43.04 & 44.56 & 3.532 & 0.0 \\
\hline 299.930 & 9.997 & 4.688 & 43.14 & 44.56 & 3.292 & 0.0 \\
\hline 300.240 & 4.999 & 2.179 & 35.13 & 38.35 & 9.166 & 0.0 \\
\hline 299.660 & 4.999 & 2.184 & 35.33 & 38.28 & 8.350 & 0.0 \\
\hline 299.870 & 4.999 & 2.182 & 35.73 & 38.30 & 7.193 & 0.0 \\
\hline 300.000 & 4.999 & 2.181 & 35.81 & 38.32 & 7.009 & 0.0 \\
\hline 320.020 & 69.996 & 18.271 & 110.32 & 110.64 & 0.290 & 0.0 \\
\hline 320.070 & 69.996 & 18.269 & 110.47 & 110.63 & 0.145 & 0.0 \\
\hline 320.000 & 59.998 & 17.227 & 101.51 & 102.54 & 1.015 & 0.0 \\
\hline 319.950 & 59.998 & 17.229 & 101.96 & 102.55 & 0.579 & 0.0 \\
\hline 319.950 & 50.001 & 15.941 & 92.80 & 93.83 & 1.110 & 0.0 \\
\hline 319.940 & 50.001 & 15.941 & 92.60 & 93.83 & 1.328 & 0.0 \\
\hline 319.980 & 40.004 & 14.271 & 83.05 & 84.24 & 1.433 & 0.0 \\
\hline 319.980 & 40.004 & 14.271 & 82.90 & 84.24 & 1.616 & 0.0 \\
\hline 319.960 & 29.999 & 11.964 & 72.75 & 73.30 & 0.756 & 0.0 \\
\hline 319.980 & 29.999 & 11.963 & 71.86 & 73.30 & 2.004 & 0.0 \\
\hline 320.060 & 20.002 & 8.610 & 59.19 & 60.10 & 1.537 & 0.0 \\
\hline 320.040 & 20.002 & 8.611 & 59.19 & 60.11 & 1.554 & 0.0 \\
\hline 319.980 & 10.004 & 4.220 & 44.92 & 46.00 & 2.404 & 0.0 \\
\hline 320.000 & 10.004 & 4.219 & 44.79 & 46.00 & 2.701 & 0.0 \\
\hline 339.800 & 69.996 & 17.451 & 106.60 & 107.14 & 0.507 & 0.0 \\
\hline 339.960 & 10.004 & 3.854 & 47.18 & 47.96 & 1.653 & 0.0 \\
\hline 339.980 & 10.004 & 3.854 & 47.67 & 47.97 & 0.629 & 0.0 \\
\hline
\end{tabular}


Data from Prasad et al. [92] (continued)

\begin{tabular}{|c|c|c|c|c|c|c|}
\hline $\mathrm{T}$ & $\mathrm{P}$ & $\rho$ & $\lambda, \exp$ & $\lambda, \mathrm{cal}$ & Dev & Wt \\
\hline $\mathrm{K}$ & $\mathrm{MPa}$ & $\mathrm{mol} \cdot \mathrm{dm}^{-3}$ & \multicolumn{2}{|c|}{$\mathrm{mW} \cdot \mathrm{m}^{-1} \cdot \mathrm{K}^{-1}$} & 8 & \\
\hline 340.000 & 10.004 & 3.853 & 47.67 & 47.97 & 0.629 & 0.0 \\
\hline 340.050 & 69.996 & 17.441 & 106.05 & 107.10 & 0.990 & 0.0 \\
\hline 340.020 & 69.996 & 17.442 & 105.66 & 107.11 & 1.372 & 0.0 \\
\hline 340.000 & 59.998 & 16.349 & 98.53 & 99.40 & 0.883 & 0.0 \\
\hline 340.020 & 59.998 & 16.348 & 98.39 & 99.40 & 1.027 & 0.0 \\
\hline 339.980 & 50.001 & 15.006 & 90.13 & 91.14 & 1.121 & 0.0 \\
\hline 339.960 & 40.004 & 13.284 & 81.33 & 82.11 & 0.959 & 0.0 \\
\hline 339.950 & 29.999 & 10.967 & 71.26 & 71.87 & 0.856 & 0.0 \\
\hline 340.230 & 20.002 & 7.782 & 58.67 & 59.93 & 2.148 & 0.0 \\
\hline 400.150 & 69.996 & 15.264 & 102.24 & 102.25 & 0.010 & 0.0 \\
\hline 400.070 & 59.998 & 14.092 & 95.35 & 95.67 & 0.336 & 0.0 \\
\hline 399.990 & 50.001 & 12.685 & 87.89 & 88.67 & 0.887 & 0.0 \\
\hline 399.950 & 40.004 & 10.956 & 79.47 & 81.10 & 2.051 & 0.0 \\
\hline 399.920 & 29.999 & 8.799 & 71.29 & 72.78 & 2.090 & 0.0 \\
\hline 399.880 & 20.002 & 6.150 & 63.87 & 63.93 & 0.094 & 0.0 \\
\hline 399.890 & 10.004 & 3.105 & 54.03 & 55.81 & 3.294 & 0.0 \\
\hline 379.920 & 69.996 & 15.945 & 102.25 & 103.07 & 0.802 & 0.0 \\
\hline 379.970 & 59.998 & 14.786 & 95.98 & 96.13 & 0.156 & 0.0 \\
\hline 379.980 & 50.001 & 13.383 & 88.08 & 88.74 & 0.749 & 0.0 \\
\hline 380.030 & 40.004 & 11.635 & 79.98 & 80.71 & 0.913 & 0.0 \\
\hline 379.970 & 40.004 & 11.637 & 79.93 & 80.71 & 0.976 & 0.0 \\
\hline 379.990 & 30.006 & 9.410 & 71.16 & 71.80 & 0.899 & 0.0 \\
\hline 380.090 & 20.002 & 6.594 & 61.76 & 62.06 & 0.486 & 0.0 \\
\hline 379.910 & 10.004 & 3.313 & 52.97 & 52.94 & -0.057 & 0.0 \\
\hline 359.900 & 69.996 & 16.670 & 104.39 & 104.65 & 0.249 & 0.0 \\
\hline 357.900 & 69.996 & 16.746 & 104.54 & 104.85 & 0.297 & 0.0 \\
\hline 359.890 & 59.998 & 15.540 & 96.62 & 97.33 & 0.735 & 0.0 \\
\hline 359.890 & 50.001 & 14.159 & 89.34 & 89.52 & 0.201 & 0.0 \\
\hline 359.900 & 40.004 & 12.412 & 80.52 & 81.00 & 0.596 & 0.0 \\
\hline 359.890 & 30.006 & 10.129 & 70.68 & 71.44 & 1.075 & 0.0 \\
\hline 359.910 & 20.002 & 7.136 & 60.69 & 60.65 & -0.066 & 0.0 \\
\hline 359.940 & 20.002 & 7.135 & 60.69 & 60.65 & -0.066 & 0.0 \\
\hline 359.940 & 10.011 & 3.561 & 50.07 & 50.31 & 0.479 & 0.0 \\
\hline 260.580 & 69.996 & 21.024 & 129.47 & 129.15 & -0.247 & 0.0 \\
\hline 260.510 & 59.998 & 20.190 & 119.32 & 120.09 & 0.645 & 0.0 \\
\hline
\end{tabular}


Data from Prasad et al. [92] (continued)

\begin{tabular}{|c|c|c|c|c|c|c|}
\hline $\mathrm{T}$ & $P$ & $\rho$ & $\lambda, \exp$ & $\lambda, \mathrm{cal}$ & Dev & Wt \\
\hline K & $\mathrm{MPa}$ & $\mathrm{mol} \cdot \mathrm{dm}^{-3}$ & \multicolumn{2}{|c|}{$\mathrm{mW} \cdot \mathrm{m}^{-1} \cdot \mathrm{K}^{-1}$} & 8 & \\
\hline 260.480 & 50.001 & 19.171 & 109.35 & 110.24 & 0.814 & 0.0 \\
\hline 260.530 & 40.004 & 17.855 & 98.79 & 99.23 & 0.445 & 0.0 \\
\hline 260.570 & 29.999 & 15.991 & 86.61 & 86.38 & -0.266 & 0.0 \\
\hline 260.710 & 20.009 & 12.803 & 71.73 & 70.01 & -2.398 & 0.0 \\
\hline 260.900 & 15.003 & 10.005 & 60.45 & 58.81 & -2.713 & 0.0 \\
\hline 261.150 & 20.009 & 12.759 & 70.62 & 69.86 & -1.076 & 0.0 \\
\hline 261.170 & 9.997 & 6.215 & 46.18 & 44.83 & -2.923 & 0.0 \\
\hline 261.690 & 4.999 & 2.666 & 32.65 & 34.40 & 5.360 & 0.0 \\
\hline 261.930 & 4.999 & 2.662 & 32.45 & 34.43 & 6.102 & 0.0 \\
\hline 277.150 & 69.996 & 20.216 & 122.16 & 122.61 & 0.368 & 0.0 \\
\hline 277.310 & 59.998 & 19.305 & 113.22 & 113.69 & 0.415 & 0.0 \\
\hline 277.430 & 49.994 & 18.188 & 103.41 & 104.04 & 0.609 & 0.0 \\
\hline 277.720 & 40.004 & 16.729 & 93.36 & 93.27 & -0.096 & 0.0 \\
\hline 277.980 & 29.999 & 14.651 & 81.06 & 80.80 & -0.321 & 0.0 \\
\hline 278.280 & 20.002 & 11.202 & 65.16 & 65.11 & -0.077 & 0.0 \\
\hline 278.520 & 15.003 & 8.531 & 55.87 & 54.89 & -1.754 & 0.0 \\
\hline 279.190 & 9.997 & 5.358 & 44.47 & 43.97 & -1.124 & 0.0 \\
\hline 279.510 & 5.006 & 2.413 & 35.72 & 36.08 & 1.008 & 0.0 \\
\hline 279.630 & 5.013 & 2.416 & 35.72 & 36.10 & 1.064 & 0.0 \\
\hline 279.990 & 9.997 & 5.327 & 44.61 & 43.97 & -1.435 & 0.0 \\
\hline
\end{tabular}

Number of Points (Ref.92) 180

$\begin{array}{llllll}\text { AAD }-8 & 1.525 & \text { BIAS }-8 & 1.280 & \text { RMS }-8 & 1.547 \\ \text { AAD } & 1.633 & \text { BIAS } & 1.474 & \text { RMS } & 1.383 \mathrm{~mW} \cdot \mathrm{m}^{-1} \cdot \mathrm{K}^{-1}\end{array}$

$\overline{\text { Data from Roder [8] }}$

$\begin{array}{ccccccc}\mathrm{T} & \mathrm{P} & \rho & \lambda, \exp & \lambda, \mathrm{cal} & \text { Dev } & \text { Wt } \\ \mathrm{K} & \mathrm{MPa} & \mathrm{mol} \cdot \mathrm{dm}^{-3} & \mathrm{~mW} \cdot \mathrm{m}^{-1} \cdot \mathrm{K}^{-1} & & \\ 111.827 & 0.561 & 26.348 & 184.27 & 184.23 & -0.022 & 9.7 \\ 111.655 & 0.556 & 26.363 & 184.76 & 184.46 & -0.162 & 9.7 \\ 111.255 & 0.551 & 26.399 & 184.75 & 185.02 & 0.146 & 9.7 \\ 110.983 & 0.546 & 26.423 & 185.12 & 185.39 & 0.146 & 9.7 \\ 111.762 & 11.644 & 26.945 & 196.81 & 195.99 & -0.417 & 9.5\end{array}$


Data from Roder [8] (continued)

\begin{tabular}{|c|c|c|c|c|c|c|}
\hline $\mathrm{T}$ & $\mathbf{P}$ & $\rho$ & $\lambda, \exp$ & $\lambda, \mathrm{cal}$ & Dev & Wt \\
\hline K & $\mathrm{MPa}$ & $\mathrm{mol} \cdot \mathrm{dm}^{-3}$ & \multicolumn{2}{|c|}{$\mathrm{mW} \cdot \mathrm{m}^{-1} \cdot \mathrm{K}^{-1}$} & 8 & \\
\hline 111.363 & 11.641 & 26.976 & 196.79 & 196.50 & -0.147 & 9.5 \\
\hline 111.215 & 11.635 & 26.988 & 197.54 & 196.68 & -0.435 & 9.5 \\
\hline 110.985 & 11.630 & 27.006 & 197.58 & 196.97 & -0.309 & 9.5 \\
\hline 111.768 & 22.698 & 27.445 & 207.04 & 206.53 & -0.246 & 9.3 \\
\hline 111.688 & 22.693 & 27.450 & 207.62 & 206.62 & -0.482 & 9.3 \\
\hline 111.301 & 22.688 & 27.479 & 207.75 & 207.07 & -0.327 & 9.3 \\
\hline 111.173 & 22.682 & 27.488 & 208.46 & 207.22 & -0.595 & 9.3 \\
\hline 111.613 & 34.119 & 27.909 & 216.81 & 216.80 & -0.005 & 9.2 \\
\hline 111.472 & 34.117 & 27.918 & 217.79 & 216.96 & -0.381 & 9.2 \\
\hline 111.231 & 34.112 & 27.935 & 217.61 & 217.22 & -0.179 & 9.2 \\
\hline 110.997 & 34.109 & 27.950 & 217.96 & 217.48 & -0.220 & 9.2 \\
\hline 111.508 & 44.939 & 28.301 & 225.69 & 225.92 & 0.102 & 9.1 \\
\hline 111.347 & 44.932 & 28.311 & 226.02 & 226.09 & 0.031 & 9.0 \\
\hline 111.101 & 44.926 & 28.326 & 226.24 & 226.34 & 0.044 & 9.0 \\
\hline 110.863 & 44.920 & 28.341 & 226.67 & 226.59 & -0.035 & 9.0 \\
\hline 111.433 & 56.744 & 28.687 & 234.94 & 235.35 & 0.175 & 8.9 \\
\hline 111.181 & 56.740 & 28.702 & 235.17 & 235.60 & 0.183 & 8.9 \\
\hline 110.963 & 56.735 & 28.715 & 235.42 & 235.81 & 0.166 & 8.9 \\
\hline 110.745 & 56.726 & 28.728 & 235.54 & 236.03 & 0.208 & 8.9 \\
\hline 111.218 & 68.635 & 29.052 & 243.25 & 244.57 & 0.543 & 8.8 \\
\hline 111.104 & 68.639 & 29.058 & 243.68 & 244.68 & 0.410 & 8.8 \\
\hline 110.894 & 68.647 & 29.070 & 243.81 & 244.88 & 0.439 & 8.8 \\
\hline 110.631 & 68.659 & 29.086 & 244.05 & 245.15 & 0.451 & 8.8 \\
\hline 134.645 & 0.316 & 0.303 & 14.83 & 14.60 & -1.551 & 14.7 \\
\hline 134.119 & 0.316 & 0.304 & 14.75 & 14.54 & -1.424 & 14.9 \\
\hline 133.606 & 0.315 & 0.305 & 14.75 & 14.49 & -1.763 & 14.4 \\
\hline 133.127 & 0.314 & 0.305 & 14.71 & 14.44 & -1.835 & 14.3 \\
\hline 135.369 & 0.778 & 24.035 & 151.36 & 150.68 & -0.449 & 10.4 \\
\hline 135.054 & 0.772 & 24.069 & 151.81 & 151.13 & -0.448 & 10.4 \\
\hline 134.663 & 0.761 & 24.110 & 152.03 & 151.69 & -0.224 & 10.4 \\
\hline 134.383 & 0.745 & 24.139 & 152.45 & 152.07 & -0.249 & 10.4 \\
\hline 135.395 & 11.718 & 24.951 & 166.43 & 165.43 & -0.601 & 10.1 \\
\hline 135.072 & 11.715 & 24.980 & 166.87 & 165.84 & -0.617 & 10.1 \\
\hline 134.724 & 11.709 & 25.010 & 167.08 & 166.28 & -0.479 & 10.1 \\
\hline 134.433 & 11.694 & 25.035 & 167.55 & 166.64 & -0.543 & 10.1 \\
\hline
\end{tabular}


Data from Roder [8] (continued)

\begin{tabular}{|c|c|c|c|c|c|c|}
\hline $\mathrm{T}$ & $\mathrm{P}$ & $\rho$ & $\lambda, \exp$ & $\lambda, \mathrm{cal}$ & Dev & Wt \\
\hline K & $\mathrm{MPa}$ & $\mathrm{mol} \cdot \mathrm{dm}^{-3}$ & \multicolumn{2}{|c|}{$\mathrm{mW} \cdot \mathrm{m}^{-1} \cdot \mathrm{K}^{-1}$} & $q$ & \\
\hline 135.255 & 22.964 & 25.687 & 179.51 & 178.46 & -0.585 & 9.8 \\
\hline 134.855 & 22.963 & 25.718 & 180.09 & 178.94 & -0.639 & 9.8 \\
\hline 134.596 & 22.961 & 25.738 & 179.96 & 179.24 & -0.400 & 9.8 \\
\hline 134.300 & 22.959 & 25.760 & 180.38 & 179.60 & -0.432 & 9.8 \\
\hline 135.304 & 34.181 & 26.279 & 190.82 & 189.87 & -0.498 & 9.6 \\
\hline 134.983 & 34.180 & 26.302 & 191.06 & 190.24 & -0.429 & 9.6 \\
\hline 134.717 & 34.177 & 26.320 & 191.53 & 190.53 & -0.522 & 9.6 \\
\hline 134.482 & 34.172 & 26.336 & 191.75 & 190.79 & -0.501 & 9.6 \\
\hline 135.055 & 45.585 & 26.817 & 201.44 & 200.89 & -0.273 & 9.4 \\
\hline 134.801 & 45.577 & 26.833 & 201.70 & 201.15 & -0.273 & 9.4 \\
\hline 134.607 & 45.574 & 26.846 & 202.24 & 201.36 & -0.435 & 9.4 \\
\hline 134.390 & 45.564 & 26.859 & 202.25 & 201.59 & -0.326 & 9.4 \\
\hline 135.242 & 56.761 & 27.257 & 210.85 & 210.56 & -0.138 & 9.3 \\
\hline 134.920 & 56.753 & 27.276 & 211.14 & 210.89 & -0.118 & 9.3 \\
\hline 134.722 & 56.743 & 27.288 & 211.86 & 211.08 & -0.368 & 9.3 \\
\hline 134.408 & 56.735 & 27.306 & 211.76 & 211.41 & -0.165 & 9.3 \\
\hline 135.053 & 67.925 & 27.675 & 219.96 & 220.11 & 0.068 & 9.1 \\
\hline 134.764 & 67.926 & 27.692 & 220.06 & 220.41 & 0.159 & 9.1 \\
\hline 134.496 & 67.926 & 27.707 & 220.36 & 220.68 & 0.145 & 9.1 \\
\hline 134.259 & 67.928 & 27.720 & 220.67 & 220.92 & 0.113 & 9.1 \\
\hline 145.151 & 0.357 & 0.315 & 15.97 & 15.80 & -1.064 & 16.1 \\
\hline 144.549 & 0.357 & 0.317 & 15.90 & 15.74 & -1.006 & 16.1 \\
\hline 144.034 & 0.357 & 0.318 & 15.84 & 15.68 & -1.010 & 16.1 \\
\hline 143.587 & 0.357 & 0.319 & 15.84 & 15.64 & -1.263 & 15.6 \\
\hline 144.746 & 0.650 & 0.612 & 16.58 & 16.58 & 0.000 & 16.7 \\
\hline 144.258 & 0.650 & 0.615 & 16.59 & 16.54 & -0.301 & 16.3 \\
\hline 143.690 & 0.650 & 0.619 & 16.60 & 16.50 & -0.602 & 15.9 \\
\hline 143.403 & 0.650 & 0.620 & 16.52 & 16.48 & -0.242 & 16.2 \\
\hline 154.710 & 0.429 & 0.355 & 17.08 & 16.99 & -0.527 & 17.5 \\
\hline 154.129 & 0.429 & 0.357 & 17.17 & 16.93 & -1.398 & 15.9 \\
\hline 153.687 & 0.429 & 0.358 & 16.97 & 16.88 & -0.530 & 17.5 \\
\hline 153.161 & 0.429 & 0.359 & 16.91 & 16.82 & -0.532 & 17.5 \\
\hline 155.000 & 0.731 & 0.635 & 17.81 & 17.70 & -0.618 & 17.0 \\
\hline 154.460 & 0.731 & 0.638 & 17.73 & 17.66 & -0.395 & 17.1 \\
\hline 153.983 & 0.731 & 0.641 & 17.71 & 17.61 & -0.565 & 17.0 \\
\hline
\end{tabular}


Data from Roder [8] (continued)

\begin{tabular}{|c|c|c|c|c|c|c|}
\hline $\mathrm{T}$ & $P$ & $\rho$ & $\lambda, \exp$ & $\lambda, \mathrm{cal}$ & Dev & Wt \\
\hline K & $\mathrm{MPa}$ & $\mathrm{mol} \cdot \mathrm{dm}^{-3}$ & \multicolumn{2}{|c|}{$\mathrm{mW} \cdot \mathrm{m}^{-1} \cdot \mathrm{K}^{-1}$} & z & \\
\hline 153.493 & 0.731 & 0.644 & 17.62 & 17.57 & -0.284 & 17.2 \\
\hline 154.611 & 1.082 & 1.014 & 18.76 & 18.80 & 0.213 & 16.6 \\
\hline 154.165 & 1.082 & 1.019 & 18.66 & 18.78 & 0.643 & 16.9 \\
\hline 153.622 & 1.082 & 1.025 & 18.64 & 18.76 & 0.644 & 16.8 \\
\hline 153.277 & 1.082 & 1.029 & 18.52 & 18.75 & 1.242 & 17.1 \\
\hline 155.515 & 1.600 & 21.645 & 121.79 & 122.07 & 0.230 & 11.3 \\
\hline 155.080 & 1.599 & 21.707 & 122.60 & 122.73 & 0.106 & 11.3 \\
\hline 154.711 & 1.598 & 21.759 & 123.06 & 123.30 & 0.195 & 11.3 \\
\hline 154.339 & 1.595 & 21.811 & 123.44 & 123.86 & 0.340 & 11.2 \\
\hline 155.315 & 6.757 & 22.476 & 131.93 & 132.27 & 0.258 & 11.0 \\
\hline 154.878 & 6.755 & 22.527 & 132.63 & 132.85 & 0.166 & 11.0 \\
\hline 154.565 & 6.754 & 22.562 & 132.94 & 133.27 & 0.248 & 11.0 \\
\hline 154.223 & 6.757 & 22.602 & 133.18 & 133.73 & 0.413 & 11.0 \\
\hline 155.484 & 14.577 & 23.343 & 143.99 & 144.11 & 0.083 & 10.7 \\
\hline 155.132 & 14.576 & 23.376 & 144.44 & 144.53 & 0.062 & 10.6 \\
\hline 154.797 & 14.575 & 23.408 & 144.87 & 144.92 & 0.035 & 10.6 \\
\hline 154.491 & 14.579 & 23.437 & 145.20 & 145.30 & 0.069 & 10.6 \\
\hline 155.595 & 25.316 & 24.239 & 157.93 & 157.73 & -0.127 & 10.3 \\
\hline 155.239 & 25.314 & 24.267 & 158.29 & 158.11 & -0.114 & 10.3 \\
\hline 154.966 & 25.310 & 24.289 & 158.67 & 158.41 & -0.164 & 10.3 \\
\hline 154.586 & 25.318 & 24.319 & 158.89 & 158.84 & -0.031 & 10.3 \\
\hline 155.724 & 36.001 & 24.941 & 169.52 & 169.51 & -0.006 & 10.1 \\
\hline 155.402 & 36.001 & 24.964 & 169.96 & 169.84 & -0.071 & 10.0 \\
\hline 154.976 & 35.997 & 24.994 & 170.56 & 170.28 & -0.164 & 10.0 \\
\hline 154.755 & 35.978 & 25.009 & 170.93 & 170.49 & -0.257 & 10.0 \\
\hline 155.563 & 46.637 & 25.547 & 180.80 & 180.44 & -0.199 & 9.8 \\
\hline 155.228 & 46.631 & 25.568 & 180.94 & 180.77 & -0.094 & 9.8 \\
\hline 154.890 & 46.626 & 25.589 & 181.48 & 181.11 & -0.204 & 9.8 \\
\hline 154.570 & 46.626 & 25.610 & 181.85 & 181.43 & -0.231 & 9.8 \\
\hline 155.419 & 56.930 & 26.055 & 190.60 & 190.28 & -0.168 & 9.6 \\
\hline 154.980 & 56.927 & 26.081 & 190.79 & 190.71 & -0.042 & 9.6 \\
\hline 154.681 & 56.921 & 26.099 & 191.18 & 190.99 & -0.099 & 9.6 \\
\hline 154.420 & 56.919 & 26.115 & 191.54 & 191.25 & -0.151 & 9.6 \\
\hline 155.151 & 67.027 & 26.508 & 199.46 & 199.53 & 0.035 & 9.5 \\
\hline 154.884 & 67.028 & 26.523 & 199.42 & 199.78 & 0.181 & 9.5 \\
\hline
\end{tabular}


Data from Roder [8] (continued)

\begin{tabular}{|c|c|c|c|c|c|c|}
\hline $\mathrm{T}$ & $\mathbf{P}$ & $\rho$ & $\lambda, \exp$ & $\lambda$, cal & Dev & Wt \\
\hline K & $\mathrm{MPa}$ & $\mathrm{mol} \cdot \mathrm{dm}^{-3}$ & \multicolumn{2}{|c|}{$\mathrm{mW} \cdot \mathrm{m}^{-1} \cdot \mathrm{K}^{-1}$} & 8 & \\
\hline 154.564 & 67.035 & 26.541 & 200.13 & 200.10 & -0.015 & 9.5 \\
\hline 154.234 & 67.043 & 26.560 & 200.37 & 200.42 & 0.025 & 9.5 \\
\hline 165.249 & 0.446 & 0.342 & 18.21 & 18.19 & -0.110 & 19.0 \\
\hline 164.627 & 0.446 & 0.344 & 18.11 & 18.12 & 0.055 & 19.5 \\
\hline 164.008 & 0.446 & 0.345 & 18.07 & 18.05 & -0.111 & 19.0 \\
\hline 163.654 & 0.446 & 0.346 & 18.03 & 18.01 & -0.111 & 19.0 \\
\hline 164.789 & 0.935 & 0.771 & 19.12 & 19.11 & -0.052 & 18.1 \\
\hline 164.336 & 0.935 & 0.774 & 19.12 & 19.07 & -0.262 & 17.8 \\
\hline 163.737 & 0.935 & 0.778 & 19.03 & 19.02 & -0.053 & 18.0 \\
\hline 163.372 & 0.935 & 0.781 & 18.91 & 18.98 & 0.370 & 18.5 \\
\hline 164.151 & 1.657 & 1.578 & 21.41 & 21.53 & 0.560 & 16.7 \\
\hline 163.770 & 1.657 & 1.587 & 21.44 & 21.53 & 0.420 & 16.5 \\
\hline 163.435 & 1.657 & 1.594 & 21.47 & 21.54 & 0.326 & 16.4 \\
\hline 163.111 & 1.657 & 1.601 & 21.41 & 21.54 & 0.607 & 16.5 \\
\hline 175.467 & 0.406 & 0.290 & 19.22 & 19.30 & 0.416 & 21.8 \\
\hline 174.780 & 0.406 & 0.291 & 19.09 & 19.22 & 0.681 & 23.3 \\
\hline 174.244 & 0.406 & 0.292 & 19.11 & 19.16 & 0.262 & 20.9 \\
\hline 173.672 & 0.406 & 0.293 & 19.08 & 19.09 & 0.052 & 20.0 \\
\hline 174.946 & 1.015 & 0.778 & 20.29 & 20.26 & -0.148 & 18.7 \\
\hline 174.370 & 1.015 & 0.782 & 20.15 & 20.21 & 0.298 & 19.2 \\
\hline 173.887 & 1.015 & 0.785 & 20.15 & 20.16 & 0.050 & 18.8 \\
\hline 173.424 & 1.015 & 0.788 & 20.10 & 20.11 & 0.050 & 18.8 \\
\hline 174.393 & 1.751 & 1.500 & 22.07 & 22.12 & 0.227 & 17.8 \\
\hline 173.984 & 1.751 & 1.507 & 22.03 & 22.10 & 0.318 & 17.8 \\
\hline 173.504 & 1.751 & 1.515 & 22.16 & 22.08 & -0.361 & 17.3 \\
\hline 173.135 & 1.752 & 1.523 & 21.94 & 22.07 & 0.593 & 17.8 \\
\hline 173.762 & 2.459 & 2.478 & 25.67 & 25.67 & 0.000 & 16.2 \\
\hline 173.415 & 2.460 & 2.497 & 25.79 & 25.74 & -0.194 & 16.1 \\
\hline 173.064 & 2.461 & 2.516 & 25.85 & 25.82 & -0.116 & 16.1 \\
\hline 172.766 & 2.462 & 2.533 & 25.28 & 25.89 & 2.413 & 16.9 \\
\hline 175.226 & 4.616 & 19.151 & 99.77 & 99.49 & -0.281 & 12.1 \\
\hline 174.785 & 4.616 & 19.236 & 100.43 & 100.18 & -0.249 & 12.1 \\
\hline 174.407 & 4.615 & 19.308 & 101.07 & 100.76 & -0.307 & 12.1 \\
\hline 174.021 & 4.616 & 19.381 & 101.29 & 101.35 & 0.059 & 12.1 \\
\hline 175.360 & 12.710 & 21.033 & 117.36 & 117.96 & 0.511 & 11.5 \\
\hline
\end{tabular}


Data from Roder [8] (continued)

\begin{tabular}{|c|c|c|c|c|c|c|}
\hline $\mathrm{T}$ & $P$ & $\rho$ & $\lambda, \exp$ & $\lambda, \mathrm{cal}$ & Dev & Wt \\
\hline K & $\mathrm{MPa}$ & $\mathrm{mol} \cdot \mathrm{dm}^{-3}$ & \multicolumn{2}{|c|}{$\mathrm{mW} \cdot \mathrm{m}^{-1} \cdot \mathrm{K}^{-1}$} & 8 & \\
\hline 175.020 & 12.708 & 21.072 & 117.69 & 118.34 & 0.552 & 11.5 \\
\hline 174.534 & 12.705 & 21.128 & 118.18 & 118.89 & 0.601 & 11.5 \\
\hline 174.088 & 12.712 & 21.180 & 118.62 & 119.41 & 0.666 & 11.5 \\
\hline 175.653 & 19.724 & 21.980 & 128.32 & 129.05 & 0.569 & 11.2 \\
\hline 175.178 & 19.722 & 22.025 & 129.01 & 129.53 & 0.403 & 11.1 \\
\hline 174.793 & 19.720 & 22.061 & 129.57 & 129.93 & 0.278 & 11.1 \\
\hline 174.357 & 19.724 & 22.103 & 129.80 & 130.38 & 0.447 & 11.1 \\
\hline 175.719 & 26.457 & 22.692 & 137.72 & 138.26 & 0.392 & 10.9 \\
\hline 175.380 & 26.455 & 22.720 & 138.11 & 138.58 & 0.340 & 10.9 \\
\hline 174.924 & 26.450 & 22.757 & 138.61 & 139.02 & 0.296 & 10.9 \\
\hline 174.570 & 26.446 & 22.787 & 138.79 & 139.36 & 0.411 & 10.9 \\
\hline 175.558 & 33.801 & 23.346 & 147.01 & 147.42 & 0.279 & 10.6 \\
\hline 175.163 & 33.796 & 23.375 & 147.43 & 147.78 & 0.237 & 10.6 \\
\hline 174.813 & 33.792 & 23.401 & 147.73 & 148.11 & 0.257 & 10.6 \\
\hline 174.483 & 33.794 & 23.425 & 148.27 & 148.42 & 0.101 & 10.6 \\
\hline 175.820 & 41.291 & 23.881 & 155.22 & 155.57 & 0.225 & 10.4 \\
\hline 175.338 & 41.288 & 23.914 & 155.73 & 156.00 & 0.173 & 10.4 \\
\hline 175.061 & 41.285 & 23.933 & 156.08 & 156.25 & 0.109 & 10.4 \\
\hline 174.654 & 41.276 & 23.960 & 156.53 & 156.61 & 0.051 & 10.4 \\
\hline 175.705 & 48.148 & 24.335 & 162.60 & 162.84 & 0.148 & 10.3 \\
\hline 175.305 & 48.149 & 24.361 & 162.91 & 163.20 & 0.178 & 10.2 \\
\hline 174.788 & 48.142 & 24.393 & 163.32 & 163.65 & 0.202 & 10.2 \\
\hline 174.596 & 48.145 & 24.406 & 163.48 & 163.83 & 0.214 & 10.2 \\
\hline 175.547 & 55.061 & 24.748 & 169.59 & 169.82 & 0.136 & 10.1 \\
\hline 175.051 & 55.062 & 24.778 & 169.93 & 170.26 & 0.194 & 10.1 \\
\hline 174.704 & 55.058 & 24.799 & 170.64 & 170.56 & -0.047 & 10.1 \\
\hline 174.439 & 55.051 & 24.815 & 170.91 & 170.79 & -0.070 & 10.1 \\
\hline 175.386 & 61.637 & 25.107 & 175.83 & 176.18 & 0.199 & 10.0 \\
\hline 175.017 & 61.642 & 25.128 & 176.36 & 176.51 & 0.085 & 9.9 \\
\hline 174.659 & 61.637 & 25.149 & 176.57 & 176.81 & 0.136 & 9.9 \\
\hline 174.273 & 61.641 & 25.171 & 177.02 & 177.15 & 0.073 & 9.9 \\
\hline 175.485 & 68.363 & 25.431 & 181.95 & 182.21 & 0.143 & 9.8 \\
\hline 175.204 & 68.360 & 25.446 & 182.24 & 182.45 & 0.115 & 9.8 \\
\hline 174.804 & 68.375 & 25.469 & 182.35 & 182.81 & 0.252 & 9.8 \\
\hline 174.506 & 68.379 & 25.486 & 182.64 & 183.07 & 0.235 & 9.8 \\
\hline
\end{tabular}


Data from Roder [8] (continued)

\begin{tabular}{|c|c|c|c|c|c|c|}
\hline $\mathrm{T}$ & $\mathrm{P}$ & $\rho$ & $\lambda, \exp$ & $\lambda, \mathrm{cal}$ & Dev & Wt \\
\hline K & $\mathrm{MPa}$ & $\mathrm{mol} \cdot \mathrm{dm}^{-3}$ & \multicolumn{2}{|c|}{$\mathrm{mW} \cdot \mathrm{m}^{-1} \cdot \mathrm{K}^{-1}$} & 8 & \\
\hline 185.421 & 0.752 & 0.520 & 20.72 & 20.96 & 1.158 & 23.4 \\
\hline 184.903 & 0.752 & 0.522 & 20.77 & 20.90 & 0.626 & 21.4 \\
\hline 184.371 & 0.752 & 0.524 & 20.79 & 20.84 & 0.241 & 20.3 \\
\hline 183.963 & 0.752 & 0.525 & 20.11 & 20.79 & 3.381 & 44.7 \\
\hline 185.602 & 1.507 & 1.124 & 22.16 & 22.33 & 0.767 & 20.0 \\
\hline 185.087 & 1.508 & 1.130 & 22.09 & 22.28 & 0.860 & 20.0 \\
\hline 184.516 & 1.508 & 1.135 & 22.06 & 22.23 & 0.771 & 19.8 \\
\hline 184.171 & 1.508 & 1.138 & 21.51 & 22.19 & 3.161 & 23.4 \\
\hline 185.078 & 2.252 & 1.856 & 24.16 & 24.28 & 0.497 & 18.6 \\
\hline 184.541 & 2.251 & 1.866 & 23.97 & 24.25 & 1.168 & 19.0 \\
\hline 184.292 & 2.252 & 1.872 & 23.74 & 24.25 & 2.148 & 19.6 \\
\hline 183.844 & 2.252 & 1.882 & 24.07 & 24.23 & 0.665 & 18.5 \\
\hline 184.688 & 2.884 & 2.664 & 26.94 & 27.11 & 0.631 & 17.7 \\
\hline 184.300 & 2.884 & 2.681 & 26.99 & 27.14 & 0.556 & 17.6 \\
\hline 183.918 & 2.884 & 2.697 & 26.92 & 27.18 & 0.966 & 17.7 \\
\hline 183.674 & 2.885 & 2.710 & 27.23 & 27.21 & -0.073 & 17.2 \\
\hline 184.243 & 3.449 & 3.735 & 32.46 & 32.48 & 0.062 & 16.5 \\
\hline 183.964 & 3.449 & 3.766 & 32.51 & 32.67 & 0.492 & 16.6 \\
\hline 183.733 & 3.450 & 3.795 & 33.64 & 32.86 & -2.319 & 15.7 \\
\hline 183.486 & 3.450 & 3.825 & 34.04 & 33.06 & -2.879 & 15.5 \\
\hline 199.676 & 1.013 & 0.654 & 22.71 & 22.86 & 0.661 & 21.5 \\
\hline 199.006 & 1.013 & 0.657 & 22.69 & 22.79 & 0.441 & 20.9 \\
\hline 199.033 & 1.014 & 0.658 & 22.60 & 22.79 & 0.841 & 22.1 \\
\hline 198.544 & 1.014 & 0.660 & 22.58 & 22.74 & 0.709 & 21.7 \\
\hline 199.330 & 1.531 & 1.032 & 23.44 & 23.58 & 0.597 & 20.6 \\
\hline 198.735 & 1.531 & 1.036 & 23.44 & 23.53 & 0.384 & 20.2 \\
\hline 198.347 & 1.532 & 1.040 & 23.24 & 23.49 & 1.076 & 21.4 \\
\hline 198.016 & 1.531 & 1.042 & 22.90 & 23.46 & 2.445 & 24.2 \\
\hline 199.379 & 2.669 & 2.000 & 25.82 & 25.88 & 0.232 & 19.3 \\
\hline 198.893 & 2.670 & 2.011 & 25.92 & 25.87 & -0.193 & 18.9 \\
\hline 198.471 & 2.671 & 2.020 & 25.86 & 25.86 & 0.000 & 19.1 \\
\hline 198.055 & 2.669 & 2.026 & 25.89 & 25.84 & -0.193 & 18.9 \\
\hline 198.901 & 3.520 & 2.942 & 28.76 & 28.69 & -0.243 & 18.4 \\
\hline 198.535 & 3.520 & 2.956 & 28.94 & 28.72 & -0.760 & 18.2 \\
\hline 198.178 & 3.520 & 2.971 & 28.84 & 28.75 & -0.312 & 18.4 \\
\hline
\end{tabular}


Data from Roder [8] (continued)

$\mathrm{T}$

K

197.847
198.920
198.545
198.129
197.856

198.576

198.243

197.957

197.623

198.546

198.321

197.947

197.737

198.059

197.769

197.600

197.361

197.928

197.711

197.542

197.323

197.897

197.733

197.549

197.367

197.769

197.637

197.424

197.318

197.894

197.711

197.560

197.396

198.115

197.914

$$
\text { P }
$$

$\mathrm{MPa} \mathrm{mol} \cdot \mathrm{dm}^{-3}$

\begin{abstract}
3.519
\end{abstract}
4.156

4.156

4.156

4.156

4.568

4.568

4.569

4.568

4.860

4.860

4.861

4.861

5.049

5.049

5.049

5.049

5.172

5.172

5.172

\subsection{2}

5.229

5.229

5.230

5.229

5.328

5.328

5.329

5.328

5.407

5.408

5.408

5.407

5.500

5.500
2.983

3. 845

3. 872

3.904

3.925

4.642

4.683

4.721

4.763

5. 348

5. 390

5.467

5.510

6.048

6.133

6.185

6.264

6.580

6.667

6.739

6.839

6.858

6.934

7.031

7.122

7.467

7.553

7.709

7.782

7.894

8.046

8.176

8.320

8.384

8.573 $\lambda, \exp$

$\lambda, \mathrm{cal}$

$$
\mathrm{mW} \cdot \mathrm{m}^{-1} \cdot \mathrm{K}^{-1}
$$

28.74

32.41

32.46

32.35

32.71

36.11

36.40

36.66

36.88

40.13

40.52

40.67

40.65

44.89

44.98

45.24

45.18

48.44

49.06

49.72

49.96

50.60

51.05

51.94

52.49

53.77

54.38

55.10

55.35

57.20

57.96

58.54

59.71

61.27

61.86
28.79

32.16

32.29

32.44

32.55

36.01

36.28

36.53

36.83

40.08

40.41

41.03

41.39

44.97

45.76

46.26

47.03

49.04

49.90

50.64

51.67

51.24

52.01

52.99

53.96

56.24

57.10

58.67

59.43

59.34

60.76

61.97

63.31

62.37

63.92
Dev wt

용

$\begin{array}{rr}0.174 & 18.6 \\ -0.771 & 17.8 \\ -0.524 & 17.8 \\ 0.278 & 18.1 \\ -0.489 & 17.8\end{array}$

$\begin{array}{ll}-0.277 & 17.6\end{array}$

$-0.330 \quad 17.5$

$\begin{array}{ll}-0.355 & 17.5\end{array}$

$-0.136 \quad 17.5$

$-0.125 \quad 17.3$

$\begin{array}{ll}-0.271 & 17.2\end{array}$

$0.885 \quad 17.5$

$1.820 \quad 17.8$

$0.178 \quad 17.0$

$1.734 \quad 17.5$

$2.255 \quad 17.6$

$4.095 \quad 18.2$

$1.239 \quad 17.1$

$1.712 \quad 17.2$

$1.850 \quad 17.2$

$3.423 \quad 17.6$

$1.265 \quad 17.0$

$1.881 \quad 17.1$

$2.022 \quad 17.1$

$2.801 \quad 17.3$

$4.594 \quad 0.0$

$5.002 \quad 0.0$

$6.479 \quad 0.0$

$7.371 \quad 0.0$

$3.741 \quad 0.0$

$4.831 \quad 0.0$

$5.859 \quad 0.0$

$6.029 \quad 0.0$

$1.795 \quad 0.0$

$3.330 \quad 0.0$ 
Data from Roder [8] (continued)

\begin{tabular}{|c|c|c|c|c|c|c|}
\hline $\mathrm{T}$ & $\mathbf{P}$ & $\rho$ & $\lambda, \exp$ & $\lambda, \mathrm{cal}$ & Dev & Wt \\
\hline K & $\mathrm{MPa}$ & $\mathrm{mol} \cdot \mathrm{dm}^{-3}$ & \multicolumn{2}{|c|}{$\mathrm{mW} \cdot \mathrm{m}^{-1} \cdot \mathrm{K}^{-1}$} & 8 & \\
\hline 197.829 & 5.500 & 8.659 & 62.56 & 64.59 & 3.245 & 0.0 \\
\hline 197.597 & 5.499 & 8.901 & 63.01 & 66.41 & 5.396 & 0.0 \\
\hline 198.226 & 5.692 & 9.855 & 68.75 & 68.57 & -0.262 & 15.4 \\
\hline 198.053 & 5.692 & 10.068 & 69.21 & 69.31 & 0.144 & 15.4 \\
\hline 197.882 & 5.692 & 10.286 & 69.19 & 69.92 & 1.055 & 15.5 \\
\hline 197.678 & 5.693 & 10.559 & 70.18 & 70.51 & 0.470 & 15.2 \\
\hline 198.474 & 5.965 & 11.521 & 71.59 & 69.54 & -2.864 & 14.4 \\
\hline 198.275 & 5.966 & 11.722 & 71.75 & 69.80 & -2.718 & 14.3 \\
\hline 198.070 & 5.966 & 11.919 & 72.03 & 70.05 & -2.749 & 14.3 \\
\hline 197.904 & 5.967 & 12.079 & 72.53 & 70.24 & -3.157 & 14.2 \\
\hline 198.471 & 6.571 & 13.638 & 74.06 & 71.86 & -2.971 & 13.7 \\
\hline 198.290 & 6.571 & 13.733 & 73.72 & 72.13 & -2.157 & 13.8 \\
\hline 198.023 & 6.572 & 13.872 & 74.24 & 72.54 & -2.290 & 13.7 \\
\hline 197.943 & 6.571 & 13.910 & 74.35 & 72.66 & -2.273 & 13.7 \\
\hline 198.650 & 7.445 & 14.955 & 77.03 & 75.94 & -1.415 & 13.5 \\
\hline 198.306 & 7.446 & 15.073 & 77.59 & 76.44 & -1.482 & 13.4 \\
\hline 197.917 & 7.447 & 15.202 & 77.58 & 77.00 & -0.748 & 13.5 \\
\hline 197.672 & 7.447 & 15.282 & 78.22 & 77.35 & -1.112 & 13.4 \\
\hline 198.998 & 8.899 & 16.143 & 82.02 & 81.47 & -0.671 & 13.2 \\
\hline 198.586 & 8.899 & 16.239 & 82.43 & 81.98 & -0.546 & 13.1 \\
\hline 198.134 & 8.900 & 16.345 & 82.95 & 82.55 & -0.482 & 13.1 \\
\hline 197.859 & 8.900 & 16.408 & 83.31 & 82.89 & -0.504 & 13.1 \\
\hline 199.382 & 11.180 & 17.276 & 88.43 & 88.28 & -0.170 & 12.8 \\
\hline 198.850 & 11.180 & 17.369 & 88.94 & 88.86 & -0.090 & 12.8 \\
\hline 198.640 & 11.180 & 17.405 & 89.21 & 89.08 & -0.146 & 12.8 \\
\hline 198.159 & 11.180 & 17.487 & 89.73 & 89.61 & -0.134 & 12.7 \\
\hline 199.233 & 14.451 & 18.412 & 96.32 & 96.48 & 0.166 & 12.4 \\
\hline 198.829 & 14.449 & 18.466 & 96.69 & 96.87 & 0.186 & 12.4 \\
\hline 198.513 & 14.449 & 18.508 & 97.01 & 97.17 & 0.165 & 12.4 \\
\hline 197.985 & 14.452 & 18.580 & 97.36 & 97.69 & 0.339 & 12.4 \\
\hline 199.647 & 19.223 & 19.463 & 105.59 & 105.42 & -0.161 & 12.0 \\
\hline 199.171 & 19.221 & 19.515 & 105.73 & 105.84 & 0.104 & 12.0 \\
\hline 198.766 & 19.217 & 19.558 & 105.77 & 106.19 & 0.397 & 12.0 \\
\hline 198.521 & 19.214 & 19.584 & 106.75 & 106.40 & -0.328 & 12.0 \\
\hline 200.860 & 26.073 & 20.469 & 114.55 & 115.34 & 0.690 & 11.7 \\
\hline
\end{tabular}


Data from Roder [8] (continued)

\begin{tabular}{|c|c|c|c|c|c|c|}
\hline $\mathrm{T}$ & $\mathbf{P}$ & $\rho$ & $\lambda, \exp$ & $\lambda, \mathrm{cal}$ & Dev & Wt \\
\hline K & $\mathrm{MPa}$ & $\mathrm{mol} \cdot \mathrm{dm}^{-3}$ & \multicolumn{2}{|c|}{$\mathrm{mW} \cdot \mathrm{m}^{-1} \cdot \mathrm{K}^{-1}$} & 8 & \\
\hline 200.358 & 26.073 & 20.514 & 115.03 & 115.74 & 0.617 & 11.7 \\
\hline 199.942 & 26.070 & 20.551 & 115.46 & 116.08 & 0.537 & 11.7 \\
\hline 199.516 & 26.067 & 20.589 & 115.52 & 116.42 & 0.779 & 11.7 \\
\hline 201.141 & 35.267 & 21.550 & 126.69 & 127.38 & 0.545 & 11.3 \\
\hline 200.642 & 35.265 & 21.588 & 127.33 & 127.76 & 0.338 & 11.3 \\
\hline 200.211 & 35.263 & 21.620 & 127.63 & 128.09 & 0.360 & 11.3 \\
\hline 199.725 & 35.263 & 21.657 & 127.79 & 128.47 & 0.532 & 11.3 \\
\hline 200.885 & 47.247 & 22.643 & 140.78 & 141.27 & 0.348 & 10.9 \\
\hline 200.367 & 47.244 & 22.677 & 141.41 & 141.65 & 0.170 & 10.8 \\
\hline 199.989 & 47.240 & 22.701 & 141.38 & 141.93 & 0.389 & 10.9 \\
\hline 199.680 & 47.238 & 22.721 & 141.74 & 142.16 & 0.296 & 10.8 \\
\hline 201.164 & 62.619 & 23.684 & 156.25 & 156.47 & 0.141 & 10.5 \\
\hline 200.687 & 62.619 & 23.711 & 156.68 & 156.82 & 0.089 & 10.4 \\
\hline 200.378 & 62.620 & 23.729 & 156.87 & 157.05 & 0.115 & 10.4 \\
\hline 200.002 & 62.621 & 23.750 & 157.19 & 157.32 & 0.083 & 10.4 \\
\hline 201.156 & 69.413 & 24.079 & 163.11 & 162.77 & -0.208 & 10.3 \\
\hline 200.706 & 69.417 & 24.104 & 163.28 & 163.10 & -0.110 & 10.3 \\
\hline 200.328 & 69.421 & 24.125 & 163.56 & 163.38 & -0.110 & 10.3 \\
\hline 200.030 & 69.426 & 24.141 & 163.91 & 163.60 & -0.189 & 10.3 \\
\hline 215.053 & 1.068 & 0.633 & 24.40 & 24.58 & 0.738 & 22.1 \\
\hline 214.489 & 1.068 & 0.635 & 24.27 & 24.52 & 1.030 & 23.0 \\
\hline 213.947 & 1.068 & 0.637 & 24.32 & 24.46 & 0.576 & 21.5 \\
\hline 213.426 & 1.068 & 0.639 & 24.11 & 24.40 & 1.203 & 23.7 \\
\hline 215.328 & 1.747 & 1.076 & 25.39 & 25.46 & 0.276 & 20.1 \\
\hline 214.790 & 1.747 & 1.080 & 25.34 & 25.41 & 0.276 & 20.1 \\
\hline 214.260 & 1.747 & 1.083 & 25.27 & 25.35 & 0.317 & 20.1 \\
\hline 213.806 & 1.748 & 1.087 & 25.12 & 25.31 & 0.756 & 20.8 \\
\hline 214.864 & 3.035 & 2.049 & 27.64 & 27.54 & -0.362 & 18.8 \\
\hline 214.326 & 3.036 & 2.059 & 27.49 & 27.51 & 0.073 & 19.1 \\
\hline 213.846 & 3.036 & 2.067 & 27.86 & 27.48 & -1.364 & 18.1 \\
\hline 213.566 & 3.038 & 2.074 & 27.61 & 27.46 & -0.543 & 18.7 \\
\hline 214.431 & 4.165 & 3.103 & 30.69 & 30.34 & -1.140 & 17.9 \\
\hline 214.021 & 4.165 & 3.117 & 30.68 & 30.34 & -1.108 & 17.9 \\
\hline 213.625 & 4.165 & 3.131 & 30.67 & 30.35 & -1.043 & 18.0 \\
\hline 213.296 & 4.165 & 3.143 & 30.69 & 30.35 & -1.108 & 17.9 \\
\hline
\end{tabular}


Data from Roder [8] (continued)

\begin{tabular}{|c|c|c|c|c|c|c|}
\hline $\mathrm{T}$ & $\mathbf{P}$ & $\rho$ & $\lambda, \exp$ & $\lambda, \mathrm{cal}$ & Dev & Wt \\
\hline $\mathrm{K}$ & $\mathrm{MPa}$ & $\mathrm{mol} \cdot \mathrm{dm}^{-3}$ & \multicolumn{2}{|c|}{$\mathrm{mW} \cdot \mathrm{m}^{-1} \cdot \mathrm{K}^{-1}$} & 8 & \\
\hline 215.344 & 5.021 & 4.028 & 33.84 & 33.41 & -1.271 & 17.5 \\
\hline 214.428 & 5.021 & 4.080 & 33.98 & 33.53 & -1.324 & 17.5 \\
\hline 213.774 & 5.021 & 4.119 & 34.21 & 33.63 & -1.695 & 17.3 \\
\hline 213.072 & 5.021 & 4.162 & 34.39 & 33.75 & -1.861 & 17.2 \\
\hline 214.675 & 5.810 & 5.168 & 38.44 & 37.76 & -1.769 & 16.9 \\
\hline 214.439 & 5.811 & 5.192 & 38.21 & 37.86 & -0.916 & 17.1 \\
\hline 214.014 & 5.813 & 5.238 & 38.06 & 38.05 & -0.026 & 17.4 \\
\hline 213.567 & 5.810 & 5.279 & 39.16 & 38.22 & -2.400 & 16.6 \\
\hline 214.369 & 6.372 & 6.164 & 42.81 & 42.14 & -1.565 & 16.5 \\
\hline 214.079 & 6.373 & 6.209 & 42.83 & 42.36 & -1.097 & 16.6 \\
\hline 213.712 & 6.370 & 6.259 & 42.67 & 42.61 & -0.141 & 16.9 \\
\hline 213.474 & 6.371 & 6.298 & 43.09 & 42.81 & -0.650 & 16.7 \\
\hline 213.934 & 6.928 & 7.386 & 47.97 & 47.87 & -0.208 & 16.4 \\
\hline 213.759 & 6.929 & 7.428 & 48.31 & 48.09 & -0.455 & 16.3 \\
\hline 213.375 & 6.929 & 7.516 & 48.62 & 48.56 & -0.123 & 16.3 \\
\hline 213.185 & 6.930 & 7.563 & 49.03 & 48.81 & -0.449 & 16.2 \\
\hline 213.566 & 7.356 & 8.495 & 52.95 & 52.91 & -0.076 & 15.9 \\
\hline 213.304 & 7.357 & 8.575 & 53.01 & 53.30 & 0.547 & 16.0 \\
\hline 213.126 & 7.358 & 8.631 & 53.68 & 53.58 & -0.186 & 15.9 \\
\hline 212.961 & 7.356 & 8.677 & 53.66 & 53.80 & 0.261 & 15.9 \\
\hline 213.712 & 7.759 & 9.444 & 56.44 & 56.66 & 0.390 & 15.6 \\
\hline 213.375 & 7.759 & 9.558 & 57.37 & 57.15 & -0.383 & 15.5 \\
\hline 213.200 & 7.760 & 9.620 & 57.56 & 57.41 & -0.261 & 15.5 \\
\hline 213.080 & 7.759 & 9.660 & 57.26 & 57.58 & 0.559 & 15.6 \\
\hline 213.798 & 8.254 & 10.564 & 60.38 & 60.44 & 0.099 & 15.2 \\
\hline 213.577 & 8.255 & 10.643 & 60.79 & 60.73 & -0.099 & 15.1 \\
\hline 213.415 & 8.255 & 10.700 & 61.04 & 60.94 & -0.164 & 15.1 \\
\hline 213.191 & 8.255 & 10.780 & 61.31 & 61.22 & -0.147 & 15.1 \\
\hline 213.742 & 8.697 & 11.473 & 63.46 & 63.22 & -0.378 & 14.8 \\
\hline 213.550 & 8.697 & 11.537 & 63.92 & 63.44 & -0.751 & 14.7 \\
\hline 213.349 & 8.698 & 11.607 & 64.11 & 63.67 & -0.686 & 14.7 \\
\hline 213.104 & 8.696 & 11.686 & 64.49 & 63.93 & -0.868 & 14.6 \\
\hline 213.965 & 9.310 & 12.406 & 66.72 & 66.07 & -0.974 & 14.4 \\
\hline 213.642 & 9.311 & 12.504 & 67.07 & 66.41 & -0.984 & 14.3 \\
\hline 213.475 & 9.312 & 12.556 & 67.04 & 66.58 & -0.686 & 14.4 \\
\hline
\end{tabular}


Data from Roder [8] (continued)

\begin{tabular}{|c|c|c|c|c|c|c|}
\hline $\mathrm{T}$ & $\mathbf{P}$ & $\rho$ & $\lambda, \exp$ & $\lambda, \mathrm{cal}$ & Dev & Wt \\
\hline K & $\mathrm{MPa}$ & $\mathrm{mol} \cdot \mathrm{dm}^{-3}$ & \multicolumn{2}{|c|}{$\mathrm{mW} \cdot \mathrm{m}^{-1} \cdot \mathrm{K}^{-1}$} & 8 & \\
\hline 213.336 & 9.309 & 12.593 & 67.24 & 66.71 & -0.788 & 14.3 \\
\hline 214.655 & 10.068 & 13.183 & 69.21 & 68.69 & -0.751 & 14.1 \\
\hline 214.386 & 10.069 & 13.255 & 69.76 & 68.96 & -1.147 & 14.1 \\
\hline 214.165 & 10.069 & 13.313 & 69.79 & 69.17 & -0.888 & 14.1 \\
\hline 213.890 & 10.070 & 13.386 & 70.16 & 69.44 & -1.026 & 14.0 \\
\hline 214.865 & 11.106 & 14.143 & 73.03 & 72.46 & -0.781 & 13.8 \\
\hline 214.535 & 11.106 & 14.216 & 73.33 & 72.76 & -0.777 & 13.8 \\
\hline 214.277 & 11.106 & 14.274 & 73.71 & 72.99 & -0.977 & 13.8 \\
\hline 213.865 & 11.107 & 14.365 & 74.08 & 73.38 & -0.945 & 13.7 \\
\hline 215.388 & 12.417 & 14.987 & 76.99 & 76.33 & -0.857 & 13.5 \\
\hline 214.771 & 12.418 & 15.104 & 77.41 & 76.86 & -0.711 & 13.5 \\
\hline 214.164 & 12.420 & 15.219 & 78.00 & 77.40 & -0.769 & 13.5 \\
\hline 213.575 & 12.422 & 15.330 & 78.22 & 77.93 & -0.371 & 13.5 \\
\hline 215.850 & 14.257 & 15.899 & 81.17 & 81.16 & -0.012 & 13.3 \\
\hline 215.227 & 14.257 & 15.997 & 81.80 & 81.67 & -0.159 & 13.3 \\
\hline 214.710 & 14.257 & 16.079 & 82.21 & 82.09 & -0.146 & 13.2 \\
\hline 214.290 & 14.256 & 16.144 & 82.28 & 82.44 & 0.194 & 13.2 \\
\hline 216.298 & 16.764 & 16.828 & 86.64 & 86.84 & 0.231 & 13.0 \\
\hline 215.635 & 16.766 & 16.917 & 87.24 & 87.35 & 0.126 & 13.0 \\
\hline 215.026 & 16.764 & 16.997 & 87.62 & 87.82 & 0.228 & 12.9 \\
\hline 214.509 & 16.767 & 17.067 & 87.84 & 88.23 & 0.444 & 12.9 \\
\hline 215.935 & 20.275 & 17.890 & 93.75 & 94.22 & 0.501 & 12.6 \\
\hline 215.396 & 20.274 & 17.950 & 94.22 & 94.62 & 0.425 & 12.6 \\
\hline 214.891 & 20.273 & 18.007 & 94.76 & 94.99 & 0.243 & 12.6 \\
\hline 214.387 & 20.270 & 18.062 & 94.91 & 95.36 & 0.474 & 12.6 \\
\hline 216.265 & 24.891 & 18.846 & 101.43 & 101.91 & 0.473 & 12.3 \\
\hline 215.719 & 24.888 & 18.898 & 101.99 & 102.29 & 0.294 & 12.3 \\
\hline 215.148 & 24.888 & 18.952 & 102.20 & 102.69 & 0.479 & 12.3 \\
\hline 214.700 & 24.886 & 18.995 & 102.68 & 103.01 & 0.321 & 12.2 \\
\hline 215.940 & 31.251 & 19.899 & 111.15 & 111.47 & 0.288 & 11.9 \\
\hline 215.396 & 31.251 & 19.944 & 111.37 & 111.84 & 0.422 & 11.9 \\
\hline 214.911 & 31.248 & 19.983 & 111.76 & 112.17 & 0.367 & 11.9 \\
\hline 214.518 & 31.245 & 20.015 & 111.99 & 112.44 & 0.402 & 11.9 \\
\hline 215.647 & 39.620 & 20.942 & 121.78 & 122.29 & 0.419 & 11.5 \\
\hline 215.127 & 39.618 & 20.979 & 122.24 & 122.63 & 0.319 & 11.5 \\
\hline
\end{tabular}


Data from Roder [8] (continued)

\begin{tabular}{|c|c|c|c|c|c|c|}
\hline $\mathrm{T}$ & $\mathbf{P}$ & $\rho$ & $\lambda, \exp$ & $\lambda, \mathrm{cal}$ & Dev & Wt \\
\hline $\mathrm{K}$ & $\mathrm{MPa}$ & $\mathrm{mol} \cdot \mathrm{dm}^{-3}$ & \multicolumn{2}{|c|}{$\mathrm{mW} \cdot \mathrm{m}^{-1} \cdot \mathrm{K}^{-1}$} & 8 & \\
\hline 214.645 & 39.614 & 21.013 & 122.54 & 122.95 & 0.335 & 11.5 \\
\hline 214.236 & 39.613 & 21.042 & 122.71 & 123.23 & 0.424 & 11.5 \\
\hline 215.862 & 48.553 & 21.785 & 131.78 & 132.20 & 0.319 & 11.2 \\
\hline 215.441 & 48.554 & 21.812 & 132.02 & 132.47 & 0.341 & 11.2 \\
\hline 214.911 & 48.549 & 21.845 & 132.38 & 132.82 & 0.332 & 11.2 \\
\hline 214.480 & 48.547 & 21.873 & 132.96 & 133.10 & 0.105 & 11.1 \\
\hline 216.114 & 57.652 & 22.492 & 141.03 & 141.36 & 0.234 & 10.9 \\
\hline 215.631 & 57.648 & 22.520 & 141.39 & 141.67 & 0.198 & 10.9 \\
\hline 215.140 & 57.648 & 22.549 & 141.70 & 141.99 & 0.205 & 10.9 \\
\hline 214.778 & 57.645 & 22.570 & 141.82 & 142.23 & 0.289 & 10.9 \\
\hline 215.841 & 66.100 & 23.084 & 149.35 & 149.62 & 0.181 & 10.7 \\
\hline 215.368 & 66.103 & 23.110 & 149.75 & 149.93 & 0.120 & 10.7 \\
\hline 214.932 & 66.101 & 23.134 & 150.16 & 150.21 & 0.033 & 10.6 \\
\hline 214.525 & 66.103 & 23.157 & 150.37 & 150.48 & 0.073 & 10.6 \\
\hline 215.706 & 69.263 & 23.289 & 152.59 & 152.61 & 0.013 & 10.6 \\
\hline 215.349 & 69.269 & 23.309 & 153.09 & 152.85 & -0.157 & 10.6 \\
\hline 214.975 & 69.277 & 23.329 & 153.29 & 153.10 & -0.124 & 10.6 \\
\hline 214.553 & 69.276 & 23.352 & 153.48 & 153.38 & -0.065 & 10.6 \\
\hline 234.956 & 0.977 & 0.520 & 26.53 & 26.75 & 0.829 & 23.5 \\
\hline 234.383 & 0.978 & 0.522 & 26.45 & 26.68 & 0.870 & 23.7 \\
\hline 233.637 & 0.978 & 0.524 & 26.34 & 26.60 & 0.987 & 24.2 \\
\hline 233.152 & 0.979 & 0.526 & 26.32 & 26.54 & 0.836 & 23.4 \\
\hline 236.032 & 3.145 & 1.834 & 29.45 & 29.44 & -0.034 & 19.2 \\
\hline 235.316 & 3.146 & 1.843 & 29.45 & 29.38 & -0.238 & 19.0 \\
\hline 234.753 & 3.147 & 1.851 & 29.35 & 29.33 & -0.068 & 19.1 \\
\hline 234.261 & 3.148 & 1.857 & 29.36 & 29.28 & -0.272 & 19.0 \\
\hline 235.158 & 5.135 & 3.346 & 33.53 & 33.08 & -1.342 & 17.7 \\
\hline 234.655 & 5.135 & 3.361 & 33.61 & 33.07 & -1.607 & 17.6 \\
\hline 234.220 & 5.135 & 3.374 & 33.61 & 33.06 & -1.636 & 17.6 \\
\hline 233.740 & 5.135 & 3.389 & 33.61 & 33.05 & -1.666 & 17.5 \\
\hline 236.400 & 7.129 & 5.155 & 38.39 & 38.91 & 1.355 & 17.8 \\
\hline 235.396 & 7.129 & 5.218 & 39.46 & 39.05 & -1.039 & 17.1 \\
\hline 234.339 & 7.131 & 5.290 & 39.52 & 39.23 & -0.734 & 17.1 \\
\hline 233.533 & 7.132 & 5.347 & 40.43 & 39.37 & -2.622 & 16.5 \\
\hline 235.044 & 9.424 & 7.925 & 49.71 & 49.39 & -0.644 & 16.0 \\
\hline
\end{tabular}


Data from Roder [8] (continued)

\begin{tabular}{|c|c|c|c|c|c|c|}
\hline $\mathrm{T}$ & $P$ & $\rho$ & $\lambda, \exp$ & $\lambda, \mathrm{cal}$ & Dev & Wt \\
\hline K & $\mathrm{MPa}$ & $\mathrm{mol} \cdot \mathrm{dm}^{-3}$ & \multicolumn{2}{|c|}{$\mathrm{mW} \cdot \mathrm{m}^{-1} \cdot \mathrm{K}^{-1}$} & 8 & \\
\hline 234.249 & 9.425 & 8.033 & 51.04 & 49.80 & -2.429 & 15.6 \\
\hline 233.554 & 9.426 & 8.130 & 50.41 & 50.17 & -0.476 & 16.0 \\
\hline 232.938 & 9.427 & 8.220 & 50.29 & 50.51 & 0.437 & 16.1 \\
\hline 235.259 & 11.469 & 10.306 & 58.58 & 58.47 & -0.188 & 15.2 \\
\hline 234.432 & 11.470 & 10.447 & 59.03 & 58.98 & -0.085 & 15.2 \\
\hline 233.788 & 11.471 & 10.560 & 59.74 & 59.38 & -0.603 & 15.1 \\
\hline 233.227 & 11.472 & 10.660 & 59.26 & 59.73 & 0.793 & 15.3 \\
\hline 235.523 & 13.600 & 12.235 & 65.54 & 65.67 & 0.198 & 14.6 \\
\hline 234.841 & 13.600 & 12.346 & 66.03 & 66.07 & 0.061 & 14.5 \\
\hline 234.078 & 13.601 & 12.471 & 66.42 & 66.51 & 0.136 & 14.5 \\
\hline 233.513 & 13.602 & 12.564 & 66.70 & 66.85 & 0.225 & 14.5 \\
\hline 235.351 & 15.750 & 13.694 & 71.73 & 71.70 & -0.042 & 14.1 \\
\hline 234.621 & 15.750 & 13.799 & 72.19 & 72.11 & -0.111 & 14.0 \\
\hline 234.028 & 15.751 & 13.885 & 72.38 & 72.45 & 0.097 & 14.0 \\
\hline 233.472 & 15.751 & 13.965 & 72.82 & 72.78 & -0.055 & 14.0 \\
\hline 235.036 & 17.692 & 14.709 & 76.44 & 76.49 & 0.065 & 13.7 \\
\hline 234.425 & 17.692 & 14.788 & 76.87 & 76.83 & -0.052 & 13.7 \\
\hline 233.812 & 17.693 & 14.868 & 77.12 & 77.18 & 0.078 & 13.7 \\
\hline 233.349 & 17.693 & 14.928 & 77.40 & 77.45 & 0.065 & 13.6 \\
\hline 234.799 & 20.536 & 15.827 & 81.88 & 82.54 & 0.806 & 13.4 \\
\hline 234.255 & 20.535 & 15.888 & 82.72 & 82.84 & 0.145 & 13.3 \\
\hline 233.698 & 20.535 & 15.951 & 83.18 & 83.15 & -0.036 & 13.3 \\
\hline 233.233 & 20.535 & 16.004 & 83.48 & 83.42 & -0.072 & 13.3 \\
\hline 235.343 & 23.411 & 16.625 & 87.34 & 87.51 & 0.195 & 13.1 \\
\hline 234.724 & 23.411 & 16.688 & 87.68 & 87.85 & 0.194 & 13.0 \\
\hline 234.109 & 23.411 & 16.750 & 87.84 & 88.19 & 0.398 & 13.0 \\
\hline 233.639 & 23.410 & 16.798 & 88.45 & 88.46 & 0.011 & 13.0 \\
\hline 235.025 & 26.932 & 17.496 & 93.32 & 93.46 & 0.150 & 12.8 \\
\hline 234.553 & 26.932 & 17.539 & 93.11 & 93.72 & 0.655 & 12.8 \\
\hline 234.168 & 26.932 & 17.575 & 93.83 & 93.93 & 0.107 & 12.7 \\
\hline 233.546 & 26.931 & 17.631 & 93.95 & 94.28 & 0.351 & 12.7 \\
\hline 235.148 & 30.368 & 18.160 & 98.44 & 98.51 & 0.071 & 12.5 \\
\hline 234.592 & 30.366 & 18.206 & 98.94 & 98.81 & -0.131 & 12.5 \\
\hline 234.248 & 30.361 & 18.234 & 99.22 & 98.99 & -0.232 & 12.5 \\
\hline 233.560 & 30.360 & 18.292 & 99.35 & 99.37 & 0.020 & 12.5 \\
\hline
\end{tabular}


Data from Roder [8] (continued)

\begin{tabular}{|c|c|c|c|c|c|c|}
\hline $\mathrm{T}$ & $\mathrm{P}$ & $\rho$ & $\lambda, \exp$ & $\lambda, \mathrm{cal}$ & Dev & Wt \\
\hline K & $\mathrm{MPa}$ & $\mathrm{mol} \cdot \mathrm{dm}^{-3}$ & \multicolumn{2}{|c|}{$\mathrm{mW} \cdot \mathrm{m}^{-1} \cdot \mathrm{K}^{-1}$} & 8 & \\
\hline 234.964 & 34.092 & 18.795 & 103.31 & 103.71 & 0.387 & 12.3 \\
\hline 234.389 & 34.091 & 18.839 & 103.59 & 104.02 & 0.415 & 12.3 \\
\hline 233.804 & 34.090 & 18.885 & 103.95 & 104.34 & 0.375 & 12.3 \\
\hline 233.473 & 34.089 & 18.910 & 103.56 & 104.52 & 0.927 & 12.3 \\
\hline 235.426 & 37.551 & 19.262 & 107.55 & 107.89 & 0.316 & 12.1 \\
\hline 234.763 & 37.547 & 19.310 & 107.94 & 108.24 & 0.278 & 12.1 \\
\hline 234.319 & 37.547 & 19.342 & 108.24 & 108.48 & 0.222 & 12.1 \\
\hline 233.849 & 37.543 & 19.376 & 108.25 & 108.73 & 0.443 & 12.1 \\
\hline 235.318 & 41.022 & 19.718 & 111.89 & 112.14 & 0.223 & 11.9 \\
\hline 234.701 & 41.021 & 19.761 & 112.12 & 112.47 & 0.312 & 11.9 \\
\hline 234.205 & 41.021 & 19.795 & 112.39 & 112.74 & 0.311 & 11.9 \\
\hline 233.707 & 41.018 & 19.829 & 112.63 & 113.01 & 0.337 & 11.9 \\
\hline 235.189 & 44.354 & 20.116 & 116.37 & 116.05 & -0.275 & 11.8 \\
\hline 234.641 & 44.354 & 20.152 & 115.99 & 116.34 & 0.302 & 11.8 \\
\hline $234.13 C$ & 44.351 & 20.186 & 116.43 & 116.62 & 0.163 & 11.8 \\
\hline 233.731 & 44.350 & 20.212 & 116.93 & 116.84 & -0.077 & 11.7 \\
\hline 235.016 & 47.931 & 20.508 & 120.00 & 120.09 & 0.075 & 11.6 \\
\hline 234.521 & 47.932 & 20.540 & 120.28 & 120.36 & 0.067 & 11.6 \\
\hline 234.030 & 47.927 & 20.570 & 120.52 & 120.62 & 0.083 & 11.6 \\
\hline 233.633 & 47.928 & 20.595 & 120.49 & 120.84 & 0.290 & 11.6 \\
\hline 234.971 & 51.244 & 20.836 & 123.41 & 123.63 & 0.178 & 11.5 \\
\hline 234.454 & 51.246 & 20.867 & 123.89 & 123.91 & 0.016 & 11.5 \\
\hline 234.029 & 51.243 & 20.893 & 124.23 & 124.14 & -0.072 & 11.5 \\
\hline 233.650 & 51.240 & 20.916 & 124.65 & 124.34 & -0.249 & 11.5 \\
\hline 235.440 & 55.105 & 21.158 & 127.30 & 127.32 & 0.016 & 11.4 \\
\hline 234.914 & 55.104 & 21.189 & 127.66 & 127.61 & -0.039 & 11.4 \\
\hline 234.391 & 55.102 & 21.219 & 128.01 & 127.89 & -0.094 & 11.4 \\
\hline 233.958 & 55.098 & 21.244 & 128.22 & 128.13 & -0.070 & 11.3 \\
\hline 235.316 & 58.418 & 21.445 & 130.73 & 130.68 & -0.038 & 11.3 \\
\hline 234.894 & 58.416 & 21.469 & 130.80 & 130.90 & 0.076 & 11.3 \\
\hline 234.436 & 58.416 & 21.495 & 131.20 & 131.15 & -0.038 & 11.3 \\
\hline 233.899 & 58.413 & 21.525 & 131.57 & 131.45 & -0.091 & 11.2 \\
\hline 235.308 & 61.887 & 21.721 & 134.15 & 134.02 & -0.097 & 11.2 \\
\hline 234.767 & 61.887 & 21.751 & 134.48 & 134.32 & -0.119 & 11.2 \\
\hline 234.277 & 61.885 & 21.778 & 134.61 & 134.59 & -0.015 & 11.1 \\
\hline
\end{tabular}


Data from Roder [8] (continued)

\begin{tabular}{|c|c|c|c|c|c|c|}
\hline $\mathrm{T}$ & $P$ & $\rho$ & $\lambda, \exp$ & $\lambda, \operatorname{cal}$ & Dev & Wt \\
\hline K & $\mathrm{MPa}$ & $\mathrm{mol} \cdot \mathrm{dm}^{-3}$ & \multicolumn{2}{|c|}{$\mathrm{mW} \cdot \mathrm{m}^{-1} \cdot \mathrm{K}^{-1}$} & 8 & \\
\hline 233.911 & 61.882 & 21.798 & 134.51 & 134.79 & 0.208 & 11.2 \\
\hline 235.226 & 65.387 & 21.987 & 137.52 & 137.36 & -0.116 & 11.1 \\
\hline 234.756 & 65.384 & 22.012 & 137.80 & 137.61 & -0.138 & 11.1 \\
\hline 234.141 & 65.382 & 22.045 & 138.34 & 137.95 & -0.282 & 11.0 \\
\hline 233.842 & 65.381 & 22.061 & 138.07 & 138.11 & 0.029 & 11.0 \\
\hline 235.239 & 68.761 & 22.225 & 140.80 & 140.44 & -0.256 & 11.0 \\
\hline 234.730 & 68.761 & 22.252 & 141.04 & 140.72 & -0.227 & 11.0 \\
\hline 234.268 & 68.757 & 22.276 & 141.56 & 140.97 & -0.417 & 10.9 \\
\hline 233.731 & 68.757 & 22.304 & 141.60 & 141.27 & -0.233 & 10.9 \\
\hline 255.835 & 1.020 & 0.495 & 29.06 & 29.29 & 0.791 & 23.8 \\
\hline 255.177 & 1.020 & 0.496 & 29.00 & 29.20 & 0.690 & 23.4 \\
\hline 254.645 & 1.019 & 0.497 & 28.87 & 29.14 & 0.935 & 24.8 \\
\hline 253.943 & 1.019 & 0.498 & 28.81 & 29.05 & 0.833 & 24.2 \\
\hline 256.518 & 2.714 & 1.384 & 30.98 & 31.00 & 0.065 & 19.6 \\
\hline 255.864 & 2.714 & 1.389 & 30.81 & 30.93 & 0.389 & 20.0 \\
\hline 255.237 & 2.716 & 1.394 & 30.80 & 30.86 & 0.195 & 19.7 \\
\hline 254.735 & 2.717 & 1.399 & 30.76 & 30.81 & 0.163 & 19.7 \\
\hline 255.777 & 4.718 & 2.587 & 33.81 & 33.50 & -0.917 & 18.2 \\
\hline 255.276 & 4.721 & 2.597 & 33.67 & 33.46 & -0.624 & 18.3 \\
\hline 254.679 & 4.726 & 2.610 & 33.65 & 33.42 & -0.684 & 18.3 \\
\hline 254.209 & 4.732 & 2.622 & 33.50 & 33.39 & -0.328 & 18.5 \\
\hline 256.067 & 6.551 & 3.825 & 37.09 & 36.72 & -0.998 & 17.6 \\
\hline 255.011 & 6.552 & 3.858 & 37.07 & 36.69 & -1.025 & 17.6 \\
\hline 254.140 & 6.555 & 3.886 & 36.97 & 36.68 & -0.784 & 17.7 \\
\hline 253.452 & 6.558 & 3.910 & 37.16 & 36.67 & -1.319 & 17.5 \\
\hline 256.771 & 6.788 & 3.975 & 37.63 & 37.23 & -1.063 & 17.5 \\
\hline 255.562 & 6.788 & 4.013 & 37.65 & 37.20 & -1.195 & 17.5 \\
\hline 254.504 & 6.789 & 4.048 & 37.68 & 37.18 & -1.327 & 17.4 \\
\hline 256.308 & 8.785 & 5.525 & 42.54 & 41.99 & -1.293 & 16.8 \\
\hline 255.358 & 8.785 & 5.576 & 42.74 & 42.07 & -1.568 & 16.7 \\
\hline 254.477 & 8.785 & 5.624 & 42.76 & 42.14 & -1.450 & 16.8 \\
\hline 253.831 & 8.786 & 5.660 & 43.15 & 42.21 & -2.178 & 16.5 \\
\hline 256.639 & 11.182 & 7.487 & 49.11 & 49.00 & -0.224 & 16.3 \\
\hline 255.633 & 11.183 & 7.570 & 49.34 & 49.23 & -0.223 & 16.3 \\
\hline 254.824 & 11.183 & 7.639 & 49.49 & 49.42 & -0.141 & 16.3 \\
\hline
\end{tabular}


Data from Roder [8] (continued)

\begin{tabular}{|c|c|c|c|c|c|c|}
\hline $\mathrm{T}$ & $\mathbf{P}$ & $\rho$ & $\lambda, \exp$ & $\lambda, \mathrm{cal}$ & Dev & Wt \\
\hline K & $\mathrm{MPa}$ & $\mathrm{mol} \cdot \mathrm{dm}^{-3}$ & \multicolumn{2}{|c|}{$\mathrm{mW} \cdot \mathrm{m}^{-1} \cdot \mathrm{K}^{-1}$} & 8 & \\
\hline 254.114 & 11.187 & 7.704 & 49.73 & 49.60 & -0.261 & 16.2 \\
\hline 256.101 & 13.268 & 9.233 & 55.38 & 55.51 & 0.235 & 15.7 \\
\hline 255.217 & 13.269 & 9.325 & 55.49 & 55.80 & 0.559 & 15.7 \\
\hline 254.473 & 13.270 & 9.405 & 55.59 & 56.04 & 0.809 & 15.7 \\
\hline 253.901 & 13.273 & 9.469 & 55.89 & 56.24 & 0.626 & 15.7 \\
\hline 256.416 & 15.396 & 10.740 & 60.85 & 61.27 & 0.690 & 15.2 \\
\hline 255.651 & 15.397 & 10.825 & 61.12 & 61.54 & 0.687 & 15.2 \\
\hline 254.967 & 15.399 & 10.904 & 61.34 & 61.79 & 0.734 & 15.2 \\
\hline 254.357 & 15.401 & 10.975 & 61.67 & 62.02 & 0.568 & 15.1 \\
\hline 256.151 & 17.518 & 12.052 & 65.96 & 66.43 & 0.713 & 14.7 \\
\hline 255.415 & 17.519 & 12.134 & 66.19 & 66.70 & 0.771 & 14.7 \\
\hline 254.730 & 17.521 & 12.212 & 66.43 & 66.96 & 0.798 & 14.7 \\
\hline 254.159 & 17.522 & 12.276 & 66.67 & 67.18 & 0.765 & 14.7 \\
\hline 255.901 & 20.351 & 13.442 & 71.95 & 72.38 & 0.598 & 14.2 \\
\hline 255.130 & 20.352 & 13.522 & 72.27 & 72.68 & 0.567 & 14.2 \\
\hline 254.542 & 20.353 & 13.584 & 72.66 & 72.90 & 0.330 & 14.1 \\
\hline 254.029 & 20.351 & 13.637 & 72.60 & 73.10 & 0.689 & 14.2 \\
\hline 256.273 & 23.422 & 14.554 & 77.46 & 77.82 & 0.465 & 13.8 \\
\hline 255.560 & 23.422 & 14.622 & 77.81 & 78.09 & 0.360 & 13.8 \\
\hline 254.902 & 23.423 & 14.686 & 77.92 & 78.35 & 0.552 & 13.8 \\
\hline 254.313 & 23.423 & 14.742 & 78.09 & 78.58 & 0.627 & 13.8 \\
\hline 255.901 & 26.850 & 15.604 & 83.05 & 83.53 & 0.578 & 13.5 \\
\hline 255.234 & 26.850 & 15.662 & 83.38 & 83.79 & 0.492 & 13.4 \\
\hline 254.549 & 26.851 & 15.722 & 83.45 & 84.07 & 0.743 & 13.4 \\
\hline 254.205 & 26.849 & 15.752 & 83.91 & 84.20 & 0.346 & 13.4 \\
\hline 255.776 & 30.401 & 16.465 & 88.33 & 88.81 & 0.543 & 13.2 \\
\hline 255.193 & 30.399 & 16.512 & 88.52 & 89.04 & 0.587 & 13.1 \\
\hline 254.584 & 30.400 & 16.561 & 89.07 & 89.29 & 0.247 & 13.1 \\
\hline 254.087 & 30.397 & 16.601 & 89.15 & 89.50 & 0.393 & 13.1 \\
\hline 255.663 & 33.905 & 17.178 & 93.23 & 93.62 & 0.418 & 12.9 \\
\hline 255.054 & 33.907 & 17.224 & 93.61 & 93.88 & 0.288 & 12.9 \\
\hline 254.579 & 33.909 & 17.260 & 93.88 & 94.08 & 0.213 & 12.8 \\
\hline 254.064 & 33.909 & 17.299 & 93.94 & 94.29 & 0.373 & 12.8 \\
\hline 255.663 & 37.376 & 17.778 & 97.80 & 98.03 & 0.235 & 12.7 \\
\hline 255.091 & 37.374 & 17.818 & 98.11 & 98.27 & 0.163 & 12.6 \\
\hline
\end{tabular}


Data from Roder [8] (continued)

\begin{tabular}{|c|c|c|c|c|c|c|}
\hline $\mathrm{T}$ & $P$ & $\rho$ & $\lambda, \exp$ & $\lambda, \mathrm{cal}$ & Dev & Wt \\
\hline K & $\mathrm{MPa}$ & $\mathrm{mol} \cdot \mathrm{dm}^{-3}$ & \multicolumn{2}{|c|}{$\mathrm{mW} \cdot \mathrm{m}^{-1} \cdot \mathrm{K}^{-1}$} & 8 & \\
\hline 254.512 & 37.373 & 17.859 & 98.20 & 98.51 & 0.316 & 12.6 \\
\hline 254.068 & 37.374 & 17.891 & 98.38 & 98.70 & 0.325 & 12.6 \\
\hline 256.199 & 40.731 & 18.254 & 101.93 & 101.83 & -0.098 & 12.5 \\
\hline 255.692 & 40.729 & 18.288 & 102.18 & 102.04 & -0.137 & 12.4 \\
\hline 255.244 & 40.727 & 18.317 & 102.49 & 102.23 & -0.254 & 12.4 \\
\hline 256.203 & 44.631 & 18.784 & 105.93 & 106.27 & 0.321 & 12.3 \\
\hline 255.607 & 44.631 & 18.822 & 106.28 & 106.52 & 0.226 & 12.3 \\
\hline 254.993 & 44.631 & 18.861 & 106.34 & 106.78 & 0.414 & 12.3 \\
\hline 254.544 & 44.632 & 18.890 & 106.82 & 106.98 & 0.150 & 12.2 \\
\hline 256.097 & 48.090 & 19.214 & 109.70 & 110.07 & 0.337 & 12.1 \\
\hline 255.479 & 48.089 & 19.252 & 110.07 & 110.33 & 0.236 & 12.1 \\
\hline 254.909 & 48.089 & 19.287 & 110.33 & 110.58 & 0.227 & 12.1 \\
\hline 254.428 & 48.087 & 19.316 & 110.59 & 110.78 & 0.172 & 12.1 \\
\hline 255.939 & 51.674 & 19.624 & 113.61 & 113.87 & 0.229 & 12.0 \\
\hline 255.358 & 51.673 & 19.658 & 113.79 & 114.12 & 0.290 & 12.0 \\
\hline 254.925 & 51.672 & 19.683 & 113.97 & 114.31 & 0.298 & 12.0 \\
\hline 254.412 & 51.673 & 19.713 & 114.22 & 114.53 & 0.271 & 11.9 \\
\hline 256.371 & 56.210 & 20.060 & 117.93 & 118.22 & 0.246 & 11.8 \\
\hline 255.786 & 56.209 & 20.093 & 118.19 & 118.48 & 0.245 & 11.8 \\
\hline 255.239 & 56.207 & 20.123 & 118.43 & 118.71 & 0.236 & 11.8 \\
\hline 254.711 & 56.208 & 20.153 & 118.39 & 118.95 & 0.473 & 11.8 \\
\hline 254.267 & 56.203 & 20.177 & 119.12 & 119.14 & 0.017 & 11.8 \\
\hline 255.471 & 59.687 & 20.434 & 121.84 & 121.97 & 0.107 & 11.7 \\
\hline 255.429 & 59.690 & 20.436 & 121.89 & 121.99 & 0.082 & 11.7 \\
\hline 254.396 & 59.684 & 20.492 & 122.09 & 122.45 & 0.295 & 11.7 \\
\hline 254.078 & 59.683 & 20.509 & 122.46 & 122.59 & 0.106 & 11.6 \\
\hline 255.957 & 63.260 & 20.719 & 125.86 & 125.11 & -0.596 & 11.5 \\
\hline 255.379 & 63.260 & 20.749 & 126.59 & 125.36 & -0.972 & 11.5 \\
\hline 254.993 & 63.255 & 20.769 & 124.68 & 125.53 & 0.682 & 11.6 \\
\hline 254.436 & 63.252 & 20.798 & 125.37 & 125.78 & 0.327 & 11.5 \\
\hline 255.922 & 66.477 & 20.984 & 128.80 & 128.06 & -0.575 & 11.4 \\
\hline 255.361 & 66.480 & 21.013 & 128.65 & 128.32 & -0.257 & 11.4 \\
\hline 254.827 & 66.474 & 21.040 & 128.88 & 128.55 & -0.256 & 11.4 \\
\hline 254.429 & 66.473 & 21.060 & 128.52 & 128.73 & 0.163 & 11.4 \\
\hline 275.820 & 1.015 & 0.453 & 31.52 & 31.79 & 0.857 & 25.6 \\
\hline
\end{tabular}


Data from Roder [8] (continued)

\begin{tabular}{|c|c|c|c|c|c|c|}
\hline $\mathrm{T}$ & P & $\rho$ & $\lambda, \exp$ & $\lambda, \mathrm{ca} 1$ & Dev & Wt \\
\hline K & $\mathrm{MPa}$ & $\mathrm{mol} \cdot \mathrm{dm}^{-3}$ & \multicolumn{2}{|c|}{$\mathrm{mW} \cdot \mathrm{m}^{-1} \cdot \mathrm{K}^{-1}$} & 8 & \\
\hline 275.218 & 1.015 & 0.454 & 31.44 & 31.71 & 0.859 & 25.6 \\
\hline 274.623 & 1.015 & 0.455 & 31.34 & 31.64 & 0.957 & 26.4 \\
\hline 274.083 & 1.016 & 0.457 & 31.34 & 31.57 & 0.734 & 24.4 \\
\hline 276.019 & 2.495 & 1.153 & 32.88 & 33.05 & 0.517 & 20.6 \\
\hline 275.382 & 2.495 & 1.156 & 32.89 & 32.98 & 0.274 & 20.1 \\
\hline 274.765 & 2.496 & 1.160 & 32.77 & 32.90 & 0.397 & 20.3 \\
\hline 274.285 & 2.497 & 1.163 & 32.74 & 32.84 & 0.305 & 20.2 \\
\hline 275.546 & 4.446 & 2.159 & 35.09 & 35.01 & -0.228 & 18.8 \\
\hline 275.066 & 4.447 & 2.165 & 34.95 & 34.96 & 0.029 & 19.0 \\
\hline 274.433 & 4.448 & 2.173 & 34.99 & 34.90 & -0.257 & 18.8 \\
\hline 274.021 & 4.449 & 2.178 & 34.89 & 34.85 & -0.115 & 18.9 \\
\hline 275.252 & 6.508 & 3.329 & 38.04 & 37.71 & -0.868 & 17.9 \\
\hline 274.818 & 6.508 & 3.338 & 38.09 & 37.68 & -1.076 & 17.8 \\
\hline 274.354 & 6.507 & 3.347 & 38.07 & 37.64 & -1.129 & 17.7 \\
\hline 274.121 & 6.507 & 3.352 & 38.16 & 37.63 & -1.389 & 17.6 \\
\hline 277.314 & 8.401 & 4.425 & 41.35 & 40.93 & -1.016 & 17.4 \\
\hline 276.746 & 8.402 & 4.443 & 41.34 & 40.92 & -1.016 & 17.3 \\
\hline 276.190 & 8.405 & 4.462 & 41.26 & 40.90 & -0.873 & 17.4 \\
\hline 275.541 & 8.407 & 4.483 & 41.20 & 40.89 & -0.752 & 17.4 \\
\hline 276.595 & 10.552 & 5.821 & 45.51 & 45.15 & -0.791 & 16.9 \\
\hline 276.054 & 10.553 & 5.846 & 45.48 & 45.17 & -0.682 & 16.9 \\
\hline 275.617 & 10.555 & 5.867 & 45.43 & 45.19 & -0.528 & 16.9 \\
\hline 275.127 & 10.559 & 5.892 & 45.47 & 45.22 & -0.550 & 16.9 \\
\hline 276.632 & 12.673 & 7.199 & 50.04 & 49.87 & -0.340 & 16.4 \\
\hline 276.126 & 12.674 & 7.230 & 50.12 & 49.92 & -0.399 & 16.4 \\
\hline 275.640 & 12.675 & 7.260 & 50.08 & 49.98 & -0.200 & 16.4 \\
\hline 275.203 & 12.678 & 7.288 & 50.07 & 50.03 & -0.080 & 16.4 \\
\hline 276.728 & 14.652 & 8.444 & 54.39 & 54.39 & 0.000 & 16.0 \\
\hline 276.201 & 14.653 & 8.481 & 54.43 & 54.47 & 0.073 & 16.0 \\
\hline 275.829 & 14.655 & 8.509 & 54.49 & 54.54 & 0.092 & 16.0 \\
\hline 275.408 & 14.656 & 8.540 & 54.52 & 54.61 & 0.165 & 16.0 \\
\hline 276.345 & 16.954 & 9.820 & 59.29 & 59.52 & 0.388 & 15.5 \\
\hline 275.929 & 16.954 & 9.853 & 59.45 & 59.60 & 0.252 & 15.5 \\
\hline 275.539 & 16.956 & 9.885 & 59.41 & 59.69 & 0.471 & 15.5 \\
\hline 275.111 & 16.957 & 9.920 & 59.39 & 59.78 & 0.657 & 15.5 \\
\hline
\end{tabular}


Data from Roder [8] (continued)

\begin{tabular}{|c|c|c|c|c|c|c|}
\hline $\mathrm{T}$ & $\mathbf{P}$ & $\rho$ & $\lambda, \exp$ & $\lambda, \mathrm{cal}$ & Dev & Wt \\
\hline K & $\mathrm{MPa}$ & $\mathrm{mol} \cdot \mathrm{dm}^{-3}$ & \multicolumn{2}{|c|}{$\mathrm{mW} \cdot \mathrm{m}^{-1} \cdot \mathrm{K}^{-1}$} & 8 & \\
\hline 276.052 & 19.153 & 10.986 & 63.56 & 64.02 & 0.724 & 15.1 \\
\hline 275.714 & 19.154 & 11.014 & 63.73 & 64.10 & 0.581 & 15.1 \\
\hline 275.280 & 19.155 & 11.051 & 63.82 & 64.20 & 0.595 & 15.1 \\
\hline 275.004 & 19.155 & 11.075 & 63.83 & 64.27 & 0.689 & 15.1 \\
\hline 276.700 & 21.322 & 11.913 & 67.46 & 67.85 & 0.578 & 14.8 \\
\hline 275.909 & 21.323 & 11.979 & 67.57 & 68.05 & 0.710 & 14.8 \\
\hline 275.069 & 21.324 & 12.051 & 67.82 & 68.26 & 0.649 & 14.7 \\
\hline 274.513 & 21.325 & 12.098 & 67.84 & 68.40 & 0.825 & 14.7 \\
\hline 276.428 & 23.529 & 12.802 & 71.33 & 71.64 & 0.435 & 14.4 \\
\hline 275.693 & 23.529 & 12.862 & 71.46 & 71.83 & 0.518 & 14.4 \\
\hline 274.982 & 23.531 & 12.921 & 71.68 & 72.02 & 0.474 & 14.4 \\
\hline 274.401 & 23.535 & 12.971 & 71.55 & 72.18 & 0.881 & 14.4 \\
\hline 276.181 & 26.983 & 13.959 & 76.78 & 77.03 & 0.326 & 14.0 \\
\hline 275.483 & 26.984 & 14.014 & 76.96 & 77.23 & 0.351 & 14.0 \\
\hline 274.770 & 26.986 & 14.070 & 77.18 & 77.43 & 0.324 & 14.0 \\
\hline 274.248 & 26.988 & 14.112 & 77.28 & 77.57 & 0.375 & 14.0 \\
\hline 276.033 & 30.486 & 14.917 & 81.88 & 82.01 & 0.159 & 13.7 \\
\hline 275.358 & 30.487 & 14.967 & 81.86 & 82.20 & 0.415 & 13.7 \\
\hline 274.715 & 30.488 & 15.015 & 82.31 & 82.39 & 0.097 & 13.6 \\
\hline 274.239 & 30.490 & 15.051 & 82.32 & 82.53 & 0.255 & 13.6 \\
\hline 276.647 & 33.987 & 15.671 & 86.53 & 86.39 & -0.162 & 13.4 \\
\hline 276.018 & 33.989 & 15.715 & 86.78 & 86.58 & -0.230 & 13.3 \\
\hline 275.248 & 33.987 & 15.768 & 86.84 & 86.81 & -0.035 & 13.3 \\
\hline 274.869 & 33.991 & 15.796 & 87.10 & 86.93 & -0.195 & 13.3 \\
\hline 276.150 & 37.531 & 16.399 & 90.67 & 90.87 & 0.221 & 13.1 \\
\hline 275.502 & 37.530 & 16.441 & 89.76 & 91.07 & 1.459 & 13.3 \\
\hline 274.848 & 37.529 & 16.485 & 91.82 & 91.27 & -0.599 & 13.0 \\
\hline 274.390 & 37.528 & 16.515 & 88.26 & 91.41 & 3.569 & 13.4 \\
\hline 276.052 & 41.154 & 17.024 & 94.92 & 95.08 & 0.169 & 12.9 \\
\hline 275.385 & 41.153 & 17.066 & 95.86 & 95.29 & -0.595 & 12.8 \\
\hline 274.737 & 41.151 & 17.107 & 95.28 & 95.50 & 0.231 & 12.9 \\
\hline 274.280 & 41.151 & 17.136 & 95.76 & 95.65 & -0.115 & 12.9 \\
\hline 275.921 & 44.660 & 17.563 & 99.29 & 98.98 & -0.312 & 12.7 \\
\hline 275.284 & 44.660 & 17.602 & 100.15 & 99.18 & -0.969 & 12.6 \\
\hline 274.679 & 44.660 & 17.639 & 99.36 & 99.38 & 0.020 & 12.7 \\
\hline
\end{tabular}


Data from Roder [8] (continued)

\begin{tabular}{|c|c|c|c|c|c|c|}
\hline $\mathrm{T}$ & $\mathbf{P}$ & $\rho$ & $\lambda, \exp$ & $\lambda, \mathrm{cal}$ & Dev & Wt \\
\hline K & $\mathrm{MPa}$ & $\mathrm{mol} \cdot \mathrm{dm}^{-3}$ & \multicolumn{2}{|c|}{$\mathrm{mW} \cdot \mathrm{m}^{-1} \cdot \mathrm{K}^{-1}$} & 8 & \\
\hline 274.171 & 44.660 & 17.670 & 99.27 & 99.55 & 0.282 & 12.7 \\
\hline 275.776 & 48.079 & 18.039 & 102.77 & 102.63 & -0.136 & 12.5 \\
\hline 275.237 & 48.080 & 18.071 & 102.90 & 102.81 & -0.087 & 12.5 \\
\hline 274.656 & 48.078 & 18.104 & 102.84 & 103.00 & 0.156 & 12.5 \\
\hline 274.170 & 48.078 & 18.132 & 103.64 & 103.16 & -0.463 & 12.5 \\
\hline 276.282 & 51.734 & 18.464 & 106.45 & 106.16 & -0.272 & 12.4 \\
\hline 275.668 & 51.733 & 18.499 & 106.52 & 106.36 & -0.150 & 12.4 \\
\hline 275.120 & 51.733 & 18.529 & 106.82 & 106.55 & -0.253 & 12.3 \\
\hline 274.592 & 51.732 & 18.559 & 106.77 & 106.73 & -0.037 & 12.3 \\
\hline 276.367 & 55.237 & 18.859 & 109.87 & 109.54 & -0.300 & 12.2 \\
\hline 275.610 & 55.235 & 18.899 & 110.00 & 109.80 & -0.182 & 12.2 \\
\hline 275.048 & 55.235 & 18.930 & 110.26 & 109.99 & -0.245 & 12.2 \\
\hline 274.537 & 55.233 & 18.957 & 110.59 & 110.17 & -0.380 & 12.2 \\
\hline 276.173 & 58.748 & 19.239 & 113.29 & 112.93 & -0.318 & 12.1 \\
\hline 275.531 & 58.747 & 19.272 & 113.47 & 113.15 & -0.282 & 12.1 \\
\hline 274.999 & 58.748 & 19.300 & 113.61 & 113.34 & -0.238 & 12.1 \\
\hline 274.471 & 58.749 & 19.328 & 113.93 & 113.52 & -0.360 & 12.0 \\
\hline 276.101 & 62.502 & 19.608 & 116.94 & 116.40 & -0.462 & 11.9 \\
\hline 275.477 & 62.503 & 19.640 & 117.10 & 116.62 & -0.410 & 11.9 \\
\hline 274.905 & 62.499 & 19.669 & 117.22 & 116.82 & -0.341 & 11.9 \\
\hline 274.457 & 62.497 & 19.692 & 117.44 & 116.98 & -0.392 & 11.9 \\
\hline 275.948 & 65.832 & 19.919 & 120.04 & 119.42 & -0.516 & 11.8 \\
\hline 275.385 & 65.838 & 19.948 & 120.10 & 119.63 & -0.391 & 11.8 \\
\hline 274.943 & 65.840 & 19.970 & 120.14 & 119.79 & -0.291 & 11.8 \\
\hline 274.423 & 65.839 & 19.996 & 120.16 & 119.98 & -0.150 & 11.8 \\
\hline 276.499 & 69.251 & 20.186 & 122.80 & 122.21 & -0.480 & 11.7 \\
\hline 275.940 & 69.252 & 20.213 & 123.06 & 122.41 & -0.528 & 11.7 \\
\hline 275.379 & 69.254 & 20.240 & 123.27 & 122.62 & -0.527 & 11.7 \\
\hline 274.798 & 69.256 & 20.268 & 123.33 & 122.83 & -0.405 & 11.7 \\
\hline 296.783 & 0.988 & 0.407 & 34.02 & 34.54 & 1.529 & 49.1 \\
\hline 296.042 & 0.988 & 0.409 & 33.89 & 34.44 & 1.623 & 57.3 \\
\hline 295.352 & 0.988 & 0.410 & 33.83 & 34.35 & 1.537 & 47.6 \\
\hline 294.797 & 0.989 & 0.411 & 33.57 & 34.27 & 2.085 & 59.8 \\
\hline 296.430 & 1.949 & 0.819 & 34.77 & 35.19 & 1.208 & 24.2 \\
\hline 295.783 & 1.949 & 0.821 & 34.65 & 35.11 & 1. 328 & 24.7 \\
\hline
\end{tabular}


Data from Roder [8] (continued)

\begin{tabular}{|c|c|c|c|c|c|c|}
\hline $\mathrm{T}$ & $\mathbf{P}$ & $\rho$ & $\lambda, \exp$ & $\lambda, \mathrm{cal}$ & Dev & Wt \\
\hline K & $\mathrm{MPa}$ & $\mathrm{mol} \cdot \mathrm{dm}^{-3}$ & \multicolumn{2}{|c|}{$\mathrm{mW} \cdot \mathrm{m}^{-1} \cdot \mathrm{K}^{-1}$} & 8 & \\
\hline 295.177 & 1.950 & 0.823 & 34.65 & 35.03 & 1.097 & 23.6 \\
\hline 294.638 & 1.950 & 0.825 & 34.51 & 34.96 & 1.304 & 24.5 \\
\hline 296.023 & 3.911 & 1.705 & 36.48 & 36.79 & 0.850 & 20.4 \\
\hline 295.413 & 3.912 & 1.710 & 36.51 & 36.72 & 0.575 & 20.0 \\
\hline 294.915 & 3.913 & 1.714 & 36.40 & 36.66 & 0.714 & 20.2 \\
\hline 294.458 & 3.914 & 1.718 & 36.38 & 36.61 & 0.632 & 20.1 \\
\hline 296.320 & 5.976 & 2.696 & 38.82 & 38.95 & 0.335 & 19.0 \\
\hline 295.741 & 5.978 & 2.705 & 38.79 & 38.89 & 0.258 & 18.9 \\
\hline 295.153 & 5.981 & 2.714 & 38.66 & 38.83 & 0.440 & 19.0 \\
\hline 294.671 & 5.974 & 2.717 & 38.62 & 38.77 & 0.388 & 19.0 \\
\hline 295.849 & 8.077 & 3.778 & 41.52 & 41.53 & 0.024 & 18.1 \\
\hline 295.213 & 8.079 & 3.792 & 41.59 & 41.48 & -0.264 & 18.0 \\
\hline 294.626 & 8.082 & 3.806 & 41.52 & 41.44 & -0.193 & 18.0 \\
\hline 294.135 & 8.087 & 3.819 & 41.46 & 41.41 & -0.121 & 18.0 \\
\hline 295.366 & 10.365 & 5.016 & 44.99 & 44.92 & -0.156 & 17.4 \\
\hline 294.726 & 10.367 & 5.037 & 45.03 & 44.90 & -0.289 & 17.4 \\
\hline 294.274 & 10.369 & 5.052 & 44.96 & 44.89 & -0.156 & 17.4 \\
\hline 293.791 & 10.373 & 5.069 & 44.98 & 44.88 & -0.222 & 17.4 \\
\hline 294.991 & 12.169 & 6.017 & 48.08 & 47.98 & -0.208 & 16.9 \\
\hline 294.453 & 12.171 & 6.039 & 47.98 & 47.98 & 0.000 & 17.0 \\
\hline 293.987 & 12.173 & 6.058 & 48.22 & 47.99 & -0.477 & 16.8 \\
\hline 293.540 & 12.176 & 6.078 & 48.13 & 48.00 & -0.270 & 16.9 \\
\hline 296.186 & 14.295 & 7.114 & 51.70 & 51.80 & 0.193 & 16.6 \\
\hline 295.091 & 14.297 & 7.168 & 51.78 & 51.85 & 0.135 & 16.5 \\
\hline 294.119 & 14.297 & 7.215 & 51.74 & 51.90 & 0.309 & 16.6 \\
\hline 293.367 & 14.299 & 7.254 & 51.59 & 51.94 & 0.678 & 16.6 \\
\hline 295.764 & 16.379 & 8.219 & 55.29 & 55.65 & 0.651 & 16.2 \\
\hline 294.721 & 16.379 & 8.277 & 55.35 & 55.74 & 0.705 & 16.2 \\
\hline 293.904 & 16.381 & 8.324 & 55.34 & 55.82 & 0.867 & 16.2 \\
\hline 293.091 & 16.382 & 8.372 & 55.32 & 55.90 & 1.048 & 16.2 \\
\hline 295.420 & 18.657 & 9.348 & 59.17 & 59.79 & 1.048 & 15.8 \\
\hline 294.415 & 18.658 & 9.411 & 59.36 & 59.91 & 0.927 & 15.8 \\
\hline 293.588 & 18.659 & 9.464 & 59.36 & 60.02 & 1.112 & 15.8 \\
\hline 292.903 & 18.662 & 9.509 & 59.52 & 60.11 & 0.991 & 15.8 \\
\hline 295.068 & 21.429 & 10.589 & 63.90 & 64.52 & 0.970 & 15.3 \\
\hline
\end{tabular}


Data from Roder [8] (continued)

\begin{tabular}{|c|c|c|c|c|c|c|}
\hline $\mathrm{T}$ & $\mathbf{P}$ & $\rho$ & $\lambda, \exp$ & $\lambda, \mathrm{cal}$ & Dev & Wt \\
\hline K & $\mathrm{MPa}$ & $\mathrm{mol} \cdot \mathrm{dm}^{-3}$ & \multicolumn{2}{|c|}{$\mathrm{mW} \cdot \mathrm{m}^{-1} \cdot \mathrm{K}^{-1}$} & 8 & \\
\hline 294.252 & 21.432 & 10.644 & 64.09 & 64.65 & 0.874 & 15.3 \\
\hline 293.482 & 21.432 & 10.696 & 64.09 & 64.77 & 1.061 & 15.3 \\
\hline 292.793 & 21.434 & 10.744 & 64.31 & 64.88 & 0.886 & 15.3 \\
\hline 295.659 & 25.128 & 11.939 & 69.21 & 70.11 & 1.300 & 14.9 \\
\hline 294.864 & 25.127 & 11.992 & 69.71 & 70.24 & 0.760 & 14.8 \\
\hline 294.012 & 25.128 & 12.050 & 69.69 & 70.39 & 1.004 & 14.8 \\
\hline 293.449 & 25.126 & 12.088 & 69.76 & 70.48 & 1.032 & 14.8 \\
\hline 295.578 & 28.719 & 13.068 & 74.60 & 75.10 & 0.670 & 14.4 \\
\hline 294.771 & 28.716 & 13.121 & 74.85 & 75.24 & 0.521 & 14.3 \\
\hline 294.080 & 28.712 & 13.166 & 74.95 & 75.37 & 0.560 & 14.3 \\
\hline 293.601 & 28.713 & 13.199 & 74.84 & 75.46 & 0.828 & 14.4 \\
\hline 296.573 & 32.484 & 13.996 & 79.14 & 79.70 & 0.708 & 14.1 \\
\hline 295.711 & 32.483 & 14.051 & 79.27 & 79.87 & 0.757 & 14.0 \\
\hline 295.042 & 32.484 & 14.094 & 79.26 & 80.01 & 0.946 & 14.1 \\
\hline 294.303 & 32.485 & 14.142 & 79.89 & 80.16 & 0.338 & 14.0 \\
\hline 296.404 & 36.134 & 14.828 & 83.42 & 84.07 & 0.779 & 13.8 \\
\hline 295.521 & 36.135 & 14.883 & 83.66 & 84.25 & 0.705 & 13.7 \\
\hline 294.862 & 36.136 & 14.924 & 83.84 & 84.40 & 0.668 & 13.7 \\
\hline 294.272 & 36.136 & 14.960 & 83.98 & 84.52 & 0.643 & 13.7 \\
\hline 296.324 & 39.442 & 15.484 & 87.33 & 87.80 & 0.538 & 13.5 \\
\hline 295.531 & 39.442 & 15.531 & 87.43 & 87.97 & 0.618 & 13.5 \\
\hline 294.765 & 39.444 & 15.577 & 87.56 & 88.15 & 0.674 & 13.5 \\
\hline 294.155 & 39.444 & 15.614 & 87.86 & 88.29 & 0.489 & 13.5 \\
\hline 296.156 & 42.826 & 16.086 & 91.03 & 91.46 & 0.472 & 13.3 \\
\hline 295.472 & 42.826 & 16.126 & 91.27 & 91.62 & 0.383 & 13.3 \\
\hline 294.753 & 42.827 & 16.167 & 91.25 & 91.79 & 0.592 & 13.3 \\
\hline 294.259 & 42.827 & 16.196 & 91.36 & 91.91 & 0.602 & 13.3 \\
\hline 296.097 & 46.333 & 16.640 & 94.70 & 95.08 & 0.401 & 13.1 \\
\hline 295.347 & 46.333 & 16.682 & 94.92 & 95.26 & 0.358 & 13.1 \\
\hline 294.739 & 46.333 & 16.716 & 94.97 & 95.41 & 0.463 & 13.1 \\
\hline 294.112 & 46.334 & 16.751 & 95.24 & 95.56 & 0.336 & 13.0 \\
\hline 296.086 & 49.998 & 17.160 & 98.47 & 98.70 & 0.234 & 12.9 \\
\hline 295.349 & 50.000 & 17.200 & 98.67 & 98.89 & 0.223 & 12.9 \\
\hline 294.672 & 50.000 & 17.236 & 98.83 & 99.06 & 0.233 & 12.8 \\
\hline 294.162 & 50.001 & 17.264 & 99.14 & 99.19 & 0.050 & 12.8 \\
\hline
\end{tabular}


Data from Roder [8] (continued)

\begin{tabular}{|c|c|c|c|c|c|c|}
\hline $\mathrm{T}$ & $P$ & $\rho$ & $\lambda, \exp$ & $\lambda, \mathrm{cal}$ & Dev & Wt \\
\hline $\mathrm{K}$ & $\mathrm{MPa}$ & $\mathrm{mol} \cdot \mathrm{dm}^{-3}$ & \multicolumn{2}{|c|}{$\mathrm{mW} \cdot \mathrm{m}^{-1} \cdot \mathrm{K}^{-1}$} & 8 & \\
\hline 295.878 & 53.680 & 17.645 & 102.13 & 102.26 & 0.127 & 12.7 \\
\hline 295.267 & 53.681 & 17.677 & 102.20 & 102.42 & 0.215 & 12.7 \\
\hline 294.552 & 53.682 & 17.714 & 102.37 & 102.60 & 0.225 & 12.7 \\
\hline 294.003 & 53.685 & 17.743 & 102.49 & 102.75 & 0.254 & 12.7 \\
\hline 295.330 & 57.061 & 18.072 & 105.41 & 105.52 & 0.104 & 12.5 \\
\hline 294.706 & 57.060 & 18.104 & 105.58 & 105.68 & 0.095 & 12.5 \\
\hline 294.110 & 57.061 & 18.134 & 105.84 & 105.85 & 0.009 & 12.5 \\
\hline 293.609 & 57.062 & 18.159 & 105.74 & 105.98 & 0.227 & 12.5 \\
\hline 295.232 & 60.576 & 18.461 & 108.65 & 108.70 & 0.046 & 12.4 \\
\hline 294.648 & 60.576 & 18.490 & 108.85 & 108.86 & 0.009 & 12.4 \\
\hline 294.036 & 60.578 & 18.520 & 108.97 & 109.03 & 0.055 & 12.4 \\
\hline 293.630 & 60.577 & 18.540 & 109.09 & 109.14 & 0.046 & 12.4 \\
\hline 295.187 & 63.953 & 18.807 & 111.77 & 111.66 & -0.098 & 12.2 \\
\hline 294.555 & 63.958 & 18.838 & 112.04 & 111.84 & -0.179 & 12.2 \\
\hline 294.012 & 63.952 & 18.864 & 111.93 & 111.99 & 0.054 & 12.2 \\
\hline 293.475 & 63.951 & 18.889 & 112.41 & 112.14 & -0.240 & 12.2 \\
\hline 295.776 & 67.319 & 19.101 & 114.71 & 114.36 & -0.305 & 12.1 \\
\hline 295.118 & 67.321 & 19.132 & 114.91 & 114.55 & -0.313 & 12.1 \\
\hline 294.631 & 67.320 & 19.155 & 115.10 & 114.69 & -0.356 & 12.1 \\
\hline 294.106 & 67.323 & 19.180 & 115.21 & 114.84 & -0.321 & 12.1 \\
\hline 309.085 & 1.076 & 0.426 & 35.91 & 36.30 & 1.086 & 31.5 \\
\hline 308.341 & 1.076 & 0.427 & 36.10 & 36.20 & 0.277 & 21.7 \\
\hline 307.755 & 1.077 & 0.428 & 35.81 & 36.11 & 0.838 & 27.3 \\
\hline 307.128 & 1.077 & 0.429 & 35.58 & 36.03 & 1.265 & 35.3 \\
\hline 309.214 & 2.675 & 1.083 & 37.42 & 37.46 & 0.107 & 19.9 \\
\hline 308.624 & 2.675 & 1.086 & 37.33 & 37.38 & 0.134 & 19.9 \\
\hline 308.033 & 2.676 & 1.089 & 37.01 & 37.30 & 0.784 & 21.5 \\
\hline 307.414 & 2.678 & 1.092 & 36.93 & 37.22 & 0.785 & 21.5 \\
\hline 309.391 & 4.743 & 1.979 & 39.36 & 39.21 & -0.381 & 18.7 \\
\hline 308.849 & 4.743 & 1.983 & 39.10 & 39.14 & 0.102 & 19.2 \\
\hline 308.250 & 4.743 & 1.988 & 39.15 & 39.07 & -0.204 & 18.9 \\
\hline 307.648 & 4.744 & 1.994 & 39.01 & 38.99 & -0.051 & 19.1 \\
\hline 309.635 & 6.732 & 2.883 & 41.48 & 41.21 & -0.651 & 18.1 \\
\hline 309.040 & 6.732 & 2.891 & 41.53 & 41.15 & -0.915 & 17.9 \\
\hline 308.464 & 6.733 & 2.899 & 41.46 & 41.08 & -0.917 & 17.9 \\
\hline
\end{tabular}


Data from Roder [8] (continued)

\begin{tabular}{|c|c|c|c|c|c|c|}
\hline $\mathrm{T}$ & $\mathbf{P}$ & $\rho$ & $\lambda, \exp$ & $\lambda, \mathrm{cal}$ & Dev & Wt \\
\hline K & $\mathrm{MPa}$ & $\mathrm{mol} \cdot \mathrm{dm}^{-3}$ & \multicolumn{2}{|c|}{$\mathrm{mW} \cdot \mathrm{m}^{-1} \cdot \mathrm{K}^{-1}$} & 8 & \\
\hline 307.941 & 6.733 & 2.906 & 41.27 & 41.03 & -0.582 & 18.2 \\
\hline 309.267 & 8.876 & 3.907 & 44.26 & 43.68 & -1.310 & 17.4 \\
\hline 308.712 & 8.876 & 3.918 & 43.95 & 43.63 & -0.728 & 17.7 \\
\hline 308.170 & 8.877 & 3.930 & 44.02 & 43.59 & -0.977 & 17.5 \\
\hline 307.710 & 8.877 & 3.939 & 44.03 & 43.55 & -1.090 & 17.5 \\
\hline 310.021 & 11.038 & 4.942 & 47.19 & 46.64 & -1.166 & 17.0 \\
\hline 308.898 & 11.039 & 4.973 & 47.15 & 46.58 & -1.209 & 17.0 \\
\hline 307.879 & 11.039 & 5.001 & 46.83 & 46.52 & -0.662 & 17.2 \\
\hline 307.031 & 11.039 & 5.025 & 46.95 & 46.48 & -1.001 & 17.1 \\
\hline 309.673 & 13.147 & 5.984 & 50.09 & 49.76 & -0.659 & 16.8 \\
\hline 308.603 & 13.147 & 6.021 & 49.96 & 49.73 & -0.460 & 16.9 \\
\hline 307.642 & 13.148 & 6.056 & 50.23 & 49.72 & -1.015 & 16.7 \\
\hline 306.841 & 13.148 & 6.084 & 50.33 & 49.70 & -1.252 & 16.6 \\
\hline 309.353 & 15.185 & 6.980 & 53.20 & 52.98 & -0.414 & 16.5 \\
\hline 308.309 & 15.186 & 7.024 & 53.23 & 53.00 & -0.432 & 16.4 \\
\hline 307.523 & 15.186 & 7.057 & 53.23 & 53.01 & -0.413 & 16.4 \\
\hline 306.746 & 15.186 & 7.090 & 53.25 & 53.02 & -0.432 & 16.4 \\
\hline 310.102 & 17.278 & 7.919 & 56.39 & 56.33 & -0.106 & 16.2 \\
\hline 308.975 & 17.278 & 7.972 & 56.47 & 56.38 & -0.159 & 16.1 \\
\hline 308.110 & 17.277 & 8.013 & 56.44 & 56.42 & -0.035 & 16.1 \\
\hline 307.320 & 17.276 & 8.051 & 56.46 & 56.45 & -0.018 & 16.1 \\
\hline 309.770 & 19.464 & 8.894 & 59.73 & 59.82 & 0.151 & 15.8 \\
\hline 308.761 & 19.463 & 8.946 & 59.86 & 59.89 & 0.050 & 15.8 \\
\hline 307.944 & 19.463 & 8.990 & 59.83 & 59.95 & 0.201 & 15.8 \\
\hline 307.225 & 19.462 & 9.028 & 60.00 & 60.00 & 0.000 & 15.7 \\
\hline 309.480 & 22.418 & 10.097 & 64.11 & 64.34 & 0.359 & 15.4 \\
\hline 308.591 & 22.418 & 10.147 & 64.22 & 64.42 & 0.311 & 15.4 \\
\hline 307.806 & 22.418 & 10.192 & 64.22 & 64.50 & 0.436 & 15.4 \\
\hline 307.109 & 22.418 & 10.233 & 64.11 & 64.57 & 0.718 & 15.4 \\
\hline 309.260 & 26.028 & 11.381 & 69.20 & 69.44 & 0.347 & 14.9 \\
\hline 308.444 & 26.028 & 11.429 & 69.41 & 69.54 & 0.187 & 14.9 \\
\hline 307.756 & 26.028 & 11.470 & 69.41 & 69.63 & 0.317 & 14.9 \\
\hline 307.136 & 26.028 & 11.508 & 69.52 & 69.70 & 0.259 & 14.9 \\
\hline 310.093 & 29.477 & 12.376 & 73.93 & 73.80 & -0.176 & 14.5 \\
\hline 309.235 & 29.477 & 12.427 & 73.95 & 73.92 & -0.041 & 14.5 \\
\hline
\end{tabular}


Data from Roder [8] (continued)

\begin{tabular}{|c|c|c|c|c|c|c|}
\hline $\mathrm{T}$ & $\mathbf{P}$ & $\rho$ & $\lambda, \exp$ & $\lambda, \mathrm{cal}$ & Dev & Wt \\
\hline K & $\mathrm{MPa}$ & $\mathrm{mol} \cdot \mathrm{dm}^{-3}$ & \multicolumn{2}{|c|}{$\mathrm{mW} \cdot \mathrm{m}^{-1} \cdot \mathrm{K}^{-1}$} & 8 & \\
\hline 308.527 & 29.476 & 12.469 & 74.17 & 74.01 & -0.216 & 14.5 \\
\hline 307.869 & 29.475 & 12.508 & 74.30 & 74.10 & -0.269 & 14.4 \\
\hline 309.694 & 33.098 & 13.346 & 78.15 & 78.21 & 0.077 & 14.2 \\
\hline 308.929 & 33.097 & 13.390 & 78.27 & 78.32 & 0.064 & 14.2 \\
\hline 308.143 & 33.098 & 13.436 & 78.33 & 78.44 & 0.140 & 14.2 \\
\hline 307.480 & 33.098 & 13.475 & 78.50 & 78.54 & 0.051 & 14.2 \\
\hline 309.498 & 36.518 & 14.135 & 81.99 & 82.10 & 0.134 & 13.9 \\
\hline 308.688 & 36.519 & 14.181 & 82.22 & 82.23 & 0.012 & 13.9 \\
\hline 308.056 & 36.520 & 14.218 & 82.15 & 82.34 & 0.231 & 13.9 \\
\hline 307.447 & 36.521 & 14.253 & 82.16 & 82.44 & 0.341 & 13.9 \\
\hline 309.222 & 39.909 & 14.831 & 85.88 & 85.79 & -0.105 & 13.7 \\
\hline 308.550 & 39.909 & 14.869 & 86.03 & 85.90 & -0.151 & 13.6 \\
\hline 307.881 & 39.911 & 14.906 & 86.21 & 86.02 & -0.220 & 13.6 \\
\hline 307.206 & 39.914 & 14.945 & 86.23 & 86.15 & -0.093 & 13.6 \\
\hline 309.943 & 43.084 & 15.364 & 89.13 & 88.92 & -0.236 & 13.5 \\
\hline 309.132 & 43.083 & 15.408 & 89.28 & 89.07 & -0.235 & 13.4 \\
\hline 308.403 & 43.081 & 15.447 & 89.18 & 89.20 & 0.022 & 13.5 \\
\hline 307.807 & 43.079 & 15.479 & 89.70 & 89.31 & -0.435 & 13.4 \\
\hline 309.716 & 46.727 & 15.968 & 93.03 & 92.56 & -0.505 & 13.2 \\
\hline 309.019 & 46.727 & 16.004 & 93.12 & 92.69 & -0.462 & 13.2 \\
\hline 308.324 & 46.726 & 16.040 & 93.05 & 92.82 & -0.247 & 13.2 \\
\hline 307.749 & 46.725 & 16.071 & 93.25 & 92.94 & -0.332 & 13.2 \\
\hline 309.703 & 50.127 & 16.468 & 96.30 & 95.79 & -0.530 & 13.0 \\
\hline 308.899 & 50.126 & 16.509 & 96.44 & 95.95 & -0.508 & 13.0 \\
\hline 308.226 & 50.126 & 16.543 & 96.38 & 96.09 & -0.301 & 13.0 \\
\hline 307.650 & 50.126 & 16.573 & 96.65 & 96.21 & -0.455 & 13.0 \\
\hline 309.618 & 53.476 & 16.924 & 99.50 & 98.89 & -0.613 & 12.9 \\
\hline 308.897 & 53.476 & 16.960 & 99.60 & 99.04 & -0.562 & 12.9 \\
\hline 308.209 & 53.476 & 16.994 & 99.74 & 99.19 & -0.551 & 12.9 \\
\hline 307.616 & 53.475 & 17.023 & 99.82 & 99.31 & -0.511 & 12.9 \\
\hline 309.416 & 57.009 & 17.372 & 102.83 & 102.09 & -0.720 & 12.7 \\
\hline 308.753 & 57.009 & 17.404 & 103.06 & 102.24 & -0.796 & 12.7 \\
\hline 308.134 & 57.009 & 17.434 & 103.01 & 102.37 & -0.621 & 12.7 \\
\hline 307.513 & 57.008 & 17.464 & 103.21 & 102.51 & -0.678 & 12.7 \\
\hline 309.315 & 60.531 & 17.781 & 106.01 & 105.17 & -0.792 & 12.6 \\
\hline
\end{tabular}


Data from Roder [8] (continued)

\begin{tabular}{ccccccc}
$\mathrm{T}$ & $\mathrm{P}$ & $\rho$ & $\lambda, \exp$ & $\lambda, \mathrm{cal}$ & Dev & Wt \\
$\mathrm{K}$ & $\mathrm{MPa}$ & $\mathrm{mo} \cdot \cdot \mathrm{dm}^{-3}$ & \multicolumn{2}{c}{$\mathrm{mW} \cdot \mathrm{m}^{-1} \cdot \mathrm{K}^{-1}$} & & \\
308.655 & & & & & & \\
308.072 & 60.530 & 17.812 & 106.23 & 105.32 & -0.857 & 12.5 \\
307.537 & 60.531 & 17.839 & 106.53 & 105.45 & -1.014 & 12.5 \\
309.320 & 60.531 & 17.864 & 106.47 & 105.57 & -0.845 & 12.5 \\
308.648 & 64.078 & 18.159 & 109.19 & 108.17 & -0.934 & 12.4 \\
308.025 & 64.075 & 18.189 & 109.26 & 108.33 & -0.851 & 12.4 \\
307.462 & 64.077 & 18.218 & 109.42 & 108.47 & -0.868 & 12.4 \\
313.129 & 66.672 & 18.248 & 1109.48 & 108.61 & -0.795 & 12.4 \\
312.494 & 66.678 & 18.277 & 110.55 & 109.46 & -0.833 & 12.4 \\
311.828 & 66.670 & 18.306 & 110.76 & 109.74 & -0.921 & 12.3 \\
311.372 & 66.685 & 18.328 & 110.71 & 109.86 & -0.768 & 12.4 \\
309.358 & 67.415 & 18.490 & 112.15 & 110.92 & -1.097 & 12.3 \\
308.758 & 67.414 & 18.516 & 112.44 & 111.06 & -1.227 & 12.2 \\
308.136 & 67.414 & 18.544 & 112.59 & 111.21 & -1.226 & 12.2 \\
307.614 & 67.416 & 18.568 & 112.78 & 111.34 & -1.277 & 12.2
\end{tabular}

Number of Points (Ref. 8) 895

$\begin{array}{llllll}\text { AAD }-8 & 0.577 & \text { BIAS }-8 & 0.069 & \text { RMS - 8 } & 0.942 \\ \text { AAD } & 0.387 & \text { BIAS } & 0.032 & \text { RMS } & 0.584 \mathrm{~mW} \cdot \mathrm{m}^{-1} \cdot \mathrm{K}^{-1}\end{array}$

Data from Sokolova and Golubev [80]

\begin{tabular}{ccclccc}
$\mathrm{T}$ & $\mathrm{P}$ & $\rho$ & $\lambda, \exp$ & $\lambda, \mathrm{cal}$ & Dev & Wt \\
$\mathrm{K}$ & $\mathrm{MPa}$ & $\mathrm{mo} \cdot \mathrm{dm}^{-3}$ & \multicolumn{2}{c}{$\mathrm{mW} \cdot \mathrm{m}^{-1} \cdot \mathrm{K}^{-1}$} & & \\
238.70 & & & & & & \\
238.80 & 0.10 & 0.051 & 26.08 & 26.40 & 1.227 & 0.0 \\
238.80 & 0.20 & 0.101 & 26.13 & 26.50 & 1.416 & 0.0 \\
238.50 & 0.26 & 0.132 & 26.16 & 26.55 & 1.491 & 0.0 \\
238.50 & 0.69 & 0.357 & 26.22 & 26.90 & 2.593 & 0.0 \\
238.80 & 1.06 & 0.557 & 26.80 & 27.24 & 1.642 & 0.0 \\
239.00 & 2.09 & 1.143 & 27.71 & 28.36 & 2.346 & 0.0 \\
238.80 & 2.09 & 1.142 & 27.90 & 28.38 & 1.720 & 0.0 \\
239.00 & 3.04 & 1.734 & 29.17 & 29.55 & 1.303 & 0.0 \\
238.80 & 3.19 & 1.829 & 29.12 & 29.78 & 2.266 & 0.0 \\
& 4.02 & 2.400 & 30.52 & 31.05 & 1.737 & 0.0
\end{tabular}


Data from Sokolova and Golubev [80] (continued)

\begin{tabular}{|c|c|c|c|c|c|c|}
\hline $\mathbf{T}$ & $\mathbf{P}$ & $\rho$ & $\lambda, \exp$ & $\lambda, \mathrm{cal}$ & Dev & Wt \\
\hline K & $\mathrm{MPa}$ & $\mathrm{mol} \cdot \mathrm{dm}^{-3}$ & \multicolumn{2}{|c|}{$\mathrm{mW} \cdot \mathrm{m}^{-1} \cdot \mathrm{K}^{-1}$} & 8 & \\
\hline 238.80 & 4.02 & 2.400 & 30.48 & 31.05 & 1.870 & 0.0 \\
\hline 239.00 & 4.33 & 2.621 & 31.19 & 31.60 & 1.315 & 0.0 \\
\hline 239.00 & 4.33 & 2.621 & 30.73 & 31.60 & 2.831 & 0.0 \\
\hline 238.80 & 5.04 & 3.167 & 32.28 & 32.98 & 2.169 & 0.0 \\
\hline 239.20 & 5.36 & 3.412 & 32.99 & 33.70 & 2.152 & 0.0 \\
\hline 239.20 & 6.49 & 4.387 & 35.76 & 36.60 & 2.349 & 0.0 \\
\hline 238.50 & 6.86 & 4.767 & 37.18 & 37.77 & 1.587 & 0.0 \\
\hline 238.30 & 8.53 & 6.515 & 43.50 & 44.04 & 1.241 & 0.0 \\
\hline 239.00 & 8.72 & 6.661 & 50.45 & 44.64 & -11.516 & 0.0 \\
\hline 238.50 & 10.01 & 8.175 & 51.61 & 50.48 & -2.189 & 0.0 \\
\hline 238.50 & 12.41 & 10.729 & 62.05 & 60.14 & -3.078 & 0.0 \\
\hline 238.50 & 12.41 & 10.729 & 61.67 & 60.14 & -2.481 & 0.0 \\
\hline 238.50 & 14.62 & 12.525 & 67.78 & 66.99 & -1.166 & 0.0 \\
\hline 238.50 & 14.81 & 12.654 & 69.04 & 67.51 & -2.216 & 0.0 \\
\hline 238.50 & 19.72 & 15.113 & 79.13 & 78.91 & -0.278 & 0.0 \\
\hline 238.50 & 19.72 & 15.113 & 78.42 & 78.91 & 0.625 & 0.0 \\
\hline 238.50 & 29.42 & 17.698 & 93.99 & 95.36 & 1.458 & 0.0 \\
\hline 238.80 & 29.53 & 17.694 & 93.66 & 95.36 & 1.815 & 0.0 \\
\hline 239.00 & 40.22 & 19.362 & 106.60 & 109.25 & 2.486 & 0.0 \\
\hline 239.00 & 50.13 & 20.481 & 116.90 & 120.32 & 2.926 & 0.0 \\
\hline 239.00 & 50.23 & 20.491 & 117.20 & 120.42 & 2.747 & 0.0 \\
\hline 231.80 & 0.10 & 0.052 & 25.25 & 25.56 & 1.228 & 0.0 \\
\hline 231.80 & 0.43 & 0.227 & 25.39 & 25.86 & 1.851 & 0.0 \\
\hline 231.80 & 1.08 & 0.586 & 26.00 & 26.48 & 1.846 & 0.0 \\
\hline 231.60 & 2.07 & 1.178 & 26.69 & 27.56 & 3.260 & 0.0 \\
\hline 231.80 & 2.07 & 1.176 & 26.77 & 27.58 & 3.026 & 0.0 \\
\hline 231.80 & 3.04 & 1.813 & 28.24 & 28.90 & 2.337 & 0.0 \\
\hline 231.80 & 4.12 & 2.606 & 30.45 & 30.76 & 1.018 & 0.0 \\
\hline 231.60 & 5.20 & 3.515 & 32.66 & 33.19 & 1.623 & 0.0 \\
\hline 231.60 & 5.20 & 3.515 & 32.57 & 33.19 & 1.904 & 0.0 \\
\hline 231.80 & 5.99 & 4.252 & 34.96 & 35.47 & 1.459 & 0.0 \\
\hline 231.80 & 5.99 & 4.252 & 35.29 & 35.47 & 0.510 & 0.0 \\
\hline 231.80 & 6.58 & 4.860 & 37.22 & 37.51 & 0.779 & 0.0 \\
\hline 232.10 & 7.16 & 5.482 & 39.82 & 39.76 & -0.151 & 0.0 \\
\hline 231.80 & 7.16 & 5.504 & 39.90 & 39.83 & -0.175 & 0.0 \\
\hline
\end{tabular}


Data from Sokolova and Golubev [80] (continued)

\begin{tabular}{|c|c|c|c|c|c|c|}
\hline$T$ & $\mathbf{P}$ & $\rho$ & $\lambda, \exp$ & $\lambda, \mathrm{cal}$ & Dev & Wt \\
\hline K & $\mathrm{MPa}$ & $\mathrm{mol} \cdot \mathrm{dm}^{-3}$ & \multicolumn{2}{|c|}{$\mathrm{mW} \cdot \mathrm{m}^{-1} \cdot \mathrm{K}^{-1}$} & 8 & \\
\hline 231.80 & 7.99 & 6.505 & 45.13 & 43.67 & -3.235 & 0.0 \\
\hline 231.80 & 7.99 & 6.505 & 44.84 & 43.67 & -2.609 & 0.0 \\
\hline 231.80 & 8.34 & 6.952 & 49.61 & 45.44 & -8.406 & 0.0 \\
\hline 231.80 & 8.84 & 7.608 & 50.58 & 48.06 & -4.982 & 0.0 \\
\hline 231.80 & 9.96 & 9.095 & 57.32 & 53.91 & -5.949 & 0.0 \\
\hline 231.80 & 9.96 & 9.095 & 57.15 & 53.91 & -5.669 & .0 \\
\hline 231.60 & 10.89 & 10.294 & 61.55 & 58.38 & -5.150 & 0.0 \\
\hline 231.60 & 10.89 & 10.294 & 62.13 & 58.38 & -6.036 & 0.0 \\
\hline 231.80 & 11.88 & 11.346 & 64.64 & 62.20 & -3.775 & 0.0 \\
\hline 231.80 & 11.88 & 11.346 & 65.69 & 62.20 & -5.313 & 0.0 \\
\hline 231.80 & 13.49 & 12.765 & 70.46 & 67.55 & -4.130 & 0 \\
\hline 231.80 & 15.30 & 13.958 & 74.40 & 72.62 & -2.392 & 0.0 \\
\hline 231.60 & 19.72 & 15.921 & 83.69 & 82.76 & -1.111 & 0.0 \\
\hline 231.60 & 19.72 & 15.921 & 83.44 & 82.76 & -0.815 & 0.0 \\
\hline 231.80 & 23.79 & 17.080 & 90.39 & 90.16 & -0.254 & 0.0 \\
\hline 231.60 & 29.53 & 18.309 & 98.56 & 99.28 & 0.731 & 0.0 \\
\hline 224.80 & 0.10 & 0.054 & 24.45 & 24.72 & 1.104 & 0.0 \\
\hline 224.80 & 0.30 & 0.163 & 24.62 & 24.91 & 1.178 & 0.0 \\
\hline 224.90 & 0.30 & 0.163 & 24.48 & 24.92 & 1.797 & 0.0 \\
\hline 224.80 & 0.61 & 0.336 & 24.80 & 25.20 & 1.613 & 0.0 \\
\hline 225.00 & 0.69 & 0.381 & 24.77 & 25.31 & 2.180 & 0.0 \\
\hline 224.90 & 1.06 & 0.596 & 25.00 & 25.67 & 2.680 & 0.0 \\
\hline 224.80 & 1.08 & 0.608 & 25.21 & 25.68 & 1.864 & 0.0 \\
\hline 224.10 & 2.11 & 1.258 & 26.26 & 26.84 & 2.209 & 0.0 \\
\hline 224.10 & 2.11 & 1.258 & 26.39 & 26.84 & 1.705 & 0.0 \\
\hline 224.60 & 3.04 & 1.905 & 27.77 & 28.28 & 1.837 & 0.0 \\
\hline 224.80 & 3.04 & 1.903 & 27.83 & 28.30 & 1.689 & 0.0 \\
\hline 224.80 & 4.00 & 2.661 & 30.20 & 30.13 & -0.232 & 0.0 \\
\hline 224.90 & 4.02 & 2.675 & 30.17 & 30.18 & 0.033 & 0.0 \\
\hline 224.60 & 4.74 & 3.327 & 32.18 & 31.95 & -0.715 & 0.0 \\
\hline 224.80 & 5.01 & 3.581 & 32.70 & 32.72 & 0.061 & 0.0 \\
\hline 224.80 & 5.01 & 3.581 & 32.62 & 32.72 & 0.307 & 0.0 \\
\hline 224.80 & 5.01 & 3.581 & 32.82 & 32.72 & -0.305 & 0.0 \\
\hline 224.90 & 6.02 & 4.658 & 37.14 & 36.28 & -2.316 & 0.0 \\
\hline 224.90 & 6.02 & 4.658 & 36.68 & 36.28 & -1.091 & 0.0 \\
\hline
\end{tabular}


Data from Sokolova and Golubev [80] (continued)

\begin{tabular}{|c|c|c|c|c|c|c|}
\hline $\mathrm{T}$ & $\mathbf{P}$ & $\rho$ & $\lambda, \exp$ & $\lambda, \mathrm{cal}$ & Dev & Wt \\
\hline K & $\mathrm{MPa}$ & $\mathrm{mol} \cdot \mathrm{dm}^{-3}$ & \multicolumn{2}{|c|}{$\mathrm{mW} \cdot \mathrm{m}^{-1} \cdot \mathrm{K}^{-1}$} & 8 & \\
\hline 224.90 & 6.86 & 5.712 & 42.50 & 40.25 & -5.294 & 0.0 \\
\hline 224.80 & 6.97 & 5.872 & 43.08 & 40.88 & -5.107 & 0.0 \\
\hline 225.40 & 7.75 & 6.927 & 53.67 & 45.23 & -15.726 & 0.0 \\
\hline 225.60 & 7.85 & 7.050 & 54.14 & 45.74 & -15.515 & 0.0 \\
\hline 224.80 & 8.83 & 8.734 & 61.63 & 52.65 & -14.571 & 0.0 \\
\hline 224.10 & 9.91 & 10.562 & 66.57 & 59.45 & -10.696 & 0.0 \\
\hline 224.80 & 9.91 & 10.402 & 65.82 & 58.86 & -10.574 & 0.0 \\
\hline 223.80 & 11.43 & 12.479 & 71.89 & 66.16 & -7.971 & 0.0 \\
\hline 223.80 & 11.43 & 12.479 & 70.51 & 66.16 & -6.169 & 0.0 \\
\hline 223.30 & 12.36 & 13.420 & 73.27 & 69.77 & -4.777 & 0.0 \\
\hline 223.30 & 12.36 & 13.420 & 72.72 & 69.77 & -4.057 & 0.0 \\
\hline 224.10 & 14.71 & 14.818 & 78.84 & 76.10 & -3.475 & 0.0 \\
\hline 223.30 & 14.81 & 14.996 & 79.09 & 76.93 & -2.731 & 0.0 \\
\hline 224.80 & 16.38 & 15.515 & 82.15 & 79.80 & -2.861 & 0.0 \\
\hline 224.10 & 20.20 & 16.944 & 89.72 & 88.40 & -1.471 & 0.0 \\
\hline 224.10 & 20.20 & 16.944 & 89.39 & 88.40 & -1.108 & 0.0 \\
\hline 224.10 & 25.21 & 18.157 & 96.84 & 97.19 & 0.361 & 0.0 \\
\hline 224.40 & 29.53 & 18.926 & 102.50 & 103.56 & 1.034 & 0.0 \\
\hline 223.40 & 38.94 & 20.310 & 113.80 & 116.52 & 2.390 & 0.0 \\
\hline 224.90 & 39.14 & 20.226 & 112.70 & 115.85 & 2.795 & 0.0 \\
\hline 223.40 & 48.36 & 21.287 & 123.00 & 127.23 & 3.439 & 0.0 \\
\hline 223.40 & 48.66 & 21.315 & 122.90 & 127.55 & 3.784 & 0.0 \\
\hline 223.40 & 48.85 & 21.332 & 123.70 & 127.76 & 3.282 & 0.0 \\
\hline 216.40 & 0.10 & 0.056 & 23.47 & 23.71 & 1.023 & 0.0 \\
\hline 216.30 & 0.42 & 0.239 & 23.61 & 24.02 & 1.737 & 0.0 \\
\hline 216.10 & 1.09 & 0.643 & 24.14 & 24.72 & 2.403 & 0.0 \\
\hline 216.10 & 1.97 & 1.225 & 25.30 & 25.85 & 2.174 & 0.0 \\
\hline 216.10 & 3.06 & 2.049 & 27.52 & 27.68 & 0.581 & 0.0 \\
\hline 216.30 & 4.07 & 2.948 & 30.41 & 30.05 & -1.184 & 0.0 \\
\hline 216.30 & 5.01 & 3.962 & 33.97 & 33.24 & -2.149 & 0.0 \\
\hline 216.30 & 5.50 & 4.584 & 37.43 & 35.49 & -5.183 & 0.0 \\
\hline 216.30 & 5.50 & 4.584 & 37.51 & 35.49 & -5.385 & 0.0 \\
\hline 216.10 & 6.18 & 5.608 & 44.00 & 39.64 & -9.909 & 0.0 \\
\hline 216.30 & 6.58 & 6.267 & 51.00 & 42.53 & -16.608 & 0.0 \\
\hline 216.30 & 6.58 & 6.267 & 50.07 & 42.53 & -15.059 & 0.0 \\
\hline
\end{tabular}


Data from Sokolova and Golubev [80] (continued)

\begin{tabular}{|c|c|c|c|c|c|c|}
\hline $\mathrm{T}$ & $\mathrm{P}$ & $\rho$ & $\lambda, \exp$ & $\lambda, \mathrm{cal}$ & Dev & Wt \\
\hline K & $\mathrm{MPa}$ & $\mathrm{mol} \cdot \mathrm{dm}^{-3}$ & \multicolumn{2}{|c|}{$\mathrm{mW} \cdot \mathrm{m}^{-1} \cdot \mathrm{K}^{-1}$} & 8 & \\
\hline 216.60 & 6.97 & 6.948 & 57.40 & 45.59 & -20.575 & 0.0 \\
\hline 216.60 & 6.97 & 6.948 & 57.99 & 45.59 & -21.383 & 0.0 \\
\hline 216.30 & 7.16 & 7.385 & 60.16 & 47.59 & -20.894 & 0.0 \\
\hline 216.30 & 7.16 & 7.385 & 59.41 & 47.59 & -19.896 & 0.0 \\
\hline 216.30 & 7.46 & 8.017 & 63.68 & 50.40 & -20.854 & 0.0 \\
\hline 216.30 & 7.94 & 9.067 & 66.78 & 54.75 & -18.014 & 0.0 \\
\hline 216.30 & 7.99 & 9.176 & 67.28 & 55.17 & -17.999 & 0.0 \\
\hline 216.30 & 8.93 & 11.074 & 71.26 & 61.70 & -13.416 & 0.0 \\
\hline 216.60 & 11.28 & 13.907 & 77.67 & 71.52 & -7.918 & 0.0 \\
\hline 216.60 & 12.85 & 15.030 & 81.27 & 76.61 & -5.734 & 0.0 \\
\hline 216.60 & 15.79 & 16.434 & 88.05 & 84.38 & -4.168 & 0.0 \\
\hline 216.60 & 19.52 & 17.620 & 94.16 & 92.30 & -1.975 & 0.0 \\
\hline 216.60 & 19.72 & 17.673 & 93.70 & 92.69 & -1.078 & 0.0 \\
\hline 216.30 & 25.41 & 18.938 & 102.50 & 102.71 & 0.205 & 0.0 \\
\hline 210.00 & 0.10 & 0.058 & 22.71 & 22.96 & 1.101 & 0.0 \\
\hline 209.80 & 1.10 & 0.673 & 23.36 & 24.04 & 2.911 & 0.0 \\
\hline 208.40 & 1.41 & 0.887 & 23.63 & 24.30 & 2.835 & 0.0 \\
\hline 209.30 & 1.77 & 1.135 & 24.13 & 24.89 & 3.150 & 0.0 \\
\hline 210.30 & 2.08 & 1.355 & 24.65 & 25.47 & 3.327 & 0.0 \\
\hline 209.80 & 2.41 & 1.615 & 25.52 & 25.98 & 1.803 & 0.0 \\
\hline 209.80 & 2.41 & 1.615 & 25.27 & 25.98 & 2.810 & 0.0 \\
\hline 207.50 & 2.91 & 2.071 & 26.65 & 26.83 & 0.675 & 0.0 \\
\hline 210.30 & 3.07 & 2.161 & 27.27 & 27.34 & 0.257 & 0.0 \\
\hline 210.30 & 3.07 & 2.161 & 27.14 & 27.34 & 0.737 & 0.0 \\
\hline 209.00 & 3.54 & 2.634 & 29.10 & 28.48 & -2.131 & 0.0 \\
\hline 209.00 & 3.54 & 2.634 & 28.48 & 28.48 & 0.000 & 0.0 \\
\hline 210.30 & 3.95 & 3.019 & 31.02 & 29.72 & -4.191 & 0.0 \\
\hline 210.30 & 3.95 & 3.019 & 30.86 & 29.72 & -3.694 & 0.0 \\
\hline 207.50 & 4.30 & 3.541 & 32.95 & 31.21 & -5.281 & 0.0 \\
\hline 207.50 & 4.30 & 3.541 & 32.74 & 31.21 & -4.673 & 0.0 \\
\hline 210.10 & 5.01 & 4.348 & 38.64 & 34.33 & -11.154 & 0.0 \\
\hline 210.10 & 5.01 & 4.348 & 38.98 & 34.33 & -11.929 & 0.0 \\
\hline 210.60 & 5.24 & 4.653 & 42.96 & 35.56 & -17.225 & 0.0 \\
\hline 210.60 & 5.24 & 4.653 & 42.62 & 35.56 & -16.565 & 0.0 \\
\hline 210.10 & 5.48 & 5.090 & 49.19 & 37.43 & -23.907 & 0.0 \\
\hline
\end{tabular}


Data from Sokolova and Golubev [80] (continued)

\begin{tabular}{|c|c|c|c|c|c|c|}
\hline $\mathrm{T}$ & $\mathbf{P}$ & $\rho$ & $\lambda, \exp$ & $\lambda, \mathrm{cal}$ & Dev & Wt \\
\hline K & $\mathrm{MPa}$ & $\mathrm{mol} \cdot \mathrm{dm}^{-3}$ & \multicolumn{2}{|c|}{$\mathrm{mW} \cdot \mathrm{m}^{-1} \cdot \mathrm{K}^{-1}$} & 8 & \\
\hline 210.10 & 5.48 & 5.090 & 50.66 & 37.43 & -26.115 & 0.0 \\
\hline 210.10 & 5.48 & 5.090 & 49.07 & 37.43 & -23.721 & 0.0 \\
\hline 209.50 & 5.72 & 5.602 & 55.98 & 39.79 & -28.921 & 0.0 \\
\hline 210.10 & 5.99 & 6.061 & 63.76 & 41.95 & -34.206 & 0.0 \\
\hline 209.60 & 6.00 & 6.166 & 68.16 & 42.52 & -37.617 & 0.0 \\
\hline 210.10 & 6.10 & 6.299 & 69.58 & 43.12 & -38.028 & 0.0 \\
\hline 210.10 & 6.10 & 6.299 & 70.80 & 43.12 & -39.096 & 0.0 \\
\hline 210.10 & 6.20 & 6.524 & 72.35 & 44.24 & -38.853 & 0.0 \\
\hline 210.10 & 6.20 & 6.524 & 75.45 & 44.24 & -41.365 & 0.0 \\
\hline 209.80 & 6.94 & 8.586 & 77.50 & 54.26 & -29.987 & 0.0 \\
\hline 209.80 & 6.94 & 8.586 & 78.21 & 54.26 & -30.623 & 0.0 \\
\hline 206.60 & 7.32 & 11.237 & 72.85 & 64.12 & -11.984 & 0.0 \\
\hline 209.60 & 7.32 & 9.815 & 73.60 & 59.08 & -19.728 & 0.0 \\
\hline 209.60 & 7.32 & 9.815 & 73.10 & 59.08 & -19.179 & 0.0 \\
\hline 209.50 & 7.35 & 9.947 & 72.35 & 59.55 & -17.692 & 0.0 \\
\hline 209.60 & 7.35 & 9.903 & 71.05 & 59.37 & -16.439 & 0.0 \\
\hline 209.60 & 7.66 & 10.773 & 72.39 & 62.00 & -14.353 & 0.0 \\
\hline 210.70 & 8.24 & 11.672 & 74.02 & 64.24 & -13.213 & 0.0 \\
\hline 210.80 & 8.24 & 11.634 & 74.90 & 64.11 & -14.406 & 0.0 \\
\hline 210.80 & 9.91 & 14.023 & 78.92 & 71.89 & -8.908 & 0.0 \\
\hline 210.10 & 12.46 & 15.983 & 83.86 & 81.19 & -3.184 & 0.0 \\
\hline 209.80 & 14.71 & 17.007 & 89.18 & 87.39 & -2.007 & 0.0 \\
\hline 210.30 & 14.92 & 17.011 & 90.02 & 87.46 & -2.844 & 0.0 \\
\hline 209.80 & 16.78 & 17.684 & 93.91 & 92.05 & -1.981 & 0.0 \\
\hline 209.80 & 16.78 & 17.684 & 93.03 & 92.05 & -1.053 & 0.0 \\
\hline 210.00 & 19.72 & 18.423 & 98.14 & 97.70 & -0.448 & 0.0 \\
\hline 210.00 & 30.50 & 20.287 & 112.50 & 114.61 & 1.876 & 0.0 \\
\hline 210.00 & 31.20 & 20.380 & 113.50 & 115.56 & 1.815 & 0.0 \\
\hline 209.60 & 39.73 & 21.385 & 122.50 & 126.55 & 3.306 & 0.0 \\
\hline 209.60 & 39.73 & 21.385 & 122.80 & 126.55 & 3.054 & 0.0 \\
\hline 209.60 & 48.95 & 22.218 & 131.10 & 136.83 & 4.371 & 0.0 \\
\hline 209.60 & 48.95 & 22.218 & 131.80 & 136.83 & 3.816 & 0.0 \\
\hline 209.60 & 50.52 & 22.344 & 133.40 & 138.48 & 3.808 & 0.0 \\
\hline 195.90 & 2.19 & 1.611 & 25.53 & 24.57 & -3.760 & 0.0 \\
\hline 195.90 & 2.19 & 1.611 & 25.23 & 24.57 & -2.616 & 0.0 \\
\hline
\end{tabular}


Data from Sokolova and Golubev [80] (continued)

\begin{tabular}{|c|c|c|c|c|c|c|}
\hline $\mathrm{T}$ & $\mathrm{P}$ & $\rho$ & $\lambda, \exp$ & $\lambda, \mathrm{cal}$ & Dev & Wt \\
\hline K & $\mathrm{MPa}$ & $\mathrm{mol} \cdot \mathrm{dm}^{-3}$ & \multicolumn{2}{|c|}{$\mathrm{mW} \cdot \mathrm{m}^{-1} \cdot \mathrm{K}^{-1}$} & 8 & \\
\hline 195.60 & 2.19 & 1.616 & 25.13 & 24.56 & -2.268 & 0.0 \\
\hline 195.90 & 3.14 & 2.587 & 28.35 & 27.37 & -3.457 & 0.0 \\
\hline 195.40 & 3.14 & 2.604 & 28.52 & 27.40 & -3.927 & 0.0 \\
\hline 195.60 & 3.14 & 2.597 & 27.92 & 27.39 & -1.898 & 0.0 \\
\hline 195.60 & 4.13 & 4.068 & 33.23 & 33.43 & 0.602 & 0.0 \\
\hline 195.40 & 4.13 & 4.087 & 32.53 & 33.55 & 3.136 & 0.0 \\
\hline 195.90 & 4.15 & 4.079 & 34.59 & 33.45 & -3.296 & 0.0 \\
\hline 194.90 & 4.15 & 4.175 & 33.90 & 34.10 & 0.590 & 0.0 \\
\hline 195.40 & 4.18 & 4.185 & 34.23 & 34.07 & -0.467 & 0.0 \\
\hline 195.60 & 4.18 & 4.166 & 33.79 & 33.93 & 0.414 & 0.0 \\
\hline 195.30 & 4.51 & 4.966 & 41.27 & 38.71 & -6.203 & 0.0 \\
\hline 195.90 & 4.66 & 5.284 & 43.71 & 40.62 & -7.069 & 0.0 \\
\hline 195.90 & 4.81 & 5.783 & 54.09 & 44.31 & -18.081 & 0.0 \\
\hline 194.90 & 4.81 & 6.102 & 54.34 & 47.84 & -11.962 & 0.0 \\
\hline 195.50 & 4.92 & 6.387 & 62.51 & 49.76 & -20.397 & 0.0 \\
\hline 195.90 & 4.93 & 6.274 & 68.54 & 48.34 & -29.472 & 0.0 \\
\hline 194.90 & 4.93 & 6.735 & 67.37 & 53.93 & -19.950 & 0.0 \\
\hline 194.90 & 4.93 & 6.735 & 67.83 & 53.93 & -20.492 & 0.0 \\
\hline 195.90 & 5.04 & 6.840 & 80.30 & 53.42 & -33.474 & 0.0 \\
\hline 195.60 & 5.04 & 7.014 & 84.20 & 55.54 & -34.038 & 0.0 \\
\hline 195.90 & 5.05 & 6.899 & 84.87 & 53.97 & -36.409 & 0.0 \\
\hline 195.90 & 5.06 & 6.959 & 86.25 & 54.53 & -36.777 & 0.0 \\
\hline 195.90 & 5.07 & 7.021 & 91.40 & 55.12 & -39.694 & 0.0 \\
\hline 195.90 & 5.08 & 7.085 & 97.89 & 55.72 & -43.079 & 0.0 \\
\hline 194.90 & 5.09 & 8.054 & 112.80 & 67.81 & -39.885 & 0.0 \\
\hline 195.90 & 5.10 & 7.218 & 114.60 & 56.98 & -50.279 & 0.0 \\
\hline 194.90 & 5.10 & 8.168 & 123.80 & 68.89 & -44.354 & 0.0 \\
\hline 194.90 & 5.11 & 8.288 & 143.00 & 70.00 & -51.049 & 0.0 \\
\hline 194.90 & 5.11 & 8.288 & 147.00 & 70.00 & -52.381 & 0.0 \\
\hline 194.90 & 5.11 & 8.288 & 143.90 & 70.00 & -51.355 & 0.0 \\
\hline 194.90 & 5.12 & 8.414 & 149.80 & 71.10 & -52.537 & 0.0 \\
\hline 194.90 & 5.12 & 8.414 & 148.70 & 71.10 & -52.186 & 0.0 \\
\hline 194.90 & 5.16 & 8.969 & 163.10 & 75.29 & -53.838 & 0.0 \\
\hline 194.90 & 5.16 & 8.969 & 174.00 & 75.29 & -56.730 & 0.0 \\
\hline 194.90 & 5.16 & 8.969 & 168.10 & 75.29 & -55.211 & 0.0 \\
\hline
\end{tabular}


Data from Sokolova and Golubev [80] (continued)

\begin{tabular}{|c|c|c|c|c|c|c|}
\hline $\mathrm{T}$ & 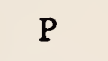 & $\rho$ & $\lambda, \exp$ & $\lambda, \mathrm{cal}$ & Dev & Wt \\
\hline K & $\mathrm{MPa}$ & $\mathrm{mol} \cdot \mathrm{dm}^{-3}$ & \multicolumn{2}{|c|}{$\mathrm{mW} \cdot \mathrm{m}^{-1} \cdot \mathrm{K}^{-1}$} & 8 & \\
\hline 194.90 & 5.16 & 8.969 & 171.60 & 75.29 & -56.125 & 0.0 \\
\hline 194.90 & 5.16 & 8.969 & 165.30 & 75.29 & -54.453 & 0.0 \\
\hline 194.90 & 5.17 & 9.121 & 171.10 & 76.18 & -55.476 & 0.0 \\
\hline 194.90 & 5.26 & 10.535 & 156.50 & 78.46 & -49.866 & 0.0 \\
\hline 194.90 & 5.28 & 10.817 & 153.30 & 77.95 & -49.152 & 0.0 \\
\hline 194.90 & 5.28 & 10.817 & 151.40 & 77.95 & -48.514 & 0.0 \\
\hline 194.90 & 5.28 & 10.817 & 148.70 & 77.95 & -47.579 & 0.0 \\
\hline 194.90 & 5.35 & 11.627 & 124.30 & 75.71 & -39.091 & 0.0 \\
\hline 194.90 & 5.35 & 11.627 & 125.80 & 75.71 & -39.817 & 0.0 \\
\hline 194.90 & 5.35 & 11.627 & 123.10 & 75.71 & -38.497 & 0.0 \\
\hline 194.90 & 5.40 & 12.064 & 117.30 & 74.57 & -36.428 & 0.0 \\
\hline 194.90 & 5.47 & 12.540 & 95.79 & 73.67 & -23.092 & 0.0 \\
\hline 194.90 & 5.48 & 12.599 & 98.89 & 73.59 & -25.584 & 0.0 \\
\hline 194.90 & 5.50 & 12.710 & 102.30 & 73.30 & -28.348 & 0.0 \\
\hline 194.90 & 5.50 & 12.710 & 99.44 & 73.30 & -26.287 & 0.0 \\
\hline 194.90 & 5.50 & 12.710 & 101.40 & 73.30 & -27.712 & 0.0 \\
\hline 194.90 & 5.50 & 12.710 & 100.20 & 73.30 & -26.846 & 0.0 \\
\hline 194.90 & 5.50 & 12.710 & 100.90 & 73.30 & -27.354 & 0.0 \\
\hline 194.90 & 5.51 & 12.764 & 100.80 & 73.25 & -27.331 & 0.0 \\
\hline 194.90 & 5.52 & 12.815 & 95.79 & 73.22 & -23.562 & 0.0 \\
\hline 194.90 & 5.52 & 12.815 & 95.42 & 73.22 & -23.266 & 0.0 \\
\hline 194.90 & 5.52 & 12.815 & 95.96 & 73.22 & -23.697 & 0.0 \\
\hline 194.90 & 5.62 & 13.258 & 88.97 & 73.15 & -17.781 & 0.0 \\
\hline 194.90 & 5.62 & 13.258 & 89.56 & 73.15 & -18.323 & 0.0 \\
\hline 194.90 & 5.62 & 13.258 & 98.64 & 73.15 & -25.841 & 0.0 \\
\hline 194.90 & 6.09 & 14.497 & 83.53 & 75.04 & -10.164 & 0.0 \\
\hline 194.90 & 6.09 & 14.497 & 83.78 & 75.04 & -10.432 & 0.0 \\
\hline 195.40 & 7.05 & 15.601 & 84.87 & 78.89 & -7.046 & 0.0 \\
\hline 195.40 & 7.05 & 15.601 & 84.20 & 78.89 & -6.306 & 0.0 \\
\hline 195.90 & 7.06 & 15.448 & 86.42 & 78.18 & -9.535 & 0.0 \\
\hline 194.90 & 7.09 & 15.798 & 84.57 & 79.82 & -5.617 & 0.0 \\
\hline 194.90 & 7.10 & 15.807 & 85.79 & 79.86 & -6.912 & 0.0 \\
\hline 194.90 & 7.10 & 15.807 & 85.96 & 79.86 & -7.096 & 0.0 \\
\hline 195.90 & 8.15 & 16.375 & 87.71 & 82.69 & -5.723 & 0.0 \\
\hline 195.40 & 8.15 & 16.497 & 87.00 & 83.36 & -4.184 & 0.0 \\
\hline
\end{tabular}


Data from Sokolova and Golubev [80] (continued)

\begin{tabular}{|c|c|c|c|c|c|c|}
\hline $\mathrm{T}$ & $\mathbf{P}$ & $\rho$ & $\lambda, \exp$ & $\lambda, \mathrm{cal}$ & Dev & Wt \\
\hline K & $\mathrm{MPa}$ & $\mathrm{mol} \cdot \mathrm{dm}^{-3}$ & \multicolumn{2}{|c|}{$\mathrm{mW} \cdot \mathrm{m}^{-1} \cdot \mathrm{K}^{-1}$} & 8 & \\
\hline 195.90 & 9.13 & 16.967 & 90.02 & 86.11 & -4.343 & 0.0 \\
\hline 195.40 & 9.13 & 17.070 & 87.71 & 86.73 & -1.117 & 0.0 \\
\hline 195.40 & 9.13 & 17.070 & 88.47 & 86.73 & -1.967 & 0.0 \\
\hline 195.90 & 10.01 & 17.393 & 91.40 & 88.83 & -2.812 & 0.0 \\
\hline 195.40 & 10.10 & 17.524 & 90.81 & 89.68 & -1.244 & 0.0 \\
\hline 194.90 & 10.10 & 17.614 & 92.57 & 90.26 & -2.495 & 0.0 \\
\hline 195.90 & 12.41 & 18.281 & 96.30 & 95.17 & -1.173 & 0.0 \\
\hline 195.40 & 12.41 & 18.355 & 94.91 & 95.69 & 0.822 & 0.0 \\
\hline 195.90 & 12.46 & 18.297 & 95.88 & 95.28 & -0.626 & 0.0 \\
\hline 195.60 & 12.46 & 18.341 & 94.91 & 95.60 & 0.727 & 0.0 \\
\hline 195.60 & 14.81 & 18.983 & 102.30 & 100.78 & -1.486 & 0.0 \\
\hline 195.40 & 14.81 & 19.009 & 103.40 & 100.98 & -2.340 & 0.0 \\
\hline 195.40 & 14.81 & 19.009 & 101.40 & 100.98 & -0.414 & 0.0 \\
\hline 195.90 & 19.37 & 19.894 & 111.30 & 109.02 & -2.049 & 0.0 \\
\hline 195.40 & 19.37 & 19.947 & 109.90 & 109.47 & -0.391 & 0.0 \\
\hline 195.40 & 22.66 & 20.481 & 113.50 & 114.80 & 1.145 & 0.0 \\
\hline 194.50 & 22.66 & 20.568 & 114.70 & 115.59 & 0.776 & 0.0 \\
\hline 195.90 & 22.66 & 20.433 & 116.20 & 114.36 & -1.583 & 0.0 \\
\hline 188.40 & 2.00 & 1.542 & 23.14 & 23.77 & 2.723 & 0.0 \\
\hline 188.40 & 2.00 & 1.542 & 22.55 & 23.77 & 5.410 & 0.0 \\
\hline 188.60 & 3.04 & 2.724 & 25.87 & 27.80 & 7.460 & 0.0 \\
\hline 188.90 & 3.04 & 2.712 & 25.41 & 27.80 & 9.406 & 0.0 \\
\hline 188.60 & 3.74 & 3.948 & 36.35 & 34.12 & -6.135 & 0.0 \\
\hline 188.90 & 3.74 & 3.916 & 35.13 & 33.97 & -3.302 & 0.0 \\
\hline 188.90 & 4.18 & 5.269 & 70.88 & 45.67 & -35.567 & 0.0 \\
\hline 188.90 & 4.20 & 5.365 & 76.91 & 46.81 & -39.137 & 0.0 \\
\hline 188.60 & 4.26 & 5.859 & 87.17 & 53.87 & -38.201 & 0.0 \\
\hline 188.60 & 4.28 & 6.019 & 94.87 & 56.64 & -40.297 & 0.0 \\
\hline 188.60 & 4.28 & 6.019 & 95.12 & 56.64 & -40.454 & 0.0 \\
\hline 188.60 & 4.28 & 6.019 & 93.45 & 56.64 & -39.390 & 0.0 \\
\hline 188.60 & 4.29 & 6.109 & 100.70 & 58.34 & -42.066 & 0.0 \\
\hline 188.60 & 4.32 & 6.439 & 106.40 & 65.72 & -38.233 & 0.0 \\
\hline 188.60 & 4.55 & 14.843 & 91.10 & 77.11 & -15.357 & 0.0 \\
\hline 188.60 & 4.56 & 14.870 & 93.70 & 77.15 & -17.663 & 0.0 \\
\hline 188.60 & 4.57 & 14.897 & 98.98 & 77.20 & -22.004 & 0.0 \\
\hline
\end{tabular}


Data from Sokolova and Golubev [80] (continued)

\begin{tabular}{|c|c|c|c|c|c|c|}
\hline $\mathrm{T}$ & $\mathbf{P}$ & $\rho$ & $\lambda, \exp$ & $\lambda, \mathrm{cal}$ & Dev & Wt \\
\hline K & $\mathrm{MPa}$ & $\mathrm{mol} \cdot \mathrm{dm}^{-3}$ & \multicolumn{2}{|c|}{$\mathrm{mW} \cdot \mathrm{m}^{-1} \cdot \mathrm{K}^{-1}$} & 8 & \\
\hline 188.60 & 5.99 & 16.736 & 94.12 & 84.36 & -10.370 & 0.0 \\
\hline 188.40 & 5.99 & 16.793 & 93.24 & 84.62 & -9.245 & 0.0 \\
\hline 188.40 & 5.99 & 16.793 & 94.58 & 84.62 & -10.531 & 0.0 \\
\hline 188.40 & 5.99 & 16.793 & 93.95 & 84.62 & -9.931 & 0.0 \\
\hline 188.40 & 7.94 & 17.907 & 94.79 & 91.69 & -3.270 & 0.0 \\
\hline 188.40 & 7.94 & 17.907 & 94.62 & 91.69 & -3.097 & 0.0 \\
\hline 188.20 & 10.10 & 18.737 & 97.43 & 97.93 & 0.513 & 0.0 \\
\hline 188.20 & 10.10 & 18.737 & 97.13 & 97.93 & 0.824 & 0.0 \\
\hline 188.20 & 10.10 & 18.737 & 98.14 & 97.93 & -0.214 & 0.0 \\
\hline 187.90 & 11.08 & 19.069 & 100.30 & 100.63 & 0.329 & 0.0 \\
\hline 187.90 & 11.08 & 19.069 & 99.06 & 100.63 & 1.585 & 0.0 \\
\hline 188.60 & 14.92 & 19.874 & 106.00 & 107.96 & 1.849 & 0.0 \\
\hline 188.60 & 14.92 & 19.874 & 104.00 & 107.96 & 3.808 & 0.0 \\
\hline 188.60 & 19.53 & 20.680 & 113.60 & 116.02 & 2.130 & 0.0 \\
\hline 188.60 & 19.53 & 20.680 & 111.80 & 116.02 & 3.775 & 0.0 \\
\hline 188.90 & 19.62 & 20.664 & 112.60 & 115.89 & 2.922 & 0.0 \\
\hline 188.90 & 19.62 & 20.664 & 111.30 & 115.89 & 4.124 & 0.0 \\
\hline 187.90 & 20.70 & 20.924 & 115.50 & 118.52 & 2.615 & 0.0 \\
\hline 187.90 & 20.89 & 20.951 & 114.50 & 118.82 & 3.773 & 0.0 \\
\hline 187.90 & 20.89 & 20.951 & 113.80 & 118.82 & 4.411 & 0.0 \\
\hline 188.40 & 29.63 & 21.964 & 127.50 & 130.69 & 2.502 & 0.0 \\
\hline 188.40 & 29.73 & 21.975 & 126.70 & 130.82 & 3.252 & 0.0 \\
\hline 188.40 & 29.73 & 21.975 & 127.50 & 130.82 & 2.604 & 0.0 \\
\hline 188.20 & 39.33 & 22.871 & 138.20 & 142.58 & 3.169 & 0.0 \\
\hline 188.20 & 39.54 & 22.888 & 138.00 & 142.82 & 3.493 & 0.0 \\
\hline 187.90 & 47.19 & 23.484 & 147.40 & 151.39 & 2.707 & 0.0 \\
\hline 187.90 & 47.19 & 23.484 & 145.50 & 151.39 & 4.048 & 0.0 \\
\hline 187.90 & 49.14 & 23.618 & 149.10 & 153.42 & 2.897 & 0.0 \\
\hline 183.20 & 0.10 & 0.066 & 19.68 & 19.81 & 0.661 & 0.0 \\
\hline 183.20 & 0.26 & 0.174 & 19.68 & 20.01 & 1.677 & 0.0 \\
\hline 183.70 & 0.26 & 0.174 & 19.95 & 20.07 & 0.602 & 0.0 \\
\hline 182.90 & 1.57 & 1.207 & 21.77 & 22.21 & 2.021 & 0.0 \\
\hline 183.20 & 1.57 & 1.204 & 21.65 & 22.24 & 2.725 & 0.0 \\
\hline 183.20 & 2.58 & 2.296 & 25.75 & 25.56 & -0.738 & 0.0 \\
\hline 183.20 & 2.58 & 2.296 & 25.46 & 25.56 & 0.393 & 0.0 \\
\hline
\end{tabular}


Data from Sokolova and Golubev [80] (continued)

\begin{tabular}{|c|c|c|c|c|c|c|}
\hline $\mathrm{T}$ & $\mathbf{P}$ & $\rho$ & $\lambda, \exp$ & $\lambda, \mathrm{cal}$ & Dev & Wt \\
\hline K & $\mathrm{MPa}$ & $\mathrm{mol} \cdot \mathrm{dm}^{-3}$ & \multicolumn{2}{|c|}{$\mathrm{mW} \cdot \mathrm{m}^{-1} \cdot \mathrm{K}^{-1}$} & 8 & \\
\hline 183.20 & 3.07 & 3.039 & 28.63 & 28.65 & 0.070 & 0.0 \\
\hline 182.90 & 3.69 & 16.486 & 87.47 & 81.78 & -6.505 & 0.0 \\
\hline 182.90 & 3.69 & 16.486 & 88.56 & 81.78 & -7.656 & 0.0 \\
\hline 182.90 & 7.46 & 18.748 & 96.66 & 97.18 & 0.538 & 0.0 \\
\hline 183.20 & 7.46 & 18.694 & 98.62 & 96.78 & -1.866 & 0.0 \\
\hline 183.50 & 10.01 & 19.425 & 105.10 & 103.06 & -1.941 & 0.0 \\
\hline 183.50 & 10.01 & 19.425 & 102.30 & 103.06 & 0.743 & 0.0 \\
\hline 183.50 & 14.92 & 20.472 & 112.50 & 113.12 & 0.551 & 0.0 \\
\hline 183.50 & 14.92 & 20.472 & 111.80 & 113.12 & 1.181 & 0.0 \\
\hline 183.70 & 19.72 & 21.200 & 118.90 & 120.98 & 1.749 & 0.0 \\
\hline 183.70 & 19.72 & 21.200 & 120.10 & 120.98 & 0.733 & 0.0 \\
\hline 183.50 & 29.53 & 22.352 & 131.70 & 134.90 & 2.430 & 0.0 \\
\hline 183.50 & 29.53 & 22.352 & 133.30 & 134.90 & 1.200 & 0.0 \\
\hline 183.50 & 39.73 & 23.234 & 142.70 & 147.03 & 3.034 & 0.0 \\
\hline 183.20 & 49.14 & 23.918 & 152.70 & 157.33 & 3.032 & 0.0 \\
\hline 183.20 & 49.14 & 23.918 & 151.50 & 157.33 & 3.848 & 0.0 \\
\hline 176.80 & 0.10 & 0.069 & 19.01 & 19.05 & 0.210 & 0.0 \\
\hline 177.00 & 0.30 & 0.210 & 19.32 & 19.33 & 0.052 & 0.0 \\
\hline 177.00 & 0.30 & 0.210 & 19.22 & 19.33 & 0.572 & 0.0 \\
\hline 175.70 & 0.91 & 0.685 & 20.23 & 20.14 & -0.445 & 0.0 \\
\hline 176.80 & 1.67 & 1.377 & 22.58 & 22.00 & -2.569 & 0.0 \\
\hline 176.50 & 2.61 & 2.605 & 28.53 & 26.33 & -7.711 & 0.0 \\
\hline 176.50 & 2.61 & 2.605 & 28.24 & 26.33 & -6.763 & 0.0 \\
\hline 176.40 & 3.83 & 18.574 & 100.40 & 94.85 & -5.528 & 0.0 \\
\hline 176.40 & 3.83 & 18.574 & 99.40 & 94.85 & -4.577 & 0.0 \\
\hline 176.70 & 5.01 & 19.010 & 102.60 & 98.49 & -4.006 & 0.0 \\
\hline 176.70 & 5.01 & 19.010 & 102.00 & 98.49 & -3.441 & 0.0 \\
\hline 177.00 & 6.24 & 19.377 & 105.40 & 101.72 & -3.491 & 0.0 \\
\hline 177.50 & 7.95 & 19.782 & 108.90 & 105.50 & -3.122 & 0.0 \\
\hline 176.70 & 7.95 & 19.902 & 108.00 & 106.54 & -1.352 & 0.0 \\
\hline 177.00 & 10.06 & 20.341 & 112.60 & 110.89 & -1.519 & 0.0 \\
\hline 177.50 & 11.68 & 20.596 & 116.40 & 113.58 & -2.423 & 0.0 \\
\hline 177.50 & 11.68 & 20.596 & 115.30 & 113.58 & -1.492 & 0.0 \\
\hline 176.50 & 19.72 & 21.899 & 127.30 & 128.17 & 0.683 & 0.0 \\
\hline 176.50 & 19.72 & 21.899 & 129.00 & 128.17 & -0.643 & 0.0 \\
\hline
\end{tabular}


Data from Sokolova and Golubev [80] (continued)

\begin{tabular}{|c|c|c|c|c|c|c|}
\hline $\mathrm{T}$ & $\mathbf{P}$ & $\rho$ & $\lambda, \exp$ & $\lambda, \mathrm{cal}$ & Dev & Wt \\
\hline K & $\mathrm{MPa}$ & $\mathrm{mol} \cdot \mathrm{dm}^{-3}$ & \multicolumn{2}{|c|}{$\mathrm{mW} \cdot \mathrm{m}^{-1} \cdot \mathrm{K}^{-1}$} & 8 & \\
\hline 176.50 & 29.53 & 22.913 & 140.60 & 141.39 & 0.562 & 0.0 \\
\hline 176.70 & 39.19 & 23.672 & 150.10 & 152.47 & 1.579 & 0.0 \\
\hline 176.70 & 39.19 & 23.672 & 149.10 & 152.47 & 2.260 & 0.0 \\
\hline 177.00 & 48.80 & 24.292 & 158.00 & 162.35 & 2.753 & 0.0 \\
\hline 177.00 & 48.80 & 24.292 & 159.00 & 162.35 & 2.107 & 0.0 \\
\hline 165.30 & 0.10 & 0.074 & 17.75 & 17.69 & -0.338 & 0.0 \\
\hline 166.00 & 0.75 & 0.596 & 18.48 & 18.83 & 1.894 & 0.0 \\
\hline 164.20 & 1.54 & 1.427 & 22.88 & 20.99 & -8.260 & 0.0 \\
\hline 164.20 & 1.54 & 1.427 & 22.65 & 20.99 & -7.329 & 0.0 \\
\hline 165.00 & 3.59 & 20.635 & 124.70 & 112.25 & -9.984 & 0.0 \\
\hline 165.50 & 3.59 & 20.555 & 125.00 & 111.49 & -10.808 & 0.0 \\
\hline 164.20 & 5.20 & 21.102 & 126.10 & 117.15 & -7.098 & 0.0 \\
\hline 165.00 & 5.40 & 21.029 & 126.80 & 116.46 & -8.155 & 0.0 \\
\hline 165.30 & 7.95 & 21.454 & 131.30 & 121.28 & -7.631 & 0.0 \\
\hline 164.70 & 9.71 & 21.803 & 134.20 & 125.29 & -6.639 & 0.0 \\
\hline 166.00 & 14.81 & 22.338 & 141.70 & 132.13 & -6.754 & 0.0 \\
\hline $164.70^{\circ}$ & 20.36 & 23.055 & 147.40 & 141.54 & -3.976 & 0.0 \\
\hline 164.70 & 20.36 & 23.055 & 148.50 & 141.54 & -4.687 & 0.0 \\
\hline 166.20 & 29.33 & 23.707 & 157.20 & 151.28 & -3.766 & 0.0 \\
\hline 164.70 & 29.53 & 23.839 & 157.20 & 153.05 & -2.640 & 0.0 \\
\hline 164.20 & 39.14 & 24.541 & 167.30 & 164.26 & -1.817 & 0.0 \\
\hline 164.20 & 49.14 & 25.127 & 175.30 & 174.45 & -0.485 & 0.0 \\
\hline 164.20 & 49.14 & 25.127 & 175.70 & 174.45 & -0.711 & 0.0 \\
\hline 152.60 & 0.10 & 0.080 & 16.39 & 16.19 & -1.220 & 0.0 \\
\hline 151.50 & 0.36 & 0.302 & 16.93 & 16.50 & -2.540 & 0.0 \\
\hline 151.50 & 0.36 & 0.302 & 17.58 & 16.50 & -6.143 & 0.0 \\
\hline 152.70 & 1.60 & 22.039 & 144.70 & 126.36 & -12.674 & 0.0 \\
\hline 152.70 & 3.07 & 22.272 & 148.20 & 129.26 & -12.780 & 0.0 \\
\hline 152.20 & 7.36 & 22.903 & 153.50 & 137.42 & -10.476 & 0.0 \\
\hline 152.20 & 7.36 & 22.903 & 156.70 & 137.42 & -12.304 & 0.0 \\
\hline 152.70 & 9.91 & 23.139 & 156.90 & 140.75 & -10.293 & 0.0 \\
\hline 151.80 & 10.60 & 23.304 & 158.60 & 142.92 & -9.887 & 0.0 \\
\hline 152.90 & 14.81 & 23.608 & 164.10 & 147.53 & -10.098 & 0.0 \\
\hline 153.20 & 14.81 & 23.580 & 164.00 & 147.17 & -10.262 & 0.0 \\
\hline 152.70 & 19.72 & 24.048 & 171.10 & 154.17 & -9.895 & 0.0 \\
\hline
\end{tabular}


Data from Sokolova and Golubev [80] (continued)

\begin{tabular}{|c|c|c|c|c|c|c|}
\hline $\mathrm{T}$ & $\mathbf{P}$ & $\rho$ & $\lambda, \exp$ & $\lambda, \mathrm{cal}$ & Dev & Wt \\
\hline K & $\mathrm{MPa}$ & $\mathrm{mol} \cdot \mathrm{dm}^{-3}$ & \multicolumn{2}{|c|}{$\mathrm{mW} \cdot \mathrm{m}^{-1} \cdot \mathrm{K}^{-1}$} & 8 & \\
\hline 152.90 & 29.13 & 24.714 & 180.60 & 165.05 & -8.610 & 0.0 \\
\hline 152.70 & 29.33 & 24.743 & 178.20 & 165.49 & -7.132 & 0.0 \\
\hline 152.90 & 39.14 & 25.321 & 185.00 & 175.70 & -5.027 & 0.0 \\
\hline 152.90 & 39.14 & 25.321 & 187.90 & 175.70 & -6.493 & 0.0 \\
\hline 152.70 & 39.24 & 25.340 & 184.80 & 176.01 & -4.756 & 0.0 \\
\hline 152.70 & 49.14 & 25.855 & 194.30 & 185.73 & -4.411 & 0.0 \\
\hline 140.30 & 0.10 & 0.087 & 15.07 & 14.75 & -2.123 & 0.0 \\
\hline 140.30 & 0.30 & 0.272 & 16.26 & 15.15 & -6.827 & 0.0 \\
\hline 140.00 & 0.30 & 0.273 & 16.36 & 15.12 & -7.579 & 0.0 \\
\hline 141.00 & 1.47 & 23.477 & 168.10 & 143.50 & -14.634 & 0.0 \\
\hline 141.00 & 3.36 & 23.683 & 170.10 & 146.50 & -13.874 & 0.0 \\
\hline 141.00 & 3.36 & 23.683 & 170.50 & 146.50 & -14.076 & 0.0 \\
\hline 141.00 & 5.10 & 23.859 & 172.90 & 149.12 & -13.754 & 0.0 \\
\hline 140.80 & 5.99 & 23.966 & 173.50 & 150.69 & -13.147 & 0.0 \\
\hline 139.90 & 7.94 & 24.233 & 177.40 & 154.63 & -12.835 & 0.0 \\
\hline 139.30 & 7.94 & 24.291 & 174.00 & 155.44 & -10.667 & 0.0 \\
\hline 139.50 & 9.32 & 24.388 & 177.40 & 157.02 & -11.488 & 0.0 \\
\hline 139.50 & 12.07 & 24.609 & 180.80 & 160.59 & -11.178 & 0.0 \\
\hline 139.50 & 14.81 & 24.813 & 183.20 & 163.99 & -10.486 & 0.0 \\
\hline 140.30 & 19.62 & 25.078 & 188.40 & 168.69 & -10.462 & 0.0 \\
\hline 140.00 & 19.72 & 25.109 & 187.10 & 169.17 & -9.583 & 0.0 \\
\hline 140.00 & 29.72 & 25.711 & 196.70 & 180.06 & -8.460 & 0.0 \\
\hline 140.00 & 39.73 & 26.227 & 201.20 & 190.05 & -5.542 & 0.0 \\
\hline 140.00 & 49.14 & 26.656 & 210.40 & 198.84 & -5.494 & 0.0 \\
\hline 118.80 & 0.10 & 0.104 & 12.71 & 12.27 & -3.462 & 0.0 \\
\hline 117.80 & 0.46 & 25.788 & 196.80 & 175.69 & -10.727 & 0.0 \\
\hline 119.00 & 6.97 & 26.087 & 201.00 & 181.57 & -9.667 & 0.0 \\
\hline 119.70 & 11.48 & 26.286 & 200.30 & 185.57 & -7.354 & 0.0 \\
\hline 119.70 & 11.48 & 26.286 & 205.40 & 185.57 & -9.654 & 0.0 \\
\hline 119.50 & 14.92 & 26.488 & 206.80 & 189.45 & -8.390 & 0.0 \\
\hline 118.00 & 19.72 & 26.844 & 211.80 & 196.15 & -7.389 & 0.0 \\
\hline 117.60 & 29.53 & 27.312 & 217.20 & 205.89 & -5.207 & 0.0 \\
\hline 117.60 & 39.24 & 27.700 & 223.40 & 214.50 & -3.984 & 0.0 \\
\hline 119.90 & 39.43 & 27.555 & 223.40 & 212.10 & -5.058 & 0.0 \\
\hline 119.90 & 48.75 & 27.902 & 229.10 & 220.06 & -3.946 & 0.0 \\
\hline
\end{tabular}


Data from Sokolova and Golubev [80] (continued)

$\begin{array}{crccccc}\mathrm{T} & \mathrm{P} & \rho & \lambda, \exp & \lambda, \mathrm{cal} & \text { Dev } & \text { Wt } \\ \mathrm{K} & \mathrm{MPa} & \mathrm{mol} \cdot \mathrm{dm}^{-3} & \mathrm{~mW} \cdot \mathrm{m}^{-1} \cdot \mathrm{K}^{-1} & & \\ 119.90 & 48.95 & 27.909 & 226.50 & 220.22 & -2.773 & 0.0 \\ 108.90 & 2.95 & 26.741 & 208.80 & 190.84 & -8.602 & 0.0 \\ 108.90 & 2.95 & 26.741 & 209.80 & 190.84 & -9.037 & 0.0 \\ 109.40 & 4.70 & 26.790 & 209.00 & 192.00 & -8.134 & 0.0 \\ 109.40 & 4.70 & 26.790 & 211.20 & 192.00 & -9.091 & 0.0 \\ 109.20 & & & & & & \\ 109.90 & 14.81 & 27.063 & 213.50 & 197.45 & -7.518 & 0.0 \\ 109.90 & 14.81 & 27.241 & 216.80 & 201.43 & -7.089 & 0.0 \\ 109.20 & 19.72 & 27.509 & 220.30 & 206.87 & -6.096 & 0.0 \\ 109.20 & 19.72 & 27.509 & 220.00 & 206.87 & -5.968 & 0.0 \\ 109.40 & 29.53 & 27.888 & 225.60 & 215.36 & -4.539 & 0.0 \\ 109.40 & 38.94 & 28.231 & 230.00 & 223.28 & -2.922 & 0.0 \\ 109.40 & 39.14 & 28.238 & 230.00 & 223.45 & -2.848 & 0.0 \\ 109.40 & 49.73 & 28.591 & 237.80 & 231.96 & -2.456 & 0.0 \\ 109.40 & 49.93 & 28.597 & 235.00 & 232.11 & -1.230 & 0.0\end{array}$

Number of Points (Ref. 80) 445

$\begin{array}{lrllll}\text { AAD }-8 & 9.654 & \text { BIAS }-8 & -8.376 & \text { RMS }-8 & 13.781 \\ \text { AAD } & 10.265 & \text { BIAS } & -9.301 & \text { RMS } & 17.521 \mathrm{~mW} \cdot \mathrm{m}^{-1} \cdot \mathrm{K}^{-1}\end{array}$

\begin{tabular}{|c|c|c|c|c|c|c|}
\hline $\mathrm{T}$ & $\mathrm{P}$ & $\rho$ & $\lambda, \exp$ & $\lambda, \mathrm{ca} 1$ & Dev & Wt \\
\hline K & $\mathrm{MPa}$ & $\mathrm{mol} \cdot \mathrm{dm}^{-3}$ & \multicolumn{2}{|c|}{$\mathrm{mW} \cdot \mathrm{m}^{-1} \cdot \mathrm{K}^{-1}$} & 8 & \\
\hline 323.35 & 0.10 & 0.037 & 37.70 & 37.74 & 0.106 & 0.0 \\
\hline 319.95 & 0.10 & 0.038 & 37.50 & 37.24 & -0.693 & 0.0 \\
\hline 316.75 & 0.10 & 0.038 & 37.30 & 36.77 & -1.421 & 0.0 \\
\hline 312.85 & 0.10 & 0.038 & 36.70 & 36.21 & -1.335 & 0.0 \\
\hline 309.35 & 0.10 & 0.039 & 36.30 & 35.71 & -1.625 & 0.0 \\
\hline 308.25 & 0.10 & 0.039 & 35.70 & 35.55 & -0.420 & 0.0 \\
\hline 303.15 & 0.10 & 0.040 & 35.10 & 34.83 & -0.769 & 0.0 \\
\hline 297.75 & 0.10 & 0.040 & 34.40 & 34.08 & -0.930 & 0.0 \\
\hline 297.75 & 2.55 & 1.077 & 36.30 & 35.84 & -1.267 & 0.0 \\
\hline 297.75 & 5.10 & 2.252 & 38.90 & 38.16 & -1.902 & 0.0 \\
\hline
\end{tabular}


Data from Yorizane et al. [82] (continued)

\begin{tabular}{|c|c|c|c|c|c|c|}
\hline $\mathrm{T}$ & $\mathbf{P}$ & $\rho$ & $\lambda, \exp$ & $\lambda, \mathrm{cal}$ & Dev & Wt \\
\hline K & $\mathrm{mPa}$ & $\mathrm{mol} \cdot \mathrm{dm}^{-3}$ & \multicolumn{2}{|c|}{$\mathrm{mW} \cdot \mathrm{m}^{-1} \cdot \mathrm{K}^{-1}$} & 8 & \\
\hline 297.75 & 7.50 & 3.442 & 42.40 & 40.92 & -3.491 & 0.0 \\
\hline 303.15 & 0.10 & 0.040 & 35.10 & 34.83 & -0.769 & 0.0 \\
\hline 303.15 & 5.30 & 2.291 & 39.90 & 38.99 & -2.281 & 0.0 \\
\hline 303.25 & 10.01 & 4.607 & 46.30 & 44.77 & -3.305 & 0.0 \\
\hline 303.25 & 14.73 & 7.017 & 55.30 & 52.33 & -5.371 & 0.0 \\
\hline 309.85 & 0.10 & 0.039 & 36.60 & 35.78 & -2.240 & 0.0 \\
\hline 312.95 & 5.07 & 2.093 & 41.10 & 39.95 & -2.798 & 0.0 \\
\hline 312.85 & 9.88 & 4.314 & 46.60 & 45.26 & -2.876 & 0.0 \\
\hline 316.75 & 0.10 & 0.038 & 37.20 & 36.77 & -1.156 & 0.0 \\
\hline 316.75 & 2.58 & 1.015 & 39.00 & 38.41 & -1.513 & 0.0 \\
\hline 316.75 & 7.60 & 3.182 & 44.30 & 42.92 & -3.115 & 0.0 \\
\hline 316.85 & 9.93 & 4.251 & 47.10 & 45.64 & -3.100 & 0.0 \\
\hline 316.65 & 12.31 & 5.366 & 50.10 & 48.79 & -2.615 & 0.0 \\
\hline 316.75 & 17.36 & 7.658 & 57.90 & 56.28 & -2.798 & 0.0 \\
\hline 317.75 & 0.10 & 0.038 & 37.20 & 36.92 & -0.753 & 0.0 \\
\hline 317.75 & 5.15 & 2.087 & 41.60 & 40.62 & -2.356 & 0.0 \\
\hline 317.75 & 10.00 & 4.264 & 47.20 & 45.81 & -2.945 & 0.0 \\
\hline 317.75 & 14.82 & 6.489 & 53.80 & 52.46 & -2.491 & 0.0 \\
\hline 317.75 & 19.71 & 8.601 & 61.20 & 59.77 & -2.337 & 0.0 \\
\hline 323.45 & 0.10 & 0.037 & 37.70 & 37.75 & 0.133 & 0.0 \\
\hline $\begin{array}{l}323.45 \\
323.45\end{array}$ & $\begin{array}{r}9.96 \\
14.80\end{array}$ & 4.130 & 47.70 & 46.26 & -3.019 & 0.0 \\
\hline
\end{tabular}

Number of Points (Ref. 82) 32

$\begin{array}{llllll}\text { AAD }-8 & 2.024 & \text { BIAS }-8 & -2.009 & \text { RMS }-8 & 1.182 \\ \text { AAD } & 0.923 & \text { BIAS } & -0.918 & \text { RMS } & 0.640 \mathrm{~mW} \cdot \mathrm{m}^{-1} \cdot \mathrm{K}^{-1}\end{array}$

$\overline{\text { Data from Zheng et al. [75] }}$

\begin{tabular}{cccccrc}
$\mathrm{T}$ & $\mathrm{P}$ & $\rho$ & $\lambda, \exp$ & $\lambda, \mathrm{cal}$ & Dev & Wt \\
$\mathrm{K}$ & $\mathrm{mPa}$ & $\mathrm{mol} \cdot \mathrm{dm}^{-3}$ & \multicolumn{2}{c}{$\mathrm{mW} \cdot \mathrm{m}^{-1} \cdot \mathrm{K}^{-1}$} & & \\
299.45 & 0.10 & 0.040 & 34.74 & 34.31 & -1.238 & 0.0 \\
299.45 & 1.07 & 0.438 & 34.92 & 34.96 & 0.115 & 0.0 \\
299.45 & 2.04 & 0.849 & 35.99 & 35.67 & -0.889 & 0.0 \\
299.45 & 3.00 & 1.269 & 36.56 & 36.43 & -0.356 & 0.0 \\
299.45 & 3.97 & 1.707 & 37.68 & 37.27 & -1.088 & 0.0
\end{tabular}


Data from Zheng et al. [75] (continued)

$\begin{array}{ccccccc}\mathrm{T} & \mathrm{P} & \rho & \lambda, \exp & \lambda, \mathrm{cal} & \text { Dev } & \text { Wt } \\ \mathrm{K} & \mathrm{mPa} & \mathrm{mol} \cdot \mathrm{dm}^{-3} & \mathrm{~mW} \cdot \mathrm{m}^{-1} \cdot \mathrm{K}^{-1} & & \\ 299.45 & 4.94 & 2.159 & 38.43 & 38.20 & -0.598 & 0.0 \\ 299.45 & 5.91 & 2.624 & 39.86 & 39.21 & -1.631 & 0.0 \\ 299.45 & 6.88 & 3.101 & 40.78 & 40.32 & -1.128 & 0.0 \\ 299.45 & 7.79 & 3.559 & 42.32 & 41.44 & -2.079 & 0.0 \\ 299.45 & 7.84 & 3.584 & 42.56 & 41.51 & -2.467 & 0.0 \\ 299.45 & & & & & & \\ 299.45 & 8.81 & 4.082 & 43.35 & 42.81 & -1.246 & 0.0 \\ 299.45 & 10.78 & 4.587 & 44.89 & 44.21 & -1.515 & 0.0 \\ 299.45 & 11.71 & 5.698 & 46.30 & 45.69 & -1.317 & 0.0 \\ 299.45 & 12.68 & 6.119 & 47.93 & 47.24 & -1.440 & 0.0 \\ 299.45 & 13.65 & 6.628 & 51.14 & 50.54 & -1.173 & 0.0 \\ 299.45 & 14.62 & 7.132 & 52.90 & 52.25 & -1.229 & 0.0 \\ 299.45 & 15.54 & 7.601 & 54.51 & 53.89 & -1.137 & 0.0 \\ 299.45 & 15.59 & 7.626 & 54.65 & 53.98 & -1.226 & 0.0\end{array}$

Number of Points (Ref. 75) 19

$\begin{array}{llllll}\text { AAD }-8 & 1.265 & \text { BIAS }-8 & -1.253 & \text { RMS }-8 & 0.586 \\ \text { AAD } & 0.565 & \text { BIAS } & -0.561 & \text { RMS } & 0.275 \mathrm{~mW} \cdot \mathrm{m}^{-1} \cdot \mathrm{K}^{-1}\end{array}$

Number of Points Total with positive weight 920

$\begin{array}{lllrll}\text { AAD }-8 & 0.518 & \text { BIAS }-8 & 0.003 & \text { RMS }-8 & 0.749 \\ \text { AAD } & 0.364 & \text { BIAS } & -0.002 & \text { RMS } & 0.500 \mathrm{~mW} \cdot \mathrm{m}^{-1} \cdot \mathrm{K}^{-1}\end{array}$

Number of Points Total excluding Ref.[80] (Sokolova and Golubev) 1447

$\begin{array}{llllll}\text { AAD }-8 & 0.805 & \text { BIAS }-8 & -0.022 & \text { RMS }-8 & 1.346 \\ \text { AAD } & 0.709 & \text { BIAS } & -0.030 & \text { RMS } & 2.020 \mathrm{~mW} \cdot \mathrm{m}^{-1} \cdot \mathrm{K}^{-1}\end{array}$

Number of Points Total 1892

$\begin{array}{llllll}\text { AAD }-8 & 2.886 & \text { BIAS }-8 & -1.985 & \text { RMS }-8 & 7.656 \\ \text { AAD } & 2.957 & \text { BIAS } & -2.208 & \text { RMS } & 9.528 \mathrm{~mW} \cdot \mathrm{m}^{-1} \cdot \mathrm{K}^{-1}\end{array}$




\section{APPENDIX B}

TABLES FOR THE THERMOPHYSICAL PROPERTIES OF METHANE

B1. PROPERTIES OF IDEAL GAS AT 0.1 MPA AND

DILUTE GAS TRANSPORT PROPERTIES .................. 288

B2. PROPERTIES ALONG SATURATION BOUNDARY.................. 298

B3. PROPERTIES IN THE SINGLE-PHASE REGION ALONG ISOBARS .......... 301

B4. PROPERTIES IN THE SINGLE-PHASE REGION ALONG ISOTHERMS . . . . . . 412

The tables in this Appendix give the values of several thermophysical properties at specified values of the independent state variables as evaluated from the correlating equations given in the main text of this report. The uncertainties in the dependent thermophysical properties are discussed in Section 4. Notes included at the head of each table should also be consulted. 


\section{PROPERTIES OF IDEAL GAS AT $0.1 \mathrm{MPa}$ AND DILUTE GAS TRANSPORT PROPERTIES}

Notes for Table B1. The ideal gas values of the Helmholtz energy, enthalpy, entropy and isobaric specific heat capacity are evaluated from eq (5). The conversion from atmospheric pressure to $0.1 \mathrm{MPa}$ affects the values of $\mathrm{A}^{\mathrm{i} d}$ and $\mathrm{S}^{i d}$. The dilute gas viscosity is from eq (9) and the dilute gas thermal conductivity is from eq (11).

\begin{tabular}{|c|c|c|c|c|c|c|}
\hline $\mathrm{T}$ & $A^{i d}$ & $\mathrm{H}^{\mathrm{id}}$ & $S^{i d}$ & $C_{p}^{i d}$ & $\eta_{0}$ & $\lambda_{0}$ \\
\hline K & $\mathrm{kJ} \cdot \mathrm{mol}^{-1}$ & $\mathrm{~kJ} \cdot \mathrm{mol}^{-1}$ & $\mathrm{~J} \cdot \mathrm{mol}$ & $\cdot K^{-1}$ & $\mu \mathrm{Pa} \cdot \mathrm{s}$ & $\mathrm{mW} \cdot \mathrm{m}^{-1} \cdot \mathrm{K}^{-1}$ \\
\hline 90.6854 & -11.023 & 3.001 & 146.33 & 33.276 & 3.61 & 8.75 \\
\hline 91. & -11.072 & 3.012 & 146.45 & 33.276 & 3.62 & 8.78 \\
\hline 92. & -11.226 & 3.045 & 146.81 & 33.276 & 3.66 & 8.90 \\
\hline 93. & -11.382 & 3.078 & 147.17 & 33.276 & 3.69 & 9.01 \\
\hline 94. & -11.537 & 3.111 & 147.52 & 33.276 & 3.73 & 9.13 \\
\hline 95. & -11.693 & 3.145 & 147.88 & 33.276 & 3.77 & 9.25 \\
\hline 96. & -11.850 & 3.178 & 148.23 & 33.277 & 3.80 & 9.36 \\
\hline 97. & -12.007 & 3.211 & 148.57 & 33.277 & 3.84 & 9.48 \\
\hline 98. & -12.164 & 3.245 & 148.91 & 33.277 & 3.88 & 9.59 \\
\hline 99. & -12.321 & 3.278 & 149.25 & 33.277 & 3.92 & 9.71 \\
\hline 100. & -12.479 & 3.311 & 149.58 & 33.277 & 3.95 & 9.83 \\
\hline 101. & -12.637 & 3.344 & 149.91 & 33.277 & 3.99 & 9.94 \\
\hline 102. & -12.795 & 3.378 & 150.24 & 33.277 & 4.03 & 10.06 \\
\hline 103. & -12.954 & 3.411 & 150.57 & 33.277 & 4.07 & 10.18 \\
\hline 104. & -13.113 & 3.444 & 150.89 & 33.277 & 4.10 & 10.29 \\
\hline 105. & -13.272 & 3.477 & 151.21 & 33.277 & 4.14 & 10.41 \\
\hline 106. & -13.432 & 3.511 & 151.52 & 33.277 & 4.18 & 10.53 \\
\hline 107. & -13.592 & 3.544 & 151.83 & 33.277 & 4.22 & 10.65 \\
\hline 108. & -13.752 & 3.577 & 152.14 & 33.277 & 4.25 & 10.76 \\
\hline 109. & -13.913 & 3.611 & 152.45 & 33.277 & 4.29 & 10.88 \\
\hline 110. & -14.074 & 3.644 & 152.76 & 33.277 & 4.33 & 11.00 \\
\hline 111. & -14.235 & 3.677 & 153.06 & 33.278 & 4.37 & 11.12 \\
\hline 112. & -14.397 & 3.710 & 153.35 & 33.278 & 4.41 & 11.23 \\
\hline 113. & -14.558 & 3.744 & 153.65 & 33.278 & 4.44 & 11.35 \\
\hline 114. & -14.720 & 3.777 & 153.94 & 33.278 & 4.48 & 11.47 \\
\hline 115. & -14.883 & 3.810 & 154.23 & 33.278 & 4.52 & 11.59 \\
\hline 116. & -15.046 & 3.843 & 154.52 & 33.278 & 4.56 & 11.70 \\
\hline 117. & -15.209 & 3.877 & 154.81 & 33.279 & 4.60 & 11.82 \\
\hline 118. & -15.372 & 3.910 & 155.09 & 33.279 & 4.63 & 11.94 \\
\hline 119. & -15.535 & 3.943 & 155.37 & 33.279 & 4.67 & 12.06 \\
\hline
\end{tabular}


Table B1 (continued)

\begin{tabular}{|c|c|c|c|c|c|c|}
\hline $\mathrm{T}$ & $A^{i d}$ & $\mathrm{H}^{i d}$ & $S^{i d}$ & $C_{p}^{i d}$ & $\eta_{0}$ & $\lambda_{0}$ \\
\hline K & $\mathrm{kJ} \cdot \mathrm{mol}^{-1}$ & $\mathrm{~kJ} \cdot \mathrm{mol}^{-1}$ & \multicolumn{2}{|c|}{$\mathrm{J} \cdot \mathrm{mol}^{-1} \cdot \mathrm{K}^{-1}$} & $\mu \mathrm{Pa} \cdot \mathrm{s}$ & $\mathrm{mW} \cdot \mathrm{m}^{-1} \cdot \mathrm{K}^{-1}$ \\
\hline 120. & -15.699 & 3.977 & 155.65 & 33.279 & 4.71 & 12.18 \\
\hline 121. & -15.863 & 4.010 & 155.93 & 33.280 & 4.75 & 12.30 \\
\hline 122. & -16.028 & 4.043 & 156.20 & 33.280 & 4.79 & 12.41 \\
\hline 123. & -16.192 & 4.076 & 156.47 & 33.280 & 4.82 & 12.53 \\
\hline 124. & -16.357 & 4.110 & 156.74 & 33.281 & 4.86 & 12.65 \\
\hline 125. & -16.522 & 4.143 & 157.01 & 33.281 & 4.90 & 12.77 \\
\hline 126. & -16.688 & 4.176 & 157.27 & 33.281 & 4.94 & 12.89 \\
\hline 127. & -16.854 & 4.210 & 157.54 & 33.282 & 4.98 & 13.01 \\
\hline 128. & -17.020 & 4.243 & 157.80 & 33.282 & 5.02 & 13.12 \\
\hline 129. & -17.186 & 4.276 & 158.06 & 33.283 & 5.05 & 13.24 \\
\hline 130. & -17.352 & 4.309 & 158.31 & 33.283 & 5.09 & 13.36 \\
\hline 131. & -17.519 & 4.343 & 158.57 & 33.284 & 5.13 & 13.48 \\
\hline 132. & -17.686 & 4.376 & 158.82 & 33.284 & 5.17 & 13.60 \\
\hline 133. & -17.853 & 4.409 & 159.07 & 33.285 & 5.21 & 13.72 \\
\hline 134. & -18.021 & 4.443 & 159.32 & 33.285 & 5.25 & 13.84 \\
\hline 135. & -18.189 & 4.476 & 159.57 & 33.286 & 5.28 & 13.95 \\
\hline 136. & -18.357 & 4.509 & 159.82 & 33.287 & 5.32 & 14.07 \\
\hline 137. & -18.525 & 4.542 & 160.06 & 33.287 & 5.36 & 14.19 \\
\hline 138. & -18.693 & 4.576 & 160.30 & 33.288 & 5.40 & 14.31 \\
\hline 139. & -18.862 & 4.609 & 160.54 & 33.289 & 5.44 & 14.43 \\
\hline 140. & -19.031 & 4.642 & 160.78 & 33.290 & 5.48 & 14.55 \\
\hline 141. & -19.200 & 4.676 & 161.02 & 33.291 & 5.51 & 14.67 \\
\hline 142. & -19.370 & 4.709 & 161.25 & 33.292 & 5.55 & 14.78 \\
\hline 143. & -19.539 & 4.742 & 161.49 & 33.293 & 5.59 & 14.90 \\
\hline 144. & -19.709 & 4.775 & 161.72 & 33.294 & 5.63 & 15.02 \\
\hline 145. & -19.880 & 4.809 & 161.95 & 33.295 & 5.67 & 15.14 \\
\hline 146. & -20.050 & 4.842 & 162.18 & 33.296 & 5.71 & 15.26 \\
\hline 147. & -20.221 & 4.875 & 162.41 & 33.297 & 5.74 & 15.38 \\
\hline 148. & -20.391 & 4.909 & 162.63 & 33.299 & 5.78 & 15.50 \\
\hline 149. & -20.562 & 4.942 & 162.86 & 33.300 & 5.82 & 15.61 \\
\hline 150. & -20.734 & 4.975 & 163.08 & 33.302 & 5.86 & 15.73 \\
\hline 151. & -20.905 & 5.009 & 163.30 & 33.303 & 5.90 & 15.85 \\
\hline 152. & -21.077 & 5.042 & 163.52 & 33.305 & 5.93 & 15.97 \\
\hline 153. & -21.249 & 5.075 & 163.74 & 33.306 & 5.97 & 16.09 \\
\hline 154. & -21.421 & 5.108 & 163.95 & 33.308 & 6.01 & 16.21 \\
\hline 155. & -21.593 & 5.142 & 164.17 & 33.310 & 6.05 & 16.32 \\
\hline 156. & -21.766 & 5.175 & 164.38 & 33.312 & 6.09 & 16.44 \\
\hline 157. & -21.939 & 5.208 & 164.60 & 33.314 & 6.13 & 16.56 \\
\hline 158. & -22.112 & 5.242 & 164.81 & 33.316 & 6.16 & 16.68 \\
\hline 159. & -22.285 & 5.275 & 165.02 & 33.318 & 6.20 & 16.80 \\
\hline
\end{tabular}


Table B1 (continued)

\begin{tabular}{|c|c|c|c|c|c|c|}
\hline $\mathrm{T}$ & $A^{i d}$ & $\mathrm{H}^{\mathrm{id}}$ & $S^{i d}$ & $C_{p}^{i d}$ & $\eta_{0}$ & $\lambda_{0}$ \\
\hline K & $\mathrm{kJ} \cdot \mathrm{mol}^{-1}$ & $\mathrm{~kJ} \cdot \mathrm{mol}^{-1}$ & $\mathrm{~J} \cdot \mathrm{mol}^{-}$ & $\cdot \mathrm{K}^{-1}$ & $\mu \mathrm{Pa} \cdot \mathrm{s}$ & $\mathrm{mW} \cdot \mathrm{m}^{-1} \cdot \mathrm{K}^{-1}$ \\
\hline 160. & -22.459 & 5.308 & 165.23 & 33.320 & 6.24 & 16.92 \\
\hline 161. & -22.632 & 5.342 & 165.44 & 33.322 & 6.28 & 17.03 \\
\hline 162 . & -22.806 & 5.375 & 165.64 & 33.325 & 6.32 & 17.15 \\
\hline 163. & -22.980 & 5.408 & 165.85 & 33.327 & 6.35 & 17.27 \\
\hline 164. & -23.154 & 5.442 & 166.05 & 33.330 & 6.39 & 17.39 \\
\hline 165. & -23.329 & 5.475 & 166.25 & 33.332 & 6.43 & 17.51 \\
\hline 166. & -23.503 & 5.508 & 166.46 & 33.335 & 6.47 & 17.63 \\
\hline 167. & -23.678 & 5.542 & 166.66 & 33.338 & 6.50 & 17.74 \\
\hline 168. & -23.853 & 5.575 & 166.85 & 33.341 & 6.54 & 17.86 \\
\hline 169. & -24.029 & 5.608 & 167.05 & 33.344 & 6.58 & 17.98 \\
\hline 170. & -24.204 & 5.642 & 167.25 & 33.348 & 6.62 & 18.10 \\
\hline 171. & -24.380 & 5.675 & 167.44 & 33.351 & 6.66 & 18.22 \\
\hline 172. & -24.556 & 5.708 & 167.64 & 33.355 & 6.69 & 18.33 \\
\hline 173. & -24.732 & 5.742 & 167.83 & 33.358 & 6.73 & 18.45 \\
\hline 174. & -24.908 & 5.775 & 168.02 & 33.362 & 6.77 & 18.57 \\
\hline 175. & -25.084 & 5.808 & 168.22 & 33.366 & 6.81 & 18.69 \\
\hline 176. & -25.261 & 5.842 & 168.41 & 33.370 & 6.84 & 18.81 \\
\hline 177. & -25.438 & 5.875 & 168.60 & 33.374 & 6.88 & 18.92 \\
\hline 178. & -25.615 & 5.909 & 168.78 & 33.379 & 6.92 & 19.04 \\
\hline 179. & -25.792 & 5.942 & 168.97 & 33.383 & 6.96 & 19.16 \\
\hline 180. & -25.969 & 5.975 & 169.16 & 33.388 & 6.99 & 19.28 \\
\hline 181. & -26.147 & 6.009 & 169.34 & 33.393 & 7.03 & 19.40 \\
\hline 182. & -26.325 & 6.042 & 169.53 & 33.397 & 7.07 & 19.52 \\
\hline 183. & -26.503 & 6.076 & 169.71 & 33.403 & 7.10 & 19.63 \\
\hline 184. & -26.681 & 6.109 & 169.89 & 33.408 & 7.14 & 19.75 \\
\hline 185. & -26.859 & 6.142 & 170.07 & 33.413 & 7.18 & 19.87 \\
\hline 186. & -27.038 & 6.176 & 170.25 & 33.419 & 7.22 & 19.99 \\
\hline 187. & -27.216 & 6.209 & 170.43 & 33.425 & 7.25 & 20.11 \\
\hline 188. & -27.395 & 6.243 & 170.61 & 33.431 & 7.29 & 20.22 \\
\hline 189. & -27.574 & 6.276 & 170.79 & 33.437 & 7.33 & 20.34 \\
\hline 190. & -27.753 & 6.309 & 170.96 & $33.44 \mathrm{~s}$ & 7.36 & 20.46 \\
\hline 190.551 & -27.852 & 6.328 & 171.06 & 33.447 & 7.38 & 20.52 \\
\hline 191. & -27.933 & 6.343 & 171.14 & 33.450 & 7.40 & 20.58 \\
\hline 192. & -28.112 & 6.376 & 171.31 & 33.457 & 7.44 & 20.70 \\
\hline 193. & -28.292 & 6.410 & 171.49 & 33.463 & 7.48 & 20.81 \\
\hline 194. & -28.472 & 6.443 & 171.66 & 33.471 & 7.51 & 20.93 \\
\hline 195. & -28.652 & 6.477 & 171.83 & 33.478 & 7.55 & 21.05 \\
\hline 196. & -28.832 & 6.510 & 172.00 & 33.485 & 7.59 & 21.17 \\
\hline 197. & -29.012 & 6.544 & 172.17 & 33.493 & 7.62 & 21.29 \\
\hline 198 & -29.193 & 6.577 & 172.34 & 33.501 & 7.66 & 21.41 \\
\hline
\end{tabular}


Table B1 (continued)

\begin{tabular}{|c|c|c|c|c|c|c|}
\hline $\mathrm{T}$ & $A^{i d}$ & $\mathrm{H}^{\mathrm{id}}$ & $S^{i d}$ & $C_{p}^{i d}$ & $\eta_{0}$ & $\lambda_{0}$ \\
\hline K & $\mathrm{kJ} \cdot \mathrm{mol}^{-1}$ & $\mathrm{~kJ} \cdot \mathrm{mol}^{-1}$ & $\mathrm{~J} \cdot \mathrm{mol}$ & $\cdot \mathrm{K}^{-1}$ & $\mu \mathrm{Pa} \cdot \mathrm{s}$ & $\mathrm{mW} \cdot \mathrm{m}^{-1} \cdot \mathrm{K}^{-1}$ \\
\hline 199. & -29.374 & 6.611 & 172.51 & 33.509 & 7.70 & 21.52 \\
\hline 200. & -29.555 & 6.644 & 172.68 & 33.518 & 7.73 & 21.64 \\
\hline 201. & -29.736 & 6.678 & 172.85 & 33.526 & 7.77 & 21.76 \\
\hline 202. & -29.917 & 6.711 & 173.01 & 33.535 & 7.81 & 21.88 \\
\hline 203. & -30.098 & 6.745 & 173.18 & 33.544 & 7.84 & 22.00 \\
\hline 204. & -30.280 & 6.778 & 173.34 & 33.553 & 7.88 & 22.12 \\
\hline 205. & -30.462 & 6.812 & 173.51 & 33.563 & 7.92 & 22.24 \\
\hline 206. & -30.644 & 6.846 & 173.67 & 33.573 & 7.95 & 22.36 \\
\hline 207. & -30.826 & 6.879 & 173.83 & 33.583 & 7.99 & 22.47 \\
\hline 208. & -31.008 & 6.913 & 174.00 & 33.593 & 8.02 & 22.59 \\
\hline 209. & -31.190 & 6.946 & 174.16 & 33.603 & 8.06 & 22.71 \\
\hline 210. & -31.373 & 6.980 & 174.32 & 33.614 & 8.10 & 22.83 \\
\hline 211. & -31.556 & 7.014 & 174.48 & 33.625 & 8.13 & 22.95 \\
\hline 212 . & -31.738 & 7.047 & 174.64 & 33.636 & 8.17 & 23.07 \\
\hline 213. & -31.921 & 7.081 & 174.79 & 33.648 & 8.21 & 23.19 \\
\hline 214 . & -32.105 & 7.114 & 174.95 & 33.659 & 8.24 & 23.31 \\
\hline 215 . & -32.288 & 7.148 & 175.11 & 33.671 & 8.28 & 23.43 \\
\hline 216. & -32.471 & 7.182 & 175.27 & 33.683 & 8.31 & 23.55 \\
\hline 217. & -32.655 & 7.215 & 175.42 & 33.696 & 8.35 & 23.67 \\
\hline 218 . & -32.839 & 7.249 & 175.58 & 33.709 & 8.38 & 23.79 \\
\hline 219. & -33.023 & 7.283 & 175.73 & 33.722 & 8.42 & 23.91 \\
\hline 220. & -33.207 & 7.317 & 175.88 & 33.735 & 8.46 & 24.03 \\
\hline 221. & -33.391 & 7.350 & 176.04 & 33.749 & 8.49 & 24.15 \\
\hline 222. & -33.576 & 7.384 & 176.19 & 33.762 & 8.53 & 24.27 \\
\hline 223. & -33.760 & 7.418 & 176.34 & 33.777 & 8.56 & 24.39 \\
\hline 224 & -33.945 & 7.452 & 176.49 & 33.791 & 8.60 & 24.51 \\
\hline 225. & -34.130 & 7.485 & 176.64 & 33.806 & 8.63 & 24.63 \\
\hline 226. & -34.315 & 7.519 & 176.79 & 33.821 & 8.67 & 24.75 \\
\hline 227. & -34.500 & 7.553 & 176.94 & 33.836 & 8.71 & 24.87 \\
\hline 228 . & -34.685 & 7.587 & 177.09 & 33.851 & 8.74 & 25.00 \\
\hline 229. & -34.871 & 7.621 & 177.24 & 33.867 & 8.78 & 25.12 \\
\hline 230. & -35.057 & 7.655 & 177.39 & 33.883 & 8.81 & 25.24 \\
\hline 231. & -35.242 & 7.689 & 177.53 & 33.900 & 8.85 & 25.36 \\
\hline 232. & -35.428 & 7.722 & 177.68 & 33.916 & 8.88 & 25.48 \\
\hline 233. & -35.614 & 7.756 & 177.83 & 33.933 & 8.92 & 25.60 \\
\hline 234. & -35.801 & 7.790 & 177.97 & 33.951 & 8.95 & 25.73 \\
\hline 235. & -35.987 & 7.824 & 178.12 & 33.968 & 8.99 & 25.85 \\
\hline 236 . & -36.173 & 7.858 & 178.26 & 33.986 & 9.02 & 25.97 \\
\hline 237. & -36.360 & 7.892 & 178.40 & 34.004 & 9.06 & 26.09 \\
\hline 238. & -36.547 & 7.926 & 178.55 & 34.023 & 9.09 & 26.22 \\
\hline
\end{tabular}


Table B1 (continued)

\begin{tabular}{|c|c|c|c|c|c|c|}
\hline $\mathrm{T}$ & $A^{i d}$ & $\mathrm{H}^{i d}$ & $S^{1 d}$ & $C_{p}^{i d}$ & $\eta_{0}$ & $\lambda_{0}$ \\
\hline K & $\mathrm{kJ} \cdot \mathrm{mol}^{-1}$ & $\mathrm{~kJ} \cdot \mathrm{mol}^{-1}$ & \multicolumn{2}{|c|}{$\mathrm{J} \cdot \mathrm{mol}^{-1} \cdot \mathrm{K}^{-1}$} & $\mu \mathrm{Pa} \cdot \mathrm{s}$ & $\mathrm{mW} \cdot \mathrm{m}^{-1} \cdot \mathrm{K}^{-1}$ \\
\hline 239. & -36.734 & 7.960 & 178.69 & 34.041 & 9.13 & 26.34 \\
\hline 240 & -36.921 & 7.994 & 178.83 & 34.061 & 9.16 & 26.46 \\
\hline 241 . & -37.108 & 8.028 & 178.97 & 34.080 & 9.20 & 26.59 \\
\hline 242 . & -37.295 & 8.063 & 179.12 & 34.100 & 9.23 & 26.71 \\
\hline 243. & -37.483 & 8.097 & 179.26 & 34.120 & 9.27 & 26.84 \\
\hline 244. & -37.671 & 8.131 & 179.40 & 34.140 & 9.30 & 26.96 \\
\hline 245 . & -37.858 & 8.165 & 179.54 & 34.161 & 9.34 & 27.08 \\
\hline 246 . & -38.046 & 8.199 & 179.67 & 34.181 & 9.37 & 27.21 \\
\hline 247. & -38.234 & 8.233 & 179.81 & 34.203 & 9.41 & 27.33 \\
\hline 248 . & -38.423 & 8.268 & 179.95 & 34.224 & 9.44 & 27.46 \\
\hline 249 . & -38.611 & 8.302 & 180.09 & 34.246 & 9.48 & 27.58 \\
\hline 250 . & -38.799 & 8.336 & 180.23 & 34.268 & 9.51 & 27.71 \\
\hline 251. & -38.988 & 8.370 & 180.36 & 34.291 & 9.54 & 27.83 \\
\hline 252. & -39.177 & 8.405 & 180.50 & 34.313 & 9.58 & 27.96 \\
\hline 253. & -39.366 & 8.439 & 180.64 & 34.337 & 9.61 & 28.09 \\
\hline 254 . & -39.555 & 8.473 & 180.77 & 34.360 & 9.65 & 28.21 \\
\hline 255. & -39.744 & 8.508 & 180.91 & 34.384 & 9.68 & 28.34 \\
\hline 256. & -39.933 & 8.542 & 181.04 & 34.408 & 9.72 & 28.47 \\
\hline 257 . & -40.122 & 8.576 & 181.18 & 34.432 & 9.75 & 28.59 \\
\hline 258. & -40.312 & 8.611 & 181.31 & 34.457 & 9.79 & 28.72 \\
\hline 259 . & -40.502 & 8.645 & 181.44 & 34.482 & 9.82 & 28.85 \\
\hline 260. & -40.692 & 8.680 & 181.58 & 34.507 & 9.85 & 28.97 \\
\hline 261. & -40.882 & 8.714 & 181.71 & 34.533 & 9.89 & 29.10 \\
\hline 262. & -41.072 & 8.749 & 181.84 & 34.559 & 9.92 & 29.23 \\
\hline 263. & -41.262 & 8.783 & 181.97 & 34.585 & 9.96 & 29.36 \\
\hline 264 . & -41.452 & 8.818 & 182.10 & 34.612 & 9.99 & 29.49 \\
\hline 265. & -41.643 & 8.853 & 182.23 & 34.638 & 10.02 & 29.62 \\
\hline 266. & -41.833 & 8.887 & 182.36 & 34.666 & 10.06 & 29.75 \\
\hline 267. & -42.024 & 8.922 & 182.49 & 34.693 & 10.09 & 29.88 \\
\hline 268 . & -42.215 & 8.957 & 182.62 & 34.721 & 10.12 & 30.01 \\
\hline 269 . & -42.406 & 8.991 & 182.75 & 34.749 & 10.16 & 30.14 \\
\hline 270 . & -42.597 & 9.026 & 182.88 & 34.778 & 10.19 & 30.27 \\
\hline 271. & -42.788 & 9.061 & 183.01 & 34.806 & 10.23 & 30.40 \\
\hline 272 . & -42.980 & 9.096 & 183.14 & 34.835 & 10.26 & 30.53 \\
\hline 273 . & -43.171 & 9.131 & 183.27 & 34.865 & 10.29 & 30.66 \\
\hline 273.15 & -43.200 & 9.136 & 183.29 & 34.869 & 10.30 & 30.68 \\
\hline 274. & -43.363 & 9.166 & 183.40 & 34.894 & 10.33 & 30.79 \\
\hline 275 . & -43.555 & 9.201 & 183.52 & 34.924 & 10.36 & 30.92 \\
\hline 276 . & -43.747 & 9.235 & 183.65 & 34.955 & 10.39 & 31.05 \\
\hline 277 . & -43.939 & 9.270 & 183.78 & 34.985 & 10.43 & 31.19 \\
\hline
\end{tabular}


Table B1 (continued)

\begin{tabular}{|c|c|c|c|c|c|c|}
\hline $\mathrm{T}$ & $A^{i d}$ & $\mathrm{H}^{\mathrm{id}}$ & $S^{i d}$ & $C_{p}^{i d}$ & $\eta_{0}$ & $\lambda_{0}$ \\
\hline K & $\mathrm{kJ} \cdot \mathrm{mol}^{-1}$ & $\mathrm{~kJ} \cdot \mathrm{mol}^{-1}$ & $\mathrm{~J} \cdot \mathrm{mol}^{-}$ & $\cdot \mathrm{K}^{-1}$ & $\mu \mathrm{Pa} \cdot \mathrm{s}$ & $\mathrm{mW} \cdot \mathrm{m}^{-1} \cdot \mathrm{K}^{-1}$ \\
\hline 278 & -44.131 & 9.305 & 183.90 & 35.016 & 10.46 & 31.32 \\
\hline 279. & -44.323 & 9.340 & 184.03 & 35.048 & 10.49 & 31.45 \\
\hline 280. & -44.515 & 9.376 & 184.15 & 35.079 & 10.53 & 31.59 \\
\hline 281 . & -44.708 & 9.411 & 184.28 & 35.111 & 10.56 & 31.72 \\
\hline 282. & -44.901 & 9.446 & 184.40 & 35.143 & 10.59 & 31.85 \\
\hline 283. & -45.093 & 9.481 & 184.53 & 35.176 & 10.63 & 31.99 \\
\hline 284. & -45.286 & 9.516 & 184.65 & 35.208 & 10.66 & 32.12 \\
\hline 285. & -45.479 & 9.551 & 184.78 & 35.241 & 10.69 & 32.26 \\
\hline 286. & -45.672 & 9.587 & 184.90 & 35.275 & 10.72 & 32.39 \\
\hline 287. & -45.866 & 9.622 & 185.02 & 35.308 & 10.76 & 32.53 \\
\hline 288 . & -46.059 & 9.657 & 185.14 & 35.342 & 10.79 & 32.66 \\
\hline 289. & -46.253 & 9.693 & 185.27 & 35.377 & 10.82 & 32.80 \\
\hline 290. & -46.446 & 9.728 & 185.39 & 35.411 & 10.86 & 32.94 \\
\hline 291. & -46.640 & 9.763 & 185.51 & 35.446 & 10.89 & 33.07 \\
\hline 292. & -46.834 & 9.799 & 185.63 & 35.481 & 10.92 & 33.21 \\
\hline 293. & -47.028 & 9.834 & 185.75 & 35.517 & 10.95 & 33.35 \\
\hline 294. & -47.222 & 9.870 & 185.88 & 35.552 & 10.99 & 33.48 \\
\hline 295 . & -47.416 & 9.905 & 186.00 & 35.588 & 11.02 & 33.62 \\
\hline 296. & -47.611 & 9.941 & 186.12 & 35.625 & 11.05 & 33.76 \\
\hline 297. & -47.805 & 9.977 & 186.24 & 35.661 & 11.08 & 33.90 \\
\hline 298. & -48.000 & 10.012 & 186.36 & 35.698 & 11.12 & 34.04 \\
\hline 298.15 & -48.029 & 10.018 & 186.38 & 35.704 & 11.12 & 34.06 \\
\hline 299. & -48.195 & 10.048 & 186.48 & 35.735 & 11.15 & 34.18 \\
\hline 300. & -48.389 & 10.084 & 186.60 & 35.773 & 11.18 & 34.32 \\
\hline 301. & -48.584 & 10.120 & 186.72 & 35.811 & 11.21 & 34.46 \\
\hline 302. & -48.779 & 10.155 & 186.83 & 35.848 & 11.25 & 34.60 \\
\hline 303. & -48.975 & 10.191 & 186.95 & 35.887 & 11.28 & 34.74 \\
\hline 304. & -49.170 & 10.227 & 187.07 & 35.925 & 11.31 & 34.88 \\
\hline 305 . & -49.365 & 10.263 & 187.19 & 35.964 & 11.34 & 35.02 \\
\hline 306 . & -49.561 & 10.299 & 187.31 & 36.003 & 11.37 & 35.16 \\
\hline 307. & -49.757 & 10.335 & 187.42 & 36.043 & 11.41 & 35.30 \\
\hline 308. & -49.952 & 10.371 & 187.54 & 36.082 & 11.44 & 35.44 \\
\hline 309. & -50.148 & 10.407 & 187.66 & 36.122 & 11.47 & 35.59 \\
\hline 310 . & -50.344 & 10.443 & 187.78 & 36.162 & 11.50 & 35.73 \\
\hline 311. & -50.541 & 10.480 & 187.89 & 36.203 & 11.53 & 35.87 \\
\hline 312 . & -50.737 & 10.516 & 188.01 & 36.243 & 11.57 & 36.02 \\
\hline 313. & -50.933 & 10.552 & 188.12 & 36.284 & 11.60 & 36.16 \\
\hline 314. & -51.130 & 10.588 & 188.24 & 36.326 & 11.63 & 36.31 \\
\hline 315. & -51.326 & 10.625 & 188.36 & 36.367 & 11.66 & 36.45 \\
\hline 316. & -51.523 & 10.661 & 188.47 & 36.409 & 11.69 & 36.59 \\
\hline
\end{tabular}


Table Bl (continued)

\begin{tabular}{|c|c|c|c|c|c|c|}
\hline $\mathrm{T}$ & $A^{i d}$ & $\mathrm{H}^{\mathrm{id}}$ & $S^{i d}$ & $C_{p}^{i d}$ & $\eta_{0}$ & $\lambda_{0}$ \\
\hline K & $\mathrm{kJ} \cdot \mathrm{mol}^{-1}$ & $\mathrm{~kJ} \cdot \mathrm{mol}^{-1}$ & $\mathrm{~J} \cdot \mathrm{mol}^{-}$ & $\cdot \mathrm{K}^{-1}$ & $\mu \mathrm{Pa} \cdot \mathrm{s}$ & $\mathrm{mW} \cdot \mathrm{m}^{-1} \cdot \mathrm{K}^{-1}$ \\
\hline $\begin{array}{l}317 . \\
318 . \\
319 . \\
320 . \\
321 .\end{array}$ & $\begin{array}{l}-51.720 \\
-51.917 \\
-52.114 \\
-52.311 \\
-52.508\end{array}$ & $\begin{array}{l}10.698 \\
10.734 \\
10.771 \\
10.807 \\
10.844\end{array}$ & $\begin{array}{l}188.59 \\
188.70 \\
188.82 \\
188.93 \\
189.04\end{array}$ & $\begin{array}{l}36.451 \\
36.493 \\
36.535 \\
36.578 \\
36.621\end{array}$ & $\begin{array}{l}11.73 \\
11.76 \\
11.79 \\
11.82 \\
11.85\end{array}$ & $\begin{array}{l}36.74 \\
36.89 \\
37.03 \\
37.18 \\
37.32\end{array}$ \\
\hline $\begin{array}{l}322 . \\
323 . \\
324 . \\
325 . \\
326 .\end{array}$ & $\begin{array}{l}-52.706 \\
-52.903 \\
-53.101 \\
-53.299 \\
-53.497\end{array}$ & $\begin{array}{l}10.880 \\
10.917 \\
10.954 \\
10.991 \\
11.027\end{array}$ & $\begin{array}{l}189.16 \\
189.27 \\
189.39 \\
189.50 \\
189.61\end{array}$ & $\begin{array}{l}36.664 \\
36.708 \\
36.751 \\
36.795 \\
36.840\end{array}$ & $\begin{array}{l}11.88 \\
11.92 \\
11.95 \\
11.98 \\
12.01\end{array}$ & $\begin{array}{l}37.47 \\
37.62 \\
37.77 \\
37.91 \\
38.06\end{array}$ \\
\hline $\begin{array}{l}327 . \\
328 . \\
329 . \\
330 . \\
331 .\end{array}$ & $\begin{array}{l}-53.695 \\
-53.893 \\
-54.091 \\
-54.289 \\
-54.488\end{array}$ & $\begin{array}{l}11.064 \\
11.101 \\
11.138 \\
11.175 \\
11.212\end{array}$ & $\begin{array}{l}189.72 \\
189.84 \\
189.95 \\
190.06 \\
190.17\end{array}$ & $\begin{array}{l}36.884 \\
36.929 \\
36.973 \\
37.018 \\
37.064\end{array}$ & $\begin{array}{l}12.04 \\
12.07 \\
12.10 \\
12.13 \\
12.17\end{array}$ & $\begin{array}{l}38.21 \\
38.36 \\
38.51 \\
38.66 \\
38.81\end{array}$ \\
\hline $\begin{array}{l}332 . \\
333 . \\
334 . \\
335 . \\
336 .\end{array}$ & $\begin{array}{l}-54.686 \\
-54.885 \\
-55.084 \\
-55.282 \\
-55.481\end{array}$ & $\begin{array}{l}11.249 \\
11.286 \\
11.324 \\
11.361 \\
11.398\end{array}$ & $\begin{array}{l}190.29 \\
190.40 \\
190.51 \\
190.62 \\
190.73\end{array}$ & $\begin{array}{l}37.109 \\
37.155 \\
37.201 \\
37.247 \\
37.294\end{array}$ & $\begin{array}{l}12.20 \\
12.23 \\
12.26 \\
12.29 \\
12.32\end{array}$ & $\begin{array}{l}38.96 \\
39.11 \\
39.26 \\
39.41 \\
39.57\end{array}$ \\
\hline $\begin{array}{l}337 . \\
338 . \\
339 . \\
340 . \\
341 .\end{array}$ & $\begin{array}{l}-55.681 \\
-55.880 \\
-56.079 \\
-56.279 \\
-56.478\end{array}$ & $\begin{array}{l}11.435 \\
11.473 \\
11.510 \\
11.548 \\
11.585\end{array}$ & $\begin{array}{l}190.84 \\
190.95 \\
191.06 \\
191.17 \\
191.28\end{array}$ & $\begin{array}{l}37.340 \\
37.387 \\
37.434 \\
37.481 \\
37.529\end{array}$ & $\begin{array}{l}12.35 \\
12.38 \\
12.41 \\
12.44 \\
12.48\end{array}$ & $\begin{array}{l}39.72 \\
39.87 \\
40.02 \\
40.18 \\
40.33\end{array}$ \\
\hline $\begin{array}{l}342 . \\
343 . \\
344 . \\
345 . \\
346 .\end{array}$ & $\begin{array}{l}-56.678 \\
-56.878 \\
-57.077 \\
-57.277 \\
-57.477\end{array}$ & $\begin{array}{l}11.623 \\
11.660 \\
11.698 \\
11.736 \\
11.773\end{array}$ & $\begin{array}{l}191.39 \\
191.50 \\
191.61 \\
191.72 \\
191.83\end{array}$ & $\begin{array}{l}37.576 \\
37.624 \\
37.672 \\
37.720 \\
37.769\end{array}$ & $\begin{array}{l}12.51 \\
12.54 \\
12.57 \\
12.60 \\
12.63\end{array}$ & $\begin{array}{l}40.48 \\
40.64 \\
40.79 \\
40.95 \\
41.10\end{array}$ \\
\hline $\begin{array}{l}347 . \\
348 . \\
349 . \\
350 . \\
351 .\end{array}$ & $\begin{array}{l}-57.678 \\
-57.878 \\
-58.078 \\
-58.279 \\
-58.480\end{array}$ & $\begin{array}{l}11.811 \\
11.849 \\
11.887 \\
11.925 \\
11.963\end{array}$ & $\begin{array}{l}191.94 \\
192.05 \\
192.16 \\
192.27 \\
192.38\end{array}$ & $\begin{array}{l}37.817 \\
37.866 \\
37.915 \\
37.964 \\
38.014\end{array}$ & $\begin{array}{l}12.66 \\
12.69 \\
12.72 \\
12.75 \\
12.78\end{array}$ & $\begin{array}{l}41.26 \\
41.41 \\
41.57 \\
41.73 \\
41.88\end{array}$ \\
\hline $\begin{array}{l}352 . \\
353 . \\
354 . \\
355 . \\
356 .\end{array}$ & $\begin{array}{l}-58.680 \\
-58.881 \\
-59.082 \\
-59.283 \\
-59.484\end{array}$ & $\begin{array}{l}12.001 \\
12.039 \\
12.077 \\
12.115 \\
12.154\end{array}$ & $\begin{array}{l}192.48 \\
192.59 \\
192.70 \\
192.81 \\
192.92\end{array}$ & $\begin{array}{l}38.063 \\
38.113 \\
38.163 \\
38.213 \\
38.263\end{array}$ & $\begin{array}{l}12.81 \\
12.84 \\
12.87 \\
12.90 \\
12.93\end{array}$ & $\begin{array}{l}42.04 \\
42.20 \\
42.36 \\
42.51 \\
42.67\end{array}$ \\
\hline
\end{tabular}


Table B1 (continued)

\begin{tabular}{|c|c|c|c|c|c|c|}
\hline $\mathrm{T}$ & $A^{i d}$ & $\mathrm{H}^{\mathrm{id}}$ & $S^{i d}$ & $C_{p}^{i d}$ & $\eta_{0}$ & $\lambda_{0}$ \\
\hline $\mathrm{K}$ & $\mathrm{kJ} \cdot \mathrm{mol}^{-1}$ & $\mathrm{~kJ} \cdot \mathrm{mol}^{-1}$ & $\mathrm{~J} \cdot \mathrm{mol}^{-}$ & $K^{-1}$ & $\mu \mathrm{Pa} \cdot \mathrm{s}$ & $\mathrm{mW} \cdot \mathrm{m}^{-1} \cdot \mathrm{K}^{-1}$ \\
\hline 357. & -59.686 & 12.192 & 193.02 & 38.314 & 12.96 & 42.83 \\
\hline 358. & -59.887 & 12.230 & 193.13 & 38.364 & 12.99 & 42.99 \\
\hline 359. & -60.089 & 12.269 & 193.24 & 38.415 & 13.02 & 43.15 \\
\hline 360. & -60.290 & 12.307 & 193.34 & 38.466 & 13.05 & 43.31 \\
\hline 361. & -60.492 & 12.345 & 193.45 & 38.517 & 13.08 & 43.47 \\
\hline 362. & -60.694 & 12.384 & 193.56 & 38.568 & 13.11 & 43.63 \\
\hline 363. & -60.896 & 12.423 & 193.66 & 38.620 & 13.14 & 43.79 \\
\hline 364. & -61.098 & 12.461 & 193.77 & 38.671 & 13.17 & 43.95 \\
\hline 365 . & -61.300 & 12.500 & 193.88 & 38.723 & 13.20 & 44.11 \\
\hline 366. & -61.502 & 12.539 & 193.98 & 38.775 & 13.23 & 44.28 \\
\hline 367 . & -61.704 & 12.577 & 194.09 & 38.827 & 13.26 & 44.44 \\
\hline 368. & -61.907 & 12.616 & 194.19 & 38.879 & 13.29 & 44.60 \\
\hline 369 . & -62.109 & 12.655 & 194.30 & 38.931 & 13.32 & 44.76 \\
\hline 370. & -62.312 & 12.694 & 194.41 & 38.984 & 13.35 & 44.93 \\
\hline 371. & -62.515 & 12.733 & 194.51 & 39.036 & 13.38 & 45.09 \\
\hline 372. & -62.718 & 12.772 & 194.62 & 39.089 & 13.41 & 45.25 \\
\hline 373. & -62.921 & 12.811 & 194.72 & 39.142 & 13.44 & 45.42 \\
\hline 374. & -63.124 & 12.851 & 194.83 & 39.195 & 13.47 & 45.58 \\
\hline 375. & -63.327 & 12.890 & 194.93 & 39.248 & 13.50 & 45.75 \\
\hline 376. & -63.530 & 12.929 & 195.03 & 39.301 & 13.53 & 45.91 \\
\hline 377 . & -63.734 & 12.968 & 195.14 & 39.354 & 13.56 & 46.08 \\
\hline 378 . & -63.937 & 13.008 & 195.24 & 39.408 & 13.59 & 46.24 \\
\hline 379. & -64.141 & 13.047 & 195.35 & 39.462 & 13.62 & 46.41 \\
\hline 380. & -64.344 & 13.087 & 195.45 & 39.515 & 13.65 & 46.57 \\
\hline 381. & -64.548 & 13.126 & 195.56 & 39.569 & 13.68 & 46.74 \\
\hline 382. & -64.752 & 13.166 & 195.66 & 39.623 & 13.71 & 46.91 \\
\hline 383. & -64.956 & 13.205 & 195.76 & 39.677 & 13.74 & 47.07 \\
\hline 384 . & -65.160 & 13.245 & 195.87 & 39.732 & 13.76 & 47.24 \\
\hline 385. & -65.365 & 13.285 & 195.97 & 39.786 & 13.79 & 47.41 \\
\hline 386. & -65.569 & 13.325 & 196.07 & 39.840 & 13.82 & 47.58 \\
\hline 387. & -65.773 & 13.365 & 196.18 & 39.895 & 13.85 & 47.74 \\
\hline 388 . & -65.978 & 13.405 & 196.28 & 39.950 & 13.88 & 47.91 \\
\hline 389 . & -66.183 & 13.445 & 196.38 & 40.004 & 13.91 & 48.08 \\
\hline 390. & -66.387 & 13.485 & 196.49 & 40.059 & 13.94 & 48.25 \\
\hline 391. & -66.592 & 13.525 & 196.59 & 40.114 & 13.97 & 48.42 \\
\hline 392. & -66.797 & 13.565 & 196.69 & 40.169 & 14.00 & 48.59 \\
\hline 393. & -67.002 & 13.605 & 196.79 & 40.224 & 14.03 & 48.76 \\
\hline 394 . & -67.207 & 13.645 & 196.90 & 40.280 & 14.06 & 48.93 \\
\hline 395. & -67.413 & 13.686 & 197.00 & 40.335 & 14.08 & 49.10 \\
\hline 396. & -67.618 & 13.726 & 197.10 & 40.391 & 14.11 & 49.27 \\
\hline
\end{tabular}


Table B1 (continued)

\begin{tabular}{|c|c|c|c|c|c|c|}
\hline $\mathrm{T}$ & $A^{i d}$ & $\mathrm{H}^{i d}$ & $S^{i d}$ & $C_{p}^{i d}$ & $\eta_{0}$ & $\lambda_{0}$ \\
\hline K & $\mathrm{kJ} \cdot \mathrm{mol}^{-1}$ & $\mathrm{~kJ} \cdot \mathrm{mol}^{-1}$ & $\mathrm{~J} \cdot \mathrm{mol}^{-1}$ & $\cdot \mathrm{K}^{-1}$ & $\mu \mathrm{Pa} \cdot \mathrm{s}$ & $\mathrm{mW} \cdot \mathrm{m}^{-1} \cdot \mathrm{K}^{-1}$ \\
\hline 397. & -67.823 & 13.766 & 197.20 & 40.446 & 14.14 & 49.44 \\
\hline 398. & -68.029 & 13.807 & 197.30 & 40.502 & 14.17 & 49.61 \\
\hline 399. & -68.235 & 13.847 & 197.40 & 40.558 & 14.20 & 49.79 \\
\hline 400. & -68.440 & 13.888 & 197.51 & 40.613 & 14.23 & 49.96 \\
\hline 405. & -69.471 & 14.092 & 198.01 & 40.894 & & 50.82 \\
\hline 410. & -70.504 & 14.297 & 198.52 & 41.176 & & 51.69 \\
\hline 415. & -71.539 & 14.503 & 199.02 & 41.460 & & 52.57 \\
\hline 420. & -72.577 & 14.711 & 199.52 & 41.745 & & 53.45 \\
\hline 425. & -73.617 & 14.921 & 200.01 & 42.031 & & 54.34 \\
\hline 430 & -74.660 & 15.132 & 200.50 & 42.319 & & 55.23 \\
\hline 435. & -75.706 & 15.344 & 201.00 & 42.608 & & 56.14 \\
\hline 440 & -76.753 & 15.558 & 201.48 & 42.897 & & 57.04 \\
\hline 445. & -77.804 & 15.773 & 201.97 & 43.187 & & 57.95 \\
\hline 450. & -78.856 & 15.990 & 202.45 & 43.477 & & 58.87 \\
\hline 455. & -79.911 & 16.208 & 202.94 & 43.767 & & 59.79 \\
\hline 460. & -80.969 & 16.427 & 203.42 & 44.057 & & 60.72 \\
\hline 465. & -82.029 & 16.648 & 203.89 & 44.348 & & 61.65 \\
\hline 470. & -83.091 & 16.871 & 204.37 & 44.638 & & 62.58 \\
\hline 475. & -84.155 & 17.095 & 204.84 & 44.928 & & 63.52 \\
\hline 480 & -85.222 & 17.320 & 205.32 & 45.217 & & 64.46 \\
\hline 485. & -86.292 & 17.547 & 205.79 & 45.506 & & 65.41 \\
\hline 490. & -87.363 & 17.775 & 206.25 & 45.794 & & 66.36 \\
\hline 495. & -88.437 & 18.005 & 206.72 & 46.082 & & 67.31 \\
\hline 500 & -89.514 & 18.236 & 207.18 & 46.368 & & 68.27 \\
\hline 505. & -90.592 & 18.469 & 207.65 & 46.654 & & 69.23 \\
\hline 510. & -91.673 & 18.703 & 208.11 & 46.938 & & 70.19 \\
\hline 515. & -92.757 & 18.938 & 208.57 & 47.222 & & 71.15 \\
\hline 520. & -93.842 & 19.175 & 209.03 & 47.504 & & 72.12 \\
\hline 525. & -94.930 & 19.413 & 209.48 & 47.785 & & 73.09 \\
\hline 530. & -96.020 & 19.653 & 209.94 & 48.065 & & 74.06 \\
\hline 535. & -97.112 & 19.894 & 210.39 & 48.343 & & 75.03 \\
\hline 540. & -98.207 & 20.136 & 210.84 & 48.620 & & 76.01 \\
\hline 545. & -99.304 & 20.380 & 211.29 & 48.895 & & 76.98 \\
\hline 550. & -100.403 & 20.625 & 211.74 & 49.169 & & 77.96 \\
\hline 555. & -101.505 & 20.872 & 212.18 & 49.441 & & 78.94 \\
\hline 560. & -102.608 & 21.119 & 212.63 & 49.712 & & 79.92 \\
\hline 565. & -103.714 & 21.369 & 213.07 & 49.981 & & 80.91 \\
\hline 570. & -104.822 & 21.619 & 213.51 & 50.248 & & 81.89 \\
\hline 575. & -105.932 & 21.871 & 213.95 & 50.514 & & 82.87 \\
\hline 580 & -107.045 & 22.124 & 214.39 & 50.778 & & 83.86 \\
\hline
\end{tabular}


Table B1 (continued)

$\mathrm{T}$

$\mathrm{K}$

585.

590 .

595 .

600 .
$A^{i d}$

$H^{i d}$

$S^{i d}$

$C_{p}^{i d}$

$\mathrm{kJ} \cdot \mathrm{mol}^{-1}$

$\mathrm{kJ} \cdot \mathrm{mol}^{-1}$

$\mathrm{J} \cdot \mathrm{mol}^{-1} \cdot \mathrm{K}^{-1}$

22.379

22.635

214.83

215.26

51.040

51.300

$\begin{array}{lll}22.892 & 215.70 & 51.558\end{array}$

$\begin{array}{lll}23.150 & 216.13 & 51.815\end{array}$ $\eta_{0} \quad \lambda_{0}$

$\mu \mathrm{Pa} \cdot \mathrm{s} \quad \mathrm{mW} \cdot \mathrm{m}^{-1} \cdot \mathrm{K}^{-1}$

84.85

85.83

86.82

87.81 


\section{PROPERTIES ALONG SATURATION BOUNDARY}

Notes for Table B2. Values of the pressure, density of the saturated 1iquid, density of the saturated vapor, specific heat capacity, sound speed, viscosity, and thermal conductivity along the two phase liquid-vapor coexistence curve. The quantities $\mathrm{P}_{\sigma \mathrm{L}}, \rho_{\sigma \mathrm{L}}$, and $\rho_{\sigma \mathrm{V}}$ are from the ancillary equations, eqs (3-5). The specific heat capacity along the saturated boundary is from the equation in Table 7; the sound speed is also taken from Table 7 but the density argument is for the saturated 1iquid and is taken from column 3 of this table. The viscosity and thermal conductivity at saturation are from eqs (8) and (9) [with the terms evaluated from eqs (10-25)]; again the density input is from column 3 of this table.

\begin{tabular}{|c|c|c|c|c|c|c|c|}
\hline $\mathrm{T}$ & $\mathrm{P}_{\sigma}$ & $\rho_{\sigma \mathrm{L}}$ & $\rho_{\sigma \mathrm{V}}$ & $\mathrm{C}_{\sigma \mathrm{L}}$ & $\mathrm{W}_{\sigma \mathrm{L}}$ & $\eta_{\sigma \mathrm{L}}$ & $\lambda_{\sigma \mathrm{L}}$ \\
\hline K & $\mathrm{MPa}$ & $\mathrm{mol} \cdot \mathrm{dm}^{-3}$ & $\mathrm{~mol} \cdot \mathrm{dm}^{-3}$ & $\mathrm{~J} \cdot \mathrm{mol}^{-1} \cdot \mathrm{K}^{-1}$ & $\mathrm{~m} \cdot \mathrm{s}^{-1}$ & $\mu \mathrm{Pa} \cdot \mathrm{s}$ & $\mathrm{mW} \cdot \mathrm{m}^{-1} \cdot \mathrm{K}^{-1}$ \\
\hline 90.6854 & 0.011694 & 28.147 & 0.01564 & 52.98 & 1549.3 & 202.20 & 211.3 \\
\hline 91. & 0.012 & 28.12 & 0.016 & 53.08 & 1545.2 & 200.42 & 210.9 \\
\hline 92. & 0.014 & 28.04 & 0.018 & 53.37 & 1532.7 & 194.89 & 209.7 \\
\hline 93. & 0.016 & 27.95 & 0.020 & 53.63 & 1520.7 & 189.55 & 208.5 \\
\hline 94. & 0.018 & 27.87 & 0.023 & 53.85 & 1509.0 & 184.39 & 207.2 \\
\hline 95. & 0.020 & 27.78 & 0.025 & 54.05 & 1497.7 & 179.41 & 206.0 \\
\hline 96. & 0.022 & 27.70 & 0.028 & 54.24 & 1486.6 & 174.60 & 204.7 \\
\hline 97. & 0.025 & 27.61 & 0.031 & 54.40 & 1475.8 & 169.95 & 203.4 \\
\hline 98. & 0.028 & 27.52 & 0.035 & 54.54 & 1465.1 & 165.47 & 202.1 \\
\hline 99. & 0.031 & 27.44 & 0.038 & 54.68 & 1454.6 & 161.14 & 200.8 \\
\hline 100. & 0.034 & 27.35 & 0.042 & 54.80 & 1444.3 & 156.97 & 199.5 \\
\hline 101. & 0.038 & 27.27 & 0.046 & 54.91 & 1434.1 & 152.94 & 198.2 \\
\hline 102. & 0.042 & 27.18 & 0.051 & 55.02 & 1424.0 & 149.04 & 196.8 \\
\hline 103. & 0.047 & 27.09 & 0.056 & 55.12 & 1413.9 & 145.29 & 195.5 \\
\hline 104. & 0.051 & 27.00 & 0.061 & 55.21 & 1403.9 & 141.66 & 194.2 \\
\hline 105. & 0.056 & 26.92 & 0.066 & 55.30 & 1394.0 & 138.16 & 192.8 \\
\hline 106. & 0.062 & 26.83 & 0.072 & 55.39 & 1384.1 & 134.79 & 191.4 \\
\hline 107. & 0.068 & 26.74 & 0.078 & 55.47 & 1374.2 & 131.52 & 190.1 \\
\hline 108. & 0.074 & 26.65 & 0.085 & 55.56 & 1364.4 & 128.37 & 188.7 \\
\hline 109. & 0.081 & 26.56 & 0.092 & 55.64 & 1354.5 & 125.33 & 187.3 \\
\hline 110. & 0.088 & 26.47 & 0.100 & 55.72 & 1344.7 & 122.39 & 186.0 \\
\hline 111. & 0.096 & 26.38 & 0.108 & 55.81 & 1334.8 & 119.55 & 184.6 \\
\hline 112. & 0.104 & 26.29 & 0.116 & 55.89 & 1325.0 & 116.80 & 183.2 \\
\hline 113. & 0.113 & 26.20 & 0.125 & 55.98 & 1315.1 & 114.15 & 181.8 \\
\hline 114. & 0.122 & 26.11 & 0.135 & 56.07 & 1305.2 & 111.59 & 180.4 \\
\hline
\end{tabular}


Table B2 (continued)

\begin{tabular}{|c|c|c|c|c|c|c|c|}
\hline $\mathrm{T}$ & $\mathrm{P}_{\sigma}$ & $\rho_{\sigma \mathrm{L}}$ & $\rho_{\sigma \mathrm{V}}$ & $\mathrm{C}_{\sigma \mathrm{L}}$ & $W_{\sigma L}$ & $\eta_{\sigma \mathrm{L}}$ & $\lambda_{\sigma \mathrm{L}}$ \\
\hline K & $\mathrm{MPa}$ & $\mathrm{mol} \cdot \mathrm{dm}^{-3}$ & $\mathrm{~mol} \cdot \mathrm{dm}^{-3}$ & $\mathrm{~J} \cdot \mathrm{mol}^{-1} \cdot \mathrm{K}^{-1}$ & $m \cdot s^{-1}$ & $\mu \mathrm{Pa} \cdot \mathrm{s}$ & $\mathrm{mW} \cdot \mathrm{m}^{-1} \cdot \mathrm{K}^{-1}$ \\
\hline 115. & 0.132 & 26.02 & 0.145 & 56.16 & 1295.3 & 109.11 & 179.0 \\
\hline 116. & 0.143 & 25.92 & 0.155 & 56.26 & 1285.4 & 106.71 & 177.6 \\
\hline 117. & 0.154 & 25.83 & 0.167 & 56.36 & 1275.4 & 104.38 & 176.2 \\
\hline 118. & 0.166 & 25.74 & 0.178 & 56.46 & 1265.4 & 102.14 & 174.8 \\
\hline 119. & 0.178 & 25.64 & 0.191 & 56.57 & 1255.4 & 99.96 & 173.4 \\
\hline 120. & 0.192 & 25.55 & 0.204 & 56.68 & 1245.3 & 97.85 & 172.0 \\
\hline 121. & 0.206 & 25.45 & 0.217 & 56.79 & 1235.2 & 95.81 & 170.6 \\
\hline 122. & 0.220 & 25.36 & 0.231 & 56.91 & 1225.1 & 93.83 & 169.2 \\
\hline 123. & 0.236 & 25.26 & 0.246 & 57.03 & 1214.9 & 91.91 & 167.8 \\
\hline 124. & 0.252 & 25.16 & 0.262 & 57.16 & 1204.7 & 90.05 & 166.3 \\
\hline 125. & 0.269 & 25.06 & 0.279 & 57.29 & 1194.4 & 88.25 & 164.9 \\
\hline 126. & 0.287 & 24.97 & 0.296 & 57.43 & 1184.0 & 86.49 & 163.5 \\
\hline 127. & 0.306 & 24.87 & 0.314 & 57.58 & 1173.7 & 84.79 & 162.1 \\
\hline 128. & 0.325 & 24.77 & 0.333 & 57.73 & 1163.2 & 83.14 & 160.7 \\
\hline 129. & 0.346 & 24.66 & 0.352 & 57.88 & 1152.7 & 81.53 & 159.2 \\
\hline 130. & 0.368 & 24.56 & 0.373 & 58.05 & 1142.2 & 79.97 & 157.8 \\
\hline 131. & 0.390 & 24.46 & 0.394 & 58.21 & 1131.6 & 78.45 & 156.4 \\
\hline 132. & 0.414 & 24.36 & 0.417 & 58.39 & 1120.9 & 76.97 & 155.0 \\
\hline 133. & 0.438 & 24.25 & 0.440 & 58.57 & 1110.2 & 75.53 & 153.6 \\
\hline 134. & 0.464 & 24.15 & 0.464 & 58.76 & 1099.4 & 74.13 & 152.1 \\
\hline 135. & 0.491 & 24.04 & 0.490 & 58.96 & 1088.6 & 72.76 & 150.7 \\
\hline 136. & 0.519 & 23.93 & 0.516 & 59.16 & 1077.7 & 71.43 & 149.3 \\
\hline 137. & 0.548 & 23.83 & 0.544 & 59.37 & 1066.7 & 70.13 & 147.9 \\
\hline 138. & 0.578 & 23.72 & 0.572 & 59.59 & 1055.6 & 68.86 & 146.4 \\
\hline 139. & 0.609 & 23.61 & 0.602 & 59.82 & 1044.5 & 67.62 & 145.0 \\
\hline 140. & 0.642 & 23.50 & 0.633 & 60.05 & 1033.3 & 66.42 & 143.6 \\
\hline 141. & 0.675 & 23.38 & 0.665 & 60.30 & 1022.1 & 65.23 & 142.2 \\
\hline 142. & 0.711 & 23.27 & 0.699 & 60.55 & 1010.7 & 64.08 & 140.7 \\
\hline 143. & 0.747 & 23.15 & 0.733 & 60.82 & 999.3 & 62.95 & 139.3 \\
\hline 144. & 0.785 & 23.04 & 0.770 & 61.09 & 987.8 & 61.85 & 137.9 \\
\hline 145. & 0.824 & 22.92 & 0.807 & 61.37 & 976.3 & 60.76 & 136.5 \\
\hline 146. & 0.864 & 22.80 & 0.846 & 61.67 & 964.6 & 59.70 & 135.0 \\
\hline 147. & 0.906 & 22.68 & 0.887 & 61.98 & 952.9 & 58.67 & 133.6 \\
\hline 148. & 0.950 & 22.56 & 0.929 & 62.30 & 941.1 & 57.65 & 132.2 \\
\hline 149. & 0.994 & 22.44 & 0.973 & 62.63 & 929.2 & 56.65 & 130.8 \\
\hline 150. & 1.041 & 22.31 & 1.018 & 62.98 & 917.2 & 55.67 & 129.3 \\
\hline 151. & 1.088 & 22.19 & 1.066 & 63.34 & 905.1 & 54.70 & 127.9 \\
\hline 152. & 1.138 & 22.06 & 1.115 & 63.72 & 892.9 & 53.76 & 126.5 \\
\hline 153. & 1.189 & 21.93 & 1.166 & 64.11 & 880.6 & 52.83 & 125.1 \\
\hline 154. & 1.242 & 21.80 & 1.219 & 64.52 & 868.2 & 51.91 & 123.6 \\
\hline
\end{tabular}


Table B2 (continued)

\begin{tabular}{|c|c|c|c|c|c|c|c|}
\hline $\mathrm{T}$ & $\mathrm{P}_{\sigma}$ & $\rho_{\sigma \mathrm{L}}$ & $\rho_{\sigma \mathrm{V}}$ & $C_{\sigma L}$ & $\mathrm{~W}_{\sigma \mathrm{L}}$ & $\eta_{\sigma L}$ & $\lambda_{\sigma \mathrm{L}}$ \\
\hline K & $\mathrm{MPa}$ & $\mathrm{mol} \cdot \mathrm{dm}^{-3}$ & $\mathrm{~mol} \cdot \mathrm{dm}^{-3}$ & $\mathrm{~J} \cdot \mathrm{mol}^{-1} \cdot \mathrm{K}^{-1}$ & $\mathrm{~m} \cdot \mathrm{s}^{-1}$ & $\mu \mathrm{Pa} \cdot \mathrm{s}$ & $\mathrm{mW} \cdot \mathrm{m}^{-1} \cdot \mathrm{K}^{-1}$ \\
\hline 155. & 1.296 & 21.66 & 1.274 & 64.95 & 855.7 & 51.01 & 122.2 \\
\hline 156. & 1.352 & 21.53 & 1.331 & 65.40 & 843.1 & 50.13 & 120.8 \\
\hline 157. & 1.409 & 21.39 & 1.391 & 65.87 & 830.4 & 49.25 & 119.3 \\
\hline 158. & 1.469 & 21.25 & 1.452 & 66.36 & 817.6 & 48.39 & 117.9 \\
\hline 159. & 1.530 & 21.11 & 1.517 & 66.88 & 804.6 & 47.54 & 116.5 \\
\hline 160. & 1.593 & 20.96 & 1.584 & 67.42 & 791.5 & 46.70 & 115.0 \\
\hline 161. & 1.658 & 20.81 & 1.654 & 68.00 & 778.3 & 45.87 & 113.6 \\
\hline 162. & 1.724 & 20.66 & 1.726 & 68.60 & 765.0 & 45.05 & 112.1 \\
\hline 163. & 1.793 & 20.51 & 1.802 & 69.24 & 751.6 & 44.24 & 110.7 \\
\hline 164. & 1.864 & 20.36 & 1.880 & 69.92 & 738.0 & 43.44 & 109.3 \\
\hline 165. & 1.936 & 20.20 & 1.963 & 70.64 & 724.2 & 42.65 & 107.8 \\
\hline 166. & 2.011 & 20.03 & 2.048 & 71.40 & 710.3 & 41.86 & 106.4 \\
\hline 167. & 2.087 & 19.87 & 2.138 & 72.22 & 696.3 & 41.08 & 104.9 \\
\hline 168. & 2.166 & 19.70 & 2.232 & 73.10 & 682.1 & 40.30 & 103.4 \\
\hline 169. & 2.246 & 19.52 & 2.330 & 74.03 & 667.7 & 39.53 & 102.0 \\
\hline 170. & 2.329 & 19.35 & 2.432 & 75.04 & 653.2 & 38.76 & 100.5 \\
\hline 171. & 2.414 & 19.16 & 2.540 & 76.14 & 638.5 & 37.99 & 99.0 \\
\hline 172. & 2.502 & 18.97 & 2.653 & 77.33 & 623.6 & 37.23 & 97.5 \\
\hline 173. & 2.591 & 18.78 & 2.772 & 78.62 & 608.5 & 36.46 & 96.1 \\
\hline 174. & 2.683 & 18.58 & 2.897 & 80.05 & 593.2 & 35.70 & 94.6 \\
\hline 175. & 2.778 & 18.37 & 3.030 & 81.62 & 577.7 & 34.93 & 93.1 \\
\hline 176. & 2.874 & 18.16 & 3.170 & 83.36 & 562.0 & 34.16 & 91.6 \\
\hline 177. & 2.974 & 17.94 & 3.318 & 85.32 & 546.0 & 33.39 & 90.1 \\
\hline 178. & 3.075 & 17.70 & 3.477 & 87.53 & 529.8 & 32.61 & 88.5 \\
\hline 179. & 3.180 & 17.46 & 3.646 & 90.05 & 513.2 & 31.82 & 87.0 \\
\hline 180. & 3.287 & 17.21 & 3.827 & 92.96 & 496.4 & 31.01 & 85.5 \\
\hline 181. & 3.396 & 16.94 & 4.023 & 96.37 & 479.1 & 30.20 & 84.0 \\
\hline 182. & 3.509 & 16.65 & 4.236 & 100.42 & 461.3 & 29.36 & 82.5 \\
\hline 183. & 3.624 & 16.35 & 4.469 & 105.34 & 443.0 & 28.49 & 81.1 \\
\hline 184. & 3.742 & 16.02 & 4.726 & 111.46 & 423.8 & 27.60 & 79.7 \\
\hline 185. & 3.864 & 15.66 & 5.015 & 119.33 & 403.7 & 26.65 & 78.4 \\
\hline 186. & 3.988 & 15.26 & 5.344 & 129.94 & 382.2 & 25.65 & 77.3 \\
\hline 187. & 4.116 & 14.81 & 5.729 & 145.23 & 358.8 & 24.56 & 76.7 \\
\hline 188. & 4.247 & 14.27 & 6.200 & 169.79 & 332.7 & 23.33 & 76.9 \\
\hline 189. & 4.382 & 13.58 & 6.821 & 218.36 & 302.4 & 21.86 & 79.8 \\
\hline 190. & 4.521 & 12.50 & 7.827 & 389.90 & 264.3 & 19.75 & 100.3 \\
\hline 190.551 & 4.5992 & 10.139 & 10.139 & & & & \\
\hline
\end{tabular}


Notes for Table B3. Values of the density, compressibility factor, enthalpy, entropy, isochoric and isobaric heat capacities, speed of sound, viscosity, and thermal conductivity in the single-phase region of the methane fluid. The independent variables were chosen to be temperature and pressure. The density was evaluated by inverting the pressure equation in Table 7; the quantities $\mathrm{H}$, $S, C_{v}, C_{p}$, and $W$ were then evaluated directly from the expressions in Table 7. The viscosity and thermal conductivity are from eqs (7) and (8) [with the terms evaluated from eqs (10-23)]; the density input is from column 3 of this table.

METHANE ISOBAR AT $\mathrm{P}=0.012 \mathrm{MPa}$

\begin{tabular}{|c|c|c|c|c|c|c|c|c|c|}
\hline $\mathrm{T}$ & $\rho$ & $Z$ & $\mathrm{H}$ & S & $\mathrm{C}_{\mathrm{v}}$ & $C_{p}$ & W & $\eta$ & $\lambda$ \\
\hline K & $\mathrm{mol} \cdot \mathrm{dm}^{-3}$ & & $\mathrm{~kJ} / \mathrm{mol}$ & $\mathrm{J} /(\mathrm{mol} \cdot \mathrm{K})$ & $\mathrm{J} /(\mathrm{mol} \cdot \mathrm{K})$ & $\mathrm{J} /(\mathrm{mol} \cdot \mathrm{K})$ & $m \cdot s^{-1}$ & $\mu \mathrm{Pa} \cdot \mathrm{s}$ & $\mathrm{mW} /(\mathrm{m} \cdot \mathrm{K})$ \\
\hline 90.685 & 28.145 & 0.001 & -5.745 & 67.80 & 33.40 & 52.57 & 1548.8 & 202.08 & 211.2 \\
\hline 90.685 & 0.0157 & 0.991 & 2.969 & 163.88 & 28.31 & 37.53 & 247.1 & 3.61 & 8.8 \\
\hline 95. & 0.0149 & 0.993 & 3.124 & 165.56 & 26.11 & 34.85 & 254.5 & 3.77 & 9.3 \\
\hline 100. & 0.0141 & 0.994 & 3.295 & 167.32 & 25.36 & 33.92 & 261.7 & 3.95 & 9.9 \\
\hline 105. & 0.0135 & 0.995 & 3.464 & 168.96 & 25.14 & 33.64 & 268.5 & 4.14 & 10.4 \\
\hline 110 & 0.0128 & 0.996 & 3.632 & 170.52 & 25.07 & 33.54 & 274.9 & 4.33 & 11.0 \\
\hline 115. & 0.0123 & 0.996 & 3.799 & 172.01 & 25.04 & 33.48 & 281.2 & 4.52 & 11.6 \\
\hline 120. & 0.0118 & 0.997 & 3.967 & 173.44 & 25.03 & 33.45 & 287.4 & 4.71 & 12.2 \\
\hline 125. & 0.0113 & 0.997 & 4.134 & 174.80 & 25.02 & 33.43 & 293.3 & 4.90 & 12.8 \\
\hline 130. & 0.0108 & 0.997 & 4.301 & 176.11 & 25.02 & 33.41 & 299.2 & 5.10 & 13.4 \\
\hline 135. & 0.0104 & 0.998 & 4.468 & 177.38 & 25.01 & 33.40 & 305.0 & 5.29 & 14.0 \\
\hline 140. & 0.0101 & 0.998 & 4.635 & 178.59 & 25.01 & 33.39 & 310.6 & 5.48 & 14.6 \\
\hline 145. & 0.0097 & 0.998 & 4.802 & 179.76 & 25.01 & 33.39 & 316.1 & 5.67 & 15.2 \\
\hline 150. & 0.0094 & 0.998 & 4.969 & 180.89 & 25.01 & 33.38 & 321.5 & 5.86 & 15.8 \\
\hline 155. & 0.0091 & 0.998 & 5.136 & 181.99 & 25.02 & 33.38 & 326.9 & 6.05 & 16.3 \\
\hline 160. & 0.0088 & 0.999 & 5.303 & 183.05 & 25.03 & 33.38 & 332.1 & 6.24 & 16.9 \\
\hline 165. & 0.0085 & 0.999 & 5.470 & 184.08 & 25.04 & 33.39 & 337.3 & 6.43 & 17.5 \\
\hline 170. & 0.0083 & 0.999 & 5.637 & 185.07 & 25.05 & 33.40 & 342.3 & 6.62 & 18.1 \\
\hline 175. & 0.0080 & 0.999 & 5.804 & 186.04 & 25.06 & 33.41 & 347.3 & 6.81 & 18.7 \\
\hline 180. & 0.0078 & 0.999 & 5.971 & 186.98 & 25.09 & 33.43 & 352.2 & 7.00 & 19.3 \\
\hline 185. & 0.0076 & 0.999 & 6.138 & 187.90 & 25.11 & 33.45 & 357.1 & 7.18 & 19.9 \\
\hline 190. & 0.0074 & 0.999 & 6.305 & 188.79 & 25.14 & 33.48 & 361.8 & 7.37 & 20.5 \\
\hline 195. & 0.0072 & 0.999 & 6.473 & 189.66 & 25.17 & 33.51 & 366.5 & 7.55 & 21.1 \\
\hline 200. & 0.0070 & 0.999 & 6.641 & 190.51 & 25.21 & 33.55 & 371.1 & 7.73 & 21.7 \\
\hline 205. & 0.0069 & 0.999 & 6.808 & 191.34 & 25.26 & 33.59 & 375.7 & 7.92 & 22.3 \\
\hline
\end{tabular}


METHANE ISOBAR AT $P=0.012 \mathrm{MPa}$ (continued)

\begin{tabular}{|c|c|c|c|c|c|c|c|c|c|}
\hline $\mathrm{T}$ & $\rho$ & $\mathrm{Z}$ & $\mathrm{H}$ & $S$ & $C_{v}$ & $C_{p}$ & W & $\eta$ & $\lambda$ \\
\hline K & $\mathrm{mol} \cdot \mathrm{dm}^{-3}$ & & $\mathrm{~kJ} / \mathrm{mol}$ & $\mathrm{J} /(\mathrm{mol} \cdot \mathrm{K})$ & $\mathrm{J} /(\mathrm{mol} \cdot \mathrm{K})$ & $\mathrm{J} /(\mathrm{mol} \cdot \mathrm{K})$ & $\mathrm{m} \cdot \mathrm{s}^{-1}$ & $\mu \mathrm{Pa} \cdot \mathrm{s}$ & $\mathrm{mW} /(\mathrm{m} \cdot \mathrm{K})$ \\
\hline 210. & 0.0067 & 0.999 & 6.977 & 192.15 & 25.31 & 33.64 & 380.1 & 8.10 & 22.9 \\
\hline 215. & 0.0065 & 0.999 & 7.145 & 192.94 & 25.36 & 33.70 & 384.5 & 8.28 & 23.5 \\
\hline 220 . & 0.0064 & 0.999 & 7.314 & 193.72 & 25.43 & 33.76 & 388.9 & 8.46 & 24.1 \\
\hline 225. & 0.0063 & 0.999 & 7.482 & 194.48 & 25.50 & 33.83 & 393.1 & 8.64 & 24.7 \\
\hline 230. & 0.0061 & 1.000 & 7.652 & 195.22 & 25.57 & 33.90 & 397.3 & 8.81 & 25.3 \\
\hline 235. & 0.0060 & 1.000 & 7.822 & 195.95 & 25.66 & 33.99 & 401.5 & 8.99 & 25.9 \\
\hline 240. & 0.0059 & 1.000 & 7.992 & 196.67 & 25.75 & 34.08 & 405.6 & 9.17 & 26.5 \\
\hline 245. & 0.0057 & 1.000 & 8.162 & 197.37 & 25.85 & 34.18 & 409.6 & 9.34 & 27.1 \\
\hline 250. & 0.0056 & 1.000 & 8.334 & 198.06 & 25.96 & 34.29 & 413.5 & 9.51 & 27.7 \\
\hline 255. & 0.0055 & 1.000 & 8.505 & 198.74 & 26.07 & 34.40 & 417.4 & 9.68 & 28.4 \\
\hline 260. & 0.0054 & 1.000 & 8.678 & 199.41 & 26.20 & 34.52 & 421.3 & 9.86 & 29.0 \\
\hline 265. & 0.0053 & 1.000 & 8.850 & 200.07 & 26.33 & 34.65 & 425.0 & 10.02 & 29.6 \\
\hline 270. & 0.0052 & 1.000 & 9.024 & 200.72 & 26.47 & 34.79 & 428.8 & 10.19 & 30.3 \\
\hline 275. & 0.0051 & 1.000 & 9.198 & 201.36 & 26.61 & 34.94 & 432.4 & 10.36 & 30.9 \\
\hline 280 . & 0.0050 & 1.000 & 9.373 & 201.99 & 26.77 & 35.09 & 436.1 & 10.53 & 31.6 \\
\hline 285. & 0.0049 & 1.000 & 9.549 & 202.61 & 26.93 & 35.25 & 439.6 & 10.69 & 32.3 \\
\hline 290. & 0.0049 & 1.000 & 9.726 & 203.23 & 27.10 & 35.42 & 443.1 & 10.86 & 32.9 \\
\hline 295. & 0.0048 & 1.000 & 9.904 & 203.83 & 27.28 & 35.60 & 446.6 & 11.02 & 33.6 \\
\hline 300. & 0.0047 & 1.000 & 10.082 & 204.43 & 27.46 & 35.78 & 450.0 & 11.18 & 34.3 \\
\hline 310. & 0.0045 & 1.000 & 10.442 & 205.61 & 27.85 & 36.17 & 456.7 & 11.50 & 35.7 \\
\hline 320. & 0.0044 & 1.000 & 10.806 & 206.77 & 28.27 & 36.59 & 463.3 & 11.82 & 37.2 \\
\hline 330. & 0.0043 & 1.000 & 11.174 & 207.90 & 28.71 & 37.03 & 469.6 & 12.14 & 38.7 \\
\hline 340. & 0.0041 & 1.000 & 11.546 & 209.01 & 29.17 & 37.49 & 475.8 & 12.45 & 40.2 \\
\hline 350. & 0.0040 & 1.000 & 11.923 & 210.11 & 29.65 & 37.97 & 481.9 & 12.75 & 41.7 \\
\hline 360. & 0.0039 & 1.000 & 12.306 & 211.18 & 30.15 & 38.47 & 487.9 & 13.05 & 43.3 \\
\hline 370 & 0.0038 & 1.000 & 12.693 & 212.24 & 30.67 & 38.99 & 493.7 & 13.35 & 44.9 \\
\hline 380. & 0.0037 & 1.000 & 13.085 & 213.29 & 31.20 & 39.52 & 499.4 & 13.65 & 46.6 \\
\hline 390. & 0.0036 & 1.000 & 13.483 & 214.33 & 31.75 & 40.06 & 505.0 & 13.94 & 48.3 \\
\hline 400. & 0.0035 & 1.000 & 13.887 & 215.35 & 32.30 & 40.62 & 510.6 & 14.23 & 50.0 \\
\hline 410. & 0.0034 & 1.000 & 14.296 & 216.36 & 32.86 & 41.18 & 516.0 & & 51.7 \\
\hline 420. & 0.0033 & 1.000 & 14.710 & 217.36 & 33.43 & 41.75 & 521.4 & & 53.5 \\
\hline 430. & 0.0033 & 1.000 & 15.131 & 218.34 & 34.01 & 42.32 & 526.6 & & 55.2 \\
\hline 440. & 0.0032 & 1.000 & 15.557 & 219.32 & 34.58 & 42.90 & 531.9 & & 57.0 \\
\hline 450. & 0.0031 & 1.000 & 15.989 & 220.29 & 35.16 & 43.48 & 537.0 & & 58.9 \\
\hline 460. & 0.0031 & 1.000 & 16.427 & 221.26 & 35.74 & 44.06 & 542.1 & & 60.7 \\
\hline 470. & 0.0030 & 1.000 & 16.870 & 222.21 & 36.32 & 44.64 & 547.1 & & 62.6 \\
\hline 480. & 0.0029 & 1.000 & 17.319 & 223.16 & 36.90 & 45.22 & 552.1 & & 64.5 \\
\hline 490. & 0.0029 & 1.000 & 17.774 & 224.09 & 37.48 & 45.80 & 557.0 & & 66.4 \\
\hline 500. & 0.0028 & 1.000 & 18.235 & 225.03 & 38.05 & 46.37 & 561.9 & & 68.3 \\
\hline 520. & 0.0027 & 1.000 & 19.174 & 226.87 & 39.19 & 47.51 & 571.6 & & 72.1 \\
\hline
\end{tabular}


METHANE ISOBAR AT $\mathrm{P}=0.012 \mathrm{MPa}$ (continued)

$\begin{array}{lllllllllll}\mathrm{T} & \rho & \mathrm{Z} & \mathrm{H} & \mathrm{S} & \mathrm{C}_{\mathrm{v}} & \mathrm{C}_{\mathrm{p}} & \mathrm{W} & \eta & \lambda\end{array}$

$\mathrm{K} \quad \mathrm{mol} \cdot \mathrm{dm}^{-3} \quad \mathrm{~kJ} / \mathrm{mol} \quad \mathrm{J} /(\mathrm{mol} \cdot \mathrm{K}) \mathrm{J} /(\mathrm{mol} \cdot \mathrm{K}) \mathrm{J} /(\mathrm{mol} \cdot \mathrm{K}) \quad \mathrm{m} \cdot \mathrm{s}^{-1} \quad \mu \mathrm{Pa} \cdot \mathrm{s} \quad \mathrm{mW} /(\mathrm{m} \cdot \mathrm{K})$

540. $\quad \begin{array}{llllllll}0.0026 & 1.000 & 20.135 & 228.68 & 40.31 & 48.62 & 581.0 & 76.0\end{array}$

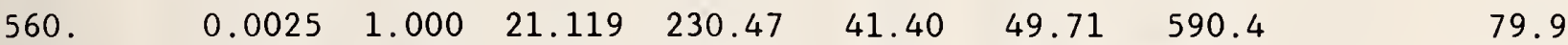

580. $\quad \begin{array}{llllllll}0.0024 & 1.000 & 22.124 & 232.23 & 42.46 & 50.78 & 599.6 & 83.9\end{array}$

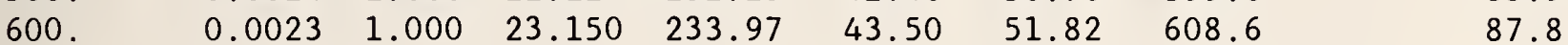

METHANE ISOBAR AT $P=0.025 \mathrm{MPa}$

\begin{tabular}{|c|c|c|c|c|c|c|c|c|c|}
\hline $\mathrm{T}$ & $\rho$ & $\mathrm{Z}$ & H & $S$ & $\mathrm{C}_{\mathrm{v}}$ & $C_{p}$ & W & $\eta$ & $\lambda$ \\
\hline K & $\mathrm{mol} \cdot \mathrm{dm}^{-3}$ & & $\mathrm{~kJ} / \mathrm{mol}$ & $\mathrm{J} /(\mathrm{mol} \cdot \mathrm{K})$ & $\mathrm{J} /(\mathrm{mol} \cdot \mathrm{K})$ & $\mathrm{J} /(\mathrm{mol} \cdot \mathrm{K})$ & $\mathrm{m} \cdot \mathrm{s}^{-1}$ & $\mu \mathrm{Pa} \cdot \mathrm{s}$ & $\mathrm{mW} /(\mathrm{m} \cdot \mathrm{K})$ \\
\hline 95. & 27.786 & 0.001 & -5.515 & 70.28 & 33.99 & 53.76 & 1498.6 & 179.63 & 206.1 \\
\hline 97.034 & 27.607 & 0.001 & -5.403 & 71.44 & 34.07 & 54.18 & 1475.4 & 169.80 & 203.4 \\
\hline 97.034 & 0.0314 & 0.986 & 3.166 & 159.75 & 28.02 & 37.36 & 254.9 & 3.85 & 9.6 \\
\hline 100. & 0.0305 & 0.987 & 3.273 & 160.84 & 26.53 & 35.53 & 259.9 & 3.96 & 9.9 \\
\hline 105. & 0.0289 & 0.989 & 3.448 & 162.54 & 25.56 & 34.32 & 267.3 & 4.15 & 10.5 \\
\hline 110. & 0.0276 & 0.991 & 3.618 & 164.12 & 25.26 & 33.91 & 274.1 & 4.33 & 11.1 \\
\hline 115. & 0.0264 & 0.992 & 3.787 & 165.63 & 25.16 & 33.75 & 280.5 & 4.53 & 11.6 \\
\hline 120. & 0.0252 & 0.993 & 3.956 & 167.06 & 25.11 & 33.67 & 286.7 & 4.72 & 12.2 \\
\hline 125. & 0.0242 & 0.994 & 4.124 & 168.43 & 25.09 & 33.61 & 292.7 & 4.91 & 12.8 \\
\hline 130. & 0.0233 & 0.994 & 4.292 & 169.75 & 25.07 & 33.57 & 298.7 & 5.10 & 13.4 \\
\hline 135. & 0.0224 & 0.995 & 4.459 & 171.02 & 25.06 & 33.53 & 304.5 & 5.29 & 14.0 \\
\hline 140. & 0.0216 & 0.996 & 4.627 & 172.24 & 25.05 & 33.51 & 310.1 & 5.48 & 14.6 \\
\hline 145. & 0.0208 & 0.996 & 4.795 & 173.41 & 25.04 & 33.49 & 315.7 & 5.67 & 15.2 \\
\hline 150. & 0.0201 & 0.996 & 4.962 & 174.55 & 25.04 & 33.47 & 321.2 & 5.86 & 15.8 \\
\hline 155. & 0.0195 & 0.997 & 5.129 & 175.64 & 25.04 & 33.46 & 326.5 & 6.05 & 16.4 \\
\hline 160. & 0.0188 & 0.997 & 5.297 & 176.71 & 25.05 & 33.46 & 331.8 & 6.24 & 17.0 \\
\hline 165. & 0.0183 & 0.997 & 5.464 & 177.74 & 25.05 & 33.46 & 337.0 & 6.43 & 17.6 \\
\hline 170. & 0.0177 & 0.997 & 5.631 & 178.73 & 25.07 & 33.46 & 342.1 & 6.62 & 18.1 \\
\hline 175. & 0.0172 & 0.998 & 5.799 & 179.70 & 25.08 & 33.47 & 347.1 & 6.81 & 18.7 \\
\hline 180. & 0.0167 & 0.998 & 5.966 & 180.65 & 25.10 & 33.48 & 352.0 & 7.00 & 19.3 \\
\hline 185. & 0.0163 & 0.998 & 6.133 & 181.57 & 25.12 & 33.50 & 356.9 & 7.18 & 19.9 \\
\hline 190. & 0.0159 & 0.998 & 6.301 & 182.46 & 25.15 & 33.52 & 361.6 & 7.37 & 20.5 \\
\hline 195. & 0.0154 & 0.998 & 6.469 & 183.33 & 25.18 & 33.55 & 366.3 & 7.55 & 21.1 \\
\hline 200 . & 0.0151 & 0.998 & 6.636 & 184.18 & 25.22 & 33.59 & 370.9 & 7.74 & 21.7 \\
\hline 205. & 0.0147 & 0.999 & 6.804 & 185.01 & 25.26 & 33.63 & 375.5 & 7.92 & 22.3 \\
\hline 210 . & 0.0143 & 0.999 & 6.973 & 185.82 & 25.31 & 33.67 & 380.0 & 8.10 & 22.9 \\
\hline 215. & 0.0140 & 0.999 & 7.141 & 186.61 & 25.37 & 33.73 & 384.4 & 8.28 & 23.5 \\
\hline 220 . & 0.0137 & 0.999 & 7.310 & 187.39 & 25.43 & 33.79 & 388.7 & 8.46 & 24.1 \\
\hline 225. & 0.0134 & 0.999 & 7.479 & 188.15 & 25.50 & 33.85 & 393.0 & 8.64 & 24.7 \\
\hline 230 . & 0.0131 & 0.999 & 7.649 & 188.89 & 25.58 & 33.93 & 397.2 & 8.82 & 25.3 \\
\hline
\end{tabular}


METHANE ISOBAR AT $\mathrm{P}=0.025 \mathrm{MPa}$ (continued)

\begin{tabular}{|c|c|c|c|c|c|c|c|c|c|}
\hline $\mathrm{T}$ & $\rho$ & $\mathrm{Z}$ & H & $S$ & $\mathrm{C}_{\mathrm{v}}$ & $\mathrm{C}_{\mathrm{p}}$ & W & $\eta$ & $\lambda$ \\
\hline K & $\mathrm{mol} \cdot \mathrm{dm}^{-3}$ & & $\mathrm{~kJ} / \mathrm{mol}$ & $\mathrm{J} /(\mathrm{mol} \cdot \mathrm{K})$ & $\mathrm{J} /(\mathrm{mol} \cdot \mathrm{K})$ & $\mathrm{J} /(\mathrm{mol} \cdot \mathrm{K})$ & $\mathrm{m} \cdot \mathrm{s}^{-1}$ & $\mu \mathrm{Pa} \cdot \mathrm{s}$ & $\mathrm{mW} /(\mathrm{m}$. \\
\hline 235 . & 0.0128 & 0.999 & 7.818 & 189.63 & 25.66 & 34.01 & 401.4 & 8.99 & 25.9 \\
\hline 240 . & 0.0125 & 0.999 & 7.989 & 190.34 & 25.75 & 34.10 & 405.5 & 9.17 & 26.5 \\
\hline 245. & 0.0123 & 0.999 & 8.159 & 191.05 & 25.85 & 34.20 & 409.5 & 9.34 & 27.1 \\
\hline 250. & 0.0120 & 0.999 & 8.331 & 191.74 & 25.96 & 34.30 & 413.4 & 9.51 & 27.7 \\
\hline 255. & 0.0118 & 0.999 & 8.502 & 192.42 & 26.08 & 34.42 & 417.3 & 9.69 & 28.4 \\
\hline 260 . & 0.0116 & 0.999 & 8.675 & 193.09 & 26.20 & 34.54 & 421.2 & 9.86 & 29.0 \\
\hline 265. & 0.0114 & 0.999 & 8.848 & 193.75 & 26.33 & 34.67 & 425.0 & 10.03 & 29.6 \\
\hline 270 . & 0.0111 & 0.999 & 9.022 & 194.40 & 26.47 & 34.81 & 428.7 & 10.20 & 30.3 \\
\hline 275 . & 0.0109 & 0.999 & 9.196 & 195.04 & 26.62 & 34.95 & 432.4 & 10.36 & 30.9 \\
\hline 280 . & 0.0107 & 0.999 & 9.371 & 195.67 & 26.77 & 35.11 & 436.0 & 10.53 & 31.6 \\
\hline 285 . & 0.0106 & 0.999 & 9.547 & 196.29 & 26.93 & 35.27 & 439.6 & 10.70 & 32.3 \\
\hline 290. & 0.0104 & 1.000 & 9.724 & 196.91 & 27.10 & 35.44 & 443.1 & 10.86 & 33.0 \\
\hline 295. & 0.0102 & 1.000 & 9.901 & 197.51 & 27.28 & 35.61 & 446.6 & 11.02 & 33.6 \\
\hline 300 . & 0.0100 & 1.000 & 10.080 & 198.11 & 27.46 & 35.80 & 450.0 & 11.19 & 34.3 \\
\hline 310. & 0.0097 & 1.000 & 10.440 & 199.29 & 27.85 & 36.18 & 456.7 & 11.51 & 35.8 \\
\hline 320. & 0.0094 & 1.000 & 10.804 & 200.45 & 28.27 & 36.60 & 463.2 & 11.82 & 37.2 \\
\hline 330. & 0.0091 & 1.000 & 11.172 & 201.58 & 28.71 & 37.04 & 469.6 & 12.14 & 38.7 \\
\hline 340 . & 0.0088 & 1.000 & 11.544 & 202.69 & 29.17 & 37.50 & 475.8 & 12.45 & 40.2 \\
\hline 350 . & 0.0086 & 1.000 & 11.922 & 203.79 & 29.65 & 37.98 & 481.9 & 12.75 & 41.7 \\
\hline 360 . & 0.0084 & 1.000 & 12.304 & 204.86 & 30.15 & 38.48 & 487.9 & 13.06 & 43.3 \\
\hline 370 . & 0.0081 & 1.000 & 12.692 & 205.93 & 30.67 & 39.00 & 493.7 & 13.36 & 44.9 \\
\hline 380 . & 0.0079 & 1.000 & 13.084 & 206.97 & 31.20 & 39.53 & 499.4 & 13.65 & 46.6 \\
\hline 390. & 0.0077 & 1.000 & 13.482 & 208.01 & 31.75 & 40.07 & 505.0 & 13.94 & 48.3 \\
\hline 400. & 0.0075 & 1.000 & 13.886 & 209.03 & 32.30 & 40.62 & 510.6 & 14.23 & 50.0 \\
\hline 410. & 0.0073 & 1.000 & 14.295 & 210.04 & 32.86 & 41.19 & 516.0 & & 51.7 \\
\hline 420. & 0.0072 & 1.000 & 14.709 & 211.04 & 33.43 & 41.76 & 521.4 & & 53.5 \\
\hline 430. & 0.0070 & 1.000 & 15.130 & 212.03 & 34.01 & 42.33 & 526.6 & & 55.2 \\
\hline 440. & 0.0068 & 1.000 & 15.556 & 213.01 & 34.58 & 42.91 & 531.9 & & 57.1 \\
\hline 450. & 0.0067 & 1.000 & 15.988 & 213.98 & 35.16 & 43.49 & 537.0 & & 58.9 \\
\hline 460. & 0.0065 & 1.000 & 16.426 & 214.94 & 35.74 & 44.07 & 542.1 & & 60.7 \\
\hline 470. & 0.0064 & 1.000 & 16.869 & 215.89 & 36.32 & 44.65 & 547.1 & & 62.6 \\
\hline 480. & 0.0063 & 1.000 & 17.319 & 216.84 & 36.90 & 45.22 & 552.1 & & 64.5 \\
\hline 490. & 0.0061 & 1.000 & 17.774 & 217.78 & 37.48 & 45.80 & 557.1 & & 66.4 \\
\hline 500. & 0.0060 & 1.000 & 18.235 & 218.71 & 38.05 & 46.37 & 561.9 & & 68.3 \\
\hline 520. & 0.0058 & 1.000 & 19.173 & 220.55 & 39.19 & 47.51 & 571.6 & & 72.1 \\
\hline 540. & 0.0056 & 1.000 & 20.135 & 222.36 & 40.31 & 48.63 & 581.1 & & 76.0 \\
\hline 560. & 0.0054 & 1.000 & 21.118 & 224.15 & 41.40 & 49.72 & 590.4 & & 79.9 \\
\hline 580. & 0.0052 & 1.000 & 22.123 & 225.92 & 42.46 & 50.78 & 599.6 & & 83.9 \\
\hline 600. & 0.0050 & 1.000 & 23.149 & 227.65 & 43.50 & 51.82 & 608.6 & & 87.8 \\
\hline
\end{tabular}


METHANE ISOBAR AT $P=0.050 \mathrm{MPa}$

\begin{tabular}{|c|c|c|c|c|c|c|c|c|c|}
\hline $\mathrm{T}$ & $\rho$ & Z & $\mathrm{H}$ & $S$ & $C_{v}$ & $C_{p}$ & W & $\eta$ & $\lambda$ \\
\hline K & $\mathrm{mol} \cdot \mathrm{dm}^{-3}$ & & $\mathrm{~kJ} / \mathrm{mol}$ & $\mathrm{J} /(\mathrm{mol} \cdot \mathrm{K})$ & $\mathrm{J} /(\mathrm{mol} \cdot \mathrm{K})$ & $\mathrm{J} /(\mathrm{mol} \cdot \mathrm{K})$ & $\mathrm{m} \cdot \mathrm{s}^{-1}$ & $\mu \mathrm{Pa} \cdot \mathrm{s}$ & $\mathrm{mW} /(\mathrm{m} \cdot \mathrm{K}$ \\
\hline 95. & 27.787 & 0.002 & -5.514 & 70.27 & 33.99 & 53.75 & 1498.8 & 179.68 & 206.1 \\
\hline 100. & 27.363 & 0.002 & -5.243 & 73.05 & 34.08 & 54.64 & 1446.4 & 157.39 & 199.7 \\
\hline 103.729 & 27.027 & 0.002 & -5.035 & 75.10 & 33.92 & 55.17 & 1406.6 & 142.63 & 194.5 \\
\hline 103.729 & 0.0593 & 0.977 & 3.367 & 156.09 & 27.52 & 37.02 & 262.5 & 4.10 & 10.4 \\
\hline 105. & 0.0586 & 0.978 & 3.413 & 156.54 & 26.94 & 36.29 & 264.7 & 4.15 & 10.6 \\
\hline 110. & 0.0557 & 0.981 & 3.590 & 158.19 & 25.80 & 34.84 & 272.2 & 4.34 & 11.1 \\
\hline 115 . & 0.0532 & 0.984 & 3.763 & 159.72 & 25.43 & 34.33 & 279.0 & 4.53 & 11.7 \\
\hline 120. & 0.0508 & 0.986 & 3.934 & 161.18 & 25.29 & 34.09 & 285.4 & 4.72 & 12.3 \\
\hline 125. & 0.0487 & 0.987 & 4.104 & 162.57 & 25.22 & 33.96 & 291.6 & 4.91 & 12.9 \\
\hline 130. & 0.0468 & 0.989 & 4.274 & 163.90 & 25.18 & 33.86 & 297.6 & 5.10 & 13.5 \\
\hline 135. & 0.0450 & 0.990 & 4.443 & 165.17 & 25.15 & 33.79 & 303.5 & 5.30 & 14.0 \\
\hline 140. & 0.0433 & 0.991 & 4.612 & 166.40 & 25.13 & 33.73 & 309.3 & 5.49 & 14.6 \\
\hline 145. & 0.0418 & 0.992 & 4.780 & 167.58 & 25.11 & 33.69 & 314.9 & 5.68 & 15.2 \\
\hline 150. & 0.0404 & 0.993 & 4.949 & 168.72 & 25.10 & 33.65 & 320.5 & 5.87 & 15.8 \\
\hline 155. & 0.0391 & 0.993 & 5.117 & 169.83 & 25.09 & 33.62 & 325.9 & 6.06 & 16.4 \\
\hline 160. & 0.0378 & 0.994 & 5.285 & 170.89 & 25.09 & 33.60 & 331.2 & 6.25 & 17.0 \\
\hline 165. & 0.0367 & 0.994 & 5.453 & 171.93 & 25.09 & 33.59 & 336.4 & 6.44 & 17.6 \\
\hline 170. & 0.0356 & 0.995 & 5.621 & 172.93 & 25.10 & 33.58 & 341.6 & 6.63 & 18.2 \\
\hline 175. & 0.0345 & 0.995 & 5.789 & 173.90 & 25.11 & 33.57 & 346.6 & 6.82 & 18.8 \\
\hline 180. & 0.0336 & 0.996 & 5.956 & 174.85 & 25.12 & 33.58 & 351.6 & 7.00 & 19.4 \\
\hline 185. & 0.0326 & 0.996 & 6.124 & 175.77 & 25.14 & 33.59 & 356.4 & 7.19 & 20.0 \\
\hline 190. & 0.0318 & 0.996 & 6.292 & 176.67 & 25.17 & 33.60 & 361.2 & 7.37 & 20.6 \\
\hline 195. & 0.0309 & 0.997 & 6.460 & 177.54 & 25.20 & 33.63 & 366.0 & 7.56 & 21.1 \\
\hline 200. & 0.0302 & 0.997 & 6.629 & 178.39 & 25.24 & 33.66 & 370.6 & 7.74 & 21.7 \\
\hline 205 . & 0.0294 & 0.997 & 6.797 & 179.22 & 25.28 & 33.69 & 375.2 & 7.92 & 22.3 \\
\hline 210 . & 0.0287 & 0.997 & 6.965 & 180.03 & 25.33 & 33.73 & 379.7 & 8.11 & 22.9 \\
\hline 215 . & 0.0280 & 0.997 & 7.134 & 180.83 & 25.38 & 33.78 & 384.1 & 8.29 & 23.5 \\
\hline 220 & 0.0274 & 0.998 & 7.303 & 181.61 & 25.44 & 33.84 & 388.5 & 8.47 & 24.1 \\
\hline 225. & 0.0268 & 0.998 & 7.473 & 182.37 & 25.51 & 33.90 & 392.8 & 8.64 & 24.7 \\
\hline 230 . & 0.0262 & 0.998 & 7.642 & 183.11 & 25.59 & 33.98 & 397.0 & 8.82 & 25.3 \\
\hline 235. & 0.0256 & 0.998 & 7.812 & 183.85 & 25.67 & 34.05 & 401.2 & 9.00 & 25.9 \\
\hline 240 & 0.0251 & 0.998 & 7.983 & 184.56 & 25.76 & 34.14 & 405.3 & 9.17 & 26.5 \\
\hline 245. & 0.0246 & 0.998 & 8.154 & 185.27 & 25.86 & 34.24 & 409.3 & 9.35 & 27.1 \\
\hline 250 . & 0.0241 & 0.998 & 8.325 & 185.96 & 25.97 & 34.34 & 413.3 & 9.52 & 27.8 \\
\hline 255 . & 0.0236 & 0.999 & 8.497 & 186.64 & 26.08 & 34.45 & 417.2 & 9.69 & 28.4 \\
\hline 260. & 0.0232 & 0.999 & 8.670 & 187.31 & 26.20 & 34.57 & 421.0 & 9.86 & 29.0 \\
\hline 265 . & 0.0227 & 0.999 & 8.843 & 187.97 & 26.34 & 34.70 & 424.8 & 10.03 & 29.7 \\
\hline 270 . & 0.0223 & 0.999 & 9.017 & 188.62 & 26.47 & 34.84 & 428.6 & 10.20 & 30.3 \\
\hline 275. & 0.0219 & 0.999 & 9.191 & 189.26 & 26.62 & 34.98 & 432.3 & 10.37 & 31.0 \\
\hline 280 . & 0.0215 & 0.999 & 9.367 & 189.89 & 26.77 & 35.13 & 435.9 & 10.53 & 31.6 \\
\hline
\end{tabular}


METHANE ISOBAR AT $\mathrm{P}=0.050 \mathrm{MPa}$ (continued)

\begin{tabular}{|c|c|c|c|c|c|c|c|c|c|}
\hline $\mathrm{T}$ & $\rho$ & $\mathrm{z}$ & $\mathrm{H}$ & S & $\mathrm{C}_{\mathrm{v}}$ & $C_{p}$ & W & $\eta$ & $\lambda$ \\
\hline K & $\mathrm{mol} \cdot \mathrm{dm}^{-3}$ & & $\mathrm{~kJ} / \mathrm{mol}$ & $\mathrm{J} /(\mathrm{mol} \cdot \mathrm{K})$ & $\mathrm{J} /(\mathrm{mol} \cdot \mathrm{K})$ & $\mathrm{J} /(\mathrm{mol} \cdot \mathrm{K})$ & $\mathrm{m} \cdot \mathrm{s}^{-1}$ & $\mu \mathrm{Pa} \cdot \mathrm{s}$ & $\mathrm{mW} /(\mathrm{m} \cdot \mathrm{K})$ \\
\hline 285. & 0.0211 & 0.999 & 9.543 & 190.52 & 26.94 & 35.29 & 439.5 & 10.70 & 32.3 \\
\hline 290. & 0.0208 & 0.999 & 9.720 & 191.13 & 27.11 & 35.46 & 443.0 & 10.86 & 33.0 \\
\hline 295. & 0.0204 & 0.999 & 9.897 & 191.74 & 27.28 & 35.64 & 446.5 & 11.03 & 33.7 \\
\hline 300 . & 0.0201 & 0.999 & 10.076 & 192.34 & 27.47 & 35.82 & 449.9 & 11.19 & 34.4 \\
\hline 310. & 0.0194 & 0.999 & 10.436 & 193.52 & 27.85 & 36.20 & 456.6 & 11.51 & 35.8 \\
\hline 320 . & 0.0188 & 0.999 & 10.800 & 194.68 & 28.27 & 36.62 & 463.2 & 11.83 & 37.2 \\
\hline 330. & 0.0182 & 0.999 & 11.169 & 195.81 & 28.71 & 37.05 & 469.6 & 12.14 & 38.7 \\
\hline 340. & 0.0177 & 0.999 & 11.541 & 196.92 & 29.17 & 37.51 & 475.8 & 12.45 & 40.2 \\
\hline 350. & 0.0172 & 1.000 & 11.919 & 198.02 & 29.66 & 38.00 & 481.9 & 12.76 & 41.8 \\
\hline 360. & 0.0167 & 1.000 & 12.301 & 199.10 & 30.16 & 38.50 & 487.8 & 13.06 & 43.3 \\
\hline 370. & 0.0163 & 1.000 & 12.689 & 200.16 & 30.67 & 39.01 & 493.7 & 13.36 & 45.0 \\
\hline 380. & 0.0158 & 1.000 & 13.082 & 201.20 & 31.20 & 39.54 & 499.4 & 13.65 & 46.6 \\
\hline 390. & 0.0154 & 1.000 & 13.480 & 202.24 & 31.75 & 40.08 & 505.0 & 13.95 & 48.3 \\
\hline 400. & 0.0150 & 1.000 & 13.883 & 203.26 & 32.30 & 40.64 & 510.6 & 14.23 & 50.0 \\
\hline 410. & 0.0147 & 1.000 & 14.292 & 204.27 & 32.86 & 41.20 & 516.0 & & 51.7 \\
\hline 420. & 0.0143 & 1.000 & 14.707 & 205.27 & 33.43 & 41.77 & 521.4 & & 53.5 \\
\hline 430. & 0.0140 & 1.000 & 15.128 & 206.26 & 34.01 & 42.34 & 526.7 & & 55.3 \\
\hline 440. & 0.0137 & 1.000 & 15.554 & 207.24 & 34.59 & 42.91 & 531.9 & & 57.1 \\
\hline 450. & 0.0134 & 1.000 & 15.986 & 208.21 & 35.16 & 43.49 & 537.0 & & 58.9 \\
\hline 460. & 0.0131 & 1.000 & 16.424 & 209.17 & 35.75 & 44.07 & 542.1 & & 60.7 \\
\hline 470. & 0.0128 & 1.000 & 16.868 & 210.13 & 36.33 & 44.65 & 547.2 & & 62.6 \\
\hline 480. & 0.0125 & 1.000 & 17.317 & 211.07 & 36.91 & 45.23 & 552.2 & & 64.5 \\
\hline 490. & 0.0123 & 1.000 & 17.772 & 212.01 & 37.48 & 45.81 & 557.1 & & 66.4 \\
\hline 500. & 0.0120 & 1.000 & 18.233 & 212.94 & 38.06 & 46.38 & 562.0 & & 68.3 \\
\hline 520. & 0.0116 & 1.000 & 19.172 & 214.78 & 39.19 & 47.52 & 571.6 & & 72.1 \\
\hline 540. & 0.0111 & 1.000 & 20.134 & 216.60 & 40.31 & 48.63 & 581.1 & & 76.0 \\
\hline 560. & 0.0107 & 1.000 & 21.117 & 218.39 & 41.40 & 49.72 & 590.4 & & 79.9 \\
\hline 580. & 0.0104 & 1.000 & 22.122 & 220.15 & 42.46 & 50.79 & 599.6 & & 83.9 \\
\hline 600. & 0.0100 & 1.000 & 23.149 & 221.89 & 43.50 & 51.82 & 608.7 & & 87.8 \\
\hline
\end{tabular}

$\overline{\text { METHANE ISOBAR AT } \mathrm{P}=0.075 \mathrm{MPa}}$

$\begin{array}{lccccccccc}\mathrm{T} & \rho & \mathrm{Z} & \mathrm{H} & \mathrm{S} & \mathrm{C} & \mathrm{C} & \mathrm{W} & \eta & \lambda \\ \mathrm{K} & \mathrm{mol} \cdot \mathrm{dm}_{\mathrm{v}}{ }^{-3} & & \mathrm{~kJ} / \mathrm{mol} & \mathrm{J} /(\mathrm{mol} \cdot \mathrm{K}) & \mathrm{J} /(\mathrm{mol} \cdot \mathrm{K}) & \mathrm{J} /(\mathrm{mol} \cdot \mathrm{K}) & \mathrm{m} \cdot \mathrm{s}^{-1} & \mu \mathrm{Pa} \cdot \mathrm{s} & \mathrm{mW} /(\mathrm{m} \cdot \mathrm{K}) \\ & & & & & & & & & \\ 95 . & 27.788 & 0.003 & -5.513 & 70.27 & 33.99 & 53.75 & 1499.0 & 179.73 & 206.1 \\ 100 . & 27.364 & 0.003 & -5.242 & 73.05 & 34.08 & 54.64 & 1446.6 & 157.43 & 199.7 \\ 105 . & 26.930 & 0.003 & -4.967 & 75.73 & 33.86 & 55.28 & 1396.6 & 138.62 & 193.1 \\ 108.127 & 26.639 & 0.003 & -4.790 & 77.39 & 33.63 & 55.68 & 1363.1 & 127.98 & 188.5 \\ 108.127 & 0.0860 & 0.970 & 3.493 & 154.00 & 27.19 & 36.85 & 267.1 & 4.28 & 11.0\end{array}$


METHANE ISOBAR AT $\mathrm{P}=0.075 \mathrm{MPa}$ (continued)

\begin{tabular}{|c|c|c|c|c|c|c|c|c|c|}
\hline $\mathrm{T}$ & $\rho$ & Z & $\mathrm{H}$ & $\mathrm{S}$ & $C_{v}$ & $C_{p}$ & W & $\eta$ & $\lambda$ \\
\hline K & $\mathrm{mol} \cdot \mathrm{dm}^{-3}$ & & $\mathrm{~kJ} / \mathrm{mol}$ & $\mathrm{J} /(\mathrm{mol} \cdot \mathrm{K})$ & $\mathrm{J} /(\mathrm{mol} \cdot \mathrm{K})$ & $\mathrm{J} /(\mathrm{mol} \cdot \mathrm{K})$ & $m \cdot s^{-1}$ & $\mu \mathrm{Pa} \cdot \mathrm{s}$ & $\mathrm{mW} /(\mathrm{m} \cdot$ \\
\hline 110. & 0.0844 & 0.971 & 3.561 & 154.63 & 26.56 & 36.04 & 270.1 & 4.35 & 11.2 \\
\hline 115. & 0.0804 & 0.975 & 3.739 & 156.20 & 25.76 & 34.98 & 277.4 & 4.54 & 11.8 \\
\hline 120. & 0.0768 & 0.978 & 3.912 & 157.68 & 25.48 & 34.55 & 284.1 & 4.73 & 12.3 \\
\hline 125. & 0.0736 & 0.981 & 4.084 & 159.09 & 25.36 & 34.32 & 290.4 & 4.92 & 12.9 \\
\hline 130. & 0.0706 & 0.983 & 4.256 & 160.43 & 25.29 & 34.17 & 296.6 & 5.11 & 13.5 \\
\hline 135. & 0.0678 & 0.985 & 4.426 & 161.72 & 25.24 & 34.06 & 302.6 & 5.30 & 14.1 \\
\hline 140. & 0.0653 & 0.986 & 4.596 & 162.96 & 25.20 & 33.97 & 308.4 & 5.49 & 14.7 \\
\hline 145. & 0.0630 & 0.988 & 4.766 & 164.15 & 25.18 & 33.89 & 314.1 & 5.68 & 15.3 \\
\hline 150. & 0.0608 & 0.989 & 4.935 & 165.29 & 25.16 & 33.83 & 319.7 & 5.88 & 15.8 \\
\hline 155. & 0.0588 & 0.990 & 5.104 & 166.40 & 25.14 & 33.78 & 325.2 & 6.07 & 16.4 \\
\hline 160. & 0.0569 & 0.991 & 5.273 & 167.47 & 25.13 & 33.74 & 330.6 & 6.26 & 17.0 \\
\hline 165. & 0.0551 & 0.992 & 5.442 & 168.51 & 25.13 & 33.71 & 335.9 & 6.45 & 17.6 \\
\hline 170. & 0.0535 & 0.992 & 5.610 & 169.52 & 25.13 & 33.69 & 341.0 & 6.63 & 18.2 \\
\hline 175. & 0.0519 & 0.993 & 5.778 & 170.50 & 25.14 & 33.68 & 346.1 & 6.82 & 18.8 \\
\hline 180. & 0.0504 & 0.993 & 5.947 & 171.44 & 25.15 & 33.68 & 351.1 & 7.01 & 19.4 \\
\hline 185. & 0.0491 & 0.994 & 6.115 & 172.37 & 25.17 & 33.68 & 356.0 & 7.19 & 20.0 \\
\hline 190. & 0.0477 & 0.994 & 6.284 & 173.26 & 25.19 & 33.69 & 360.9 & 7.38 & 20.6 \\
\hline 195. & 0.0465 & 0.995 & 6.452 & 174.14 & 25.22 & 33.70 & 365.6 & 7.56 & 21.2 \\
\hline 200 . & 0.0453 & 0.995 & 6.621 & 174.99 & 25.25 & 33.72 & 370.3 & 7.75 & 21.8 \\
\hline 205 . & 0.0442 & 0.996 & 6.789 & 175.83 & 25.29 & 33.76 & 374.9 & 7.93 & 22.3 \\
\hline 210. & 0.0431 & 0.996 & 6.958 & 176.64 & 25.34 & 33.79 & 379.4 & 8.11 & 22.9 \\
\hline 215. & 0.0421 & 0.996 & 7.127 & 177.44 & 25.39 & 33.84 & 383.9 & 8.29 & 23.5 \\
\hline 220 . & 0.0411 & 0.996 & 7.297 & 178.21 & 25.45 & 33.89 & 388.2 & 8.47 & 24.1 \\
\hline 225 . & 0.0402 & 0.997 & 7.466 & 178.98 & 25.52 & 33.95 & 392.6 & 8.65 & 24.7 \\
\hline 230 . & 0.0393 & 0.997 & 7.636 & 179.72 & 25.60 & 34.02 & 396.8 & 8.83 & 25.3 \\
\hline 235 . & 0.0385 & 0.997 & 7.806 & 180.46 & 25.68 & 34.10 & 401.0 & 9.00 & 25.9 \\
\hline 240 . & 0.0377 & 0.997 & 7.977 & 181.18 & 25.77 & 34.18 & 405.1 & 9.18 & 26.5 \\
\hline 245 . & 0.0369 & 0.997 & 8.148 & 181.88 & 25.87 & 34.28 & 409.1 & 9.35 & 27.2 \\
\hline 250 . & 0.0362 & 0.998 & 8.320 & 182.57 & 25.97 & 34.38 & 413.1 & 9.52 & 27.8 \\
\hline 255 . & 0.0355 & 0.998 & 8.492 & 183.26 & 26.09 & 34.49 & 417.0 & 9.70 & 28.4 \\
\hline 260 . & 0.0348 & 0.998 & 8.665 & 183.93 & 26.21 & 34.61 & 420.9 & 9.87 & 29.0 \\
\hline 265. & 0.0341 & 0.998 & 8.838 & 184.59 & 26.34 & 34.73 & 424.7 & 10.04 & 29.7 \\
\hline 270 . & 0.0335 & 0.998 & 9.012 & 185.24 & 26.48 & 34.87 & 428.5 & 10.20 & 30.3 \\
\hline 275 . & 0.0329 & 0.998 & 9.187 & 185.88 & 26.63 & 35.01 & 432.2 & 10.37 & 31.0 \\
\hline 280. & 0.0323 & 0.998 & 9.362 & 186.51 & 26.78 & 35.16 & 435.8 & 10.54 & 31.6 \\
\hline 285 . & 0.0317 & 0.998 & 9.539 & 187.14 & 26.94 & 35.32 & 439.4 & 10.70 & 32.3 \\
\hline 290 . & 0.0311 & 0.999 & 9.716 & 187.75 & 27.11 & 35.49 & 442.9 & 10.87 & 33.0 \\
\hline 295. & 0.0306 & 0.999 & 9.893 & 188.36 & 27.29 & 35.66 & 446.4 & 11.03 & 33.7 \\
\hline 300 . & 0.0301 & 0.999 & 10.072 & 188.96 & 27.47 & 35.84 & 449.8 & 11.19 & 34.4 \\
\hline 310 . & 0.0291 & 0.999 & 10.432 & 190.14 & 27.86 & 36.23 & 456.6 & 11.51 & 35.8 \\
\hline
\end{tabular}


METHANE ISOBAR AT $\mathrm{P}=0.075 \mathrm{MPa}$ (continued)

\begin{tabular}{|c|c|c|c|c|c|c|c|c|c|}
\hline $\mathrm{T}$ & $\rho$ & Z & $\mathrm{H}$ & $S$ & $C_{v}$ & $C_{p}$ & W & $\eta$ & $\lambda$ \\
\hline K & $\mathrm{mol} \cdot \mathrm{dm}^{-3}$ & & $\mathrm{~kJ} / \mathrm{mo} 1$ & $\mathrm{~J} /(\mathrm{mol} \cdot \mathrm{K})$ & $\mathrm{J} /(\mathrm{mol} \cdot \mathrm{K})$ & $\mathrm{J} /(\mathrm{mol} \cdot \mathrm{K})$ & $\mathrm{m} \cdot \mathrm{s}^{-1}$ & $\mu \mathrm{Pa} \cdot \mathrm{s}$ & $\mathrm{mW} /(\mathrm{m} \cdot \mathrm{K}$ \\
\hline 320 . & 0.0282 & 0.999 & 10.797 & 191.30 & 28.27 & 36.64 & 463.1 & 11.83 & 37.2 \\
\hline 330. & 0.0274 & 0.999 & 11.165 & 192.43 & 28.71 & 37.07 & 469.5 & 12.15 & 38.7 \\
\hline 340 . & 0.0266 & 0.999 & 11.538 & 193.55 & 29.17 & 37.53 & 475.7 & 12.45 & 40.2 \\
\hline 350 . & 0.0258 & 0.999 & 11.916 & 194.64 & 29.66 & 38.01 & 481.8 & 12.76 & 41.8 \\
\hline 360 . & 0.0251 & 0.999 & 12.299 & 195.72 & 30.16 & 38.51 & 487.8 & 13.06 & 43.4 \\
\hline 370. & 0.0244 & 0.999 & 12.686 & 196.78 & 30.68 & 39.02 & 493.6 & 13.36 & 45.0 \\
\hline 380 . & 0.0237 & 1.000 & 13.079 & 197.83 & 31.21 & 39.55 & 499.4 & 13.66 & 46.6 \\
\hline 390 . & 0.0231 & 1.000 & 13.477 & 198.86 & 31.75 & 40.10 & 505.0 & 13.95 & 48.3 \\
\hline 400. & 0.0226 & 1.000 & 13.881 & 199.88 & 32.30 & 40.65 & 510.6 & 14.24 & 50.0 \\
\hline 410. & 0.0220 & 1.000 & 14.290 & 200.89 & 32.87 & 41.21 & 516.0 & & 51.7 \\
\hline 420. & 0.0215 & 1.000 & 14.705 & 201.89 & 33.44 & 41.78 & 521.4 & & 53.5 \\
\hline 430. & 0.0210 & 1.000 & 15.126 & 202.88 & 34.01 & 42.35 & 526.7 & & 55.3 \\
\hline 440. & 0.0205 & 1.000 & 15.552 & 203.86 & 34.59 & 42.92 & 531.9 & & 57.1 \\
\hline 450. & 0.0200 & 1.000 & 15.984 & 204.84 & 35.17 & 43.50 & 537.0 & & 58.9 \\
\hline 460. & 0.0196 & 1.000 & 16.422 & 205.80 & 35.75 & 44.08 & 542.1 & & 60.7 \\
\hline 470. & 0.0192 & 1.000 & 16.866 & 206.75 & 36.33 & 44.66 & 547.2 & & 62.6 \\
\hline 480. & 0.0188 & 1.000 & 17.315 & 207.70 & 36.91 & 45.24 & 552.2 & & 64.5 \\
\hline 490. & 0.0184 & 1.000 & 17.771 & 208.64 & 37.48 & 45.82 & 557.1 & & 66.4 \\
\hline 500 . & 0.0180 & 1.000 & 18.232 & 209.57 & 38.06 & 46.39 & 562.0 & & 68.3 \\
\hline 520. & 0.0173 & 1.000 & 19.171 & 211.41 & 39.19 & 47.52 & 571.7 & & 72.1 \\
\hline 540 . & 0.0167 & 1.000 & 20.133 & 213.22 & 40.31 & 48.64 & 581.1 & & 76.0 \\
\hline 560. & 0.0161 & 1.000 & 21.116 & 215.01 & 41.40 & 49.73 & 590.5 & & 79.9 \\
\hline 580 . & 0.0156 & 1.000 & 22.121 & 216.78 & 42.47 & 50.79 & 599.7 & & 83.9 \\
\hline 600. & 0.0150 & 1.000 & 23.148 & 218.52 & 43.50 & 51.83 & 608.7 & & 87.8 \\
\hline
\end{tabular}

$\overline{\text { METHANE ISOBAR AT } \mathrm{P}=0.100 \mathrm{MPa}}$

$\begin{array}{lccccccccc}\mathrm{T} & \rho & \mathrm{Z} & \mathrm{H} & \mathrm{S} & \mathrm{C}_{\mathrm{v}} & \mathrm{C}_{\mathrm{p}} & \mathrm{W} & \eta & \lambda \\ \mathrm{K} & \mathrm{mol} \cdot \mathrm{dm}^{-3} & & \mathrm{~kJ} / \mathrm{mol} & \mathrm{J} /(\mathrm{mol} \cdot \mathrm{K}) & \mathrm{J} /(\mathrm{mol} \cdot \mathrm{K}) & \mathrm{J} /(\mathrm{mol} \cdot \mathrm{K}) & \mathrm{m} \cdot \mathrm{s}^{-1} & \mu \mathrm{Pa} \cdot \mathrm{s} & \mathrm{mW} /(\mathrm{m} \cdot \mathrm{K}) \\ & & & & & & & & & \\ 95 . & 27.789 & 0.005 & -5.513 & 70.27 & 33.99 & 53.75 & 1499.2 & 179.78 & 206.1 \\ 100 . & 27.366 & 0.004 & -5.242 & 73.05 & 34.08 & 54.64 & 1446.8 & 157.48 & 199.8 \\ 105 . & 26.932 & 0.004 & -4.967 & 75.73 & 33.86 & 55.28 & 1396.9 & 138.67 & 193.1 \\ 110 . & 26.486 & 0.004 & -4.689 & 78.32 & 33.49 & 55.84 & 1347.4 & 122.80 & 186.3 \\ 111.498 & 26.336 & 0.004 & -4.601 & 79.10 & 33.36 & 56.06 & 1329.9 & 118.17 & 183.9 \\ 111.498 & 0.1120 & 0.963 & 3.587 & 152.54 & 26.97 & 36.80 & 270.3 & 4.41 & 11.5 \\ 115 . & 0.1082 & 0.967 & 3.713 & 153.66 & 26.15 & 35.72 & 275.7 & 4.54 & 11.8 \\ 120 . & 0.1032 & 0.971 & 3.890 & 155.17 & 25.68 & 35.03 & 282.7 & 4.73 & 12.4 \\ 125 . & 0.0987 & 0.974 & 4.064 & 156.59 & 25.49 & 34.69 & 289.2 & 4.92 & 13.0 \\ 130 . & 0.0947 & 0.977 & 4.237 & 157.95 & 25.39 & 34.48 & 295.5 & 5.12 & 13.5\end{array}$


METHANE ISOBAR AT P $=0.100 \mathrm{MPa}$ (continued)

\begin{tabular}{|c|c|c|c|c|c|c|c|c|c|}
\hline $\mathrm{T}$ & $\rho$ & $Z$ & $\mathrm{H}$ & $S$ & $C_{v}$ & $C_{p}$ & W & $\eta$ & $\lambda$ \\
\hline K & $\mathrm{mol} \cdot \mathrm{dm}^{-3}$ & & $\mathrm{~kJ} / \mathrm{mol}$ & $\mathrm{J} /(\mathrm{mol} \cdot \mathrm{K})$ & $\mathrm{J} /(\mathrm{mol} \cdot \mathrm{K})$ & $\mathrm{J} /(\mathrm{mol} \cdot \mathrm{K})$ & $\mathrm{m} \cdot \mathrm{s}^{-1}$ & $\mu \mathrm{Pa} \cdot \mathrm{s}$ & $\mathrm{mW} /(\mathrm{m} \cdot \mathrm{l}$ \\
\hline 135. & 0.0909 & 0.980 & 4.409 & 159.24 & 25.33 & 34.33 & 301.6 & 5.31 & 14.1 \\
\hline 140. & 0.0875 & 0.982 & 4.580 & 160.49 & 25.28 & 34.20 & 307.5 & 5.50 & 14.7 \\
\hline 145. & 0.0843 & 0.984 & 4.751 & 161.69 & 25.24 & 34.10 & 313.3 & 5.69 & 15.3 \\
\hline 150. & 0.0814 & 0.985 & 4.921 & 162.84 & 25.21 & 34.01 & 319.0 & 5.88 & 15.9 \\
\hline 155. & 0.0787 & 0.987 & 5.091 & 163.96 & 25.19 & 33.95 & 324.5 & 6.07 & 16.5 \\
\hline 160. & 0.0761 & 0.988 & 5.261 & 165.03 & 25.18 & 33.89 & 330.0 & 6.26 & 17.1 \\
\hline 165. & 0.0737 & 0.989 & 5.430 & 166.08 & 25.17 & 33.85 & 335.3 & 6.45 & 17.7 \\
\hline 170. & 0.0715 & 0.990 & 5.599 & 167.09 & 25.16 & 33.81 & 340.5 & 6.64 & 18.2 \\
\hline 175. & 0.0694 & 0.991 & 5.768 & 168.07 & 25.17 & 33.79 & 345.6 & 6.83 & 18.8 \\
\hline 180. & 0.0674 & 0.991 & 5.937 & 169.02 & 25.18 & 33.77 & 350.7 & 7.01 & 19.4 \\
\hline 185. & 0.0655 & 0.992 & 6.106 & 169.94 & 25.19 & 33.77 & 355.6 & 7.20 & 20.0 \\
\hline 190. & 0.0638 & 0.993 & 6.275 & 170.84 & 25.21 & 33.77 & 360.5 & 7.39 & 20.6 \\
\hline 195. & 0.0621 & 0.993 & 6.444 & 171.72 & 25.24 & 33.78 & 365.3 & 7.57 & 21.2 \\
\hline 200. & 0.0605 & 0.994 & 6.613 & 172.58 & 25.27 & 33.80 & 370.0 & 7.75 & 21.8 \\
\hline 205. & 0.0590 & 0.994 & 6.782 & 173.41 & 25.31 & 33.82 & 374.6 & 7.93 & 22.4 \\
\hline 210 . & 0.0576 & 0.995 & 6.951 & 174.23 & 25.35 & 33.85 & 379.1 & 8.12 & 23.0 \\
\hline 215. & 0.0562 & 0.995 & 7.120 & 175.02 & 25.41 & 33.90 & 383.6 & 8.30 & 23.5 \\
\hline 220. & 0.0549 & 0.995 & 7.290 & 175.80 & 25.47 & 33.94 & 388.0 & 8.48 & 24.1 \\
\hline 225. & 0.0537 & 0.996 & 7.460 & 176.57 & 25.53 & 34.00 & 392.3 & 8.65 & 24.7 \\
\hline 230. & 0.0525 & 0.996 & 7.630 & 177.31 & 25.61 & 34.07 & 396.6 & 8.83 & 25.3 \\
\hline 235 & 0.0514 & 0.996 & 7.801 & 178.05 & 25.69 & 34.14 & 400.8 & 9.01 & 26.0 \\
\hline 240. & 0.0503 & 0.996 & 7.971 & 178.77 & 25.78 & 34.22 & 404.9 & 9.18 & 26.6 \\
\hline 245. & 0.0493 & 0.997 & 8.143 & 179.47 & 25.88 & 34.32 & 409.0 & 9.36 & 27.2 \\
\hline 250. & 0.0483 & 0.997 & 8.315 & 180.17 & 25.98 & 34.42 & 413.0 & 9.53 & 27.8 \\
\hline 255. & 0.0473 & 0.997 & 8.487 & 180.85 & 26.10 & 34.52 & 416.9 & 9.70 & 28.4 \\
\hline 260. & 0.0464 & 0.997 & 8.660 & 181.52 & 26.22 & 34.64 & 420.8 & 9.87 & 29.1 \\
\hline 265. & 0.0455 & 0.997 & 8.833 & 182.18 & 26.35 & 34.76 & 424.6 & 10.04 & 29.7 \\
\hline 270. & 0.0447 & 0.998 & 9.008 & 182.83 & 26.48 & 34.90 & 428.3 & 10.21 & 30.3 \\
\hline 275. & 0.0438 & 0.998 & 9.182 & 183.48 & 26.63 & 35.04 & 432.0 & 10.38 & 31.0 \\
\hline 280. & 0.0430 & 0.998 & 9.358 & 184.11 & 26.78 & 35.19 & 435.7 & 10.54 & 31.7 \\
\hline 285. & 0.0423 & 0.998 & 9.534 & 184.73 & 26.95 & 35.35 & 439.3 & 10.71 & 32.3 \\
\hline 290. & 0.0416 & 0.998 & 9.711 & 185.35 & 27.11 & 35.51 & 442.8 & 10.87 & 33.0 \\
\hline 295. & 0.0408 & 0.998 & 9.889 & 185.96 & 27.29 & 35.68 & 446.3 & 11.03 & 33.7 \\
\hline 300. & 0.0402 & 0.998 & 10.068 & 186.56 & 27.47 & 35.86 & 449.7 & 11.20 & 34.4 \\
\hline 310. & 0.0389 & 0.998 & 10.429 & 187.74 & 27.86 & 36.25 & 456.5 & 11.52 & 35.8 \\
\hline 320. & 0.0376 & 0.999 & 10.793 & 188.90 & 28.28 & 36.66 & 463.1 & 11.84 & 37.2 \\
\hline 330. & 0.0365 & 0.999 & 11.162 & 190.03 & 28.72 & 37.09 & 469.5 & 12.15 & 38.7 \\
\hline 340. & 0.0354 & 0.999 & 11.535 & 191.15 & 29.18 & 37.55 & 475.7 & 12.46 & 40.2 \\
\hline 350. & 0.0344 & 0.999 & 11.913 & 192.24 & 29.66 & 38.03 & 481.8 & 12.76 & 41.8 \\
\hline 360. & 0.0334 & 0.999 & 12.296 & 193.32 & 30.16 & 38.52 & 487.8 & 13.07 & 43.4 \\
\hline
\end{tabular}


METHANE ISOBAR AT $\mathrm{P}=0.100 \mathrm{MPa}$ (continued)

\begin{tabular}{|c|c|c|c|c|c|c|c|c|c|}
\hline $\mathrm{T}$ & $\rho$ & $\mathrm{z}$ & $\mathrm{H}$ & S & $C_{v}$ & $C_{p}$ & W & $\eta$ & $\lambda$ \\
\hline K & $\mathrm{mol} \cdot \mathrm{dm}^{-3}$ & & $\mathrm{~kJ} / \mathrm{mol}$ & $\mathrm{J} /(\mathrm{mol} \cdot \mathrm{K})$ & $\mathrm{J} /(\mathrm{mol} \cdot \mathrm{K})$ & $\mathrm{J} /(\mathrm{mol} \cdot \mathrm{K})$ & $\mathrm{m} \cdot \mathrm{s}^{-1}$ & $\mu \mathrm{Pa} \cdot \mathrm{s}$ & $\mathrm{mW} /(\mathrm{m} \cdot \mathrm{K})$ \\
\hline 370. & 0.0325 & 0.999 & 12.684 & 194.38 & 30.68 & 39.04 & 493.6 & 13.37 & 45.0 \\
\hline 380 . & 0.0317 & 0.999 & 13.077 & 195.43 & 31.21 & 39.57 & 499.4 & 13.66 & 46.6 \\
\hline 390. & 0.0309 & 0.999 & 13.475 & 196.46 & 31.75 & 40.11 & 505.0 & 13.95 & 48.3 \\
\hline 400. & 0.0301 & 1.000 & 13.879 & 197.49 & 32.31 & 40.66 & 510.5 & 14.24 & 50.0 \\
\hline 410. & 0.0293 & 1.000 & 14.288 & 198.50 & 32.87 & 41.22 & 516.0 & & 51.7 \\
\hline 420. & 0.0286 & 1.000 & 14.703 & 199.50 & 33.44 & 41.79 & 521.4 & & 53.5 \\
\hline 430. & 0.0280 & 1.000 & 15.124 & 200.49 & 34.01 & 42.36 & 526.7 & & 55.3 \\
\hline 440. & 0.0273 & 1.000 & 15.550 & 201.47 & 34.59 & 42.93 & 531.9 & & 57.1 \\
\hline 450. & 0.0267 & 1.000 & 15.983 & 202.44 & 35.17 & 43.51 & 537.1 & & 58.9 \\
\hline 460. & 0.0262 & 1.000 & 16.421 & 203.40 & 35.75 & 44.09 & 542.2 & & 60.8 \\
\hline 470. & 0.0256 & 1.000 & 16.864 & 204.36 & 36.33 & 44.67 & 547.2 & & 62.6 \\
\hline 480. & 0.0251 & 1.000 & 17.314 & 205.30 & 36.91 & 45.25 & 552.2 & & 64.5 \\
\hline 490. & 0.0245 & 1.000 & 17.769 & 206.24 & 37.48 & 45.82 & 557.1 & & 66.4 \\
\hline 500. & 0.0241 & 1.000 & 18.230 & 207.17 & 38.06 & 46.39 & 562.0 & & 68.3 \\
\hline 520. & 0.0231 & 1.000 & 19.170 & 209.02 & 39.19 & 47.53 & 571.7 & & 72.2 \\
\hline 540. & 0.0223 & 1.000 & 20.131 & 210.83 & 40.31 & 48.64 & 581.2 & & 76.0 \\
\hline 560. & 0.0215 & 1.000 & 21.115 & 212.62 & 41.40 & 49.73 & 590.5 & & 80.0 \\
\hline 580. & 0.0207 & 1.000 & 22.120 & 214.38 & 42.47 & 50.80 & 599.7 & & 83.9 \\
\hline 600. & 0.0200 & 1.000 & 23.147 & 216.12 & 43.50 & 51.83 & 608.8 & & 87.8 \\
\hline
\end{tabular}

$\overline{\text { METHANE ISOBAR AT } \mathrm{P}=0.150 \mathrm{MPa}}$

$\begin{array}{lccccccccc}\mathrm{T} & \mathrm{N} & \mathrm{Z} & \mathrm{H} & \mathrm{S} & \mathrm{C} & \mathrm{C} & \mathrm{W} & \eta & \lambda \\ \mathrm{K} & \mathrm{mol} \cdot \mathrm{dm}^{-3} & & \mathrm{~kJ} / \mathrm{mol} & \mathrm{J} /(\mathrm{mol} \cdot \mathrm{K}) & \mathrm{J} /(\mathrm{mol} \cdot \mathrm{K}) & \mathrm{J} /(\mathrm{mol} \cdot \mathrm{K}) & \mathrm{m} \cdot \mathrm{s}^{-1} & \mu \mathrm{Pa} \cdot \mathrm{s} & \mathrm{mW} /(\mathrm{m} \cdot \mathrm{K}) \\ & & & & & & & & & \\ 95 . & 27.792 & 0.007 & -5.511 & 70.26 & 33.99 & 53.74 & 1499.6 & 179.88 & 206.2 \\ 100 . & 27.368 & 0.007 & -5.240 & 73.04 & 34.08 & 54.63 & 1447.2 & 157.57 & 199.8 \\ 105 . & 26.934 & 0.006 & -4.966 & 75.72 & 33.86 & 55.27 & 1397.3 & 138.75 & 193.2 \\ 110 . & 26.489 & 0.006 & -4.688 & 78.31 & 33.49 & 55.83 & 1347.9 & 122.87 & 186.3 \\ 115 . & 26.031 & 0.006 & -4.407 & 80.80 & 33.07 & 56.40 & 1298.0 & 109.44 & 179.3 \\ 116.645 & 25.863 & 0.006 & -4.311 & 81.64 & 32.92 & 56.66 & 1279.0 & 105.20 & 176.7 \\ 116.645 & 0.1625 & 0.952 & 3.723 & 150.51 & 26.74 & 36.94 & 274.7 & 4.62 & 12.2 \\ 120 . & 0.1573 & 0.956 & 3.845 & 151.54 & 26.14 & 36.08 & 279.8 & 4.75 & 12.6 \\ 125 . & 0.1502 & 0.961 & 4.023 & 153.00 & 25.77 & 35.46 & 286.8 & 4.94 & 13.1 \\ 130 . & 0.1437 & 0.966 & 4.200 & 154.38 & 25.61 & 35.12 & 293.3 & 5.13 & 13.7 \\ & & & & & & & & & \\ 135 . & 0.1379 & 0.969 & 4.375 & 155.70 & 25.51 & 34.88 & 299.7 & 5.32 & 14.2 \\ 140 . & 0.1325 & 0.972 & 4.549 & 156.97 & 25.44 & 34.68 & 305.8 & 5.51 & 14.8 \\ 145 . & 0.1276 & 0.975 & 4.722 & 158.18 & 25.38 & 34.52 & 311.7 & 5.70 & 15.4 \\ 150 . & 0.1230 & 0.978 & 4.894 & 159.35 & 25.33 & 34.39 & 317.5 & 5.89 & 16.0 \\ 155 . & 0.1188 & 0.980 & 5.066 & 160.48 & 25.29 & 34.28 & 323.2 & 6.08 & 16.5\end{array}$


METHANE ISOBAR AT $\mathrm{P}=0.150 \mathrm{MPa}$ (continued)

\begin{tabular}{|c|c|c|c|c|c|c|c|c|c|}
\hline $\mathrm{T}$ & $\rho$ & 2 & $\mathrm{H}$ & S & $\mathrm{C}_{\mathrm{v}}$ & $C_{p}$ & W & $\eta$ & $\lambda$ \\
\hline K & $\mathrm{mol} \cdot \mathrm{dm}^{-3}$ & & $\mathrm{~kJ} / \mathrm{mo} 1$ & $\mathrm{~J} /(\mathrm{mol} \cdot \mathrm{K})$ & $\mathrm{J} /(\mathrm{mol} \cdot \mathrm{K})$ & $\mathrm{J} /(\mathrm{mol} \cdot \mathrm{K})$ & $\mathrm{m} \cdot \mathrm{s}^{-1}$ & $\mu \mathrm{Pa} \cdot \mathrm{s}$ & $\mathrm{mW} /(\mathrm{m} \cdot \mathrm{K})$ \\
\hline 160. & 0.1149 & 0.981 & 5.237 & 161.56 & 25.26 & 34.19 & 328.7 & 6.27 & 17.1 \\
\hline 165. & 0.1112 & 0.983 & 5.408 & 162.61 & 25.24 & 34.11 & 334.2 & 6.46 & 17.7 \\
\hline 170. & 0.1078 & 0.985 & 5.578 & 163.63 & 25.23 & 34.05 & 339.5 & 6.65 & 18.3 \\
\hline 175. & 0.1046 & 0.986 & 5.748 & 164.62 & 25.23 & 34.01 & 344.7 & 6.84 & 18.9 \\
\hline 180. & 0.1016 & 0.987 & 5.918 & 165.58 & 25.23 & 33.97 & 349.8 & 7.02 & 19.5 \\
\hline 185. & 0.0987 & 0.988 & 6.088 & 166.51 & 25.24 & 33.95 & 354.8 & 7.21 & 20.1 \\
\hline 190. & 0.0960 & 0.989 & 6.258 & 167.41 & 25.25 & 33.94 & 359.7 & 7.40 & 20.7 \\
\hline 195. & 0.0935 & 0.990 & 6.427 & 168.29 & 25.27 & 33.93 & 364.5 & 7.58 & 21.3 \\
\hline 200. & 0.0911 & 0.990 & 6.597 & 169.15 & 25.30 & 33.94 & 369.3 & 7.76 & 21.8 \\
\hline 205. & 0.0888 & 0.991 & 6.767 & 169.99 & 25.34 & 33.95 & 374.0 & 7.95 & 22.4 \\
\hline 210 . & 0.0866 & 0.992 & 6.936 & 170.81 & 25.38 & 33.98 & 378.5 & 8.13 & 23.0 \\
\hline 215 . & 0.0846 & 0.992 & 7.106 & 171.61 & 25.43 & 34.01 & 383.1 & 8.31 & 23.6 \\
\hline 220. & 0.0826 & 0.993 & 7.277 & 172.39 & 25.49 & 34.05 & 387.5 & 8.49 & 24.2 \\
\hline 225. & 0.0807 & 0.993 & 7.447 & 173.16 & 25.55 & 34.10 & 391.9 & 8.66 & 24.8 \\
\hline 230. & 0.0789 & 0.994 & 7.618 & 173.91 & 25.63 & 34.16 & 396.1 & 8.84 & 25.4 \\
\hline 235 . & 0.0772 & 0.994 & 7.789 & 174.64 & 25.71 & 34.23 & 400.4 & 9.02 & 26.0 \\
\hline 240 . & 0.0756 & 0.995 & 7.960 & 175.36 & 25.79 & 34.31 & 404.5 & 9.19 & 26.6 \\
\hline 245. & 0.0740 & 0.995 & 8.132 & 176.07 & 25.89 & 34.39 & 408.6 & 9.36 & 27.2 \\
\hline 250. & 0.0725 & 0.995 & 8.304 & 176.77 & 26.00 & 34.49 & 412.6 & 9.54 & 27.8 \\
\hline 255. & 0.0711 & 0.996 & 8.477 & 177.45 & 26.11 & 34.59 & 416.6 & 9.71 & 28.5 \\
\hline 260. & 0.0697 & 0.996 & 8.650 & 178.12 & 26.23 & 34.71 & 420.5 & 9.88 & 29.1 \\
\hline 265. & 0.0683 & 0.996 & 8.824 & 178.79 & 26.36 & 34.83 & 424.3 & 10.05 & 29.7 \\
\hline 270 . & 0.0671 & 0.996 & 8.998 & 179.44 & 26.50 & 34.96 & 428.1 & 10.22 & 30.4 \\
\hline 275 . & 0.0658 & 0.997 & 9.173 & 180.08 & 26.64 & 35.10 & 431.8 & 10.38 & 31.0 \\
\hline 280 . & 0.0646 & 0.997 & 9.349 & 180.71 & 26.79 & 35.24 & 435.5 & 10.55 & 31.7 \\
\hline 285. & 0.0635 & 0.997 & 9.526 & 181.34 & 26.95 & 35.40 & 439.1 & 10.72 & 32.4 \\
\hline 290. & 0.0624 & 0.997 & 9.703 & 181.96 & 27.12 & 35.56 & 442.6 & 10.88 & 33.0 \\
\hline 295. & 0.0613 & 0.997 & 9.881 & 182.57 & 27.30 & 35.73 & 446.1 & 11.04 & 33.7 \\
\hline 300 . & 0.0603 & 0.997 & 10.060 & 183.17 & 27.48 & 35.91 & 449.6 & 11.20 & 34.4 \\
\hline 310 . & 0.0583 & 0.998 & 10.421 & 184.35 & 27.87 & 36.29 & 456.4 & 11.53 & 35.8 \\
\hline 320. & 0.0565 & 0.998 & 10.786 & 185.51 & 28.28 & 36.70 & 462.9 & 11.84 & 37.3 \\
\hline 330. & 0.0548 & 0.998 & 11.155 & 186.65 & 28.72 & 37.13 & 469.4 & 12.16 & 38.8 \\
\hline 340 . & 0.0531 & 0.998 & 11.529 & 187.76 & 29.18 & 37.58 & 475.6 & 12.47 & 40.3 \\
\hline 350. & 0.0516 & 0.999 & 11.907 & 188.86 & 29.67 & 38.06 & 481.7 & 12.77 & 41.8 \\
\hline 360. & 0.0502 & 0.999 & 12.290 & 189.94 & 30.17 & 38.55 & 487.7 & 13.07 & 43.4 \\
\hline 370. & 0.0488 & 0.999 & 12.678 & 191.00 & 30.68 & 39.07 & 493.6 & 13.37 & 45.0 \\
\hline 380 . & 0.0475 & 0.999 & 13.072 & 192.05 & 31.21 & 39.59 & 499.3 & 13.67 & 46.7 \\
\hline 390. & 0.0463 & 0.999 & 13.470 & 193.08 & 31.76 & 40.13 & 505.0 & 13.96 & 48.3 \\
\hline 400. & 0.0451 & 0.999 & 13.874 & 194.11 & 32.31 & 40.68 & 510.5 & 14.25 & 50.0 \\
\hline 410. & 0.0440 & 0.999 & 14.284 & 195.12 & 32.87 & 41.24 & 516.0 & & 51.8 \\
\hline
\end{tabular}


METHANE ISOBAR AT $\mathrm{P}=0.150 \mathrm{MPa}$ (continued)

\begin{tabular}{|c|c|c|c|c|c|c|c|c|c|}
\hline $\mathrm{T}$ & $\rho$ & $\mathrm{Z}$ & $\mathrm{H}$ & $S$ & $C_{v}$ & $C_{p}$ & W & $\eta$ & $\lambda$ \\
\hline K & $\mathrm{mol} \cdot \mathrm{dm}^{-3}$ & & $\mathrm{~kJ} / \mathrm{mol}$ & $\mathrm{J} /(\mathrm{mol} \cdot \mathrm{K})$ & $\mathrm{J} /(\mathrm{mol} \cdot \mathrm{K})$ & $\mathrm{J} /(\mathrm{mol} \cdot \mathrm{K})$ & $\mathrm{m} \cdot \mathrm{s}^{-1}$ & $\mu \mathrm{Pa} \cdot \mathrm{s}$ & $\mathrm{mW} /(\mathrm{m} \cdot \mathrm{K})$ \\
\hline 420. & 0.0430 & 0.999 & 14.699 & 196.12 & 33.44 & 41.81 & 521.4 & & 53.5 \\
\hline 430. & 0.0420 & 1.000 & 15.120 & 197.11 & 34.01 & 42.38 & 526.7 & & 55.3 \\
\hline 440 . & 0.0410 & 1.000 & 15.547 & 198.09 & 34.59 & 42.95 & 531.9 & & 57.1 \\
\hline 450. & 0.0401 & 1.000 & 15.979 & 199.06 & 35.17 & 43.53 & 537.1 & & 58.9 \\
\hline 460. & 0.0392 & 1.000 & 16.417 & 200.02 & 35.75 & 44.11 & 542.2 & & 60.8 \\
\hline 470. & 0.0384 & 1.000 & 16.861 & 200.98 & 36.33 & 44.68 & 547.3 & & 62.6 \\
\hline 480 . & 0.0376 & 1.000 & 17.311 & 201.93 & 36.91 & 45.26 & 552.2 & & 64.5 \\
\hline 490. & 0.0368 & 1.000 & 17.766 & 202.87 & 37.49 & 45.84 & 557.2 & & 66.4 \\
\hline 500 . & 0.0361 & 1.000 & 18.228 & 203.80 & 38.06 & 46.41 & 562.1 & & 68.3 \\
\hline 520. & 0.0347 & 1.000 & 19.167 & 205.64 & 39.20 & 47.54 & 571.8 & & 72.2 \\
\hline 540 . & 0.0334 & 1.000 & 20.129 & 207.45 & 40.31 & 48.65 & 581.2 & & 76.1 \\
\hline 560. & 0.0322 & 1.000 & 21.113 & 209.24 & 41.40 & 49.74 & 590.6 & & 80.0 \\
\hline 580. & 0.0311 & 1.000 & 22.119 & 211.01 & 42.47 & 50.81 & 599.8 & & 83.9 \\
\hline 600 . & 0.0301 & 1.000 & 23.145 & 212.75 & 43.50 & 51.84 & 608.9 & & 87.9 \\
\hline
\end{tabular}

$\overline{\text { METHANE ISOBAR AT } \mathrm{P}=0.200 \mathrm{MPa}}$

$\begin{array}{lccccccccc}\mathrm{T} & \rho & \mathrm{Z} & \mathrm{H} & \mathrm{S} & \mathrm{C}_{\mathrm{v}} & \mathrm{C}_{\mathrm{p}} & \mathrm{W} & \eta & \lambda \\ \mathrm{K} & \mathrm{mol} \cdot \mathrm{dm}^{-3} & & \mathrm{~kJ} / \mathrm{mol} & \mathrm{J} /(\mathrm{mol} \cdot \mathrm{K}) & \mathrm{J} /(\mathrm{mol} \cdot \mathrm{K}) & \mathrm{J} /(\mathrm{mol} \cdot \mathrm{K}) & \mathrm{m} \cdot \mathrm{s}^{-1} & \mu \mathrm{Pa} \cdot \mathrm{s} & \mathrm{mW} /(\mathrm{m} \cdot \mathrm{K}) \\ & & & & & & & & & \\ \text { 95. } & 27.794 & 0.009 & -5.510 & 70.26 & 33.99 & 53.74 & 1500.0 & 179.97 & 206.2 \\ 100 . & 27.370 & 0.009 & -5.239 & 73.04 & 34.09 & 54.63 & 1447.7 & 157.66 & 199.9 \\ 105 . & 26.937 & 0.009 & -4.964 & 75.72 & 33.86 & 55.27 & 1397.8 & 138.83 & 193.2 \\ 110 . & 26.492 & 0.008 & -4.687 & 78.30 & 33.49 & 55.82 & 1348.4 & 122.95 & 186.4 \\ 115 . & 26.034 & 0.008 & -4.406 & 80.80 & 33.07 & 56.39 & 1298.5 & 109.51 & 179.4 \\ 120 . & 25.561 & 0.008 & -4.123 & 83.21 & 32.64 & 57.04 & 1247.6 & 98.10 & 172.2 \\ 120.611 & 25.489 & 0.008 & -4.085 & 83.53 & 32.59 & 57.18 & 1239.2 & 96.60 & 171.1 \\ 120.611 & 0.2118 & 0.942 & 3.821 & 149.07 & 26.65 & 37.23 & 277.7 & 4.78 & 12.8 \\ 125 . & 0.2031 & 0.948 & 3.982 & 150.38 & 26.08 & 36.30 & 284.2 & 4.95 & 13.2 \\ 130 . & 0.1940 & 0.954 & 4.162 & 151.79 & 25.82 & 35.78 & 291.1 & 5.14 & 13.8 \\ 135 . & 0.1859 & 0.959 & 4.340 & 153.14 & 25.68 & 35.45 & 297.7 & 5.33 & 14.3 \\ 140 . & 0.1784 & 0.963 & 4.516 & 154.42 & 25.59 & 35.18 & 304.0 & 5.52 & 14.9 \\ 145 . & 0.1716 & 0.967 & 4.692 & 155.65 & 25.51 & 34.97 & 310.1 & 5.71 & 15.5 \\ 150 . & 0.1653 & 0.970 & 4.866 & 156.83 & 25.45 & 34.78 & 316.0 & 5.91 & 16.0 \\ 155 . & 0.1595 & 0.973 & 5.040 & 157.97 & 25.40 & 34.63 & 321.8 & 6.10 & 16.6 \\ 160 . & 0.1542 & 0.975 & 5.212 & 159.07 & 25.35 & 34.50 & 327.5 & 6.28 & 17.2 \\ 165 . & 0.1492 & 0.977 & 5.385 & 160.13 & 25.32 & 34.39 & 333.0 & 6.47 & 17.8 \\ 170 . & 0.1445 & 0.979 & 5.556 & 161.15 & 25.30 & 34.30 & 338.4 & 6.66 & 18.4 \\ 175 . & 0.1401 & 0.981 & 5.728 & 162.15 & 25.29 & 34.23 & 343.7 & 6.85 & 19.0 \\ 180 . & 0.1360 & 0.983 & 5.899 & 163.11 & 25.28 & 34.18 & 348.9 & 7.04 & 19.6\end{array}$


METHANE ISOBAR AT $\mathrm{P}=0.200 \mathrm{MPa}$ (continued)

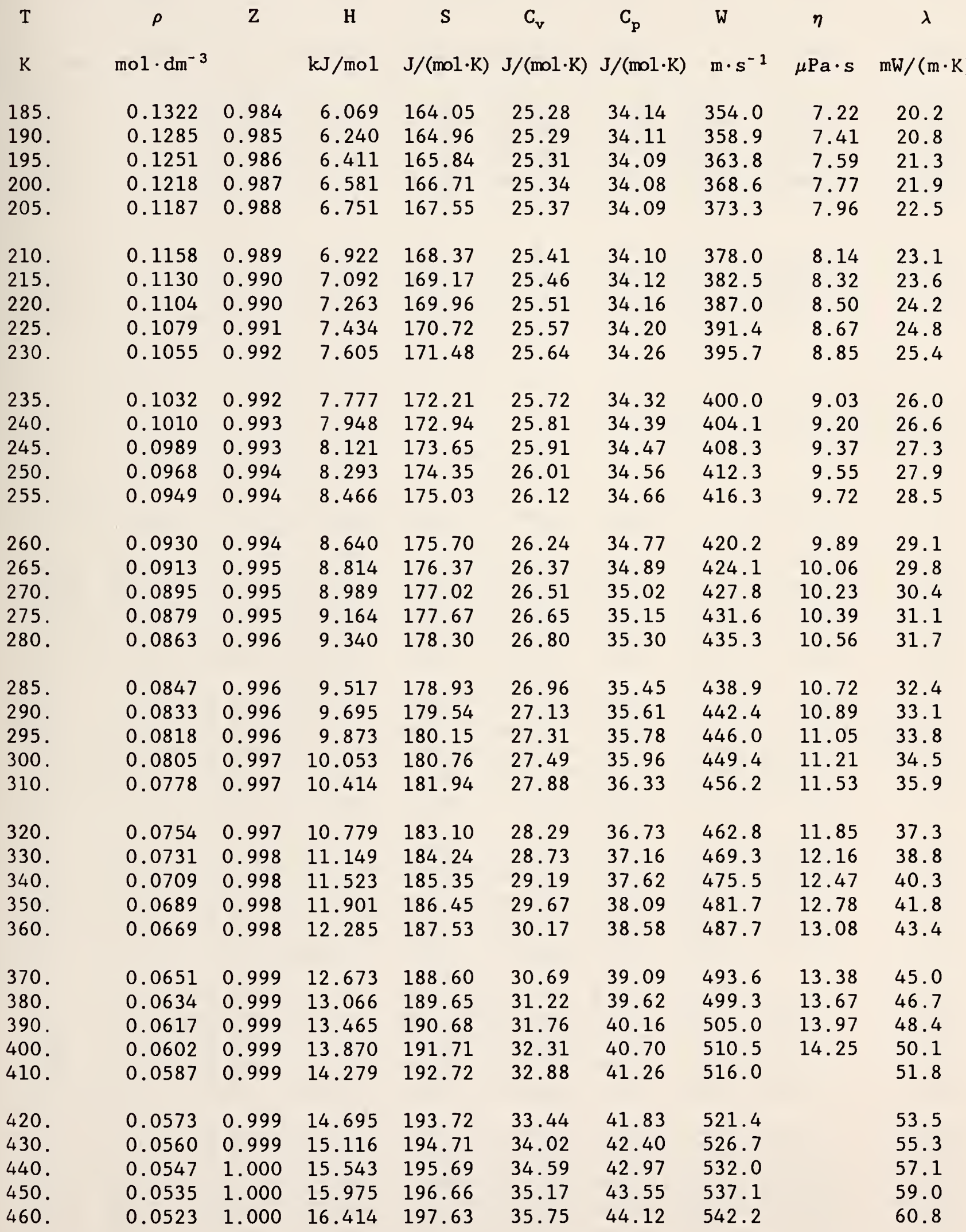


METHANE ISOBAR AT $\mathrm{P}=0.200 \mathrm{MPa}$ (continued)

\begin{tabular}{|c|c|c|c|c|c|c|c|c|c|}
\hline $\mathrm{T}$ & $\rho$ & $\mathrm{z}$ & $\mathrm{H}$ & S & $C_{v}$ & $C_{p}$ & W & $\eta$ & $\lambda$ \\
\hline K & $\mathrm{mol} \cdot \mathrm{dm}^{-3}$ & & $\mathrm{~kJ} / \mathrm{mol}$ & $\mathrm{J} /(\mathrm{mol} \cdot \mathrm{K})$ & $\mathrm{J} /(\mathrm{mol} \cdot \mathrm{K})$ & $\mathrm{J} /(\mathrm{mol} \cdot \mathrm{K})$ & $\mathrm{m} \cdot \mathrm{s}^{-1}$ & $\mu \mathrm{Pa} \cdot \mathrm{s}$ & $\mathrm{mW} /(\mathrm{m} \cdot \mathrm{K})$ \\
\hline 470. & 0.0512 & 1.000 & 16.858 & 198.58 & 36.33 & 44.70 & 547.3 & & 62.7 \\
\hline 480. & 0.0501 & 1.000 & 17.308 & 199.53 & 36.91 & 45.28 & 552.3 & & 64.5 \\
\hline 490. & 0.0491 & 1.000 & 17.763 & 200.47 & 37.49 & 45.85 & 557.3 & & 66.4 \\
\hline 500. & 0.0481 & 1.000 & 18.225 & 201.40 & 38.06 & 46.42 & 562.2 & & 68.3 \\
\hline 520. & 0.0463 & 1.000 & 19.164 & 203.24 & 39.20 & 47.55 & 571.8 & & 72.2 \\
\hline 540. & 0.0445 & 1.000 & 20.127 & 205.06 & 40.31 & 48.66 & 581.3 & & 76.1 \\
\hline 560. & 0.0429 & 1.000 & 21.111 & 206.85 & 41.40 & 49.75 & 590.7 & & 80.0 \\
\hline 580. & 0.0415 & 1.000 & 22.117 & 208.61 & 42.47 & 50.82 & 599.9 & & 83.9 \\
\hline 600. & 0.0401 & 1.000 & 23.143 & 210.35 & 43.51 & 51.85 & 609.0 & & 87.9 \\
\hline
\end{tabular}

METHANE ISOBAR AT $\mathrm{P}=0.250 \mathrm{MPa}$

\begin{tabular}{|c|c|c|c|c|c|c|c|c|c|}
\hline $\mathrm{T}$ & $\rho$ & Z & $\mathrm{H}$ & S & $C_{v}$ & $C_{p}$ & W & $\eta$ & $\lambda$ \\
\hline K & $\mathrm{mol} \cdot \mathrm{dm}^{-3}$ & & $\mathrm{~kJ} / \mathrm{mol}$ & $\mathrm{J} /(\mathrm{mol} \cdot \mathrm{K})$ & $\mathrm{J} /(\mathrm{mol} \cdot \mathrm{K})$ & $\mathrm{J} /(\mathrm{mol} \cdot \mathrm{K})$ & $\mathrm{m} \cdot \mathrm{s}^{-1}$ & $\mu \mathrm{Pa} \cdot \mathrm{s}$ & $\mathrm{mW} /(\mathrm{m} \cdot \mathrm{K})$ \\
\hline 95. & 27.796 & 0.011 & -5.509 & 70.25 & 33.99 & 53.73 & 1500.4 & 180.07 & 206.3 \\
\hline 100. & 27.373 & 0.011 & -5.238 & 73.03 & 34.09 & 54.62 & 1448.1 & 157.75 & 199.9 \\
\hline 105. & 26.940 & 0.011 & -4.963 & 75.71 & 33.86 & 55.26 & 1398.3 & 138.91 & 193.3 \\
\hline 110. & 26.495 & 0.010 & -4.685 & 78.30 & 33.50 & 55.81 & 1348.9 & 123.02 & 186.4 \\
\hline 115. & 26.037 & 0.010 & -4.405 & 80.79 & 33.07 & 56.38 & 1299.0 & 109.58 & 179.4 \\
\hline 120. & 25.564 & 0.010 & -4.121 & 83.20 & 32.65 & 57.03 & 1248.1 & 98.16 & 172.3 \\
\hline 123.886 & 25.173 & 0.010 & -3.896 & 85.05 & 32.32 & 57.66 & 1205.8 & 90.26 & 166.5 \\
\hline 123.886 & 0.2603 & 0.932 & 3.897 & 147.96 & 26.65 & 37.63 & 279.8 & 4.92 & 13.3 \\
\hline 125. & 0.2576 & 0.934 & 3.939 & 148.29 & 26.47 & 37.32 & 281.5 & 4.96 & 13.4 \\
\hline 130. & 0.2457 & 0.941 & 4.123 & 149.74 & 26.04 & 36.50 & 288.8 & 5.15 & 13.9 \\
\hline 135. & 0.2350 & 0.948 & 4.304 & 151.10 & 25.85 & 36.04 & 295.6 & 5.35 & 14.5 \\
\hline 140. & 0.2253 & 0.953 & 4.483 & 152.41 & 25.74 & 35.70 & 302.1 & 5.54 & 15.0 \\
\hline 145. & 0.2164 & 0.958 & 4.661 & 153.66 & 25.64 & 35.42 & 308.4 & 5.73 & 15.6 \\
\hline 150. & 0.2083 & 0.962 & 4.838 & 154.85 & 25.56 & 35.19 & 314.5 & 5.92 & 16.1 \\
\hline 155. & 0.2009 & 0.966 & 5.013 & 156.00 & 25.50 & 34.99 & 320.4 & 6.11 & 16.7 \\
\hline 160. & 0.1940 & 0.969 & 5.188 & 157.11 & 25.44 & 34.82 & 326.2 & 6.30 & 17.3 \\
\hline 165. & 0.1876 & 0.972 & 5.361 & 158.18 & 25.40 & 34.68 & 331.8 & 6.49 & 17.9 \\
\hline 170 . & 0.1816 & 0.974 & 5.534 & 159.21 & 25.37 & 34.56 & 337.3 & 6.67 & 18.4 \\
\hline 175. & 0.1760 & 0.976 & 5.707 & 160.21 & 25.35 & 34.46 & 342.7 & 6.86 & 19.0 \\
\hline 180. & 0.1708 & 0.978 & 5.879 & 161.18 & 25.33 & 34.39 & 348.0 & 7.05 & 19.6 \\
\hline 185. & 0.1659 & 0.980 & 6.051 & 162.13 & 25.33 & 34.33 & 353.1 & 7.23 & 20.2 \\
\hline 190. & 0.1613 & 0.981 & 6.222 & 163.04 & 25.33 & 34.28 & 358.2 & 7.42 & 20.8 \\
\hline 195. & 0.1569 & 0.983 & 6.394 & 163.93 & 25.35 & 34.25 & 363.1 & 7.60 & 21.4 \\
\hline 200 . & 0.1528 & 0.984 & 6.565 & 164.80 & 25.37 & 34.23 & 368.0 & 7.78 & 22.0 \\
\hline 205. & 0.1489 & 0.985 & 6.736 & 165.64 & 25.40 & 34.22 & 372.7 & 7.97 & 22.5 \\
\hline
\end{tabular}


METHANE ISOBAR AT $\mathrm{P}=0.250 \mathrm{MPa}$ (continued)

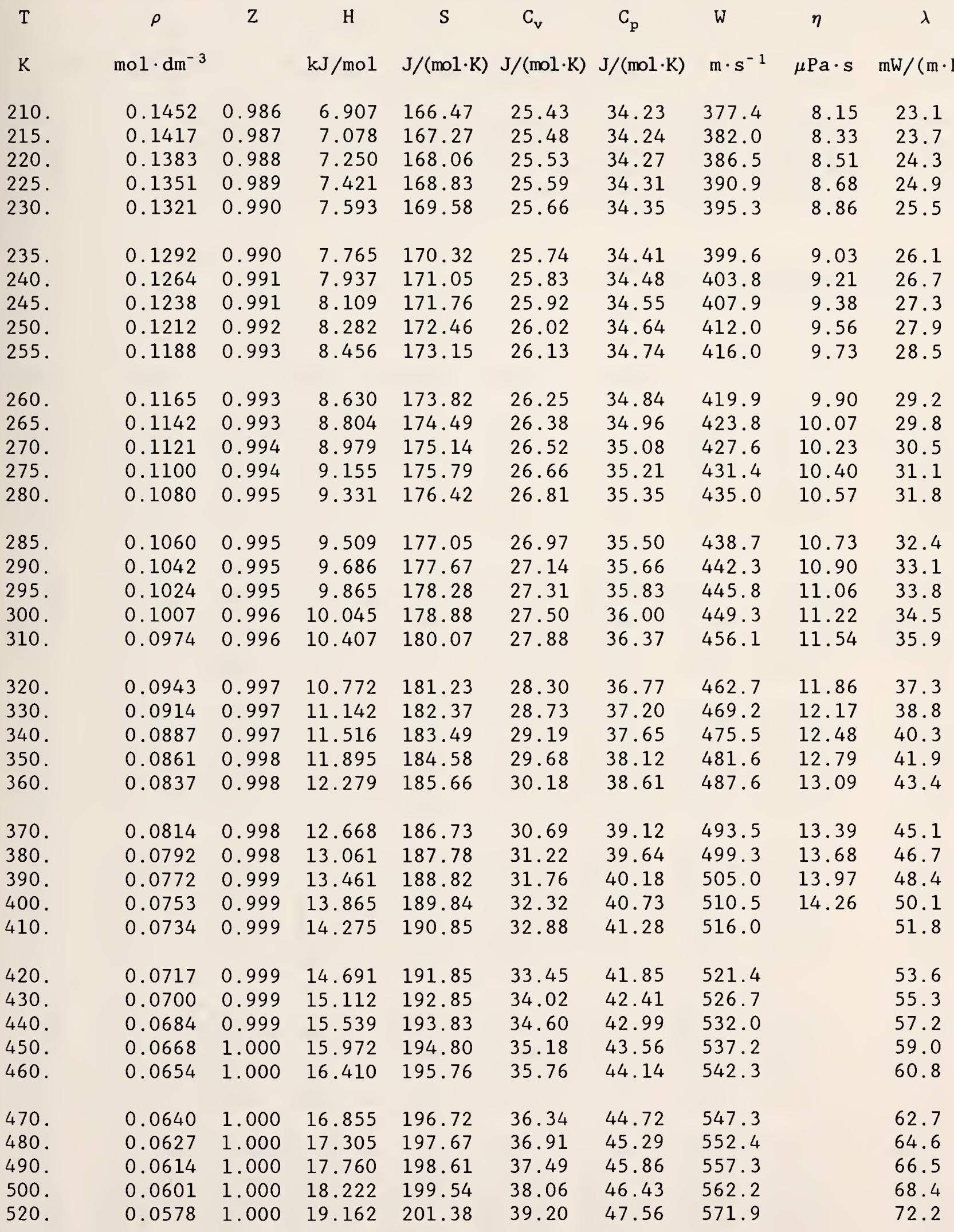


METHANE ISOBAR AT $\mathrm{P}=0.250 \mathrm{MPa}$ (continued)

$\begin{array}{cccccccccc}\mathrm{T} & \rho & \mathrm{Z} & \mathrm{H} & \mathrm{S} & \mathrm{C}_{\mathrm{v}} & \mathrm{C}_{\mathrm{p}} & \mathrm{W} & \eta & \lambda \\ \mathrm{K} & \mathrm{mol} \cdot \mathrm{dm}^{-3} & & \mathrm{~kJ} / \mathrm{mol} & \mathrm{J} /(\mathrm{mol} \cdot \mathrm{K}) & \mathrm{J} /(\mathrm{mol} \cdot \mathrm{K}) & \mathrm{J} /(\mathrm{mol} \cdot \mathrm{K}) & \mathrm{m} \cdot \mathrm{s}^{-1} & \mu \mathrm{Pa} \cdot \mathrm{s} & \mathrm{mW} /(\mathrm{m} \cdot \mathrm{K}) \\ & & & & & & & & & \\ 540 . & 0.0557 & 1.000 & 20.124 & 203.20 & 40.31 & 48.68 & 581.4 & 76.1 \\ 560 . & 0.0537 & 1.000 & 21.109 & 204.99 & 41.41 & 49.76 & 590.8 & 80.0 \\ 580 . & 0.0518 & 1.000 & 22.115 & 206.75 & 42.47 & 50.82 & 600.0 & 83.9 \\ 600 . & 0.0501 & 1.000 & 23.142 & 208.49 & 43.51 & 51.86 & 609.0 & 87.9\end{array}$

$\overline{\text { METHANE ISOBAR AT } P=0.300 \mathrm{MPa}}$

\begin{tabular}{|c|c|c|c|c|c|c|c|c|c|}
\hline $\mathrm{T}$ & $\rho$ & $z$ & $\mathrm{H}$ & S & $\mathrm{C}_{\mathrm{v}}$ & $C_{p}$ & W & $\eta$ & $\lambda$ \\
\hline K & $\mathrm{mol} \cdot \mathrm{dm}^{-3}$ & & $\mathrm{~kJ} / \mathrm{mol}$ & $\mathrm{J} /(\mathrm{mol} \cdot \mathrm{K})$ & $\mathrm{J} /(\mathrm{mol} \cdot \mathrm{K})$ & $\mathrm{J} /(\mathrm{mol} \cdot \mathrm{K})$ & $\mathrm{m} \cdot \mathrm{s}^{-1}$ & $\mu \mathrm{Pa} \cdot \mathrm{s}$ & $\mathrm{mW} /(\mathrm{m} \cdot \mathrm{K})$ \\
\hline 95. & 27.798 & 0.014 & -5.508 & 70.25 & 34.00 & 53.73 & 1500.8 & 180.17 & 206.3 \\
\hline 100. & 27.375 & 0.013 & -5.237 & 73.03 & 34.09 & 54.61 & 1448.5 & 157.83 & 200.0 \\
\hline 105. & 26.942 & 0.013 & -4.962 & 75.71 & 33.87 & 55.25 & 1398.7 & 138.99 & 193.3 \\
\hline 110. & 26.498 & 0.012 & -4.684 & 78.29 & 33.50 & 55.80 & 1349.4 & 123.10 & 186.5 \\
\hline 115. & 26.040 & 0.012 & -4.404 & 80.78 & 33.08 & 56.37 & 1299.6 & 109.65 & 179.5 \\
\hline 120. & 25.568 & 0.012 & -4.120 & 83.19 & 32.65 & 57.01 & 1248.7 & 98.23 & 172.3 \\
\hline 125. & 25.077 & 0.012 & -3.833 & 85.54 & 32.24 & 57.78 & 1196.4 & 88.44 & 165.1 \\
\hline 126.702 & 24.896 & 0.011 & -3.732 & 86.34 & 32.10 & 58.12 & 1176.8 & 85.29 & 162.5 \\
\hline 126.702 & 0.3084 & 0.923 & 3.960 & 147.05 & 26.70 & 38.07 & 281.4 & 5.04 & 13.8 \\
\hline 130. & 0.2988 & 0.929 & 4.083 & 148.01 & 26.30 & 37.31 & 286.4 & 5.17 & 14.1 \\
\hline 135. & 0.2853 & 0.937 & 4.268 & 149.41 & 26.03 & 36.67 & 293.5 & 5.36 & 14.6 \\
\hline 140. & 0.2732 & 0.943 & 4.450 & 150.73 & 25.88 & 36.24 & 300.3 & 5.55 & 15.1 \\
\hline 145. & 0.2621 & 0.949 & 4.630 & 152.00 & 25.77 & 35.90 & 306.7 & 5.74 & 15.7 \\
\hline 150. & 0.2521 & 0.954 & 4.809 & 153.21 & 25.68 & 35.61 & 313.0 & 5.93 & 16.2 \\
\hline 155. & 0.2428 & 0.959 & 4.986 & 154.37 & 25.60 & 35.36 & 319.0 & 6.12 & 16.8 \\
\hline 160. & 0.2343 & 0.962 & 5.163 & 155.49 & 25.53 & 35.15 & 324.9 & 6.31 & 17.4 \\
\hline 165. & 0.2264 & 0.966 & 5.338 & 156.57 & 25.48 & 34.97 & 330.7 & 6.50 & 17.9 \\
\hline 170. & 0.2191 & 0.969 & 5.512 & 157.61 & 25.44 & 34.82 & 336.3 & 6.69 & 18.5 \\
\hline 175. & 0.2123 & 0.971 & 5.686 & 158.62 & 25.41 & 34.70 & 341.7 & 6.87 & 19.1 \\
\hline 180. & 0.2059 & 0.974 & 5.859 & 159.60 & 25.39 & 34.60 & 347.1 & 7.06 & 19.7 \\
\hline 185. & 0.1999 & 0.976 & 6.032 & 160.54 & 25.38 & 34.52 & 352.3 & 7.24 & 20.3 \\
\hline 190. & 0.1943 & 0.978 & 6.205 & 161.46 & 25.38 & 34.46 & 357.4 & 7.43 & 20.9 \\
\hline 195. & 0.1890 & 0.979 & 6.377 & 162.36 & 25.39 & 34.41 & 362.4 & 7.61 & 21.4 \\
\hline 200 . & 0.1839 & 0.981 & 6.549 & 163.23 & 25.40 & 34.38 & 367.3 & 7.79 & 22.0 \\
\hline 205. & 0.1792 & 0.982 & 6.721 & 164.08 & 25.43 & 34.36 & 372.1 & 7.98 & 22.6 \\
\hline 210 . & 0.1747 & 0.983 & 6.892 & 164.90 & 25.46 & 34.35 & 376.8 & 8.16 & 23.2 \\
\hline 215 . & 0.1704 & 0.985 & 7.064 & 165.71 & 25.51 & 34.36 & 381.5 & 8.34 & 23.8 \\
\hline 220 . & 0.1664 & 0.986 & 7.236 & 166.50 & 25.56 & 34.38 & 386.0 & 8.52 & 24.3 \\
\hline 225. & 0.1625 & 0.987 & 7.408 & 167.28 & 25.62 & 34.41 & 390.5 & 8.69 & 24.9 \\
\hline 230. & 0.1589 & 0.988 & 7.580 & 168.03 & 25.68 & 34.45 & 394.8 & 8.87 & 25.5 \\
\hline
\end{tabular}


METHANE ISOBAR AT $\mathrm{P}=0.300 \mathrm{MPa}$ (continued)

\begin{tabular}{|c|c|c|c|c|c|c|c|c|c|}
\hline $\mathrm{T}$ & $\rho$ & 2 & $\mathrm{H}$ & $S$ & $\mathrm{C}_{\mathrm{v}}$ & $C_{p}$ & $\mathrm{~W}$ & $\eta$ & $\lambda$ \\
\hline K & $\mathrm{mol} \cdot \mathrm{dm}^{-3}$ & & $\mathrm{~kJ} / \mathrm{mol}$ & $\mathrm{J} /(\mathrm{mol} \cdot \mathrm{K})$ & $\mathrm{J} /(\mathrm{mol} \cdot \mathrm{K})$ & $\mathrm{J} /(\mathrm{mol} \cdot \mathrm{K})$ & $m \cdot s^{-1}$ & $\mu \mathrm{Pa} \cdot \mathrm{s}$ & $\mathrm{mW} /(\mathrm{m} \cdot \mathrm{l}$ \\
\hline 235. & 0.1554 & 0.988 & 7.753 & 168.77 & 25.76 & 34.50 & 399.2 & 9.04 & 26.1 \\
\hline 240 . & 0.1520 & 0.989 & 7.925 & 169.50 & 25.84 & 34.56 & 403.4 & 9.22 & 26.7 \\
\hline 245 . & 0.1488 & 0.990 & 8.098 & 170.21 & 25.94 & 34.63 & 407.6 & 9.39 & 27.3 \\
\hline 250 . & 0.1457 & 0.990 & 8.272 & 170.91 & 26.04 & 34.72 & 411.6 & 9.56 & 28.0 \\
\hline 255 . & 0.1428 & 0.991 & 8.445 & 171.60 & 26.15 & 34.81 & 415.7 & 9.74 & 28.6 \\
\hline 60. & 0.1400 & 0.992 & 8.620 & 172.28 & 26.27 & 34.91 & 419.6 & 9.91 & 29.2 \\
\hline 265. & 0.1372 & 0.992 & 8.794 & 172.95 & 26.39 & 35.02 & 423.5 & 10.07 & 29.9 \\
\hline 270 & 0.1346 & 0.993 & 8.970 & 173.60 & 26.53 & 35.14 & 427.4 & 10.24 & 30.5 \\
\hline 275 . & 0.1321 & 0.993 & 9.146 & 174.25 & 26.67 & 35.27 & 431.1 & 10.41 & 31.1 \\
\hline 280 & 0.1297 & 0.993 & 9.323 & 174.88 & 26.82 & 35.41 & 434.8 & 10.58 & 31.8 \\
\hline 285. & 0.1274 & 0.994 & 9.500 & 175.51 & 26.98 & 35.56 & 438.5 & 10.74 & 32.5 \\
\hline 290. & 0.1251 & 0.994 & 9.678 & 176.13 & 27.15 & 35.71 & 442.1 & 10.90 & 33.1 \\
\hline 295. & 0.1230 & 0.995 & 9.857 & 176.74 & 27.32 & 35.88 & 445.6 & 11.07 & 33.8 \\
\hline 300 . & 0.1209 & 0.995 & 10.037 & 177.35 & 27.51 & 36.05 & 449.1 & 11.23 & 34.5 \\
\hline 310 . & 0.1169 & 0.995 & 10.399 & 178.54 & 27.89 & 36.42 & 456.0 & 11.55 & 35.9 \\
\hline 20. & 0.1132 & 0.996 & 10.765 & 179.70 & 28.30 & 36.81 & 462.6 & 11.87 & 37.4 \\
\hline 330. & 0.1097 & 0.996 & 11.136 & 180.84 & 28.74 & 37.24 & 469.1 & 12.18 & 38.8 \\
\hline 340 . & 0.1065 & 0.997 & 11.510 & 181.96 & 29.20 & 37.68 & 475.4 & 12.49 & 40.4 \\
\hline 350 . & 0.1034 & 0.997 & 11.889 & 183.05 & 29.68 & 38.15 & 481.6 & 12.79 & 41.9 \\
\hline 360 . & 0.1005 & 0.998 & 12.273 & 184.14 & 30.18 & 38.64 & 487.6 & 13.09 & 43.5 \\
\hline 370. & 0.0 & 0.998 & 12.6 & 185.20 & & & & & 45.1 \\
\hline 380. & 0.0951 & 0.998 & 13.056 & 186.25 & 31.23 & 39.67 & 499.3 & 13.69 & 46.7 \\
\hline 390. & 0.0927 & 0.998 & 13.456 & 187.29 & 31.77 & 40.20 & 505.0 & 13.98 & 48.4 \\
\hline 400 & 0.0903 & 0.999 & 13.860 & 188.31 & 32.32 & 40.75 & 510.5 & 14.27 & 50.1 \\
\hline 410. & 0.0881 & 0.999 & 14.271 & 189.33 & 32.88 & 41.30 & 516.0 & & 51.8 \\
\hline 420. & 0.0860 & 0.999 & 14.687 & 190.33 & 33.45 & 41 & 521.4 & & 53.6 \\
\hline 430. & 0.0840 & 0.999 & 15.108 & 191.32 & 34.02 & 42.43 & 526.8 & & 55.4 \\
\hline 440. & 0.0821 & 0.999 & 15.535 & 192.30 & 34.60 & 43.01 & 532.0 & & 57.2 \\
\hline 450 & 0.0802 & 0.999 & 15.968 & 193.28 & 35.18 & 43.58 & 537.2 & & 59.0 \\
\hline 460. & 0.0785 & 1.000 & 16.407 & 194.24 & 35.76 & 44.16 & 542.3 & & 60.8 \\
\hline 470. & 0.0768 & 1.000 & 16.851 & 195.20 & 36.34 & 44.73 & 547.4 & & 62.7 \\
\hline 480. & 0.0752 & 1.000 & 17.301 & 196.14 & 36.92 & 45.31 & 552.4 & & 64.6 \\
\hline 490. & 0.0736 & 1.000 & 17.757 & 197.08 & 37.49 & 45.88 & 557.4 & & 66.5 \\
\hline 500 & 0.0722 & 1.000 & 18.219 & 198.02 & 38.07 & 46.45 & 562.3 & & 68.4 \\
\hline 520. & 0.0694 & 1.000 & 19.159 & 199.86 & 39.20 & 47.58 & 572.0 & & 72.2 \\
\hline 540. & 0.0668 & 1.000 & 20.122 & 201.68 & 40.32 & 48.69 & 581.5 & & 76.1 \\
\hline 560. & 0.0644 & 1.000 & 21.107 & 203.47 & 41.41 & 49.77 & 590.8 & & 80.0 \\
\hline 580. & 0.0622 & 1.000 & 22.113 & 205.23 & 42.47 & 50.83 & 600.1 & & 84.0 \\
\hline 600. & 0.0601 & 1.000 & 23.140 & 206.97 & 43.51 & 51.87 & 609.1 & & 87.9 \\
\hline
\end{tabular}


METHANE ISOBAR AT $\mathrm{P}=0.350 \mathrm{MPa}$

\begin{tabular}{|c|c|c|c|c|c|c|c|c|c|}
\hline $\mathrm{T}$ & $\rho$ & $\mathrm{Z}$ & H & $S$ & $\mathrm{C}_{\mathrm{v}}$ & $C_{p}$ & W & $\eta$ & $\lambda$ \\
\hline K & $\mathrm{mol} \cdot \mathrm{dm}^{-3}$ & & $\mathrm{~kJ} / \mathrm{mol}$ & $\mathrm{J} /(\mathrm{mol} \cdot \mathrm{K})$ & $\mathrm{J} /(\mathrm{mol} \cdot \mathrm{K})$ & $\mathrm{J} /(\mathrm{mol} \cdot \mathrm{K})$ & $m \cdot s^{-1}$ & $\mu \mathrm{Pa} \cdot \mathrm{s}$ & $\mathrm{mW} /(\mathrm{m} \cdot \mathrm{K})$ \\
\hline 95. & 27.800 & 0.016 & -5.506 & 70.24 & 34.00 & 53.72 & 1501.2 & 180.27 & 206.4 \\
\hline 100. & 27.377 & 0.015 & -5.235 & 73.02 & 34.09 & 54.61 & 1449.0 & 157.92 & 200.0 \\
\hline 105. & 26.945 & 0.015 & -4.961 & 75.70 & 33.87 & 55.24 & 1399.2 & 139.07 & 193.4 \\
\hline 110. & 26.501 & 0.014 & -4.683 & 78.28 & 33.50 & 55.80 & 1349.9 & 123.17 & 186.5 \\
\hline 115. & 26.044 & 0.014 & -4.403 & 80.78 & 33.08 & 56.36 & 1300.1 & 109.72 & 179.5 \\
\hline 120. & 25.571 & 0.014 & -4.119 & 83.19 & 32.65 & 57.00 & 1249.3 & 98.29 & 172.4 \\
\hline 125. & 25.081 & 0.013 & -3.832 & 85.53 & 32.24 & 57.77 & 1197.1 & 88.51 & 165.2 \\
\hline 129.188 & 24.646 & 0.013 & -3.587 & 87.46 & 31.92 & 58.57 & 1150.7 & 81.23 & 159.0 \\
\hline 129.188 & 0.3562 & 0.915 & 4.011 & 146.28 & 26.78 & 38.56 & 282.7 & 5.15 & 14.2 \\
\hline 130. & 0.3535 & 0.916 & 4.042 & 146.51 & 26.65 & 38.32 & 283.9 & 5.18 & 14.3 \\
\hline 135. & 0.3369 & 0.925 & 4.231 & 147.94 & 26.22 & 37.35 & 291.4 & 5.37 & 14.7 \\
\hline 140. & 0.3221 & 0.933 & 4.416 & 149.28 & 26.03 & 36.80 & 298.3 & 5.56 & 15.2 \\
\hline 145. & 0.3087 & 0.940 & 4.599 & 150.57 & 25.90 & 36.39 & 305.0 & 5.75 & 15.8 \\
\hline 150. & 0.2966 & 0.946 & 4.780 & 151.80 & 25.80 & 36.04 & 311.4 & 5.94 & 16.3 \\
\hline 155. & 0.2855 & 0.951 & 4.959 & 152.97 & 25.71 & 35.74 & 317.6 & 6.13 & 16.9 \\
\hline 160. & 0.2752 & 0.956 & 5.137 & 154.10 & 25.63 & 35.49 & 323.6 & 6.32 & 17.4 \\
\hline 165. & 0.2658 & 0.960 & 5.314 & 155.19 & 25.56 & 35.27 & 329.5 & 6.51 & 18.0 \\
\hline 170. & 0.2571 & 0.963 & 5.490 & 156.24 & 25.51 & 35.09 & 335.2 & 6.70 & 18.6 \\
\hline 175. & 0.2489 & 0.966 & 5.665 & 157.26 & 25.47 & 34.94 & 340.7 & 6.88 & 19.2 \\
\hline 180. & 0.2413 & 0.969 & 5.840 & 158.24 & 25.44 & 34.82 & 346.1 & 7.07 & 19.8 \\
\hline 185. & 0.2342 & 0.972 & 6.013 & 159.19 & 25.43 & 34.72 & 351.4 & 7.26 & 20.3 \\
\hline 190. & 0.2275 & 0.974 & 6.187 & 160.12 & 25.42 & 34.64 & 356.6 & 7.44 & 21.0 \\
\hline 195. & 0.2212 & 0.976 & 6.360 & 161.02 & 25.42 & 34.58 & 361.7 & 7.62 & 21.5 \\
\hline 200 . & 0.2153 & 0.978 & 6.533 & 161.89 & 25.44 & 34.53 & 366.6 & 7.81 & 22.1 \\
\hline 205. & 0.2097 & 0.979 & 6.705 & 162.74 & 25.46 & 34.50 & 371.5 & 7.99 & 22.6 \\
\hline 210 . & 0.2044 & 0.981 & 6.878 & 163.57 & 25.49 & 34.48 & 376.2 & 8.17 & 23.2 \\
\hline 215 . & 0.1994 & 0.982 & 7.050 & 164.39 & 25.53 & 34.48 & 380.9 & 8.35 & 23.8 \\
\hline 220 . & 0.1946 & 0.983 & 7.222 & 165.18 & 25.58 & 34.49 & 385.5 & 8.53 & 24.4 \\
\hline 225. & 0.1901 & 0.984 & 7.395 & 165.95 & 25.64 & 34.51 & 390.0 & 8.70 & 25.0 \\
\hline 230 . & 0.1857 & 0.985 & 7.568 & 166.71 & 25.70 & 34.55 & 394.4 & 8.88 & 25.6 \\
\hline 235. & 0.1816 & 0.986 & 7.740 & 167.46 & 25.78 & 34.59 & 398.8 & 9.05 & 26.2 \\
\hline 240 . & 0.1777 & 0.987 & 7.914 & 168.19 & 25.86 & 34.65 & 403.0 & 9.23 & 26.8 \\
\hline 245 . & 0.1739 & 0.988 & 8.087 & 168.90 & 25.95 & 34.71 & 407.2 & 9.40 & 27.4 \\
\hline 250 . & 0.1703 & 0.989 & 8.261 & 169.60 & 26.05 & 34.79 & 411.3 & 9.57 & 28.0 \\
\hline 255. & 0.1668 & 0.990 & 8.435 & 170.29 & 26.16 & 34.88 & 415.4 & 9.74 & 28.6 \\
\hline 260 . & 0.1635 & 0.990 & 8.610 & 170.97 & 26.28 & 34.98 & 419.3 & 9.91 & 29.3 \\
\hline 265. & 0.1603 & 0.991 & 8.785 & 171.64 & 26.40 & 35.09 & 423.3 & 10.08 & 29.9 \\
\hline 270 & 0.1573 & 0.991 & 8.960 & 172.29 & 26.54 & 35.20 & 427.1 & 10.25 & 30.5 \\
\hline 275 . & 0.1543 & 0.992 & 9.137 & 172.94 & 26.68 & 35.33 & 430.9 & 10.42 & 31.2 \\
\hline 280 . & 0.1515 & 0.992 & 9.314 & 173.58 & 26.83 & 35.47 & 434.6 & 10.58 & 31.8 \\
\hline
\end{tabular}


METHANE ISOBAR AT $\mathrm{P}=0.350 \mathrm{MPa}$ (continued)

\begin{tabular}{|c|c|c|c|c|c|c|c|c|c|}
\hline $\mathrm{T}$ & $\rho$ & $\mathrm{z}$ & H & S & $\mathrm{C}_{\mathrm{v}}$ & $C_{p}$ & W & $\eta$ & $\lambda$ \\
\hline K & $\mathrm{mol} \cdot \mathrm{dm}^{-3}$ & & $\mathrm{~kJ} / \mathrm{mo} 1$ & $\mathrm{~J} /(\mathrm{mol} \cdot \mathrm{K})$ & $\mathrm{J} /(\mathrm{mol} \cdot \mathrm{K})$ & $\mathrm{J} /(\mathrm{mol} \cdot \mathrm{K})$ & $m \cdot s^{-1}$ & $\mu \mathrm{Pa} \cdot \mathrm{s}$ & $\mathrm{mW} /(\mathrm{m} \cdot \mathrm{K})$ \\
\hline 285. & 0.1488 & 0.993 & 9.491 & 174.21 & 26.99 & 35.61 & 438.3 & 10.75 & 32.5 \\
\hline 290. & 0.1461 & 0.993 & 9.670 & 174.83 & 27.16 & 35.76 & 441.9 & 10.91 & 33.2 \\
\hline 295. & 0.1436 & 0.994 & 9.849 & 175.44 & 27.33 & 35.93 & 445.5 & 11.07 & 33.9 \\
\hline 300 . & 0.1412 & 0.994 & 10.029 & 176.05 & 27.51 & 36.10 & 449.0 & 11.24 & 34.6 \\
\hline 310 . & 0.1365 & 0.995 & 10.392 & 177.24 & 27.90 & 36.46 & 455.8 & 11.56 & 36.0 \\
\hline 320. & 0.1322 & 0.995 & 10.758 & 178.40 & 28.31 & 36.85 & 462.5 & 11.87 & 37.4 \\
\hline 330. & 0.1281 & 0.996 & 11.129 & 179.54 & 28.75 & 37.27 & 469.0 & 12.19 & 38.9 \\
\hline 340. & 0.1243 & 0.996 & 11.504 & 180.66 & 29.21 & 37.72 & 475.3 & 12.49 & 40.4 \\
\hline 350. & 0.1207 & 0.997 & 11.883 & 181.76 & 29.69 & 38.18 & 481.5 & 12.80 & 41.9 \\
\hline 360. & 0.1173 & 0.997 & 12.268 & 182.84 & 30.18 & 38.67 & 487.5 & 13.10 & 43.5 \\
\hline 370 . & 0.1141 & 0.998 & 12.657 & 183.91 & 30.70 & 39.18 & 493.5 & 13.40 & 45.1 \\
\hline 380. & 0.1110 & 0.998 & 13.051 & 184.96 & 31.23 & 39.70 & 499.3 & 13.69 & 46.8 \\
\hline 390. & 0.1081 & 0.998 & 13.451 & 186.00 & 31.77 & 40.23 & 504.9 & 13.98 & 48.4 \\
\hline 400. & 0.1054 & 0.998 & 13.856 & 187.02 & 32.32 & 40.77 & 510.5 & 14.27 & 50.1 \\
\hline 410. & 0.1028 & 0.999 & 14.266 & 188.04 & 32.89 & 41.33 & 516.0 & & 51.9 \\
\hline 420. & 0.1003 & 0.999 & 14.682 & 189.04 & 33.45 & 41.89 & 521.4 & & 53.6 \\
\hline 430. & 0.0980 & 0.999 & 15.104 & 190.03 & 34.03 & 42.45 & 526.8 & & 55.4 \\
\hline 440. & 0.0957 & 0.999 & 15.532 & 191.01 & 34.60 & 43.02 & 532.0 & & 57.2 \\
\hline 450. & 0.0936 & 0.999 & 15.965 & 191.99 & 35.18 & 43.60 & 537.2 & & 59.0 \\
\hline 460. & 0.0916 & 0.999 & 16.403 & 192.95 & 35.76 & 44.17 & 542.4 & & 60.9 \\
\hline 470. & 0.0896 & 1.000 & 16.848 & 193.91 & 36.34 & 44.75 & 547.4 & & 62.7 \\
\hline 480. & 0.0877 & 1.000 & 17.298 & 194.86 & 36.92 & 45.32 & 552.5 & & 64.6 \\
\hline 490. & 0.0859 & 1.000 & 17.754 & 195.80 & 37.50 & 45.89 & 557.4 & & 66.5 \\
\hline 500. & 0.0842 & 1.000 & 18.216 & 196.73 & 38.07 & 46.46 & 562.3 & & 68.4 \\
\hline 520. & 0.0809 & 1.000 & 19.157 & 198.57 & 39.20 & 47.59 & 572.0 & & 72.2 \\
\hline 540. & 0.0779 & 1.000 & 20.120 & 200.39 & 40.32 & 48.70 & 581.5 & & 76.1 \\
\hline 560. & 0.0751 & 1.000 & 21.105 & 202.18 & 41.41 & 49.78 & 590.9 & & 80.0 \\
\hline 580. & 0.0725 & 1.000 & 22.111 & 203.95 & 42.47 & 50.84 & 600.1 & & 84.0 \\
\hline 600 . & 0.0701 & 1.001 & 23.138 & 205.69 & 43.51 & 51.88 & 609.2 & & 87.9 \\
\hline
\end{tabular}

$\overline{\text { METHANE ISOBAR AT } \mathrm{P}=0.400 \mathrm{MPa}}$

$\begin{array}{cccccccccc}\mathrm{T} & \rho & \mathrm{Z} & \mathrm{H} & \mathrm{S} & \mathrm{C}_{\mathrm{v}} & \mathrm{C}_{\mathrm{p}} & \mathrm{W} & \eta & \lambda \\ \mathrm{K} & \mathrm{mol} \cdot \mathrm{dm}^{-3} & & \mathrm{~kJ} / \mathrm{mol} & \mathrm{J} /(\mathrm{mol} \cdot \mathrm{K}) & \mathrm{J} /(\mathrm{mol} \cdot \mathrm{K}) & \mathrm{J} /(\mathrm{mol} \cdot \mathrm{K}) & \mathrm{m} \cdot \mathrm{s}^{-1} & \mu \mathrm{Pa} \cdot \mathrm{s} & \mathrm{mW} /(\mathrm{m} \cdot \mathrm{K}) \\ & & & & & & & & & \\ \text { 95. } & 27.803 & 0.018 & -5.505 & 70.24 & 34.00 & 53.72 & 1501.6 & 180.36 & 206.4 \\ 100 . & 27.380 & 0.018 & -5.234 & 73.01 & 34.09 & 54.60 & 1449.4 & 158.01 & 200.1 \\ 105 . & 26.947 & 0.017 & -4.959 & 75.69 & 33.87 & 55.24 & 1399.6 & 139.15 & 193.4 \\ 110 . & 26.504 & 0.017 & -4.682 & 78.28 & 33.50 & 55.79 & 1350.4 & 123.25 & 186.6 \\ 115 . & 26.047 & 0.016 & -4.402 & 80.77 & 33.08 & 56.35 & 1300.6 & 109.79 & 179.6\end{array}$


METHANE ISOBAR AT $\mathrm{P}=0.400 \mathrm{MPa}$ (continued)

\begin{tabular}{|c|c|c|c|c|c|c|c|c|c|}
\hline $\mathrm{T}$ & $\rho$ & $\mathrm{Z}$ & $\mathrm{H}$ & $S$ & $C_{v}$ & $C_{p}$ & W & $\eta$ & $\lambda$ \\
\hline $\mathrm{K}$ & $\mathrm{mol} \cdot \mathrm{dm}^{-3}$ & & $\mathrm{~kJ} / \mathrm{mol}$ & $\mathrm{J} /(\mathrm{mol} \cdot \mathrm{K})$ & $\mathrm{J} /(\mathrm{mol} \cdot \mathrm{K})$ & $\mathrm{J} /(\mathrm{mol} \cdot \mathrm{K})$ & $\mathrm{m} \cdot \mathrm{s}^{-1}$ & $\mu \mathrm{Pa} \cdot \mathrm{s}$ & $\mathrm{mW} /(\mathrm{m}$. \\
\hline 120 . & 25.575 & 0.016 & -4.118 & 83.18 & 32.65 & 56.99 & 1249.9 & 98.36 & 172.5 \\
\hline 125. & 25.085 & 0.015 & -3.831 & 85.52 & 32.24 & 57.75 & 1197.7 & 88.57 & 165.3 \\
\hline 130. & 24.574 & 0.015 & -3.540 & 87.80 & 31.86 & 58.68 & 1143.9 & 80.11 & 158.0 \\
\hline 131.423 & 24.417 & 0.015 & -3.455 & 88.46 & 31.76 & 59.02 & 1127.1 & 77.82 & 155.8 \\
\hline 131.423 & 0.4037 & 0.907 & 4.055 & 145.60 & 26.88 & 39.07 & 283.6 & 5.25 & 14.6 \\
\hline 135. & 0.3900 & 0.914 & 4.192 & 146.63 & 26.45 & 38.14 & 289.1 & 5.39 & 14.9 \\
\hline 140. & 0.3722 & 0.923 & 4.381 & 148.01 & 26.18 & 37.41 & 296.4 & 5.58 & 15.4 \\
\hline 145. & 0.3563 & 0.931 & 4.567 & 149.31 & 26.03 & 36.90 & 303.2 & 5.77 & 15.9 \\
\hline 150. & 0.3419 & 0.938 & 4.750 & 150.55 & 25.91 & 36.49 & 309.8 & 5.96 & 16.4 \\
\hline 155. & 0.3288 & 0.944 & 4.932 & 151.74 & 25.81 & 36.14 & 316.2 & 6.15 & 17.0 \\
\hline 160. & 0.3167 & 0.949 & 5.112 & 152.89 & 25.72 & 35.84 & 322.3 & 6.33 & 17.5 \\
\hline 165. & 0.3057 & 0.954 & 5.290 & 153.98 & 25.64 & 35.59 & 328.3 & 6.52 & 18.1 \\
\hline 170. & 0.2954 & 0.958 & 5.468 & 155.04 & 25.58 & 35.37 & 334.1 & 6.71 & 18.7 \\
\hline 175. & 0.2859 & 0.961 & 5.644 & 156.07 & 25.53 & 35.19 & 339.7 & 6.90 & 19.2 \\
\hline 180. & 0.2771 & 0.965 & 5.820 & 157.06 & 25.50 & 35.04 & 345.2 & 7.08 & 19.8 \\
\hline 185. & 0.2688 & 0.967 & 5.995 & 158.01 & 25.48 & 34.92 & 35 & 7.27 & 20.4 \\
\hline 190. & 0.2611 & 0.970 & 6.169 & 158.94 & 25.46 & 34.82 & 355.8 & 7.45 & 21.0 \\
\hline 195. & 0.2538 & 0.972 & 6.343 & 159.85 & 25.46 & 34.74 & 360.9 & 7.63 & 21.6 \\
\hline 200. & 0.2469 & 0.974 & 6.516 & 160.73 & 25.47 & 34.68 & 365.9 & 7.82 & 22.1 \\
\hline 205. & 0.2404 & 0.976 & 6.690 & 161.58 & 25.49 & 34.64 & 370.8 & 8.00 & 22.7 \\
\hline 210 . & 0.2343 & 0.978 & 6.863 & 162.42 & 25.52 & 34.61 & 375.7 & 8.18 & 23.3 \\
\hline 215. & 0.2285 & 0.979 & 7.036 & 163.23 & 25.56 & 34.60 & 380.4 & 8.36 & 23.9 \\
\hline 220 . & 0.2230 & 0.981 & 7.209 & 164.03 & 25.60 & 34.60 & 385.0 & 8.54 & 24.4 \\
\hline 225 . & 0.2177 & 0.982 & 7.382 & 164.80 & 25.66 & 34.62 & 389.5 & 8.71 & 25.0 \\
\hline 230 . & 0.2127 & 0.983 & 7.555 & 165.57 & 25.72 & 34.65 & 394.0 & 8.89 & 25.6 \\
\hline 235 . & 0.2080 & 0.984 & 7.728 & 166.31 & 25.79 & 34.68 & 398.4 & 9.06 & 26.2 \\
\hline 240 . & 0.2034 & 0.985 & 7.902 & 167.04 & 25.88 & 34.73 & 402.6 & 9.24 & 26.8 \\
\hline 245 . & 0.1991 & 0.986 & 8.076 & 167.76 & 25.97 & 34.80 & 406.9 & 9.41 & 27.4 \\
\hline 250. & 0.1949 & 0.987 & 8.250 & 168.46 & 26.07 & 34.87 & 411.0 & 9.58 & 28.0 \\
\hline 255. & 0.1909 & 0.988 & 8.424 & 169.15 & 26.17 & 34.95 & 415.1 & 9.75 & 28.7 \\
\hline 260 . & 0.1871 & 0.989 & 8.599 & 169.83 & 26.29 & 35.05 & 419.1 & 9.92 & 29.3 \\
\hline 265. & 0.1835 & 0.989 & 8.775 & 170.50 & 26.42 & 35.15 & 423.0 & 10.09 & 29.9 \\
\hline 270. & 0.1800 & 0.990 & 8.951 & 171.16 & 26.55 & 35.27 & 426.9 & 10.26 & 30.6 \\
\hline 275. & 0.1766 & 0.991 & 9.128 & 171.81 & 26.69 & 35.39 & 430.7 & 10.43 & 31.2 \\
\hline 280 . & 0.1733 & 0.991 & 9.305 & 172.45 & 26.84 & 35.52 & 434.4 & 10.59 & 31.9 \\
\hline 285 . & 0.1702 & 0.992 & 9.483 & 173.08 & 27.00 & 35.66 & 438.1 & 10.76 & 32.5 \\
\hline 290. & 0.1672 & 0.992 & 9.662 & 173.70 & 27.17 & 35.82 & 441.7 & 10.92 & 33.2 \\
\hline 295. & 0.1643 & 0.993 & 9.841 & 174.31 & 27.34 & 35.98 & 445.3 & 11.08 & 33.9 \\
\hline 300 . & 0.1615 & 0.993 & 10.021 & 174.92 & 27.52 & 36.14 & 448.8 & 11.24 & 34.6 \\
\hline 310. & 0.1561 & 0.994 & 10.384 & 176.11 & 27.90 & 36.50 & 455.7 & 11.56 & 36.0 \\
\hline
\end{tabular}


METHANE ISOBAR AT $\mathrm{P}=0.400 \mathrm{MPa}$ (continued)

$\begin{array}{lccccccccc}\mathrm{T} & \rho & \mathrm{Z} & \mathrm{H} & \mathrm{S} & \mathrm{C}_{\mathrm{v}} & \mathrm{C}_{\mathrm{p}} & \mathrm{W} & \eta & \lambda \\ \mathrm{K} & \mathrm{mol} \cdot \mathrm{dm}^{-3} & & \mathrm{~kJ} / \mathrm{mol} & \mathrm{J} /(\mathrm{mol} \cdot \mathrm{K}) & \mathrm{J} /(\mathrm{mol} \cdot \mathrm{K}) & \mathrm{J} /(\mathrm{mol} \cdot \mathrm{K}) & \mathrm{m} \cdot \mathrm{s}^{-1} & \mu \mathrm{Pa} \cdot \mathrm{s} & \mathrm{mW} /(\mathrm{m} \cdot \mathrm{K}) \\ & & & & & & & & & \\ 320 . & 0.1511 & 0.995 & 10.751 & 177.27 & 28.32 & 36.89 & 462.4 & 11.88 & 37.4 \\ 330 . & 0.1465 & 0.995 & 11.122 & 178.42 & 28.75 & 37.31 & 468.9 & 12.19 & 38.9 \\ 340 . & 0.1421 & 0.996 & 11.498 & 179.54 & 29.21 & 37.75 & 475.2 & 12.50 & 40.4 \\ 350 . & 0.1380 & 0.996 & 11.878 & 180.64 & 29.69 & 38.22 & 481.4 & 12.81 & 42.0 \\ 360 . & 0.1341 & 0.997 & 12.262 & 181.72 & 30.19 & 38.70 & 487.5 & 13.11 & 43.5 \\ & & & & & & & & & \\ 370 . & 0.1304 & 0.997 & 12.652 & 182.79 & 30.70 & 39.20 & 493.4 & 13.41 & 45.1 \\ 380 . & 0.1269 & 0.998 & 13.046 & 183.84 & 31.23 & 39.72 & 499.2 & 13.70 & 46.8 \\ 390 . & 0.1236 & 0.998 & 13.446 & 184.88 & 31.78 & 40.25 & 504.9 & 13.99 & 48.5 \\ 400 . & 0.1205 & 0.998 & 13.851 & 185.90 & 32.33 & 40.80 & 510.5 & 14.28 & 50.2 \\ 410 . & 0.1175 & 0.998 & 14.262 & 186.92 & 32.89 & 41.35 & 516.0 & & 51.9 \\ & & & & & & & & & \\ 420 . & 0.1147 & 0.999 & 14.678 & 187.92 & 33.46 & 41.91 & 521.5 & & 53.6 \\ 430 . & 0.1120 & 0.999 & 15.100 & 188.91 & 34.03 & 42.47 & 526.8 & 55.4 \\ 440 . & 0.1094 & 0.999 & 15.528 & 189.90 & 34.61 & 43.04 & 532.1 & & 57.2 \\ 450 . & 0.1070 & 0.999 & 15.961 & 190.87 & 35.18 & 43.61 & 537.3 & & 59.0 \\ 460 . & 0.1046 & 0.999 & 16.400 & 191.84 & 35.76 & 44.19 & 542.4 & & 60.9 \\ & & & & & & & & \\ 470 . & 0.1024 & 1.000 & 16.845 & 192.79 & 36.34 & 44.76 & 547.5 & & 62.7 \\ 480 . & 0.1003 & 1.000 & 17.295 & 193.74 & 36.92 & 45.33 & 552.5 & & 64.6 \\ 490 . & 0.0982 & 1.000 & 17.751 & 194.68 & 37.50 & 45.91 & 557.5 & 66.5 \\ 500 . & 0.0962 & 1.000 & 18.213 & 195.61 & 38.07 & 46.47 & 562.4 & & 68.4 \\ 520 . & 0.0925 & 1.000 & 19.154 & 197.46 & 39.21 & 47.60 & 572.1 & & 72.3 \\ & & & & & & & & \\ 540 . & 0.0891 & 1.000 & 20.117 & 199.28 & 40.32 & 48.71 & 581.6 & & 76.1 \\ 560 . & 0.0859 & 1.000 & 21.102 & 201.07 & 41.41 & 49.79 & 591.0 & & 80.1 \\ 580 . & 0.0829 & 1.001 & 22.109 & 202.83 & 42.48 & 50.85 & 600.2 & & 84.0 \\ 600 . & 0.0801 & 1.001 & 23.136 & 204.57 & 43.51 & 51.88 & 609.3 & & 87.9\end{array}$

METHANE ISOBAR AT $\mathrm{P}=0.450 \mathrm{MPa}$

$\begin{array}{lccccccccc}\mathrm{T} & \rho & \mathrm{Z} & \mathrm{H} & \mathrm{S} & \mathrm{C}_{\mathrm{v}} & \mathrm{C}_{\mathrm{p}} & \mathrm{W} & \eta & \lambda \\ \mathrm{K} & \mathrm{mol} \cdot \mathrm{dm}^{-3} & & \mathrm{~kJ} / \mathrm{mol} & \mathrm{J} /(\mathrm{mol} \cdot \mathrm{K}) & \mathrm{J} /(\mathrm{mol} \cdot \mathrm{K}) & \mathrm{J} /(\mathrm{mol} \cdot \mathrm{K}) & \mathrm{m} \cdot \mathrm{s}^{-1} & \mu \mathrm{Pa} \cdot \mathrm{s} & \mathrm{mW} /(\mathrm{m} \cdot \mathrm{K}) \\ & & & & & & & & & \\ 95 . & 27.805 & 0.020 & -5.504 & 70.23 & 34.00 & 53.71 & 1502.0 & 180.46 & 206.5 \\ 100 . & 27.382 & 0.020 & -5.233 & 73.01 & 34.09 & 54.59 & 1449.8 & 158.10 & 200.1 \\ 105 . & 26.950 & 0.019 & -4.958 & 75.69 & 33.87 & 55.23 & 1400.1 & 139.24 & 193.5 \\ 110 . & 26.506 & 0.019 & -4.681 & 78.27 & 33.50 & 55.78 & 1350.9 & 123.32 & 186.6 \\ 115 . & 26.050 & 0.018 & -4.400 & 80.76 & 33.08 & 56.34 & 1301.2 & 109.86 & 179.6 \\ & & & & & & & & & \\ 120 . & 25.578 & 0.018 & -4.117 & 83.17 & 32.65 & 56.98 & 1250.5 & 98.42 & 172.5 \\ 125 . & 25.088 & 0.017 & -3.830 & 85.51 & 32.24 & 57.73 & 1198.4 & 88.63 & 165.3 \\ 130 . & 24.578 & 0.017 & -3.540 & 87.80 & 31.86 & 58.66 & 1144.6 & 80.17 & 158.1 \\ 133.461 & 24.205 & 0.017 & -3.334 & 89.36 & 31.62 & 59.46 & 1105.2 & 74.88 & 152.9 \\ 133.461 & 0.4511 & 0.899 & 4.093 & 145.01 & 26.99 & 39.60 & 284.4 & 5.35 & 14.9\end{array}$


METHANE ISOBAR AT $\mathrm{P}=0.450 \mathrm{MPa}$ (continued)

\begin{tabular}{|c|c|c|c|c|c|c|c|c|c|}
\hline $\mathrm{T}$ & $\rho$ & $\mathrm{Z}$ & $\mathrm{H}$ & $\mathrm{S}$ & $\mathrm{C}_{\mathrm{v}}$ & $C_{p}$ & W & $\eta$ & $\lambda$ \\
\hline K & $\mathrm{mol} \cdot \mathrm{dm}^{-3}$ & & $\mathrm{~kJ} / \mathrm{mol}$ & $\mathrm{J} /(\mathrm{mol} \cdot \mathrm{K})$ & $\mathrm{J} /(\mathrm{mol} \cdot \mathrm{K})$ & $\mathrm{J} /(\mathrm{mol} \cdot \mathrm{K})$ & $m \cdot s^{-1}$ & $\mu \mathrm{Pa} \cdot \mathrm{s}$ & $\mathrm{mW} /(\mathrm{m}$. \\
\hline 135. & 0.4445 & 0.902 & 4.153 & 145.45 & 26.75 & 39.10 & 286.8 & 5.40 & 15.1 \\
\hline 140. & 0.4235 & 0.913 & 4.345 & 146.85 & 26.35 & 38.07 & 294.4 & 5.59 & 15.5 \\
\hline 145. & 0.4048 & 0.922 & 4.534 & 148.18 & 26.17 & 37.45 & 301.5 & 5.78 & 16.0 \\
\hline 150. & 0.3880 & 0.930 & 4.720 & 149.44 & 26.03 & 36.96 & 308.2 & 5.97 & 16.5 \\
\hline 155. & 0.3728 & 0.937 & 4.904 & 150.64 & 25.91 & 36.55 & 314.7 & 6.16 & 17.1 \\
\hline 160. & 0.3589 & 0.943 & 5.086 & 151.80 & 25.81 & 36.20 & 321.0 & 6.35 & 17.6 \\
\hline 165. & 0.3461 & 0.948 & 5.266 & 152.91 & 25.73 & 35.91 & 327.1 & 6.54 & 18.2 \\
\hline 170. & 0.3343 & 0.952 & 5.445 & 153.97 & 25.65 & 35.66 & 333.0 & 6.72 & 18.7 \\
\hline 175. & 0.3234 & 0.956 & 5.623 & 155.00 & 25.60 & 35.45 & 338.7 & 6.91 & 19.3 \\
\hline 180. & 0.3132 & 0.960 & 5.799 & 156.00 & 25.55 & 35.27 & 344.3 & 7.09 & 19.9 \\
\hline 185. & 0.3037 & 0.963 & 5.975 & 156.97 & 25.52 & 35.13 & 349.7 & 7.28 & 20.5 \\
\hline 190. & 0.2949 & 0.966 & 6.151 & 157.90 & 25.51 & 35.01 & 355.0 & 7.46 & 21.1 \\
\hline 195. & 0.2865 & 0.969 & 6.326 & 158.81 & 25.50 & 34.92 & 360.2 & 7.65 & 21.6 \\
\hline 200. & 0.2787 & 0.971 & 6.500 & 159.69 & 25.51 & 34.84 & 365.3 & 7.83 & 22.2 \\
\hline 205 . & 0.2713 & 0.973 & 6.674 & 160.55 & 25.52 & 34.79 & 370.2 & 8.01 & 22.8 \\
\hline 210 . & 0.2643 & 0.975 & 6.848 & 161.39 & 25.55 & 34.75 & 375.1 & 8.19 & 23.3 \\
\hline 215. & 0.2577 & 0.977 & 7.022 & 162.21 & 25.58 & 34.73 & 379.8 & 8.37 & 23.9 \\
\hline 220 . & 0.2514 & 0.978 & 7.195 & 163.00 & 25.63 & 34.72 & 384.5 & 8.55 & 24.5 \\
\hline 225 . & 0.2455 & 0.980 & 7.369 & 163.78 & 25.68 & 34.72 & 389.1 & 8.72 & 25.1 \\
\hline 230. & 0.2398 & 0.981 & 7.542 & 164.55 & 25.74 & 34.74 & 393.6 & 8.90 & 25.7 \\
\hline 235. & 0.2344 & 0.982 & 7.716 & 165.30 & 25.81 & 34.78 & 398.0 & 9.07 & 26.3 \\
\hline 240 . & 0.2293 & 0.984 & 7.890 & 166.03 & 25.89 & 34.82 & 402.3 & 9.25 & 26.9 \\
\hline 245 . & 0.2243 & 0.985 & 8.064 & 166.75 & 25.98 & 34.88 & 406.5 & 9.42 & 27.5 \\
\hline 250 . & 0.2196 & 0.986 & 8.239 & 167.45 & 26.08 & 34.95 & 410.7 & 9.59 & 28.1 \\
\hline 255. & 0.2151 & 0.987 & 8.414 & 168.15 & 26.19 & 35.03 & 414.8 & 9.76 & 28.7 \\
\hline 260 . & 0.2108 & 0.987 & 8.589 & 168.83 & 26.30 & 35.12 & 418.8 & 9.93 & 29.3 \\
\hline 265. & 0.2067 & 0.988 & 8.765 & 169.50 & 26.43 & 35.22 & 422.7 & 10.10 & 30.0 \\
\hline 270. & 0.2027 & 0.989 & 8.941 & 170.16 & 26.56 & 35.33 & 426.6 & 10.27 & 30.6 \\
\hline 275. & 0.1989 & 0.990 & 9.118 & 170.80 & 26.70 & 35.45 & 430.4 & 10.44 & 31.3 \\
\hline 280. & 0.1952 & 0.990 & 9.296 & 171.44 & 26.85 & 35.58 & 434.2 & 10.60 & 31.9 \\
\hline 285. & 0.1917 & 0.991 & 9.474 & 172.08 & 27.01 & 35.72 & 437.9 & 10.77 & 32.6 \\
\hline 290. & 0.1883 & 0.991 & 9.653 & 172.70 & 27.17 & 35.87 & 441.5 & 10.93 & 33.3 \\
\hline 295. & 0.1850 & 0.992 & 9.833 & 173.31 & 27.35 & 36.02 & 445.1 & 11.09 & 33.9 \\
\hline 300. & 0.1818 & 0.992 & 10.013 & 173.92 & 27.53 & 36.19 & 448.7 & 11.25 & 34.6 \\
\hline 310. & 0.1758 & 0.993 & 10.377 & 175.11 & 27.91 & 36.55 & 455.6 & 11.57 & 36.0 \\
\hline 320 . & 0.1701 & 0.994 & 10.744 & 176.28 & 28.32 & 36.93 & 462.3 & 11.89 & 37.5 \\
\hline 330. & 0.1649 & 0.995 & 11.116 & 177.42 & 28.76 & 37.35 & 468.8 & 12.20 & 38.9 \\
\hline 340. & 0.1599 & 0.995 & 11.491 & 178.54 & 29.22 & 37.79 & 475.2 & 12.51 & 40.4 \\
\hline 350. & 0.1553 & 0.996 & 11.872 & 179.64 & 29.70 & 38.25 & 481.4 & 12.81 & 42.0 \\
\hline 360. & 0.1509 & 0.996 & 12.257 & 180.73 & 30.19 & 38.73 & 487.5 & 13.11 & 43.6 \\
\hline
\end{tabular}


METHANE ISOBAR AT $\mathrm{P}=0.450 \mathrm{MPa}$ (continued)

\begin{tabular}{|c|c|c|c|c|c|c|c|c|c|}
\hline $\mathrm{T}$ & $\rho$ & $\mathrm{z}$ & $\mathrm{H}$ & S & $C_{v}$ & $C_{p}$ & W & $\eta$ & $\lambda$ \\
\hline K & $\mathrm{mol} \cdot \mathrm{dm}^{-3}$ & & $\mathrm{~kJ} / \mathrm{mol}$ & $\mathrm{J} /(\mathrm{mol} \cdot \mathrm{K})$ & $\mathrm{J} /(\mathrm{mol} \cdot \mathrm{K})$ & $\mathrm{J} /(\mathrm{mol} \cdot \mathrm{K})$ & $\mathrm{m} \cdot \mathrm{s}^{-1}$ & $\mu \mathrm{Pa} \cdot \mathrm{s}$ & $\mathrm{mW} /(\mathrm{m} \cdot \mathrm{K})$ \\
\hline 370 . & 0.1467 & 0.997 & 12.646 & 181.80 & 30.71 & 39.23 & 493.4 & 13.41 & 45.2 \\
\hline 380. & 0.1428 & 0.997 & 13.041 & 182.85 & 31.24 & 39.75 & 499.2 & 13.71 & 46.8 \\
\hline 390. & 0.1391 & 0.998 & 13.441 & 183.89 & 31.78 & 40.28 & 504.9 & 14.00 & 48.5 \\
\hline 400. & 0.1356 & 0.998 & 13.847 & 184.92 & 32.33 & 40.82 & 510.5 & 14.28 & 50.2 \\
\hline 410. & 0.1322 & 0.998 & 14.258 & 185.93 & 32.89 & 41.37 & 516.0 & & 51.9 \\
\hline 420 & 0.1291 & 0.998 & 14.674 & 186.93 & 33.46 & 41.93 & 521.5 & & 53.7 \\
\hline 430. & 0.1260 & 0.999 & 15.096 & 187.93 & 34.03 & 42.49 & 526.8 & & 55.4 \\
\hline 440. & 0.1231 & 0.999 & 15.524 & 188.91 & 34.61 & 43.06 & 532.1 & & 57.2 \\
\hline 450. & 0.1204 & 0.999 & 15.957 & 189.88 & 35.19 & 43.63 & 537.3 & & 59.1 \\
\hline 460. & 0.1177 & 0.999 & 16.397 & 190.85 & 35.77 & 44.20 & 542.4 & & 60.9 \\
\hline 470. & 0.1152 & 0.999 & 16.842 & 191.81 & 36.35 & 44.78 & 547.5 & & 62.8 \\
\hline 480. & 0.1128 & 1.000 & 17.292 & 192.75 & 36.92 & 45.35 & 552.6 & & 64.6 \\
\hline 490 & 0.1105 & 1.000 & 17.749 & 193.70 & 37.50 & 45.92 & 557.5 & & 66.5 \\
\hline 500. & 0.1083 & 1.000 & 18.211 & 194.63 & 38.07 & 46.49 & 562.5 & & 68.4 \\
\hline 520. & 0.1041 & 1.000 & 19.152 & 196.47 & 39.21 & 47.61 & 572.2 & & 72.3 \\
\hline 540. & 0.1002 & 1.000 & 20.115 & 198.29 & 40.32 & 48.72 & 581.7 & & 76.2 \\
\hline 560. & 0.0966 & 1.000 & 21.100 & 200.08 & 41.41 & 49.80 & 591.1 & & 80.1 \\
\hline 580. & 0.0933 & 1.001 & 22.107 & 201.85 & 42.48 & 50.86 & 600.3 & & 84.0 \\
\hline 600. & 0.0901 & 1.001 & 23.135 & 203.59 & 43.51 & 51.89 & 609.4 & & 87.9 \\
\hline
\end{tabular}

\footnotetext{
$\overline{\text { METHANE ISOBAR AT } \mathrm{P}=0.500 \mathrm{MPa}}$
}

$\begin{array}{lccccccccc}\mathrm{T} & \rho & \mathrm{Z} & \mathrm{H} & \mathrm{S} & \mathrm{C}_{\mathrm{v}} & \mathrm{C}_{\mathrm{p}} & \mathrm{W} & \eta & \lambda \\ \mathrm{K} & \mathrm{mol} \cdot \mathrm{dm}^{-3} & & \mathrm{~kJ} / \mathrm{mol} & \mathrm{J} /(\mathrm{mol} \cdot \mathrm{K}) & \mathrm{J} /(\mathrm{mol} \cdot \mathrm{K}) & \mathrm{J} /(\mathrm{mol} \cdot \mathrm{K}) & \mathrm{m} \cdot \mathrm{s}^{-1} & \mu \mathrm{Pa} \cdot \mathrm{s} & \mathrm{mW} /(\mathrm{m} \cdot \mathrm{K}) \\ & & & & & & & & & \\ \text { 95. } & 27.807 & 0.023 & -5.502 & 70.22 & 34.00 & 53.71 & 1502.4 & 180.56 & 206.5 \\ 100 . & 27.385 & 0.022 & -5.232 & 73.00 & 34.10 & 54.59 & 1450.3 & 158.19 & 200.2 \\ 105 . & 26.953 & 0.021 & -4.957 & 75.68 & 33.87 & 55.22 & 1400.6 & 139.32 & 193.5 \\ 110 . & 26.509 & 0.021 & -4.679 & 78.26 & 33.50 & 55.77 & 1351.4 & 123.40 & 186.7 \\ 115 . & 26.053 & 0.020 & -4.399 & 80.76 & 33.08 & 56.33 & 1301.7 & 109.93 & 179.7 \\ & & & & & & & & & \\ 120 . & 25.581 & 0.020 & -4.116 & 83.17 & 32.66 & 56.96 & 1251.0 & 98.49 & 172.6 \\ 125 . & 25.092 & 0.019 & -3.829 & 85.51 & 32.25 & 57.72 & 1199.0 & 88.69 & 165.4 \\ 130 . & 24.582 & 0.019 & -3.539 & 87.79 & 31.87 & 58.64 & 1145.3 & 80.22 & 158.1 \\ 135 . & 24.047 & 0.019 & -3.243 & 90.02 & 31.52 & 59.79 & 1089.5 & 72.83 & 150.8 \\ 135.338 & 24.005 & 0.019 & -3.221 & 90.18 & 31.50 & 59.90 & 1084.9 & 72.31 & 150.2 \\ & & & & & & & & & \\ 135.338 & 0.4985 & 0.891 & 4.126 & 144.47 & 27.11 & 40.15 & 284.9 & 5.43 & 15.3 \\ 140 . & 0.4761 & 0.902 & 4.309 & 145.80 & 26.55 & 38.82 & 292.3 & 5.61 & 15.7 \\ 145 . & 0.4545 & 0.913 & 4.501 & 147.14 & 26.31 & 38.03 & 299.6 & 5.80 & 16.1 \\ 150 . & 0.4351 & 0.922 & 4.689 & 148.42 & 26.15 & 37.45 & 306.6 & 5.99 & 16.6 \\ 155 . & 0.4175 & 0.929 & 4.875 & 149.64 & 26.02 & 36.98 & 313.2 & 6.17 & 17.2\end{array}$


METHANE ISOBAR AT $\mathrm{P}=0.500 \mathrm{MPa}$ (continued)

\begin{tabular}{|c|c|c|c|c|c|c|c|c|c|}
\hline $\mathrm{T}$ & $\rho$ & $\mathrm{Z}$ & H & S & $C_{v}$ & $C_{p}$ & $\mathrm{~W}$ & $\eta$ & $\lambda$ \\
\hline K & $\mathrm{mol} \cdot \mathrm{dm}^{-3}$ & & $\mathrm{~kJ} / \mathrm{mol}$ & $\mathrm{J} /(\mathrm{mol} \cdot \mathrm{K})$ & $\mathrm{J} /(\mathrm{mol} \cdot \mathrm{K})$ & $\mathrm{J} /(\mathrm{mol} \cdot \mathrm{K})$ & $\mathrm{m} \cdot \mathrm{s}^{-1}$ & $\mu \mathrm{Pa} \cdot \mathrm{s}$ & $\mathrm{mW} /(\mathrm{m} \cdot$ \\
\hline 160. & 0.4016 & 0.936 & 5.059 & 150.81 & 25.91 & 36.58 & 319.6 & 6.36 & 17.7 \\
\hline 165. & 0.3870 & 0.942 & 5.241 & 151.93 & 25.81 & 36.24 & 325.8 & 6.55 & 18.3 \\
\hline 170. & 0.3736 & 0.947 & 5.422 & 153.01 & 25.73 & 35.95 & 331.8 & 6.74 & 18.8 \\
\hline 175. & 0.3612 & 0.951 & 5.601 & 154.05 & 25.66 & 35.71 & 337.7 & 6.92 & 19.4 \\
\hline 180. & 0.3497 & 0.955 & 5.779 & 155.05 & 25.61 & 35.51 & 343.3 & 7.11 & 20.0 \\
\hline 185. & 0.3390 & 0.959 & 5.956 & 156.02 & 25.58 & 35.34 & 348.8 & 7.29 & 20.5 \\
\hline 190. & 0.3289 & 0.962 & 6.133 & 156.96 & 25.55 & 35.20 & 354.2 & 7.47 & 21.2 \\
\hline 195. & 0.3195 & 0.965 & 6.308 & 157.87 & 25.54 & 35.09 & 359.5 & 7.66 & 21.7 \\
\hline 200 . & 0.3107 & 0.968 & 6.483 & 158.76 & 25.54 & 35.00 & 364.6 & 7.84 & 22.2 \\
\hline 205. & 0.3024 & 0.970 & 6.658 & 159.62 & 25.55 & 34.93 & 369.6 & 8.02 & 22.8 \\
\hline 210 . & 0.2945 & 0.972 & 6.833 & 160.46 & 25.58 & 34.88 & 374.5 & 8.20 & 23.4 \\
\hline 215 . & 0.2871 & 0.974 & 7.007 & 161.29 & 25.61 & 34.85 & 379.3 & 8.38 & 24.0 \\
\hline 220. & 0.2801 & 0.976 & 7.181 & 162.09 & 25.65 & 34.83 & 384.0 & 8.56 & 24.5 \\
\hline 225 . & 0.2734 & 0.978 & 7.356 & 162.87 & 25.70 & 34.83 & 388.6 & 8.73 & 25.1 \\
\hline 230. & 0.2670 & 0.979 & 7.530 & 163.63 & 25.76 & 34.85 & 393.1 & 8.91 & 25.7 \\
\hline 235 . & 0.2610 & 0.980 & 7.704 & 164.38 & 25.83 & 34.87 & 397.5 & 9.08 & 26.3 \\
\hline 240 . & 0.2552 & 0.982 & 7.878 & 165.12 & 25.91 & 34.91 & 401.9 & 9.26 & 26.9 \\
\hline 245. & 0.2497 & 0.983 & 8.053 & 165.84 & 26.00 & 34.96 & 406.2 & 9.43 & 27.5 \\
\hline 250. & 0.2444 & 0.984 & 8.228 & 166.55 & 26.09 & 35.03 & 410.4 & 9.60 & 28.1 \\
\hline 255. & 0.2394 & 0.985 & 8.403 & 167.24 & 26.20 & 35.10 & 414.5 & 9.77 & 28.7 \\
\hline 260 . & 0.2346 & 0.986 & 8.579 & 167.92 & 26.32 & 35.19 & 418.5 & 9.94 & 29.4 \\
\hline 265. & 0.2300 & 0.987 & 8.755 & 168.59 & 26.44 & 35.28 & 422.5 & 10.11 & 30.0 \\
\hline 270. & 0.2255 & 0.988 & 8.932 & 169.25 & 26.57 & 35.39 & 426.4 & 10.28 & 30.6 \\
\hline 275. & 0.2212 & 0.988 & 9.109 & 169.91 & 26.71 & 35.51 & 430.2 & 10.44 & 31.3 \\
\hline 280 . & 0.2171 & 0.989 & 9.287 & 170.55 & 26.86 & 35.64 & 434.0 & 10.61 & 32.0 \\
\hline 285. & 0.2132 & 0.990 & 9.466 & 171.18 & 27.02 & 35.77 & 437.7 & 10.77 & 32.6 \\
\hline 290. & 0.2094 & 0.990 & 9.645 & 171.80 & 27.18 & 35.92 & 441.4 & 10.94 & 33.3 \\
\hline 295. & 0.2057 & 0.991 & 9.825 & 172.42 & 27.36 & 36.07 & 445.0 & 11.10 & 34.0 \\
\hline 300 . & 0.2022 & 0.992 & 10.006 & 173.02 & 27.54 & 36.24 & 448.5 & 11.26 & 34.7 \\
\hline 310. & 0.1955 & 0.993 & 10.370 & 174.22 & 27.92 & 36.59 & 455.4 & 11.58 & 36.1 \\
\hline 320. & 0.1892 & 0.993 & 10.737 & 175.39 & 28.33 & 36.97 & 462.2 & 11.90 & 37.5 \\
\hline 330. & 0.1833 & 0.994 & 11.109 & 176.53 & 28.76 & 37.38 & 468.7 & 12.21 & 39.0 \\
\hline 340 . & 0.1778 & 0.995 & 11.485 & 177.65 & 29.22 & 37.82 & 475.1 & 12.52 & 40.5 \\
\hline 350. & 0.1726 & 0.995 & 11.866 & 178.76 & 29.70 & 38.28 & 481.3 & 12.82 & 42.0 \\
\hline 360. & 0.1677 & 0.996 & 12.251 & 179.84 & 30.20 & 38.76 & 487.4 & 13.12 & 43.6 \\
\hline 370. & 0.1631 & 0.996 & 12.641 & 180.91 & 30.71 & 39.26 & 493.4 & 13.42 & 45.2 \\
\hline 380. & 0.1587 & 0.997 & 13.036 & 181.96 & 31.24 & 39.77 & 499.2 & 13.71 & 46.8 \\
\hline 390. & 0.1546 & 0.997 & 13.437 & 183.00 & 31.78 & 40.30 & 504.9 & 14.00 & 48.5 \\
\hline 400. & 0.1507 & 0.998 & 13.842 & 184.03 & 32.34 & 40.84 & 510.5 & 14.29 & 50.2 \\
\hline 410. & 0.1470 & 0.998 & 14.253 & 185.04 & 32.90 & 41.39 & 516.1 & & 51.9 \\
\hline
\end{tabular}


METHANE ISOBAR AT $\mathrm{P}=0.500 \mathrm{MPa}$ (continued)

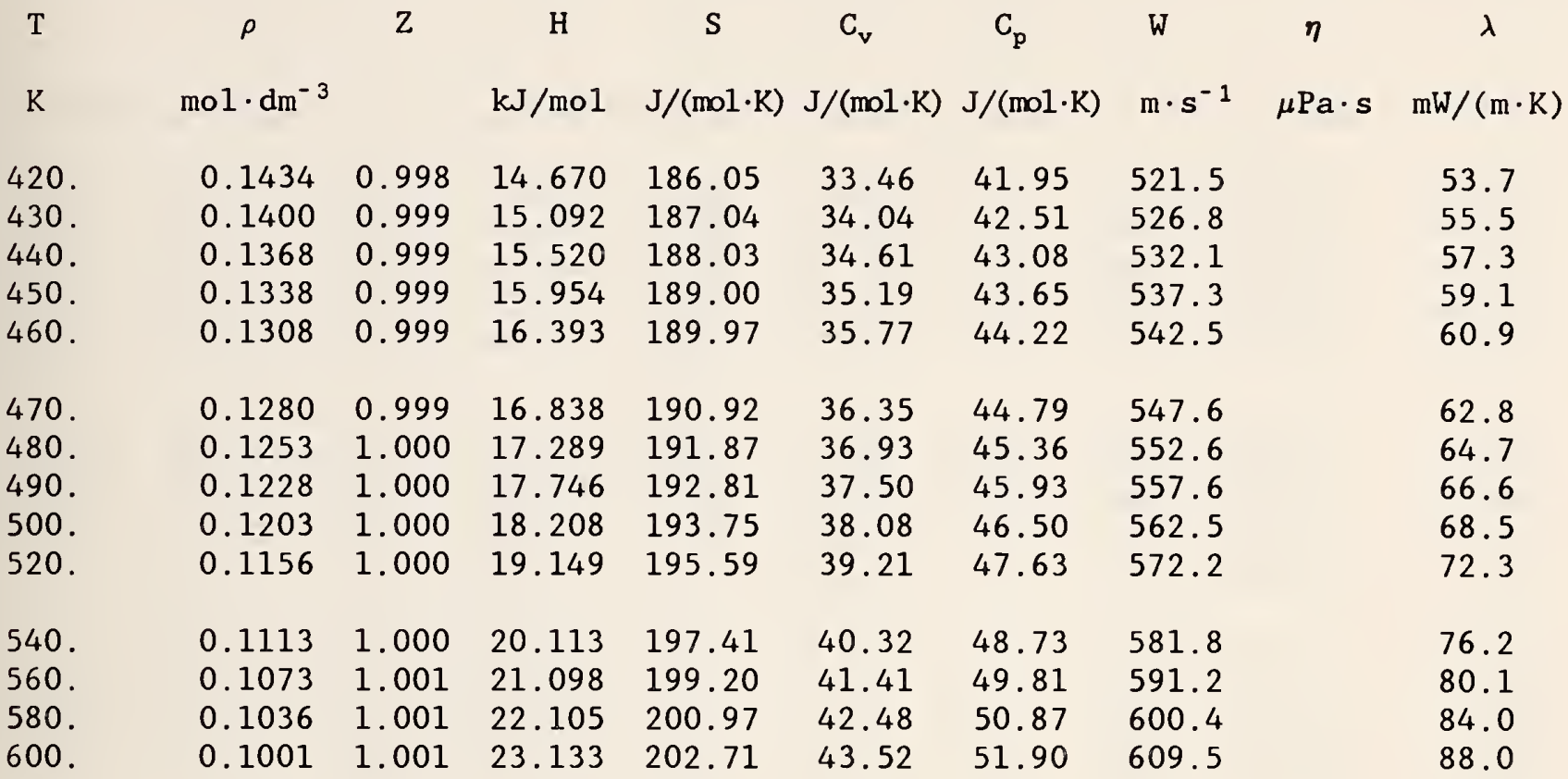

\footnotetext{
$\overline{\text { METHANE ISOBAR AT } \mathrm{P}=0.550 \mathrm{MPa}}$
}

$\begin{array}{lccccccccc}\text { T } & \rho & \mathrm{Z} & \mathrm{H} & \mathrm{S} & \mathrm{C} & \mathrm{C} & \mathrm{W} & \eta & \lambda \\ \mathrm{K} & \mathrm{mo1} \cdot \mathrm{dm}^{-3} & & \mathrm{~kJ} / \mathrm{mol} & \mathrm{J} /(\mathrm{mol} \cdot \mathrm{K}) & \mathrm{J} /(\mathrm{mol} \cdot \mathrm{K}) & \mathrm{J} /(\mathrm{mol} \cdot \mathrm{K}) & \mathrm{m} \cdot \mathrm{s}^{-1} & \mu \mathrm{Pa} \cdot \mathrm{s} & \mathrm{mW} /(\mathrm{m} \cdot \mathrm{K}) \\ & & & & & & & & & \\ \text { 95. } & 27.809 & 0.025 & -5.501 & 70.22 & 34.00 & 53.70 & 1502.8 & 180.66 & 206.5 \\ 100 . & 27.387 & 0.024 & -5.230 & 73.00 & 34.10 & 54.58 & 1450.7 & 158.28 & 200.2 \\ 105 . & 26.955 & 0.023 & -4.956 & 75.68 & 33.87 & 55.21 & 1401.0 & 139.40 & 193.6 \\ 110 . & 26.512 & 0.023 & -4.678 & 78.26 & 33.51 & 55.76 & 1351.9 & 123.47 & 186.8 \\ 115 . & 26.056 & 0.022 & -4.398 & 80.75 & 33.08 & 56.32 & 1302.2 & 110.00 & 179.8 \\ & & & & & & & & & \\ 120 . & 25.585 & 0.022 & -4.115 & 83.16 & 32.66 & 56.95 & 1251.6 & 98.55 & 172.7 \\ 125 . & 25.096 & 0.021 & -3.828 & 85.50 & 32.25 & 57.70 & 1199.6 & 88.75 & 165.5 \\ 130 . & 24.587 & 0.021 & -3.538 & 87.78 & 31.87 & 58.62 & 1145.9 & 80.28 & 158.2 \\ 135 . & 24.052 & 0.020 & -3.242 & 90.01 & 31.52 & 59.76 & 1090.3 & 72.88 & 150.9 \\ 137.082 & 23.817 & 0.020 & -3.116 & 90.94 & 31.39 & 60.34 & 1065.8 & 70.02 & 147.7 \\ 137.082 & 0.5459 & 0.884 & 4.155 & 143.98 & 27.23 & 40.71 & 285.4 & 5.52 & 15.6 \\ 140 . & 0.5302 & 0.891 & 4.271 & 144.82 & 26.80 & 39.69 & 290.1 & 5.62 & 15.9 \\ 145 . & 0.5052 & 0.903 & 4.467 & 146.19 & 26.46 & 38.66 & 297.7 & 5.81 & 16.3 \\ 150 . & 0.4830 & 0.913 & 4.658 & 147.49 & 26.27 & 37.97 & 304.9 & 6.00 & 16.8 \\ 155 . & 0.4631 & 0.922 & 4.847 & 148.72 & 26.13 & 37.43 & 311.7 & 6.19 & 17.3 \\ 160 . & 0.4450 & 0.929 & 5.033 & 149.90 & 26.00 & 36.97 & 318.3 & 6.38 & 17.8 \\ 165 . & 0.4285 & 0.936 & 5.217 & 151.03 & 25.89 & 36.59 & 324.6 & 6.56 & 18.3 \\ 170 . & 0.4134 & 0.941 & 5.399 & 152.12 & 25.80 & 36.26 & 330.7 & 6.75 & 18.9 \\ 175 . & 0.3995 & 0.946 & 5.579 & 153.17 & 25.73 & 35.98 & 336.6 & 6.93 & 19.5 \\ 180 . & 0.3865 & 0.951 & 5.759 & 154.18 & 25.67 & 35.75 & 342.4 & 7.12 & 20.0\end{array}$


METHANE ISOBAR AT $\mathrm{P}=0.550 \mathrm{MPa}$ (continued)

\begin{tabular}{|c|c|c|c|c|c|c|c|c|c|}
\hline $\mathrm{T}$ & $\rho$ & Z & $\mathrm{H}$ & $\mathrm{S}$ & $c_{v}$ & $C_{p}$ & W & $\eta$ & $\lambda$ \\
\hline K & $\mathrm{mol} \cdot \mathrm{dm}^{-3}$ & & $\mathrm{~kJ} / \mathrm{mol}$ & $\mathrm{J} /(\mathrm{mol} \cdot \mathrm{K})$ & $\mathrm{J} /(\mathrm{mol} \cdot \mathrm{K})$ & $\mathrm{J} /(\mathrm{mol} \cdot \mathrm{K})$ & $\mathrm{m} \cdot \mathrm{s}^{-1}$ & $\mu \mathrm{Pa} \cdot \mathrm{s}$ & $\mathrm{mW} /(\mathrm{m} \cdot \mathrm{K})$ \\
\hline 185. & 0.3745 & 0.955 & 5.937 & 155.16 & 25.63 & 35.56 & 348.0 & 7.30 & 20.6 \\
\hline 190. & 0.3633 & 0.958 & 6.114 & 156.10 & 25.60 & 35.40 & 353.4 & 7.49 & 21.2 \\
\hline 195. & 0.3528 & 0.962 & 6.291 & 157.02 & 25.58 & 35.27 & 358.7 & 7.67 & 21.8 \\
\hline 200 . & 0.3430 & 0.964 & 6.467 & 157.91 & 25.58 & 35.16 & 363.9 & 7.85 & 22.3 \\
\hline 205. & 0.3337 & 0.967 & 6.643 & 158.78 & 25.59 & 35.08 & 369.0 & 8.03 & 22.9 \\
\hline 210 . & 0.3249 & 0.969 & 6.818 & 159.62 & 25.60 & 35.02 & 373.9 & 8.21 & 23.4 \\
\hline 215. & 0.3167 & 0.972 & 6.993 & 160.45 & 25.63 & 34.98 & 378.8 & 8.39 & 24.0 \\
\hline 220 . & 0.3089 & 0.974 & 7.168 & 161.25 & 25.67 & 34.95 & 383.5 & 8.57 & 24.6 \\
\hline 225. & 0.3014 & 0.975 & 7.342 & 162.04 & 25.72 & 34.94 & 388.1 & 8.74 & 25.2 \\
\hline 230 . & 0.2944 & 0.977 & 7.517 & 162.80 & 25.78 & 34.95 & 392.7 & 8.92 & 25.8 \\
\hline 235. & 0.2877 & 0.979 & 7.692 & 163.56 & 25.85 & 34.97 & 397.1 & 9.09 & 26.4 \\
\hline 240 . & 0.2813 & 0.980 & 7.867 & 164.29 & 25.93 & 35.00 & 401.5 & 9.27 & 27.0 \\
\hline 245. & 0.2752 & 0.981 & 8.042 & 165.01 & 26.01 & 35.05 & 405.8 & 9.44 & 27.6 \\
\hline 250. & 0.2693 & 0.982 & 8.217 & 165.72 & 26.11 & 35.10 & 410.0 & 9.61 & 28.2 \\
\hline 255 . & 0.2638 & 0.984 & 8.393 & 166.42 & 26.21 & 35.18 & 414.2 & 9.78 & 28.8 \\
\hline 260. & 0.2584 & 0.985 & 8.569 & 167.10 & 26.33 & 35.26 & 418.2 & 9.95 & 29.4 \\
\hline 265. & 0.2533 & 0.986 & 8.745 & 167.78 & 26.45 & 35.35 & 422.2 & 10.12 & 30.0 \\
\hline 270 . & 0.2484 & 0.986 & 8.922 & 168.44 & 26.58 & 35.45 & 426.1 & 10.29 & 30.7 \\
\hline 275 . & 0.2436 & 0.987 & 9.100 & 169.09 & 26.72 & 35.57 & 430.0 & 10.45 & 31.3 \\
\hline 280. & 0.2391 & 0.988 & 9.278 & 169.73 & 26.87 & 35.69 & 433.8 & 10.62 & 32.0 \\
\hline 285. & 0.2347 & 0.989 & 9.457 & 170.36 & 27.03 & 35.83 & 437.5 & 10.78 & 32.6 \\
\hline 290. & 0.2305 & 0.989 & 9.636 & 170.99 & 27.19 & 35.97 & 441.2 & 10.95 & 33.3 \\
\hline 295. & 0.2265 & 0.990 & 9.817 & 171.60 & 27.36 & 36.12 & 444.8 & 11.11 & 34.0 \\
\hline 300 . & 0.2226 & 0.991 & 9.998 & 172.21 & 27.54 & 36.29 & 448.4 & 11.27 & 34.7 \\
\hline 310. & 0.2152 & 0.992 & 10.362 & 173.41 & 27.93 & 36.63 & 455.3 & 11.59 & 36.1 \\
\hline 320 . & 0.2082 & 0.993 & 10.731 & 174.58 & 28.34 & 37.01 & 462.1 & 11.90 & 37.5 \\
\hline 330. & 0.2018 & 0.994 & 11.103 & 175.72 & 28.77 & 37.42 & 468.6 & 12.22 & 39.0 \\
\hline 340 . & 0.1957 & 0.994 & 11.479 & 176.85 & 29.23 & 37.85 & 475.0 & 12.52 & 40.5 \\
\hline 350. & 0.1899 & 0.995 & 11.860 & 177.95 & 29.71 & 38.31 & 481.3 & 12.83 & 42.0 \\
\hline 360. & 0.1846 & 0.996 & 12.245 & 179.04 & 30.20 & 38.79 & 487.4 & 13.13 & 43.6 \\
\hline 370. & 0.1795 & 0.996 & 12.636 & 180.11 & 30.72 & 39.29 & 493.3 & 13.43 & 45.2 \\
\hline 380 . & 0.1747 & 0.997 & 13.031 & 181.16 & 31.25 & 39.80 & 499.2 & 13.72 & 46.9 \\
\hline 390. & 0.1701 & 0.997 & 13.432 & 182.20 & 31.79 & 40.33 & 504.9 & 14.01 & 48.5 \\
\hline 400. & 0.1658 & 0.997 & 13.838 & 183.23 & 32.34 & 40.86 & 510.5 & 14.30 & 50.2 \\
\hline 410. & 0.1617 & 0.998 & 14.249 & 184.24 & 32.90 & 41.41 & 516.1 & & 51.9 \\
\hline 420 . & 0.1578 & 0.998 & 14.666 & 185.25 & 33.47 & 41.97 & 521.5 & & 53.7 \\
\hline 430. & 0.1541 & 0.998 & 15.088 & 186.24 & 34.04 & 42.53 & 526.9 & & 55.5 \\
\hline 440. & 0.1505 & 0.999 & 15.517 & 187.23 & 34.61 & 43.10 & 532.2 & & 57.3 \\
\hline 450. & 0.1472 & 0.999 & 15.950 & 188.20 & 35.19 & 43.67 & 537.4 & & 59.1 \\
\hline 460. & 0.1439 & 0.999 & 16.390 & 189.17 & 35.77 & 44.24 & 542.5 & & 60.9 \\
\hline
\end{tabular}


METHANE ISOBAR AT $\mathrm{P}=0.550 \mathrm{MPa}$ (continued)

\begin{tabular}{|c|c|c|c|c|c|c|c|c|c|}
\hline$T$ & $\rho$ & Z & $\mathrm{H}$ & S & $C_{v}$ & $C_{p}$ & W & $\eta$ & $\lambda$ \\
\hline K & $\mathrm{mol} \cdot \mathrm{dm}^{-3}$ & & $\mathrm{~kJ} / \mathrm{mol}$ & $\mathrm{J} /(\mathrm{mol} \cdot \mathrm{K})$ & $\mathrm{J} /(\mathrm{mol} \cdot \mathrm{K})$ & $\mathrm{J} /(\mathrm{mol} \cdot \mathrm{K})$ & $\mathrm{m} \cdot \mathrm{s}^{-1}$ & $\mu \mathrm{Pa} \cdot \mathrm{s}$ & $\mathrm{mW} /(\mathrm{m} \cdot \mathrm{K})$ \\
\hline 470. & 0.1408 & 0.999 & 16.835 & 190.12 & 36.35 & 44.81 & 547.6 & & 62.8 \\
\hline 480 & 0.1379 & 1.000 & 17.286 & 191.07 & 36.93 & 45.38 & 552.7 & & 64.7 \\
\hline 490. & 0.1350 & 1.000 & 17.743 & 192.02 & 37.51 & 45.95 & 557.6 & & 66.6 \\
\hline 500 & 0.1323 & 1.000 & 18.205 & 192.95 & 38.08 & 46.51 & 562.6 & & 68.5 \\
\hline 520. & 0.1272 & 1.000 & 19.147 & 194.80 & 39.21 & 47.64 & 572.3 & & 72.3 \\
\hline 540 & 0.1224 & 1.000 & 20.110 & 196.61 & 40.33 & 48.74 & 581.8 & & 76.2 \\
\hline 560. & 0.1181 & 1.001 & 21.096 & 198.41 & 41.42 & 49.82 & 591.2 & & 80.1 \\
\hline 580. & 0.1140 & 1.001 & 22.103 & 200.17 & 42.48 & 50.88 & 600.5 & & 84.0 \\
\hline 600 & 0.1102 & 1.001 & 23.131 & 201.92 & 43.52 & 51.91 & 609.6 & & 88.0 \\
\hline
\end{tabular}

$\overline{\text { METHANE ISOBAR AT } \mathrm{P}=0.600 \mathrm{MPa}}$

\begin{tabular}{|c|c|c|c|c|c|c|c|c|c|}
\hline $\mathrm{T}$ & $\rho$ & $\mathrm{Z}$ & $\mathrm{H}$ & $S$ & $C_{v}$ & $\mathrm{C}_{\mathrm{p}}$ & W & $\eta$ & $\lambda$ \\
\hline K & $\mathrm{mol} \cdot \mathrm{dm}^{-3}$ & & $\mathrm{~kJ} / \mathrm{mol}$ & $\mathrm{J} /(\mathrm{mol} \cdot \mathrm{K})$ & $\mathrm{J} /(\mathrm{mol} \cdot \mathrm{K})$ & $\mathrm{J} /(\mathrm{mol} \cdot \mathrm{K})$ & $\mathrm{m} \cdot \mathrm{s}^{-1}$ & $\mu \mathrm{Pa} \cdot \mathrm{s}$ & $\mathrm{mW} /(\mathrm{m} \cdot \mathrm{K})$ \\
\hline 95. & 27.811 & 0.027 & -5.500 & 70.21 & 34.01 & 53.70 & 1503.3 & 180.75 & 206.6 \\
\hline 100. & 27.389 & 0.026 & -5.229 & 72.99 & 34.10 & 54.58 & 1451.1 & 158.37 & 200.3 \\
\hline 105. & 26.958 & 0.025 & -4.955 & 75.67 & 33.87 & 55.21 & 1401.5 & 139.48 & 193.6 \\
\hline 110. & 26.515 & 0.025 & -4.677 & 78.25 & 33.51 & 55.75 & 1352.4 & 123.55 & 186.8 \\
\hline 115. & 26.059 & 0.024 & -4.397 & 80.74 & 33.09 & 56.31 & 1302.8 & 110.07 & 179.8 \\
\hline 120. & 25.588 & 0.024 & -4.114 & 83.15 & 32.66 & 56.94 & 1252.2 & 98.62 & 172.7 \\
\hline 125. & 25.100 & 0.023 & -3.827 & 85.49 & 32.25 & 57.69 & 1200.3 & 88.81 & 165.5 \\
\hline 130. & 24.591 & 0.023 & -3.537 & 87.77 & 31.87 & 58.60 & 1146.6 & 80.34 & 158.3 \\
\hline 135. & 24.057 & 0.022 & -3.241 & 90.00 & 31.52 & 59.73 & 1091.0 & 72.94 & 150.9 \\
\hline 138.715 & 23.638 & 0.022 & -3.016 & 91.64 & 31.29 & 60.78 & 1047.7 & 67.97 & 145.4 \\
\hline 138.715 & 0.5933 & 0.877 & 4.181 & 143.53 & 27.36 & 41.28 & 285.7 & 5.59 & 15.9 \\
\hline 140 & 0.5858 & 0.880 & 4.232 & 143.90 & 27.12 & 40.73 & 287.9 & 5.64 & 16.0 \\
\hline 145. & 0.5572 & 0.893 & 4.432 & 145.30 & 26.63 & 39.35 & 295.8 & 5.83 & 16.4 \\
\hline 150. & 0.5320 & 0.904 & 4.626 & 146.62 & 26.40 & 38.52 & 303.2 & 6.02 & 16.9 \\
\hline 155. & 0.5094 & 0.914 & 4.817 & 147.87 & 26.23 & 37.90 & 310.2 & 6.20 & 17.4 \\
\hline 160. & 0.4891 & 0.922 & 5.006 & 149.06 & 26.10 & 37.38 & 316.9 & 6.39 & 17.9 \\
\hline 165. & 0.4706 & 0.929 & 5.191 & 150.21 & 25.98 & 36.94 & 323.4 & 6.58 & 18.4 \\
\hline 170. & 0.4537 & 0.936 & 5.375 & 151.30 & 25.88 & 36.57 & 329.6 & 6.76 & 19.0 \\
\hline 175. & 0.4381 & 0.941 & 5.557 & 152.36 & 25.79 & 36.26 & 335.6 & 6.95 & 19.5 \\
\hline 180. & 0.4238 & 0.946 & 5.738 & 153.38 & 25.73 & 36.00 & 341.4 & 7.13 & 20.1 \\
\hline 185. & 0.4104 & 0.950 & 5.917 & 154.36 & 25.68 & 35.78 & 347.1 & 7.32 & 20.7 \\
\hline 190. & 0.3980 & 0.954 & 6.096 & 155.31 & 25.64 & 35.60 & 352.6 & 7.50 & 21.3 \\
\hline 195. & 0.3863 & 0.958 & 6.273 & 156.24 & 25.62 & 35.45 & 358.0 & 7.68 & 21.8 \\
\hline 200. & 0.3754 & 0.961 & 6.450 & 157.13 & 25.61 & 35.33 & 363.2 & 7.86 & 22.4 \\
\hline 205 . & 0.3652 & 0.964 & 6.627 & 158.00 & 25.62 & 35.23 & 368.3 & 8.04 & 22.9 \\
\hline
\end{tabular}


METHANE ISOBAR AT $\mathrm{P}=0.600 \mathrm{MPa}$ (continued)

\begin{tabular}{|c|c|c|c|c|c|c|c|c|c|}
\hline $\mathrm{T}$ & $\rho$ & $\mathrm{Z}$ & $\mathrm{H}$ & $S$ & $C_{v}$ & $C_{p}$ & W & $\eta$ & $\lambda$ \\
\hline K & $\mathrm{mol} \cdot \mathrm{dm}^{-3}$ & & $\mathrm{~kJ} / \mathrm{mol}$ & $\mathrm{J} /(\mathrm{mol} \cdot \mathrm{K})$ & $\mathrm{J} /(\mathrm{mol} \cdot \mathrm{K})$ & $\mathrm{J} /(\mathrm{mol} \cdot \mathrm{K})$ & $\mathrm{m} \cdot \mathrm{s}^{-1}$ & $\mu \mathrm{Pa} \cdot \mathrm{s}$ & $\mathrm{mW} /(\mathrm{m} \cdot \mathrm{K})$ \\
\hline 210 . & 0.3555 & 0.967 & 6.803 & 158.85 & 25.63 & 35.16 & 373.3 & 8.22 & 23.5 \\
\hline 215 . & 0.3464 & 0.969 & 6.978 & 159.68 & 25.66 & 35.11 & 378.2 & 8.40 & 24.1 \\
\hline 220 . & 0.3378 & 0.971 & 7.154 & 160.48 & 25.70 & 35.07 & 383.0 & 8.58 & 24.6 \\
\hline 225. & 0.3296 & 0.973 & 7.329 & 161.27 & 25.74 & 35.05 & 387.7 & 8.75 & 25.2 \\
\hline 230 . & 0.3218 & 0.975 & 7.504 & 162.04 & 25.80 & 35.05 & 392.3 & 8.93 & 25.8 \\
\hline 235 . & 0.3145 & 0.977 & 7.680 & 162.80 & 25.87 & 35.06 & 396.7 & 9.10 & 26.4 \\
\hline 240 . & 0.3074 & 0.978 & 7.855 & 163.54 & 25.94 & 35.09 & 401.1 & 9.28 & 27.0 \\
\hline 245 . & 0.3007 & 0.980 & 8.030 & 164.26 & 26.03 & 35.13 & 405.5 & 9.45 & 27.6 \\
\hline 250 . & 0.2943 & 0.981 & 8.206 & 164.97 & 26.12 & 35.18 & 409.7 & 9.62 & 28.2 \\
\hline 255. & 0.2882 & 0.982 & 8.382 & 165.67 & 26.23 & 35.25 & 413.9 & 9.79 & 28.8 \\
\hline 260 . & 0.2823 & 0.983 & 8.559 & 166.35 & 26.34 & 35.33 & 417.9 & 9.96 & 29.5 \\
\hline 265 . & 0.2767 & 0.984 & 8.736 & 167.03 & 26.46 & 35.42 & 422.0 & 10.13 & 30.1 \\
\hline 270 . & 0.2713 & 0.985 & 8.913 & 167.69 & 26.59 & 35.52 & 425.9 & 10.30 & 30.7 \\
\hline 275 . & 0.2661 & 0.986 & 9.091 & 168.34 & 26.73 & 35.63 & 429.8 & 10.46 & 31.4 \\
\hline 280 . & 0.2611 & 0.987 & 9.269 & 168.98 & 26.88 & 35.75 & 433.6 & 10.63 & 32.0 \\
\hline 285. & 0.2563 & 0.988 & 9.448 & 169.62 & 27.04 & 35.88 & 437.3 & 10.79 & 32.7 \\
\hline 290 . & 0.2517 & 0.988 & 9.628 & 170.24 & 27.20 & 36.02 & 441.0 & 10.95 & 33.4 \\
\hline 295. & 0.2473 & 0.989 & 9.809 & 170.86 & 27.37 & 36.17 & 444.6 & 11.12 & 34.0 \\
\hline 300 . & 0.2430 & 0.990 & 9.990 & 171.47 & 27.55 & 36.33 & 448.2 & 11.28 & 34.7 \\
\hline 310 . & 0.2349 & 0.991 & 10.355 & 172.67 & 27.93 & 36.68 & 455.2 & 11.60 & 36.1 \\
\hline 320 . & 0.2273 & 0.992 & 10.724 & 173.84 & 28.34 & 37.05 & 462.0 & 11.91 & 37.6 \\
\hline 330 . & 0.2202 & 0.993 & 11.096 & 174.98 & 28.78 & 37.46 & 468.5 & 12.22 & 39.0 \\
\hline 340 . & 0.2136 & 0.994 & 11.473 & 176.11 & 29.23 & 37.89 & 475.0 & 12.53 & 40.5 \\
\hline 350 . & 0.2073 & 0.995 & 11.854 & 177.21 & 29.71 & 38.34 & 481.2 & 12.84 & 42.1 \\
\hline 360 . & 0.2014 & 0.995 & 12.240 & 178.30 & 30.21 & 38.82 & 487.3 & 13.14 & 43.6 \\
\hline 370. & 0.1959 & 0.996 & 12.630 & 179.37 & 30.72 & 39.31 & 493.3 & 13.43 & 45.2 \\
\hline 380. & 0.1906 & 0.996 & 13.026 & 180.43 & 31.25 & 39.83 & 499.2 & 13.73 & 46.9 \\
\hline 390. & 0.1856 & 0.997 & 13.427 & 181.47 & 31.79 & 40.35 & 504.9 & 14.02 & 48.5 \\
\hline 400. & 0.1809 & 0.997 & 13.833 & 182.49 & 32.34 & 40.89 & 510.5 & 14.30 & 50.2 \\
\hline 410. & 0.1764 & 0.998 & 14.245 & 183.51 & 32.90 & 41.43 & 516.1 & & 52.0 \\
\hline 420. & 0.1722 & 0.998 & 14.662 & 184.52 & 33.47 & 41.99 & 521.5 & & 53.7 \\
\hline 430. & 0.1681 & 0.998 & 15.084 & 185.51 & 34.04 & 42.55 & 526.9 & & 55.5 \\
\hline 440. & 0.1642 & 0.999 & 15.513 & 186.50 & 34.62 & 43.11 & 532.2 & & 57.3 \\
\hline 450. & 0.1605 & 0.999 & 15.947 & 187.47 & 35.20 & 43.68 & 537.4 & & 59.1 \\
\hline 460. & 0.1570 & 0.999 & 16.386 & 188.44 & 35.77 & 44.25 & 542.6 & & 61.0 \\
\hline 470. & 0.1536 & 0.999 & 16.832 & 189.39 & 36.35 & 44.82 & 547.7 & & 62.8 \\
\hline 480. & 0.1504 & 1.000 & 17.283 & 190.34 & 36.93 & 45.39 & 552.7 & & 64.7 \\
\hline 490. & 0.1473 & 1.000 & 17.740 & 191.29 & 37.51 & 45.96 & 557.7 & & 66.6 \\
\hline 500. & 0.1443 & 1.000 & 18.202 & 192.22 & 38.08 & 46.53 & 562.6 & & 68.5 \\
\hline 520. & 0.1387 & 1.000 & 19.144 & 194.07 & 39.21 & 47.65 & 572.4 & & 72.3 \\
\hline
\end{tabular}


METHANE ISOBAR AT $P=0.600 \mathrm{MPa}$ (continued)

$\mathrm{T}$

$\mathrm{K} \quad \mathrm{mol} \cdot \mathrm{dm}^{-3}$

540.

560 .

580

600 .
Z

0.1336

0.1288

0.1243

0.1202
1.000

1.001

1.001

1.001
H

$\mathrm{kJ} / \mathrm{mol}$

20.108

21.094

22.101

23.129
S $\quad C_{\mathrm{v}}$

$\mathrm{C}_{\mathrm{p}}$

$\mathrm{J} /(\mathrm{mol} \cdot \mathrm{K}) \mathrm{J} /(\mathrm{mol} \cdot \mathrm{K}) \mathrm{J} /(\mathrm{mol} \cdot \mathrm{K})$

195.89

197.68

199.45

201.19
40.33

41.42

42.48

43.52
$48.75 \quad 581.9$

$49.83 \quad 591.3$

$50.89 \quad 600.6$

$51.92 \quad 609.7$ $\eta$

$\lambda$

$\mu \mathrm{Pa} \cdot \mathrm{s} \quad \mathrm{mW} /(\mathrm{m} \cdot \mathrm{K})$

76.2

80.1

84.1

88.0

\section{METHANE ISOBAR AT P $=0.650 \mathrm{MPa}$}

\begin{tabular}{|c|c|c|c|c|c|c|c|c|c|}
\hline $\mathrm{T}$ & $\rho$ & $\mathrm{Z}$ & H & $S$ & $C_{v}$ & $C_{p}$ & W & $\eta$ & $\lambda$ \\
\hline K & $\mathrm{mol} \cdot \mathrm{dm}^{-3}$ & & $\mathrm{~kJ} / \mathrm{mol}$ & $\mathrm{J} /(\mathrm{mol} \cdot \mathrm{K})$ & $\mathrm{J} /(\mathrm{mol} \cdot \mathrm{K})$ & $\mathrm{J} /(\mathrm{mol} \cdot \mathrm{K})$ & $\mathrm{m} \cdot \mathrm{s}^{-1}$ & $\mu \mathrm{Pa} \cdot \mathrm{s}$ & $\mathrm{mW} /(\mathrm{m} \cdot \mathrm{K}$ \\
\hline 95. & 27.814 & 0.030 & -5.499 & 70.21 & 34.01 & 53.69 & 1503.7 & 180.85 & 206.6 \\
\hline 100. & 27.392 & 0.029 & -5.228 & 72.99 & 34.10 & 54.57 & 1451.6 & 158.46 & 200.3 \\
\hline 05. & 26.960 & 0.028 & -4.953 & 75.66 & 33.88 & 55.20 & 1401.9 & 139.56 & 193.7 \\
\hline 10. & 26.518 & 0.027 & -4.676 & 78.24 & 33.51 & 55.74 & 1352.8 & 123.62 & 186.9 \\
\hline 5. & 26.062 & 0.026 & -4.396 & 80.73 & 33.09 & 56.30 & 1303.3 & 110.14 & 179.9 \\
\hline 0. & 25.592 & 0.025 & -4.113 & 83.14 & 32.66 & 56.92 & 1252.8 & 98.68 & 172.8 \\
\hline 5. & 25.104 & 0.025 & -3.826 & 85.48 & 32.25 & 57.67 & 1200.9 & 88.87 & 165.6 \\
\hline 0. & 24.595 & 0.024 & -3.536 & 87.76 & 31.87 & 58.58 & 1147.3 & 80.40 & 158.3 \\
\hline 5. & 24.062 & 0.024 & -3.240 & 89.99 & 31.53 & 59.71 & 1091.8 & 73.00 & 151.0 \\
\hline+0. & 23.499 & 0.024 & -2.938 & 92.19 & 31.22 & 61.13 & 1033.9 & 66.45 & 143.6 \\
\hline 0.251 & 23.468 & 0.024 & -2.922 & 92.30 & 31.21 & 61.23 & 1030.5 & 66.12 & 143.2 \\
\hline 140.251 & 0.6408 & 0.870 & 4.203 & 143.11 & 27.49 & 41.86 & 286.0 & 5.67 & 16.3 \\
\hline 145. & 0.6105 & 0.883 & 4.396 & 144.46 & 26.82 & 40.13 & 293.8 & 5.85 & 16.6 \\
\hline 150. & 0.5820 & 0.895 & 4.594 & 145.80 & 26.53 & 39.11 & 301.4 & 6.03 & 17.0 \\
\hline 55. & 0.5567 & 0.906 & 4.788 & 147.07 & 26.34 & 38.39 & 308.6 & 6.22 & 17.5 \\
\hline 160. & 0.5339 & 0.915 & 4.978 & 148.28 & 26.19 & 37.80 & 315.5 & 6.41 & 18.0 \\
\hline 165. & 0.5133 & 0.923 & 5.166 & 149.44 & 26.06 & 37.31 & 322.1 & 6.59 & 18.5 \\
\hline 170 . & 0.4945 & 0.930 & 5.351 & 150.54 & 25.95 & 36.90 & 328.4 & 6.78 & 19.1 \\
\hline 175. & 0.4773 & 0.936 & 5.535 & 151.61 & 25.86 & 36.55 & 334.5 & 6.96 & 19.6 \\
\hline 180 & 0.4614 & 0.941 & 5.717 & 152.63 & 25.79 & 36.26 & 340.5 & 7.15 & 20.2 \\
\hline 185. & 0.4466 & 0.946 & 5.898 & 153.62 & 25.73 & 36.01 & 346.2 & 7.33 & 20.8 \\
\hline 190. & 0.4329 & 0.950 & 6.077 & 154.58 & 25.69 & 35.81 & 351.8 & 7.51 & 21.4 \\
\hline 195 & 0.4201 & 0.954 & 6.256 & 155.51 & 25.66 & 35.64 & 357.2 & 7.69 & 21.9 \\
\hline 200. & 0.4081 & 0.958 & 6.434 & 156.41 & 25.65 & 35.50 & 362.5 & 7.87 & 22.4 \\
\hline 205 & 0.3969 & 0.961 & 6.611 & 157.28 & 25.65 & 35.39 & 367.7 & 8.05 & 23.0 \\
\hline 21 & 0.3863 & 0.964 & 6.787 & 158.14 & 25.66 & 35.30 & 372.7 & 8.23 & 23.5 \\
\hline 215 . & 0.3763 & 0.966 & 6.964 & 158.97 & 25.69 & 35.24 & 377.7 & 8.41 & 24.1 \\
\hline 220 & 0.3669 & 0.969 & 7.140 & 159.78 & 25.72 & 35.19 & 382.5 & 8.59 & 24.7 \\
\hline 22 & 0.3579 & 0.971 & 7.316 & 160.57 & 25.76 & 35.16 & 387.2 & 8.76 & 25.3 \\
\hline 230. & 0.3494 & 0.973 & 7.491 & 161.34 & 25.82 & 35.15 & 391.8 & 8.94 & 25.9 \\
\hline
\end{tabular}


METHANE ISOBAR AT $\mathrm{P}=0.650 \mathrm{MPa}$ (continued)

\begin{tabular}{|c|c|c|c|c|c|c|c|c|c|}
\hline $\mathrm{T}$ & $\rho$ & $\mathrm{z}$ & $\mathrm{H}$ & $S$ & $C_{v}$ & $C_{p}$ & W & $\eta$ & $\lambda$ \\
\hline K & $\mathrm{mol} \cdot \mathrm{dm}^{-3}$ & & $\mathrm{~kJ} / \mathrm{mol}$ & $\mathrm{J} /(\mathrm{mol} \cdot \mathrm{K})$ & $\mathrm{J} /(\mathrm{mol} \cdot \mathrm{K})$ & $\mathrm{J} /(\mathrm{mol} \cdot \mathrm{K})$ & $\mathrm{m} \cdot \mathrm{s}^{-1}$ & $\mu \mathrm{Pa} \cdot \mathrm{s}$ & $\mathrm{mW} /(\mathrm{m} \cdot \mathrm{K})$ \\
\hline 235. & 0.3413 & 0.975 & 7.667 & 162.10 & 25.88 & 35.16 & 396.3 & 9.11 & 26.4 \\
\hline 240 . & 0.3337 & 0.976 & 7.843 & 162.84 & 25.96 & 35.18 & 400.8 & 9.29 & 27.0 \\
\hline 245. & 0.3263 & 0.978 & 8.019 & 163.56 & 26.04 & 35.22 & 405.1 & 9.46 & 27.6 \\
\hline 250. & 0.3193 & 0.979 & 8.195 & 164.27 & 26.14 & 35.26 & 409.4 & 9.63 & 28.3 \\
\hline 255. & 0.3127 & 0.981 & 8.372 & 164.97 & 26.24 & 35.33 & 413.6 & 9.80 & 28.9 \\
\hline 260 . & 0.3063 & 0.982 & 8.549 & 165.66 & 26.35 & 35.40 & 417.7 & 9.97 & 29.5 \\
\hline 265 . & 0.3001 & 0.983 & 8.726 & 166.33 & 26.47 & 35.49 & 421.7 & 10.14 & 30.1 \\
\hline 270. & 0.2943 & 0.984 & 8.903 & 167.00 & 26.60 & 35.58 & 425.7 & 10.30 & 30.8 \\
\hline 275 . & 0.2886 & 0.985 & 9.082 & 167.65 & 26.74 & 35.69 & 429.6 & 10.47 & 31.4 \\
\hline 280 . & 0.2832 & 0.986 & 9.260 & 168.30 & 26.89 & 35.81 & 433.4 & 10.64 & 32.1 \\
\hline 285. & 0.2780 & 0.987 & 9.440 & 168.93 & 27.05 & 35.94 & 437.1 & 10.80 & 32.7 \\
\hline 290. & 0.2730 & 0.988 & 9.620 & 169.56 & 27.21 & 36.08 & 440.8 & 10.96 & 33.4 \\
\hline 295. & 0.2681 & 0.988 & 9.801 & 170.18 & 27.38 & 36.22 & 444.5 & 11.12 & 34.1 \\
\hline 300. & 0.2635 & 0.989 & 9.982 & 170.79 & 27.56 & 36.38 & 448.1 & 11.29 & 34.8 \\
\hline 310 . & 0.2547 & 0.990 & 10.348 & 171.98 & 27.94 & 36.72 & 455.1 & 11.60 & 36.1 \\
\hline 320. & 0.2464 & 0.991 & 10.717 & 173.16 & 28.35 & 37.09 & 461.8 & 11.92 & 37.6 \\
\hline 330. & 0.2387 & 0.992 & 11.089 & 174.30 & 28.78 & 37.49 & 468.5 & 12.23 & 39.0 \\
\hline 340. & 0.2315 & 0.993 & 11.467 & 175.43 & 29.24 & 37.92 & 474.9 & 12.54 & 40.6 \\
\hline 350. & 0.2247 & 0.994 & 11.848 & 176.53 & 29.72 & 38.38 & 481.2 & 12.84 & 42.1 \\
\hline 360 . & 0.2183 & 0.995 & 12.234 & 177.62 & 30.21 & 38.85 & 487.3 & 13.14 & 43.7 \\
\hline 370. & 0.2123 & 0.995 & 12.625 & 178.69 & 30.73 & 39.34 & 493.3 & 13.44 & 45.3 \\
\hline 380. & 0.2065 & 0.996 & 13.021 & 179.75 & 31.25 & 39.85 & 499.1 & 13.73 & 46.9 \\
\hline 390. & 0.2011 & 0.997 & 13.422 & 180.79 & 31.80 & 40.37 & 504.9 & 14.02 & 48.6 \\
\hline 400. & 0.1960 & 0.997 & 13.829 & 181.82 & 32.35 & 40.91 & 510.5 & 14.31 & 50.3 \\
\hline 410. & 0.1912 & 0.997 & 14.240 & 182.84 & 32.91 & 41.46 & 516.1 & & 52.0 \\
\hline 420. & 0.1865 & 0.998 & 14.658 & 183.84 & 33.47 & 42.01 & 521.5 & & 53.7 \\
\hline 430. & 0.1821 & 0.998 & 15.081 & 184.84 & 34.04 & 42.57 & 526.9 & & 55.5 \\
\hline 440. & 0.1779 & 0.998 & 15.509 & 185.82 & 34.62 & 43.13 & 532.2 & & 57.3 \\
\hline 450. & 0.1739 & 0.999 & 15.943 & 186.80 & 35.20 & 43.70 & 537.5 & & 59.1 \\
\hline 460. & 0.1701 & 0.999 & 16.383 & 187.76 & 35.78 & 44.27 & 542.6 & & 61.0 \\
\hline 470. & 0.1665 & 0.999 & 16.829 & 188.72 & 36.36 & 44.84 & 547.7 & & 62.8 \\
\hline 480. & 0.1629 & 0.999 & 17.280 & 189.67 & 36.93 & 45.41 & 552.8 & & 64.7 \\
\hline 490. & 0.1596 & 1.000 & 17.737 & 190.62 & 37.51 & 45.98 & 557.8 & & 66.6 \\
\hline 500. & 0.1564 & 1.000 & 18.199 & 191.55 & 38.08 & 46.54 & 562.7 & & 68.5 \\
\hline 520. & 0.1503 & 1.000 & 19.141 & 193.40 & 39.22 & 47.66 & 572.4 & & 72.4 \\
\hline 540. & 0.1447 & 1.000 & 20.106 & 195.22 & 40.33 & 48.76 & 582.0 & & 76.2 \\
\hline 560. & 0.1395 & 1.001 & 21.092 & 197.01 & 41.42 & 49.84 & 591.4 & & 80.1 \\
\hline 580. & 0.1347 & 1.001 & 22.099 & 198.78 & 42.48 & 50.90 & 600.7 & & 84.1 \\
\hline 600. & 0.1302 & 1.001 & 23.128 & 200.52 & 43.52 & 51.93 & 609.8 & & 88.0 \\
\hline
\end{tabular}


METHANE ISOBAR AT $P=0.700 \mathrm{MPa}$

\begin{tabular}{|c|c|c|c|c|c|c|c|c|c|}
\hline $\mathrm{T}$ & $\rho$ & $z$ & $\mathrm{H}$ & S & $C_{v}$ & $C_{p}$ & $\mathrm{~W}$ & $\eta$ & $\lambda$ \\
\hline K & $\mathrm{mol} \cdot \mathrm{dm}^{-3}$ & & $\mathrm{~kJ} / \mathrm{mol}$ & $\mathrm{J} /(\mathrm{mol} \cdot \mathrm{K})$ & $\mathrm{J} /(\mathrm{mol} \cdot \mathrm{K})$ & $\mathrm{J} /(\mathrm{mol} \cdot \mathrm{K})$ & $\mathrm{m} \cdot \mathrm{s}^{-1}$ & $\mu \mathrm{Pa} \cdot \mathrm{s}$ & $\mathrm{mW} /(\mathrm{m} \cdot \mathrm{K}$ \\
\hline 95. & 27.816 & 0.032 & -5.497 & 70.20 & 34.01 & 53.69 & 1504.1 & 180.95 & 206.7 \\
\hline 100. & 27.394 & 0.031 & -5.227 & 72.98 & 34.10 & 54.56 & 1452.0 & 158.55 & 200.4 \\
\hline 05. & 26.963 & 0.030 & -4.952 & 75.66 & 33.88 & 55.19 & 1402.4 & 139.65 & 193.7 \\
\hline 110 . & 26.521 & 0.029 & -4.675 & 78.24 & 33.51 & 55.73 & 1353.3 & 123.70 & 186.9 \\
\hline 15 . & 26.065 & 0.028 & -4.395 & 80.73 & 33.09 & 56.29 & 1303.8 & 110.21 & 179.9 \\
\hline 20. & 25.595 & 0.027 & -4.112 & 83.14 & 32.66 & 56.91 & 1253.3 & 98.75 & 172.8 \\
\hline 25. & 25.108 & 0.027 & -3.825 & 85.47 & 32.25 & 57.65 & 1201.5 & 88.93 & 165.7 \\
\hline 130. & 24.600 & 0.026 & -3.535 & 87.75 & 31.87 & 58.56 & 1148.0 & 80.46 & 158.4 \\
\hline 35. & 24.067 & 0.026 & -3.239 & 89.98 & 31.53 & 59.68 & 1092.6 & 73.06 & 151.1 \\
\hline 140. & 23.505 & 0.026 & -2.938 & 92.18 & 31.22 & 61.10 & 1034.8 & 66.51 & 143.7 \\
\hline 141.703 & 23.303 & 0.025 & -2.833 & 92.92 & 31.13 & 61.68 & 1014.1 & 64.42 & 141.2 \\
\hline 141.703 & 0.6885 & 0.863 & 4.223 & 142.71 & 27.62 & 42.46 & 286.2 & 5.74 & 16.6 \\
\hline 145. & 0.6653 & 0.873 & 4.359 & 143.66 & 27.06 & 41.02 & 291.8 & 5.86 & 16.8 \\
\hline 150. & 0.6331 & 0.886 & 4.561 & 145.03 & 26.67 & 39.75 & 299.7 & 6.05 & 17.2 \\
\hline 155. & 0.6048 & 0.898 & 4.757 & 146.32 & 26.46 & 38.91 & 307.1 & 6.23 & 17.6 \\
\hline 160. & 0.5795 & 0.908 & 4.950 & 147.55 & 26.29 & 38.24 & 314.1 & 6.42 & 18.1 \\
\hline 165. & 0.5566 & 0.917 & 5.140 & 148.71 & 26.15 & 37.69 & 320.8 & 6.61 & 18.6 \\
\hline 170 . & 0.5359 & 0.924 & 5.327 & 149.83 & 26.03 & 37.23 & 327.3 & 6.79 & 19.2 \\
\hline 175. & 0.5169 & 0.931 & 5.512 & 150.91 & 25.93 & 36.84 & 333.5 & 6.98 & 19.7 \\
\hline 180. & 0.4994 & 0.937 & 5.696 & 151.94 & 25.84 & 36.52 & 339.5 & 7.16 & 20.3 \\
\hline 185. & 0.4832 & 0.942 & 5.878 & 152.94 & 25.78 & 36.24 & 345.3 & 7.34 & 20.8 \\
\hline 190. & 0.4682 & 0.946 & 6.058 & 153.90 & 25.73 & 36.02 & 351.0 & 7.52 & 21.4 \\
\hline 195. & 0.4542 & 0.951 & 6.238 & 154.83 & 25.70 & 35.83 & 356.5 & 7.71 & 21.9 \\
\hline 200 . & 0.4411 & 0.954 & 6.417 & 155.74 & 25.69 & 35.67 & 361.8 & 7.89 & 22.5 \\
\hline 205. & 0.4288 & 0.958 & 6.595 & 156.62 & 25.68 & 35.54 & 367.1 & 8.07 & 23.0 \\
\hline 210 . & 0.4172 & 0.961 & 6.772 & 157.47 & 25.69 & 35.44 & 372.1 & 8.24 & 23.6 \\
\hline 215 . & 0.4064 & 0.964 & 6.949 & 158.30 & 25.71 & 35.37 & 377.1 & 8.42 & 24.2 \\
\hline 220 . & 0.3961 & 0.966 & 7.126 & 159.12 & 25.74 & 35.31 & 382.0 & 8.60 & 24.7 \\
\hline 225 . & 0.3864 & 0.968 & 7.302 & 159.91 & 25.79 & 35.28 & 386.7 & 8.78 & 25.3 \\
\hline 230 . & 0.3771 & 0.971 & 7.479 & 160.68 & 25.84 & 35.26 & 391.4 & 8.95 & 25.9 \\
\hline 235 . & 0.3684 & 0.973 & 7.655 & 161.44 & 25.90 & 35.26 & 39 & 9.12 & 26.5 \\
\hline 240 & 0.3600 & 0.974 & 7.831 & 162.19 & 25.98 & 35.27 & 400.4 & 9.30 & 27.1 \\
\hline 245 . & 0.3521 & 0.976 & 8.008 & 162.91 & 26.06 & 35.30 & 404.8 & 9.47 & 27.7 \\
\hline 250 . & 0.3445 & 0.978 & 8.184 & 163.63 & 26.15 & 35.35 & 409.1 & 9.64 & 28.3 \\
\hline 255. & 0.3372 & 0.979 & 8.361 & 164.33 & 26.25 & 35.40 & 413.3 & 9.81 & 28.9 \\
\hline 260 . & 0.3303 & 0.980 & 8.538 & 165.02 & 26.37 & 35.47 & 417.4 & 9.98 & 29.5 \\
\hline 265 . & 0.3237 & 0.982 & 8.716 & 165.69 & 26.49 & 35.55 & 421.4 & 10.15 & 30.2 \\
\hline 270 . & 0.3173 & 0.983 & 8.894 & 166.36 & 26.62 & 35.65 & 425.4 & 10.31 & 30.8 \\
\hline 27 & 0.3112 & 0.984 & 9.072 & 167.01 & 26.75 & 35.75 & 429.3 & 10.48 & 31.4 \\
\hline & 0.3053 & 0.985 & 9.251 & 167.66 & 26.90 & 35.87 & 433.2 & 10.64 & 32.1 \\
\hline
\end{tabular}


METHANE ISOBAR AT $\mathrm{P}=0.700 \mathrm{MPa}$ (continued)

\begin{tabular}{|c|c|c|c|c|c|c|c|c|c|}
\hline $\mathrm{T}$ & $\rho$ & $\mathrm{z}$ & H & $\mathrm{S}$ & $\mathrm{C}_{\mathrm{v}}$ & $C_{p}$ & $\mathrm{~W}$ & $\eta$ & $\lambda$ \\
\hline $\mathrm{K}$ & $\mathrm{mol} \cdot \mathrm{dm}^{-3}$ & & $\mathrm{~kJ} / \mathrm{mol}$ & $\mathrm{J} /(\mathrm{mol} \cdot \mathrm{K})$ & $\mathrm{J} /(\mathrm{mol} \cdot \mathrm{K})$ & $\mathrm{J} /(\mathrm{mol} \cdot \mathrm{K})$ & $m \cdot s^{-1}$ & $\mu \mathrm{Pa} \cdot \mathrm{s}$ & $\mathrm{mW} /(\mathrm{m} \cdot \mathrm{K})$ \\
\hline 285. & 0.2997 & 0.986 & 9.431 & 168.29 & 27.05 & 35.99 & 436.9 & 10.81 & 32.8 \\
\hline 290. & 0.2943 & 0.987 & 9.611 & 168.92 & 27.22 & 36.13 & 440.7 & 10.97 & 33.4 \\
\hline 295. & 0.2890 & 0.987 & 9.792 & 169.54 & 27.39 & 36.27 & 444.3 & 11.13 & 34.1 \\
\hline 300 . & 0.2840 & 0.988 & 9.974 & 170.15 & 27.57 & 36.43 & 447.9 & 11.29 & 34.8 \\
\hline 310 . & 0.2745 & 0.990 & 10.340 & 171.35 & 27.95 & 36.76 & 454.9 & 11.61 & 36.2 \\
\hline 320. & 0.2656 & 0.991 & 10.710 & 172.52 & 28.35 & 37.13 & 461.7 & 11.93 & 37.6 \\
\hline 330. & 0.2572 & 0.992 & 11.083 & 173.67 & 28.79 & 37.53 & 468.4 & 12.24 & 39.1 \\
\hline 340 . & 0.2494 & 0.993 & 11.460 & 174.80 & 29.24 & 37.96 & 474.8 & 12.55 & 40.6 \\
\hline 350 . & 0.2421 & 0.994 & 11.842 & 175.91 & 29.72 & 38.41 & 481.1 & 12.85 & 42.1 \\
\hline 360 . & 0.2352 & 0.994 & 12.229 & 176.99 & 30.22 & 38.88 & 487.2 & 13.15 & 43.7 \\
\hline 370 . & 0.2287 & 0.995 & 12.620 & 178.07 & 30.73 & 39.37 & 493.2 & 13.45 & 45.3 \\
\hline 380 . & 0.2225 & 0.996 & 13.016 & 179.12 & 31.26 & 39.88 & 499.1 & 13.74 & 46.9 \\
\hline 390. & 0.2167 & 0.996 & 13.417 & 180.16 & 31.80 & 40.40 & 504.9 & 14.03 & 48.6 \\
\hline 400. & 0.2112 & 0.997 & 13.824 & 181.19 & 32.35 & 40.93 & 510.5 & 14.32 & 50.3 \\
\hline 410. & 0.2059 & 0.997 & 14.236 & 182.21 & 32.91 & 41.48 & 516.1 & & 52.0 \\
\hline 420. & 0.2009 & 0.998 & 14.654 & 183.22 & 33.48 & 42.03 & 521.6 & & 53.8 \\
\hline 430. & 0.1962 & 0.998 & 15.077 & 184.21 & 34.05 & 42.59 & 526.9 & & 55.5 \\
\hline 440. & 0.1917 & 0.998 & 15.505 & 185.20 & 34.62 & 43.15 & 532.3 & & 57.3 \\
\hline 450. & 0.1873 & 0.999 & 15.940 & 186.17 & 35.20 & 43.72 & 537.5 & & 59.2 \\
\hline 460. & 0.1832 & 0.999 & 16.380 & 187.14 & 35.78 & 44.29 & 542.7 & & 61.0 \\
\hline 470. & 0.1793 & 0.999 & 16.825 & 188.10 & 36.36 & 44.85 & 547.8 & & 62.9 \\
\hline 480. & 0.1755 & 0.999 & 17.277 & 189.05 & 36.94 & 45.42 & 552.8 & & 64.7 \\
\hline 490. & 0.1719 & 1.000 & 17.734 & 189.99 & 37.51 & 45.99 & 557.8 & & 66.6 \\
\hline 500. & 0.1684 & 1.000 & 18.197 & 190.93 & 38.08 & 46.55 & 562.8 & & 68.5 \\
\hline 520. & 0.1619 & 1.000 & 19.139 & 192.78 & 39.22 & 47.67 & 572.5 & & 72.4 \\
\hline 540. & 0.1558 & 1.001 & 20.103 & 194.60 & 40.33 & 48.78 & 582.1 & & 76.3 \\
\hline 560. & 0.1502 & 1.001 & 21.090 & 196.39 & 41.42 & 49.85 & 591.5 & & 80.2 \\
\hline 580. & 0.1450 & 1.001 & 22.097 & 198.16 & 42.49 & 50.91 & 600.7 & & 84.1 \\
\hline 600. & 0.1402 & 1.001 & 23.126 & 199.90 & 43.52 & 51.94 & 609.9 & & 88.0 \\
\hline
\end{tabular}

$\overline{\text { METHANE ISOBAR AT } \mathrm{P}=0.750 \mathrm{MPa}}$

$\begin{array}{cccccccccc}\mathrm{T} & \rho & \mathrm{Z} & \mathrm{H} & \mathrm{S} & \mathrm{C}_{\mathrm{v}} & \mathrm{C}_{\mathrm{p}} & \mathrm{W} & \eta & \lambda \\ \mathrm{K} & \mathrm{mol} \cdot \mathrm{dm}^{-3} & & \mathrm{~kJ} / \mathrm{mol} & \mathrm{J} /(\mathrm{mol} \cdot \mathrm{K}) & \mathrm{J} /(\mathrm{mol} \cdot \mathrm{K}) & \mathrm{J} /(\mathrm{mol} \cdot \mathrm{K}) & \mathrm{m} \cdot \mathrm{s}^{-1} & \mu \mathrm{Pa} \cdot \mathrm{s} & \mathrm{mW} /(\mathrm{m} \cdot \mathrm{K}) \\ & & & & & & & & & \\ 95 . & 27.818 & 0.034 & -5.496 & 70.20 & 34.01 & 53.68 & 1504.5 & 181.05 & 206.7 \\ 100 . & 27.396 & 0.033 & -5.225 & 72.97 & 34.10 & 54.56 & 1452.4 & 158.64 & 200.4 \\ 105 . & 26.965 & 0.032 & -4.951 & 75.65 & 33.88 & 55.18 & 1402.9 & 139.73 & 193.8 \\ 110 . & 26.523 & 0.031 & -4.674 & 78.23 & 33.51 & 55.73 & 1353.8 & 123.77 & 187.0 \\ 115 . & 26.069 & 0.030 & -4.394 & 80.72 & 33.09 & 56.28 & 1304.4 & 110.28 & 180.0\end{array}$


METHANE ISOBAR AT $\mathrm{P}=0.750 \mathrm{MPa}$ (continued)

\begin{tabular}{|c|c|c|c|c|c|c|c|c|c|}
\hline $\mathrm{T}$ & $\rho$ & Z & $\mathrm{H}$ & $S$ & $\mathrm{C}_{\mathrm{v}}$ & $C_{p}$ & W & $\eta$ & $\lambda$ \\
\hline K & $\mathrm{mol} \cdot \mathrm{dm}^{-3}$ & & $\mathrm{~kJ} / \mathrm{mol}$ & $\mathrm{J} /(\mathrm{mol} \cdot \mathrm{K})$ & $\mathrm{J} /(\mathrm{mol} \cdot \mathrm{K})$ & $\mathrm{J} /(\mathrm{mol} \cdot \mathrm{K})$ & $\mathrm{m} \cdot \mathrm{s}^{-1}$ & $\mu \mathrm{Pa} \cdot \mathrm{s}$ & $\mathrm{mW} /(\mathrm{m}$ \\
\hline 120 . & 25.599 & 0.029 & -4.111 & 83.13 & 32.66 & 56.90 & 1253.9 & 98.81 & 172.9 \\
\hline 125. & 25.112 & 0.029 & -3.824 & 85.47 & 32.25 & 57.64 & 1202.1 & 88.99 & 165.7 \\
\hline 130 & 24.604 & 0.028 & -3.534 & 87.74 & 31.87 & 58.54 & 1148.7 & 80.52 & 158.5 \\
\hline 135. & 24.072 & 0.028 & -3.239 & 89.97 & 31.53 & 59.66 & 1093.4 & 73.11 & 151.2 \\
\hline 140 & 23.511 & 0.027 & -2.937 & 92.17 & 31.23 & 61.06 & 1035.6 & 66.56 & 143.8 \\
\hline 143.082 & 23.145 & 0.027 & -2.747 & 93.51 & 31.06 & 62.13 & 998.4 & 62.86 & 139.2 \\
\hline 143.082 & 0.7363 & 0.856 & 4.241 & 142.35 & 27.75 & 43.06 & 286.3 & 5.81 & 16.9 \\
\hline 145. & 0.7216 & 0.862 & 4.321 & 142.90 & 27.36 & 42.07 & 289.6 & 5.88 & 17.0 \\
\hline 150. & 0.6855 & 0.877 & 4.527 & 144.30 & 26.84 & 40.46 & 297.8 & 6.07 & 17.3 \\
\hline 155. & 0.6539 & 0.890 & 4.727 & 145.61 & 26.58 & 39.46 & 305.4 & 6.25 & 17.8 \\
\hline 160. & 0.6258 & 0.901 & 4.922 & 146.85 & 26.39 & 38.71 & 312.6 & 6.44 & 18.2 \\
\hline 165. & 0.6006 & 0.910 & 5.114 & 148.03 & 26.24 & 38.09 & 319.5 & 6.62 & 18.7 \\
\hline 170. & 0.5778 & 0.918 & 5.303 & 149.16 & 26.11 & 37.58 & 326.1 & 6.81 & 19.2 \\
\hline 175. & 0.5569 & 0.926 & 5.490 & 150.24 & 26.00 & 37.15 & 332.4 & 6.99 & 19.8 \\
\hline 180 . & 0.5378 & 0.932 & 5.675 & 151.28 & 25.90 & 36.79 & 338.5 & 7.17 & 20.3 \\
\hline 185. & 0.5201 & 0.937 & 5.858 & 152.29 & 25.83 & 36.48 & 344.4 & 7.36 & 20.9 \\
\hline 190. & 0.5037 & 0.942 & 6.039 & 153.26 & 25.78 & 36.23 & 350.2 & 7.54 & 21.5 \\
\hline 195. & 0.4885 & 0.947 & 6.220 & 154.20 & 25.74 & 36.02 & 355.7 & 7.72 & 22.0 \\
\hline 200 . & 0.4743 & 0.951 & 6.400 & 155.11 & 25.72 & 35.85 & 361.1 & 7.90 & 22.5 \\
\hline 205 . & 0.4609 & 0.955 & 6.579 & 155.99 & 25.71 & 35.70 & 366.4 & 8.08 & 23.1 \\
\hline 210 & 0.4484 & 0.958 & 6.757 & 156.85 & 25.72 & 35.59 & 371.6 & 8.26 & 23.7 \\
\hline 215 . & 0.4366 & 0.961 & 6.935 & 157.68 & 25.74 & 35.50 & 376.6 & 8.43 & 24.2 \\
\hline 220 . & 0.4255 & 0.964 & 7.112 & 158.50 & 25.77 & 35.44 & 381.5 & 8.61 & 24.8 \\
\hline 225 . & 0.4149 & 0.966 & 7.289 & 159.30 & 25.81 & 35.39 & 386.3 & 8.79 & 25.4 \\
\hline 230 & 0.4049 & 0.968 & 7.466 & 160.07 & 25.86 & 35.37 & 391.0 & 8.96 & 25.9 \\
\hline 235. & 0.3955 & 0.971 & 7.643 & 160.83 & 25.92 & 35 & & 9.14 & 26.5 \\
\hline 240. & 0.3865 & 0.973 & 7.819 & 161.58 & 25.99 & 35.37 & 400.0 & 9.31 & 27.1 \\
\hline 245. & 0.3779 & 0.974 & 7.996 & 162.31 & 26.07 & 35.39 & 404.4 & 9.48 & 27.7 \\
\hline 250 . & 0.3697 & 0.976 & 8.173 & 163.02 & 26.17 & 35.43 & 408.7 & 9.65 & 28.3 \\
\hline 255. & 0.3619 & 0.978 & 8.351 & 163.72 & 26.27 & 35.48 & 413.0 & 9.82 & 29.0 \\
\hline 260. & 0.3544 & 0.979 & 8.528 & 164.41 & 26.38 & & 417.1 & 9.99 & 29.6 \\
\hline 265. & 0.3472 & 0.980 & 8.706 & 165.09 & 26.50 & 35.62 & 421.2 & 10.16 & 30.2 \\
\hline 270. & 0.3404 & 0.981 & 8.884 & 165.76 & 26.63 & 35.71 & 425.2 & 10.32 & 30.8 \\
\hline 275 . & 0.3338 & 0.983 & 9.063 & 166.41 & 26.76 & 35.81 & 429.1 & 10.49 & 31.5 \\
\hline 280 & 0.3275 & 0.984 & 9.243 & 167.06 & 26.91 & 35.92 & 433.0 & 10.65 & 32.1 \\
\hline 285. & 0.3214 & 0.985 & 9.422 & 167.70 & 27.06 & 36.05 & 436.8 & 10.82 & 32.8 \\
\hline 290. & 0.3156 & 0.986 & 9.603 & 168.33 & 27.23 & 36.18 & 440.5 & 10.98 & 33.5 \\
\hline 295. & 0.3100 & 0.986 & 9.784 & 168.95 & 27.40 & 36.32 & 444.2 & 11.14 & 34.1 \\
\hline 300 & 0.3045 & 0.987 & 9.966 & 169.56 & 27.58 & 36.48 & 447.8 & 11.30 & 34.8 \\
\hline 310. & 0.2943 & 0.989 & 10.333 & 170.76 & 27.95 & 36.81 & 454.8 & 11.62 & 36.2 \\
\hline
\end{tabular}


METHANE ISOBAR AT $\mathrm{P}=0.750 \mathrm{MPa}$ (continued)

\begin{tabular}{|c|c|c|c|c|c|c|c|c|c|}
\hline $\mathrm{T}$ & $\rho$ & 2 & $\mathrm{H}$ & S & $c_{v}$ & $C_{p}$ & W & $\eta$ & $\lambda$ \\
\hline K & $\mathrm{mol} \cdot \mathrm{dm}^{-3}$ & & $\mathrm{~kJ} / \mathrm{mol}$ & $\mathrm{J} /(\mathrm{mol} \cdot \mathrm{K})$ & $\mathrm{J} /(\mathrm{mol} \cdot \mathrm{K})$ & $\mathrm{J} /(\mathrm{mol} \cdot \mathrm{K})$ & $\mathrm{m} \cdot \mathrm{s}^{-1}$ & $\mu \mathrm{Pa} \cdot \mathrm{s}$ & $\mathrm{mW} /(\mathrm{m} \cdot \mathrm{K})$ \\
\hline 320 . & 0.2847 & 0.990 & 10.703 & 171.93 & 28.36 & 37.17 & 461.6 & 11.94 & 37.6 \\
\hline 330. & 0.2758 & 0.991 & 11.076 & 173.08 & 28.79 & 37.57 & 468.3 & 12.25 & 39.1 \\
\hline 340 . & 0.2674 & 0.992 & 11.454 & 174.21 & 29.25 & 37.99 & 474.7 & 12.55 & 40.6 \\
\hline 350. & 0.2595 & 0.993 & 11.836 & 175.32 & 29.73 & 38.44 & 481.0 & 12.86 & 42.1 \\
\hline 360. & 0.2521 & 0.994 & 12.223 & 176.41 & 30.22 & 38.91 & 487.2 & 13.16 & 43.7 \\
\hline 370 . & 0.2451 & 0.995 & 12.614 & 177.48 & 30.74 & 39.40 & 493.2 & 13.45 & 45.3 \\
\hline 380 . & 0.2385 & 0.995 & 13.011 & 178.54 & 31.26 & 39.90 & 499.1 & 13.75 & 47.0 \\
\hline 390. & 0.2322 & 0.996 & 13.413 & 179.58 & 31.80 & 40.42 & 504.9 & 14.04 & 48.6 \\
\hline 400. & 0.2263 & 0.997 & 13.819 & 180.61 & 32.35 & 40.96 & 510.5 & 14.32 & 50.3 \\
\hline 410. & 0.2207 & 0.997 & 14.232 & 181.63 & 32.91 & 41.50 & 516.1 & & 52.0 \\
\hline 420. & 0.2153 & 0.997 & 14.649 & 182.64 & 33.48 & 42.05 & 521.6 & & 53.8 \\
\hline 430. & 0.2102 & 0.998 & 15.073 & 183.63 & 34.05 & 42.61 & 527.0 & & 55.6 \\
\hline 440. & 0.2054 & 0.998 & 15.502 & 184.62 & 34.63 & 43.17 & 532.3 & & 57.4 \\
\hline 450. & 0.2007 & 0.999 & 15.936 & 185.59 & 35.20 & 43.73 & 537.5 & & 59.2 \\
\hline 460. & 0.1963 & 0.999 & 16.376 & 186.56 & 35.78 & 44.30 & 542.7 & & 61.0 \\
\hline 470. & 0.1921 & 0.999 & 16.822 & 187.52 & 36.36 & 44.87 & 547.8 & & 62.9 \\
\hline 480. & 0.1880 & 0.999 & 17.274 & 188.47 & 36.94 & 45.44 & 552.9 & & 64.8 \\
\hline 490. & 0.1842 & 1.000 & 17.731 & 189.41 & 37.51 & 46.00 & 557.9 & & 66.6 \\
\hline 500. & 0.1804 & 1.000 & 18.194 & 190.35 & 38.09 & 46.57 & 562.8 & & 68.5 \\
\hline 520. & 0.1734 & 1.000 & 19.136 & 192.20 & 39.22 & 47.69 & 572.6 & & 72.4 \\
\hline 540. & 0.1670 & 1.001 & 20.101 & 194.02 & 40.33 & 48.79 & 582.2 & & 76.3 \\
\hline 560. & 0.1609 & 1.001 & 21.088 & 195.81 & 41.42 & 49.86 & 591.6 & & 80.2 \\
\hline 580. & 0.1554 & 1.001 & 22.095 & 197.58 & 42.49 & 50.92 & 600.8 & & 84.1 \\
\hline 600. & 0.1502 & 1.001 & 23.124 & 199.32 & 43.52 & 51.94 & 610.0 & & 88.0 \\
\hline
\end{tabular}

$\overline{\text { METHANE ISOBAR AT } \mathrm{P}=0.800 \mathrm{MPa}}$

$\begin{array}{rccccccccc}\mathrm{T} & \rho & \mathrm{Z} & \mathrm{H} & \mathrm{S} & \mathrm{C}_{\mathrm{v}} & \mathrm{C}_{\mathrm{p}} & \mathrm{W} & \eta & \lambda \\ \mathrm{K} & \mathrm{mol} \cdot \mathrm{dm}^{-3} & & \mathrm{~kJ} / \mathrm{mol} & \mathrm{J} /(\mathrm{mol} \cdot \mathrm{K}) & \mathrm{J} /(\mathrm{mol} \cdot \mathrm{K}) & \mathrm{J} /(\mathrm{mol} \cdot \mathrm{K}) & \mathrm{m} \cdot \mathrm{s}^{-1} & \mu \mathrm{Pa} \cdot \mathrm{s} & \mathrm{mW} /(\mathrm{m} \cdot \mathrm{K}) \\ & & & & & & & & & \\ \text { 95. } & 27.820 & 0.036 & -5.495 & 70.19 & 34.01 & 53.68 & 1504.9 & 181.15 & 206.8 \\ 100 . & 27.399 & 0.035 & -5.224 & 72.97 & 34.11 & 54.55 & 1452.8 & 158.73 & 200.5 \\ 105 . & 26.968 & 0.034 & -4.950 & 75.65 & 33.88 & 55.18 & 1403.3 & 139.81 & 193.9 \\ 110 . & 26.526 & 0.033 & -4.672 & 78.23 & 33.51 & 55.72 & 1354.3 & 123.85 & 187.0 \\ 115 . & 26.072 & 0.032 & -4.392 & 80.71 & 33.09 & 56.26 & 1304.9 & 110.35 & 180.1 \\ & & & & & & & & & \\ 120 . & 25.602 & 0.031 & -4.110 & 83.12 & 32.67 & 56.88 & 1254.5 & 98.87 & 173.0 \\ 125 . & 25.116 & 0.031 & -3.823 & 85.46 & 32.26 & 57.62 & 1202.8 & 89.06 & 165.8 \\ 130 . & 24.609 & 0.030 & -3.533 & 87.74 & 31.88 & 58.52 & 1149.4 & 80.58 & 158.5 \\ 135 . & 24.077 & 0.030 & -3.238 & 89.96 & 31.53 & 59.63 & 1094.1 & 73.17 & 151.2 \\ 140 . & 23.516 & 0.029 & -2.936 & 92.16 & 31.23 & 61.03 & 1036.5 & 66.62 & 143.9\end{array}$


METHANE ISOBAR AT $\mathrm{P}=0.800 \mathrm{MPa}$ (continued)$$
\mathrm{T}
$$

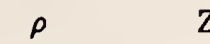

$\mathrm{K} \quad \mathrm{mol} \cdot \mathrm{dm}^{-3}$

Z

H

S

$\mathrm{C}_{\mathrm{v}} \quad \mathrm{C}_{\mathrm{p}}$

W

$\mathrm{kJ} / \mathrm{mol}$

$\mathrm{J} /(\mathrm{mol} \cdot \mathrm{K}) \mathrm{J} /(\mathrm{mol} \cdot \mathrm{K}) \mathrm{J} /(\mathrm{H}$

$\mathrm{J} /(\mathrm{mol} \cdot \mathrm{K})$

$\mathrm{m} \cdot \mathrm{s}^{-1}$

$\mu \mathrm{Pa} \cdot \mathrm{s} \quad \mathrm{mW} /(\mathrm{m} \cdot \mathrm{K})$

144.396

22.992

0.029

$\begin{array}{lll}-2.664 & 94.07 & 31.00\end{array}$

62.59

983.3

$61.41 \quad 137.3$

144.396

0.7843

0.850

$4.256 \quad 142.00$

27.88

43.68

286.3

5.88

17.1

145 .

0.7796

0.851

$\begin{array}{ll}4.281 & 142.17\end{array}$

27.74

43.31

287.4

5.90

17.2

155.

0.7040

0.882

41.23

296.0

6.08

17.5

$\begin{array}{lll}4.695 & 144.93 & 26.70\end{array}$

40.05

303.8

6.27

17.9

160

0.6730

0.894

$\begin{array}{lll}4.893 & 146.19\end{array}$

26.50

39.19

311.2

6.45

18.3

165.

0.6453

0.904

$5.087 \quad 147.38$

26.33

38.51

318.2

6.64

18.8

37.94

324.9

6.82

19.3

175 .

0.5975

0.920

$5.467 \quad 149.62$

26.06

37.46

331.3

7.00

19.9

337.5

7.19

20.4

185

0.5574

0.933

$5.838 \quad 151.68$

25.89

36.73

343.5

7.37

21.0

36.45

349.3

7.55

21.6

195.

0.5231

0.943

$6.202 \quad 153.60$

25.79

36.22

355.0

7.73

22.1

36.02

360.5

7.91

22.6

205.

0.4933

0.952

$6.562 \quad 155.40$

25.75

35.87

365.8

8.09

23.2

210

$$
0.4797
$$

0.955

$6.741 \quad 156.26$

25.75

35.74

371.0

8.27

23.7

220 .

0.4670

$\begin{array}{lll}6.920 & 157.10\end{array}$

25.76

35.64

376.0

8.45

24.3

35.56

381.0

8.62

24.8

25.83

35.51

385.8

8.80

25.4

230 .

0.4329

0.966

$7.453 \quad 159.50$

25.88

35.47

390.5

8.97

26.0

235.

$$
0.4227
$$

0.969

$\begin{array}{ll}7.630 & 160.26\end{array}$

25.94

35.46

395.1

9.15

35.46

399.7

9.32

26.6

35.48

404.1

9.49

27.2

35.51

408.4

9.66

27.8

35.56

412.7

9.83

28.4

29.0

260

0.3786

0.978

$8.518 \quad 163.85$

26.39

35.62

416.8

10.00

420.9

10.17

29.6

35.69

424.9

10.33

30.2

26.64

26.77

35.78

428.9

10.50

30.9

35.98

432.8

10.66

31.5

280 .

0.3565

0.983

$9.234 \quad 166.50$

26.92

36.10

436.6

10.83

36.23

440.3

10.99

11.15

36.53

444.0

11.31

27.58

36.53

447.6

11.31

11.63

$37.21 \quad 461.5$

11.94

12.25

12.56

474.7

12.56

481.0

12.86

13.16

32.2

350 .

0.2769

0.993

$11.830 \quad 174.77$

29.73

38.47

487.2

30.23

38.94 
METHANE ISOBAR AT $\mathrm{P}=0.800 \mathrm{MPa}$ (continued)

\begin{tabular}{|c|c|c|c|c|c|c|c|c|c|}
\hline $\mathrm{T}$ & $\rho$ & $z$ & $\mathrm{H}$ & S & $C_{v}$ & $C_{p}$ & W & $\eta$ & $\lambda$ \\
\hline K & $\mathrm{mol} \cdot \mathrm{dm}^{-3}$ & & $\mathrm{~kJ} / \mathrm{mol}$ & $\mathrm{J} /(\mathrm{mol} \cdot \mathrm{K})$ & $\mathrm{J} /(\mathrm{mol} \cdot \mathrm{K})$ & $\mathrm{J} /(\mathrm{mol} \cdot \mathrm{K})$ & $\mathrm{m} \cdot \mathrm{s}^{-1}$ & $\mu \mathrm{Pa} \cdot \mathrm{s}$ & $\mathrm{mW} /(\mathrm{m} \cdot \mathrm{K})$ \\
\hline 370. & 0.2615 & 0.994 & 12.609 & 176.93 & 30.74 & 39.43 & 493.2 & 13.46 & 45.3 \\
\hline 380 . & 0.2544 & 0.995 & 13.006 & 177.99 & 31.27 & 39.93 & 499.1 & 13.75 & 47.0 \\
\hline 390. & 0.2478 & 0.996 & 13.408 & 179.03 & 31.81 & 40.45 & 504.9 & 14.04 & 48.6 \\
\hline 400. & 0.2414 & 0.996 & 13.815 & 180.06 & 32.36 & 40.98 & 510.5 & 14.33 & 50.3 \\
\hline 410. & 0.2354 & 0.997 & 14.227 & 181.08 & 32.92 & 41.52 & 516.1 & & 52.1 \\
\hline 420. & 0.2297 & 0.997 & 14.645 & 182.09 & 33.48 & 42.07 & 521.6 & & 53.8 \\
\hline 430. & 0.2243 & 0.998 & 15.069 & 183.09 & 34.05 & 42.63 & 527.0 & & 55.6 \\
\hline 440. & 0.2191 & 0.998 & 15.498 & 184.07 & 34.63 & 43.19 & 532.3 & & 57.4 \\
\hline 450. & 0.2141 & 0.999 & 15.933 & 185.05 & 35.21 & 43.75 & 537.6 & & 59.2 \\
\hline 460. & 0.2094 & 0.999 & 16.373 & 186.02 & 35.79 & 44.32 & 542.7 & & 61.0 \\
\hline 470. & 0.2049 & 0.999 & 16.819 & 186.98 & 36.36 & 44.89 & 547.9 & & 62.9 \\
\hline 480. & 0.2006 & 0.999 & 17.271 & 187.93 & 36.94 & 45.45 & 552.9 & & 64.8 \\
\hline 490. & 0.1964 & 1.000 & 17.728 & 188.87 & 37.52 & 46.02 & 557.9 & & 66.7 \\
\hline 500. & 0.1925 & 1.000 & 18.191 & 189.81 & 38.09 & 46.58 & 562.9 & & 68.6 \\
\hline 520. & 0.1850 & 1.000 & 19.134 & 191.66 & 39.22 & 47.70 & 572.6 & & 72.4 \\
\hline 540. & 0.1781 & 1.001 & 20.099 & 193.48 & 40.33 & 48.80 & 582.2 & & 76.3 \\
\hline 560. & 0.1717 & 1.001 & 21.086 & 195.27 & 41.42 & 49.87 & 591.7 & & 80.2 \\
\hline 580. & 0.1657 & 1.001 & 22.094 & 197.04 & 42.49 & 50.93 & 600.9 & & 84.1 \\
\hline 600. & 0.1602 & 1.001 & 23.122 & 198.78 & 43.52 & 51.95 & 610.1 & & 88.1 \\
\hline
\end{tabular}

$\overline{\text { METHANE ISOBAR AT } \mathrm{P}=0.850 \mathrm{MPa}}$

$\begin{array}{lccccccccc}\mathrm{T} & \rho & \mathrm{Z} & \mathrm{H} & \mathrm{S} & \mathrm{C}_{\mathrm{v}} & \mathrm{C}_{\mathrm{p}} & \mathrm{W} & \eta & \lambda \\ \mathrm{K} & \mathrm{mol} \cdot \mathrm{dm}^{-3} & & \mathrm{~kJ} / \mathrm{mol} & \mathrm{J} /(\mathrm{mol} \cdot \mathrm{K}) & \mathrm{J} /(\mathrm{mol} \cdot \mathrm{K}) & \mathrm{J} /(\mathrm{mol} \cdot \mathrm{K}) & \mathrm{m} \cdot \mathrm{s}^{-1} & \mu \mathrm{Pa} \cdot \mathrm{s} & \mathrm{mW} /(\mathrm{m} \cdot \mathrm{K}) \\ & & & & & & & & & \\ 95 . & 27.822 & 0.039 & -5.493 & 70.19 & 34.01 & 53.67 & 1505.3 & 181.24 & 206.8 \\ 100 . & 27.401 & 0.037 & -5.223 & 72.96 & 34.11 & 54.55 & 1453.3 & 158.82 & 200.5 \\ 105 . & 26.971 & 0.036 & -4.948 & 75.64 & 33.88 & 55.17 & 1403.8 & 139.89 & 193.9 \\ 110 . & 26.529 & 0.035 & -4.671 & 78.22 & 33.52 & 55.71 & 1354.8 & 123.92 & 187.1 \\ 115 . & 26.075 & 0.034 & -4.391 & 80.71 & 33.10 & 56.25 & 1305.4 & 110.42 & 180.1 \\ & & & & & & & & & \\ 120 . & 25.606 & 0.033 & -4.109 & 83.11 & 32.67 & 56.87 & 1255.1 & 98.94 & 173.0 \\ 125 . & 25.119 & 0.033 & -3.822 & 85.45 & 32.26 & 57.61 & 1203.4 & 89.12 & 165.9 \\ 130 . & 24.613 & 0.032 & -3.532 & 87.73 & 31.88 & 58.50 & 1150.1 & 80.63 & 158.6 \\ 135 . & 24.082 & 0.031 & -3.237 & 89.95 & 31.53 & 59.61 & 1094.9 & 73.23 & 151.3 \\ 140 . & 23.522 & 0.031 & -2.936 & 92.15 & 31.23 & 61.00 & 1037.4 & 66.68 & 144.0 \\ & & & & & & & & & \\ 145 . & 22.926 & 0.031 & -2.626 & 94.32 & 30.97 & 62.78 & 977.0 & 60.80 & 136.5 \\ 145.651 & 22.844 & 0.031 & -2.585 & 94.60 & 30.94 & 63.06 & 968.7 & 60.07 & 135.5 \\ 145.651 & 0.8324 & 0.843 & 4.270 & 141.67 & 28.01 & 44.30 & 286.3 & 5.94 & 17.4 \\ 150 . & 0.7941 & 0.858 & 4.456 & 142.93 & 27.23 & 42.10 & 294.0 & 6.10 & 17.7 \\ 155 . & 0.7552 & 0.873 & 4.663 & 144.28 & 26.84 & 40.68 & 302.1 & 6.29 & 18.0\end{array}$


METHANE ISOBAR AT $\mathrm{P}=0.850 \mathrm{MPa}$ (continued)

$\mathrm{T}$

$\rho \quad z$

$\mathrm{K}$

$\mathrm{mol} \cdot \mathrm{dm}^{-3}$

$\mathrm{z}$

$\mathrm{H} \quad \mathrm{S}$

$\mathrm{kJ} / \mathrm{mol} \mathrm{J} /(\mathrm{mol} \cdot \mathrm{K}) \mathrm{J} /(\mathrm{mol} \cdot \mathrm{K})$

$\mathrm{C}_{\mathrm{p}} \quad \mathrm{W}$

$\mathrm{J} /(\mathrm{mol} \cdot \mathrm{K})$

$\mathrm{m} \cdot \mathrm{s}^{-1}$

$\mu \mathrm{Pa} \cdot \mathrm{s} \quad \mathrm{mW} /(\mathrm{m} \cdot \mathrm{K})$

160 .

0.7211

0.886

$4.864 \quad 145.56$

26.60

$5.060 \quad 146.77$

26.42

0.6907

0.897

5.253

147.92

170.

0.6385

0.907

5.444

149.02

180 .

0.6159

0.922

$5.631 \quad 150.08$

26.26

26.13

26.03

185

0.5950

0.929

$5.817 \quad 151.10$

25.94

$6.001 \quad 152.08$

190.

0.5758

0.934

6.184

153.03

200.

0.5580

0.940

6.366

153.95

0.5258

0.948

6.546

154.84

25.88

25.83

25.80

25.78

210.

215.

0.5113

0.952

$\begin{array}{lll}6.726 & 155.71\end{array}$

25.78

0.4976

0.956

$6.905 \quad 156.55$

220 .

225.

230 .

0.4847

0.959

7.084

157.37

0.4725

0.962

7.262

158.17

0.4610

0.964

$7.440 \quad 158.95$

235

0.4500

0.967

$\begin{array}{ll}7.618 & 159.72\end{array}$

0.4397

0.969

7.796

160.47

245.

0.4298

0.971

7.973

161.20

0.4204

0.973

8.151

161.92

255.

0.4114

0.974

8.329

162.63

260.

0.4028

0.976

8.508

163.32

8.686

164.00

0.3868

0.978

8.865

164.67

$9.045 \quad 165.33$

$9.225 \quad 165.97$

0.3720

0.980

0.982

0.3650

0.983

$9.405 \quad 166.61$

$9.586 \quad 167.24$

290.

0.3584

0.984

$9.768 \quad 167.86$

300 .

0.3519

0.985

$9.951 \quad 168.48$

310 .

0.987

$10.318 \quad 169.68$

320 .

330 .

340 .

350 .

360 .

370 .

380 .

390 .

400.

410 .

0.3340

0.989

$10.689 \quad 170.86$

28.37

37.26

37.64

38.06

29.26

38.50

$\begin{array}{lll}0.992 & 11.824 & 174.25\end{array}$

29.74

38.97

0.2859

0.993

$12.212 \quad 175.34$

30.23

30.74

39.45

39.96

40.47

31.81

32.36

41.00

41.54

$\begin{array}{ll}14.223 & 180.57\end{array}$

32.92

0.2502

0.996
$6.47 \quad 18.5$

$6.65 \quad 18.9$

$\begin{array}{ll}6.84 & 19.4\end{array}$

$7.02 \quad 20.0$
7.20

$7.20 \quad 20.5$

$7.38 \quad 21.1$

$\begin{array}{ll}7.56 & 21.7\end{array}$

$\begin{array}{ll}7.74 & 22.2\end{array}$

$\begin{array}{ll}7.92 & 22.7\end{array}$

$8.10 \quad 23.2$

$8.28 \quad 23.8$

$8.46 \quad 24.3$

$8.63 \quad 24.9$

$8.81 \quad 25.5$

$8.98 \quad 26.0$

390.1

$9.16 \quad 26.6$

394.7

9.33

27.2

9.50

27.8

408.1

9.67

28.4

412.4

9.84

29.0

416.6

10.01

29.7

420.7

10.18

30.3

424.7

10.34

30.9

428.7

10.51

31.6

432.6

10.67

32.2

$\begin{array}{lll}436.4 & 10.84 & 32.9\end{array}$

$\begin{array}{lll}440.1 & 11.00 & 33.5\end{array}$

$\begin{array}{lll}443.8 & 11.16 & 34.2\end{array}$

$\begin{array}{lll}447.5 & 11.32 & 34.9\end{array}$

$\begin{array}{lll}454.6 & 11.64 & 36.3\end{array}$

$\begin{array}{lll}461.4 & 11.95 & 37.7\end{array}$

$\begin{array}{lll}468.1 & 12.26 & 39.2\end{array}$

$\begin{array}{lll}474.6 & 12.57 & 40.7\end{array}$

$\begin{array}{lll}480.9 & 12.87 & 42.2\end{array}$

$\begin{array}{lll}487.1 & 13.17 & 43.8\end{array}$

$493.2 \quad 13.47 \quad 45.4$

$499.1 \quad 13.76 \quad 47.0$

$\begin{array}{lll}504.9 & 14.05 & 48.7\end{array}$

$\begin{array}{lll}510.5 & 14.34 & 50.4\end{array}$

516.1

52.1 
METHANE ISOBAR AT $\mathrm{P}=0.850 \mathrm{MPa}$ (continued)

\begin{tabular}{|c|c|c|c|c|c|c|c|c|c|}
\hline $\mathrm{T}$ & $\rho$ & $z$ & $\mathrm{H}$ & $S$ & $\mathrm{C}_{\mathrm{v}}$ & $C_{p}$ & W & $\eta$ & $\lambda$ \\
\hline K & $\mathrm{mol} \cdot \mathrm{dm}^{-3}$ & & $\mathrm{~kJ} / \mathrm{mol}$ & $\mathrm{J} /(\mathrm{mol} \cdot \mathrm{K})$ & $\mathrm{J} /(\mathrm{mol} \cdot \mathrm{K})$ & $\mathrm{J} /(\mathrm{mol} \cdot \mathrm{K})$ & $\mathrm{m} \cdot \mathrm{s}^{-1}$ & $\mu \mathrm{Pa} \cdot \mathrm{s}$ & $\mathrm{mW} /(\mathrm{m} \cdot \mathrm{K})$ \\
\hline 420. & 0.2441 & 0.997 & 14.641 & 181.58 & 33.49 & 42.09 & 521.6 & & 53.8 \\
\hline 430. & 0.2383 & 0.998 & 15.065 & 182.58 & 34.06 & 42.64 & 527.0 & & 55.6 \\
\hline 440. & 0.2328 & 0.998 & 15.494 & 183.56 & 34.63 & 43.20 & 532.3 & & 57.4 \\
\hline 450. & 0.2275 & 0.998 & 15.929 & 184.54 & 35.21 & 43.77 & 537.6 & & 59.2 \\
\hline 460. & 0.2225 & 0.999 & 16.369 & 185.51 & 35.79 & 44.33 & 542.8 & & 61.1 \\
\hline 470. & 0.2177 & 0.999 & 16.816 & 186.47 & 36.37 & 44.90 & 547.9 & & 62.9 \\
\hline 480. & 0.2131 & 0.999 & 17.268 & 187.42 & 36.94 & 45.47 & 553.0 & & 64.8 \\
\hline 490. & 0.2087 & 1.000 & 17.725 & 188.36 & 37.52 & 46.03 & 558.0 & & 66.7 \\
\hline 500. & 0.2045 & 1.000 & 18.188 & 189.30 & 38.09 & 46.59 & 563.0 & & 68.6 \\
\hline 520 . & 0.1965 & 1.000 & 19.131 & 191.15 & 39.22 & 47.71 & 572.7 & & 72.4 \\
\hline 540. & 0.1892 & 1.001 & 20.096 & 192.97 & 40.34 & 48.81 & 582.3 & & 76.3 \\
\hline 560. & 0.1824 & 1.001 & 21.083 & 194.76 & 41.43 & 49.88 & 591.7 & & 80.2 \\
\hline 580. & 0.1761 & 1.001 & 22.092 & 196.53 & 42.49 & 50.94 & 601.0 & & 84.1 \\
\hline 600. & 0.1701 & 1.001 & 23.121 & 198.28 & 43.53 & 51.96 & 610.1 & & 88.1 \\
\hline
\end{tabular}

$\overline{\text { METHANE ISOBAR AT } \mathrm{P}=0.900 \mathrm{MPa}}$

$\begin{array}{lccccccccc}\mathrm{T} & \rho & \mathrm{Z} & \mathrm{H} & \mathrm{S} & \mathrm{C} & \mathrm{C} & \mathrm{C} & \eta & \lambda \\ \mathrm{K} & \mathrm{mol} \cdot \mathrm{dm}^{-3} & & \mathrm{~kJ} / \mathrm{mol} & \mathrm{J} /(\mathrm{mol} \cdot \mathrm{K}) & \mathrm{J} /(\mathrm{mol} \cdot \mathrm{K}) & \mathrm{J} /(\mathrm{mol} \cdot \mathrm{K}) & \mathrm{m} \cdot \mathrm{s}^{-1} & \mu \mathrm{Pa} \cdot \mathrm{s} & \mathrm{mW} /(\mathrm{m} \cdot \mathrm{K}) \\ & & & & & & & & & \\ \text { 95. } & 27.824 & 0.041 & -5.492 & 70.18 & 34.02 & 53.67 & 1505.7 & 181.34 & 206.9 \\ 100 . & 27.403 & 0.040 & -5.222 & 72.96 & 34.11 & 54.54 & 1453.7 & 158.91 & 200.6 \\ 105 . & 26.973 & 0.038 & -4.947 & 75.63 & 33.88 & 55.16 & 1404.2 & 139.97 & 194.0 \\ 110 . & 26.532 & 0.037 & -4.670 & 78.21 & 33.52 & 55.70 & 1355.3 & 124.00 & 187.1 \\ 115 . & 26.078 & 0.036 & -4.390 & 80.70 & 33.10 & 56.24 & 1305.9 & 110.49 & 180.2 \\ & & & & & & & & & \\ 120 . & 25.609 & 0.035 & -4.107 & 83.11 & 32.67 & 56.86 & 1255.6 & 99.00 & 173.1 \\ 125 . & 25.123 & 0.034 & -3.821 & 85.44 & 32.26 & 57.59 & 1204.0 & 89.18 & 165.9 \\ 130 . & 24.617 & 0.034 & -3.531 & 87.72 & 31.88 & 58.48 & 1150.8 & 80.69 & 158.7 \\ 135 . & 24.087 & 0.033 & -3.236 & 89.95 & 31.53 & 59.58 & 1095.7 & 73.28 & 151.4 \\ 140 . & 23.528 & 0.033 & -2.935 & 92.14 & 31.23 & 60.97 & 1038.2 & 66.73 & 144.0 \\ 145 . & 22.932 & 0.033 & -2.626 & 94.30 & 30.97 & 62.74 & 978.0 & 60.86 & 136.6 \\ 146.855 & 22.699 & 0.032 & -2.509 & 95.11 & 30.89 & 63.53 & 954.6 & 58.81 & 133.8 \\ 146.855 & 0.8808 & 0.837 & 4.282 & 141.35 & 28.15 & 44.94 & 286.2 & 6.01 & 17.7 \\ 150 . & 0.8506 & 0.848 & 4.419 & 142.27 & 27.49 & 43.09 & 292.0 & 6.12 & 17.9 \\ 155 . & 0.8076 & 0.865 & 4.630 & 143.66 & 26.98 & 41.36 & 300.4 & 6.30 & 18.2 \\ & & & & & & & & & \\ 160 . & 0.7701 & 0.879 & 4.834 & 144.95 & 26.71 & 40.24 & 308.2 & 6.49 & 18.6 \\ 165 . & 0.7368 & 0.890 & 5.033 & 146.18 & 26.51 & 39.39 & 315.5 & 6.67 & 19.0 \\ 170 . & 0.7070 & 0.901 & 5.228 & 147.34 & 26.35 & 38.69 & 322.5 & 6.85 & 19.5 \\ 175 . & 0.6801 & 0.909 & 5.420 & 148.45 & 26.21 & 38.12 & 329.1 & 7.03 & 20.0 \\ 180 . & 0.6556 & 0.917 & 5.609 & 149.52 & 26.09 & 37.64 & 335.5 & 7.22 & 20.6\end{array}$


METHANE ISOBAR AT $\mathrm{P}=0.900 \mathrm{MPa}$ (continued)

$\mathrm{T}$

$\mathrm{K} \quad \mathrm{mol} \cdot \mathrm{dm}^{-3}$

$\begin{array}{lll}185 . & 0.6331 & 0.924 \\ 190 . & 0.6123 & 0.930 \\ 195 . & 0.5931 & 0.936 \\ 200 . & 0.5753 & 0.941 \\ 205 . & 0.5586 & 0.945\end{array}$

210 .

215 .

220 .

225 .

230 .

235 .

240 .

245 .

250 .

255 .

260

265 .

270 .

275 .

280 .

285

290 .

295.

300 .

310 .

320.

330 .

340 .

350 .

360 .

370

380 .

390 .

400 .

410 .

420.

430 .

440 .

450 .

460 .

Z

$\begin{array}{lll}0.5430 & 0.949\end{array}$

0.5283

0.953

0.5145

0.956

0.5015

0.959

0.4892

0.962

0.4775

0.965

0.4664

0.967

0.4559

0.969

0.4458

0.971

0.4363

0.973

0.4271

0.975

0.4100

0.978

0.3943

0.980

0. 3869

0.982

0.3730

0.3664

0.984

0.3539

0.985

0.3423

0.988

0.3315

0.990

0.3213

0.991

0. 3118

0.992

0.3028

0.993

0.2944

0.994

0.2864

0.995

0.2789

0.995

0.2717

0.996

0.996

0.2585

0.997

0.2524

0.997

0.2465

0.998

0.2409

0.998

0.999
H

S

$\mathrm{C}_{\mathrm{v}}$

$C_{p}$

W

$\mathrm{kJ} / \mathrm{mol} \mathrm{J} /(\mathrm{mol} \cdot \mathrm{K}) \mathrm{J} /(\mathrm{mol} \cdot \mathrm{K})$

$5.797 \quad 150.55$

5.982151 .53

$6.166 \quad 152.49$

$6.348 \quad 153.41$

$6.530 \quad 154.31$

$6.710 \quad 155.18$

6.890

156.03

7.070

156.85

7.248

157.66

7.427

158.44

7.605

159.21

7.784

159.96

7.962

160.69

8.140

161.41

8.319

162.12

8.497

162.81

8.676

163.50

8.856

164.17

9.035

164.83

9.216

165.48

9.396

166.12

$9.578 \quad 166.75$

$9.760 \quad 167.37$

$9.943 \quad 167.98$

$10.310 \quad 169.19$

$\begin{array}{ll}10.682 & 170.37\end{array}$

11.056

171.52

11.435

172.65

$11.818 \quad 173.76$

$12.206 \quad 174.86$

$12.599 \quad 175.93$

12.996

176.99

13.398

178.03

13.806

179.07

0.2649

$14.219 \quad 180.09$

$14.637 \quad 181.09$

15.061

182.09

15.490

183.08

15.925

184.06

$16.366 \quad 185.03$

26.00
25.92
25.87
25.83
25.81

25.81

25.82

25.84

25.87

25.92

25.98

26.04

26.12

26.21

26.31

26.42

26.53

26.66

26.79

26.94

27.09

27.25

27.42

27.60

27.98

28.38

28.81

29.27

29.74

30.24

30.75

31.28

31.81

32.36

32.92

33.49

34.06

34.63

35.21

35.79

\author{
$\mathrm{J} /(\mathrm{mol} \cdot \mathrm{K})$
}

37.24
36.90
36.62
36.39
36.20

341.7
347.7
353.5
359.1
364.5

$\begin{array}{ll}36.04 & 369.8\end{array}$

374.9

380.0

384.9

389.7

35.66

35.65

35.65

35.68

394.3

398.9

403.4

407.8

412.1

35.76

416.3

35.83

420.4

424.5

428.5

36.10

432.4

36.22

436.2

36.34

36.48

36.62

36.94

440.0

443.7

447.3

454.4

37.30

461.3

37.68

38.10

38.54

39.00

468.0

474.5

480.9

487.1

39.48

39.98

493.1

499.0

504.8

40.50

41.02

510.5

41.56

516.1

42.11

42.66

43.22

43.79

44.35
521.6

527.0

532.4

537.6

542.8

7.40

7.58

7.76

7.94

8.11

8.29

8.47

8.65

8.82

8.99

9.17

9. 34

9.51

9.68 
METHANE ISOBAR AT $\mathrm{P}=0.900 \mathrm{MPa}$ (continued)

\begin{tabular}{|c|c|c|c|c|c|c|c|c|c|}
\hline $\mathrm{T}$ & $\rho$ & $\mathrm{Z}$ & H & $\mathrm{S}$ & $C_{v}$ & $C_{p}$ & W & $\eta$ & $\lambda$ \\
\hline K & $\mathrm{mol} \cdot \mathrm{dm}^{-3}$ & & $\mathrm{~kJ} / \mathrm{mol}$ & $\mathrm{J} /(\mathrm{mol} \cdot \mathrm{K})$ & $\mathrm{J} /(\mathrm{mol} \cdot \mathrm{K})$ & $\mathrm{J} /(\mathrm{mol} \cdot \mathrm{K})$ & $\mathrm{m} \cdot \mathrm{s}^{-1}$ & $\mu \mathrm{Pa} \cdot \mathrm{s}$ & $\mathrm{mW} /(\mathrm{m}$ \\
\hline 470. & 0.2305 & 0.999 & 16.812 & 185.99 & 36.37 & 44.92 & 548.0 & & 62.9 \\
\hline 480. & 0.2257 & 0.999 & 17.264 & 186.94 & 36.95 & 45.48 & 553.0 & & 64.8 \\
\hline 490. & 0.2210 & 1.000 & 17.722 & 187.88 & 37.52 & 46.05 & 558.1 & & 66.7 \\
\hline 500. & 0.2165 & 1.000 & 18.185 & 188.82 & 38.09 & 46.61 & 563.0 & & 68.6 \\
\hline 520. & 0.2081 & 1.000 & 19.129 & 190.67 & 39.23 & 47.72 & 572.8 & & 72.4 \\
\hline 540. & 0.2003 & 1.001 & 20.094 & 192.49 & 40.34 & 48.82 & 582.4 & & 76.3 \\
\hline 560. & 0.1931 & 1.001 & 21.081 & 194.28 & 41.43 & 49.90 & 591.8 & & 80.2 \\
\hline 580. & 0.1864 & 1.001 & 22.090 & 196.05 & 42.49 & 50.95 & 601.1 & & 84.1 \\
\hline 600. & 0.1801 & 1.001 & 23.119 & 197.80 & 43.53 & 51.97 & 610.2 & & 88.1 \\
\hline
\end{tabular}

$\overline{\text { METHANE ISOBAR AT } \mathrm{P}=0.950 \mathrm{MPa}}$

\begin{tabular}{|c|c|c|c|c|c|c|c|c|c|}
\hline $\mathrm{T}$ & $\rho$ & $\mathrm{Z}$ & $\mathrm{H}$ & $S$ & $\mathrm{C}_{\mathrm{v}}$ & $C_{p}$ & W & $\eta$ & $\lambda$ \\
\hline K & $\mathrm{mol} \cdot \mathrm{dm}^{-3}$ & & $\mathrm{~kJ} / \mathrm{mol}$ & $\mathrm{J} /(\mathrm{mol} \cdot \mathrm{K})$ & $\mathrm{J} /(\mathrm{mol} \cdot \mathrm{K})$ & $\mathrm{J} /(\mathrm{mol} \cdot \mathrm{K})$ & $m \cdot s^{-1}$ & $\mu \mathrm{Pa} \cdot \mathrm{s}$ & $\mathrm{mW} /(\mathrm{m} \cdot \mathrm{K})$ \\
\hline 95. & 27.827 & 0.043 & -5.491 & 70.18 & 34.02 & 53.66 & 1506.1 & 181.44 & 206.9 \\
\hline 100. & 27.406 & 0.042 & -5.220 & 72.95 & 34.11 & 54.53 & 1454.1 & 159.00 & 200.6 \\
\hline 105. & 26.976 & 0.040 & -4.946 & 75.63 & 33.89 & 55.16 & 1404.7 & 140.05 & 194.0 \\
\hline 110. & 26.535 & 0.039 & -4.669 & 78.21 & 33.52 & 55.69 & 1355.8 & 124.07 & 187.2 \\
\hline 115. & 26.081 & 0.038 & -4.389 & 80.69 & 33.10 & 56.23 & 1306.5 & 110.55 & 180.2 \\
\hline 120. & 25.613 & 0.037 & -4.106 & 83.10 & 32.67 & 56.85 & 1256.2 & 99.07 & 173.1 \\
\hline 125. & 25.127 & 0.036 & -3.820 & 85.43 & 32.26 & 57.57 & 1204.6 & 89.24 & 166.0 \\
\hline 130. & 24.621 & 0.036 & -3.530 & 87.71 & 31.88 & 58.46 & 1151.5 & 80.75 & 158.8 \\
\hline 135. & 24.092 & 0.035 & -3.235 & 89.94 & 31.54 & 59.56 & 1096.4 & 73.34 & 151.5 \\
\hline 140. & 23.533 & 0.035 & -2.934 & 92.13 & 31.23 & 60.93 & 1039.1 & 66.79 & 144.1 \\
\hline 145. & 22.939 & 0.034 & -2.625 & 94.29 & 30.97 & 62.69 & 978.9 & 60.92 & 136.7 \\
\hline 148.011 & 22.559 & 0.034 & -2.435 & 95.60 & 30.84 & 64.01 & 940.9 & 57.64 & 132.2 \\
\hline 148.011 & 0.9295 & 0.831 & 4.293 & 141.05 & 28.28 & 45.59 & 286.1 & 6.07 & 18.0 \\
\hline 150. & 0.9088 & 0.838 & 4.381 & 141.64 & 27.79 & 44.23 & 289.9 & 6.14 & 18.1 \\
\hline 155. & 0.8611 & 0.856 & 4.597 & 143.05 & 27.15 & 42.11 & 298.6 & 6.32 & 18.4 \\
\hline 160. & 0.8200 & 0.871 & 4.804 & 144.37 & 26.83 & 40.82 & 306.6 & 6.50 & 18.7 \\
\hline 165. & 0.7837 & 0.884 & 5.005 & 145.61 & 26.61 & 39.86 & 314.1 & 6.69 & 19.2 \\
\hline 170. & 0.7514 & 0.894 & 5.203 & 146.79 & 26.43 & 39.09 & 321.2 & 6.87 & 19.6 \\
\hline 175. & 0.7222 & 0.904 & 5.396 & 147.91 & 26.28 & 38.46 & 328.0 & 7.05 & 20.1 \\
\hline 180. & 0.6957 & 0.912 & 5.587 & 148.99 & 26.15 & 37.94 & 334.5 & 7.23 & 20.7 \\
\hline 185. & 0.6715 & 0.920 & 5.776 & 150.02 & 26.05 & 37.50 & 340.8 & 7.41 & 21.2 \\
\hline 190. & 0.6492 & 0.926 & 5.963 & 151.02 & 25.97 & 37.14 & 346.8 & 7.59 & 21.8 \\
\hline 195. & 0.6286 & 0.932 & 6.147 & 151.98 & 25.91 & 36.83 & 352.7 & 7.77 & 22.3 \\
\hline 200. & 0.6094 & 0.937 & 6.331 & 152.90 & 25.87 & 36.58 & 358.4 & 7.95 & 22.8 \\
\hline 205. & 0.5916 & 0.942 & 6.513 & 153.81 & 25.85 & 36.37 & 363.8 & 8.13 & 23.3 \\
\hline
\end{tabular}


METHANE ISOBAR AT $P=0.950 \mathrm{MPa}$ (continued)

\begin{tabular}{|c|c|c|c|c|c|c|c|c|c|}
\hline $\mathrm{T}$ & $\rho$ & Z & H & $S$ & $C_{v}$ & $C_{p}$ & W & $\eta$ & $\lambda$ \\
\hline K & $\mathrm{mol} \cdot \mathrm{dm}^{-3}$ & & $\mathrm{~kJ} / \mathrm{mol}$ & $\mathrm{J} /(\mathrm{mol} \cdot \mathrm{K})$ & $\mathrm{J} /(\mathrm{mol} \cdot \mathrm{K})$ & $\mathrm{J} /(\mathrm{mol} \cdot \mathrm{K})$ & $\mathrm{m} \cdot \mathrm{s}^{-1}$ & $\mu \mathrm{Pa} \cdot \mathrm{s}$ & $\mathrm{mW} /(\mathrm{m} \cdot \mathrm{K}$ \\
\hline 210 . & 0.5749 & 0.946 & 6.695 & 154.68 & 25.84 & 36.20 & 369.2 & 8.30 & 23.9 \\
\hline 215 . & 0.5593 & 0.950 & 6.875 & 155.53 & 25.85 & 36.06 & 374.4 & 8.48 & 24.4 \\
\hline 220 . & 0.5445 & 0.954 & 7.055 & 156.36 & 25.86 & 35.95 & 379.5 & 8.66 & 25.0 \\
\hline 225 . & 0.5306 & 0.957 & 7.235 & 157.16 & 25.90 & 35.86 & 384.4 & 8.83 & 25.6 \\
\hline 230. & 0.5175 & 0.960 & 7.414 & 157.95 & 25.94 & 35.80 & 389.2 & 9.01 & 26.1 \\
\hline 35. & 0.5051 & 0.963 & 7.593 & 158.72 & 25.99 & 35.76 & 393.9 & 9.18 & 26.7 \\
\hline 240. & 0.4933 & 0.965 & 7.772 & 159.47 & 26.06 & 35.74 & 398.5 & 9.35 & 27.3 \\
\hline 245. & 0.4821 & 0.967 & 7.950 & 160.21 & 26.14 & 35.74 & 403.0 & 9.52 & 27.9 \\
\hline 250 . & 0.4714 & 0.970 & 8.129 & 160.93 & 26.22 & 35.76 & 407.5 & 9.69 & 28.5 \\
\hline 255. & 0.4612 & 0.971 & 8.308 & 161.64 & 26.32 & 35.79 & 411.8 & 9.86 & 29.1 \\
\hline 260 . & 0.4515 & 0.973 & 8.487 & 162.34 & 26.43 & 35.84 & 416.0 & 10.03 & 29.7 \\
\hline 265. & 0.4422 & 0.975 & 8.666 & 163.02 & 26.54 & 35.90 & 420.2 & 10.19 & 30.4 \\
\hline 270 . & 0.4333 & 0.977 & 8.846 & 163.69 & 26.67 & 35.97 & 424.2 & 10.36 & 31.0 \\
\hline 275 . & 0.4248 & 0.978 & 9.026 & 164.35 & 26.80 & 36.06 & 428.2 & 10.53 & 31.6 \\
\hline 280 & 0.4167 & 0.979 & 9.207 & 165.00 & 26.95 & 36.16 & 432.2 & 10.69 & 32.3 \\
\hline 285 . & 0.4088 & 0.981 & 9.388 & 165.64 & 27.10 & 36.27 & 436.0 & 10.85 & 32.9 \\
\hline 290. & 0.4013 & 0.982 & 9.569 & 166.28 & 27.26 & 36.40 & 439.8 & 11.02 & 33.6 \\
\hline 295. & 0.3941 & 0.983 & 9.752 & 166.90 & 27.43 & 36.53 & 443.5 & 11.18 & 34.3 \\
\hline 300 . & 0.3871 & 0.984 & 9.935 & 167.51 & 27.61 & 36.67 & 447.2 & 11.34 & 35.0 \\
\hline 310. & 0.3739 & 0.986 & 10.303 & 168.72 & 27.98 & 36.99 & 454.3 & 11.65 & 36.3 \\
\hline 320. & 0.3616 & 0.987 & 10.675 & 169.90 & 28.39 & 37.34 & 461.2 & 11.97 & 37.8 \\
\hline 330. & 0.3501 & 0.989 & 11.050 & 171.06 & 28.82 & 37.72 & 467.9 & 12.28 & 39.2 \\
\hline 340 . & 0.3394 & 0.990 & 11.429 & 172.19 & 29.27 & 38.13 & 474.5 & 12.58 & 40.7 \\
\hline 350. & 0.3293 & 0.991 & 11.813 & 173.30 & 29.75 & 38.57 & 480.8 & 12.89 & 42.3 \\
\hline 360. & 0.3198 & 0.992 & 12.201 & 174.39 & 30.24 & 39.03 & 487.0 & 13.19 & 43.8 \\
\hline 370. & 0.3109 & 0.993 & 12.593 & 175.47 & 30.75 & 39.51 & 493.1 & 13.48 & 45.4 \\
\hline 380. & 0.3024 & 0.994 & 12.991 & 176.53 & 31.28 & 40.01 & 499.0 & 13.77 & 47.1 \\
\hline 390. & 0.2944 & 0.995 & 13.393 & 177.58 & 31.82 & 40.52 & 504.8 & 14.06 & 48.7 \\
\hline 400. & 0.2869 & 0.996 & 13.801 & 178.61 & 32.37 & 41.05 & 510.5 & 14.35 & 50.4 \\
\hline 410 & 0.2797 & 0.996 & 14.214 & 179.63 & 32.93 & 41.58 & 516.1 & & 52.1 \\
\hline 420. & 0.2729 & 0.997 & 14.633 & 180.64 & 33.49 & 42.13 & 521.7 & & 53.9 \\
\hline 430. & 0.2664 & 0.997 & 15.057 & 181.63 & 34.06 & 42.68 & 527.1 & & 55.7 \\
\hline 440 & 0.2602 & 0.998 & 15.487 & 182.62 & 34.64 & 43.24 & 532.4 & & 57.5 \\
\hline 450. & 0.2544 & 0.998 & 15.922 & 183.60 & 35.21 & 43.80 & 537.7 & & 59.3 \\
\hline 460. & 0.2487 & 0.999 & 16.363 & 184.57 & 35.79 & 44.37 & 542.9 & & 61.1 \\
\hline 470. & 0.2434 & 0.999 & 16.809 & 185.53 & 36.37 & 44.93 & 548.0 & & 63.0 \\
\hline 480. & 0.2382 & 0.999 & 17.261 & 186.48 & 36.95 & 45.50 & 553.1 & & 64.8 \\
\hline 490. & 0.2333 & 1.000 & 17.719 & 187.42 & 37.52 & 46.06 & 558.1 & & 66.7 \\
\hline 500. & 0.2285 & 1.000 & 18.183 & 188.36 & 38.10 & 46.62 & 563.1 & & 68.6 \\
\hline 520. & 0.2197 & 1.000 & 19.126 & 190.21 & 39.23 & 47.73 & 572.9 & & 72.5 \\
\hline
\end{tabular}


METHANE ISOBAR AT $\mathrm{P}=0.950 \mathrm{MPa}$ (continued)

$\begin{array}{cccccccccc}\mathrm{T} & \rho & \mathrm{Z} & \mathrm{H} & \mathrm{S} & \mathrm{C}_{\mathrm{v}} & \mathrm{C}_{\mathrm{p}} & \mathrm{W} & \eta & \lambda \\ \mathrm{K} & \mathrm{mol} \cdot \mathrm{dm}^{-3} & & \mathrm{~kJ} / \mathrm{mol} & \mathrm{J} /(\mathrm{mol} \cdot \mathrm{K}) & \mathrm{J} /(\mathrm{mol} \cdot \mathrm{K}) & \mathrm{J} /(\mathrm{mol} \cdot \mathrm{K}) & \mathrm{m} \cdot \mathrm{s}^{-1} & \mu \mathrm{Pa} \cdot \mathrm{s} & \mathrm{mW} /(\mathrm{m} \cdot \mathrm{K}) \\ & & & & & & & & & \\ 540 . & 0.2114 & 1.001 & 20.092 & 192.03 & 40.34 & 48.83 & 582.5 & & 76.3 \\ 560 . & 0.2038 & 1.001 & 21.079 & 193.83 & 41.43 & 49.91 & 591.9 & 80.2 \\ 580 . & 0.1967 & 1.001 & 22.088 & 195.60 & 42.49 & 50.96 & 601.2 & 84.2 \\ 600 . & 0.1901 & 1.002 & 23.117 & 197.34 & 43.53 & 51.98 & 610.3 & 88.1\end{array}$

$\overline{\text { METHANE ISOBAR AT } \mathrm{P}=1.0 \mathrm{MPa}}$

\begin{tabular}{|c|c|c|c|c|c|c|c|c|c|}
\hline $\mathrm{T}$ & $\rho$ & Z & H & S & $C_{v}$ & $C_{p}$ & W & $\eta$ & $\lambda$ \\
\hline K & $\mathrm{mol} \cdot \mathrm{dm}^{-3}$ & & $\mathrm{~kJ} / \mathrm{mol}$ & $\mathrm{J} /(\mathrm{mol} \cdot \mathrm{K})$ & $\mathrm{J} /(\mathrm{mol} \cdot \mathrm{K})$ & $\mathrm{J} /(\mathrm{mol} \cdot \mathrm{K})$ & $\mathrm{m} \cdot \mathrm{s}^{-1}$ & $\mu \mathrm{Pa} \cdot \mathrm{s}$ & $\mathrm{mW} /(\mathrm{m} \cdot \mathrm{K})$ \\
\hline 95. & 27.829 & 0.045 & -5.490 & 70.17 & 34.02 & 53.66 & 1506.5 & 181.54 & 207.0 \\
\hline 100. & 27.408 & 0.044 & -5.219 & 72.95 & 34.11 & 54.53 & 1454.5 & 159.09 & 200.7 \\
\hline 105. & 26.978 & 0.042 & -4.945 & 75.62 & 33.89 & 55.15 & 1405.1 & 140.14 & 194.1 \\
\hline 110. & 26.538 & 0.041 & -4.668 & 78.20 & 33.52 & 55.68 & 1356.3 & 124.15 & 187.2 \\
\hline 115. & 26.084 & 0.040 & -4.388 & 80.69 & 33.10 & 56.22 & 1307.0 & 110.62 & 180.3 \\
\hline 120. & 25.616 & 0.039 & -4.105 & 83.09 & 32.67 & 56.83 & 1256.8 & 99.13 & 173.2 \\
\hline 125. & 25.131 & 0.038 & -3.819 & 85.43 & 32.26 & 57.56 & 1205.3 & 89.30 & 166.1 \\
\hline 130. & 24.626 & 0.038 & -3.529 & 87.70 & 31.88 & 58.44 & 1152.2 & 80.81 & 158.8 \\
\hline 135. & 24.097 & 0.037 & -3.235 & 89.93 & 31.54 & 59.53 & 1097.2 & 73.40 & 151.5 \\
\hline 140. & 23.539 & 0.036 & -2.934 & 92.12 & 31.23 & 60.90 & 1040.0 & 66.84 & 144.2 \\
\hline 145. & 22.945 & 0.036 & -2.625 & 94.28 & 30.97 & 62.65 & 979.9 & 60.97 & 136.8 \\
\hline 149.124 & 22.422 & 0.036 & -2.363 & 96.06 & 30.80 & 64.50 & 927.7 & 56.53 & 130.6 \\
\hline 149.124 & 0.9783 & 0.824 & 4.302 & 140.76 & 28.41 & 46.26 & 286.0 & 6.13 & 18.2 \\
\hline 150. & 0.9687 & 0.828 & 4.342 & 141.02 & 28.16 & 45.57 & 287.7 & 6.16 & 18.3 \\
\hline 155. & 0.9160 & 0.847 & 4.562 & 142.47 & 27.33 & 42.94 & 296.8 & 6.34 & 18.5 \\
\hline 160. & 0.8709 & 0.863 & 4.773 & 143.80 & 26.95 & 41.43 & 305.0 & 6.52 & 18.9 \\
\hline 165. & 0.8315 & 0.877 & 4.977 & 145.06 & 26.71 & 40.35 & 312.7 & 6.70 & 19.3 \\
\hline 170. & 0.7964 & 0.888 & 5.177 & 146.25 & 26.51 & 39.51 & 320.0 & 6.88 & 19.7 \\
\hline 175. & 0.7649 & 0.898 & 5.372 & 147.39 & 26.35 & 38.82 & 326.9 & 7.06 & 20.2 \\
\hline 180. & 0.7364 & 0.907 & 5.565 & 148.47 & 26.22 & 38.25 & 333.5 & 7.24 & 20.8 \\
\hline 185. & 0.7103 & 0.915 & 5.755 & 149.52 & 26.11 & 37.77 & 339.9 & 7.43 & 21.3 \\
\hline 190. & 0.6864 & 0.922 & 5.943 & 150.52 & 26.02 & 37.38 & 346.0 & 7.60 & 21.9 \\
\hline 195. & 0.6644 & 0.928 & 6.129 & 151.48 & 25.96 & 37.05 & 351.9 & 7.78 & 22.4 \\
\hline 200 . & 0.6439 & 0.934 & 6.313 & 152.42 & 25.91 & 36.77 & 357.6 & 7.96 & 22.9 \\
\hline 205. & 0.6248 & 0.939 & 6.497 & 153.32 & 25.88 & 36.54 & 363.2 & 8.14 & 23.4 \\
\hline 210. & 0.6070 & 0.943 & 6.679 & 154.20 & 25.87 & 36.35 & 368.6 & 8.32 & 23.9 \\
\hline 215. & 0.5904 & 0.948 & 6.860 & 155.06 & 25.87 & 36.20 & 373.8 & 8.49 & 24.5 \\
\hline 220 . & 0.5747 & 0.951 & 7.041 & 155.89 & 25.89 & 36.08 & 378.9 & 8.67 & 25.1 \\
\hline 225. & 0.5599 & 0.955 & 7.221 & 156.70 & 25.92 & 35.98 & 383.9 & 8.84 & 25.6 \\
\hline 230. & 0.5460 & 0.958 & 7.401 & 157.49 & 25.96 & 35.91 & 388.8 & 9.02 & 26.2 \\
\hline
\end{tabular}


METHANE ISOBAR AT $\mathrm{P}=1.0 \mathrm{MPa}$ (continued)

\begin{tabular}{|c|c|c|c|c|c|c|c|c|c|}
\hline $\mathrm{T}$ & $\rho$ & $\mathrm{Z}$ & $\mathrm{H}$ & s & $C_{v}$ & $C_{p}$ & W & $\eta$ & $\lambda$ \\
\hline K & $\mathrm{mol} \cdot \mathrm{dm}^{-3}$ & & $\mathrm{~kJ} / \mathrm{mol}$ & $\mathrm{J} /(\mathrm{mol} \cdot \mathrm{K})$ & $\mathrm{J} /(\mathrm{mol} \cdot \mathrm{K})$ & $\mathrm{J} /(\mathrm{mol} \cdot \mathrm{K})$ & $\mathrm{m} \cdot \mathrm{s}^{-1}$ & $\mu \mathrm{Pa} \cdot \mathrm{s}$ & $\mathrm{mW} /(\mathrm{m} \cdot \mathrm{K})$ \\
\hline 235. & 0.5328 & 0.961 & 7.580 & 158.26 & 26.01 & 35.87 & 393.5 & 9.19 & 26.8 \\
\hline 240 . & 0.5202 & 0.963 & 7.760 & 159.01 & 26.08 & 35.84 & 398.2 & 9.36 & 27.4 \\
\hline 245 . & 0.5084 & 0.966 & 7.939 & 159.75 & 26.15 & 35.83 & 402.7 & 9.53 & 28.0 \\
\hline 250 . & 0.4970 & 0.968 & 8.118 & 160.48 & 26.24 & 35.84 & 407.1 & 9.70 & 28.6 \\
\hline 255. & 0.4863 & 0.970 & 8.297 & 161.19 & 26.33 & 35.87 & 411.5 & 9.87 & 29.2 \\
\hline 260 . & 0.4760 & 0.972 & 8.477 & 161.88 & 26.44 & 35.91 & 415.7 & 10.04 & 29.8 \\
\hline 265. & 0.4661 & 0.974 & 8.656 & 162.57 & 26.56 & 35.97 & 419.9 & 10.20 & 30.4 \\
\hline 270 . & 0.4567 & 0.975 & 8.836 & 163.24 & 26.68 & 36.04 & 424.0 & 10.37 & 31.0 \\
\hline 275 . & 0.4477 & 0.977 & 9.017 & 163.90 & 26.82 & 36.12 & 428.0 & 10.54 & 31.7 \\
\hline 280 . & 0.4391 & 0.978 & 9.198 & 164.55 & 26.96 & 36.22 & 432.0 & 10.70 & 32.3 \\
\hline 285. & 0.4308 & 0.980 & 9.379 & 165.20 & 27.11 & 36.33 & 435.8 & 10.86 & 33.0 \\
\hline 290. & 0.4228 & 0.981 & 9.561 & 165.83 & 27.27 & 36.45 & 439.6 & 11.02 & 33.6 \\
\hline 295. & 0.4152 & 0.982 & 9.744 & 166.45 & 27.44 & 36.58 & 443.4 & 11.19 & 34.3 \\
\hline 300 . & 0.4078 & 0.983 & 9.927 & 167.07 & 27.61 & 36.72 & 447.0 & 11.35 & 35.0 \\
\hline 310. & 0.3939 & 0.985 & 10.296 & 168.28 & 27.99 & 37.03 & 454.2 & 11.66 & 36.4 \\
\hline 320 . & 0.3809 & 0.987 & 10.668 & 169.46 & 28.39 & 37.38 & 461.1 & 11.98 & 37.8 \\
\hline 330. & 0.3687 & 0.988 & 11.043 & 170.62 & 28.82 & 37.76 & 467.9 & 12.29 & 39.3 \\
\hline 340 . & 0.3574 & 0.990 & 11.423 & 171.75 & 29.28 & 38.17 & 474.4 & 12.59 & 40.8 \\
\hline 350. & 0.3468 & 0.991 & 11.807 & 172.86 & 29.75 & 38.60 & 480.8 & 12.89 & 42.3 \\
\hline 360 . & 0.3368 & 0.992 & 12.195 & 173.95 & 30.25 & 39.06 & 487.0 & 13.19 & 43.9 \\
\hline 370. & 0.3273 & 0.993 & 12.588 & 175.03 & 30.76 & 39.54 & 493.1 & 13.49 & 45.5 \\
\hline 380 . & 0.3184 & 0.994 & 12.986 & 176.09 & 31.28 & 40.03 & 499.0 & 13.78 & 47.1 \\
\hline 390. & 0.3100 & 0.995 & 13.389 & 177.14 & 31.82 & 40.55 & 504.8 & 14.07 & 48.7 \\
\hline 400. & 0.3021 & 0.995 & 13.797 & 178.17 & 32.37 & 41.07 & 510.5 & 14.36 & 50.4 \\
\hline 410. & 0.2945 & 0.996 & 14.210 & 179.19 & 32.93 & 41.61 & 516.2 & & 52.2 \\
\hline 420. & 0.2873 & 0.997 & 14.629 & 180.20 & 33.50 & 42.15 & 521.7 & & 53.9 \\
\hline 430. & 0.2805 & 0.997 & 15.053 & 181.20 & 34.07 & 42.70 & 527.1 & & 55.7 \\
\hline 440. & 0.2740 & 0.998 & 15.483 & 182.19 & 34.64 & 43.26 & 532.4 & & 57.5 \\
\hline 450. & 0.2678 & 0.998 & 15.918 & 183.17 & 35.22 & 43.82 & 537.7 & & 59.3 \\
\hline 460. & 0.2618 & 0.999 & 16.359 & 184.14 & 35.80 & 44.38 & 542.9 & & 61.1 \\
\hline 470. & 0.2562 & 0.999 & 16.806 & 185.10 & 36.37 & 44.95 & 548.1 & & 63.0 \\
\hline 480. & 0.2508 & 0.999 & 17.258 & 186.05 & 36.95 & 45.51 & 553.1 & & 64.9 \\
\hline 490. & 0.2456 & 1.000 & 17.716 & 186.99 & 37.53 & 46.07 & 558.2 & & 66.7 \\
\hline 500. & 0.2406 & 1.000 & 18.180 & 187.93 & 38.10 & 46.63 & 563.1 & & 68.6 \\
\hline 520. & 0.2312 & 1.000 & 19.124 & 189.78 & 39.23 & 47.75 & 572.9 & & 72.5 \\
\hline 540 . & 0.2226 & 1.001 & 20.090 & 191.60 & 40.34 & 48.84 & 582.5 & & 76.4 \\
\hline 560. & 0.2145 & 1.001 & 21.077 & 193.40 & 41.43 & 49.92 & 592.0 & & 80.3 \\
\hline 580. & 0.2071 & 1.001 & 22.086 & 195.17 & 42.49 & 50.96 & 601.3 & & 84.2 \\
\hline 600 . & 0.2001 & 1.002 & 23.116 & 196.91 & 43.53 & 51.99 & 610.4 & & 88.1 \\
\hline
\end{tabular}


METHANE ISOBAR AT $P=1.5 \mathrm{MPa}$

\begin{tabular}{|c|c|c|c|c|c|c|c|c|c|}
\hline $\mathrm{T}$ & $\rho$ & $\mathrm{Z}$ & $\mathrm{H}$ & $S$ & $\mathrm{C}_{\mathrm{v}}$ & $\mathrm{C}_{\mathrm{p}}$ & W & $\eta$ & $\lambda$ \\
\hline K & $\mathrm{mol} \cdot \mathrm{dm}^{-3}$ & & $\mathrm{~kJ} / \mathrm{mol}$ & $\mathrm{J} /(\mathrm{mol} \cdot \mathrm{K})$ & $\mathrm{J} /(\mathrm{mol} \cdot \mathrm{K})$ & $\mathrm{J} /(\mathrm{mol} \cdot \mathrm{K})$ & $m \cdot s^{-1}$ & $\mu \mathrm{Pa} \cdot \mathrm{s}$ & $\mathrm{mW} /(\mathrm{m} \cdot \mathrm{K})$ \\
\hline 95. & 27.850 & 0.068 & -5.477 & 70.12 & 34.04 & 53.61 & 1510.4 & 182.52 & 207.4 \\
\hline 100. & 27.432 & 0.066 & -5.206 & 72.89 & 34.13 & 54.47 & 1458.8 & 159.98 & 201.1 \\
\hline 105. & 27.004 & 0.064 & -4.932 & 75.56 & 33.90 & 55.08 & 1409.7 & 140.96 & 194.6 \\
\hline 110. & 26.566 & 0.062 & -4.656 & 78.14 & 33.54 & 55.60 & 1361.1 & 124.90 & 187.8 \\
\hline 115. & 26.115 & 0.060 & -4.376 & 80.62 & 33.12 & 56.12 & 1312.2 & 111.32 & 180.9 \\
\hline 120. & 25.650 & 0.059 & -4.094 & 83.02 & 32.69 & 56.71 & 1262.4 & 99.78 & 173.8 \\
\hline 125. & 25.169 & 0.057 & -3.809 & 85.35 & 32.28 & 57.41 & 1211.4 & 89.91 & 166.7 \\
\hline 130. & 24.668 & 0.056 & -3.520 & 87.62 & 31.90 & 58.25 & 1159.0 & 81.39 & 159.5 \\
\hline 135. & 24.145 & 0.055 & -3.226 & 89.83 & 31.55 & 59.29 & 1104.7 & 73.96 & 152.3 \\
\hline 140. & 23.595 & 0.055 & -2.927 & 92.01 & 31.25 & 60.59 & 1048.4 & 67.40 & 145.0 \\
\hline 145. & 23.010 & 0.054 & -2.620 & 94.17 & 30.98 & 62.23 & 989.6 & 61.53 & 137.7 \\
\hline 150. & 22.383 & 0.054 & -2.304 & 96.31 & 30.77 & 64.37 & 927.4 & 56.20 & 130.2 \\
\hline 155. & 21.700 & 0.054 & -1.975 & 98.47 & 30.61 & 67.25 & 861.0 & 51.26 & 122.6 \\
\hline 158.514 & 21.176 & 0.054 & -1.734 & 100.00 & 30.55 & 69.98 & 810.9 & 47.95 & 117.2 \\
\hline 158.514 & 1.485 & 0.766 & 4.339 & 138.32 & 29.70 & 53.79 & 283.4 & 6.70 & 20.8 \\
\hline 160. & 1.454 & 0.775 & 4.417 & 138.81 & 29.11 & 51.72 & 286.9 & 6.75 & 20.8 \\
\hline 165. & 1.365 & 0.801 & 4.664 & 140.32 & 28.02 & 47.41 & 297.3 & 6.91 & 20.8 \\
\hline 170. & 1.291 & 0.822 & 4.894 & 141.70 & 27.50 & 44.95 & 306.6 & 7.08 & 21.1 \\
\hline 175. & 1.227 & 0.840 & 5.115 & 142.98 & 27.16 & 43.26 & 315.0 & 7.25 & 21.4 \\
\hline 180. & 1.172 & 0.855 & 5.328 & 144.18 & 26.91 & 41.98 & 322.9 & 7.42 & 21.8 \\
\hline 185. & 1.123 & 0.868 & 5.535 & 145.31 & 26.71 & 40.97 & 330.3 & 7.59 & 22.3 \\
\hline 190. & 1.080 & 0.879 & 5.738 & 146.39 & 26.55 & 40.15 & 337.3 & 7.76 & 22.8 \\
\hline 195. & 1.040 & 0.890 & 5.937 & 147.43 & 26.42 & 39.48 & 344.0 & 7.93 & 23.2 \\
\hline 200. & 1.004 & 0.899 & 6.133 & 148.42 & 26.32 & 38.92 & 350.5 & 8.11 & 23.6 \\
\hline 205. & 0.9708 & 0.906 & 6.326 & 149.38 & 26.24 & 38.46 & 356.6 & 8.28 & 24.1 \\
\hline 210 . & 0.9403 & 0.914 & 6.517 & 150.30 & 26.19 & 38.08 & 362.6 & 8.45 & 24.6 \\
\hline 215. & 0.9120 & 0.920 & 6.707 & 151.19 & 26.16 & 37.76 & 368.3 & 8.62 & 25.1 \\
\hline 220 . & 0.8856 & 0.926 & 6.895 & 152.06 & 26.15 & 37.50 & 373.9 & 8.80 & 25.6 \\
\hline 225 . & 0.8610 & 0.931 & 7.082 & 152.90 & 26.15 & 37.28 & 379.2 & 8.97 & 26.2 \\
\hline 230 . & 0.8380 & 0.936 & 7.268 & 153.71 & 26.17 & 37.11 & 384.5 & 9.14 & 26.7 \\
\hline 235. & 0.8163 & 0.940 & 7.453 & 154.51 & 26.20 & 36.97 & 389.5 & 9.31 & 27.3 \\
\hline 240 . & 0.7959 & 0.945 & 7.638 & 155.29 & 26.25 & 36.86 & 394.5 & 9.47 & 27.9 \\
\hline 245 . & 0.7766 & 0.948 & 7.822 & 156.05 & 26.31 & 36.78 & 399.3 & 9.64 & 28.4 \\
\hline 250 . & 0.7583 & 0.952 & 8.006 & 156.79 & 26.39 & 36.73 & 404.0 & 9.81 & 29.0 \\
\hline 255. & 0.7410 & 0.955 & 8.189 & 157.52 & 26.47 & 36.70 & 408.6 & 9.98 & 29.6 \\
\hline 260. & 0.7245 & 0.958 & 8.373 & 158.23 & 26.57 & 36.69 & 413.1 & 10.14 & 30.2 \\
\hline 265. & 0.7089 & 0.960 & 8.556 & 158.93 & 26.67 & 36.70 & 417.4 & 10.31 & 30.8 \\
\hline 270 . & 0.6939 & 0.963 & 8.740 & 159.61 & 26.79 & 36.73 & 421.7 & 10.47 & 31.4 \\
\hline 275 . & 0.6797 & 0.965 & 8.923 & 160.29 & 26.92 & 36.77 & 425.9 & 10.63 & 32.1 \\
\hline 280 . & 0.6660 & 0.967 & 9.107 & 160.95 & 27.06 & 36.84 & 430.0 & 10.80 & 32.7 \\
\hline
\end{tabular}


METHANE ISOBAR AT $\mathrm{P}=1.5 \mathrm{MPa}$ (continued)

\begin{tabular}{|c|c|c|c|c|c|c|c|c|c|}
\hline $\mathrm{T}$ & $\rho$ & 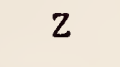 & $\mathrm{H}$ & S & $C_{v}$ & $C_{p}$ & W & $\eta$ & $\lambda$ \\
\hline K & $\mathrm{mol} \cdot \mathrm{dm}^{-3}$ & & $\mathrm{~kJ} / \mathrm{mol}$ & $\mathrm{J} /(\mathrm{mol} \cdot \mathrm{K})$ & $\mathrm{J} /(\mathrm{mol} \cdot \mathrm{K})$ & $\mathrm{J} /(\mathrm{mol} \cdot \mathrm{K})$ & $m \cdot s^{-1}$ & $\mu \mathrm{Pa} \cdot \mathrm{s}$ & $\mathrm{mW} /(\mathrm{m} \cdot \mathrm{K})$ \\
\hline 285. & 0.6530 & 0.969 & 9.292 & 161.60 & 27.20 & 36.91 & 434.0 & 10.96 & 33.4 \\
\hline 290. & 0.6405 & 0.971 & 9.476 & 162.25 & 27.36 & 37.00 & 438.0 & 11.12 & 34.0 \\
\hline 295. & 0.6285 & 0.973 & 9.662 & 162.88 & 27.52 & 37.11 & 441.8 & 11.28 & 34.7 \\
\hline 300 . & 0.6170 & 0.975 & 9.848 & 163.50 & 27.69 & 37.22 & 445.6 & 11.43 & 35.3 \\
\hline 310. & 0.5952 & 0.978 & 10.221 & 164.73 & 28.06 & 37.49 & 453.0 & 11.75 & 36.7 \\
\hline 320 . & 0.5751 & 0.980 & 10.597 & 165.92 & 28.46 & 37.80 & 460.2 & 12.06 & 38.1 \\
\hline 330. & 0.5563 & 0.983 & 10.977 & 167.09 & 28.88 & 38.14 & 467.1 & 12.37 & 39.6 \\
\hline 340 . & 0.5388 & 0.985 & 11.360 & 168.24 & 29.33 & 38.52 & 473.8 & 12.67 & 41.1 \\
\hline 350 . & 0.5225 & 0.987 & 11.748 & 169.36 & 29.80 & 38.93 & 480.3 & 12.97 & 42.6 \\
\hline 360. & 0.5071 & 0.988 & 12.139 & 170.46 & 30.29 & 39.36 & 486.7 & 13.27 & 44.1 \\
\hline 370. & 0.4927 & 0.990 & 12.535 & 171.55 & 30.80 & 39.82 & 492.9 & 13.56 & 45.7 \\
\hline 380 . & 0.4791 & 0.991 & 12.935 & 172.61 & 31.32 & 40.30 & 498.9 & 13.85 & 47.3 \\
\hline 390. & 0.4662 & 0.992 & 13.341 & 173.67 & 31.86 & 40.79 & 504.8 & 14.14 & 49.0 \\
\hline 400. & 0.4541 & 0.993 & 13.751 & 174.71 & 32.41 & 41.30 & 510.6 & 14.42 & 50.7 \\
\hline 410. & 0.4426 & 0.994 & 14.167 & 175.73 & 32.96 & 41.82 & 516.3 & & 52.4 \\
\hline 420. & 0.4316 & 0.995 & 14.588 & 176.75 & 33.53 & 42.36 & 521.9 & & 54.1 \\
\hline 430 . & 0.4213 & 0.996 & 15.014 & 177.75 & 34.10 & 42.90 & 527.4 & & 55.9 \\
\hline 440. & 0.4114 & 0.997 & 15.446 & 178.74 & 34.67 & 43.44 & 532.8 & & 57.7 \\
\hline 450. & 0.4020 & 0.997 & 15.883 & 179.72 & 35.25 & 43.99 & 538.2 & & 59.5 \\
\hline 460. & 0.3930 & 0.998 & 16.326 & 180.70 & 35.82 & 44.55 & 543.4 & & 61.3 \\
\hline 470. & 0.3844 & 0.998 & 16.774 & 181.66 & 36.40 & 45.10 & 548.6 & & 63.2 \\
\hline 480 . & 0.3762 & 0.999 & 17.228 & 182.62 & 36.97 & 45.66 & 553.7 & & 65.1 \\
\hline 490. & 0.3684 & 0.999 & 17.687 & 183.56 & 37.55 & 46.21 & 558.8 & & 66.9 \\
\hline 500. & 0.3609 & 1.000 & 18.152 & 184.50 & 38.12 & 46.77 & 563.8 & & 68.8 \\
\hline 520. & 0.3467 & 1.001 & 19.098 & 186.36 & 39.25 & 47.87 & 573.7 & & 72.7 \\
\hline 540. & 0.3337 & 1.001 & 20.067 & 188.19 & 40.36 & 48.95 & 583.3 & & 76.5 \\
\hline 560 . & 0.3216 & 1.002 & 21.056 & 189.99 & 41.45 & 50.02 & 592.8 & & 80.4 \\
\hline 580. & 0.3104 & 1.002 & 22.067 & 191.76 & 42.51 & 51.06 & 602.2 & & 84.3 \\
\hline 600. & 0.2999 & 1.003 & 23.098 & 193.51 & 43.54 & 52.07 & 611.4 & & 88.3 \\
\hline
\end{tabular}

$\overline{\text { METHANE ISOBAR AT } \mathrm{P}=2.0 \mathrm{MPa}}$

$\begin{array}{rccccccccc}\text { T } & \rho & \mathrm{Z} & \mathrm{H} & \mathrm{S} & \mathrm{C}_{\mathrm{v}} & \mathrm{C}_{\mathrm{p}} & \mathrm{W} & \eta & \lambda \\ \mathrm{K} & \mathrm{mol} \cdot \mathrm{dm}^{-3} & & \mathrm{~kJ} / \mathrm{mol} & \mathrm{J} /(\mathrm{mol} \cdot \mathrm{K}) & \mathrm{J} /(\mathrm{mol} \cdot \mathrm{K}) & \mathrm{J} /(\mathrm{mol} \cdot \mathrm{K}) & \mathrm{m} \cdot \mathrm{s}^{-1} & \mu \mathrm{Pa} \cdot \mathrm{s} & \mathrm{mW} /(\mathrm{m} \cdot \mathrm{K}) \\ & & & & & & & & & \\ 95 . & 27.872 & 0.091 & -5.464 & 70.06 & 34.05 & 53.56 & 1514.4 & 183.50 & 207.9 \\ 100 . & 27.455 & 0.088 & -5.194 & 72.83 & 34.14 & 54.41 & 1463.0 & 160.88 & 201.6 \\ 105 . & 27.029 & 0.085 & -4.920 & 75.50 & 33.92 & 55.01 & 1414.1 & 141.78 & 195.1 \\ 110 . & 26.593 & 0.082 & -4.644 & 78.07 & 33.56 & 55.51 & 1365.9 & 125.65 & 188.3 \\ 115 . & 26.145 & 0.080 & -4.365 & 80.55 & 33.14 & 56.02 & 1317.4 & 112.02 & 181.4\end{array}$


METHANE ISOBAR AT $\mathrm{P}=2.0 \mathrm{MPa}$ (continued)

\begin{tabular}{|c|c|c|c|c|c|c|c|c|c|}
\hline $\mathrm{T}$ & $\rho$ & $\mathrm{Z}$ & $\mathrm{H}$ & $S$ & $\mathrm{C}_{\mathrm{v}}$ & $C_{p}$ & W & $\eta$ & $\lambda$ \\
\hline K & $\mathrm{mol} \cdot \mathrm{dm}^{-3}$ & & $\mathrm{~kJ} / \mathrm{mol}$ & $\mathrm{J} /(\mathrm{mol} \cdot \mathrm{K})$ & $\mathrm{J} /(\mathrm{mol} \cdot \mathrm{K})$ & $\mathrm{J} /(\mathrm{mol} \cdot \mathrm{K})$ & $\mathrm{m} \cdot \mathrm{s}^{-1}$ & $\mu \mathrm{Pa} \cdot \mathrm{s}$ & $\mathrm{mW} /(\mathrm{m}$ \\
\hline 120. & 25.684 & 0.078 & -4.084 & 82.95 & 32.71 & 56.59 & 1268.0 & 100.43 & 174.4 \\
\hline 125. & 25.206 & 0.076 & -3.799 & 85.27 & 32.30 & 57.26 & 1217.5 & 90.52 & 167.3 \\
\hline 130. & 24.711 & 0.075 & -3.511 & 87.53 & 31.92 & 58.07 & 1165.6 & 81.97 & 160.2 \\
\hline 135. & 24.193 & 0.074 & -3.218 & 89.74 & 31.57 & 59.06 & 1112.1 & 74.52 & 153.0 \\
\hline 140. & 23.649 & 0.073 & -2.920 & 91.91 & 31.26 & 60.29 & 1056.7 & 67.96 & 145.8 \\
\hline 145. & 23.073 & 0.072 & -2.615 & 94.05 & 30.99 & 61.84 & 998.9 & 62.09 & 138.5 \\
\hline 150. & 22.458 & 0.071 & -2.301 & 96.18 & 30.77 & 63.83 & 938.2 & 56.76 & 131.2 \\
\hline 155. & 21.791 & 0.071 & -1.975 & 98.32 & 30.61 & 66.48 & 873.7 & 51.85 & 123.7 \\
\hline 160. & 21.054 & 0.071 & -1.634 & 100.48 & 30.52 & 70.18 & 804.0 & 47.23 & 116.0 \\
\hline 165. & 20.215 & 0.072 & -1.270 & 102.72 & 30.54 & 75.82 & 726.6 & 42.74 & 108.0 \\
\hline 165.860 & 20.057 & 0.072 & -1.205 & 103.12 & 30 . & 77.11 & 712.3 & 41.97 & 106.6 \\
\hline 165.860 & 2.036 & 0.712 & 4.303 & 136.32 & 30.99 & 63.76 & 279.2 & 7.24 & 23.5 \\
\hline 170. & 1.904 & 0.743 & 4.548 & 137.78 & 29.24 & 55.59 & 290.3 & 7.35 & 23.1 \\
\hline 175. & 1.779 & 0.773 & 4.812 & 139.32 & 28.30 & 50.57 & 301.4 & 7.49 & 23.1 \\
\hline 180. & 1.678 & 0.796 & 5.057 & 140.70 & 27.78 & 47.53 & 311.1 & 7.64 & 23.2 \\
\hline 185. & 1.593 & 0.816 & 5.289 & 141.97 & 27.42 & 45.42 & 319.9 & 7.79 & 23.5 \\
\hline 190. & 1.519 & 0.833 & 5.512 & 143.16 & 27.15 & 43.83 & 328.1 & 7.95 & 24.0 \\
\hline 195. & 1.454 & 0.848 & 5.728 & 144.28 & 26.94 & 42.59 & 335.8 & 8.11 & 24.1 \\
\hline 200 . & 1.397 & 0.861 & 5.939 & 145.34 & 26.77 & 41.60 & 343.0 & 8.28 & 24.5 \\
\hline 205. & 1.345 & 0.872 & 6.144 & 146.36 & 26.63 & 40.79 & 349.9 & 8.44 & 24.9 \\
\hline 210. & 1.298 & 0.883 & 6.347 & 147.34 & 26 & 40.13 & & & 25.3 \\
\hline 215. & 1.255 & 0.892 & 6.546 & 148.27 & 26.46 & 39.58 & 362.7 & 8.77 & 25.8 \\
\hline 220. & 1.215 & 0.900 & 6.743 & 149.18 & 26.42 & 39.13 & 368.8 & 8.94 & 26.3 \\
\hline 225. & 1.178 & 0.907 & 6.937 & 150.05 & 26.39 & 38.76 & 374.6 & 9.10 & 26.8 \\
\hline 230 . & 1.144 & 0.914 & 7.130 & 150.90 & 26.39 & 38.45 & 380.2 & 9.27 & 27.3 \\
\hline 235 . & 1.113 & 0.920 & 1.322 & (1. & 26 & 38. & & 9.44 & 27.8 \\
\hline 240 . & 1.083 & 0.925 & 7.512 & 152.53 & 26.43 & 37.98 & 390.9 & 9.60 & 28.4 \\
\hline 245. & 1.055 & 0.931 & 7.702 & 153.31 & 26.48 & 37.82 & 396.0 & 9.76 & 28.9 \\
\hline 250. & 1.029 & 0.935 & 7.891 & 154.07 & 26.54 & 37.69 & 400.9 & 9.93 & 29.5 \\
\hline 255. & 1.004 & 0.940 & 8.079 & 154.82 & 26.61 & 37.59 & 405.8 & 10.09 & 30.1 \\
\hline 260. & 0.98 & 0.94 & 8.2 & & & 37 & & & 30.7 \\
\hline 265. & 0.9584 & 0.947 & 8.454 & 156.26 & 26 . & 37.48 & 415.1 & 10.42 & 31.3 \\
\hline 270 . & 0.9373 & 0.951 & 8.641 & 156.96 & 26.90 & 37.46 & 419.5 & 10.58 & 31.9 \\
\hline 275 . & 0.9172 & 0.954 & 8.829 & 157.65 & 27.02 & 37.46 & 423.9 & 10.74 & 32.5 \\
\hline 280 . & 0.8981 & 0.957 & 9.016 & 158.32 & 27.15 & 37.48 & 428.2 & 10.90 & 33.1 \\
\hline 285. & 0.8798 & 0.959 & 9.204 & 158.99 & 27 . & 37.52 & 432.3 & 11.06 & 33.7 \\
\hline 290. & 0.8624 & 0.962 & 9.391 & 159.64 & 27.44 & 37.58 & 436.4 & 11.22 & 34.4 \\
\hline 295. & 0.8457 & 0.964 & 9.579 & 160.28 & 27.60 & 37.65 & 440.4 & 11.37 & 35.0 \\
\hline 300. & 0.8297 & 0.966 & 9.768 & 160.92 & 27.77 & 37.74 & 444.3 & 11.53 & 35.7 \\
\hline 310. & 0.7996 & 0.970 & 10.146 & 162.16 & 28.13 & 37.96 & 452.0 & 11.84 & 37.1 \\
\hline
\end{tabular}


METHANE ISOBAR AT $\mathrm{P}=2.0 \mathrm{MPa}$ (continued)

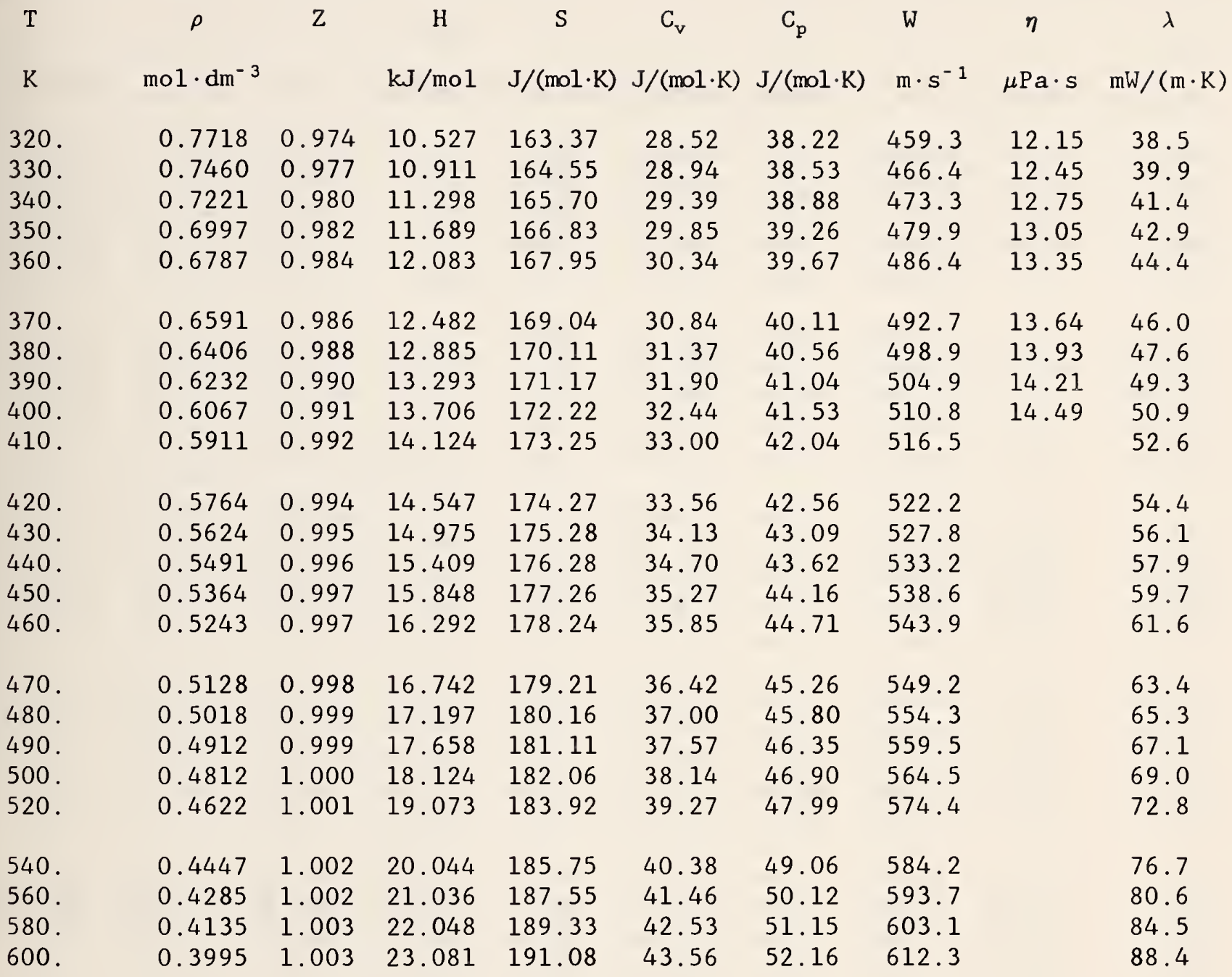

\section{METHANE ISOBAR AT $\mathrm{P}=2.5 \mathrm{MPa}$}

$\begin{array}{rccccccccc}\mathrm{T} & \rho & \mathrm{Z} & \mathrm{H} & \mathrm{S} & \mathrm{C}_{\mathrm{v}} & \mathrm{C}_{\mathrm{p}} & \mathrm{W} & \eta & \lambda \\ \mathrm{K} & \mathrm{mol} \cdot \mathrm{dm}^{-3} & & \mathrm{~kJ} / \mathrm{mol} & \mathrm{J} /(\mathrm{mol} \cdot \mathrm{K}) & \mathrm{J} /(\mathrm{mol} \cdot \mathrm{K}) & \mathrm{J} /(\mathrm{mol} \cdot \mathrm{K}) & \mathrm{m} \cdot \mathrm{s}^{-1} & \mu \mathrm{Pa} \cdot \mathrm{s} & \mathrm{mW} /(\mathrm{m} \cdot \mathrm{K}) \\ & & & & & & & & & \\ 95 . & 27.893 & 0.113 & -5.451 & 70.01 & 34.07 & 53.51 & 1518.2 & 184.49 & 208.3 \\ 100 . & 27.478 & 0.109 & -5.181 & 72.78 & 34.16 & 54.35 & 1467.1 & 161.78 & 202.1 \\ 105 . & 27.054 & 0.106 & -4.908 & 75.44 & 33.94 & 54.94 & 1418.6 & 142.60 & 195.6 \\ 110 . & 26.621 & 0.103 & -4.632 & 78.01 & 33.57 & 55.43 & 1370.6 & 126.41 & 188.9 \\ 115 . & 26.176 & 0.100 & -4.354 & 80.49 & 33.16 & 55.92 & 1322.4 & 112.71 & 182.0 \\ 120 . & 25.717 & 0.097 & -4.073 & 82.88 & 32.73 & 56.47 & 1273.5 & 101.08 & 175.0 \\ 125 . & 25.243 & 0.095 & -3.789 & 85.20 & 32.32 & 57.11 & 1223.5 & 91.13 & 168.0 \\ 130 . & 24.752 & 0.093 & -3.501 & 87.45 & 31.94 & 57.89 & 1172.2 & 82.55 & 160.9 \\ 135 . & 24.240 & 0.092 & -3.210 & 89.65 & 31.59 & 58.84 & 1119.3 & 75.08 & 153.8 \\ 140 . & 23.702 & 0.091 & -2.913 & 91.81 & 31.28 & 60.01 & 1064.8 & 68.50 & 146.6\end{array}$


METHANE ISOBAR AT $\mathrm{P}=2.5 \mathrm{MPa}$ (continued)

\begin{tabular}{|c|c|c|c|c|c|c|c|c|c|}
\hline $\mathrm{T}$ & $\rho$ & $\mathrm{Z}$ & H & S & $\mathrm{C}_{\mathrm{v}}$ & $C_{p}$ & W & $\eta$ & $\lambda$ \\
\hline K & $\mathrm{mol} \cdot \mathrm{dm}^{-3}$ & & $\mathrm{~kJ} / \mathrm{mol}$ & $\mathrm{J} /(\mathrm{mol} \cdot \mathrm{K})$ & $\mathrm{J} /(\mathrm{mol} \cdot \mathrm{K})$ & $\mathrm{J} /(\mathrm{mol} \cdot \mathrm{K})$ & $m \cdot s^{-1}$ & $\mu \mathrm{Pa} \cdot \mathrm{s}$ & $\mathrm{mW} /(\mathrm{m} \cdot \mathrm{K})$ \\
\hline 145. & 23.135 & 0.090 & -2.609 & 93.94 & 31.01 & 61.47 & 1008.0 & 62.63 & 139.4 \\
\hline 150. & 22.530 & 0.089 & -2.297 & 96.06 & 30.78 & 63.33 & 948.7 & 57.32 & 132.1 \\
\hline 155. & 21.878 & 0.089 & -1.975 & 98.17 & 30.60 & 65.77 & 885.9 & 52.44 & 124.8 \\
\hline 160. & 21.162 & 0.089 & -1.638 & 100.31 & 30.49 & 69.11 & 818.6 & 47.85 & 117.2 \\
\hline 165. & 20.357 & 0.090 & -1.281 & 102.51 & 30.48 & 74.02 & 744.8 & 43.45 & 109.4 \\
\hline 170. & 19.412 & 0.091 & -0.893 & 104.82 & 30.63 & 82.14 & 661.1 & 39.04 & 101.1 \\
\hline 171.980 & 18.978 & 0.092 & -0.726 & 105.80 & 30.77 & 87.13 & 623.9 & 37.24 & 97.6 \\
\hline 171.980 & 2.651 & 0.660 & 4.211 & 134.51 & 32.36 & 78.20 & 274.3 & 7.80 & 26.5 \\
\hline 175. & 2.490 & 0.690 & 4.428 & 135.76 & 30.48 & 66.54 & 284.3 & 7.84 & 25.8 \\
\hline 180. & 2.293 & 0.728 & 4.735 & 137.48 & 29.05 & 57.23 & 297.4 & 7.93 & 25.3 \\
\hline 185. & 2.143 & 0.758 & 5.007 & 138.98 & 28.33 & 52.26 & 308.4 & 8.06 & 25.2 \\
\hline 190. & 2.021 & 0.783 & 5.260 & 140.33 & 27.87 & 49.05 & 318.2 & 8.19 & 25.5 \\
\hline 195. & 1.919 & 0.804 & 5.499 & 141.57 & 27.53 & 46.76 & 327.0 & 8.33 & 25.3 \\
\hline 200 & 1.831 & 0.821 & 5.728 & 142.73 & 27.27 & 45.03 & 335.3 & 8.48 & 25.5 \\
\hline 205. & 1.753 & 0.837 & 5.950 & 143.83 & 27.07 & 43.69 & 343.0 & 8.63 & 25.8 \\
\hline 210 . & 1.684 & 0.850 & 6.166 & 144.86 & 26.91 & 42.61 & 350.2 & 8.79 & 26.2 \\
\hline 215 . & 1.622 & 0.862 & 6.377 & 145.86 & 26.79 & 41.74 & 357.1 & 8.94 & 26.6 \\
\hline 220 . & 1.566 & 0.873 & 6.583 & 146.81 & 26.71 & 41.03 & 363.7 & 9.10 & 27.0 \\
\hline 225 . & 1.514 & 0.883 & 6.787 & 147.72 & 26.65 & 40.44 & 370.0 & 9.26 & 27.5 \\
\hline 230 . & 1.467 & 0.891 & 6.988 & 148.61 & 26.62 & 39.96 & 376.0 & 9.42 & 28.0 \\
\hline 235 . & 1.423 & 0.899 & 7.187 & 149.46 & 26.61 & 39.56 & 381.8 & 9.58 & 28.5 \\
\hline 240 & 1.382 & 0.906 & 7.384 & 150.29 & 26.62 & 39.22 & 387.4 & 9.74 & 29.0 \\
\hline 245. & 1.345 & 0.913 & 7.579 & 151.10 & 26.65 & 38.95 & 392.8 & 9.90 & 29.5 \\
\hline 250 . & 1.309 & 0.919 & 7.773 & 151.88 & 26.69 & 38.73 & 398.0 & 10.06 & 30.0 \\
\hline 255. & 1.276 & 0.924 & 7.967 & 152.65 & 26.75 & 38.55 & 403.1 & 10.22 & 30.6 \\
\hline 260. & 1.245 & 0.929 & 8.159 & 153.39 & 26.83 & 38.41 & 408.0 & 10.38 & 31.2 \\
\hline 265. & 1.215 & 0.934 & 8.351 & 154.12 & 26.92 & 38.30 & 412.8 & 10.54 & 31.7 \\
\hline 270 . & 1.187 & 0.938 & 8.542 & 154.84 & 27.02 & 38.23 & 417.5 & 10.69 & 32.3 \\
\hline 275 . & 1.161 & 0.942 & 8.733 & 155.54 & 27.13 & 38.18 & 422.0 & 10.85 & 32.9 \\
\hline 280. & 1.135 & 0.946 & 8.924 & 156.23 & 27.25 & 38.16 & 426.4 & 11.01 & 33.5 \\
\hline 285. & 1.111 & 0.949 & 9.115 & 156.90 & 27.39 & 38.16 & 430.7 & 11.16 & 34.2 \\
\hline 290 . & 1.089 & 0.952 & 9.305 & 157.57 & 27.53 & 38.18 & 435.0 & 11.32 & 34.8 \\
\hline 295. & 1.067 & 0.955 & 9.496 & 158.22 & 27.69 & 38.22 & 439.1 & 11.48 & 35.4 \\
\hline 300 . & 1.046 & 0.958 & 9.688 & 158.86 & 27.85 & 38.28 & 443.1 & 11.63 & 36.1 \\
\hline 310. & 1.007 & 0.963 & 10.071 & 160.12 & 28.20 & 38.44 & 451.0 & 11.94 & 37.4 \\
\hline 320. & 0.9711 & 0.968 & 10.457 & 161.34 & 28.59 & 38.66 & 458.5 & 12.24 & 38.8 \\
\hline 330. & 0.9379 & 0.972 & 10.845 & 162.54 & 29.00 & 38.93 & 465.8 & 12.54 & 40.2 \\
\hline 340 . & 0.9070 & 0.975 & 11.235 & 163.70 & 29.44 & 39.25 & 472.8 & 12.84 & 41.7 \\
\hline 350 . & 0.8784 & 0.978 & 11.630 & 164.85 & 29.90 & 39.60 & 479.6 & 13.13 & 43.2 \\
\hline 360 . & 0.8516 & 0.981 & 12.027 & 165.97 & 30.39 & 39.98 & 486.2 & 13.43 & 44.7 \\
\hline
\end{tabular}


METHANE ISOBAR AT $\mathrm{P}=2.5 \mathrm{MPa}$ (continued)

\begin{tabular}{|c|c|c|c|c|c|c|c|c|c|}
\hline $\mathrm{T}$ & $\rho$ & Z & $\mathrm{H}$ & $S$ & $C_{v}$ & $C_{p}$ & $\mathrm{~W}$ & $\eta$ & $\lambda$ \\
\hline K & $\mathrm{mol} \cdot \mathrm{dm}^{-3}$ & & $\mathrm{~kJ} / \mathrm{mol}$ & $\mathrm{J} /(\mathrm{mol} \cdot \mathrm{K})$ & $\mathrm{J} /(\mathrm{mol} \cdot \mathrm{K})$ & $\mathrm{J} /(\mathrm{mol} \cdot \mathrm{K})$ & $\mathrm{m} \cdot \mathrm{s}^{-1}$ & $\mu \mathrm{Pa} \cdot \mathrm{s}$ & $\mathrm{mW} /(\mathrm{m} \cdot \mathrm{K})$ \\
\hline 370. & 0.8265 & 0.983 & 12.429 & 167.07 & 30.89 & 40.39 & 492.6 & 13.71 & 46.3 \\
\hline 380. & 0.8030 & 0.985 & 12.835 & 168.15 & 31.41 & 40.83 & 498.9 & 14.00 & 47.9 \\
\hline 390. & 0.7808 & 0.987 & 13.246 & 169.22 & 31.94 & 41.29 & 505.0 & 14.28 & 49.5 \\
\hline 400 & 0.7599 & 0.989 & 13.661 & 170.27 & 32.48 & 41.77 & 511.0 & 14.56 & 51.2 \\
\hline 410 & 0.7402 & 0.991 & 14.081 & 171.31 & 33.03 & 42.26 & 516.8 & & 52.9 \\
\hline 420. & 0.7215 & 0.992 & 14.507 & 172.33 & 33.59 & 42.77 & 522.5 & & 54.6 \\
\hline 430. & 0.7038 & 0.994 & 14.937 & 173.34 & 34.16 & 43.28 & 528.2 & & 56.4 \\
\hline 440 & 0.6870 & 0.995 & 15.372 & 174.35 & 34.73 & 43.81 & 533.7 & & 58.1 \\
\hline 450. & 0.6710 & 0.996 & 15.813 & 175.34 & 35.30 & 44.34 & 539.1 & & 59.9 \\
\hline 460 . & 0.6558 & 0.997 & 16.259 & 176.32 & 35.87 & 44.87 & 544.5 & & 61.8 \\
\hline 470. & 0.6412 & 0.998 & 16.710 & 177.29 & 36.45 & 45.41 & 549.8 & & 63.6 \\
\hline 480. & 0.6274 & 0.998 & 17.167 & 178.25 & 37.02 & 45.95 & 555.0 & & 65.5 \\
\hline 490. & 0.6141 & 0.999 & 17.629 & 179.20 & 37.59 & 46.49 & 560.1 & & 67.3 \\
\hline 500. & 0.6014 & 1.000 & 18.097 & 180.15 & 38.16 & 47.03 & 565.2 & & 69.2 \\
\hline 520. & 0.5776 & 1.001 & 19.048 & 182.01 & 39.29 & 48.11 & 575.2 & & 73.0 \\
\hline 540. & 0.5556 & 1.002 & 20.021 & 183.85 & 40.40 & 49.17 & 585.0 & & 76.9 \\
\hline 560. & 0.5353 & 1.003 & 21.015 & 185.65 & 41.48 & 50.22 & 594.6 & & 80.8 \\
\hline 580. & 0.5165 & 1.004 & 22.030 & 187.43 & 42.54 & 51.24 & 604.1 & & 84.7 \\
\hline 600. & 0.4990 & 1.004 & 23.065 & 189.19 & 43.57 & 52.24 & 613.3 & & 88.6 \\
\hline
\end{tabular}

METHANE ISOBAR AT $P=3.0 \mathrm{MPa}$

\begin{tabular}{|c|c|c|c|c|c|c|c|c|c|}
\hline $\mathrm{T}$ & $\rho$ & Z & $\mathrm{H}$ & S & $\mathrm{C}_{\mathrm{v}}$ & $C_{p}$ & W & $\eta$ & $\lambda$ \\
\hline K & $\mathrm{mol} \cdot \mathrm{dm}^{-3}$ & & $\mathrm{~kJ} / \mathrm{mol}$ & $\mathrm{J} /(\mathrm{mol} \cdot \mathrm{K})$ & $\mathrm{J} /(\mathrm{mol} \cdot \mathrm{K})$ & $\mathrm{J} /(\mathrm{mol} \cdot \mathrm{K})$ & $\mathrm{m} \cdot \mathrm{s}^{-1}$ & $\mu \mathrm{Pa} \cdot \mathrm{s}$ & $\mathrm{mW} /(\mathrm{m} \cdot \mathrm{K})$ \\
\hline 95. & 27.914 & 0.136 & -5.438 & 69.96 & 34.09 & 53.47 & 1522.1 & 185.48 & 208.8 \\
\hline 100. & 27.501 & 0.131 & -5.168 & 72.72 & 34.18 & 54.30 & 1471.2 & 162.69 & 202.6 \\
\hline 105. & 27.079 & 0.127 & -4.895 & 75.39 & 33.96 & 54.87 & 1422.9 & 143.43 & 196.1 \\
\hline 110. & 26.648 & 0.123 & -4.620 & 77.95 & 33.59 & 55.35 & 1375.3 & 127.17 & 189.4 \\
\hline 115. & 26.206 & 0.120 & -4.342 & 80.42 & 33.17 & 55.83 & 1327.5 & 113.41 & 182.6 \\
\hline 120 & 25.750 & 0.117 & -4.062 & 82.81 & 32.75 & 56.35 & 1278.9 & 101.73 & 175.6 \\
\hline 125. & 25.280 & 0.114 & -3.778 & 85.12 & 32.34 & 56.97 & 1229.3 & 91.74 & 168.6 \\
\hline 130. & 24.793 & 0.112 & -3.492 & 87.37 & 31.96 & 57.72 & 1178.6 & 83.13 & 161.6 \\
\hline 135. & 24.285 & 0.110 & -3.201 & 89.56 & 31.61 & 58.62 & 1126.4 & 75.64 & 154.5 \\
\hline 140. & 23.754 & 0.108 & -2.905 & 91.72 & 31.30 & 59.74 & 1072.6 & 69.05 & 147.4 \\
\hline 145. & 23.195 & 0.107 & -2.603 & 93.83 & 31.02 & 61.12 & 1016.9 & 63.18 & 140.2 \\
\hline 150 & 22.600 & 0.106 & -2.293 & 95.93 & 30.79 & 62.86 & 958.7 & 57.87 & 133.0 \\
\hline 155 & 21.962 & 0.106 & -1.974 & 98.03 & 30.60 & 65.12 & 897.6 & 53.01 & 125.8 \\
\hline 160 & 21.265 & 0.106 & -1.641 & 100.14 & 30.48 & 68.15 & 832.4 & 48.46 & 118.4 \\
\hline 165. & 20.489 & 0.107 & -1.290 & 102.30 & 30.44 & 72.48 & 761.8 & 44.13 & 110.7 \\
\hline
\end{tabular}


METHANE ISOBAR AT $\mathrm{P}=3.0 \mathrm{MPa}$ (continued)

\begin{tabular}{|c|c|c|c|c|c|c|c|c|c|}
\hline $\mathrm{T}$ & $\rho$ & Z & $\mathrm{H}$ & $S$ & $C_{v}$ & $C_{p}$ & W & $\eta$ & $\lambda$ \\
\hline K & $\mathrm{mol} \cdot \mathrm{dm}^{-3}$ & & $\mathrm{~kJ} / \mathrm{mol}$ & $\mathrm{J} /(\mathrm{mol} \cdot \mathrm{K})$ & $\mathrm{J} /(\mathrm{mol} \cdot \mathrm{K})$ & $\mathrm{J} /(\mathrm{mol} \cdot \mathrm{K})$ & $m \cdot s^{-1}$ & $\mu \mathrm{Pa} \cdot \mathrm{s}$ & $\mathrm{mW} /(\mathrm{m} \cdot \mathrm{K})$ \\
\hline 170. & 19.593 & 0.108 & -0.912 & 104.56 & 30.53 & 79.26 & 683.1 & 39.85 & 102.7 \\
\hline 175. & 18.493 & 0.111 & -0.488 & 107.02 & 30.87 & 91.95 & 590.9 & 35.39 & 94.0 \\
\hline 177.262 & 17.876 & 0.114 & -0.270 & 108.26 & 31.19 & 102.68 & 541.8 & 33.18 & 89.7 \\
\hline 177.262 & 3.359 & 0.606 & 4.064 & 132.70 & 33.91 & 101.61 & 268.7 & 8.43 & 30.3 \\
\hline 180 & 3.120 & 0.642 & 4.309 & 134.08 & 31.56 & 80.48 & 280.0 & 8.39 & 28.9 \\
\hline 185. & 2.825 & 0.690 & 4.666 & 136.03 & 29.68 & 64.62 & 295.2 & 8.42 & 27.8 \\
\hline 190. & 2.615 & 0.726 & 4.969 & 137.65 & 28.79 & 57.18 & 307.3 & 8.50 & 27.6 \\
\hline 195. & 2.452 & 0.755 & 5.243 & 139.07 & 28.24 & 52.68 & 317.8 & 8.61 & 26.9 \\
\hline 200. & 2.317 & 0.779 & 5.498 & 140.36 & 27.85 & 49.62 & 327.2 & 8.73 & 26.8 \\
\hline 205 . & 2.203 & 0.799 & 5.740 & 141.56 & 27.55 & 47.38 & 335.9 & 8.86 & 26.9 \\
\hline 210 . & 2.105 & 0.816 & 5.973 & 142.68 & 27.32 & 45.67 & 344.0 & 9.00 & 27.2 \\
\hline 215. & 2.018 & 0.832 & 6.197 & 143.74 & 27.15 & 44.33 & 351.5 & 9.14 & 27.5 \\
\hline 220 . & 1.940 & 0.845 & 6.416 & 144.75 & 27.02 & 43.26 & 358.7 & 9.29 & 27.8 \\
\hline 225. & 1.870 & 0.857 & 6.630 & 145.71 & 26.92 & 42.39 & 365.4 & 9.44 & 28.2 \\
\hline 230 . & 1.807 & 0.868 & 6.840 & 146.63 & 26.86 & 41.67 & 371.9 & 9.59 & 28.7 \\
\hline 235. & 1.749 & 0.878 & 7.047 & 147.52 & 26.83 & 41.08 & 378.1 & 9.74 & 29.1 \\
\hline 240 . & 1.695 & 0.887 & 7.251 & 148.38 & 26.81 & 40.59 & 384.0 & 9.89 & 29.6 \\
\hline 245. & 1.646 & 0.895 & 7.453 & 149.21 & 26.82 & 40.19 & 389.7 & 10.05 & 30.1 \\
\hline 250. & 1.600 & 0.902 & 7.653 & 150.02 & 26.85 & 39.86 & 395.2 & 10.20 & 30.6 \\
\hline 255 . & 1.557 & 0.909 & 7.852 & 150.81 & 26.90 & 39.58 & 400.5 & 10.35 & 31.2 \\
\hline 260. & 1.517 & 0.915 & 8.049 & 151.57 & 26.96 & 39.36 & 405.7 & 10.51 & 31.7 \\
\hline 265 . & 1.479 & 0.921 & 8.246 & 152.32 & 27.04 & 39.18 & 410.7 & 10.66 & 32.3 \\
\hline 270 . & 1.443 & 0.926 & 8.441 & 153.05 & 27.13 & 39.04 & 415.5 & 10.82 & 32.8 \\
\hline 275. & 1.410 & 0.931 & 8.636 & 153.77 & 27.24 & 38.94 & 420.2 & 10.97 & 33.4 \\
\hline 280 . & 1.378 & 0.935 & 8.831 & 154.47 & 27.35 & 38.87 & 424.8 & 11.13 & 34.0 \\
\hline 285. & 1.348 & 0.939 & 9.025 & 155.16 & 27.48 & 38.82 & 429.3 & 11.28 & 34.6 \\
\hline 290. & 1.319 & 0.943 & 9.219 & 155.83 & 27.62 & 38.80 & 433.6 & 11.43 & 35.2 \\
\hline 295. & 1.292 & 0.947 & 9.413 & 156.50 & 27.77 & 38.81 & 437.9 & 11.58 & 35.9 \\
\hline 300 . & 1.266 & 0.950 & 9.607 & 157.15 & 27.93 & 38.83 & 442.1 & 11.74 & 36.5 \\
\hline 310. & 1.217 & 0.956 & 9.996 & 158.42 & 28.27 & 38.94 & 450.1 & 12.04 & 37.8 \\
\hline 320 . & 1.173 & 0.961 & 10.386 & 159.66 & 28.65 & 39.11 & 457.8 & 12.34 & 39.2 \\
\hline 330. & 1.132 & 0.966 & 10.778 & 160.87 & 29.06 & 39.34 & 465.3 & 12.63 & 40.6 \\
\hline 340 . & 1.094 & 0.970 & 11.173 & 162.05 & 29.49 & 39.62 & 472.4 & 12.93 & 42.0 \\
\hline 350. & 1.059 & 0.974 & 11.571 & 163.20 & 29.95 & 39.94 & 479.4 & 13.22 & 43.5 \\
\hline 360. & 1.026 & 0.977 & 11.972 & 164.33 & 30.43 & 40.30 & 486.1 & 13.51 & 45.0 \\
\hline 370. & 0.9950 & 0.980 & 12.377 & 165.44 & 30.93 & 40.69 & 492.6 & 13.80 & 46.6 \\
\hline 380 . & 0.9662 & 0.983 & 12.786 & 166.53 & 31.45 & 41.10 & 499.0 & 14.08 & 48.2 \\
\hline 390. & 0.9392 & 0.985 & 13.199 & 167.60 & 31.97 & 41.54 & 505.2 & 14.36 & 49.8 \\
\hline 400. & 0.9137 & 0.987 & 13.617 & 168.66 & 32.51 & 42.00 & 511.2 & 14.64 & 51.5 \\
\hline 410. & 0.8897 & 0.989 & 14.039 & 169.70 & 33.06 & 42.48 & 517.1 & & 53.1 \\
\hline
\end{tabular}


METHANE ISOBAR AT $\mathrm{P}=3.0 \mathrm{MPa}$ (continued)

\begin{tabular}{|c|c|c|c|c|c|c|c|c|c|}
\hline $\mathrm{T}$ & $\rho$ & Z & H & $S$ & $\mathrm{C}_{\mathrm{v}}$ & $C_{p}$ & W & $\eta$ & $\lambda$ \\
\hline K & $\mathrm{mol} \cdot \mathrm{dm}^{-3}$ & & $\mathrm{~kJ} / \mathrm{mol}$ & $\mathrm{J} /(\mathrm{mol} \cdot \mathrm{K})$ & $\mathrm{J} /(\mathrm{mol} \cdot \mathrm{K})$ & $\mathrm{J} /(\mathrm{mol} \cdot \mathrm{K})$ & $m \cdot s^{-1}$ & $\mu \mathrm{Pa} \cdot \mathrm{s}$ & $\mathrm{mW} /(\mathrm{m} \cdot \mathrm{K})$ \\
\hline 420. & 0.8670 & 0.991 & 14.466 & 170.73 & 33.62 & 42.97 & 522.9 & & 54.9 \\
\hline 430. & 0.8455 & 0.992 & 14.899 & 171.75 & 34.19 & 43.48 & 528.6 & & 56.6 \\
\hline 440. & 0.8251 & 0.994 & 15.336 & 172.76 & 34.75 & 43.99 & 534.2 & & 58.4 \\
\hline 450. & 0.8058 & 0.995 & 15.778 & 173.75 & 35.33 & 44.51 & 539.7 & & 60.2 \\
\hline 460. & 0.7873 & 0.996 & 16.226 & 174.73 & 35.90 & 45.03 & 545.1 & & 62.0 \\
\hline 470. & 0.7697 & 0.997 & 16.679 & 175.71 & 36.47 & 45.56 & 550.4 & & 63.8 \\
\hline 480. & 0.7530 & 0.998 & 17.137 & 176.67 & 37.04 & 46.10 & 555.7 & & 65.7 \\
\hline 490. & 0.7369 & 0.999 & 17.601 & 177.63 & 37.61 & 46.63 & 560.9 & & 67.5 \\
\hline 500 . & 0.7216 & 1.000 & 18.070 & 178.58 & 38.18 & 47.16 & 566.0 & & 69.4 \\
\hline 520. & 0.6929 & 1.001 & 19.024 & 180.45 & 39.31 & 48.23 & 576.1 & & 73.2 \\
\hline 540. & 0.6664 & 1.003 & 19.999 & 182.29 & 40.41 & 49.28 & 585.9 & & 77.1 \\
\hline 560. & 0.6420 & 1.004 & 20.995 & 184.10 & 41.50 & 50.32 & 595.6 & & 80.9 \\
\hline 580. & 0.6193 & 1.005 & 22.011 & 185.88 & 42.56 & 51.33 & 605.0 & & 84.8 \\
\hline 600. & 0.5982 & 1.005 & 23.048 & 187.64 & 43.59 & 52.33 & 614.3 & & 88.8 \\
\hline
\end{tabular}

\section{METHANE ISOBAR AT P $=4.0 \mathrm{MPa}$}

\begin{tabular}{|c|c|c|c|c|c|c|c|c|c|}
\hline $\mathrm{T}$ & $\rho$ & Z & $\mathrm{H}$ & S & $\mathrm{C}_{\mathrm{v}}$ & $C_{p}$ & W & $\eta$ & $\lambda$ \\
\hline K & $\mathrm{mol} \cdot \mathrm{dm}^{-3}$ & & $\mathrm{~kJ} / \mathrm{mol}$ & $\mathrm{J} /(\mathrm{mol} \cdot \mathrm{K})$ & $\mathrm{J} /(\mathrm{mol} \cdot \mathrm{K})$ & $\mathrm{J} /(\mathrm{mol} \cdot \mathrm{K})$ & $\mathrm{m} \cdot \mathrm{s}^{-1}$ & $\mu \mathrm{Pa} \cdot \mathrm{s}$ & $\mathrm{mW} /(\mathrm{m} \cdot \mathrm{K})$ \\
\hline 95. & 27.956 & 0.181 & -5.412 & 69.85 & 34.12 & 53.37 & 1529.7 & 187.47 & 209.7 \\
\hline 100. & 27.546 & 0.175 & -5.143 & 72.61 & 34.21 & 54.19 & 1479.3 & 164.51 & 203.5 \\
\hline 105. & 27.129 & 0.169 & -4.871 & 75.27 & 33.99 & 54.74 & 1431.6 & 145.09 & 197.1 \\
\hline 110. & 26.702 & 0.164 & -4.596 & 77.83 & 33.63 & 55.20 & 1384.5 & 128.69 & 190.5 \\
\hline 115 . & 26.264 & 0.159 & -4.319 & 80.29 & 33.21 & 55.64 & 1337.3 & 114.81 & 183.7 \\
\hline 120. & 25.815 & 0.155 & -4.039 & 82.67 & 32.79 & 56.13 & 1289.5 & 103.03 & 176.8 \\
\hline 125. & 25.352 & 0.152 & -3.757 & 84.97 & 32.38 & 56.71 & 1240.8 & 92.96 & 169.9 \\
\hline 130. & 24.873 & 0.149 & -3.472 & 87.21 & 32.00 & 57.39 & 1191.1 & 84.29 & 162.9 \\
\hline 135. & 24.375 & 0.146 & -3.183 & 89.39 & 31.65 & 58.22 & 1140.2 & 76.75 & 155.9 \\
\hline 140. & 23.856 & 0.144 & -2.890 & 91.53 & 31.33 & 59.23 & 1087.9 & 70.13 & 148.9 \\
\hline 145. & 23.311 & 0.142 & -2.590 & 93.62 & 31.05 & 60.47 & 1033.9 & 64.25 & 141.9 \\
\hline 150. & 22.735 & 0.141 & -2.284 & 95.70 & 30.81 & 62.01 & 978.0 & 58.95 & 134.8 \\
\hline 155. & 22.121 & 0.140 & -1.970 & 97.76 & 30.61 & 63.95 & 919.7 & 54.12 & 127.7 \\
\hline 160. & 21.458 & 0.140 & -1.644 & 99.83 & 30.46 & 66.50 & 858.3 & 49.64 & 120.6 \\
\hline 165. & 20.730 & 0.141 & -1.303 & 101.93 & 30.38 & 69.95 & 792.8 & 45.40 & 113.3 \\
\hline 170 & 19.913 & 0.142 & -0.942 & 104.09 & 30.39 & 74.95 & 721.8 & 41.31 & 105.7 \\
\hline 175. & 18.958 & 0.145 & -0.549 & 106.36 & 30.55 & 82.98 & 642.7 & 37.21 & 97.8 \\
\hline 180. & 17.758 & 0.151 & -0.100 & 108.89 & 31.00 & 98.73 & 550.0 & 32.84 & 89.2 \\
\hline 185. & 15.923 & 0.163 & 0.494 & 112.14 & 32.37 & 154.98 & 424.3 & 27.36 & 79.5 \\
\hline 186.094 & 15.222 & 0.170 & 0.691 & 113.20 & 33.27 & 208.48 & 380.1 & 25.55 & 77.3 \\
\hline
\end{tabular}


METHANE ISOBAR AT $\mathrm{P}=4.0 \mathrm{MPa}$ (continued)

\begin{tabular}{|c|c|c|c|c|c|c|c|c|c|}
\hline $\mathrm{T}$ & $\rho$ & $\mathrm{Z}$ & $\mathrm{H}$ & $S$ & $\mathrm{C}_{\mathrm{v}}$ & $C_{p}$ & W & $\eta$ & $\lambda$ \\
\hline K & $\mathrm{mol} \cdot \mathrm{dm}^{-3}$ & & $\mathrm{~kJ} / \mathrm{mol}$ & $\mathrm{J} /(\mathrm{mol} \cdot \mathrm{K})$ & $\mathrm{J} /(\mathrm{mol} \cdot \mathrm{K})$ & $\mathrm{J} /(\mathrm{mol} \cdot \mathrm{K})$ & $m \cdot s^{-1}$ & $\mu \mathrm{Pa} \cdot \mathrm{s}$ & $\mathrm{mW} /(\mathrm{m} \cdot$ \\
\hline 186.094 & 5.377 & 0.481 & 3.518 & 128.40 & 38.57 & 271.14 & 254.9 & 10.25 & 47.9 \\
\hline 190. & 4.416 & 0.573 & 4.132 & 131.67 & 32.58 & 110.14 & 279.6 & 9.66 & 37.9 \\
\hline 195. & 3.877 & 0.636 & 4.588 & 134.04 & 30.40 & 78.39 & 297.0 & 9.48 & 32.6 \\
\hline 200 . & 3.535 & 0.680 & 4.945 & 135.84 & 29.38 & 65.92 & 310.2 & 9.45 & 30.9 \\
\hline 205 . & 3.283 & 0.715 & 5.256 & 137.38 & 28.74 & 59.01 & 321.5 & 9.49 & 30.2 \\
\hline 210 . & 3.083 & 0.743 & 5.539 & 138.75 & 28.30 & 54.54 & 331.5 & 9.55 & 29.9 \\
\hline 215 . & 2.918 & 0.767 & 5.803 & 139.99 & 27.96 & 51.40 & 340.6 & 9.65 & 29.8 \\
\hline 220 . & 2.778 & 0.787 & 6.054 & 141.14 & 27.71 & 49.07 & 348.9 & 9.75 & 29.9 \\
\hline 225 . & 2.656 & 0.805 & 6.295 & 142.23 & 27.52 & 47.27 & 356.8 & 9.87 & 30.1 \\
\hline 230 . & 2.549 & 0.821 & 6.528 & 143.25 & 27.38 & 45.86 & 364.1 & 9.99 & 30.4 \\
\hline 235 . & 2.453 & 0.835 & 6.754 & 144.22 & 27.29 & 44.72 & 371.1 & 10.12 & 30.7 \\
\hline 240 . & 2.366 & 0.847 & 6.975 & 145.15 & 27.22 & 43.80 & 377.7 & 10.25 & 31.1 \\
\hline 245 . & 2.287 & 0.858 & 7.192 & 146.05 & 27.19 & 43.04 & 384.0 & 10.39 & 31.5 \\
\hline 250 & 2.215 & 0.869 & 7.406 & 146.91 & 27.18 & 42.41 & 390.1 & 10.52 & 31.9 \\
\hline 255. & 2.149 & 0.878 & 7.617 & 147.75 & 27.20 & 41.89 & 395.9 & 10.67 & 32.4 \\
\hline 260 . & 2.087 & 0.886 & 7.825 & 148.56 & 27.24 & 41.46 & 401.5 & 10.81 & 32.9 \\
\hline 265 . & 2.030 & 0.894 & 8.031 & 149.34 & 27.29 & 41.11 & 406.9 & 10.95 & 33.4 \\
\hline 270 . & 1.977 & 0.901 & 8.236 & 150.11 & 27.36 & 40.81 & 412.1 & 11.09 & 33.9 \\
\hline 275 . & 1.927 & 0.908 & 8.440 & 150.85 & 27.45 & 40.57 & 417.1 & 11.24 & 34.4 \\
\hline 280 . & 1.880 & 0.914 & 8.642 & 151.58 & 27.55 & 40.38 & 422.0 & 11.38 & 35.0 \\
\hline 285 . & 1.836 & 0.920 & 8.844 & 152.30 & 27.67 & 40.24 & 426.8 & 11.53 & 35.6 \\
\hline 290. & 1.794 & 0.925 & 9.044 & 153.00 & 27.80 & 40.12 & 431.4 & 11.67 & 36.2 \\
\hline 295. & 1.754 & 0.930 & 9.245 & 153.68 & 27.93 & 40.04 & 435.9 & 11.82 & 36.8 \\
\hline 300 & 1.717 & 0.934 & 9.445 & 154.35 & 28.08 & 39.99 & 440.3 & 11.97 & 37.4 \\
\hline 310. & 1.647 & 0.942 & 9.845 & 155.66 & 28.41 & 39.97 & 448.8 & 12.26 & 38.6 \\
\hline 320 . & 1.584 & 0.949 & 10.245 & 156.93 & 28.78 & 40.04 & 456.8 & 12.55 & 39.9 \\
\hline 330. & 1.526 & 0.956 & 10.646 & 158.17 & 29.17 & 40.18 & 464.6 & 12.83 & 41.3 \\
\hline 340 . & 1.472 & 0.961 & 11.048 & 159.37 & 29.60 & 40.38 & 472.0 & 13.12 & 42.7 \\
\hline 350. & 1.423 & 0.966 & 11.454 & 160.54 & 30.05 & 40.64 & 479.2 & 13.40 & 44.2 \\
\hline 360. & 1.377 & 0.970 & 11.861 & 161.69 & 30.52 & 40.94 & 486.1 & 13.69 & 45.7 \\
\hline 370 . & 1.335 & 0.974 & 12.272 & 162.82 & 31.01 & 41. & 492.8 & 13.97 & 47.2 \\
\hline 380 . & 1.295 & 0.978 & 12.687 & 163.92 & 31.52 & 41.65 & 499.4 & 14.24 & 48.8 \\
\hline 390. & 1.258 & 0.981 & 13.105 & 165.01 & 32.05 & 42.05 & 505.7 & 14.52 & 50.4 \\
\hline 400 & 1.223 & 0.984 & 13.528 & 166.08 & 32.58 & 42.48 & 511.9 & 14.79 & 52.0 \\
\hline 410 & 1.190 & 0.986 & 13.955 & 167.14 & 33.13 & 42.92 & 518.0 & & 53.7 \\
\hline 420. & 1.159 & 0.988 & 14.387 & 168.18 & 33.68 & 43.39 & 523.9 & & 55.4 \\
\hline 430. & 1.130 & 0.990 & 14.823 & 169.20 & 34.24 & 43.86 & 529.7 & & 57.1 \\
\hline 440 & 1.102 & 0.992 & 15.264 & 170.22 & 34.81 & 44.35 & 535.4 & & 58.9 \\
\hline 450 & 1.076 & 0.994 & 15.710 & 171.22 & 35.38 & 44.85 & 541.0 & & 60.6 \\
\hline 460 & 1.051 & 0.995 & 16.161 & 172.21 & 35.95 & 45.36 & 546.5 & & 62.4 \\
\hline
\end{tabular}


METHANE ISOBAR AT $\mathrm{P}=4.0 \mathrm{MPa}$ (continued)

\begin{tabular}{|c|c|c|c|c|c|c|c|c|c|}
\hline $\mathrm{T}$ & $\rho$ & $\mathrm{z}$ & H & $S$ & $\mathrm{C}_{\mathrm{v}}$ & $C_{p}$ & W & $\eta$ & $\lambda$ \\
\hline K & $\mathrm{mol} \cdot \mathrm{dm}^{-3}$ & & $\mathrm{~kJ} / \mathrm{mol}$ & $\mathrm{J} /(\mathrm{mol} \cdot \mathrm{K})$ & $\mathrm{J} /(\mathrm{mol} \cdot \mathrm{K})$ & $\mathrm{J} /(\mathrm{mol} \cdot \mathrm{K})$ & $\mathrm{m} \cdot \mathrm{s}^{-1}$ & $\mu \mathrm{Pa} \cdot \mathrm{s}$ & $\mathrm{mW} /(\mathrm{m} \cdot \mathrm{K})$ \\
\hline 470. & 1.027 & 0.997 & 16.617 & 173.19 & 36.52 & 45.87 & 551.9 & & 64.3 \\
\hline 480. & 1.004 & 0.998 & 17.078 & 174.16 & 37.09 & 46.39 & 557.2 & & 66.1 \\
\hline 490. & 0.9825 & 0.999 & 17.545 & 175.12 & 37.66 & 46.91 & 562.4 & & 68.0 \\
\hline 500 . & 0.9618 & 1.000 & 18.016 & 176.08 & 38.22 & 47.43 & 567.6 & & 69.8 \\
\hline 520. & 0.9231 & 1.002 & 18.975 & 177.96 & 39.34 & 48.46 & 577.8 & & 73.6 \\
\hline 540. & 0.8875 & 1.004 & 19.955 & 179.81 & 40.45 & 49.50 & 587.8 & & 77.4 \\
\hline 560. & 0.8547 & 1.005 & 20.955 & 181.62 & 41.53 & 50.51 & 597.5 & & 81.3 \\
\hline 580 . & 0.8243 & 1.006 & 21.975 & 183.41 & 42.58 & 51.51 & 607.0 & & 85.2 \\
\hline 600. & 0.7961 & 1.007 & 23.016 & 185.18 & 43.61 & 52.49 & 616.4 & & 89.1 \\
\hline
\end{tabular}

\section{METHANE ISOBAR AT $P=5.0 \mathrm{MPa}$}

\begin{tabular}{|c|c|c|c|c|c|c|c|c|c|}
\hline $\mathrm{T}$ & $\rho$ & $\mathrm{z}$ & H & $\mathbf{S}$ & $C_{v}$ & $C_{p}$ & W & $\eta$ & $\lambda$ \\
\hline K & $\mathrm{mol} \cdot \mathrm{dm}^{-}$ & & $\mathrm{kJ} / \mathrm{mol}$ & $\mathrm{J} /(\mathrm{mol} \cdot \mathrm{K})$ & $\mathrm{J} /(\mathrm{mol} \cdot \mathrm{K})$ & $\mathrm{J} /(\mathrm{mol} \cdot \mathrm{K})$ & $m \cdot s^{-1}$ & $\mu \mathrm{Pa} \cdot \mathrm{s}$ & $\mathrm{mW} /(\mathrm{m} \cdot \mathrm{K})$ \\
\hline 95. & 27.998 & 0.226 & -5.386 & 69.75 & 34.16 & 53.29 & 1537.2 & 189.48 & 210.6 \\
\hline 100. & 27.591 & 0.218 & -5.118 & 72.50 & 34.25 & 54.08 & 1487.3 & 166.34 & 204.5 \\
\hline 105. & 27.177 & 0.211 & -4.846 & 75.16 & 34.02 & 54.62 & 1440.0 & 146.76 & 198.1 \\
\hline 110. & 26.755 & 0.204 & -4.572 & 77.71 & 33.66 & 55.05 & 1393.5 & 130.21 & 191.5 \\
\hline 115. & 26.322 & 0.199 & -4.295 & 80.16 & 33.25 & 55.47 & 1346.9 & 116.21 & 184.8 \\
\hline 120. & 25.879 & 0.194 & -4.017 & 82.53 & 32.83 & 55.92 & 1299.8 & 104.33 & 178.0 \\
\hline 125. & 25.422 & 0.189 & -3.736 & 84.83 & 32.42 & 56.45 & 1252.0 & 94.18 & 171.1 \\
\hline 130. & 24.950 & 0.185 & -3.452 & 87.05 & 32.04 & 57.09 & 1203.2 & 85.44 & 164.2 \\
\hline 135. & 24.462 & 0.182 & -3.165 & 89.22 & 31.69 & 57.85 & 1153.4 & 77.86 & 157.3 \\
\hline 140. & 23.954 & 0.179 & -2.874 & 91.34 & 31.37 & 58.76 & 1102.5 & 71.20 & 150.4 \\
\hline 145. & 23.422 & 0.177 & -2.577 & 93.42 & 31.09 & 59.88 & 1050.1 & 65.30 & 143.4 \\
\hline 150. & 22.863 & 0.175 & -2.274 & 95.48 & 30.84 & 61.25 & 996.2 & 60.01 & 136.5 \\
\hline 155. & 22.270 & 0.174 & -1.964 & 97.51 & 30.63 & 62.95 & 940.3 & 55.19 & 129.6 \\
\hline 160. & 21.635 & 0.174 & -1.644 & 99.54 & 30.46 & 65.11 & 882.0 & 50.75 & 122.6 \\
\hline 165. & 20.948 & 0.174 & -1.312 & 101.59 & 30.35 & 67.95 & 820.7 & 46.60 & 115.6 \\
\hline 170. & 20.189 & 0.175 & -0.963 & 103.67 & 30.30 & 71.82 & 755.4 & 42.64 & 108.4 \\
\hline 175. & 19.330 & 0.178 & -0.591 & 105.83 & 30.36 & 77.50 & 684.7 & 38.76 & 101.0 \\
\hline 180. & 18.316 & 0.182 & -0.182 & 108.13 & 30.58 & 86.75 & 606.4 & 34.82 & 93.3 \\
\hline 185. & 17.023 & 0.191 & 0.292 & 110.72 & 31.11 & 105.36 & 515.9 & 30.57 & 85.2 \\
\hline 190. & 14.994 & 0.211 & 0.939 & 114.17 & 32.57 & 172.39 & 396.8 & 25.12 & 78.2 \\
\hline 195. & 7.142 & 0.432 & 3.274 & 126.27 & 36.88 & 311.74 & 267.3 & 12.29 & 58.0 \\
\hline 200 & 5.459 & 0.551 & 4.144 & 130.69 & 32.02 & 116.34 & 292.0 & 10.90 & 40.4 \\
\hline 205. & 4.776 & 0.614 & 4.632 & 133.10 & 30.42 & 84.31 & 307.4 & 10.54 & 36.1 \\
\hline 210 . & 4.340 & 0.660 & 5.015 & 134.94 & 29.53 & 70.53 & 319.8 & 10.40 & 34.3 \\
\hline 215. & 4.021 & 0.696 & 5.347 & 136.50 & 28.94 & 62.71 & 330.6 & 10.37 & 33.4 \\
\hline
\end{tabular}


METHANE ISOBAR AT $\mathrm{P}=5.0 \mathrm{MPa}$ (continued)

\begin{tabular}{|c|c|c|c|c|c|c|c|c|c|}
\hline $\mathrm{T}$ & $\rho$ & Z & $\mathrm{H}$ & $S$ & $\mathrm{C}_{\mathrm{v}}$ & $C_{p}$ & W & $\eta$ & $\lambda$ \\
\hline K & $\mathrm{mol} \cdot \mathrm{dm}^{-3}$ & & $\mathrm{~kJ} / \mathrm{mol}$ & $\mathrm{J} /(\mathrm{mol} \cdot \mathrm{K})$ & $\mathrm{J} /(\mathrm{mol} \cdot \mathrm{K})$ & $\mathrm{J} /(\mathrm{mol} \cdot \mathrm{K})$ & $\mathrm{m} \cdot \mathrm{s}^{-1}$ & $\mu \mathrm{Pa} \cdot \mathrm{s}$ & $\mathrm{mW} /(\mathrm{m}$ \\
\hline 220 . & 3.770 & 0.725 & 5.647 & 137.88 & 28.52 & 57.62 & 340.3 & 10.38 & 32.9 \\
\hline 225 . & 3.563 & 0.750 & 5.925 & 139.14 & 28.20 & 54.04 & 349.2 & 10.43 & 32.7 \\
\hline 230. & 3.389 & 0.772 & 6.189 & 140.29 & 27.96 & 51.39 & 357.5 & 10.51 & 32.7 \\
\hline 235. & 3.237 & 0.790 & 6.440 & 141.38 & 27.79 & 49.36 & 365.2 & 10.59 & 32.8 \\
\hline 240 . & 3.104 & 0.807 & 6.683 & 142.40 & 27.66 & 47.76 & 372.5 & 10.69 & 33.0 \\
\hline 245. & 2.986 & 0.822 & 6.918 & 143.37 & 27.58 & 46.47 & 379.4 & 10.80 & 33.2 \\
\hline 250 . & 2.880 & 0.835 & 7.148 & 144.30 & 27.53 & 45.43 & 385.9 & 10.91 & 33.5 \\
\hline 255. & 2.783 & 0.847 & 7.373 & 145.19 & 27.51 & 44.57 & 392.2 & 11.03 & 33.9 \\
\hline 260. & 2.695 & 0.858 & 7.594 & 146.05 & 27.52 & 43.86 & 398.2 & 11.16 & 34.3 \\
\hline 265. & 2.614 & 0.868 & 7.812 & 146.88 & 27.55 & 43.27 & 403.9 & 11.28 & 34.7 \\
\hline 270 . & 2.539 & 0.877 & 8.027 & 147.68 & 27.60 & 42.78 & 409.5 & 11.41 & 35.1 \\
\hline 275 . & 2.469 & 0.886 & 8.240 & 148.46 & 27.67 & 42.37 & 414.8 & 11.54 & 35.6 \\
\hline 280 & 2.404 & 0.893 & 8.451 & 149.22 & 27.75 & 42.04 & 420.0 & 11.68 & 36.1 \\
\hline 285 . & 2.343 & 0.901 & 8.660 & 149.96 & 27.86 & 41.76 & 425.0 & 11.81 & 36.6 \\
\hline 290. & 2.286 & 0.907 & 8.868 & 150.69 & 27.97 & 41.54 & 429.8 & 11.95 & 37.2 \\
\hline 295. & 2.232 & 0.913 & 9.076 & 151.40 & 28.10 & 41.36 & 434.5 & 12.08 & 37.7 \\
\hline 300 . & 2.182 & 0.919 & 9.282 & 152.09 & 28.24 & 41.23 & 439.1 & 12.22 & 38.3 \\
\hline 310 . & 2.088 & 0.929 & 9.693 & 153.44 & 28.55 & 41.06 & 447.9 & 12.50 & 39.5 \\
\hline 320. & 2.004 & 0.938 & 10.104 & 154.74 & 28.90 & 41.00 & 456.3 & 12.77 & 40.8 \\
\hline 330. & 1.927 & 0.946 & 10.514 & 156.00 & 29.29 & 41.04 & 464.3 & 13.05 & 42.1 \\
\hline 340 . & 1.857 & 0.952 & 10.925 & 157.23 & 29.70 & 41.16 & 472.0 & 13.33 & 43.5 \\
\hline 350. & 1.793 & 0.958 & 11.337 & 158.43 & 30.14 & 41.35 & 479.4 & 13.60 & 44.9 \\
\hline 360. & 1.733 & 0.964 & 11.752 & 159.59 & 30.61 & 41.59 & 486.5 & 13.88 & 46.3 \\
\hline 370 . & 1.678 & 0.969 & 12.169 & 160.74 & 31.10 & 41.87 & 493.4 & 14.15 & 47.8 \\
\hline 380 & 1.627 & 0.973 & 12.589 & 161.86 & 31.60 & 42.20 & 500.1 & 14.42 & 49.4 \\
\hline 390. & 1.579 & 0.977 & 13.013 & 162.96 & 32.12 & 42.56 & 506.6 & 14.69 & 51.0 \\
\hline 400 & 1.534 & 0.980 & 13.441 & 164.04 & 32.65 & 42.95 & 512.9 & 14.96 & 52.6 \\
\hline 410 & 1.492 & 0.983 & 13.872 & 165.11 & 33.19 & 43.36 & 519.0 & & 54.2 \\
\hline 420 & 1.452 & 0.986 & 14.308 & 166.16 & 33.74 & 43.80 & 525.1 & & 55.9 \\
\hline 430. & 1.415 & 0.989 & 14.748 & 167.19 & 34.30 & 44.25 & 531.0 & & 57.6 \\
\hline 440. & 1.379 & 0.991 & 15.193 & 168.22 & 34.86 & 44.72 & 536.7 & & 59.4 \\
\hline 450 & 1.346 & 0.993 & 15.643 & 169.23 & 35.43 & 45.19 & 542.4 & & 61.1 \\
\hline 460 & 1.314 & 0.995 & 16.097 & 170.22 & 36.00 & 45.68 & 548.0 & & 62.9 \\
\hline 470 & 1.284 & 0.997 & 16.556 & 171.21 & 36.56 & 46.18 & 553.5 & & 64.7 \\
\hline 480 & 1.255 & 0.998 & 17.020 & 172.19 & 37.13 & 46.68 & 558.9 & & 66.5 \\
\hline 490. & 1.228 & 1.000 & 17.490 & 173.16 & 37.70 & 47.18 & 564.2 & & 68.4 \\
\hline 500 & 1.202 & 1.001 & 17.964 & 174.12 & 38.26 & 47.69 & 569.4 & & 70.3 \\
\hline 520 & 1.153 & 1.003 & 18.928 & 176.01 & 39.38 & 48.70 & 579.7 & & 74.0 \\
\hline 540. & 1.108 & 1.005 & 19.912 & 177.86 & 40.48 & 49.71 & 589.7 & & 77.8 \\
\hline 560. & 1.067 & 1.007 & 20.916 & 179.69 & 41.56 & 50.71 & 599.5 & & 81.7 \\
\hline
\end{tabular}


METHANE ISOBAR AT $\mathrm{P}=5.0 \mathrm{MPa}$ (continued)

$\mathrm{T}$

$\rho$

$\mathrm{K}$

$\mathrm{K} \quad \mathrm{mol} \cdot \mathrm{dm}^{-3} \quad \mathrm{~kJ} / \mathrm{mol} \quad \mathrm{J} /(\mathrm{mol} \cdot \mathrm{K}) \quad \mathrm{J} /(\mathrm{mol} \cdot \mathrm{K}) \quad \mathrm{J} /(\mathrm{mol} \cdot \mathrm{K}) \quad \mathrm{m} \cdot \mathrm{s}^{-1} \quad \mu \mathrm{Pa} \cdot \mathrm{s} \quad \mathrm{mW} /(\mathrm{m} \cdot \mathrm{K})$

Z $\quad \mathrm{H}$

S $\quad \mathrm{C}_{\mathrm{v}}$

$\mathrm{C}_{\mathrm{p}}$

W

$\eta$

$\lambda$

580 .

1.029

1.008

21.940

181.48

42.61

51.69

609.2

85.5

600 .

0.9931

1.009

$22.984 \quad 183.25$

43.64

52.66

618.6

89.4

\section{METHANE ISOBAR AT $\mathrm{P}=6.0 \mathrm{MPa}$}

\begin{tabular}{|c|c|c|c|c|c|c|c|c|c|}
\hline $\mathrm{T}$ & $\rho$ & Z & $\mathrm{H}$ & $S$ & $\mathrm{C}_{\mathrm{v}}$ & $\mathrm{C}_{\mathrm{p}}$ & W & $\eta$ & $\lambda$ \\
\hline K & $\mathrm{mol} \cdot \mathrm{dm}^{-3}$ & & $\mathrm{~kJ} / \mathrm{mol}$ & $\mathrm{J} /(\mathrm{mol} \cdot \mathrm{K})$ & $\mathrm{J} /(\mathrm{mol} \cdot \mathrm{K})$ & $\mathrm{J} /(\mathrm{mol} \cdot \mathrm{K})$ & $\mathrm{m} \cdot \mathrm{s}^{-1}$ & $\mu \mathrm{Pa} \cdot \mathrm{s}$ & $\mathrm{mW} /(\mathrm{m} \cdot \mathrm{K})$ \\
\hline 95. & 28.038 & 0.271 & -5.360 & 69.65 & 34.19 & 53.20 & 1544.6 & 191.49 & 211.5 \\
\hline 100. & 27.635 & 0.261 & -5.092 & 72.40 & 34.28 & 53.98 & 1495.1 & 168.18 & 205.4 \\
\hline 105. & 27.225 & 0.252 & -4.821 & 75.04 & 34.06 & 54.50 & 1448.3 & 148.44 & 199.1 \\
\hline 110. & 26.807 & 0.245 & -4.547 & 77.59 & 33.70 & 54.91 & 1402.3 & 131.75 & 192.6 \\
\hline 115. & .26 .379 & 0.238 & -4.272 & 80.04 & 33.29 & 55.30 & 1356.3 & 117.62 & 185.9 \\
\hline 120. & 25.941 & 0.232 & -3.994 & 82.40 & 32.87 & 55.72 & 1309.9 & 105.63 & 179.1 \\
\hline 125. & 25.490 & 0.226 & -3.715 & 84.69 & 32.47 & 56.22 & 1262.8 & 95.40 & 172.3 \\
\hline 130. & 25.026 & 0.222 & -3.432 & 86.90 & 32.09 & 56.80 & 1215.0 & 86.59 & 165.5 \\
\hline 135. & 24.546 & 0.218 & -3.146 & 89.06 & 31.74 & 57.50 & 1166.2 & 78.95 & 158.6 \\
\hline 140. & 24.048 & 0.214 & -2.857 & 91.16 & 31.41 & 58.34 & 1116.5 & 72.26 & 151.8 \\
\hline 145. & 23.529 & 0.212 & -2.563 & 93.23 & 31.13 & 59.35 & 1065.6 & 66.34 & 145.0 \\
\hline 150. & 22.985 & 0.209 & -2.263 & 95.26 & 30.87 & 60.57 & 1013.4 & 61.04 & 138.2 \\
\hline 155. & 22.411 & 0.208 & -1.957 & 97.27 & 30.65 & 62.07 & 959.6 & 56.24 & 131.4 \\
\hline 160. & 21.801 & 0.207 & -1.642 & 99.27 & 30.47 & 63.93 & 904.0 & 51.83 & 124.6 \\
\hline 165. & 21.146 & 0.207 & -1.316 & 101.27 & 30.33 & 66.31 & 846.0 & 47.73 & 117.8 \\
\hline 170. & 20.434 & 0.208 & -0.977 & 103.29 & 30.25 & 69.43 & 785.2 & 43.86 & 110.9 \\
\hline 175. & 19.644 & 0.210 & -0.620 & 105.36 & 30.25 & 73.71 & 720.7 & 40.14 & 103.9 \\
\hline 180. & 18.746 & 0.214 & -0.237 & 107.52 & 30.35 & 79.99 & 651.4 & 36.46 & 96.7 \\
\hline 185. & 17.681 & 0.221 & 0.186 & 109.84 & 30.61 & 90.21 & 575.6 & 32.70 & 89.5 \\
\hline 190. & 16.319 & 0.233 & 0.681 & 112.48 & 31.15 & 110.35 & 490.3 & 28.62 & 82.8 \\
\hline 195. & 14.264 & 0.259 & 1.346 & 115.93 & 32.39 & 167.24 & 388.7 & 23.58 & 74.4 \\
\hline 200 . & 10.203 & 0.354 & 2.572 & 122.13 & 35.35 & 314.49 & 293.7 & 16.33 & 66.5 \\
\hline 205. & 7.243 & 0.486 & 3.720 & 127.81 & 32.84 & 156.54 & 298.3 & 12.81 & 49.3 \\
\hline 210 . & 6.099 & 0.563 & 4.345 & 130.82 & 31.10 & 103.21 & 311.6 & 11.87 & 42.1 \\
\hline 215 . & 5.437 & 0.617 & 4.801 & 132.97 & 30.10 & 81.96 & 323.4 & 11.48 & 38.9 \\
\hline 220 . & 4.977 & 0.659 & 5.180 & 134.71 & 29.43 & 70.62 & 334.0 & 11.29 & 37.2 \\
\hline 225 . & 4.629 & 0.693 & 5.515 & 136.22 & 28.95 & 63.55 & 343.7 & 11.20 & 36.2 \\
\hline 230 . & 4.350 & 0.721 & 5.820 & 137.56 & 28.59 & 58.73 & 352.6 & 11.18 & 35.6 \\
\hline 23 & 4.118 & 0.746 & 6.104 & 138.78 & 28.32 & 55.24 & 360.9 & 11.20 & 35.3 \\
\hline 240 . & 3.921 & 0.767 & 6.374 & 139.92 & 28.12 & 52.61 & 368.7 & 11.24 & 35.2 \\
\hline
\end{tabular}


METHANE ISOBAR AT $\mathrm{P}=6.0 \mathrm{MPa}$ (continued)

\begin{tabular}{|c|c|c|c|c|c|c|c|c|c|}
\hline $\mathrm{T}$ & $\rho$ & $Z$ & $\mathrm{H}$ & S & $C_{v}$ & $C_{p}$ & W & $\eta$ & $\lambda$ \\
\hline K & $\mathrm{mol} \cdot \mathrm{dm}^{-3}$ & & $\mathrm{~kJ} / \mathrm{mol}$ & $\mathrm{J} /(\mathrm{mol} \cdot \mathrm{K})$ & $\mathrm{J} /(\mathrm{mol} \cdot \mathrm{K})$ & $\mathrm{J} /(\mathrm{mol} \cdot \mathrm{K})$ & $m \cdot s^{-1}$ & $\mu \mathrm{Pa} \cdot \mathrm{s}$ & $\mathrm{mW} /(\mathrm{m} \cdot$ \\
\hline 245 . & 3.749 & 0.786 & 6.631 & 140.98 & 27.98 & 50.57 & 376.0 & 11.30 & 35.3 \\
\hline 250. & 3.598 & 0.802 & 6.880 & 141.98 & 27.89 & 48.94 & 383.0 & 11.38 & 35.4 \\
\hline 255. & 3.463 & 0.817 & 7.121 & 142.94 & 27.83 & 47.63 & 389.6 & 11.47 & 35.6 \\
\hline 260. & 3.341 & 0.831 & 7.357 & 143.85 & 27.80 & 46.56 & 395.9 & 11.57 & 35.9 \\
\hline 265. & 3.231 & 0.843 & 7.587 & 144.73 & 27.81 & 45.67 & 401.9 & 11.67 & 36.2 \\
\hline 270 & 3.129 & 0.854 & 7.814 & 145.58 & 27.84 & 44.94 & 407.7 & 11.78 & 36.6 \\
\hline 275 . & 3.036 & 0.864 & 8.037 & 146.40 & 27.89 & 44.33 & 413.3 & 11.89 & 37.0 \\
\hline 280 . & 2.950 & 0.874 & 8.257 & 147.19 & 27.95 & 43.82 & 418.7 & 12.01 & 37.4 \\
\hline 285 . & 2.870 & 0.882 & 8.475 & 147.96 & 28.04 & 43.39 & 423.9 & 12.13 & 37.8 \\
\hline 290. & 2.796 & 0.890 & 8.691 & 148.71 & 28.14 & 43.04 & 429.0 & 12.25 & 38.3 \\
\hline 295. & 2.726 & 0.897 & 8.906 & 149.45 & 28.26 & 42.75 & 433.8 & 12.38 & 38.8 \\
\hline 300 & 2.660 & 0.904 & 9.119 & 150.16 & 28.39 & 42.52 & 438.6 & 12.50 & 39.4 \\
\hline 310 . & 2.540 & 0.916 & 9.542 & 151.55 & 28.68 & 42.18 & 447.7 & 12.76 & 40.5 \\
\hline 320 . & 2.433 & 0.927 & 9.963 & 152.89 & 29.02 & 42.00 & 456.3 & 13.02 & 41.7 \\
\hline 330. & 2.336 & 0.936 & 10.382 & 154.18 & 29.40 & 41.93 & 464.5 & 13.28 & 42.9 \\
\hline 340 . & 2.248 & 0.944 & 10.802 & 155.43 & 29.80 & 41.96 & 472.4 & 13.55 & 44.3 \\
\hline 350. & 2.167 & 0.952 & 11.222 & 156.65 & 30.24 & 42.07 & 480.0 & 13.81 & 45.6 \\
\hline 360. & 2.093 & 0.958 & 11.643 & 157.84 & 30.70 & 42.24 & 487.3 & 14.08 & 47.0 \\
\hline 370. & 2.024 & 0.964 & 12.067 & 159.00 & 31.18 & 42.47 & 494.3 & 14.34 & 48.5 \\
\hline 380 . & 1.961 & 0.969 & 12.493 & 160.13 & 31.67 & 42.75 & 501.1 & 14.60 & 50.0 \\
\hline 390. & 1.901 & 0.973 & 12.922 & 161.25 & 32.19 & 43.07 & 507.7 & 14.87 & 51.6 \\
\hline 400. & 1.846 & 0.977 & 13.354 & 162.34 & 32.72 & 43.42 & 514.1 & 15.13 & 53.2 \\
\hline 410 & 1.794 & 0.981 & 13.791 & 163.42 & 33.25 & 43.80 & 520.4 & & 54.8 \\
\hline 420 & 1.746 & 0.984 & 14.231 & 164.48 & 33.80 & 44.21 & 526.5 & & 56.5 \\
\hline 430. & 1.700 & 0.987 & 14.675 & 165.52 & 34.36 & 44.63 & 532.5 & & 58.2 \\
\hline 440. & 1.657 & 0.990 & 15.123 & 166.56 & 34.92 & 45.08 & 538.3 & & 59.9 \\
\hline 450 & 1.616 & 0.992 & 15.576 & 167.57 & 35.48 & 45.53 & 544.1 & & 61.6 \\
\hline 460. & 1.577 & 0.995 & 16.034 & 168.58 & 36.04 & 46.00 & 549.7 & & 63.4 \\
\hline 470 & 1.541 & 0.997 & 16.496 & 169.57 & 36.61 & 46.48 & 555.2 & & 65.2 \\
\hline 480. & 1.506 & 0.998 & 16.964 & 170.56 & 37.18 & 46.96 & 560.7 & & 67.0 \\
\hline 490. & 1.473 & 1.000 & 17.436 & 171.53 & 37.74 & 47.45 & 566.1 & & 68.8 \\
\hline 500. & 1.441 & 1.002 & 17.913 & 172.49 & 38.30 & 47.94 & 571.3 & & 70.7 \\
\hline 520. & 1.382 & 1.004 & 18.881 & 174.39 & 39.42 & 48.93 & 581.7 & & 74.4 \\
\hline 540. & 1.328 & 1.007 & 19.870 & 176.26 & 40.51 & 49.92 & 591.8 & & 78.2 \\
\hline 560 & 1.278 & 1.008 & 20.878 & 178.09 & 41.59 & 50.90 & 601.7 & & 82.0 \\
\hline 580 & 1.232 & 1.010 & 21.906 & 179.90 & 42.64 & 51.87 & 611.4 & & 85.9 \\
\hline 600 & 1.189 & 1.011 & 22.953 & 181.67 & 43.67 & 52.82 & 620.8 & & 89.8 \\
\hline
\end{tabular}


METHANE ISOBAR AT $\mathrm{P}=7.0 \mathrm{MPa}$

\begin{tabular}{|c|c|c|c|c|c|c|c|c|}
\hline $\mathrm{T}$ & $\mathrm{Z}$ & $\mathrm{H}$ & S & $\mathrm{C}_{\mathrm{v}}$ & $C_{p}$ & W & $\eta$ & $\lambda$ \\
\hline K & $\mathrm{mol} \cdot \mathrm{dm}^{-3}$ & $\mathrm{~kJ} / \mathrm{mol}$ & $\mathrm{J} /(\mathrm{mol} \cdot \mathrm{K})$ & $\mathrm{J} /(\mathrm{mol} \cdot \mathrm{K})$ & $\mathrm{J} /(\mathrm{mol} \cdot \mathrm{K})$ & $\mathrm{m} \cdot \mathrm{s}^{-1}$ & $\mu \mathrm{Pa} \cdot \mathrm{s}$ & $\mathrm{mW} /(\mathrm{m} \cdot$ \\
\hline 95. & 28.079 & -5.334 & 69.55 & 34.23 & 53.12 & 1551.8 & 193.53 & 212.4 \\
\hline 100. & 27.679 & -5.067 & 72.29 & 34.32 & 53.88 & 1502.8 & 170.04 & 206.4 \\
\hline 105. & 27.273 & -4.796 & 74.93 & 34.10 & 54.38 & 1456.4 & 150.13 & 200.1 \\
\hline 110. & 26.858 & -4.523 & 77.47 & 33.74 & 54.77 & 1410.9 & 133.29 & 193.6 \\
\hline 115. & 26.435 & -4.248 & 79.92 & 33.33 & 55.14 & 1365.5 & 119.04 & 187.0 \\
\hline 120. & 26.002 & -3.972 & 82.27 & 32.91 & 55.53 & 1319.7 & 106.94 & 180.3 \\
\hline 125. & 25.557 & -3.693 & 84.55 & 32.51 & 55.99 & 1273.4 & 96.62 & 173.5 \\
\hline 130. & 25.100 & -3.411 & 86.75 & 32.13 & 56.53 & 1226.4 & 87.74 & 166.7 \\
\hline 135. & 24.628 & -3.127 & 88.90 & 31.78 & 57.17 & 1178.6 & 80.05 & 160.0 \\
\hline 140. & 24.139 & -2.840 & 90.99 & 31.46 & 57.94 & 1130.0 & 73.32 & 153.2 \\
\hline 145. & 23.632 & -2.548 & 93.04 & 31.17 & 58.86 & 1080.4 & 67.37 & 146.5 \\
\hline 150. & 23.101 & -2.251 & 95.05 & 30.91 & 59.96 & 1029.8 & 62.06 & 139.8 \\
\hline 155. & 22.545 & -1.948 & 97.04 & 30.68 & 61.29 & 977.9 & 57.26 & 133.1 \\
\hline 160. & 21.956 & -1.637 & 99.01 & 30.49 & 62.91 & 924.5 & 52.87 & 126.5 \\
\hline 165. & 21.329 & -1.318 & 100.98 & 30.33 & 64.94 & 869.4 & 48.82 & 119.8 \\
\hline 170. & 20.655 & -0.987 & 102.95 & 30.23 & 67.51 & 812.2 & 45.01 & 113.2 \\
\hline 175. & 19.919 & -0.641 & 104.95 & 30.18 & 70.88 & 752.4 & 41.39 & 106.5 \\
\hline 180. & 19.101 & -0.276 & 107.01 & 30.21 & 75.50 & 689.4 & 37.89 & 99.7 \\
\hline 185. & 18.170 & 0.117 & 109.17 & 30.34 & 82.20 & 622.5 & 34.41 & 93.0 \\
\hline 190. & 17.067 & 0.552 & 111.49 & 30.61 & 92.85 & 551.0 & 30.85 & 86.7 \\
\hline 195. & 15.679 & 1.059 & 114.12 & 31.10 & 112.08 & 473.8 & 27.02 & 79.3 \\
\hline 200. & 13.764 & 1.704 & 117.38 & 31.98 & 149.65 & 393.4 & 22.66 & 71.8 \\
\hline 205. & 11.100 & 2.579 & 121.70 & 33.07 & 195.46 & 331.6 & 17.91 & 64.4 \\
\hline 210. & 8.693 & 3.489 & 126.09 & 32.44 & 156.19 & 317.7 & 14.68 & 54.7 \\
\hline 215. & 0.535 & 4.149 & 129.20 & 31.25 & 112.40 & 323.7 & 13.28 & 47.4 \\
\hline 220. & 0.591 & 4.648 & 131.49 & 30.36 & 89.52 & 332.7 & 12.62 & 43.3 \\
\hline 225 . & 5.893 & 5.060 & 133.34 & 29.71 & 76.48 & 341.9 & 12.27 & 41.0 \\
\hline 230. & 5.455 & 5.420 & 134.93 & 29.23 & 68.21 & 350.7 & 12.07 & 39.5 \\
\hline 235. & 5.108 & 5.746 & 136.33 & 28.86 & 62.54 & 359.1 & 11.97 & 38.6 \\
\hline 240 . & 4.822 & 6.048 & 137.60 & 28.59 & 58.44 & 367.0 & 11.92 & 38.1 \\
\hline 245. & 0.750 & 6.332 & 138.77 & 28.39 & 55.36 & 374.6 & 11.92 & 37.8 \\
\hline 250. & 0.770 & 6.603 & 139.87 & 28.24 & 52.97 & 381.7 & 11.94 & 37.6 \\
\hline 255. & 4.189 & 6.863 & 140.90 & 28.15 & 51.08 & 388.5 & 11.98 & 37.6 \\
\hline 260 . & 4.027 & 7.114 & 141.87 & 28.09 & 49.55 & 395.0 & 12.04 & 37.7 \\
\hline 265. & 3.881 & 7.359 & 142.81 & 28.06 & 48.31 & 401.2 & 12.11 & 37.9 \\
\hline 270. & 0.832 & 7.598 & 143.70 & 28.07 & 47.28 & 407.2 & 12.20 & 38.2 \\
\hline 275. & 3.629 & 7.832 & 144.56 & 28.10 & 46.43 & 412.9 & 12.29 & 38.5 \\
\hline 280. & 0.855 & 8.062 & 145.39 & 28.15 & 45.71 & 418.4 & 12.38 & 38.8 \\
\hline 285. & 0.865 & 8.289 & 146.19 & 28.22 & 45.12 & 423.8 & 12.49 & 39.2 \\
\hline 290. & 0.874 & 8.514 & 146.97 & 28.31 & 44.62 & 428.9 & 12.59 & 39.6 \\
\hline
\end{tabular}


METHANE ISOBAR AT $P=7.0 \mathrm{MPa}$ (continued)

\begin{tabular}{|c|c|c|c|c|c|c|c|c|c|}
\hline $\mathrm{T}$ & $\rho$ & Z & $\mathrm{H}$ & $S$ & $\mathrm{C}_{\mathrm{v}}$ & $C_{p}$ & W & $\eta$ & $\lambda$ \\
\hline K & $\mathrm{mol} \cdot \mathrm{dm}^{-}$ & & $\mathrm{kJ} / \mathrm{mol}$ & $\mathrm{J} /(\mathrm{mol} \cdot \mathrm{K})$ & $\mathrm{J} /(\mathrm{mol} \cdot \mathrm{K})$ & $\mathrm{J} /(\mathrm{mol} \cdot \mathrm{K})$ & $m \cdot s^{-1}$ & $\mu \mathrm{Pa} \cdot \mathrm{s}$ & $\mathrm{mW} /(\mathrm{m} \cdot \mathrm{K}$ \\
\hline 295. & 3.234 & 0.882 & 8.736 & 147.73 & 28.42 & 44.20 & 434.0 & 12.70 & 40.0 \\
\hline 300 . & 3.152 & 0.890 & 8.956 & 148.47 & 28.54 & 43.86 & 438.8 & 12.82 & 40.5 \\
\hline 310. & 3.003 & 0.905 & 9.392 & 149.90 & 28.82 & 43.34 & 448.1 & 13.05 & 41.5 \\
\hline 320 . & 2.870 & 0.917 & 9.823 & 151.27 & 29.14 & 43.01 & 456.9 & 13.29 & 42.7 \\
\hline 330 . & 2.751 & 0.927 & 10.252 & 152.59 & 29.50 & 42.83 & 465.3 & 13.54 & 43.8 \\
\hline 340 . & 2.643 & 0.937 & 10.680 & 153.87 & 29.90 & 42.76 & 473.3 & 13.79 & 45.1 \\
\hline 350 . & 2.545 & 0.945 & 11.108 & 155.11 & 30.33 & 42.79 & 481.0 & 14.04 & 46.4 \\
\hline 360 . & 2.455 & 0.952 & 11.536 & 156.32 & 30.78 & 42.90 & 488.4 & 14.29 & 47.8 \\
\hline 370. & 2.373 & 0.959 & 11.966 & 157.49 & 31.25 & 43.07 & 495.6 & 14.55 & 49.2 \\
\hline 380. & 2.297 & 0.965 & 12.398 & 158.64 & 31.75 & 43.30 & 502.5 & 14.80 & 50.7 \\
\hline 390. & 2.226 & 0.970 & 12.832 & 159.77 & 32.26 & 43.58 & 509.2 & 15.05 & 52.2 \\
\hline 400. & 2.160 & 0.975 & 13.270 & 160.88 & 32.78 & 43.89 & 515.7 & 15.31 & 53.8 \\
\hline 410. & 2.098 & 0.979 & 13.710 & 161.97 & 33.31 & 44.24 & 522.0 & & 55.4 \\
\hline 420. & 2.040 & 0.983 & 14.155 & 163.04 & 33.86 & 44.62 & 528.2 & & 57.0 \\
\hline 430. & 1.986 & 0.986 & 14.603 & 164.09 & 34.41 & 45.02 & 534.2 & & 58.7 \\
\hline 440. & 1.935 & 0.989 & 15.055 & 165.13 & 34.97 & 45.43 & 540.1 & & 60.4 \\
\hline 450. & 1.886 & 0.992 & 15.512 & 166.16 & 35.53 & 45.87 & 545.9 & & 62.1 \\
\hline 460. & 1.840 & 0.994 & 15.972 & 167.17 & 36.09 & 46.32 & 551.6 & & 63.9 \\
\hline 470. & 1.797 & 0.997 & 16.438 & 168.17 & 36.65 & 46.78 & 557.2 & & 65.7 \\
\hline 480. & 1.756 & 0.999 & 16.908 & 169.16 & 37.22 & 47.24 & 562.7 & & 67.5 \\
\hline 490. & 1.717 & 1.001 & 17.383 & 170.14 & 37.78 & 47.72 & 568.1 & & 69.3 \\
\hline 500. & 1.680 & 1.003 & 17.862 & 171.11 & 38.34 & 48.19 & 573.4 & & 71.1 \\
\hline 520. & 1.610 & 1.006 & 18.836 & 173.02 & 39.45 & 49.16 & 583.9 & & 74.8 \\
\hline 540. & 1.546 & 1.008 & 19.829 & 174.89 & 40.55 & 50.13 & 594.0 & & 78.6 \\
\hline 560. & 1.488 & 1.010 & 20.841 & 176.73 & 41.62 & 51.09 & 603.9 & & 82.4 \\
\hline 580. & 1.434 & 1.012 & 21.872 & 178.54 & 42.67 & 52.04 & 613.7 & & 86.3 \\
\hline 600. & 1.384 & 1.014 & 22.922 & 180.32 & 43.69 & 52.98 & 623.2 & & 90.1 \\
\hline
\end{tabular}

$\overline{\text { METHANE ISOBAR AT } \mathrm{P}=8.0 \mathrm{MPa}}$

\begin{tabular}{|c|c|c|c|c|c|c|c|c|c|}
\hline $\mathrm{T}$ & $\rho$ & $Z$ & $\mathrm{H}$ & $S$ & $\mathrm{C}_{\mathrm{v}}$ & $C_{p}$ & W & $\eta$ & $\lambda$ \\
\hline K & $\mathrm{mol} \cdot \mathrm{dm}^{-3}$ & & $\mathrm{~kJ} / \mathrm{mo} 1$ & $\mathrm{~J} /(\mathrm{mol} \cdot \mathrm{K})$ & $\mathrm{J} /(\mathrm{mol} \cdot \mathrm{K})$ & $\mathrm{J} /(\mathrm{mol} \cdot \mathrm{K})$ & $m \cdot s^{-1}$ & $\mu \mathrm{Pa} \cdot \mathrm{s}$ & $\mathrm{mW} /(\mathrm{m} \cdot \mathrm{K})$ \\
\hline 95. & 28.119 & 0.360 & -5.308 & 69.45 & 34.27 & 53.04 & 1558.9 & 195.57 & 213.2 \\
\hline 100. & 27.722 & 0.347 & -5.041 & 72.19 & 34.35 & 53.79 & 1510.3 & 171.91 & 207.3 \\
\hline 105. & 27.319 & 0.335 & -4.771 & 74.82 & 34.13 & 54.27 & 1464.4 & 151.83 & 201.0 \\
\hline 110. & 26.909 & 0.325 & -4.498 & 77.36 & 33.78 & 54.64 & 1419.4 & 134.85 & 194.6 \\
\hline 115. & 26.490 & 0.316 & -4.224 & 79.79 & 33.37 & 54.98 & 1374.5 & 120.47 & 188.0 \\
\hline 120. & 26.062 & 0.308 & -3.949 & 82.14 & 32.96 & 55.35 & 1329.3 & 108.26 & 181.4 \\
\hline
\end{tabular}


METHANE ISOBAR AT $\mathrm{P}=8.0 \mathrm{MPa}$ (continued)

\begin{tabular}{|c|c|c|c|c|c|c|c|c|c|}
\hline $\mathrm{T}$ & $\rho$ & $\mathrm{Z}$ & $\mathrm{H}$ & $S$ & $C_{v}$ & $C_{p}$ & W & $\eta$ & $\lambda$ \\
\hline K & $\mathrm{mol} \cdot \mathrm{dm}^{-3}$ & & $\mathrm{~kJ} / \mathrm{mol}$ & $\mathrm{J} /(\mathrm{mol} \cdot \mathrm{K})$ & $\mathrm{J} /(\mathrm{mol} \cdot \mathrm{K})$ & $\mathrm{J} /(\mathrm{mol} \cdot \mathrm{K})$ & $\mathrm{m} \cdot \mathrm{s}^{-1}$ & $\mu \mathrm{Pa} \cdot \mathrm{s}$ & $\mathrm{mW} /(\mathrm{m}$ \\
\hline 25 . & 25.623 & 0.300 & -3.671 & 84.41 & 32.55 & 55.78 & 1283.7 & 97.84 & 174.7 \\
\hline 30. & 25.172 & 0.294 & -3.391 & 86.61 & 32.18 & 56.28 & 1237.5 & 88.89 & 168.0 \\
\hline 135. & 24.708 & 0.288 & -3.108 & 88.74 & 31.83 & 56.87 & 1190.6 & 81.14 & 161.3 \\
\hline 140. & 24.228 & 0.284 & -2.822 & 90.82 & 31.50 & 57.57 & 1143.0 & 74.37 & 154.6 \\
\hline 145. & 23.731 & 0.280 & -2.532 & 92.86 & 31.21 & 58.41 & 1094.7 & 68.39 & 147.9 \\
\hline 50. & 23.213 & 0.276 & -2.237 & 94.85 & 30.95 & 59.40 & 1045.4 & 63.06 & 141.3 \\
\hline 55. & 22.672 & 0.274 & -1.938 & 96.82 & 30.71 & 60.59 & 995.2 & 58.26 & 134.8 \\
\hline 160. & 22.102 & 0.272 & -1.631 & 98.76 & 30.51 & 62.02 & 943.8 & 53.89 & 128.3 \\
\hline 165. & 21.500 & 0.271 & -1.317 & 100.70 & 30.35 & 63.77 & 891.1 & 49.86 & 121.8 \\
\hline 170 . & 20.857 & 0.271 & -0.993 & 102.63 & 30.22 & 65.93 & 836.9 & 46.11 & 115.3 \\
\hline 75. & 20.164 & 0.273 & -0.657 & 104.58 & 30.14 & 68.67 & 780.8 & 42.56 & 108.8 \\
\hline 180. & 19.407 & 0.275 & -0.305 & 106.56 & 30.12 & 72.24 & 722.5 & 39.17 & 102.4 \\
\hline 185. & 18.567 & 0.280 & 0.068 & 108.61 & 30.17 & 77.06 & 661.9 & 35.88 & 96.0 \\
\hline 190. & 17.613 & 0.288 & 0.469 & 110.75 & 30.31 & 83.89 & 598.6 & 32.60 & 90.1 \\
\hline 195. & 16.494 & 0.299 & 0.912 & 113.05 & 30.58 & 94.18 & 532.7 & 29.27 & 83.3 \\
\hline 200. & 15.130 & 0.318 & 1.420 & 115.62 & 30.98 & 110.16 & 465.7 & 25.79 & 76.6 \\
\hline 05. & 13.426 & 0.350 & 2.025 & 118.60 & 31.55 & 132.18 & 403.5 & 22.13 & 70.0 \\
\hline 210. & 11.433 & 0.401 & 2.735 & 122.03 & 32.00 & 148.93 & 359.5 & 18.64 & 63.7 \\
\hline 215. & 9.592 & 0.467 & 3.459 & 125.43 & 31.74 & 135.46 & 342.0 & 16.06 & 56.9 \\
\hline 220 . & 8.273 & 0.529 & 4.071 & 128.25 & 31.05 & 110.05 & 341.4 & 14.56 & 51.1 \\
\hline 225 . & 7.368 & 0.580 & 4.572 & 130.50 & 30.37 & 91.50 & 346.6 & 13.73 & 47.1 \\
\hline 230 . & 6.712 & 0.623 & 4.997 & 132.37 & 29.82 & 79.27 & 353.5 & 13.24 & 44.4 \\
\hline 235. & 6.211 & 0.659 & 5.371 & 133.98 & 29.38 & 70.97 & 360.9 & 12.95 & 42.7 \\
\hline 240. & 5.811 & 0.690 & 5.711 & 135.41 & 29.04 & 65.08 & 368.3 & 12.76 & 41.5 \\
\hline 245 . & 5.481 & 0.717 & 6.025 & 136.70 & 28.78 & 60.74 & 375.5 & 12.66 & 40.8 \\
\hline 250 . & 5.202 & 0.740 & 6.320 & 137.90 & 28.59 & 57.43 & 382.5 & 12.60 & 40.3 \\
\hline 255 . & 4.961 & 0.761 & 6.600 & 139.01 & 28.46 & 54.85 & 389.2 & 12.58 & 40.0 \\
\hline 260 . & 4.750 & 0.779 & 6.869 & 140.05 & 28.37 & 52.79 & 395.7 & 12.59 & 39.9 \\
\hline 265 . & 4.563 & 0.796 & 7.129 & 141.04 & 28.32 & 51.13 & 401.9 & 12.62 & 39.9 \\
\hline 270 . & 4.395 & 0.811 & 7.381 & 141.98 & 28.30 & 49.77 & 407.9 & 12.67 & 40.0 \\
\hline 275 . & 4.244 & 0.824 & 7.627 & 142.89 & 28.31 & 48.64 & 413.7 & 12.73 & 40.1 \\
\hline 280. & 4.106 & 0.837 & 7.867 & 143.75 & 28.34 & 47.70 & 419.3 & 12.80 & 40.4 \\
\hline 285 . & 3.979 & 0.848 & 8.104 & 144.59 & 28.40 & 46.91 & 424.7 & 12.88 & 40.6 \\
\hline 290 . & 3.863 & 0.859 & 8.337 & 145.40 & 28.48 & 46.25 & 429.9 & 12.97 & 41.0 \\
\hline 295. & 3.755 & 0.869 & 8.567 & 146.19 & 28.57 & 45.70 & 434.9 & 13.06 & 41.4 \\
\hline 300 . & 3.655 & 0.878 & 8.794 & 146.95 & 28.68 & 45.24 & 439.8 & 13.16 & 41.8 \\
\hline 310 . & 3.474 & 0.894 & 9.243 & 148.42 & 28.94 & 44.52 & 449.2 & 13.36 & 42.7 \\
\hline 320 . & 3.314 & 0.907 & 9.685 & 149.83 & 29.25 & 44.04 & 458.2 & 13.58 & 43.7 \\
\hline 33 & 3.171 & 0.919 & 10.124 & 151.18 & 29.61 & 43.74 & 466.6 & 13.81 & 44.8 \\
\hline 34 & 3.043 & 0.930 & 10.560 & 152.48 & 30.00 & 43.57 & 474.8 & 14.04 & 46.0 \\
\hline
\end{tabular}


METHANE ISOBAR AT $\mathrm{P}=8.0 \mathrm{MPa}$ (continued)

$\begin{array}{lccccccccc}\mathrm{T} & \rho & \mathrm{Z} & \mathrm{H} & \mathrm{S} & \mathrm{C} & \mathrm{C} & \mathrm{W} & \eta & \lambda \\ \mathrm{K} & \mathrm{mo} \cdot \mathrm{dm}^{-3} & & \mathrm{~kJ} / \mathrm{mo} 1 & \mathrm{~J} /(\mathrm{mol} \cdot \mathrm{K}) & \mathrm{J} /(\mathrm{mol} \cdot \mathrm{K}) & \mathrm{J} /(\mathrm{mol} \cdot \mathrm{K}) & \mathrm{m} \cdot \mathrm{s}^{-1} & \mu \mathrm{Pa} \cdot \mathrm{s} & \mathrm{mW} /(\mathrm{m} \cdot \mathrm{K}) \\ & & & & & & & & & \\ 350 . & 2.927 & 0.939 & 10.996 & 153.74 & 30.41 & 43.52 & 482.5 & 14.28 & 47.3 \\ 360 . & 2.821 & 0.947 & 11.431 & 154.97 & 30.86 & 43.56 & 490.0 & 14.52 & 48.6 \\ 370 . & 2.724 & 0.955 & 11.867 & 156.16 & 31.33 & 43.67 & 497.2 & 14.76 & 50.0 \\ 380 . & 2.634 & 0.961 & 12.305 & 157.33 & 31.82 & 43.85 & 504.2 & 15.01 & 51.4 \\ 390 . & 2.551 & 0.967 & 12.744 & 158.47 & 32.32 & 44.08 & 510.9 & 15.25 & 52.9 \\ & & & & & & & & & \\ 400 . & 2.474 & 0.972 & 13.187 & 159.59 & 32.84 & 44.36 & 517.5 & 15.50 & 54.4 \\ 410 . & 2.402 & 0.977 & 13.632 & 160.69 & 33.37 & 44.67 & 523.9 & & 56.0 \\ 420 . & 2.335 & 0.981 & 14.080 & 161.77 & 33.91 & 45.02 & 530.1 & & 57.6 \\ 430 . & 2.271 & 0.985 & 14.532 & 162.83 & 34.46 & 45.39 & 536.2 & & 59.3 \\ 440 . & 2.212 & 0.989 & 14.988 & 163.88 & 35.02 & 45.79 & 542.2 & & 61.0 \\ & & & & & & & & \\ 450 . & 2.156 & 0.992 & 15.448 & 164.92 & 35.57 & 46.20 & 548.0 & & 62.7 \\ 460 . & 2.103 & 0.995 & 15.912 & 165.94 & 36.13 & 46.63 & 553.7 & & 64.4 \\ 470 . & 2.053 & 0.997 & 16.381 & 166.94 & 36.70 & 47.07 & 559.3 & & 66.2 \\ 480 . & 2.006 & 0.999 & 16.854 & 167.94 & 37.26 & 47.52 & 564.9 & & 68.0 \\ 490 . & 1.960 & 1.002 & 17.331 & 168.92 & 37.82 & 47.98 & 570.3 & & 69.8 \\ & & & & & & & & & \\ 500 . & 1.917 & 1.004 & 17.813 & 169.90 & 38.38 & 48.44 & 575.6 & & 71.6 \\ 520 . & 1.837 & 1.007 & 18.791 & 171.82 & 39.49 & 49.38 & 586.1 & & 75.3 \\ 540 . & 1.764 & 1.010 & 19.789 & 173.70 & 40.58 & 50.33 & 596.3 & & 79.0 \\ 560 . & 1.697 & 1.012 & 20.805 & 175.55 & 41.65 & 51.27 & 606.3 & & 82.8 \\ 580 . & 1.636 & 1.014 & 21.840 & 177.36 & 42.70 & 52.21 & 616.1 & & 86.6 \\ & & & & & & & & \\ 600 . & 1.578 & 1.016 & 22.893 & 179.15 & 43.72 & 53.13 & 625.6 & & 90.5\end{array}$

\section{METHANE ISOBAR AT P $=9.0 \mathrm{MPa}$}

$\begin{array}{cccccccccc}\mathrm{T} & \rho & \mathrm{Z} & \mathrm{H} & \mathrm{S} & \mathrm{C}_{\mathrm{v}} & \mathrm{C}_{\mathrm{p}} & \mathrm{W} & \eta & \lambda \\ \mathrm{K} & \mathrm{mol} \cdot \mathrm{dm}^{-3} & & \mathrm{~kJ} / \mathrm{mol} & \mathrm{J} /(\mathrm{mol} \cdot \mathrm{K}) & \mathrm{J} /(\mathrm{mol} \cdot \mathrm{K}) & \mathrm{J} /(\mathrm{mol} \cdot \mathrm{K}) & \mathrm{m} \cdot \mathrm{s}^{-1} & \mu \mathrm{Pa} \cdot \mathrm{s} & \mathrm{mW} /(\mathrm{m} \cdot \mathrm{K}) \\ & & & & & & & & & \\ \text { 95. } & 28.158 & 0.405 & -5.282 & 69.35 & 34.30 & 52.96 & 1565.9 & 197.64 & 214.1 \\ 100 . & 27.765 & 0.390 & -5.015 & 72.08 & 34.39 & 53.69 & 1517.7 & 173.79 & 208.2 \\ 105 . & 27.365 & 0.377 & -4.746 & 74.72 & 34.17 & 54.16 & 1472.2 & 153.55 & 202.0 \\ 110 . & 26.958 & 0.365 & -4.474 & 77.24 & 33.82 & 54.51 & 1427.7 & 136.41 & 195.6 \\ 115 . & 26.544 & 0.355 & -4.200 & 79.67 & 33.41 & 54.84 & 1383.3 & 121.90 & 189.1 \\ & & & & & & & & & \\ 120 . & 26.120 & 0.345 & -3.925 & 82.02 & 33.00 & 55.18 & 1338.7 & 109.58 & 182.5 \\ 125 . & 25.687 & 0.337 & -3.649 & 84.28 & 32.60 & 55.57 & 1293.7 & 99.06 & 175.8 \\ 130 . & 25.242 & 0.330 & -3.370 & 86.46 & 32.22 & 56.03 & 1248.2 & 90.04 & 169.2 \\ 135 . & 24.785 & 0.324 & -3.088 & 88.59 & 31.87 & 56.58 & 1202.2 & 82.23 & 162.5 \\ 140 . & 24.314 & 0.318 & -2.804 & 90.66 & 31.55 & 57.23 & 1155.6 & 75.41 & 155.9\end{array}$


METHANE ISOBAR AT $P=9.0 \mathrm{MPa}$ (continued)

\begin{tabular}{|c|c|c|c|c|c|c|c|c|c|}
\hline $\mathrm{T}$ & $\rho$ & $\mathrm{Z}$ & $\mathrm{H}$ & $S$ & $C_{v}$ & $C_{p}$ & W & $\eta$ & $\lambda$ \\
\hline K & $\mathrm{mol} \cdot \mathrm{dm}^{-3}$ & & $\mathrm{~kJ} / \mathrm{mo} 1$ & $\mathrm{~J} /(\mathrm{mol} \cdot \mathrm{K})$ & $\mathrm{J} /(\mathrm{mol} \cdot \mathrm{K})$ & $\mathrm{J} /(\mathrm{mol} \cdot \mathrm{K})$ & $\mathrm{m} \cdot \mathrm{s}^{-1}$ & $\mu \mathrm{Pa} \cdot \mathrm{s}$ & $\mathrm{mW} /(\mathrm{m} \cdot \mathrm{K})$ \\
\hline 145. & 23.826 & 0.313 & -2.516 & 92.68 & 31.25 & 57.99 & 1108.4 & 69.40 & 149.4 \\
\hline 150. & 23.320 & 0.309 & -2.223 & 94.66 & 30.99 & 58.89 & 1060.4 & 64.05 & 142.9 \\
\hline 155. & 22.793 & 0.306 & -1.926 & 96.61 & 30.75 & 59.96 & 1011.7 & 59.24 & 136.4 \\
\hline 160. & 22.241 & 0.304 & -1.624 & 98.53 & 30.54 & 61.23 & 962.1 & 54.87 & 130.0 \\
\hline 165. & 21.660 & 0.303 & -1.314 & 100.44 & 30.37 & 62.75 & 911.5 & 50.87 & 123.6 \\
\hline 170 . & 21.044 & 0.303 & -0.995 & 102.34 & 30.22 & 64.60 & 859.7 & 47.15 & 117.3 \\
\hline 175. & 20.386 & 0.303 & -0.667 & 104.24 & 30.12 & 66.88 & 806.7 & 43.67 & 111.1 \\
\hline 180. & 19.678 & 0.306 & -0.326 & 106.16 & 30.06 & 69.74 & 752.2 & 40.36 & 104.9 \\
\hline 185. & 18.905 & 0.310 & 0.032 & 108.12 & 30.06 & 73.41 & 696.1 & 37.19 & 98.8 \\
\hline 190. & 18.049 & 0.316 & 0.410 & 110.14 & 30.13 & 78.25 & 638.6 & 34.10 & 93.1 \\
\hline 195. & 17.084 & 0.325 & 0.817 & 112.25 & 30.27 & 84.85 & 579.8 & 31.04 & 86.8 \\
\hline 200 . & 15.974 & 0.339 & 1.263 & 114.51 & 30.50 & 93.94 & 520.7 & 27.97 & 80.6 \\
\hline 205. & 14.678 & 0.360 & 1.761 & 116.97 & 30.81 & 105.72 & 464.1 & 24.87 & 74.6 \\
\hline 210 . & 13.188 & 0.391 & 2.321 & 119.67 & 31.17 & 117.98 & 415.7 & 21.84 & 68.9 \\
\hline 215 . & 11.600 & 0.434 & 2.932 & 122.54 & 31.36 & 124.35 & 382.1 & 19.11 & 63.5 \\
\hline 220 . & 10.135 & 0.485 & 3.541 & 125.34 & 31.17 & 117.27 & 365.5 & 17.01 & 58.2 \\
\hline 225 . & 8.967 & 0.537 & 4.092 & 127.82 & 30.73 & 102.72 & 361.2 & 15.62 & 53.6 \\
\hline 230 . & 8.084 & 0.582 & 4.571 & 129.93 & 30.24 & 89.44 & 363.1 & 14.73 & 50.0 \\
\hline 235. & 7.408 & 0.622 & 4.992 & 131.74 & 29.80 & 79.35 & 367.7 & 14.16 & 47.4 \\
\hline 240 . & 6.874 & 0.656 & 5.369 & 133.33 & 29.43 & 71.89 & 373.5 & 13.79 & 45.5 \\
\hline 245 . & 6.441 & 0.686 & 5.714 & 134.75 & 29.14 & 66.32 & 379.6 & 13.54 & 44.2 \\
\hline 250 . & 6.080 & 0.712 & 6.034 & 136.04 & 28.91 & 62.08 & 385.9 & 13.38 & 43.3 \\
\hline 255. & 5.772 & 0.735 & 6.336 & 137.24 & 28.75 & 58.78 & 392.2 & 13.28 & 42.7 \\
\hline 260. & 5.506 & 0.756 & 6.623 & 138.35 & 28.63 & 56.16 & 398.3 & 13.23 & 42.3 \\
\hline 265. & 5.273 & 0.775 & 6.898 & 139.40 & 28.55 & 54.05 & 404.3 & 13.20 & 42.1 \\
\hline 270 . & 5.065 & 0.792 & 7.164 & 140.40 & 28.51 & 52.33 & 410.2 & 13.21 & 42.0 \\
\hline 275. & 4.879 & 0.807 & 7.422 & 141.34 & 28.51 & 50.91 & 415.8 & 13.23 & 42.0 \\
\hline 280. & 4.711 & 0.821 & 7.674 & 142.25 & 28.53 & 49.73 & 421.3 & 13.27 & 42.1 \\
\hline 285. & 4.557 & 0.833 & 7.920 & 143.12 & 28.57 & 48.75 & 426.7 & 13.32 & 42.3 \\
\hline 290. & 4.417 & 0.845 & 8.162 & 143.96 & 28.63 & 47.92 & 431.8 & 13.38 & 42.5 \\
\hline 295. & 4.287 & 0.856 & 8.399 & 144.77 & 28.72 & 47.22 & 436.9 & 13.45 & 42.8 \\
\hline 300 . & 4.168 & 0.866 & 8.634 & 145.56 & 28.82 & 46.63 & 441.7 & 13.53 & 43.1 \\
\hline 310. & 3.952 & 0.883 & 9.095 & 147.08 & 29.07 & 45.71 & 451.1 & 13.70 & 43.9 \\
\hline 320 . & 3.763 & 0.899 & 9.549 & 148.52 & 29.36 & 45.08 & 460.1 & 13.90 & 44.8 \\
\hline 330 . & 3.596 & 0.912 & 9.998 & 149.90 & 29.71 & 44.64 & 468.6 & 14.10 & 45.8 \\
\hline 340 . & 3.446 & 0.924 & 10.443 & 151.23 & 30.09 & 44.38 & 476.7 & 14.31 & 47.0 \\
\hline 350. & 3.311 & 0.934 & 10.886 & 152.51 & 30.50 & 44.24 & 484.5 & 14.53 & 48.2 \\
\hline 360. & 3.188 & 0.943 & 11.328 & 153.76 & 30.94 & 44.21 & 492.0 & 14.76 & 49.4 \\
\hline 370. & 3.076 & 0.951 & 11.770 & 154.97 & 31.40 & 44.27 & 499.3 & 14.99 & 50.8 \\
\hline 380 . & 2.972 & 0.958 & 12.213 & 156.15 & 31.89 & 44.40 & 506.3 & 15.22 & 52.2 \\
\hline
\end{tabular}


METHANE ISOBAR AT $\mathrm{P}=9.0 \mathrm{MPa}$ (continued)

\begin{tabular}{|c|c|c|c|c|c|c|c|c|c|}
\hline $\mathrm{T}$ & $\rho$ & 2 & $\mathrm{H}$ & $S$ & $C_{v}$ & $C_{p}$ & W & $\eta$ & $\lambda$ \\
\hline K & $\mathrm{mol} \cdot \mathrm{dm}^{-3}$ & & $\mathrm{~kJ} / \mathrm{mol}$ & $\mathrm{J} /(\mathrm{mol} \cdot \mathrm{K})$ & $\mathrm{J} /(\mathrm{mol} \cdot \mathrm{K})$ & $\mathrm{J} /(\mathrm{mol} \cdot \mathrm{K})$ & $\mathrm{m} \cdot \mathrm{s}^{-1}$ & $\mu \mathrm{Pa} \cdot \mathrm{s}$ & $\mathrm{mW} /(\mathrm{m} \cdot \mathrm{K})$ \\
\hline 390. & 2.877 & 0.965 & 12.658 & 157.30 & 32.39 & 44.58 & 513.0 & 15.46 & 53.6 \\
\hline 400. & 2.788 & 0.971 & 13.105 & 158.44 & 32.90 & 44.82 & 519.6 & 15.70 & 55.1 \\
\hline 410. & 2.706 & 0.976 & 13.555 & 159.55 & 33.43 & 45.10 & 526.1 & & 56.7 \\
\hline 420. & 2.629 & 0.980 & 14.007 & 160.64 & 33.97 & 45.42 & 532.3 & & 58.3 \\
\hline 430. & 2.557 & 0.985 & 14.463 & 161.71 & 34.51 & 45.76 & 538.4 & & 59.9 \\
\hline 440 . & 2.489 & 0.988 & 14.923 & 162.77 & 35.06 & 46.13 & 544.4 & & 61.5 \\
\hline 450. & 2.425 & 0.992 & 15.386 & 163.81 & 35.62 & 46.53 & 550.3 & & 63.2 \\
\hline 460. & 2.365 & 0.995 & 15.853 & 164.83 & 36.18 & 46.93 & 556.0 & & 64.9 \\
\hline 470. & 2.308 & 0.998 & 16.325 & 165.85 & 36.74 & 47.36 & 561.7 & & 66.7 \\
\hline 480. & 2.254 & 1.000 & 16.800 & 166.85 & 37.30 & 47.79 & 567.2 & & 68.5 \\
\hline 490. & 2.203 & 1.003 & 17.281 & 167.84 & 37.86 & 48.24 & 572.7 & & 70.2 \\
\hline 500. & 2.154 & 1.005 & 17.765 & 168.82 & 38.41 & 48.69 & 578.0 & & 72.0 \\
\hline 520. & 2.064 & 1.009 & 18.748 & 170.75 & 39.52 & 49.60 & 588.5 & & 75.7 \\
\hline 540. & 1.981 & 1.012 & 19.749 & 172.64 & 40.61 & 50.53 & 598.8 & & 79.4 \\
\hline 560. & 1.906 & 1.014 & 20.769 & 174.49 & 41.68 & 51.46 & 608.8 & & 83.2 \\
\hline 580 & 1.836 & 1.017 & 21.808 & 176.31 & 42.72 & 52.38 & 618.6 & & 87.0 \\
\hline 600 . & 1.771 & 1.018 & 22.864 & 178.10 & 43.74 & 53.29 & 628.1 & & 90.8 \\
\hline
\end{tabular}

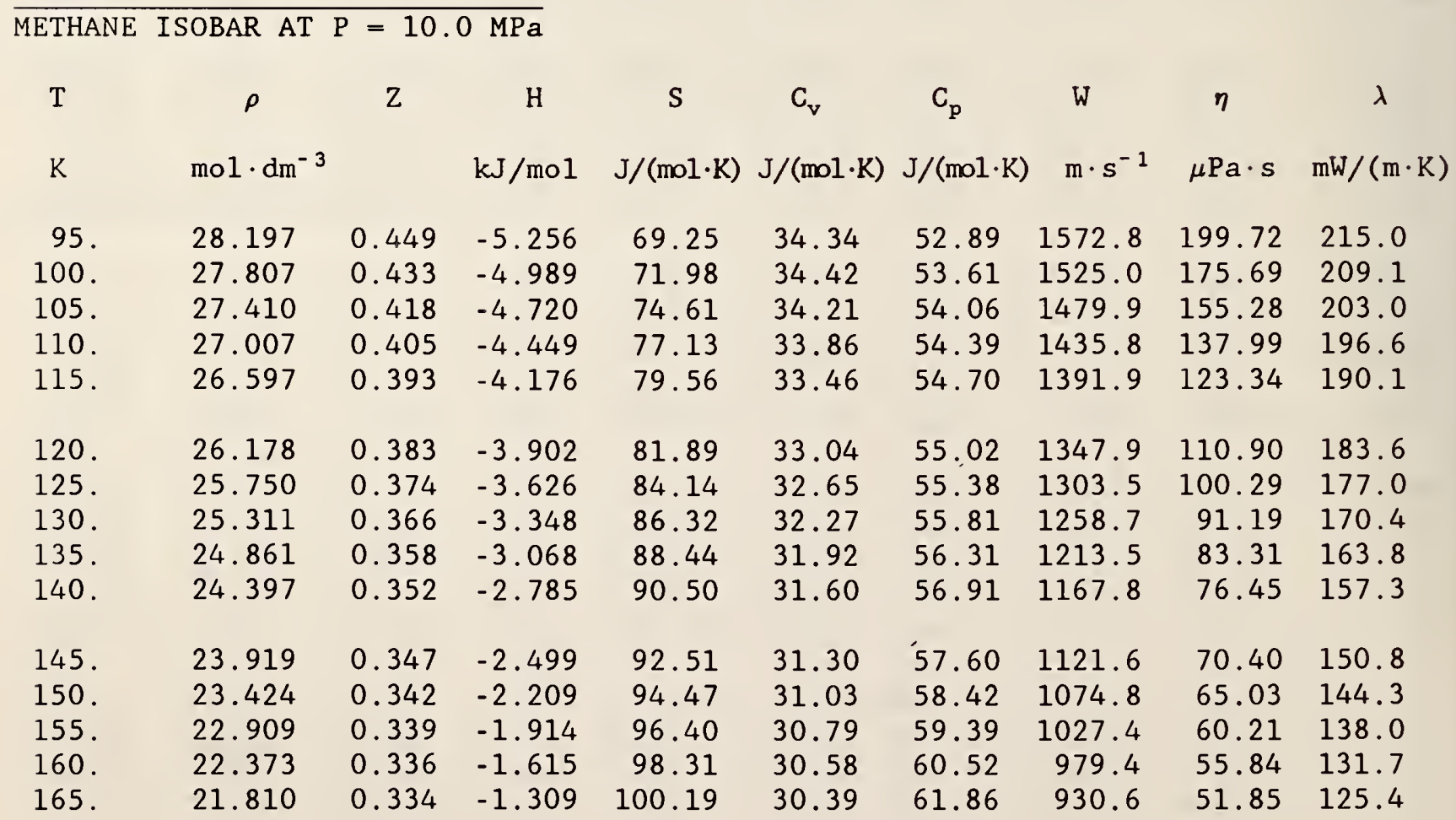


METHANE ISOBAR AT $P=10.0 \mathrm{MPa}$ (continued)

\begin{tabular}{|c|c|c|c|c|c|c|c|c|}
\hline$\rho$ & Z & $\mathrm{H}$ & S & $\mathrm{C}_{\mathrm{v}}$ & $C_{p}$ & W & $\eta$ & $\lambda$ \\
\hline $\mathrm{mol} \cdot \mathrm{dm}^{-3}$ & & $\mathrm{~kJ} / \mathrm{mol}$ & $\mathrm{J} /(\mathrm{mol} \cdot \mathrm{K})$ & $\mathrm{J} /(\mathrm{mol} \cdot \mathrm{K})$ & $\mathrm{J} /(\mathrm{mol} \cdot \mathrm{K})$ & $m \cdot s^{-1}$ & $\mu \mathrm{Pa} \cdot \mathrm{s}$ & $\mathrm{mW} /(\mathrm{m} \cdot$ \\
\hline 21.218 & 0.333 & -0.995 & 102.06 & 30.24 & 63.46 & 881.0 & 48.16 & 119.3 \\
\hline 20.591 & 0.334 & -0.674 & 103.93 & 30.12 & 65.39 & 830.5 & 44.72 & 113.2 \\
\hline 19.921 & 0.335 & -0.341 & 105.80 & 30.04 & 67.74 & 779.0 & 41.48 & 107.2 \\
\hline 19.200 & 0.339 & 0.005 & 107.69 & 30.00 & 70.65 & 726.6 & 38.39 & 101.3 \\
\hline 18.416 & 0.344 & 0.367 & 109.62 & 30.01 & 74.30 & 673.4 & 35.43 & 95.8 \\
\hline 17.554 & 0.351 & 0.750 & 111.61 & 30.08 & 78.97 & 619.6 & 32.54 & 89.9 \\
\hline 16.594 & 0.362 & 1.159 & 113.68 & 30.21 & 84.94 & 566.0 & 29.72 & 84.1 \\
\hline 15.518 & 0.378 & 1.601 & 115.87 & 30.40 & 92.29 & 514.1 & 26.93 & 78.4 \\
\hline 14.315 & 0.400 & 2.083 & 118.19 & 30.62 & 100.26 & 467.0 & 24.22 & 73.1 \\
\hline 13.014 & 0.430 & 2.602 & 120.63 & 30.83 & 106.87 & 428.7 & 21.68 & 68.1 \\
\hline 11.697 & 0.467 & 3.144 & 123.12 & 30.89 & 108.93 & 401.9 & 19.47 & 63.5 \\
\hline 10.485 & 0.510 & 3.679 & 125.53 & 30.73 & 104.07 & 386.9 & 17.72 & 59.1 \\
\hline 9.464 & 0.553 & 4.177 & 127.72 & 30.42 & 94.96 & 381.1 & 16.46 & 55.3 \\
\hline 8.641 & 0.592 & 4.628 & 129.66 & 30.06 & 85.63 & 380.8 & 15.59 & 52.2 \\
\hline 7.980 & 0.628 & 5.036 & 131.38 & 29.72 & 77.75 & 383.4 & 14.99 & 49.8 \\
\hline 7.440 & 0.660 & 5.409 & 132.91 & 29.42 & 71.46 & 387.5 & 14.57 & 48.0 \\
\hline 6.992 & 0.688 & 5.753 & 134.31 & 29.19 & 66.52 & 392.4 & 14.28 & 46.6 \\
\hline 6.613 & 0.713 & 6.075 & 135.58 & 29.00 & 62.61 & 397.7 & 14.08 & 45.7 \\
\hline 6.288 & 0.736 & 6.380 & 136.77 & 28.86 & 59.48 & 403.2 & 13.94 & 45.0 \\
\hline 6.004 & 0.756 & 6.671 & 137.87 & 28.77 & 56.95 & 408.7 & 13.85 & 44.5 \\
\hline 5.753 & 0.774 & 6.951 & 138.92 & 28.71 & 54.89 & 414.1 & 13.80 & 44.2 \\
\hline 5.530 & 0.791 & 7.221 & 139.91 & 28.69 & 53.18 & 419.5 & 13.78 & 44.0 \\
\hline 5.329 & 0.806 & 7.483 & 140.86 & 28.70 & 51.76 & 424.7 & 13.78 & 44.0 \\
\hline 5.147 & 0.820 & 7.739 & 141.76 & 28.73 & 50.58 & 429.9 & 13.80 & 44.0 \\
\hline 4.981 & 0.833 & 7.989 & 142.63 & 28.78 & 49.58 & 434.9 & 13.83 & 44.1 \\
\hline 4.828 & 0.844 & 8.235 & 143.47 & 28.86 & 48.73 & 439.8 & 13.88 & 44.3 \\
\hline 4.688 & 0.855 & 8.477 & 144.28 & 28.95 & 48.02 & 444.6 & 13.93 & 44.6 \\
\hline 4.436 & 0.875 & 8.951 & 145.84 & 29.18 & 46.90 & 453.8 & 14.07 & 45.2 \\
\hline 4.217 & 0.891 & 9.416 & 147.32 & 29.47 & 46.10 & 462.7 & 14.23 & 46.0 \\
\hline 4.024 & 0.906 & 9.874 & 148.73 & 29.80 & 45.54 & 471.1 & 14.41 & 46.9 \\
\hline 3.852 & 0.918 & 10.327 & 150.08 & 30.17 & 45.18 & 479.2 & 14.60 & 48.0 \\
\hline 3.697 & 0.930 & 10.778 & 151.39 & 30.58 & 44.96 & 487.0 & 14.80 & 49.1 \\
\hline 3.556 & 0.939 & 11.227 & 152.65 & 31.01 & 44.86 & 494.5 & 15.01 & 50.3 \\
\hline 3.428 & 0.948 & 11.675 & 153.88 & 31.47 & 44.85 & 501.7 & 15.23 & 51.6 \\
\hline 3.311 & 0.956 & 12.124 & 155.08 & 31.95 & 44.93 & 508.7 & 15.45 & 53.0 \\
\hline 3.203 & 0.963 & 12.574 & 156.24 & 32.45 & 45.07 & 515.5 & 15.67 & 54.4 \\
\hline 3.103 & 0.969 & 13.026 & 157.39 & 32.96 & 45.27 & 522.1 & 15.90 & 55.8 \\
\hline 3.010 & 0.975 & 13.480 & 158.51 & 33.48 & 45.52 & 528.5 & & 57.3 \\
\hline 2.923 & 0.980 & 13.936 & 159.61 & 34.02 & 45.81 & 534.8 & & 58.9 \\
\hline 2.842 & 0.984 & 14.396 & 160.69 & 34.56 & 46.13 & 540.9 & & 60.5 \\
\hline
\end{tabular}


METHANE ISOBAR AT $\mathrm{P}=10.0 \mathrm{MPa}$ (continued)

\begin{tabular}{|c|c|c|c|c|c|c|c|c|c|}
\hline $\mathrm{T}$ & $\rho$ & $\mathrm{Z}$ & $\mathrm{H}$ & $\mathrm{S}$ & $\mathrm{C}_{\mathrm{v}}$ & $C_{p}$ & W & $\eta$ & $\lambda$ \\
\hline K & $\mathrm{mol} \cdot \mathrm{dm}^{-3}$ & & $\mathrm{~kJ} / \mathrm{mol}$ & $\mathrm{J} /(\mathrm{mol} \cdot \mathrm{K})$ & $\mathrm{J} /(\mathrm{mol} \cdot \mathrm{K})$ & $\mathrm{J} /(\mathrm{mol} \cdot \mathrm{K})$ & $\mathrm{m} \cdot \mathrm{s}^{-1}$ & $\mu \mathrm{Pa} \cdot \mathrm{s}$ & $\mathrm{mW} /(\mathrm{m} \cdot \mathrm{K})$ \\
\hline 440 & 2.765 & 0.988 & 14.859 & 161.76 & 35.11 & 46.47 & 546.9 & & 62.1 \\
\hline 450. & 2.694 & 0.992 & 15.325 & 162.80 & 35.66 & 46.84 & 552.7 & & 63.8 \\
\hline 460. & 2.626 & 0.996 & 15.796 & 163.84 & 36.22 & 47.23 & 558.5 & & 65.5 \\
\hline 470 & 2.562 & 0.999 & 16.270 & 164.86 & 36.78 & 47.64 & 564.2 & & 67.2 \\
\hline 480 & 2.502 & 1.001 & 16.749 & 165.87 & 37.34 & 48.06 & 569.7 & & 69.0 \\
\hline 490 & 2.445 & 1.004 & 17.231 & 166.86 & 37.89 & 48.49 & 575.2 & & 70.7 \\
\hline 500 & 2.390 & 1.006 & 17.718 & 167.84 & 38.45 & 48.93 & 580.6 & & 72.5 \\
\hline 520. & 2.289 & 1.010 & 18.706 & 169.78 & 39.55 & 49.82 & 591.1 & & 76.2 \\
\hline 540. & 2.197 & 1.014 & 19.711 & 171.68 & 40.64 & 50.73 & 601.4 & & 79.9 \\
\hline 560. & 2.113 & 1.017 & 20.735 & 173.54 & 41.71 & 51.64 & 611.4 & & 83.6 \\
\hline 580. & 2.035 & 1.019 & 21.777 & 175.37 & 42.75 & 52.54 & 621.2 & & 87.4 \\
\hline 600 . & 1.963 & 1.021 & 22.837 & 177.16 & 43.77 & 53.44 & 630.7 & & 91.2 \\
\hline
\end{tabular}

$\overline{\text { METHANE ISOBAR AT } \mathrm{P}=11.0 \mathrm{MPa}}$

$\begin{array}{rccccccccc}\mathrm{T} & \rho & \mathrm{Z} & \mathrm{H} & \mathrm{S} & \mathrm{C}_{\mathrm{v}} & \mathrm{C}_{\mathrm{p}} & \mathrm{W} & \eta & \lambda \\ \mathrm{K} & \mathrm{mol} \cdot \mathrm{dm}^{-3} & & \mathrm{~kJ} / \mathrm{mol} & \mathrm{J} /(\mathrm{mol} \cdot \mathrm{K}) & \mathrm{J} /(\mathrm{mol} \cdot \mathrm{K}) & \mathrm{J} /(\mathrm{mol} \cdot \mathrm{K}) & \mathrm{m} \cdot \mathrm{s}^{-1} & \mu \mathrm{Pa} \cdot \mathrm{s} & \mathrm{mW} /(\mathrm{m} \cdot \mathrm{K}) \\ & & & & & & & & & \\ \text { 95. } & 28.236 & 0.493 & -5.230 & 69.15 & 34.38 & 52.81 & 1579.6 & 201.81 & 215.8 \\ 100 . & 27.848 & 0.475 & -4.964 & 71.88 & 34.46 & 53.52 & 1532.2 & 177.60 & 210.0 \\ 105 . & 27.455 & 0.459 & -4.695 & 74.50 & 34.25 & 53.96 & 1487.5 & 157.02 & 203.9 \\ 110 . & 27.055 & 0.445 & -4.424 & 77.02 & 33.90 & 54.28 & 1443.8 & 139.57 & 197.6 \\ 115 . & 26.649 & 0.432 & -4.152 & 79.44 & 33.50 & 54.56 & 1400.4 & 124.79 & 191.2 \\ & & & & & & & & & \\ 120 . & 26.234 & 0.420 & -3.879 & 81.77 & 33.09 & 54.86 & 1356.9 & 112.23 & 184.7 \\ 125 . & 25.811 & 0.410 & -3.604 & 84.01 & 32.69 & 55.20 & 1313.1 & 101.53 & 178.1 \\ 130 . & 25.378 & 0.401 & -3.327 & 86.19 & 32.32 & 55.59 & 1269.0 & 92.34 & 171.6 \\ 135 . & 24.935 & 0.393 & -3.048 & 88.29 & 31.97 & 56.06 & 1224.5 & 84.40 & 165.1 \\ 140 . & 24.479 & 0.386 & -2.766 & 90.34 & 31.64 & 56.61 & 1179.6 & 77.48 & 158.6 \\ & & & & & & & & & \\ 145 . & 24.009 & 0.380 & -2.481 & 92.34 & 31.35 & 57.25 & 1134.4 & 71.40 & 152.1 \\ 150 . & 23.524 & 0.375 & -2.193 & 94.29 & 31.08 & 57.99 & 1088.7 & 66.00 & 145.8 \\ 155 . & 23.021 & 0.371 & -1.901 & 96.21 & 30.83 & 58.87 & 1042.5 & 61.16 & 139.5 \\ 160 . & 22.498 & 0.368 & -1.604 & 98.09 & 30.62 & 59.88 & 995.9 & 56.79 & 133.3 \\ 165 . & 21.953 & 0.365 & -1.302 & 99.95 & 30.42 & 61.07 & 948.7 & 52.80 & 127.2 \\ & & & & & & & & & \\ 170 . & 21.382 & 0.364 & -0.993 & 101.80 & 30.26 & 62.47 & 901.0 & 49.13 & 121.1 \\ 175 . & 20.780 & 0.364 & -0.677 & 103.63 & 30.13 & 64.12 & 852.6 & 45.72 & 115.2 \\ 180 . & 20.142 & 0.365 & -0.352 & 105.46 & 30.03 & 66.10 & 803.7 & 42.53 & 109.3 \\ 185 . & 19.463 & 0.367 & -0.015 & 107.30 & 29.96 & 68.47 & 754.2 & 39.51 & 103.6 \\ 190 . & 18.735 & 0.372 & 0.334 & 109.17 & 29.94 & 71.34 & 704.4 & 36.63 & 98.3\end{array}$


METHANE ISOBAR AT $\mathrm{P}=11.0 \mathrm{MPa}$ (continued)

\begin{tabular}{|c|c|c|c|c|c|c|c|c|c|}
\hline $\mathrm{T}$ & $\rho$ & $\mathrm{Z}$ & $\mathrm{H}$ & $S$ & $C_{v}$ & $C_{p}$ & W & $\eta$ & $\lambda$ \\
\hline K & $\mathrm{mol} \cdot \mathrm{dm}^{-}$ & & $\mathrm{kJ} / \mathrm{mol}$ & $\mathrm{J} /(\mathrm{mol} \cdot \mathrm{K})$ & $\mathrm{J} /(\mathrm{mol} \cdot \mathrm{K})$ & $\mathrm{J} /(\mathrm{mol} \cdot \mathrm{K})$ & $\mathrm{m} \cdot \mathrm{s}^{-1}$ & $\mu \mathrm{Pa} \cdot \mathrm{s}$ & $\mathrm{mW} /(\mathrm{m} \cdot \mathrm{K})$ \\
\hline 195. & 17.947 & 0.378 & 0.699 & 111.06 & 29.96 & 74.85 & 654.4 & 33.87 & 92.6 \\
\hline 200. & 17.089 & 0.387 & 1.084 & 113.01 & 30.03 & 79.12 & 604.9 & 31.20 & 87.1 \\
\hline 205. & 16.150 & 0.400 & 1.492 & 115.03 & 30.14 & 84.18 & 556.8 & 28.61 & 81.8 \\
\hline 210 . & 15.124 & 0.417 & 1.926 & 117.12 & 30.28 & 89.68 & 512.0 & 26.11 & 76.7 \\
\hline 215 . & 14.022 & 0.439 & 2.388 & 119.29 & 30.43 & 94.76 & 472.9 & 23.75 & 72.0 \\
\hline 220. & 12.881 & 0.467 & 2.871 & 121.51 & 30.54 & 98.12 & 441.8 & 21.60 & 67.6 \\
\hline 225 . & 11.759 & 0.500 & 3.364 & 123.73 & 30.53 & 98.25 & 419.8 & 19.76 & 63.6 \\
\hline 230 . & 10.726 & 0.536 & 3.847 & 125.85 & 30.39 & 94.46 & 406.5 & 18.28 & 59.9 \\
\hline 235. & 9.829 & 0.573 & 4.304 & 127.82 & 30.15 & 88.20 & 400.2 & 17.15 & 56.7 \\
\hline 240 . & 9.074 & 0.607 & 4.728 & 129.60 & 29.88 & 81.48 & 398.4 & 16.32 & 54.0 \\
\hline 245. & 8.445 & 0.639 & 5.120 & 131.22 & 29.62 & 75.39 & 399.5 & 15.72 & 51.8 \\
\hline 250. & 7.916 & 0.669 & 5.484 & 132.69 & 29.39 & 70.24 & 402.3 & 15.29 & 50.1 \\
\hline 255 . & 7.467 & 0.695 & 5.824 & 134.04 & 29.21 & 66.00 & 406.1 & 14.97 & 48.8 \\
\hline 260 & 7.082 & 0.718 & 6.145 & 135.28 & 29.06 & 62.53 & 410.4 & 14.74 & 47.8 \\
\hline 265. & 6.747 & 0.740 & 6.450 & 136.45 & 28.96 & 59.67 & 415.0 & 14.57 & 47.1 \\
\hline 70 . & 6.452 & 0.759 & 6.742 & 137.54 & 28.89 & 57.32 & 419.8 & 14.46 & 46.6 \\
\hline 275 . & 6.190 & 0.777 & 7.024 & 138.57 & 28.86 & 55.36 & 424.7 & 14.38 & 46.2 \\
\hline 280 & 5.955 & 0.793 & 7.297 & 139.56 & 28.85 & 53.73 & 429.5 & 14.34 & 46.0 \\
\hline 285 . & 5.744 & 0.808 & 7.562 & 140.50 & 28.88 & 52.35 & 434.3 & 14.32 & 45.9 \\
\hline 290. & 5.551 & 0.822 & 7.821 & 141.40 & 28.92 & 51.19 & 439.1 & 14.32 & 45.9 \\
\hline 295. & 5.375 & 0.834 & 8.074 & 142.26 & 28.99 & 50.21 & 443.8 & 14.34 & 46.0 \\
\hline 300 . & 5.213 & 0.846 & 8.323 & 143.10 & 29.07 & 49.37 & 448.4 & 14.37 & 46.1 \\
\hline 310. & 4.924 & 0.867 & 8.810 & 144.69 & 29.29 & 48.06 & 457.4 & 14.46 & 46.6 \\
\hline 320 . & 4.674 & 0.885 & 9.285 & 146.20 & 29.57 & 47.10 & 466.0 & 14.59 & 47.2 \\
\hline 330. & 4.454 & 0.900 & 9.753 & 147.64 & 29.89 & 46.42 & 474.3 & 14.74 & 48.1 \\
\hline 340 . & 4.259 & 0.914 & 10.214 & 149.02 & 30.26 & 45.96 & 482.2 & 14.90 & 49.0 \\
\hline 350 . & 4.084 & 0.926 & 10.672 & 150.35 & 30.66 & 45.65 & 489.9 & 15.09 & 50.1 \\
\hline 360 . & 3.925 & 0.936 & 11.128 & 151.63 & 31.09 & 45.49 & 497.4 & 15.28 & 51.2 \\
\hline 370 . & 3.781 & 0.946 & 11.582 & 152.88 & 31.54 & 45.43 & 504.5 & 15.48 & 52.5 \\
\hline 380 & 3.650 & 0.954 & 12.037 & 154.09 & 32.01 & 45.45 & 511.5 & 15.69 & 53.8 \\
\hline 390 . & 3.528 & 0.961 & 12.492 & 155.27 & 32.51 & 45.55 & 518.3 & 15.90 & 55.1 \\
\hline 400 & 3.416 & 0.968 & 12.948 & 156.43 & 33.02 & 45.72 & 524.8 & 16.12 & 56.6 \\
\hline 410 & 3.312 & 0.974 & 13.406 & 157.56 & 33.54 & 45.93 & 531.2 & & 58.0 \\
\hline 420. & 3.216 & 0.980 & 13.867 & 158.67 & 34.07 & 46.19 & 537.5 & & 59.6 \\
\hline 130 & 3.125 & 0.984 & 14.330 & 159.76 & 34.61 & 46.48 & 543.6 & & 61.1 \\
\hline 440. & 3.041 & 0.989 & 14.797 & 160.83 & 35.16 & 46.81 & 549.6 & & 62.7 \\
\hline 45 & 2.961 & 0.993 & 15.266 & 161.89 & 35.71 & 47.16 & 555.4 & & 64.4 \\
\hline 460. & 2.886 & 0.996 & 15.740 & 162.93 & 36.26 & 47.53 & 561.2 & & 66.0 \\
\hline 470. & 2.816 & 1.000 & 16.217 & 163.95 & 36.82 & 47.92 & 566.8 & & 67.8 \\
\hline 480. & 2.749 & 1.003 & 16.698 & 164.97 & 37.37 & 48.32 & 572.4 & & 69.5 \\
\hline
\end{tabular}


METHANE ISOBAR AT $\mathrm{P}=11.0 \mathrm{MPa}$ (continued)

$\begin{array}{cccccccccc}\mathrm{T} & \rho & \mathrm{Z} & \mathrm{H} & \mathrm{S} & \mathrm{C}_{\mathrm{v}} & \mathrm{C}_{\mathrm{p}} & \mathrm{W} & \eta & \lambda \\ \mathrm{K} & \mathrm{mol} \cdot \mathrm{dm}^{-3} & & \mathrm{~kJ} / \mathrm{mol} & \mathrm{J} /(\mathrm{mol} \cdot \mathrm{K}) & \mathrm{J} /(\mathrm{mol} \cdot \mathrm{K}) & \mathrm{J} /(\mathrm{mol} \cdot \mathrm{K}) & \mathrm{m} \cdot \mathrm{s}^{-1} & \mu \mathrm{Pa} \cdot \mathrm{s} & \mathrm{mW} /(\mathrm{m} \cdot \mathrm{K}) \\ & & & & & & & & & \\ \text { 490. } & 2.685 & 1.005 & 17.183 & 165.97 & 37.93 & 48.74 & 577.9 & & 71.2 \\ 500 . & 2.625 & 1.008 & 17.673 & 166.96 & 38.48 & 49.16 & 583.2 & 73.0 \\ 520 . & 2.513 & 1.012 & 18.665 & 168.90 & 39.58 & 50.03 & 593.8 & 76.6 \\ 540 . & 2.412 & 1.016 & 19.674 & 170.81 & 40.67 & 50.92 & 604.0 & 80.3 \\ 560 . & 2.318 & 1.019 & 20.702 & 172.67 & 41.73 & 51.81 & 614.1 & & 84.0 \\ & & & & & & & & \\ 580 . & 2.233 & 1.022 & 21.747 & 174.51 & 42.77 & 52.70 & 623.9 & 87.8 \\ 600 . & 2.154 & 1.024 & 22.810 & 176.31 & 43.79 & 53.59 & 633.4 & & 91.6\end{array}$

$\overline{\text { METHANE ISOBAR AT } \mathrm{P}=12.0 \mathrm{MPa}}$

\begin{tabular}{|c|c|c|c|c|c|c|c|c|c|}
\hline $\mathrm{T}$ & $\rho$ & Z & $\mathrm{H}$ & S & $C_{v}$ & $C_{p}$ & W & $\eta$ & $\lambda$ \\
\hline K & $\mathrm{mol} \cdot \mathrm{dm}^{-3}$ & & $\mathrm{~kJ} / \mathrm{mol}$ & $\mathrm{J} /(\mathrm{mol} \cdot \mathrm{K})$ & $\mathrm{J} /(\mathrm{mol} \cdot \mathrm{K})$ & $\mathrm{J} /(\mathrm{mol} \cdot \mathrm{K})$ & $m \cdot s^{-1}$ & $\mu \mathrm{Pa} \cdot \mathrm{s}$ & $\mathrm{mW} /(\mathrm{m} \cdot \mathrm{K})$ \\
\hline 95. & 28.274 & 0.537 & -5.203 & 69.06 & 34.41 & 52.74 & 1586.3 & 203.92 & 216.7 \\
\hline 100. & 27.889 & 0.517 & -4.938 & 71.78 & 34.50 & 53.44 & 1539.3 & 179.53 & 210.9 \\
\hline 105. & 27.499 & 0.500 & -4.669 & 74.40 & 34.28 & 53.86 & 1494.9 & 158.78 & 204.8 \\
\hline 110. & 27.103 & 0.484 & -4.399 & 76.91 & 33.94 & 54.16 & 1451.7 & 141.17 & 198.6 \\
\hline 115. & 26.700 & 0.470 & -4.128 & 79.32 & 33.54 & 54.43 & 1408.7 & 126.25 & 192.2 \\
\hline 120. & 26.290 & 0.457 & -3.855 & 81.65 & 33.13 & 54.71 & 1365.7 & 113.58 & 185.7 \\
\hline 125. & 25.872 & 0.446 & -3.581 & 83.89 & 32.74 & 55.02 & 1322.5 & 102.77 & 179.2 \\
\hline 130. & 25.444 & 0.436 & -3.305 & 86.05 & 32.36 & 55.39 & 1279.0 & 93.50 & 172.8 \\
\hline 135. & 25.007 & 0.428 & -3.027 & 88.15 & 32.01 & 55.82 & 1235.2 & 85.49 & 166.3 \\
\hline 140. & 24.558 & 0.420 & -2.746 & 90.19 & 31.69 & 56.32 & 1191.1 & 78.51 & 159.9 \\
\hline 145. & 24.096 & 0.413 & -2.463 & 92.18 & 31.40 & 56.91 & 1146.7 & 72.39 & 153.5 \\
\hline 150. & 23.620 & 0.407 & -2.177 & 94.12 & 31.13 & 57.60 & 1102.0 & 66.96 & 147.2 \\
\hline 155. & 23.128 & 0.403 & -1.887 & 96.02 & 30.88 & 58.39 & 1057.0 & 62.10 & 141.0 \\
\hline 160. & 22.619 & 0.399 & -1.593 & 97.88 & 30.66 & 59.30 & 1011.6 & 57.72 & 134.9 \\
\hline 165. & 22.089 & 0.396 & -1.294 & 99.73 & 30.46 & 60.37 & 965.9 & 53.74 & 128.8 \\
\hline 170. & 21.536 & 0.394 & -0.989 & 101.55 & 30.29 & 61.60 & 919.8 & 50.08 & 122.9 \\
\hline 175. & 20.956 & 0.394 & -0.678 & 103.35 & 30.15 & 63.03 & 873.4 & 46.69 & 117.1 \\
\hline 180. & 20.347 & 0.394 & -0.358 & 105.15 & 30.03 & 64.71 & 826.6 & 43.53 & 111.4 \\
\hline 185. & 19.702 & 0.396 & -0.030 & 106.95 & 29.95 & 66.69 & 779.5 & 40.56 & 105.8 \\
\hline 190. & 19.018 & 0.399 & 0.309 & 108.76 & 29.90 & 69.02 & 732.4 & 37.75 & 100.6 \\
\hline 195. & 18.287 & 0.405 & 0.661 & 110.58 & 29.88 & 71.77 & 685.4 & 35.08 & 95.1 \\
\hline 200. & 17.503 & 0.412 & 1.028 & 112.44 & 29.91 & 75.00 & 639.1 & 32.52 & 89.9 \\
\hline 205. & 16.659 & 0.423 & 1.412 & 114.34 & 29.96 & 78.71 & 594.1 & 30.06 & 84.8 \\
\hline 210. & 15.753 & 0.436 & 1.815 & 116.28 & 30.05 & 82.72 & 551.6 & 27.70 & 79.9 \\
\hline 215. & 14.789 & 0.454 & 2.239 & 118.28 & 30.15 & 86.59 & 513.2 & 25.48 & 75.4 \\
\hline
\end{tabular}


METHANE ISOBAR AT $\mathrm{P}=12.0 \mathrm{MPa}$ (continued)

T

$\begin{array}{ccc}\mathrm{K} & \mathrm{mol} \cdot \mathrm{dm}^{-3} & \\ & & \\ 220 . & 13.786 & 0.476 \\ 225 . & 12.776 & 0.502 \\ 230 . & 11.800 & 0.532 \\ 235 . & 10.901 & 0.563 \\ 240 . & 10.105 & 0.595\end{array}$

245

250 .

255.

260.

265 .

270 .

275 .

280 .

285.

290 .

295

300 .

310 .

320 .

330 .

340.

350 .

360 .

370 .

380 .

390.

400.

410 .

420.

430 .

440 .

450 .

460 .

470 .

480 .

490

500 .

520 .

540 .

560 .
9.415

8.823

8.315

7.875

7.491

0.626

0.654

0.681

0.705

0.727

7.153

0.747

6.853

0.766

0.783

6.343

0.798

6.124

0.813

5.923

0.826

5.740

0.838

0.860

0.879

5.131

4.884

0.895

4.666

0.910

0.923

0.934

0.944

4.294

4.134

3.987

0.953

6.542

6.834

7.116

7.390

7.657

$7.918 \quad 141.13$

$8.174 \quad 141.99$

$8.672 \quad 143.63$

$9.158 \quad 145.17$

$9.635 \quad 146.64$

$10.105 \quad 148.04$

$10.570 \quad 149.39$

$11.032 \quad 150.69$

$11.492 \quad 151.95$

$11.952 \quad 153.18$

3.853

3.729

0.960

$12.412 \quad 154.37$

$\begin{array}{llll}0.968 & 12.872 & 155.54\end{array}$

3.614

0.974

13.335

156.68

3.508

3.408

0.980

13.799

157.80

0.985

14.266

158.90

3.315

0.990

$14.736 \quad 159.98$

3.227

0.994

15.209

161.04

3.145

0.998

15.685

162.09

3.067

1.001

16.165

163.12

2.994

1.004

$16.649 \quad 164.14$

2.924

$\begin{array}{lll}1.007 & 17.137 & 165.14\end{array}$

2.858

1.010

17.62

165.14
166.14

$\begin{array}{lll}1.014 & 18.625 & 168.09\end{array}$

$\begin{array}{lll}1.018 & 19.638 & 170.00\end{array}$

2.625

2.523

$\begin{array}{lll}1.021 & 20.669 & 171.88\end{array}$
30.34

30.73

31.16

31.60

32.08

32.57

33.07

33.59

34.12

34.66

35.20

35.75

36.30

36.86

37.41

37.97

38.52

39.62

40.70

41.76
$\mathrm{C}_{\mathrm{p}}$

$\mathrm{J} /(\mathrm{mol} \cdot \mathrm{K})$
W

$\mathrm{m} \cdot \mathrm{s}^{-1}$

89.65

91.14

90.36

87.23

82.61

77.58

72.84

68.65

65.07

62.05

59.51

57.36

55.56

54.03

52.73

51.62

50.67

49.17

48.07

47.28

46.71

46.33

46.10

45.98

45.96

46.02

46.15

46.33

46.56

46.83

47.13

47.46

47.82

48.19

48.58

48.98

49.39

50.24

51.11

51.98

480.6

454.9

436.4

424.6

418.2

415.6

415.7

417.4

420.1

423.6

427.4

431.5

435.8

440.1

444.5

448.9

453.2

461.7

470.0

478.1

485.9

493.4

500.7

507.8

514.7

521.4

527.

534.2

540.5

546.5

569.7

575.2
16.96

16.37

15.93

15.60

15.35

15.17

15.04

14.94

14.88

14.84

14.83

14.83

14.87

14.96

15.08

15.22

15.38

15.56

15.74

15.9

16.13

16.34

55.9

57.3

58.7

60.2

61.8

63.4

65.0

66.6

68.3

70.0

71.8

73.5

77.1

80.7

84.4 
METHANE ISOBAR AT $\mathrm{P}=12.0 \mathrm{MPa}$ (continued)

$\begin{array}{cccccccccc}\mathrm{T} & \rho & \mathrm{Z} & \mathrm{H} & \mathrm{S} & \mathrm{C}_{\mathrm{v}} & \mathrm{C}_{\mathrm{p}} & \mathrm{W} & \eta & \lambda \\ \mathrm{K} & \mathrm{mol} \cdot \mathrm{dm}^{-3} & & \mathrm{~kJ} / \mathrm{mol} & \mathrm{J} /(\mathrm{mol} \cdot \mathrm{K}) & \mathrm{J} /(\mathrm{mol} \cdot \mathrm{K}) & \mathrm{J} /(\mathrm{mol} \cdot \mathrm{K}) & \mathrm{m} \cdot \mathrm{s}^{-1} & \mu \mathrm{Pa} \cdot \mathrm{s} & \mathrm{mW} /(\mathrm{m} \cdot \mathrm{K}) \\ & & & & & & & & & \\ 580 . & 2.430 & 1.024 & 21.718 & 173.72 & 42.80 & 52.86 & 626.6 & & 88.2 \\ 600 . & 2.343 & 1.026 & 22.784 & 175.52 & 43.81 & 53.73 & 636.2 & & 91.9\end{array}$

$\overline{\text { METHANE ISOBAR AT P }=13.0 \mathrm{MPa}}$

\begin{tabular}{|c|c|c|c|c|c|c|c|c|c|}
\hline $\mathrm{T}$ & $\rho$ & $\mathrm{Z}$ & $\mathrm{H}$ & $S$ & $C_{v}$ & $C_{p}$ & W & $\eta$ & $\lambda$ \\
\hline K & $\mathrm{mol} \cdot \mathrm{dm}^{-}$ & & $\mathrm{kJ} / \mathrm{mol}$ & $\mathrm{J} /(\mathrm{mol} \cdot \mathrm{K})$ & $\mathrm{J} /(\mathrm{mol} \cdot \mathrm{K})$ & $\mathrm{J} /(\mathrm{mol} \cdot \mathrm{K})$ & $\mathrm{m} \cdot \mathrm{s}^{-1}$ & $\mu \mathrm{Pa} \cdot \mathrm{s}$ & $\mathrm{mW} /(\mathrm{m} \cdot \mathrm{K})$ \\
\hline 95. & 28.312 & 0.581 & -5.177 & 68.96 & 34.45 & 52.67 & 1592.9 & 206.06 & 217.5 \\
\hline 100. & 27.930 & 0.560 & -4.912 & 71.68 & 34.53 & 53.36 & 1546.2 & 181.48 & 211.8 \\
\hline 105. & 27.543 & 0.541 & -4.644 & 74.30 & 34.32 & 53.77 & 1502.3 & 160.55 & 205.8 \\
\hline 110. & 27.150 & 0.524 & -4.374 & 76.80 & 33.98 & 54.06 & 1459.4 & 142.78 & 199.6 \\
\hline 115 . & 26.751 & 0.508 & -4.104 & 79.21 & 33.58 & 54.31 & 1416.8 & 127.72 & 193.2 \\
\hline 120. & 26.345 & 0.495 & -3.831 & 81.53 & 33.18 & 54.56 & 1374.3 & 114.92 & 186.8 \\
\hline 125. & 25.931 & 0.482 & -3.558 & 83.76 & 32.78 & 54.85 & 1331.6 & 104.01 & 180.4 \\
\hline 130. & 25.509 & 0.471 & -3.283 & 85.92 & 32.41 & 55.19 & 1288.7 & 94.66 & 173.9 \\
\hline 135. & 25.077 & 0.462 & -3.006 & 88.01 & 32.06 & 55.59 & 1245.6 & 86.58 & 167.5 \\
\hline 140 . & 24.635 & 0.453 & -2.727 & 90.04 & 31.74 & 56.06 & 1202.3 & 79.55 & 161.1 \\
\hline 145. & 24.181 & 0.446 & -2.445 & 92.02 & 31.44 & 56.60 & 1158.7 & 73.38 & 154.8 \\
\hline 150. & 23.714 & 0.440 & -2.161 & 93.94 & 31.17 & 57.23 & 1115.0 & 67.91 & 148.6 \\
\hline 155. & 23.232 & 0.434 & -1.873 & 95.83 & 30.92 & 57.95 & 1071.0 & 63.04 & 142.4 \\
\hline 160. & 22.734 & 0.430 & -1.581 & 97.69 & 30.70 & 58.78 & 1026.7 & 58.64 & 136.4 \\
\hline 165. & 22.218 & 0.426 & -1.285 & 99.51 & 30.50 & 59.73 & 982.3 & 54.65 & 130.5 \\
\hline 170. & 21.682 & 0.424 & -0.983 & 101.31 & 30.32 & 60.82 & 937.7 & 51.00 & 124.6 \\
\hline 175. & 21.122 & 0.423 & -0.676 & 103.09 & 30.17 & 62.08 & 892.9 & 47.63 & 118.9 \\
\hline 180. & 20.537 & 0.423 & -0.362 & 104.86 & 30.04 & 63.53 & 847.9 & 44.50 & 113.3 \\
\hline 185. & 19.922 & 0.424 & -0.041 & 106.62 & 29.95 & 65.20 & 802.9 & 41.56 & 107.9 \\
\hline 190. & 19.273 & 0.427 & 0.290 & 108.38 & 29.88 & 67.14 & 758.1 & 38.80 & 102.8 \\
\hline 195. & 18.588 & 0.431 & 0.631 & 110.16 & 29.84 & 69.36 & 713.6 & 36.19 & 97.5 \\
\hline 200 . & 17.860 & 0.438 & 0.984 & 111.94 & 29.83 & 71.91 & 669.8 & 33.71 & 92.4 \\
\hline 205. & 17.087 & 0.446 & 1.351 & 113.75 & 29.85 & 74.75 & 627.4 & 31.34 & 87.5 \\
\hline 210. & 16.267 & 0.458 & 1.732 & 115.59 & 29.89 & 77.81 & 586.9 & 29.09 & 82.8 \\
\hline 215 . & 15.403 & 0.472 & 2.129 & 117.46 & 29.96 & 80.81 & 549.7 & 26.97 & 78.4 \\
\hline 220 . & 14.505 & 0.490 & 2.540 & 119.35 & 30.02 & 83.37 & 516.8 & 24.99 & 74.3 \\
\hline 225 . & 13.593 & 0.511 & 2.961 & 121.24 & 30.07 & 85.07 & 489.3 & 23.19 & 70.6 \\
\hline 230 . & 12.691 & 0.536 & 3.388 & 123.12 & 30.07 & 85.47 & 467.8 & 21.60 & 67.2 \\
\hline 235. & 11.830 & 0.562 & 3.813 & 124.95 & 30.01 & 84.28 & 452.0 & 20.25 & 64.1 \\
\hline 240. & 11.034 & 0.590 & 4.228 & 126.69 & 29.90 & 81.62 & 441.4 & 19.12 & 61.3 \\
\hline
\end{tabular}


METHANE ISOBAR AT $\mathrm{P}=13.0 \mathrm{MPa}$ (continued)

\begin{tabular}{|c|c|c|c|c|c|c|c|c|}
\hline$T$ & $\rho$ & $\mathrm{H}$ & $S$ & $C_{v}$ & $C_{p}$ & W & $\eta$ & $\lambda$ \\
\hline K & $\mathrm{mol} \cdot \mathrm{dm}^{-3}$ & $\mathrm{~kJ} / \mathrm{mol}$ & $\mathrm{J} /(\mathrm{mol} \cdot \mathrm{K})$ & $\mathrm{J} /(\mathrm{mol} \cdot \mathrm{K})$ & $\mathrm{J} /(\mathrm{mol} \cdot \mathrm{K})$ & $\mathrm{m} \cdot \mathrm{s}^{-1}$ & $\mu \mathrm{Pa} \cdot \mathrm{s}$ & $\mathrm{m} W /(\mathrm{m} \cdot \mathrm{K})$ \\
\hline 245. & 10.319 & 4.628 & 128.34 & 29.75 & 78.02 & 435.2 & 18.22 & 58.9 \\
\hline 250. & 9.688 & 5.008 & 129.88 & 29.60 & 74.13 & 432.2 & 17.51 & 56.8 \\
\hline 255. & 9.134 & 5.369 & 131.31 & 29.46 & 70.37 & 431.4 & 16.95 & 55.1 \\
\hline 260. & 8.650 & 5.712 & 132.64 & 29.34 & 66.95 & 432.3 & 16.52 & 53.6 \\
\hline 265. & 8.223 & 6.039 & 133.89 & 29.24 & 63.94 & 434.2 & 16.19 & 52.4 \\
\hline 270 . & 7.847 & 6.352 & 135.06 & 29.17 & 61.34 & 436.9 & 15.93 & 51.5 \\
\hline 275 . & 7.511 & 6.653 & 136.16 & 29.13 & 59.10 & 440.1 & 15.73 & 50.8 \\
\hline 280 . & 7.211 & 6.944 & 137.21 & 29.12 & 57.19 & 443.6 & 15.58 & 50.3 \\
\hline 285. & 6.940 & 7.226 & 138.21 & 29.13 & 55.54 & 447.3 & 15.47 & 49.9 \\
\hline 290. & 6.695 & 7.500 & 139.16 & 29.16 & 54.14 & 451.1 & 15.40 & 49.6 \\
\hline 295. & 6.471 & 7.767 & 140.07 & 29.22 & 52.93 & 455.0 & 15.35 & 49.5 \\
\hline 300. & 6.265 & 8.029 & 140.96 & 29.29 & 51.89 & 459.0 & 15.32 & 49.4 \\
\hline 310 . & 5.901 & 8.540 & 142.63 & 29.50 & 50.23 & 466.9 & 15.31 & 49.5 \\
\hline 320 . & 5.588 & 9.035 & 144.20 & 29.76 & 49.00 & 474.8 & 15.36 & 49.9 \\
\hline 330 . & 5.314 & 9.521 & 145.70 & 30.06 & 48.10 & 482.5 & 15.44 & 50.5 \\
\hline 340 . & 5.071 & 9.998 & 147.12 & 30.42 & 47.44 & 490.0 & 15.56 & 51.3 \\
\hline 350 . & 4.855 & 10.470 & 148.49 & 30.80 & 46.99 & 497.4 & 15.69 & 52.2 \\
\hline 360. & 4.661 & 10.938 & 149.81 & 31.22 & 46.69 & 504.5 & 15.84 & 53.2 \\
\hline 370. & 4.484 & 11.404 & 151.09 & 31.67 & 46.52 & 511.4 & 16.01 & 54.3 \\
\hline 380 . & 4.324 & 11.869 & 152.33 & 32.13 & 46.46 & 518.2 & 16.19 & 55.5 \\
\hline 390 . & 4.176 & 12.334 & 153.53 & 32.62 & 46.48 & 524.8 & 16.37 & 56.7 \\
\hline 400. & 4.041 & 12.799 & 154.71 & 33.12 & 46.57 & 531.2 & 16.57 & 58.1 \\
\hline 410. & 3.915 & 13.265 & 155.86 & 33.64 & 46.72 & 537.5 & & 59.5 \\
\hline 420. & 3.798 & 13.734 & 156.99 & 34.17 & 46.92 & 543.7 & & 60.9 \\
\hline 430. & 3.689 & 14.204 & 158.10 & 34.70 & 47.17 & 549.7 & & 62.4 \\
\hline 440. & 3.587 & 14.677 & 159.18 & 35.24 & 47.45 & 555.6 & & 64.0 \\
\hline 450. & 3.492 & 15.153 & 160.25 & 35.79 & 47.76 & 561.4 & & 65.6 \\
\hline 460. & 3.402 & 15.632 & 161.31 & 36.34 & 48.09 & 567.1 & & 67.2 \\
\hline 470. & 3.318 & 16.115 & 162.35 & 36.89 & 48.45 & 572.8 & & 68.9 \\
\hline 480. & 3.238 & 16.601 & 163.37 & 37.45 & 48.82 & 578.3 & & 70.6 \\
\hline 490. & 3.162 & 17.092 & 164.38 & 38.00 & 49.21 & 583.7 & & 72.3 \\
\hline 500. & 3.090 & 17.586 & 165.38 & 38.55 & 49.61 & 589.1 & & 74.0 \\
\hline 520 . & 2.957 & 18.586 & 167.34 & 39.65 & 50.44 & 599.6 & & 77.6 \\
\hline 540. & 2.837 & 19.603 & 169.26 & 40.73 & 51.29 & 609.8 & & 81.2 \\
\hline & 2.726 & 20.638 & 171.14 & 41.79 & 52.15 & 619.8 & & 84.9 \\
\hline 200. & 2.625 & 21.689 & 172.99 & 42.82 & 53.01 & 629.5 & & 88.6 \\
\hline 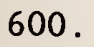 & 2.532 & 22.758 & 174.80 & 43.83 & 53.87 & 639.1 & & 92.3 \\
\hline
\end{tabular}


METHANE ISOBAR AT $\mathrm{P}=14.0 \mathrm{MPa}$

\begin{tabular}{|c|c|c|c|c|c|c|c|c|c|}
\hline $\mathrm{T}$ & $\rho$ & $\mathrm{Z}$ & $\mathrm{H}$ & $S$ & $C_{v}$ & $C_{p}$ & W & $\eta$ & $\lambda$ \\
\hline K & $\mathrm{mol} \cdot \mathrm{dm}^{-}$ & & $\mathrm{kJ} / \mathrm{mol}$ & $\mathrm{J} /(\mathrm{mol} \cdot \mathrm{K})$ & $\mathrm{J} /(\mathrm{mol} \cdot \mathrm{K})$ & $\mathrm{J} /(\mathrm{mol} \cdot \mathrm{K})$ & $\mathrm{m} \cdot \mathrm{s}^{-1}$ & $\mu \mathrm{Pa} \cdot \mathrm{s}$ & $\mathrm{mW} /(\mathrm{m} \cdot \mathrm{K})$ \\
\hline 95. & 28.349 & 0.625 & -5.151 & 68.87 & 34.49 & 52.61 & 1599.4 & 208.21 & 218.4 \\
\hline 100. & 27.970 & 0.602 & -4.886 & 71.58 & 34.57 & 53.28 & 1553.1 & 183.44 & 212.7 \\
\hline 105. & 27.586 & 0.581 & -4.618 & 74.19 & 34.36 & 53.68 & 1509.5 & 162.33 & 206.7 \\
\hline 110. & 27.196 & 0.563 & -4.349 & 76.70 & 34.02 & 53.95 & 1467.0 & 144.41 & 200.5 \\
\hline 115. & 26.801 & 0.546 & -4.079 & 79.10 & 33.62 & 54.19 & 1424.8 & 129.20 & 194.2 \\
\hline 120. & 26.399 & 0.532 & -3.807 & 81.41 & 33.22 & 54.42 & 1382.8 & 116.28 & 187.9 \\
\hline 125. & 25.989 & 0.518 & -3.535 & 83.64 & 32.83 & 54.69 & 1340.6 & 105.26 & 181.5 \\
\hline 130. & 25.572 & 0.507 & -3.260 & 85.79 & 32.46 & 55.01 & 1298.3 & 95.82 & 175.1 \\
\hline 135. & 25.146 & 0.496 & -2.985 & 87.87 & 32.11 & 55.37 & 1255.8 & 87.67 & 168.7 \\
\hline 140. & 24.710 & 0.487 & -2.707 & 89.89 & 31.79 & 55.81 & 1213.1 & 80.58 & 162.4 \\
\hline 145. & 24.263 & 0.479 & -2.426 & 91.86 & 31.49 & 56.30 & 1170.4 & 74.36 & 156.1 \\
\hline 150. & 23.804 & 0.472 & -2.143 & 93.78 & 31.22 & 56.88 & 1127.5 & 68.86 & 150.0 \\
\hline 155. & 23.332 & 0.466 & -1.857 & 95.65 & 30.97 & 57.54 & 1084.4 & 63.96 & 143.9 \\
\hline 160 . & 22.845 & 0.461 & -1.568 & 97.49 & 30.75 & 58.29 & 1041.3 & 59.55 & 137.9 \\
\hline 165. & 22.342 & 0.457 & -1.274 & 99.30 & 30.54 & 59.15 & 998.0 & 55.55 & 132.0 \\
\hline 170 . & 21.820 & 0.454 & -0.976 & 101.08 & 30.36 & 60.13 & 954.7 & 51.90 & 126.3 \\
\hline 175. & 21.279 & 0.452 & -0.673 & 102.84 & 30.20 & 61.24 & 911.3 & 48.54 & 120.6 \\
\hline 180. & 20.714 & 0.452 & -0.364 & 104.58 & 30.06 & 62.50 & 868.0 & 45.43 & 115.2 \\
\hline 185. & 20.124 & 0.452 & -0.048 & 106.31 & 29.95 & 63.94 & 824.8 & 42.52 & 109.8 \\
\hline 190. & 19.507 & 0.454 & 0.276 & 108.04 & 29.87 & 65.57 & 781.8 & 39.80 & 104.8 \\
\hline 195. & 18.858 & 0.458 & 0.609 & 109.76 & 29.81 & 67.41 & 739.4 & 37.24 & 99.7 \\
\hline 200 . & 18.176 & 0.463 & 0.951 & 111.50 & 29.78 & 69.47 & 697.8 & 34.81 & 94.7 \\
\hline 205. & 17.458 & 0.470 & 1.304 & 113.24 & 29.77 & 71.74 & 657.4 & 32.51 & 90.0 \\
\hline 210 . & 16.704 & 0.480 & 1.668 & 115.00 & 29.79 & 74.14 & 618.8 & 30.34 & 85.5 \\
\hline 215 . & 15.915 & 0.492 & 2.045 & 116.77 & 29.82 & 76.52 & 582.8 & 28.29 & 81.2 \\
\hline 220 . & 15.097 & 0.507 & 2.433 & 118.55 & 29.86 & 78.64 & 550.3 & 26.38 & 77.2 \\
\hline 225 . & 14.264 & 0.525 & 2.830 & 120.34 & 29.90 & 80.23 & 522.1 & 24.62 & 73.5 \\
\hline 230 . & 13.432 & 0.545 & 3.234 & 122.11 & 29.92 & 81.03 & 498.7 & 23.04 & 70.2 \\
\hline 235. & 12.621 & 0.568 & 3.639 & 123.86 & 29.90 & 80.80 & 480.3 & 21.65 & 67.2 \\
\hline 240 . & 11.851 & 0.592 & 4.040 & 125.55 & 29.83 & 79.45 & 466.6 & 20.46 & 64.4 \\
\hline 245 . & 11.138 & 0.617 & 4.432 & 127.16 & 29.74 & 77.14 & 457.1 & 19.47 & 62.0 \\
\hline 250 . & 10.490 & 0.642 & 4.811 & 128.69 & 29.63 & 74.24 & 451.2 & 18.65 & 59.9 \\
\hline 255. & 9.910 & 0.666 & 5.174 & 130.13 & 29.51 & 71.13 & 447.9 & 18.00 & 58.0 \\
\hline 260. & 9.393 & 0.689 & 5.522 & 131.48 & 29.41 & 68.09 & 446.7 & 17.47 & 56.4 \\
\hline 265. & 8.933 & 0.711 & 5.855 & 132.75 & 29.33 & 65.28 & 446.9 & 17.05 & 55.1 \\
\hline 270 . & 8.523 & 0.732 & 6.175 & 133.95 & 29.27 & 62.75 & 448.2 & 16.72 & 54.0 \\
\hline 275. & 8.157 & 0.751 & 6.483 & 135.08 & 29.23 & 60.52 & 450.2 & 16.46 & 53.2 \\
\hline 280. & 7.827 & 0.768 & 6.781 & 136.15 & 29.22 & 58.56 & 452.8 & 16.26 & 52.5 \\
\hline 285. & 7.530 & 0.785 & 7.069 & 137.17 & 29.23 & 56.86 & 455.7 & 16.10 & 52.0 \\
\hline 290. & 7.259 & 0.800 & 7.350 & 138.15 & 29.27 & 55.39 & 458.9 & 15.98 & 51.6 \\
\hline
\end{tabular}


METHANE ISOBAR AT $\mathrm{P}=14.0 \mathrm{MPa}$ (continued)

$\mathrm{T}$

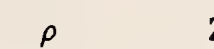

K $\mathrm{mol} \cdot \mathrm{dm}^{-3}$

Z

H

$\mathrm{S} \quad \mathrm{C}_{\mathrm{v}}$

$\mathrm{C}_{\mathrm{p}}$

W

$\eta$

$\lambda$ $\mathrm{kJ} / \mathrm{mol} \mathrm{J} /(\mathrm{mol} \cdot \mathrm{K}) \mathrm{J} /(\mathrm{mol} \cdot \mathrm{K}) \mathrm{J} /(\mathrm{mol} \cdot \mathrm{K}) \quad \mathrm{m} \cdot \mathrm{s}^{-1} \quad \mu \mathrm{Pa} \cdot \mathrm{s} \quad \mathrm{mW} /(\mathrm{m} \cdot \mathrm{K})$

$\begin{array}{llllllllll}295 . & 7.013 & 0.814 & 7.624 & 139.08 & 29.32 & 54.11 & 462.3 & 15.89 & 51.3 \\ 300 . & 6.787 & 0.827 & 7.891 & 139.98 & 29.39 & 53.00 & 465.8 & 15.83 & 51.1 \\ 310 . & 6.386 & 0.851 & 8.412 & 141.69 & 29.59 & 51.21 & 473.0 & 15.77 & 51.1 \\ 320 . & 6.041 & 0.871 & 8.917 & 143.30 & 29.84 & 49.87 & 480.3 & 15.77 & 51.3 \\ 330 . & 5.741 & 0.889 & 9.410 & 144.81 & 30.14 & 48.87 & 487.6 & 15.82 & 51.8 \\ & & & & & & & & & \\ 340 . & 5.475 & 0.905 & 9.895 & 146.26 & 30.49 & 48.14 & 494.8 & 15.90 & 52.4 \\ 350 . & 5.238 & 0.918 & 10.374 & 147.65 & 30.87 & 47.61 & 501.8 & 16.01 & 53.2 \\ 360 . & 5.026 & 0.931 & 10.848 & 148.98 & 31.29 & 47.26 & 508.7 & 16.14 & 54.2 \\ 370 . & 4.833 & 0.942 & 11.320 & 150.28 & 31.73 & 47.04 & 515.5 & 16.29 & 55.2 \\ 380 . & 4.658 & 0.951 & 11.789 & 151.53 & 32.19 & 46.93 & 522.1 & 16.45 & 56.3 \\ & & & & & & & & & \\ 390 . & 4.498 & 0.960 & 12.258 & 152.75 & 32.67 & 46.91 & 528.6 & 16.62 & 57.6 \\ 400 . & 4.350 & 0.968 & 12.728 & 153.94 & 33.17 & 46.97 & 534.9 & 16.80 & 58.9 \\ 410 . & 4.213 & 0.975 & 13.198 & 155.10 & 33.69 & 47.10 & 541.1 & & 60.2 \\ 420 . & 4.087 & 0.981 & 13.670 & 156.23 & 34.21 & 47.27 & 547.2 & & 61.6 \\ 430 . & 3.969 & 0.987 & 14.144 & 157.35 & 34.75 & 47.49 & 553.2 & & 63.1 \\ 440 . & 3.858 & 0.992 & 14.620 & 158.44 & 35.29 & 47.75 & 559.0 & & 64.6 \\ 450 . & 3.755 & 0.996 & 15.099 & 159.52 & 35.83 & 48.05 & 564.8 & & 66.2 \\ 460 . & 3.658 & 1.001 & 15.581 & 160.58 & 36.38 & 48.37 & 570.4 & & 67.8 \\ 470 . & 3.566 & 1.005 & 16.066 & 161.62 & 36.93 & 48.71 & 576.0 & & 69.4 \\ 480 . & 3.480 & 1.008 & 16.555 & 162.65 & 37.48 & 49.07 & 581.5 & & 71.1 \\ 490 . & 3.398 & 1.011 & 17.048 & 163.67 & 38.03 & 49.44 & 586.9 & & 72.8 \\ 500 . & 3.321 & 1.014 & 17.544 & 164.67 & 38.58 & 49.83 & 592.2 & & 74.5 \\ 520 . & 3.177 & 1.019 & 18.549 & 166.64 & 39.68 & 50.64 & 602.6 & & 78.1 \\ 540 . & 3.047 & 1.023 & 19.570 & 168.57 & 40.75 & 51.47 & 612.8 & & 81.6 \\ 560 . & 2.928 & 1.027 & 20.607 & 170.45 & 41.81 & 52.31 & 622.8 & & 85.3 \\ 580 . & 2.819 & 1.030 & 21.662 & 172.30 & 42.85 & 53.16 & 632.5 & & 89.0 \\ 600 . & 2.719 & 1.032 & 22.734 & 174.12 & 43.86 & 54.01 & 642.1 & & 92.7\end{array}$

$\overline{\text { METHANE ISOBAR AT } \mathrm{P}=15.0 \mathrm{MPa}}$

$\begin{array}{cccccccccc}\mathrm{T} & \rho & \mathrm{Z} & \mathrm{H} & \mathrm{S} & \mathrm{C}_{\mathrm{v}} & \mathrm{C}_{\mathrm{p}} & \mathrm{W} & \eta & \lambda \\ \mathrm{K} & \mathrm{mol} \cdot \mathrm{dm}^{-3} & & \mathrm{~kJ} / \mathrm{mol} & \mathrm{J} /(\mathrm{mol} \cdot \mathrm{K}) & \mathrm{J} /(\mathrm{mol} \cdot \mathrm{K}) & \mathrm{J} /(\mathrm{mol} \cdot \mathrm{K}) & \mathrm{m} \cdot \mathrm{s}^{-1} & \mu \mathrm{Pa} \cdot \mathrm{s} & \mathrm{mW} /(\mathrm{m} \cdot \mathrm{K}) \\ & & & & & & & & & \\ 95 . & 28.386 & 0.669 & -5.124 & 68.77 & 34.52 & 52.54 & 1605.8 & 210.37 & 219.2 \\ 100 . & 28.009 & 0.644 & -4.860 & 71.49 & 34.61 & 53.20 & 1559.9 & 185.42 & 213.6 \\ 105 . & 27.628 & 0.622 & -4.593 & 74.09 & 34.40 & 53.59 & 1516.6 & 164.14 & 207.6 \\ 110 . & 27.242 & 0.602 & -4.324 & 76.59 & 34.06 & 53.85 & 1474.4 & 146.05 & 201.5 \\ 115 . & 26.850 & 0.584 & -4.054 & 78.99 & 33.67 & 54.07 & 1432.7 & 130.69 & 195.2\end{array}$


METHANE ISOBAR AT $P=15.0 \mathrm{MPa}$ (continued)

\begin{tabular}{|c|c|c|c|c|c|c|c|c|c|}
\hline $\mathrm{T}$ & $\rho$ & $Z$ & $\mathrm{H}$ & $S$ & $C_{v}$ & $C_{p}$ & $\mathrm{~W}$ & $\eta$ & $\lambda$ \\
\hline K & $\mathrm{mol} \cdot \mathrm{dm}^{-3}$ & & $\mathrm{~kJ} / \mathrm{mol}$ & $\mathrm{J} /(\mathrm{mol} \cdot \mathrm{K})$ & $\mathrm{J} /(\mathrm{mol} \cdot \mathrm{K})$ & $\mathrm{J} /(\mathrm{mol} \cdot \mathrm{K})$ & $\mathrm{m} \cdot \mathrm{s}^{-1}$ & $\mu \mathrm{Pa} \cdot \mathrm{s}$ & $\mathrm{mW} /(\mathrm{m} \cdot \mathrm{K}$ \\
\hline 120. & 26.452 & 0.568 & -3.784 & 81.30 & 33.27 & 54.29 & 1391.1 & 117.65 & 188.9 \\
\hline 125. & 26.047 & 0.554 & -3.511 & 83.52 & 32.88 & 54.54 & 1349.4 & 106.52 & 182.5 \\
\hline 130. & 25.634 & 0.541 & -3.238 & 85.66 & 32.51 & 54.83 & 1307.6 & 96.99 & 176.2 \\
\hline 135. & 25.213 & 0.530 & -2.963 & 87.74 & 32.16 & 55.17 & 1265.7 & 88.76 & 169.9 \\
\hline 140 . & 24.784 & 0.520 & -2.686 & 89.75 & 31.84 & 55.57 & 1223.7 & 81.61 & 163.6 \\
\hline 145. & 24.344 & 0.511 & -2.407 & 91.71 & 31.54 & 56.03 & 1181.7 & 75.35 & 157.4 \\
\hline 150. & 23.892 & 0.503 & -2.126 & 93.62 & 31.27 & 56.56 & 1139.6 & 69.81 & 151.3 \\
\hline 155. & 23.429 & 0.497 & -1.842 & 95.48 & 31.02 & 57.16 & 1097.4 & 64.88 & 145.3 \\
\hline 160. & 22.952 & 0.491 & -1.554 & 97.31 & 30.79 & 57.85 & 1055.2 & 60.45 & 139.4 \\
\hline 165. & 22.461 & 0.487 & -1.263 & 99.10 & 30.58 & 58.62 & 1013.1 & 56.44 & 133.6 \\
\hline 170. & 21.953 & 0.483 & -0.968 & 100.86 & 30.40 & 59.50 & 970.9 & 52.79 & 127.9 \\
\hline 175. & 21.427 & 0.481 & -0.668 & 102.60 & 30.23 & 60.49 & 928.8 & 49.43 & 122.3 \\
\hline 180. & 20.881 & 0.480 & -0.363 & 104.32 & 30.09 & 61.60 & 886.9 & 46.33 & 116.9 \\
\hline 185. & 20.314 & 0.480 & -0.052 & 106.02 & 29.97 & 62.85 & 845.3 & 43.45 & 111.7 \\
\hline 190. & 19.722 & 0.481 & 0.266 & 107.72 & 29.87 & 64.24 & 804.0 & 40.75 & 106.8 \\
\hline 195. & 19.105 & 0.484 & 0.591 & 109.41 & 29.80 & 65.80 & 763.3 & 38.22 & 101.8 \\
\hline 200. & 18.460 & 0.489 & 0.924 & 111.09 & 29.75 & 67.50 & 723.5 & 35.84 & 96.9 \\
\hline 205. & 17.786 & 0.495 & 1.266 & 112.78 & 29.72 & 69.35 & 684.9 & 33.60 & 92.3 \\
\hline 210 . & 17.083 & 0.503 & 1.618 & 114.48 & 29.72 & 71.29 & 647.9 & 31.48 & 87.9 \\
\hline 215. & 16.353 & 0.513 & 1.979 & 116.18 & 29.73 & 73.22 & 613.1 & 29.49 & 83.7 \\
\hline 220 . & 15.599 & 0.526 & 2.350 & 117.88 & 29.75 & 74.98 & 581.2 & 27.63 & 79.8 \\
\hline 225 . & 14.831 & 0.541 & 2.728 & 119.58 & 29.77 & 76.39 & 552.8 & 25.91 & 76.2 \\
\hline 230 . & 14.059 & 0.558 & 3.113 & 121.27 & 29.79 & 77.28 & 528.5 & 24.35 & 72.9 \\
\hline 235 . & 13.298 & 0.577 & 3.500 & 122.94 & 29.79 & 77.49 & 508.4 & 22.95 & 69.9 \\
\hline 240 . & 12.563 & 0.598 & 3.886 & 124.56 & 29.75 & 76.90 & 492.5 & 21.72 & 67.2 \\
\hline 245 . & 11.867 & 0.620 & 4.268 & 126.14 & 29.70 & 75.52 & 480.5 & 20.67 & 64.8 \\
\hline 250. & 11.221 & 0.643 & 4.641 & 127.64 & 29.62 & 73.50 & 471.9 & 19.78 & 62.6 \\
\hline 255. & 10.629 & 0.666 & 5.002 & 129.07 & 29.54 & 71.10 & 466.3 & 19.04 & 60.7 \\
\hline 260 . & 10.093 & 0.687 & 5.351 & 130.43 & 29.46 & 68.55 & 462.9 & 18.43 & 59.1 \\
\hline 265. & 9.610 & 0.708 & 5.688 & 131.71 & 29.39 & 66.06 & 461.3 & 17.94 & 57.7 \\
\hline 270 . & 9.175 & 0.728 & 6.012 & 132.93 & 29.35 & 63.71 & 461.1 & 17.54 & 56.5 \\
\hline 275 . & 8.782 & 0.747 & 6.325 & 134.07 & 29.32 & 61.57 & 461.9 & 17.22 & 55.5 \\
\hline 280 . & 8.428 & 0.765 & 6.628 & 135.17 & 29.31 & 59.66 & 463.4 & 16.96 & 54.7 \\
\hline 285. & 8.106 & 0.781 & 6.922 & 136.21 & 29.32 & 57.96 & 465.4 & 16.75 & 54.0 \\
\hline 290 . & 7.814 & 0.796 & 7.208 & 137.20 & 29.35 & 56.46 & 467.8 & 16.59 & 53.5 \\
\hline 295. & 7.546 & 0.810 & 7.487 & 138.16 & 29.41 & 55.14 & 470.6 & 16.46 & 53.2 \\
\hline 300 . & 7.301 & 0.824 & 7.760 & 139.07 & 29.48 & 53.99 & 473.5 & 16.36 & 52.9 \\
\hline 310 . & 6.865 & 0.848 & 8.290 & 140.81 & 29.67 & 52.11 & 479.8 & 16.25 & 52.7 \\
\hline 320 . & 6.490 & 0.869 & 8.803 & 142.44 & 29.92 & 50.67 & 486.5 & 16.20 & 52.8 \\
\hline 330. & 6.163 & 0.887 & 9.304 & 143.98 & 30.22 & 49.59 & 493.2 & 16.21 & 53.1 \\
\hline
\end{tabular}


METHANE ISOBAR AT $\mathrm{P}=15.0 \mathrm{MPa}$ (continued)

\begin{tabular}{|c|c|c|c|c|c|c|c|c|c|}
\hline $\mathrm{T}$ & $\rho$ & $\mathrm{Z}$ & $\mathrm{H}$ & $\mathrm{S}$ & $C_{v}$ & $C_{p}$ & W & $\eta$ & $\lambda$ \\
\hline K & $\mathrm{mol} \cdot \mathrm{dm}^{-3}$ & & $\mathrm{~kJ} / \mathrm{mol}$ & $\mathrm{J} /(\mathrm{mol} \cdot \mathrm{K})$ & $\mathrm{J} /(\mathrm{mol} \cdot \mathrm{K})$ & $\mathrm{J} /(\mathrm{mol} \cdot \mathrm{K})$ & $m \cdot s^{-1}$ & $\mu \mathrm{Pa} \cdot \mathrm{s}$ & $\mathrm{mW} /(\mathrm{m} \cdot \mathrm{K})$ \\
\hline 340. & 5.875 & 0.903 & 9.796 & 145.45 & 30.56 & 48.79 & 500.0 & 16.26 & 53.6 \\
\hline 350. & 5.619 & 0.917 & 10.281 & 146.86 & 30.94 & 48.21 & 506.8 & 16.35 & 54.4 \\
\hline 360. & 5.388 & 0.930 & 10.761 & 148.21 & 31.35 & 47.80 & 513.4 & 16.45 & 55.2 \\
\hline 370 . & 5.180 & 0.941 & 11.237 & 149.51 & 31.79 & 47.53 & 519.9 & 16.58 & 56.2 \\
\hline 380. & 4.990 & 0.951 & 11.712 & 150.78 & 32.25 & 47.38 & 526.4 & 16.72 & 57.3 \\
\hline 390. & 4.817 & 0.960 & 12.186 & 152.01 & 32.73 & 47.33 & 532.7 & 16.88 & 58.4 \\
\hline 400. & 4.657 & 0.968 & 12.659 & 153.21 & 33.22 & 47.36 & 538.9 & 17.05 & 59.7 \\
\hline 410. & 4.510 & 0.976 & 13.133 & 154.38 & 33.73 & 47.46 & 545.0 & & 61.0 \\
\hline 420. & 4.373 & 0.982 & 13.608 & 155.52 & 34.26 & 47.61 & 550.9 & & 62.4 \\
\hline 430. & 4.246 & 0.988 & 14.085 & 156.65 & 34.79 & 47.81 & 556.8 & & 63.8 \\
\hline 440. & 4.127 & 0.993 & 14.565 & 157.75 & 35.33 & 48.05 & 562.6 & & 65.3 \\
\hline 450. & 4.016 & 0.998 & 15.046 & 158.83 & 35.87 & 48.32 & 568.3 & & 66.8 \\
\hline 460. & 3.912 & 1.003 & 15.531 & 159.90 & 36.42 & 48.63 & 573.9 & & 68.4 \\
\hline 470. & 3.813 & 1.007 & 16.019 & 160.95 & 36.97 & 48.95 & 579.4 & & 70.0 \\
\hline 480. & 3.720 & 1.010 & 16.510 & 161.98 & 37.52 & 49.30 & 584.8 & & 71.7 \\
\hline 490. & 3.633 & 1.014 & 17.005 & 163.00 & 38.07 & 49.66 & 590.2 & & 73.4 \\
\hline 500. & 3.549 & 1.017 & 17.504 & 164.01 & 38.61 & 50.04 & 595.5 & & 75.1 \\
\hline 520. & 3.395 & 1.022 & 18.512 & 165.99 & 39.70 & 50.83 & 605.8 & & 78.6 \\
\hline 540. & 3.256 & 1.026 & 19.537 & 167.92 & 40.78 & 51.64 & 616.0 & & 82.1 \\
\hline 560. & 3.128 & 1.030 & 20.578 & 169.81 & 41.83 & 52.47 & 625.9 & & 85.7 \\
\hline 580. & 3.012 & 1.033 & 21.636 & 171.67 & 42.87 & 53.31 & 635.6 & & 89.4 \\
\hline 600. & 2.904 & 1.035 & 22.710 & 173.49 & 43.88 & 54.14 & 645.1 & & 93.1 \\
\hline
\end{tabular}

\section{$\overline{\text { METHANE ISOBAR AT } \mathrm{P}=16.0 \mathrm{MPa}}$}

$\begin{array}{cccccccccc}\mathrm{T} & \rho & \mathrm{Z} & \mathrm{H} & \mathrm{S} & \mathrm{C}_{\mathrm{v}} & \mathrm{C}_{\mathrm{p}} & \mathrm{W} & \eta & \lambda \\ \mathrm{K} & \mathrm{mol} \cdot \mathrm{dm}^{-3} & & \mathrm{~kJ} / \mathrm{mol} & \mathrm{J} /(\mathrm{mol} \cdot \mathrm{K}) & \mathrm{J} /(\mathrm{mol} \cdot \mathrm{K}) & \mathrm{J} /(\mathrm{mol} \cdot \mathrm{K}) & \mathrm{m} \cdot \mathrm{s}^{-1} & \mu \mathrm{Pa} \cdot \mathrm{s} & \mathrm{mW} /(\mathrm{m} \cdot \mathrm{K}) \\ & & & & & & & & & \\ \text { 95. } & 28.423 & 0.713 & -5.098 & 68.68 & 34.56 & 52.48 & 1612.2 & 212.56 & 220.1 \\ 100 . & 28.048 & 0.686 & -4.834 & 71.39 & 34.64 & 53.13 & 1566.5 & 187.42 & 214.4 \\ 105 . & 27.670 & 0.662 & -4.567 & 73.99 & 34.44 & 53.50 & 1523.6 & 165.95 & 208.5 \\ 110 . & 27.287 & 0.641 & -4.299 & 76.49 & 34.10 & 53.75 & 1481.8 & 147.70 & 202.4 \\ 115 . & 26.898 & 0.622 & -4.030 & 78.88 & 33.71 & 53.96 & 1440.5 & 132.20 & 196.2 \\ & & & & & & & & & \\ 120 . & 26.504 & 0.605 & -3.759 & 81.18 & 33.31 & 54.16 & 1399.2 & 119.02 & 189.9 \\ 125 . & 26.103 & 0.590 & -3.488 & 83.40 & 32.92 & 54.39 & 1358.0 & 107.79 & 183.6 \\ 130 . & 25.695 & 0.576 & -3.215 & 85.54 & 32.55 & 54.66 & 1316.7 & 98.16 & 177.3 \\ 135 . & 25.280 & 0.564 & -2.941 & 87.60 & 32.21 & 54.98 & 1275.4 & 89.86 & 171.0 \\ 140 . & 24.855 & 0.553 & -2.666 & 89.61 & 31.89 & 55.34 & 1234.1 & 82.64 & 164.8\end{array}$


METHANE ISOBAR AT $\mathrm{P}=16.0 \mathrm{MPa}$ (continued)

\begin{tabular}{|c|c|c|c|c|c|c|c|c|c|}
\hline $\mathrm{T}$ & $\rho$ & Z & $\mathrm{H}$ & S & $C_{v}$ & $C_{p}$ & W & $\eta$ & $\lambda$ \\
\hline K & $\mathrm{mol} \cdot \mathrm{dm}^{-3}$ & & $\mathrm{~kJ} / \mathrm{mol}$ & $\mathrm{J} /(\mathrm{mol} \cdot \mathrm{K})$ & $\mathrm{J} /(\mathrm{mol} \cdot \mathrm{K})$ & $\mathrm{J} /(\mathrm{mol} \cdot \mathrm{K})$ & $\mathrm{m} \cdot \mathrm{s}^{-1}$ & $\mu \mathrm{Pa} \cdot \mathrm{s}$ & $\mathrm{mW} /(\mathrm{m} \cdot \mathrm{K})$ \\
\hline 145. & 24.422 & 0.543 & -2.388 & 91.56 & 31.59 & 55.77 & 1192.7 & 76.33 & 158.7 \\
\hline 150. & 23.978 & 0.535 & -2.108 & 93.46 & 31.32 & 56.26 & 1151.3 & 70.75 & 152.6 \\
\hline 155. & 23.523 & 0.528 & -1.825 & 95.31 & 31.07 & 56.81 & 1110.0 & 65.79 & 146.6 \\
\hline 160. & 23.056 & 0.522 & -1.540 & 97.12 & 30.84 & 57.44 & 1068.7 & 61.34 & 140.8 \\
\hline 165. & 22.575 & 0.517 & -1.251 & 98.90 & 30.63 & 58.14 & 1027.5 & 57.32 & 135.1 \\
\hline 170. & 22.079 & 0.513 & -0.958 & 100.65 & 30.44 & 58.93 & 986.4 & 53.66 & 129.5 \\
\hline 175. & 21.568 & 0.510 & -0.661 & 102.37 & 30.27 & 59.81 & 945.5 & 50.30 & 124.0 \\
\hline 180. & 21.039 & 0.508 & -0.360 & 104.07 & 30.12 & 60.80 & 904.9 & 47.21 & 118.7 \\
\hline 185. & 20.491 & 0.508 & -0.053 & 105.75 & 30.00 & 61.89 & 864.6 & 44.34 & 113.5 \\
\hline 190. & 19.922 & 0.508 & 0.259 & 107.42 & 29.89 & 63.10 & 824.8 & 41.67 & 108.7 \\
\hline 195. & 19.332 & 0.510 & 0.578 & 109.07 & 29.80 & 64.43 & 785.6 & 39.16 & 103.7 \\
\hline 200. & 18.718 & 0.514 & 0.904 & 110.72 & 29.74 & 65.87 & 747.3 & 36.82 & 99.0 \\
\hline 205. & 18.081 & 0.519 & 1.237 & 112.37 & 29.70 & 67.40 & 710.2 & 34.61 & 94.5 \\
\hline 210 . & 17.420 & 0.526 & 1.578 & 114.01 & 29.67 & 69.00 & 674.6 & 32.54 & 90.2 \\
\hline 215 . & 16.736 & 0.535 & 1.927 & 115.65 & 29.66 & 70.59 & 641.0 & 30.59 & 86.1 \\
\hline 220 . & 16.034 & 0.546 & 2.284 & 117.29 & 29.67 & 72.07 & 609.8 & 28.77 & 82.3 \\
\hline 225 . & 15.318 & 0.558 & 2.647 & 118.93 & 29.68 & 73.31 & 581.6 & 27.09 & 78.8 \\
\hline 230 . & 14.598 & 0.573 & 3.016 & 120.55 & 29.69 & 74.17 & 556.8 & 25.54 & 75.5 \\
\hline 235. & 13.883 & 0.590 & 3.388 & 122.15 & 29.69 & 74.56 & 535.7 & 24.14 & 72.5 \\
\hline 240 . & 13.185 & 0.608 & 3.761 & 123.72 & 29.68 & 74.38 & 518.2 & 22.90 & 69.8 \\
\hline 245 . & 12.514 & 0.628 & 4.131 & 125.24 & 29.65 & 73.60 & 504.3 & 21.81 & 67.4 \\
\hline 250 . & 11.880 & 0.648 & 4.496 & 126.72 & 29.60 & 72.26 & 493.6 & 20.87 & 65.2 \\
\hline 255 . & 11.289 & 0.668 & 4.853 & 128.13 & 29.54 & 70.48 & 485.9 & 20.06 & 63.3 \\
\hline 260 . & 10.745 & 0.689 & 5.200 & 129.48 & 29.48 & 68.45 & 480.5 & 19.39 & 61.6 \\
\hline 265 . & 10.248 & 0.709 & 5.537 & 130.76 & 29.44 & 66.33 & 477.2 & 18.83 & 60.1 \\
\hline 270 . & 9.795 & 0.728 & 5.864 & 131.98 & 29.40 & 64.25 & 475.4 & 18.37 & 58.8 \\
\hline 275 . & 9.383 & 0.746 & 6.180 & 133.15 & 29.38 & 62.28 & 474.8 & 17.99 & 57.7 \\
\hline 280 . & 9.008 & 0.763 & 6.487 & 134.25 & 29.38 & 60.46 & 475.2 & 17.67 & 56.8 \\
\hline 285. & 8.666 & 0.779 & 6.785 & 135.31 & 29.40 & 58.81 & 476.2 & 17.42 & 56.1 \\
\hline 290 . & 8.354 & 0.794 & 7.075 & 136.32 & 29.43 & 57.33 & 477.8 & 17.21 & 55.5 \\
\hline 295. & 8.067 & 0.809 & 7.358 & 137.28 & 29.49 & 56.01 & 479.8 & 17.05 & 55.0 \\
\hline 300 . & 7.804 & 0.822 & 7.635 & 138.22 & 29.56 & 54.84 & 482.2 & 16.92 & 54.7 \\
\hline 310 . & 7.336 & 0.846 & 8.174 & 139.98 & 29.74 & 52.90 & 487.5 & 16.74 & 54.3 \\
\hline 320 . & 6.933 & 0.867 & 8.695 & 141.64 & 29.99 & 51.41 & 493.4 & 16.64 & 54.2 \\
\hline 330. & 6.581 & 0.886 & 9.203 & 143.20 & 30.28 & 50.26 & 499.5 & 16.61 & 54.4 \\
\hline 340 . & 6.271 & 0.903 & 9.701 & 144.69 & 30.62 & 49.40 & 505.8 & 16.63 & 54.9 \\
\hline 350 . & 5.995 & 0.917 & 10.192 & 146.11 & 31.00 & 48.76 & 512.2 & 16.69 & 55.5 \\
\hline 360. & 5.747 & 0.930 & 10.677 & 147.48 & 31.41 & 48.31 & 518.5 & 16.77 & 56.3 \\
\hline 370 . & 5.523 & 0.942 & 11.158 & 148.80 & 31.84 & 48.00 & 524.8 & 16.88 & 57.2 \\
\hline 380. & 5.319 & 0.952 & 11.637 & 150.07 & 32.30 & 47.82 & 531.0 & 17.00 & 58.2 \\
\hline
\end{tabular}


METHANE ISOBAR AT $\mathrm{P}=16.0 \mathrm{MPa}$ (continued)

\begin{tabular}{|c|c|c|c|c|c|c|c|c|c|}
\hline $\mathrm{T}$ & $\rho$ & $\mathrm{Z}$ & $\mathrm{H}$ & S & $\mathrm{C}_{\mathrm{v}}$ & $C_{p}$ & W & $\eta$ & $\lambda$ \\
\hline K & $\mathrm{mol} \cdot \mathrm{dm}^{-3}$ & & $\mathrm{~kJ} / \mathrm{mol}$ & $\mathrm{J} /(\mathrm{mol} \cdot \mathrm{K})$ & $\mathrm{J} /(\mathrm{mol} \cdot \mathrm{K})$ & $\mathrm{J} /(\mathrm{mol} \cdot \mathrm{K})$ & $m \cdot s^{-1}$ & $\mu \mathrm{Pa} \cdot \mathrm{s}$ & $\mathrm{mW} /(\mathrm{m} \cdot \mathrm{K})$ \\
\hline 390. & 5.133 & 0.961 & 12.115 & 151.31 & 32.78 & 47.73 & 537.1 & 17.14 & 59.3 \\
\hline 400. & 4.962 & 0.970 & 12.592 & 152.52 & 33.27 & 47.73 & 543.1 & 17.29 & 60.5 \\
\hline 410. & 4.804 & 0.977 & 13.070 & 153.70 & 33.78 & 47.80 & 549.1 & & 61.8 \\
\hline 420. & 4.657 & 0.984 & 13.549 & 154.85 & 34.30 & 47.93 & 554.9 & & 63.1 \\
\hline 430. & 4.521 & 0.990 & 14.029 & 155.98 & 34.83 & 48.11 & 560.7 & & 64.5 \\
\hline 440. & 4.394 & 0.995 & 14.511 & 157.09 & 35.36 & 48.34 & 566.4 & & 66.0 \\
\hline 450. & 4.275 & 1.000 & 14.996 & 158.18 & 35.91 & 48.59 & 572.0 & & 67.5 \\
\hline 460. & 4.163 & 1.005 & 15.483 & 159.25 & 36.45 & 48.88 & 577.5 & & 69.0 \\
\hline 470. & 4.058 & 1.009 & 15.973 & 160.31 & 37.00 & 49.19 & 583.0 & & 70.6 \\
\hline 480. & 3.959 & 1.013 & 16.467 & 161.35 & 37.55 & 49.53 & 588.3 & & 72.3 \\
\hline 490. & 3.865 & 1.016 & 16.964 & 162.37 & 38.10 & 49.88 & 593.6 & & 73.9 \\
\hline 500. & 3.776 & 1.019 & 17.465 & 163.38 & 38.64 & 50.25 & 598.9 & & 75.6 \\
\hline 520. & 3.612 & 1.025 & 18.477 & 165.37 & 39.73 & 51.01 & 609.2 & & 79.1 \\
\hline 540. & 3.463 & 1.029 & 19.505 & 167.31 & 40.80 & 51.81 & 619.2 & & 82.6 \\
\hline 560. & 3.327 & 1.033 & 20.550 & 169.21 & 41.86 & 52.63 & 629.1 & & 86.2 \\
\hline 580 & 3.203 & 1.036 & 21.610 & 171.07 & 42.89 & 53.45 & 638.8 & & 89.8 \\
\hline 600 & 3.088 & 1.039 & 22.688 & 172.89 & 43.90 & 54.28 & 648.2 & & 93.5 \\
\hline
\end{tabular}

\section{METHANE ISOBAR AT P $=17.0 \mathrm{MPa}$}

$\begin{array}{rccccccccc}\mathrm{T} & \rho & \mathrm{Z} & \mathrm{H} & \mathrm{S} & \mathrm{C}_{\mathrm{v}} & \mathrm{C}_{\mathrm{p}} & \mathrm{W} & \eta & \lambda \\ \mathrm{K} & \mathrm{mol} \cdot \mathrm{dm}^{-3} & & \mathrm{~kJ} / \mathrm{mol} & \mathrm{J} /(\mathrm{mol} \cdot \mathrm{K}) & \mathrm{J} /(\mathrm{mol} \cdot \mathrm{K}) & \mathrm{J} /(\mathrm{mol} \cdot \mathrm{K}) & \mathrm{m} \cdot \mathrm{s}^{-1} & \mu \mathrm{Pa} \cdot \mathrm{s} & \mathrm{mW} /(\mathrm{m} \cdot \mathrm{K}) \\ & & & & & & & & & \\ 95 . & 28.459 & 0.756 & -5.072 & 68.59 & 34.60 & 52.42 & 1618.4 & 214.77 & 220.9 \\ 100 . & 28.087 & 0.728 & -4.808 & 71.29 & 34.68 & 53.06 & 1573.1 & 189.44 & 215.3 \\ 105 . & 27.711 & 0.703 & -4.541 & 73.89 & 34.48 & 53.42 & 1530.5 & 167.79 & 209.4 \\ 110 . & 27.331 & 0.680 & -4.274 & 76.38 & 34.14 & 53.66 & 1489.0 & 149.37 & 203.4 \\ 115 . & 26.946 & 0.660 & -4.005 & 78.77 & 33.75 & 53.85 & 1448.1 & 133.71 & 197.2 \\ & & & & & & & & & \\ 120 . & 26.555 & 0.642 & -3.735 & 81.07 & 33.35 & 54.04 & 1407.3 & 120.41 & 190.9 \\ 125 . & 26.158 & 0.625 & -3.465 & 83.28 & 32.97 & 54.25 & 1366.5 & 109.06 & 184.7 \\ 130 . & 25.755 & 0.611 & -3.193 & 85.41 & 32.60 & 54.50 & 1325.7 & 99.34 & 178.4 \\ 135 . & 25.344 & 0.598 & -2.919 & 87.47 & 32.26 & 54.79 & 1284.9 & 90.96 & 172.2 \\ 140 . & 24.926 & 0.586 & -2.645 & 89.47 & 31.94 & 55.13 & 1244.2 & 83.68 & 166.0 \\ & & & & & & & & & \\ 145 . & 24.498 & 0.576 & -2.368 & 91.41 & 31.64 & 55.52 & 1203.4 & 77.31 & 159.9 \\ 150 . & 24.061 & 0.567 & -2.089 & 93.30 & 31.37 & 55.97 & 1162.8 & 71.69 & 153.9 \\ 155 . & 23.614 & 0.559 & -1.808 & 95.15 & 31.12 & 56.48 & 1122.2 & 66.70 & 148.0 \\ 160 . & 23.156 & 0.552 & -1.524 & 96.95 & 30.89 & 57.05 & 1081.8 & 62.23 & 142.2 \\ 165 . & 22.685 & 0.546 & -1.238 & 98.71 & 30.68 & 57.69 & 1041.5 & 58.19 & 136.5\end{array}$


METHANE ISOBAR AT $\mathrm{P}=17.0 \mathrm{MPa}$ (continued)

\begin{tabular}{|c|c|c|c|c|c|c|c|c|c|}
\hline $\mathrm{T}$ & $\rho$ & $\mathrm{Z}$ & $\mathrm{H}$ & $S$ & $C_{v}$ & $C_{p}$ & W & $\eta$ & $\lambda$ \\
\hline K & $\mathrm{mol} \cdot \mathrm{dm}^{-}$ & & $\mathrm{kJ} / \mathrm{mol}$ & $\mathrm{J} /(\mathrm{mol} \cdot \mathrm{K})$ & $\mathrm{J} /(\mathrm{mol} \cdot \mathrm{K})$ & $\mathrm{J} /(\mathrm{mol} \cdot \mathrm{K})$ & $\mathrm{m} \cdot \mathrm{s}^{-1}$ & $\mu \mathrm{Pa} \cdot \mathrm{s}$ & $\mathrm{mW} /(\mathrm{m} \cdot \mathrm{K})$ \\
\hline 170. & 22.201 & 0.542 & -0.947 & 100.45 & 30.48 & 58.41 & 1001.4 & 54.52 & 131.0 \\
\hline 175. & 21.703 & 0.538 & -0.653 & 102.15 & 30.31 & 59.20 & 961.5 & 51.16 & 125.6 \\
\hline 180. & 21.189 & 0.536 & -0.355 & 103.83 & 30.16 & 60.08 & 922.0 & 48.07 & 120.3 \\
\hline 185. & 20.658 & 0.535 & -0.052 & 105.49 & 30.02 & 61.05 & 882.9 & 45.21 & 115.3 \\
\hline 190. & 20.110 & 0.535 & 0.255 & 107.13 & 29.91 & 62.10 & 844.4 & 42.55 & 110.5 \\
\hline 195. & 19.543 & 0.537 & 0.569 & 108.76 & 29.82 & 63.25 & 806.5 & 40.07 & 105.6 \\
\hline 200. & 18.956 & 0.539 & 0.888 & 110.38 & 29.74 & 64.48 & 769.6 & 37.75 & 101.0 \\
\hline 205. & 18.349 & 0.544 & 1.214 & 111.98 & 29.69 & 65.78 & 733.8 & 35.57 & 96.6 \\
\hline 210 . & 17.723 & 0.549 & 1.546 & 113.59 & 29.65 & 67.12 & 699.4 & 33.53 & 92.4 \\
\hline 215. & 17.078 & 0.557 & 1.885 & 115.18 & 29.63 & 68.45 & 666.8 & 31.61 & 88.4 \\
\hline 220 . & 16.418 & 0.566 & 2.230 & 116.77 & 29.62 & 69.70 & 636.4 & 29.83 & 84.6 \\
\hline 225. & 15.746 & 0.577 & 2.582 & 118.35 & 29.62 & 70.78 & 608.5 & 28.17 & 81.1 \\
\hline 230 . & 15.069 & 0.590 & 2.938 & 119.91 & 29.62 & 71.59 & 583.6 & 26.64 & 77.9 \\
\hline 235 . & 14.395 & 0.604 & 3.297 & 121.46 & 29.62 & 72.04 & 561.8 & 25.25 & 74.9 \\
\hline 240 . & 13.732 & 0.620 & 3.657 & 122.98 & 29.61 & 72.08 & 543.3 & 24.00 & 72.2 \\
\hline 245 . & 13.088 & 0.638 & 4.017 & 124.46 & 29.60 & 71.66 & 528.0 & 22.89 & 69.8 \\
\hline 250 . & 12.472 & 0.656 & 4.373 & 125.90 & 29.57 & 70.79 & 515.7 & 21.91 & 67.6 \\
\hline 255 . & 11.890 & 0.674 & 4.724 & 127.29 & 29.53 & 69.51 & 506.2 & 21.06 & 65.7 \\
\hline 260 . & 11.347 & 0.693 & 5.068 & 128.62 & 29.50 & 67.95 & 499.1 & 20.33 & 63.9 \\
\hline 265. & 10.843 & 0.712 & 5.403 & 129.90 & 29.46 & 66.21 & 494.1 & 19.72 & 62.4 \\
\hline 270 . & 10.379 & 0.730 & 5.730 & 131.12 & 29.44 & 64.42 & 490.8 & 19.20 & 61.1 \\
\hline 275 . & 9.953 & 0.747 & 6.048 & 132.29 & 29.43 & 62.65 & 488.9 & 18.76 & 59.9 \\
\hline 280. & 9.563 & 0.764 & 6.357 & 133.40 & 29.44 & 60.98 & 488.0 & 18.40 & 58.9 \\
\hline 285. & 9.205 & 0.779 & 6.658 & 134.47 & 29.46 & 59.42 & 488.1 & 18.10 & 58.1 \\
\hline 290. & 8.876 & 0.794 & 6.951 & 135.49 & 29.50 & 57.99 & 488.8 & 17.85 & 57.4 \\
\hline 295. & 8.574 & 0.808 & 7.238 & 136.47 & 29.55 & 56.70 & 490.0 & 17.65 & 56.9 \\
\hline 300 . & 8.295 & 0.822 & 7.518 & 137.41 & 29.62 & 55.55 & 491.7 & 17.48 & 56.4 \\
\hline 310. & 7.798 & 0.846 & 8.064 & 139.20 & 29.81 & 53.59 & 495.9 & 17.24 & 55.9 \\
\hline 320. & 7.368 & 0.867 & 8.592 & 140.88 & 30.06 & 52.06 & 500.9 & 17.10 & 55.7 \\
\hline 330 . & 6.992 & 0.886 & 9.106 & 142.46 & 30.35 & 50.87 & 506.4 & 17.03 & 55.8 \\
\hline 340 . & 6.661 & 0.903 & 9.610 & 143.96 & 30.68 & 49.96 & 512.1 & 17.01 & 56.1 \\
\hline 350 . & 6.366 & 0.918 & 10.106 & 145.40 & 31.06 & 49.28 & 518.0 & 17.04 & 56.7 \\
\hline 360 . & 6.101 & 0.931 & 10.596 & 146.78 & 31.46 & 48.79 & 524.0 & 17.10 & 57.3 \\
\hline 370 . & 5.862 & 0.943 & 11.082 & 148.11 & 31.89 & 48.45 & 529.9 & 17.18 & 58.2 \\
\hline 380. & 5.645 & 0.953 & 11.566 & 149.40 & 32.35 & 48.23 & 535.9 & 17.29 & 59.1 \\
\hline 390 . & 5.446 & 0.963 & 12.047 & 150.65 & 32.82 & 48.12 & 541.8 & 17.41 & 60.2 \\
\hline 400. & 5.264 & 0.971 & 12.528 & 151.87 & 33.32 & 48.09 & 547.7 & 17.55 & 61.3 \\
\hline 410. & 5.095 & 0.979 & 13.009 & 153.06 & 33.82 & 48.14 & 553.4 & & 62.6 \\
\hline 420 . & 4.939 & 0.986 & 13.491 & 154.22 & 34.34 & 48.25 & 559.2 & & 63.9 \\
\hline 430. & 4.794 & 0.992 & 13.974 & 155.36 & 34.87 & 48.41 & 564.8 & & 65.3 \\
\hline
\end{tabular}


METHANE ISOBAR AT $\mathrm{P}=17.0 \mathrm{MPa}$ (continued)

$\begin{array}{lllllllllll}\mathrm{T} & \rho & \mathrm{Z} & \mathrm{H} & \mathrm{S} & \mathrm{C}_{\mathrm{v}} & \mathrm{C}_{\mathrm{p}} & \mathrm{W} & \eta & \lambda\end{array}$

$\mathrm{K} \quad \mathrm{mol} \cdot \mathrm{dm}^{-3} \quad \mathrm{~kJ} / \mathrm{mol} \quad \mathrm{J} /(\mathrm{mol} \cdot \mathrm{K}) \mathrm{J} /(\mathrm{mol} \cdot \mathrm{K}) \mathrm{J} /(\mathrm{mol} \cdot \mathrm{K}) \quad \mathrm{m} \cdot \mathrm{s}^{-1} \quad \mu \mathrm{Pa} \cdot \mathrm{s} \quad \mathrm{mW} /(\mathrm{m} \cdot \mathrm{K})$

$\begin{array}{lllllllll}440 . & 4.658 & 0.998 & 14.459 & 156.47 & 35.40 & 48.61 & 570.4 & 66.7 \\ 450 . & 4.532 & 1.003 & 14.947 & 157.57 & 35.94 & 48.85 & 575.9 & 68.2 \\ 460 . & 4.413 & 1.007 & 15.437 & 158.65 & 36.49 & 49.13 & 581.3 & 69.7 \\ 470 . & 4.301 & 1.011 & 15.929 & 159.70 & 37.03 & 49.43 & 586.7 & 71.2 \\ 480 . & 4.195 & 1.015 & 16.425 & 160.75 & 37.58 & 49.75 & 592.0 & 72.8 \\ 490 . & 4.096 & 1.019 & 16.924 & 161.78 & 38.13 & 50.09 & 597.3 & 74.5 \\ 500 . & 4.001 & 1.022 & 17.427 & 162.79 & 38.67 & 50.44 & 602.4 & 76.2 \\ 520 . & 3.827 & 1.027 & 18.443 & 164.79 & 39.76 & 51.19 & 612.6 & 79.6 \\ 540 . & 3.669 & 1.032 & 19.475 & 166.73 & 40.83 & 51.97 & 622.6 & 83.1 \\ 560 . & 3.524 & 1.036 & 20.522 & 168.64 & 41.88 & 52.78 & 632.4 & 86.6 \\ 580 . & 3.392 & 1.039 & 21.586 & 170.50 & 42.91 & 53.59 & 642.0 & 90.3 \\ 600 . & 3.271 & 1.042 & 22.666 & 172.33 & 43.92 & 54.40 & 651.4 & 93.9\end{array}$

\section{METHANE ISOBAR AT $\mathrm{P}=18.0 \mathrm{MPa}$}

$\begin{array}{lccccccccc}\text { T } & \rho & \mathrm{Z} & \mathrm{H} & \mathrm{S} & \mathrm{C}_{\mathrm{v}} & \mathrm{C}_{\mathrm{p}} & \mathrm{W} & \eta & \lambda \\ \mathrm{K} & \mathrm{mol} \cdot \mathrm{dm}^{-3} & & \mathrm{~kJ} / \mathrm{mol} & \mathrm{J} /(\mathrm{mol} \cdot \mathrm{K}) & \mathrm{J} /(\mathrm{mol} \cdot \mathrm{K}) & \mathrm{J} /(\mathrm{mol} \cdot \mathrm{K}) & \mathrm{m} \cdot \mathrm{s}^{-1} & \mu \mathrm{Pa} \cdot \mathrm{s} & \mathrm{mW} /(\mathrm{m} \cdot \mathrm{K}) \\ & & & & & & & & & \\ 100 . & 28.126 & 0.770 & -4.782 & 71.20 & 34.72 & 52.99 & 1579.6 & 191.48 & 216.2 \\ 105 . & 27.752 & 0.743 & -4.516 & 73.79 & 34.51 & 53.34 & 1537.3 & 169.64 & 210.3 \\ 110 . & 27.375 & 0.719 & -4.248 & 76.28 & 34.18 & 53.57 & 1496.2 & 151.05 & 204.3 \\ 115 . & 26.993 & 0.697 & -3.980 & 78.67 & 33.80 & 53.75 & 1455.6 & 135.24 & 198.2 \\ 120 . & 26.606 & 0.678 & -3.711 & 80.96 & 33.40 & 53.92 & 1415.1 & 121.80 & 192.0 \\ & & & & & & & & & \\ 125 . & 26.213 & 0.661 & -3.441 & 83.16 & 33.01 & 54.12 & 1374.8 & 110.34 & 185.7 \\ 130 . & 25.814 & 0.645 & -3.170 & 85.29 & 32.65 & 54.34 & 1334.5 & 100.52 & 179.5 \\ 135 . & 25.408 & 0.631 & -2.897 & 87.35 & 32.31 & 54.61 & 1294.2 & 92.06 & 173.3 \\ 140 . & 24.994 & 0.619 & -2.623 & 89.34 & 31.99 & 54.93 & 1254.0 & 84.72 & 167.2 \\ 145 . & 24.573 & 0.608 & -2.348 & 91.27 & 31.69 & 55.29 & 1213.9 & 78.29 & 161.1 \\ & & & & & & & & & \\ 150 . & 24.143 & 0.598 & -2.070 & 93.15 & 31.42 & 55.70 & 1173.9 & 72.63 & 155.2 \\ 155 . & 23.703 & 0.589 & -1.791 & 94.99 & 31.17 & 56.17 & 1134.0 & 67.60 & 149.3 \\ 160 . & 23.253 & 0.582 & -1.509 & 96.78 & 30.94 & 56.70 & 1094.4 & 63.10 & 143.6 \\ 165 . & 22.791 & 0.576 & -1.224 & 98.53 & 30.72 & 57.28 & 1054.9 & 59.05 & 138.0 \\ 170 . & 22.318 & 0.571 & -0.936 & 100.25 & 30.53 & 57.93 & 1015.7 & 55.36 & 132.5 \\ & & & & & & & & & \\ 175 . & 21.831 & 0.567 & -0.644 & 101.94 & 30.35 & 58.65 & 976.9 & 52.00 & 127.1 \\ 180 . & 21.331 & 0.564 & -0.349 & 103.60 & 30.20 & 59.43 & 938.4 & 48.91 & 122.0 \\ 185 . & 20.816 & 0.562 & -0.050 & 105.24 & 30.06 & 60.29 & 900.4 & 46.05 & 116.9 \\ 190 . & 20.286 & 0.562 & 0.254 & 106.86 & 29.94 & 61.22 & 863.0 & 43.40 & 112.2 \\ 195 . & 19.739 & 0.562 & 0.562 & 108.47 & 29.84 & 62.22 & 826.3 & 40.94 & 107.5\end{array}$


METHANE ISOBAR AT $\mathrm{P}=18.0 \mathrm{MPa}$ (continued)

\begin{tabular}{|c|c|c|c|c|c|c|c|c|c|}
\hline $\mathrm{T}$ & $\rho$ & $\mathrm{Z}$ & $\mathrm{H}$ & $S$ & $\mathrm{C}_{\mathrm{v}}$ & $C_{p}$ & W & $\eta$ & $\lambda$ \\
\hline K & $\mathrm{mol} \cdot \mathrm{dm}^{-3}$ & & $\mathrm{~kJ} / \mathrm{mol}$ & $\mathrm{J} /(\mathrm{mol} \cdot \mathrm{K})$ & $\mathrm{J} /(\mathrm{mol} \cdot \mathrm{K})$ & $\mathrm{J} /(\mathrm{mol} \cdot \mathrm{K})$ & $\mathrm{m} \cdot \mathrm{s}^{-1}$ & $\mu \mathrm{Pa} \cdot \mathrm{s}$ & $\mathrm{mW} /(\mathrm{m} \cdot \mathrm{K})$ \\
\hline 200 . & 19.176 & 0.564 & 0.876 & 110.06 & 29.75 & 63.29 & 790.5 & 38.63 & 102.9 \\
\hline 205. & 18.595 & 0.568 & 1.195 & 111.63 & 29.69 & 64.40 & 755.9 & 36.48 & 98.5 \\
\hline 210 . & 17.999 & 0.573 & 1.520 & 113.20 & 29.64 & 65.55 & 722.6 & 34.46 & 94.4 \\
\hline 215 . & 17.387 & 0.579 & 1.851 & 114.75 & 29.60 & 66.68 & 690.9 & 32.58 & 90.5 \\
\hline 220 . & 16.762 & 0.587 & 2.187 & 116.30 & 29.58 & 67.74 & 661.2 & 30.82 & 86.8 \\
\hline 225. & 16.127 & 0.597 & 2.528 & 117.83 & 29.57 & 68.68 & 633.7 & 29.18 & 83.3 \\
\hline 230 . & 15.487 & 0.608 & 2.873 & 119.35 & 29.57 & 69.41 & 608.9 & 27.67 & 80.1 \\
\hline 235. & 14.848 & 0.620 & 3.222 & 120.85 & 29.57 & 69.88 & 586.8 & 26.29 & 77.2 \\
\hline 240 . & 14.217 & 0.634 & 3.572 & 122.32 & 29.56 & 70.04 & 567.6 & 25.03 & 74.5 \\
\hline 245 . & 13.600 & 0.650 & 3.922 & 123.76 & 29.56 & 69.84 & 551.3 & 23.90 & 72.1 \\
\hline 250 . & 13.005 & 0.666 & 4.270 & 125.17 & 29.54 & 69.28 & 537.7 & 22.90 & 69.9 \\
\hline 255. & 12.436 & 0.683 & 4.614 & 126.53 & 29.52 & 68.37 & 526.8 & 22.01 & 67.9 \\
\hline 260 . & 11.899 & 0.700 & 4.953 & 127.85 & 29.50 & 67.18 & 518.2 & 21.25 & 66.1 \\
\hline 265. & 11.395 & 0.717 & 5.285 & 129.12 & 29.48 & 65.79 & 511.7 & 20.58 & 64.6 \\
\hline 270 . & 10.927 & 0.734 & 5.611 & 130.33 & 29.47 & 64.29 & 507.0 & 20.02 & 63.2 \\
\hline 275. & 10.493 & 0.750 & 5.928 & 131.50 & 29.47 & 62.75 & 503.8 & 19.54 & 62.0 \\
\hline 280 . & 10.091 & 0.766 & 6.238 & 132.62 & 29.48 & 61.25 & 501.8 & 19.13 & 60.9 \\
\hline 285. & 9.721 & 0.781 & 6.541 & 133.69 & 29.51 & 59.81 & 500.7 & 18.78 & 60.0 \\
\hline 290 . & 9.379 & 0.796 & 6.836 & 134.72 & 29.56 & 58.47 & 500.5 & 18.50 & 59.3 \\
\hline 295. & 9.063 & 0.810 & 7.126 & 135.70 & 29.61 & 57.24 & 501.0 & 18.26 & 58.7 \\
\hline 300 . & 8.771 & 0.823 & 7.409 & 136.66 & 29.69 & 56.11 & 501.9 & 18.06 & 58.2 \\
\hline 310. & 8.247 & 0.847 & 7.960 & 138.46 & 29.88 & 54.18 & 504.9 & 17.76 & 57.5 \\
\hline 320 . & 7.793 & 0.868 & 8.494 & 140.16 & 30.12 & 52.63 & 509.0 & 17.57 & 57.2 \\
\hline 330. & 7.396 & 0.887 & 9.014 & 141.76 & 30.41 & 51.42 & 513.7 & 17.45 & 57.2 \\
\hline 340. & 7.045 & 0.904 & 9.523 & 143.28 & 30.74 & 50.48 & 518.9 & 17.40 & 57.4 \\
\hline 350. & 6.732 & 0.919 & 10.024 & 144.73 & 31.11 & 49.76 & 524.3 & 17.40 & 57.8 \\
\hline 360. & 6.451 & 0.932 & 10.519 & 146.13 & 31.51 & 49.24 & 529.8 & 17.43 & 58.4 \\
\hline 370. & 6.197 & 0.944 & 11.009 & 147.47 & 31.94 & 48.86 & 535.5 & 17.50 & 59.2 \\
\hline 380 & 5.966 & 0.955 & 11.497 & 148.77 & 32.40 & 48.62 & 541.1 & 17.58 & 60.1 \\
\hline 390. & 5.755 & 0.964 & 11.982 & 150.03 & 32.87 & 48.48 & 546.8 & 17.69 & 61.1 \\
\hline 400. & 5.562 & 0.973 & 12.466 & 151.26 & 33.36 & 48.43 & 552.5 & 17.81 & 62.2 \\
\hline 410. & 5.383 & 0.981 & 12.951 & 152.45 & 33.86 & 48.45 & 558.1 & & 63.4 \\
\hline 420. & 5.218 & 0.988 & 13.436 & 153.62 & 34.38 & 48.54 & 563.6 & & 64.7 \\
\hline 430. & 5.064 & 0.994 & 13.922 & 154.76 & 34.91 & 48.68 & 569.1 & & 66.0 \\
\hline 440. & 4.920 & 1.000 & 14.410 & 155.89 & 35.44 & 48.87 & 574.6 & & 67.4 \\
\hline 450. & 4.786 & 1.005 & 14.899 & 156.99 & 35.98 & 49.10 & 580.0 & & 68.8 \\
\hline 460. & 4.660 & 1.010 & 15.392 & 158.07 & 36.52 & 49.36 & 585.3 & & 70.3 \\
\hline 470. & 4.541 & 1.014 & 15.887 & 159.13 & 37.06 & 49.65 & 590.6 & & 71.9 \\
\hline 480. & 4.430 & 1.018 & 16.385 & 160.18 & 37.61 & 49.96 & 595.8 & & 73.4 \\
\hline 490. & 4.324 & 1.022 & 16.886 & 161.21 & 38.16 & 50.29 & 601.0 & & 75.1 \\
\hline
\end{tabular}


METHANE ISOBAR AT $\mathrm{P}=18.0 \mathrm{MPa}$ (continued)

$\begin{array}{lccccccccc}\mathrm{T} & \rho & \mathrm{Z} & \mathrm{H} & \mathrm{S} & \mathrm{C}_{\mathrm{v}} & \mathrm{C}_{\mathrm{p}} & \mathrm{W} & \eta & \lambda \\ \mathrm{K} & \mathrm{mol} \cdot \mathrm{dm}^{-3} & & \mathrm{~kJ} / \mathrm{mol} & \mathrm{J} /(\mathrm{mol} \cdot \mathrm{K}) & \mathrm{J} /(\mathrm{mol} \cdot \mathrm{K}) & \mathrm{J} /(\mathrm{mol} \cdot \mathrm{K}) & \mathrm{m} \cdot \mathrm{s}^{-1} & \mu \mathrm{Pa} \cdot \mathrm{s} & \mathrm{mW} /(\mathrm{m} \cdot \mathrm{K}) \\ & & & & & & & & & \\ 500 . & 4.224 & 1.025 & 17.391 & 162.23 & 38.70 & 50.64 & 606.1 & 76.7 \\ 520 . & 4.040 & 1.031 & 18.410 & 164.23 & 39.79 & 51.37 & 616.2 & 80.1 \\ 540 . & 3.872 & 1.035 & 19.445 & 166.19 & 40.85 & 52.13 & 626.1 & 83.5 \\ 560 . & 3.720 & 1.039 & 20.496 & 168.10 & 41.91 & 52.92 & 635.8 & 87.1 \\ 580 . & 3.580 & 1.042 & 21.562 & 169.97 & 42.93 & 53.72 & 645.4 & & 90.7 \\ 600 . & 3.452 & 1.045 & 22.645 & 171.80 & 43.94 & 54.53 & 654.7 & & 94.3\end{array}$

\section{METHANE ISOBAR AT $\mathrm{P}=19.0 \mathrm{MPa}$}

$\begin{array}{llllllllllll}\mathrm{T} & \rho & \mathrm{Z} & \mathrm{H} & \mathrm{S} & \mathrm{C}_{\mathrm{v}} & \mathrm{C}_{\mathrm{p}} & \mathrm{W} & \eta & \lambda\end{array}$

$\mathrm{K} \quad \mathrm{mol} \cdot \mathrm{dm}^{-3} \quad \mathrm{~kJ} / \mathrm{mol} \quad \mathrm{J} /(\mathrm{mol} \cdot \mathrm{K}) \mathrm{J} /(\mathrm{mol} \cdot \mathrm{K}) \quad \mathrm{J} /(\mathrm{mol} \cdot \mathrm{K}) \quad \mathrm{m} \cdot \mathrm{s}^{-1} \quad \mu \mathrm{Pa} \cdot \mathrm{s} \quad \mathrm{mW} /(\mathrm{m} \cdot \mathrm{K})$

$\begin{array}{lrllllllll}100 . & 28.163 & 0.811 & -4.755 & 71.11 & 34.76 & 52.92 & 1586.0 & 193.53 & 217.0 \\ 105 . & 27.793 & 0.783 & -4.490 & 73.70 & 34.55 & 53.26 & 1544.0 & 171.51 & 211.2 \\ 110 . & 27.418 & 0.758 & -4.223 & 76.18 & 34.22 & 53.48 & 1503.2 & 152.75 & 205.2 \\ 115 . & 27.040 & 0.735 & -3.955 & 78.56 & 33.84 & 53.65 & 1462.9 & 136.79 & 199.1 \\ 120 . & 26.656 & 0.714 & -3.686 & 80.85 & 33.44 & 53.81 & 1422.9 & 123.21 & 193.0 \\ & & & & & & & & & \\ 125 . & 26.267 & 0.696 & -3.417 & 83.05 & 33.06 & 53.99 & 1383.0 & 111.63 & 186.8 \\ 130 . & 25.872 & 0.679 & -3.147 & 85.17 & 32.70 & 54.20 & 1343.1 & 101.72 & 180.6 \\ 135 . & 25.470 & 0.665 & -2.875 & 87.22 & 32.36 & 54.44 & 1303.3 & 93.17 & 174.4 \\ 140 . & 25.062 & 0.651 & -2.602 & 89.21 & 32.04 & 54.73 & 1263.7 & 85.76 & 168.3 \\ 145 . & 24.646 & 0.639 & -2.328 & 91.13 & 31.74 & 55.07 & 1224.1 & 79.28 & 162.3 \\ & & & & & & & & & \\ 150 . & 24.222 & 0.629 & -2.051 & 93.01 & 31.47 & 55.45 & 1184.7 & 73.57 & 156.4 \\ 155 . & 23.789 & 0.620 & -1.773 & 94.83 & 31.22 & 55.88 & 1145.6 & 68.50 & 150.6 \\ 160 . & 23.347 & 0.612 & -1.492 & 96.61 & 30.98 & 56.37 & 1106.6 & 63.98 & 144.9 \\ 165 . & 22.894 & 0.605 & -1.209 & 98.35 & 30.77 & 56.90 & 1068.0 & 59.90 & 139.4 \\ 170 . & 22.430 & 0.599 & -0.923 & 100.06 & 30.57 & 57.49 & 1029.6 & 56.20 & 133.9 \\ & & & & & & & & & \\ 175 . & 21.955 & 0.595 & -0.634 & 101.74 & 30.40 & 58.14 & 991.6 & 52.83 & 128.6 \\ 180 . & 21.468 & 0.591 & -0.342 & 103.39 & 30.24 & 58.85 & 954.1 & 49.73 & 123.5 \\ 185 . & 20.967 & 0.589 & -0.046 & 105.01 & 30.09 & 59.61 & 917.0 & 46.88 & 118.6 \\ 190 . & 20.452 & 0.588 & 0.254 & 106.61 & 29.97 & 60.44 & 880.6 & 44.24 & 113.9 \\ 195 . & 19.924 & 0.588 & 0.559 & 108.19 & 29.36 & 61.32 & 845.0 & 41.78 & 109.2 \\ & & & & & & & & & \\ 200 . & 19.381 & 0.590 & 0.868 & 109.75 & 29.77 & 62.25 & 810.3 & 39.49 & 104.7 \\ 205 . & 18.824 & 0.592 & 1.181 & 111.30 & 29.70 & 63.22 & 776.7 & 37.35 & 100.4 \\ 210 . & 18.252 & 0.596 & 1.500 & 112.84 & 29.64 & 64.20 & 744.3 & 35.36 & 96.3 \\ 215 . & 17.668 & 0.602 & 1.823 & 114.36 & 29.60 & 65.17 & 713.5 & 33.49 & 92.5 \\ 220 . & 17.073 & 0.608 & 2.152 & 115.87 & 29.57 & 66.09 & 684.5 & 31.75 & 88.8\end{array}$


METHANE ISOBAR AT $\mathrm{P}=19.0 \mathrm{MPa}$ (continued)

\begin{tabular}{|c|c|c|c|c|c|c|c|c|c|}
\hline $\mathrm{T}$ & $\rho$ & $\mathrm{z}$ & H & $\mathrm{S}$ & $C_{v}$ & $C_{p}$ & W & $\eta$ & $\lambda$ \\
\hline K & $\mathrm{mol} \cdot \mathrm{dm}^{-}$ & & $\mathrm{kJ} / \mathrm{mol}$ & $\mathrm{J} /(\mathrm{mol} \cdot \mathrm{K})$ & $\mathrm{J} /(\mathrm{mol} \cdot \mathrm{K})$ & $\mathrm{J} /(\mathrm{mol} \cdot \mathrm{K})$ & $\mathrm{m} \cdot \mathrm{s}^{-1}$ & $\mu \mathrm{Pa} \cdot \mathrm{s}$ & $\mathrm{mW} /(\mathrm{m} \cdot \mathrm{K})$ \\
\hline 225 . & 16.470 & 0.617 & 2.484 & 117.36 & 29.55 & 66.91 & 657.5 & 30.13 & 85.4 \\
\hline 230. & 15.862 & 0.626 & 2.820 & 118.84 & 29.54 & 67.57 & 632.8 & 28.63 & 82.3 \\
\hline 235. & 15.254 & 0.637 & 3.159 & 120.30 & 29.53 & 68.03 & 610.5 & 27.26 & 79.3 \\
\hline 240. & 14.652 & 0.650 & 3.500 & 121.73 & 29.53 & 68.24 & 590.9 & 26.00 & 76.7 \\
\hline 245. & 14.061 & 0.663 & 3.841 & 123.14 & 29.52 & 68.17 & 573.8 & 24.86 & 74.2 \\
\hline 250. & 13.486 & 0.678 & 4.181 & 124.52 & 29.52 & 67.82 & 559.4 & 23.84 & 72.0 \\
\hline 255. & 12.933 & 0.693 & 4.519 & 125.85 & 29.51 & 67.18 & 547.3 & 22.93 & 70.0 \\
\hline 260. & 12.405 & 0.708 & 4.853 & 127.15 & 29.50 & 66.28 & 537.5 & 22.13 & 68.2 \\
\hline 265. & 11.906 & 0.724 & 5.182 & 128.40 & 29.49 & 65.18 & 529.7 & 21.43 & 66.6 \\
\hline 270. & 11.437 & 0.740 & 5.504 & 129.61 & 29.49 & 63.95 & 523.7 & 20.82 & 65.2 \\
\hline 275 . & 10.999 & 0.755 & 5.821 & 130.77 & 29.50 & 62.63 & 519.3 & 20.30 & 64.0 \\
\hline 280. & 10.591 & 0.771 & 6.131 & 131.89 & 29.52 & 61.31 & 516.2 & 19.85 & 62.9 \\
\hline 285. & 10.212 & 0.785 & 6.434 & 132.96 & 29.56 & 60.01 & 514.1 & 19.47 & 61.9 \\
\hline 290. & 9.860 & 0.799 & 6.731 & 133.99 & 29.60 & 58.77 & 513.0 & 19.14 & 61.1 \\
\hline 295. & 9.534 & 0.813 & 7.022 & 134.99 & 29.67 & 57.61 & 512.6 & 18.87 & 60.4 \\
\hline 300 . & 9.230 & 0.825 & 7.307 & 135.95 & 29.74 & 56.54 & 512.8 & 18.64 & 59.9 \\
\hline 310. & 8.684 & 0.849 & 7.863 & 137.77 & 29.93 & 54.66 & 514.6 & 18.28 & 59.1 \\
\hline 320. & 8.208 & 0.870 & 8.402 & 139.48 & 30.18 & 53.12 & 517.6 & 18.04 & 58.6 \\
\hline 330. & 7.791 & 0.889 & 8.926 & 141.09 & 30.47 & 51.90 & 521.6 & 17.89 & 58.5 \\
\hline 340. & 7.421 & 0.906 & 9.440 & 142.63 & 30.80 & 50.94 & 526.1 & 17.80 & 58.7 \\
\hline 350. & 7.091 & 0.921 & 9.946 & 144.09 & 31.16 & 50.20 & 531.0 & 17.77 & 59.0 \\
\hline 360. & 6.795 & 0.934 & 10.445 & 145.50 & 31.56 & 49.65 & 536.1 & 17.77 & 59.5 \\
\hline 370. & 6.527 & 0.946 & 10.939 & 146.86 & 31.99 & 49.25 & 541.4 & 17.81 & 60.2 \\
\hline 380. & 6.283 & 0.957 & 11.430 & 148.16 & 32.44 & 48.98 & 546.7 & 17.88 & 61.1 \\
\hline 390. & 6.061 & 0.967 & 11.919 & 149.43 & 32.91 & 48.82 & 552.1 & 17.97 & 62.0 \\
\hline 400. & 5.856 & 0.976 & 12.407 & 150.67 & 33.40 & 48.75 & 557.5 & 18.08 & 63.1 \\
\hline 410. & 5.668 & 0.983 & 12.895 & 151.87 & 33.90 & 48.75 & 562.9 & & 64.2 \\
\hline 420. & 5.493 & 0.991 & 13.382 & 153.05 & 34.42 & 48.82 & 568.3 & & 65.4 \\
\hline 430. & 5.331 & 0.997 & 13.871 & 154.20 & 34.94 & 48.95 & 573.7 & & 66.7 \\
\hline 440. & 5.179 & 1.003 & 14.362 & 155.33 & 35.47 & 49.13 & 579.0 & & 68.1 \\
\hline 450. & 5.037 & 1.008 & 14.854 & 156.43 & 36.01 & 49.34 & 584.2 & & 69.5 \\
\hline 460. & 4.905 & 1.013 & 15.348 & 157.52 & 36.55 & 49.59 & 589.5 & & 71.0 \\
\hline 470. & 4.780 & 1.017 & 15.846 & 158.59 & 37.10 & 49.86 & 594.7 & & 72.5 \\
\hline 480. & 4.662 & 1.021 & 16.346 & 159.64 & 37.64 & 50.16 & 599.8 & & 74.0 \\
\hline 490. & 4.551 & 1.025 & 16.849 & 160.68 & 38.19 & 50.48 & 604.9 & & 75.6 \\
\hline 500. & 4.445 & 1.028 & 17.355 & 161.70 & 38.73 & 50.82 & 610.0 & & 77.3 \\
\hline 520. & 4.251 & 1.034 & 18.379 & 163.71 & 39.81 & 51.54 & 619.9 & & 80.6 \\
\hline 540. & 4.074 & 1.039 & 19.417 & 165.67 & 40.88 & 52.29 & 629.7 & & 84.0 \\
\hline 560. & 3.914 & 1.043 & 20.471 & 167.58 & 41.93 & 53.07 & 639.3 & & 87.6 \\
\hline 580. & 3.767 & 1.046 & 21.540 & 169.46 & 42.95 & 53.85 & 648.8 & & 91.1 \\
\hline 600. & 3.632 & 1.049 & 22.625 & 171.30 & 43.96 & 54.65 & 658.1 & & 94.7 \\
\hline
\end{tabular}


METHANE ISOBAR AT $\mathrm{P}=20.0 \mathrm{MPa}$

\begin{tabular}{|c|c|c|c|c|c|c|c|c|c|}
\hline $\mathrm{T}$ & $\rho$ & Z & $\mathrm{H}$ & $S$ & $\mathrm{C}_{\mathrm{v}}$ & $C_{p}$ & W & $\eta$ & $\lambda$ \\
\hline K & $\mathrm{mol} \cdot \mathrm{dm}^{-3}$ & & $\mathrm{~kJ} / \mathrm{mol}$ & $\mathrm{J} /(\mathrm{mol} \cdot \mathrm{K})$ & $\mathrm{J} /(\mathrm{mol} \cdot \mathrm{K})$ & $\mathrm{J} /(\mathrm{mol} \cdot \mathrm{K})$ & $m \cdot s^{-1}$ & $\mu \mathrm{Pa} \cdot \mathrm{s}$ & $\mathrm{mW} /(\mathrm{m} \cdot \mathrm{K})$ \\
\hline 100. & 28.201 & 0.853 & -4.729 & 71.01 & 34.79 & 52.85 & 1592.3 & 195.61 & 217.9 \\
\hline 105. & 27.833 & 0.823 & -4.464 & 73.60 & 34.59 & 53.19 & 1550.6 & 173.40 & 212.1 \\
\hline 110. & 27.461 & 0.796 & -4.197 & 76.08 & 34.26 & 53.40 & 1510.1 & 154.46 & 206.2 \\
\hline 115. & 27.085 & 0.772 & -3.930 & 78.46 & 33.88 & 53.55 & 1470.2 & 138.35 & 200.1 \\
\hline 120. & 26.705 & 0.751 & -3.662 & 80.74 & 33.49 & 53.70 & 1430.5 & 124.63 & 194.0 \\
\hline 125. & 26.319 & 0.731 & -3.393 & 82.94 & 33.11 & 53.86 & 1391.0 & 112.94 & 187.8 \\
\hline 130. & 25.928 & 0.714 & -3.123 & 85.05 & 32.74 & 54.05 & 1351.6 & 102.92 & 181.7 \\
\hline 135. & 25.531 & 0.698 & -2.852 & 87.10 & 32.40 & 54.28 & 1312.3 & 94.28 & 175.5 \\
\hline 140. & 25.128 & 0.684 & -2.580 & 89.08 & 32.09 & 54.55 & 1273.1 & 86.80 & 169.5 \\
\hline 145. & 24.717 & 0.671 & -2.307 & 90.99 & 31.79 & 54.86 & 1234.1 & 80.26 & 163.5 \\
\hline 150. & 24.299 & 0.660 & -2.032 & 92.86 & 31.52 & 55.21 & 1195.3 & 74.51 & 157.7 \\
\hline 155. & 23.873 & 0.650 & -1.755 & 94.68 & 31.27 & 55.61 & 1156.8 & 69.40 & 151.9 \\
\hline 160. & 23.438 & 0.641 & -1.476 & 96.45 & 31.03 & 56.05 & 1118.5 & 64.84 & 146.2 \\
\hline 165. & 22.993 & 0.634 & -1.194 & 98.18 & 30.82 & 56.54 & 1080.6 & 60.74 & 140.7 \\
\hline 170. & 22.539 & 0.628 & -0.910 & 99.88 & 30.62 & 57.08 & 1043.0 & 57.03 & 135.4 \\
\hline 175. & 22.074 & 0.623 & -0.623 & 101.54 & 30.44 & 57.67 & 1005.8 & 53.64 & 130.1 \\
\hline 180. & 21.598 & 0.619 & -0.333 & 103.17 & 30.28 & 58.31 & 969.1 & 50.54 & 125.1 \\
\hline 185. & 21.110 & 0.616 & -0.040 & 104.78 & 30.13 & 59.00 & 933.0 & 47.69 & 120.2 \\
\hline 190. & 20.610 & 0.614 & 0.257 & 106.36 & 30.00 & 59.74 & 897.5 & 45.05 & 115.5 \\
\hline 195. & 20.098 & 0.614 & 0.557 & 107.93 & 29.89 & 60.52 & 862.8 & 42.60 & 110.9 \\
\hline 200 . & 19.573 & 0.614 & 0.862 & 109.47 & 29.79 & 61.34 & 829.1 & 40.32 & 106.5 \\
\hline 205. & 19.036 & 0.616 & 1.171 & 110.99 & 29.71 & 62.18 & 796.4 & 38.20 & 102.2 \\
\hline 210 . & 18.487 & 0.620 & 1.484 & 112.50 & 29.65 & 63.03 & 764.9 & 36.21 & 98.2 \\
\hline 215 . & 17.927 & 0.624 & 1.801 & 114.00 & 29.60 & 63.88 & 734.8 & 34.36 & 94.4 \\
\hline 220 . & 17.358 & 0.630 & 2.123 & 115.47 & 29.56 & 64.68 & 706.4 & 32.64 & 90.8 \\
\hline 225 . & 16.782 & 0.637 & 2.448 & 116.93 & 29.53 & 65.39 & 679.9 & 31.03 & 87.4 \\
\hline 230 . & 16.201 & 0.646 & 2.776 & 118.38 & 29.52 & 65.99 & 655.4 & 29.54 & 84.3 \\
\hline 235. & 15.621 & 0.655 & 3.107 & 119.80 & 29.51 & 66.42 & 633.1 & 28.17 & 81.4 \\
\hline 240 . & 15.045 & 0.666 & 3.440 & 121.20 & 29.50 & 66.66 & 613.2 & 26.92 & 78.7 \\
\hline 245. & 14.477 & 0.678 & 3.774 & 122.58 & 29.50 & 66.67 & 595.7 & 25.77 & 76.3 \\
\hline 250 . & 13.923 & 0.691 & 4.107 & 123.92 & 29.50 & 66.46 & 580.5 & 24.74 & 74.0 \\
\hline 255. & 13.386 & 0.705 & 4.438 & 125.24 & 29.50 & 66.00 & 567.6 & 23.81 & 72.0 \\
\hline 260. & 12.870 & 0.719 & 4.766 & 126.51 & 29.50 & 65.33 & 556.7 & 22.98 & 70.2 \\
\hline 265. & 12.378 & 0.733 & 5.091 & 127.75 & 29.50 & 64.46 & 547.9 & 22.25 & 68.6 \\
\hline 270 . & 11.913 & 0.748 & 5.411 & 128.94 & 29.51 & 63.45 & 540.8 & 21.61 & 67.2 \\
\hline 275. & 11.474 & 0.762 & 5.725 & 130.10 & 29.53 & 62.35 & 535.3 & 21.05 & 65.9 \\
\hline 280. & 11.063 & 0.777 & 6.034 & 131.21 & 29.56 & 61.20 & 531.1 & 20.57 & 64.7 \\
\hline 285 . & 10.678 & 0.790 & 6.337 & 132.28 & 29.60 & 60.05 & 528.1 & 20.15 & 63.7 \\
\hline 290. & 10.319 & 0.804 & 6.635 & 133.32 & 29.65 & 58.92 & 526.0 & 19.79 & 62.9 \\
\hline 295. & 9.984 & 0.817 & 6.926 & 134.32 & 29.71 & 57.85 & 524.8 & 19.48 & 62.2 \\
\hline
\end{tabular}


METHANE ISOBAR AT $\mathrm{P}=20.0 \mathrm{MPa}$ (continued)

\begin{tabular}{|c|c|c|c|c|c|c|c|c|c|}
\hline $\mathrm{T}$ & $\rho$ & $\mathrm{Z}$ & $\mathrm{H}$ & $\mathbf{S}$ & $\mathrm{C}_{\mathrm{v}}$ & $C_{p}$ & $\mathrm{~W}$ & $\eta$ & $\lambda$ \\
\hline K & $\mathrm{mol} \cdot \mathrm{dm}^{-3}$ & & $\mathrm{~kJ} / \mathrm{mol}$ & $\mathrm{J} /(\mathrm{mol} \cdot \mathrm{K})$ & $\mathrm{J} /(\mathrm{mol} \cdot \mathrm{K})$ & $\mathrm{J} /(\mathrm{mol} \cdot \mathrm{K})$ & $\mathrm{m} \cdot \mathrm{s}^{-1}$ & $\mu \mathrm{Pa} \cdot \mathrm{s}$ & $\mathrm{mW} /(\mathrm{m} \cdot \mathrm{K})$ \\
\hline 300 . & 9.672 & 0.829 & 7.213 & 135.28 & 29.79 & 56.84 & 524.3 & 19.22 & 61.5 \\
\hline 310 . & 9.107 & 0.852 & 7.772 & 137.11 & 29.99 & 55.04 & 524.8 & 18.81 & 60.6 \\
\hline 320. & 8.612 & 0.873 & 8.315 & 138.84 & 30.23 & 53.54 & 526.8 & 18.52 & 60.1 \\
\hline 330. & 8.176 & 0.892 & 8.844 & 140.47 & 30.52 & 52.32 & 529.9 & 18.33 & 59.9 \\
\hline 340 . & 7.789 & 0.908 & 9.362 & 142.01 & 30.85 & 51.35 & 533.7 & 18.20 & 59.9 \\
\hline 350 . & 7.444 & 0.923 & 9.872 & 143.49 & 31.21 & 50.60 & 538.0 & 18.14 & 60.2 \\
\hline 360 . & 7.133 & 0.937 & 10.375 & 144.91 & 31.61 & 50.02 & 542.7 & 18.12 & 60.7 \\
\hline 370. & 6.852 & 0.949 & 10.873 & 146.27 & 32.04 & 49.61 & 547.5 & 18.14 & 61.3 \\
\hline 380. & 6.596 & 0.960 & 11.367 & 147.59 & 32.49 & 49.32 & 552.6 & 18.18 & 62.0 \\
\hline 390. & 6.362 & 0.969 & 11.859 & 148.87 & 32.96 & 49.14 & 557.7 & 18.26 & 62.9 \\
\hline 400. & 6.147 & 0.978 & 12.350 & 150.11 & 33.44 & 49.05 & 562.8 & 18.35 & 63.9 \\
\hline 410. & 5.949 & 0.986 & 12.841 & 151.32 & 33.94 & 49.04 & 568.0 & & 65.0 \\
\hline 420. & 5.765 & 0.993 & 13.331 & 152.50 & 34.46 & 49.09 & 573.2 & & 66.2 \\
\hline 430. & 5.594 & 1.000 & 13.823 & 153.66 & 34.98 & 49.21 & 578.4 & & 67.5 \\
\hline 440 . & 5.435 & 1.006 & 14.315 & 154.79 & 35.51 & 49.37 & 583.5 & & 68.8 \\
\hline 450. & 5.286 & 1.011 & 14.810 & 155.90 & 36.04 & 49.57 & 588.7 & & 70.2 \\
\hline 460. & 5.147 & 1.016 & 15.307 & 157.00 & 36.58 & 49.80 & 593.8 & & 71.6 \\
\hline 470. & 5.015 & 1.020 & 15.806 & 158.07 & 37.13 & 50.07 & 598.9 & & 73.1 \\
\hline 480. & 4.891 & 1.024 & 16.308 & 159.13 & 37.67 & 50.36 & 603.9 & & 74.7 \\
\hline 490. & 4.775 & 1.028 & 16.813 & 160.17 & 38.21 & 50.67 & 608.9 & & 76.2 \\
\hline 500. & 4.664 & 1.031 & 17.322 & 161.20 & 38.76 & 51.00 & 613.9 & & 77.8 \\
\hline 520. & 4.460 & 1.037 & 18.349 & 163.21 & 39.84 & 51.70 & 623.7 & & 81.1 \\
\hline 540. & 4.274 & 1.042 & 19.390 & 165.17 & 40.90 & 52.44 & 633.4 & & 84.5 \\
\hline 560. & 4.106 & 1.046 & 20.446 & 167.10 & 41.95 & 53.20 & 642.9 & & 88.0 \\
\hline 580. & 3.952 & 1.049 & 21.518 & 168.98 & 42.97 & 53.98 & 652.3 & & 91.6 \\
\hline 600. & 3.810 & 1.052 & 22.606 & 170.82 & 43.98 & 54.77 & 661.5 & & 95.2 \\
\hline
\end{tabular}

$\overline{\text { METHANE ISOBAR AT } \mathrm{P}=25.0 \mathrm{MPa}}$

$\begin{array}{lccccccccc}\mathrm{T} & \rho & \mathrm{Z} & \mathrm{H} & \mathrm{S} & \mathrm{C}_{\mathrm{v}} & \mathrm{C}_{\mathrm{p}} & \mathrm{W} & \eta & \lambda \\ \mathrm{K} & \mathrm{mol} \cdot \mathrm{dm}^{-3} & & \mathrm{~kJ} / \mathrm{mol} & \mathrm{J} /(\mathrm{mol} \cdot \mathrm{K}) & \mathrm{J} /(\mathrm{mol} \cdot \mathrm{K}) & \mathrm{J} /(\mathrm{mol} \cdot \mathrm{K}) & \mathrm{m} \cdot \mathrm{s}^{-1} & \mu \mathrm{Pa} \cdot \mathrm{s} & \mathrm{mW} /(\mathrm{m} \cdot \mathrm{K}) \\ & & & & & & & & & \\ 100 . & 28.383 & 1.059 & -4.597 & 70.56 & 34.98 & 52.55 & 1622.8 & 206.34 & 222.0 \\ 105 . & 28.027 & 1.022 & -4.334 & 73.14 & 34.79 & 52.85 & 1582.4 & 183.16 & 216.5 \\ 110 . & 27.668 & 0.988 & -4.069 & 75.60 & 34.47 & 53.01 & 1543.4 & 163.31 & 210.7 \\ 115 . & 27.306 & 0.958 & -3.804 & 77.96 & 34.09 & 53.11 & 1505.0 & 146.37 & 204.8 \\ 120 . & 26.941 & 0.930 & -3.538 & 80.22 & 33.71 & 53.20 & 1467.0 & 131.93 & 198.8\end{array}$


METHANE ISOBAR AT $\mathrm{P}=25.0 \mathrm{MPa}$ (continued)

\begin{tabular}{|c|c|c|c|c|c|c|c|c|}
\hline$T$ & $\rho$ & $\mathrm{H}$ & $S$ & $C_{v}$ & $C_{p}$ & W & $\eta$ & $\lambda$ \\
\hline K & $\mathrm{mol} \cdot \mathrm{dm}^{-3}$ & $\mathrm{~kJ} / \mathrm{mol}$ & $\mathrm{J} /(\mathrm{mol} \cdot \mathrm{K})$ & $\mathrm{J} /(\mathrm{mol} \cdot \mathrm{K})$ & $\mathrm{J} /(\mathrm{mol} \cdot \mathrm{K})$ & $\mathrm{m} \cdot \mathrm{s}^{-1}$ & $\mu \mathrm{Pa} \cdot \mathrm{s}$ & $\mathrm{mW} /(\mathrm{m} \cdot \mathrm{K}$ \\
\hline 25. & 26.572 & -3.272 & 82.39 & 33.34 & 53.30 & 1429.2 & 119.61 & 192.8 \\
\hline 130. & 26.199 & -3.005 & 84.49 & 32.98 & 53.43 & 1391.8 & 109.05 & 186.9 \\
\hline 135. & 25.822 & -2.738 & 86.51 & 32.65 & 53.57 & 1354.5 & 99.96 & 180.9 \\
\hline 140. & 25.440 & -2.469 & 88.46 & 32.33 & 53.75 & 1317.6 & 92.09 & 175.1 \\
\hline 45 & 25.053 & -2.200 & 90.35 & 32.04 & 53.96 & 1281.0 & 85.23 & 169.3 \\
\hline . & 24.661 & -1.930 & 92.18 & 31.77 & 54.19 & 1244.8 & 79.21 & 163.6 \\
\hline 5 & 24.263 & -1.658 & 93.96 & 31.52 & 54.46 & 1208.9 & 73.89 & 158.0 \\
\hline 160. & 23.859 & -1.385 & 95.69 & 31.28 & 54.75 & 1173.5 & 69.15 & 152.6 \\
\hline 165. & 23.450 & -1.110 & 97.38 & 31.06 & 55.08 & 1138.6 & 64.91 & 147.2 \\
\hline 70. & 23.033 & -0.834 & 99.03 & 30.86 & 55.43 & 1104.2 & 61.08 & 142.1 \\
\hline 5. & 22.611 & -0.556 & 100.65 & 30.67 & 55.80 & 1070.3 & 57.61 & 137.1 \\
\hline 0 & 22.181 & -0.276 & 102.22 & 30.50 & 56.20 & 1037.0 & 54.45 & 132.2 \\
\hline 35. & 21.744 & 0.006 & 103.77 & 30.34 & 56.62 & 1004.4 & 51.55 & 127.5 \\
\hline 0 & 21.301 & 0.290 & 105.28 & 30.20 & 57.07 & 972.5 & 48.89 & 123.0 \\
\hline 5. & 20.851 & 0.577 & 106.77 & 30.07 & 57.52 & 941.5 & 46.43 & 118.7 \\
\hline 0 & 20.394 & 0.865 & 108.24 & 29.96 & 57.99 & 911.3 & 44.16 & 114.5 \\
\hline 05. & 19.930 & 1.157 & 109.67 & 29.86 & 58.47 & 882.0 & 42.05 & 110.4 \\
\hline 0 & 19.461 & 1.450 & 111.09 & 29.77 & 58.94 & 853.8 & 40.09 & 106.6 \\
\hline 5 & 18.987 & 1.746 & 112.48 & 29.70 & 59.40 & 826.8 & 38.27 & 103.0 \\
\hline 0 & 18.509 & 2.044 & 113.85 & 29.64 & 59.83 & 801.0 & 36.57 & 99.5 \\
\hline 5. & 18.027 & 2.344 & 115.20 & 29.59 & 60.23 & 776.5 & 35.00 & 96.3 \\
\hline 30. & 17.544 & 2.646 & 116.53 & 29.55 & 60.58 & 753.4 & 33.53 & 93.2 \\
\hline 35. & 17.061 & 2.950 & 117.83 & 29.53 & 60.87 & 731.9 & 32.17 & 90.4 \\
\hline 0 & 16.580 & 3.255 & 119.12 & 29.51 & 61.08 & 711.9 & 30.91 & 87.7 \\
\hline 5 & 16.102 & 3.560 & 120.38 & 29.51 & 61.20 & 693.5 & 29.75 & 85.3 \\
\hline 50. & 15.631 & 3.867 & 121.61 & 29.51 & 61.23 & 676.7 & 28.68 & 83.0 \\
\hline 5. & 15.167 & 4.173 & 122.83 & 29.52 & 61.15 & 661.5 & 27.69 & 80.9 \\
\hline 50 & 14.714 & 4.478 & 124.01 & 29.54 & 60.98 & 648.0 & 26.80 & 79.1 \\
\hline 265. & 14.272 & 4.782 & 125.17 & 29.56 & 60.71 & 635.9 & 25.98 & 77.3 \\
\hline 270 & 13.844 & 5.085 & 126.30 & 29.60 & 60.34 & 625.3 & 25.23 & 75.8 \\
\hline 275. & 13.430 & 5.385 & 127.41 & 29.64 & 59.90 & 616.1 & 24.56 & 74.4 \\
\hline 280 & 13.033 & 5.684 & 128.48 & 29.69 & 59.38 & 608.1 & 23.95 & 73.1 \\
\hline 5 & 12.651 & 5.979 & 129.53 & 29.75 & 58.81 & 601.3 & 23.41 & 71.9 \\
\hline 290 & 12.287 & 6.272 & 130.54 & 29.82 & 58.21 & 595.6 & 22.92 & 70.9 \\
\hline 205 & 11.939 & 6.561 & 131.53 & 29.90 & 57.58 & 590.8 & 22.49 & 70.0 \\
\hline 300 . & 11.608 & 6.848 & 132.50 & 29.99 & 56.95 & 586.9 & 22.10 & 69.2 \\
\hline 310 . & 10.995 & 7.411 & 134.34 & 30.20 & 55.71 & 581.3 & 21.46 & 68.0 \\
\hline 20 & 10.443 & 7.962 & 136.09 & 30.45 & 54.56 & 578.2 & 20.95 & 67.0 \\
\hline 33 & 0.916 & 8.502 & 137.76 & 30.74 & 53.55 & 576.9 & 20.57 & 66.4 \\
\hline & 0.931 & 9.034 & 139.34 & 31.07 & 52.69 & 576.9 & 20.28 & 66.1 \\
\hline
\end{tabular}


METHANE ISOBAR AT $\mathrm{P}=25.0 \mathrm{MPa}$ (continued)

\begin{tabular}{|c|c|c|c|c|c|c|c|c|c|}
\hline $\mathrm{T}$ & $\rho$ & 2 & $\mathrm{H}$ & S & $C_{v}$ & $C_{p}$ & W & $\eta$ & $\lambda$ \\
\hline K & $\mathrm{mol} \cdot \mathrm{dm}^{-3}$ & & $\mathrm{~kJ} / \mathrm{mol}$ & $\mathrm{J} /(\mathrm{mol} \cdot \mathrm{K})$ & $\mathrm{J} /(\mathrm{mol} \cdot \mathrm{K})$ & $\mathrm{J} /(\mathrm{mol} \cdot \mathrm{K})$ & $\mathrm{m} \cdot \mathrm{s}^{-1}$ & $\mu \mathrm{Pa} \cdot \mathrm{s}$ & $\mathrm{mW} /(\mathrm{m} \cdot \mathrm{K})$ \\
\hline 350 . & 9.090 & 0.945 & 9.557 & 140.86 & 31.43 & 51.98 & 578.1 & 20.06 & 66.0 \\
\hline 360 . & 8.721 & 0.958 & 10.074 & 142.32 & 31.82 & 51.41 & 580.0 & 19.91 & 66.2 \\
\hline 370. & 8.384 & 0.969 & 10.585 & 143.72 & 32.24 & 50.97 & 582.6 & 19.82 & 66.5 \\
\hline 380. & 8.077 & 0.980 & 11.093 & 145.07 & 32.68 & 50.64 & 585.6 & 19.76 & 67.0 \\
\hline 390. & 7.794 & 0.989 & 11.599 & 146.38 & 33.15 & 50.41 & 589.0 & 19.74 & 67.6 \\
\hline 400. & 7.534 & 0.998 & 12.102 & 147.66 & 33.63 & 50.28 & 592.6 & 19.75 & 68.4 \\
\hline 410. & 7.293 & 1.006 & 12.604 & 148.90 & 34.12 & 50.21 & 596.5 & & 69.3 \\
\hline 420. & 7.069 & 1.013 & 13.107 & 150.11 & 34.63 & 50.22 & 600.5 & & 70.3 \\
\hline 430. & 6.861 & 1.019 & 13.609 & 151.29 & 35.14 & 50.28 & 604.7 & & 71.3 \\
\hline 440. & 6.666 & 1.025 & 14.112 & 152.45 & 35.66 & 50.39 & 608.9 & & 72.5 \\
\hline 450. & 6.484 & 1.030 & 14.617 & 153.58 & 36.19 & 50.55 & 613.2 & & 73.7 \\
\hline 460. & 6.313 & 1.035 & 15.123 & 154.70 & 36.73 & 50.74 & 617.6 & & 75.0 \\
\hline 470. & 6.153 & 1.040 & 15.632 & 155.79 & 37.27 & 50.97 & 622.0 & & 76.4 \\
\hline 480. & 6.001 & 1.044 & 16.143 & 156.87 & 37.80 & 51.22 & 626.5 & & 77.8 \\
\hline 490. & 5.858 & 1.047 & 16.656 & 157.92 & 38.34 & 51.49 & 631.0 & & 79.2 \\
\hline 500. & 5.723 & 1.051 & 17.173 & 158.97 & 38.88 & 51.79 & 635.4 & & 80.7 \\
\hline 520 . & 5.472 & 1.057 & 18.215 & 161.01 & 39.95 & 52.43 & 644.4 & & 83.8 \\
\hline 540. & 5.246 & 1.061 & 19.270 & 163.00 & 41.01 & 53.11 & 653.3 & & 87.1 \\
\hline 560. & 5.039 & 1.065 & 20.340 & 164.95 & 42.05 & 53.83 & 662.2 & & 90.4 \\
\hline 580. & 4.850 & 1.069 & 21.423 & 166.85 & 43.07 & 54.56 & 671.0 & & 93.8 \\
\hline 600. & 4.677 & 1.071 & 22.522 & 168.71 & 44.06 & 55.31 & 679.8 & & 97.3 \\
\hline
\end{tabular}

$\overline{\text { METHANE ISOBAR AT } \mathrm{P}=30.0 \mathrm{MPa}}$

$\begin{array}{lccccccccc}\mathrm{T} & \rho & \mathrm{Z} & \mathrm{H} & \mathrm{S} & \mathrm{C} & \mathrm{C}_{\mathrm{p}} & \mathrm{W} & \eta & \lambda \\ \mathrm{K} & \mathrm{mol} \cdot \mathrm{dm}^{-3} & & \mathrm{~kJ} / \mathrm{mol} & \mathrm{J} /(\mathrm{mol} \cdot \mathrm{K}) & \mathrm{J} /(\mathrm{mol} \cdot \mathrm{K}) & \mathrm{J} /(\mathrm{mol} \cdot \mathrm{K}) & \mathrm{m} \cdot \mathrm{s}^{-1} & \mu \mathrm{Pa} \cdot \mathrm{s} & \mathrm{mW} /(\mathrm{m} \cdot \mathrm{K}) \\ & & & & & & & & & \\ 100 . & 28.557 & 1.263 & -4.465 & 70.13 & 35.17 & 52.29 & 1651.5 & 217.69 & 226.1 \\ 105 . & 28.212 & 1.218 & -4.203 & 72.69 & 34.98 & 52.55 & 1612.4 & 193.49 & 220.7 \\ 110 . & 27.864 & 1.177 & -3.940 & 75.14 & 34.67 & 52.67 & 1574.6 & 172.67 & 215.1 \\ 115 . & 27.515 & 1.140 & -3.676 & 77.48 & 34.30 & 52.74 & 1537.6 & 154.85 & 209.3 \\ 120 . & 27.163 & 1.107 & -3.412 & 79.73 & 33.93 & 52.79 & 1501.0 & 139.62 & 203.5 \\ 125 . & 26.808 & 1.077 & -3.148 & 81.88 & 33.56 & 52.84 & 1464.8 & 126.60 & 197.7 \\ 130 . & 26.451 & 1.049 & -2.884 & 83.96 & 33.21 & 52.90 & 1428.9 & 115.44 & 191.9 \\ 135 . & 26.090 & 1.024 & -2.619 & 85.96 & 32.88 & 52.99 & 1393.5 & 105.85 & 186.1 \\ 140 . & 25.726 & 1.002 & -2.354 & 87.88 & 32.57 & 53.10 & 1358.4 & 97.54 & 180.3 \\ 145 . & 25.359 & 0.981 & -2.088 & 89.75 & 32.28 & 53.23 & 1323.7 & 90.32 & 174.7\end{array}$


ETHANE ISOBAR AT $\mathrm{P}=30.0 \mathrm{MPa}$ (continued)

\begin{tabular}{|c|c|c|c|c|c|c|c|c|c|}
\hline$T$ & $\rho$ & 2 & $\mathrm{H}$ & $S$ & $\mathrm{C}_{\mathrm{v}}$ & $C_{p}$ & W & $\eta$ & $\lambda$ \\
\hline K & $\mathrm{mol} \cdot \mathrm{dm}^{-}$ & & $\mathrm{kJ} / \mathrm{mol}$ & $\mathrm{J} /(\mathrm{mol} \cdot \mathrm{K})$ & $\mathrm{J} /(\mathrm{mol} \cdot \mathrm{K})$ & $\mathrm{J} /(\mathrm{mol} \cdot \mathrm{K})$ & $m \cdot s^{-1}$ & $\mu \mathrm{Pa} \cdot \mathrm{s}$ & $\mathrm{mW} /(\mathrm{m} \cdot \mathrm{K})$ \\
\hline 150. & 24.988 & 0.963 & -1.822 & 91.56 & 32.01 & 53.39 & 1289.5 & 83.99 & 169.2 \\
\hline 155. & 24.613 & 0.946 & -1.554 & 93.31 & 31.76 & 53.56 & 1255.8 & 78.41 & 163.8 \\
\hline 160. & 24.234 & 0.931 & -1.286 & 95.01 & 31.52 & 53.76 & 1222.6 & 73.46 & 158.5 \\
\hline 165. & 23.851 & 0.917 & -1.017 & 96.67 & 31.30 & 53.97 & 1190.0 & 69.03 & 153.3 \\
\hline 170. & 23.464 & 0.905 & -0.746 & 98.29 & 31.10 & 54.21 & 1157.9 & 65.06 & 148.3 \\
\hline 175. & 23.073 & 0.894 & -0.475 & 99.86 & 30.91 & 54.45 & 1126.5 & 61.46 & 143.4 \\
\hline 180. & 22.677 & 0.884 & -0.202 & 101.40 & 30.73 & 54.71 & 1095.7 & 58.20 & 138.7 \\
\hline 185. & 22.277 & 0.876 & 0.073 & 102.90 & 30.57 & 54.99 & 1065.6 & 55.22 & 134.2 \\
\hline 190. & 21.873 & 0.868 & 0.348 & 104.37 & 30.42 & 55.27 & 1036.2 & 52.49 & 129.8 \\
\hline 195. & 21.465 & 0.862 & 0.625 & 105.81 & 30.28 & 55.55 & 1007.6 & 49.99 & 125.6 \\
\hline 200. & 21.053 & 0.857 & 0.904 & 107.22 & 30.16 & 55.85 & 979.9 & 47.67 & 121.5 \\
\hline 205. & 20.638 & 0.853 & 1.184 & 108.60 & 30.05 & 56.14 & 953.0 & 45.53 & 117.6 \\
\hline 210 . & 20.219 & 0.850 & 1.465 & 109.96 & 29.95 & 56.42 & 927.0 & 43.55 & 113.9 \\
\hline 215 . & 19.799 & 0.848 & 1.748 & 111.29 & 29.87 & 56.70 & 902.1 & 41.71 & 110.4 \\
\hline 220 . & 19.376 & 0.846 & 2.032 & 112.60 & 29.80 & 56.96 & 878.1 & 40.00 & 107.0 \\
\hline 225 . & 18.953 & 0.846 & 2.318 & 113.88 & 29.74 & 57.20 & 855.2 & 38.41 & 103.9 \\
\hline 230 . & 18.529 & 0.847 & 2.604 & 115.14 & 29.69 & 57.42 & 833.5 & 36.94 & 100.9 \\
\hline 235 . & 18.106 & 0.848 & 2.892 & 116.38 & 29.66 & 57.60 & 812.9 & 35.56 & 98.1 \\
\hline 240. & 17.684 & 0.850 & 3.180 & 117.59 & 29.63 & 57.75 & 793.5 & 34.29 & 95.4 \\
\hline 245. & 17.265 & 0.853 & 3.469 & 118.78 & 29.62 & 57.86 & 775.3 & 33.10 & 93.0 \\
\hline 250 . & 16.850 & 0.857 & 3.759 & 119.95 & 29.62 & 57.91 & 758.4 & 32.00 & 90.7 \\
\hline 255 . & 16.440 & 0.861 & 4.048 & 121.10 & 29.63 & 57.92 & 742.7 & 30.98 & 88.6 \\
\hline 260 . & 16.037 & 0.865 & 4.338 & 122.22 & 29.64 & 57.88 & 728.2 & 30.04 & 86.6 \\
\hline 265. & 15.640 & 0.871 & 4.627 & 123.33 & 29.67 & 57.78 & 714.9 & 29.17 & 84.8 \\
\hline 270 . & 15.252 & 0.876 & 4.915 & 124.40 & 29.71 & 57.64 & 702.8 & 28.37 & 83.1 \\
\hline 275 . & 14.873 & 0.882 & 5.203 & 125.46 & 29.76 & 57.44 & 691.8 & 27.64 & 81.6 \\
\hline 280. & 14.504 & 0.888 & 5.490 & 126.49 & 29.82 & 57.21 & 681.9 & 26.96 & 80.3 \\
\hline 285 . & 14.146 & 0.895 & 5.775 & 127.50 & 29.88 & 56.93 & 673.0 & 26.35 & 79.0 \\
\hline 290. & 13.798 & 0.902 & 6.059 & 128.49 & 29.96 & 56.62 & 665.0 & 25.78 & 77.9 \\
\hline 295. & 13.463 & 0.909 & 6.341 & 129.46 & 30.05 & 56.29 & 658.0 & 25.27 & 76.9 \\
\hline 300 . & 13.139 & 0.915 & 6.622 & 130.40 & 30.14 & 55.93 & 651.8 & 24.80 & 76.0 \\
\hline 310 . & 12.527 & 0.929 & 7.177 & 132.22 & 30.36 & 55.19 & 641.7 & 23.99 & 74.5 \\
\hline 320 . & 11.963 & 0.943 & 7.726 & 133.96 & 30.62 & 54.45 & 634.2 & 23.32 & 73.3 \\
\hline 330. & 11.443 & 0.956 & 8.267 & 135.63 & 30.92 & 53.74 & 628.9 & 22.79 & 72.4 \\
\hline 340. & 10.965 & 0.968 & 8.801 & 137.22 & 31.25 & 53.11 & 625.4 & 22.35 & 71.9 \\
\hline 350. & 10.527 & 0.979 & 9.329 & 138.75 & 31.61 & 52.55 & 623.4 & 22.01 & 71.5 \\
\hline 360 & 10.123 & 0.990 & 9.852 & 140.23 & 32.00 & 52.09 & 622.5 & 21.74 & 71.4 \\
\hline $37 c$ & 9.752 & 1.000 & 10.371 & 141.65 & 32.41 & 51.71 & 622.5 & 21.54 & 71.5 \\
\hline 380. & 9.409 & 1.009 & 10.887 & 143.02 & 32.85 & 51.42 & 623.3 & 21.38 & 71.8 \\
\hline 390 & 9.091 & 1.018 & 11.400 & 144.36 & 33.31 & 51.22 & 624.8 & 21.27 & 72.2 \\
\hline
\end{tabular}


METHANE ISOBAR AT $\mathrm{P}=30.0 \mathrm{MPa}$ (continued)

\begin{tabular}{|c|c|c|c|c|c|c|c|c|c|}
\hline $\mathrm{T}$ & $\rho$ & $\mathrm{Z}$ & $\mathrm{H}$ & $S$ & $\mathrm{C}_{\mathrm{v}}$ & $C_{p}$ & W & $\eta$ & $\lambda$ \\
\hline K & $\mathrm{mol} \cdot \mathrm{dm}^{-3}$ & & $\mathrm{~kJ} / \mathrm{mol}$ & $\mathrm{J} /(\mathrm{mol} \cdot \mathrm{K})$ & $\mathrm{J} /(\mathrm{mol} \cdot \mathrm{K})$ & $\mathrm{J} /(\mathrm{mol} \cdot \mathrm{K})$ & $\mathrm{m} \cdot \mathrm{s}^{-1}$ & $\mu \mathrm{Pa} \cdot \mathrm{s}$ & $\mathrm{mW} /(\mathrm{m} \cdot \mathrm{K})$ \\
\hline 400 & 8.797 & 1.025 & 11.911 & 145.65 & 33.78 & 51.08 & 626.7 & 21.20 & 72.8 \\
\hline 410. & 8.524 & 1.032 & 12.422 & 146.91 & 34.27 & 51.02 & 629.0 & & 73.5 \\
\hline 420. & 8.269 & 1.039 & 12.932 & 148.14 & 34.77 & 51.01 & 631.7 & & 74.3 \\
\hline 430. & 8.031 & 1.045 & 13.442 & 149.34 & 35.28 & 51.06 & 634.6 & & 75.2 \\
\hline 440. & 7.808 & 1.050 & 13.953 & 150.52 & 35.80 & 51.16 & 637.7 & & 76.2 \\
\hline 450. & 7.599 & 1.055 & 14.465 & 151.67 & 36.33 & 51.29 & 641.0 & & 77.3 \\
\hline 460. & 7.402 & 1.060 & 14.979 & 152.80 & 36.85 & 51.46 & 644.5 & & 78.5 \\
\hline 470. & 7.216 & 1.064 & 15.495 & 153.90 & 37.39 & 51.67 & 648.1 & & 79.7 \\
\hline 480. & 7.041 & 1.068 & 16.012 & 154.99 & 37.92 & 51.90 & 651.8 & & 81.0 \\
\hline 490 & 6.876 & 1.071 & 16.533 & 156.07 & 38.46 & 52.15 & 655.6 & & 82.3 \\
\hline 500 & 6.719 & 1.074 & 17.055 & 157.12 & 38.99 & 52.42 & 659.5 & & 83.7 \\
\hline 520. & 6.428 & 1.079 & 18.110 & 159.19 & 40.05 & 53.02 & 667.3 & & 86.6 \\
\hline 540. & 6.165 & 1.084 & 19.177 & 161.20 & 41.10 & 53.67 & 675.3 & & 89.7 \\
\hline 560. & 5.925 & 1.088 & 20.257 & 163.17 & 42.14 & 54.35 & 683.4 & & 92.9 \\
\hline 580. & 5.705 & 1.091 & 21.351 & 165.09 & 43.15 & 55.05 & 691.5 & & 96.2 \\
\hline 600 & 5.502 & 1.093 & 22.459 & 166.97 & 44.14 & 55.76 & 699.7 & & 99.5 \\
\hline
\end{tabular}

$\overline{\text { METHANE ISOBAR AT } P=35.0 \mathrm{MPa}}$

$\begin{array}{lccccccccc}\mathrm{T} & \rho & \mathrm{Z} & \mathrm{H} & \mathrm{S} & \mathrm{C} & \mathrm{C} & \mathrm{W} & \eta & \lambda \\ \mathrm{K} & \mathrm{mol} \cdot \mathrm{dm}^{-3} & & \mathrm{~kJ} / \mathrm{mol} & \mathrm{J} /(\mathrm{mol} \cdot \mathrm{K}) & \mathrm{J} /(\mathrm{mol} \cdot \mathrm{K}) & \mathrm{J} /(\mathrm{mol} \cdot \mathrm{K}) & \mathrm{m} \cdot \mathrm{s}^{-1} & \mu \mathrm{Pa} \cdot \mathrm{s} & \mathrm{mW} /(\mathrm{m} \cdot \mathrm{K}) \\ & & & & & & & & & \\ 100 . & 28.723 & 1.466 & -4.332 & 69.72 & 35.35 & 52.05 & 1678.9 & 229.75 & 230.1 \\ 105 . & 28.388 & 1.412 & -4.071 & 72.27 & 35.17 & 52.29 & 1640.9 & 204.48 & 224.8 \\ 110 . & 28.051 & 1.364 & -3.809 & 74.70 & 34.87 & 52.39 & 1604.2 & 182.63 & 219.3 \\ 115 . & 27.712 & 1.321 & -3.547 & 77.03 & 34.51 & 52.42 & 1568.3 & 163.86 & 213.7 \\ 120 . & 27.372 & 1.282 & -3.285 & 79.26 & 34.14 & 52.43 & 1533.0 & 147.76 & 208.1 \\ & & & & & & & & & \\ 125 . & 27.030 & 1.246 & -3.023 & 81.40 & 33.78 & 52.44 & 1498.1 & 133.99 & 202.4 \\ 130 . & 26.686 & 1.213 & -2.761 & 83.46 & 33.44 & 52.47 & 1463.7 & 122.17 & 196.7 \\ 135 . & 26.340 & 1.184 & -2.498 & 85.44 & 33.11 & 52.51 & 1429.7 & 112.01 & 191.0 \\ 140 . & 25.992 & 1.157 & -2.235 & 87.35 & 32.80 & 52.56 & 1396.2 & 103.22 & 185.4 \\ 145 . & 25.641 & 1.132 & -1.972 & 89.20 & 32.52 & 52.64 & 1363.2 & 95.59 & 179.9 \\ & & & & & & & & & \\ 150 . & 25.288 & 1.110 & -1.709 & 90.98 & 32.25 & 52.74 & 1330.6 & 88.91 & 174.5 \\ 155 . & 24.932 & 1.089 & -1.445 & 92.71 & 31.99 & 52.85 & 1298.7 & 83.03 & 169.2 \\ 160 . & 24.573 & 1.071 & -1.180 & 94.39 & 31.76 & 52.97 & 1267.3 & 77.83 & 164.0 \\ 165 . & 24.212 & 1.054 & -0.915 & 96.03 & 31.54 & 53.11 & 1236.5 & 73.19 & 159.0 \\ 170 . & 23.848 & 1.038 & -0.649 & 97.61 & 31.33 & 53.26 & 1206.3 & 69.03 & 154.1\end{array}$


METHANE ISOBAR AT $\mathrm{P}=35.0 \mathrm{MPa}$ (continued)

\begin{tabular}{|c|c|c|c|c|c|c|c|c|c|}
\hline $\mathrm{T}$ & $\rho$ & $\mathrm{Z}$ & $\mathrm{H}$ & $S$ & $\mathrm{C}_{\mathrm{v}}$ & $C_{p}$ & W & $\eta$ & $\lambda$ \\
\hline K & $\mathrm{mol} \cdot \mathrm{dm}^{-3}$ & & $\mathrm{~kJ} / \mathrm{mol}$ & $\mathrm{J} /(\mathrm{mol} \cdot \mathrm{K})$ & $\mathrm{J} /(\mathrm{mol} \cdot \mathrm{K})$ & $\mathrm{J} /(\mathrm{mol} \cdot \mathrm{K})$ & $\mathrm{m} \cdot \mathrm{s}^{-1}$ & $\mu \mathrm{Pa} \cdot \mathrm{s}$ & $\mathrm{mW} /(\mathrm{m} \cdot$ \\
\hline 175. & 23.481 & 1.024 & -0.383 & 99.16 & 31.14 & 53.43 & 1176.8 & 65.28 & 149.3 \\
\hline 180. & 23.112 & 1.012 & -0.115 & 100.67 & 30.96 & 53.60 & 1147.9 & 61.89 & 144.7 \\
\hline 185. & 22.739 & 1.001 & 0.153 & 102.14 & 30.79 & 53.77 & 1119.7 & 58.80 & 140.3 \\
\hline 190. & 22.365 & 0.991 & 0.423 & 103.57 & 30.64 & 53.96 & 1092.2 & 55.98 & 136.0 \\
\hline 195. & 21.988 & 0.982 & 0.693 & 104.98 & 30.50 & 54.14 & 1065.5 & 53.39 & 131.9 \\
\hline 200. & 21.609 & 0.974 & 0.964 & 106.35 & 30.37 & 54.33 & 1039.6 & 51.01 & 127.9 \\
\hline 205 . & 21.228 & 0.967 & 1.236 & 107.70 & 30.25 & 54.52 & 1014.4 & 48.81 & 124.1 \\
\hline 210 . & 20.846 & 0.962 & 1.509 & 109.01 & 30.15 & 54.70 & 990.1 & 46.78 & 120.5 \\
\hline 215. & 20.463 & 0.957 & 1.783 & 110.30 & 30.06 & 54.87 & 966.7 & 44.90 & 117.0 \\
\hline 220 . & 20.079 & 0.953 & 2.058 & 111.56 & 29.98 & 55.04 & 944.2 & 43.15 & 113.8 \\
\hline 225 . & 19.695 & 0.950 & 2.334 & 112.80 & 29.91 & 55.19 & 922.5 & 41.53 & 110.6 \\
\hline 230 . & 19.312 & 0.948 & 2.610 & 114.02 & 29.86 & 55.33 & 901.9 & 40.02 & 107.7 \\
\hline 235. & 18.930 & 0.946 & 2.887 & 115.21 & 29.82 & 55.45 & 882.2 & 38.61 & 104.9 \\
\hline 240 . & 18.549 & 0.946 & 3.164 & 116.38 & 29.79 & 55.55 & 863.5 & 37.31 & 102.3 \\
\hline 245. & 18.171 & 0.946 & 3.442 & 117.52 & 29.77 & 55.63 & 845.8 & 36.09 & 99.8 \\
\hline 50. & 17.796 & 0.946 & 3.721 & 118.65 & 29.76 & 55.68 & 829.0 & 34.96 & 97.5 \\
\hline 255. & 17.425 & 0.947 & 3.999 & 119.75 & 29.77 & 55.71 & 813.3 & 33.91 & 95.3 \\
\hline 260 . & 17.058 & 0.949 & 4.278 & 120.83 & 29.79 & 55.70 & 798.6 & 32.93 & 93.3 \\
\hline 265. & 16.697 & 0.951 & 4.556 & 121.89 & 29.81 & 55.67 & 784.9 & 32.02 & 91.5 \\
\hline 270 & 16.342 & 0.954 & 4.834 & 122.93 & 29.85 & 55.61 & 772.1 & 31.17 & 89.7 \\
\hline 275. & 15.993 & 0.957 & 5.112 & 123.95 & 29.90 & 55.52 & 760.3 & 30.39 & 88.1 \\
\hline 280 . & 15.651 & 0.961 & 5.389 & 124.95 & 29.96 & 55.40 & 749.4 & 29.67 & 86.7 \\
\hline 285. & 15.317 & 0.964 & 5.666 & 125.93 & 30.03 & 55.26 & 739.4 & 29.00 & 85.4 \\
\hline 290. & 14.991 & 0.968 & 5.942 & 126.89 & 30.10 & 55.10 & 730.2 & 28.38 & 84.1 \\
\hline 295. & 14.673 & 0.972 & 6.217 & 127.83 & 30.19 & 54.92 & 721.8 & 27.81 & 83.0 \\
\hline 300. & 14.364 & 0.977 & 6.491 & 128.75 & 30.29 & 54.73 & 714.2 & 27.28 & 82.0 \\
\hline 310. & 13.773 & 0.986 & 7.036 & 130.54 & 30.52 & 54.30 & 701.0 & 26.35 & 80.3 \\
\hline 320 . & 13.218 & 0.995 & 7.577 & 132.26 & 30.78 & 53.85 & 690.5 & 25.57 & 79.0 \\
\hline 330. & 12.699 & 1.004 & 8.113 & 133.91 & 31.07 & 53.41 & 682.1 & 24.91 & 77.9 \\
\hline 340. & 12.215 & 1.014 & 8.645 & 135.50 & 31.40 & 52.99 & 675.7 & 24.37 & 77.2 \\
\hline 350 . & 11.764 & 1.022 & 9.173 & 137.03 & 31.76 & 52.61 & 670.9 & 23.92 & 76.7 \\
\hline 360. & 11.345 & 1.031 & 9.698 & 138.50 & 32.15 & 52.28 & 667.4 & 23.55 & 76.4 \\
\hline 370. & 10.954 & 1.039 & 10.219 & 139.93 & 32.56 & 52.01 & 665.1 & 23.24 & 76.3 \\
\hline 380. & 10.591 & 1.046 & 10.738 & 141.32 & 32.99 & 51.80 & 663.8 & 23.00 & 76.4 \\
\hline 390. & 10.251 & 1.053 & 11.255 & 142.66 & 33.45 & 51.65 & 663.3 & 22.81 & 76.6 \\
\hline 400 . & 9.935 & 1.059 & 11.771 & 143.97 & 33.92 & 51.56 & 663.5 & 22.66 & 77.0 \\
\hline 410 . & 9.639 & 1.065 & 12.287 & 145.24 & 34.40 & 51.52 & 664.2 & & 77.6 \\
\hline 420. & 9.361 & 1.071 & 12.802 & 146.48 & 34.90 & 51.53 & 665.4 & & 78.2 \\
\hline 430. & 9.101 & 1.076 & 13.317 & 147.69 & 35.40 & 51.59 & 667.0 & & 79.0 \\
\hline 440. & 8.856 & 1.080 & 13.834 & 148.88 & 35.92 & 51.69 & 669.0 & & 79.9 \\
\hline
\end{tabular}


METHANE ISOBAR AT $\mathrm{P}=35.0 \mathrm{MPa}$ (continued)

\begin{tabular}{|c|c|c|c|c|c|c|c|c|c|}
\hline $\mathrm{T}$ & $\rho$ & $\mathrm{Z}$ & $\mathrm{H}$ & S & $C_{v}$ & $C_{p}$ & W & $\eta$ & $\lambda$ \\
\hline K & $\mathrm{mol} \cdot \mathrm{dm}^{-3}$ & & $\mathrm{~kJ} / \mathrm{mol}$ & $\mathrm{J} /(\mathrm{mol} \cdot \mathrm{K})$ & $\mathrm{J} /(\mathrm{mol} \cdot \mathrm{K})$ & $\mathrm{J} /(\mathrm{mol} \cdot \mathrm{K})$ & $\mathrm{m} \cdot \mathrm{s}^{-1}$ & $\mu \mathrm{Pa} \cdot \mathrm{s}$ & $\mathrm{mW} /(\mathrm{m} \cdot \mathrm{K})$ \\
\hline 450. & 8.626 & 1.084 & 14.351 & 150.04 & 36.44 & 51.82 & 671.2 & & 80.8 \\
\hline 460. & 8.408 & 1.088 & 14.870 & 151.18 & 36.97 & 51.99 & 673.7 & & 81.9 \\
\hline 470. & 8.203 & 1.092 & 15.391 & 152.30 & 37.49 & 52.19 & 676.4 & & 83.0 \\
\hline 480. & 8.009 & 1.095 & 15.914 & 153.40 & 38.03 & 52.41 & 679.2 & & 84.2 \\
\hline 490. & 7.824 & 1.098 & 16.439 & 154.49 & 38.56 & 52.65 & 682.3 & & 85.4 \\
\hline 500. & 7.650 & 1.101 & 16.967 & 155.55 & 39.09 & 52.92 & 685.4 & & 86.7 \\
\hline 520. & 7.325 & 1.105 & 18.031 & 157.64 & 40.14 & 53.49 & 692.0 & & 89.4 \\
\hline 540. & 7.030 & 1.109 & 19.107 & 159.67 & 41.19 & 54.12 & 698.9 & & 92.4 \\
\hline 560. & 6.760 & 1.112 & 20.196 & 161.65 & 42.22 & 54.77 & 706.1 & & 95.4 \\
\hline 580. & 6.513 & 1.114 & 21.298 & 163.58 & 43.23 & 55.45 & 713.4 & & 98.5 \\
\hline 600. & 6.285 & 1.116 & 22.414 & 165.48 & 44.21 & 56.15 & 720.8 & & 101.8 \\
\hline
\end{tabular}

\begin{tabular}{|c|c|c|c|c|c|c|c|c|c|}
\hline $\mathrm{T}$ & $\rho$ & Z & $\mathrm{H}$ & $\mathrm{S}$ & $C_{v}$ & $C_{p}$ & W & $\eta$ & $\lambda$ \\
\hline K & $\mathrm{mol} \cdot \mathrm{dm}^{-3}$ & & $\mathrm{~kJ} / \mathrm{mol}$ & $\mathrm{J} /(\mathrm{mol} \cdot \mathrm{K})$ & $\mathrm{J} /(\mathrm{mol} \cdot \mathrm{K})$ & $\mathrm{J} /(\mathrm{mol} \cdot \mathrm{K})$ & $\mathrm{m} \cdot \mathrm{s}^{-1}$ & $\mu \mathrm{Pa} \cdot \mathrm{s}$ & $\mathrm{mW} /(\mathrm{m} \cdot \mathrm{K})$ \\
\hline 105. & 28.556 & 1.604 & -3.938 & 71.86 & 35.36 & 52.06 & 1667.9 & 216.24 & 228.8 \\
\hline 110. & 28.229 & 1.549 & -3.678 & 74.28 & 35.06 & 52.13 & 1632.3 & 193.30 & 223.5 \\
\hline 115. & 27.900 & 1.499 & -3.417 & 76.60 & 34.71 & 52.14 & 1597.4 & 173.48 & 218.0 \\
\hline 120. & 27.571 & 1.454 & -3.156 & 78.82 & 34.35 & 52.12 & 1563.2 & 156.46 & 212.5 \\
\hline 125. & 27.240 & 1.413 & -2.896 & 80.94 & 33.99 & 52.10 & 1529.6 & 141.85 & 206.9 \\
\hline 130. & 26.908 & 1.375 & -2.635 & 82.99 & 33.65 & 52.09 & 1496.4 & 129.30 & 201.3 \\
\hline 135. & 26.575 & 1. 341 & -2.375 & 84.95 & 33.33 & 52.10 & 1463.7 & 118.51 & 195.8 \\
\hline 140 & 26.240 & 1.310 & -2.114 & 86.85 & 33.03 & 52.11 & 1431.5 & 109.18 & 190.3 \\
\hline 145. & 25.903 & 1. 281 & -1.854 & 88.68 & 32.74 & 52.15 & 1399.9 & 101.08 & 184.9 \\
\hline 150. & 25.565 & 1.255 & -1.593 & 90.45 & 32.47 & 52.20 & 1368.8 & 94.01 & 179.6 \\
\hline 155. & 25.225 & 1.230 & -1.332 & 92.16 & 32.22 & 52.26 & 1338.3 & 87.80 & 174.4 \\
\hline 160. & 24.884 & 1.208 & -1.070 & 93.82 & 31.98 & 52.33 & 1308.5 & 82.31 & 169.3 \\
\hline 165. & 24.540 & 1.188 & -0.808 & 95.43 & 31.76 & 52.42 & 1279.2 & 77.42 & 164.4 \\
\hline 170. & 24.195 & 1.170 & -0.546 & 97.00 & 31.55 & 52.51 & 1250.5 & 73.05 & 159.6 \\
\hline 175. & 23.848 & 1.153 & -0.283 & 98.52 & 31.36 & 52.62 & 1222.5 & 69.13 & 154.9 \\
\hline 180. & 23.500 & 1.137 & -0.020 & 100.00 & 31.18 & 52.73 & 1195.2 & 65.58 & 150.4 \\
\hline 185. & 23.150 & 1.123 & 0.244 & 101.45 & 31.01 & 52.84 & 1168.5 & 62.35 & 146.0 \\
\hline 190. & 22.799 & 1.111 & 0.509 & 102.86 & 30.85 & 52.96 & 1142.6 & 59.41 & 141.9 \\
\hline 195. & 22.446 & 1.099 & 0.774 & 104.24 & 30.71 & 53.08 & 1117.3 & 56.73 & 137.8 \\
\hline 200 & 22.092 & 1.089 & 1.039 & 105.58 & 30.57 & 53.20 & 1092.8 & 54.26 & 133.9 \\
\hline
\end{tabular}


METHANE ISOBAR AT $\mathrm{P}=40.0 \mathrm{MPa}$ (continued)

\begin{tabular}{|c|c|c|c|c|c|c|c|c|}
\hline $\mathrm{T}$ & $\rho$ & $\mathrm{H}$ & $S$ & $c_{v}$ & $C_{p}$ & W & $\eta$ & $\lambda$ \\
\hline K & $\mathrm{mol} \cdot \mathrm{dm}^{-3}$ & $\mathrm{~kJ} / \mathrm{mol}$ & $\mathrm{J} /(\mathrm{mol} \cdot \mathrm{K})$ & $\mathrm{J} /(\mathrm{mol} \cdot \mathrm{K})$ & $\mathrm{J} /(\mathrm{mol} \cdot \mathrm{K})$ & $\mathrm{m} \cdot \mathrm{s}^{-1}$ & $\mu \mathrm{Pa} \cdot \mathrm{s}$ & $\mathrm{mW} /(\mathrm{m} \cdot \mathrm{K})$ \\
\hline 205. & 21.738 & 1.306 & 106.90 & 30.45 & 53.32 & 1069.1 & 51.98 & 130.2 \\
\hline 210 & 21.383 & 1.573 & 108.18 & 30.35 & 53.43 & 1046.1 & 49.88 & 126.6 \\
\hline 215 . & 21.028 & 1.840 & 109.44 & 30.25 & 53.54 & 1023.9 & 47.94 & 123.2 \\
\hline 220 & 20.673 & 2.108 & 110.68 & 30.17 & 53.65 & 1002.4 & 46.14 & 119.9 \\
\hline 225. & 20.319 & 2.377 & 111.88 & 30.10 & 53.75 & 981.9 & 44.46 & 116.8 \\
\hline 230 . & 19.966 & 2.645 & 113.06 & 30.04 & 53.84 & 962.1 & 42.91 & 113.9 \\
\hline 235. & 19.613 & 2.915 & 114.22 & 29.99 & 53.92 & 943.2 & 41.46 & 111.1 \\
\hline 240 . & 19.263 & 3.185 & 115.36 & 29.96 & 53.99 & 925.1 & 40.11 & 108.5 \\
\hline 245 . & 18.915 & 3.455 & 116.47 & 29.94 & 54.04 & 907.9 & 38.86 & 106.0 \\
\hline 250 . & 18.570 & 3.725 & 117.57 & 29.93 & 54.08 & 891.5 & 37.68 & 103.7 \\
\hline 255. & 18.228 & 3.996 & 118.64 & 29.93 & 54.11 & 876.0 & 36.59 & 101.5 \\
\hline 260. & 17.890 & 4.266 & 119.69 & 29.94 & 54.12 & 861.3 & 35.58 & 99.4 \\
\hline 265. & 17.556 & 4.537 & 120.72 & 29.96 & 54.11 & 847.5 & 34.63 & 97.5 \\
\hline 270 & 17.227 & 4.807 & 121.73 & 30.00 & 54.08 & 834.5 & 33.74 & 95.8 \\
\hline 275 . & 16.903 & 5.077 & 122.72 & 30.05 & 54.04 & 822.3 & 32.92 & 94.1 \\
\hline 280 & 16.584 & 5.348 & 123.69 & 30.10 & 53.99 & 810.9 & 32.15 & 92.6 \\
\hline 285. & 16.271 & 5.617 & 124.65 & 30.17 & 53.92 & 800.2 & 31.44 & 91.2 \\
\hline 290. & 15.965 & 5.887 & 125.59 & 30.25 & 53.83 & 790.3 & 30.78 & 89.9 \\
\hline 295. & 15.665 & 6.156 & 126.51 & 30.34 & 53.74 & 781.1 & 30.16 & 88.7 \\
\hline 300 & 15.371 & 6.424 & 127.41 & 30.43 & 53.63 & 772.6 & 29.59 & 87.6 \\
\hline 310. & 14.806 & 6.959 & 129.16 & 30.66 & 53.39 & 757.5 & 28.56 & 85.8 \\
\hline 320 . & 14.269 & 7.492 & 130.85 & 30.92 & 53.14 & 744.8 & 27.69 & 84.2 \\
\hline 330. & 13.761 & 8.022 & 132.49 & 31.22 & 52.88 & 734.3 & 26.94 & 83.0 \\
\hline 340 . & 13.282 & 8.549 & 134.06 & 31.54 & 52.62 & 725.6 & 26.30 & 82.1 \\
\hline 350. & 12.831 & 9.074 & 135.58 & 31.90 & 52.39 & 718.5 & 25.75 & 81.4 \\
\hline 360. & 12.407 & 9.597 & 137.06 & 32.28 & 52.19 & 713.0 & 25.30 & 81.0 \\
\hline 370. & 12.009 & 10.118 & 138.48 & 32.69 & 52.03 & 708.7 & 24.91 & 80.8 \\
\hline 380. & 11.635 & 10.638 & 139.87 & 33.12 & 51.90 & 705.4 & 24.59 & 80.7 \\
\hline 390. & 11.284 & 11.157 & 141.22 & 33.57 & 51.82 & 703.2 & 24.33 & 80.8 \\
\hline 400 & 10.953 & 11.675 & 142.53 & 34.04 & 51.79 & 701.7 & 24.11 & 81.1 \\
\hline 410 & 10.643 & 12.193 & 143.81 & 34.52 & 51.79 & 700.9 & & 81.5 \\
\hline 420. & 10.350 & 12.711 & 145.05 & 35.01 & 51.84 & 700.7 & & 82.0 \\
\hline 430. & 10.074 & 13.229 & 146.28 & 35.52 & 51.92 & 701.0 & & 82.7 \\
\hline 440. & 1.114 & 13.749 & 147.47 & 36.03 & 52.04 & 701.8 & & 83.4 \\
\hline 450 & 1.117 & 14.270 & 148.64 & 36.54 & 52.18 & 702.9 & & 84.3 \\
\hline 460. & 1.120 & 14.793 & 149.79 & 37.07 & 52.36 & 704.4 & & 85.2 \\
\hline 470. & 1.123 & 15.317 & 150.92 & 37.59 & 52.56 & 706.1 & & 86.2 \\
\hline 480. & 1.126 & 15.844 & 152.03 & 38.12 & 52.79 & 708.1 & & 87.3 \\
\hline 490 & 1.128 & 16.373 & 153.12 & 38.65 & 53.03 & 710.4 & & 88.5 \\
\hline 500. & 1.130 & 16.905 & 154.19 & 39.17 & 53.29 & 712.8 & & 89.7 \\
\hline
\end{tabular}


METHANE ISOBAR AT $\mathrm{P}=40.0 \mathrm{MPa}$ (continued)

$\begin{array}{cccccccccc}\mathrm{T} & \rho & \mathrm{Z} & \mathrm{H} & \mathrm{S} & \mathrm{C} & \mathrm{C} & \mathrm{W} & \eta & \lambda \\ \mathrm{K} & \mathrm{mol} \cdot \mathrm{dm}_{\mathrm{p}}{ }^{-3} & & \mathrm{~kJ} / \mathrm{mol} & \mathrm{J} /(\mathrm{mol} \cdot \mathrm{K}) & \mathrm{J} /(\mathrm{mol} \cdot \mathrm{K}) & \mathrm{J} /(\mathrm{mol} \cdot \mathrm{K}) & \mathrm{m} \cdot \mathrm{s}^{-1} & \mu \mathrm{Pa} \cdot \mathrm{s} & \mathrm{mW} /(\mathrm{m} \cdot \mathrm{K}) \\ & & & & & & & & & \\ 520 . & 8.163 & 1.133 & 17.976 & 156.29 & 40.23 & 53.86 & 718.0 & & 92.2 \\ 540 . & 7.841 & 1.136 & 19.060 & 158.34 & 41.27 & 54.47 & 723.8 & 95.0 \\ 560 . & 7.547 & 1.138 & 20.155 & 160.33 & 42.29 & 55.12 & 730.0 & 97.9 \\ 580 . & 7.276 & 1.140 & 21.265 & 162.28 & 43.29 & 55.79 & 736.4 & 100.9 \\ 600 . & 7.026 & 1.141 & 22.387 & 164.18 & 44.27 & 56.47 & 743.0 & 104.0\end{array}$

$\overline{\text { METHANE ISOBAR AT } \mathrm{P}=45.0 \mathrm{MPa}}$

\begin{tabular}{|c|c|c|c|c|c|c|c|c|c|}
\hline $\mathrm{T}$ & $\rho$ & $\mathrm{Z}$ & $\mathrm{H}$ & S & $\mathrm{C}_{\mathrm{v}}$ & $C_{p}$ & $\mathrm{~W}$ & $\eta$ & $\lambda$ \\
\hline K & $\mathrm{mol} \cdot \mathrm{dm}^{-3}$ & & $\mathrm{~kJ} / \mathrm{mol}$ & $\mathrm{J} /(\mathrm{mol} \cdot \mathrm{K})$ & $\mathrm{J} /(\mathrm{mol} \cdot \mathrm{K})$ & $\mathrm{J} /(\mathrm{mol} \cdot \mathrm{K})$ & $\mathrm{m} \cdot \mathrm{s}^{-1}$ & $\mu \mathrm{Pa} \cdot \mathrm{s}$ & $\mathrm{mW} /(\mathrm{m} \cdot \mathrm{K})$ \\
\hline 105. & 28.718 & 1.795 & -3.805 & 71.46 & 35.54 & 51.86 & 1693.8 & 228.89 & 232.8 \\
\hline 110. & 28.399 & 1.733 & -3.545 & 73.88 & 35.25 & 51.91 & 1659.1 & 204.77 & 227.6 \\
\hline 115. & 28.079 & 1.676 & -3.286 & 76.18 & 34.91 & 51.90 & 1625.2 & 183.84 & 222.2 \\
\hline 120. & 27.760 & 1.625 & -3.027 & 78.39 & 34.55 & 51.86 & 1592.0 & 165.79 & 216.8 \\
\hline 125. & 27.439 & 1.578 & -2.767 & 80.51 & 34.20 & 51.81 & 1559.4 & 150.26 & 211.3 \\
\hline 130. & 27.118 & 1.535 & -2.508 & 82.54 & 33.86 & 51.77 & 1527.3 & 136.91 & 205.9 \\
\hline 135. & 26.796 & 1.496 & -2.250 & 84.49 & 33.54 & 51.74 & 1495.8 & 125.42 & 200.4 \\
\hline 140. & 26.473 & 1.460 & -1.991 & 86.37 & 33.24 & 51.73 & 1464.8 & 115.49 & 195.0 \\
\hline 145. & 26.149 & 1.427 & -1.732 & 88.19 & 32.95 & 51.73 & 1434.4 & 106.87 & 189.7 \\
\hline 150. & 25.824 & 1.397 & -1.474 & 89.94 & 32.69 & 51.74 & 1404.6 & 99.36 & 184.5 \\
\hline 155. & 25.498 & 1.369 & -1.215 & 91.64 & 32.43 & 51.77 & 1375.4 & 92.76 & 179.4 \\
\hline 160. & 25.171 & 1.344 & -0.956 & 93.28 & 32.20 & 51.81 & 1346.8 & 86.95 & 174.4 \\
\hline 165. & 24.843 & 1.320 & -0.697 & 94.88 & 31.97 & 51.85 & 1318.8 & 81.78 & 169.6 \\
\hline 170. & 24.513 & 1.299 & -0.437 & 96.43 & 31.76 & 51.90 & 1291.5 & 77.17 & 164.8 \\
\hline 175. & 24.183 & 1.279 & -0.178 & 97.93 & 31.57 & 51.96 & 1264.8 & 73.04 & 160.2 \\
\hline 180. & 23.852 & 1.261 & 0.082 & 99.40 & 31.38 & 52.03 & 1238.7 & 69.31 & 155.8 \\
\hline 185. & 23.520 & 1.244 & 0.343 & 100.82 & 31.21 & 52.09 & 1213.4 & 65.93 & 151.5 \\
\hline 190. & 23.188 & 1.228 & 0.603 & 102.21 & 31.05 & 52.17 & 1188.6 & 62.85 & 147.4 \\
\hline 195. & 22.855 & 1.214 & 0.864 & 103.57 & 30.91 & 52.24 & 1164.6 & 60.04 & 143.4 \\
\hline 200 . & 22.522 & 1.202 & 1.126 & 104.89 & 30.77 & 52.31 & 1141.3 & 57.47 & 139.5 \\
\hline 205. & 22.189 & 1.190 & 1.387 & 106.19 & 30.65 & 52.39 & 1118.6 & 55.10 & 135.8 \\
\hline 210 . & 21.855 & 1.179 & 1.649 & 107.45 & 30.54 & 52.46 & 1096.7 & 52.91 & 132.3 \\
\hline 215. & 21.522 & 1.170 & 1.912 & 108.68 & 30.44 & 52.53 & 1075.5 & 50.89 & 128.9 \\
\hline 220 & 21.190 & 1.161 & 2.175 & 109.89 & 30.35 & 52.60 & 1055.0 & 49.03 & 125.7 \\
\hline 225 . & 20.859 & 1.153 & 2.438 & 111.08 & 30.28 & 52.66 & 1035.3 & 47.29 & 122.6 \\
\hline
\end{tabular}


METHANE ISOBAR AT $\mathrm{P}=45.0 \mathrm{MPa}$ (continued)

\begin{tabular}{|c|c|c|c|c|c|c|c|c|c|}
\hline $\mathrm{T}$ & $\rho$ & $\mathrm{Z}$ & H & S & $\mathrm{C}_{\mathrm{v}}$ & $C_{p}$ & W & $\eta$ & $\lambda$ \\
\hline K & $\mathrm{mol} \cdot \mathrm{dm}^{-3}$ & & $\mathrm{~kJ} / \mathrm{mol}$ & $\mathrm{J} /(\mathrm{mol} \cdot \mathrm{K})$ & $\mathrm{J} /(\mathrm{mol} \cdot \mathrm{K})$ & $\mathrm{J} /(\mathrm{mol} \cdot \mathrm{K})$ & $\mathrm{m} \cdot \mathrm{s}^{-1}$ & $\mu \mathrm{Pa} \cdot \mathrm{s}$ & $\mathrm{mW} /(\mathrm{m} \cdot \mathrm{l}$ \\
\hline 230 . & 20.529 & 1.146 & 2.701 & 112.23 & 30.22 & 52.72 & 1016.3 & 45.68 & 119.7 \\
\hline 235 . & 20.200 & 1.140 & 2.965 & 113.37 & 30.17 & 52.77 & 998.1 & 44.18 & 116.9 \\
\hline 240 . & 19.873 & 1.135 & 3.229 & 114.48 & 30.13 & 52.81 & 980.6 & 42.78 & 114.2 \\
\hline 245 . & 19.549 & 1.130 & 3.493 & 115.57 & 30.10 & 52.85 & 963.8 & 41.48 & 111.7 \\
\hline 250 . & 19.227 & 1.126 & 3.757 & 116.64 & 30.09 & 52.88 & 947.8 & 40.26 & 109.4 \\
\hline 255. & 18.908 & 1.123 & 4.022 & 117.68 & 30.09 & 52.90 & 932.6 & 39.13 & 107.2 \\
\hline 260 . & 18.592 & 1.120 & 4.286 & 118.71 & 30.10 & 52.91 & 918.1 & 38.07 & 105.1 \\
\hline 265 . & 18.280 & 1.117 & 4.551 & 119.72 & 30.12 & 52.92 & 904.3 & 37.08 & 103.2 \\
\hline 270. & 17.972 & 1.115 & 4.816 & 120.71 & 30.15 & 52.91 & 891.2 & 36.15 & 101.3 \\
\hline 275 . & 17.668 & 1.114 & 5.080 & 121.68 & 30.19 & 52.90 & 878.9 & 35.29 & 99.6 \\
\hline 280. & 17.368 & 1.113 & 5.345 & 122.63 & 30.25 & 52.87 & 867.2 & 34.48 & 98.1 \\
\hline 285. & 17.073 & 1.112 & 5.609 & 123.57 & 30.31 & 52.84 & 856.2 & 33.73 & 96.6 \\
\hline 290. & 16.784 & 1.112 & 5.873 & 124.49 & 30.39 & 52.80 & 845.9 & 33.02 & 95.2 \\
\hline 295. & 16.499 & 1.112 & 6.137 & 125.39 & 30.48 & 52.75 & 836.2 & 32.37 & 94.0 \\
\hline 300 . & 16.221 & 1.112 & 6.400 & 126.28 & 30.57 & 52.70 & 827.1 & 31.75 & 92.8 \\
\hline 310. & 15.680 & 1.113 & 6.927 & 128.00 & 30.80 & 52.58 & 810.7 & 30.65 & 90.8 \\
\hline 320. & 15.163 & 1.115 & 7.452 & 129.67 & 31.05 & 52.44 & 796.6 & 29.69 & 89.2 \\
\hline 330. & 14.670 & 1.118 & 7.976 & 131.28 & 31.35 & 52.31 & 784.4 & 28.86 & 87.8 \\
\hline 340. & 14.202 & 1.121 & 8.498 & 132.84 & 31.67 & 52.18 & 774.0 & 28.14 & 86.7 \\
\hline 350. & 13.757 & 1.124 & 9.019 & 134.35 & 32.03 & 52.06 & 765.3 & 27.52 & 85.9 \\
\hline 360. & 13.336 & 1.127 & 9.539 & 135.82 & 32.41 & 51.96 & .0 & 26.99 & 85.4 \\
\hline 370. & 12.937 & 1.131 & 10.059 & 137.24 & 32.81 & 51.89 & 752.0 & 26.53 & 85.0 \\
\hline 380. & 12.560 & 1.134 & 10.577 & 138.62 & 33.24 & 51.85 & 747.2 & 26.14 & 84.8 \\
\hline 390. & 12.203 & 1.137 & 11.096 & 139.97 & 33.69 & 51.84 & 743.3 & 25.81 & 84.8 \\
\hline 400. & 11.865 & 1.140 & 11.614 & 141.28 & 34.15 & 51.86 & 740.4 & 25.54 & 85.0 \\
\hline 410. & 11.546 & 1.143 & 12.133 & 142.56 & 34.63 & 51.91 & 738 & & 85.3 \\
\hline 420. & 11.244 & 1.146 & 12.653 & 143.81 & 35.12 & 51.99 & 736.7 & & 85.7 \\
\hline 430. & 10.958 & 1.149 & 13.173 & 145.04 & 35.61 & 52.11 & 735.8 & & 86.2 \\
\hline 440. & 10.687 & 1.151 & 13.695 & 146.24 & 36.12 & 52.25 & 735.4 & & 86.9 \\
\hline 450. & 10.430 & 1.153 & 14.218 & 147.41 & 36.64 & 52.42 & 735.5 & & 87.6 \\
\hline 460. & 10.185 & 1.155 & 14.743 & 148.57 & 37.16 & 52.61 & 736.0 & & 88.5 \\
\hline 470. & 9.953 & 1.157 & 15.270 & 149.70 & 37.68 & 52.82 & 736.8 & & 89.4 \\
\hline 480. & 9.732 & 1.159 & 15.800 & 150.82 & 38.20 & 53.05 & 738.0 & & 90.4 \\
\hline 490. & 9.521 & 1.160 & 16.331 & 151.91 & 38.73 & 53.31 & 739.4 & & 91.5 \\
\hline 500. & 9.320 & 1.161 & 16.866 & 152.99 & 39.25 & 53.57 & 741.1 & & 92.6 \\
\hline 520. & 8.945 & 1.164 & 17.943 & 155.10 & 40.30 & 54.14 & 745.0 & & 95.0 \\
\hline 540 . & 8.601 & 1.165 & 19.032 & 157.16 & 41.33 & 54.76 & 749.6 & & 97.6 \\
\hline 560. & 8.286 & 1.166 & 20.133 & 159.16 & 42.35 & 55.40 & 754.7 & & 100.4 \\
\hline 580. & 7.995 & 1.167 & 21.248 & 161.12 & 43.35 & 56.06 & 760.2 & & 103.3 \\
\hline 600. & 7.726 & 1.168 & 22.376 & 163.03 & 44.33 & 56.74 & 766.0 & & 106.3 \\
\hline
\end{tabular}


METHANE ISOBAR AT P $=50.0 \mathrm{MPa}$

\begin{tabular}{|c|c|c|c|c|c|c|c|c|c|}
\hline $\mathrm{T}$ & $\rho$ & $Z$ & $\mathrm{H}$ & S & $\mathrm{C}_{\mathrm{v}}$ & $C_{p}$ & W & $\eta$ & $\lambda$ \\
\hline K & $\mathrm{mol} \cdot \mathrm{dm}^{-3}$ & & $\mathrm{~kJ} / \mathrm{mol}$ & $\mathrm{J} /(\mathrm{mol} \cdot \mathrm{K})$ & $\mathrm{J} /(\mathrm{mol} \cdot \mathrm{K})$ & $\mathrm{J} /(\mathrm{mol} \cdot \mathrm{K})$ & $\mathrm{m} \cdot \mathrm{s}^{-1}$ & $\mu \mathrm{Pa} \cdot \mathrm{s}$ & $\mathrm{mW} /(\mathrm{m} \cdot \mathrm{K})$ \\
\hline 105. & 28.873 & 1.984 & -3.671 & 71.08 & 35.72 & 51.68 & 1718.6 & 242.56 & 236.6 \\
\hline 110. & 28.562 & 1.914 & -3.413 & 73.49 & 35.44 & 51.72 & 1684.7 & 217.19 & 231.5 \\
\hline 115. & 28.251 & 1.851 & -3.154 & 75.79 & 35.10 & 51.68 & 1651.8 & 195.05 & 226.3 \\
\hline 120. & 27.940 & 1.794 & -2.896 & 77.99 & 34.75 & 51.62 & 1619.5 & 175.88 & 221.0 \\
\hline 125. & 27.629 & 1.741 & -2.638 & 80.09 & 34.40 & 51.55 & 1587.8 & 159.34 & 215.6 \\
\hline 130. & 27.317 & 1.693 & -2.380 & 82.11 & 34.06 & 51.49 & 1556.8 & 145.09 & 210.3 \\
\hline 135. & 27.005 & 1.650 & -2.123 & 84.05 & 33.74 & 51.44 & 1526.3 & 132.82 & 204.9 \\
\hline 140. & 26.693 & 1.609 & -1.866 & 85.92 & 33.44 & 51.40 & 1496.4 & 122.21 & 199.6 \\
\hline 145. & 26.380 & 1.572 & -1.609 & 87.73 & 33.16 & 51.37 & 1467.1 & 113.02 & 194.4 \\
\hline 150. & 26.066 & 1.538 & -1.352 & 89.47 & 32.89 & 51.36 & 1438.4 & 105.00 & 189.3 \\
\hline 155. & 25.752 & 1.507 & -1.095 & 91.15 & 32.64 & 51.35 & 1410.3 & 97.98 & 184.2 \\
\hline 160. & 25.438 & 1.478 & -0.839 & 92.78 & 32.40 & 51.36 & 1382.8 & 91.80 & 179.3 \\
\hline 165. & 25.123 & 1.451 & -0.582 & 94.36 & 32.18 & 51.37 & 1356.0 & 86.32 & 174.5 \\
\hline 170. & 24.807 & 1.426 & -0.325 & 95.90 & 31.97 & 51.39 & 1329.7 & 81.43 & 169.9 \\
\hline 175. & 24.492 & 1.403 & -0.068 & 97.39 & 31.77 & 51.42 & 1304.2 & 77.06 & 165.3 \\
\hline 180. & 24.175 & 1.382 & 0.189 & 98.84 & 31.58 & 51.45 & 1279.2 & 73.13 & 160.9 \\
\hline 185. & 23.859 & 1.362 & 0.447 & 100.25 & 31.41 & 51.48 & 1254.9 & 69.57 & 156.7 \\
\hline 190. & 23.542 & 1.344 & 0.704 & 101.62 & 31.25 & 51.52 & 1231.3 & 66.33 & 152.6 \\
\hline 195. & 23.226 & 1.328 & 0.962 & 102.96 & 31.10 & 51.56 & 1208.3 & 63.38 & 148.6 \\
\hline 200 . & 22.909 & 1.312 & 1.220 & 104.26 & 30.96 & 51.60 & 1185.9 & 60.68 & 144.8 \\
\hline 205. & 22.593 & 1.298 & 1.478 & 105.54 & 30.83 & 51.64 & 1164.2 & 58.21 & 141.2 \\
\hline 210 . & 22.278 & 1.285 & 1.736 & 106.78 & 30.72 & 51.68 & 1143.2 & 55.92 & 137.7 \\
\hline 215 . & 21.963 & 1.274 & 1.995 & 108.00 & 30.62 & 51.73 & 1122.9 & 53.82 & 134.3 \\
\hline 220 . & 21.649 & 1.263 & 2.254 & 109.19 & 30.53 & 51.76 & 1103.2 & 51.87 & 131.1 \\
\hline 225 . & 21.336 & 1.253 & 2.512 & 110.35 & 30.45 & 51.80 & 1084.2 & 50.06 & 128.0 \\
\hline 230 . & 21.025 & 1.244 & 2.772 & 111.49 & 30.39 & 51.84 & 1065.9 & 48.38 & 125.1 \\
\hline 235 . & 20.715 & 1.235 & 3.031 & 112.61 & 30.33 & 51.87 & 1048.2 & 46.82 & 122.3 \\
\hline 240 . & 20.407 & 1.228 & 3.290 & 113.70 & 30.29 & 51.90 & 1031.2 & 45.36 & 119.7 \\
\hline 245 . & 20.101 & 1.221 & 3.550 & 114.77 & 30.26 & 51.92 & 1014.9 & 44.01 & 117.2 \\
\hline 250 . & 19.798 & 1.215 & 3.809 & 115.82 & 30.25 & 51.95 & 999.3 & 42.74 & 114.8 \\
\hline 255. & 19.497 & 1.210 & 4.069 & 116.85 & 30.24 & 51.96 & 984.3 & 41.56 & 112.5 \\
\hline 260. & 19.200 & 1.205 & 4.329 & 117.86 & 30.25 & 51.98 & 970.0 & 40.45 & 110.4 \\
\hline 265 . & 18.905 & 1.200 & 4.589 & 118.85 & 30.27 & 51.98 & 956.3 & 39.42 & 108.5 \\
\hline 270 . & 18.614 & 1.197 & 4.849 & 119.82 & 30.30 & 51.99 & 943.3 & 38.45 & 106.6 \\
\hline 275 . & 18.327 & 1.193 & 5.109 & 120.77 & 30.34 & 51.98 & 930.9 & 37.54 & 104.8 \\
\hline 280 . & 18.044 & 1.190 & 5.369 & 121.71 & 30.39 & 51.98 & 919.2 & 36.70 & 103.2 \\
\hline 285 & 17.764 & 1.188 & 5.629 & 122.63 & 30.45 & 51.97 & 908.0 & 35.90 & 101.7 \\
\hline 290. & 17.489 & 1.186 & 5.888 & 123.53 & 30.53 & 51.96 & 897.5 & 35.16 & 100.3 \\
\hline 295. & 17.219 & 1.184 & 6.148 & 124.42 & 30.61 & 51.94 & 887.5 & 34.46 & 99.0 \\
\hline 300 . & 16.953 & 1.182 & 6.408 & 125.30 & 30.71 & 51.92 & 878.0 & 33.81 & 97.8 \\
\hline
\end{tabular}


METHANE ISOBAR AT $\mathrm{P}=50.0 \mathrm{MPa}$ (continued)

\begin{tabular}{|c|c|c|c|c|c|c|c|c|c|}
\hline $\mathrm{T}$ & $\rho$ & Z & $\mathrm{H}$ & S & $\mathrm{C}_{\mathrm{v}}$ & $C_{p}$ & W & $\eta$ & $\lambda$ \\
\hline K & $\mathrm{mol} \cdot \mathrm{dm}^{-3}$ & & $\mathrm{~kJ} / \mathrm{mol}$ & $\mathrm{J} /(\mathrm{mol} \cdot \mathrm{K})$ & $\mathrm{J} /(\mathrm{mol} \cdot \mathrm{K})$ & $\mathrm{J} /(\mathrm{mol} \cdot \mathrm{K})$ & $\mathrm{m} \cdot \mathrm{s}^{-1}$ & $\mu \mathrm{Pa} \cdot \mathrm{s}$ & $\mathrm{mW} /(\mathrm{m} \cdot \mathrm{K})$ \\
\hline 310. & 16.435 & 1.180 & 6.927 & 127.00 & 30.93 & 51.87 & 860.8 & 32.63 & 95.6 \\
\hline 320 . & 15.938 & 1.179 & 7.445 & 128.64 & 31.18 & 51.82 & 845.5 & 31.60 & 93.8 \\
\hline 330. & 15.461 & 1.179 & 7.963 & 130.24 & 31.47 & 51.77 & 832.2 & 30.70 & 92.3 \\
\hline 340 . & 15.005 & 1.179 & 8.481 & 131.78 & 31.79 & 51.73 & 820.5 & 29.91 & 91.1 \\
\hline 350 . & 14.569 & 1.179 & 8.998 & 133.28 & 32.14 & 51.69 & 810.5 & 29.22 & 90.2 \\
\hline 360. & 14.154 & 1.180 & 9.515 & 134.74 & 32.52 & 51.68 & 801.8 & 28.62 & 89.5 \\
\hline 370. & 13.759 & 1.181 & 10.031 & 136.15 & 32.92 & 51.68 & 794.5 & 28.10 & 89.0 \\
\hline 380 . & 13.382 & 1.183 & 10.548 & 137.53 & 33.35 & 51.70 & 788.3 & 27.65 & 88.7 \\
\hline 390. & 13.025 & 1.184 & 11.066 & 138.87 & 33.79 & 51.75 & 783.2 & 27.26 & 88.6 \\
\hline 400. & 12.685 & 1.185 & 11.583 & 140.19 & 34.25 & 51.82 & 778.9 & 26.93 & 88.7 \\
\hline 410. & 12.361 & 1.187 & 12.102 & 141.47 & 34.72 & 51.92 & 775.5 & & 88.9 \\
\hline 420. & 12.054 & 1.188 & 12.622 & 142.72 & 35.21 & 52.04 & 772.9 & & 89.2 \\
\hline 430. & 11.762 & 1.189 & 13.143 & 143.95 & 35.71 & 52.19 & 770.9 & & 89.6 \\
\hline 440. & 11.483 & 1.190 & 13.666 & 145.15 & 36.21 & 52.36 & 769.5 & & 90.2 \\
\hline 450. & 11.219 & 1.191 & 14.190 & 146.33 & 36.72 & 52.55 & 768.6 & & 90.9 \\
\hline 460. & 10.966 & 1.192 & 14.717 & 147.48 & 37.24 & 52.76 & 768.1 & & 91.6 \\
\hline 470. & 10.725 & 1.193 & 15.246 & 148.62 & 37.76 & 52.99 & 768.1 & & 92.5 \\
\hline 480. & 10.495 & 1.194 & 15.777 & 149.74 & 38.28 & 53.24 & 768.4 & & 93.4 \\
\hline 490. & 10.276 & 1.194 & 16.311 & 150.84 & 38.80 & 53.50 & 769.0 & & 94.4 \\
\hline 500. & 10.066 & 1.195 & 16.847 & 151.92 & 39.32 & 53.78 & 770.0 & & 95.4 \\
\hline 520. & 9.673 & 1.196 & 17.928 & 154.04 & 40.36 & 54.36 & 772.6 & & 97.7 \\
\hline 540. & 9.312 & 1.196 & 19.021 & 156.11 & 41.40 & 54.98 & 776.0 & & 100.2 \\
\hline 560. & 8.979 & 1.196 & 20.127 & 158.12 & 42.41 & 55.62 & 780.1 & & 102.8 \\
\hline 580. & 8.672 & 1.196 & 21.246 & 160.08 & 43.41 & 56.28 & 784.6 & & 105.6 \\
\hline 600 . & 8.386 & 1.195 & 22.379 & 162.00 & 44.38 & 56.96 & 789.6 & & 108.5 \\
\hline
\end{tabular}

METHANE ISOBAR AT $\mathrm{P}=55.0 \mathrm{MPa}$

$\begin{array}{lccccccccc}\mathrm{T} & \rho & \mathrm{Z} & \mathrm{H} & \mathrm{S} & \mathrm{C}_{\mathrm{v}} & \mathrm{C}_{\mathrm{p}} & \mathrm{W} & \eta & \lambda \\ \mathrm{K} & \mathrm{mol} \cdot \mathrm{dm}^{-3} & & \mathrm{~kJ} / \mathrm{mol} & \mathrm{J} /(\mathrm{mol} \cdot \mathrm{K}) & \mathrm{J} /(\mathrm{mol} \cdot \mathrm{K}) & \mathrm{J} /(\mathrm{mol} \cdot \mathrm{K}) & \mathrm{m} \cdot \mathrm{s}^{-1} & \mu \mathrm{Pa} \cdot \mathrm{s} & \mathrm{mW} /(\mathrm{m} \cdot \mathrm{K}) \\ & & & & & & & & & \\ 105 . & 29.023 & 2.171 & -3.537 & 70.72 & 35.89 & 51.52 & 1742.5 & 257.43 & 240.4 \\ 110 . & 28.720 & 2.094 & -3.279 & 73.11 & 35.62 & 51.54 & 1709.4 & 230.72 & 235.4 \\ 115 . & 28.416 & 2.024 & -3.022 & 75.40 & 35.29 & 51.50 & 1677.3 & 207.27 & 230.3 \\ 120 . & 28.113 & 1.961 & -2.764 & 77.59 & 34.94 & 51.42 & 1645.8 & 186.85 & 225.1 \\ 125 . & 27.810 & 1.903 & -2.507 & 79.69 & 34.59 & 51.33 & 1615.1 & 169.19 & 219.8\end{array}$


METHANE ISOBAR AT $\mathrm{P}=55.0 \mathrm{MPa}$ (continued)

\begin{tabular}{|c|c|c|c|c|c|c|c|c|c|}
\hline $\mathrm{T}$ & $\rho$ & z & H & $\mathrm{s}$ & $\mathrm{C}_{\mathrm{v}}$ & $C_{p}$ & W & $\eta$ & $\lambda$ \\
\hline K & $\mathrm{mol} \cdot \mathrm{dm}^{-3}$ & & $\mathrm{~kJ} / \mathrm{mol}$ & $\mathrm{J} /(\mathrm{mol} \cdot \mathrm{K})$ & $\mathrm{J} /(\mathrm{mol} \cdot \mathrm{K})$ & $\mathrm{J} /(\mathrm{mol} \cdot \mathrm{K})$ & $\mathrm{m} \cdot \mathrm{s}^{-1}$ & $\mu \mathrm{Pa} \cdot \mathrm{s}$ & $\mathrm{mW} /(\mathrm{n}$ \\
\hline 130. & 27.507 & 1.850 & -2.251 & 81.70 & 34.26 & 51.25 & 1584.9 & 153.94 & 214.5 \\
\hline 135. & 27.204 & 1.801 & -1.995 & 83.64 & 33.94 & 51.18 & 1555.4 & 140.79 & 209.3 \\
\hline 140. & 26.901 & 1.756 & -1.739 & 85.50 & 33.64 & 51.11 & 1526.4 & 129.43 & 204.1 \\
\hline 145. & 26.598 & 1.715 & -1.484 & 87.29 & 33.35 & 51.06 & 1498.1 & 119.58 & 198.9 \\
\hline 150. & 26.295 & 1.677 & -1.229 & 89.02 & 33.09 & 51.03 & 1470.4 & 111.00 & 193.9 \\
\hline 155. & 25.992 & 1.642 & -0.974 & 90.69 & 32.83 & 51.00 & 1443.3 & 103.50 & 188.9 \\
\hline 160. & 25.688 & 1.609 & -0.719 & 92.31 & 32.59 & 50.98 & 1416.8 & 96.90 & 184.1 \\
\hline 165. & 25.385 & 1.579 & -0.464 & 93.88 & 32.37 & 50.97 & 1391.0 & 91.06 & 179.3 \\
\hline 170. & 25.081 & 1.551 & -0.209 & 95.40 & 32.16 & 50.96 & 1365.8 & 85.87 & 174.7 \\
\hline 175. & 24.778 & 1.526 & 0.046 & 96.88 & 31.96 & 50.96 & 1341.2 & 81.23 & 170.2 \\
\hline 180. & 24.474 & 1.502 & 0.301 & 98.31 & 31.77 & 50.97 & 1317.2 & 77.07 & 165.9 \\
\hline 185. & 24.171 & 1.479 & 0.556 & 99.71 & 31.59 & 50.98 & 1293.9 & 73.30 & 161.7 \\
\hline 190. & 23.868 & 1.459 & 0.810 & 101.07 & 31.43 & 50.99 & 1271.1 & 69.89 & 157.6 \\
\hline 195. & 23.565 & 1.440 & 1.065 & 102.39 & 31.28 & 51.00 & 1249.0 & 66.78 & 153.7 \\
\hline 200 . & 23.263 & 1.422 & 1.320 & 103.69 & 31.14 & 51.02 & 1227.5 & 63.94 & 149.9 \\
\hline 205. & 22.961 & 1.405 & 1.576 & 04.95 & 31.01 & 51.04 & 120 & 61.34 & 146.3 \\
\hline 210 . & 22.661 & 1.390 & 1.831 & 106.18 & 30.89 & 51.05 & 1186.4 & 58.95 & 142.8 \\
\hline 215. & 22.361 & 1.376 & 2.086 & 107.38 & 30.79 & 51.07 & 1166.8 & 56.74 & 139.4 \\
\hline 220. & 22.062 & 1.363 & 2.342 & 108.55 & 30.69 & 51.09 & 1147.8 & 54.70 & 136.2 \\
\hline 225. & 21.765 & 1.351 & 2.597 & 109.70 & 30.61 & 51.11 & 1129.5 & 52.81 & 133.2 \\
\hline 230 . & 1.469 & 1.340 & 2.853 & 110.82 & 30.55 & 51.13 & 111 & 05 & 130.2 \\
\hline 235 . & 21.175 & 1.329 & 3.108 & 111.92 & 30.49 & 51.15 & 1094.6 & 49.42 & 127.5 \\
\hline 240 . & 20.882 & 1.320 & 3.364 & 113.00 & 30.45 & 51.16 & 1078.1 & 47.90 & 124.8 \\
\hline 245 . & 20.592 & 1.311 & 3.620 & 114.06 & 30.41 & 51.18 & 1062.2 & 46.49 & 122.3 \\
\hline 250 . & 20.304 & 1.303 & 3.876 & 115.09 & 30.40 & 51.20 & 1046.9 & 45.16 & 119.9 \\
\hline 255. & 20.019 & 1.296 & 4.132 & 116.10 & 30.39 & 51.21 & 103 & 43.93 & 117.6 \\
\hline 260 . & 19.736 & 1.289 & 4.388 & 117.10 & 30.39 & 51.22 & 1018.1 & 42.77 & 115.5 \\
\hline 265 . & 19.457 & 1.283 & 4.644 & 118.07 & 30.41 & 51.23 & 1004.6 & 41.69 & 113.5 \\
\hline 270. & 19.180 & 1.277 & 4.900 & 119.03 & 30.44 & 51.24 & 991.7 & 40.68 & 111.6 \\
\hline 275. & 18.907 & 1.272 & 5.157 & 119.97 & 30.47 & 51.25 & 979.3 & 39.73 & 109.8 \\
\hline 280. & 18.637 & 1.268 & 5.413 & 120.89 & 30.53 & 51.25 & & 84 & 108.1 \\
\hline 285. & 18.371 & 1.263 & 5.669 & 121.80 & 30.59 & 51.26 & 956.3 & 38.00 & 106.5 \\
\hline 290. & 18.108 & 1.260 & 5.925 & 122.69 & 30.66 & 51.26 & 945.6 & 37.22 & 105.1 \\
\hline 295. & 17.850 & 1.256 & 6.182 & 123.57 & 30.74 & 51.26 & 935.4 & 36.48 & 103.7 \\
\hline 300 . & 17.595 & 1.253 & 6.438 & 124.43 & 30.83 & 51.26 & 925.8 & 35.80 & 102.4 \\
\hline 310. & 17.099 & 1.248 & 6.951 & 126.11 & 31.05 & 51.27 & & & 100.2 \\
\hline 320 . & 16.620 & 1.244 & 7.463 & 127.74 & 31.30 & 51.27 & 891.9 & 33.44 & 98.3 \\
\hline 330. & 16.158 & 1. 241 & 7.976 & 129.32 & 31.59 & 51.28 & 877.7 & 32.47 & 96.7 \\
\hline 340 . & 15.715 & 1.238 & 8.489 & 130.85 & 31.91 & 51.30 & 865.1 & 31.61 & 95.4 \\
\hline 350 . & 15.289 & 1.236 & 9.002 & 132.34 & 32.25 & 51.33 & 854.0 & 30.86 & 94.3 \\
\hline
\end{tabular}


METHANE ISOBAR AT $\mathrm{P}=55.0 \mathrm{MPa}$ (continued)

\begin{tabular}{|c|c|c|c|c|c|c|c|c|c|}
\hline $\mathrm{T}$ & $\rho$ & Z & H & $S$ & $C_{v}$ & $C_{p}$ & W & $\eta$ & $\lambda$ \\
\hline K & $\mathrm{mol} \cdot \mathrm{dm}^{-3}$ & & $\mathrm{~kJ} / \mathrm{mol}$ & $\mathrm{J} /(\mathrm{mol} \cdot \mathrm{K})$ & $\mathrm{J} /(\mathrm{mol} \cdot \mathrm{K})$ & $\mathrm{J} /(\mathrm{mol} \cdot \mathrm{K})$ & $\mathrm{m} \cdot \mathrm{s}^{-1}$ & $\mu \mathrm{Pa} \cdot \mathrm{s}$ & $\mathrm{mW} /(\mathrm{m} \cdot \mathrm{K})$ \\
\hline 360. & 14.882 & 1.235 & 9.516 & 133.78 & 32.63 & 51.38 & 844.3 & 30.20 & 93.5 \\
\hline 370 . & 14.492 & 1.234 & 10.030 & 135.19 & 33.03 & 51.44 & 835.8 & 29.62 & 92.9 \\
\hline 380. & 14.120 & 1.233 & 10.545 & 136.56 & 33.45 & 51.52 & 828.5 & 29.11 & 92.5 \\
\hline 390. & 13.764 & 1.232 & 11.060 & 137.90 & 33.89 & 51.61 & 822.3 & 28.67 & 92.3 \\
\hline 400. & 13.424 & 1.232 & 11.577 & 139.21 & 34.34 & 51.73 & 817.0 & 28.29 & 92.2 \\
\hline 410. & 13.100 & 1.232 & 12.095 & 140.49 & 34.81 & 51.87 & 812.5 & & 92.3 \\
\hline 420. & 12.790 & 1.231 & 12.614 & 141.74 & 35.30 & 52.03 & 808.8 & & 92.6 \\
\hline 430. & 12.494 & 1.231 & 13.136 & 142.97 & 35.79 & 52.21 & 805.8 & & 92.9 \\
\hline 440 & 12.212 & 1.231 & 13.659 & 144.17 & 36.29 & 52.41 & 803.5 & & 93.4 \\
\hline 450 & 11.942 & 1.231 & 14.184 & 145.35 & 36.80 & 52.62 & 801.7 & & 94.0 \\
\hline 460. & 11.684 & 1.231 & 14.711 & 146.51 & 37.31 & 52.85 & 800.4 & & 94.7 \\
\hline 470 & 11.438 & 1.231 & 15.241 & 147.65 & 37.83 & 53.10 & 799.5 & & 95.4 \\
\hline 480 & 11.202 & 1.230 & 15.773 & 148.77 & 38.35 & 53.36 & 799.0 & & 96.3 \\
\hline 490. & 10.976 & 1.230 & 16.308 & 149.87 & 38.87 & 53.63 & 799.0 & & 97.2 \\
\hline 500 & 10.759 & 1.230 & 16.846 & 150.96 & 39.39 & 53.92 & 799.2 & & 98.2 \\
\hline 520. & 10.352 & 1.229 & 17.930 & 153.09 & 40.43 & 54.52 & 800.5 & & 100.3 \\
\hline 540. & 9.977 & 1. 228 & 19.027 & 155.16 & 41.45 & 55.15 & 802.8 & & 102.7 \\
\hline 560. & 9.630 & 1.227 & 20.136 & 157.17 & 42.46 & 55.80 & 805.8 & & 105.2 \\
\hline 580. & 9.309 & 1.225 & 21.259 & 159.14 & 43.46 & 56.46 & 809.5 & & 107.9 \\
\hline 600 & 9.010 & 1.224 & 22.395 & 161.07 & 44.43 & 57.14 & 813.6 & & 110.8 \\
\hline
\end{tabular}

$\overline{\text { METHANE ISOBAR AT } \mathrm{P}=60.0 \mathrm{MPa}}$

\begin{tabular}{|c|c|c|c|c|c|c|c|c|c|}
\hline $\mathrm{T}$ & $\rho$ & Z & $\mathrm{H}$ & S & $\mathrm{C}_{\mathrm{v}}$ & $C_{p}$ & W & $\eta$ & $\lambda$ \\
\hline K & $\mathrm{mol} \cdot \mathrm{dm}^{-3}$ & & $\mathrm{~kJ} / \mathrm{mol}$ & $\mathrm{J} /(\mathrm{mol} \cdot \mathrm{K})$ & $\mathrm{J} /(\mathrm{mol} \cdot \mathrm{K})$ & $\mathrm{J} /(\mathrm{mol} \cdot \mathrm{K})$ & $\mathrm{m} \cdot \mathrm{s}^{-1}$ & $\mu \mathrm{Pa} \cdot \mathrm{s}$ & $\mathrm{mW} /(\mathrm{m} \cdot \mathrm{K})$ \\
\hline 110. & 28.871 & 2.272 & -3.145 & 72.75 & 35.79 & 51.39 & 1733.2 & & 239.3 \\
\hline 115. & 28.575 & 2.196 & -2.889 & 75.03 & 35.47 & 51.33 & 1701.8 & & 234.3 \\
\hline 120. & 28.279 & 2.127 & -2.632 & 77.22 & 35.12 & 51.23 & 1671.2 & & 229.1 \\
\hline 125. & 27.983 & 2.063 & -2.376 & 79.31 & 34.78 & 51.13 & 1641.2 & & 223.9 \\
\hline 130. & 27.688 & 2.005 & -2.121 & 81.31 & 34.45 & 51.03 & 1611.9 & & 218.7 \\
\hline 135. & 27.394 & 1.951 & -1.866 & 83.23 & 34.13 & 50.94 & 1583.2 & & 213.6 \\
\hline 140. & 27.099 & 1.902 & -1.611 & 85.09 & 33.83 & 50.86 & 1555.2 & & 208.4 \\
\hline 145. & 26.805 & 1.857 & -1.357 & 86.87 & 33.54 & 50.80 & 1527.7 & & 203.4 \\
\hline 150. & 26.511 & 1.815 & -1.103 & 88.59 & 33.27 & 50.74 & 1500.9 & & 198.4 \\
\hline 155. & 26.218 & 1.776 & -0.850 & 90.25 & 33.02 & 50.69 & 1474.7 & & 193.5 \\
\hline
\end{tabular}


METHANE ISOBAR AT $\mathrm{P}=60.0 \mathrm{MPa}$ (continued)

\begin{tabular}{|c|c|c|c|c|c|c|c|c|c|}
\hline $\mathrm{T}$ & $\rho$ & $\mathrm{Z}$ & $\mathrm{H}$ & $S$ & $C_{v}$ & $C_{p}$ & W & $\eta$ & $\lambda$ \\
\hline K & $\mathrm{mol} \cdot \mathrm{dm}^{-3}$ & & $\mathrm{~kJ} / \mathrm{mol}$ & $\mathrm{J} /(\mathrm{mol} \cdot \mathrm{K})$ & $\mathrm{J} /(\mathrm{mol} \cdot \mathrm{K})$ & $\mathrm{J} /(\mathrm{mol} \cdot \mathrm{K})$ & $\mathrm{m} \cdot \mathrm{s}^{-1}$ & $\mu \mathrm{Pa} \cdot \mathrm{s}$ & $\mathrm{mW} /(\mathrm{m} \cdot \mathrm{K})$ \\
\hline 160. & 25.924 & 1.740 & -0.597 & 91.86 & 32.78 & 50.65 & 1449.2 & & 188.7 \\
\hline 165. & 25.631 & 1.706 & -0.343 & 93.42 & 32.55 & 50.62 & 1424.2 & & 184.0 \\
\hline 170 . & 25.338 & 1.675 & -0.090 & 94.93 & 32.34 & 50.59 & 1399.9 & & 179.4 \\
\hline 175. & 25.045 & 1.646 & 0.163 & 96.40 & 32.13 & 50.57 & 1376.2 & & 175.0 \\
\hline 180. & 24.753 & 1.620 & 0.415 & 97.82 & 31.94 & 50.56 & 1353.1 & & 170.7 \\
\hline 185. & 24.461 & 1.595 & 0.668 & 99.21 & 31.77 & 50.55 & 1330.6 & & 166.5 \\
\hline 190. & 24.170 & 1.571 & 0.921 & 100.55 & 31.60 & 50.54 & 1308.6 & & 162.5 \\
\hline 195. & 23.879 & 1.550 & 1.174 & 101.87 & 31.45 & 50.53 & 1287.3 & & 158.6 \\
\hline 200 . & 23.589 & 1.530 & 1.426 & 103.15 & 31.30 & 50.53 & 1266.6 & & 154.8 \\
\hline 205. & 23.300 & 1.511 & 1.679 & 104.39 & 31.17 & 50.53 & 1246.5 & & 151.2 \\
\hline 210 . & 23.011 & 1.493 & 1.931 & 105.61 & 31.05 & 50.53 & 1226.9 & & 147.7 \\
\hline 215 . & 22.724 & 1.477 & 2.184 & 106.80 & 30.95 & 50.53 & 1208.0 & & 144.4 \\
\hline 220 . & 22.439 & 1.462 & 2.437 & 107.96 & 30.85 & 50.54 & 1189.6 & & 141.2 \\
\hline 225. & 22.154 & 1.448 & 2.689 & 109.10 & 30.77 & 50.54 & 1171.8 & & 138.1 \\
\hline 230 . & 21.872 & 1.435 & 2.942 & 110.21 & 30.70 & 50.55 & 1154.6 & & 135.2 \\
\hline 235. & 21.591 & 1.422 & 3.195 & 111.30 & 30.64 & 50.55 & 1137.9 & & 132.4 \\
\hline 240 . & 21.312 & 1.411 & 3.448 & 112.36 & 30.59 & 50.56 & 1121.8 & & 129.7 \\
\hline 245 . & 21.035 & 1.400 & 3.701 & 113.40 & 30.56 & 50.57 & 1106.3 & & 127.2 \\
\hline 250 . & 20.760 & 1.390 & 3.953 & 114.43 & 30.54 & 50.58 & 1091.3 & & 124.8 \\
\hline 255 . & 20.487 & 1.381 & 4.206 & 115.43 & 30.53 & 50.59 & 1076.9 & & 122.5 \\
\hline 260 . & 20.217 & 1.373 & 4.459 & 116.41 & 30.53 & 50.60 & 1063.0 & & 120.3 \\
\hline 265 . & 19.950 & 1.365 & 4.712 & 117.37 & 30.54 & 50.61 & 1049.7 & & 118.3 \\
\hline 270. & 19.686 & 1.358 & 4.965 & 118.32 & 30.57 & 50.62 & 1036.9 & & 116.3 \\
\hline 275 . & 19.425 & 1.351 & 5.219 & 119.25 & 30.61 & 50.64 & 1024.6 & & 114.5 \\
\hline 280 . & 19.166 & 1.345 & 5.472 & 120.16 & 30.65 & 50.65 & 1012.9 & & 112.8 \\
\hline 285. & 18.911 & 1.339 & 5.725 & 121.06 & 30.71 & 50.66 & 1001.6 & & 111.2 \\
\hline 290 . & 18.660 & 1.334 & 5.978 & 121.94 & 30.78 & 50.68 & 990.8 & & 109.7 \\
\hline 295. & 18.412 & 1.329 & 6.232 & 122.81 & 30.86 & 50.69 & 980.6 & & 108.2 \\
\hline 300 . & 18.167 & 1.324 & 6.485 & 123.66 & 30.95 & 50.71 & 970.7 & & 106.9 \\
\hline 310 . & 17.689 & 1.316 & 6.993 & 125.32 & 31.16 & 50.75 & 952.5 & & 104.6 \\
\hline 320 . & 17.227 & 1.309 & 7.500 & 126.93 & 31.41 & 50.79 & 936.0 & & 102.5 \\
\hline 330 . & 16.780 & 1.303 & 8.009 & 128.50 & 31.70 & 50.85 & 921.1 & & 100.8 \\
\hline 340 . & 16.349 & 1.298 & 8.517 & 130.02 & 32.01 & 50.91 & 907.7 & & 99.4 \\
\hline 350 . & 15.934 & 1.294 & 9.027 & 131.49 & 32.36 & 50.99 & 895.8 & & 98.2 \\
\hline 360 . & 15.536 & 1.290 & 9.537 & 132.93 & 32.73 & 51.08 & 885.2 & & 97.3 \\
\hline 370. & 15.153 & 1.287 & 10.049 & 134.33 & 33.12 & 51.19 & 875.9 & & 96.6 \\
\hline 380 . & 14.785 & 1.284 & 10.561 & 135.70 & 33.54 & 51.31 & 867.7 & & 96.1 \\
\hline 390 . & 14.433 & 1.282 & 11.075 & 137.03 & 33.98 & 51.45 & 860.5 & & 95.8 \\
\hline 400. & 14.095 & 1.280 & 11.590 & 138.34 & 34.43 & 51.61 & 854.2 & & 95.7 \\
\hline 410. & 13.771 & 1.278 & 12.107 & 139.61 & 34.90 & 51.78 & 848.9 & & 95.7 \\
\hline
\end{tabular}


METHANE ISOBAR AT $\mathrm{P}=60.0 \mathrm{MPa}$ (continued)

\begin{tabular}{|c|c|c|c|c|c|c|c|c|c|}
\hline $\mathrm{T}$ & $\rho$ & Z & $\mathrm{H}$ & $S$ & $C_{v}$ & $C_{p}$ & W & $\eta$ & $\lambda$ \\
\hline K & $\mathrm{mol} \cdot \mathrm{dm}^{-3}$ & & $\mathrm{~kJ} / \mathrm{mol}$ & $\mathrm{J} /(\mathrm{mol} \cdot \mathrm{K})$ & $\mathrm{J} /(\mathrm{mol} \cdot \mathrm{K})$ & $\mathrm{J} /(\mathrm{mol} \cdot \mathrm{K})$ & $\mathrm{m} \cdot \mathrm{s}^{-1}$ & $\mu \mathrm{Pa} \cdot \mathrm{s}$ & $\mathrm{mW} /(\mathrm{m} \cdot \mathrm{K})$ \\
\hline 420 & 13.462 & 1.276 & 12.626 & 140.86 & 35.38 & 51.97 & 844.3 & & 95.8 \\
\hline 430. & 13.165 & 1.275 & 13.147 & 142.09 & 35.87 & 52.18 & 840.4 & & 96.1 \\
\hline 440 & 12.880 & 1.273 & 13.670 & 143.29 & 36.37 & 52.40 & 837.2 & & 96.5 \\
\hline 450 & 12.608 & 1.272 & 14.195 & 144.47 & 36.87 & 52.64 & 834.6 & & 97.0 \\
\hline 460 & 12.347 & 1.271 & 14.722 & 145.63 & 37.38 & 52.89 & 832.5 & & 97.6 \\
\hline 470 & 12.096 & 1.269 & 15.253 & 146.77 & 37.90 & 53.16 & 830.9 & & 98.3 \\
\hline 480. & 11.856 & 1.268 & 15.786 & 147.89 & 38.41 & 53.43 & 829.7 & & 99.1 \\
\hline 490 & 11.625 & 1.267 & 16.321 & 149.00 & 38.93 & 53.72 & 828.9 & & 100.0 \\
\hline 500 & 11.404 & 1.266 & 16.860 & 150.09 & 39.45 & 54.02 & 828.5 & & 100.9 \\
\hline 520. & 10.986 & 1.263 & 17.946 & 152.22 & 40.48 & 54.63 & 828.6 & & 102.9 \\
\hline 540. & 10.600 & 1.261 & 19.045 & 154.29 & 41.50 & 55.28 & 829.8 & & 105.2 \\
\hline 560. & 10.242 & 1.258 & 20.158 & 156.31 & 42.51 & 55.93 & 831.8 & & 107.6 \\
\hline 580. & 9.909 & 1.256 & 21.283 & 158.29 & 43.50 & 56.61 & 834.5 & & 110.2 \\
\hline 600 & 9.598 & 1.253 & 22.422 & 160.22 & 44.47 & 57.28 & 837.9 & & 112.9 \\
\hline
\end{tabular}

METHANE ISOBAR AT P $=65.0 \mathrm{MPa}$

\begin{tabular}{|c|c|c|c|c|c|c|c|c|c|}
\hline $\mathrm{T}$ & $\rho$ & $z$ & $\mathrm{H}$ & S & $C_{v}$ & $C_{p}$ & W & $\eta$ & $\lambda$ \\
\hline K & $\mathrm{mol} \cdot \mathrm{dm}^{-}$ & & $\mathrm{kJ} / \mathrm{mol}$ & $\mathrm{J} /(\mathrm{mol} \cdot \mathrm{K})$ & $\mathrm{J} /(\mathrm{mol} \cdot \mathrm{K})$ & $\mathrm{J} /(\mathrm{mol} \cdot \mathrm{K})$ & $\mathrm{m} \cdot \mathrm{s}^{-1}$ & $\mu \mathrm{Pa} \cdot \mathrm{s}$ & $\mathrm{mW} /(\mathrm{m} \cdot \mathrm{r}$ \\
\hline 110. & 29.018 & 2.449 & -3.011 & 72.40 & 35.96 & 51.25 & 1756.1 & & 243.0 \\
\hline 115. & 28.728 & 2.366 & -2.755 & 74.68 & 35.64 & 51.18 & 1725.5 & & 238.1 \\
\hline 120 . & 28.439 & 2.291 & -2.499 & 76.85 & 35.30 & 51.07 & 1695.6 & & 233.1 \\
\hline 125. & 28.150 & 2.222 & -2.244 & 78.94 & 34.96 & 50.96 & 1666.4 & & 228.0 \\
\hline 130. & 27.863 & 2.158 & -1.990 & 80.93 & 34.63 & 50.84 & 1637.9 & & 222.9 \\
\hline 35. & 27.575 & 2.100 & -1.736 & 82.85 & 34.31 & 50.74 & 1610.0 & & 217.8 \\
\hline 140 . & 27.289 & 2.046 & -1.482 & 84.69 & 34.01 & 50.64 & 1582.7 & & 212.7 \\
\hline 145. & 27.003 & 1.997 & -1.229 & 86.47 & 33.72 & 50.56 & 1556.1 & & 207.7 \\
\hline 150 . & 26.717 & 1.951 & -0.977 & 88.18 & 33.45 & 50.49 & 1530.1 & & 202.8 \\
\hline 155. & 26.432 & 1.908 & -0.725 & 89.84 & 33.19 & 50.42 & 1504.8 & & 197.9 \\
\hline 60. & 26.147 & 1.869 & -0.473 & 91.44 & 32.95 & 50.36 & 1480.0 & & 193.2 \\
\hline 165. & 25.863 & 1.832 & -0.221 & 92.99 & 32.72 & 50.32 & 1455.9 & & 188.5 \\
\hline 170 . & 25.580 & 1.798 & 0.031 & 94.49 & 32.51 & 50.27 & 1432.4 & & 184.0 \\
\hline 175. & 25.296 & 1.766 & 0.282 & 95.94 & 32.30 & 50.24 & 1409.5 & & 179.6 \\
\hline 180. & 25.014 & 1.736 & 0.533 & 97.36 & 32.11 & 50.20 & 1387.1 & & 175.3 \\
\hline 185. & 24.732 & 1.709 & 0.784 & 98.73 & 31.93 & 50.18 & 1365.4 & & 171.2 \\
\hline 90. & 24.451 & 1.683 & 1.035 & 100.07 & 31.76 & 50.15 & 1344.2 & & 167.2 \\
\hline 195. & 24.171 & 1.659 & 1.285 & 101.37 & 31.61 & 50.13 & 1323.6 & & 163.3 \\
\hline 200. & 23.891 & 1.636 & 1.536 & 102.64 & 31.46 & 50.11 & 1303.5 & & 159.6 \\
\hline 205. & 23.613 & 1.615 & 1.787 & 103.88 & 31.33 & 50.10 & 1284.0 & & 155.9 \\
\hline
\end{tabular}


METHANE ISOBAR AT $\mathrm{P}=65.0 \mathrm{MPa}$ (continued)

\begin{tabular}{|c|c|c|c|c|c|c|c|c|c|}
\hline $\mathrm{T}$ & $\rho$ & $\mathrm{z}$ & $\mathrm{H}$ & s & $\mathrm{C}_{\mathrm{v}}$ & $C_{p}$ & W & $\eta$ & $\lambda$ \\
\hline K & $\mathrm{mol} \cdot \mathrm{dm}^{-3}$ & & $\mathrm{~kJ} / \mathrm{mol}$ & $\mathrm{J} /(\mathrm{mol} \cdot \mathrm{K})$ & $\mathrm{J} /(\mathrm{mol} \cdot \mathrm{K})$ & $\mathrm{J} /(\mathrm{mol} \cdot \mathrm{K})$ & $\mathrm{m} \cdot \mathrm{s}^{-1}$ & $\mu \mathrm{Pa} \cdot \mathrm{s}$ & $\mathrm{mW} /(\mathrm{m} \cdot \mathrm{K})$ \\
\hline 210 . & 23.336 & 1.595 & 2.037 & 105.09 & 31.21 & 50.09 & 1265.1 & & 152.5 \\
\hline 215 . & 23.060 & 1.577 & 2.287 & 106.27 & 31.10 & 50.08 & 1246.7 & & 149.1 \\
\hline 220 . & 22.785 & 1.560 & 2.538 & 107.42 & 31.00 & 50.07 & 1228.9 & & 145.9 \\
\hline 225 . & 22.512 & 1.543 & 2.788 & 108.54 & 30.92 & 50.06 & 1211.6 & & 142.9 \\
\hline 230 . & 22.241 & 1.528 & 3.038 & 109.64 & 30.84 & 50.06 & 1194.9 & & 139.9 \\
\hline 235. & 21.971 & 1.514 & 3.289 & 110.72 & 30.78 & 50.06 & 1178.6 & & 137.1 \\
\hline 240 . & 21.703 & 1.501 & 3.539 & 111.77 & 30.73 & 50.06 & 1162.9 & & 134.4 \\
\hline 245 . & 21.438 & 1.488 & 3.789 & 112.80 & 30.70 & 50.06 & 1147.8 & & 131.9 \\
\hline 250 . & 21.174 & 1.477 & 4.040 & 113.82 & 30.67 & 50.07 & 1133.1 & & 129.4 \\
\hline 255. & 20.913 & 1.466 & 4.290 & 114.81 & 30.66 & 50.07 & 1119.0 & & 127.1 \\
\hline 260 . & 20.654 & 1.456 & 4.540 & 115.78 & 30.66 & 50.08 & 1105.3 & & 124.9 \\
\hline 265. & 20.397 & 1.446 & 4.791 & 116.73 & 30.67 & 50.09 & 1092.2 & & 122.9 \\
\hline 270 . & 20.144 & 1.437 & 5.041 & 117.67 & 30.69 & 50.11 & 1079.5 & & 120.9 \\
\hline 275 . & 19.893 & 1.429 & 5.292 & 118.59 & 30.73 & 50.12 & 1067.3 & & 119.0 \\
\hline 280 . & 19.645 & 1.421 & 5.542 & 119.49 & 30.77 & 50.14 & 1055.6 & & 117.3 \\
\hline 285. & 19.399 & 1.414 & 5.793 & 120.38 & 30.83 & 50.16 & 1044.4 & & 115.6 \\
\hline 290. & 19.157 & 1.407 & 6.044 & 121.25 & 30.90 & 50.19 & 1033.6 & & 114.1 \\
\hline 295. & 18.918 & 1.401 & 6.295 & 122.11 & 30.98 & 50.21 & 1023.2 & & 112.6 \\
\hline 300 . & 18.683 & 1.395 & 6.546 & 122.96 & 31.07 & 50.24 & 1013.3 & & 111.2 \\
\hline 310. & 18.222 & 1.384 & 7.049 & 124.60 & 31.27 & 50.30 & 994.8 & & 108.8 \\
\hline 320 . & 17.774 & 1.374 & 7.552 & 126.20 & 31.52 & 50.38 & 977.9 & & 106.7 \\
\hline 330. & 17.341 & 1.366 & 8.056 & 127.75 & 31.80 & 50.46 & 962.5 & & 104.8 \\
\hline 340 . & 16.922 & 1.359 & 8.562 & 129.26 & 32.11 & 50.56 & 948.6 & & 103.3 \\
\hline 350 . & 16.517 & 1.352 & 9.068 & 130.73 & 32.45 & 50.68 & 936.0 & & 102.1 \\
\hline 360. & 16.127 & 1.347 & 9.575 & 132.16 & 32.82 & 50.81 & 924.7 & & 101.0 \\
\hline 370 . & 15.751 & 1.341 & 10.084 & 133.55 & 33.21 & 50.95 & 914.6 & & 100.2 \\
\hline 380 . & 15.390 & 1.337 & 10.594 & 134.91 & 33.63 & 51.11 & 905.6 & & 99.6 \\
\hline 390. & 15.042 & 1.333 & 11.106 & 136.24 & 34.06 & 51.28 & 897.7 & & 99.2 \\
\hline 400. & 14.707 & 1.329 & 11.620 & 137.54 & 34.51 & 51.47 & 890.6 & & 99.0 \\
\hline 410. & 14.386 & 1.325 & 12.136 & 138.82 & 34.97 & 51.67 & 884.5 & & 98.9 \\
\hline 420. & 14.077 & 1.322 & 12.653 & 140.06 & 35.45 & 51.89 & 879.1 & & 99.0 \\
\hline 430. & 13.781 & 1.319 & 13.173 & 141.29 & 35.94 & 52.12 & 874.5 & & 99.2 \\
\hline 440. & 13.496 & 1.317 & 13.696 & 142.49 & 36.44 & 52.37 & 870.5 & & 99.5 \\
\hline 450. & 13.222 & 1.314 & 14.221 & 143.67 & 36.94 & 52.63 & 867.1 & & 100.0 \\
\hline 460. & 12.959 & 1.311 & 14.748 & 144.83 & 37.45 & 52.90 & 864.3 & & 100.5 \\
\hline 470. & 12.706 & 1.309 & 15.279 & 145.97 & 37.96 & 53.18 & 862.0 & & 101.1 \\
\hline 480. & 12.463 & 1.307 & 15.812 & 147.09 & 38.47 & 53.47 & 860.2 & & 101.8 \\
\hline 490. & 12.229 & 1.305 & 16.348 & 148.20 & 38.99 & 53.77 & 858.8 & & 102.6 \\
\hline 500. & 12.004 & 1.302 & 16.887 & 149.29 & 39.50 & 54.08 & 857.7 & & 103.5 \\
\hline 520. & 11.579 & 1.298 & 17.975 & 151.42 & 40.53 & 54.71 & 856.7 & & 105.4 \\
\hline
\end{tabular}


METHANE ISOBAR AT $\mathrm{P}=65.0 \mathrm{MPa}$ (continued)

$\begin{array}{cccccccccc}\mathrm{T} & \rho & \mathrm{Z} & \mathrm{H} & \mathrm{S} & \mathrm{C}_{\mathrm{v}} & \mathrm{C}_{\mathrm{p}} & \mathrm{W} & \eta & \lambda \\ \mathrm{K} & \mathrm{mol} \cdot \mathrm{dm}^{-3} & & \mathrm{~kJ} / \mathrm{mol} & \mathrm{J} /(\mathrm{mol} \cdot \mathrm{K}) & \mathrm{J} /(\mathrm{mol} \cdot \mathrm{K}) & \mathrm{J} /(\mathrm{mol} \cdot \mathrm{K}) & \mathrm{m} \cdot \mathrm{s}^{-1} & \mu \mathrm{Pa} \cdot \mathrm{s} & \mathrm{mW} /(\mathrm{m} \cdot \mathrm{K}) \\ & & & & & & & & & \\ 540 . & 11.184 & 1.294 & 19.076 & 153.50 & 41.55 & 55.37 & 856.8 & 107.6 \\ 560 . & 10.817 & 1.291 & 20.190 & 155.52 & 42.56 & 56.04 & 857.9 & & 109.9 \\ 580 . & 10.474 & 1.287 & 21.318 & 157.50 & 43.54 & 56.72 & 859.7 & 112.4 \\ 600 . & 10.154 & 1.283 & 22.459 & 159.44 & 44.51 & 57.40 & 862.3 & 115.1\end{array}$

$\overline{\text { METHANE ISOBAR AT } P=70.0 \mathrm{MPa}}$

\begin{tabular}{|c|c|c|c|c|c|c|c|c|c|}
\hline $\mathrm{T}$ & $\rho$ & $\mathrm{Z}$ & $\mathrm{H}$ & $\mathrm{S}$ & $C_{v}$ & $C_{p}$ & W & $\eta$ & $\lambda$ \\
\hline K & $\mathrm{mol} \cdot \mathrm{dm}^{-}$ & & $\mathrm{kJ} / \mathrm{mol}$ & $\mathrm{J} /(\mathrm{mol} \cdot \mathrm{K})$ & $\mathrm{J} /(\mathrm{mol} \cdot \mathrm{K})$ & $\mathrm{J} /(\mathrm{mol} \cdot \mathrm{K})$ & $\mathrm{m} \cdot \mathrm{s}^{-1}$ & $\mu \mathrm{Pa} \cdot \mathrm{s}$ & $\mathrm{mW} /(\mathrm{m} \cdot \mathrm{K})$ \\
\hline 110. & 29.159 & 2.625 & -2.877 & 72.06 & 36.13 & 51.13 & 1778.4 & & 246.7 \\
\hline 115. & 28.876 & 2.535 & -2.621 & 74.33 & 35.81 & 51.05 & 1748.4 & & 241.9 \\
\hline 120. & 28.593 & 2.454 & -2.366 & 76.50 & 35.47 & 50.93 & 1719.2 & & 237.0 \\
\hline 125. & 28.311 & 2.379 & -2.112 & 78.58 & 35.13 & 50.80 & 1690.7 & & 231.9 \\
\hline 130. & 28.030 & 2.310 & -1.858 & 80.57 & 34.80 & 50.68 & 1662.9 & & 226.9 \\
\hline 135. & 27.750 & 2.247 & -1.605 & 82.48 & 34.48 & 50.56 & 1635.8 & & 221.9 \\
\hline 140. & 27.470 & 2.189 & -1.353 & 84.32 & 34.18 & 50.45 & 1609.3 & & 216.9 \\
\hline 145. & 27.192 & 2.135 & -1.101 & 86.09 & 33.89 & 50.35 & 1583.4 & & 211.9 \\
\hline 150. & 26.914 & 2.085 & -0.849 & 87.79 & 33.62 & 50.26 & 1558.2 & & 207.0 \\
\hline 155. & 26.636 & 2.039 & -0.598 & 89.44 & 33.36 & 50.18 & 1533.6 & & 202.2 \\
\hline 160. & 26.360 & 1.996 & -0.347 & 91.03 & 33.12 & 50.11 & 1509.6 & & 197.5 \\
\hline 165. & 26.084 & 1.956 & -0.097 & 92.57 & 32.89 & 50.05 & 1486.2 & & 192.9 \\
\hline 170. & 25.808 & 1.919 & 0.153 & 94.06 & 32.67 & 49.99 & 1463.4 & & 188.5 \\
\hline 175. & 25.534 & 1.884 & 0.403 & 95.51 & 32.46 & 49.94 & 1441.2 & & 184.1 \\
\hline 180. & 25.260 & 1.852 & 0.653 & 96.92 & 32.27 & 49.90 & 1419.6 & & 179.8 \\
\hline 185. & 24.987 & 1.821 & 0.902 & 98.29 & 32.09 & 49.86 & 1398.5 & & 175.7 \\
\hline 190. & 24.715 & 1.793 & 1.151 & 99.61 & 31.92 & 49.82 & 1378.0 & & 171.7 \\
\hline 195. & 24.444 & 1.766 & 1.400 & 100.91 & 31.76 & 49.79 & 1358.0 & & 167.9 \\
\hline 200. & 24.174 & 1.741 & 1.649 & 102.17 & 31.61 & 49.76 & 1338.6 & & 164.1 \\
\hline 205. & 23.905 & 1.718 & 1.898 & 103.40 & 31.48 & 49.73 & 1319.7 & & 160.5 \\
\hline 210. & 23.638 & 1.696 & 2.146 & 104.59 & 31.35 & 49.71 & 1301.4 & & 157.1 \\
\hline 215 . & 23.372 & 1.675 & 2.395 & 105.76 & 31.24 & 49.69 & 1283.5 & & 153.7 \\
\hline 220. & 23.107 & 1.656 & 2.643 & 106.91 & 31.14 & 49.67 & 1266.2 & & 150.5 \\
\hline 225 . & 22.843 & 1.638 & 2.892 & 108.02 & 31.05 & 49.65 & 1249.4 & & 147.5 \\
\hline 230 . & 22.582 & 1.621 & 3.140 & 109.11 & 30.98 & 49.64 & 1233.0 & & 144.5 \\
\hline 235. & 22.322 & 1.605 & 3.388 & 110.18 & 30.91 & 49.64 & 1217.2 & & 141.7 \\
\hline 240. & 22.064 & 1.590 & 3.636 & 111.23 & 30.86 & 49.63 & 1201.9 & & 139.0 \\
\hline 245. & 21.808 & 1.576 & 3.884 & 112.25 & 30.82 & 49.63 & 1187.0 & & 136.4 \\
\hline 250. & 21.554 & 1.562 & 4.132 & 113.25 & 30.80 & 49.63 & 1172.6 & & 134.0 \\
\hline 255. & 21.303 & 1.550 & 4.381 & 114.23 & 30.78 & 49.64 & 1158.7 & & 131.6 \\
\hline
\end{tabular}


METHANE ISOBAR AT $\mathrm{P}=70.0 \mathrm{MPa}$ (continued)

\begin{tabular}{|c|c|c|c|c|c|c|c|c|c|}
\hline $\mathrm{T}$ & $\rho$ & $\mathrm{z}$ & $\mathrm{H}$ & $S$ & $\mathrm{C}_{\mathrm{v}}$ & $C_{p}$ & W & $\eta$ & $\lambda$ \\
\hline K & $\mathrm{mol} \cdot \mathrm{dm}^{-3}$ & & $\mathrm{~kJ} / \mathrm{mol}$ & $\mathrm{J} /(\mathrm{mol} \cdot \mathrm{K})$ & $\mathrm{J} /(\mathrm{mol} \cdot \mathrm{K})$ & $\mathrm{J} /(\mathrm{mol} \cdot \mathrm{K})$ & $m \cdot s^{-1}$ & $\mu \mathrm{Pa} \cdot \mathrm{s}$ & $\mathrm{mW} /(\mathrm{m} \cdot \mathrm{K})$ \\
\hline 260 . & 21.053 & 1.538 & 4.629 & 115.20 & 30.78 & 49.64 & 1145.3 & & 129.4 \\
\hline 265 . & 20.806 & 1.527 & 4.877 & 116.14 & 30.79 & 49.66 & 1132.3 & & 127.3 \\
\hline 270 . & 20.562 & 1.516 & 5.125 & 117.07 & 30.81 & 49.67 & 1119.8 & & 125.3 \\
\hline 275 . & 20.320 & 1.507 & 5.374 & 117.98 & 30.84 & 49.69 & 1107.7 & & 123.4 \\
\hline 280 . & 20.081 & 1.497 & 5.622 & 118.88 & 30.89 & 49.71 & 1096.1 & & 121.6 \\
\hline 285. & 19.844 & 1.489 & 5.871 & 119.76 & 30.94 & 49.74 & 1084.9 & & 119.9 \\
\hline 290. & 19.610 & 1.480 & 6.120 & 120.62 & 31.01 & 49.76 & 1074.1 & & 118.3 \\
\hline 295. & 19.380 & 1.473 & 6.369 & 121.48 & 31.09 & 49.80 & 1063.8 & & 116.8 \\
\hline 300 . & 19.152 & 1.465 & 6.618 & 122.31 & 31.17 & 49.83 & 1053.8 & & 115.4 \\
\hline 310 . & 18.706 & 1.452 & 7.116 & 123.95 & 31.38 & 49.92 & 1035.1 & & 112.9 \\
\hline 320. & 18.272 & 1.440 & 7.616 & 125.53 & 31.62 & 50.01 & 1017.9 & & 110.6 \\
\hline 330. & 17.851 & 1.429 & 8.117 & 127.08 & 31.89 & 50.12 & 1002.1 & & 108.7 \\
\hline 340 . & 17.443 & 1.420 & 8.619 & 128.57 & 32.20 & 50.25 & 987.7 & & 107.1 \\
\hline 350 . & 17.048 & 1.411 & 9.122 & 130.03 & 32.54 & 50.39 & 974.7 & & 105.8 \\
\hline 360 . & 16.667 & 1.403 & 9.626 & 131.45 & 32.91 & 50.55 & 962.8 & & 104.6 \\
\hline 370 . & 16.298 & 1.396 & 10.133 & 132.84 & 33.29 & 50.72 & 952.1 & & 103.8 \\
\hline 380 . & 15.943 & 1.390 & 10.641 & 134.20 & 33.71 & 50.91 & 942.5 & & 103.1 \\
\hline 390. & 15.600 & 1.384 & 11.151 & 135.52 & 34.14 & 51.11 & 933.8 & & 102.6 \\
\hline 400. & 15.269 & 1.378 & 11.663 & 136.82 & 34.58 & 51.32 & 926.1 & & 102.3 \\
\hline 410. & 14.951 & 1.373 & 12.177 & 138.09 & 35.05 & 51.55 & 919.2 & & 102.1 \\
\hline 420. & 14.644 & 1.369 & 12.694 & 139.33 & 35.52 & 51.79 & 913.2 & & 102.1 \\
\hline 430. & 14.349 & 1.364 & 13.213 & 140.55 & 36.01 & 52.05 & 907.9 & & 102.2 \\
\hline 440. & 14.065 & 1.360 & 13.735 & 141.75 & 36.50 & 52.32 & 903.2 & & 102.5 \\
\hline 450. & 13.791 & 1.357 & 14.260 & 142.93 & 37.00 & 52.59 & 899.2 & & 102.8 \\
\hline 460. & 13.527 & 1.353 & 14.787 & 144.09 & 37.51 & 52.88 & 895.7 & & 103.3 \\
\hline 470. & 13.273 & 1.350 & 15.317 & 145.23 & 38.02 & 53.18 & 892.8 & & 103.9 \\
\hline 480. & 13.028 & 1.346 & 15.851 & 146.36 & 38.53 & 53.48 & 890.4 & & 104.5 \\
\hline 490. & 12.792 & 1.343 & 16.387 & 147.46 & 39.04 & 53.80 & 888.4 & & 105.3 \\
\hline 500. & 12.565 & 1.340 & 16.927 & 148.55 & 39.56 & 54.12 & 886.8 & & 106.1 \\
\hline 520. & 12.134 & 1.334 & 18.015 & 150.69 & 40.58 & 54.77 & 884.6 & & 107.9 \\
\hline 540. & 11.733 & 1.329 & 19.117 & 152.77 & 41.60 & 55.44 & 883.8 & & 109.9 \\
\hline 560. & 11.358 & 1.324 & 20.233 & 154.79 & 42.60 & 56.12 & 883.9 & & 112.2 \\
\hline 580. & 11.008 & 1.319 & 21.362 & 156.78 & 43.58 & 56.81 & 885.0 & & 114.6 \\
\hline 600. & 10.680 & 1.314 & 22.506 & 158.71 & 44.54 & 57.50 & 886.7 & & 117.2 \\
\hline
\end{tabular}


METHANE ISOBAR AT $P=75.0 \mathrm{MPa}$

\begin{tabular}{|c|c|c|c|c|c|c|c|c|c|}
\hline $\mathrm{T}$ & $\rho$ & Z & $\mathrm{H}$ & $S$ & $C_{v}$ & $C_{p}$ & W & $\eta$ & $\lambda$ \\
\hline K & $\mathrm{mol} \cdot \mathrm{dm}^{-3}$ & & $\mathrm{~kJ} / \mathrm{mol}$ & $\mathrm{J} /(\mathrm{mol} \cdot \mathrm{K})$ & $\mathrm{J} /(\mathrm{mol} \cdot \mathrm{K})$ & $\mathrm{J} /(\mathrm{mol} \cdot \mathrm{K})$ & $\mathrm{m} \cdot \mathrm{s}^{-1}$ & $\mu \mathrm{Pa} \cdot \mathrm{s}$ & $\mathrm{mW} /(\mathrm{m} \cdot \mathrm{K})$ \\
\hline 110. & 29.297 & 2.799 & -2.742 & 71.73 & 36.29 & 51.02 & 1799.9 & & 250.4 \\
\hline 115. & 29.019 & 2.703 & -2.487 & 74.00 & 35.98 & 50.93 & 1770.6 & & 245.6 \\
\hline 120. & 28.742 & 2.615 & -2.233 & 76.16 & 35.64 & 50.80 & 1742.0 & & 240.8 \\
\hline 125. & 28.466 & 2.535 & -1.979 & 78.23 & 35.30 & 50.66 & 1714.2 & & 235.8 \\
\hline 130. & 28.191 & 2.461 & -1.726 & 80.22 & 34.97 & 50.53 & 1687.1 & & 230.9 \\
\hline 135. & 27.917 & 2.393 & -1.474 & 82.12 & 34.65 & 50.40 & 1660.7 & & 225.9 \\
\hline 140. & 27.644 & 2.331 & -1.222 & 83.95 & 34.35 & 50.27 & 1634.9 & & 220.9 \\
\hline 145. & 27.372 & 2.273 & -0.971 & 85.72 & 34.06 & 50.17 & 1609.7 & & 216.1 \\
\hline 150. & 27.101 & 2.219 & -0.720 & 87.41 & 33.78 & 50.07 & 1585.2 & & 211.2 \\
\hline 155. & 26.831 & 2.169 & -0.470 & 89.05 & 33.52 & 49.97 & 1561.3 & & 206.5 \\
\hline 160. & 26.562 & 2.123 & -0.221 & 90.64 & 33.28 & 49.89 & 1538.0 & & 201.8 \\
\hline 165. & 26.293 & 2.079 & 0.029 & 92.17 & 33.05 & 49.82 & 1515.3 & & 197.3 \\
\hline 170. & 26.025 & 2.039 & 0.278 & 93.66 & 32.82 & 49.75 & 1493.2 & & 192.8 \\
\hline 175. & 25.759 & 2.001 & 0.526 & 95.10 & 32.62 & 49.69 & 1471.7 & & 188.5 \\
\hline 180. & 25.493 & 1.966 & 0.774 & 96.50 & 32.42 & 49.63 & 1450.7 & & 184.2 \\
\hline 185. & 25.228 & 1.933 & 1.022 & 97.86 & 32.24 & 49.58 & 1430.3 & & 180.1 \\
\hline 190. & 24.964 & 1.902 & 1.270 & 99.18 & 32.06 & 49.53 & 1410.3 & & 176.2 \\
\hline 195. & 24.701 & 1.873 & 1.518 & 100.47 & 31.90 & 49.49 & 1391.0 & & 172.3 \\
\hline 200 . & 24.440 & 1.845 & 1.765 & 101.72 & 31.75 & 49.45 & 1372.1 & & 168.6 \\
\hline 205. & 24.179 & 1.820 & 2.012 & 102.94 & 31.61 & 49.41 & 1353.8 & & 165.0 \\
\hline 210 . & 23.920 & 1.796 & 2.259 & 104.13 & 31.49 & 49.38 & 1335.9 & & 161.5 \\
\hline 215. & 23.663 & 1.773 & 2.506 & 105.29 & 31.37 & 49.35 & 1318.5 & & 158.2 \\
\hline 220 . & 23.407 & 1.752 & 2.753 & 106.43 & 31.27 & 49.32 & 1301.7 & & 155.0 \\
\hline 225. & 23.152 & 1.732 & 2.999 & 107.53 & 31.18 & 49.30 & 1285.3 & & 151.9 \\
\hline 230 . & 22.899 & 1.713 & 3.246 & 108.62 & 31.11 & 49.29 & 1269.3 & & 149.0 \\
\hline 235. & 22.648 & 1.695 & 3.492 & 109.68 & 31.04 & 49.27 & 1253.9 & & 146.1 \\
\hline 240 . & 22.399 & 1.678 & 3.738 & 110.71 & 30.99 & 49.26 & 1238.9 & & 143.4 \\
\hline 245 . & 22.151 & 1.662 & 3.985 & 111.73 & 30.95 & 49.26 & 1224.3 & & 140.8 \\
\hline 250 . & 21.906 & 1.647 & 4.231 & 112.73 & 30.92 & 49.26 & 1210.2 & & 138.3 \\
\hline 255. & 21.663 & 1.633 & 4.477 & 113.70 & 30.90 & 49.26 & 1196.6 & & 136.0 \\
\hline 260. & 21.422 & 1.620 & 4.724 & 114.66 & 30.90 & 49.27 & 1183.4 & & 133.7 \\
\hline 265. & 21.183 & 1.607 & 4.970 & 115.60 & 30.90 & 49.28 & 1170.6 & & 131.6 \\
\hline 270 . & 20.947 & 1.595 & 5.216 & 116.52 & 30.92 & 49.29 & 1158.2 & & 129.6 \\
\hline 275. & 20.713 & 1.584 & 5.463 & 117.42 & 30.95 & 49.32 & 1146.2 & & 127.6 \\
\hline 280. & 20.481 & 1.573 & 5.710 & 118.31 & 31.00 & 49.34 & 1134.7 & & 125.8 \\
\hline 285. & 20.253 & 1.563 & 5.956 & 119.18 & 31.05 & 49.37 & 1123.5 & & 124.1 \\
\hline 290. & 20.027 & 1.553 & 6.203 & 120.04 & 31.11 & 49.40 & 1112.8 & & 122.4 \\
\hline 295. & 19.803 & 1.544 & 6.450 & 120.89 & 31.19 & 49.44 & 1102.4 & & 120.9 \\
\hline 300 . & 19.582 & 1.535 & 6.698 & 121.72 & 31.27 & 49.48 & 1092.4 & & 119.5 \\
\hline 310 . & 19.149 & 1.520 & 7.193 & 123.34 & 31.47 & 49.58 & 1073.6 & & 116.8 \\
\hline
\end{tabular}


METHANE ISOBAR AT $\mathrm{P}=75.0 \mathrm{MPa}$ (continued)

\begin{tabular}{|c|c|c|c|c|c|c|c|c|c|}
\hline $\mathrm{T}$ & $\rho$ & Z & $\mathrm{H}$ & S & $C_{v}$ & $C_{p}$ & W & $\eta$ & $\lambda$ \\
\hline K & $\mathrm{mol} \cdot \mathrm{dm}^{-3}$ & & $\mathrm{~kJ} / \mathrm{mol}$ & $\mathrm{J} /(\mathrm{mol} \cdot \mathrm{K})$ & $\mathrm{J} /(\mathrm{mol} \cdot \mathrm{K})$ & $\mathrm{J} /(\mathrm{mol} \cdot \mathrm{K})$ & $\mathrm{m} \cdot \mathrm{s}^{-1}$ & $\mu \mathrm{Pa} \cdot \mathrm{s}$ & $\mathrm{mW} /(\mathrm{m} \cdot \mathrm{K})$ \\
\hline 320 . & 18.728 & 1.505 & 7.689 & 124.92 & 31.71 & 49.69 & 1056.2 & & 114.5 \\
\hline 330. & 18.319 & 1.492 & 8.187 & 126.45 & 31.98 & 49.82 & 1040.1 & & 112.5 \\
\hline 340 . & 17.921 & 1.480 & 8.686 & 127.94 & 32.29 & 49.97 & 1025.4 & & 110.8 \\
\hline 350. & 17.536 & 1.470 & 9.186 & 129.39 & 32.62 & 50.13 & 1011.9 & & 109.4 \\
\hline 360 . & 17.162 & 1.460 & 9.688 & 130.81 & 32.99 & 50.31 & 999.6 & & 108.2 \\
\hline 370. & 16.801 & 1.451 & 10.193 & 132.19 & 33.37 & 50.51 & 988.3 & & 107.2 \\
\hline 380. & 16.452 & 1.443 & 10.699 & 133.54 & 33.78 & 50.72 & 978.1 & & 106.4 \\
\hline 390. & 16.114 & 1.435 & 11.207 & 134.86 & 34.21 & 50.94 & 968.9 & & 105.8 \\
\hline 400. & 15.788 & 1.428 & 11.718 & 136.15 & 34.65 & 51.18 & 960.6 & & 105.4 \\
\hline 410. & 15.473 & 1.422 & 12.231 & 137.42 & 35.11 & 51.43 & 953.2 & & 105.2 \\
\hline 420. & 15.169 & 1.416 & 12.746 & 138.66 & 35.59 & 51.69 & 946.5 & & 105.1 \\
\hline 430. & 14.876 & 1.410 & 13.264 & 139.88 & 36.07 & 51.97 & 940.6 & & 105.2 \\
\hline 440. & 14.593 & 1.405 & 13.786 & 141.08 & 36.56 & 52.25 & 935.3 & & 105.3 \\
\hline 450. & 14.319 & 1.400 & 14.309 & 142.25 & 37.06 & 52.54 & 930.7 & & 105.6 \\
\hline 460. & 14.056 & 1.395 & 14.836 & 143.41 & 37.56 & 52.85 & 926.7 & & 106.1 \\
\hline 470. & 13.801 & 1.391 & 15.366 & 144.55 & 38.07 & 53.16 & 923.2 & & 106.6 \\
\hline 480. & 13.556 & 1.386 & 15.900 & 145.67 & 38.58 & 53.48 & 920.2 & & 107.2 \\
\hline 490. & 13.319 & 1.382 & 16.436 & 146.78 & 39.09 & 53.80 & 917.6 & & 107.8 \\
\hline 500. & 13.090 & 1.378 & 16.976 & 147.87 & 39.60 & 54.13 & 915.5 & & 108.6 \\
\hline 520. & 12.655 & 1.371 & 18.065 & 150.01 & 40.62 & 54.81 & 912.4 & & 110.3 \\
\hline 540. & 12.249 & 1.364 & 19.168 & 152.09 & 41.64 & 55.49 & 910.6 & & 112.3 \\
\hline 560. & 11.869 & 1.357 & 20.285 & 154.12 & 42.64 & 56.19 & 909.9 & & 114.4 \\
\hline 580. & 11.513 & 1.351 & 21.415 & 156.10 & 43.62 & 56.88 & 910.1 & & 116.8 \\
\hline 600. & 11.178 & 1.345 & 22.560 & 158.04 & 44.58 & 57.58 & 911.1 & & 119.2 \\
\hline
\end{tabular}

METHANE ISOBAR AT $\mathrm{P}=80.0 \mathrm{MPa}$

$\begin{array}{cccccccccc}\mathrm{T} & \rho & \mathrm{Z} & \mathrm{H} & \mathrm{S} & \mathrm{C}_{\mathrm{v}} & \mathrm{C}_{\mathrm{p}} & \mathrm{W} & \eta & \lambda \\ \mathrm{K} & \mathrm{mol} \cdot \mathrm{dm}^{-3} & & \mathrm{~kJ} / \mathrm{mol} & \mathrm{J} /(\mathrm{mol} \cdot \mathrm{K}) & \mathrm{J} /(\mathrm{mol} \cdot \mathrm{K}) & \mathrm{J} /(\mathrm{mol} \cdot \mathrm{K}) & \mathrm{m} \cdot \mathrm{s}^{-1} & \mu \mathrm{Pa} \cdot \mathrm{s} & \mathrm{mW} /(\mathrm{m} \cdot \mathrm{K}) \\ & & & & & & & & & \\ 110 . & 29.430 & 2.972 & -2.607 & 71.41 & 36.45 & 50.92 & 1820.8 & 254.0 \\ 115 . & 29.157 & 2.870 & -2.352 & 73.67 & 36.14 & 50.82 & 1792.1 & 249.3 \\ 120 . & 28.886 & 2.776 & -2.099 & 75.83 & 35.80 & 50.68 & 1764.2 & 244.5 \\ 125 . & 28.615 & 2.690 & -1.846 & 77.90 & 35.46 & 50.54 & 1737.0 & 239.7 \\ 130 . & 28.346 & 2.611 & -1.593 & 79.88 & 35.13 & 50.39 & 1710.5 & & 234.8 \\ 135 . & 28.079 & 2.538 & -1.342 & 81.78 & 34.81 & 50.25 & 1684.8 & & 229.8 \\ 140 . & 27.812 & 2.471 & -1.091 & 83.60 & 34.51 & 50.12 & 1659.6 & 225.0 \\ 145 . & 27.546 & 2.409 & -0.840 & 85.36 & 34.22 & 50.00 & 1635.2 & 220.1 \\ 150 . & 27.281 & 2.351 & -0.591 & 87.05 & 33.94 & 49.89 & 1611.3 & 215.3 \\ 155 . & 27.018 & 2.298 & -0.342 & 88.69 & 33.68 & 49.79 & 1588.1 & & 210.6\end{array}$


METHANE ISOBAR AT $\mathrm{P}=80.0 \mathrm{MPa}$ (continued)

\begin{tabular}{|c|c|c|c|c|c|c|c|c|c|}
\hline $\mathrm{T}$ & $\rho$ & Z & $\mathrm{H}$ & $S$ & $\mathrm{C}_{\mathrm{v}}$ & $C_{p}$ & W & $\eta$ & $\lambda$ \\
\hline K & $\mathrm{mol} \cdot \mathrm{dm}^{-3}$ & & $\mathrm{~kJ} / \mathrm{mol}$ & $\mathrm{J} /(\mathrm{mol} \cdot \mathrm{K})$ & $\mathrm{J} /(\mathrm{mol} \cdot \mathrm{K})$ & $\mathrm{J} /(\mathrm{mol} \cdot \mathrm{K})$ & $\mathrm{m} \cdot \mathrm{s}^{-1}$ & $\mu \mathrm{Pa} \cdot \mathrm{s}$ & $\mathrm{mW} /(\mathrm{m} \cdot \mathrm{K})$ \\
\hline 160. & 26.755 & 2.248 & -0.093 & 90.27 & 33.43 & 49.70 & 1565.4 & & 206.0 \\
\hline 165. & 26.493 & 2.201 & 0.155 & 91.79 & 33.20 & 49.61 & 1543.4 & & 201.5 \\
\hline 170. & 26.232 & 2.158 & 0.403 & 93.27 & 32.97 & 49.53 & 1521.9 & & 197.1 \\
\hline 175. & 25.973 & 2.117 & 0.651 & 94.71 & 32.76 & 49.46 & 1501.0 & & 192.7 \\
\hline 180. & 25.714 & 2.079 & 0.898 & 96.10 & 32.56 & 49.39 & 1480.6 & & 188.5 \\
\hline 185. & 25.456 & 2.043 & 1.145 & 97.45 & 32.38 & 49.33 & 1460.7 & & 184.5 \\
\hline 190. & 25.200 & 2.010 & 1.391 & 98.77 & 32.20 & 49.27 & 1441.4 & & 180.5 \\
\hline 195. & 24.944 & 1.978 & 1.637 & 100.05 & 32.04 & 49.22 & 1422.5 & & 176.7 \\
\hline 200 . & 24.690 & 1.948 & 1.883 & 101.29 & 31.89 & 49.17 & 1404.2 & & 173.0 \\
\hline 205. & 24.438 & 1.921 & 2.129 & 102.51 & 31.75 & 49.13 & 1386.3 & & 169.4 \\
\hline 210 . & 24.186 & 1.894 & 2.375 & 103.69 & 31.62 & 49.09 & 1369.0 & & 165.9 \\
\hline 215. & 23.936 & 1.870 & 2.620 & 104.85 & 31.50 & 49.05 & 1352.0 & & 162.6 \\
\hline 220 . & 23.688 & 1.846 & 2.865 & 105.97 & 31.40 & 49.02 & 1335.6 & & 159.3 \\
\hline 225. & 23.441 & 1.824 & 3.110 & 107.07 & 31.31 & 49.00 & 1319.6 & & 156.3 \\
\hline 230 . & 23.196 & 1.803 & 3.355 & 108.15 & 31.23 & 48.97 & 1304.0 & & 153.3 \\
\hline 235 . & 22.952 & 1.784 & 3.600 & 109.20 & 31.16 & 48.96 & 1288.9 & & 150.4 \\
\hline 240 . & 22.711 & 1.765 & 3.845 & 110.23 & 31.10 & 48.94 & 1274.2 & & 147.7 \\
\hline 245. & 22.471 & 1.748 & 4.090 & 111.24 & 31.06 & 48.94 & 1260.0 & & 145.1 \\
\hline 250 . & 22.234 & 1.731 & 4.334 & 112.23 & 31.03 & 48.93 & 1246.1 & & 142.6 \\
\hline 255. & 21.998 & 1.715 & 4.579 & 113.20 & 31.01 & 48.93 & 1232.7 & & 140.2 \\
\hline 260. & 21.764 & 1.700 & 4.824 & 114.15 & 31.00 & 48.94 & 1219.7 & & 137.9 \\
\hline 265. & 21.533 & 1.686 & 5.068 & 115.08 & 31.01 & 48.95 & 1207.1 & & 135.8 \\
\hline 270 . & 21.304 & 1.673 & 5.313 & 116.00 & 31.03 & 48.97 & 1194.8 & & 133.7 \\
\hline 275 . & 21.077 & 1.660 & 5.558 & 116.90 & 31.06 & 48.99 & 1183.0 & & 131.7 \\
\hline 280. & 20.853 & 1.648 & 5.803 & 117.78 & 31.10 & 49.02 & 1171.6 & & 129.9 \\
\hline 285. & 20.631 & 1.636 & 6.048 & 118.65 & 31.15 & 49.05 & 1160.5 & & 128.1 \\
\hline 290. & 20.411 & 1.625 & 6.293 & 119.50 & 31.21 & 49.09 & 1149.8 & & 126.4 \\
\hline 295. & 20.194 & 1.615 & 6.539 & 120.34 & 31.28 & 49.13 & 1139.4 & & 124.9 \\
\hline 300 . & 19.980 & 1.605 & 6.785 & 121.17 & 31.37 & 49.17 & 1129.4 & & 123.4 \\
\hline 310. & 19.559 & 1.587 & 7.277 & 122.78 & 31.56 & 49.28 & 1110.5 & & 120.7 \\
\hline 320. & 19.149 & 1.570 & 7.770 & 124.35 & 31.80 & 49.41 & 1093.0 & & 118.3 \\
\hline 330. & 18.750 & 1.555 & 8.265 & 125.87 & 32.07 & 49.55 & 1076.7 & & 116.2 \\
\hline 340 . & 18.363 & 1.541 & 8.762 & 127.35 & 32.37 & 49.72 & 1061.6 & & 114.4 \\
\hline 350. & 17.986 & 1.528 & 9.260 & 128.80 & 32.70 & 49.90 & 1047.8 & & 112.9 \\
\hline 360. & 17.620 & 1.517 & 9.760 & 130.20 & 33.06 & 50.10 & 1035.1 & & 111.6 \\
\hline 370. & 17.266 & 1.506 & 10.262 & 131.58 & 33.45 & 50.31 & 1023.4 & & 110.5 \\
\hline 380 . & 16.923 & 1.496 & 10.766 & 132.92 & 33.85 & 50.54 & 1012.8 & & 109.7 \\
\hline 390 . & 16.590 & 1.487 & 11.272 & 134.24 & 34.28 & 50.78 & 1003.0 & & 109.0 \\
\hline 400. & 16.269 & 1.479 & 11.782 & 135.53 & 34.72 & 51.04 & 994.2 & & 108.5 \\
\hline 410. & 15.958 & 1.471 & 12.293 & 136.79 & 35.18 & 51.31 & 986.3 & & 108.2 \\
\hline
\end{tabular}


METHANE ISOBAR AT $\mathrm{P}=80.0 \mathrm{MPa}$ (continued)

$\begin{array}{lccccccccc}\mathrm{T} & \rho & \mathrm{Z} & \mathrm{H} & \mathrm{S} & \mathrm{C}_{\mathrm{v}} & \mathrm{C}_{\mathrm{p}} & \mathrm{W} & \eta & \lambda \\ \mathrm{K} & \mathrm{mol} \cdot \mathrm{dm}^{-3} & & \mathrm{~kJ} / \mathrm{mol} & \mathrm{J} /(\mathrm{mol} \cdot \mathrm{K}) & \mathrm{J} /(\mathrm{mol} \cdot \mathrm{K}) & \mathrm{J} /(\mathrm{mol} \cdot \mathrm{K}) & \mathrm{m} \cdot \mathrm{s}^{-1} & \mu \mathrm{Pa} \cdot \mathrm{s} & \mathrm{mW} /(\mathrm{m} \cdot \mathrm{K}) \\ & & & & & & & & & \\ \text { 420. } & 15.657 & 1.463 & 12.808 & 138.03 & 35.65 & 51.59 & 979.1 & 108.1 \\ \text { 430. } & 15.366 & 1.456 & 13.325 & 139.25 & 36.13 & 51.88 & 972.6 & 108.1 \\ 440 . & 15.084 & 1.450 & 13.845 & 140.45 & 36.62 & 52.18 & 966.8 & 108.2 \\ 450 . & 14.812 & 1.443 & 14.369 & 141.62 & 37.11 & 52.49 & 961.7 & 108.4 \\ 460 . & 14.550 & 1.438 & 14.895 & 142.78 & 37.61 & 52.80 & 957.1 & 108.7 \\ & & & & & & & & \\ 470 . & 14.296 & 1.432 & 15.425 & 143.92 & 38.12 & 53.13 & 953.1 & 109.2 \\ 480 . & 14.050 & 1.427 & 15.958 & 145.04 & 38.63 & 53.46 & 949.6 & 109.7 \\ 490 . & 13.812 & 1.422 & 16.494 & 146.15 & 39.14 & 53.79 & 946.5 & 110.3 \\ 500 . & 13.583 & 1.417 & 17.034 & 147.24 & 39.65 & 54.13 & 943.9 & 111.0 \\ 520 . & 13.145 & 1.408 & 18.123 & 149.37 & 40.67 & 54.83 & 939.9 & 112.7 \\ 540 . & 12.735 & 1.399 & 19.227 & 151.45 & 41.67 & 55.53 & 937.2 & 114.5 \\ 560 . & 12.351 & 1.391 & 20.344 & 153.49 & 42.67 & 56.23 & 935.7 & 116.6 \\ 580 . & 11.990 & 1.384 & 21.476 & 155.47 & 43.65 & 56.94 & 935.2 & 118.9 \\ 600 . & 11.651 & 1.376 & 22.622 & 157.41 & 44.61 & 57.64 & 935.5 & 121.3\end{array}$

$\overline{\text { METHANE ISOBAR AT } \mathrm{P}=85.0 \mathrm{MPa}}$

$\begin{array}{lccccccccc}\mathrm{T} & \rho & \mathrm{Z} & \mathrm{H} & \mathrm{S} & \mathrm{C}_{\mathrm{v}} & \mathrm{C}_{\mathrm{p}} & \mathrm{W} & \eta & \lambda \\ \mathrm{K} & \mathrm{mol} \cdot \mathrm{dm}^{-3} & & \mathrm{~kJ} / \mathrm{mol} & \mathrm{J} /(\mathrm{mol} \cdot \mathrm{K}) & \mathrm{J} /(\mathrm{mol} \cdot \mathrm{K}) & \mathrm{J} /(\mathrm{mol} \cdot \mathrm{K}) & \mathrm{m} \cdot \mathrm{s}^{-1} & \mu \mathrm{Pa} \cdot \mathrm{s} & \mathrm{mW} /(\mathrm{m} \cdot \mathrm{K}) \\ & & & & & & & & & \\ 115 . & 29.292 & 3.035 & -2.218 & 73.36 & 36.29 & 50.72 & 1813.0 & 252.9 \\ 120 . & 29.025 & 2.935 & -1.964 & 75.51 & 35.96 & 50.58 & 1785.7 & 248.2 \\ 125 . & 28.760 & 2.844 & -1.712 & 77.58 & 35.62 & 50.42 & 1759.1 & 243.4 \\ 130 . & 28.497 & 2.760 & -1.460 & 79.55 & 35.29 & 50.27 & 1733.3 & 238.6 \\ 135 . & 28.234 & 2.682 & -1.209 & 81.44 & 34.97 & 50.12 & 1708.1 & 233.7 \\ 140 . & 27.973 & 2.610 & -0.959 & 83.26 & 34.66 & 49.98 & 1683.6 & 228.9 \\ 145 . & 27.713 & 2.544 & -0.709 & 85.02 & 34.37 & 49.85 & 1659.8 & 224.1 \\ 150 . & 27.454 & 2.482 & -0.460 & 86.70 & 34.09 & 49.73 & 1636.5 & 219.4 \\ 155 . & 27.197 & 2.425 & -0.212 & 88.33 & 33.83 & 49.62 & 1613.9 & 214.7 \\ 160 . & 26.940 & 2.372 & 0.036 & 89.91 & 33.58 & 49.52 & 1591.9 & 210.1 \\ 165 . & 26.685 & 2.322 & 0.283 & 91.43 & 33.34 & 49.42 & 1570.4 & \\ 170 . & 26.430 & 2.275 & 0.530 & 92.90 & 33.11 & 49.34 & 1549.5 & 205.6 \\ 175 . & 26.177 & 2.232 & 0.777 & 94.33 & 32.90 & 49.26 & 1529.2 & 201.2 \\ 180 . & 25.924 & 2.191 & 1.023 & 95.72 & 32.70 & 49.18 & 1509.3 & 196.9 \\ 185 . & 25.673 & 2.152 & 1.268 & 97.07 & 32.51 & 49.11 & 1490.0 & 192.8 \\ & & & & & & & & 188.7 \\ 190 . & 25.424 & 2.116 & 1.514 & 98.37 & 32.33 & 49.05 & 1471.2 & \\ 195 . & 25.175 & 2.082 & 1.759 & 99.65 & 32.17 & 48.99 & 1452.9 & 184.7 \\ 200 . & 24.928 & 2.051 & 2.004 & 100.89 & 32.01 & 48.93 & 1435.0 & 180.9 \\ 205 . & 24.682 & 2.020 & 2.248 & 102.09 & 31.87 & 48.88 & 1417.6 & 177.2 \\ 210 . & 24.437 & 1.992 & 2.493 & 103.27 & 31.74 & 48.84 & 1400.7 & 173.6 \\ & & & & & & & & & 170.1\end{array}$


METHANE ISOBAR AT $\mathrm{P}=85.0 \mathrm{MPa}$ (continued)

\begin{tabular}{|c|c|c|c|c|c|c|c|c|c|}
\hline $\mathrm{T}$ & $\rho$ & $z$ & $\mathrm{H}$ & $S$ & $C_{v}$ & $C_{p}$ & W & $\eta$ & $\lambda$ \\
\hline K & $\mathrm{mol} \cdot \mathrm{dm}^{-3}$ & & $\mathrm{~kJ} / \mathrm{mol}$ & $\mathrm{J} /(\mathrm{mol} \cdot \mathrm{K})$ & $\mathrm{J} /(\mathrm{mol} \cdot \mathrm{K})$ & $\mathrm{J} /(\mathrm{mol} \cdot \mathrm{K})$ & $\mathrm{m} \cdot \mathrm{s}^{-1}$ & $\mu \mathrm{Pa} \cdot \mathrm{s}$ & $\mathrm{mW} /(\mathrm{m} \cdot \mathrm{K})$ \\
\hline 215 . & 24.194 & 1.965 & 2.737 & 104.42 & 31.62 & 48.80 & 1384.2 & & 166.8 \\
\hline 220 . & 23.953 & 1.940 & 2.981 & 105.54 & 31.52 & 48.76 & 1368.1 & & 163.6 \\
\hline 225 . & 23.713 & 1.916 & 3.224 & 106.64 & 31.42 & 48.73 & 1352.5 & & 160.5 \\
\hline 230 . & 23.475 & 1.893 & 3.468 & 107.71 & 31.34 & 48.70 & 1337.3 & & 157.5 \\
\hline 235 . & 23.238 & 1.872 & 3.711 & 108.76 & 31.27 & 48.68 & 1322.5 & & 154.6 \\
\hline 240 . & 23.004 & 1.852 & 3.955 & 109.78 & 31.21 & 48.67 & 1308.1 & & 151.9 \\
\hline 245 . & 22.771 & 1.832 & 4.198 & 110.78 & 31.17 & 48.65 & 1294.1 & & 149.3 \\
\hline 250 . & 22.540 & 1.814 & 4.441 & 111.77 & 31.14 & 48.65 & 1280.5 & & 146.7 \\
\hline 255. & 22.311 & 1.797 & 4.684 & 112.73 & 31.12 & 48.65 & 1267.3 & & 144.3 \\
\hline 260. & 22.084 & 1.780 & 4.928 & 113.67 & 31.11 & 48.66 & 1254.5 & & 142.0 \\
\hline 265. & 21.860 & 1.765 & 5.171 & 114.60 & 31.11 & 48.67 & 1242.1 & & 139.8 \\
\hline 270. & 21.637 & 1.750 & 5.414 & 115.51 & 31.13 & 48.68 & 1230.0 & & 137.7 \\
\hline 275 . & 21.417 & 1.736 & 5.658 & 116.40 & 31.15 & 48.71 & 1218.3 & & 135.7 \\
\hline 280. & 21.199 & 1.722 & 5.901 & 117.28 & 31.19 & 48.73 & 1206.9 & & 133.8 \\
\hline 285. & 20.983 & 1.710 & 6.145 & 118.15 & 31.24 & 48.77 & 1195.9 & & 132.1 \\
\hline 290. & 20.769 & 1.697 & 6.389 & 118.99 & 31.30 & 48.81 & 1185.3 & & 130.4 \\
\hline 295. & 20.558 & 1.686 & 6.633 & 119.83 & 31.37 & 48.85 & 1175.0 & & 128.7 \\
\hline 300 . & 20.350 & 1.675 & 6.878 & 120.65 & 31.45 & 48.90 & 1165.0 & & 127.2 \\
\hline 310. & 19.940 & 1.654 & 7.367 & 122.26 & 31.65 & 49.02 & 1146.0 & & 124.4 \\
\hline 320 . & 19.540 & 1.635 & 7.858 & 123.81 & 31.88 & 49.16 & 1128.3 & & 122.0 \\
\hline 330. & 19.151 & 1.618 & 8.350 & 125.33 & 32.15 & 49.32 & 1111.9 & & 119.8 \\
\hline 340 . & 18.772 & 1.602 & 8.844 & 126.80 & 32.45 & 49.49 & 1096.6 & & 117.9 \\
\hline 350. & 18.404 & 1.587 & 9.340 & 128.24 & 32.78 & 49.69 & 1082.5 & & 116.3 \\
\hline 360. & 18.046 & 1.574 & 9.838 & 129.64 & 33.13 & 49.90 & 1069.4 & & 114.9 \\
\hline 370. & 17.698 & 1.561 & 10.338 & 131.01 & 33.51 & 50.13 & 1057.4 & & 113.8 \\
\hline 380. & 17.361 & 1.550 & 10.841 & 132.35 & 33.92 & 50.37 & 1046.4 & & 112.9 \\
\hline 390. & 17.033 & 1.539 & 11.346 & 133.67 & 34.34 & 50.63 & 1036.2 & & 112.1 \\
\hline 400. & 16.716 & 1.529 & 11.854 & 134.95 & 34.78 & 50.91 & 1027.0 & & 111.6 \\
\hline 410. & 16.409 & 1.520 & 12.364 & 136.21 & 35.24 & 51.19 & 1018.5 & & 111.2 \\
\hline 420. & 16.111 & 1.511 & 12.877 & 137.45 & 35.70 & 51.48 & 1010.9 & & 111.0 \\
\hline 430. & 15.823 & 1.503 & 13.394 & 138.66 & 36.18 & 51.79 & 1003.9 & & 110.9 \\
\hline 440. & 15.544 & 1.495 & 13.913 & 139.86 & 36.67 & 52.10 & 997.7 & & 110.9 \\
\hline 450. & 15.274 & 1.487 & 14.436 & 141.03 & 37.16 & 52.43 & 992.0 & & 111.1 \\
\hline 460. & 15.012 & 1.480 & 14.962 & 142.19 & 37.66 & 52.75 & 987.0 & & 111.4 \\
\hline 470. & 14.759 & 1.474 & 15.491 & 143.33 & 38.17 & 53.09 & 982.5 & & 111.8 \\
\hline 480 & 14.514 & 1.467 & 16.024 & 144.45 & 38.67 & 53.43 & 978.5 & & 112.2 \\
\hline 490. & 14.276 & 1.461 & 16.560 & 145.55 & 39.18 & 53.78 & 975.0 & & 112.8 \\
\hline 500 & 14.046 & 1.456 & 17.099 & 146.64 & 39.69 & 54.13 & 972.0 & & 113.5 \\
\hline 520. & 13.607 & 1.445 & 18.189 & 148.78 & 40.70 & 54.83 & 967.1 & & 115.0 \\
\hline 540. & 13.195 & 1.435 & 19.293 & 150.86 & 41.71 & 55.55 & 963.6 & & 116.8 \\
\hline
\end{tabular}


METHANE ISOBAR AT $P=85.0 \mathrm{MPa}$ (continued)

$\begin{array}{lccccccccc}\mathrm{T} & \rho & \mathrm{Z} & \mathrm{H} & \mathrm{S} & \mathrm{C}_{\mathrm{v}} & \mathrm{C}_{\mathrm{p}} & \mathrm{W} & \eta & \lambda \\ \mathrm{K} & \mathrm{mol} \cdot \mathrm{dm}^{-3} & & \mathrm{~kJ} / \mathrm{mol} & \mathrm{J} /(\mathrm{mol} \cdot \mathrm{K}) & \mathrm{J} /(\mathrm{mol} \cdot \mathrm{K}) & \mathrm{J} /(\mathrm{mol} \cdot \mathrm{K}) & \mathrm{m} \cdot \mathrm{s}^{-1} & \mu \mathrm{Pa} \cdot \mathrm{s} & \mathrm{mW} /(\mathrm{m} \cdot \mathrm{K}) \\ & & & & & & & & & \\ 560 . & 12.808 & 1.425 & 20.411 & 152.90 & 42.70 & 56.26 & 961.3 & 118.8 \\ 580 . & 12.443 & 1.417 & 21.543 & 154.88 & 43.68 & 56.98 & 960.0 & 120.9 \\ 600 . & 12.099 & 1.408 & 22.690 & 156.83 & 44.63 & 57.69 & 959.7 & \end{array}$

\section{$\overline{\text { METHANE ISOBAR AT P }=90.0 \mathrm{MPa}}$}

\begin{tabular}{|c|c|c|c|c|c|c|c|c|c|}
\hline $\mathrm{T}$ & $\rho$ & Z & H & $\mathrm{S}$ & $C_{v}$ & $C_{p}$ & W & $\eta$ & $\lambda$ \\
\hline K & $\mathrm{mol} \cdot \mathrm{dm}^{-3}$ & & $\mathrm{~kJ} / \mathrm{mol}$ & $\mathrm{J} /(\mathrm{mol} \cdot \mathrm{K})$ & $\mathrm{J} /(\mathrm{mol} \cdot \mathrm{K})$ & $\mathrm{J} /(\mathrm{mol} \cdot \mathrm{K})$ & $m \cdot s^{-1}$ & $\mu \mathrm{Pa} \cdot \mathrm{s}$ & $\mathrm{mW} /(\mathrm{m} \cdot \mathrm{K})$ \\
\hline 115. & 29.422 & 3.199 & -2.083 & 73.05 & 36.45 & 50.64 & 1833.4 & & 256.5 \\
\hline 120. & 29.161 & 3.093 & -1.830 & 75.20 & 36.11 & 50.49 & 1806.6 & & 251.9 \\
\hline 125. & 28.901 & 2.996 & -1.578 & 77.26 & 35.77 & 50.32 & 1780.6 & & 247.1 \\
\hline 130. & 28.642 & 2.907 & -1.327 & 79.23 & 35.44 & 50.16 & 1755.4 & & 242.4 \\
\hline 135. & 28.385 & 2.825 & -1.076 & 81.12 & 35.12 & 50.00 & 1730.8 & & 237.6 \\
\hline 140. & 28.129 & 2.749 & -0.827 & 82.94 & 34.81 & 49.85 & 1706.9 & & 232.8 \\
\hline 145. & 27.874 & 2.678 & -0.578 & 84.68 & 34.51 & 49.72 & 1683.6 & & 228.0 \\
\hline 150. & 27.621 & 2.613 & -0.329 & 86.37 & 34.23 & 49.59 & 1661.0 & & 223.3 \\
\hline 155. & 27.369 & 2.552 & -0.082 & 87.99 & 33.97 & 49.47 & 1639.0 & & 218.7 \\
\hline 160. & 27.118 & 2.495 & 0.165 & 89.56 & 33.71 & 49.36 & 1617.5 & & 214.2 \\
\hline 165. & 26.868 & 2.442 & 0.412 & 91.08 & 33.47 & 49.26 & 1596.6 & & 209.7 \\
\hline 170. & 26.619 & 2.392 & 0.658 & 92.55 & 33.25 & 49.16 & 1576.3 & & 205.3 \\
\hline 175. & 26.372 & 2.345 & 0.904 & 93.97 & 33.03 & 49.08 & 1556.4 & & 201.0 \\
\hline 180. & 26.126 & 2.302 & 1.149 & 95.35 & 32.83 & 48.99 & 1537.1 & & 196.9 \\
\hline 185. & 25.881 & 2.261 & 1.394 & 96.69 & 32.64 & 48.92 & 1518.3 & & 192.8 \\
\hline 190. & 25.637 & 2.222 & 1.638 & 98.00 & 32.46 & 48.85 & 1500.0 & & 188.9 \\
\hline 195. & 25.395 & 2.186 & 1.882 & 99.26 & 32.29 & 48.78 & 1482.1 & & 185.1 \\
\hline 200 . & 25.153 & 2.152 & 2.126 & 100.50 & 32.13 & 48.72 & 1464.7 & & 181.4 \\
\hline 205. & 24.914 & 2.119 & 2.369 & 101.70 & 31.99 & 48.66 & 1447.8 & & 177.8 \\
\hline 210 . & 24.676 & 2.089 & 2.612 & 102.87 & 31.86 & 48.61 & 1431.2 & & 174.3 \\
\hline 215 . & 24.439 & 2.060 & 2.855 & 104.02 & 31.74 & 48.57 & 1415.1 & & 171.0 \\
\hline 220 . & 24.204 & 2.033 & 3.098 & 105.13 & 31.63 & 48.53 & 1399.5 & & 167.7 \\
\hline 225 . & 23.970 & 2.007 & 3.341 & 106.22 & 31.53 & 48.49 & 1384.2 & & 164.6 \\
\hline 230. & 23.738 & 1.983 & 3.583 & 107.29 & 31.45 & 48.46 & 1369.3 & & 161.6 \\
\hline 235. & 23.508 & 1.959 & 3.825 & 108.33 & 31.38 & 48.44 & 1354.8 & & 158.7 \\
\hline 240 . & 23.280 & 1.937 & 4.067 & 109.35 & 31.32 & 48.42 & 1340.7 & & 156.0 \\
\hline 245 . & 23.053 & 1.917 & 4.309 & 110.35 & 31.27 & 48.41 & 1327.0 & & 153.3 \\
\hline 250 . & 22.828 & 1.897 & 4.551 & 111.33 & 31.24 & 48.40 & 1313.6 & & 150.8 \\
\hline 255. & 22.605 & 1.878 & 4.793 & 112.28 & 31.21 & 48.40 & 1300.6 & & 148.3 \\
\hline 260. & 22.385 & 1.860 & 5.035 & 113.22 & 31.20 & 48.40 & 1288.0 & & 146.0 \\
\hline
\end{tabular}


METHANE ISOBAR AT $\mathrm{P}=90.0 \mathrm{MPa}$ (continued)

\begin{tabular}{|c|c|c|c|c|c|c|c|c|c|}
\hline $\mathrm{T}$ & $\rho$ & $z$ & $\mathrm{H}$ & $S$ & $C_{v}$ & $C_{p}$ & W & $\eta$ & $\lambda$ \\
\hline K & $\mathrm{mol} \cdot \mathrm{dm}^{-3}$ & & $\mathrm{~kJ} / \mathrm{mol}$ & $\mathrm{J} /(\mathrm{mol} \cdot \mathrm{K})$ & $\mathrm{J} /(\mathrm{mol} \cdot \mathrm{K})$ & $\mathrm{J} /(\mathrm{mol} \cdot \mathrm{K})$ & $\mathrm{m} \cdot \mathrm{s}^{-1}$ & $\mu \mathrm{Pa} \cdot \mathrm{s}$ & $\mathrm{mW} /(\mathrm{m} \cdot \mathrm{K})$ \\
\hline 265. & 22.166 & 1.843 & 5.277 & 114.15 & 31.21 & 48.41 & 1275.7 & & 143.8 \\
\hline 270 . & 21.949 & 1.827 & 5.520 & 115.05 & 31.22 & 48.43 & 1263.8 & & 141.7 \\
\hline 275 . & 21.735 & 1.811 & 5.762 & 115.94 & 31.24 & 48.46 & 1252.2 & & 139.7 \\
\hline 280 . & 21.522 & 1.796 & 6.004 & 116.81 & 31.28 & 48.48 & 1240.9 & & 137.7 \\
\hline 285 . & 21.312 & 1.782 & 6.247 & 117.67 & 31.33 & 48.52 & 1230.0 & & 135.9 \\
\hline 290. & 21.104 & 1.769 & 6.489 & 118.52 & 31.39 & 48.56 & 1219.4 & & 134.2 \\
\hline 295. & 20.899 & 1.756 & 6.732 & 119.35 & 31.46 & 48.61 & 1209.2 & & 132.5 \\
\hline 300. & 20.695 & 1.743 & 6.975 & 120.16 & 31.54 & 48.66 & 1199.2 & & 131.0 \\
\hline 310 . & 20.296 & 1.720 & 7.463 & 121.76 & 31.73 & 48.79 & 1180.2 & & 128.1 \\
\hline 320 . & 19.906 & 1.699 & 7.951 & 123.31 & 31.96 & 48.93 & 1162.5 & & 125.6 \\
\hline 330. & 19.525 & 1.680 & 8.441 & 124.82 & 32.22 & 49.10 & 1145.9 & & 123.3 \\
\hline 340. & 19.154 & 1.662 & 8.933 & 126.29 & 32.52 & 49.29 & 1130.5 & & 121.4 \\
\hline 350. & 18.794 & 1.646 & 9.427 & 127.72 & 32.85 & 49.50 & 1116.1 & & 119.7 \\
\hline 360 . & 18.442 & 1.630 & 9.923 & 129.12 & 33.20 & 49.72 & 1102.8 & & 118.2 \\
\hline 370 . & 18.101 & 1.616 & 10.422 & 130.48 & 33.58 & 49.96 & 1090.4 & & 117.0 \\
\hline 380. & 17.770 & 1.603 & 10.923 & 131.82 & 33.98 & 50.22 & 1079.0 & & 116.0 \\
\hline 390. & 17.448 & 1.591 & 11.426 & 133.13 & 34.40 & 50.49 & 1068.5 & & 115.2 \\
\hline 400. & 17.135 & 1.579 & 11.933 & 134.41 & 34.84 & 50.78 & 1058.9 & & 114.6 \\
\hline 410. & 16.832 & 1.569 & 12.442 & 135.67 & 35.29 & 51.08 & 1050.0 & & 114.1 \\
\hline 420. & 16.537 & 1.558 & 12.954 & 136.90 & 35.76 & 51.38 & 1041.9 & & 113.8 \\
\hline 430. & 16.252 & 1.549 & 13.470 & 138.12 & 36.23 & 51.70 & 1034.6 & & 113.7 \\
\hline 440. & 15.975 & 1.540 & 13.988 & 139.31 & 36.72 & 52.03 & 1027.9 & & 113.6 \\
\hline 450. & 15.707 & 1.531 & 14.510 & 140.48 & 37.21 & 52.36 & 1021.8 & & 113.8 \\
\hline 460. & 15.447 & 1.523 & 15.035 & 141.64 & 37.71 & 52.70 & 1016.3 & & 114.0 \\
\hline 470. & 15.195 & 1.516 & 15.564 & 142.77 & 38.21 & 53.05 & 1011.4 & & 114.3 \\
\hline 480. & 14.950 & 1.508 & 16.096 & 143.89 & 38.71 & 53.40 & 1007.0 & & 114.7 \\
\hline 490. & 14.713 & 1.501 & 16.632 & 145.00 & 39.22 & 53.75 & 1003.1 & & 115.2 \\
\hline 500. & 14.483 & 1.495 & 17.172 & 146.09 & 39.73 & 54.11 & 999.6 & & 115.8 \\
\hline 520. & 14.044 & 1.482 & 18.261 & 148.22 & 40.74 & 54.83 & 993.9 & & 117.3 \\
\hline 540. & 13.630 & 1.471 & 19.365 & 150.31 & 41.74 & 55.56 & 989.6 & & 119.0 \\
\hline 560. & 13.241 & 1.460 & 20.483 & 152.34 & 42.73 & 56.29 & 986.6 & & 120.9 \\
\hline 580. & 12.873 & 1.450 & 21.616 & 154.33 & 43.71 & 57.01 & 984.7 & & 123.0 \\
\hline 600. & 12.526 & 1.440 & 22.764 & 156.27 & 44.66 & 57.73 & 983.7 & & 125.2 \\
\hline
\end{tabular}


METHANE ISOBAR AT $\mathrm{P}=95.0 \mathrm{MPa}$

\begin{tabular}{|c|c|c|c|c|c|c|c|c|c|}
\hline $\mathrm{T}$ & $\rho$ & $\mathrm{Z}$ & $\mathrm{H}$ & S & $C_{v}$ & $C_{p}$ & W & $\eta$ & $\lambda$ \\
\hline $\mathrm{K}$ & $\mathrm{mol} \cdot \mathrm{dm}^{-}$ & & $\mathrm{kJ} / \mathrm{mol}$ & $\mathrm{J} /(\mathrm{mol} \cdot \mathrm{K})$ & $\mathrm{J} /(\mathrm{mol} \cdot \mathrm{K})$ & $\mathrm{J} /(\mathrm{mol} \cdot \mathrm{K})$ & $\mathrm{m} \cdot \mathrm{s}^{-1}$ & $\mu \mathrm{Pa} \cdot \mathrm{s}$ & $\mathrm{mW} /(\mathrm{m} \cdot \mathrm{K})$ \\
\hline 115. & 29.549 & 3.362 & -1.947 & 72.75 & 36.59 & 50.56 & 1853.2 & & 260.1 \\
\hline 120. & 29.292 & 3.251 & -1.695 & 74.90 & 36.26 & 50.40 & 1827.0 & & 255.5 \\
\hline 125. & 29.037 & 3.148 & -1.443 & 76.95 & 35.92 & 50.23 & 1801.6 & & 250.8 \\
\hline 130. & 28.783 & 3.054 & -1.193 & 78.92 & 35.59 & 50.06 & 1776.9 & & 246.1 \\
\hline 135. & 28.531 & 2.966 & -0.943 & 80.81 & 35.26 & 49.90 & 1752.9 & & 241.4 \\
\hline 140. & 28.280 & 2.886 & -0.694 & 82.62 & 34.95 & 49.74 & 1729.5 & & 236.6 \\
\hline 145. & 28.030 & 2.811 & -0.445 & 84.36 & 34.65 & 49.60 & 1706.8 & & 231.9 \\
\hline 150. & 27.782 & 2.742 & -0.198 & 86.04 & 34.37 & 49.46 & 1684.7 & & 227.2 \\
\hline 155. & 27.535 & 2.677 & 0.049 & 87.66 & 34.10 & 49.33 & 1663.2 & & 222.6 \\
\hline 160. & 27.289 & 2.617 & 0.296 & 89.23 & 33.85 & 49.22 & 1642.3 & & 218.1 \\
\hline 165. & 27.045 & 2.560 & 0.541 & 90.74 & 33.60 & 49.11 & 1622.0 & & 213.7 \\
\hline 170. & 26.801 & 2.508 & 0.787 & 92.20 & 33.37 & 49.01 & 1602.1 & & 209.3 \\
\hline 175. & 26.559 & 2.458 & 1.032 & 93.62 & 33.16 & 48.91 & 1582.8 & & 205.1 \\
\hline 180. & 26.318 & 2.412 & 1.276 & 95.00 & 32.95 & 48.82 & 1564.0 & & 200.9 \\
\hline 185. & 26.079 & 2.368 & 1.520 & 96.34 & 32.76 & 48.74 & 1545.7 & & 196.9 \\
\hline 190. & 25.841 & 2.327 & 1.763 & 97.63 & 32.57 & 48.66 & 1527.8 & & 193.0 \\
\hline 195. & 25.604 & 2.288 & 2.006 & 98.90 & 32.40 & 48.59 & 1510.4 & & 189.1 \\
\hline 200 . & 25.369 & 2.252 & 2.249 & 100.13 & 32.25 & 48.53 & 1493.4 & & 185.4 \\
\hline 205. & 25.135 & 2.217 & 2.492 & 101.32 & 32.10 & 48.47 & 1476.9 & & 181.8 \\
\hline 210 . & 24.902 & 2.185 & 2.734 & 102.49 & 31.97 & 48.41 & 1460.7 & & 178.4 \\
\hline 215 . & 24.671 & 2.154 & 2.976 & 103.63 & 31.84 & 48.36 & 1445.0 & & 175.0 \\
\hline 220 . & 24.442 & 2.125 & 3.218 & 104.74 & 31.73 & 48.32 & 1429.7 & & 171.8 \\
\hline 225. & 24.214 & 2.097 & 3.459 & 105.83 & 31.64 & 48.28 & 1414.7 & & 168.7 \\
\hline 230 . & 23.988 & 2.071 & 3.700 & 106.89 & 31.55 & 48.25 & 1400.2 & & 165.6 \\
\hline 235. & 23.763 & 2.046 & 3.941 & 107.92 & 31.48 & 48.22 & 1386.0 & & 162.7 \\
\hline 240 . & 23.540 & 2.022 & 4.183 & 108.94 & 31.42 & 48.20 & 1372.1 & & 160.0 \\
\hline 245 . & 23.320 & 2.000 & 4.424 & 109.93 & 31.37 & 48.19 & 1358.6 & & 157.3 \\
\hline 250 . & 23.100 & 1.978 & 4.664 & 110.91 & 31.33 & 48.18 & 1345.5 & & 154.7 \\
\hline 255. & 22.883 & 1.958 & 4.905 & 111.86 & 31.31 & 48.18 & 1332.7 & & 152.3 \\
\hline 260. & 22.668 & 1.939 & 5.146 & 112.80 & 31.29 & 48.18 & 1320.3 & & 149.9 \\
\hline 265 . & 22.455 & 1.920 & 5.387 & 113.71 & 31.30 & 48.19 & 1308.1 & & 147.7 \\
\hline 270 . & 22.243 & 1.902 & 5.628 & 114.62 & 31.31 & 48.21 & 1296.4 & & 145.5 \\
\hline 275 . & 22.034 & 1.886 & 5.869 & 115.50 & 31.33 & 48.23 & 1284.9 & & 143.5 \\
\hline 280. & 21.827 & 1.870 & 6.110 & 116.37 & 31.37 & 48.26 & 1273.7 & & 141.5 \\
\hline 285 . & 21.622 & 1.854 & 6.352 & 117.22 & 31.41 & 48.30 & 1262.9 & & 139.7 \\
\hline 290. & 21.419 & 1.839 & 6.593 & 118.06 & 31.47 & 48.34 & 1252.4 & & 137.9 \\
\hline 295. & 21.218 & 1.825 & 6.835 & 118.89 & 31.54 & 48.39 & 1242.2 & & 136.2 \\
\hline 300. & 21.020 & 1.812 & 7.077 & 119.71 & 31.62 & 48.45 & 1232.3 & & 134.6 \\
\hline 310. & 20.629 & 1.787 & 7.563 & 121.30 & 31.80 & 48.58 & 1213.3 & & 131.7 \\
\hline 320. & 20.248 & 1.763 & 8.049 & 122.84 & 32.03 & 48.73 & 1195.5 & & 129.1 \\
\hline
\end{tabular}


METHANE ISOBAR AT P $=95.0 \mathrm{MPa}$ (continued)

$\begin{array}{lccccccccc}\mathrm{T} & \mathrm{P} & \mathrm{Z} & \mathrm{H} & \mathrm{S} & \mathrm{C}_{\mathrm{v}} & \mathrm{C}_{\mathrm{p}} & \mathrm{W} & \eta & \lambda \\ \mathrm{K} & \mathrm{mol} \cdot \mathrm{dm}^{-3} & & \mathrm{~kJ} / \mathrm{mol} & \mathrm{J} /(\mathrm{mol} \cdot \mathrm{K}) & \mathrm{J} /(\mathrm{mol} \cdot \mathrm{K}) & \mathrm{J} /(\mathrm{mol} \cdot \mathrm{K}) & \mathrm{m} \cdot \mathrm{s}^{-1} & \mu \mathrm{Pa} \cdot \mathrm{s} & \mathrm{mW} /(\mathrm{m} \cdot \mathrm{K}) \\ & & & & & & & & \\ 330 . & 19.876 & 1.742 & 8.537 & 124.34 & 32.29 & 48.91 & 1178.8 & 126.8 \\ 340 . & 19.513 & 1.722 & 9.027 & 125.81 & 32.59 & 49.11 & 1163.2 & 124.7 \\ 350 . & 19.159 & 1.704 & 9.519 & 127.23 & 32.91 & 49.32 & 1148.7 & 123.0 \\ 360 . & 18.814 & 1.687 & 10.014 & 128.62 & 33.26 & 49.56 & 1135.1 & 121.4 \\ 370 . & 18.479 & 1.671 & 10.511 & 129.99 & 33.64 & 49.81 & 1122.5 & 120.2 \\ & & & & & & & & \\ 380 . & 18.153 & 1.656 & 11.010 & 131.32 & 34.04 & 50.08 & 1110.8 & 119.1 \\ 390 . & 17.836 & 1.643 & 11.512 & 132.62 & 34.46 & 50.36 & 1099.9 & 118.2 \\ 400 . & 17.528 & 1.630 & 12.017 & 133.90 & 34.89 & 50.66 & 1089.9 & 117.5 \\ 410 . & 17.228 & 1.618 & 12.526 & 135.16 & 35.34 & 50.97 & 1080.7 & 117.0 \\ 420 . & 16.938 & 1.606 & 13.037 & 136.39 & 35.81 & 51.29 & 1072.3 & 116.6 \\ 430 . & 16.655 & 1.595 & 13.551 & 137.60 & 36.28 & 51.62 & 1064.5 & 116.4 \\ 440 . & 16.381 & 1.585 & 14.069 & 138.79 & 36.76 & 51.95 & 1057.5 & 116.3 \\ 450 . & 16.115 & 1.576 & 14.590 & 139.96 & 37.25 & 52.30 & 1051.0 & 116.4 \\ 460 . & 15.857 & 1.566 & 15.115 & 141.11 & 37.75 & 52.65 & 1045.1 & 116.5 \\ 470 . & 15.606 & 1.558 & 15.643 & 142.25 & 38.25 & 53.00 & 1039.8 & 116.8 \\ 480 . & 15.362 & 1.549 & 16.175 & 143.37 & 38.75 & 53.36 & 1035.0 & \\ 490 . & 15.126 & 1.542 & 16.711 & 144.47 & 39.26 & 53.72 & 1030.7 & 117.2 \\ 500 . & 14.896 & 1.534 & 17.250 & 145.56 & 39.76 & 54.09 & 1026.9 & 117.6 \\ 520 . & 14.457 & 1.520 & 18.339 & 147.70 & 40.77 & 54.83 & 1020.4 & 118.2 \\ 540 . & 14.043 & 1.507 & 19.443 & 149.78 & 41.77 & 55.57 & 1015.4 & 119.5 \\ 560 . & 13.652 & 1.495 & 20.561 & 151.82 & 42.76 & 56.30 & 1011.7 & 121.1 \\ 580 . & 13.282 & 1.483 & 21.695 & 153.80 & 43.73 & 57.04 & 1009.2 & 123.0 \\ 600 . & 12.932 & 1.473 & 22.843 & 155.75 & 44.68 & 57.76 & 1007.6 & 125.0 \\ & & & & & & & & & 127.2\end{array}$

\section{$\overline{\text { METHANE ISOBAR AT } \mathrm{P}=100.0 \mathrm{MPa}}$}

$\begin{array}{lccccccccc}\mathrm{T} & \rho & \mathrm{Z} & \mathrm{H} & \mathrm{S} & \mathrm{C}_{\mathrm{v}} & \mathrm{C}_{\mathrm{p}} & \mathrm{W} & \eta & \lambda \\ \mathrm{K} & \mathrm{mol} \cdot \mathrm{dm}^{-3} & & \mathrm{~kJ} / \mathrm{mol} & \mathrm{J} /(\mathrm{mol} \cdot \mathrm{K}) & \mathrm{J} /(\mathrm{mol} \cdot \mathrm{K}) & \mathrm{J} /(\mathrm{mol} \cdot \mathrm{K}) & \mathrm{m} \cdot \mathrm{s}^{-1} & \mu \mathrm{Pa} \cdot \mathrm{s} & \mathrm{mW} /(\mathrm{m} \cdot \mathrm{K}) \\ & & & & & & & & & \\ 115 . & 29.673 & 3.525 & -1.812 & 72.46 & 36.74 & 50.49 & 1872.6 & 263.6 \\ 120 . & 29.420 & 3.407 & -1.560 & 74.61 & 36.40 & 50.32 & 1846.9 & 259.0 \\ 125 . & 29.169 & 3.299 & -1.309 & 76.66 & 36.06 & 50.15 & 1822.0 & 254.4 \\ 130 . & 28.920 & 3.199 & -1.059 & 78.62 & 35.73 & 49.97 & 1797.8 & 249.8 \\ 135 . & 28.672 & 3.107 & -0.809 & 80.50 & 35.40 & 49.80 & 1774.4 & 245.1 \\ & & & & & & & & \\ 140 . & 28.426 & 3.022 & -0.561 & 82.31 & 35.09 & 49.64 & 1751.6 & 240.4 \\ 145 . & 28.181 & 2.943 & -0.313 & 84.05 & 34.79 & 49.49 & 1729.4 & 235.7 \\ 150 . & 27.937 & 2.870 & -0.066 & 85.73 & 34.50 & 49.34 & 1707.8 & 231.1 \\ 155 . & 27.695 & 2.802 & 0.181 & 87.34 & 34.23 & 49.21 & 1686.9 & 226.5 \\ 160 . & 27.454 & 2.738 & 0.426 & 88.90 & 33.97 & 49.09 & 1666.5 & 222.0\end{array}$


METHANE ISOBAR AT $P=100.0 \mathrm{MPa}$ (continued)

\begin{tabular}{|c|c|c|c|c|c|c|c|c|c|}
\hline $\mathrm{T}$ & $\rho$ & Z & $\mathrm{H}$ & S & $\mathrm{C}_{\mathrm{v}}$ & $C_{p}$ & W & $\eta$ & $\lambda$ \\
\hline K & $\mathrm{mol} \cdot \mathrm{dm}^{-3}$ & & $\mathrm{~kJ} / \mathrm{mol}$ & $\mathrm{J} /(\mathrm{mol} \cdot \mathrm{K})$ & $\mathrm{J} /(\mathrm{mol} \cdot \mathrm{K})$ & $\mathrm{J} /(\mathrm{mol} \cdot \mathrm{K})$ & $\mathrm{m} \cdot \mathrm{s}^{-1}$ & $\mu \mathrm{Pa} \cdot \mathrm{s}$ & $\mathrm{mW} /(\mathrm{m} \cdot \mathrm{K})$ \\
\hline 165. & 27.215 & 2.678 & 0.672 & 90.41 & 33.73 & 48.97 & 1646.6 & & 217.6 \\
\hline 170. & 26.976 & 2.623 & 0.916 & 91.87 & 33.50 & 48.87 & 1627.3 & & 213.3 \\
\hline 175. & 26.739 & 2.570 & 1.160 & 93.29 & 33.28 & 48.77 & 1608.4 & & 209.0 \\
\hline 180. & 26.504 & 2.521 & 1.404 & 94.66 & 33.07 & 48.67 & 1590.1 & & 204.9 \\
\hline 185. & 26.269 & 2.475 & 1.647 & 95.99 & 32.87 & 48.58 & 1572.2 & & 200.9 \\
\hline 190. & 26.036 & 2.431 & 1.890 & 97.29 & 32.69 & 48.50 & 1554.8 & & 196.9 \\
\hline 195. & 25.805 & 2.390 & 2.132 & 98.54 & 32.51 & 48.43 & 1537.8 & & 193.1 \\
\hline 200 . & 25.574 & 2.351 & 2.374 & 99.77 & 32.35 & 48.36 & 1521.2 & & 189.4 \\
\hline 205. & 25.346 & 2.315 & 2.616 & 100.96 & 32.21 & 48.29 & 1505.0 & & 185.8 \\
\hline 210 . & 25.118 & 2.280 & 2.857 & 102.13 & 32.07 & 48.23 & 1489.3 & & 182.4 \\
\hline 215 . & 24.893 & 2.247 & 3.098 & 103.26 & 31.95 & 48.18 & 1473.9 & & 179.0 \\
\hline 220 . & 24.668 & 2.216 & 3.339 & 104.37 & 31.83 & 48.13 & 1458.9 & & 175.8 \\
\hline 225 . & 24.446 & 2.187 & 3.579 & 105.45 & 31.73 & 48.09 & 1444.3 & & 172.6 \\
\hline 230. & 24.225 & 2.159 & 3.820 & 106.50 & 31.65 & 48.06 & 1430.0 & & 169.6 \\
\hline 235. & 24.006 & 2.132 & 4.060 & 107.54 & 31.57 & 48.03 & 1416.0 & & 166.7 \\
\hline 240 . & 23.788 & 2.107 & 4.300 & 108.55 & 31.51 & 48.00 & 1402.5 & & 163.9 \\
\hline 245 . & 23.572 & 2.083 & 4.540 & 109.54 & 31.46 & 47.99 & 1389.2 & & 161.2 \\
\hline 250. & 23.358 & 2.060 & 4.780 & 110.51 & 31.42 & 47.98 & 1376.3 & & 158.6 \\
\hline 255. & 23.146 & 2.038 & 5.020 & 111.46 & 31.39 & 47.98 & 1363.7 & & 156.1 \\
\hline 260 . & 22.936 & 2.017 & 5.260 & 112.39 & 31.38 & 47.98 & 1351.4 & & 153.7 \\
\hline 265 . & 22.727 & 1.997 & 5.499 & 113.30 & 31.38 & 47.99 & 1339.5 & & 151.5 \\
\hline 270 . & 22.521 & 1.978 & 5.739 & 114.20 & 31.39 & 48.01 & 1327.8 & & 149.3 \\
\hline 275 . & 22.317 & 1.960 & 5.980 & 115.08 & 31.41 & 48.03 & 1316.5 & & 147.2 \\
\hline 280. & 22.114 & 1.942 & 6.220 & 115.95 & 31.45 & 48.07 & 1305.5 & & 145.2 \\
\hline 285. & 21.914 & 1.926 & 6.460 & 116.80 & 31.49 & 48.10 & 1294.7 & & 143.4 \\
\hline 290. & 21.715 & 1.910 & 6.701 & 117.64 & 31.55 & 48.15 & 1284.3 & & 141.6 \\
\hline 295. & 21.519 & 1.895 & 6.942 & 118.46 & 31.61 & 48.20 & 1274.1 & & 139.8 \\
\hline 300 . & 21.325 & 1.880 & 7.183 & 119.27 & 31.69 & 48.26 & 1264.2 & & 138.2 \\
\hline 310. & 20.943 & 1.853 & 7.666 & 120.85 & 31.88 & 48.39 & 1245.3 & & 135.2 \\
\hline 320. & 20.570 & 1.827 & 8.151 & 122.39 & 32.10 & 48.55 & 1227.5 & & 132.5 \\
\hline 330. & 20.205 & 1.804 & 8.637 & 123.89 & 32.36 & 48.74 & 1210.7 & & 130.2 \\
\hline 340 . & 19.849 & 1.782 & 9.126 & 125.35 & 32.65 & 48.94 & 1195.0 & & 128.0 \\
\hline 350. & 19.502 & 1.762 & 9.616 & 126.77 & 32.97 & 49.17 & 1180.3 & & 126.2 \\
\hline 360. & 19.164 & 1.743 & 10.109 & 128.16 & 33.32 & 49.41 & 1166.5 & & 124.6 \\
\hline 370 . & 18.835 & 1.726 & 10.604 & 129.52 & 33.70 & 49.67 & 1153.6 & & 123.2 \\
\hline 380. & 18.514 & 1.710 & 11.102 & 130.84 & 34.09 & 49.95 & 1141.7 & & 122.1 \\
\hline 390. & 18.201 & 1.694 & 11.603 & 132.14 & 34.51 & 50.24 & 1130.6 & & 121.2 \\
\hline 400. & 17.898 & 1.680 & 12.107 & 133.42 & 34.94 & 50.55 & 1120.3 & & 120.4 \\
\hline 410. & 17.602 & 1.667 & 12.614 & 134.67 & 35.39 & 50.87 & 1110.8 & & 119.8 \\
\hline 420. & 17.315 & 1.654 & 13.125 & 135.90 & 35.85 & 51.20 & 1102.0 & & 119.4 \\
\hline
\end{tabular}




\begin{tabular}{|c|c|c|c|c|c|c|c|c|c|}
\hline $\mathrm{T}$ & $\rho$ & $\mathrm{Z}$ & H & $\mathrm{s}$ & $\mathrm{C}_{\mathrm{v}}$ & $C_{p}$ & W & $\eta$ & $\lambda$ \\
\hline K & $\mathrm{mol} \cdot \mathrm{dm}^{-3}$ & & $\mathrm{~kJ} / \mathrm{mol}$ & $\mathrm{J} /(\mathrm{mol} \cdot \mathrm{K})$ & $\mathrm{J} /(\mathrm{mol} \cdot \mathrm{K})$ & $\mathrm{J} /(\mathrm{mol} \cdot \mathrm{K})$ & $\mathrm{m} \cdot \mathrm{s}^{-1}$ & $\mu \mathrm{Pa} \cdot \mathrm{s}$ & $\mathrm{mW} /(\mathrm{m} \cdot \mathrm{K})$ \\
\hline 430. & 17.035 & 1.642 & 13.638 & 137.11 & 36.33 & 51.53 & 1093.9 & & 119.1 \\
\hline 440. & 16.764 & 1.631 & 14.156 & 138.30 & 36.81 & 51.88 & 1086.4 & & 119.0 \\
\hline 450. & 16.500 & 1.620 & 14.676 & 139.47 & 37.30 & 52.23 & 1079.6 & & 118.9 \\
\hline 460 & 16.244 & 1.610 & 15.200 & 140.62 & 37.79 & 52.59 & 1073.4 & & 119.1 \\
\hline 470. & 15.994 & 1.600 & 15.728 & 141.76 & 38.29 & 52.95 & 1067.7 & & 119.3 \\
\hline 480 . & 15.752 & 1.591 & 16.259 & 142.88 & 38.79 & 53.32 & 1062.6 & & 119.6 \\
\hline 490. & 15.517 & 1.582 & 16.794 & 143.98 & 39.29 & 53.69 & 1057.9 & & 120.0 \\
\hline 500. & 15.288 & 1.573 & 17.333 & 145.07 & 39.80 & 54.06 & 1053.7 & & 120.5 \\
\hline 520 . & 14.850 & 1.558 & 18.422 & 147.20 & 40.81 & 54.81 & 1046.5 & & 121.7 \\
\hline 540 & 14.435 & 1.543 & 19.526 & 149.28 & 41.80 & 55.56 & 1040.9 & & 123.3 \\
\hline 560. & 14.043 & 1.529 & 20.645 & 151.32 & 42.79 & 56.31 & 1036.6 & & 125.0 \\
\hline 580. & 13.672 & 1.517 & 21.778 & 153.31 & 43.76 & 57.06 & 1033.4 & & 127.0 \\
\hline 600 . & 13.320 & 1.505 & 22.927 & 155.25 & 44.71 & 57.79 & 1031.2 & & 129.1 \\
\hline
\end{tabular}


Notes for Table B4. Values of the pressure, isothermal density derivative of the pressure, isochoric temperature derivative of the pressure, isochoric specific heat capacity, speed of sound, viscosity, and thermal conductivity in the single-phase region of the methane fluid. The independent variables were chosen to be temperature and density. The quantities $\mathrm{P}, \mathrm{dP} /\left.\mathrm{d} \rho\right|_{\mathrm{T}}, \mathrm{dP} /\left.\mathrm{dT}\right|_{\rho}, \mathrm{C}_{\mathrm{v}}$, and $\mathrm{W}$ were evaluated directly from the expressions in Table 7. The viscosity and thermal conductivity are from eqs (7) and (8) [with the terms evaluated from eqs (10-23)].

METHANE ISOTHERM AT $\mathrm{T}=95 . \mathrm{K}$ Second Virial Coefficient is $-446.4 \mathrm{~cm}^{3} / \mathrm{mol}$

$$
\begin{array}{llllllll}
\rho & \mathrm{P} & \mathrm{dP} /\left.\mathrm{d} \rho\right|_{\mathrm{T}} & \mathrm{dP} /\left.\mathrm{dT}\right|_{\rho} & \mathrm{C}_{\mathrm{v}} & \mathrm{W} & \eta & \lambda
\end{array}
$$

$\mathrm{mol} / \mathrm{dm}^{3} \quad \mathrm{MPa} \quad \mathrm{MPa} \cdot \mathrm{dm}^{3} / \mathrm{mol} \quad \mathrm{MPa} / \mathrm{K} \quad \mathrm{J} /(\mathrm{mol} \cdot \mathrm{k}) \quad \mathrm{m} / \mathrm{s} \quad \mu \mathrm{Pa} \cdot \mathrm{s} \quad \mathrm{mW} /(\mathrm{m} \cdot \mathrm{K})$

$\begin{array}{rrrrrrrr}27.8 & 0.3399 & 22.8777 & 1.91608 & 34.00 & 1501.16 & 180.25 & 206.4 \\ 27.9 & 2.6635 & 23.5946 & 1.93770 & 34.08 & 1519.51 & 184.81 & 208.5 \\ 28.0 & 5.0591 & 24.3186 & 1.95897 & 34.16 & 1537.64 & 189.59 & 210.6 \\ 28.1 & 7.5274 & 25.0497 & 1.97990 & 34.25 & 1555.56 & 194.60 & 212.8 \\ 28.2 & 10.0693 & 25.7882 & 2.00053 & 34.34 & 1573.28 & 199.86 & 215.0 \\ & & & & & & & \\ 28.3 & 12.6853 & 26.5343 & 2.02085 & 34.44 & 1590.83 & 205.38 & 217.3 \\ 28.4 & 15.3764 & 27.2882 & 2.04090 & 34.54 & 1608.21 & 211.19 & 219.5\end{array}$

METHANE ISOTHERM AT $\mathrm{T}=100, \mathrm{~K}$

Second Virial Coefficient is $-403.3 \mathrm{~cm}^{3} / \mathrm{mol}$

$\begin{array}{llllllll}\rho & \mathrm{P} & \mathrm{dP} /\left.\mathrm{d} \rho\right|_{\mathrm{T}} & \mathrm{dP} /\left.\mathrm{dT}\right|_{\rho} & \mathrm{C}_{\mathrm{v}} & \mathrm{W} & \eta & \lambda\end{array}$

$\mathrm{mol} / \mathrm{dm}^{3} \quad \mathrm{MPa} \quad \mathrm{MPa} \cdot \mathrm{dm}^{3} / \mathrm{mol} \quad \mathrm{MPa} / \mathrm{K} \quad \mathrm{J} /(\mathrm{mol} \cdot \mathrm{k}) \quad \mathrm{m} / \mathrm{s} \quad \mu \mathrm{Pa} \cdot \mathrm{s} \quad \mathrm{mW} /(\mathrm{m} \cdot \mathrm{K})$

$\begin{array}{llllllll}27.4 & 0.8262 & 21.1787 & 1.80290 & 34.11 & 1453.06 & 158.77 & 200.5 \\ 27.5 & 2.9777 & 21.8514 & 1.82354 & 34.18 & 1471.06 & 162.65 & 202.6 \\ 27.6 & 5.1968 & 22.5312 & 1.84390 & 34.25 & 1488.85 & 166.70 & 204.7 \\ 27.7 & 7.4842 & 23.2183 & 1.86400 & 34.33 & 1506.45 & 170.94 & 206.8 \\ 27.8 & 9.8407 & 23.9130 & 1.88384 & 34.42 & 1523.88 & 175.38 & 209.0 \\ & & & & & & & \\ 27.9 & 12.2670 & 24.6152 & 1.90343 & 34.51 & 1541.15 & 180.05 & 211.1 \\ 28.0 & 14.7640 & 25.3253 & 1.92281 & 34.60 & 1558.27 & 184.95 & 213.3 \\ 28.1 & 17.3324 & 26.0434 & 1.94196 & 34.69 & 1575.26 & 190.11 & 215.6 \\ 28.2 & 19.9729 & 26.7696 & 1.96092 & 34.79 & 1592.13 & 195.56 & 217.8 \\ 28.3 & 22.6865 & 27.5040 & 1.97968 & 34.89 & 1608.90 & 201.30 & 220.1\end{array}$


METHANE ISOTHERM AT $\mathrm{T}=100 . \mathrm{K}$ (continued)

$\begin{array}{cccccccc}\rho & \mathrm{P} & \mathrm{dP} /\left.\mathrm{d} \rho\right|_{\mathrm{T}} & \mathrm{dP} /\left.\mathrm{dT}\right|_{\rho} & \mathrm{C}_{\mathrm{v}} & \mathrm{W} & \eta & \lambda \\ \mathrm{mol} / \mathrm{dm}^{3} & \mathrm{MPa} & \mathrm{MPa} \cdot \mathrm{dm}^{3} / \mathrm{mol} & \mathrm{MPa} / \mathrm{K} & \mathrm{J} /(\mathrm{mol} \cdot \mathrm{k}) & \mathrm{m} / \mathrm{s} & \mu \mathrm{Pa} \cdot \mathrm{s} & \mathrm{mW} /(\mathrm{m} \cdot \mathrm{K}) \\ & & & & & & & \\ 28.4 & 25.4740 & 28.2468 & 1.99826 & 35.00 & 1625.56 & 207.39 & 222.4 \\ 28.5 & 28.3362 & 28.9982 & 2.01667 & 35.10 & 1642.14 & 213.84 & 224.8 \\ 28.6 & 31.2739 & 29.7583 & 2.03493 & 35.21 & 1658.64 & 220.69 & 227.1 \\ 28.7 & 34.2881 & 30.5272 & 2.05303 & 35.32 & 1675.06 & 227.98 & 229.5 \\ 28.8 & 37.3797 & 31.3050 & 2.07098 & 35.43 & 1691.43 & 235.77 & 231.9\end{array}$

$\begin{aligned} & \text { METHANE } \text { ISOTHERM AT } T=105 . \mathrm{K} \\ & \text { Second Virial Coefficient is }-365.9 \mathrm{~cm}^{3} / \mathrm{mol}\end{aligned}$

$\begin{array}{ccccccccc}\rho & \mathrm{P} & \mathrm{dP} /\left.\mathrm{d} \rho\right|_{\mathrm{T}} & \mathrm{dP} /\left.\mathrm{dT}\right|_{\rho} & \mathrm{C}_{\mathrm{v}} & \mathrm{W} & \eta & \lambda \\ \mathrm{mol} / \mathrm{dm}^{3} & \mathrm{MPa} & \mathrm{MPa} \cdot \mathrm{dm}^{3} / \mathrm{mol} & \mathrm{MPa} / \mathrm{K} & \mathrm{J} /(\mathrm{mol} \cdot \mathrm{k}) & \mathrm{m} / \mathrm{s} & \mu \mathrm{Pa} \cdot \mathrm{s} & \mathrm{mW} /(\mathrm{m} \cdot \mathrm{K}) \\ & & & & & & & \\ 27.0 & 1.4235 & 19.5999 & 1.69794 & 33.90 & 1408.97 & 140.83 & 194.5 \\ 27.1 & 3.4150 & 20.2310 & 1.71757 & 33.97 & 1426.53 & 144.12 & 196.5 \\ 27.2 & 5.4699 & 20.8692 & 1.73698 & 34.04 & 1443.91 & 147.55 & 198.6 \\ 27.3 & 7.5891 & 21.5149 & 1.75617 & 34.12 & 1461.13 & 151.13 & 200.6 \\ 27.4 & 9.7731 & 22.1680 & 1.77515 & 34.20 & 1478.19 & 154.89 & 202.7 \\ & & & & & & & \\ 27.5 & 12.0229 & 22.8289 & 1.79394 & 34.28 & 1495.11 & 158.82 & 204.9 \\ 27.6 & 14.3392 & 23.4975 & 1.81255 & 34.37 & 1511.90 & 162.94 & 207.0 \\ 27.7 & 16.7227 & 24.1740 & 1.83098 & 34.47 & 1528.58 & 167.28 & 209.2 \\ 27.8 & 19.1743 & 24.8587 & 1.84925 & 34.56 & 1545.14 & 171.84 & 211.4 \\ 27.9 & 21.6947 & 25.5515 & 1.86737 & 34.66 & 1561.61 & 176.65 & 213.6 \\ & & & & & & & \\ 28.0 & 24.2848 & 26.2527 & 1.88533 & 34.76 & 1578.00 & 181.73 & 215.8 \\ 28.1 & 26.9455 & 26.9623 & 1.90317 & 34.86 & 1594.31 & 187.11 & 218.1 \\ 28.2 & 29.6776 & 27.6806 & 1.92087 & 34.97 & 1610.54 & 192.81 & 220.4 \\ 28.3 & 32.4819 & 28.4076 & 1.93846 & 35.07 & 1626.72 & 198.86 & 222.8 \\ 28.4 & 35.3594 & 29.1434 & 1.95593 & 35.18 & 1642.84 & 205.30 & 225.1 \\ & & & & & & & \\ 28.5 & 38.3109 & 29.8882 & 1.97329 & 35.29 & 1658.91 & 212.18 & 227.5 \\ 28.6 & 41.3373 & 30.6420 & 1.99055 & 35.41 & 1674.95 & 219.53 & 229.9 \\ 28.7 & 44.4396 & 31.4050 & 2.00773 & 35.52 & 1690.94 & 227.42 & 232.3 \\ 28.8 & 47.6186 & 32.1773 & 2.02481 & 35.63 & 1706.91 & 235.91 & 234.8 \\ 28.9 & 50.8754 & 32.9589 & 2.04181 & 35.75 & 1722.86 & 245.07 & 237.3 \\ & & & & & & & \\ 29.0 & 54.2107 & 33.7500 & 2.05873 & 35.87 & 1738.78 & 254.99 & 239.8 \\ 29.1 & 57.6257 & 34.5506 & 2.07558 & 35.98 & 1754.69 & 265.78 & 242.4\end{array}$


METHANE ISOTHERM AT $\mathrm{T}=110 . \mathrm{K}$

Second Virial Coefficient is $-333.6 \mathrm{~cm}^{3} / \mathrm{mol}$

$\begin{array}{cccccccc}\rho & \mathrm{P} & \mathrm{dP} /\left.\mathrm{d} \rho\right|_{\mathrm{T}} & \mathrm{dP} / \mathrm{dT} \mid \rho & \mathrm{C}_{\mathrm{v}} & \mathrm{W} & \eta & \lambda \\ \mathrm{mol} / \mathrm{dm}^{3} & \mathrm{MPa} & \mathrm{MPa} \cdot \mathrm{dm}^{3} / \mathrm{mol} & \mathrm{MPa} / \mathrm{K} & \mathrm{J} /(\mathrm{mol} \cdot \mathrm{k}) & \mathrm{m} / \mathrm{s} & \mu \mathrm{Pa} \cdot \mathrm{s} & \mathrm{mW} /(\mathrm{m} \cdot \mathrm{K}) \\ & & & & & & & \\ 26.5 & 0.3377 & 17.5472 & 1.58053 & 33.50 & 1349.77 & 123.15 & 186.5 \\ 26.6 & 2.1216 & 18.1320 & 1.59939 & 33.56 & 1367.06 & 125.84 & 188.5 \\ 26.7 & 3.9643 & 18.7238 & 1.61806 & 33.63 & 1384.18 & 128.63 & 190.4 \\ 26.8 & 5.8666 & 19.3228 & 1.63655 & 33.70 & 1401.14 & 131.54 & 192.4 \\ 26.9 & 7.8291 & 19.9292 & 1.65486 & 33.77 & 1417.95 & 134.58 & 194.4 \\ & & & & & & & \\ 27.0 & 9.8526 & 20.5431 & 1.67300 & 33.85 & 1434.63 & 137.75 & 196.5 \\ 27.1 & 11.9380 & 21.1646 & 1.69098 & 33.93 & 1451.18 & 141.07 & 198.5 \\ 27.2 & 14.0858 & 21.7938 & 1.70882 & 34.02 & 1467.62 & 144.55 & 200.6 \\ 27.3 & 16.2970 & 22.4309 & 1.72652 & 34.11 & 1483.95 & 148.19 & 202.7 \\ 27.4 & 18.5722 & 23.0759 & 1.74409 & 34.20 & 1500.20 & 152.02 & 204.8 \\ 27.5 & 20.9124 & 23.7291 & 1.76153 & 34.30 & 1516.35 & 156.04 & 207.0 \\ 27.6 & 23.3184 & 24.3906 & 1.77885 & 34.40 & 1532.43 & 160.28 & 209.2 \\ 27.7 & 25.7908 & 25.0604 & 1.79607 & 34.50 & 1548.45 & 164.76 & 211.4 \\ 27.8 & 28.3307 & 25.7387 & 1.81319 & 34.60 & 1564.40 & 169.49 & 213.6 \\ 27.9 & 30.9388 & 26.4256 & 1.83021 & 34.70 & 1580.30 & 174.50 & 215.9 \\ 28.0 & 33.6161 & 27.1212 & 1.84714 & 34.81 & 1596.16 & 179.81 & 218.2 \\ 28.1 & 36.3634 & 27.8256 & 1.86399 & 34.92 & 1611.97 & 185.47 & 220.5 \\ 28.2 & 39.1815 & 28.5390 & 1.88077 & 35.03 & 1627.75 & 191.50 & 222.8 \\ 28.3 & 42.0715 & 29.2614 & 1.89747 & 35.14 & 1643.51 & 197.94 & 225.2 \\ 28.4 & 45.0341 & 29.9929 & 1.91410 & 35.25 & 1659.24 & 204.85 & 227.6 \\ 28.5 & 48.0704 & 30.7337 & 1.93068 & 35.36 & 1674.95 & 212.27 & 230.0 \\ 28.6 & 51.1812 & 31.4838 & 1.94720 & 35.48 & 1690.65 & 220.28 & 232.5 \\ 28.7 & 54.3674 & 32.2434 & 1.96366 & 35.59 & 1706.33 & 228.94 & 235.0 \\ 28.8 & 57.6301 & 33.0124 & 1.98008 & 35.71 & 1722.02 & 238.34 & 237.5 \\ 28.9 & 60.9702 & 33.7910 & 1.99645 & 35.83 & 1737.70 & 248.59 & 240.0 \\ 29.0 & 64.3887 & 34.5792 & 2.01278 & 35.94 & 1753.37 & 259.81 & 242.6 \\ 29.1 & 67.8864 & 35.3771 & 2.02907 & 36.06 & 1769.06 & 272.16 & 245.2 \\ 29.2 & 71.4644 & 36.1848 & 2.04532 & 36.18 & 1784.74 & 285.81 & 247.8 \\ 29.3 & 75.1237 & 37.0022 & 2.06155 & 36.30 & 1800.44 & 300.99 & 250.5 \\ 29.4 & 78.8652 & 37.8296 & 2.07774 & 36.42 & 1816.14 & 317.99 & 253.2\end{array}$


METHANE ISOTHERM AT $\mathrm{T}=115 . \mathrm{K}$

Second Virial Coefficient is $-305.4 \mathrm{~cm}^{3} / \mathrm{mol}$

$\begin{array}{llllllll}\rho & \mathrm{P} & \mathrm{dP} /\left.\mathrm{d} \rho\right|_{\mathrm{T}} & \mathrm{dP} /\left.\mathrm{dT}\right|_{\rho} & \mathrm{C}_{\mathrm{v}} & \mathrm{W} & \eta & \lambda\end{array}$

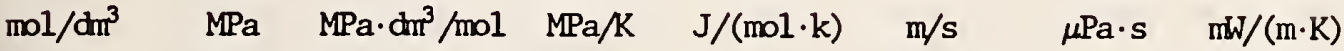

$\begin{array}{rrrrrrrr}0.1 & 0.0927 & 0.8977 & 0.00086 & 26.03 & 276.21 & 4.54 & 11.8\end{array}$

$\begin{array}{llllllll}26.1 & 1.2578 & 16.2212 & 1.48854 & 33.11 & 1309.70 & 110.98 & 180.6\end{array}$

$\begin{array}{llllllll}26.2 & 2.9072 & 16.7688 & 1.50646 & 33.17 & 1326.53 & 113.28 & 182.5\end{array}$

$\begin{array}{lllllllll}26.3 & 4.6118 & 17.3235 & 1.52423 & 33.24 & 1343.22 & 115.67 & 184.4\end{array}$

$\begin{array}{llllllll}26.4 & 6.3721 & 17.8854 & 1.54184 & 33.31 & 1359.76 & 118.15 & 186.3\end{array}$

$\begin{array}{lllllllll}26.5 & 8.1891 & 18.4545 & 1.55932 & 33.38 & 1376.17 & 120.74 & 188.2\end{array}$

$\begin{array}{lllllllll}26.6 & 10.0633 & 19.0311 & 1.57666 & 33.46 & 1392.47 & 123.43 & 190.2\end{array}$

$\begin{array}{llllllll}26.7 & 11.9956 & 19.6152 & 1.59388 & 33.54 & 1408.65 & 126.24 & 192.2\end{array}$

$\begin{array}{llllllll}26.8 & 13.9866 & 20.2070 & 1.61098 & 33.62 & 1424.74 & 129.18 & 194.2\end{array}$

$\begin{array}{llllllll}26.9 & 16.0372 & 20.8065 & 1.62797 & 33.71 & 1440.74 & 132.25 & 196.3\end{array}$

$\begin{array}{lllllllll}27.0 & 18.1482 & 21.4139 & 1.64485 & 33.80 & 1456.65 & 135.47 & 198.3\end{array}$

$\begin{array}{llllllll}27.1 & 20.3202 & 22.0292 & 1.66163 & 33.89 & 1472.49 & 138.85 & 200.4\end{array}$

$\begin{array}{llllllll}27.2 & 22.5543 & 22.6527 & 1.67833 & 33.99 & 1488.26 & 142.40 & 202.5\end{array}$

$\begin{array}{llllllll}27.3 & 24.8511 & 23.2844 & 1.69493 & 34.09 & 1503.98 & 146.13 & 204.7\end{array}$

$\begin{array}{llllllll}27.4 & 27.2114 & 23.9245 & 1.71146 & 34.19 & 1519.65 & 150.06 & 206.8\end{array}$

$\begin{array}{lllllllll}27.5 & 29.6362 & 24.5730 & 1.72792 & 34.29 & 1535.27 & 154.22 & 209.0\end{array}$

$\begin{array}{llllllll}27.6 & 32.1263 & 25.2300 & 1.74430 & 34.39 & 1550.85 & 158.61 & 211.2\end{array}$

$\begin{array}{llllllll}27.7 & 34.6825 & 25.8958 & 1.76062 & 34.50 & 1566.40 & 163.27 & 213.5\end{array}$

$\begin{array}{llllllll}27.8 & 37.3058 & 26.5703 & 1.77689 & 34.60 & 1581.92 & 168.21 & 215.7\end{array}$

$\begin{array}{llllllll}27.9 & 39.9969 & 27.2537 & 1.79310 & 34.71 & 1597.42 & 173.48 & 218.0\end{array}$

$\begin{array}{llllllllll}28.0 & 42.7568 & 27.9460 & 1.80927 & 34.82 & 1612.91 & 179.10 & 220.4\end{array}$

$\begin{array}{llllllll}28.1 & 45.5864 & 28.6475 & 1.82539 & 34.93 & 1628.38 & 185.11 & 222.7\end{array}$

$\begin{array}{lllllllll}28.2 & 48.4866 & 29.3581 & 1.84147 & 35.04 & 1643.84 & 191.56 & 225.1\end{array}$

$\begin{array}{lllllllll}28.3 & 51.4583 & 30.0780 & 1.85751 & 35.15 & 1659.30 & 198.50 & 227.5\end{array}$

$\begin{array}{llllllll}28.4 & 54.5025 & 30.8072 & 1.87353 & 35.27 & 1674.76 & 206.00 & 229.9\end{array}$

$\begin{array}{llllllll}28.5 & 57.6201 & 31.5459 & 1.88951 & 35.38 & 1690.23 & 214.12 & 232.4\end{array}$

$\begin{array}{lllllllll}28.6 & 60.8120 & 32.2941 & 1.90547 & 35.50 & 1705.70 & 222.95 & 234.9\end{array}$

$\begin{array}{lllllllll}28.7 & 64.0792 & 33.0519 & 1.92141 & 35.61 & 1721.17 & 232.60 & 237.4\end{array}$

$\begin{array}{llllllll}28.8 & 67.4227 & 33.8193 & 1.93733 & 35.73 & 1736.66 & 243.19 & 240.0\end{array}$

$\begin{array}{llllllll}28.9 & 70.8434 & 34.5965 & 1.95323 & 35.84 & 1752.16 & 254.86 & 242.5\end{array}$

$\begin{array}{lllllllll}29.0 & 74.3423 & 35.3834 & 1.96911 & 35.96 & 1767.68 & 267.79 & 245.2\end{array}$

$\begin{array}{lllllllll}29.1 & 77.9204 & 36.1801 & 1.98499 & 36.07 & 1783.21 & 282.23 & 247.8\end{array}$

$\begin{array}{llllllll}29.2 & 81.5787 & 36.9867 & 2.00085 & 36.19 & 1798.76 & 298.44 & 250.5\end{array}$

$\begin{array}{lllllllll}29.3 & 85.3181 & 37.8033 & 2.01671 & 36.30 & 1814.33 & 316.80 & 253.2\end{array}$

$\begin{array}{llllllll}29.4 & 89.1397 & 38.6297 & 2.03256 & 36.42 & 1829.92 & 337.76 & 255.9\end{array}$

$\begin{array}{llllllll}29.5 & 93.0444 & 39.4662 & 2.04841 & 36.54 & 1845.53 & 361.94 & 258.7\end{array}$ $\begin{array}{llllllll}29.6 & 97.0332 & 40.3126 & 2.06425 & 36.65 & 1861.16 & 390.15 & 261.5\end{array}$ 
METHANE ISOTHERM AT $\mathrm{T}=120 . \mathrm{K}$

Second Virial Coefficient is $-280.9 \mathrm{~cm}^{3} / \mathrm{mol}$

\begin{tabular}{|c|c|c|c|c|c|c|c|}
\hline$\rho$ & $\mathrm{P}$ & $\mathrm{dP} /\left.\mathrm{d} \rho\right|_{\mathrm{T}}$ & $\mathrm{dP} /\left.\mathrm{dT}\right|_{\rho}$ & $\mathrm{C}_{\mathrm{v}}$ & W & $\eta$ & $\lambda$ \\
\hline $1 / \mathrm{dm}^{3}$ & $\mathrm{MPa}$ & $\mathrm{MPa} \cdot \mathrm{dm}^{3} / \mathrm{mol}$ & $\mathrm{MPa} / \mathrm{K}$ & $\mathrm{J} /(\mathrm{mol} \cdot \mathrm{k})$ & $\mathrm{m} / \mathrm{s}$ & $\mu \mathrm{Pa} \cdot \mathrm{s}$ & $\mathrm{mW} /(\mathrm{m} \cdot \mathrm{K})$ \\
\hline 0.1 & 0.0970 & 0.9418 & 0.00085 & 25.66 & 282.86 & 4.73 & 12.4 \\
\hline 0.2 & 0.1884 & 0.8864 & 0.00176 & 26.61 & 277.46 & 4.76 & 12.7 \\
\hline 25.6 & 0.7676 & 14.4874 & 1.38452 & 32.66 & 1254.12 & 98.83 & 172.9 \\
\hline 25.7 & 2.2416 & 14.9930 & 1.40170 & 32.72 & 1270.66 & 100.74 & 174.7 \\
\hline 25.8 & 3.7665 & 15.5056 & 1.41875 & 32.78 & 1287.05 & 102.72 & 176.5 \\
\hline 25 . & 5.3 & 51 & 1.43 & 32 & 1303.31 & 104.78 & 178.4 \\
\hline 26. & 6.9717 & 16 & 1.45248 & 32.91 & 1319.45 & 106.91 & 180.2 \\
\hline 26. & 8.6535 & 56 & 1.46917 & 32.99 & 1335.48 & 109.12 & 182.1 \\
\hline 26.2 & 10.3891 & 17.6267 & 1.48576 & 33.06 & 1351.40 & 111.42 & 184.0 \\
\hline 26.3 & 12.1791 & 18.1753 & 1.50225 & 33.14 & 1367.23 & 113.82 & 185.9 \\
\hline 26. & 4 & 13 & 1.5 & 2 & 97 & 116 & 101.7 \\
\hline 26. & 15.9 & 19 & 1.53496 & 33.31 & 139 & 118.92 & 189.9 \\
\hline 26.6 & 17.8837 & 19. & 1.55119 & 33.39 & 1414.22 & 121.64 & 191.8 \\
\hline 26.7 & 19.8992 & 20.4457 & 1.56734 & 33.48 & 1429.75 & 124.49 & 193.9 \\
\hline 26.8 & 21.9731 & 21.0329 & 1.58342 & 33.58 & 1445.22 & 127.47 & 195.9 \\
\hline 26. & 24. & & 3 & & & & \\
\hline 27.0 & 26.2 & 22 & $1.6]$ & 33 & 14 & 133 & 200.1 \\
\hline 27.1 & 28.5 & 34 & 1.63129 & 33. & 1491.36 & 137.35 & 202.2 \\
\hline 27.2 & 30.8 & 635 & 1.64713 & 33.97 & 1506.68 & 141.00 & 204.3 \\
\hline 27.3 & 33.2457 & 24.0921 & 1.66294 & 34.07 & 1521.96 & 144.85 & 206.5 \\
\hline 27. & J & & 1.0 & & & & \\
\hline 27 . & 38 & 2 & 1.6 & 34 & 4 & 15 & 210.9 \\
\hline 27.6 & 40.7 & 99 & 1.7 & 34.38 & 15 & 157.83 & 213.2 \\
\hline 27.7 & 43.3981 & 935 & 1.72577 & 34.49 & 1582.94 & 162.72 & 215.4 \\
\hline 27.8 & 46.1010 & 27.3660 & 1.74140 & 34.60 & 1598.17 & 167.94 & 217.7 \\
\hline 27.9 & 48. & & 1.1 & & & $17:$ & \\
\hline 28.0 & 51.7 & 28 & 1.7 & 34 & 16 & 179 & 222.4 \\
\hline 28.1 & 54.6196 & 29.4387 & 1.78819 & 34.92 & 1643.87 & 185.98 & 224.8 \\
\hline 28.2 & 57.5989 & 30.1481 & 1.80376 & 35.03 & 1659.12 & 192.96 & 227.2 \\
\hline 28.3 & 60.6495 & 30.8670 & 1.81931 & 35.15 & 1674.39 & 200.52 & 229.6 \\
\hline 28.4 & 63. & & 1.83 & & & $20 \varepsilon$ & 232.1 \\
\hline & & & & & 1704 & 217 & 234.6 \\
\hline 28.6 & 70.2396 & 33.0810 & 1.86596 & 35.48 & 1720.29 & 227.64 & 237.1 \\
\hline 28.7 & 73.5855 & 33.8383 & 1.88151 & 35.59 & 1735.63 & 238.55 & 239.7 \\
\hline 28.8 & 77.0076 & 34.6054 & 1.89706 & 35.71 & 1750.99 & 250.67 & 242.3 \\
\hline
\end{tabular}


METHANE ISOTHERM AT $\mathrm{T}=120 . \mathrm{K}$ (continued)

$\begin{array}{cccccccc}\rho & \mathrm{P} & \mathrm{dP} /\left.\mathrm{d} \rho\right|_{\mathrm{T}} & \mathrm{dP} /\left.\mathrm{dT}\right|_{\rho} & \mathrm{C}_{\mathrm{v} .} & \mathrm{W} & \eta & \lambda \\ \mathrm{mol} / \mathrm{dm}^{3} & \mathrm{MPa} & \mathrm{MPa} \cdot \mathrm{dm}^{3} / \mathrm{mol} & \mathrm{MPa} / \mathrm{K} & \mathrm{J} /(\mathrm{mol} \cdot \mathrm{k}) & \mathrm{m} / \mathrm{s} & \mu \mathrm{Pa} \cdot \mathrm{s} & \mathrm{mW} /(\mathrm{m} \cdot \mathrm{K}) \\ 28.9 & 80.5069 & 35.3824 & 1.91262 & 35.82 & 1766.38 & 264.20 & 244.9 \\ 29.0 & 84.0844 & 36.1692 & 1.92818 & 35.93 & 1781.80 & 279.43 & 247.6 \\ 29.1 & 87.7410 & 36.9659 & 1.94376 & 36.04 & 1797.24 & 296.71 & 250.2 \\ 29.2 & 91.4779 & 37.7726 & 1.95934 & 36.16 & 1812.71 & 316.49 & 253.0 \\ 29.3 & 95.2959 & 38.5893 & 1.97494 & 36.27 & 1828.21 & 339.36 & 255.7 \\ 29.4 & 99.1961 & 39.4160 & 1.99055 & 36.38 & 1843.74 & 366.14 & 258.5\end{array}$

\footnotetext{
METHANE ISOTHERM AT $\mathrm{T}=130 . \mathrm{K}$

Second Virial Coefficient is $-240.4 \mathrm{~cm}^{3} / \mathrm{mol}$
}

\begin{tabular}{rrrrrrrrr}
$\rho$ & \multicolumn{1}{c}{$\mathrm{P}$} & $\mathrm{dP} /\left.\mathrm{d} \rho\right|_{\mathrm{T}}$ & $\mathrm{dP} /\left.\mathrm{dT}\right|_{\rho}$ & $\mathrm{C}_{\mathrm{v}}$ & $\mathrm{W}$ & $\eta$ & $\lambda$ \\
$\mathrm{mol} / \mathrm{dm}^{3}$ & $\mathrm{MPa}$ & $\mathrm{MPa} \cdot \mathrm{dm}^{3} / \mathrm{mol}$ & $\mathrm{MPa} / \mathrm{K}$ & $\mathrm{J} /(\mathrm{mol} \cdot \mathrm{k})$ & $\mathrm{m} / \mathrm{s}$ & $\mu \mathrm{Pa} \cdot \mathrm{s}$ & $\mathrm{mW} /(\mathrm{m} \cdot \mathrm{K})$ \\
& & & & & & & & \\
0.1 & 0.1055 & 1.0291 & 0.00085 & 25.42 & 295.28 & 5.12 & 13.6 \\
0.2 & 0.2058 & 0.9780 & 0.00174 & 25.84 & 290.85 & 5.14 & 13.8 \\
0.3 & 0.3011 & 0.9271 & 0.00266 & 26.30 & 286.38 & 5.17 & 14.1 \\
24.6 & 0.7020 & 11.5089 & 1.19572 & 31.87 & 1148.06 & 80.46 & 158.4 \\
24.7 & 1.8742 & 11.9369 & 1.21142 & 31.92 & 1163.96 & 81.83 & 160.0 \\
& & & & & & & \\
24.8 & 3.0896 & 12.3714 & 1.22704 & 31.96 & 1179.73 & 83.24 & 161.7 \\
24.9 & 4.3487 & 12.8126 & 1.24258 & 32.02 & 1195.39 & 84.69 & 163.3 \\
25.0 & 5.6523 & 13.2604 & 1.25805 & 32.07 & 1210.94 & 86.19 & 165.0 \\
25.1 & 7.0010 & 13.7151 & 1.27344 & 32.13 & 1226.39 & 87.74 & 166.7 \\
25.2 & 8.3956 & 14.1765 & 1.28877 & 32.19 & 1241.76 & 89.34 & 168.5 \\
& & & & & & & \\
25.3 & 9.8366 & 14.6450 & 1.30403 & 32.26 & 1257.04 & 91.00 & 170.2 \\
25.4 & 11.3248 & 15.1204 & 1.31924 & 32.33 & 1272.24 & 92.72 & 172.0 \\
25.5 & 12.8609 & 15.6030 & 1.33438 & 32.40 & 1287.38 & 94.49 & 173.8 \\
25.6 & 14.4456 & 16.0927 & 1.34948 & 32.48 & 1302.45 & 96.34 & 175.6 \\
25.7 & 16.0797 & 16.5898 & 1.36452 & 32.56 & 1317.46 & 98.25 & 177.4 \\
25.8 & 17.7638 & 17.0943 & 1.37952 & 32.64 & 1332.42 & 100.24 & 179.2 \\
25.9 & 19.4988 & 17.6062 & 1.39448 & 32.72 & 1347.34 & 102.31 & 181.1 \\
26.0 & 21.2853 & 18.1257 & 1.40940 & 32.81 & 1362.22 & 104.47 & 183.0 \\
26.1 & 23.1242 & 18.6529 & 1.42429 & 32.89 & 1377.06 & 106.72 & 184.9 \\
26.2 & 25.0162 & 19.1879 & 1.43915 & 32.98 & 1391.88 & 109.07 & 186.9 \\
26.3 & 26.9620 & 19.7307 & 1.45398 & 33.07 & 1406.67 & 111.52 & 188.8 \\
26.4 & 28.9626 & 20.2815 & 1.46879 & 33.16 & 1421.44 & 114.09 & 190.8 \\
26.5 & 31.0186 & 20.8404 & 1.48358 & 33.26 & 1436.20 & 116.79 & 192.9 \\
26.6 & 33.1309 & 21.4075 & 1.49835 & 33.35 & 1450.95 & 119.61 & 194.9 \\
26.7 & 35.3004 & 21.9828 & 1.51311 & 33.45 & 1465.69 & 122.59 & 197.0
\end{tabular}


METHANE ISOTHERM AT $\mathrm{T}=130 . \mathrm{K}$ (continued)

$\begin{array}{ccccccccc}\rho & \mathrm{P} & \mathrm{dP} /\left.\mathrm{d} \rho\right|_{\mathrm{T}} & \mathrm{dP} /\left.\mathrm{dT}\right|_{\rho} & \mathrm{C}_{\mathrm{v}} & \mathrm{W} & \eta & \lambda \\ \mathrm{mol} / \mathrm{dm}^{3} & \mathrm{MPa} & \mathrm{MPa} \cdot \mathrm{dm}^{3} / \mathrm{mol} & \mathrm{MPa} / \mathrm{K} & \mathrm{J} /(\mathrm{mol} \cdot \mathrm{k}) & \mathrm{m} / \mathrm{s} & \mu \mathrm{Pa} \cdot \mathrm{s} & \mathrm{mW} /(\mathrm{m} \cdot \mathrm{K}) \\ & & & & & & & \\ 26.8 & 37.5278 & 22.5665 & 1.52786 & 33.55 & 1480.44 & 125.72 & 199.0 \\ 26.9 & 39.8140 & 23.1587 & 1.54260 & 33.65 & 1495.18 & 129.03 & 201.2 \\ 27.0 & 42.1598 & 23.7594 & 1.55734 & 33.74 & 1509.93 & 132.53 & 203.3 \\ 27.1 & 44.5661 & 24.3688 & 1.57209 & 33.84 & 1524.69 & 136.23 & 205.5 \\ 27.2 & 47.0338 & 24.9869 & 1.58683 & 33.95 & 1539.46 & 140.17 & 207.7 \\ & & & & & & & \\ 27.3 & 49.5638 & 25.6139 & 1.60158 & 34.05 & 1554.25 & 144.36 & 209.9 \\ 27.4 & 52.1569 & 26.2498 & 1.61633 & 34.15 & 1569.06 & 148.82 & 212.1 \\ 27.5 & 54.8141 & 26.8947 & 1.63110 & 34.25 & 1583.89 & 153.60 & 214.4 \\ 27.6 & 57.5362 & 27.5488 & 1.64588 & 34.35 & 1598.74 & 158.73 & 216.7 \\ 27.7 & 60.3241 & 28.2120 & 1.66068 & 34.46 & 1613.62 & 164.24 & 219.0 \\ & & & & & & & \\ 27.8 & 63.1789 & 28.8845 & 1.67550 & 34.56 & 1628.52 & 170.20 & 221.4 \\ 27.9 & 66.1013 & 29.5664 & 1.69033 & 34.67 & 1643.46 & 176.65 & 223.8 \\ 28.0 & 69.0925 & 30.2578 & 1.70519 & 34.77 & 1658.42 & 183.67 & 226.2 \\ 28.1 & 72.1532 & 30.9586 & 1.72007 & 34.87 & 1673.42 & 191.34 & 228.6 \\ 28.2 & 75.2845 & 31.6691 & 1.73498 & 34.98 & 1688.46 & 199.76 & 231.1 \\ & & & & & & & \\ 28.3 & 78.4873 & 32.3892 & 1.74992 & 35.08 & 1703.53 & 209.04 & 233.6 \\ 28.4 & 81.7627 & 33.1190 & 1.76489 & 35.19 & 1718.64 & 219.34 & 236.1 \\ 28.5 & 85.1115 & 33.8586 & 1.77988 & 35.29 & 1733.79 & 230.83 & 238.7 \\ 28.6 & 88.5347 & 34.6080 & 1.79492 & 35.40 & 1748.97 & 243.76 & 241.3 \\ 28.7 & 92.0334 & 35.3674 & 1.80998 & 35.50 & 1764.20 & 258.40 & 243.9 \\ 28.8 & 95.6085 & 36.1367 & 1.82509 & 35.60 & 1779.46 & 275.15 & 246.5 \\ 28.9 & 99.2611 & 36.9159 & 1.84023 & 35.71 & 1794.77 & 294.50 & 249.2\end{array}$

$\begin{array}{llllllll}\rho & \mathrm{P} & \mathrm{dP} /\left.\mathrm{d} \rho\right|_{\mathrm{T}} & \mathrm{dP} /\left.\mathrm{dT}\right|_{\rho} & \mathrm{C}_{\mathrm{v}} & \mathrm{W} & \eta & \lambda\end{array}$

$\begin{array}{llllllll}\mathrm{mol} / \mathrm{dm}^{3} & \mathrm{MPa} & \mathrm{MPa} \cdot \mathrm{dm}^{3} / \mathrm{mol} & \mathrm{MPa} / \mathrm{K} & \mathrm{J} /(\mathrm{mol} \cdot \mathrm{k}) \quad \mathrm{m} / \mathrm{s} \quad \mu \mathrm{Pa} \cdot \mathrm{s} \quad \mathrm{mW} /(\mathrm{m} \cdot \mathrm{K})\end{array}$

$\begin{array}{rrrrrrrr}0.1 & 0.1140 & 1.1157 & 0.00085 & 25.32 & 307.06 & 5.50 & 14.7 \\ 0.2 & 0.2232 & 1.0678 & 0.00173 & 25.66 & 303.13 & 5.53 & 14.9 \\ 0.3 & 0.3276 & 1.0204 & 0.00263 & 25.96 & 299.21 & 5.56 & 15.2 \\ 0.4 & 0.4272 & 0.9734 & 0.00357 & 26.27 & 295.30 & 5.59 & 15.5 \\ 0.5 & 0.5222 & 0.9263 & 0.00455 & 26.66 & 291.35 & 5.62 & 15.8 \\ & & & & & & & \\ 0.6 & 0.6125 & 0.8786 & 0.00559 & 27.22 & 287.31 & 5.65 & 16.1 \\ 23.5 & 0.6559 & 8.7615 & 1.01661 & 31.22 & 1034.01 & 66.46 & 143.6 \\ 23.6 & 1.5496 & 9.1140 & 1.03088 & 31.25 & 1049.26 & 67.46 & 145.1 \\ 23.7 & 2.4789 & 9.4726 & 1.04512 & 31.28 & 1064.42 & 68.48 & 146.6 \\ 23.8 & 3.4443 & 9.8374 & 1.05931 & 31.31 & 1079.49 & 69.53 & 148.0\end{array}$


METHANE ISOTHERM AT $T=140 . \mathrm{K}$ (continued)

\begin{tabular}{|c|c|c|c|c|c|c|c|}
\hline$\rho$ & $P$ & $\mathrm{dP} /\left.\mathrm{d} \rho\right|_{\mathrm{T}}$ & $\mathrm{dP} /\left.\mathrm{dT}\right|_{\rho}$ & $\mathrm{C}_{\mathrm{v}}$ & W & $\eta$ & $\lambda$ \\
\hline $\mathrm{mol} / \mathrm{cm}^{3}$ & $\mathrm{MPa}$ & $\mathrm{MPa} \cdot \mathrm{cm}^{3} / \mathrm{mol}$ & $\mathrm{MPa} / \mathrm{K}$ & $\mathrm{J} /(\mathrm{mol} \cdot \mathrm{k})$ & $\mathrm{m} / \mathrm{s}$ & $\mu \mathrm{Pa} \cdot \mathrm{s}$ & $\mathrm{mW} /(\mathrm{m} \cdot \mathrm{K})$ \\
\hline 23.9 & 4.4466 & 10.2084 & 1.07346 & 31.35 & 1094.46 & 70.61 & 149.5 \\
\hline 24.0 & 5.4862 & 10.5856 & 1.08758 & 31.39 & 1109.35 & 71.72 & 151.1 \\
\hline 24.1 & 6.5639 & 10.9692 & 1.10166 & 31.44 & 1124.16 & 72.86 & 152.6 \\
\hline 24.2 & 7.6803 & 11.3591 & 1.11571 & 31.49 & 1138.91 & 74.03 & 154.1 \\
\hline 24.3 & 8.8359 & 11.7556 & 1.12972 & 31.54 & 1153.58 & 75.24 & 155.7 \\
\hline 24.4 & 10.0316 & 585 & 1.14371 & 31.60 & 1168.19 & 76.48 & 157.3 \\
\hline 24.5 & 11.2679 & 12.5680 & 1.15767 & 31.66 & 1182.74 & 77.76 & 158.9 \\
\hline 24.6 & 12.5454 & 12.9843 & 1.17160 & 31.72 & 1197.24 & 79.08 & 160.6 \\
\hline 24.7 & 13.8649 & 13.4072 & 1.18551 & 31.78 & 1211.69 & 80.44 & 162.2 \\
\hline 24.8 & 15.2271 & 13.8370 & 1.19940 & 31.85 & 1226.10 & 81.84 & 163.9 \\
\hline 24.9 & 16.6326 & 14 & 1.21327 & 31.92 & 1240.47 & 83.30 & 165.6 \\
\hline 25.0 & 18.0821 & 14.7174 & 1.22712 & 31.99 & 1254.81 & 84.80 & 167.3 \\
\hline 25.1 & 19.5763 & 15.1682 & 1.24096 & 32.07 & 1269.12 & 86.36 & 169.0 \\
\hline 25.2 & 21.1159 & 15.6261 & 1.25479 & 32.14 & 1283.41 & 87.97 & 170.8 \\
\hline 25.3 & 22.7017 & 16.0913 & 1.26861 & 32.22 & 1297.68 & 89.64 & 172.5 \\
\hline 25.4 & 24.3 & 16 & 1.2 & 32.30 & 1.93 & 91.38 & 174.3 \\
\hline 25. & 26.0148 & 17. & 1.29 & 32.38 & 1326.17 & 93.18 & 176.1 \\
\hline 25.6 & 27.7434 & 17. & 1.31003 & 32.47 & 1340.40 & 95.06 & 178.0 \\
\hline 25.7 & 29.5212 & 18.0262 & 1.32384 & 32.55 & 1354.62 & 97.01 & 179.9 \\
\hline 25.8 & 31.3489 & 18.5290 & 1.33766 & 32.64 & 1368.85 & 99.05 & 181.7 \\
\hline 25 . & 33.22 & 19 & 1.3 & 32.72 & 13 & 8 & 183.6 \\
\hline 26. & 35.1571 & 19. & 1.36530 & 32.81 & 1397.32 & 103.40 & 185.6 \\
\hline 26.1 & 37.1392 & 20. & 1.37914 & 32.90 & 1411.57 & 105.73 & 187.5 \\
\hline 26.2 & 39.1742 & 20.6188 & 1. 39299 & 32.99 & 1425.83 & 108.18 & 189.5 \\
\hline 26.3 & 41.2632 & 21.1614 & 1.40686 & 33.08 & 1440.11 & 110.74 & 191.5 \\
\hline 26. & 43.4 & 23 & 1.4 & 33 & 1454.40 & & 193.5 \\
\hline 26.5 & 45.6059 & 22 . & 1.43465 & 33 & 1468.72 & 116.28 & 195.6 \\
\hline 26.6 & 47.8614 & 22.8393 & 1.44 & 33.36 & 1483.07 & 119.28 & 197.7 \\
\hline 26.7 & 50.1741 & 23.4155 & 1.46254 & 33.45 & 1497.44 & 122.46 & 199.8 \\
\hline 26.8 & 52.5448 & 24.0003 & 1.47652 & 33.54 & 1511.84 & 125.82 & 201.9 \\
\hline 26.9 & 54.97 & 39 & 1.4 & 33 & 15 & 39 & 204.1 \\
\hline 27.0 & 57.46 & 25 . & 1.5 & 33.73 & 1540.74 & 133.19 & 206.2 \\
\hline 27.1 & 60.0140 & 25.8 & 1.51 & 33.83 & 1555.24 & 137.25 & 208.4 \\
\hline 27.2 & 62.6257 & 26.4279 & 1.53276 & 33.92 & 1569.78 & 141.60 & 210.7 \\
\hline 27.3 & 65.2999 & 27.0572 & 1.54691 & 34.02 & 1584.35 & 146.26 & 212.9 \\
\hline 27.4 & 68.03 & 27 . & 1.5 & 34.11 & 1598.97 & 101 & 215.2 \\
\hline 27.5 & 70.8 & 28. & 1.57 & 34.21 & 1613.63 & 71 & 217.6 \\
\hline 27.6 & 73.7064 & 29.0005 & 1.58960 & 34.30 & 1628.34 & 162.59 & 219.9 \\
\hline 27.7 & 76.6397 & 29.6669 & 1.60391 & 34.40 & 1643.09 & 169.00 & 222.3 \\
\hline 27.8 & 79.6401 & 30.3429 & 1.61828 & 34.50 & 1657.88 & 176.00 & 224.7 \\
\hline
\end{tabular}


METHANE ISOTHERM AT $\mathrm{T}=140 . \mathrm{K}$ (continued)

$\begin{array}{cccccccc}\rho & \mathrm{P} & \mathrm{dP} /\left.\mathrm{d} \rho\right|_{\mathrm{T}} & \mathrm{dP} /\left.\mathrm{dT}\right|_{\rho} & \mathrm{C}_{\mathrm{v}} & \mathrm{W} & \eta & \lambda \\ \mathrm{mol} / \mathrm{dm}^{3} & \mathrm{MPa} & \mathrm{MPa} \cdot \mathrm{dm}^{3} / \mathrm{mol} & \mathrm{MPa} / \mathrm{K} & \mathrm{J} /(\mathrm{mol} \cdot \mathrm{k}) & \mathrm{m} / \mathrm{s} & \mu \mathrm{Pa} \cdot \mathrm{s} & \mathrm{mW} /(\mathrm{m} \cdot \mathrm{K}) \\ & & & & & & & \\ 27.9 & 82.7086 & 31.0283 & 1.63269 & 34.59 & 1672.72 & 183.70 & 227.1 \\ 28.0 & 85.8461 & 31.7234 & 1.64715 & 34.69 & 1687.61 & 192.20 & 229.6 \\ 28.1 & 89.0536 & 32.4282 & 1.66166 & 34.78 & 1702.55 & 201.64 & 232.1 \\ 28.2 & 92.3321 & 33.1428 & 1.67622 & 34.88 & 1717.53 & 212.20 & 234.6 \\ 28.3 & 95.6825 & 33.8671 & 1.69084 & 34.97 & 1732.57 & 224.10 & 237.1 \\ 28.4 & 99.1058 & 34.6013 & 1.70551 & 35.06 & 1747.66 & 237.61 & 239.7\end{array}$

METHANE ISOTHERM AT $\mathrm{T}=150 . \mathrm{K}$

Second Virial Coefficient is $-182.8 \mathrm{~cm}^{3} / \mathrm{mol}$

\begin{tabular}{rrrllllll}
$\rho$ & $\mathrm{P}$ & $\mathrm{dP} /\left.\mathrm{d} \rho\right|_{\mathrm{T}}$ & $\mathrm{dP} /\left.\mathrm{dT}\right|_{\rho}$ & \multicolumn{2}{l}{$\mathrm{C}_{\mathrm{v}}$} & $W$ & $\eta$ & $\lambda$ \\
$\mathrm{mol} / \mathrm{dm}^{3}$ & $\mathrm{MPa}$ & $\mathrm{MPa} \cdot \mathrm{dm}^{3} / \mathrm{mol}$ & $\mathrm{MPa} / \mathrm{K}$ & $\mathrm{J} /(\mathrm{mol} \cdot \mathrm{k})$ & $\mathrm{m} / \mathrm{s}$ & $\mu \mathrm{Pa} \cdot \mathrm{s}$ & $\mathrm{mW} /(\mathrm{m} \cdot \mathrm{K})$ \\
& & & & & & & \\
0.2 & 0.2404 & 1.1568 & 0.00172 & 25.54 & 314.82 & 5.92 & 16.1 \\
0.4 & 0.4628 & 1.0681 & 0.00354 & 26.06 & 307.80 & 5.98 & 16.6 \\
0.6 & 0.6677 & 0.9810 & 0.00548 & 26.58 & 300.82 & 6.04 & 17.1 \\
0.8 & 0.8553 & 0.8945 & 0.00755 & 27.26 & 293.81 & 6.10 & 17.7 \\
1.0 & 1.0254 & 0.8069 & 0.00983 & 28.38 & 286.57 & 6.17 & 18.4 \\
& & & & & & & \\
22.4 & 1.6116 & 6.6435 & 0.86253 & 30.77 & 929.89 & 56.32 & 130.4 \\
22.6 & 2.9977 & 7.2213 & 0.88808 & 30.79 & 958.69 & 57.87 & 133.0 \\
22.8 & 4.5016 & 7.8218 & 0.91361 & 30.82 & 987.23 & 59.48 & 135.7 \\
23.0 & 6.1279 & 8.4452 & 0.93913 & 30.87 & 1015.53 & 61.17 & 138.4 \\
23.2 & 7.8813 & 9.0921 & 0.96463 & 30.94 & 1043.61 & 62.94 & 141.2 \\
& & & & & & & \\
23.4 & 9.7664 & 9.7628 & 0.99011 & 31.02 & 1071.51 & 64.80 & 144.0 \\
23.6 & 11.7880 & 10.4578 & 1.01559 & 31.12 & 1099.25 & 66.76 & 146.9 \\
23.8 & 13.9511 & 11.1776 & 1.04108 & 31.22 & 1126.85 & 68.82 & 149.9 \\
24.0 & 16.2607 & 11.9228 & 1.06657 & 31.33 & 1154.34 & 71.00 & 152.9 \\
24.2 & 18.7220 & 12.6939 & 1.09208 & 31.46 & 1181.76 & 73.31 & 156.1 \\
& & & & & & & \\
24.4 & 21.3401 & 13.4916 & 1.11763 & 31.59 & 1209.12 & 75.76 & 159.3 \\
24.6 & 24.1204 & 14.3163 & 1.14322 & 31.73 & 1236.46 & 78.38 & 162.6 \\
24.8 & 27.0685 & 15.1689 & 1.16886 & 31.87 & 1263.80 & 81.17 & 165.9 \\
25.0 & 30.1899 & 16.0499 & 1.19458 & 32.02 & 1291.16 & 84.17 & 169.4 \\
25.2 & 33.4904 & 16.9600 & 1.22038 & 32.18 & 1318.57 & 87.41 & 172.9 \\
25.4 & 36.9759 & 17.9000 & 1.24628 & 32.34 & 1346.05 & 90.90 & 176.5 \\
25.6 & 40.6524 & 18.8705 & 1.27230 & 32.50 & 1373.62 & 94.69 & 180.3 \\
25.8 & 44.5261 & 19.8723 & 1.29844 & 32.67 & 1401.31 & 98.84 & 184.1 \\
26.0 & 48.6034 & 20.9060 & 1.32473 & 32.83 & 1429.11 & 103.39 & 188.0 \\
26.2 & 52.8907 & 21.9726 & 1.35117 & 33.00 & 1457.07 & 108.42 & 192.0
\end{tabular}


METHANE ISOTHERM AT $\mathrm{T}=150 . \mathrm{K}$ (continued)

$\begin{array}{llllllll}26.4 & 57.3947 & 23.0726 & 1.37778 & 33.18 & 1485.18 & 114.02 & 196.0 \\ 26.6 & 62.1220 & 24.2067 & 1.40458 & 33.35 & 1513.47 & 120.31 & 200.2 \\ 26.8 & 67.0797 & 25.3758 & 1.43158 & 33.52 & 1541.94 & 127.42 & 204.5 \\ 27.0 & 72.2747 & 26.5805 & 1.45879 & 33.70 & 1570.61 & 135.56 & 208.9 \\ 27.2 & 77.7143 & 27.8215 & 1.48623 & 33.87 & 1599.49 & 144.98 & 213.5 \\ & & & & & & & \\ 27.4 & 83.4058 & 29.0994 & 1.51390 & 34.04 & 1628.58 & 156.04 & 218.1 \\ 27.6 & 89.3566 & 30.4149 & 1.54181 & 34.22 & 1657.89 & 169.25 & 222.8 \\ 27.8 & 95.5743 & 31.7686 & 1.56999 & 34.39 & 1687.42 & 185.32 & 227.7\end{array}$

\begin{aligned} \hline METHANE & ISOTHERM AT $\mathrm{T}=160 . \mathrm{K} \\ &$ Second Virial Coefficient is $-161.7 \mathrm{~cm}^{3} / \mathrm{mol}\end{aligned}$

$\begin{array}{rrrllllll}\rho & \mathrm{P} & \mathrm{dP} /\left.\mathrm{d} \rho\right|_{\mathrm{T}} & \mathrm{dP} /\left.\mathrm{dT}\right|_{\rho} & \mathrm{C}_{\mathrm{v}} & \mathrm{W} & \eta & \lambda \\ \mathrm{mol} / \mathrm{dm}^{3} & \mathrm{MPa} & \mathrm{MPa} \cdot \mathrm{dm}^{3} / \mathrm{mol} & \mathrm{MPa} / \mathrm{K} & \mathrm{J} /(\mathrm{mol} \cdot \mathrm{k}) & \mathrm{m} / \mathrm{s} & \mu \mathrm{Pa} \cdot \mathrm{s} & \mathrm{mW} /(\mathrm{m} \cdot \mathrm{K}) \\ & & & & & & & \\ 0.2 & 0.2575 & 1.2451 & 0.00171 & 25.46 & 326.03 & 6.30 & 17.3 \\ 0.4 & 0.4981 & 1.1615 & 0.00352 & 25.90 & 319.70 & 6.36 & 17.7 \\ 0.6 & 0.7222 & 1.0796 & 0.00542 & 26.34 & 313.44 & 6.43 & 18.2 \\ 0.8 & 0.9301 & 0.9993 & 0.00743 & 26.78 & 307.23 & 6.50 & 18.7 \\ 1.0 & 1.1220 & 0.9203 & 0.00954 & 27.29 & 301.07 & 6.57 & 19.2 \\ & & & & & & & \\ 1.2 & 1.2983 & 0.8421 & 0.01179 & 27.93 & 294.88 & 6.65 & 19.9 \\ 1.4 & 1.4589 & 0.7643 & 0.01421 & 28.81 & 288.61 & 6.73 & 20.6 \\ 21.0 & 1.7596 & 4.3943 & 0.69792 & 30.53 & 796.71 & 46.92 & 115.4 \\ 21.2 & 2.6814 & 4.8266 & 0.72026 & 30.49 & 823.69 & 48.08 & 117.6 \\ 21.4 & 3.6916 & 5.2790 & 0.74273 & 30.46 & 850.53 & 49.28 & 119.9 \\ & & & & & & & \\ 21.6 & 4.7944 & 5.7517 & 0.76530 & 30.46 & 877.25 & 50.53 & 122.2 \\ 21.8 & 5.9937 & 6.2450 & 0.78797 & 30.47 & 903.84 & 51.82 & 124.6 \\ 22.0 & 7.2938 & 6.7594 & 0.81074 & 30.49 & 930.32 & 53.17 & 127.0 \\ 22.2 & 8.6989 & 7.2951 & 0.83360 & 30.53 & 956.70 & 54.58 & 129.5 \\ 22.4 & 10.2133 & 7.8527 & 0.85655 & 30.59 & 982.99 & 56.04 & 132.0 \\ & & & & & & & \\ 22.6 & 11.8414 & 8.4324 & 0.87958 & 30.65 & 1009.20 & 57.58 & 134.6 \\ 22.8 & 13.5877 & 9.0347 & 0.90270 & 30.73 & 1035.34 & 59.18 & 137.3 \\ 23.0 & 15.4568 & 9.6601 & 0.92590 & 30.81 & 1061.45 & 60.86 & 140.0 \\ 23.2 & 17.4533 & 10.3091 & 0.94920 & 30.91 & 1087.53 & 62.62 & 142.8 \\ 23.4 & 19.5821 & 10.9822 & 0.97259 & 31.01 & 1113.59 & 64.48 & 145.7 \\ 23.6 & 21.8479 & 11.6800 & 0.99608 & 31.13 & 1139.67 & 66.44 & 148.6 \\ 23.8 & 24.2557 & 12.4029 & 1.01968 & 31.24 & 1165.77 & 68.51 & 151.6 \\ 24.0 & 26.8108 & 13.1517 & 1.04339 & 31.37 & 1191.92 & 70.71 & 154.7 \\ 24.2 & 29.5182 & 13.9268 & 1.06723 & 31.50 & 1218.13 & 73.04 & 157.9 \\ 24.4 & 32.3833 & 14.7290 & 1.09120 & 31.64 & 1244.42 & 75.53 & 161.1\end{array}$


METHANE ISOTHERM AT $\mathrm{T}=160 . \mathrm{K}$ (continued)

$\begin{array}{ccccccccc}\rho & \mathrm{P} & \mathrm{dP} /\left.\mathrm{d} \rho\right|_{\mathrm{T}} & \mathrm{dP} /\left.\mathrm{dT}\right|_{\rho} & \mathrm{C}_{\mathrm{v}} & \mathrm{W} & \eta & \lambda \\ \mathrm{mol} / \mathrm{dm}^{3} & \mathrm{MPa} & \mathrm{MPa} \cdot \mathrm{dm}^{3} / \mathrm{mol} & \mathrm{MPa} / \mathrm{K} & \mathrm{J} /(\mathrm{mol} \cdot \mathrm{k}) & \mathrm{m} / \mathrm{s} & \mu \mathrm{Pa} \cdot \mathrm{s} & \mathrm{mW} /(\mathrm{m} \cdot \mathrm{K}) \\ & & & & & & & \\ 24.6 & 35.4116 & 15.5590 & 1.11532 & 31.78 & 1270.81 & 78.19 & 164.5 \\ 24.8 & 38.6088 & 16.4172 & 1.13959 & 31.92 & 1297.31 & 81.04 & 167.9 \\ 25.0 & 41.9805 & 17.3046 & 1.16402 & 32.07 & 1323.95 & 84.12 & 171.4 \\ 25.2 & 45.5326 & 18.2217 & 1.18863 & 32.22 & 1350.74 & 87.45 & 174.9 \\ 25.4 & 49.2712 & 19.1693 & 1.21343 & 32.37 & 1377.68 & 91.07 & 178.6 \\ & & & & & & & \\ 25.6 & 53.2024 & 20.1481 & 1.23842 & 32.52 & 1404.80 & 95.03 & 182.4 \\ 25.8 & 57.3326 & 21.1588 & 1.26363 & 32.68 & 1432.11 & 99.38 & 186.2 \\ 26.0 & 61.6681 & 22.2022 & 1.28906 & 32.84 & 1459.62 & 104.20 & 190.2 \\ 26.2 & 66.2157 & 23.2789 & 1.31473 & 32.99 & 1487.34 & 109.57 & 194.2 \\ 26.4 & 70.9820 & 24.3897 & 1.34063 & 33.15 & 1515.28 & 115.60 & 198.4 \\ & & & & & & & \\ 26.6 & 75.9739 & 25.5354 & 1.36680 & 33.31 & 1543.45 & 122.44 & 202.6 \\ 26.8 & 81.1985 & 26.7165 & 1.39323 & 33.47 & 1571.85 & 130.29 & 207.0 \\ 27.0 & 86.6629 & 27.9338 & 1.41994 & 33.62 & 1600.49 & 139.39 & 211.5 \\ 27.2 & 92.3745 & 29.1880 & 1.44695 & 33.78 & 1629.38 & 150.11 & 216.0 \\ 27.4 & 98.3406 & 30.4796 & 1.47425 & 33.93 & 1658.53 & 162.95 & 220.7\end{array}$

METHANE ISOTHERM AT $\mathrm{T}=170 . \mathrm{K}$

Second Virial Coefficient is $-144.1 \mathrm{~cm}^{3} / \mathrm{mol}$

\begin{tabular}{rcrllllll}
$\rho$ & $\mathrm{P}$ & $\mathrm{dP} /\left.\mathrm{d} \rho\right|_{\mathrm{T}}$ & $\mathrm{dP} /\left.\mathrm{dT}\right|_{\rho}$ & $\mathrm{C}_{\mathrm{v}}$ & $\mathrm{W}$ & $\eta$ & $\lambda$ \\
$\mathrm{mol} / \mathrm{dm}^{3}$ & $\mathrm{MPa}$ & $\mathrm{MPa} \cdot \mathrm{dm}^{3} / \mathrm{mol}$ & $\mathrm{MPa} / \mathrm{K}$ & $\mathrm{J} /(\mathrm{mol} \cdot \mathrm{k})$ & \multicolumn{1}{l}{$\mathrm{m} / \mathrm{s}$} & $\mu \mathrm{Pa} \cdot \mathrm{s}$ & $\mathrm{mW} /(\mathrm{m} \cdot \mathrm{K})$ \\
& & & & & & & \\
0.2 & 0.2746 & 1.3328 & 0.00171 & 25.40 & 336.81 & 6.68 & 18.5 \\
0.4 & 0.5332 & 1.2538 & 0.00350 & 25.78 & 331.10 & 6.74 & 18.9 \\
0.6 & 0.7762 & 1.1765 & 0.00538 & 26.15 & 325.45 & 6.81 & 19.3 \\
0.8 & 1.0040 & 1.1009 & 0.00735 & 26.52 & 319.89 & 6.88 & 19.8 \\
1.0 & 1.2167 & 1.0270 & 0.00940 & 26.90 & 314.40 & 6.96 & 20.3 \\
& & & & & & & \\
1.2 & 1.4149 & 0.9546 & 0.01155 & 27.30 & 308.99 & 7.04 & 20.8 \\
1.4 & 1.5987 & 0.8838 & 0.01380 & 27.75 & 303.64 & 7.12 & 21.4 \\
1.6 & 1.7685 & 0.8143 & 0.01616 & 28.26 & 298.33 & 7.21 & 22.0 \\
1.8 & 1.9245 & 0.7460 & 0.01864 & 28.87 & 293.04 & 7.30 & 22.7 \\
2.0 & 2.0670 & 0.6789 & 0.02127 & 29.62 & 287.73 & 7.39 & 23.5 \\
& & & & & & & \\
2.2 & 2.1961 & 0.6127 & 0.02407 & 30.54 & 282.37 & 7.49 & 24.3 \\
2.4 & 2.3121 & 0.5473 & 0.02707 & 31.67 & 276.92 & 7.59 & 25.2 \\
19.4 & 2.4699 & 2.5986 & 0.54533 & 30.64 & 659.74 & 38.99 & 101.0 \\
19.6 & 3.0189 & 2.8938 & 0.56393 & 30.53 & 683.91 & 39.87 & 102.8 \\
19.8 & 3.6286 & 3.2060 & 0.58278 & 30.43 & 708.15 & 40.78 & 104.7
\end{tabular}


METHANE ISOTHERM AT $\mathrm{T}=170 . \mathrm{K}$ (continued)

\begin{tabular}{|c|c|c|c|c|c|c|c|}
\hline$\rho$ & $\mathrm{P}$ & $\mathrm{dP} /\left.\mathrm{d} \rho\right|_{\mathrm{T}}$ & $\mathrm{dP} /\left.\mathrm{dT}\right|_{\rho}$ & $\mathrm{C}_{\mathrm{v}}$ & W & $\eta$ & $\lambda$ \\
\hline $\mathrm{mol} / \mathrm{dm}^{3}$ & $\mathrm{MPa}$ & $\mathrm{MPa} \cdot \mathrm{dm}^{3} / \mathrm{mol}$ & $\mathrm{MPa} / \mathrm{K}$ & $\mathrm{J} /(\mathrm{mol} \cdot \mathrm{k})$ & $\mathrm{m} / \mathrm{s}$ & $\mu \mathrm{Pa} \cdot \mathrm{s}$ & $\mathrm{mW} /(\mathrm{m} \cdot \mathrm{K})$ \\
\hline 20.0 & 4.3024 & 3.5353 & 0.60186 & 30.36 & 732.43 & 41.72 & 106.6 \\
\hline 20.2 & 5.0439 & 3.8823 & 0.62116 & 30.30 & 756.76 & 42.69 & 108.5 \\
\hline 20.4 & 5.8566 & 4.2472 & 0.64068 & 30.26 & 781.12 & 43.69 & 110.5 \\
\hline 20.6 & 6.7440 & 4.6302 & 0.66040 & 30.23 & 805.51 & 44.72 & 112.6 \\
\hline 20.8 & 7.7099 & 5.0318 & 0.68031 & 30.22 & 829.92 & 45.79 & 114.7 \\
\hline 21.0 & 30 & 24 & .70040 & 30 & 854.34 & 46.90 & 116.8 \\
\hline 21.2 & 9.8921 & 921 & 0.72066 & 30. & 878.77 & 48.05 & 119.1 \\
\hline 21.4 & 11.1161 & 6.3515 & 0.74109 & 30.26 & 903.22 & 49.24 & 121.3 \\
\hline 21.6 & 12.4340 & 6.8308 & 0.76168 & 30.30 & 927.69 & 50.48 & 123.6 \\
\hline 21.8 & 13.8498 & 06 & 0.78242 & 30.35 & 952.18 & 51.77 & 126.0 \\
\hline 22.0 & 15.3677 & 12 & 0.80 & 30 . & 976.69 & 53.11 & 128.5 \\
\hline 22.2 & 16.9917 & 8.3930 & 0.82437 & 30 . & 1001.25 & 54 & 131.0 \\
\hline 22.4 & 18.7263 & 8.9566 & 0.84557 & 30.56 & 1025.85 & 55.97 & 133.5 \\
\hline 22.6 & 20.5758 & 23 & 0.86693 & 30.65 & 1050.50 & 57.50 & 136.2 \\
\hline 22.8 & 22.5448 & 10.1 & 0.88843 & 30.74 & 1075.22 & 59.11 & 138.8 \\
\hline 23.0 & 7 & & 0 & & 110 & & 141.6 \\
\hline 23.2 & 26.8594 & 11.6 & 0.93192 & 30 . & 1124.91 & 62.56 & 144.4 \\
\hline 23.4 & 29.2146 & 12.1179 & 0.95392 & 31.06 & 1149.90 & 64.43 & 147.3 \\
\hline 23.6 & 31.7082 & 12.8228 & 0.97608 & 31.18 & 1175.00 & 66.41 & 150.3 \\
\hline 23.8 & 34.3454 & 13.5532 & 0.99842 & 31.30 & 1200.24 & 68.51 & 153.3 \\
\hline 24.0 & 37. & 8 & 5 & & 1 & & 15 \\
\hline 24.2 & 40.0711 & 15 & 1.0 & 31. & 1251.14 & 11 & 159.7 \\
\hline 24.4 & 43.1703 & 15.9041 & 1.06659 & 31.69 & 1276.84 & 75.65 & 162.9 \\
\hline 24.6 & 46.4346 & 16.7432 & 1.08973 & 31.82 & 1302.71 & 78.38 & 166.3 \\
\hline 24.8 & 49.8695 & 17.6112 & 1.11308 & 31.96 & 1328.78 & 81.32 & 169.7 \\
\hline 25.0 & 53.4 & & 1.1 & & 4 & & 173.3 \\
\hline 25.2 & 57.2751 & 19.4366 & 1.16049 & 32.24 & 1381.52 & 87.97 & 176.9 \\
\hline 25.4 & 61.2578 & 20.3955 & 1.18456 & 32.38 & 1408.21 & 91.75 & 180.6 \\
\hline 25.6 & 65.4354 & 21.3 & 1.20889 & 32.52 & 1435.14 & 95 & 184.4 \\
\hline 25.8 & 69.8144 & 22.4092 & 1.23349 & 32.66 & 1462.31 & 100.52 & 188.3 \\
\hline 26.0 & 74.4013 & 23 & 1.2 & & & & 192.3 \\
\hline 26.2 & 79.2028 & 24.5555 & 1.28353 & 32.95 & 1517.38 & 111.43 & 196.4 \\
\hline 26. & 84.2258 & 25.6803 & 1.30900 & 33.09 & 1545.31 & 117.98 & 200.6 \\
\hline 26 & 89.4773 & 304 & 1.33478 & 33. & 1573.50 & 125.50 & 204.9 \\
\hline 26.8 & 94.9644 & 28.0365 & 1.36087 & 33.37 & 1601.96 & 134.23 & 209.3 \\
\hline
\end{tabular}


METHANE ISOTHERM AT $\mathrm{T}=180 . \mathrm{K}$

Second Virial Coefficient is $-129.2 \mathrm{~cm}^{3} / \mathrm{mol}$

\begin{tabular}{rrrllllll}
\multicolumn{1}{l}{$\boldsymbol{\rho}$} & $\mathrm{P}$ & $\mathrm{dP} /\left.\mathrm{d} \rho\right|_{\mathrm{T}}$ & $\mathrm{dP} /\left.\mathrm{dT}\right|_{\rho}$ & $\mathrm{C}_{\mathrm{v}}$ & $\mathrm{W}$ & $\eta$ & $\lambda$ \\
$\mathrm{mol} / \mathrm{dm}^{3}$ & $\mathrm{MPa}$ & $\mathrm{MPa} \cdot \mathrm{dm}^{3} / \mathrm{mol}$ & $\mathrm{MPa} / \mathrm{K}$ & $\mathrm{J} /(\mathrm{mol} \cdot \mathrm{k})$ & $\mathrm{m} / \mathrm{s}$ & $\mu \mathrm{Pa} \cdot \mathrm{s}$ & $\mathrm{mW} /(\mathrm{m} \cdot \mathrm{K})$ \\
& & & & & & & \\
0.5 & 0.7008 & 1.3085 & 0.00440 & 25.85 & 339.48 & 7.16 & 20.3 \\
1.0 & 1.3102 & 1.1309 & 0.00930 & 26.63 & 327.03 & 7.35 & 21.4 \\
1.5 & 1.8335 & 0.9640 & 0.01470 & 27.46 & 315.15 & 7.56 & 22.7 \\
2.0 & 2.2760 & 0.8075 & 0.02062 & 28.40 & 303.84 & 7.79 & 24.2 \\
2.5 & 2.6428 & 0.6612 & 0.02712 & 29.57 & 293.01 & 8.04 & 26.1 \\
& & & & & & & \\
3.0 & 2.9388 & 0.5246 & 0.03429 & 31.12 & 282.50 & 8.32 & 28.3 \\
3.5 & 3.1690 & 0.3978 & 0.04225 & 33.21 & 272.09 & 8.63 & 31.1 \\
17.5 & 3.6359 & 1.3001 & 0.40692 & 31.24 & 524.61 & 31.97 & 87.4 \\
18.0 & 4.3957 & 1.7522 & 0.44286 & 30.80 & 574.20 & 33.68 & 91.0 \\
18.5 & 5.4019 & 2.2872 & 0.48087 & 30.48 & 625.55 & 35.51 & 94.8 \\
& & & & & & & \\
19.0 & 6.6978 & 2.9121 & 0.52085 & 30.24 & 678.47 & 37.47 & 98.9 \\
19.5 & 8.3299 & 3.6327 & 0.56263 & 30.10 & 732.65 & 39.58 & 103.2 \\
20.0 & 10.3473 & 4.4543 & 0.60602 & 30.03 & 787.82 & 41.85 & 107.9 \\
20.5 & 12.8020 & 5.3824 & 0.65086 & 30.04 & 843.79 & 44.31 & 112.9 \\
21.0 & 15.7485 & 6.4228 & 0.69704 & 30.11 & 900.46 & 46.99 & 118.2 \\
& & & & & & & \\
21.5 & 19.2445 & 7.5818 & 0.74447 & 30.25 & 957.79 & 49.93 & 123.9 \\
22.0 & 23.3512 & 8.8662 & 0.79312 & 30.43 & 1015.84 & 53.18 & 129.9 \\
22.5 & 28.1329 & 10.2836 & 0.84299 & 30.65 & 1074.67 & 56.81 & 136.3 \\
23.0 & 33.6583 & 11.8422 & 0.89410 & 30.90 & 1134.42 & 60.90 & 143.1 \\
23.5 & 40.0002 & 13.5511 & 0.94654 & 31.18 & 1195.21 & 65.58 & 150.4 \\
& & & & & & & \\
24.0 & 47.2361 & 15.4199 & 1.00037 & 31.47 & 1257.19 & 71.00 & 158.1 \\
24.5 & 55.4485 & 17.4590 & 1.05572 & 31.78 & 1320.50 & 77.43 & 166.3 \\
25.0 & 64.7252 & 19.6791 & 1.11272 & 32.10 & 1385.29 & 85.21 & 175.1 \\
25.5 & 75.1596 & 22.0914 & 1.17149 & 32.42 & 1451.66 & 94.95 & 184.4 \\
26.0 & 86.8505 & 24.7074 & 1.23219 & 32.75 & 1519.74 & 107.63 & 194.3 \\
& & & & & & & \\
26.5 & 99.9028 & 27.5383 & 1.29496 & 33.07 & 1589.59 & 125.03 & 204.8
\end{tabular}

METHANE ISOTHERM AT $\mathrm{T}=190 . \mathrm{K}$

Second Virial Coefficient is $-116.4 \mathrm{~cm}^{3} / \mathrm{mol}$

$\begin{array}{llllllll}\rho & \mathrm{P} & \mathrm{dP} /\left.\mathrm{d} \rho\right|_{\mathrm{T}} & \mathrm{dP} /\left.\mathrm{dT}\right|_{\rho} & \mathrm{C}_{\mathrm{v}} & \mathrm{W} & \eta & \lambda\end{array}$

$\mathrm{mol} / \mathrm{dm}^{3} \quad \mathrm{MPa} \quad \mathrm{MPa} \cdot \mathrm{dm}^{3} / \mathrm{mol} \quad \mathrm{MPa} / \mathrm{K} \quad \mathrm{J} /(\mathrm{mol} \cdot \mathrm{k}) \quad \mathrm{m} / \mathrm{s} \quad \mu \mathrm{Pa} \cdot \mathrm{s} \quad \mathrm{mW} /(\mathrm{m} \cdot \mathrm{K})$

$\begin{array}{llllllll}0.5 & 0.7448 & 1.4011 & 0.00439 & 25.78 & 350.25 & 7.54 & 21.5 \\ 1.0 & 1.4029 & 1.2330 & 0.00922 & 26.44 & 339.06 & 7.73 & 22.6 \\ 1.5 & 1.9795 & 1.0755 & 0.01451 & 27.12 & 328.48 & 7.94 & 23.9 \\ 2.0 & 2.4802 & 0.9288 & 0.02024 & 27.84 & 318.57 & 8.18 & 25.4 \\ 2.5 & 2.9102 & 0.7929 & 0.02643 & 28.61 & 309.34 & 8.44 & 27.2\end{array}$


METHANE ISOTHERM AT $\mathrm{T}=190 . \mathrm{K}$ (continued)

\begin{tabular}{|c|c|c|c|c|c|c|c|}
\hline$\rho$ & $\mathbf{P}$ & $\mathrm{dP} /\left.\mathrm{d} \rho\right|_{\mathrm{T}}$ & $\mathrm{dP} /\left.\mathrm{dT}\right|_{\rho}$ & $\mathrm{C}_{\mathrm{v}}$ & W & $\eta$ & $\lambda$ \\
\hline $\mathrm{mol} / \mathrm{dm}^{3}$ & $\mathrm{MPa}$ & $\mathrm{MPa} \cdot \mathrm{dm}^{3} / \mathrm{mol}$ & $\mathrm{MPa} / \mathrm{K}$ & $\mathrm{J} /(\mathrm{mol} \cdot \mathrm{k})$ & $\mathrm{m} / \mathrm{s}$ & $\mu \mathrm{Pa} \cdot \mathrm{s}$ & $\mathrm{mW} /(\mathrm{m} \cdot \mathrm{K})$ \\
\hline 3.0 & 3.2748 & 0.6676 & 0.03307 & 29.46 & 300.77 & 8.72 & 29.3 \\
\hline 3.5 & 3.5795 & 0.5530 & 0.04017 & 30.42 & 292.81 & 9.03 & 31.8 \\
\hline 4.0 & 3.8296 & 0.4491 & 0.04772 & 31.53 & 285.40 & 9.36 & 34.8 \\
\hline 4.5 & 4.0304 & 0.3562 & 0.05571 & 32.80 & 278.47 & 9.72 & 38.5 \\
\hline 5.0 & 4.1876 & 0.2743 & 0.06412 & 34.26 & 271.92 & 10.11 & 43.1 \\
\hline 5.5 & 4.3066 & 0.2036 & 0.07286 & 35.89 & 265.70 & 10.52 & 48.8 \\
\hline 6.0 & 4.3930 & 0.1441 & 0.08183 & 37.66 & 259.75 & 10.96 & 56.0 \\
\hline 6.5 & 4.4525 & 0.0958 & 0.09088 & 39.53 & 254.04 & 11.43 & 65.3 \\
\hline 7. & 07 & 0 . & 982 & 41 & 248.59 & 11.93 & 78.1 \\
\hline 7.5 & 4.5127 & 0.0311 & 0.10844 & 43.20 & 243.41 & 12.46 & 97.1 \\
\hline 12.5 & 4.5192 & 0. & 0.18683 & 38. & 264.32 & 19.75 & 100.3 \\
\hline 13.0 & 4.5413 & 0.0646 & 0.20194 & 37.08 & 284.79 & 20.72 & 85.1 \\
\hline 13.5 & 4.5875 & 0.1242 & 0.21921 & 35.58 & 309.04 & 21.74 & 80.0 \\
\hline 14.0 & 4.6699 & 03 & 0.23833 & 34 & 24 & 22.81 & 78.0 \\
\hline 14.5 & 4.8029 & 272 & 0.25905 & 33.35 & 365.72 & 23.94 & 77.6 \\
\hline 15.0 & 5.0030 & 93 & 0.28125 & 32. & 397.16 & 25.13 & 78.2 \\
\hline 15.5 & 5.2889 & 0.6715 & 0.30499 & 31.94 & 430.62 & 26.39 & 79.5 \\
\hline 16.0 & 5.6822 & 0.9098 & 0.33045 & 31.43 & 466.31 & 27.73 & 81.3 \\
\hline 16.5 & 6.2076 & 1.2012 & 0.35783 & 31.01 & 504.49 & 29.14 & 83.6 \\
\hline 17.0 & 6.8935 & 530 & 0.38728 & 30.66 & 545.26 & 30.64 & 86.3 \\
\hline 17.5 & 7.7719 & 1.9725 & 0.41885 & 3 & 588.52 & 32.23 & 89.4 \\
\hline 18.0 & 8.8784 & 2.4665 & 0.45250 & 30 & 634.03 & 33.93 & 92.7 \\
\hline 18.5 & 10.2518 & 3.0412 & 0.48810 & 29.99 & 681.50 & 35.74 & 96.4 \\
\hline 19.0 & 11.9340 & 3.7025 & 0.52552 & 29.90 & 730.62 & 37.68 & 100.4 \\
\hline 19.5 & 13.9697 & 4.4563 & 0.56461 & 29.87 & 781.14 & 39.77 & 104.8 \\
\hline 20.0 & 16.4066 & 5.3 & 0.60 & 29 & 832.87 & 42.03 & 109.4 \\
\hline 20.5 & 19.2954 & 6.2646 & 0.64739 & 29.98 & 885.71 & 44.48 & 114.4 \\
\hline 21.0 & 22.6897 & 7.3318 & 0.69092 & 30.10 & 939.61 & 47.15 & 119.7 \\
\hline 21.5 & 26.6469 & 8.5169 & 0.73583 & 30.27 & 994.55 & 50.10 & 125.3 \\
\hline 22.0 & 31.2276 & 74 & 0.78212 & 30. & 1050.60 & 53.36 & 131.4 \\
\hline 22.5 & 36.4965 & 11. & 0.82982 & 30 & 97.82 & 01 & 137.8 \\
\hline 23.0 & 42.5227 & 12. & 0.87897 & 30 & 1166 & 61 & 144.7 \\
\hline 23.5 & 49.3797 & 14.5963 & 0.92966 & 31.22 & 1226.15 & 65.90 & 152.0 \\
\hline 24.0 & 57.1460 & 16.4966 & 0.98198 & 31.50 & 1287.49 & 71.45 & 159.7 \\
\hline 24.5 & 65.9051 & 18.5695 & 1.03604 & 31.79 & 1350.42 & 78.07 & 168.0 \\
\hline 25 & 75.7461 & 20.8261 & 1.09197 & 32.08 & 1415.05 & 86.18 & 176.8 \\
\hline & 86 & 23.27 & 1.14988 & 32 & 1481.48 & 96.44 & 186.2 \\
\hline 26 & 582 & 5.9357 & 20993 & 32.67 & 1549 & 110.01 & 19 \\
\hline
\end{tabular}


METHANE ISOTHERM AT $\mathrm{T}=200 . \mathrm{K}$

Second Virial Coefficient is $-105.2 \mathrm{~cm}^{3} / \mathrm{mol}$

\begin{tabular}{|c|c|c|c|c|c|c|c|}
\hline$\rho$ & $\mathbf{P}$ & $\mathrm{dP} /\left.\mathrm{d} \rho\right|_{\mathrm{T}}$ & $\mathrm{dP} /\left.\mathrm{dT}\right|_{\rho}$ & $\mathrm{C}_{\mathrm{v}}$ & W & $\eta$ & $\lambda$ \\
\hline $\mathrm{mol} / \mathrm{dm}^{3}$ & $\mathrm{MPa}$ & $\mathrm{MPa} \cdot \mathrm{dm}^{3} / \mathrm{mol}$ & $\mathrm{MPa} / \mathrm{K}$ & $\mathrm{J} /(\mathrm{mol} \cdot \mathrm{k})$ & $\mathrm{m} / \mathrm{s}$ & $\mu \mathrm{Pa} \cdot \mathrm{s}$ & $\mathrm{mW} /(\mathrm{m} \cdot \mathrm{K})$ \\
\hline 0.5 & 0.7886 & 1.4930 & 0.00437 & 25.75 & 360.61 & 7.91 & 22.6 \\
\hline 1.0 & 1.4948 & 1.3336 & 0.00916 & 26.31 & 350.54 & 8.10 & 23.6 \\
\hline 1.5 & 2.1239 & 1.1847 & 0.01436 & 26.89 & 341.12 & 8.32 & 24.7 \\
\hline 2.0 & 2.6812 & 1.0465 & 0.01997 & 27.47 & 332.40 & 8.56 & 25.9 \\
\hline 2.5 & 3.1721 & 0.9189 & 0.02598 & 28.07 & 324.41 & 8.83 & 27.3 \\
\hline 3.0 & 3.6018 & 0.8018 & 0.03238 & 28.69 & 317.17 & 9.12 & 28.9 \\
\hline 3.5 & 3.9757 & 0.6952 & 0.03915 & 29.33 & 310.66 & 9.43 & 30.7 \\
\hline 4.0 & 4.2988 & 0.5990 & 0.04627 & 29.99 & 304.89 & 9.77 & 32.8 \\
\hline 4.5 & 4.5764 & 131 & 0.05372 & 30.68 & 299.84 & 10.13 & 35.1 \\
\hline 5.0 & 4.8136 & 0.4374 & 0.06146 & 31.37 & 295.47 & 10.52 & 37.7 \\
\hline 5.5 & 5.0 & 16 & 0.06945 & 32 & 291.76 & 10.93 & 40.6 \\
\hline 6.0 & 5.1868 & 154 & 0.07764 & 32.78 & 288.68 & 11.37 & 43.7 \\
\hline 6.5 & 5.3323 & 0.2684 & 0.08598 & 33.46 & 286.22 & 11.85 & 47.1 \\
\hline 7.0 & 5.4566 & 0 & 0.09442 & 34.09 & 284.39 & 12.35 & 50.5 \\
\hline 7.5 & 5.5637 & 0.1997 & 0.10292 & 34.64 & 283.21 & 12.88 & 54.0 \\
\hline 12. & 6.4856 & & 0.20 & & 343.82 & 20.14 & 69.2 \\
\hline 13. & 6.6563 & 789 & 0.21779 & 32.68 & 361.51 & 21.10 & 70.0 \\
\hline 13.5 & 6.8680 & 0.4716 & 0.23401 & 32.21 & 381.70 & 22.10 & 71.1 \\
\hline 14.0 & 7.1322 & 0 . & 0.25 & 31. & 404.44 & 23.17 & 72.5 \\
\hline 14.5 & 7.4629 & 0.7386 & 0.27105 & 31.41 & 429.82 & 24.29 & 74.1 \\
\hline 15. & 7.8768 & 37 & 0.29 & 31 & 92 & 25.47 & 76.0 \\
\hline 15.5 & 8.3937 & 1.1514 & 0.31480 & 30.76 & 488.82 & 26.72 & 78.3 \\
\hline 16.0 & 9.0363 & 1.4278 & 0.33942 & 30.49 & 522.50 & 28.04 & 80.7 \\
\hline 16.5 & 9.8306 & & 0.36 & 30 & 558.87 & 29.44 & 83.5 \\
\hline 17.0 & 10.8056 & 2.1514 & 0.39437 & 30.06 & 597.75 & 30.93 & 86.5 \\
\hline 17.5 & .9932 & & 0.42 & 29 & & & 89.8 \\
\hline $18 . \mathrm{C}$ & 13.4282 & 3.1424 & 0.45682 & 29.80 & 682.12 & 34.19 & 93.4 \\
\hline 18. & 15.1486 & 528 & 0.49069 & 29 & 727.13 & 35.99 & 97.3 \\
\hline 19.0 & 17.1 & & 0.5 & & 773 & & 101.4 \\
\hline 19.5 & 19.6114 & 5.2330 & 0.56332 & 29.78 & 821.88 & 40.00 & 105.8 \\
\hline 20.0 & 22.4442 & 6.1151 & 0.60195 & 29.87 & 871.37 & 42.25 & 110.5 \\
\hline 20.5 & 25.7437 & 7.1006 & 0.64207 & 29.99 & 922.18 & 44.70 & 115.6 \\
\hline 21 . & 29.5632 & 8.1964 & 0.68366 & 30.14 & 974.29 & 47.37 & 120.9 \\
\hline 21 . & 33.9597 & 9.4101 & 0.72672 & 30.33 & 1027.73 & 50.32 & 126.6 \\
\hline 22.0 & 38.9942 & 10.7497 & 0.77129 & 30.53 & 1082.54 & 53.61 & 132.7 \\
\hline
\end{tabular}


METHANE ISOTHERM AT $\mathrm{T}=200 . \mathrm{K}$ (continued)

$\begin{array}{cccccccc}\rho & \mathrm{P} & \mathrm{dP} /\left.\mathrm{d} \rho\right|_{\mathrm{T}} & \mathrm{dP} /\left.\mathrm{dT}\right|_{\rho} & \mathrm{C}_{\mathrm{v}} & \mathrm{W} & \eta & \lambda \\ \mathrm{mol} / \mathrm{dm}^{3} & \mathrm{MPa} & \mathrm{MPa} \cdot \mathrm{dm}^{3} / \mathrm{mol} & \mathrm{MPa} / \mathrm{K} & \mathrm{J} /(\mathrm{mol} \cdot \mathrm{k}) & \mathrm{m} / \mathrm{s} & \mu \mathrm{Pa} \cdot \mathrm{s} & \mathrm{mW} /(\mathrm{m} \cdot \mathrm{K}) \\ & & & & & & & \\ 22.5 & 44.7318 & 12.2239 & 0.81740 & 30.76 & 1138.78 & 57.29 & 139.2 \\ 23.0 & 51.2421 & 13.8419 & 0.86512 & 31.00 & 1196.53 & 61.49 & 146.1 \\ 23.5 & 58.5993 & 15.6135 & 0.91454 & 31.26 & 1255.89 & 66.33 & 153.5 \\ 24.0 & 66.8830 & 17.5493 & 0.96576 & 31.52 & 1316.95 & 72.02 & 161.3 \\ 24.5 & 76.1777 & 19.6600 & 1.01889 & 31.78 & 1379.78 & 78.87 & 169.6 \\ 25.0 & 86.5740 & 21.9569 & 1.07405 & 32.05 & 1444.49 & 87.33 & 178.5 \\ 25.5 & 98.1676 & 24.4515 & 1.13137 & 32.32 & 1511.13 & 98.16 & 188.0\end{array}$

METHANE ISOTHERM AT T $=210 . \mathrm{K}$
Second Virial Coefficient is $-95.5 \mathrm{~cm}^{3} / \mathrm{mol}$

$\begin{array}{llllllll}\rho & \mathrm{P} & \mathrm{dP} /\left.\mathrm{d} \rho\right|_{\mathrm{T}} & \mathrm{dP} /\left.\mathrm{dT}\right|_{\rho} & \mathrm{C}_{\mathrm{v}} & \mathrm{W} & \eta & \lambda\end{array}$

$\mathrm{mol} / \mathrm{dm}^{3} \quad \mathrm{MPa} \quad \mathrm{MPa} \cdot \mathrm{dm}^{3} / \mathrm{mol} \quad \mathrm{MPa} / \mathrm{K} \quad \mathrm{J} /(\mathrm{mol} \cdot \mathrm{k}) \quad \mathrm{m} / \mathrm{s} \quad \mu \mathrm{Pa} \cdot \mathrm{s} \quad \mathrm{mW} /(\mathrm{m} \cdot \mathrm{K})$

$\begin{array}{rrrrrrrr}0.5 & 0.8322 & 1.5844 & 0.00436 & 25.77 & 370.59 & 8.28 & 23.7 \\ 1.0 & 1.5861 & 1.4330 & 0.00911 & 26.25 & 361.53 & 8.48 & 24.7 \\ 1.5 & 2.2669 & 1.2920 & 0.01425 & 26.73 & 353.14 & 8.70 & 25.7 \\ 2.0 & 2.8799 & 1.1615 & 0.01976 & 27.22 & 345.48 & 8.94 & 26.9 \\ 2.5 & 3.4302 & 1.0415 & 0.02565 & 27.72 & 338.56 & 9.21 & 28.2 \\ & & & & & & & \\ 3.0 & 3.9231 & 0.9317 & 0.03189 & 28.21 & 332.41 & 9.50 & 29.6 \\ 3.5 & 4.3636 & 0.8321 & 0.03847 & 28.71 & 327.04 & 9.82 & 31.2 \\ 4.0 & 4.7568 & 0.7424 & 0.04538 & 29.20 & 322.45 & 10.16 & 33.0 \\ 4.5 & 5.1076 & 0.6625 & 0.05260 & 29.69 & 318.63 & 10.52 & 34.9 \\ 5.0 & 5.4208 & 0.5920 & 0.06009 & 30.16 & 315.59 & 10.92 & 37.0 \\ & & & & & & & \\ 5.5 & 5.7012 & 0.5308 & 0.06784 & 30.60 & 313.30 & 11.33 & 39.3 \\ 6.0 & 5.9532 & 0.4786 & 0.07582 & 31.02 & 311.78 & 11.78 & 41.7 \\ 6.5 & 6.1812 & 0.4349 & 0.08401 & 31.40 & 311.03 & 12.25 & 44.1 \\ 7.0 & 6.3895 & 0.3995 & 0.09240 & 31.74 & 311.06 & 12.75 & 46.6 \\ 7.5 & 6.5820 & 0.3718 & 0.10099 & 32.02 & 311.92 & 13.28 & 49.1 \\ & & & & & & & \\ 12.5 & 8.5466 & 0.5920 & 0.20845 & 31.51 & 390.43 & 20.52 & 66.7 \\ 13.0 & 8.8662 & 0.6897 & 0.22348 & 31.26 & 408.35 & 21.47 & 68.2 \\ 13.5 & 9.2402 & 0.8106 & 0.23981 & 31.01 & 428.63 & 22.47 & 69.9 \\ 14.0 & 9.6814 & 0.9590 & 0.25755 & 30.77 & 451.38 & 23.52 & 71.8 \\ 14.5 & 10.2047 & 1.1400 & 0.27680 & 30.54 & 476.70 & 24.64 & 73.9 \\ & & & & & & & \\ 15.0 & 10.8277 & 1.3590 & 0.29766 & 30.33 & 504.65 & 25.81 & 76.1 \\ 15.5 & 11.5709 & 1.6218 & 0.32019 & 30.14 & 535.20 & 27.05 & 78.6 \\ 16.0 & 12.4578 & 1.9341 & 0.34441 & 29.97 & 568.27 & 28.36 & 81.3 \\ 16.5 & 13.5143 & 2.3016 & 0.37033 & 29.83 & 603.72 & 29.75 & 84.2 \\ 17.0 & 14.7694 & 2.7295 & 0.39794 & 29.73 & 641.39 & 31.23 & 87.4\end{array}$


METHANE ISOTHERM AT $\mathrm{T}=210 . \mathrm{K}$ (continued)

$\begin{array}{cccccccc}\rho & \mathrm{P} & \mathrm{dP} /\left.\mathrm{d} \rho\right|_{\mathrm{T}} & \mathrm{dP} / \mathrm{dT} \mid \rho & \mathrm{C}_{\mathrm{v}} & \mathrm{W} & \eta & \lambda \\ \mathrm{mol} / \mathrm{dm}^{3} & \mathrm{MPa} & \mathrm{MPa} \cdot \mathrm{dm}^{3} / \mathrm{mol} & \mathrm{MPa} / \mathrm{K} & \mathrm{J} /(\mathrm{mol} \cdot \mathrm{k}) & \mathrm{m} / \mathrm{s} & \mu \mathrm{Pa} \cdot \mathrm{s} & \mathrm{mW} /(\mathrm{m} \cdot \mathrm{K}) \\ & & & & & & & \\ 17.5 & 16.2548 & 3.2235 & 0.42722 & 29.67 & 681.10 & 32.79 & 90.8 \\ 18.0 & 18.0049 & 3.7892 & 0.45813 & 29.64 & 722.69 & 34.47 & 94.4 \\ 18.5 & 20.0569 & 4.4322 & 0.49063 & 29.65 & 766.02 & 36.26 & 98.3 \\ 19.0 & 22.4510 & 5.1586 & 0.52469 & 29.70 & 810.98 & 38.18 & 102.5 \\ 19.5 & 25.2304 & 5.9746 & 0.56028 & 29.78 & 857.49 & 40.26 & 107.0 \\ & & & & & & & \\ 20.0 & 28.4416 & 6.8868 & 0.59738 & 29.89 & 905.50 & 42.50 & 111.7 \\ 20.5 & 32.1343 & 7.9021 & 0.63599 & 30.04 & 954.99 & 44.95 & 116.8 \\ 21.0 & 36.3621 & 9.0279 & 0.67612 & 30.20 & 1005.97 & 47.63 & 122.2 \\ 21.5 & 41.1820 & 10.2722 & 0.71780 & 30.39 & 1058.46 & 50.60 & 128.0 \\ 22.0 & 46.6554 & 11.6432 & 0.76107 & 30.60 & 1112.52 & 53.91 & 134.1 \\ 22.5 & 52.8479 & 13.1501 & 0.80599 & 30.82 & 1168.21 & 57.64 & 140.6 \\ 23.0 & 59.8297 & 14.8025 & 0.85264 & 31.05 & 1225.59 & 61.91 & 147.6 \\ 23.5 & 67.6762 & 16.6104 & 0.90112 & 31.29 & 1284.74 & 66.85 & 155.0 \\ 24.0 & 76.4678 & 18.5845 & 0.95152 & 31.53 & 1345.75 & 72.70 & 162.8 \\ 24.5 & 86.2903 & 20.7360 & 1.00397 & 31.77 & 1408.68 & 79.78 & 171.2 \\ 25.0 & 97.2352 & 23.0763 & 1.05859 & 32.01 & 1473.62 & 88.62 & 180.2\end{array}$

METHANE ISOTHERM AT $\mathrm{T}=220 . \mathrm{K}$
Second Virial Coefficient is $-86.8 \mathrm{~cm}^{3} / \mathrm{mol}$

$\begin{array}{llllllll}\rho & \mathrm{P} & \mathrm{dP} /\left.\mathrm{d} \rho\right|_{\mathrm{T}} & \mathrm{dP} / \mathrm{dT} \mid \rho & \mathrm{C}_{\mathrm{v}} & \mathrm{W} & \eta & \lambda\end{array}$

$\mathrm{mol} / \mathrm{dm}^{3} \quad \mathrm{MPa} \quad \mathrm{MPa} \cdot \mathrm{dm}^{3} / \mathrm{mol} \quad \mathrm{MPa} / \mathrm{K} \quad \mathrm{J} /(\mathrm{mol} \cdot \mathrm{k}) \quad \mathrm{m} / \mathrm{s} \quad \mu \mathrm{Pa} \cdot \mathrm{s} \quad \mathrm{mW} /(\mathrm{m} \cdot \mathrm{K})$

$\begin{array}{rrrrrrrr}0.5 & 0.8757 & 1.6753 & 0.00435 & 25.83 & 380.20 & 8.64 & 24.9 \\ 1.0 & 1.6770 & 1.5315 & 0.00907 & 26.24 & 372.06 & 8.84 & 25.9 \\ 1.5 & 2.4089 & 1.3979 & 0.01415 & 26.65 & 364.61 & 9.07 & 26.9 \\ 2.0 & 3.0766 & 1.2747 & 0.01959 & 27.07 & 357.89 & 9.32 & 28.0 \\ 2.5 & 3.6853 & 1.1617 & 0.02538 & 27.48 & 351.93 & 9.59 & 29.2 \\ & & & & & & & \\ 3.0 & 4.2400 & 1.0587 & 0.03151 & 27.89 & 346.74 & 9.88 & 30.6 \\ 3.5 & 4.7457 & 0.9657 & 0.03796 & 28.30 & 342.35 & 10.20 & 32.0 \\ 4.0 & 5.2072 & 0.8822 & 0.04473 & 28.70 & 338.75 & 10.55 & 33.6 \\ 4.5 & 5.6294 & 0.8081 & 0.05180 & 29.08 & 335.94 & 10.91 & 35.4 \\ 5.0 & 6.0168 & 0.7431 & 0.05916 & 29.45 & 333.93 & 11.31 & 37.3 \\ & & & & & & & \\ 5.5 & 6.3740 & 0.6870 & 0.06678 & 29.79 & 332.72 & 11.73 & 39.2 \\ 6.0 & 6.7053 & 0.6396 & 0.07467 & 30.10 & 332.31 & 12.17 & 41.3 \\ 6.5 & 7.0150 & 0.6005 & 0.08282 & 30.38 & 332.73 & 12.65 & 43.5 \\ 7.0 & 7.3072 & 0.5697 & 0.09122 & 30.62 & 334.00 & 13.15 & 45.6 \\ 7.5 & 7.5860 & 0.5469 & 0.09988 & 30.82 & 336.16 & 13.68 & 47.8\end{array}$


METHANE ISOTHERM AT $T=220 . K$ (continued)

$\begin{array}{cccccccc}\rho & \mathrm{P} & \mathrm{dP} /\left.\mathrm{d} \rho\right|_{\mathrm{T}} \mathrm{dP} / \mathrm{dT} \mid \rho & \mathrm{C}_{\mathrm{v}} & \mathrm{W} & \eta & \lambda \\ & & & & & & \\ \mathrm{m} / \mathrm{dm}^{3} & \mathrm{MPa} & \mathrm{MPa} \cdot \mathrm{dm}^{3} / \mathrm{mol} & \mathrm{MPa} / \mathrm{K} & \mathrm{J} /(\mathrm{mol} \cdot \mathrm{k}) & \mathrm{m} / \mathrm{s} & \mu \mathrm{Pa} \cdot \mathrm{s} & \mathrm{mW} /(\mathrm{m} \cdot \mathrm{K}) \\ 12.5 & 10.6465 & 0.8844 & 0.21129 & 30.66 & 427.69 & 20.89 & 66.2 \\ 13.0 & 11.1181 & 1.0058 & 0.22665 & 30.50 & 446.50 & 21.83 & 68.0 \\ 13.5 & 11.6566 & 1.1528 & 0.24321 & 30.34 & 467.52 & 22.83 & 70.0 \\ 14.0 & 12.2759 & 1.3296 & 0.26109 & 30.18 & 490.84 & 23.87 & 72.1 \\ 14.5 & 12.9919 & 1.5406 & 0.28037 & 30.02 & 516.51 & 24.98 & 74.3 \\ 15.0 & 13.8230 & 1.7909 & 0.30111 & 29.89 & 544.54 & 26.15 & 76.7 \\ 15.5 & 14.7902 & 2.0858 & 0.32337 & 29.77 & 574.90 & 27.38 & 79.3 \\ 16.0 & 15.9171 & 2.4304 & 0.34715 & 29.67 & 607.49 & 28.68 & 82.1 \\ 16.5 & 17.2298 & 2.8301 & 0.37248 & 29.61 & 642.22 & 30.06 & 85.1 \\ 17.0 & 18.7572 & 3.2901 & 0.39934 & 29.57 & 678.97 & 31.53 & 88.3 \\ & & & & & & & \\ 17.5 & 20.5308 & 3.8157 & 0.42773 & 29.56 & 717.62 & 33.09 & 91.8 \\ 18.0 & 22.5848 & 4.4125 & 0.45763 & 29.58 & 758.06 & 34.76 & 95.5 \\ 18.5 & 24.9562 & 5.0862 & 0.48904 & 29.64 & 800.23 & 36.54 & 99.5 \\ 19.0 & 27.6849 & 5.8430 & 0.52195 & 29.72 & 844.06 & 38.46 & 103.7 \\ 19.5 & 30.8141 & 6.6892 & 0.55636 & 29.83 & 889.51 & 40.53 & 108.2 \\ & & & & & & & \\ 20.0 & 34.3901 & 7.6316 & 0.59229 & 29.96 & 936.56 & 42.78 & 113.0 \\ 20.5 & 38.4629 & 8.6775 & 0.62974 & 30.11 & 985.21 & 45.23 & 118.1 \\ 21.0 & 43.0861 & 9.8345 & 0.66875 & 30.28 & 1035.49 & 47.93 & 123.5 \\ 21.5 & 48.3173 & 11.1107 & 0.70936 & 30.47 & 1087.42 & 50.91 & 129.3 \\ 22.0 & 54.2182 & 12.5150 & 0.75164 & 30.67 & 1141.06 & 54.26 & 135.4 \\ & & & & & & & \\ 22.5 & 60.8552 & 14.0566 & 0.79566 & 30.88 & 1196.47 & 58.04 & 142.0 \\ 23.0 & 68.2994 & 15.7454 & 0.84150 & 31.09 & 1253.72 & 62.37 & 149.0 \\ 23.5 & 76.6269 & 17.5919 & 0.88926 & 31.31 & 1312.87 & 67.43 & 156.4 \\ 24.0 & 85.9193 & 19.6068 & 0.93906 & 31.54 & 1373.99 & 73.45 & 164.3 \\ 24.5 & 96.2637 & 21.8015 & 0.99100 & 31.76 & 1437.15 & 80.79 & 172.8\end{array}$

METHANE ISOTHERM AT $\mathrm{T}=240 . \mathrm{K}$

Second Virial Coefficient is $-72.3 \mathrm{~cm}^{3} / \mathrm{mol}$

$\begin{array}{llllllll}\rho & \mathrm{P} & \mathrm{dP} /\left.\mathrm{d} \rho\right|_{\mathrm{T}} & \mathrm{dP} /\left.\mathrm{dT}\right|_{\rho} & \mathrm{C}_{\mathrm{v}} & \mathrm{W} & \eta & \lambda\end{array}$

$\mathrm{mol} / \mathrm{cm}^{3} \quad \mathrm{MPa} \quad \mathrm{MPa} \cdot \mathrm{dm}^{3} / \mathrm{mol} \quad \mathrm{MPa} / \mathrm{K} \quad \mathrm{J} /(\mathrm{mol} \cdot \mathrm{k}) \quad \mathrm{m} / \mathrm{s} \quad \mu \mathrm{Pa} \cdot \mathrm{s} \quad \mathrm{mW} /(\mathrm{m} \cdot \mathrm{K})$

$\begin{array}{rrrrrrrr}0.5 & 0.9625 & 1.8560 & 0.00433 & 26.07 & 398.45 & 9.35 & 27.3 \\ 1.0 & 1.8577 & 1.7263 & 0.00900 & 26.38 & 391.90 & 9.56 & 28.2 \\ 1.5 & 2.6904 & 1.6065 & 0.01401 & 26.69 & 386.06 & 9.80 & 29.2 \\ 2.0 & 3.4659 & 1.4968 & 0.01935 & 27.00 & 380.97 & 10.05 & 30.3 \\ 2.5 & 4.1889 & 1.3969 & 0.02500 & 27.30 & 376.64 & 10.33 & 31.4\end{array}$


METHANE ISOTHERM AT $\mathrm{T}=240 . \mathrm{K}$ (continued)

\begin{tabular}{|c|c|c|c|c|c|c|c|}
\hline$\rho$ & $P$ & $\mathrm{dP} /\left.\mathrm{d} \rho\right|_{\mathrm{T}}$ & $\mathrm{dP} /\left.\mathrm{dT}\right|_{\rho}$ & $\mathrm{C}_{\mathrm{v}}$ & W & $\eta$ & $\lambda$ \\
\hline $\mathrm{mol} / \mathrm{cm}^{3}$ & $\mathrm{MPa}$ & $\mathrm{MPa} \cdot \mathrm{cm}^{3} / \mathrm{mol}$ & $\mathrm{MPa} / \mathrm{K}$ & $\mathrm{J} /(\mathrm{mol} \cdot \mathrm{k})$ & $\mathrm{m} / \mathrm{s}$ & $\mu \mathrm{Pa} \cdot \mathrm{s}$ & $\mathrm{mW} /(\mathrm{m} \cdot \mathrm{K})$ \\
\hline 3.0 & 4.8644 & 1.3068 & 0.03097 & 27.60 & 373.11 & 10.63 & 32.7 \\
\hline 3.5 & 5.4972 & 1.2262 & 0.03724 & 27.89 & 370.37 & 10.95 & 34.0 \\
\hline 4.0 & 6.0921 & 1.1549 & 0.04382 & 28.16 & 368.44 & 11.30 & 35.5 \\
\hline 4.5 & 6.6537 & 1.0927 & 0.05071 & 28.43 & 367.32 & 11.67 & 37.0 \\
\hline 5.0 & 7.1863 & 1.0393 & 0.05789 & 28.68 & 367.02 & 12.07 & 38.7 \\
\hline 5.5 & 7.6944 & 946 & 0.06537 & 28.91 & 367.55 & 12.49 & 40.4 \\
\hline 6.0 & 8.1823 & 0.9585 & 0.07315 & 29.12 & 368.93 & 12.94 & 42.2 \\
\hline 6.5 & 8.6543 & 0.9310 & 0.08124 & 29.31 & 371.19 & 13.41 & 44.1 \\
\hline 7.0 & 9.1147 & 0.9122 & 0.08966 & 29.47 & 374.35 & 13.92 & 46.0 \\
\hline 7.5 & 9.5679 & 0.9022 & 0.09842 & 29.61 & 378.47 & 14.45 & 47.9 \\
\hline 12.5 & .9054 & 363 & 0.21412 & 29. & 490.03 & 21.61 & 67.0 \\
\hline 13.0 & 15.6885 & 1.6508 & 0.22984 & 29.70 & 510.24 & 22.54 & 69.0 \\
\hline 13.5 & 16.5610 & 1.8448 & 0.24663 & 29.64 & 532.38 & 23.53 & 71.2 \\
\hline 14.0 & 17.5387 & 2.0717 & 0.26454 & 29.59 & 556.49 & 24.56 & 73.5 \\
\hline 14.5 & 18.6389 & 2.3356 & 0.28365 & 29.54 & 582.57 & 25.66 & 75.9 \\
\hline 15.0 & 19.8 & 408 & 0.3 & 29 & 62 & & 78.5 \\
\hline 15.5 & 21.2873 & 2.9917 & 0.32563 & 29.49 & 640.62 & 28.03 & 81.2 \\
\hline 16.0 & 22.8813 & 3.3931 & 0.34858 & 29.49 & 672.53 & 29.32 & 84.1 \\
\hline 16.5 & 24.6897 & 3.8500 & 0.37286 & 29.51 & 706.31 & 30.69 & 87.2 \\
\hline 17.0 & 26.7415 & 4.3675 & 0.39849 & 29.55 & 741.92 & 32.14 & 90.5 \\
\hline 17.5 & 29. & & 0 & & 119.32 & & 94.1 \\
\hline 18.0 & 31.7 & 57 & 0.4 & 29.68 & 818.48 & 35.35 & 97.8 \\
\hline 18.5 & 34.6868 & 6.3379 & 0.48373 & 29.78 & 859.37 & 37.13 & 101.8 \\
\hline 19.0 & 38.0560 & 7.1537 & 0.51499 & 29.89 & 901.98 & 39.04 & 106.1 \\
\hline 19.5 & 41.8555 & 8.0598 & 0.54773 & 30.02 & 946.32 & 41.11 & 110.7 \\
\hline 20.0 & 46.1321 & & 0.5 & 30.17 & 992.41 & 43.37 & 115.5 \\
\hline 20.5 & 50.9364 & 10.1720 & 0.61781 & 30.32 & 1040.27 & 45.84 & 120.6 \\
\hline 21.0 & 56.3229 & 11.3937 & 0.65525 & 30.49 & 1089.93 & 48.57 & 126.1 \\
\hline 21.5 & 62.3504 & 12.7373 & 0.69439 & 30.66 & 1141.45 & 51.61 & 132.0 \\
\hline 22.0 & 69.0821 & 14.2120 & 0.73529 & 30.84 & 1194.88 & 55.02 & 138.2 \\
\hline 22.5 & 76.5859 & & 0.77806 & 31.02 & 1250.27 & & 144.8 \\
\hline & 84.9348 & 17.5942 & 0.82279 & 31.21 & 1307.69 & 63.42 & 151.8 \\
\hline 23.5 & 94.2071 & 19.5229 & 0.86958 & 31.40 & 1367.21 & 68.71 & 159.3 \\
\hline
\end{tabular}


METHANE ISOTHERM AT $\mathrm{T}=260 . \mathrm{K}$

Second Virial Coefficient is $-60.4 \mathrm{~cm}^{3} / \mathrm{mol}$

\begin{tabular}{|c|c|c|c|c|c|c|c|}
\hline$\rho$ & $\mathbf{P}$ & $\mathrm{dP} /\left.\mathrm{d} \rho\right|_{\mathrm{T}}$ & $\mathrm{dP} /\left.\mathrm{dT}\right|_{\rho}$ & $\mathrm{C}_{\mathrm{v}}$ & W & $\eta$ & $\lambda$ \\
\hline $\mathrm{nol} / \mathrm{dm}^{3}$ & $\mathrm{MPa}$ & $\mathrm{MPa} \cdot \mathrm{dm}^{3} / \mathrm{mol}$ & $\mathrm{MPa} / \mathrm{K}$ & $\mathrm{J} /(\mathrm{mol} \cdot \mathrm{k})$ & $\mathrm{m} / \mathrm{s}$ & $\mu \mathrm{Pa} \cdot \mathrm{s}$ & $\mathrm{mW} /(\mathrm{m} \cdot \mathrm{K})$ \\
\hline 0.5 & 1.0490 & 2.0357 & 0.00432 & 26.45 & 415.48 & 10.05 & 29.8 \\
\hline 1.0 & 2.0373 & 1.9190 & 0.00896 & 26.71 & 410.29 & 10.26 & 30.7 \\
\hline 1.5 & 2.9696 & 1.8120 & 0.01391 & 26.96 & 405.80 & 10.50 & 31.7 \\
\hline 2.0 & 3.8510 & 1.7148 & 0.01917 & 27.20 & 402.06 & 10.76 & 32.7 \\
\hline 2.5 & 4.6861 & 1.6273 & 0.02474 & 27.43 & 399.10 & 11.04 & 33.8 \\
\hline 3.0 & 5.4798 & 1.5494 & 0.03060 & 27.66 & 396.92 & 11.35 & 35.0 \\
\hline 3.5 & 6.2370 & 1.4809 & 0.03676 & 27.87 & 395.55 & 11.67 & 36.3 \\
\hline 4.0 & 6.9623 & 1.4217 & 0.04323 & 28.08 & 394.99 & 12.02 & 37.7 \\
\hline 4.5 & 7.6602 & 1.3715 & 0.04999 & 28.27 & 395.26 & 12.40 & 39.1 \\
\hline 5.0 & 8.3353 & 1.3303 & 0.05706 & 28.46 & 396.36 & 12.80 & 40.7 \\
\hline 5.5 & 8.9920 & 1.2981 & 0.06445 & 28.63 & 398.32 & 13.22 & 42.3 \\
\hline 6.0 & 9.6348 & 1.2748 & 0.07217 & 28.78 & 401.14 & 13.67 & 44.0 \\
\hline 6.5 & 10.2683 & 1.2606 & 0.08022 & 28.92 & 404.88 & 14.15 & 45.7 \\
\hline 7.0 & 10.8970 & 1.2559 & 0.08863 & 29.04 & 409.55 & 14.65 & 47.5 \\
\hline 7.5 & 11.5258 & 1.2610 & 0.09742 & 29.15 & 415.21 & 15.18 & 49.3 \\
\hline 12.5 & 19.1971 & 2.0979 & 0.21481 & 29.50 & 541.30 & 22.30 & 68.6 \\
\hline 13.0 & 20.2956 & 2.3013 & 0.23059 & 29.50 & 562.39 & 23.23 & 70.8 \\
\hline 13.5 & 21.5037 & 2.5367 & 0.24732 & 29.50 & 585.22 & 24.20 & 73.1 \\
\hline 14.0 & 22.8382 & 2.8075 & 0.26507 & 29.51 & 609.80 & 25.23 & 75.4 \\
\hline 14.5 & 24.3177 & 3.1173 & 0.28389 & 29.53 & 636.15 & 26.31 & 77.9 \\
\hline 15.0 & 25.9627 & 3.4701 & 0.30381 & 29.55 & 664.27 & 27.46 & 80.6 \\
\hline 15.5 & 27.7957 & 3.8702 & 0.32488 & 29.59 & 694.16 & 28.67 & 83.4 \\
\hline 16.0 & 29.8415 & 4.3221 & 0.34715 & 29.64 & 725.81 & 29.95 & 86.4 \\
\hline 16.5 & 32.1272 & 4.8306 & 0.37064 & 29.70 & 759.23 & 31.31 & 89.5 \\
\hline 17.0 & 34.6824 & 5.4007 & 0.39539 & 29.78 & 794.40 & 32.75 & 92.9 \\
\hline 17.5 & 37.5391 & 6.0379 & 0.42144 & 29.86 & 831.32 & 34.30 & 96.5 \\
\hline 18.0 & 40.7324 & 6.7477 & 0.44882 & 29.96 & 869.99 & 35.95 & 100.3 \\
\hline 18.5 & 44.3000 & 7.5363 & 0.47758 & 30.07 & 910.42 & 37.72 & 104.3 \\
\hline 19.0 & 48.2829 & 8.4102 & 0.50775 & 30.20 & 952.64 & 39.64 & 108.6 \\
\hline 19.5 & 52.7255 & 9.3761 & 0.53939 & 30.33 & 996.66 & 41.72 & 113.2 \\
\hline 20.0 & 57.6756 & 10.4417 & 0.57257 & 30.47 & 1042.51 & 43.99 & 118.1 \\
\hline 20.5 & 63.1851 & 11.6148 & 0.60734 & 30.61 & 1090.25 & 46.49 & 123.3 \\
\hline 21.0 & 69.3098 & 12.9040 & 0.64378 & 30.76 & 1139.92 & 49.26 & 128.8 \\
\hline 21.5 & 76.1099 & 14.3182 & 0.68197 & 30.92 & 1191.56 & 52.35 & 134.7 \\
\hline 22.0 & 83.6504 & 15.8671 & 0.72201 & 31.08 & 1245.25 & 55.85 & 140.9 \\
\hline 22.5 & 92.0011 & 17.5608 & .76399 & 31.24 & 1301.04 & 59.86 & 47.6 \\
\hline
\end{tabular}


METHANE ISOTHERM AT $\mathrm{T}=280 . \mathrm{K}$

Second Virial Coefficient is $-50.6 \mathrm{~cm}^{3} / \mathrm{mol}$

\begin{tabular}{|c|c|c|c|c|c|c|c|}
\hline$\rho$ & $P$ & $\mathrm{dP} /\left.\mathrm{d} \rho\right|_{\mathrm{T}}$ & $\mathrm{dP} /\left.\mathrm{dT}\right|_{\rho}$ & $\mathrm{C}_{\mathrm{v}}$ & W & $\eta$ & $\lambda$ \\
\hline $1 / \mathrm{dm}^{3}$ & $\mathrm{MPa}$ & $\mathrm{MPa} \cdot \mathrm{dm}^{3} / \mathrm{mol}$ & $\mathrm{MPa} / \mathrm{K}$ & $\mathrm{J} /(\mathrm{mol} \cdot \mathrm{k})$ & $\mathrm{m} / \mathrm{s}$ & $\mu \mathrm{Pa} \cdot \mathrm{s}$ & $\mathrm{mW} /(\mathrm{m} \cdot \mathrm{K})$ \\
\hline 0.5 & 1.1353 & 2.2146 & 0.00431 & 26.98 & 431.42 & 10.72 & 32.4 \\
\hline 1.0 & 2.2161 & 2.1103 & 0.00893 & 27.20 & 427.39 & 10.94 & 33.3 \\
\hline 1.5 & 3.2471 & 2.0154 & 0.01384 & 27.40 & 424.07 & 11.19 & 34.2 \\
\hline 2.0 & 4.2331 & 1.9301 & 0.01905 & 27.60 & 421.49 & 11.45 & 35.3 \\
\hline 2.5 & 5.1788 & 1.8544 & 0.02455 & 27.79 & 419.68 & 11.73 & 36.3 \\
\hline 3.0 & 6.0891 & 883 & 0.03034 & 27.97 & 418.65 & 12.04 & 37.5 \\
\hline 3.5 & 6.9687 & 1.7317 & 0.03642 & 28.15 & 418.44 & 12.37 & 38.7 \\
\hline 4.0 & 7.8223 & 1.6845 & 0.04280 & 28.31 & 419.04 & 12.73 & 40.1 \\
\hline 4.5 & 8.6547 & 1.6466 & 0.04949 & 28.46 & 420.47 & 13.10 & 41.5 \\
\hline 5.0 & 9.4705 & 1.6180 & 0.05648 & 28.61 & 422.74 & 13.50 & 43.0 \\
\hline 5.5 & .2742 & 87 & 0.0 & 28.74 & 425 & 13.93 & 44.5 \\
\hline 6.0 & 11.0708 & 90 & 0.07146 & 28.87 & 429.91 & 14.38 & 46.1 \\
\hline 6.5 & 11.8649 & 1.5892 & 0.07948 & 28.98 & 434.86 & 14.86 & 47.8 \\
\hline 7.0 & 12.6617 & 1.5996 & 0.08787 & 29.08 & 440.77 & 15.36 & 49.5 \\
\hline 7.5 & 13.4663 & 1.6209 & 0.09666 & 29.17 & 447.69 & 15.89 & 51.3 \\
\hline 12.5 & 23.4 & 98 & 0.2 & 29.65 & 584 & 97 & 70.7 \\
\hline 13.0 & 24.9037 & 2 . & 0.23008 & 29.69 & 606.61 & 23.89 & 72.9 \\
\hline 13.5 & 26.4450 & 3.2224 & 0.24666 & 29.72 & 629.98 & 24.86 & 75.3 \\
\hline 14.0 & 28.1323 & 3.5331 & 0.26418 & 29.77 & 654.99 & 25.88 & 77.7 \\
\hline 14.5 & 29.9850 & 3.8848 & 0.28269 & 29.82 & 681.65 & 26.95 & 80.2 \\
\hline 15.0 & 32.0 & & 0.3 & 29 . & 709 & & 82.9 \\
\hline 15.5 & 34.2744 & 4.7 & 0.32286 & 29.94 & 739.98 & 29.29 & 85.8 \\
\hline 16.0 & 36.7601 & 5.2254 & 0.34460 & 30.01 & 771.69 & 30.56 & 88.8 \\
\hline 16.5 & 39.5094 & 5.7823 & 0.36750 & 30.09 & 805.10 & 31.92 & 92.0 \\
\hline 17.0 & 42.5529 & 6.4024 & 0.39161 & 30.18 & 840.23 & 33.36 & 95.4 \\
\hline 17.5 & 45.9233 & & 0.41 & 30 & 877.10 & 34.90 & 99.0 \\
\hline 18.0 & 49.6565 & 7.8 & 0.44363 & 30.38 & 915.73 & 36.55 & 102.9 \\
\hline 18.5 & 53.7914 & 8.6987 & 0.47165 & 30.49 & 956.15 & 38.32 & 106.9 \\
\hline 19.0 & 58.3698 & 9.6302 & 0.50107 & 30.61 & 998.40 & 40.25 & 111.3 \\
\hline 19.5 & 63.4374 & 10.6564 & 0.53196 & 30.74 & 1042.50 & 42.34 & 115.9 \\
\hline 20.0 & 69.0432 & & 0.56 & 30.87 & 1088.52 & 44.63 & 120.8 \\
\hline 20.5 & 75.2406 & 13.0238 & 0.59847 & 31.00 & 1136.50 & 47.15 & 126.0 \\
\hline 21.0 & 82.0869 & 14.3822 & 0.63423 & 31.14 & 1186.49 & 49.96 & 131.5 \\
\hline 21.5 & 89.6442 & 15.8693 & 0.67180 & 31.28 & 1238.56 & 53.12 & 137.5 \\
\hline 22.0 & 97.9794 & 17.4952 & 0.71126 & 31.41 & 1292.76 & 56.70 & 143.7 \\
\hline
\end{tabular}


METHANE ISOTHERM AT $\mathrm{T}=300 . \mathrm{K}$

Second Virial Coefficient is $-42.4 \mathrm{~cm}^{3} / \mathrm{mol}$

\begin{tabular}{|c|c|c|c|c|c|c|c|}
\hline$\rho$ & $P$ & $\mathrm{dP} /\left.\mathrm{d} \rho\right|_{\mathrm{T}}$ & $\mathrm{dP} /\left.\mathrm{dT}\right|_{\rho}$ & $C_{v}$ & W & $\eta$ & $\lambda$ \\
\hline $\mathrm{mol} / \mathrm{dm}^{3}$ & $\mathrm{MPa}$ & $\mathrm{MPa} \cdot \mathrm{dm}^{3} / \mathrm{mol}$ & $\mathrm{MPa} / \mathrm{K}$ & $\mathrm{J} /(\mathrm{mol} \cdot \mathrm{k})$ & $\mathrm{m} / \mathrm{s}$ & $\mu \mathrm{Pa} \cdot \mathrm{s}$ & $\mathrm{mW} /(\mathrm{m} \cdot \mathrm{K})$ \\
\hline 0.5 & 1.2214 & 2.3929 & 0.00430 & 27.65 & 446.40 & 11.38 & 35.1 \\
\hline 1.0 & 2.3944 & 2.3003 & 0.00890 & 27.83 & 443.39 & 11.61 & 36.0 \\
\hline 1.5 & 3.5233 & 2.2170 & 0.01378 & 28.01 & 441.08 & 11.85 & 36.9 \\
\hline 2.0 & 4.6130 & 2.1433 & 0.01895 & 28.18 & 439.50 & 12.12 & 37.9 \\
\hline 2.5 & 5.6682 & 2.0791 & 0.02440 & 28.34 & 438.69 & 12.41 & 39.0 \\
\hline 3.0 & 6.6937 & 2.0246 & 0.03013 & 28.49 & 438.66 & 12.72 & 40.2 \\
\hline 3.5 & 7.6944 & 1.9797 & 0.03616 & 28.64 & 439.43 & 13.05 & 41.4 \\
\hline 4.0 & 8.6750 & 1.9445 & 0.04248 & 28.77 & 441.03 & 13.41 & 42.7 \\
\hline 4.5 & 9.6405 & 1.9189 & 0.04911 & 28.90 & 443.45 & 13.79 & 44.0 \\
\hline 5.0 & 10.5956 & 1.9031 & 0.05605 & 29.02 & 446.73 & 14.19 & 45.5 \\
\hline 5.5 & 11.5452 & 1.8972 & 0.06331 & 29.14 & 450.89 & 14.62 & 47.0 \\
\hline 6.0 & 12.4945 & 1.9015 & 0.07092 & 29.24 & 455.94 & 15.07 & 48.6 \\
\hline 6.5 & 13.4485 & 1.9164 & 0.07890 & 29.34 & 461.92 & 15.55 & 50.2 \\
\hline 7.0 & 14.4128 & 1.9426 & 0.08726 & 29.43 & 468.87 & 16.05 & 51.9 \\
\hline 7.5 & 15.3931 & 1.9807 & 0.09603 & 29.51 & 476.82 & 16.58 & 53.6 \\
\hline 12.5 & 27.7692 & 3.3176 & 0.21345 & 30.07 & 622.98 & 23.62 & 73.1 \\
\hline 13.0 & 29.4950 & 3.5909 & 0.22900 & 30.13 & 645.31 & 24.54 & 75.3 \\
\hline 13.5 & 31.3662 & 3.9001 & 0.24540 & 30.18 & 669.18 & 25.50 & 77.7 \\
\hline 14.0 & 33.4016 & 4.2485 & 0.26269 & 30.24 & 694.63 & 26.51 & 80.2 \\
\hline 14.5 & 35.6218 & 4.6396 & 0.28093 & 30.31 & 721.66 & 27.58 & 82.8 \\
\hline 15.0 & 38.0489 & 5.0772 & 0.30015 & 30.38 & 750.30 & 28.71 & 85.5 \\
\hline 15.5 & 40.7074 & 5.5655 & 0.32041 & 30.45 & 780.57 & 29.90 & 88.4 \\
\hline 16.0 & 43.6236 & 6.1090 & 0.34174 & 30.54 & 812.50 & 31.17 & 91.4 \\
\hline 16.5 & 46.8264 & 6.7125 & 0.36420 & 30.62 & 846.11 & 32.52 & 94.7 \\
\hline 17.0 & 50.3470 & 7.3811 & 0.38783 & 30.72 & 881.44 & 33.95 & 98.1 \\
\hline 17.5 & 54.2193 & 8.1205 & 0.41268 & 30.81 & 918.50 & 35.49 & 101.7 \\
\hline 18.0 & 58.4802 & 8.9366 & 0.43882 & 30.92 & 957.34 & 37.14 & 105.6 \\
\hline 18.5 & 63.1697 & 9.8357 & 0.46629 & 31.03 & 998.00 & 38.92 & 109.7 \\
\hline 19.0 & 68.3310 & 10.8249 & 0.49518 & 31.14 & 1040.51 & 40.85 & 114.0 \\
\hline 19.5 & 74.0108 & 11.9115 & 0.52555 & 31.25 & 1084.94 & 42.95 & 118.7 \\
\hline 20.0 & 80.2600 & 13.1036 & 0.55747 & 31.37 & 1131.33 & 45.26 & 123.6 \\
\hline 20.5 & 87.1334 & 14.4096 & 0.59104 & 31.49 & 1179.74 & 47.82 & 128.8 \\
\hline 21.0 & 94.6902 & 15.8387 & 0.62634 & 31.61 & 1230.24 & 50.67 & 134.4 \\
\hline
\end{tabular}


METHANE ISOTHERM AT $\mathrm{T}=320 . \mathrm{K}$

Second Virial Coefficient is $-35.4 \mathrm{~cm}^{3} / \mathrm{mol}$

$\begin{array}{llllllll}\rho & \mathrm{P} & \mathrm{dP} /\left.\mathrm{d} \rho\right|_{\mathrm{T}} & \mathrm{dP} /\left.\mathrm{dT}\right|_{\rho} & \mathrm{C}_{\mathrm{v}} & \mathrm{W} & \eta & \lambda\end{array}$

$\mathrm{mol} / \mathrm{dm}^{3} \quad \mathrm{MPa} \quad \mathrm{MPa} \cdot \mathrm{dm}^{3} / \mathrm{mol} \quad \mathrm{MPa} / \mathrm{K} \quad \mathrm{J} /(\mathrm{mol} \cdot \mathrm{k}) \quad \mathrm{m} / \mathrm{s} \quad \mu \mathrm{Pa} \cdot \mathrm{s} \quad \mathrm{mW} /(\mathrm{m} \cdot \mathrm{K})$

$\begin{array}{llllllll}0.5 & 1.3075 & 2.5707 & 0.00430 & 28.43 & 460.53 & 12.03 & 38 .\end{array}$

$\begin{array}{llllllll}1.0 & 2.5721 & 2.4895 & 0.00888 & 28.60 & 458.41 & 12.25 & 38.9\end{array}$

$\begin{array}{llllllll}1.5 & 3.7985 & 2.4174 & 0.01373 & 28.75 & 457.00 & 12.50 & 39.8\end{array}$

$\begin{array}{llllllll}2.0 & 4.9911 & 2.3549 & 0.01887 & 28.90 & 456.31 & 12.77 & 40.8\end{array}$

$\begin{array}{llllllll}2.5 & 6.1549 & 2.3020 & 0.02428 & 29.04 & 456.37 & 13.06 & 41.8\end{array}$

$\begin{array}{llllllll}3.0 & 7.2947 & 2.2589 & 0.02997 & 29.17 & 457.22 & 13.37 & 43.0\end{array}$

$\begin{array}{llllllll}3.5 & 8.4154 & 2.2256 & 0.03595 & 29.30 & 458.87 & 13.71 & 44.2\end{array}$

$\begin{array}{llllllll}4.0 & 9.5220 & 2.2023 & 0.04222 & 29.42 & 461.33 & 14.07 & 45.4\end{array}$

$\begin{array}{llllllll}4.5 & 10.6194 & 2.1891 & 0.04880 & 29.53 & 464.63 & 14.45 & 46.8\end{array}$

$\begin{array}{llllllll}5.0 & 11.7128 & 2.1862 & 0.05569 & 29.64 & 468.79 & 14.85 & 48.2\end{array}$

$\begin{array}{llllllll}5.5 & 12.8074 & 2.1938 & 0.06291 & 29.74 & 473.82 & 15.28 & 49.7\end{array}$

$\begin{array}{llllllll}6.0 & 13.9084 & 2.2123 & 0.07048 & 29.83 & 479.76 & 15.73 & 51.2\end{array}$

$\begin{array}{llllllll}6.5 & 15.0216 & 2.2423 & 0.07842 & 29.92 & 486.63 & 16.21 & 52.8\end{array}$

$\begin{array}{llllllll}7.0 & 16.1527 & 2.2844 & 0.08674 & 30.00 & 494.46 & 16.71 & 54.5\end{array}$

$\begin{array}{llllllll}7.5 & 17.3081 & 2.3395 & 0.09548 & 30.08 & 503.31 & 17.24 & 56.2\end{array}$

$\begin{array}{llllllll}12.5 & 32.0270 & 3.9202 & 0.21231 & 30.69 & 657.16 & 24.25 & 75.7\end{array}$

$\begin{array}{llllllll}13.0 & 34.0621 & 4.2262 & 0.22770 & 30.75 & 680.03 & 25.16 & 77.9\end{array}$

$\begin{array}{llllllll}13.5 & 36.2595 & 4.5698 & 0.24392 & 30.81 & 704.39 & 26.12 & 80.3\end{array}$

$\begin{array}{llllllll}14.0 & 38.6387 & 4.9543 & 0.26101 & 30.88 & 730.28 & 27.12 & 82.8\end{array}$

$\begin{array}{llllllll}14.5 & 41.2212 & 5.3833 & 0.27901 & 30.95 & 757.72 & 28.19 & 85.5\end{array}$

$\begin{array}{llllllll}15.0 & 44.0301 & 5.8606 & 0.29796 & 31.03 & 786.74 & 29.31 & 88.2\end{array}$

$\begin{array}{llllllll}15.5 & 47.0906 & 6.3905 & 0.31792 & 31.11 & 817.37 & 30.50 & 91.1\end{array}$

$\begin{array}{llllllll}16.0 & 50.4301 & 6.9774 & 0.33893 & 31.19 & 849.63 & 31.76 & 94.2\end{array}$

$\begin{array}{rrrrrrrr}16.5 & 54.0783 & 7.6264 & 0.36104 & 31.28 & 883.57 & 33.10 & 97.5\end{array}$

$\begin{array}{llllllll}17.0 & 58.0677 & 8.3426 & 0.38430 & 31.37 & 919.21 & 34.54 & 100.9\end{array}$

$\begin{array}{lllllllll}17.5 & 62.4331 & 9.1318 & 0.40877 & 31.46 & 956.61 & 36.07 & 104.6\end{array}$

$\begin{array}{llllllll}18.0 & 67.2127 & 10.0002 & 0.43452 & 31.56 & 995.80 & 37.72 & 108.4\end{array}$

$\begin{array}{llllllll}18.5 & 72.4476 & 10.9543 & 0.46160 & 31.66 & 1036.83 & 39.51 & 112.6\end{array}$

$\begin{array}{llllllll}19.0 & 78.1825 & 12.0013 & 0.49010 & 31.77 & 1079.75 & 41.45 & 116.9\end{array}$

$\begin{array}{llllllll}19.5 & 84.4656 & 13.1488 & 0.52008 & 31.87 & 1124.63 & 43.56 & 121.6\end{array}$

$\begin{array}{lllllllll}20.0 & 91.3494 & 14.4052 & 0.55164 & 31 . y 8 & 1171.51 & 45.89 & 126.5\end{array}$

$\begin{array}{llllllll}20.5 & 98.8904 & 15.7792 & 0.58485 & 32.08 & 1220.48 & 48.47 & 131.8\end{array}$ 
METHANE ISOTHERM AT $\mathrm{T}=340 . \mathrm{K}$

Second Virial Coefficient is $-29.4 \mathrm{~cm}^{3} / \mathrm{mol}$

$\begin{array}{rccccccc}\rho & \mathrm{P} & \mathrm{dP} /\left.\mathrm{d} \rho\right|_{\mathrm{T}} & \mathrm{dP} /\left.\mathrm{dT}\right|_{\rho} & \mathrm{C}_{\mathrm{v}} & \mathrm{W} & \eta & \lambda \\ \mathrm{mol} / \mathrm{dm}^{3} & \mathrm{MPa} & \mathrm{MPa} \cdot \mathrm{dm}^{3} / \mathrm{mol} & \mathrm{MPa} / \mathrm{K} & \mathrm{J} /(\mathrm{mol} \cdot \mathrm{k}) & \mathrm{m} / \mathrm{s} & \mu \mathrm{Pa} \cdot \mathrm{s} & \mathrm{mW} /(\mathrm{m} \cdot \mathrm{K}) \\ & & & & & & & \\ 0.5 & 1.3934 & 2.7480 & 0.00429 & 29.32 & 473.92 & 12.65 & 41.0 \\ 1.0 & 2.7495 & 2.6778 & 0.00886 & 29.47 & 472.61 & 12.88 & 41.8 \\ 1.5 & 4.0727 & 2.6168 & 0.01369 & 29.61 & 471.99 & 13.13 & 42.8 \\ 2.0 & 5.3678 & 2.5652 & 0.01880 & 29.74 & 472.10 & 13.41 & 43.7 \\ 2.5 & 6.6395 & 2.5233 & 0.02418 & 29.87 & 472.95 & 13.70 & 44.8 \\ 3.0 & 7.8928 & 2.4915 & 0.02984 & 29.99 & 474.58 & 14.01 & 45.9 \\ 3.5 & 9.1327 & 2.4698 & 0.03578 & 30.10 & 477.00 & 14.35 & 47.1 \\ 4.0 & 10.3643 & 2.4584 & 0.04201 & 30.21 & 480.24 & 14.71 & 48.3 \\ 4.5 & 11.5928 & 2.4575 & 0.04855 & 30.31 & 484.32 & 15.09 & 49.7 \\ 5.0 & 12.8236 & 2.4675 & 0.05540 & 30.40 & 489.25 & 15.50 & 51.1 \\ 5.5 & 14.0622 & 2.4886 & 0.06258 & 30.49 & 495.07 & 15.92 & 52.5 \\ 6.0 & 15.3142 & 2.5215 & 0.07011 & 30.58 & 501.79 & 16.38 & 54.0 \\ 6.5 & 16.5857 & 2.5666 & 0.07800 & 30.66 & 509.44 & 16.85 & 55.6 \\ 7.0 & 17.8830 & 2.6248 & 0.08629 & 30.74 & 518.06 & 17.36 & 57.2 \\ 7.5 & 19.2128 & 2.6969 & 0.09499 & 30.81 & 527.69 & 17.89 & 58.9 \\ 12.5 & 36.2612 & 4.5172 & 0.21111 & 31.44 & 688.36 & 24.86 & 78.4 \\ 13.0 & 38.6027 & 4.8551 & 0.22636 & 31.51 & 711.75 & 25.77 & 80.8 \\ 13.5 & 41.1228 & 5.2322 & 0.24242 & 31.57 & 736.60 & 26.72 & 83.2 \\ 14.0 & 43.8420 & 5.6518 & 0.25932 & 31.64 & 762.95 & 27.72 & 85.7 \\ 14.5 & 46.7823 & 6.1177 & 0.27712 & 31.72 & 790.82 & 28.78 & 88.3 \\ 15.0 & 49.9680 & 6.6337 & 0.29585 & 31.79 & 820.25 & 29.89 & 91.1 \\ 15.5 & 53.4251 & 7.2042 & 0.31557 & 31.87 & 851.28 & 31.08 & 94.0 \\ 16.0 & 57.1821 & 7.8339 & 0.33631 & 31.95 & 883.94 & 32.34 & 97.1 \\ 16.5 & 61.2697 & 8.5276 & 0.35815 & 32.04 & 918.28 & 33.68 & 100.4 \\ 17.0 & 65.7213 & 9.2909 & 0.38113 & 32.12 & 954.32 & 35.11 & 103.9 \\ & & & & & & & \\ 17.5 & 70.5732 & 10.1297 & 0.40531 & 32.21 & 992.13 & 36.64 & 107.5 \\ 18.0 & 75.8646 & 11.0501 & 0.43076 & 32.30 & 1031.75 & 38.30 & 111.4 \\ 18.5 & 81.6381 & 12.0592 & 0.45755 & 32.40 & 1073.24 & 40.08 & 115.6 \\ 19.0 & 87.9398 & 13.1643 & 0.48575 & 32.49 & 1116.65 & 42.03 & 120.0 \\ 19.5 & 94.8197 & 14.3732 & 0.51545 & 32.58 & 1162.06 & 44.16 & 124.6\end{array}$


METHANE ISOTHERM AT $\mathrm{T}=360 . \mathrm{K}$

Second Virial Coefficient is $-24.2 \mathrm{~cm}^{3} / \mathrm{mol}$

$\begin{array}{rrrllllll}\rho & \mathrm{P} & \mathrm{dP} /\left.\mathrm{d} \rho\right|_{\mathrm{T}} & \mathrm{dP} / \mathrm{dT} \mid \rho & \mathrm{C}_{\mathrm{v}} & \mathrm{W} & \eta & \lambda \\ \mathrm{mol} / \mathrm{dm}^{3} & \mathrm{MPa} & \mathrm{MPa} \cdot \mathrm{dm}^{3} / \mathrm{mol} & \mathrm{MPa} / \mathrm{K} & \mathrm{J} /(\mathrm{mol} \cdot \mathrm{k}) & \mathrm{m} / \mathrm{s} & \mu \mathrm{Pa} \cdot \mathrm{s} & \mathrm{mW} /(\mathrm{m} \cdot \mathrm{K}) \\ & & & & & & & \\ 0.5 & 1.4792 & 2.9251 & 0.00429 & 30.29 & 486.67 & 13.26 & 44.1 \\ 1.0 & 2.9265 & 2.8655 & 0.00884 & 30.42 & 486.10 & 13.50 & 45.0 \\ 1.5 & 4.3463 & 2.8152 & 0.01366 & 30.55 & 486.21 & 13.75 & 45.9 \\ 2.0 & 5.7433 & 2.7744 & 0.01875 & 30.67 & 487.03 & 14.02 & 46.9 \\ 2.5 & 7.1223 & 2.7435 & 0.02410 & 30.79 & 488.59 & 14.32 & 47.9 \\ & & & & & & & \\ 3.0 & 8.4884 & 2.7227 & 0.02972 & 30.90 & 490.93 & 14.64 & 49.0 \\ 3.5 & 9.8468 & 2.7125 & 0.03563 & 31.00 & 494.06 & 14.97 & 50.2 \\ 4.0 & 11.2027 & 2.7129 & 0.04183 & 31.10 & 498.00 & 15.33 & 51.4 \\ 4.5 & 12.5615 & 2.7244 & 0.04833 & 31.19 & 502.78 & 15.72 & 52.7 \\ 5.0 & 13.9289 & 2.7472 & 0.05514 & 31.28 & 508.41 & 16.12 & 54.1 \\ & & & & & & & \\ 5.5 & 15.3107 & 2.7820 & 0.06228 & 31.37 & 514.93 & 16.55 & 55.5 \\ 6.0 & 16.7130 & 2.8291 & 0.06978 & 31.45 & 522.35 & 17.00 & 57.0 \\ 6.5 & 18.1420 & 2.8893 & 0.07764 & 31.52 & 530.71 & 17.48 & 58.6 \\ 7.0 & 19.6047 & 2.9636 & 0.08588 & 31.59 & 540.04 & 17.98 & 60.2 \\ 7.5 & 21.1081 & 3.0527 & 0.09454 & 31.66 & 550.36 & 18.51 & 61.9 \\ & & & & & & & \\ 12.5 & 40.4717 & 5.1090 & 0.20995 & 32.30 & 717.26 & 25.46 & 81.4 \\ 13.0 & 43.1168 & 5.4781 & 0.22507 & 32.36 & 741.14 & 26.36 & 83.7 \\ 13.5 & 45.9566 & 5.8880 & 0.24098 & 32.43 & 766.48 & 27.30 & 86.2 \\ 14.0 & 49.0122 & 6.3421 & 0.25772 & 32.50 & 793.29 & 28.30 & 88.7 \\ 14.5 & 52.3067 & 6.8443 & 0.27535 & 32.57 & 821.61 & 29.35 & 91.4 \\ 15.0 & 55.8652 & 7.3984 & 0.29389 & 32.65 & 851.49 & 30.47 & 94.2 \\ 15.5 & 59.7145 & 8.0090 & 0.31341 & 32.72 & 882.95 & 31.65 & 97.1 \\ 16.0 & 63.8843 & 8.6808 & 0.33395 & 32.80 & 916.05 & 32.90 & 100.2 \\ 16.5 & 68.4064 & 9.4189 & 0.35557 & 32.88 & 950.82 & 34.24 & 103.5 \\ 17.0 & 73.3152 & 10.2290 & 0.37832 & 32.96 & 987.31 & 35.67 & 107.0 \\ & & & & & & & \\ 17.5 & 78.6484 & 11.1171 & 0.40228 & 33.04 & 1025.58 & 37.20 & 110.7 \\ 18.0 & 84.4464 & 12.0897 & 0.42750 & 33.13 & 1065.69 & 38.86 & 114.6 \\ 18.5 & 90.7533 & 13.1539 & 0.45406 & 33.21 & 1107.68 & 40.65 & 118.7 \\ 19.0 & 97.6168 & 14.3172 & 0.48205 & 33.29 & 1151.63 & 42.60 & 123.1\end{array}$


METHANE ISOTHERM AT $\mathrm{T}=380 . \mathrm{K}$

Second Virial Coefficent is $-19.6 \mathrm{~cm}^{3} / \mathrm{mol}$

$\begin{array}{rrrrrrrr}\rho & \mathrm{P} & \mathrm{dP} /\left.\mathrm{d} \rho\right|_{\mathrm{T}} & \mathrm{dP} / \mathrm{dT} \mid \rho & \mathrm{C}_{\mathrm{v}} & \mathrm{W} & \eta & \lambda \\ \mathrm{mol} / \mathrm{dm}^{3} & \mathrm{MPa} & \mathrm{MPa} \cdot \mathrm{dm}^{3} / \mathrm{mol} & \mathrm{MPa} / \mathrm{K} & \mathrm{J} /(\mathrm{mol} \cdot \mathrm{k}) & \mathrm{m} / \mathrm{s} & \mu \mathrm{Pa} \cdot \mathrm{s} & \mathrm{mW} /(\mathrm{m} \cdot \mathrm{K}) \\ & & & & & & & \\ 0.5 & 1.5650 & 3.1018 & 0.00429 & 31.33 & 498.90 & 13.86 & 47.4 \\ 1.0 & 3.1032 & 3.0527 & 0.00883 & 31.45 & 499.00 & 14.10 & 48.2 \\ 1.5 & 4.6192 & 3.0128 & 0.01363 & 31.57 & 499.77 & 14.35 & 49.1 \\ 2.0 & 6.1177 & 2.9826 & 0.01870 & 31.68 & 501.24 & 14.63 & 50.1 \\ 2.5 & 7.6035 & 2.9625 & 0.02402 & 31.79 & 503.46 & 14.92 & 51.1 \\ & & & & & & & \\ 3.0 & 9.0819 & 2.9528 & 0.02962 & 31.89 & 506.44 & 15.24 & 52.2 \\ 3.5 & 10.5581 & 2.9539 & 0.03550 & 31.99 & 510.21 & 15.58 & 53.4 \\ 4.0 & 12.0376 & 2.9661 & 0.04167 & 32.08 & 514.80 & 15.94 & 54.6 \\ 4.5 & 13.5261 & 2.9899 & 0.04814 & 32.16 & 520.22 & 16.33 & 55.9 \\ 5.0 & 15.0295 & 3.0256 & 0.05492 & 32.25 & 526.50 & 16.73 & 57.3 \\ & & & & & & & \\ 5.5 & 16.5538 & 3.0738 & 0.06203 & 32.33 & 533.66 & 17.16 & 58.7 \\ 6.0 & 18.1055 & 3.1353 & 0.06948 & 32.40 & 541.72 & 17.61 & 60.2 \\ 6.5 & 19.6914 & 3.2106 & 0.07730 & 32.47 & 550.72 & 18.09 & 61.7 \\ 7.0 & 21.3186 & 3.3008 & 0.08552 & 32.54 & 560.69 & 18.59 & 63.3 \\ 7.5 & 22.9948 & 3.4069 & 0.09414 & 32.61 & 571.66 & 19.12 & 65.0 \\ 12.5 & 44.6595 & 5.6962 & 0.20885 & 33.23 & 744.33 & 26.04 & 84.5 \\ 13.0 & 47.6059 & 6.0960 & 0.22385 & 33.30 & 768.70 & 26.93 & 86.9 \\ 13.5 & 50.7626 & 6.5382 & 0.23964 & 33.36 & 794.51 & 27.87 & 89.3 \\ 14.0 & 54.1517 & 7.0263 & 0.25625 & 33.43 & 821.79 & 28.87 & 91.9 \\ 14.5 & 57.7972 & 7.5642 & 0.27373 & 33.50 & 850.57 & 29.92 & 94.5 \\ 15.0 & 61.7250 & 8.1561 & 0.29212 & 33.57 & 880.90 & 31.02 & 97.4 \\ 15.5 & 65.9630 & 8.8064 & 0.31147 & 33.64 & 912.82 & 32.20 & 100.3 \\ 16.0 & 70.5419 & 9.5200 & 0.33185 & 33.71 & 946.37 & 33.45 & 103.4 \\ 16.5 & 75.4945 & 10.3023 & 0.35329 & 33.79 & 981.61 & 34.79 & 106.7 \\ 17.0 & 80.8566 & 11.1591 & 0.37587 & 33.86 & 1018.58 & 36.22 & 110.2 \\ 17.5 & 86.6670 & 12.0965 & 0.39965 & 33.94 & 1057.34 & 37.75 & 113.9 \\ 18.0 & 92.9676 & 13.1212 & 0.42469 & 34.02 & 1097.96 & 39.41 & 117.8 \\ 18.5 & 99.8040 & 14.2407 & 0.45108 & 34.09 & 1140.49 & 41.20 & 122.0\end{array}$


METHANE ISOTHERM AT $\mathrm{T}=400 . \mathrm{K}$

Second Virial Coefficient is $-15.5 \mathrm{~cm}^{3} / \mathrm{mol}$

$\begin{array}{rcrlllll}\rho & \mathrm{P} & \mathrm{dP} /\left.\mathrm{d} \rho\right|_{\mathrm{T}} & \mathrm{dP} /\left.\mathrm{dT}\right|_{\rho} & \mathrm{C}_{\mathrm{v}} & \mathrm{W} & \eta & \lambda \\ \mathrm{mol} / \mathrm{dm}^{3} & \mathrm{MPa} & \mathrm{MPa} \cdot \mathrm{dm}^{3} / \mathrm{mol} & \mathrm{MPa} / \mathrm{K} & \mathrm{J} /(\mathrm{mol} \cdot \mathrm{k}) & \mathrm{m} / \mathrm{s} & \mu \mathrm{Pa} \cdot \mathrm{s} & \mathrm{mW} /(\mathrm{m} \cdot \mathrm{K}) \\ & & & & & & & \\ 0.5 & 1.6507 & 3.2783 & 0.00428 & 32.42 & 510.66 & 14.44 & 50.8 \\ 1.0 & 3.2797 & 3.2394 & 0.00882 & 32.53 & 511.38 & 14.68 & 51.6 \\ 1.5 & 4.8916 & 3.2098 & 0.01361 & 32.64 & 512.76 & 14.94 & 52.5 \\ 2.0 & 6.4912 & 3.1900 & 0.01865 & 32.75 & 514.85 & 15.22 & 53.5 \\ 2.5 & 8.0834 & 3.1805 & 0.02396 & 32.85 & 517.67 & 15.51 & 54.5 \\ & & & & & & & \\ 3.0 & 9.6735 & 3.1818 & 0.02954 & 32.94 & 521.24 & 15.83 & 55.6 \\ 3.5 & 11.2670 & 3.1941 & 0.03539 & 33.03 & 525.61 & 16.17 & 56.7 \\ 4.0 & 12.8696 & 3.2181 & 0.04153 & 33.12 & 530.79 & 16.54 & 58.0 \\ 4.5 & 14.4871 & 3.2541 & 0.04797 & 33.20 & 536.81 & 16.92 & 59.3 \\ 5.0 & 16.1257 & 3.3027 & 0.05472 & 33.28 & 543.68 & 17.33 & 60.6 \\ & & & & & & & \\ 5.5 & 17.7919 & 3.3644 & 0.06179 & 33.35 & 551.43 & 17.76 & 62.0 \\ 6.0 & 19.4925 & 3.4400 & 0.06922 & 33.42 & 560.10 & 18.21 & 63.5 \\ 6.5 & 21.2344 & 3.5304 & 0.07701 & 33.49 & 569.69 & 18.68 & 65.0 \\ 7.0 & 23.0255 & 3.6365 & 0.08518 & 33.56 & 580.25 & 19.18 & 66.6 \\ 7.5 & 24.8738 & 3.7594 & 0.09376 & 33.62 & 591.81 & 19.71 & 68.3 \\ 12.5 & 48.8263 & 6.2791 & 0.20784 & 34.23 & 769.92 & 26.60 & 87.8 \\ 13.0 & 52.0716 & 6.7094 & 0.22274 & 34.29 & 794.78 & 27.49 & 90.2 \\ 13.5 & 55.5430 & 7.1836 & 0.23842 & 34.35 & 821.06 & 28.43 & 92.6 \\ 14.0 & 59.2631 & 7.7054 & 0.25491 & 34.42 & 848.80 & 29.42 & 95.2 \\ 14.5 & 63.2569 & 8.2787 & 0.27227 & 34.48 & 878.05 & 30.46 & 97.9 \\ 15.0 & 67.5512 & 8.9080 & 0.29053 & 34.55 & 908.84 & 31.57 & 100.7 \\ 15.5 & 72.1750 & 9.5977 & 0.30975 & 34.62 & 941.23 & 32.74 & 103.6 \\ 16.0 & 77.1598 & 10.3531 & 0.32999 & 34.68 & 975.25 & 33.99 & 106.8 \\ 16.5 & 82.5399 & 11.1794 & 0.35129 & 34.75 & 1010.97 & 35.32 & 110.1 \\ 17.0 & 88.3521 & 12.0828 & 0.37373 & 34.82 & 1048.44 & 36.75 & 113.6 \\ 17.5 & 94.6366 & 13.0695 & 0.39737 & 34.89 & 1087.71 & 38.29 & 117.3\end{array}$


METHANE ISOTHERM AT $\mathrm{T}=420 . \mathrm{K}$

Second Virial Coefficient is $-11.9 \mathrm{~cm}^{3} / \mathrm{mol}$

\begin{tabular}{|c|c|c|c|c|c|c|c|}
\hline$\rho$ & $P$ & $\mathrm{dP} /\left.\mathrm{d} \rho\right|_{\mathrm{T}}$ & $\mathrm{dP} /\left.\mathrm{dT}\right|_{\rho}$ & $C_{v}$ & W & $\eta$ & $\lambda$ \\
\hline $\mathrm{mol} / \mathrm{dm}^{3}$ & $\mathrm{MPa}$ & $\mathrm{MPa} \cdot \mathrm{dm}^{3} / \mathrm{mol}$ & $\mathrm{MPa} / \mathrm{K}$ & $\mathrm{J} /(\mathrm{mol} \cdot \mathrm{k})$ & $\mathrm{m} / \mathrm{s}$ & $\mu \mathrm{Pa} \cdot \mathrm{S}$ & $\mathrm{mW} /(\mathrm{m} \cdot \mathrm{K})$ \\
\hline 0.5 & 1.7363 & 3.4545 & 0.00428 & 33.54 & 522.04 & & 54.3 \\
\hline 1.0 & 3.4560 & 3.4257 & 0.00881 & 33.65 & 523.33 & & 55.1 \\
\hline 1.5 & 5.1635 & 3.4062 & 0.01358 & 33.75 & 525.29 & & 56.0 \\
\hline 2.0 & 6.8638 & 3.3967 & 0.01861 & 33.85 & 527.94 & & 57.0 \\
\hline 2.5 & 8.5620 & 3.3978 & 0.02390 & 33.94 & 531.32 & & 58.0 \\
\hline 3.0 & 10.2634 & 3.4098 & 0.02946 & 34.03 & 535.46 & & 59.1 \\
\hline 3.5 & 11.9737 & 3.4334 & 0.03529 & 34.12 & 540.38 & & 60.2 \\
\hline 4.0 & 13.6988 & 3.4690 & 0.04140 & 34.20 & 546.12 & & 61.4 \\
\hline 4.5 & 15.4448 & 3.5172 & 0.04781 & 34.28 & 552.69 & & 62.7 \\
\hline 5.0 & 17.2182 & 3.5785 & 0.05453 & 34.35 & 560.11 & & 64.1 \\
\hline 5.5 & 19.0257 & 3.6537 & 0.06158 & 34.42 & 568.42 & & 65.5 \\
\hline 6.0 & 20.8744 & 3.7435 & 0.06898 & 34.49 & 577.64 & & 66.9 \\
\hline 6.5 & 22.7718 & 3.8489 & 0.07673 & 34.55 & 587.79 & & 68.4 \\
\hline 7.0 & 24.7260 & 3.9708 & 0.08487 & 34.62 & 598.91 & & 70.0 \\
\hline 7.5 & 26.7456 & 4.1105 & 0.09342 & 34.68 & 611.02 & & 71.7 \\
\hline 12.5 & 52.9736 & 6.8585 & 0.20691 & 35.26 & 794.32 & & 91.2 \\
\hline 13.0 & 56.5161 & 7.3188 & 0.22173 & 35.32 & 819.64 & & 93.6 \\
\hline 13.5 & 60.3001 & 7.8248 & 0.23731 & 35.38 & 846.39 & & 96.0 \\
\hline 14.0 & 64.3491 & 8.3800 & 0.25371 & 35.44 & 874.60 & & 98.6 \\
\hline 14.5 & 68.6890 & 8.9887 & 0.27096 & 35.50 & 904.32 & & 101.3 \\
\hline 15.0 & 73.3474 & 9.6551 & 0.28912 & 35.57 & 935.58 & & 104.1 \\
\hline 15.5 & 78.3545 & 10.3842 & 0.30823 & 35.63 & 968.43 & & 107.1 \\
\hline 16.0 & 83.7429 & 11.1812 & 0.32836 & 35.69 & 1002.94 & & 110.2 \\
\hline 16.5 & 89.5479 & 12.0515 & 0.34955 & 35.75 & 1039.15 & & 113.6 \\
\hline 17.0 & 95.8078 & 13.0015 & 0.37188 & 35.81 & 1077.13 & & 117.1 \\
\hline
\end{tabular}

METHANE ISOTHERM AT $\mathrm{T}=450 . \mathrm{K}$
Second Virial Coefficient is $-7.3 \mathrm{~cm}^{3} / \mathrm{mol}$

$\begin{array}{llllllll}\rho & \mathrm{P} & \mathrm{dP} /\left.\mathrm{d} \rho\right|_{\mathrm{T}} & \mathrm{dP} /\left.\mathrm{dT}\right|_{\rho} & \mathrm{C}_{\mathrm{v}} & \mathrm{W} & \eta & \lambda\end{array}$

$\mathrm{mol} / \mathrm{dm}^{3} \quad \mathrm{MPa} \quad \mathrm{MPa} \cdot \mathrm{dm}^{3} / \mathrm{mol} \quad \mathrm{MPa} / \mathrm{K} \quad \mathrm{J} /(\mathrm{mol} \cdot \mathrm{k}) \quad \mathrm{m} / \mathrm{s} \quad \mu \mathrm{Pa} \cdot \mathrm{s} \quad \mathrm{mW} /(\mathrm{m} \cdot \mathrm{K})$

$\begin{array}{lllllll}0.5 & 1.8646 & 3.7185 & 0.00428 & 35.26 & 538.49 & 59.7 \\ 1.0 & 3.7200 & 3.7043 & 0.00879 & 35.36 & 540.59 & 60.5 \\ 1.5 & 5.5706 & 3.6998 & 0.01355 & 35.46 & 543.33 & 61.4 \\ 2.0 & 7.4215 & 3.7055 & 0.01856 & 35.55 & 546.77 & 62.4 \\ 2.5 & 9.2779 & 3.7222 & 0.02383 & 35.63 & 550.93 & 63.4\end{array}$


METHANE ISOTHERM AT $\mathrm{T}=450 . \mathrm{K}$ (continued)

\begin{tabular}{|c|c|c|c|c|c|c|c|}
\hline$\rho$ & $P$ & $\mathrm{dP} /\left.\mathrm{d} \rho\right|_{\mathrm{T}}$ & $\mathrm{dP} /\left.\mathrm{dT}\right|_{\rho}$ & $\mathrm{C}_{\mathrm{v}}$ & W & $\eta$ & $\lambda$ \\
\hline $\mathrm{mol} / \mathrm{dm}^{3}$ & $\mathrm{MPa}$ & $\mathrm{MPa} \cdot \mathrm{dm}^{3} / \mathrm{mol}$ & $\mathrm{MPa} / \mathrm{K}$ & $\mathrm{J} /(\mathrm{mol} \cdot \mathrm{k})$ & $\mathrm{m} / \mathrm{s}$ & $\mu \mathrm{Pa} \cdot \mathrm{s}$ & $\mathrm{mW} /(\mathrm{m} \cdot \mathrm{K})$ \\
\hline 3.0 & 11.1456 & 3.7503 & 0.02935 & 35.71 & 555.85 & & 64.5 \\
\hline 3.5 & 13.0303 & 3.7906 & 0.03515 & 35.79 & 561.55 & & 65.6 \\
\hline 4.0 & 14.9383 & 3.8436 & 0.04123 & 35.87 & 568.05 & & 66.8 \\
\hline 4.5 & 16.8761 & 3.9099 & 0.04761 & 35.94 & 575.40 & & 68.1 \\
\hline 5.0 & 18.8506 & 3.9903 & 0.05429 & 36.01 & 583.60 & & 69.4 \\
\hline 5.5 & 20.8689 & 4.0856 & 0.06130 & 36.07 & 592.68 & & 70.8 \\
\hline 6.0 & 22.9388 & 4.1965 & 0.06866 & 36.14 & 602.68 & & 72.3 \\
\hline 6.5 & 25.0682 & 4.3242 & 0.07637 & 36.20 & 613.61 & & 73.8 \\
\hline 7.0 & 27.2660 & 4.4697 & 0.08446 & 36.26 & 625.50 & & 75.3 \\
\hline 7.5 & 29.5411 & 4.6343 & 0.09296 & 36.31 & 638.39 & & 77.0 \\
\hline 12.5 & 59.1623 & 7.7217 & 0.20569 & 36.86 & 829.08 & & 96.5 \\
\hline 13.0 & 63.1475 & 8.2269 & 0.22040 & 36.91 & 855.11 & & 98.9 \\
\hline 13.5 & 67.3972 & 8.7801 & 0.23587 & 36.97 & 882.55 & & 101.4 \\
\hline 14.0 & 71.9363 & 9.3852 & 0.25215 & 37.02 & 911.46 & & 103.9 \\
\hline 14.5 & 76.7918 & 10.0466 & 0.26928 & 37.08 & 941.87 & & 106.6 \\
\hline 15.0 & 81.9930 & 10.7687 & 0.28731 & 37.13 & 973.84 & & 109.5 \\
\hline 15.5 & 87.5715 & 11.5567 & 0.30629 & 37.19 & 1007.42 & & 112.5 \\
\hline 16.0 & 93.5616 & 12.4160 & 0.32629 & 37.24 & 1042.66 & & 115.6 \\
\hline
\end{tabular}

METHANE ISOTHERM AT $\mathrm{T}=500 . \mathrm{K}$
Second Virial Coefficient is $-1.0 \mathrm{~cm}^{3} / \mathrm{mol}$

$\begin{array}{llllllll}\rho & \mathrm{P} & \mathrm{dP} /\left.\mathrm{d} \rho\right|_{\mathrm{T}} & \mathrm{dP} /\left.\mathrm{dT}\right|_{\rho} & \mathrm{C}_{\mathrm{v}} & \mathrm{W} & \eta & \lambda\end{array}$

$\mathrm{mol} / \mathrm{dm}^{3} \quad \mathrm{MPa} \quad \mathrm{MPa} \cdot \mathrm{dm}^{3} / \mathrm{mol} \quad \mathrm{MPa} / \mathrm{K} \quad \mathrm{J} /(\mathrm{mol} \cdot \mathrm{k}) \quad \mathrm{m} / \mathrm{s} \quad \mu \mathrm{Pa} \cdot \mathrm{s} \quad \mathrm{mW} /(\mathrm{m} \cdot \mathrm{K})$

$\begin{array}{rrrrrrr}0.5 & 2.0783 & 4.1576 & 0.00427 & 38.14 & 564.61 & 69.1 \\ 1.0 & 4.1592 & 4.1672 & 0.00877 & 38.23 & 567.91 & 69.9 \\ 1.5 & 6.2472 & 4.1869 & 0.01351 & 38.31 & 571.84 & 70.8 \\ 2.0 & 8.3479 & 4.2175 & 0.01849 & 38.39 & 576.46 & 71.7 \\ 2.5 & 10.4666 & 4.2597 & 0.02372 & 38.47 & 581.79 & 72.8 \\ 3.0 & 12.6096 & 4.3143 & 0.02921 & 38.54 & 587.88 & 73.8 \\ 3.5 & 14.7831 & 4.3821 & 0.03497 & 38.61 & 594.75 & 75.0 \\ 4.0 & 16.9940 & 4.4637 & 0.04100 & 38.67 & 602.42 & 76.1 \\ 4.5 & 19.2493 & 4.5601 & 0.04733 & 38.74 & 610.93 & 77.4 \\ 5.0 & 21.5567 & 4.6720 & 0.05396 & 38.80 & 620.31 & 78.7\end{array}$


METHANE ISOTHERM AT $\mathrm{T}=500 . \mathrm{K}$ (continued)

$\begin{array}{llllllll}\rho & \mathrm{P} & \mathrm{dP} /\left.\mathrm{d} \rho\right|_{\mathrm{T}} & \mathrm{dP} /\left.\mathrm{dT}\right|_{\rho} & \mathrm{C}_{\mathrm{v}} & \mathrm{W} & \eta & \lambda\end{array}$

$\mathrm{mol} / \mathrm{dm}^{3} \quad \mathrm{MPa} \quad \mathrm{MPa} \cdot \mathrm{dm}^{3} / \mathrm{mol} \quad \mathrm{MPa} / \mathrm{K} \quad \mathrm{J} /(\mathrm{mol} \cdot \mathrm{k}) \quad \mathrm{m} / \mathrm{s} \quad \mu \mathrm{Pa} \cdot \mathrm{s} \quad \mathrm{mW} /(\mathrm{m} \cdot \mathrm{K})$

$\begin{array}{rrrrrrr}5.5 & 23.9241 & 4.8004 & 0.06092 & 38.86 & 630.57 & 80.1 \\ 6.0 & 26.3600 & 4.9464 & 0.06821 & 38.91 & 641.74 & 81.5 \\ 6.5 & 28.8736 & 5.1110 & 0.07586 & 38.97 & 653.85 & 83.0 \\ 7.0 & 31.4743 & 5.2954 & 0.08389 & 39.02 & 666.93 & 84.6 \\ 7.5 & 34.1726 & 5.5012 & 0.09232 & 39.07 & 681.00 & 86.2 \\ & & & & & & \\ 12.5 & 69.4038 & 9.1484 & 0.20404 & 39.55 & 883.30 & 105.8 \\ 13.0 & 74.1207 & 9.7278 & 0.21862 & 39.59 & 910.47 & 108.1 \\ 13.5 & 79.1403 & 10.3593 & 0.23395 & 39.64 & 939.06 & 110.6 \\ 14.0 & 84.4895 & 11.0473 & 0.25009 & 39.69 & 969.13 & 113.2 \\ 14.5 & 90.1977 & 11.7961 & 0.26708 & 39.73 & 1000.71 & 115.9 \\ 15.0 & 96.2966 & 12.6110 & 0.28497 & 39.77 & 1033.87 & 118.8\end{array}$

METHANE ISOTHERM AT $T=550 . \mathrm{K}$
Second Virial Coefficient is $4.0 \mathrm{~cm}^{3} / \mathrm{mol}$

$\begin{array}{llllllll}\rho & \mathrm{P} & \mathrm{dP} /\left.\mathrm{d} \rho\right|_{\mathrm{T}} & \mathrm{dP} / \mathrm{dT} \mid \rho & \mathrm{C}_{\mathrm{v}} & \mathrm{W} & \eta & \lambda\end{array}$

$\mathrm{mol} / \mathrm{dm}^{3} \quad \mathrm{MPa} \quad \mathrm{MPa} \cdot \mathrm{dm}^{3} / \mathrm{mol} \quad \mathrm{MPa} / \mathrm{K} \quad \mathrm{J} /(\mathrm{mol} \cdot \mathrm{k}) \quad \mathrm{m} / \mathrm{s} \quad \mu \mathrm{Pa} \cdot \mathrm{s} \quad \mathrm{mW} /(\mathrm{m} \cdot \mathrm{K})$

$\begin{array}{rrrrrrr}0.5 & 2.2918 & 4.5959 & 0.00427 & 40.93 & 589.48 & 78.8 \\ 1.0 & 4.5975 & 4.6285 & 0.00876 & 41.01 & 593.84 & 79.6 \\ 1.5 & 6.9221 & 4.6717 & 0.01348 & 41.08 & 598.83 & 80.5 \\ 2.0 & 9.2711 & 4.7266 & 0.01844 & 41.15 & 604.50 & 81.4 \\ 2.5 & 11.6507 & 4.7939 & 0.02364 & 41.22 & 610.89 & 82.4 \\ 3.0 & 14.0673 & 4.8746 & 0.02910 & 41.29 & 618.03 & 83.5 \\ 3.5 & 16.5277 & 4.9695 & 0.03482 & 41.35 & 625.95 & 84.6 \\ 4.0 & 19.0393 & 5.0795 & 0.04082 & 41.41 & 634.69 & 85.8 \\ 4.5 & 21.6099 & 5.2057 & 0.04710 & 41.46 & 644.26 & 87.1 \\ 5.0 & 24.2478 & 5.3489 & 0.05369 & 41.52 & 654.70 & 88.4 \\ & & & & & & \\ 5.5 & 26.9618 & 5.5102 & 0.06060 & 41.57 & 666.03 & 89.7 \\ 6.0 & 29.7612 & 5.6908 & 0.06785 & 41.62 & 678.27 & 91.2 \\ 6.5 & 32.6560 & 5.8920 & 0.07545 & 41.67 & 691.46 & 94.2 \\ 7.0 & 35.6569 & 6.1151 & 0.08343 & 41.72 & 705.63 & 95.8 \\ 7.5 & 38.7751 & 6.3617 & 0.09180 & 41.76 & 720.78 & \\ & & & & & & 115.4 \\ 12.5 & 79.5735 & 10.5644 & 0.20280 & 42.17 & 934.07 & 117.7 \\ 13.0 & 85.0167 & 11.2177 & 0.21729 & 42.21 & 962.36 & 120.2 \\ 13.5 & 90.8006 & 11.9274 & 0.23254 & 42.25 & 992.09 & 122.8 \\ 14.0 & 96.9543 & 12.6981 & 0.24859 & 42.28 & 1023.30 & \end{array}$


METHANE ISOTHERM AT $\mathrm{T}=600 . \mathrm{K}$

Second Virial Coefficient is $8.0 \mathrm{~cm}^{3} / \mathrm{mol}$

$\begin{array}{lllllllll}\rho & \mathrm{P} & \mathrm{dP} /\left.\mathrm{d} \rho\right|_{\mathrm{T}} & \mathrm{dP} /\left.\mathrm{dT}\right|_{\rho} & \mathrm{C}_{\mathrm{v}} & \mathrm{W} & \eta & \lambda\end{array}$

$\begin{array}{llllllll}\mathrm{mol} / \mathrm{dm}^{3} & \mathrm{MPa} & \mathrm{MPa} \cdot \mathrm{dm}^{3} / \mathrm{mol} & \mathrm{MPa} / \mathrm{K} & \mathrm{J} /(\mathrm{mol} \cdot \mathrm{k}) & \mathrm{m} / \mathrm{s} & \mu \mathrm{Pa} \cdot \mathrm{s} & \mathrm{mW} /(\mathrm{m} \cdot \mathrm{K})\end{array}$

$\begin{array}{rrrrrrr}0.5 & 2.5051 & 5.0334 & 0.00426 & 43.57 & 613.34 & 88.6 \\ 1.0 & 5.0351 & 5.0884 & 0.00875 & 43.64 & 618.66 & 89.4 \\ 1.5 & 7.5954 & 5.1547 & 0.01345 & 43.71 & 624.62 & 90.3 \\ 2.0 & 10.1919 & 5.2334 & 0.01839 & 43.77 & 631.26 & 91.3 \\ 2.5 & 12.8311 & 5.3254 & 0.02357 & 43.83 & 638.61 & 92.3 \\ & & & & & & \\ 3.0 & 15.5198 & 5.4318 & 0.02900 & 43.89 & 646.71 & 93.3 \\ 3.5 & 18.2655 & 5.5536 & 0.03470 & 43.94 & 655.60 & 94.4 \\ 4.0 & 21.0761 & 5.6918 & 0.04066 & 44.00 & 665.31 & 95.6 \\ 4.5 & 23.9602 & 5.8475 & 0.04692 & 44.05 & 675.86 & 96.9 \\ 5.0 & 26.9267 & 6.0218 & 0.05347 & 44.10 & 687.28 & 98.2 \\ & & & & & & \\ 5.5 & 29.9853 & 6.2158 & 0.06035 & 44.14 & 699.61 & 99.5 \\ 6.0 & 33.1460 & 6.4310 & 0.06756 & 44.19 & 712.85 & 100.9 \\ 6.5 & 36.4200 & 6.6686 & 0.07512 & 44.23 & 727.05 & 102.4 \\ 7.0 & 39.8186 & 6.9301 & 0.08305 & 44.27 & 742.22 & 104.0 \\ 7.5 & 43.3543 & 7.2172 & 0.09138 & 44.31 & 758.40 & 105.6 \\ & & & & & & \\ 12.5 & 89.6893 & 11.9735 & 0.20188 & 44.66 & 982.23 & 125.1 \\ 13.0 & 95.8555 & 12.7008 & 0.21631 & 44.69 & 1011.62 & 127.5\end{array}$




\section{APPENDIX C}

LISTING OF A FORTRAN 77 PROGRAM TO CALCULATE THE THERMOPHYSICAL PROPERTIES OF METHANE 
In this Appendix, we give the complete listing for a FORTRAN 77 (FORTRAN V) program to calculate the thermophysical properties of methane. Several aspects of this code must be mentioned. The program was written for a mainframe computer with very high precision; implementations for many personal computers will need to convert constants, functions, etc. to double precision. The program reflects the correlations as written in the body of the text; no systematic attempt to optimize the code was undertaken. The comments interspersed throughout the code are meant to assist a programmer; however these are not complete. The program should be used in conjunction with this manuscript and Ref. [1], to assist in judging the uncertainties of the resulting properties and the applicable ranges of the correlations. 


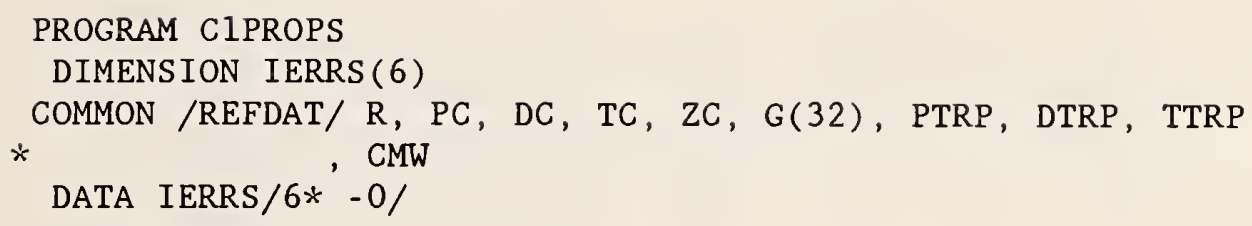




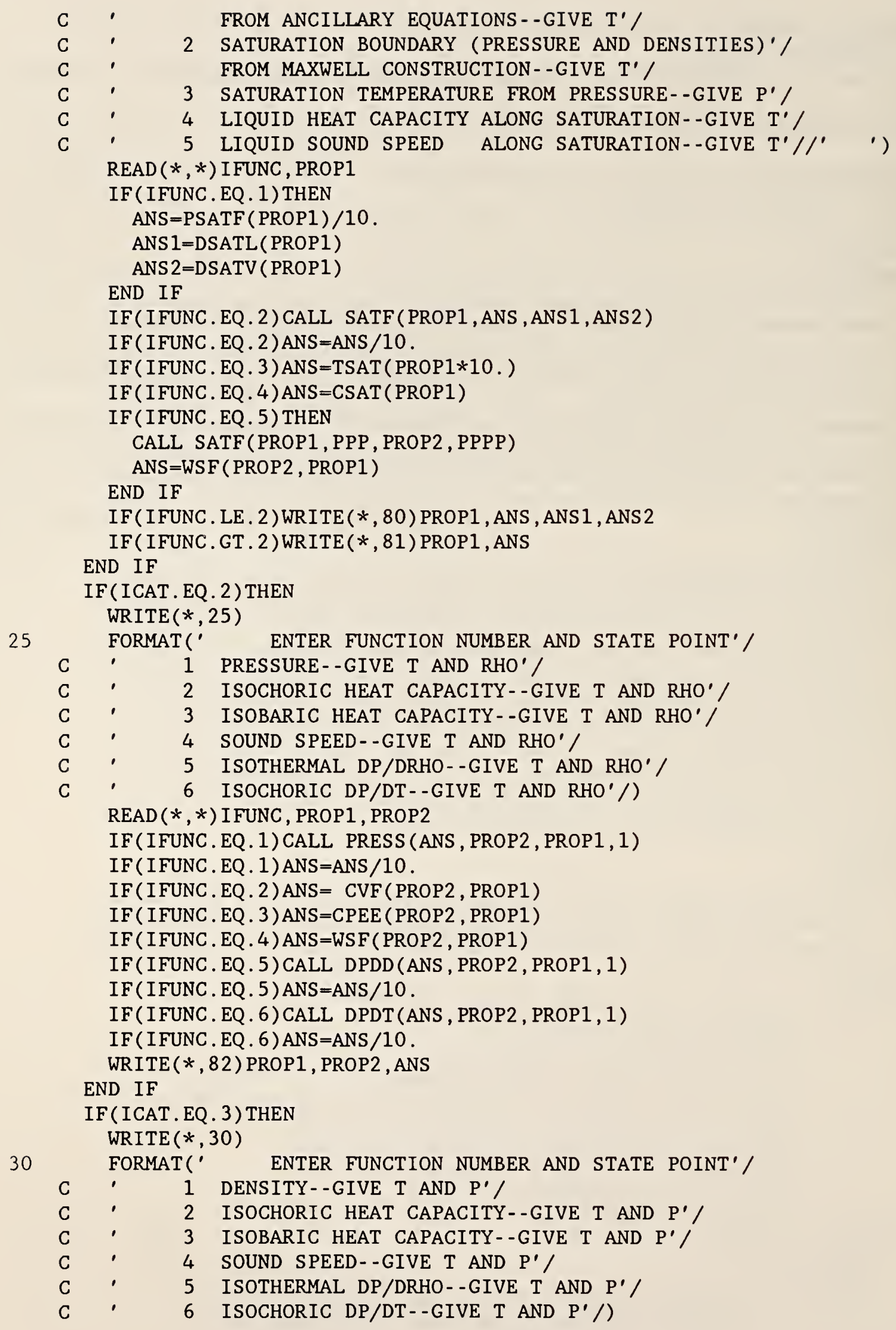


$\operatorname{READ}(*, *)$ IFUNC , PROP 1, PROP2

RHO $=$ RHOF $($ PROP $2 * 10 .$, PROP $1,0.0)$

IF (IFUNC. EQ . 1) ANS $=$ RHO

IF (IFUNC.EQ. 2$)$ ANS $=$ CVF $($ RHO,$P R O P 1)$

I F ( IFUNC . EQ . 3) ANS =CPEE (RHO, PROP1)

IF (IFUNC . EQ . 4) ANS=WSF (RHO, PROP1)

IF (I FUNC . EQ . 5) CALL DPDD (ANS , RHO , PROP1 , 1)

IF (IFUNC. EQ . 6) CALL DPDT (ANS , RHO, PROP1, 1)

IF (IFUNC. GE . 5) ANS=ANS $/ 10$.

WRITE $(*, 82)$ PROP1, PROP2, ANS

END IF

IF (ICAT . EQ . 4) THEN

$\operatorname{WRITE}(*, 35)$

35

FORMAT (' ENTER FUNCTION NUMBER AND STATE POINT' $/$

$C$ ' 1 ENTROPY--GIVE T AND RHO' /

C ' 2 HELMHOLTZ ENERGY--GIVE T AND RHO'/

C ' 3 INTERNAL ENERGY--GIVE T AND RHO'/

C ' 4 GIBBS ENERGY--GIVE T AND RHO'/

C ' 5 ENTHALPY--GIVE T AND RHO' $/$ )

$\operatorname{READ}(*, *)$ IFUNC , PROP 1 , PROP2

IF ( IFUNC. EQ . 1) ANS=ENTROPY (PROP2, PROP1)

I F ( I FUNC. EQ . 2) ANS=HELM (PROP2 , PROP1)

IF (IFUNC . EQ . 3) ANS=UNTERN (PROP2, PROP1)

IF (IFUNC.GE . 4) CALI. PRESS (PPP, PROP2, PROP1, 1)

IF (IFUNC . EQ . 4) ANS=GIBBS (PROP2, PROP1, PPP)

I $F$ ( I FUNC . EQ . 5) ANS=ENTHALP ( PROP2, PROP1, PPP)

WRITE $(*, 82)$ PROP1, PROP 2 , ANS

END IF

IF (ICAT . EQ . 5) THEN

$\operatorname{WRITE}(*, 40)$

40

FORMAT (' ENTER FUNCTION NUMBER AND STATE POINT' $/$

C ' 1 VISCOSITY--GIVE T AND RHO'/

C ' 2 THERMAL CONDUCTIVITY--GIVE T AND RHO'/

C ' 3 VISCOSITY--GIVE T AND P' /

C ' 4 THERMAL CONDUCTIVITY--GIVE T AND $\mathrm{P}^{\prime} /$ )

$\operatorname{READ}(*, *)$ IFUNC , PROP1, PROP2

IF (IFUNC. EQ . 1) ANS=VSCTY (PROP2, PROP1)

IF (IFUNC . EQ. 2) ANS=XLAMF ( PROP2, PROP1)

IF (IFUNC. GE . 3) PROO=RHOF (PROP $2 * 10$. , PROP $1,0.0$ )

IF (IFUNC . EQ . 3) ANS =VSCTY (PROO, PROP1)

IF (I FUNC . EQ . 4) ANS $=$ XLAMF ( PROO, PROP1)

WRITE $(*, 82)$ PROP1, PROP2, ANS

END IF

IF (ICAT . EQ . 6) THEN

$\operatorname{WRITE}(*, 45)$

FORMAT (' ENTER FUNCTION NUMBER AND STATE POINT'/

C ' 1 SECOND VIRIAL COEFFICIENT--GIVE T AND 0'/

C ' 2 IDEAL GAS ISCHORIC HEAT CAPACITY--GIVE T AND $0 \prime$

$C$ ' 3 IDEAL GAS ISOBARIC HEAT CAPACITY--GIVE T AND $0 \prime \prime$

C, 4 IDEAL GAS ENTHALPY--GIVE T AND $0 \%$

C ' 5 IDEAL GAS INTERNAL ENERGY--GIVE T AND 0'/ 


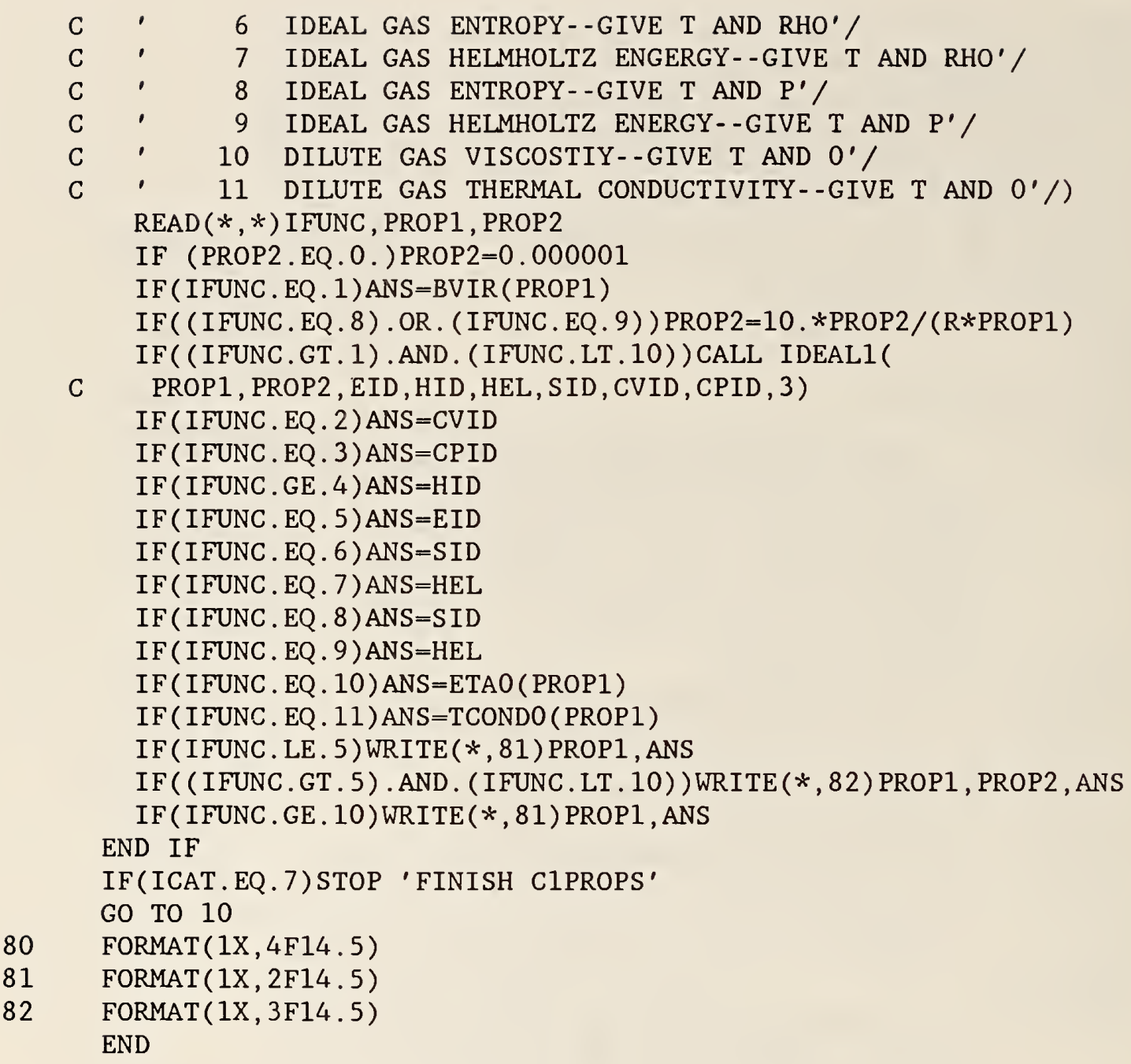


C

FUNCTION CSAT(T)

C

C***********THIS FUNCTION CALCULATES THE HEAT CAPACITY OF THE LIQUID C $x^{2} x^{2} x+x+x+x+x$ ALONG THE SATURATION BOUNDARY

C

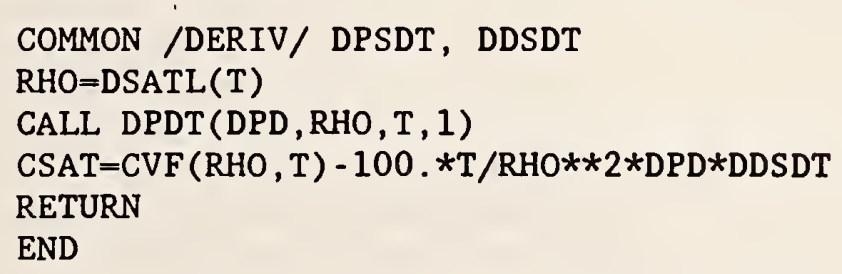

C

FUNCTION BVIR(T)

C

C***********THIS FUNCION CALCULATES THE SECOND VIRIAL COEFFICIENT C $\dot{x} \dot{x} \dot{x} \dot{x} \dot{x} \dot{x} \dot{x} \dot{x} \times$ USING THE SCHMIDT-WAGNER EQUATION OF STATE

C

COMMON /REFDAT/ R, PC, DC, TC, ZC, G(32), PTRP, DTRP, TTRP

C , CMW

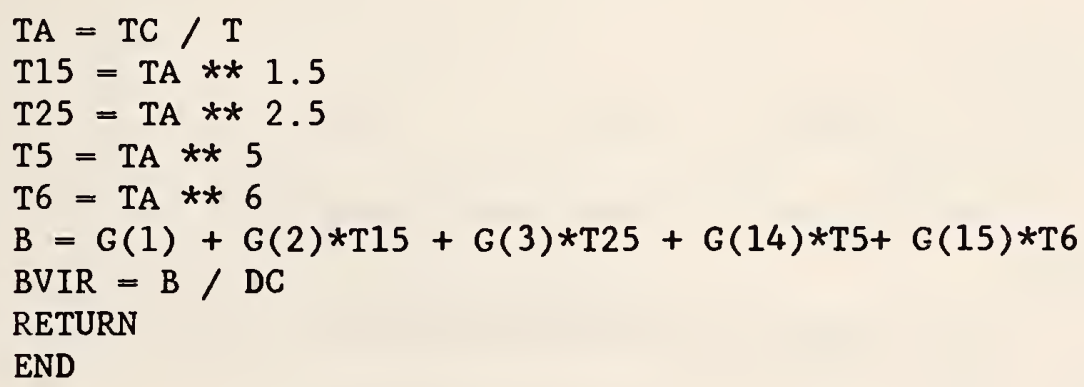




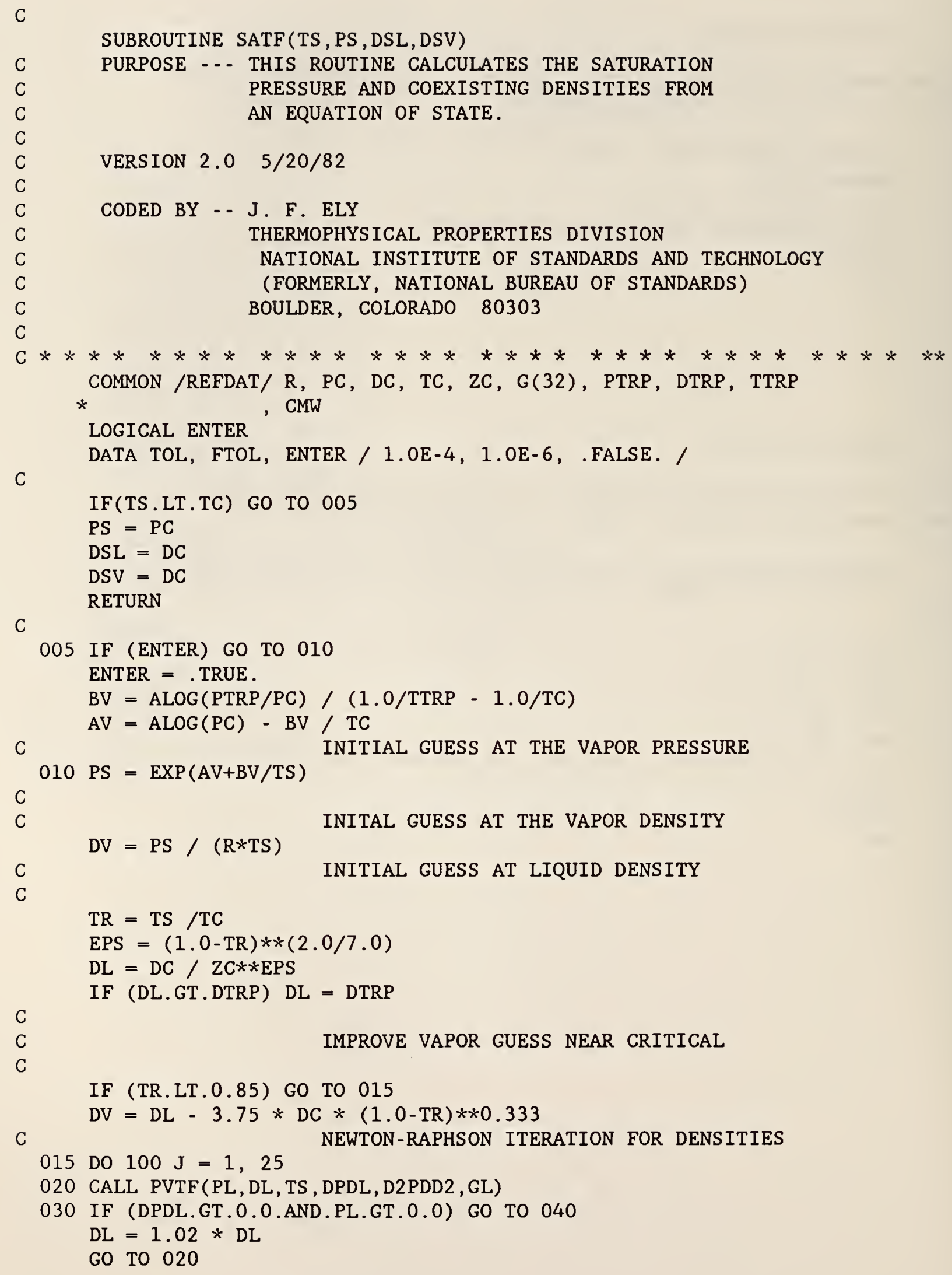


040 CALL PVTF(PV, DV, TS, DPDV, D2PDD2, GV)

IF (DPDV.GT.0.0) GO TO 060

$\mathrm{DV}=0.98 * \mathrm{DV}$

GO TO 040

$060 \mathrm{Fl}=\mathrm{GL}-\mathrm{GV}$

$\mathrm{F} 2=\mathrm{PL}-\mathrm{PV}$

$\mathrm{F} 2 \mathrm{~L}=\mathrm{DPDL}$

$\mathrm{F} 2 \mathrm{~V}=-\mathrm{DPDV}$

$F 1 L=F 2 L / D L$

$\mathrm{F} 1 \mathrm{~V}=\mathrm{F} 2 \mathrm{~V} / \mathrm{DV}$

DENOM $=F 1 L * F 2 V-F 2 L * F 1 V$

IF (ABS(DENOM).LE.1.0E-10) GO TO 120

$\mathrm{DDL}=-(\mathrm{F} 1 * \mathrm{~F} 2 \mathrm{~V}-\mathrm{F} 2 * \mathrm{~F} 1 \mathrm{~V}) / \mathrm{DENOM}$

$\mathrm{DDV}=-(\mathrm{F} 1+\mathrm{DDL} * \mathrm{~F} 1 \mathrm{~L}) / \mathrm{F} 1 \mathrm{~V}$

$\mathrm{DL}=\mathrm{DL}+\mathrm{DDL}$

IF(DL.LT.DC) DL $=\mathrm{DC}$

$\mathrm{DVS}=\mathrm{DV}$

$\mathrm{DV}=\mathrm{DV}+\mathrm{DDV}$

$I F(D V . G T . D C) \quad D V=D C$

IF (DV.LE.0.0) $\quad$ DV $=D V S / 2.0$

IF $(A B S(D D L / D L)$. LT. TOL .AND. ABS (DDV/DV).LE.TOL) GO TO 110

FNORM $=F 1 * F 1+F 2 * F 2$

IF(TR.LT.0.99 .AND. FNORM.LE.FTOL) GO TO 110

100 CONTINUE

110 PS $=$ PV

$\mathrm{DSL}=\mathrm{DL}$

$\mathrm{DSV}=\mathrm{DV}$

RETURN

120 WRITE $(6,340)$ DENOM

GO TO 110

300 FORMAT (I $3,8 \mathrm{G} 10.4)$

310 FORMAT (I3, 2F12.8,G13.6)

330 FORMAT (F8.2,G13.6, 2F10.6, 2G13.6)

340 FORMAT('DENOM IS TOO SMALL IN SATF',G13.6)

END 


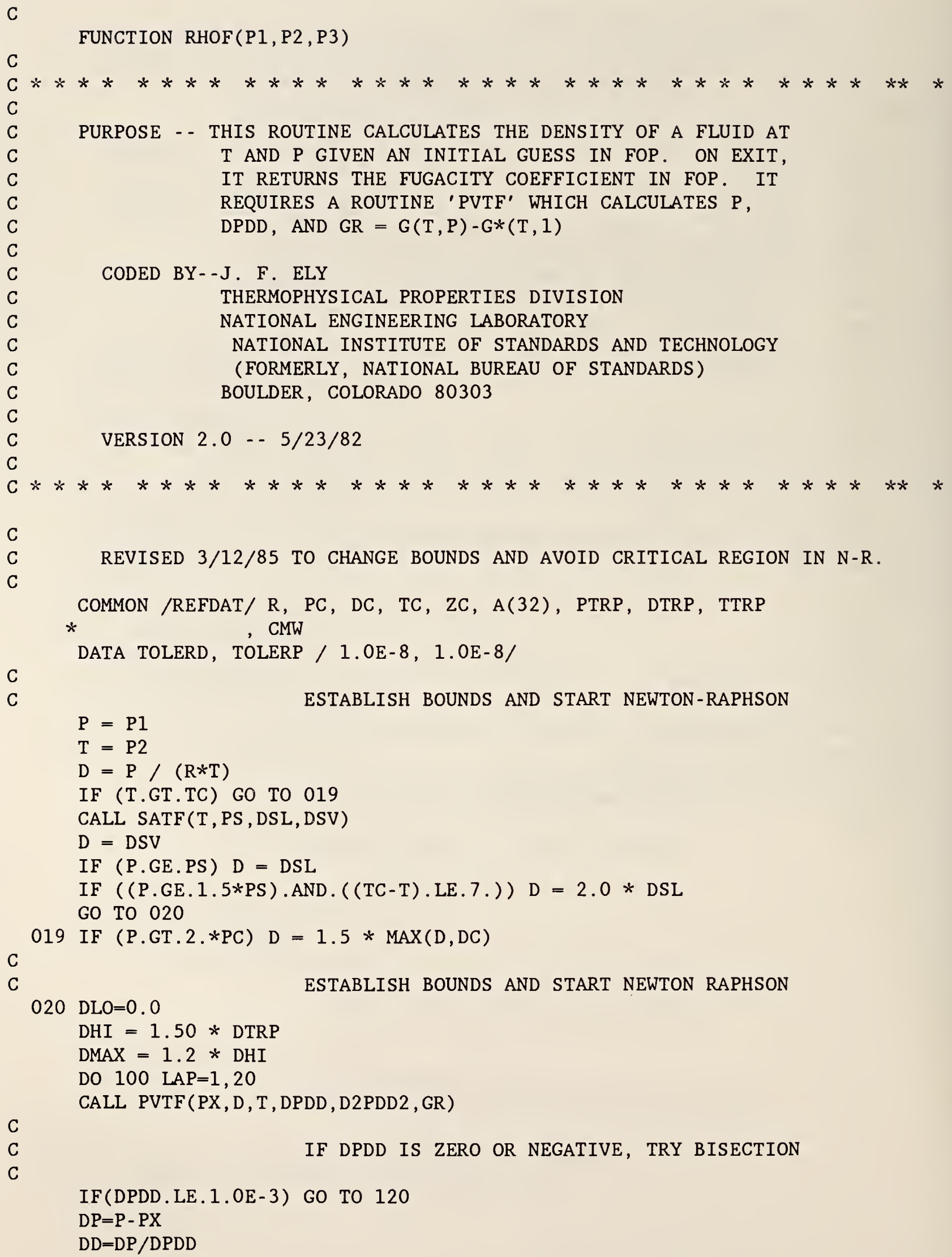


C
$040 \mathrm{DHI}=\mathrm{D}$
GO TO 080
060 DLO=D
$080 \mathrm{DN}=\mathrm{D}+\mathrm{DD}$

IF (DP) $040,300,060^{\circ}$

C

C

KEEP D WITHIN BOUNDS OR GO TO BISECTION

IF (DN.LT.0.0 .OR. DN.GT.DMAX) GO TO 120

$\mathrm{D}=\mathrm{DN}$

IF (LAP.EQ. 1) GO TO 100

IF (ABS (DP/P). LE. TOLERP) GO TO 300

$\operatorname{IF}(\mathrm{ABS}(\mathrm{DD} / \mathrm{D})$. LE. TOLERD) GO TO 300

100 CONTINUE

C

120 IF(T.GT.TC) GO TO 160

NEWTON-RAPHSON FAILURE. TRY BISECTION

C

C

SUB-CRITICAL. MAKE SURE THAT WE HAVE THE PROPER BOUNDS ON THE DENSITY.

C

C

IF (P.LT.PS) GO TO 140

$\mathrm{DLO}=\mathrm{DSL}$

IF (DHI. LE. DSL) DHI=DMAX

GO TO 160

C

140 IF(DLO.GE.DSV) DLO $=0.0$

$\mathrm{DHI}=\mathrm{DSV}$

C

C START THE BISECTION

$160 \mathrm{D}=0.50 *(\mathrm{DLO}+\mathrm{DHI})$

CALL PVTF(PX,D, T, DPDD, D2PDD2,GR)

$\mathrm{DP}=\mathrm{PX}-\mathrm{P}$

IF (DP) $200,300,220$

$200 \mathrm{DLO}=\mathrm{D}$

GO TO 240

220 DHI $=$ D

$240 \operatorname{IF}(A B S(D P / P)$. LE. TOLERP) GO TO 300

IF (ABS (DLO/DHI-1.0).GT. TOLERD) GO TO 160

C

C BISECTION FAILED. GIVE UP

260 WRITE $(6,400) \mathrm{T}, \mathrm{P}, \mathrm{D}, \mathrm{DPDD}, \mathrm{DLO}, \mathrm{DHI}, \mathrm{LAP}, \mathrm{PX}$

C

300 RHOF $=\mathrm{D}$

RETURN

400 FORMAT('RHOF FAILED AT $\mathrm{T}=$ ', F9.3,' $\mathrm{P}=$ ' , G14.7, 4G12.6, I4, G12.6)

END 
C

SUBROUTINE PVTF(PO,DO, T0, DPDD, D2PD2, G0)

$C$

C $* * * * * * * * * * * * * * * * * * * * * * * * * * * * * * * * * * *$

$\mathrm{C}$

C PURPOSE - - THIS ROUTINE CALCULATES THE PRESSURE, ITS FIRST

C TWO DENSITY DERIVATIVES AND THE GIBBS ENERGY

C RELATIVE TO THE IDEAL GAS AT 1 BAR

C THIS ROUTINE IS FOR THE WAGNER EQUATION OF STATE

C VERSION $1.0-2 / 18 / 85$

C

C CODED BY - - J. F. ELY

C

$\mathrm{C}$

C

C

C

C $* * * * * * * * * * * * * * * * * * * * * * * * * * * * * * * * * *$

$\mathrm{C}$

C

DIMENSION $S(4), P(10,3), Q(3), \operatorname{DQDD}(3), \operatorname{D} 2 \mathrm{QDD} 2(3), \mathrm{D} 3 \mathrm{QDD} 3(3)$

CHEMICAL ENGINEERING SCIENCE DIVISION

NATIONAL INSTITUTE OF STANDARDS AND TECHNOLOGY

(FORMERLY, NATIONAL BUREAU OF STANDARDS)

BOULDER, COLORADO 80303

C

COMMON /REFDAT/ RG, PC, DC, TC, ZC, G(32), PTRP, DTRP, TTRP

* $\quad$, CMW

C

DATA P / 30*0. /, TLAST / $-1.0 /$

C

$\mathrm{T}=\mathrm{TC} / \mathrm{T} 0$

$\mathrm{D}=\mathrm{D} 0 / \mathrm{DC}$

IF(T.EQ.TLAST) GO TO 040

TLAST $=\mathrm{T}$

$\mathrm{RT}=\mathrm{RG} * \mathrm{~T} 0$

$\mathrm{TS}=\mathrm{SQRT}(\mathrm{T})$

TTS $=\mathrm{T} * \mathrm{TS}$

$\mathrm{T} 2=\mathrm{T} * \mathrm{~T}$

$\mathrm{T} 3=\mathrm{T} * \mathrm{~T} 2$

$\mathrm{T} 4=\mathrm{T} * \mathrm{~T} 3$

$\mathrm{T} 5=\mathrm{T} * \mathrm{~T} 4$

$\mathrm{T} 6=\mathrm{T} * \mathrm{~T} 5$

$\mathrm{T} 7=\mathrm{T} * \mathrm{~T} 6$

$\mathrm{T} 8=\mathrm{T} 2 * \mathrm{~T} 6$

$\mathrm{T} 11=\mathrm{T} 8 * \mathrm{~T} 3$

$\mathrm{T} 17=\mathrm{T} 6 * \mathrm{~T} 11$

$\mathrm{T} 18=\mathrm{T} * \mathrm{~T} 17$

C

$P(1,1)=G(1)+(G(2)+G(3) * T) * T T S$

$P(2,1)=G(4) / T S+G(5) * T T S+G(6) * T 2$

$P(3,1)=G(7)+(G(8)+G(9) * T T S) * T$

$P(6,1)=G(10)$

$P(7,1)=G(11) * \mathrm{~T} 2+G(12) * \mathrm{~T} 5$

$P(8,1)=G(13) * T 2$ 
C

$$
\begin{aligned}
& \mathrm{P}(1,2)=G(14) * \mathrm{~T} 5+\mathrm{G}(15) * \mathrm{~T} 6 \\
& \mathrm{P}(2,2)=(\mathrm{G}(16)+\mathrm{G}(17) * \mathrm{~T} 2) * \mathrm{~T} 2 * \mathrm{TTS} \\
& \mathrm{P}(3,2)=\mathrm{T}(18) * \mathrm{~T} 3+\mathrm{G}(19) * \mathrm{~T} 7 \\
& \mathrm{P}(5,2)=\mathrm{G}(20) * \mathrm{~T} 6 \\
& \mathrm{P}(6,2)=\mathrm{G}(21) * \mathrm{~T} 8 * \mathrm{TS} \\
& \mathrm{P}(7,2)=\mathrm{G}(22) * \mathrm{~T} 4 \\
& \mathrm{P}(8,2)=\mathrm{G}(23) * \mathrm{~T} 6 * \mathrm{TS} \\
& \mathrm{P}(10,2)=\mathrm{G}(24) * \mathrm{~T} 5 * \mathrm{TS}
\end{aligned}
$$

C

C

$$
\begin{aligned}
& \mathrm{P}(2,3)=\mathrm{G}(25) * \mathrm{~T} 11 * \mathrm{~T} 11 \\
& \mathrm{P}(3,3)=\mathrm{G}(26) * \mathrm{~T} 11+\mathrm{G}(27) * \mathrm{~T} 18 \\
& \mathrm{P}(4,3)=(\mathrm{G}(28)+\mathrm{G}(29) * \mathrm{~T} 11 * \mathrm{~T}) * \mathrm{~T} 11 \\
& \mathrm{P}(5,3)=(\mathrm{G}(30)+\mathrm{T} *(\mathrm{G}(31)+\mathrm{G}(32) * \mathrm{~T} 5)) * \mathrm{~T} 17
\end{aligned}
$$

$$
\begin{aligned}
040 \text { DO } 070 \mathrm{~J}=1,3 \\
\text { DO } 050 \mathrm{~K}=1,4
\end{aligned}
$$

$$
050 \mathrm{~S}(\mathrm{~K})=0 \text {. }
$$$$
\text { DO } 060 I=1,10
$$$$
\mathrm{S}(1)=\mathrm{S}(1) * \mathrm{D}+\mathrm{P}(11-\mathrm{I}, \mathrm{J})
$$$$
S(2)=S(2) * D+S(1)
$$

IF (I.EQ.10) GO TO 060

$$
S(3)=S(3) * D+S(2)
$$

IF (I.EQ.9) GO TO 060

060 CONTINUE

$S(4)=S(4) * D+S(3)$

$\mathrm{Q}(\mathrm{J})=\mathrm{S}(1) * \mathrm{D}$

$\operatorname{DQDD}(\mathrm{J})=\mathrm{S}(2)$

$$
\operatorname{D2QDD} 2(\mathrm{~J})=\mathrm{S}(3) * 2.0
$$

C

$070 \mathrm{D} 3 Q \mathrm{DD} 3(\mathrm{~J})=\mathrm{S}(4) * 6.0$

$$
\begin{aligned}
& \mathrm{D} 2=\mathrm{D} * \mathrm{D} \\
& \mathrm{D} 3=\mathrm{D} * \mathrm{D} 2 \\
& \mathrm{~F} 2=\operatorname{EXP}(-\mathrm{D} 2) \\
& \mathrm{F} 3=\operatorname{EXP}(-\mathrm{D} * \mathrm{D} 3) \\
& \text { TWOD }=2.0 * \mathrm{D} \\
& \text { FORD }=4.0 * \mathrm{D} 3 \\
& \text { DRT }=\mathrm{D} 0 * \mathrm{RT}
\end{aligned}
$$

C

$$
\mathrm{PHI}=\mathrm{Q}(1)+\mathrm{Q}(2) * \mathrm{~F} 2+\mathrm{Q}(3) * \mathrm{~F} 3
$$

C

$$
\begin{aligned}
& \text { TRM2 }=\text { DQDD }(2)-\text { TWOD } * \mathrm{Q}(2) \\
& \text { TRM3 }=\text { DQDD (3) }- \text { FORD } * \mathrm{Q}(3) \\
& \mathrm{DPHDD}=\mathrm{DQDD}(1)+\mathrm{TRM} 2 * \mathrm{~F} 2+\mathrm{TRM} 3 * \mathrm{~F} 3 \\
& \mathrm{PO}=\mathrm{DRT} *(1.0+\mathrm{D} * \mathrm{DPHDD})
\end{aligned}
$$

C

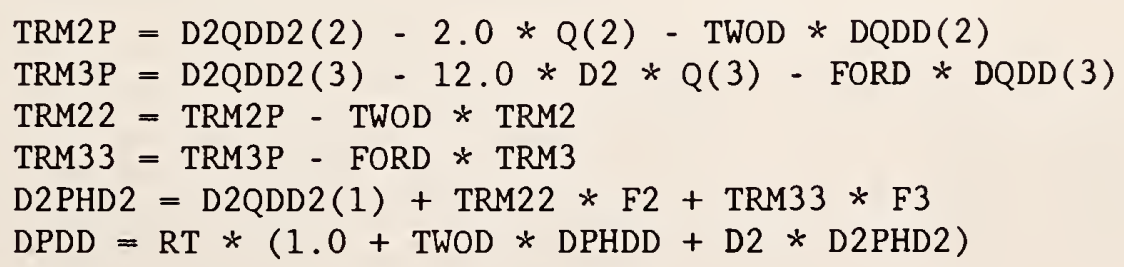


C

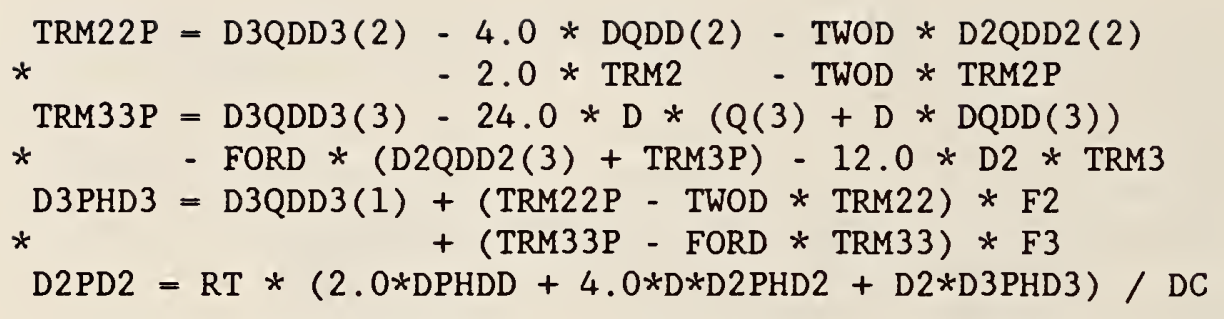

C

$\mathrm{ZO}=\mathrm{PO} / \mathrm{DRT}$

$\mathrm{GO}=\mathrm{RT} *(\mathrm{PHI}+\mathrm{ZO}-1.0+\mathrm{ALOG}(\mathrm{DRT}))$

RETURN

END

C

FUNCTION $\operatorname{CVF}(D, T)$

C

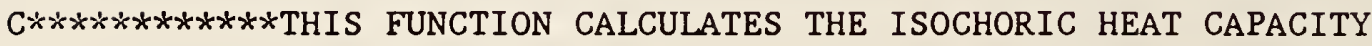

C

CALL CVR(CVD, D, T, 1)

CALL IDEAL(T, CVI, SI)

$\mathrm{CVF}=\mathrm{CVI}+100.0 * \mathrm{CVD}$

RETURN

END

C

FUNCTION $\operatorname{WSF}(D, T)$

C

C

C

C

C

COMMON /REFDAT/ R, PC, DC, TC, ZC, G(32), PTRP, DTRP, TTRP

*

THIS ROUTINE CALCULATES THE SOUND VELOCITY GIVEN THE DENSITY AND TEMPERATURE

COMMON /DPDCAL/ CP, CV, DPD, DPT

C

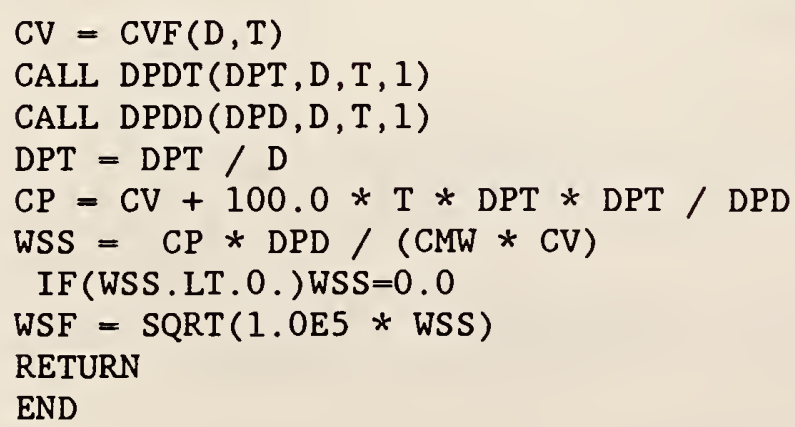


C

FUNCTION DSATL(T)

$\mathrm{C}$

C THIS ROUTINE CALCULATES THE SATURATED LIQUid

C DENSITY OF METHANE

C

C REVISED 2/27/85 TO REJECT CALLS IF T .GT. TC

C

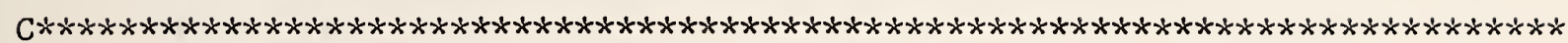

$\mathrm{C}$

C REVISED COEFFICIENTS FROM 6/03/86

COMMON/SATBDY/TC, PC , DC , ZC, R, EPP, BETA

COMMON /DERIV/ DPSDT, DDSDT

DIMENSION G(4)

DATA G/ . $1838981951 \mathrm{E}+01$,

C $-.7727452180 \mathrm{E}+00$,

C. $5592446346 \mathrm{E}+00$,

C- $.3807792546 \mathrm{E}+00 /$

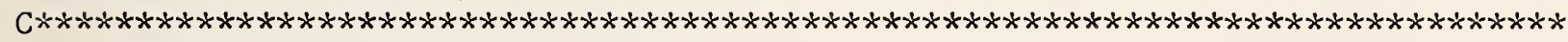

$\mathrm{X}=1.0-\mathrm{T} / \mathrm{TC}$

IF (X) $1,2,3$

1 PRINT 4

4 FORMAT (10X,' DSATL $=0$, T EXCEEDS TCRT. ' / )

STOP

2 DSATL $=$ DC

DDSDT $=-1.0 \mathrm{E}+10$

RETURN

3 DENOM $=1.0+G(4) * X * *(1.0-B E T A)$

$\mathrm{Y}=(\mathrm{G}(1) * \mathrm{X} * * \mathrm{BETA}+\mathrm{G}(2) * \mathrm{X} * \mathrm{X}+\mathrm{G}(3) * \mathrm{X} * \mathrm{X} * \mathrm{X}) /$ DENOM

DYDX $=\operatorname{BETA} * G(1) * X * *(B E T A-1.0)+2.0 * G(2) * X+3.0 * G(3) * X * X$

DYDX $=($ DYDX $-\mathrm{Y} * \mathrm{G}(4) *(1.0-\mathrm{BETA}) / \mathrm{X} * * \mathrm{BETA}) / \mathrm{DENOM}$

DSATL $=D C *(Y+1.0)$

DDSDT $=-\mathrm{DC} * \mathrm{DYDX} / \mathrm{TC}$

RETURN

END 
C

FUNCTION DSATV(T)

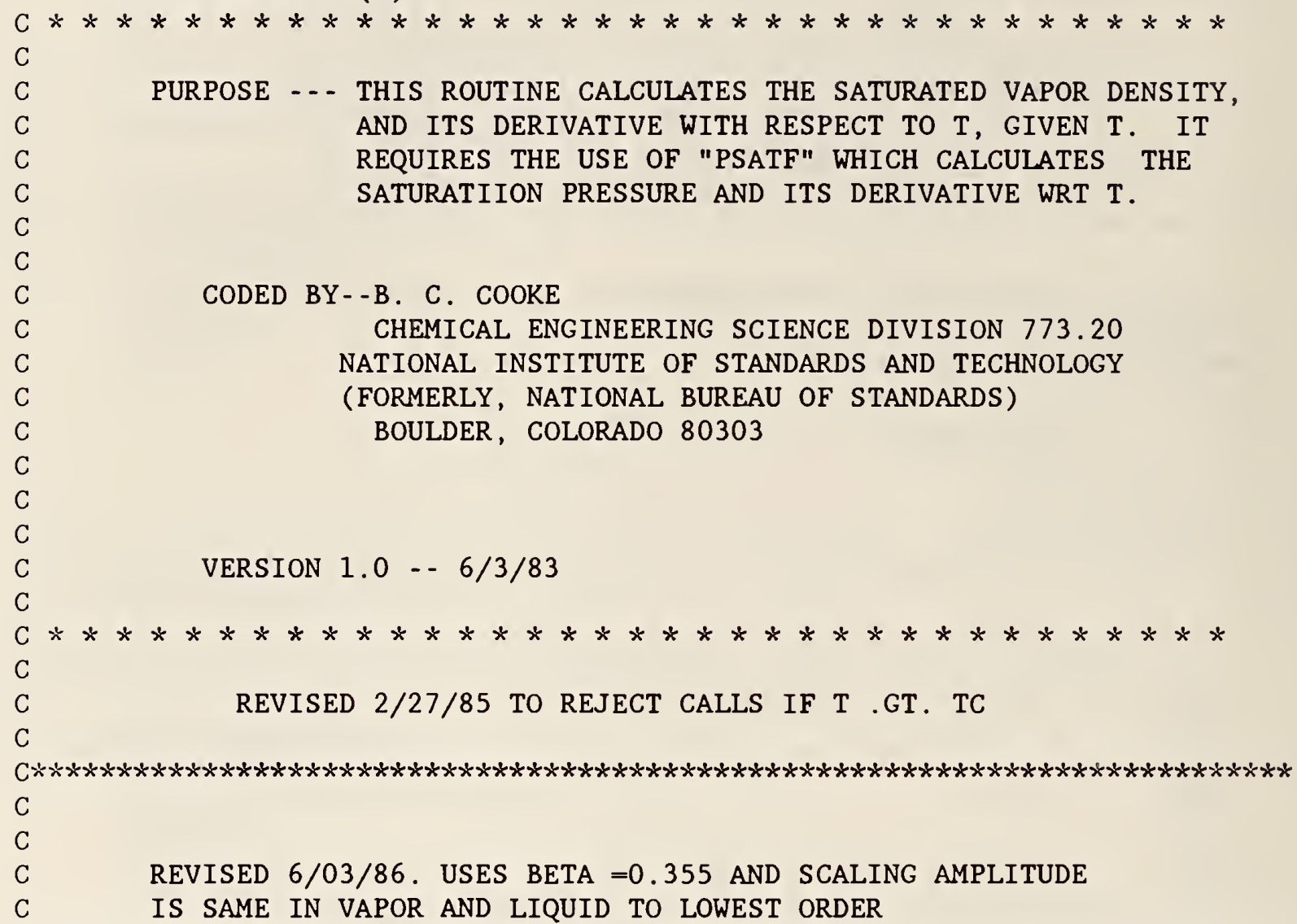

C THIS COEFFICIENT IS DIRECTLY FROM SAT LIQUID FIT DATA G00/.1838981951E+01/ 


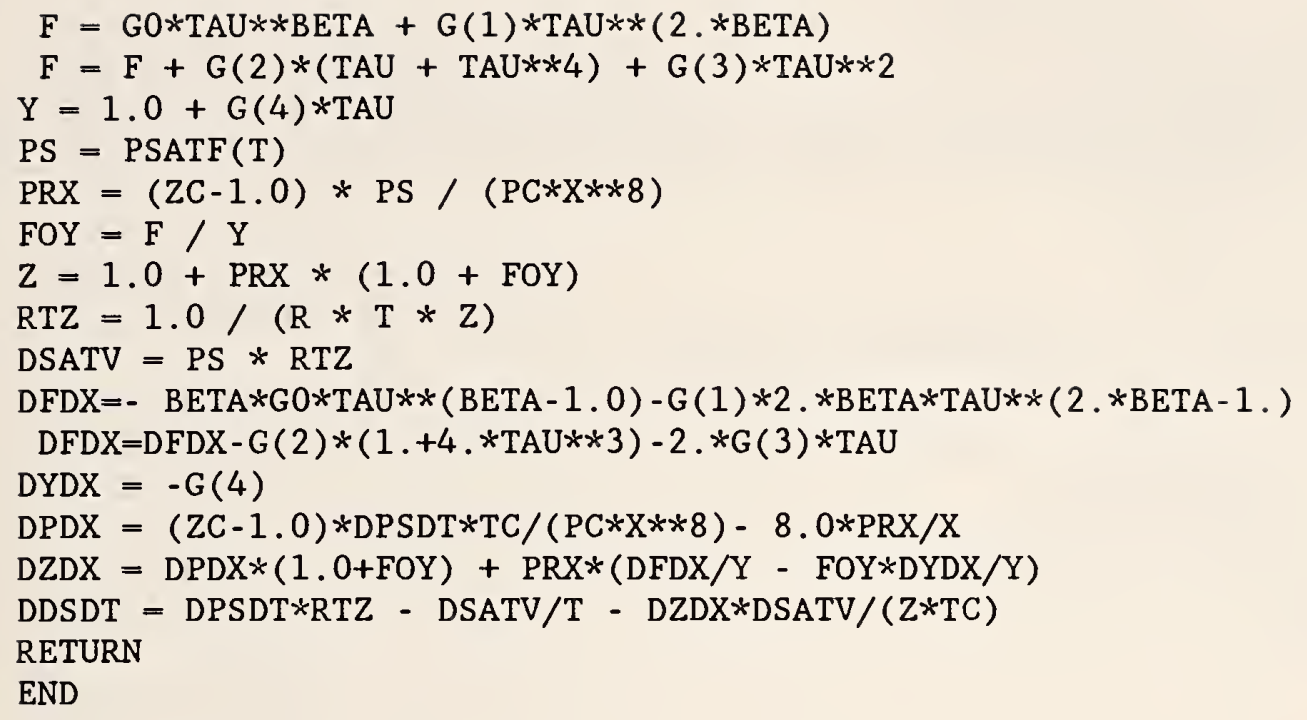




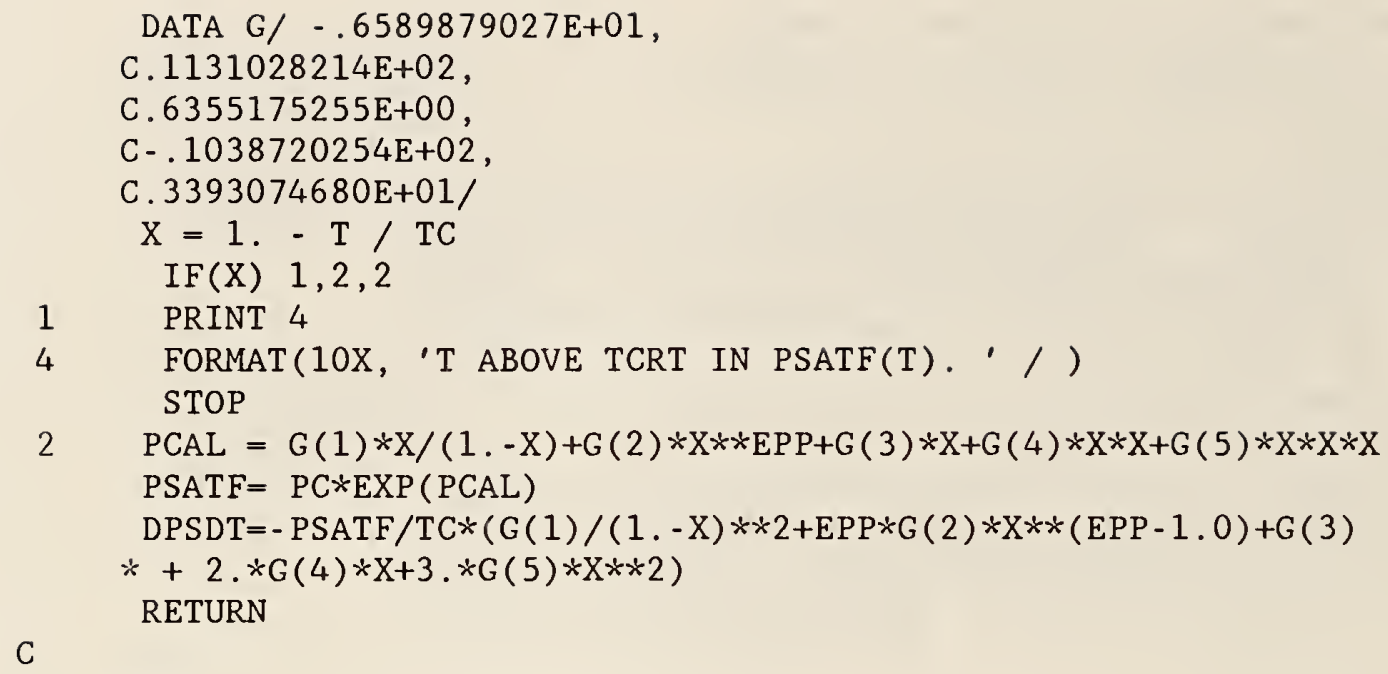

END

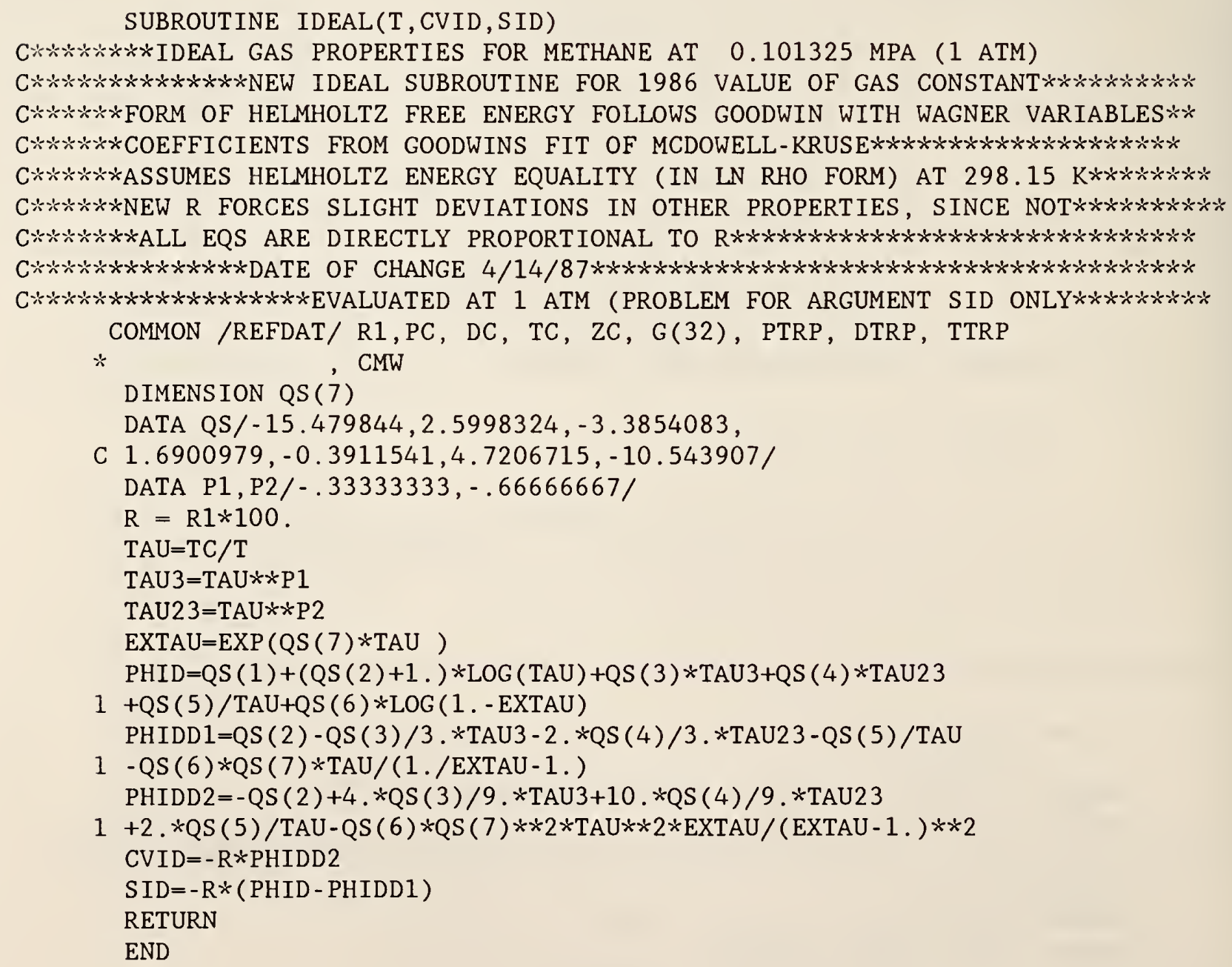


C

SUBROUTINE PROPSS (PP, DD, TT, IDD)

C $* * * * * * * * x * x * x * *$ THIS SUBROUTINE CALCULATES MANY OF THE THERMODYNAMIC C $* \dot{*} * \dot{*} * * * x * * x * * x$ PROPERTIES FROM THE SCHMIDT-WAGNER EQUATION OF STATE $\mathrm{C}$

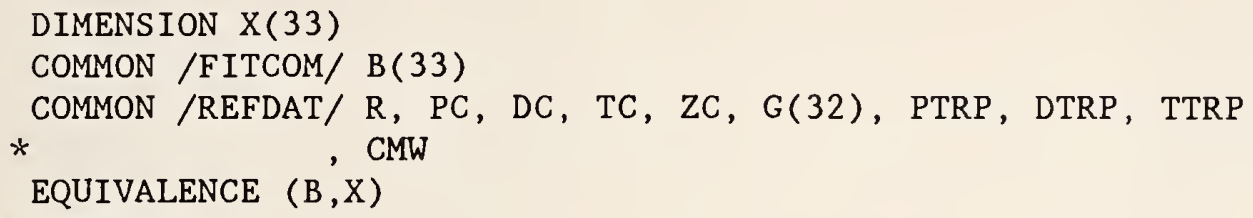

1 CONTINUE

$\mathrm{D}=\mathrm{DD} / \mathrm{DC}$

$\mathrm{DEN}=\mathrm{DD}$

$\mathrm{T}=\mathrm{TC} / \mathrm{TT}$

$\mathrm{TEMP}=\mathrm{TT}$

$\mathrm{RT}=\mathrm{R} * \mathrm{TEMP}$

$\mathrm{DRT}=\mathrm{DEN} * \mathrm{RT}$

$\mathrm{DDRT}=\mathrm{D} * \mathrm{DRT}$

$\mathrm{RTD}=\mathrm{RT} / \mathrm{DC}$

$\mathrm{RD}=\mathrm{DEN} * \mathrm{R}$

$\mathrm{RTT}=\mathrm{RD} / \mathrm{TC}$

$\mathrm{D} 2=\mathrm{D} * \mathrm{D}$

$\mathrm{D} 3=\mathrm{D} 2 * \mathrm{D}$

$\mathrm{D} 4=\mathrm{D} 3 * \mathrm{D}$

$\mathrm{D} 5=\mathrm{D} 4 * \mathrm{D}$

$\mathrm{D} 6=\mathrm{D} 5 * \mathrm{D}$

$\mathrm{D} 7=\mathrm{D} 6 * \mathrm{D}$

$\mathrm{D} 8=\mathrm{D} 7 * \mathrm{D}$

$\mathrm{D} 9=\mathrm{D} 8 * \mathrm{D}$

$\mathrm{D} 10=\mathrm{D} 9 * \mathrm{D}$

$\mathrm{D} 11=\mathrm{D} 10 * \mathrm{D}$

$\mathrm{D} 12=\mathrm{D} 11 * \mathrm{D}$

$\mathrm{D} 13=\mathrm{D} 12 * \mathrm{D}$

$\mathrm{D} 14=\mathrm{D} 13 * \mathrm{D}$

$\mathrm{D} 15=\mathrm{D} 14 * \mathrm{D}$

$\mathrm{D} 16=\mathrm{D} 15 * \mathrm{D}$

$\mathrm{TS}=\mathrm{SQRT} \quad(\mathrm{T})$

TMS $=1.0 / \mathrm{TS}$

$\mathrm{T} 15=\mathrm{T} * \mathrm{TS}$

$\mathrm{T} 2=\mathrm{T} * \mathrm{~T}$

$\mathrm{T} 25=\mathrm{T} 2 * \mathrm{TS}$

$\mathrm{T} 3=\mathrm{T} 2 * \mathrm{~T}$

$\mathrm{T} 35=\mathrm{T} 3 * \mathrm{TS}$

$\mathrm{T} 4=\mathrm{T} 3 * \mathrm{~T}$

$\mathrm{T} 45=\mathrm{T} 4 * \mathrm{TS}$

$\mathrm{T} 5=\mathrm{T} 4 * \mathrm{~T}$

$\mathrm{T} 55=\mathrm{T} 5 * \mathrm{TS}$

$\mathrm{T} 6=\mathrm{T} 5 * \mathrm{~T}$

$\mathrm{T} 65=\mathrm{T} 6 * \mathrm{TS}$

$\mathrm{T} 7=\mathrm{T} 6 * \mathrm{~T}$

$\mathrm{T} 75=\mathrm{T} 7 * \mathrm{TS}$

$\mathrm{T} 8=\mathrm{T} 7 * \mathrm{~T}$

$\mathrm{T} 85=\mathrm{T} 8 * \mathrm{TS}$ 


$$
\begin{aligned}
& \mathrm{T} 9=\mathrm{T} 8 * \mathrm{~T} \\
& \mathrm{~T} 95=\mathrm{T} 9 * \mathrm{TS} \\
& \mathrm{T} 11=\mathrm{T} 8 * \mathrm{~T} 3 \\
& \mathrm{~T} 12=\mathrm{T} 11 * \mathrm{~T} \\
& \mathrm{~T} 17=\mathrm{T} 12 * \mathrm{~T} 5 \\
& \mathrm{~T} 18=\mathrm{T} 17 * \mathrm{~T} \\
& \mathrm{~T} 19=\mathrm{T} 18 * \mathrm{~T} \\
& \mathrm{~T} 22=\mathrm{T} 18 * \mathrm{~T} 4 \\
& \mathrm{~T} 23=\mathrm{T} 22 * \mathrm{~T} \\
& \mathrm{~T} 24=\mathrm{T} 23 * \mathrm{~T} \\
& \mathrm{~F} 1=\mathrm{EXP}(-\mathrm{D} 2) \\
& \mathrm{F} 2=\mathrm{EXP}(-\mathrm{D} 4) \\
& \mathrm{G} 0 \mathrm{TO}(100,200,300,400,500,600,700,800), \mathrm{K}
\end{aligned}
$$

C

ENTRY PRESS (PP, DD, TT, IDD)

\section{C*********ENTRY TO CALCULATE PRESSURE}

C

$\mathrm{K}=1$

GO TO 1

100 CONTINUE

$$
\begin{aligned}
& \mathrm{B}(1)=\mathrm{DDRT} * 1.0 \\
& \mathrm{~B}(2)=\mathrm{DDRT} * \mathrm{~T} 15 \\
& \mathrm{~B}(3)=\mathrm{DDRT} * \mathrm{~T} 25 \\
& \mathrm{~B}(4)=\mathrm{DDRT} * 2 . * \mathrm{D} * \mathrm{TMS} \\
& \mathrm{B}(5)=\mathrm{DDRT} * 2 . * \mathrm{D} * \mathrm{~T} 15 \\
& \mathrm{~B}(6)=\mathrm{DDRT} * 2 . * \mathrm{D} * \mathrm{~T} 2 \\
& \mathrm{~B}(7)=\mathrm{DDRT} * 3 . * \mathrm{D} 2 \\
& \mathrm{~B}(8)=\mathrm{DDRT} * 3 . * \mathrm{D} 2 * \mathrm{~T} \\
& \mathrm{~B}(9)=\mathrm{DDRT} * 3 . * \mathrm{D} 2 * \mathrm{~T} 25 \\
& \mathrm{~B}(10)=\mathrm{DDRT} * 6 . * \mathrm{D} 5 \\
& \mathrm{~B}(11)=\mathrm{DDRT} * 7 . * \mathrm{D} 6 * \mathrm{~T} 2 \\
& \mathrm{~B}(12)=\mathrm{DDRT} * 7 . * \mathrm{D} 6 * \mathrm{~T} 5 \\
& \mathrm{~B}(13)=\mathrm{DDRT} * 8 . * \mathrm{D} 7 * \mathrm{~T} 2 \\
& \mathrm{~B}(14)=\mathrm{DDRT} *(1,-2, * \mathrm{D} 2) * \mathrm{~T} 5 * \mathrm{~F} 1 \\
& \mathrm{~B}(15)=\mathrm{DDRT} *(1 .-2 . * \mathrm{D} 2) * \mathrm{~T} 6 * \mathrm{~F} 1 \\
& \mathrm{~B}(16)=\mathrm{DDRT} *(2 . * \mathrm{D}-2 . * \mathrm{D} 3) * \mathrm{~T} 35 * \mathrm{~F} 1 \\
& \mathrm{~B}(17)=\mathrm{DDRT} *(2 . * \mathrm{D}-2 * \mathrm{D} 3) * \mathrm{~T} 55 * \mathrm{~F} 1 \\
& \mathrm{~B}(18)=\mathrm{DDRT} *(3 . * \mathrm{D} 2-2 . * \mathrm{D} 4) * \mathrm{~T} 3 * \mathrm{~F} 1 \\
& \mathrm{~B}(19)=\mathrm{DDRT} *(3 . * \mathrm{D} 2-2 . * \mathrm{D} 4) * \mathrm{~T} 7 * \mathrm{~F} 1 \\
& \mathrm{~B}(20)=\mathrm{DDRT} *(5 . * \mathrm{D} 4-2 . * \mathrm{D} 6) * \mathrm{~T} 6 * \mathrm{~F} 1 \\
& \mathrm{~B}(21)=\mathrm{DDRT} *(6 . * \mathrm{D} 5-2 . * \mathrm{D} 7) * \mathrm{~T} 85 * \mathrm{~F} 1 \\
& \mathrm{~B}(22)=\mathrm{DDRT} *(7 . * \mathrm{D} 6-2 . * \mathrm{D} 8) * \mathrm{~T} 4 * \mathrm{~F} 1 \\
& \mathrm{~B}(23)=\mathrm{DDRT} *(8 . * \mathrm{D} 7-2 . * \mathrm{D} 9) * \mathrm{~T} 65 * \mathrm{~F} 1 \\
& \mathrm{~B}(24)=\mathrm{DDRT} *(10 . * \mathrm{D} 9-2 . * \mathrm{D} 11) * \mathrm{~T} 55 * \mathrm{~F} 1 \\
& \mathrm{~B}(25)=\mathrm{DDRT} *(2 . * \mathrm{D}-4 . * \mathrm{D} 5) * \mathrm{~T} 22 * \mathrm{~F} 2 \\
& \mathrm{~B}(26)=\mathrm{DDRT} *(3 . * \mathrm{D} 2-4 * \mathrm{D} 6) * \mathrm{~T} 11 * \mathrm{~F} 2 \\
& \mathrm{~B}(27)=\mathrm{DDRT} *(3 . * \mathrm{D} 2-4 * \mathrm{D} 6) * \mathrm{~T} 18 * \mathrm{~F} 2 \\
& \mathrm{~B}(28)=\mathrm{DDRT} *(4 . * \mathrm{D} 3-4 * \text { D } 7) * \mathrm{~T} 11 * \mathrm{~F} 2 \\
& \mathrm{~B}(29)=\mathrm{DDRT} *(4 . * \mathrm{D} 3-4 . * \mathrm{D} 7) * \mathrm{~T} 23 * \mathrm{~F} 2 \\
& \mathrm{~B}(30)=\mathrm{DDRT} *(5 . * \mathrm{D} 4-4 * \mathrm{D} 8) * \mathrm{~T} 17 * \mathrm{~F} 2 \\
& \mathrm{~B}(31)=\mathrm{DDRT} *(5 . * \mathrm{D} 4-4 * \text { D } 8) * \mathrm{~T} 18 * \mathrm{~F} 2 \\
& \mathrm{~B}(32)=\mathrm{DDRT} *(5 . * \mathrm{D} 4-4 * \mathrm{D} 8) * \mathrm{~T} 23 * \mathrm{~F} 2 \\
& \text { IF (IDD.GT.0)GO TO } 102
\end{aligned}
$$




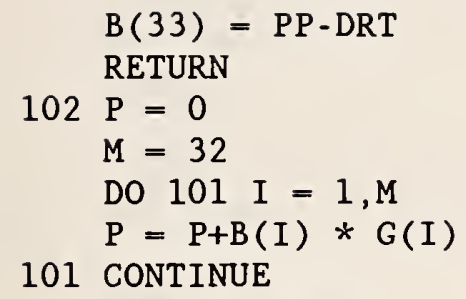

101 CONTINUE

$\mathrm{P}=\mathrm{P}+\mathrm{DRT}$

$\mathrm{PP}=\mathrm{P}$

RETURN

C

ENTRY DPDD (PP, DD , TT, IDD)

C $* * * * * * * * * * * *$ ENTRY TO CALCULATE ISOTHERMAL DENSITY DERIVATIVE OF PRESSURE C
$\mathrm{K}=2$
GO TO 1

200 CONTINUE

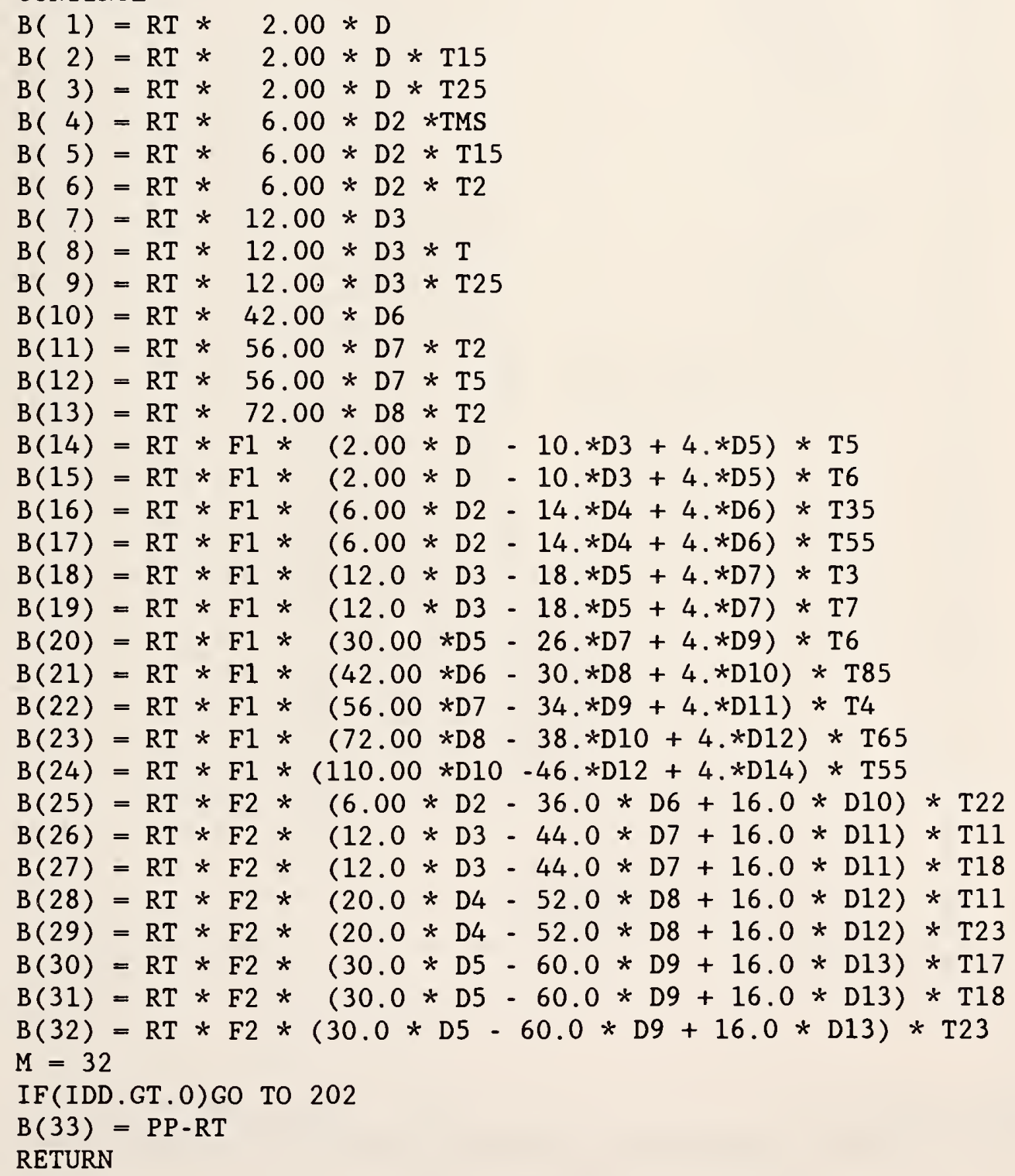




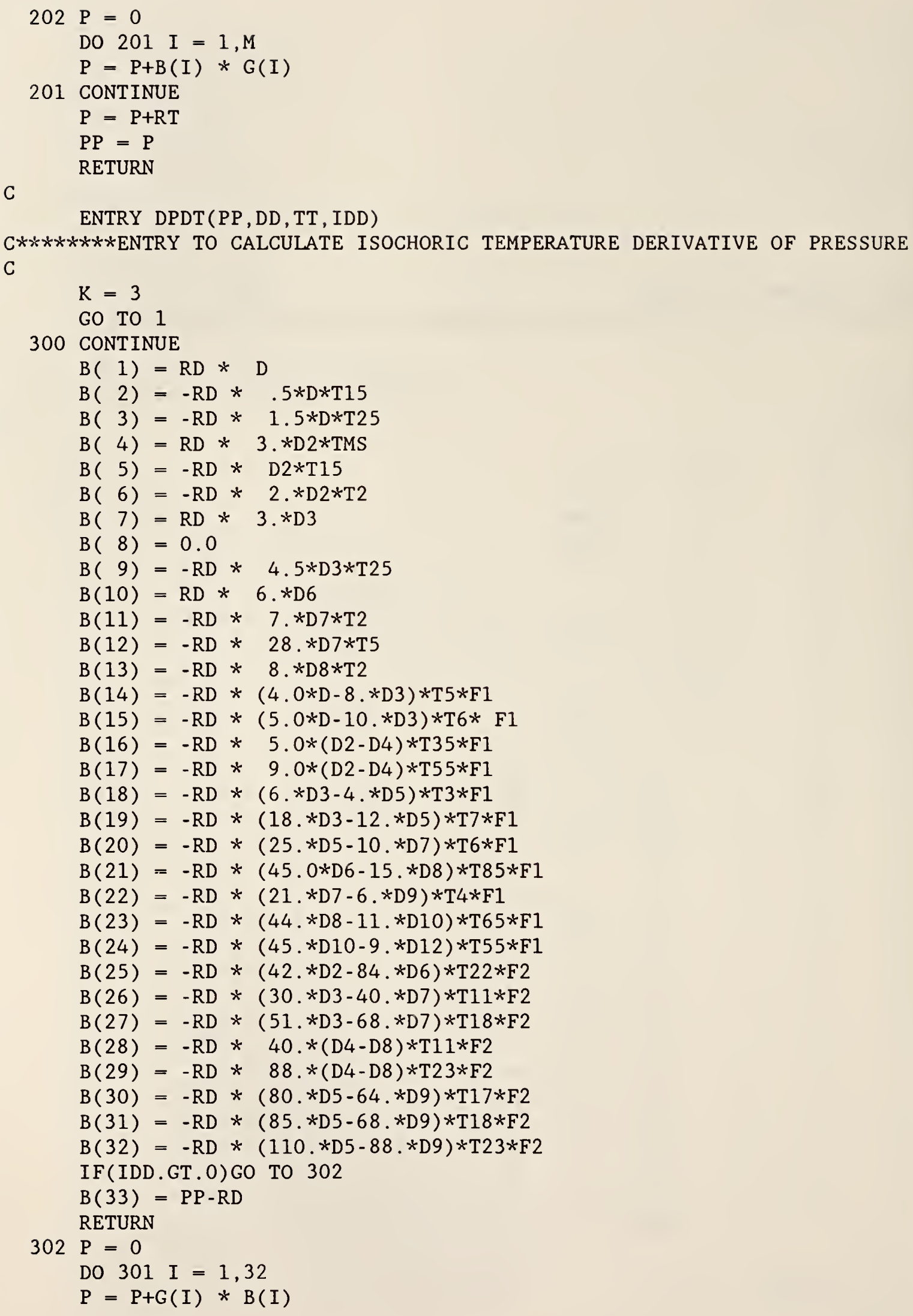


301 CONTINUE

$\mathrm{PP}=\mathrm{P}+\mathrm{RD}$

RETURN

C

ENTRY SR(PP, DD , TT, IDD)

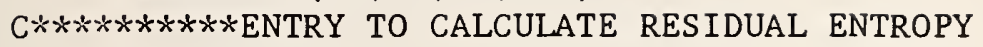

$\mathrm{C}$

$\mathrm{K}=4$

GO TO 1

400 CONTINUE

$\mathrm{X}(1)=-\mathrm{R} * \mathrm{D}$

$\mathrm{X}(2)=\mathrm{R} * 0.50 * \mathrm{D} * \mathrm{~T} 15$

$\mathrm{X}(3)=\mathrm{R} * 1.50 * \mathrm{D} * \mathrm{~T} 25$

$\mathrm{X}(4)=-\mathrm{R} * 1.50 * \mathrm{D} 2 * \mathrm{TMS}$

$\mathrm{X}(5)=\mathrm{R} * 0.50 * \mathrm{D} 2 * \mathrm{~T} 15$

$\mathrm{X}(6)=\mathrm{R} * \mathrm{D} 2 * \mathrm{~T} 2$

$\mathrm{X}(7)=-\mathrm{R} * \mathrm{D} 3$

$\mathrm{X}(8)=0.0$

$\mathrm{X}(9)=\mathrm{R} * 1.5 * \mathrm{D} 3 * \mathrm{~T} 25$

$\mathrm{X}(10)=-\mathrm{R} * \mathrm{D} 6$

$\mathrm{X}(11)=\mathrm{R} * \mathrm{D} 7 * \mathrm{~T} 2$

$\mathrm{X}(12)=\mathrm{R} * 4.00 * \mathrm{D} 7 * \mathrm{~T} 5$

$\mathrm{X}(13)=\mathrm{R} * \mathrm{D} 8 * \mathrm{~T} 2$

$\mathrm{X}(14)=\mathrm{R} * 4.00 * \mathrm{D} * \mathrm{~T} 5 * \mathrm{~F} 1$

$\mathrm{X}(15)=\mathrm{R} * 5.00 * \mathrm{D} * \mathrm{~T} 6 * \mathrm{~F} 1$

$\mathrm{X}(16)=\mathrm{R} * 2.5 * \mathrm{D} 2 * \mathrm{~T} 35 * \mathrm{~F} 1$

$\mathrm{X}(17)=\mathrm{R} * 4.5 * \mathrm{D} 2 * \mathrm{~T} 55 * \mathrm{~F} 1$

$\mathrm{X}(18)=\mathrm{R} * 2.00 * \mathrm{D} 3 * \mathrm{~T} 3 * \mathrm{~F} 1$

$\mathrm{X}(19)=\mathrm{R} * 6.00 * \mathrm{D} 3 * \mathrm{~T} 7 * \mathrm{~F} 1$

$\mathrm{X}(20)=\mathrm{R} * 5.00 * \mathrm{D} 5 * \mathrm{~T} 6 * \mathrm{~F} 1$

$\mathrm{X}(21)=\mathrm{R} * 7.5 * \mathrm{D} 6 * \mathrm{~T} 85 * \mathrm{~F} 1$

$\mathrm{X}(22)=\mathrm{R} * 3.0 * \mathrm{D} 7 * \mathrm{~T} 4 * \mathrm{~F} 1$

$\mathrm{X}(23)=\mathrm{R} * 5.5 * \mathrm{D} 8 * \mathrm{~T} 65 * \mathrm{~F} 1$

$\mathrm{X}(24)=\mathrm{R} * 4.5 * \mathrm{D} 10 * \mathrm{~T} 55 * \mathrm{~F} 1$

$\mathrm{X}(25)=\mathrm{R} * 21.0 * \mathrm{D} 2 * \mathrm{~T} 22 * \mathrm{~F} 2$

$\mathrm{X}(26)=\mathrm{R} * 10.0 * \mathrm{D} 3 * \mathrm{~T} 11 * \mathrm{~F} 2$

$\mathrm{X}(27)=\mathrm{R} * 17.0 * \mathrm{D} 3 * \mathrm{~T} 18 * \mathrm{~F} 2$

$\mathrm{X}(28)=\mathrm{R} * 10.0 * \mathrm{D} 4 * \mathrm{~T} 11 * \mathrm{~F} 2$

$\mathrm{X}(29)=\mathrm{R} * 22.0 * \mathrm{D} 4 * \mathrm{~T} 23 * \mathrm{~F} 2$

$\mathrm{X}(30)=\mathrm{R} * 16.0 * \mathrm{D} 5 * \mathrm{~T} 17 * \mathrm{~F} 2$

$\mathrm{X}(31)=\mathrm{R} * 17.0 * \mathrm{D} 5 * \mathrm{~T} 18 * \mathrm{~F} 2$

$\mathrm{X}(32)=\mathrm{R} * 22.0 * \mathrm{D} 5 * \mathrm{~T} 23 * \mathrm{~F} 2$

IF(IDD.GT.0)GO TO 402

RETURN

$402 P=0$

DO $401 \mathrm{I}=1,32$

$P=P+G(I) * X(I)$

401 CONTINUE

$P P=P$

RETURN

C

ENTRY AR(PP, DD , TT, IDD)

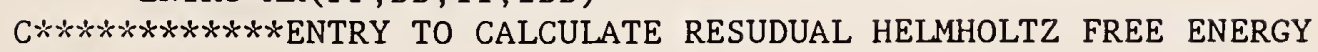




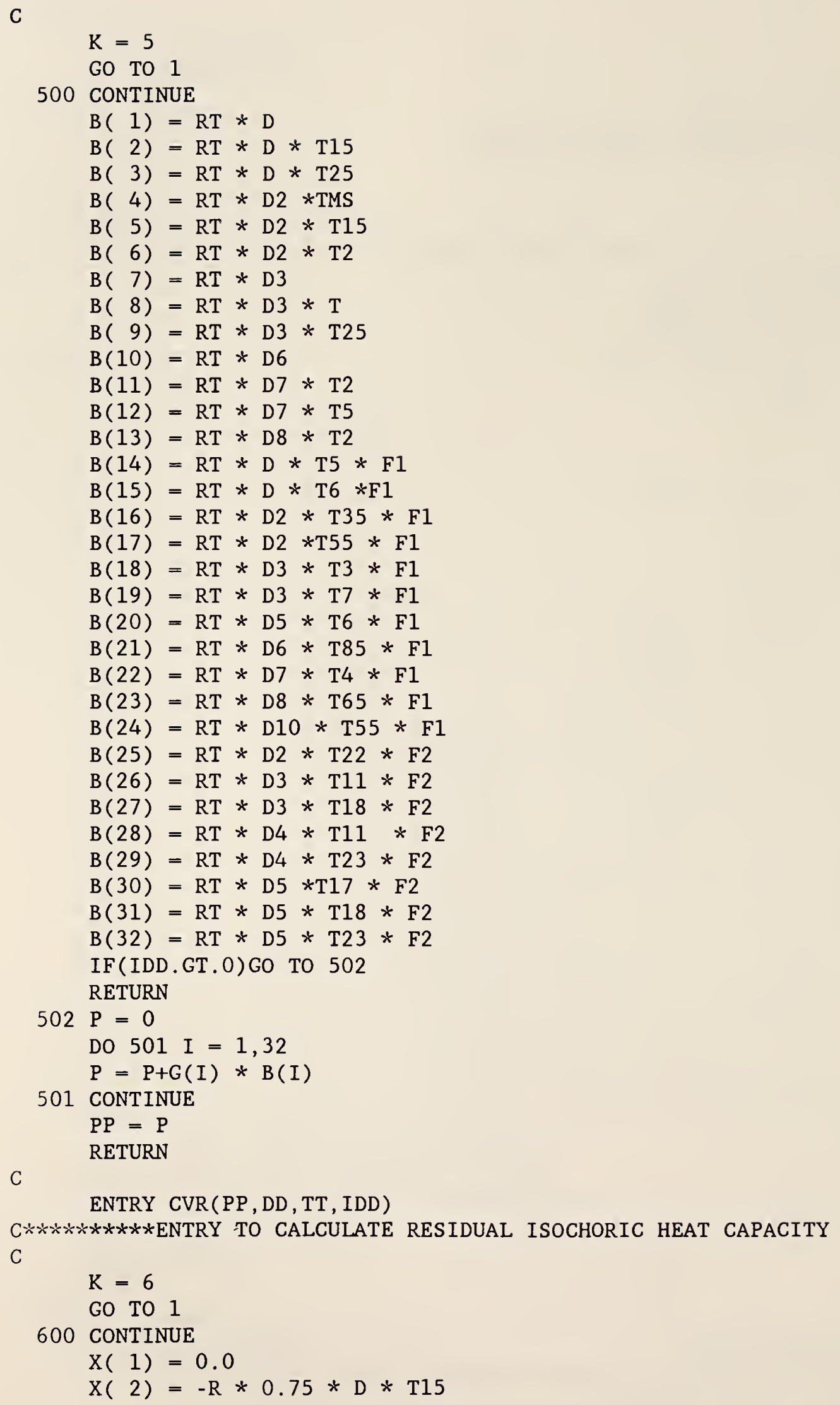




$$
\begin{aligned}
& \mathrm{X}(3)=-\mathrm{R} * 3.75 * \mathrm{D} * \mathrm{~T} 25 \\
& \mathrm{X}(4)=-\mathrm{R} * 0.75 * \mathrm{D} 2 * \mathrm{TMS} \\
& \mathrm{X}(5)=-\mathrm{R} * 0.75 * \mathrm{D} 2 * \mathrm{~T} 15 \\
& \mathrm{X}(6)=-\mathrm{R} * 2.00 * \mathrm{D} 2 * \mathrm{~T} 2 \\
& X(7)=0.0 \\
& X(8)=0.0 \\
& \mathrm{X}(9)=-\mathrm{R} * 3.75 * \mathrm{D} 3 * \mathrm{~T} 25 \\
& X(10)=0.0 \\
& \mathrm{X}(11)=-\mathrm{R} * 2.00 * \mathrm{D} 7 * \mathrm{~T} 2 \\
& \mathrm{X}(12)=-\mathrm{R} * 20.00 * \mathrm{D} 7 * \mathrm{~T} 5 \\
& \mathrm{X}(13)=-\mathrm{R} * 2.00 * \mathrm{D} 8 * \mathrm{~T} 2 \\
& \mathrm{X}(14)=-\mathrm{R} * 20.00 * \mathrm{D} * \mathrm{~T} 5 * \mathrm{~F} 1 \\
& \mathrm{X}(15)=-\mathrm{R} * 30.00 * \mathrm{D} * \mathrm{~T} 6 * \mathrm{~F} 1 \\
& \mathrm{X}(16)=-\mathrm{R} * 8.75 * \mathrm{D} 2 * \mathrm{~T} 35 * \mathrm{~F} 1 \\
& \mathrm{X}(17)=-\mathrm{R} * 24.75 * \mathrm{D} 2 * \mathrm{~T} 55 * \mathrm{~F} 1 \\
& \mathrm{X}(18)=-\mathrm{R} * 6.00 * \mathrm{D} 3 * \mathrm{~T} 3 * \mathrm{~F} 1 \\
& \mathrm{X}(19)=-\mathrm{R} * 42.00 * \mathrm{D} 3 * \mathrm{~T} 7 * \mathrm{~F} 1 \\
& \mathrm{X}(20)=-\mathrm{R} * 30.00 * \mathrm{D} 5 * \mathrm{~T} 6 * \mathrm{~F} 1 \\
& \mathrm{X}(21)=-\mathrm{R} * 63.75 * \mathrm{D} 6 * \mathrm{~T} 85 * \mathrm{~F} 1 \\
& \mathrm{X}(22)=-\mathrm{R} * 12.0 * \mathrm{D} 7 * \mathrm{~T} 4 * \mathrm{~F} 1 \\
& \mathrm{X}(23)=-\mathrm{R} * 35.75 * \mathrm{D} 8 * \mathrm{~T} 65 * \mathrm{~F} 1 \\
& \mathrm{X}(24)=-\mathrm{R} * 24.75 * \mathrm{D} 10 * \mathrm{~T} 55 * \mathrm{~F} 1 \\
& \mathrm{X}(25)=-\mathrm{R} * 462.0 * \mathrm{D} 2 * \mathrm{~T} 22 * \mathrm{~F} 2 \\
& \mathrm{X}(26)=-\mathrm{R} * 110.0 * \mathrm{D} 3 * \mathrm{~T} 11 * \mathrm{~F} 2 \\
& \mathrm{X}(27)=-\mathrm{R} * 306.0 * \mathrm{D} 3 * \mathrm{~T} 18 * \mathrm{~F} 2 \\
& \mathrm{X}(28)=-\mathrm{R} * 110.0 * \mathrm{D} 4 * \mathrm{~T} 11 * \mathrm{~F} 2 \\
& \mathrm{X}(29)=-\mathrm{R} * 506.0 * \mathrm{D} 4 * \mathrm{~T} 23 * \mathrm{~F} 2 \\
& \mathrm{X}(30)=-\mathrm{R} * 272.0 * \mathrm{D} 5 * \mathrm{~T} 17 * \mathrm{~F} 2 \\
& \mathrm{X}(31)=-\mathrm{R} * 306.0 * \mathrm{D} 5 * \mathrm{~T} 18 * \mathrm{~F} 2 \\
& \mathrm{X}(32)=-\mathrm{R} * 506.0 * \mathrm{D} 5 * \mathrm{~T} 23 * \mathrm{~F} 2 \\
& \text { IF(IDD.GT.0)GO TO } 602 \\
& \text { RETURN } \\
& 602 \mathrm{P}=0 \\
& \text { DO } 601 \mathrm{I}=1,32 \\
& P=P+G(I) * X(I)
\end{aligned}
$$

C $* \dot{*} * \dot{x} * \dot{*} * * * * *$ ENTRY TO CALCULATE SECOND ISOTHERMAL DENSITY DERIVATIVE C************OF PRESSURE

$\mathrm{C}$

$\mathrm{K}=7$

GO TO 1

700 CONTINUE

$\mathrm{B}(1)=\mathrm{RTD} * 2.0$

$\mathrm{B}(2)=\mathrm{RTD} * 2.0 * \mathrm{~T} 15$

$\mathrm{B}(3)=\mathrm{RTD} * 2.0 * \mathrm{~T} 25$

$\mathrm{B}(4)=\mathrm{RTD} * 12.0 * \mathrm{D} *$ TMS

$\mathrm{B}(5)=\mathrm{RTD} * 12.0 * \mathrm{D} * \mathrm{~T} 15$

$\mathrm{B}(6)=\mathrm{RTD} * 12.0 * \mathrm{D} * \mathrm{~T} 2$

$\mathrm{B}(7)=\mathrm{RTD} * 36.0 * \mathrm{D} 2$ 


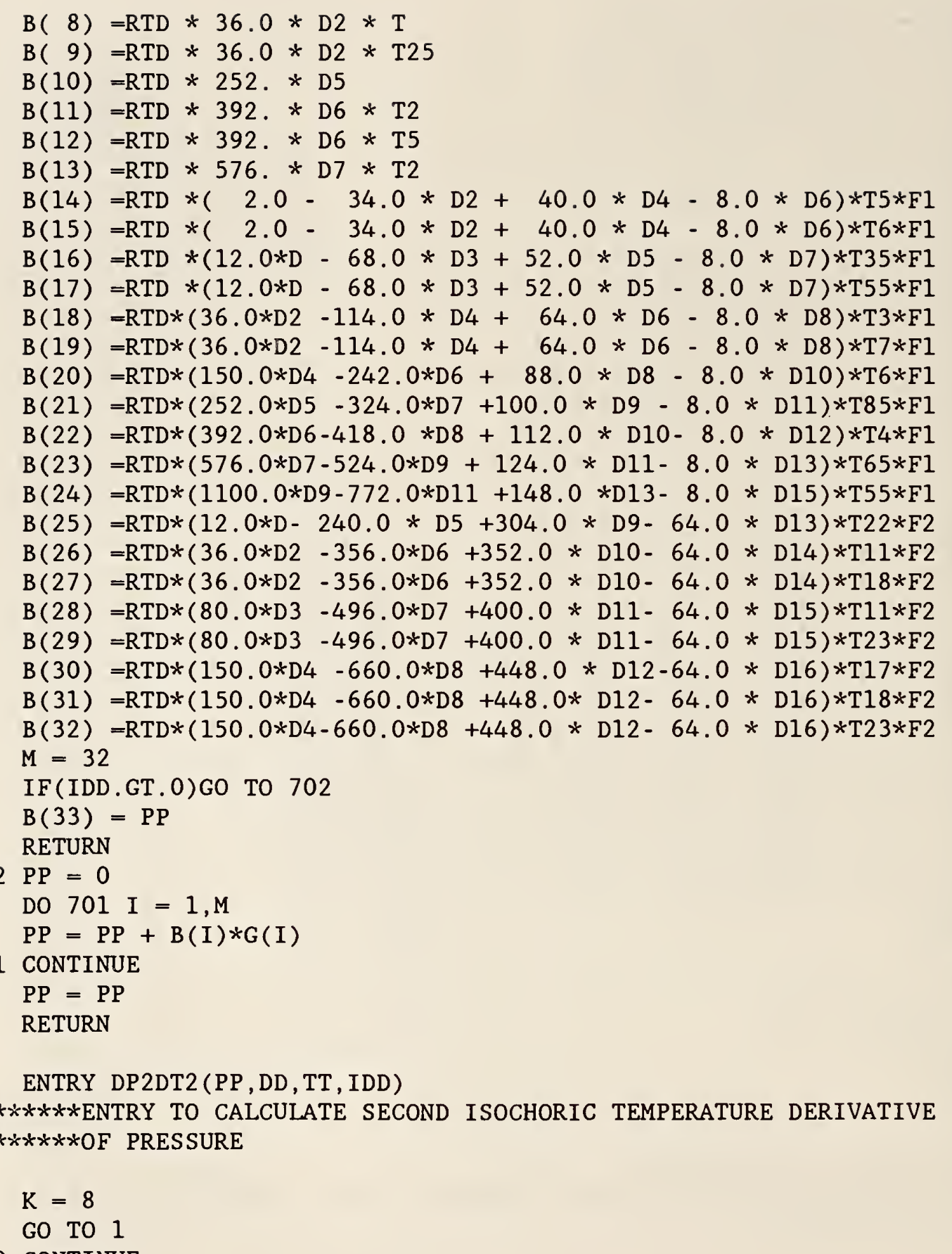

800 CONTINUE
$B(1)=0.0$
$\mathrm{B}(2)=\mathrm{RTT} * \mathrm{D} * 0.75 * \mathrm{~T} 25$
$\mathrm{B}(3)=\mathrm{RTT} * \mathrm{D} * 3.75 * \mathrm{~T} 35$
$B(4)=\operatorname{RTT} * 1.5 * \mathrm{D} 2 * \mathrm{TS}$
$\mathrm{B}(5)=\mathrm{RTT} * 1.5 * \mathrm{D} 2 * \mathrm{~T} 25$
$\mathrm{B}(6)=\mathrm{RTT} * 4.0 * \mathrm{D} 2 * \mathrm{~T} 3$
$B(7)=0.0$
$B(8)=0.0$
$\mathrm{B}(9)=\mathrm{RTT} * 11.25 * \mathrm{D} 3 * \mathrm{~T} 35$
$B(10)=0.0$ 


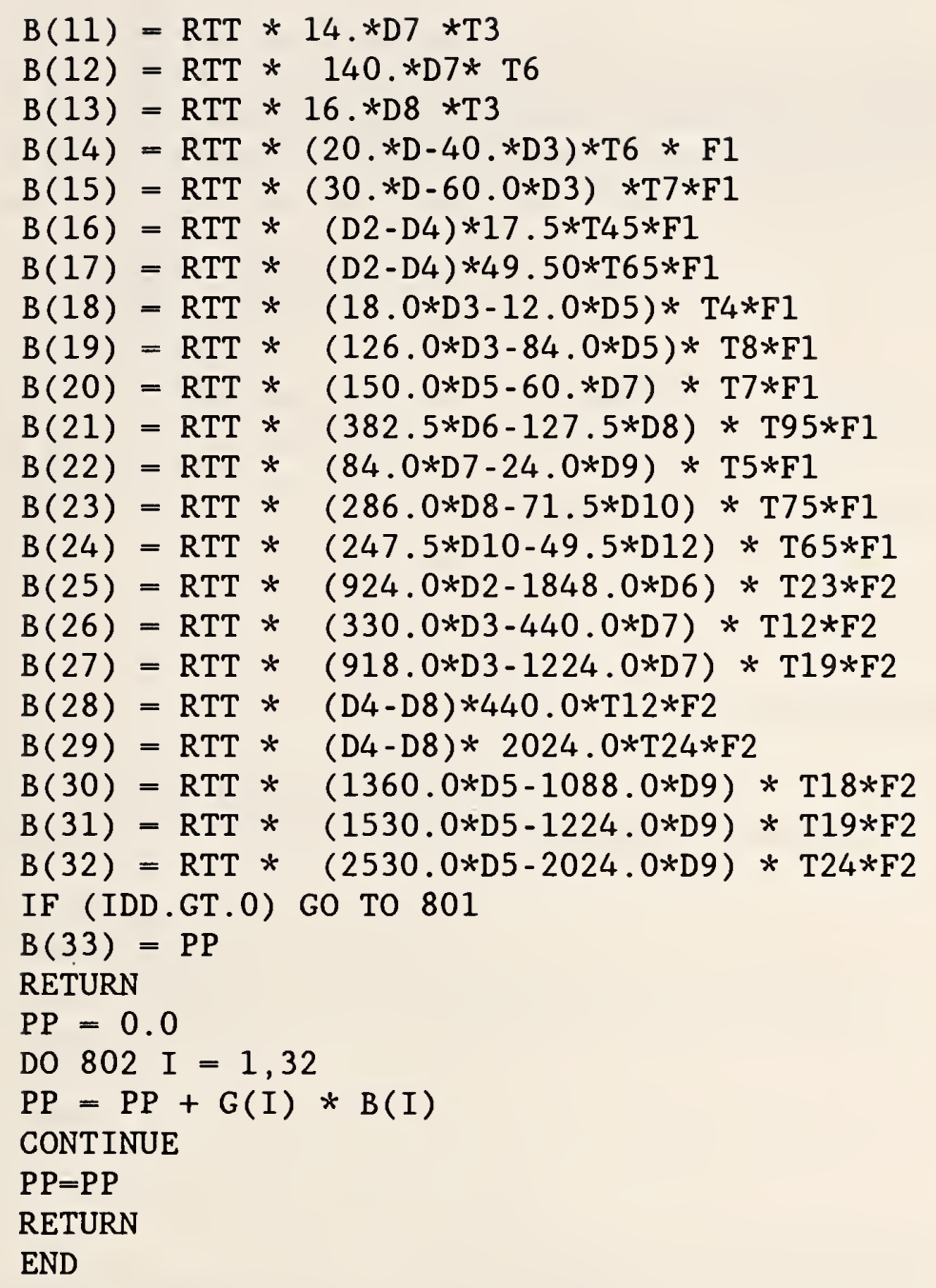

C

FUNCTION ENTROPY(DENS, TEMP)

C**********FUNCTION TO CALCULATE ENTROPY FROM DENSITY AND TEMPERATURE C

CALL SR (EN, DENS, TEMP , 1)

CALL IDEAL1 (TEMP , DENS , EID , HID , HEL , SID , CVID , CPID , 1)

ENTROPY $=100 * *$ EN + SID

RETURN

END 
C

SUBROUTINE IDEAL1( T , D , EID , HID , HEL , SID , CVID , CPID , IATM)

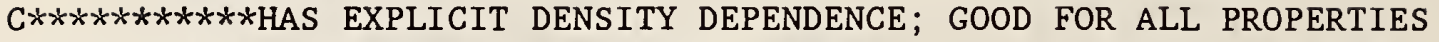
C********AT ANY PRESSURE (OR DENSITY RELATED TO P VIA IDEAL GAS EOS) $* * * * *$ C******THIS IS FOR METHANE AT ANY DENSITY AND TEMPERATURE

C

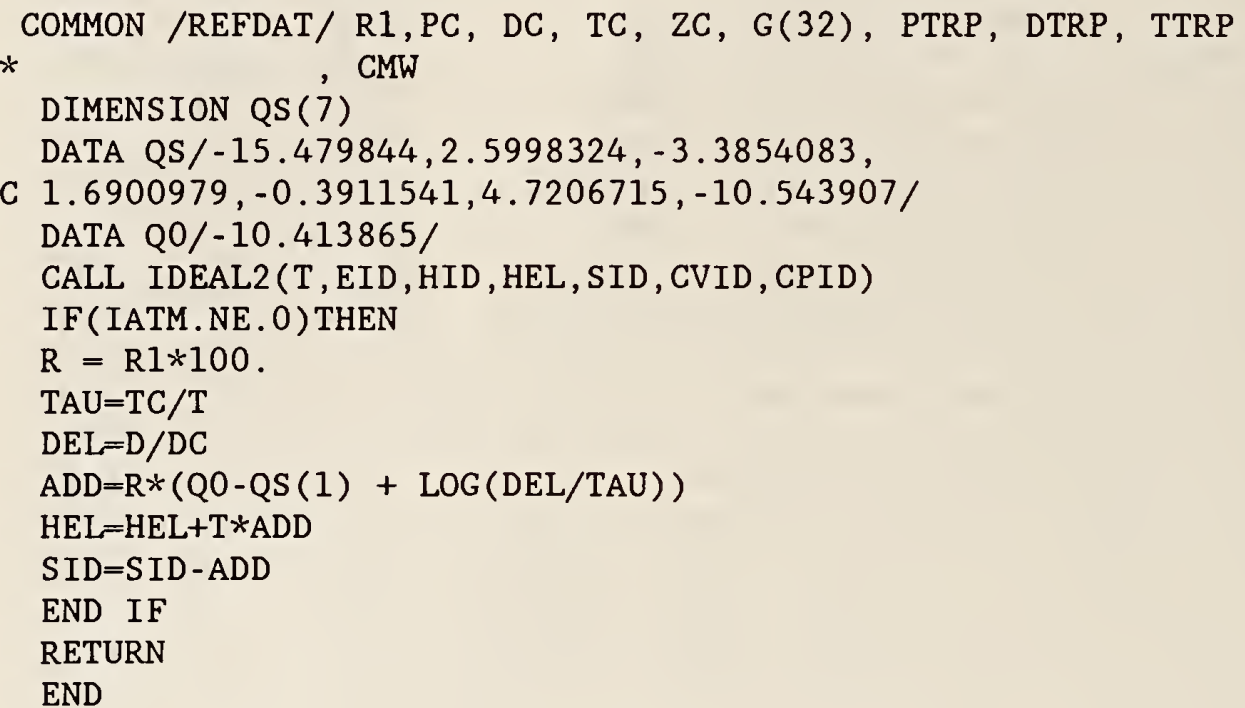

C

SUBROUTINE IDEAL2(T, EID, HID, HEL, SID , CVID , CPID)

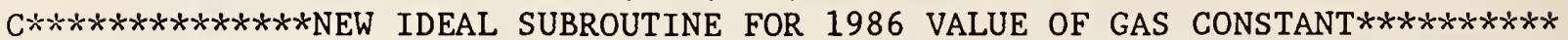
C********FORM OF HELMHOLTZ FREE ENERGY FOLLOWS GOODWIN WITH WAGNER VARIABLES $* *$

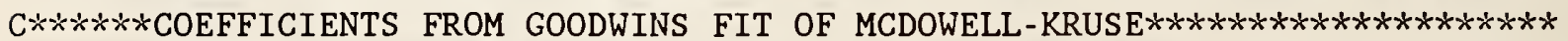
C********ASSUMES HELMHOLTZ ENERGY EQUALITY (IN LN RHO FORM) AT $298.15 \mathrm{~K} * * * * * * * * *$

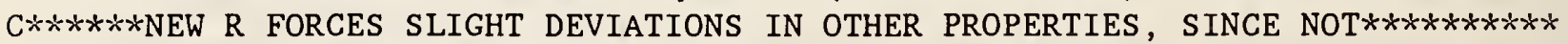

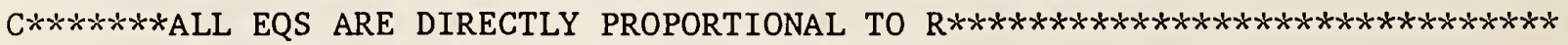

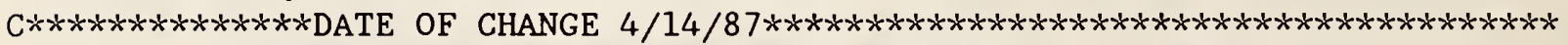
C $* * * * * * * * * * * *$ GOOD ONLY AT 1 ATM; PROBLEM FOR HEL AND SID--SEE IDEAL1 $* * * * * * * * *$ C

COMMON /REFDAT/ R1,PC, DC, TC, ZC, G(32), PTRP, DTRP, TTRP

$*$

DIMENSION QS (7) $\mathrm{CMW}$

DATA QS/-15.479844,2.5998324, -3.3854083,

C $1.6900979,-0.3911541,4.7206715,-10.543907 /$

DATA P1,P2/-.33333333, $-.66666667 /$

$\mathrm{R}=\mathrm{R} 1 * 100$.

$\mathrm{TAU}=\mathrm{TC} / \mathrm{T}$

TAU $3=\mathrm{TAU} * * \mathrm{P} 1$

TAU23 $=$ TAU $* * P 2$

$\operatorname{EXTAU}=\operatorname{EXP}(\mathrm{QS}(7) * \mathrm{TAU})$

$\mathrm{PHID}=\mathrm{QS}(1)+(\mathrm{QS}(2)+1). * \mathrm{LOG}(\mathrm{TAU})+\mathrm{QS}(3) * \mathrm{TAU} 3+\mathrm{QS}(4) * \mathrm{TAU} 23$ 
$1+\mathrm{QS}(5) / \mathrm{TAU}+\mathrm{QS}(6) * \mathrm{LOG}(1$. - EXTAU)

$\mathrm{PHIDD} 1=\mathrm{QS}(2)-\mathrm{QS}(3) / 3 . * \mathrm{TAU} 3-2 . * \mathrm{QS}(4) / 3 . * \mathrm{TAU} 23-\mathrm{QS}(5) / \mathrm{TAU}$

$1-\mathrm{QS}(6) * \mathrm{QS}(7) * \mathrm{TAU} /(1 . / \mathrm{EXTAU}-1$.

PHIDD $2=-\mathrm{QS}(2)+4 . * \mathrm{QS}(3) / 9 . * \mathrm{TAU} 3+10 . * \mathrm{QS}(4) / 9 . * \mathrm{TAU} 23$

$1+2 . * \mathrm{QS}(5) / \mathrm{TAU}-\mathrm{QS}(6) * \mathrm{QS}(7) * * 2 * \mathrm{TAU} * * 2 * \operatorname{EXTAU} /(\operatorname{EXTAU}-1) * *$.

$\mathrm{HEL}=\mathrm{R} * \mathrm{~T} * \mathrm{PHID}$

$\mathrm{CPID}=\mathrm{R} *(1$. $-\mathrm{PHIDD} 2)$

$\mathrm{EID}=\mathrm{R} * \mathrm{~T} * \mathrm{PHIDD} 1$

$\mathrm{HID}=\mathrm{R} * \mathrm{~T}+\mathrm{EID}$

CVID $=-\mathrm{R} * \mathrm{PHIDD} 2$

$S I D=-R *(P H I D-P H I D D 1)$

RETURN

END

C

FUNCTION HELM(DENS, TEMP)

C***********THIS FUNCTION IS FOR HELMHOLTZ FREE ENERGY

C

CALL AR(HE, DENS , TEMP , 1)

$\mathrm{HE}=100 . * \mathrm{HE}$

CALL IDEAL1 (TEMP , DENS , EID , HID , HEL , SID , CVID , CPID , 1)

HELM $=$ HE+HEL

RETURN

END

C

FUNCTION GIBBS (DENS, TEMP, PRES)

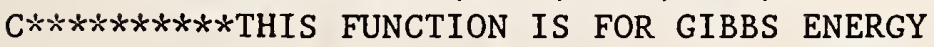

C

HEL=HELM (DENS , TEMP)

GIBBS $=$ HEL+PRES $* 1 . E 05 /($ DENS $* 1000$. )

RETURN

END

C

FUNCTION UNTERN(DENS, TEMP)

C************THIS FUNCTION IS FOR INTERNAL ENERGY

C

HEL=HELM (DENS , TEMP)

$S S=E N T R O P Y(D E N S, T E M P)$

UNTERN=HEL+SS*TEMP

RETURN

END 
C

FUNCTION ENTHALP(DENS, TEMP, PRES)

C******x*****THIS FUNCTION IS FOR ENTHALPY

C

HEL=HELM(DENS , TEMP)

UNT $=$ UNTERN (DENS , TEMP)

$G I B=G I B B S$ (DENS, TEMP , PRES)

ENTHALP $=$ GIB+UNT - HEL

RETURN

END

C

FUNCTION CPEE (RHO, TEE)

C***********THIS FUNCTION IS FOR ISOBARIC HEAT CAPACITY

C

$\mathrm{D}=\mathrm{RHO}$

CVCAL $=\operatorname{CVF}(\mathrm{D}, \mathrm{TEE})$

CALL DPDT (P1, D, TEE, 1)

CALL DPDD (P2, D, TEE, 1)

CPCAL $=$ CVCAL + 100.0* TEE *P1*P1 / $(D * D * P 2)$

CPEE $=$ CPCAL

RETURN

END

C

FUNCTION XLAMF $(D, T)$

C

C********THIS FUNCTION IS FOR THE THERMAL CONDUCTIVITY OF METHANE

C

COMMON /REFDAT/ R, PC, DC, TC, ZC, A(32), PTRP, DTRP, TTRP * , CMW

$\mathrm{C}$

$\mathrm{C}$

C

DIMENSION $G(7), F(7)$

DATA G,ES /.241492073721E+01, .551663305903E+00,

$1-.528377342545 \mathrm{E}+00, .738095527532 \mathrm{E}-01, .244655067073 \mathrm{E}+00$,

C

$2-.476136258746 \mathrm{E}-01, .155546119921 \mathrm{E}+01,6.29638 /$

C

020 DELTA $=\mathrm{D} / \mathrm{DC}$

$\mathrm{TAU}=\mathrm{TC} / \mathrm{T}$

$\mathrm{TCO}=\mathrm{TCONDO}(\mathrm{T})$

TCCRIT $=\operatorname{TCRIT} 1(\mathrm{D}, \mathrm{T})$

C 
C

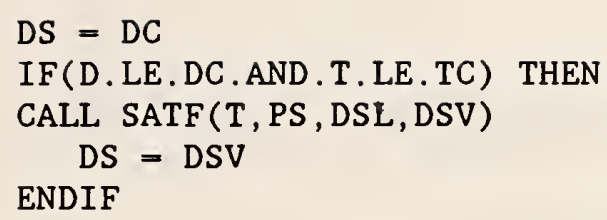

C

$$
\begin{aligned}
& F(1)=\text { DELTA } \\
& F(2)=\text { DELTA } * * 3 \\
& F(3)=\text { DELTA } * * 4 \\
& F(4)=\text { DELTA } * * 4 * \text { TAU } \\
& F(5)=\text { DELTA } * * 5 \\
& F(6)=\text { DELTA } * * 5 * \text { TAU } \\
& F(7)=\text { D DS } * \text { DELTA }
\end{aligned}
$$

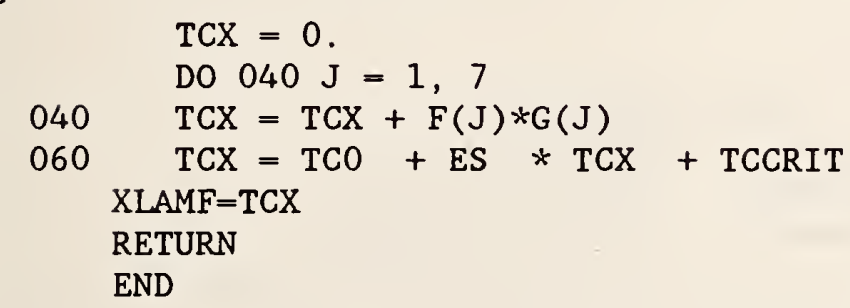

C

FUNCTION OM22S(TS)

C $* \dot{*} * \dot{x} * x * x+x+x$ THIS FUNCTION IS FOR THE 11-6-8 GAMMMA EQUALS 3

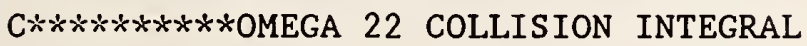

$\mathrm{C}$

C

$$
\text { DIMENSION CO(9) }
$$

DATA C0/-3.0328138281E+00, 1.6918880086E+01, $-3.7189364917 \mathrm{E}+01$, * 4.1288861858E+01, $-2.4615921140 \mathrm{E}+01,8.9488430959 \mathrm{E}+00$,

$*$ $-1.8739245042 \mathrm{E}+00,2.0966101390 \mathrm{E}-01,-9.6570437074 \mathrm{E}-03 /$

$\mathrm{TI}=1.0 / \mathrm{TS}$

$\mathrm{T} 1=\mathrm{TS} * *(1.0 / 3.0)$

$\mathrm{ETA}=0.0$

DO $020 \mathrm{~J}=1,9$

$\mathrm{ETA}=\mathrm{ETA}+\mathrm{CO}(\mathrm{J}) * \mathrm{TI}$

$020 \mathrm{TI}=\mathrm{TI} * \mathrm{~T} 1$

$\mathrm{OM} 22 \mathrm{~S}=1.0 / \mathrm{ETA}$

RETURN

END 
C

FUNCTION VSCTY (D, T)

$\mathrm{C} * * * * * * * * * * * * * * * * * * * * * * * * * * * * * * * * * * *$

C PURPOSE - - THIS ROUTINE CALCULATES THE VISCOSITY OF METHANE

$\mathrm{C}$

C VERSION $4.05 / 17 / 85$ - CODED BY J . F. ELY

C WITH SUBSEQUENT REVISIONS

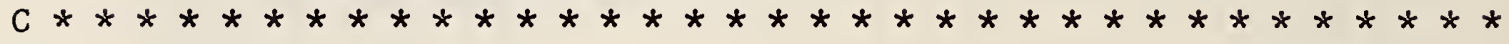
$\mathrm{C}$

$\mathrm{C}$

COMMON /REFDAT/ R, PC, DC, TC, ZC, A(32), PTRP, DTRP, TTRP

*

, CMW

DIMENSION GV(11), $F(11)$

C

C COEF FROM VP3, FILE ETAWT

C UNITS ARE IN MICRO PA SEC MUST BE $* 10$.

DATA GV, ETAS/.412501370594E+00, -.143909116635E+00,

$1.103669933465 \mathrm{E}+00, .402874641440 \mathrm{E}+00,-.249035243650 \mathrm{E}+00$,

$2-.129531310621 \mathrm{E}+00, .657577550033 \mathrm{E}-01, .256662763171 \mathrm{E}-01$,

$3-.371652573606 \mathrm{E}-01,-.387983411200 \mathrm{E}+00, .353381454207 \mathrm{E}-01$,

C

$412.149 /$

020 DELTA $=\mathrm{D} / \mathrm{DC}$

$\mathrm{TAU}=\mathrm{TC} / \mathrm{T}$

$F(1)=$ DELTA

$F(2)=$ TAU $*$ DELTA

C

C

$F(3)=$ DELTA $* * 2$

$F(4)=$ TAU $*$ DELTA $* * 2$

C

$F(5)=\operatorname{DELTA} * * 2 * \mathrm{TAU} * \operatorname{SQRT}(\mathrm{TAU})$

$F(6)=$ DELTA $* * 3$

$F(7)=$ DELTA $* * 3 *$ TAU $* * 2$

C

$F(8)=$ DELTA $* * 4$

$F(9)=$ DELTA $* * 4 *$ TAU

C

$F(10)=$ DELTA

$F(11)=$ DELTA $*$ TAU

C

$\mathrm{ETA}=0$.

DO $040 \mathrm{~J}=1,9$

$040 \quad \mathrm{ETA}=\mathrm{ETA}+\mathrm{F}(\mathrm{J}) * \mathrm{GV}(\mathrm{J})$

DENOM $=1.0+F(10) * G V(10)+F(11) * G V(11)$

$\operatorname{ETAG}=\operatorname{ETAO}(\mathrm{T})$

060 ETA $=$ ETAG + ETAS * ETA $/$ DENOM

VSCTY $=$ ETA

RETURN

END 
C

FUNCTION ETAO(T)

C $* * * * * * *$ THIS FUNCTION EVALUATES THE ZERO DENSITY VISCOSITY

C $x^{2} \cdot x^{2} \cdot x^{2} \times$ OF METHANE

$\mathrm{C}$

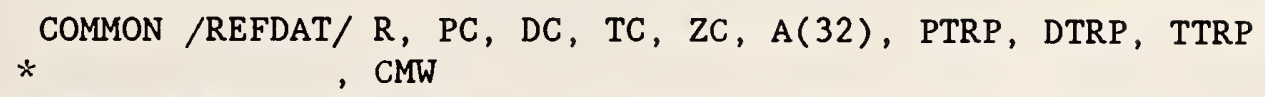

C SIGMA AS $11 / 7 / 86$

$\mathrm{TS}=\mathrm{T} / \mathrm{EOK}$

$\mathrm{ETAO}=10.50 * \mathrm{SQRT}(\mathrm{TS}) / \mathrm{OM} 22 \mathrm{~S}(\mathrm{TS})$

RETURN

END

C

FUNCTION TCONDO(T)

C $* * * * * * * * * * * *$ THIS FUNCTION EVALUATES THE ZERO DENSITY

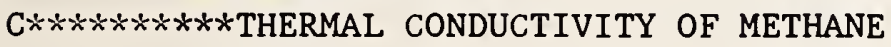

$\mathrm{C}$

COMMON /REFDAT/ RT, PC, DC, TC, ZC, A9(32), PTRP, DTRP, TTRF $*$ , CMW

DATA CON, EOK/0.51826, 174.0/

C CORRECTED VERSION OF LAMZERO GIVES THE FOLLOWING 12/29/87

DATA G1,G2/ $1.4588497685,-0.43771624308 /$

CALL IDEAL2 ( $T, \mathrm{X} 1, \mathrm{X} 2, \mathrm{X} 3, \mathrm{X} 4, \mathrm{CV}, \mathrm{X} 5)$

$\mathrm{CVDIM}=-\mathrm{CV} /(100 \cdot * \mathrm{RT})$

$\mathrm{TS}=\mathrm{T} / \mathrm{EOK}$

$\mathrm{TCC}=-(\mathrm{G} 1+\mathrm{G} 2 /(\mathrm{TS})) *(\mathrm{CVDIM}+1.5)$

$\mathrm{ETAO}=\mathrm{ETAO}(\mathrm{T})$

TCONDO $=$ CON $*$ ETAO $*(3.75+$ TCC $)$

RETURN

END

C

FUNCTION TCRIT1(D,T)

COMMON /REFDAT/ R, PC, DC, TC, ZC, A(32), PTRP, DTRP, TTRP * , CMW

C

DIMENSION G1(4)

C CRIT. ENHANC. AS IN SENGERS ET AL 1981 U MARYL. REPORT

C DERIVATIVES FROM BWR, ... 26 JAN 82

C UNITS, IN MOL/L, K, INTERNAL ALSO BAR, OUT W/M-K, ETA PA-S,BK J/K

C 
C

DATA GAM, BETA, AEX, BEX, ECON/1.19,0.355, 3. 352,0.732,0.287/

DATA RCON, QCON, SCON, WCON/0.535,0.1133, -6.098, -1.401/

DATA CAPGAM/0.0801/

C

COEFS FOR DFACT AS OF $1 / 04 / 88$

DATA Gl/ .264609797168E+01, .267792744757E+01,

C $-.637034076544 \mathrm{E}+00, .223519888946 \mathrm{E}+01 /$

DELTA $=\mathrm{D} / \mathrm{DC}$

$\mathrm{TAU}=\mathrm{TC} / \mathrm{T}$

DELD $=$ ABS $($ DELTA -1.0$)$

$\mathrm{DELT}=\mathrm{ABS}(\mathrm{T}-\mathrm{TC}) / \mathrm{TC}$

DELA $=(D / D C-1.0)$

$\mathrm{DFACT}=\operatorname{EXP}(-(\mathrm{Gl}(1) * \operatorname{DELT} * *(1 . / 2)+.\mathrm{Gl}(2) * \mathrm{DELD} * * 2.0)+$

C

C

$$
\mathrm{Gl}(3) * \mathrm{DELA})
$$

$\mathrm{ETA}=\operatorname{VSCTY}(\mathrm{D}, \mathrm{T})$

CALL DPDT (DPT, D , T , 1)

$\mathrm{DPTDIM}=\mathrm{DPT} /(\mathrm{D} * \mathrm{R})$

CALL DPDD (DPD, $, T, 1)$

$D P D D I M=D P D /(T * R)$

IF(DELD.EQ. 0. AND. DELT.LT. .03) GO TO 3

IF(DELD. LE. 0.25.AND.DELT.LT.0.03) GO TO 8

CHISTAR $=0.28631 *$ DELTA $*$ TAU/DPDDIM

GO TO 12

$8 \mathrm{XX}=(\mathrm{TC}-\mathrm{T}) / \mathrm{TC} / \mathrm{DELD} * *(1.0 / \mathrm{BETA})$

$\mathrm{Y}=1 .+\mathrm{SCON} * \mathrm{XX}$

IF(Y.LT.0.) THEN

PRINT*,' $\mathrm{T}$ ' , T, ' $\mathrm{D}^{\prime}, \mathrm{\prime}^{\prime} \mathrm{Y}$ ', $\mathrm{Y}$,'IN TCRIT1'

$\mathrm{Y}=0$.

ENDIF

THETA $=1 .+E C O N * Y * *(2, * B E T A)$

OMEGA $=$ WCON $* X X$

TOP $=Q C O N * D E L D * *(-A E X) *$ THETA** $(B E X)$

DIV $=$ THETA+OMEGA* $($ THETA+RCON $)$

CHISTAR $=$ TOP $/$ DIV

$12 \mathrm{CHI}=\mathrm{CHISTAR} * * 0.4681$

SENG $=91.855 *$ DPTDIM $* * 2 *$ CHI $*$ DFACT $/($ ETA $*$ TAU $* * 2)$

TCRIT1 = SENG

RETURN

3 CHISTAR $=$ CAPGAM $*$ DELT $* *(-$ GAM $)$

GO TO 12

END 
C

FUNCTION TSAT(PRESS)

C**********THIS FUNCTION CALCULATES THE SATURATION TEMPERATURE

C $* * * * * * * * * *$ GIVEN A PRESSURE BELOW ITS CRITICAL VALUE

$\mathrm{C}$

COMMON /DERIV/DF, JUNK

COMMON /REFDAT/ R, PC, DC, TC, ZC, G(32), PTRP, DTRP, TTRP

* , CMW

IF (PRESS . GT . PC) THEN

PAUSE 'PRESSURE GREATER THAN CRITICAL IN TSAT'

RETURN

END IF

IF (PRESS . LT . PTRP) THEN

PAUSE 'PRESSURE LESS THAN TRIPLE POINT IN TSAT'

RETURN

END IF

$\mathrm{XI}=\mathrm{TTRP}$

$\mathrm{XH}=\mathrm{TC}$

TSAT $=.5 *(X L+X H)$

$\mathrm{DXOLD}=\mathrm{ABS}(\mathrm{XH}-\mathrm{XL})$

$\mathrm{DX}=\mathrm{DXOLD}$

$\mathrm{F}=$ PSATF $($ TSAT $)-$ PRESS

DO $11 \mathrm{~J}=1,100$

$\operatorname{IF}(((T S A T-X H) * D F-F) *((T S A T-X L) * D F-F), G E .0$.

C .OR. ABS $(2, * F) . G T \cdot A B S(D X O L D * D F))$ THEN

$\mathrm{DXOLD}=\mathrm{DX}$

$\mathrm{DX}=0.5 *(\mathrm{XH}-\mathrm{XL})$

TSAT $=X L+D X$

IF (XL. EQ. TSAT) RETURN

ELSE

$D X O L D=D X$

$\mathrm{DX}=\mathrm{F} / \mathrm{DF}$

TEMP $=$ TSAT

TSAT $=$ TSAT - DX

IF (TEMP.EQ . TSAT) RETURN

ENDIF

IF (ABS (DX) . LT . . 00005) RETURN

$F=$ PSATF $($ TSAT) - PRESS

IF (F. LT . O.) THEN

$\mathrm{XL}=\mathrm{TSAT}$

ELSE

$\mathrm{XH}=\mathrm{TSAT}$

ENDIF

11 CONTINUE

PAUSE 'IN TSAT: EXCEEDES MAXIMUM ITERATIONS'

RETURN

END 
C

BLOCK DATA C1INIT

C $* * * * * * *$ THIS BLOCK DATA SUBROUTINE SUPPLIES THE FUNDAMENTAL CONSTANTS

C********AND PARAMETERS NECESSARY FOR THE METHANE FLUID IN

C********ACCORDANCE WITH THE CORRELATIONS OF

C*******FRIEND, ELY, AND INGHAM, 'THERMOPHYSICAL PROPERTIES OF METHANE'

COMMON /REFDAT/ R, PC, DC, TC, ZC, G(32), PTRP, DTRP, TTRP

*

, CMW

C

COMMON/SATBDY/TCC , PCC , DCC , ZCC , RC , EPP , BETA

C

DATA TC, PC, DC, PTRP, DTRP, TTRP, CMW

C $/ 190.551,45.9920,10.139,0.11696,28.145,90.6854,16.043 /$

DATA TCC, PCC, DCC

C/190.551, 45.9920, 10.139/

DATA G/ $\quad .384436099659 \mathrm{E}+00,-.179692598800 \mathrm{E}+01$,

$1.329444947369 \mathrm{E}+00, .226312728442 \mathrm{E}-01, .759236768798 \mathrm{E}-01$,

$2.693758447259 \mathrm{E}-01, .241163263947 \mathrm{E}-01, .107009920854 \mathrm{E}-01$,

$3-.380933275164 \mathrm{E}-01, .471537561143 \mathrm{E}-03, .556607678805 \mathrm{E}-03$,

$4.548759346533 \mathrm{E}-06,-.999632699967 \mathrm{E}-04,-.128087979280 \mathrm{E}+00$,

$5 \quad .380198873377 \mathrm{E}-01, .139226650551 \mathrm{E}+00,-.874996348859 \mathrm{E}-01$,

$6-.334894165760 \mathrm{E}-02,-.517576297122 \mathrm{E}-01, .252835179116 \mathrm{E}-01$,

$7 \quad .518703205947 \mathrm{E}-03,-.166770594525 \mathrm{E}-02,-.607401927389 \mathrm{E}-03$,

$8 \quad-.972915359991 \mathrm{E}-04,-.298844010462 \mathrm{E}-04,-.130940111242 \mathrm{E}-01$,

$9.198175833798 \mathrm{E}-01, .208465762327 \mathrm{E}-01,-.358025052631 \mathrm{E}-01$,

A $\quad-.203486851741 \mathrm{E}+00, .215964755088 \mathrm{E}+00,-.429340628249 \mathrm{E}-02 /$

C

DATA R, EPP, BETA, ZC/0.08314510, 1.9, $0.355,0.28631182 /$

DATA RC, ZCC/0.08314510,0.28631182/

C

END 
NBS-114A (REV. 2-8C)

U.S. DEPT. OF COMM.

BIBLIOGRAPHIC DATA

SHEET (See instructions)

1. PUBLICATION OR REPORT NO.

NIST/TN-1325

2. Performing Organ. Report No.

3. Publication Date

Apri1 1989

4. TITLE AND SUBTITLE

Tables for the Thermophysical Properties of Methane

5. AUTHOR(S)

Daniel G. Friend, James F. Ely, and Hepburn Ingham

6. PERFORMING ORGANIZATION (If joint or other than NBS, see instructions) National Institute of Standards and Technology

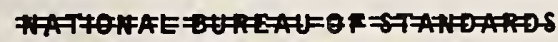

DEPARTMENT OF COMMERCE

WASHINGTON, D.C. 20234

9. SPONSORINC ORGANIZATION NAME AND COMPLETE ADDRESS (Stregt, City, State, ZIF)

7. Contract/Grant No.

8. Type of Report \& Period Covered

10. SUPPLEMENTARY NOTES

Document describes a computer program; SF-185, FIPS Software Summary, is attached.

11. ABSTRACT (A 200-word or less factual summary of most significant information. If document includes a significant bibliography or literature survey. mention it here)

The thermophysical properties of methane are tabulated for a large range of fluid states based on recently formulated correlations. For the thermodynamic properties, temperatures from 91 to $600 \mathrm{~K}$ at pressures less than $100 \mathrm{MPa}$ are included. For the viscosity, the corresponding range is $91-400 \mathrm{~K}$ with pressures to 55MPa, while for the thermal conductivity the range is $91-600 \mathrm{~K}$ with pressures to $100 \mathrm{MPa}$. In addition to the tables of properties, algebraic expressions and associated tables of coefficients are given to allow additional property calculations. Tables of comparisons between experimental property determinations and the correlations are also given both for primary data used in the formulation of the correlations and for additional data. A listing of a FORTRAN program for the evaluation of methane thermophysical properties using the Schmidt-Wagner equation of state is included.

12. KEY WORDS (Six to twelve entries; alphabetical order: capitalize only proper names; and separate key words by semicolons) correlation; density; equation of state; heat capacity; methane; phase boundary; pressure; speed of sound; thermal conductivity; thermophysical properties; transport properties; virial coefficients; viscosity.

\section{AVAILABILITY}

X Unlimited

For Official Distribution. Do Not Release to NTIS

[X] Order From Superintendent of Documents, U.S. Government Printing Office, Washington, D.C. 20402.

Order From National Technical Information Service (NTIS), Springfield, VA. 2216I
14. NO. OF PRINTED PAGES

$$
490
$$

15. Price 



Journal of Research of the National Institute of Standards and Technology-Reports NIST research and development in those disciplines of the physical and engineering sciences in which the Institute is active. These include physics, chemistry, engineering, mathematics, and computer sciences. Papers cover a broad range of subjects, with major emphasis on measurement methodology and the basic technology underlying standardization. Also included from time to time are survey articles on topics closely related to the Institute's technical and scientific programs. Issued six times a year.

\section{Nonperiodicals}

Monographs-Major contributions to the technical literature on various subjects related to the Institute's scientific and technical activities.

Handbooks-Recommended codes of engineering and industrial practice (including safety codes) developed in cooperation with interested industries, professional organizations, and regulatory bodies.

Special Publications-Include proceedings of conferences sponsored by NIST, NIST annual reports, and other special publications appropriate to this grouping such as wall charts, pocket cards, and bibliographies.

Applied Mathematics Series-Mathematical tables, manuals, and studies of special interest to physicists, engineers, chemists, biologists, mathematicians, computer programmers, and others engaged in scientific and technical work.

National Standard Reference Data Series-Provides quantitative data on the physical and chemical properties of materials, compiled from the world's literature and critically evaluated. Developed under a worldwide program coordinated by NIST under the authority of the National Standard Data Act (Public Law 90-396). NOTE: The Journal of Physical and Chemical Reference Data (JPCRD) is published quarterly for NIST by the American Chemical Society (ACS) and the American Institute of Physics (AIP). Subscriptions, reprints, and supplements are available from ACS, 1155 Sixteenth St., NW., Washington, DC 20056.

Building Science Series-Disseminates technical information developed at the Institute on building materials, components, systems, and whole structures. The series presents research results, test methods, and performance criteria related to the structural and environmental functions and the durability and safety characteristics of building elements and systems.

Technical Notes-Studies or reports which are complete in themselves but restrictive in their treatment of a subject. Analogous to monographs but not so comprehensive in scope or definitive in treatment of the subject area. Often serve as a vehicle for final reports of work performed at NIST under the sponsorship of other government agencies.

Voluntary Product Standards-Developed under procedures published by the Department of Commerce in Part 10, Title 15, of the Code of Federal Regulations. The standards establish nationally recognized requirements for products, and provide all concerned interests with a basis for common understanding of the characteristics of the products. NIST administers this program as a supplement to the activities of the private sector standardizing organizations.

Consumer Information Series-Practical information, based on NIST research and experience, covering areas of interest to the consumer. Easily understandable language and illustrations provide useful background knowledge for shopping in today's technological marketplace.

Order the above NIST publications from: Superintendent of Documents, Government Printing Office, Washington, DC 20402.

Order the following NIST publications-FIPS and NISTIRs-from the National Technical Information Service, Springfield, VA 22161.

Federal Information Processing Standards Publications (FIPS PUB)-Publications in this series collectively constitute the Federal Information Processing Standards Register. The Register serves as the official source of information in the Federal Government regarding standards issued by NIST pursuant to the Federal Property and Administrative Services Act of 1949 as amended, Public Law 89-306 (79 Stat. 1127), and as implemented by Executive Order 11717 (38 FR 12315, dated May 11, 1973) and Part 6 of Title 15 CFR (Code of Federal Regulations).

NIST Interagency Reports (NISTIR)-A special series of interim or final reports on work performed by NIST for outside sponsors (both government and non-government). In general, initial distribution is handled by the sponsor; public distribution is by the National Technical Information Service, Springfield, VA 22161, in paper copy or microfiche form. 


\section{U.S. DEPARTMENT OF COMMERCE}

National Institute of Standards and Technology

(formerly National Bureau of Standards)

325 Broadway

Boulder, Colorado 80303-3328

\section{OFFICIAL BUSINESS}

PENALTY FOR PRIVATE USE, $\$ 300$ 